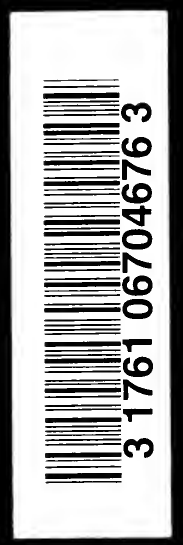




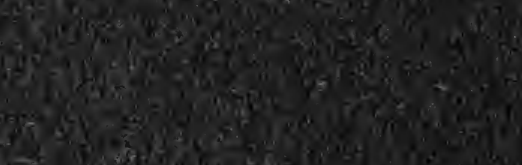

2. 36x

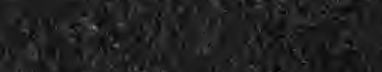

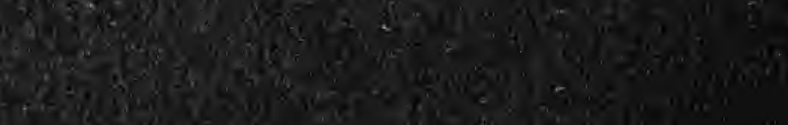

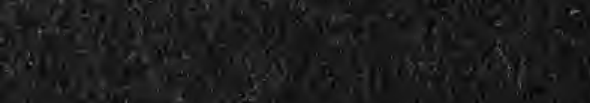

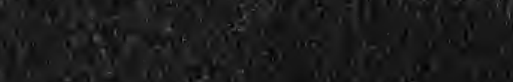




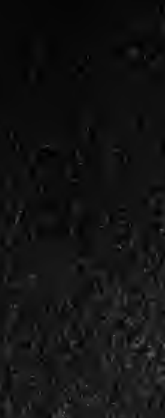

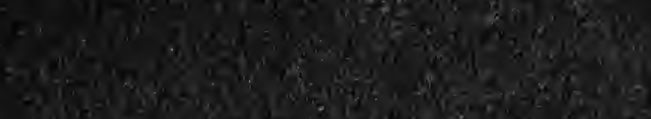

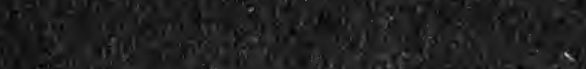

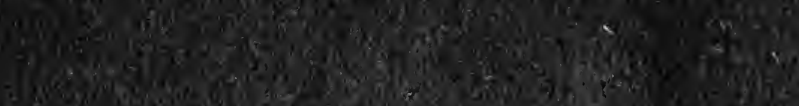
4.5.

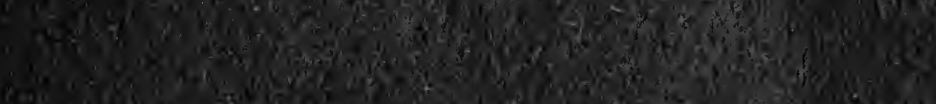

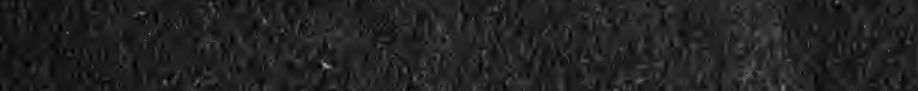

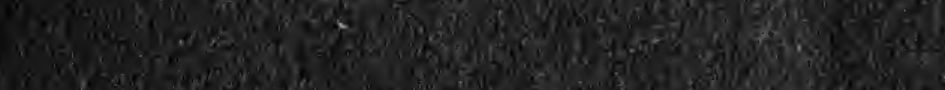

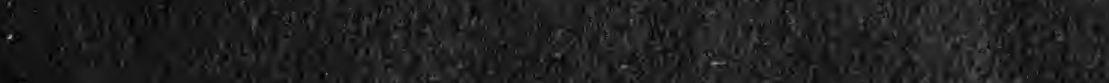

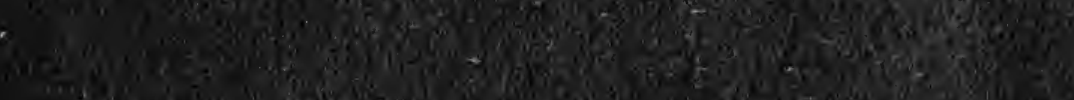
2
4

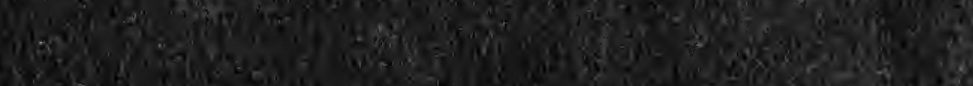
2. Wa

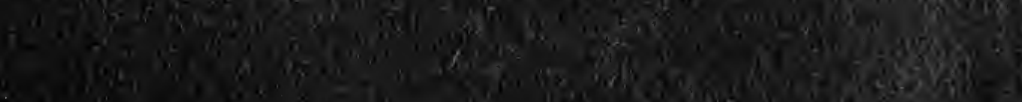





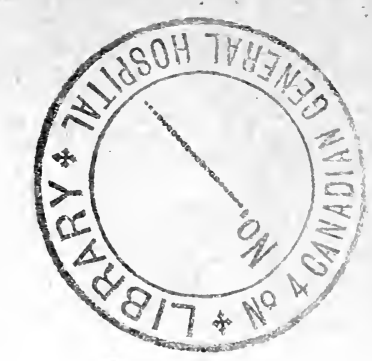

Digitized by the Internet Archive in 2007 with funding from Microsoft Corporation 



\title{
Diseases and SuRgery
}

OF THE

\section{GENITO-URINARY SYSTEM}

\author{
BY \\ FRANCIS SedWATSON, M.D.
}

SENIOR VISITING SURGEON TO THE BOSTON CITY HOSPITAL; LECTURER ON GENITO-URINARY SURGERY, HARVARD MEDICAI SCHOOL; MEMBER OF THE AMERICAN SURGiCAL ASSOCIATION, OF THE AMERICAN ASSOCIATION OF GENITOURINARY SURGEONS, OF THE AMERICAN UROLOGICAL ASSOCIATION, OF THE INTERNATIONAL SURGICAL

ASSOCIATION, OF L'ASSOCiation FRANÇAISE D'UROLOGIE, OF THE INTERNATIONAL

ASSOCIATION OF UROLOGY AND ITS FIRST VICE-PRESIDENT; CORRESPONDING

MEMBER OF THE SURGICAL SOCIETY OF MOSCOW

ASSISTED BY

JOHN H. CUNNINGHAM, Jr., M.D.

VISITING SURGEON TO THE LONG ISLAND HOSPITAL, BOSTON; ASSISTANT VISITING SURGEON TO THE BOSTON CITY HOSPITAL; MEMBER OF THE AMERICAN ASSOCIATION OF GENITO-URINARY SURGEONS

AND OF THE AMERICAN UROLOGICAL ASSOCIATION

VOLUME I

THE EXTERNAL GENITALS

THE PROSTATE AND BLADDER

W:ITH 339 ENGRAVINGS AND 23 COLORED PLATES
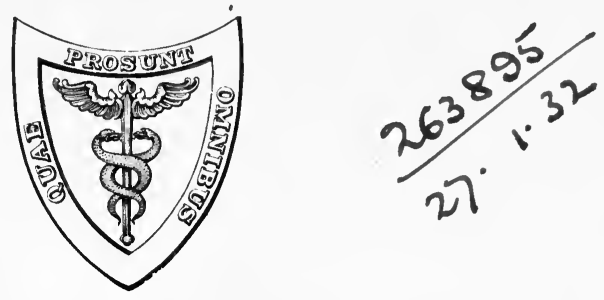

PHILADELPHIA AND NEW YORK

LEA \& FEBIGER 
Entered according to Act of Congress, in the year 1908, by

\section{LEA \& FEBIGER,}

in the Office of the Librarian of Congress at Washington. All rights reserved. 


\title{
EDWARD L. KEYES
}

\begin{abstract}
SURGEON, AUTHOR, TEACHER, AND MASTER IN HIS FIELD OF THE PROFESSION
\end{abstract}

\section{THE AUTHOR}

\section{DEDICATES THIS WORK AS A TOKEN OF HIS RESPECT AND ESTEEM}





\section{P R E F A C E.}

In undertaking this work it was the author's desire to cover both the medical and surgical aspects of genito-urinary diseases according to the present state of knowledge, and to give a prominent place to certain features which have received but comparatively slight attention hitherto in the literature of this department. These are, first, the operative surgery of the genito-urinary tract; secondly, very full series of statistical data, more especially such as relate to the results of surgical treatment; and thirdly, as complete and artistic illustration for the work as the author could secure.

Apart from these particular characteristics, there are some characteristics in the arrangement of the work which have seemed to the author to be advantageous. For example, all clinical cases have been excluded from the text, and grouped at the ends of the chapters. This has been done for the sake of avoiding the interruption in the reading which these cases always involve when they are scattered through the body of the text. With a similar object in view, all references to the literature which have been quoted are also omitted from the text and placed in the bibliography at the ends of the chapters. They are indicated for the reader merely by a number following the name of the author quoted.

With regard to the illustrations, it should be said that the original drawings from which the reproductions were made in many instances do not attempt to reproduce accurately the anatomical conditions of the parts represented. They are rather partly anatomical and partly diagrammatic. This has oftentimes been done with intention, owing to the impossibility of portraying the exact anatomy and at the same time of showing the step or steps of the operation that it is desired to demonstrate.

The high grade of the illustrations is due to the excellence of the original drawings made for the author by the three.artists from whose hands they issued, Mr. H. F. Aitken, Miss Florence Byrnes, and Miss Florence Spaulding, and to the knowledge and cordial coöperation of the publishers, who have spared no pains in securing the best quality of reproduction.

An important number of the illustrations in the chapter on the Technique of Operations upon the Prostate were made from the original draw- 
ings used in the classic treatise upon the same subject by Dr. John B. Deaver, to whose kindness and generosity the author is greatly indebted for the use of the figures for this present work. To Dr. Deaver and to the others mentioned above the author wishes to offer his most hearty thanks.

With reference to the statistical compilations and their analysis, to which the author devoted much time and labor, a word of explanation is appropriate. He would not wish it to be thought that he is unconscious of the fallacies to which statistics are conspicuously exposed, and would make it clear that such inferences as are drawn from the data of this sort occurring in the work are not regarded by him as being in any sense final. He has been led to make such data a marked feature largely for the purpose of saving future writers the trouble and time involved in the search for them. He has sought as conscientiously as possible to avoid that frequent error of the statistician, namely, making figures so appear as to support the writer's preconceived ideas, and has, on the contrary, in every instance wholly put aside any beliefs that he may have held with regard to matters upon which the data bear, and has allowed the figures to lead to their own conclusion, whether it contradicted his own prior ideas or not.

The most important innovation included in this publication is that relating to the operative technique. As a rule, such operative surgery as is included in the literature of this special field is inadequate, and is so scattered as to make it difficult to find. In the present work the descriptions of the technique of operations are given in full detail and will be found in special sections following the chapters dealing with the particular parts under consideration. By no means all the operations that are in vogue are described or illustrated. To do this would have been to exceed the proper limits of such a work as this, and would demand a separate volume. The author has sought to include here only such of the operations as, in his experience and judgment, are the best.

When the author had been occupied for four years in the preparation of this work he realized that if it were to be published within the time which he had allotted for its completion, he must have the aid of a collaborator. He was then so fortunate as to secure the coöperation and invaluable assistance of his younger colleague and former house surgeon, Dr. John H. Cunningham, Jr., to whose ability and disinterested and untiring industry it is due that the work has been finished within the seven years which the author had allowed for its completion.

The Chapter on Tumors of the Kidney and Adrenal Gland has been generously contributed by Dr. Edgar Garceau, of Boston, to whom the 
author is greatly indebted for what seems to him and to his collaborator, Dr. Cunningham, to be one of the most complete and best parts of the work. The photomicrographs illustrating this chapter were taken at the Laboratory of the Massachusetts General Hospital.

In conclusion, indulgence is asked for such errors as may inadvertently have been made in quoting from other writers, or for any failure to note many excellent volumes that are extant relating to one or another of the subject matters with which the present work is concerned.

FRANCIS S. WATSON.

92 MARLBOROUGH STREET, BOSTON, IgO8. 



\title{
CONTENTS OF VOLUME I.
}

\author{
PART I. \\ THE EXTERNAL GENITALS.
}

CHAPTER I.

THE PENIS.

Anatomy of the Penis-Physiology of the Penis-Abnormalities of the Penis -Injuries of the Penis-Diseases of the Penis-Cutaneous and Mucous Membrane Affections of the Penis . . . . . . . . . . . I7-53

\section{CHAPTER II.}

THE URETHRA.

Acute and Chronic Urethritis-Endoscopy-Polyp and Papilloma . . . . 54-83

\section{CHA P T E R I I I.}

STRICTURE OF THE URETHRA.

Spasmodic Stricture - Inflammatory Stricture - Organic Stricture - Traumatic Stricture-Obliteration and Stricture of the Urethra (Congenital) . . . . 84-I 38

\section{CHAPTER I V.}

TECHNIQUE OF OPERATIONS FOR STRICTURE OF THE URETHRA.

Divulsion-Internal Urethrotomy-External Perineal Urethrotomy, Perineal Section,

The Boutonnière-Exposure of the Urethra behind the Stricture by Open

Dissection-Suture of the Urethra after Perineal Urethrotomy-Resection of

Stricture . . . . . . . . . . . . . . . . . I39-I55

\section{CHAPTER V.}

TECHNIQUE OF OPERATIONS UPON THE PENIS.

Operations for Phimosis-Operations for Paraphimosis-Amputation of the Penis -Extirpation of the Penis-Total Emasculation of the Penis-Plastic Operations on the Penis-Operations for Hypospadias-Operations for EpispadiasOperations for the Purpose of Closing Urethral Fistulæ . . . . . 156-190 


\section{CHAPTER VI.}

THE SCROTUM.

Anatomy of the Scrotum-Abnormalities of the Scrotum-Injuries of the Scrotum -Cutaneous Diseases of the Scrotum-Inflammations of the Scrotum . I9I-I99

\section{CHAPTER VII.}

THE VAS DEFERENS AND SPERMATIC CORD.

Anatomy-Abnormalities - Injuries - Inflammation-Tumors-Varicocele • • 200-206

\section{CHAPTER VIII.}

THE TESTICLES.

Anatomy of the Testicles-Physiology of the Testicle-Descent of the TesticleAbnormalities of the Testicle-Injuries of the Testicles-Neuralgia of the Testicle-Inflammations of the Testicle and Epididymis-Cysts and Newgrowths of the Testicles-Solid Tumors of the Testicle and EpididymisHydrocele-Hematocele-Spermatocele-Galactocele . . . . . . 207-257

\section{CHAPTER IX.}

THE SEMINAL VESICLES.

Anatomy of the Seminal Vesicles-Physiology of the Seminal Vesicles-Abnormalities of the Seminal Vesicles-Injuries of the Seminal Vesicles-Diseases of the Seminal Vesicles-Calcification and Concretions of the Vesicles and Ducts . . . . . . . . . . . . . . . . $25^{8-272}$

\section{CHA PTER X.}

TECHNIQUE OF OPERATIONS ON THE TESTICLE, EPIDIDYMIS, SPERMATIC CORD, AND SEMINAL VESICLES.

Orchidopexy-Orchidectomy (Castration)-Operations for Hydrocele-Epididymectomy-Vasectomy-Incision of the Epididymis - Operations for Varicocele -Incision of Abscess of the Seminal Vesicles-Resection of the ScrotumExcision of the Scrotum . . . . . . . . . . . . 273-298 


\section{PART II. \\ THE PROSTATE AND BLADDER. \\ CHAPTER XI. \\ THE PROSTATE.}

Anatomy of the Prostate-Hypertrophy of the Prostate-Malignant Disease of the Prostate-Acute Prostatitis, Non-tuberculous Suppuration of the Prostate

-Prostatic Calculus . . . . . . . . . . . . . . 299-37I

\section{CHAPTER XII.}

TECHNIQUE OF OPERATIONS UPON THE PROSTATE.

Prostatectomy-Prostatotomy-Perineal Prostatotomy for Abscess of the Prostate -Operations for Malignant Disease of the Prostate. . . . . . 372-420

\section{CHAPTER XIII.}

\section{CYSTOSCOPY.}

Photographic Attachment to the Cystoscope-Operating Cystoscopes-Cleansing of the Cystoscope-Manner of Making Cystoscopic Examinations-Difficulties Encountered in Cystoscopy-Cystoscopic Pictures Presented by the Normal Bladder and by Certain of the Pathological Conditions of the Organ . . 42I-430

\section{CHAPTER XIV.}

\section{THE BLADDER.}

Anatomy of the Bladder-Anomalies of the Bladder-Injuries of the BladderCystitis (Inflammation of the Bladder). . . . . . . . . . 43-467

\section{CHAPTER XV.}

VESICAL CALCULUS AND DIVERTICULUM OF THE BLADDER.

Vesical Calculus-Diverticula of the Bladder . . . . . . . . . ${ }_{468-493}^{66}$

\section{CHA P TER XVI.}

TUMORS OF THE BLADDER.

The Origin of Tumors-Structural Classification of Vesical Tumors-Subdivisions and Synonyms-Special Characters of Different Neoplasms of the BladderPropagation of Malignant Tumors . . . . . . . . . . . 494-543 


\section{CHAPTER XVII.}

TECHNIQUE OF OPERATIONS ON THE BLADDER.

Suprapubic Aspiration of the Bladder-Suprapubic Cystotomy (Epicystotomy, Sectio Alta)-Operations for Vesical Calculus-Perineal Lithotomy-Suprapubic Lithotomy-Litholapaxy (Rapid Lithotrity, Lithotrity in one Sitting) Operations for Removal of Vesical Tumors-Total Extirpation of the Bladder

-Operations upon Diverticula of the Bladder . . . . . . . . 544-599

Index to Authors . . . . . . . . . . . . . . . . . . . . . 60I

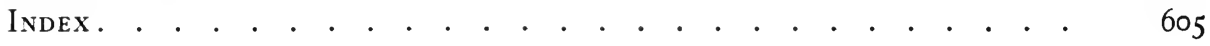




\title{
DISEASES AND SURGERY
}

OF THE

\section{GENITO-URINARY SYSTEM.}

\author{
PAR T I. \\ THE EXTERNAL GENITALS.
}

CHAPTER I.

THE PENIS.

ANATOMY OF THE PENIS.

THE penis is primarily the organ of copulation, secondarily it is concerned in the function of urination. It is from three to four inches in length when flaccid, and from five to seven when erect. The circumference of the average penis, when flaccid, is three and one-quarter inches. The size of the penis bears no relation to that of the individual, it being often noticeable that small and weakly men have large organs, while those who are strong and well-developed have small ones.

Structure.-The penis is composed of three cylindrical, parallel bodies, two of which lie side by side and are called the corpora cavernosa; the third is placed beneath and between these, and is termed the corpus spongiosum.

The corpora cavernosa begin in two cone-shaped bodies, which are closely united one to each ramus of the os pubis. From these points they converge and become joined together a little anterior to the arch of the symphysis pubis. Anteriorly they again separate slightly and terminate in two conical ends, which fit into corresponding hollows upon the under side of the glans penis. The parts of the corpora cavernosa between the point at which they separate posteriorly and their insertions upon the rami of the os pubis are called the crura of the penis. 
The corpus spongiosum contains the urethra, which terminates in a slit-like opening upon the tip of the glans, called the meatus urinarius.

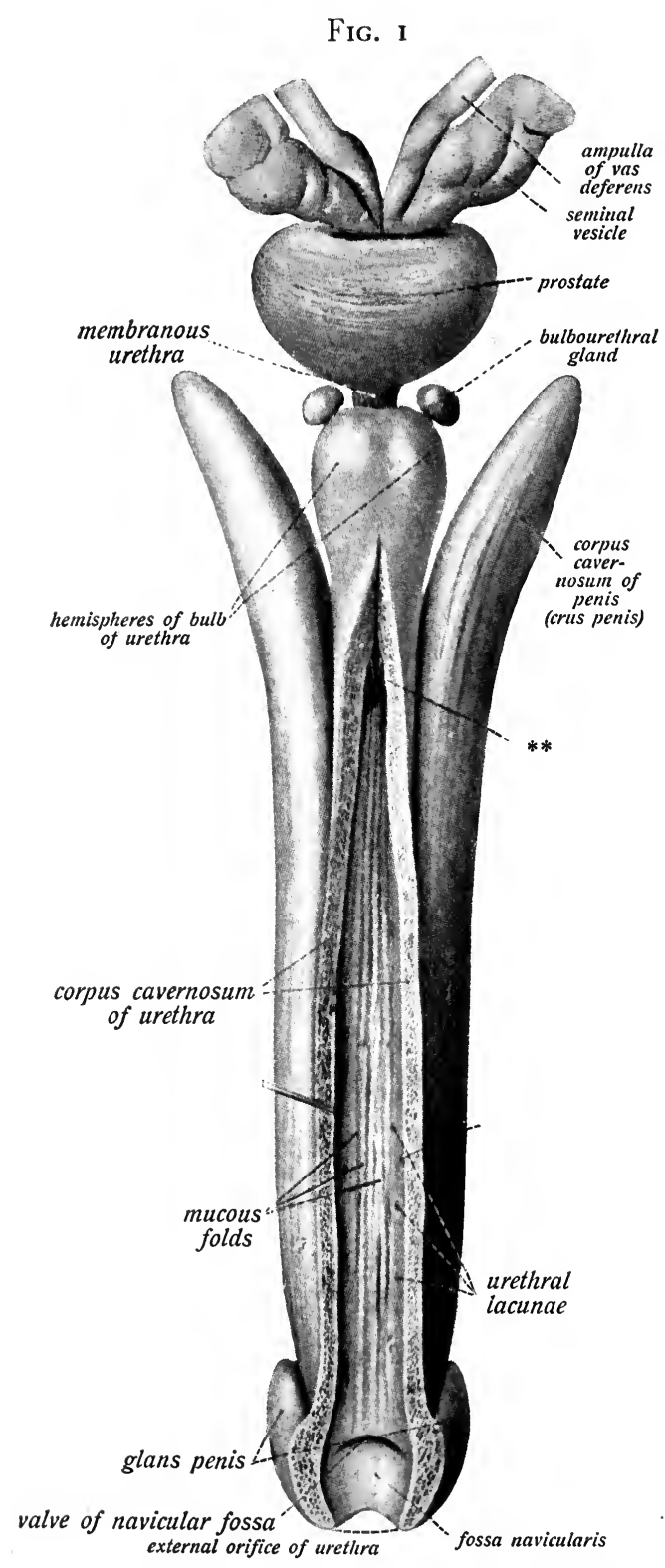

The male urethra, with the corpora cavernosa of the penis, the bulbo-urethral glands, and the prostate. (Sobotta.)
The corpus spongiosum presents two expansions in its course. One is just beneath the point of separation of the two corpora cavernosa, and is called the bulb of the corpus spongiosum; the other expansion forms the head of the penis, and is termed the glans. (Plate I and Fig. I.)

All three of the corpora are composed of erectile tissue, the fibrous partitions of which are derived from their connectivetissue envelopes. The corpora cavernosa are enveloped in a single fibrous covering, which, however, is extended in the form of a septum between the two, and serves to separate one from the other. This septum is least distinct anteriorly and allows intercommunication between the corpora in front. It is called the septum pectiniforme. The fibrous envelope of the corpus spongiosum is distinct from that of the corpora cavernosa. The mutual relations of these fibrous coverings are seen in Fig. 2, which represents a cross-section of the penis.

The fibrous bands of the corpora which are derived from these envelopes pass in all directions in the interior of the bodies, and make in so doing a varied series of spaces, which contain the bloodvessels that supply the corpora; the fibrous trabeculæ also give support to the small branches of the arteries of the corpora 


\section{PLATE I}

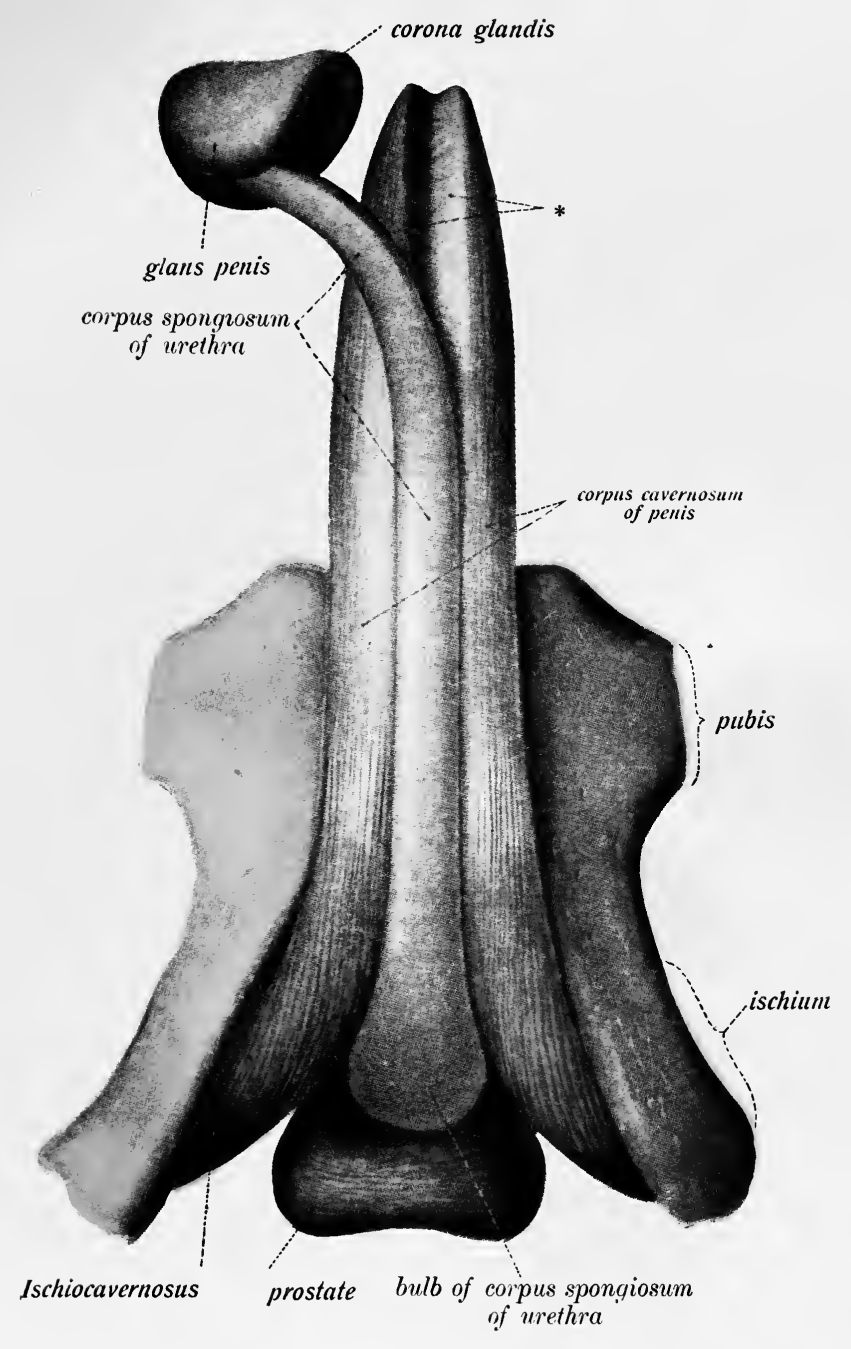

THE CORPORA CAVERNOSA AND CORPUS SPONGIOSUM OF THE PENIS. (Sobotta.) 
cavernosa, the main trunks of which pass through the middle of each of their bodies respectively, and give off the minor twigs over the walls of the fibrous structure just described. The whole structure represents typical erectile tissue.

The structure of the corpus spongiosum is similar in character to that of the corpora cavernosa. The bulb, already referred to, is covered externally by the accelerator urinæ muscle.

All three of the corpora are surrounded by a second fibrous covering known as Buck's fascia. This fascia blends with the suspensory ligament of the penis, which is the fibrous band that connects it to the symphysis pubis above.

\section{FIG. 2}

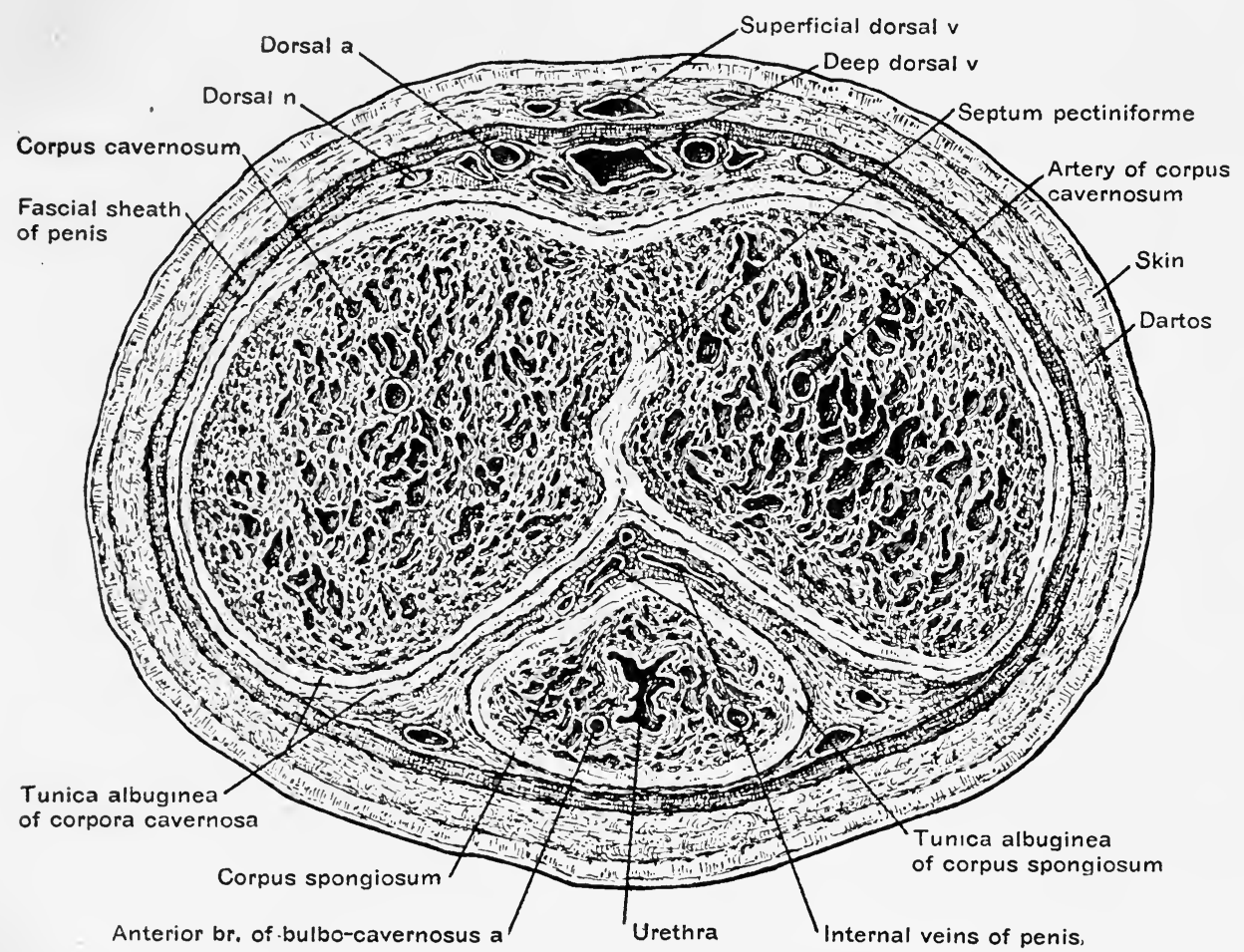

Transverse section through body of the penis. (Deaver.)

Still another covering to the organ is supplied by the dartos, which is immediately beneath the skin and continuous with the dartos of the scrotum. It is on account of the loose arrangement of the fibers of this structure that the excessive œdema of the penis which occurs under certain conditions is permitted to take place. The fibrous walls of the corpora cavernosa contain elastic fibers, which allow for the distention of the bodies. 
Cruveilhier $^{1}$ states that these sheaths are strong enough to sustain the weight of the cadaver.

The prepuce is composed of a cutaneous and a mucous layer, between which there is a loose elastic connective-tissue structure devoid of adipose tissue. The mucous layer is attached just behind the corona of the glans, posteriorly; on its ventral surface it is bound to the meatus by a small triangular fold of mucous membrane, the frenum preputii (Fig. 3 ).

Arteries of the Penis (Plate II).-The arteries of the penis are derived from the internal pudic, and are: (1) The artery of the bulb. (2) The artery of the corpus cavernosum. (3) The dorsal artery of the penis.

The Artery of The Bulb.-The artery of the bulb is given off from the internal pudic just after that vessel penetrates the posterior leaf of the triangular ligament. It passes forward through the compressor urethræ muscle, enters the bulb of the corpus spongiosum, and supplies the latter

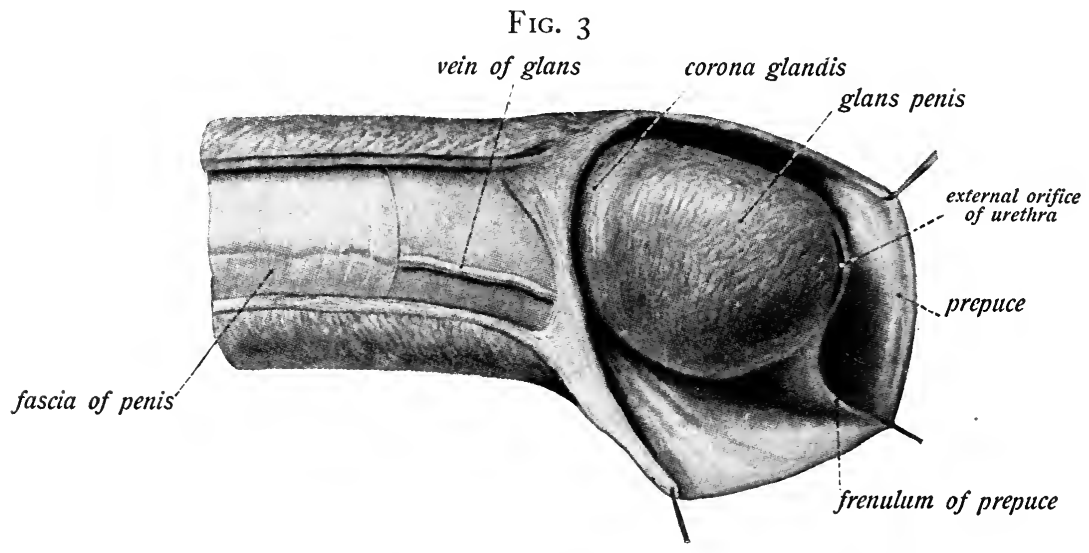

The distal end of the penis with the prepuce. (Sobotta.)

with blood. It also sends small branches to Cowper's glands. It is a vessel of some size, and when cut in the operation of lateral lithotomy, may give rise to troublesome hemorrhage. 'On this account care should be taken to avoid it.

The Artery of the Corpus Cavernosum.-On leaving the internal pudic, this vessel divides and sends a branch to each of the corpora which enter the crura and run forward through the body of each corpus cavernosum.

The Dorsal Artery of the Penis.-This vessel arises as a branch of the internal pudic, passes through the anterior layer of the triangular ligament, runs forward between the crura and the layers of the suspensory ligament, and is continued to the glans in the groove between the corpora upon the dorsum of the penis, in company with the dorsal vein and nerve. At the base of the glans it divides and encircles it. 


\section{PLATE II}

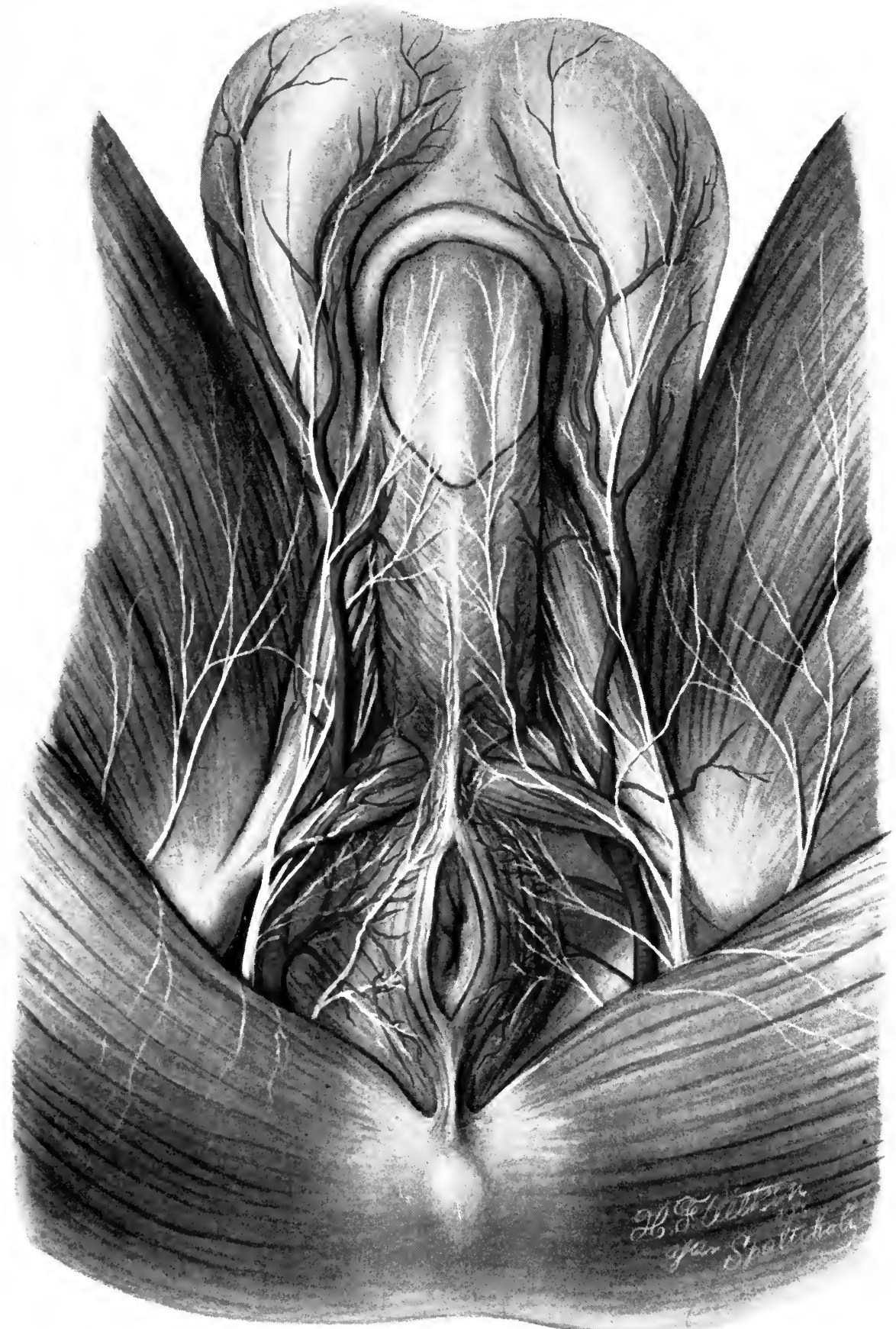

THE INTERNAL PUDIC ARTERIES AND THEIR BRANCHES.

The arteries of the bulb of the urethra, the arteries of the penis supplying the corpora cavernosa, the transverse perineal arteries, and posterior scrotal arteries. Also, the crura of the penis, the transverse perineal muscles, the external sphincter ani, the levator ani, the accelerator urinæ muscles, and the tendinous centre of the perineum. 

Veins of the Penis (Plate III).-The veins correspond with the arteries. The dorsal vein has two branches, which unite near the root of the organ into a single trunk. From here it passes between the layers of the suspensory ligament, beneath the arch of the symphysis, between the subpubic and triangular ligaments, and joins the prostatic plexus of veins.

Lymphatics.-These vessels run along the dorsum of the penis. They are in two sets, a deep and a superficial one. The former passes to the pelvic nodes, the latter to the inguinal ones.

Nerves.-The coverings of the penis receive their nerve supply from the genital branch of the genitocrural nerve and from the inferior perineal branch of the internal pudic nerve. The erectile structures are under control of sympathetic branches from the hypogastric plexus, of spinal branches, the dorsal nerve of the penis, and of the superficial perineal nerve.

The Urethra.-The urethra is the passage for the exit of urine from the bladder, and extends from its outlet to the tip of the glans penis. It is divided for descriptive purposes into the prostatic, the membranous, and the spongy or pendulous portions.

The prostatic urethra is one and one-quarter inches in length, the membranous three-quarters of an inch, and the spongy urethra from five to six inches. The prostatic urethra is that part of the canal which is surrounded by the prostate gland; the membranous portion is that which extends from the apex of the prostate to the slight enlargement of the urethra which occurs in that part of it corresponding to and enclosed in the bulb of the corpus spongiosum. The spongy urethra extends from the end of the membranous part to the termination of the canal at the tip of the glans.

The largest part of the urethra is the prostatic portion, the narrowest is its membranous part. There are two points in the course of the canal at which it expands into a larger size than those which are immediately adjacent to them. These are the bulbous portion and the fossa navicularis.

The size of the meatus is, with but few exceptions, less than that of the urethra; the average size of the former being $24 \mathrm{~mm}$. and of the latter $32 \mathrm{~mm}$. of the French scale.

The membranous urethra is included between the anterior and the posterior layers of the triangular ligament. It is surrounded by the compressor urethræ muscle. The former of these facts is of importance with reference to the direction taken by an extravasation of urine which originates in a rent in this part of the canal, and which is determined by the triangular ligament. The anterior leaf of this membrane limits the passage of the fluid forward, and the posterior leaf arrests its backward progress, so that its first appearance is in the perineum. 
The compressor urethræ muscle is important in two respects: the first being that it is the most efficient of the two sphincters which control the exit of the urine from the bladder, and the second because it closes the membranous part of the urethra and retains inflammatory exudations which originate in that part of the canal which lies behind it, so that their free escape is thereby prevented and insufficient drainage results.

How far the action of the compressor urethræ muscle may prevent the backward extension of infections of the anterior urethra it is difficult to say, but it is quite probable that such an influence is exercised by it.

The interior of the urethra is lined with mucous membrane, upon the surface of which are numerous small openings, which represent the mouths of the ducts of Littre's and Cowper's glands, of the ejaculatory ducts, of the utricle, and of the lacuna magna, the last named of which is a wide orifice upon the roof of the fossa navicularis.

The orifices of the ducts of Cowper's glands are placed one on either side of the floor of the bulbous urethra, about one inch in front of the anterior leaf of the triangular ligament (see Fig. I **).

\section{PHYSIOLOGY OF THE PENIS.}

Erection.-Erection of the penis is a complex, reflex phenomenon. The nerve centre controlling it lies in the lumbar region of the spinal cord. It responds to nerve impulses coming from different sources. The stimulus may originate in the ducts of the testes, owing to the pressure of semen in them; in the penis, from stimulation of the terminal nerves of the glans and the skin; or in the brain, as sexual emotions.

Erection is essentially a vascular phenomenon, and is produced by distention of the cavernous spaces of the corpora by the blood.

This distention is produced by complex nervous and muscular activities. The arteries supplying the corpora dilate under the influence of the vasodilator nerves, and allow the blood to flow into the corpora cavernosa and spongiosum, while, simultaneously, the involuntary muscle fibers in the erectile tissue relax, thus increasing the capacity of the blood spaces. The erector penis and the bulbocavernous muscles are brought into spastic contraction and compress the veins as they pass from the cavernous bodies. The free entrance of blood into the corpora and the obstruction to its outflow thus produced, cause distention of the erectile tissue, which further compresses the venous outlet, and the organ becomes hard and erect.

The Secondary Function of the Penis.-In its secondary functional capacity the penis is concerned in the passing of the urine from the bladder by expelling it through the urethra. As the last drops pass by 


\section{PLATE III}

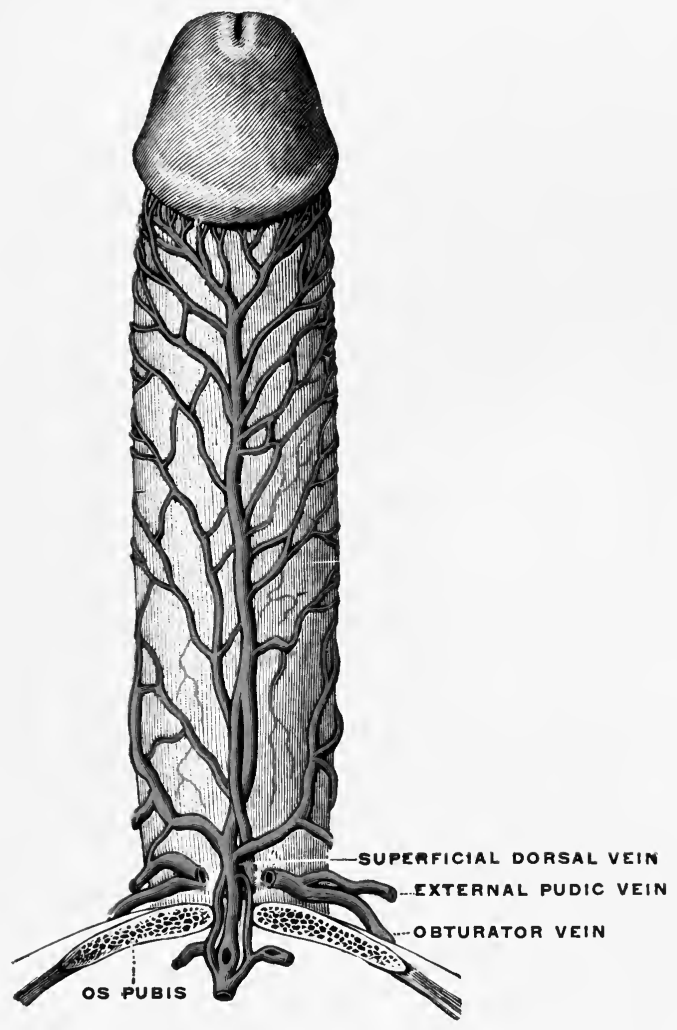

VEINS OF THE PENIS. (Testut.) 
the compressor urethræ muscle into the anterior urethra, the fibers of the accelerator urinæ muscle surrounding the bulb contract, forcing the blood contained in the bulbous urethra forward, thus distending the corpus spongiosum and driving onward the fluid contained in the passage by creating a sort of peristaltic movement along the canal.

\section{ABNORMALITIES OF THE PENIS.}

The congenital abnormalities of the penis consist chiefly in absence or duplication of certain parts, and in variations or abnormal positions of the external urinary orifice, with or without other associated changes in the organ.

Absence of the Penis.-Complete absence of the penis is very rare, and occurs only in association with other developmental defects of the external genitals. The parts may closely resemble those of the female, the scrotum being bifurcated and often so small as to be considered absent. The external urinary orifice opens in the perineum, or a true cloaca exists communicating with the rectum through its anterior wall.

Extreme dwarfing of the penis, so that it somewhat resembles the clitoris, is more common than complete absence. It is usually associated with hypospadias, but the condition should not be regarded as absence of the penis.

Boutelier ${ }^{2}$ records a case in which the penis lay buried beneath the skin of the pubes. Other instances are on record where the extremely small penis has been retained beneath the integument. When occurring in children, the condition has been satisfactorily treated by liberating the organ from its bed.

Complete absence and extreme dwarfing of the penis are so uncommon as to have been given but slight mention in literature, and no surgical procedures have been proposed for the relief of the conditions. Preston, ${ }^{3}$ Revolat, ${ }^{4}$ Dumarquay, ${ }^{5}$ Wright, ${ }^{6}$ and Harris have each recorded a case, and the latter in addition to his own has collected six others from the literature.

Goschler's ${ }^{7}$ case of congenital absence of the organ is one of the most remarkable. This individual was an otherwise normal, fully developed man, aged twenty-seven years. The scrotum was natural and contained the testes and cords. The penis was entirely absent, and the bladder communicated with the rectum through its anterior wall, about four inches from the anus, by an orifice large enough to allow the passage from the bowel into the bladder, of a sound of moderately large caliber. Just anterior to the anus was a small fold of skin, beneath which was a body of erectile tissue which responded to sexual stimulus. 
Double Penis.-This condition is also extremely rare. It is usually found in connection with other congenital malformations, or fœtal inclusions, $e . g$. , double bladder. Smith ${ }^{8}$ records a case in which the patient with this congenital defect had a stone removed from one of his bladders. Another remarkable feature of the case was that the patient had control of both bladders, being able to empty one at a time. Other cases are recorded by Beck $^{9}$ and Lorthior. ${ }^{10}$ The illustration here presented is taken from one of the most remarkable cases in the literature. This man was exhibited in many clinics on the European continent. (Plate IV.)

Hermaphroditism.-This name is applied to individuals who have one or more of the sexual organs of both sexes. Strictly speaking, the term should be limited to the possession by one individual of all the essential parts of both sexes. This, however, with one possible exception, has never been seen, and the greater number of the cases of reported hermaphroditism have been either women with very large clitorises, or men with diminutive penises, cleft scrotums, and undescended testes. In some instances of the latter conformation a vagina has been closely simulated, or actually present in rudimentary form, and the individual has had an unusual development of the breasts for the male, and a feminine voice and manner.

Lydston $^{11}$ states that pseudohermaphroditism is by no means rare, and that true hermaphroditism does not exist, meaning that no individual has yet been shown to have the essential sexual organs of the male and the female and the functional capabilities of both sexes. Most authors are in accordance with this view, and such cases as are recorded ${ }^{12}-e . g$., in which the individual lived for thirty years having sexual intercourse as a female and became a mother, and later assumed the role of the male and in that capacity became the father of a family-are generally discredited.

Hypospadias.-Hypospadias is a congenital penile defect in which the urethra terminates on the under side of the organ at some point posterior to its normal location at the end of the glans penis.

Varieties and Frequency.-According to Rennes, Kauffmann, and others, hypospadias occurs in a proportion of one in three hundred males.

Loumeau and Kaufmann describe eleven varieties of hypospadias (Fig. 4). They may, however, for surgical consideration, be divided into three classes:

i. Balanitic or Glandular Hypospadias.-Here the urethral opening is either in the glans or just behind it.

2. Penile Hypospadias.- The urethral outlet may be at any part of the under surface of the penis. It is usually situated at one of the three 
PLATE IV

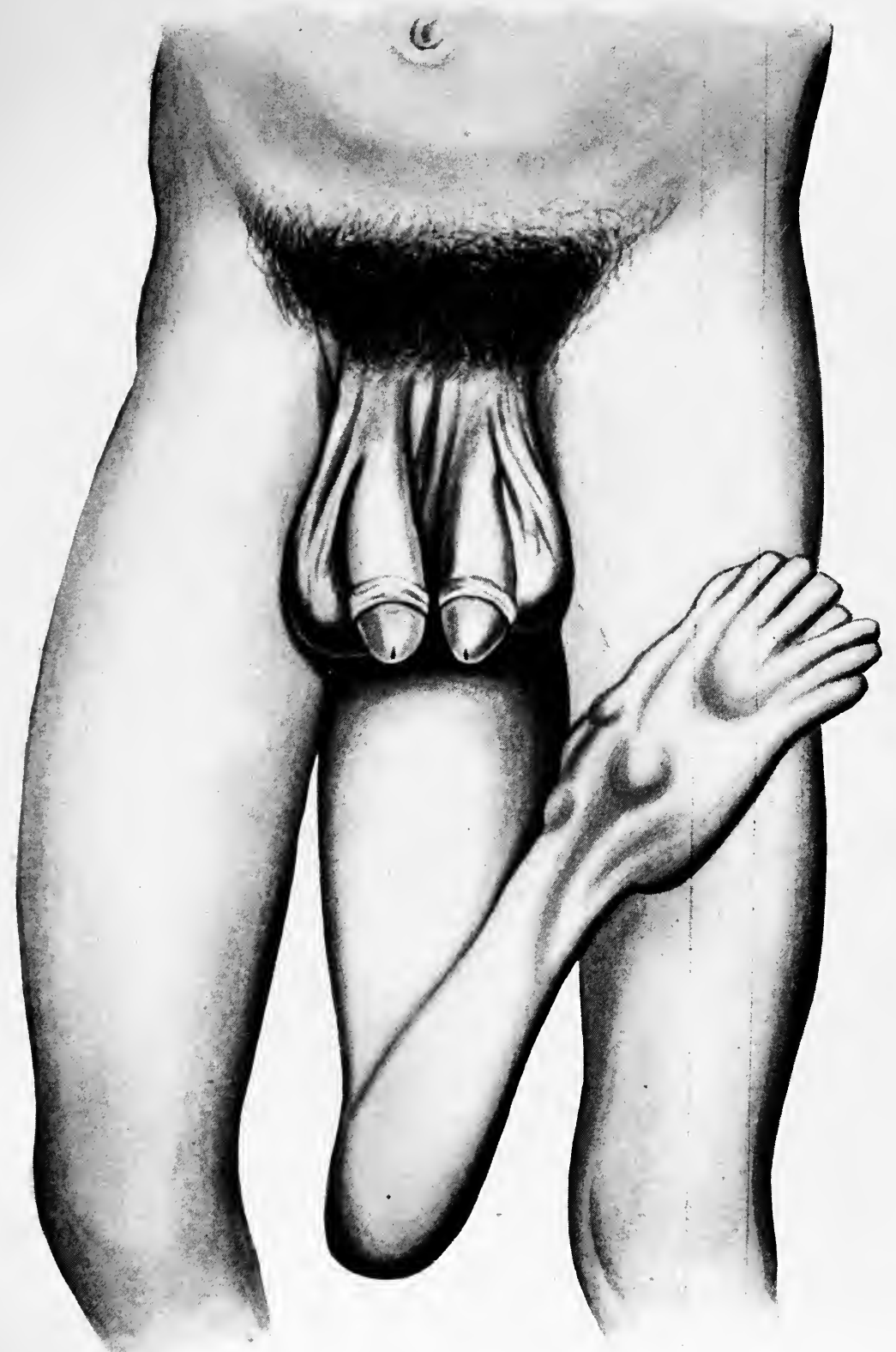

DOUBLE PENIS. (Taylor.) 

following points: (I) A short distance behind the base of the frenum. (2) Midway between the tip of the glans and the penoscrotal angle. At the penoscrotal angle.

3. Scrotal Hypospadias.-In this form the urethral opening is either at the junction of the scrotum with the penis in front, or at its junction

Fig. 4

1.

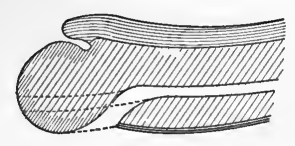

4.

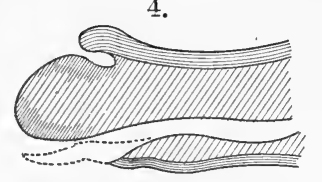

$\%$

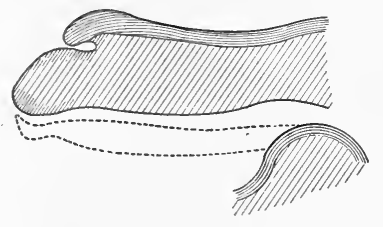

2.

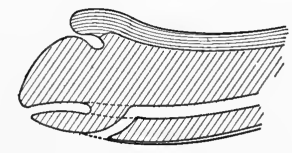

5.

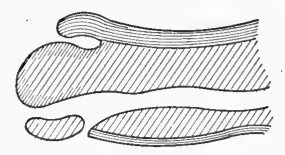

8.

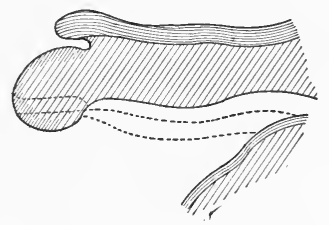

3.

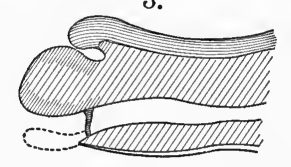

6.

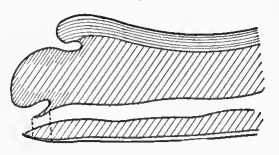

9.

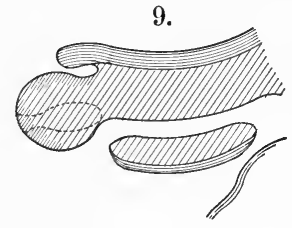

10.

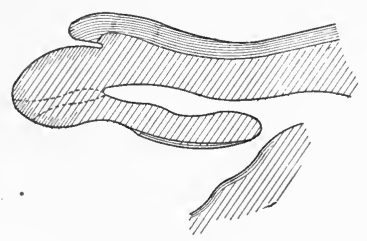

11.

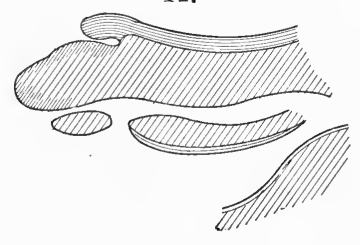

Diagrammatic sections showing different varieties of hypospadias: I, hypospadias with imperforate glans; 2, hypospadias with blind canal in glans; 3, with barrier placed between penile urethra and balanitic groove; 4, typical case of hypospadias; 5, hypospadias with normal meatus; 6 , penile urethra opening below the glans; 7 , absence of the whole inferior part of the penile urethra; 8 , hypospadias with absence of urethra through glans; 9, case of D’Arnaud; Io, case of Lacroix; I I, case of Lippert with normal meatus. (Kauffmann.)

with the perineum behind. The varieties are called respectively penoscrotal and perineoscrotal.

In order of frequency the above-named varieties stand as follows: (I) The glandular. (2) The penile. (3) The perineal.

In all but the slightly marked cases the penis is small and deformed, 
the glans flattened, the prepuce faultily attached, or it may present a condition of partial phimosis. The organ itself is sharply incurved upon the scrotum, to which it is drawn down by a strong fibrous band upon its under surface, which represents the undeveloped urethra. In the extreme cases the incurving is so great that the glans is not visible.

Consequences of Hypospadias.-There are two serious consequences involved in the condition of hypospadias. One is the interference with or impossibility of coitus due to the incurving of the penis upon the scrotum, the other the distress and eczema attending and resulting from the inability to urinate in a natural manner, owing to which the legs are wet and the skin is inflamed.

Atrophy of the corresponding half of the scrotum is usually. present in association with the condition of hypospadias. Retained testis is also a commonly associated abnormality.

Treatment.-The treatment of hypospadias is wholly surgical. (The best of the many surgical procedures which have been devised to relieve the condition are described in the chapter on the Technique of Operations on the Penis.)

To be successful, operations for the relief of this condition should be performed during childhood, between the ages of three and six years preferably, although some successful results have been reported when the surgical procedures were undertaken in adult life, $e . g$., Lucke's case and Duplay's case, in which the patients were aged, respectively, nineteen and twenty-one years, and in which cure was obtained by operations, in the course of one year, and one year and a half.

In the less well-marked forms of the balanitic variety of the condition operative measures may not be required. Their adoption will be largely determined in all cases by the degree of disability or disturbance in the act of urination or copulation.

The following factors enter into the decision of the question of operation: (I) the degree of deformity; (2) whether the penis is of fair size; (3) whether the testicles have descended, or if they are atrophied; (4) whether eczema of the skin, if present, is likely to interfere with the success of the operation.

Epispadias.-Epispadias is a congenital penile defect, in which the urethra opens upon the upper aspect of the penis at some point posterior to its normal termination at the end of the glans.

There is no satisfactory embryological explanation of the manner in which the urethra becomes transferred to this position above the corpora cavernosa.

Frequency and Varieties.-This condition, less common than hypospadias, is of much more serious consequence, and more difficult to treat. 
Keyes quotes Dolbeau, ${ }^{13}$ who says that its relative frequency to that of hypospadias is about I to I50. Rennes records Io cases in 3000 conscripts. Marshall did not find a case in 60,000 conscripts. Czerny ${ }^{14}$ treated 6 cases in his Heidelberg clinic from 1878 to i892. The ages of Czerny's patients varied from twenty-two months to twenty-nine years.

Epispadias may exist quite independently of exstrophy of the bladder, but the latter malformation is always accompanied by epispadias.

Various grades of epispadias are somewhat arbitrarily divided by Czerny into those of the first, second, and third degree. The latter is the complete form, and that to which the operative procedures are applied. Here the penis is shortened and flattened, curved upward, and held against the abdomen by a band of connective tissue which replaces the urethra. The prepuce is usually large and has an apron-like form.

Symptoms. - The condition produces disorders of micturition and erection. The most distressing of the symptoms is incontinence of urine and the constant wetting of the surface of the neighboring parts with urine. Eczema frequently results as a consequence of this condition, and adds to the trials of the patient. Even when the opening is placed well forward, incontinence, nevertheless, results. The short, flattened, and deformed penis has but little power of erection, and coitus is impossible or incapable of being completely carried out. For the same reasons the individual is generally both sterile and impotent, the former because of the impossibility of directing the seminal fluid effectively.

Treatment.-The treatment of epispadias is entirely surgical, and the surgical procedures are described in the chapter on the Technique of Operations on the Penis.

\section{INJURIES OF THE PENIS.}

The penis may be accidentally or purposely injured. Insane persons not infrequently mutilate the organ intentionally, and sometimes with fatal result.

Wounds.-Wounds involving the corpora give rise to sharp hemorrhage, which sometimes is fatal, and if not properly cared for they may become infected and give rise to acute septic phlegmon and gangrene, from which deformity of the organ or fistula may follow, and cause painful or imperfect erections.

Treatment.-Superficial wounds heal under simple antiseptic applications and dressings. In the case of deep wounds, hemorrhage is usually the condition which calls for prompt treatment. It may be checked by packing the wound or by closing it by suture. Of the two, the second is 
the better. Care should be taken to assure one's self that hemorrhage does not continue beneath the surface after the application of the suture. A firm bandage should be applied to the penis. The sutures should be of absorbable material, and should include the severed edge of the sheath of the wounded corpus. An ice-bag may be applied outside the bandage, and will often lessen the tendency to erection, the occurrence of which it is desirable to avoid during the healing process.

An attempt should always be made to save the organ, even if the degree of mutilation makes success doubtful.

Contusions.-The term contusion should be limited to the cases in which there is no actual rupture of the fibrous sheaths of the corpora or of the urethra; that of fracture is applicable to those of which the contrary is true.

The usual form of contusion produces a moderate rupture of the superficial bloodvessels and the escape of blood from them into the lax areolar tissue immediately beneath the skin. The more severe degrees of the injury are such as involve the bodies of the corpora. In these cases there is a greater or less localized fluctuating swelling. If infection occurs, suppuration may result. Gangrene occasionally follows such injuries.

Treatment.-Even pressure applied by a well-adapted bandage, and cold, are all that is required for the milder forms of the injury. For those which are more severe, or when infection occurs, evacuation of extravasated blood and of pus may be required. This is accomplished by external incision.

Fracture of the Penis.-In this accident the fibrous sheaths of one or all of the corpora are lacerated. The urethra is frequently torn through, but sometimes escapes injury.

Etiology.-The injury is produced either by direct blows upon the surface of the penis or from the sudden bending of the organ while erect, usually caused by striking the head of it against the parts adjacent to the vulva during an attempted coitus.

Symptoms.-Pain immediately follows the accident, and is very shortly succeeded by hemorrhage. When the urethra is torn through, blood always flows from the meatus. If the urethra and corpus spongiosum alone are involved, which is seldom the case, the blood may almost entirely escape from the meatus, and but little swelling then results. If the corpora cavernosa are involved, an extensive extravasation of blood immediately takes place and appears as a diffuse hematoma beneath the integument of the organ. The swelling is often very great in these cases and the discoloration is marked.

With the first urination following the injury, in the cases in which the 
urethra is torn through, urinary extravasation takes place, and when this happens the condition becomes a much more serious one, owing to the fact that infection supervenes.

Prognosis.-The prognosis is usually good, but if extravasation of urine has occurred and is not promptly relieved, the patient is very likely to die. The prognosis with regard to the functional capability of the organ will depend upon whether or not, in the course of healing, there has been formed a sufficient quantity of connective tissue to interfere with the free flow of blood into the corpora and prevent normal erection. If the connective-tissue deposit is in the corpus spongiosum, chordee will result; and if in one or both of the corpora cavernosa, curvature of the penislaterally or upward-will occur. Stricture of the urethra always follows the accident if the urethra has been involved in the injury.

Treatment.-Hemorrhage from the urethra is best arrested and the chance of extravasation of urine avoided by passing a catheter into the bladder, securing it there, and then applying pressure upon the surface of the penis by a bandage. The further progress of the bleeding into the corpora cavernosa is thus also checked. Occasionally the hematoma may have to be evacuated by an incision. When this is the case, and if after removing the blood clot there is no further bleeding, the incision may be closed at once. When extravasation of urine takes place, it should be treated as described in the chapter on Stricture of the Urethra.

Dislocation of the Penis.-This condition results from an injury such as to separate the body of the penis from its integument and to force it into the scrotum, the perineum, or beneath the skin of the symphysis pubis. In order to have the condition produced, the mucous membrane of the prepuce must be torn from its attachment to the base of the glans or from the preputial orifice. As a rule, the dislocation of the penis is one of a number of injuries received by the patient.

Symptoms.-The displacement of the organ and the formation of an extensive hematoma beneath the skin are the two objective evidences of the condition. Retention of urine is a conspicuous symptom. Extravasated urine contributes to the size of the tumor formed by the bleeding in the cases in which the urethra is lacerated.

Treatment.-When the blood can be removed from beneath the integument by irrigation and breaking up the clot, this should be done. When this cannot be accomplished, the preputial orifice should be incised freely and the penis drawn back into its natural position. If urinary extravasation has occurred, external perineal urethrotomy should be performed promptly, and a catheter should be tied into the bladder through this incision. If the blood supply of the penis has been cut off, the organ must be amputated. 


\section{DISEASES OF THE PENIS.}

Phimosis.-Phimosis is a condition of the glans penis or of the prepuce which prevents the retraction of the latter over the former. The condition is often congenital, but may be acquired.

Congenital Phimosis.-In the congenital form it is the narrowness of the preputial orifice which usually constitutes the phimosis. Adhesions between the inner lining of the prepuce and the surface of the glans are often met with in cases of congenital phimosis. In some instances the orifice of the prepuce is large enough to permit a partial exposure of the glans. In others it is extremely narrow, and when this is the case the urine distends the prepuce with each act of micturition.

Moderate phimosis may undergo spontaneous cure, but the prepuce remains long and pendulous even when this occurs. The condition exposes its possessor to greater liability to venereal contagion than is the case with those with whom it does not exist. Hutchinson ${ }^{15}$ showed that syphilis was less common among Jews than among Christians.

Acquired Phimosis. - The condition is produced most frequently by repeated attacks of inflammation. The resulting connective-tissue formation and induration about the orifice of the prepuce prevent its being retracted. Such inflammatory conditions as are productive of phimosis are sometimes seen in cases of diabetes, also in connection with herpes and balanitis, cicatricial contraction resulting from chancroids, and malignant disease of the prepuce.

Symptoms.-The symptoms of congenital phimosis are: (I) Reflex nervous disturbances. (2) Inflammatory conditions of the glans and prepuce. (3) Possibly, and in a very few instances, such as are due to the changes produced in the bladder, ureters, and the kidneys by obstructed micturition.

I. Reflex Nervous Disturbances.-These are manifested in the form of excitability of the sexual organs, producing frequent erections; neuralgias; incontinence of urine, and the simulated signs of hip disease. Muscular incoördination, spasms, or spastic contractions of the legs have also been attributed to phimosis.

2. Inflammatory Conditions.-These àre productive of sexual stimulation and more or less itching of the glans and prepuce. The inflammatory conditions are perpetuated by the retention, within the preputial sac, of urine and smegma. Occasionally the smegma secretion becomes calcified and gives rise to preputial calculus. The constant irritation which results from such a foreign body, the filling of the sac with urine during the acts of micturition, and the presence of organisms add to the inflammatory disturbance. 
Congenital phimosis sometimes prevents the normal ejaculation of semen during coitus, and is then productive of pain. Slight lacerations of the preputial orifice are sometimes caused during coitus because of its constriction.

Treatment.-Gradual dilatation of the preputial orifice and the separation of adhesions sometimes relieves the milder forms of the congenital condition. It is objectionable, however, owing to the pain which it involves and because of the length of time required for its execution.

Circumcision is the best treatment to apply at the outset, and is invariably successful if properly performed. When due to acquired conditions, complete circumcision is not always required.

In inflammatory phimosis the patient should be kept quiet. The preputial cavity should be irrigated several times a day with any of the mild antiseptic solutions, and the organ should be soaked for ten minutes every three or four hours in water as hot as can be comfortably borne.

The treatment of phimosis due to gonorrhœa will be discussed in the chapter on that subject.

Diabetic phimosis requires constitutional treatment, and the glans should be kept dry by lightly dusting it with aristol or stearate of zinc. Circumcision in this class of cases should not be practised, as the procedure is frequently followed by gangrene of the part, and in some instances death has resulted.

Gicatricial phimosis is best treated by circumcision. The first step in the operation should be the breaking down of all adhesions between the glans and the prepuce, if such are present. After this, the circumcision is carried out in the ordinary manner, or may be limited to a dorsal incision only, inasmuch as the prepuce is not redundant in this form of the condition, but is, on the contrary, small, contracted, and tight.

Complications of Phimosis.-Total destruction of the glans may take place in cases of rapidly progressing chancroidal ulceration, when it is concealed beneath a phimosis.

Inflammation of the lymph glands of the groin sometimes occurs in connection with the inflammatory form of phimosis. Suppuration may then result, giving rise to bubo. The lymphatics of the dorsum of the penis may also become inflamed, but suppuration does not take place in them.

Treatment of Complications.-In the cases of chancroidal inflammation and ulceration in connection with phimosis, the treatment must be prompt and energetic if the process is an acute and violent one. There should be no hesitation with regard to operating by laying open the prepuce by a dorsal incision, thus giving free access to the lesion, which should thereafter be submitted to cleansing applications, but not to cauterization. 
The fear of chancroidal infection of the cut edges of the wound is not without ground, but it can be controlled when it does happen, and is far less an evil than the continuance of the inflammation beneath the prepuce.

Paraphimosis.-Paraphimosis is the condition in which the foreskin, having been drawn back behind the corona, it cannot be brought forward again; it is produced either by the existence of a congenitally small preputial orifice, or by an acquired constriction of it due to inflammation or to cicatricial contraction.

Inflammatory paraphimosis is a condition produced by an already existing inflammation of the glans-balanitis, herpes, chancroid, gonorrhœa.

The prepuce, which is often retracted for the purpose of cleansing and treating such conditions, becomes inflamed and swollen, producing a tight constricting ring behind the corona glandis. As pressure is exerted by this ring, the glans and soft parts distal to the constriction become infiltrated, œdematous, and swollen. (Plate V.)

Symptoms.-If the paraphimosis does not receive prompt attention, the constricting ring gradually becomes ulcerated and gangrenous from lack of blood supply. The ulcerations involve the skin and subjacent cellular tissue. There may be several fissures at varying intervals in the constricting ring, which are points at which it has split. The ulcerative process, which finally releases the distal part by destroying the constriction, is nature's method of relieving this distressing situation. If, however, the paraphimosis is of an inflammatory character, the ulceration may spread, resulting in a gangrene involving the constricted area and some distance about it, the glans usually being involved. (Plate VI.)

Hemorrhage from ulceration of an artery, or from the deep tissues of the penis, may have grave and even fatal consequences. Inflammation appearing as cellulitis, phlebitis, lymphangitis, inguinal adenitis, and erysipelas may result.

If none of these sequelæ of the ulceration occur, the swelling subsides after the ring has ulcerated, and adhesions between the skin and the upper surfaces of the corpora cavernosa may take place, making it often impossible to subsequently reduce the paraphimosis, and the organ then remains deformed.

Treatment.-The determining feature of the condition, with reference to treatment, is the absence or presence of strangulation. Its presence is made evident by the dark color of the glans, by absence of warmth and sensibility, and by swelling. If these evidences are present, the condition must be promptly relieved, otherwise gangrene and possibly the loss of the entire glans will result. If the contrary of the above-named conditions is true, palliative measures may be employed. 


\section{PLATE V}

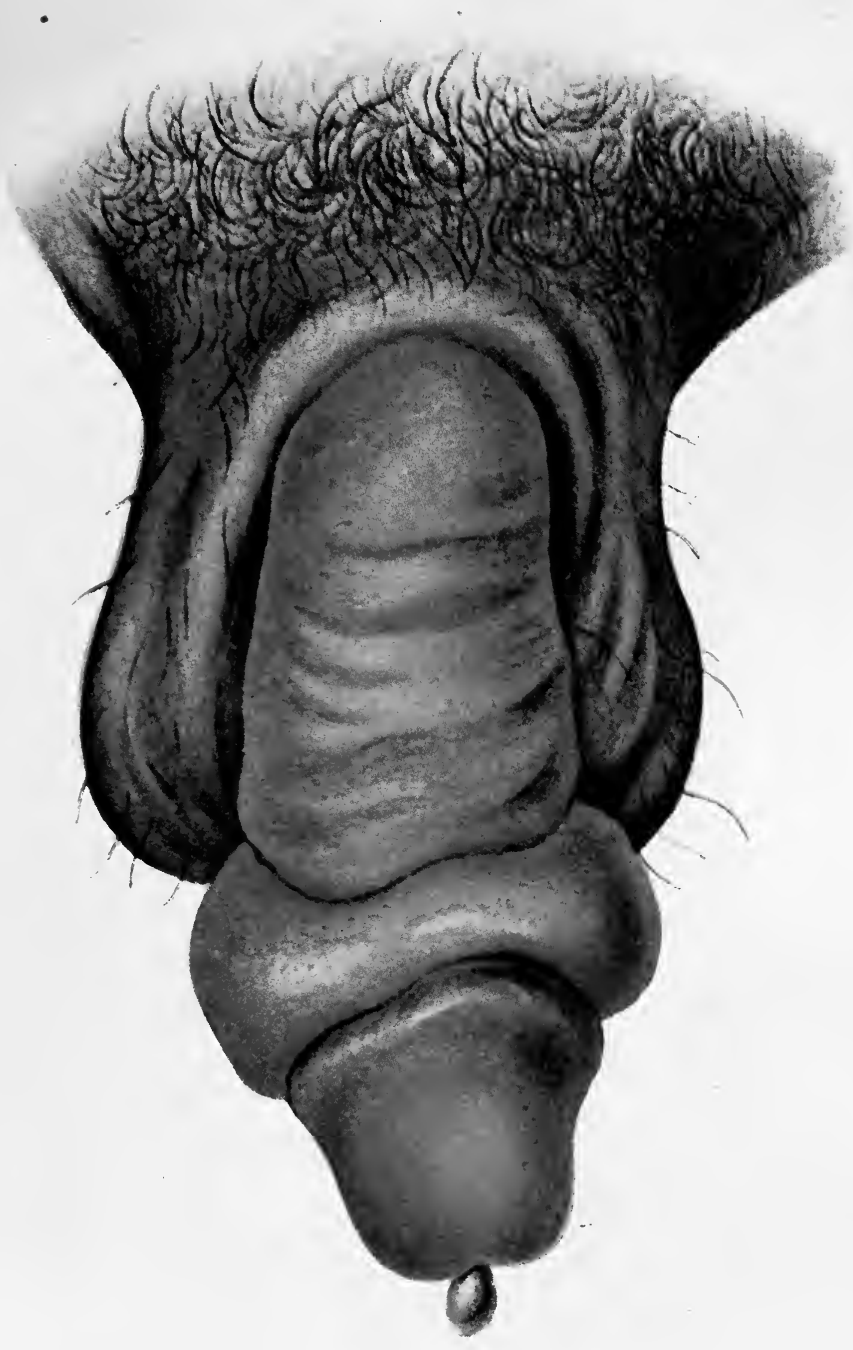

PARAPHIMOSIS. (Kaposi.)

Showing the constricting band and œdema of prepuce. 



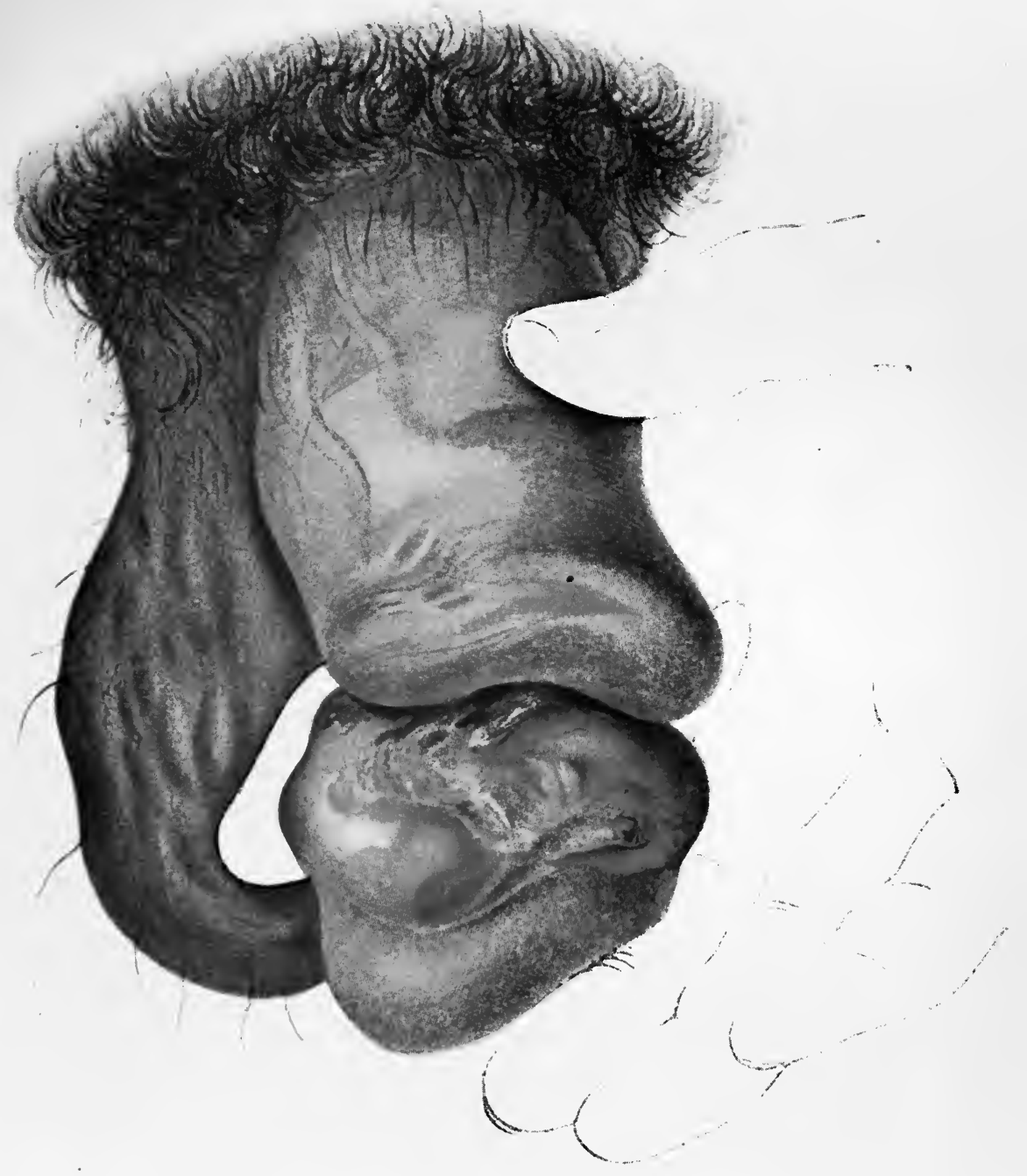

PARAPHIMOSIS WITH STRANGULATION. (Kaposi.) Showing linear gangrene and ulcerated areas. 

Palliative.-This consists in the application of cold, and compression with a well-applied bandage to the anterior part of the organ. If the swollen glans can be reduced by pressing it backward through the constricting ring made by the tight foreskin, and simultaneously drawing the latter forward, the condition will be relieved without any further measures being taken. The manœuver is accomplished by holding the organ with the two first fingers of each hand in the manner shown in Fig. 5, pressing the thumbs upon the head of the glans, and at the same time drawing forward upon the constricting prepuce with the fingers.

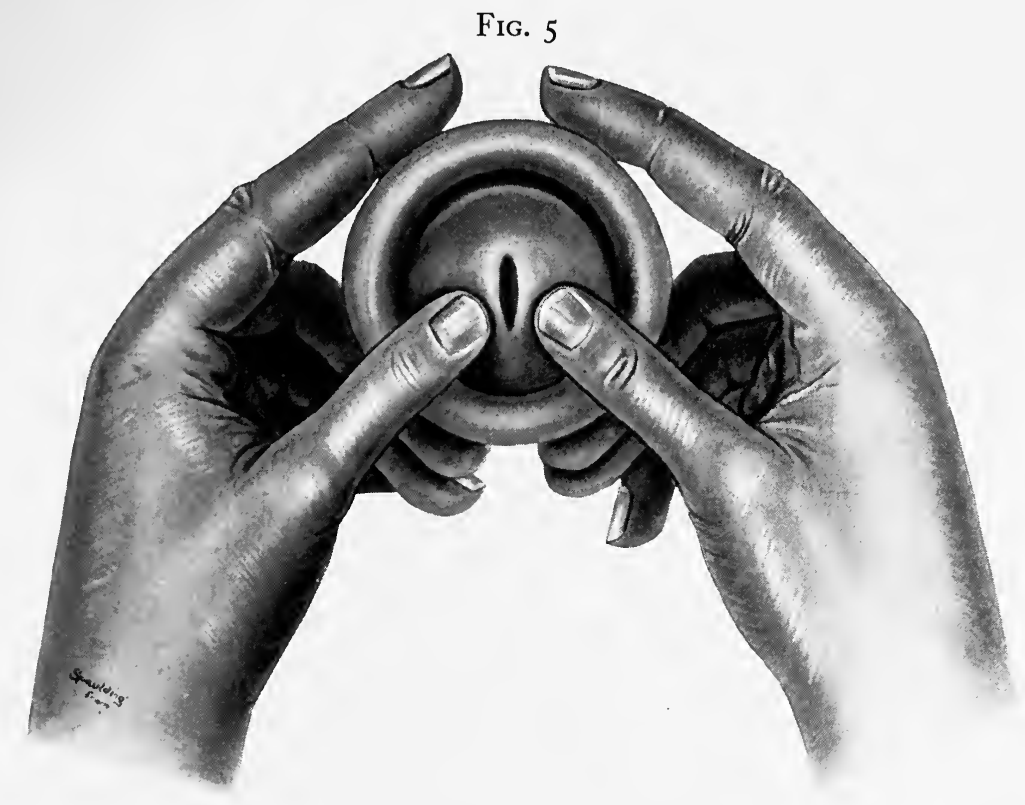

Showing method of reducing paraphimosis.

Surgical.-Relief is best obtained operatively by passing a tenotomy knife from before backward, through the swollen ring of the prepuce, beneath its constricting band, and by dividing the latter. The knife blade should be entered horizontally, then turning the cutting edge of it upward against the band, a small nick is made in it. Reduction can sometimes be effected by first reducing the œdematous swelling of the prepuce by making numerous punctures in it with a glover's needle and squeezing the fluid from the tissues.

Inflammations of the Penis.-The acute inflammations of the penis, apart from gonorrhœa, which is considered by itself, are secondary to other lesions. The loose cellular tissue of the penis, the abundant blood supply, and numerous lymphatics encourage rapid spreading of inflammatory products in this situation.

vol. I-3 
Cellulitis.-This form of inflammation does not differ in its characteristics from cellulitis elsewhere. It usually arises from chancroidal ulcers or from balanitis and accompanying posthitis, which so frequently results from phimosis. Superficial wounds which become infected, and periurethral inflammation from gonorrhœa, are less frequently the sources of it. (For description of acute diffuse phlegmon of the penis, see chapter on Stricture of the Urethra, under the heading of Extravasation of Urine.)

The extent of the inflammation varies considerably. It may spread from the penis over the scrotum and thighs, and even high on to the abdomen. The corpora may also become infected, thrombi may be formed in the vascular erectile tissue, and death may result from emboli being carried into the general circulation.

Treatment.-The patient should be kept in bed and the diet should be restricted to liquids. The local treatment consists chiefly in soaking the organ in hot, mild solutions, such as creoline, or corrosive sublimate, I to I0,000. The source of infection, if beneath the prepuce, should be directly exposed to the medication, and a dorsal or lateral incision of the prepuce may be necessary to render this possible. If the inflammation does not subside readily and promptly, superficial incisions should be made in the inflamed areas to drain the infected tissues. When phimosis, paraphimosis, or infiltration of urine occur, they require prompt surgical interference. (See chapter on Stricture of the Urethra, Extravasation of Urine.) The general infection accompanying the more severe forms of cellulitis requires constitutional treatment to eliminate the toxin from the circulation. The bowels should be kept open and stimulants freely but judiciously given. Liquid food should be given at short intervals in the acute stage, but later the diet should be generous.

Lymphangitis.-This is invariably associated with the inflammatory process, the products of which are absorbed by the capillary lymphatics of this region. It also originates from superficial wounds and virulent urethritis.

The large dorsal lymphatics appear as a reddened line. This condition may be differentiated from phlebitis of the dorsal vein by the fact that it can be traced to the enlarged inguinal nodes, instead of disappearing beneath the symphysis.

The process usually remains superficial and discreet, but when the infection is virulent, perilymphangitis may cause considerable swelling of the organ, phimosis or paraphimosis may develop, and suppuration may then occur in the inguinal nodes.

Treatment.-The treatment is directed to the lesion from which the lymphangitis originates; in addition, flaxseed poultices should be employed 
over the inguinal nodes to increase their circulation and enable them to cope with the inflammatory products which are being deposited in them.

Cavernositis.-Acute inflammation of the corpora cavernosa, or corpus spongiosum, may result primarily from the infection of wounds involving these bodies, or from infected lesions of the tissues adjacent to them.

Inflammation of the corpus spongiosum is comparatively common. It is inflammation of this body which produces the condition known as chordee. Except in the latter form, acute cavernositis is rare. It presents the usual symptoms of inflammation; œdema of the skin is associated with it, and prolonged priapism often occurs. Septic thrombosis and embolism may result.

Treatment.-The infected area should be freely incised, the products of inflammation removed by irrigations with mild antiseptic solutions, and the wound drained. If hemorrhage results, it may be necessary to pack the wound with gauze and apply compression, which should be evenly and firmly made over the whole penis as well as upon the wound itself.

Chronic Cavernositis or Fibrous Sclerosis of the Corpora.-This condition is extremely rare. It occurs secondarily from acute inflammation of the corpora, from injury, or as a complication of chronic urethritis and stricture. The corpora cavernosa are more often the site of this condition than is the corpus spongiosum. It has also been attributed to syphilitic proliferation of the connective tissue. The condition is first recognized by the patient as small, single, or as multiple, hard, discreet lumps in the sheath of the corpus cavernosum. As a rule, erection is defective. The penis curves in one or another direction, and the entrance of the blood into it is more or less impeded.

Treatment.-Antisyphilitic treatment should be employed if the induration is believed to be of specific origin. Some authors advise electricity, while others regard it as useless.

Gout.-This condition is occasionally reported in literature. Paget and also Duckworth ${ }^{16}$ record such cases. It is difficult to differentiate this condition from a long-forgotten injury.

Gangrene.-Gangrene of the penis is almost invariably limited to a part of the organ. The glans may be involved, as has been mentioned, in connection with paraphimoses, or chancroidal ulceration beneath a phimosis. More commonly than from any other cause, gangrene of either the deep or superficial tissues of the perineal part of the urethra, or of the skin of the body of the penis or scrotum, is due to extravasation of urine and diffuse phlegmon. Occasionally it may be produced by senile endarteritis of the dorsal artery of the penis, or thrombosis of the iliac vessels, and now and then it is met with in cases of diabetes. 
Treatment.-The treatment consists in free incisions through the involved parts, removal of as much of the gangrenous tissue as possible, and the application of antiseptic wet dressings of one kind or another afterward. Alcoholic stimulants and frequent feeding with small quantities of easily digested and nourishing food are of much importance.

\section{CUTANEOUS AND MUCOUS MEMBRANE AFFECTIONS OF THE PENIS.}

The integument of the penis is the site of such diseases as acne, eczema, lupus erythematosus, psoriasis, scabies, and herpes zoster. They do not exhibit, in this situation, characteristics which differ from those which they have elsewhere on the body, and they belong properly to the sphere of dermatology, hence they are not included in this work.

Papillomata (Synonyms: verruce, condylomata acuminata, venereal warts).-The so-called venereal warts are not necessarily of venereal origin; they are termed venereal because of their having been confused with venereal conditions and on account of their location.

Etiology.-Venereal warts are usually seen in connection with conditions which produce local irritations, such as gonorrhœea, chancroids, syphilis, leucorrhœa, and balanitis. They are especially frequent in persons of uncleanly habits, although they are occasionally seen in those who are scrupulously clean.

Pathology.-The warts are papillary outgrowths originating in the papillæ and capillaries of the epidermis, and with stems composed largely of connective tissue. They occur especially at the junction of the skin and mucous membrane.

Papillomata may be divided into two classes: (I) Those which are soft and originate on the mucous membrane of the glans, or at the mucocutaneous junction. (2) Hard and firm warty growths situated on the skin at some distance from the mucous membrane.

I. Soft Warts.- These occur most often between the age of puberty and adult life, and are equally common in both sexes. Moisture, heat, and uncleanliness are the common predisposing factors. They are most frequent with persons who have long foreskins which retain the secretions and urine.

They begin as small reddened papules, and become eroded as they develop. Before the papillæ become well-defined, the condition somewhat resembles chancre, and may be mistaken for it. The progress is rapid, and fresh papillæ develop about the original growth. As they advance they become more or less pedunculated. Sometimes the pedicles 
are slender, sometimes the growth is sessile. When massed together they have the appearance shown in Fig. 6.

They occasionally attain an enormous size, presenting an irregular gray or dirty gray surface, which often becomes infected and ulcerates, giving rise to a copious discharge of pus. This is especially true if the preputial orifice is small and phimosis has resulted as a consequence. Paraphimosis may also be produced because of the growth preventing the foreskin from being drawn forward.

FIG. 6

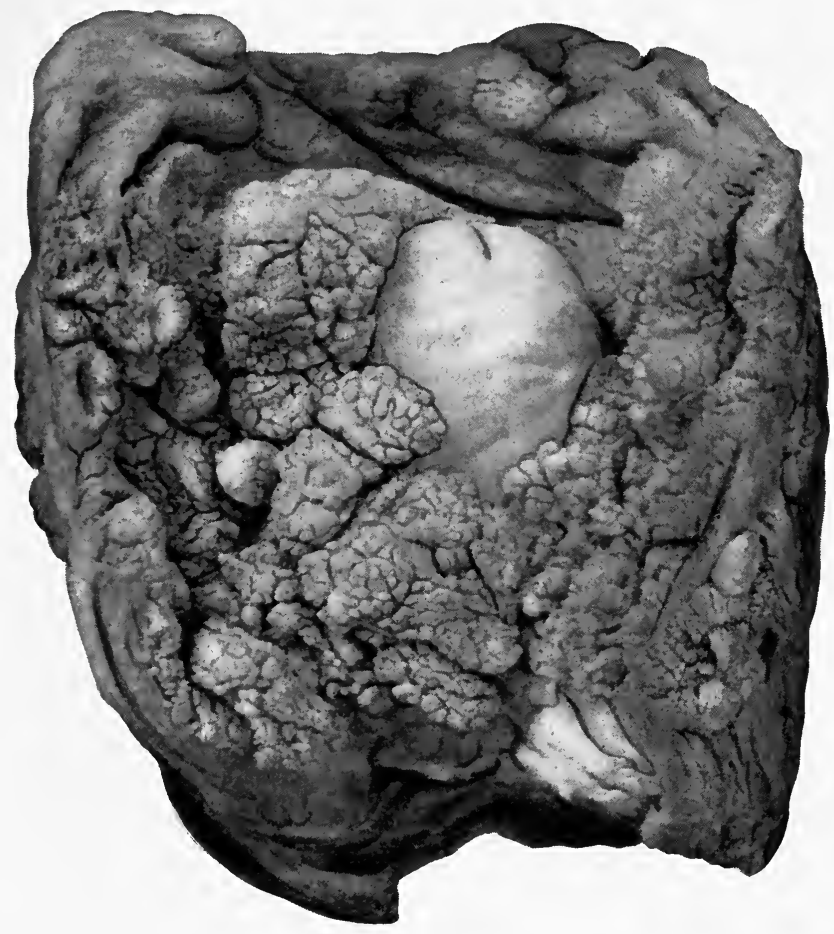

Large, soft, exuberant warts situated on the glans, inner layer of the prepuce and sulcus. (Warren Museum.)

In the female the vegetations are usually situated in the vestibule of the vagina near the meatus of the urethra, and often become ulcerated. When they are situated in the urethral orifice, or in its canal, frequent and painful micturition is a prominent symptom and the urethra may be more or less obstructed.

2. Hard Warts. - This form of growth occurs upon the skin, and although occasionally existing alone, is more frequently associated with soft vegetations which begin on the mucous membrane. They are like the soft vegetations, but their progress is slower; they are also more sessile and rounded in contour. 
Morphologically, they are the same as the soft growths, except that they are less vascular, the outer covering is harder, and there is a larger proportion of connective tissue in them. They are often found on the scrotum and perineum, and especially about the anus.

With persons of uncleanly habits, the growth shows greater activity and often ulcerates; if the parts are kept dry, it frequently remains single, and is slow in its progress.

Treatment.-The same treatment is appropriate to both forms of the disease. It consists in destroying the growths either by means of repeated applications of caustics, by galvanocautery, or by excision and burning the bases.

Treatment by Topical Applications.-This should be carried out as follows: cleanse the growths by washing them with a solution of I to 4000 of bichloride of mercury, then apply strong nitric acid or full strength carbolic acid by touching the warts with a swab of absorbent cotton merely moistened with these fluids. The contact should be continued for about a minute, then a solution of sodium hydrate should be applied in order to prevent a too extended action of the acid. The parts are then dried and dusted with either calomel, aristol, or bismuth powder. The procedure must be repeated every four days or so until the warts have disappeared. The parts should be kept dry and clean subsequently, as there is a marked tendency to recurrence.

Operative Treatment.-Ligation of the base or bases of the growths, cutting them off outside the ligatures, and cauterizing the base later if necessary, to destroy the growth completely, is the most thorough and satisfactory manner of treating the condition, in our experience.

If bleeding of consequence takes place in connection with this method of treatment, it can be readily arrested by applying a solution of adrenalin, I to I000, or by pressure.

Horny Growths.- Horny growths of the penis are rare. They appear in two forms: as horny plaques and as horny outgrowths. The glans is their most frequent seat. Plate VII, for which we are indebted to our colleague, Dr. J. Bapst Blake, illustrates this condition.

In Pick's case the horn was two and a half inches in length. Gould ${ }^{17}$ mentions the case of a man, aged fifty-two years, who was circumcised at forty-eight years of age, and upon the margin of the resulting cicatrix a horn subsequently developed. The case of another patient with whom the condition was apparently congenital is reported..$^{18}$

Treatment.-This consists in excision of the growth and of as much of the part of the penis on which it is located as may be found necessary.

Elephantiasis.-This disease occurs in epidemic form in tropical countries. It is found occasionally in sporadic form in the temperate 


\section{PLATE VII}

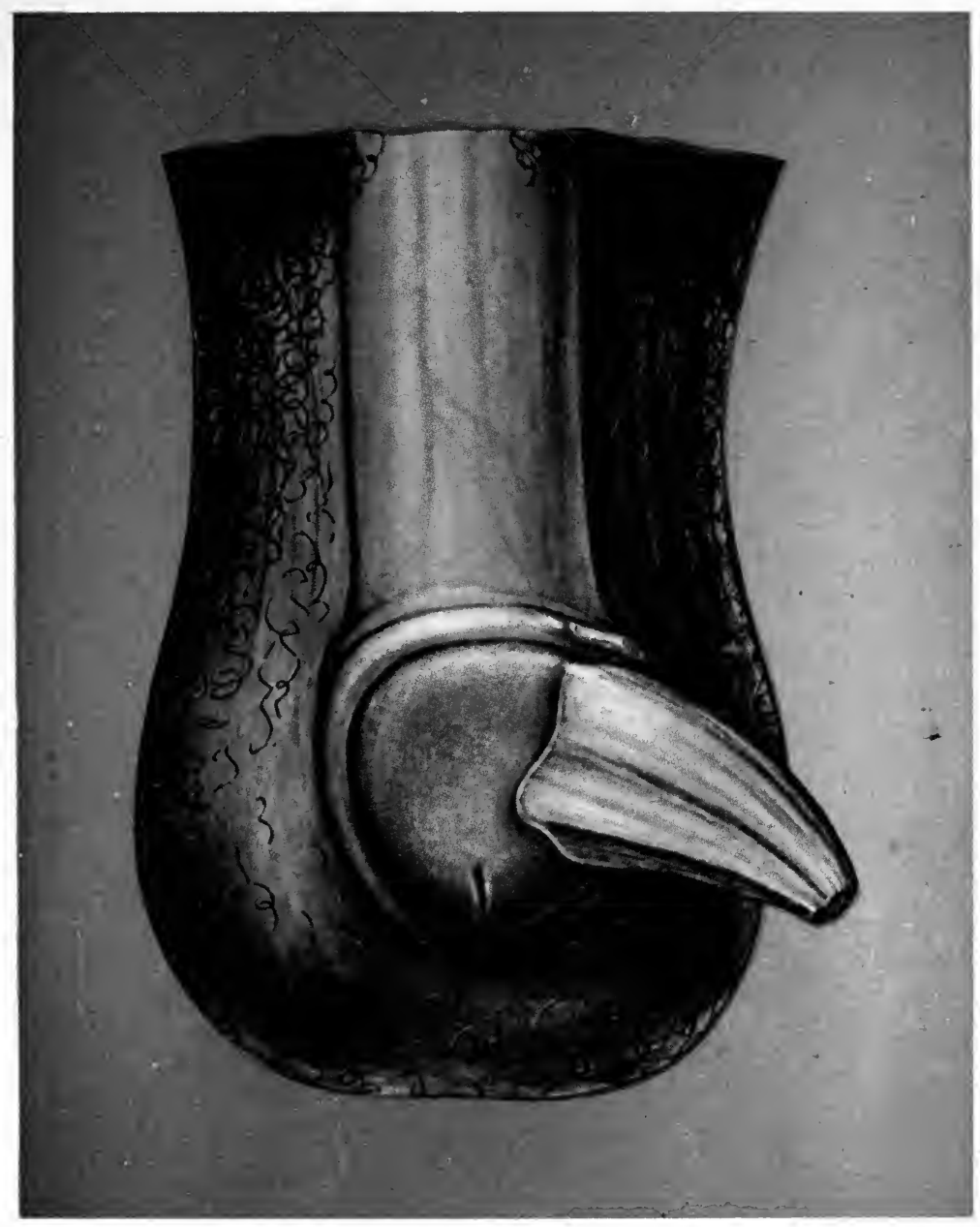

HORNY GROWTH OF THE PENIS. (By courtesy of L). J. Bapst Blake.) 

and northern zones. It is sometimes confined to the penis alone, but is oftener primary in the scrotum and subsequently involves the penis. Its etiology is not entirely clear, but it probably is due, in the majority of cases, to the fllaria sanguinis hominis. (The pathology and further details of this disease will be found in the chapter on Diseases of the Scrotum.) When affected, the penis is often enormously enlarged, and may reach to the knees.

The disease ordinarily begins in the form of hard bodies situated beneath the skin. These enlarge, and as they progress the skin becomes much thickened, furrowed, indurated, and discolored, and somewhat resembles that of the elephant (Fig. 7). The prepuce is elongated, and the meatus is situated at some distance behind the preputial orifice.

Treatment.-Amputation is, as a rule, the best treatment to apply, for if the disease is due to filariasis the parent worms will probably be contained in the mass removed. It is established that when the greater part of the diseased tissue has been removed, even if that which remains still contains some of the elements of the disease, further development of it does not follow.

Tuberculosis.-Primary tuberculosis of the meatus, urethra, or glans probably does not occur. When the disease appears at

FIG. 7

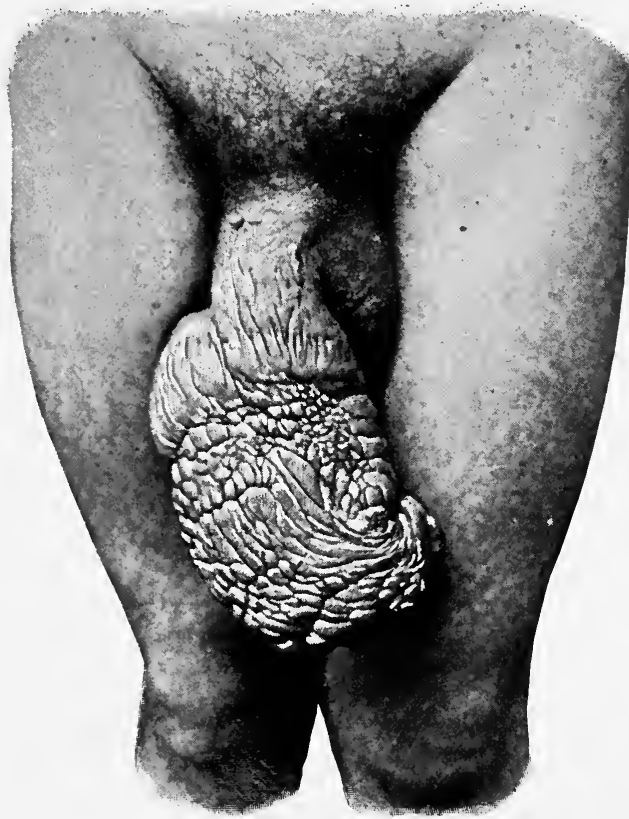

Elephantiasis of the penis. (Taylor.) these points it is secondary to other foci higher up in the urinary tract. A few cases have been reported in which the lesions have been situated at the points mentioned. Malecot ${ }^{19}$ records one in which the meatus was surrounded by a tuberculous lesion the size of a dime. Senn ${ }^{20}$ also describes two cases. It may be difficult to differentiate the lesion from those of syphilis and of cancer.

Treatment.-Radical treatment is not likely to be of any important benefit, because of the existence of the disease in other parts of the tract. Surgical interference might be demanded because of the obstruction to urination that might be caused if the disease occupied the meatus and produced contraction of it. 
Balanitis and Balanoposthitis.-These terms are applied to inflammation of the mucous membrane of the prepuce and of the glans penis. Inasmuch as they rarely exist independently of each other, they are usually considered together under the term balanoposthitis.

Etiology.-The condition is produced by the irritation due to the presence beneath the prepuce of retained smegma and urine, or of diabetic urine, and in certain individuals, more especially such as are of gouty diathesis, even if no retention of these products occurs, and in whom the etiological factors are of uncertain nature.

A special form, in which there is produced a diphtheritic membrane, occasionally is seen in connection with the acute exanthemata. Gonorrhœa is often productive of the condition.

Symptoms.-Subjectively there is more or less burning and itching of the glans and a purulent discharge from beneath the prepuce. Objectively the appearances are redness and erosion of the mucous surfaces in irregular areas. The ulcerations are very superficial and have sharply defined but irregular outlines. They are covered by a yellowish discharge which is of a purulent nature, and which varies in amount. They often become confluent. When acute, there may be a good deal of pain with erections, and erections are frequent.

Chronic Balanitis.- In the chronic form the prepuce becomes thickened, and its orifice is often constricted by the deposit of connective tissue and its contraction. Adhesions are apt to form between the surfaces of the prepuce and the glans.

Diagnosis.-Balanitis, as a rule, is easily recognized. When the prepuce cannot be retracted, the discharge may be mistaken for that of gonorrhœa, and vice versa. If the meatus can be brought into view, the source of the purulent discharge can be determined by wiping it away and then seeing whether or not more pus can be made to flow from the meatus by pressure upon the urethra from behind forward, or if the pus issues from beneath the prepuce only.

The pus of chancroidal ulcers which are concealed beneath the prepuce can be traced to its true source by the presence of the somewhat indurated area which the chancroid produces and by the œdema and more severe local manifestations associated with that condition than appear in connection with balanitis.

Syphilitic chancre is recognizable by the induration of the lesion, and in typical cases, by its discreet character and by the involvement of the lymphatic glands of the groin, which takes place in the form of round, hard, nodular bodies, of shot-like character. When chancre is secondarily infected by an acute process, it may be more difficult to determine the nature of the glandular involvement in the groin, and reliance must then 
be placed upon the characteristic features presented by the syphilitic lesion upon the penis and elsewhere upon the body.

Balanitis closely resembles herpes when the latter is ulcerated and its vesicles have been destroyed.

Treatment.-When the foreskin can be retracted, the parts should be gently washed with soap and water and the glans and prepuce soaked in a solution of corrosive sublimate of the strength of $\mathrm{I}$ to 6000 . When the secretion becomes less, mild astringent washes, such as liquor plumbi acetatis, or acetate of alum, 2.0 per cent., should be applied. These may be advantageously alternated, day and night, with such powders as aristol, stearate of zinc, or calomel dusted lightly upon the parts. If necessary, a lint dressing, moistened with any of the solutions mentioned, may be worn, changing it for a new one each night and morning.

When phimosis is present and the surface cannot be exposed for direct medication, the preputial pouch should be thoroughly cleansed several times daily by irrigation with peroxide of hydrogen, one-half strength, followed by a. I to 6000 solution of corrosive sublimate.

If the prepuce is very much swollen and irrigation of the preputial sac is difficult and painful, the phimosis should be relieved by a dorsal incision, and when the inflammation has subsided, circumcision should be advised, except in the cases of diabetics, as it is the only procedure which will prevent recurrence. As stated elsewhere, diabetics do not bear the operation well, and death has occurred from operation.

Complications.-Phimosis, paraphimosis, lymphangitis, and inguinal adenitis often complicate the condition. The moisture of the parts predisposes to the formation of venereal warts.

Herpes Progenitalis.-This condition is the development, upon the mucous membrane or skin of the genitals, of single or multiple small vesicles containing a watery fluid.

It occurs more frequently in males, and is usually situated on the prepuce; less frequently on the glans and integument of the penis. Phimosis is a predisposing factor. In the female the vesicles occur on the inner aspect of the labia majora, about the labia minora, or other parts of the mucous surface, but are seldom observed on the surrounding integument.

The condition is a harmless one, but it is of importance because of its frequent occurrence, the secondary changes which may take place in the vesicles, which often make it difficult to differentiate it from a beginning syphilitic chancre or chancroid, and the fear that it may excite in the patient's mind lest it be of syphilitic nature.

Etiology.-Herpes is usually the result of one or another of various kinds of irritants applied to the glans or prepuce, such as friction against the garments, masturbation, and the contact with leucorrhœal discharges. 
The condition is also seen to occur in some persons of highly nervous temperament who exhibit a peculiar tendency to recurring attacks of herpes. Unna regards it as a form of herpes zoster, and calls attention to the correspondence of the lesions with the peripheral nerve terminations, especially with the distribution of the ramus dorsalis penis nerve, a branch of the pudic nerve.

In nervous women there is often a crop of herpes just before menstruation, and they have been frequently observed to occur in connection with pregnancy. Herpes occurs most frequently in early and in late adult life, and affects males rather more often than females.

Lydston $^{21}$ states that in many cases of herpes progenitalis it seems to be directly dependent upon a syphilitic cachexia, and that the condition is not amenable to local treatment, other than with mercurials. This author attributes the herpes in such cases to local irritation incidental to a chancre or a mixed sore, or to disturbed innervation and consequent trophic changes incidental to the effects of syphilitic infection, excessive medication, especially with mercury, or to nervous influences affecting the sympathetic system.

Symptoms. - The first evidence of the disease is a small reddened spot upon which a vesicle develops in the course of a few hours. It varies in size from a pin's point to a pin's head; occasionally it is slightly larger. It may be single, but is more often multiple. The vesicles, when multiple, are usually arranged in a circular or crescentic form. Individually, they are rounded, translucent, and generally contain clear serum, sometimes a seropurulent fluid. (Plate VIII.)

The vesicle soon ruptures and a somewhat elevated, pinkish spot, with a central depression, takes its place. When the vesicles become infected, ulceration occurs, and the lesion may then resemble chancroid. The subjective symptoms are not marked, and consist of a slight burning and itching of the glans soon after the vesicle begins to develop. When it has ruptured and the urine comes in contact with the open surface, there is more or less pain.

In the simple cases herpes disappears in a few days, if cleanliness is observed. In neglected cases, however, the ulceration, which is sometimes chancroidal in appearance, may occur, and inflammatory reaction and suppuration occasionally involve the inguinal lymph nodes under these circumstances.

Diagnosis.-A simple case of herpes progenitalis is easily recognized when the characteristics are such as have already been described, but when ulceration takes place from secondary infection with pyogenic organisms or syphilitic virus, the diagnosis is obscured. The differential diagnosis will be given in tabular form later. 


\section{PLATE VIII}

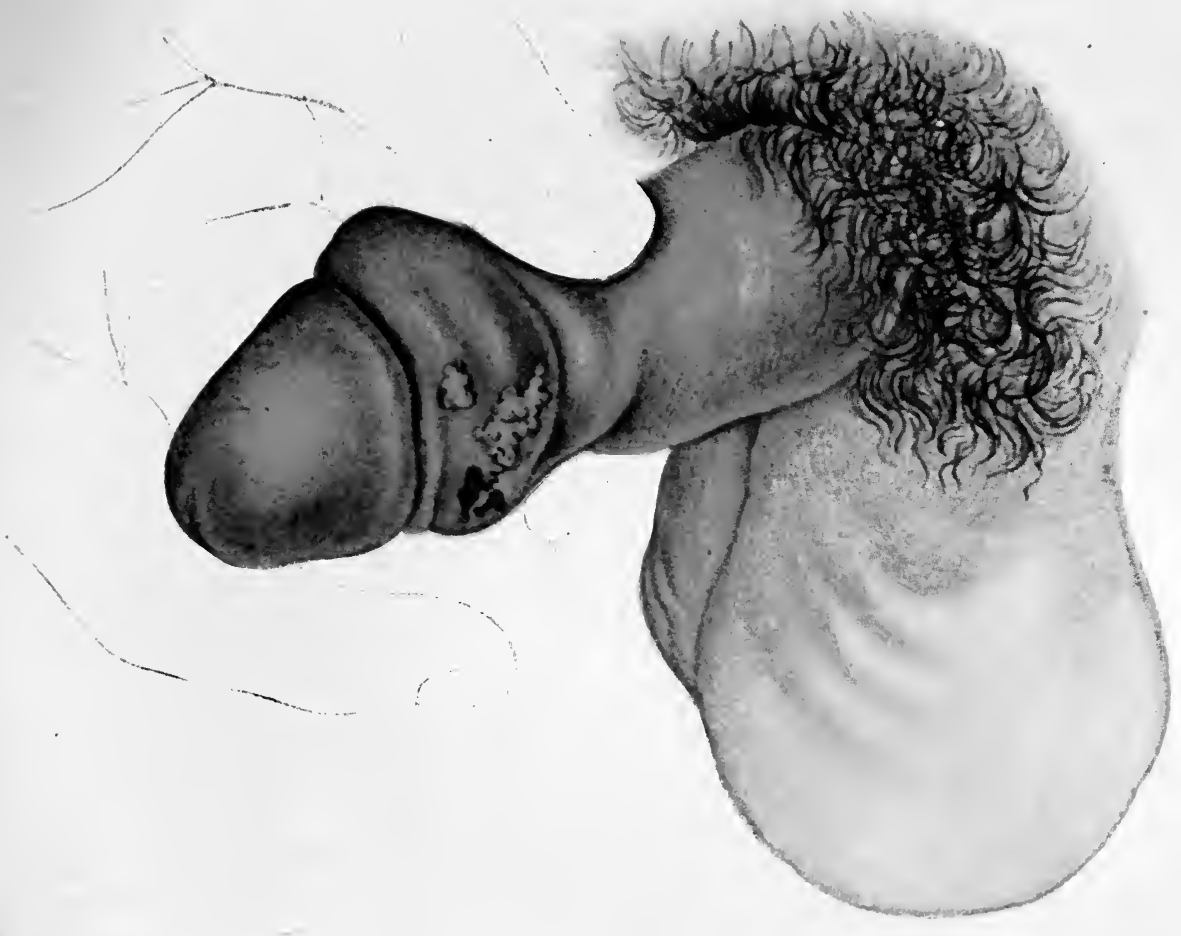

HERPES PROGENITALIS. (Kaposi.) 

Treatment.-General measures should be applied to cure the causal or associated conditions.

Locally.-Non-infected herpes responds readily to treatment, but the tendency to recurrence is marked, and treatment should be promptly resumed if it occurs. It consists in applying mild astringent washes and keeping the parts perfectly dry with powder. A I per cent. solution of zinc sulphate, or acetate of lead, or alum, may be applied to the lesions by placing beneath the foreskin a piece of cotton soaked in one of these solutions. These medicaments may be advantageously alternated with stearate of zinc, aristol, or bismuth powder. If there is a long or tightly fitting prepuce, circumcision should be advised, the performance of which may prevent any further recurrence of the disease.

Chancroid.-The term chancroid is applied to an ulcer, usually occurring on the penis, and especially upon the prepuce and glans. It is a local disease, secreting a virulent, auto-inoculable pus, and is usually due to a specific infection by a streptobacillus described by Ducrey and Unna. Various staphylococci, streptococci, and bacilli have been found in the secretions of the ulcerations, and have led to the conclusion that the auto-inoculable destructive chancroidal ulcerations occurring on the penis are not essentially different, bacteriologically, from others, such as impetigo, and certain auto-inoculable lesions which occur elsewhere upon the integument.

Ducrey, in the year I 889 , described a special rod-like bacillus, with rounded ends, occurring in the secretions of the ulcerations, and also in the pus of buboes obtained under aseptic precautions.

Etiology.-Chancroidal infection may take place directly, from contact with a chancroidal lesion, as in coitus, or indirectly, by being carried by some intermediary object which serves as the transferring agent. Taylor states that indirect infection of chancroid is less common than of the syphilitic virus. Both lesions are quite frequently associated together.

Location of Chancroidal Ulcerations.-Chancroids occur commonly on the genitals of both sexes, but are occasionally observed on the face and fingers, usually from auto-inoculation. In males, by far the greater number of chancroids occur on the glans and prepuce, and when seen on the scrotum, pubes, anus, and thighs, usually arise from auto-inoculation.

The various sites at which they occur in males, and also their relative frequency, is given in Fournier's table (see next page). ${ }^{22}$ 


\section{Fournier's Table (Men).}

Chancroids of the glans or prepuce

Chancroids on the sheath of the penis

Chancroids on various parts of the penis, as, for instance, occupying the prepuce and sheath, the sheath and the glans, etc. . . . . . . . 24

Chancroids on the penis (exact situation not recorded) . . . . . . 25

Chancroids on the meatus . . . . . . . . . . . . . . . II

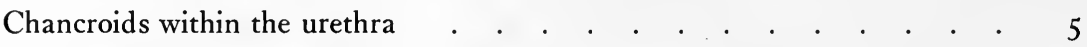

Chancroids of the scrotum . . . . . . . . . . . . . . . 3

Chancroids on the pubes . . . . . . . . . . . . . . . . . . 3

Chancroids on the fingers . . . . . . . . . . . . . . . . 2

Chancroids on the upper and inner portions of the thighs . . . . . . . 2

Chancroids of the anus . . . . . . . . . . . . . . . . . . I

Chancroids of the anterior thoracic region . . . . . . . . . . . $\quad$ I

Total . . . . . . . . . . . . . . . . . . . 445

In females, the common site is also on the mucous surfaces of the genitals, and the lesions existing on the surrounding parts are usually due to auto-inoculation. These lesions in the female are often much inflamed and very painful from contact with irritating urine and the vaginal secretions.

Development of the Chancroid.-There is no definite period of incubation. If the contagion is inoculated upon a fissure or an abrasion, the ulcer begins to develop at once, while if no such opening exists, and the virus is deposited upon sound mucous membrane or skin, infection can take place only by the destruction of the surface by the virus, in which case the appearance of the chancroid will be delayed, or the inoculation may fail to take place at all. Fournier tabulated 52 cases in which the patients had had but a single connection for a long time (several months).

The patients stated the time between coitus and the date when they first noticed the lesions to have been as follows:

The first day after exposure

Cases.

The second day after exposure . . . . . . . . . . . . . . . . 2

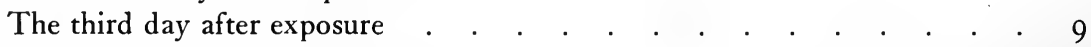

From the third to the fourth day . . . . . . . . . . . . . 4

The fourth day . . . . . . . . . . . . . . . . . . 3

The fifth day . . . . . . . . . . . . . . . . . . . . . .

The sixth day . . . . . . . . . . . . . . . . . . . . . 3

From the seventh to the eighth day . . . . . . . . . . . . I3

The ninth day.

The tenth day

The eleventh day .

The thirteenth day

From the thirteenth to the fourteenth day

From the seventeenth to the twentieth day 
Appearances of Chancroid.-The initial pustule of chancroid is not often observed, the patients usually coming under observation only after ulceration has taken place. The chancroid begins as a small, yellow pustule, surrounded by a red areola, which is less pronounced at its edges. The pustule soon ruptures, and leaves a small round or oval ulcer, which grows rapidly at its periphery. The tendency to destructive tissue changes is most noticeable along existing furrows, as, for example, in the sulcus behind the glans penis. The edges of the ulceration are clean cut, but undermined, rough, and irregular. The floor of the ulcer is eroded and uneven, and is covered with a grayish-yellow membrane of disorganized tissue. There is a varying amount of inflammatory œdema about the ulcer, often sufficient to produce phimosis or paraphimosis.

The discharge from the chancroidal ulcer is abundant, purulent, and often brownish in color from the presence of blood. The pus is autoinoculable, and other similar ulcers appear upon surfaces lying over or in contact with that of the original one.

The duration and progress of chancroidal ulcerations depend much upon the time at which treatment is begun. If the patient comes under observation early, the development may be quickly arrested, while if untreated, the ulcer may last for months, often involving the whole mucous membrane of the preputial fossa, which is covered with a grayish-white membrane and bathed in a purulent discharge. Lymphangitis and cellulitis may result, and the inguinal nodes usually become enlarged and often suppurate.

When the ulcer begins to repair, the inflammatory areola surrounding it disappears; the œdema, if present, subsides, and the tissues about the ulcer assume a healthy appearance. The discharge becomes more serous, and fresh granulation tissue replaces the grayish-yellow membrane upon the floor of the ulcer; diminution in the size of the ulcer begins only when the surface and edges are entirely covered by it.

Some of the more important varieties of chancroid are illustrated in Plate IX, I to 6 inclusive.

To these and still other varieties, descriptive names, such as follicular, serpiginous, phagedenic, etc., are given. They are employed merely to denote one or another special feature or quality of the sores, and their meaning is sufficiently indicated without a word description, by which, moreover, their actual appearance cannot be adequately conveyed.

Diagnosis.-The diagnosis of chancroidal ulceration is not always easy. Under.ordinary circumstances, it is generally and correctly recognized, but a differential diagnosis between it and infected herpes progenitalis and some forms of syphilitic chancre may be difficult or impossible. It 
may also be mistaken for severe balanitis, mucous patches, late syphilitic lesions, and epithelioma.

The property of auto-inoculation, which was formerly believed to be an essential characteristic of chancroidal ulcers, is no longer regarded as wholly reliable.

Differential Diagnosis between Chancre, Chancroid, and Herpes.-The appended table, showing the features of the above-named conditions, is taken from White and Martin $:^{23}$

\section{Chancre.}

Origin.-Due to inoculation with the blood or lesion discharges of a syphilitic.

Incubation.-From ten days to eight weeks. Average about three weeks.

Situation.-Generally on the genitalia. Often on lips, nipples, and hands.

Number.-Single; at times simultaneously multiple.

Beginning.-Begins as an erosion, papule, tubercle, or ulcer. May remain without ulceration through its entire course.

Shape.-Round, oval, or symmetrically irregular.

Depth.-Usually superficial, cup-shaped or saucer-shaped, or may be elevated.

\section{Chancroid.}

Due to inoculation with the discharge of a chancroidal sore. Possibly caused by pus from other sources.

No definite period. It may not be noticed for two or three days.

Generally on the glans penis and the prepuce. Rarely on other genital surfaces. Hardly ever on other parts of the body.

Frequently multiple, of ten on apposing surfaces by auto-inoculation.

Begins as a pustule or an ulcer. Always ulcerates.

Round, oval, or unsymmetrically irregular, with border described by segments of large circles.

Hollow, excavated, or "punched out."

\section{Herpes.}

Due to-

(I) Mechanical irritation, as in sexual intercourse.

(2) Chemical irritation, such as is produced by acrid discharges or by uncleanliness.

(3) To neuroses; often following fever, and particularly occurring in syphilitics.

None.

Generally on the glans penis and the inner layer of the prepuce.

Multiple. Ultimately often confluent.

Begins as a group of vesicles, which may coalesce or may ulcerate singly.

Irregular, circinate borders, representing segments of small circles; sometimes serrated.

Superficial. 
PLATE IX

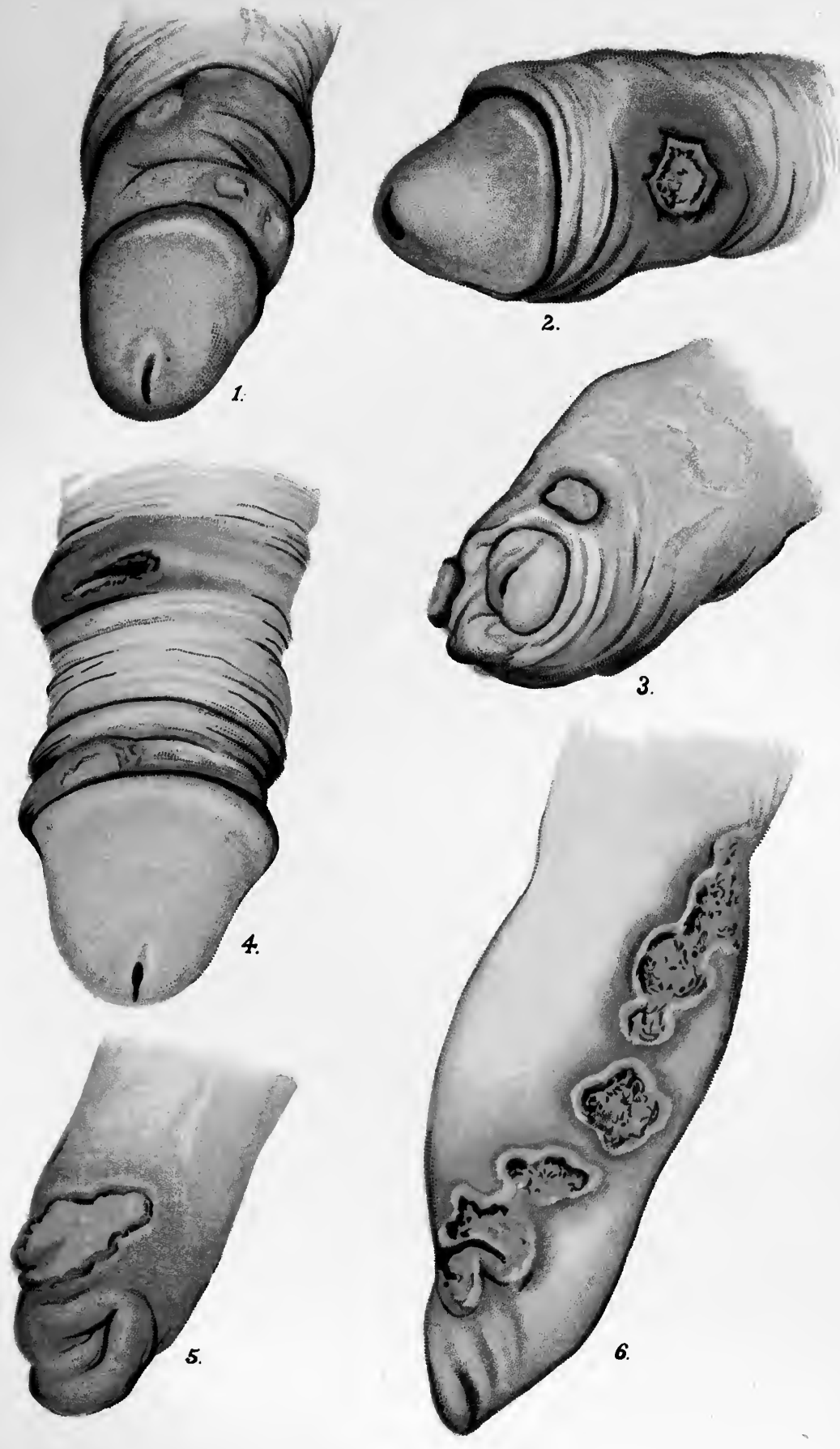

CHANCROIDS. (Taylor.) 


\section{Chancre.}

Surface.-Smooth, shining, dusky red, glazed; diphtheritic membrane, or scab, or epithelial crusts.

Secretion.-Scanty, serous, hardly ever auto-inoculable, except in cases of mixed infection, when a chancroidal sore may be produced. On squeezing cannot press out a discharge.

Induration.-Almost always present; firm, cartilaginous, or parchmentlike; sharply circumscribed; movable upon subjacent parts. Prolonged pressure by the examining fingers does not produce any change in it; usually persistent; disappears under specific treatment.

Sensibility. - Very rarely painful.

Course.-Progressively toward cure, the sore often healing spontaneously. Relapses and phagedena uncommon.
Chancroid.

Rough, uneven, "wormeaten," warty, grayish, pultaceous slough.

Abundant, purulent, readily auto-inoculable.

Only exceptionally present. Due to caustics or other irritants, or to simple inflammation; boggy, inelastic, shades off into surrounding parts, to which it is adherent; disappears soon after cicatrization. Prolonged pressure causes changes in shape, such as are noted in œdema.

Often painful.

Irregular; may cicatrize rapidly or may extend. Relapses and phagedena not uncommon.

\section{Herpes.}

Bright-red superficial granulations, sometimes covered by diphtheritic membrane.

Moderate secretion, autoinoculable with difficulty. On squeezing, a small serous drop exudes. When this is wiped away, another drop can be pressed out. This can be repeated several times.

Same as local ulcer.

\section{Often painful.}

Easily and quickly cured. Sometimes spreads by the appearance of successive crops of vesicles. Lesions preserve the polycyclic form. Likely to recur, especially in syphilitics and in uncleanly patients with long foreskins.

\section{Subpreputial Chancre.}

Discharge.-Moderate, thin, at times blood-stained. Not readily auto-inoculable.

Preputial Orifice.-Not markedly ulcerated.

Buboes.-Non-inflammatory, bilateral, inguinal buboes always develop.

\section{Non-syphilitic Subpreputial}

\section{Ulceration.}

Discharge.-Often produces auto-inoculation by accidental contact.

Preputial Orifice.-Almost invariably ulcerated.

Buboes.-Single, inflammatory, suppurating buboes often develop. 
Prophylaxis.-The use of a condom protects the part which it covers from contagion, but the adjacent parts of the genitals and the remaining portion of the body are still exposed. Ricord's expression upon this point illustrates this very well: "Carrying an umbrella over the head in a rainstorm will not prevent the feet from getting wet."

Cleansing the genitals after suspicious intercourse, taking special care not to abrade the surfaces, often averts infection. Taylor ${ }^{24}$ summarizes the essentials of prophylaxis with characteristic terseness thus: "The most efficient prophylactic measure is thorough cleansing of every fold and recess of the genitals."

Treatment.-With regard to the negative side, he says: "In the treatment of chancroid it is important to know what not to do-namely, not to give mercury and treat the case as one of syphilis; not to cauterize injudiciously and indiscriminately; not to use ointments and fatty preparations; and never to resort to excision. Nothing but harm can follow any of these procedures."

Taylor's instructions (op. cit.) with regard to general measures are to keep the patient absolutely at rest, to avoid friction and compression of the penis. "Alcoholics should be uncompromisingly interdicted, and plain, digestible food taken.

"The most rigid attention to cleanliness and to keeping the parts dry is necessary during the course of chancroids."

With regard to destructive cauterization, he says that it should be used only in the early stages of the disease. He counsels the employment of strong nitric acid and liquefied carbolic acid, applied with an absorbent cotton swab on the end of a cotton stick, the cotton being but slightly moistened with the liquids, and the parts to be touched having been cleansed with a solution of peroxide of hydrogen or a hot solution of boroformalin ( $\mathrm{I}$ per cent. to 3 per cent. in water). Care is to be taken not to let the fluid touch any of the surrounding parts. The undermined edges should be thoroughly touched with the acid. The treatment should never be applied to any chancroid which cannot be subsequently exposed.

The subsequent treatment should consist in frequent irrigations and applications, often renewed, of iodoform. Its objectionable odor may be disguised by cumarin, the active principle of tonka beans. Aristol, bismuth, and similar mild remedies may be advantageously employed later. In the phagedenic and necrotic forms of the ulcer, wet, chlorinated soda, bichloride of mercury, or creolin dressings are sometimes preferable to the treatment just outlined.

Various forms of chancroid respond differently to treatment. If the ulcer has eaten deeply into the tissues, as in the phagedenic form, or if it has extended over a wide area, as in the serpiginous variety, the cure is 
slow, and it may be months before healing takes place. General treatment is an important element in securing healing in the cases of debilitated patients and of extensive ulcerations.

Complications of Chancroids.-Lymphangitis appears on the dorsum, or side of the penis, in the form of painful, red, hot, and tender lines. The inflamed lymphatics are situated on the same side as the ulcer. Occasionally the lymphatics ulcerate and a new chancroid is thus formed.

Chancroidal Bubo.-Inflammation of the inguinal glands, on one or both sides-usually the side upon which the chancroid is located-is brought about by the passage of the septic products from the ulceration through the lymphatics of the penis. Suppuration may or may not result, and is determined by the degree of virulence of the septic products absorbed, and by the degree of resistance of the tissúes attacked. Adenitis is often severe. (Plate X.) Kaposi figures in his classic work a suppurating bubo which destroyed the integument from the middle of Scarpa's triangle to the free border of the ribs. Taylor ${ }^{25}$ describes four distinct varieties, or gradations of inflammatory bubo, as follows:

"I. Simple hyperplasia of one or more ganglia-monoganglionic and polyganglionic adenitis-which may be acute or chronic. These lesions are due to a mild irritative process which shows itself by enlargement of the inguinal ganglia and swelling of the parts, which may be of normal color or of a more or less deep red. Pain may or may not be present. In these cases spontaneous resolution may or may not occur. Not uncommonly the ganglia are left in a permanently phlegmasic or hyperplastic condition. In some instances these swellings run a chronic course, and later on break down and suppurate, in which conditions they were once called strumous buboes.

"2. Suppuration of one or many ganglia and of the ambient connective tissue, while some ganglia still remain in a hyperplastic condition. A red and painful swelling is found in the groin, and digital examination shows a combination of fluctuation, doughy sensation, and nodulation. This mixed form of bubo may be as large as an egg, or even larger. In this form of bubo the morbid process just falls short of that which occurs in the suppurating bubo.

"3. Suppuration of the whole mass of ganglia and the formation of an abscess cavity. The true suppurating bubo shows itself by a round or oval, red and painful swelling, which is much elevated and has an area of one to four inches, or even larger, its long axis usually corresponding to the fold of the groin. In this form an abscess cavity is soon formed, and fluctuation is readily discovered.

"All of the foregoing forms of bubo may be observed in cases of chancroid of the genitals and of ulcerative lesions of the legs and toes. vol. I-4 
"4. In contradistinction to these comparatively mild forms of bubo, the true chancroidal or virulent bubo, which nowadays is quite rare, stands out with marked features. These virulent buboes are generally caused by pus from very active and destructive chancroids and from mixed chancres, in which the initial lesion has become infected with chancroidal pus. The chancroidal bubo gives evidence from the first of an actively destructive process. The groin becomes red and swollen, and a perceptible tumor is soon developed. The skin becomes red, tense, and the seat of much pain. Redness gives place to a brownish-red tint, and then the swelling, which is considerably salient, presents decided fluctuation. The abscess either bursts from ulceration of the skin or it is incised. The roof of this cavity, which consists of thinned and inflamed skin, then promptly melts away, and the typical chancroidal bubo cavity is left. This cavity is usually deep; its base is anfractuous, covered with sloughy tissue of a dirty brown color, over which is a layer of unhealthy pus. The edges of this ulcer (since it really is one) are of a deep red, thickened, and decidedly undermined. When untreated, this condition leads to serious destruction of the penis, and may threaten the integrity of the vessels of the groin."

Chancroidal phimosis occasionally results from marked œedema, dependent upon the infection, or severe cauterization of the ulcer. The end of the organ becomes much enlarged, reddened, and painful, and a purulent secretion exudes from the constricted meatus. Destruction of the prepuce is not uncommon, provided the condition is left untreated. It results in the formation of an ulcerated hole behind the preputial orifice, through which the glans may protrude. Phagedena may be formed, and inguinal adenitis or bubo are common.

Chancroidal Paraphimosis.-This condition is rather uncommon. It is usually produced by drawing back the prepuce in order to apply medication to the ulceration. The foreskin is left behind the corona, swells and cannot be reduced. Gangrene of the whole glans may result if the constriction is not soon relieved.

Cancer of the Penis.-Barney ${ }^{26}$ has written an excellent paper upon this subject, in which he gives the result of his study of Ioo unselected cases of epithelioma of the penis. We have made the following extracts from his communication.

Etiology.-AgE.-In Barney's series the youngest patient was aged twenty-five years, and the oldest eighty-two years. The average age was fifty-six years.

Occupation.-Almost all trades and professions were represented in the series, and no particular influence seems to have been exercised by the occupation of the patients, with respect to the production of the disease. 


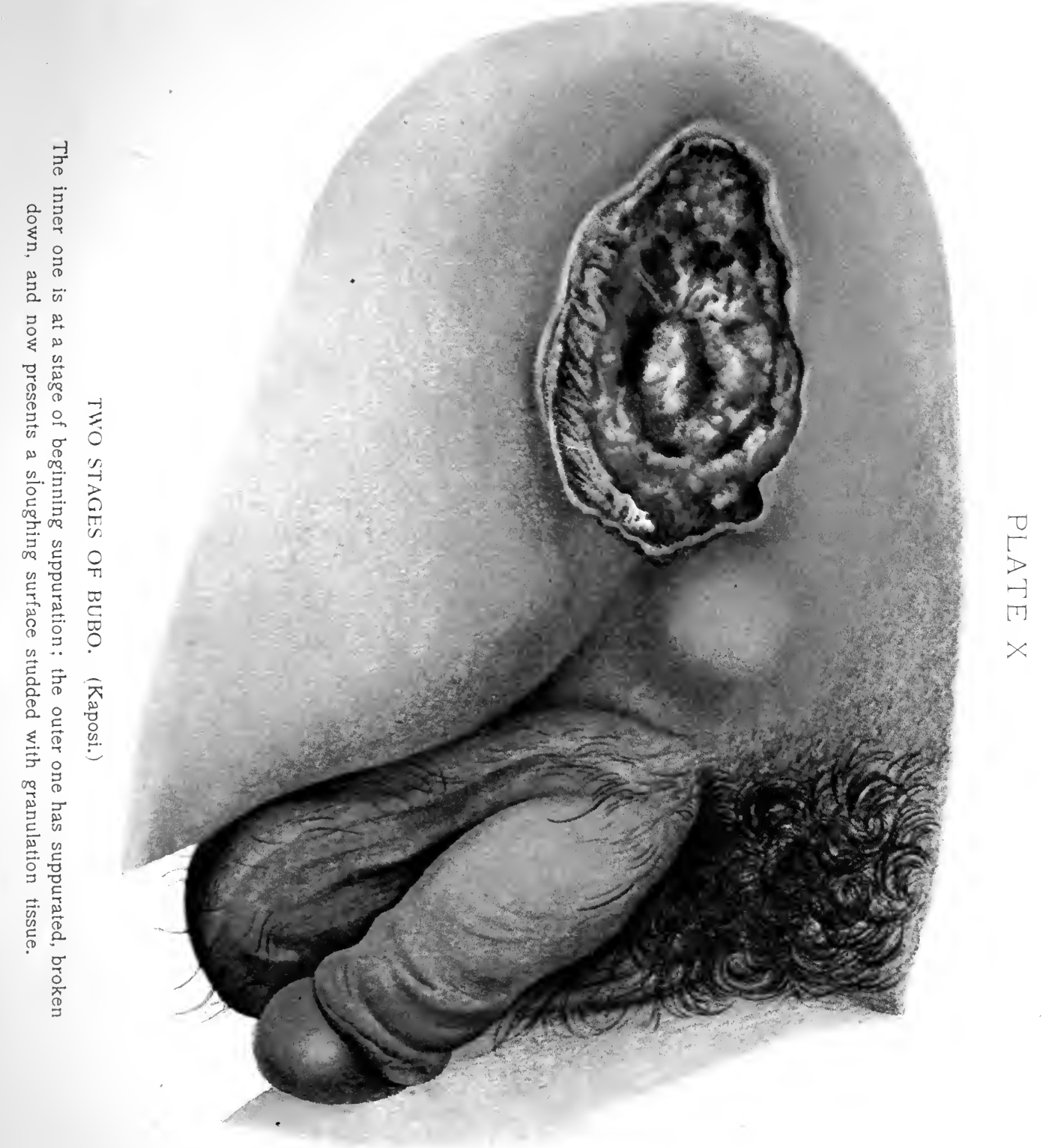



Contact with Cancerous Cervix.-There was no evidence whatever that contact with a cancerous cervix had any etiological importance. It was noticeable that there were no Jews among the patients of this series. Barney calls attention to previous observations of -other writers, which indicate that the Jews exhibit an apparent immunity with respect to this malady.

Phimosis.-There were but 42 patients with regard to whom the presence or absence of phimosis was noted. Of these, it was present in 85 per cent. This observation is in accord with those of other writers.

FIG. 8

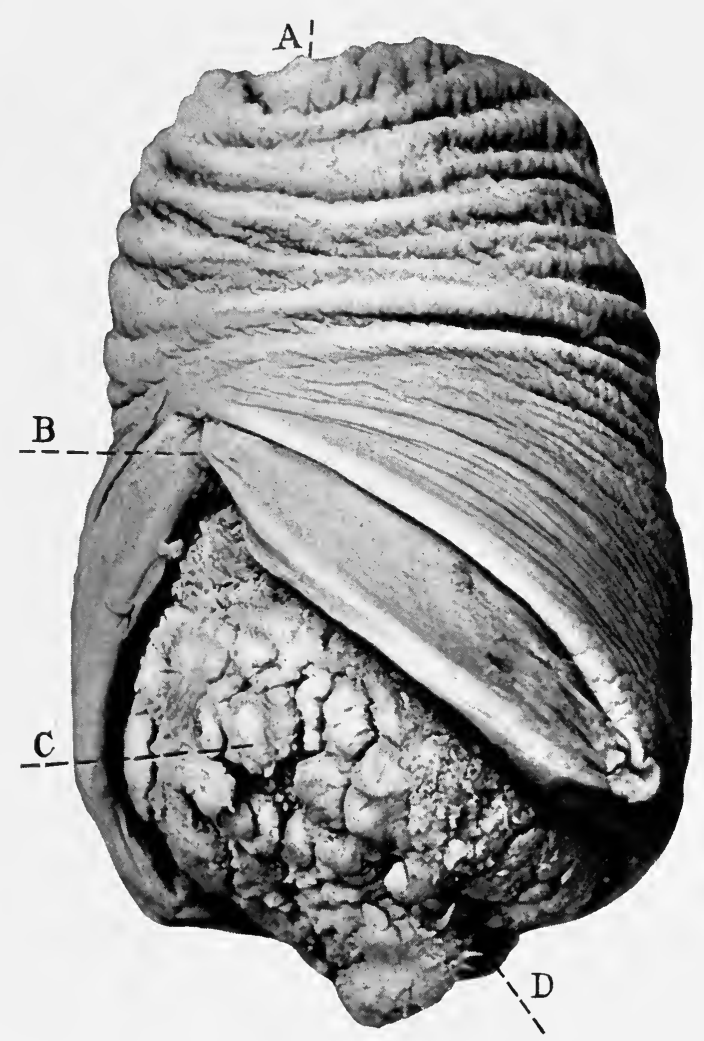

Cauliflower growth involving glans at $C$. Amputated extremity of penis at $A$. Dorsal incision through prepuce at $B$. Meatus at $D$. (Barney.)

Venereal Disease.-Barney found two patients out of the whole number who had had a venereal ulcer upon the glans.

Point of Origin.-In 69 per cent. of Barney's cases the original lesion was on the glans; in about 36 per cent. it began on the prepuce.

Duration.-Barney gives the following figures with regard to the duration of the disease prior to the time at which the patients came under observation, as follows: 
Primary Cases.-Under one year, $3^{8}$ cases; one to two years, i 8 cases; two to three years, 5 cases; three to four years, 3 cases; four to five years, 2 cases; over five years, 4 cases; unknown, 4 cases. Total, 74 cases.

RecurRent CASEs.-Under one year, 4 cases; one to two years, 7 cases; two to three years, 6 cases; three to four years, I case; four to five years, I case; over five years, 6 cases; unknown, I case. Total, 26 cases.

Glandular Involvement.-Of the 66 cases in which this point was noted, there was glandular involvement in over 75 per cent.; in 16 per cent. no enlargement was present. In those cases in which it occurred it was mostly unilateral.

Metastases.-In this series the more distant metastases were all limited to the pelvic organs. Barney quotes other writers who have reported metastatic nodules of various internal organs-nervous system, lungs, heart, liver, and stomach.

Pathology.- "Epithelioma of the penis may begin in one of several ways:

"I. As a simple wart.

"2. As a pimple, in which case it is usually situated near the lymphatics.

"3. As a superficial excoriation or raw patch.

“4. More rarely as a true ulcer.

"5. Very rarely begins in the urethra (i.e., meatus)."

End Results of Treatment.-In one group of I I cases, Barney reports 3 patients who were probably cured. Two patients were seen at four years and at eight years after operation, and were found to be free from all evidence of cancer. A third patient died nine years after operation, and without evidence of recurrence. The average length of life in the remain-. ing 8 cases was about twenty-four years after operation. In one instance the patient is still living thirty years after it.

These results are sufficiently encouraging to warrant the hope that, if operations of a radical character are done in the early stages of the disease, a considerable proportion of the patients will be cured. 


\section{BiBLIOGRAPHY.}

I. Cruveilhier. Traité d'anatomie descriptive, Paris, I865, ii, I, 386.

2. Boutelier. Union méd. de la sieur infir., I875, xi, 27.

3. Preston. Medical Record, I898, liv, 315 .

4. Revolat. H. de Sedillot, xxxii, 370.

5. Dumarquay. Maladies chir. du penis, Paris, I879, p. 538.

6. Wright. Ashby and Wright, Diseases of Children, p. $53 \mathrm{I}$.

7. Goschler. Vierteljahrschrift f. prakt. Heilk., Prague, i 857 .

8. Smith. Transactions of the Medico-Chirurgical Faculty of Maryland, April, I878.

9. Beck. Medical News, I90 I, lxxix, 45I.

ıo. Lorthior. Centralbl. f. d. Krankh. d. Harn. u. Sex.-Org., I9o I, xii, 38 I

I I. Lydston. Genito-urinary, Venereal, and Sexual Diseases.

12. British Medical Journal, I889, i, I038.

I3. Dolbeau. De l'epispadias, Paris, I86 I.

I4. Czerny. Annals of Surgery, I894, xix, I I I.

I5. Hutchinson. Medical Times and Gazette, London, December I, I855.

I6. Duckworth. Trans. Clin. Soc., London, I89I-I892, xxv, 97.

17. Gould. Lancet, I887, i, 42 I.

I8. British Medical Journal, August I3, I887.

19. Malecot. Ann. des malad. des org. gen.-urin., November, I893.

20. Senn. Tuberculosis of the Genito-urinary Organs, I898, p. 22.

2I. Lydston. Genito-urinary, Venereal, and Sexual Disorders, p. 86.

22. Fournier. N. Dict. de med. et clin. prat., Paris, vii, 72.

23. White and Martin. Genito-urinary and Venereal Diseases, 6th edition, I907.

24. Taylor. Genito-urinary and Venereal Diseases, 3d edition, I904, p. 45I.

25. Taylor. Genito-urinary and Venereal Diseases, p. 448.

26. Barney. Annals of Surgery, December, 1907. 


\section{CHAPTER II.}

\section{THE URETHRA.}

\section{ACUTE AND CHRONIC URETHRITIS.}

Acute Urethritis.-Definition.-An acute inflammation of the urethra, accompanied by a spontaneous purulent discharge from the meatus urinarius.

Etiology.-Acute urethritis results, in the great majority of cases, from the infection of the urethral mucous membrane with the diplococcus discovered by Neisser, and called by him the gonococcus, which is the specific microörganism found in the urethral discharge of patients having gonorrhœa and believed to be its essential etiological factor. In a minority of the cases of acute urethritis the causal agents are of a nature other than gonorrhœal infection. Acute urethritis may be produced by mechanical irritation, $e . g$., through the rough use of instruments. The application of certain drugs in too strong doses is another way in which the condition may be produced, e. g., injections of corrosive sublimate, carbolic acid, and nitrate of silver.

Gout occasionally has urethritis as one of its manifestations.

Now and then there occurs a case of urethritis in the male in which there is a well-marked urethral discharge, which contains diplococci, responding, in most respects, so far as can be determined, to the tests by which the nature of the gonococcus is established, and in which, nevertheless, the patient has not had sexual connection, and could not have had it for so long a time previous to the appearance of the disease as to make a gonorrhœal contagion possible. These cases are difficult or impossible to explain, and we do not attempt to explain them, but the writer has seen two such instances, in which there can have been no gonorrhœal element and yet in which there was present an infecting organism indistinguishable from the gonococcus.

The urethritis which is seen in connection with neoplasms and tuberculosis of the urethra is a secondary condition and does not enter into consideration in this part of our work.

Pathology.-The pathological changes which occur in the urethra, in the cases of urethritis of a nature other than gonorrhœal, are those which are characteristic of inflammations of mucous membranes ordinarily and do not need to be described here. Those which are incidental to 
gonorrhœal infection will be described after the section in which the gonococcus is discussed.

Gonorrhœal infection of the urethra does not require an abrasion or other preëxisting lesion of the urethra in order to effect a foothold in its tissues. It is sufficient to have a gonococcus-bearing discharge come in contact with the urethral mucous membrane.

The Gonococcus. - Neisser ${ }^{1}$ described the infecting organism of gonorrhœa in 1879 , and, although its etiological relation to gonorrhœa, as well as its actual identity, have been questioned, we must accept it as an established scientific fact that this microörganism is the active agent in the production of this disease, although, in the writer's belief, there may be another diplococcus which has not, as yet, been differentiated from the gonococcus, and which is capable of creating an acute urethritis differing from gonorrhœa in its clinical course, and which has not, as yet, been subjected to a test by which it can be distinguished from the gonococcus.

That there are other diplococci, as well as various kinds of microorganisms in the normal urethra, is well known, and it was impossible to pronounce absolutely with regard to the distinctive nature of some of the former and the gonococcus, until Gram's test was known, which test demonstrated the fact that diplococci had more or less frequently been pronounced to be gonococci when such was not the case.

The identity of the specific diplococcus which is responsible for the production of gonorrhœa should not rest upon the microscopic tests alone. An essential factor in the demonstration is the microbe's power to create a specific infection. This demonstration was made by Welander. ${ }^{2}$ Keyes $^{3}$ refers to Welander's experiments with respect to the infectious properties of the urethral microbes and of the gonococcus as follows:

"But in spite of other microbes and other secretions, the specificity of gonorrhøal pus is splendidly demonstrated by Welander, who inoculated the male urethra five times without success, using pus containing small bacilli, and derived from putrid balanitis; several times with vaginal secretions, containing a variety of microbes; three times with vaginal secretions containing several rounded bacillary microbic forms; three times from a putrid and purulent vaginal flow containing moving bacilli; three times with vaginal secretion containing no gonococci from women whose urethræ did contain these microbes; and then he gave a typical gonorrhœa to these last three subjects by inoculating them with the above-mentioned urethral pus from the same women, which pus did contain gonococci.

"It may be taken for granted, then, that the discovery of the diplo- 
coccus which appears in a urethral discharge of an individual whose urethritis has followed a sexual connection, and which answers to the requirements of the Gram test, establishes beyond peradventure the specific gonorrhœal nature of the malady."

Tests of THE Gonococcus.-For the purpose of examination, a small smear of the urethral discharge is made between two coverglasses. The specimen, after being dried, is stained with an aniline dye and fixed, and shows the gonococci, under an oil-immersion lens of high power, with the following features: They appear in pairs, each half of which is convex upon its outer side and straight upon the inner side which faces that of its fellow, being separated from it by a narrow space. The individual pairs lie in somewhat irregularly formed groups or in lines with relation to each other. They occupy the epithelial and pus cells, and are also seen outside of them. Those which are confined within the cells show a greater tendency than the extracellular ones to be grouped in the form of squares, this fact being probably due to their being retained within the limited space imposed upon them by the cells, which tend to keep them in the form of square grouping, into which they naturally fall by virtue of their mode of reproduction, which is by transverse segmentation of each microbian pair.

In form, a pair of these microörganisms may be likened to a French roll.

The fact of the invasion of the pus and epithelial cells by the gonococcus has been asserted to be one of its distinctive features with relation to other urethral microörganisms bearing a likeness to it morphologically. It cannot be assumed to be more than suggestive, however, since others do penetrate the cells, as well as lie outside them, though their tendency to do this is probably less well-marked than with the gonococcus.

The Gram Test.- The method by which this test is to be applied to the specimens selected for the purpose of determining the nature of the diplococcus which is present in the urethral discharge in any given case is as follows:

\section{Reagents:}

No. I. The staining fluid.

A saturated alcoholic solution of gentian violet

I part.

2 per cent. carbolated water

9 parts.

No. 2. The Gram solution.

Iodine

I part.

Potassium iodide

2 parts.

Distilled water

300 parts.

No.3. Carbolic acid

2 parts.

Saturated solution of Bismarck brown in water . . . . . 98 parts.

No. 4. Alcohol.

No. 5. Distilled water. 
Besides the above-named articles, there should be an alcohol lamp, Canada balsam, coverglasses, and a microscope and oil-immersion lens of high power.

The test consists in staining a smear made from the urethral discharge and examining it under a high-power oil-immersion lens. In the microscopic field there will be seen several kinds of microörganisms, and among them probably a diplococcus which will look like the gonococcus. Alcohol is applied. This renders all but pseudogonococci invisible. The next steps of the test are for the purpose of determining whether or not these diplococci are gonococci. They consist in staining a second specimen as was done with the first one examined, and then adding the Gram stain to it, after which it is washed with alcohol, then with water, and then counterstained with another color. This will cause the gonococci which were made invisible in the first specimen to become visible, if they are present.

Manner of Making the Test.-Make a very thin smear with a small quantity of the discharge placed upon one coverglass and by then rubbing a second glass upon it, in order to spread the matter evenly over both. Separate the glasses. Take each of them with a pair of fine smooth forceps, by its edge, and pass them two or three times through an alcohol flame, after having let them dry over a very mild degree of heat.

Cover the smear on one coverglass with solution No. I, and allow it to remain thus for thirty seconds. Wash off the excess of the staining fluid with water. Dry this glass in the alcohol flame, passing it through it several times. Mount the specimen in Canada balsam and examine it with an oil-immersion lens. If diplococci are found in it which resemble gonococci, the other coverglass is submitted to the Gram test as follows:

Proceed as with the first coverglass up to the point of washing off the first stain. Instead of doing this, shake off the surplus staining fluid from the glass. Dry and fix it in the flame as before, then let the coverglass lie-smear side down-upon the Gram fluid for five minutes.

Wash with alcohol until all color has apparently left the smear. Wash with water, dry the film, and stain with the No. 3 fluid for five minutes. Wash with water and dry the coverglass again. Mount the specimen as before, and examine with the oil-immersion lens. (Plate XI.)

The first specimen will have had the color taken from all microorganisms except the pseudogonococcus, which remains deeply colored, as seen in Plate XII. The true gonococcus appears in the second specimen stained brown, as in Plate XIII, and in contrast with the darkly stained false coccus, which will appear as it shows in the second specimen in Plate XII. 
It is to be remembered that the failure to find gonococci by the above test is not necessarily a proof that the patient has not gonorrhœa, for they may fail to be demonstrable if the urethra is under the influence of active ocal treatment; nor can the test be considered as positive in cases in which there is only a mild chronic discharge.

The Culture Test.-Still another test of the identity of the gonococcus is by making cultures of it. With regard to the culture of this micrococcus, White and Martin ${ }^{4}$ speak as follows:

They say that the growth reaches its greatest extent in three days, after which time the germs lose their virulence and can no longer be transplanted with success. The growth is of small extent.

The best medium for the culture is the human-blood serum, and the best temperature for the growth of the germ is $34^{\circ}$ to $37^{\circ} \mathrm{C}$.

The growth of the gonococcus is interfered with by the development of other pyogenic germs, because of its slow growth, owing to which fact the cultivation of the former is not always successfully accomplished.

White and Martin refer to the statements of Torro, who asserted that the difficulty that has been experienced in growing gonococci lies in the fact that alkaline media have been employed.

The pure cultures retain their virulence for many weeks if kept in acid media. Torro stated that he reproduced the disease from pure cultures obtained by inoculating dogs.

Pathological Anatomy.-The tissue changes have been described by Finger ${ }^{5}$ and others, who obtained the opportunity to inoculate the urethræ of certain criminals under death sentence, and by subsequent postmortem examinations. Keyes gives the results of their work as follows: "Thirtysix hours after inoculation the gonococci had only just begun to effect an entrance between the epithelial cells. The lacunæ of Morgagni were crowded with the cocci, diapedesis had begun, and intracellular gonococci were found among the few leukocytes on the surface of the epithelium.

"At the end of three days the inflammatory process was well under way. The surface of the mucous membrane was covered with pus, the epithelium infiltrated by bacteria from one side, and by leukocytes from the other. The inflammation showed four striking characteristics, viz., (I) the pavement epithelium of the fossa navicularis, although swollen with leukocytes, resisted the invasion of the gonococci almost absolutely; (2) the cylindrical epithelium of the penile urethra was generally invaded; (3) this invasion was most marked about the crypts and glands, which were packed with pus and gonococci; and (4) the subepithelial tissue, though showing every evidence of inflammation, contained few gonococci except in the neighborhood of the crypts and glands and in all places not covered by epithelium." 


\section{PLATE XI}

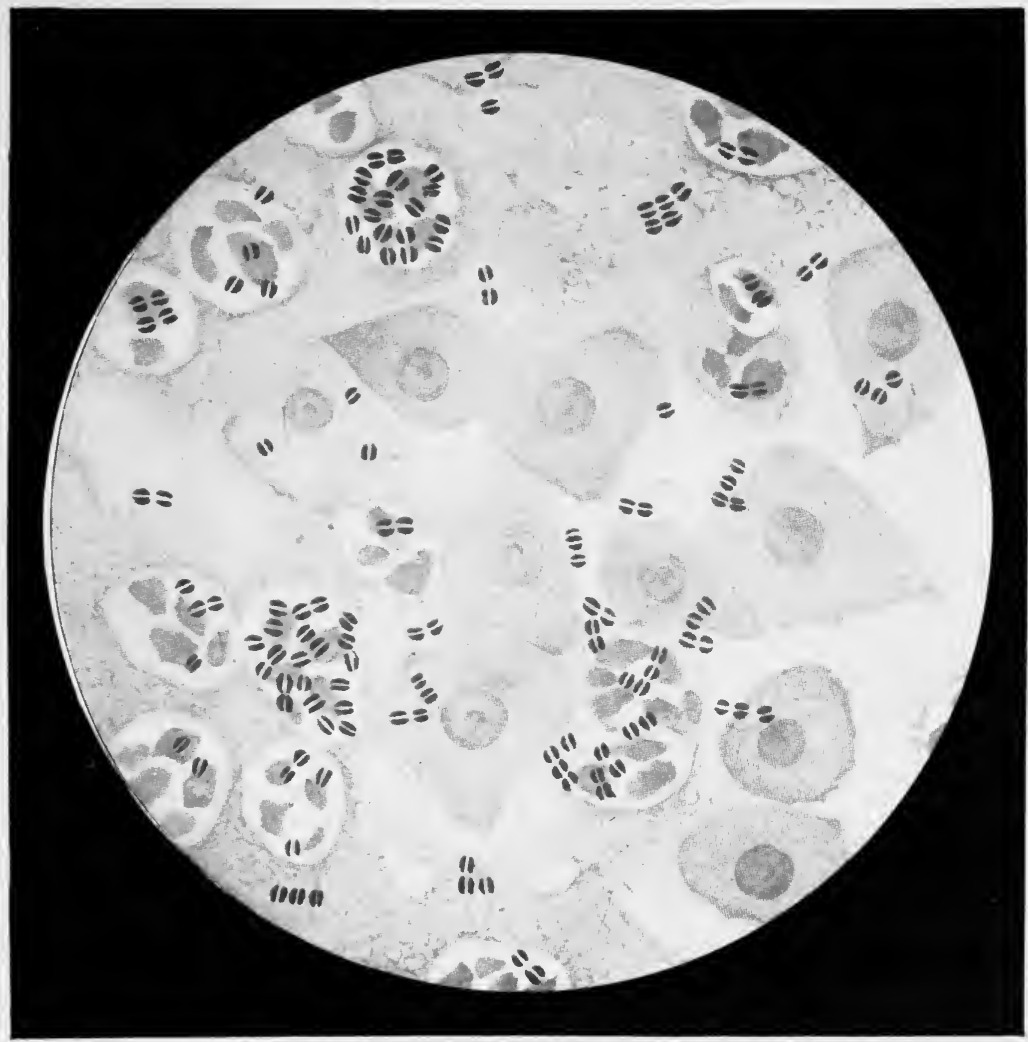

GONORRHOEAL PUS. (Keyes.)

First stain of Gram's test. 

PLATE XII

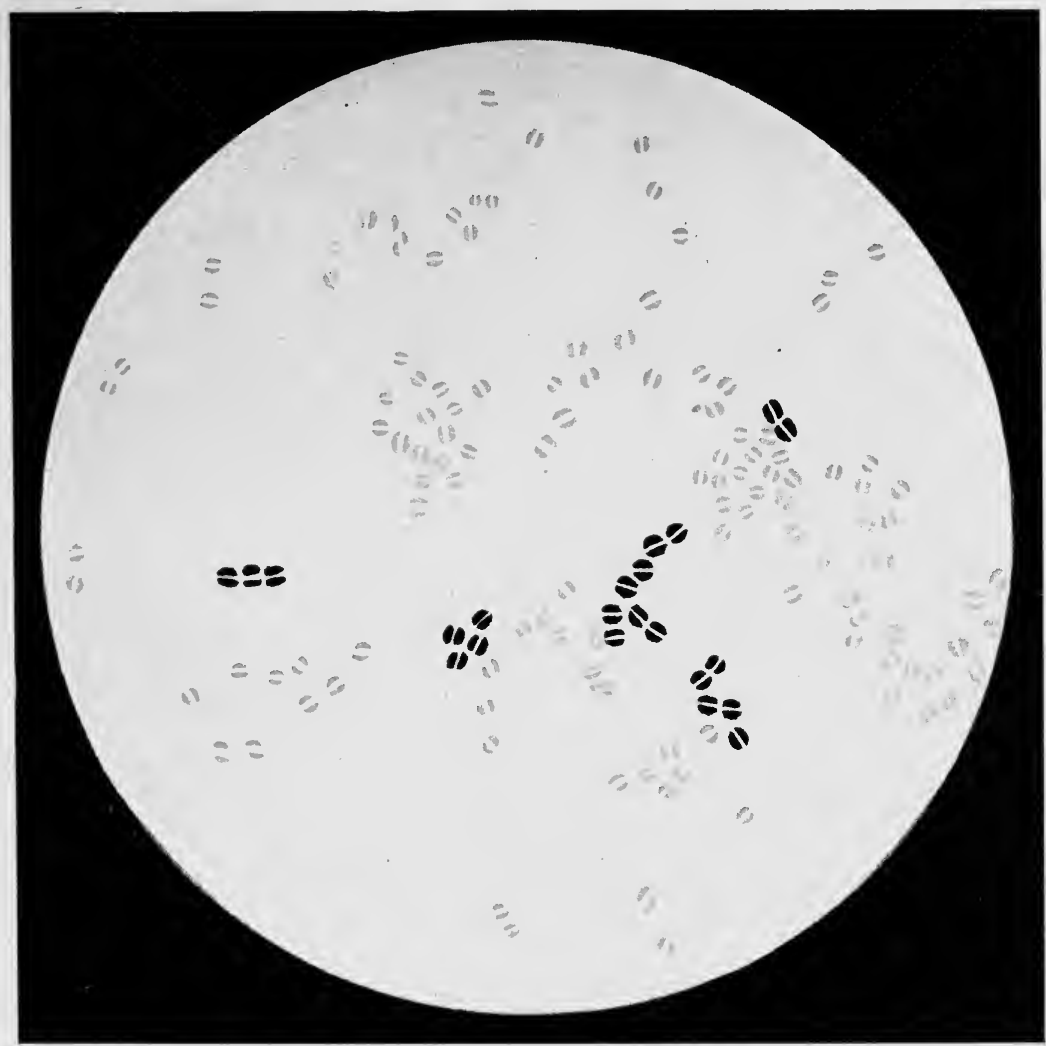

GONOCOCCI AS SEEN UPON MAKING THE CONTRAST STAIN WITH BISMARCK BROWN. (Keyes.)

Gram's test. 


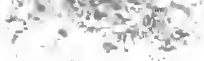




\section{PLATE XIII}

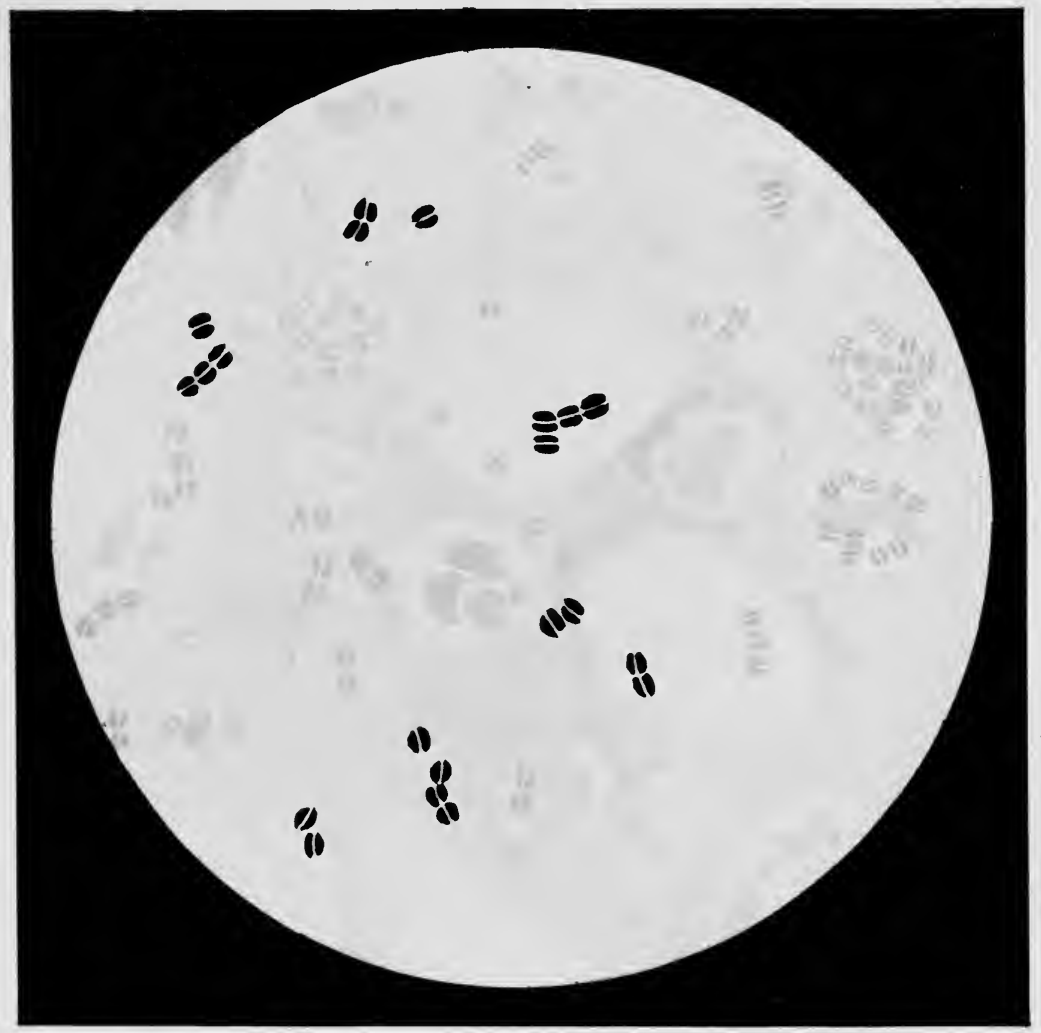

GONORRHOEAL PUS. (Keyes.)

Gram's stain, after washing with alcohol, showing pseudogonococci. 

Under the influence of the above-described invasion by the gonococci, the more superficial epithelial cells are cast off with abnormal rapidity, and those which are beneath them do not receive their habitual protection. In consequence of this there ensues presently, in the course of the malady, a superficial erosion of the mucous membrane, which is more or less extended in different cases, according to the virulence, or lack of it, of the attacking germs, and to the degree of cellular resistance opposed to their advance by the epithelium. (The changes in the tissues which take place as the process becomes chronic in character will be described in the chapter on Stricture of the Urethra.)

Incubation.-The periods of incubation in gonorrhœa vary markedly. The average time of appearance of the first symptoms after exposure to the contagion is about five to six days. It is distinctly shorter in recurrent attacks.

It is with the rarest exceptions, if ever, seen within the first day following exposure in a first attack, unless the individual has taken violent exercise or drunk a large quantity of alcoholic stimulant, especially of beer.

The writer has seen one patient whose gonorrhœa had an incubation of twenty days, so far as could be determined by the most rigid crossexamination, but it is very rare to have the period prolonged beyond the tenth day, and it is seldom that it exceeds the seventh day.

Symptoms.-The first symptoms of gonorrhœa are a slight tingling or tickling at the end of the urethra, which is more noticeable during micturition, and the appearance of redness and slight swelling of the meatus urinarius. Within a few hours after these have become evident, sometimes synchronously with them, there appears the first sign of the urethral discharge, which has the character of a sticky, somewhat cloudy, pearly gray fluid.

Within the next twenty-four hours these evidences of the malady have all become much more marked, and in addition, the prepuce has become involved in the swelling; the tingling sensation at the end of the urethra has been converted into a most violent burning, so intense that the patient involuntarily shrinks from urinating, and postpones the act as long as possible. The discharge will have changed from the character mentioned above, and will have become thick, profuse, and of a creamy yellow color. In the more acute cases a fawn color is sometimes seen, owing to the admixture of blood with the discharge; in other instances the discharge will be streaked with blood.

If left to itself, the disease continues to present the symptoms just described for from eight to fourteen days, at the end of which time the period of decline begins. From this point there is a more or less pro- 
gressive recession of the symptoms mentioned; the burning lessens; the discharge diminishes in quantity and becomes thinner; the redness and swelling of the meatus and prepuce gradually disappear.

What may be called a typical gonorrhœa will cease to show any outward, or to produce any subjective, signs by which its presence may be recognized, at about the eighth week after its inception in the cases which go on to spontaneous cure, a rare and unusually fortunate phenomenon. Thereafter, with these happy individuals, there will be left merely a few comma-like shreds-tripperfaden of the Germans-to testify to the former presence of the enemy. During this time the territory occupied by the gonococcus in its march will have extended from its starting point at the meatus to the bulbomembranous junction of the urethra. Here it will have been arrested; whether because of the guardianship which may, perhaps, be exercised by the compressor urethræ muscle over the posterior urethra, or for some occult reason as yet unknown, we do not pretend to judge.

Treatment.-There are two lines along which the treatment of acute gonorrhœa may be conducted. The first consists in allowing the malady to progress to the end of its acute period without attempting to check its virulence by the application of any local measures, and to devote the attention to lessening the violence of the symptoms by purely palliative means, and to begin with the declining stage the endeavor to shorten the course of the malady by local as well as internal medicinal treatment. The second method of treatment, which is the modern one, aims at destroying the germs of the disease, or at so inhibiting their activity and virulence as to materially shorten the time during which they shall continue to be harmful in the urethra, and this method begins at once with vigorous local treatment and with comparatively little treatment of other kinds.

It may be said at once as a broad principle that the second method of treatment is that which should always be applied and continued, unless it becomes evident that it is not benefiting the patient, or unless it is evidently making his condition worse. We make this last statement for the reason that there are patients who are made worse by the employment of the local measures which constitute the modern treatment.

In speaking thus unreservedly in favor of the adoption of the local treatment of gonorrhœa, we do not wish to be understood as minimizing the importance of the general remedies and hygienic measures, for these are an essential part of the treatment of the disease. We will deal with them first, and with the local methods afterward.

General Medicinal and Hygienic Measures.-These may be briefly stated as follows: 
I. Rest during the early part of the acute stage of the disease.

2. Cleanliness of the parts.

3. The avoidance of all alcoholic stimulants and of all sexual excitement.

4. When possible to enforce it, a milk diet for the first three or four days, and, in any case, avoidance of stimulating articles of food, e. $g$., spices, peppers, and highly seasoned sauces.

5. The administration of diluents and of an alkaline diuretic, for the purpose of rendering the urine unirritating and to flush the urethra.

6. The administration of sandalwood oil, for the sake of securing such inhibitory action as it possesses upon the activity of the gonococci.

7. Anodynes.

Of the steps to be taken which have just been enumerated, we need not refer in detail to the first four or to the last one, since they do not require to be explained.

Of the alkaline diuretics, we have always preferred the citrate or acetate of potash, in 10 to 20 grain doses, thrice daily, well diluted with water. Plenty of water should be drunk throughout the acute stage of the disease.

With regard to the employment of sandalwood oil, we have decided views. It is of distinct value in all the stages of acute and of the declining stage of the malady, provided that it is not given in too large doses; that a pure preparation of the drug is used, and that it does not disturb the stomach or digestion.

There are a good many individuals who cannot take this drug because of its ill effects upon the stomach, and a number with whom it is unwise to continue its administration, because of the pain in the kidneys which it produces. It is a drug the use of which in more than very moderate doses is not free from danger. The writer has more than once seen wellmarked renal congestion, if not a temporary glomerulonephritis, follow the administration of 20 minim doses thrice daily. It is a remedy which is often sold by druggists upon the request of persons suffering from gonorrhœa, and patients are frequently advised by them to take as much as 30 minims of it thrice daily.

In the writer's experience, this remedy is far superior in all respects to copaiba, turpentine, oil of wintergreen, or any of the other innumerable "specifics" which have been in use in the treatment of gonorrhœa.

Sandalwood oil should not be given in larger doses than 10 minims thrice daily; plenty of water should be drunk while it is being employed, and it should be discontinued at once upon evidence of its disagreeing with the stomach, or if it causes pain in the renal region. If these signs of the inability of the patient to tolerate the drug do not occur, its use 
may be beneficially continued for three weeks, or even more, but the urine should be examined for albumin and blood corpuscles, in order to be certain that it is not causing renal congestion.

One word with regard to the use of alcoholic stimulants: It is our rule to absolutely interdict them until at least one month has passed after the complete cessation of all discharge from the urethra and from the urine, and after all symptoms have disappeared. A longer time of abstinence is just so much the safer; but, as a rule, a completely cured gonorrhœa will not be made to recur by the use of wines and liquors one month after its entire cessation.

The question of resuming sexual intercourse is a more complex one, for it has to do not only with the patient's well-being, but that of his partner in the connection as well. The sanction of the physician must be made dependent upon what is shown by the tests to determine the presence or absence of the gonococcus, and also upon the absence of all signs of the disease.

We are in the habit of interdicting sexual congress for a period of two months after the disappearance of all evidence of the existence of the disease. In which statement the failure to demonstrate the presence of the gonococcus, it need not be said, assumes the first place.

Local Measures.--No disease which attacks the human organism has had brought to bear upon it so many "specifics" as has gonorrhœa. It is an implied tribute to the capabilities of this most prevalent little germ that this should have been the case, since it is clearly an evidence that none of the means which have been used to destroy it have been wholly successful, and in most instances they have been almost or wholly futile.

We do not propose to let ourselves stray from the narrow limits defined by the few really efficacious remedies which have earned a right to be seriously accepted as having an established value. Those which are worthy to be thus classed are the following: the silver preparationsprotargol, argyrol, and nitrate of silver-permanganate of potash, and the astringent remedies, zinc and lead.

The first three and permanganate of potash aim at the destruction of the gonococcus, or at inhibiting its activity, to the degree that the urethral membrane shall have the power to repair sufficiently to repel its further attacks. The nitrate is of special value in the more chronic stages of the disease.

Manner of Employing Local Remedies.-The two remedies that are suitable for the first (acute) stage of the disease are permanganate of potash and argyrol. The second is the less irritating of the two, and may be used in from 5 per cent. to Io per cent. solutions without causing any irritation. 
Argyrol.-The simplest manner of applying this $m$ is with the ordinary blunt-pointed urethral syringe (Fig. 9), wrich holds half an ounce. The patient sits on the edge of a chair with a basin conveniently placed upon a stool a little below the level of the knees, and between them. (The bladder must be emptied immediately before taking the injection.) The syringe is filled with the solution, and is to be held between the thumb and middle finger of the right hand, with the forefinger tip passed through the ring at the end of the piston handle. The penis is held between the middle and forefinger, or thumb and forefinger, of the left hand placed immediately behind the corona on either side, and should be drawn forward in order to make the urethra straight, so that it is open throughout its anterior part. The blunt nozzle of the syringe is then pushed into the meatus until the lumen of the latter is entirely filled by it and the fluid cannot escape around it while the injection is being given. The piston of the syringe is then pushed forward by the forefinger of the right hand, and the fluid is very gently and slowly urged into the urethra. The syringe is removed from the meatus and the latter is compressed by

FIG. 9

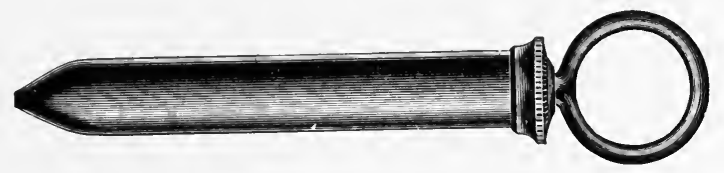

Urethral syringe.

the fingers of the left hand at the same instant. The injection should be held in the urethra for ten minutes, and then allowed to escape, and should be repeated every three hours.

The discharge rapidly diminishes under this treatment, and at the end of a week or a fortnight has usually almost disappeared. At this time the patient is very likely to consider himself cured, and to stop further treatment. If he does, the discharge and the other symptoms characterizing the acute stage of the process are certain to return, though in less violent form than at first.

In some cases the drug may be continued with benefit during the next fortnight or month in a smaller number of daily injections, and may be alternated with a mild zinc sulphate injection ( 0.5 per cent. to I per cent.).

Moderate doses of sandalwood oil and of acetate of potash should be continued, if tolerated, throughout the first three weeks of the treatment.

It is our rule to insist upon the continuance of the treatment for one week after the disappearance of all discharge from the urethra and of pus from the urine. We then reduce the injections throughout the next 
week, until at the end of it they are entirely omitted. The patient is then cautioned in the manner already stated with regard to the use of alcoholic stimulants and sexual excitement. If there has been no sign of any return of the disease at the end of three weeks after this, the patient will generally be found to be wholly cured.

The Janet Method.-The following description of the Janet method of treatment is taken from Keyes. ${ }^{3}$

"The instruments required are a suspended tank capable of holding a quart of fluid, with a rubber tube attached, a set of two-way nozzles of different sizes, and the scissors shut-off.

"The alternating shut-off instrument clasps the rubber tubes attached to the nozzle, and, by a scissors-like motion, impeding the outflow as the fluid enters the urethra, distends the canal, and, impeding the flow when the urethra is full, allows the canal to empty itself. A proper distention of the urethra is secured by raising the reservoir two or three feet. Such elevation will not force the membranous urethra.

"A hot I to 6000 solution of potassium permanganate is now made by crushing and dissolving three $\mathrm{I}$ grain tablets in a quart of water at $110^{\circ} \mathrm{F}$. (for the solution does not keep, and should be made fresh daily). This is poured into the tank, which is raised to a height of three feet; nozzle and scissors are attached; the patient (having urinated) stands, holding a wide basin to catch the outflow. The anterior urethra is irrigated by filling the nozzle with solution, introducing it within the meatus, then slowly opening the scissors until the urethra is filled, then closing them until it is empty, and so proceeding to and fro until the tank is empty. After the first two or three treatments the strength of the solution is increased to I to 4000 ; beyond this it is unnecessary to go."

Irrigation of the Urethra by Catheter.-Instead of applying the injection in the manner above described, it may be done by passing a small catheter into the urethra as far as the bulbomembranous junction, attaching the tube of the tank, containing the same solution of permanganate of potash as that already spoken of, to the outer end of the catheter-an eye-dropper or fine-pointed nozzle may be used for connecting the tube with it-and letting the fluid flow through the catheter and back again from the urethra around it. When the discharge is copious, the irrigation should be repeated twice daily; otherwise, once is sufficient.

Of the three methods of applying the solutions to the diseased mucous membrane of the urethra, we personally prefer the ordinary urethral syringe for the acute stage of the disease. The forcible stretching of the urethra by the expanding injections, used in the second of the ways described, we believe to be objectionable, and there is with its employment always the danger of carrying the infection to the posterior urethra, 
more especially with those patients whose cut-off muscle has been weakened by preceding attacks of gonorrhœa.

The disadvantage of the irrigation method lies in the placing of a foreign body-catheter-in the acutely inflamed urethra, and the absence of any expansion of the canal prevents the medicament reaching all parts of its surface. The latter feature, however, may be secured by momentary compression of the meatus and the prevention thereby of the escape of the fluid. If this is gently done by a skilled hand, a moderate expansion can be produced with less force than is the case with the nozzle method of employing hydrostatic pressure.

Of the drugs, we have procured more strikingly favorable and quicker results with argyrol.

Argyrol is not nearly so efficacious in the more chronic phases of the malady as in the acute ones. In the chronic stage, it should be replaced by nitrate of silver, which is best applied through the endoscopic tubes. (See section on Endoscopy.) Protargol, in our experience, is more efficient than argyrol in the chronic phases of the malady, but less so in the latter than the nitrate of silver. It therefore occupies a position somewhat between the two other remedies.

One frequently hears the claim that gonorrhœa can be cured within a week or ten days, or at most a fortnight; personally, we have no sense of shame in frankly confessing our inability to accomplish such favorable results, as a rule, although we sometimes succeed in so doing. We consider ourselves and the patient fortunate if we obtain a cure at any time less than six weeks; by cure we mean not merely the cessation of the discharge, but its non-recurrence upon omitting the treatment and upon resuming an ordinary manner of living.

Complications.-The above description applies to the more fortunate only of the cases of gonorrhœa. Unfortunately, these do not, at least if the disease be judged from the specialist's point of view, constitute anything like the majority of them. Instead of the easy career of the malady we have pictured, we see it extending to one or another or all of the urethral adnexa, beginning with the follicles and glands of the canal in some cases, and forming there abscesses of the type of the so-called peri-urethral abscess; or again, in others, devoting its attention to the lymphatics of the penis and of the groin, producing in the former the condition which is termed lymphangitis, and in the latter that which is known as bubo. To continue our inventory, by returning to the internal part of the tract, the next structures to be involved, in the case of the male, are the glands of Cowper, in which the process may produce a quickly forming and violent suppuration and abscess, or may take on a milder form and continue to inhabit these dwellings, in the guise of vol. $\mathrm{I}-5$ 
a chronic, more or less suppurating process, which may, on the one hand, never attract the patient's or the physician's attention during life; and, on the other hand, may, without apparent warning, give rise to a sudden and life-endangering suppuration that manifests itself in the form of the acute phlegmonous process, which was formerly, in all instances, regarded as urinary infiltration or extravasation. (See chapter on Stricture of the Urethra.)

Accompanying the germ and its manifestations still farther along the tract, we come next in order to its invasion of the ejaculatory ducts, seminal vesicles, vasa deferentia, epididymes, and testicles.

Proceeding to the posterior urethra, the next part to suffer from the active microörganism is the prostate. Here, again, the type of the process varies with respect to its degree of virulence, or the contrary, sometimes going no farther than to create a moderate and temporary swelling of the gland; at others, producing a violent inflammation of it and of the periprostatic tissue, which may or may not end in suppuration, and which, in a few instances, has implicated the peritoneum with fatal result.

The next step takes the disease through the posterior urethra and into the bladder, in both of which it has an unfortunate tendency to prolong its stay in the form of chronic posterior urethritis and cystitis. Finally, the process may force the entrances of the ureters, mount to the renal pelvis, and perhaps to its parenchyma. It is to be noted, however, that there have been but very few cases reported in which a pure gonococcus infection of the kidney has taken place, with the presence of the specific organism definitely proved. (See chapter on Suppurative Diseases of the Kidney.)

The last step which this malicious micrococcus takes, as though to make it evident that his sphere is not limited to the genito-urinary tract, if he chooses to journey beyond it, is by metastatic migration into the serous membranes of the joints and other structures of the body, in which he announces his arrival by creating severe and obstinate inflammation.

The conditions just referred to constitute the complications of acute gonorrhœa. They are discussed separately, each under its own heading: Peri-urethral abscess, page 65; abscess of Cowper's glands and diffuse phlegmon, urinary infiltration, in the chapter on Stricture of the Urethra; acute prostatitis and prostatic abscess, in the section which deals with diseases of the prostate; gonorrhœal cystitis, in the chapter on Cystitis; the invasion of the renal pelvis and the kidney, in the chapter on Suppurative Conditions of the Kidney.

The only complications of gonorrhœa not discussed under other head- 
ings in the course of this work, and which we therefore consider here, are: lymphangitis, bubo, acute posterior urethritis, gonorrhœal rheumatism, and gonorrhœal ophthalmia.

Lymphangitis.-Inflammation of the lymphatics of the penis and groin are conspicuous complications of the acute stage of some cases of gonorrhœa. Under the influence of this process the chain of lymphatic vessels upon the dorsum of the penis become indurated, enlarged, and present the ordinary signs of acute inflammation-redness, tenderness on pressure, swelling, and pain. The entire skin of the organ may become œdematous.

By its extension to the lymphatic glands immediately above Poupart's ligament, these structures become involved in the inflammation. In the larger number of cases, resolution takes place under the employment of appropriate treatment, in a minority of them abscess formation occurs, and, if not subjected to surgical treatment, the burrowing of pus beneath the integument of the region may extend over a wide area. Ordinarily, however, before this has taken place, the matter makes its escape through the outlet afforded it by the localized necrosis of a small area of the skin immediately overlying the abscess.

These complications are usually indicative of an especially virulent infection, or of another sort of infection superadded to that of gonorrhœa, by virtue of which the latter process appears to be intensified. Constitutional disturbance, manifested by the occurrence of fever and considerable bodily depression, accompanies the local lesion in these instances.

Treatment.-The two methods most commonly employed to subdue the inflammation of the lymphatics are applications of cold or of heat.

Personally, we do not believe in cold applications, and have much faith in hot ones as a means of promoting resolution of the process which we are discussing. The best manner of applying heat is by flaxseed poultice. In order that it shall be effective, the poultice must be thick, large, and frequently changed. In the majority of cases bubo will not go on to suppuration if the patient is confined to bed, and if poultices are thoroughly employed in this manner.

If suppuration does occur, the sooner the pus is evacuated by a free incision, the sooner will the local process be terminated.

It must be remembered that the glands of the groin are numerous, and that some of them are fairly deep-seated when the tissues are acutely inflamed, as in the condition under consideration. It also is to be borne in mind that glands which are advanced nearly to the point of suppuration are very likely to proceed to it if they are allowed to remain when an abscess involves adjacent glands. It has, therefore, been our custom 
to excise the entire inflamed mass of tissue-the inflamed but not yet suppurating glands, as well as all those which have already advanced to suppuration-rather than to rest content with simple incision of the abscess.

The writer has obtained about 50 per cent. of healing of such wounds per primam in the cases in which the skin overlying the abscess has not become too seriously involved in the inflammatory process and still retains its vitality. The operative method employed by him has been to expose the whole of the inflamed tissue by turning back a crescentic skin flap from below upward, and dissecting out the whole of the mass of inflamed glands. The field of operation is then thoroughly swabbed out with peroxide of hydrogen, and the flap turned back and sutured in place, leaving only a small space open to allow the passage through it of several strands of catgut, as a drain, during the first forty-eight hours. Firm pressure is put upon the cavity left by the operation.

Acute Posterior Urethritis.-The passage backward to the posterior urethra of the gonorrhœal inflammation is infallibly announced by one symptom, viz., a very frequent desire to urinate. This will not be present in a marked degree unless the posterior urethra has been invaded by the process. Associated with this eminently characteristic sign of the nature of the trouble, there occur also an increase of constitutional disturbance; in many instances, a feeling of more or less tenderness in the rectum on defecation, and the appearance of pus in the second as well as in the first half of the urinary stream, when it has been passed into separate vessels for the purpose of determining this fact.

The prostate and the vesical outlet, one or both, usually share in the inflammation of the prostatic urethra. The tendency of the process to become localized and chronic in the deep part of the urethra is very marked, and is probably referable to the obstacle to the free forward passage of the discharge constituted by the compressor urethræ muscle.

Treatment.-The treatment of acute inflammation of the posterior urethra is conducted on the same principle as that which is employed in the anterior urethra. The choice lies between the palliative measures and active local treatment. The former consists in: (I) Frequently repeated hot hip baths. (2) Absolute rest in bed. (3) Elevation of the hips. (4) The administration of morphine and belladonna suppositories. (5) Keeping the bowels freely open.

The local treatment consists in copious applications of the milder medicaments-3 per cent. to 5 per cent. argyrol solutions, or I to 6000 permanganate of potash solution-to the inflamed part. This is best done by forcing the shut-off muscle by the hydrostatic pressure in the manner already described, after having irrigated the anterior urethra 
with a solution of permanganate of potash, immediately beforehand. The patient may be allowed to spontaneously empty the bladder of the first forced injection thus made, but should retain a second one, that is to be made immediately after the first has been passed out.

Under this regimen the active inflammation usually recedes within three or four days.

The objection to the employment of this method of treatment of acute posterior urethritis is on account of the liability to spreading the infection by forcing it into the bladder or into the ejaculatory ducts and having it proceed from there to the epididymis and testis.

Systemic Gonorrhœal Infection and Gonorrhœal Rheumatism.-In a certain proportion of cases of gonorrhœa, at any time after the inflammation has reached the deeper parts of the urethra, there occurs a migration of the gonococcus from the genito-urinary tract to other parts of the body, these parts being any of the following structures: the synovial membranes of the joints, the bursæ, muscle sheaths, pleuræ, cardiac valves, nerves, conjunctivæ of the eyes, the periosteum, the veins, and the fasciæ. Preference is shown by the microörganism for the serous membranes of the joints, and they are consequently the parts most frequently attacked among those named.

The gonococcus gains entrance to the blood current by entering the capillaries of the venous system at some one or another part of the genitourinary tract, and in due course arrives through the blood current at some point or other of the structures named. Whether or not the germ effects an entrance to the lymphatics is not clear, but its presence in the venous capillaries immediately adjacent to an area of gonorrhœal infection in the genito-urinary tract and in the synovial membranes of a joint of the same individual has been demonstrated, and in the lastnamed situation it has often been found. It may occur there alone, or in association with other microörganisms; in the latter case, the condition having become one of mixed infection.

It has no, apparent connection with the ordinary articular rheumatism, and persons who are the subjects of the latter malady or of gout are not more prone than others to be attacked by gonorrhœal rheumatism.

It is peculiarly not amenable to the usual remedies by which the ordinary form of rheumatism is overcome. What the nature of the element in the production of gonorrhœal systemic infections may be that is conspicuous in certain individuals, and which appears in them oftentimes with every successive attack of gonorrhœa, and which predisposes them to this invasion of infection, we do not pretend to know, but that there is a well-marked tendency in some persons to be thus attacked is well known. 
Course of Gonorrhœal Rheumatism.-This complication of gonorrhœa may occur at any time after the first week of the disease. It is more commonly seen to take place after the end of the second week, and in some cases it is not manifested until very late in the course of the malady. The duration of gonorrhœal infection of the joints is variable, but it may be said in general that it is more prolonged than is the case with either articular rheumatism or rheumatic gout, so-called. It may continue for months in a somewhat less pronounced form than during the first part of the attack.

Character of the Local Manifestations.-The infection may be confined to one joint or may implicate a large number of them. We have seen a patient whose elbows, wrists, ankles, knees, and vertebral joints were involved in greater or less degree in four attacks of gonorrhœa, or exacerbations of a chronic process.

The inflammation of the joint or joints involved may be acute or mild in character. The redness which attends the ordinary form of articular rheumatism is absent in the gonorrhœal infection. Pain is, however, present, and this, too, varies according to the degree of acuteness of the process, in different cases. Hydrarthrosis is frequently present, and may come on late in the course of the condition. It is absent in some instances. The knee is more frequently the seat of the collection of serous fluid than other joints.

In the acute cases there is more or less febrile disturbance, and for a short time it may be quite severe. Ordinarily, however, the constitutional manifestations are less pronounced than is the case with the usual form of rheumatism affecting the joints. Pain, which is often severe at the outset, becomes much less marked when the patient is put at rest, which is not the case with the ordinary articular rheumatism.

The gonorrhœal process undergoes resolution in the joints more tardily than does that of articular rheumatism.

When there is a mixed infection in a joint, suppuration often takes place and serious destructive lesions may then be established.

Diagnosis.--The following table, taken from Keyes, ${ }^{3}$ is serviceable:

Gonorrheal Rheumatism.

I. Cause: Gonorrhœa. No influence of cold in the production of the rheumatism.

2. Very rarely observed in women.

3. Non-febrile, or much less so than simple rheumatism. Even in acute cases reaction never attains the habitual intensity of rheumatic fever.

\section{Simple Rheumatism.}

I. No etiological relation with the state of the urethra. Habitual causes: Cold, inheritance, rheumatic diathesis, etc.

2. Common in the female, although less frequent than in the male.

3. Reactional phenomena much more intense and prolonged than in gonorrhœal rheumatism. 
Gonorrhoeal Rheumatism.

4. Symptoms habitually limited to a small number of joints. The affection never becomes general to the same extent as does simple rheumatism.

5. Less movable than simple rheumatism, going from one joint to another less quickly. No delitescence; no real jumping from one joint to another.

6. Local pains generally moderate, always less than in simple rheumatism; sometimes remarkably indolent.

7. Frequently a tendency to hydrarthrosis following the acute fluxion.

8. No sweating.

9. Urine not modified.

Io. Blood not furnishing a marked buffy coat.

II. Cardiac complications exceptional.

I2. Frequent coincidence with a special ophthalmia, inflammation of the synovial sheaths of the tendons, inflammation of the bursæ, etc. The latter localities may be exclusively implicated.

13. Relapse in the course of successive gonorrhœas very frequent.

\section{Simple Rheumatism.}

4. Symptoms usually involve a number of the articulations; sometimes nearly all of them.

5. Symptoms: Movable, ambulatory fluxions; rapid delitescence, jumping from one joint to another.

6. Pains always rather intense, sometimes excessive, disappearing less rapidly than those of gonorrhœal rheumatism.

7. Little or no tendency to consecutive hydrarthrosis.

8. Abundant sweats, constituting a symptom almost essential to the malady.

9. Urine specially modified.

Io. Blood forming a firm, concave clot with buffy coat.

II. Cardiac complications frequent.

I2. Acute rheumatism does not affect the eye; the bursæ escape, as do usually the sheaths of the tendons.

13. Relapse frequent, but always independent of the state of the urethra.

Treatment.-Absolute rest, the application of heat in the form of douches, poultices, or wet absorbent cotton dressings impregnated with a small quantity of oil of eucalyptus, or of spirits of turpentine in order to obtain counterirritation will usually reduce the inflammation and relieve the pain in the affected joints.

Internal remedies, so far as any specifie action is concerned, such as that which the salicylates exercise in ordinary articular rheumatism, are powerless to benefit the gonorrhœal condition. Reliance must be placed principally upon local applications, such as those named above. In the acute and subacute conditions, and in the more chronic form of the malady as well, we must confess that there is very little benefit to be derived from any form of treatment other than the measures which tend to increase the patient's general strength and health, such as nourishing food, abundance of fresh air, avoidance of wet and cold. Moderate massage and the local application, in the form of prolonged inunctions, of iodoform ointment, are the only ones which, in our experience, have seemed to be of any real worth.

In the cases in which suppuration in the joint occurs, surgical treat- 
ment is demanded. This consists in either a free laying open of the joint affected, and in drainage or cleansing it, closing the incision at once; or, what in our judgment is a better way of treating the joint, and which is recommended by Keyes, viz., thorough irrigation, which is done by thrusting a good-sized trocar into each side of the kneejoint, which is the only one to which the method is applicable, and by passing a current of hot I to 5000 corrosive sublimate through the joint. The cavity of the joint should be moderately distended by the inflowing fluid. After it has been thoroughly flushed and all of the fluid drawn off, the punctures may be closed by suture, if they have been large enough to require it.

Gonorrhœal Infection of the Eye.-The eye may become infected with the gonococcus by (I) direct contagion through pus from the urethra being transferred to the conjunctiva by the fingers, or (2) indirectly by the blood current. The second variety of the infection is usually associated with gonorrhœal rheumatism.

The distinction between the types of the ocular infection, corresponding to the manner in which the invasion takes place, is well-marked, and should, as Keyes says, be clearly recognized.

When conveyed to the eye by direct contact, it is the conjunctiva which is involved. In the metastatic variety, the conjunctiva, the iris, or the membrane of Descemet may one or all be implicated. Keyes ${ }^{7}$ makes the following statements with regard to the latter variety of the infection: "It is generally associated with the polyarticular variety of gonorrhoal rheumatism. It may precede or follow the development of the disease elsewhere. Contagion will not produce it. Its essential cause is the urethral gonorrhœa. According to Fournier, it is fourteen times more frequent than gonorrhœal conjunctivitis. . . . Cold, fatigue of the eye, etc., have no power to produce it. An individual idiosyncrasy seems to preside over its appearance. Should it occur with one urethral inflammation, the chances are that it will reappear with the next."

Symptoms.-We again quote Keyes with respect to this part of the subject: "Inflammation of the membrane of Descemet is the most common form of attack. Here the conjunctiva is only moderately injected. The cornea is transparent. A cloudy, smoky appearance of the fluid of the anterior chamber is the most characteristic objective symptom. Sight is slightly troubled, objects looking misty. There is no pain, but sometimes a sensation of uneasiness about the eye. Photophobia is absent or very mild. Sometimes there is a slight flocculent deposit on the posterior face of the cornea, with escape of a little blood into the aqueous humor. The iris is unaffected, perhaps a little slow in its movements. There is no deformity of the pupil, no change in the 
color of the iris, no other sign of iritis-points strongly insisted on by Cullerier.

"When the iris is attacked, the symptoms do not differ from those of simple iritis; there are redness of the cornea, radiate pericorneal injection, contracted, deformed pupil, sluggishness or abolition of the movements of the iris, change of color, effusion of lymph into the pupil, plastic deposits in the anterior chamber, gelatinous or spongy iritis, more abundant in gonorrhœal than in ordinary iritis, obscurity of vision, photophobia, lacrymation, and peri-orbital and ocular pains.

"Fournier has described a rare conjunctival form. There is simple conjunctivitis, injection of the conjunctiva, uniform or marked at certain points, the secretion scanty and mucopurulent. There is sometimes absolutely no pain, no photophobia, no alteration of vision, no symptom of iritis or of aquocapsulitis."

This picture is a very different one from the violent course pursued by the virulent acute gonorrheal conjunctivitis which arises from direct contact of gonorrhœal pus with the conjunctiva. In the latter form the progress of the malady is exceedingly rapid, its manifestations are intense, and its destructive action is great.

The symptoms are: the characteristic burning of the under surface of the eyelid and eyeball similar to that produced by the presence of gritty substances beneath the lid. A few hours later, or almost at the same time with the pain, there occurs a mucopurulent, and soon a purulent, and profuse discharge. The conjunctiva is infiltrated and thickened, the eyelids œdematous and reddened, and if the process is not promptly checked, ulcerations form upon the cornea. Perforation into the anterior chamber of the eye and loss of sight may occur. There is marked constitutional disturbance in many of the cases.

Treatment.-The treatment of the first variety of the ocular infection consists in mild cleansing solutions applied to the conjunctiva with an eye-dropper, and of evaporating lotions to the exterior of the eyelids, combined with local treatment of the gonorrhœal lesions in the genitourinary tract, and if the symptoms are at all acute, liquid diet, rest, and moderate catharsis.

The conduct toward the other or violent form of the infection is very different. It must be attacked energetically. Keyes quotes Knapp with respect to the treatment, as follows: "He [Knapp] totally condemns the old-fashioned nitrate-of-silver treatment in the early stages of the malady, believing that this always does harm, and never aborts the disease, but, by adding a new irritative element, only kindles the flame and adds to the danger.

The nitrate is a coagulating agent. It does not penetrate.

The only instance in which it is per- 
fectly proper to apply nitrate of silver early in this malady is immediately after known infection, before any redness is seen or any irritation felt, as, for instance, when a little pus from an infected eye is accidentally spattered and gets into the eye of the surgeon or the nurse. In such a contingency, a 2 per cent. solution of nitrate may be once freely instilled into the contaminated eye with fair hope of aborting the malady. After three hours it is hopeless."

Keyes states the essentials of the treatment to be the three following things: (I) Antiphlogosis (cold); (2) cleanliness (irrigation); (3) antiseptics (bactericides).

"Cold applications are of the utmost importance, and their application must be unremitting night and day."

The manner of applying cold which is counselled by Keyes is by laying upon the eyelids bits of thin linen which are taken immediately beforehand from a piece of ice upon which they are to be laid when not in use. The application of these light bits of material should be renewed every four or five minutes. Keyes advises that the applications be continued as long as they feel grateful to the patient, and warns that the cold must not be too intense, else they may become harmful by interfering with the nutrition of the cornea, the evidence of which is the appearance of a misty area, which commences at the centre of this structure. If this appears, the application of cold must be immediately stopped. Irrigation beneath the lid should be frequently repeated, and the fluids advised by Keyes as best for the purpose are chlorine water, 4 per cent. boric acid solution, or permanganate of potash i to 6000 . These irrigations to be repeated every two hours. They should be supplemented by painting the entire conjunctiva with 5 per cent. to 15 per cent. argyrol solution, three times daily.

Chronic Urethritis.-Chronic urethritis appears in the form of areas of congestion and as so-called granulation tissue, and is also manifest in the orifices of the follicles of the urethral gland ducts.

(The changes in the tissues are described in the chapter on Stricture of the Urethra. The treatment of the condition is placed under the heading of Endoscopy.)

\section{ENDOSCOPY.}

Endoscopy consists in the examination and treatment of urethral lesions by means of tubes passed into the urethral canal and by the application of medicaments or of other local measures which are applied through the tubes by applicators, by means of delicate knives, probes, 
and other appropriate instruments, the operator being enabled to employ them by illuminating the urethral surfaces by reflected or direct light.

Instruments.-Many forms of endoscopic or urethroscopic instruments have been devised for the above-named purposes. The tubes which carry the electric lamps at their ends are preferable because of the better illumination which they afford. These tubes have the further advantage of allowing a curved instrument to be employed without in any way interfering with the lighting of the parts to be treated. Figs. ro and I I illustrate the tubes which we ordinarily use.

FIG. IO

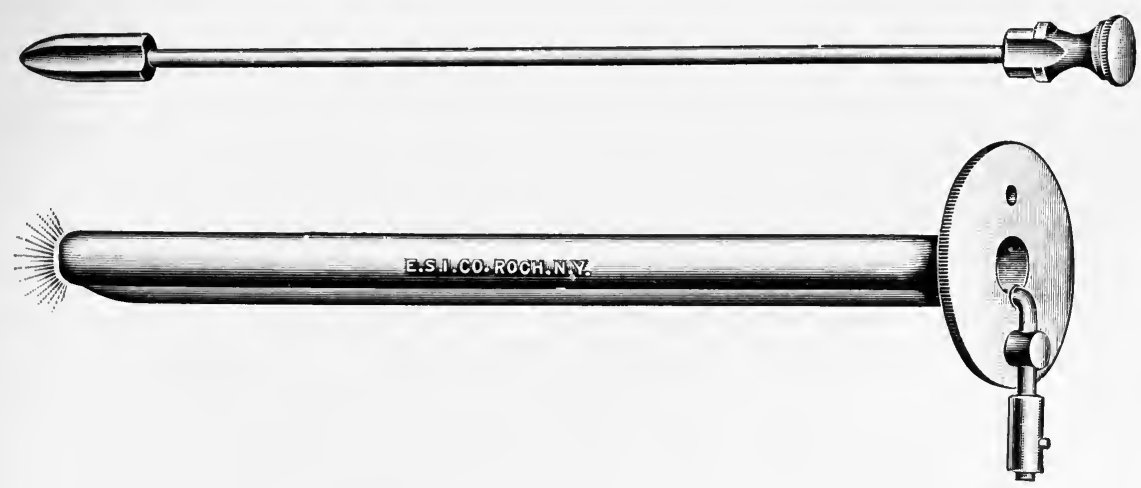

Electric endoscopic tube for the anterior urethra.

FIG. II

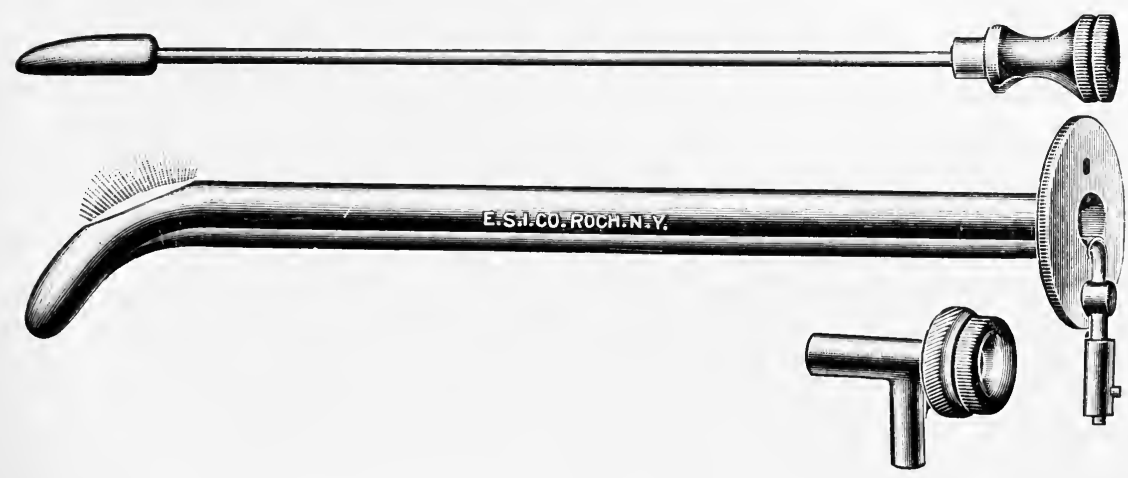

Electric endoscopic tube for the prostatic urethra.

Manner of Using the Instruments.- The examination can be made with the patient recumbent or in a semirecumbent position, as the operator may prefer. In the latter case the surgeon seats himself between the patient's knees, which are bent over the end of a chair or table, with the feet resting upon foot supports. The examiner's eyes should be on a level with the mouth of the tube when it is in place. 
The patient being properly placed for making the examination, the largest sized tube that the meatus will readily admit is passed gently into the urethra as far as the bulbomembranous junction of the canal.

From this point the instrument is slowly withdrawn, and the appearances of the different parts of the surface are noted as the end of the tube successively passes over them. It must be borne in mind that the picture presented is of circular form and slightly funnel-shaped, the latter character being given to it by the elastic quality of the urethra, which causes it to assume this shape as it closes again beyond the mouth of the tube while it is being withdrawn.

If the patient has urinated just before the examination is made, the flakes of pus and exudations which may have been adhering to the surface of the mucous membrane beforehand will, for the most part, be washed out by the urinary stream; if the examination is made without the patient having emptied the bladder, these elements will be found attached to the surface. They must then be removed by the cotton stick before the appearances of the urethra beneath them can be judged.

The cone-like form of the picture seen at the end of the tube is changed if the tube is canted up or down or to either side. The point which lies in the centre of the tip of the cone when the tube is held in the line of the natural axis of the urethra is carried to one side or the other of the field, and becomes eccentric instead of central, when the direction of the tube is changed in the manner described.

Appearances of the Normal Urethra.-The features of the urethra to be noted in making endoscopic examinations are: (I) the color; (2) the degree of elasticity of the walls of the canal; (3) the form, size, and shape of the lumen of the urethra beyond the end of the tube-that is to say, at the central part of the tip of the cone or funnel which has already been described.

Beginning at the bulbomembranous junction, the following appearances are seen as the tube is withdrawn:

The funnel shape of the canal beyond the end of the tube is lost and becomes a vertical opening, owing to the pressure exercised upon the sides of the canal at this point by its surrounding external muscles. Anterior to the bulb, the funnel shape is resumed, and its opening, or the continuation of the lumen of the canal at the tip of the cone, occupies a horizontal plane. The sides of the funnel are marked by radiating lines, which represent the partly smoothed-out folds of the mucous membrane, which are resuming their natural positions and character as the tube passes and permits the walls of the canal once more to fall together. This character is preserved until the fossa navicularis is reached, when the lumen becomes triangular in shape. 
The mucous membrane bulges but very slightly into the lumen of the end of the examining tube while it is being withdrawn, but does so very markedly if the tube is advanced into the urethra.

Color.-The color of the mucous membrane varies somewhat in the different parts of the urethra. It is also changed and becomes unlike its natural tone if pressure is made by the tube upon sides of the canal, or if the tube used is one that fits the urethra tightly.

Under natural conditions the color at the bulb is somewhat darker than in the portions immediately anterior to it. It is of a rather pronounced red tone. Throughout the rest of the anterior urethra, as far as the fossa navicularis, the color is about the same, and is of a pinkishred shade. (Plate XIV, Fig. I.) In the fossa navicularis it again becomes darker, until its anterior end is reached, when it changes suddenly to a much paler shade, and immediately behind the meatus becomes a pale yellow or almost white. (Plate XIV, Fig. 2.)

At either side of the floor of the canal, rather less than an inch anterior to the bulbomembranous junction of the urethra, are to be seen the openings of the ducts of Cowper's glands.

Throughout the rest of the anterior part of the urethra its walls are marked by numerous dot-like points of a somewhat darker shade than the surrounding surface, and these represent the orifices of the follicles which open onto the surface of the mucous membrane.

The lacuna magna is also to be seen upon the roof of the fossa navicularis.

The Examination of the Prostatic Urethra.-This can be made with a straight tube, but it is apt to cause a good deal of discomfort to the patient when that form of instrument is employed, and inasmuch as almost all the lesions which involve the deep urethra are situated upon its floor, the curved tube, with its window upon the convex aspect of the curve, which gives a view of the floor of the canal, is perfectly satisfactory for the purpose.

As this tube is drawn forward over the surface of the deep urethra, the caput gallinaginis may be recognized as a body of a darker red color than the parts which have preceded it and rises prominently into the lumen of the fenestrum of the tube.

If the tip of the tube be lifted upward, so as to remove pressure from the upper surface of the caput, the sinus pocularis can be seen as a slitlike depression upon its summit.

The orifices of the prostatic ducts can be made out on either side of the central ridge of the prostatic urethra. They are more clearly made visible if the magnifying lens, which is called the megaloscope, is used in making the examination. If this attachment to the instrument is employed, it 
must be borne in mind that all the details of the picture are exaggerated, and due allowance must be made for that fact in judging of their significance when studying urethral lesions in any part of the canal. The color of the deep urethra is a medium tone of red, except over the caput gallinaginis, where it becomes intensified into a much more pronounced shade.

The color of the surface of the membranous part of the canal is paler than that of the parts immediately before or behind it.

The Appearances Seen in Chronic Inflammation of the Urethra.The more important and characteristic of these appearances are as follows: the inflammatory process results in changes in the character of the urethral walls, and the loss of elasticity in them and the œdema which occurs are manifested by a change of the characteristic cone-like form of the lumen of the canal beyond the tube, and in changes in color as well. The canal is more open in the cases in which there is an infiltration of the submucosa, and this causes the cone-like process beyond the end of the tube to be much longer than in the normal urethra. One can see that the walls of the canal are stiff and inelastic. In the cases marked by œdema or congestion of the mucous membrane itself, the sides of the canal bulge into the lumen of the tube, and the funnel shape of the urethral lumen is wholly lost. (Plate XIV, Fig. 3.)

The Orifices of the Follicles and Gland Ducts.-In some cases these present the appearances shown in Plate XIV, Figs. 4 and 5. In others the orifices are concealed by an overlying film of exudate, and appear, when this is removed, as small depressions with elevated, reddened edges.

Granular Urethritis.--One of the most characteristic of the appearances of chronic inflammation of the urethra and of the stage of the process which precedes the formation of connective tissue, which constitutes stricture of the urethra, is the so-called granulation tissue, which occupies more or less well-marked areas of the surface, above which it rises slightly, and presents a surface which is sometimes of an uneven red color throughout, sometimes marked by yellow dots on the summits of numerous little elevations, giving to the part a resemblance to the surface of a strawberry. (Plate XIV, Figs. 6 and 7.)

Treatment of Chronic Urethritis.-Local application of nitrate of silver, administered through the endoscopic tubes, or by means of the distending air endoscope, is the most important of the remedial agents in the treatment of chronic inflammatory conditions of the anterior urethra, and of the posterior urethra as well.

The meatus, when it is too small to admit the larger sizes of the endoscopic tubes, should be cut, as a preliminary step in the treatment. 

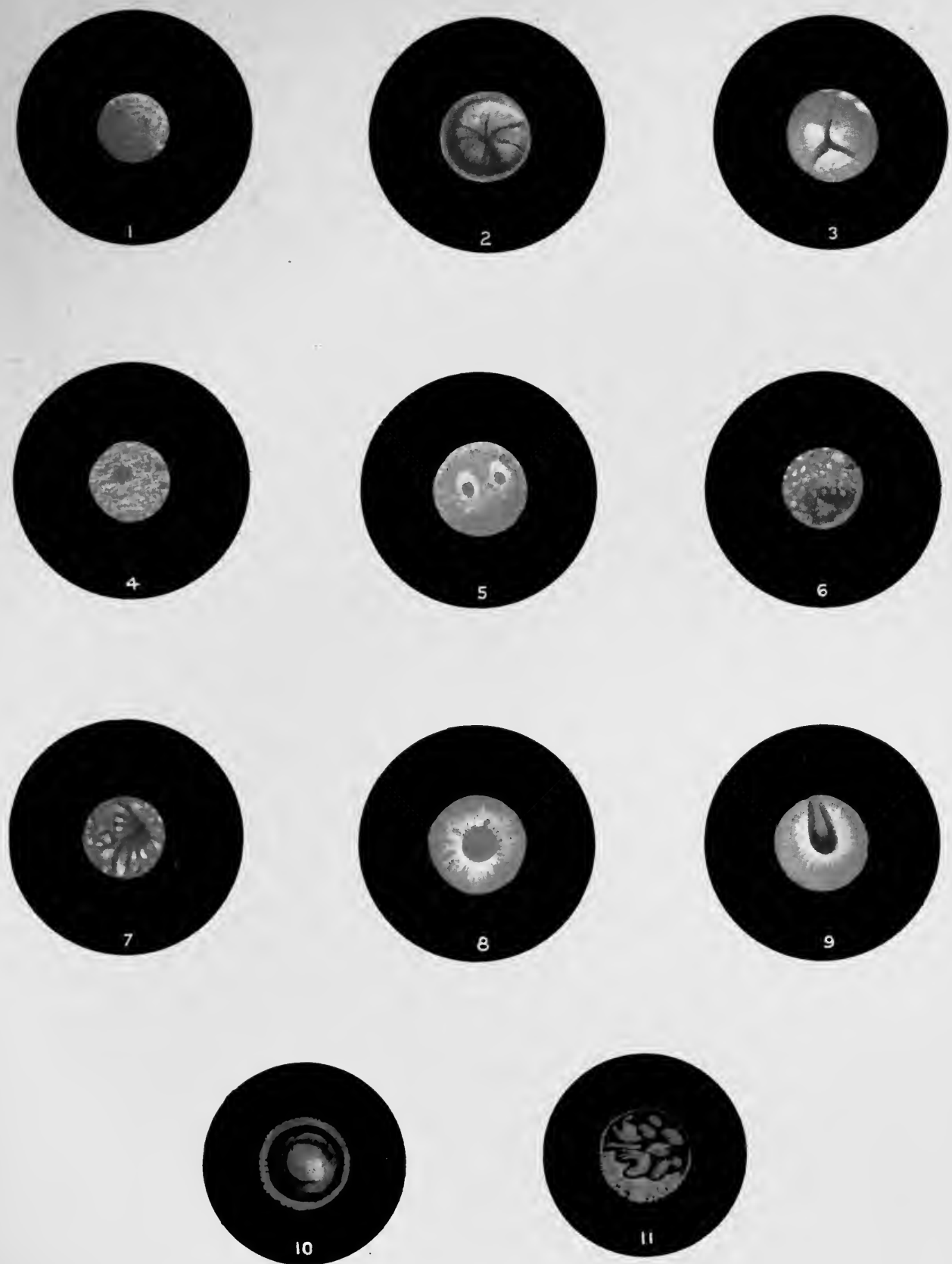

ENDOSCOPIC PICTURES OF THE URETHRA.

1, normal urethral mucous membrane; 2, normal opening of membranous urethra; 3 , œdematous folds at opening of membranous urethra; 4,5 , urethral glands in chronic urethritis; 6, granular urethritis; 7, granular urethritis, severe grade; 8 , stricture of medium grade, central lumen; 9, stricture after internal urethrotomy upward; 10, polyp; 11, papilloma. 


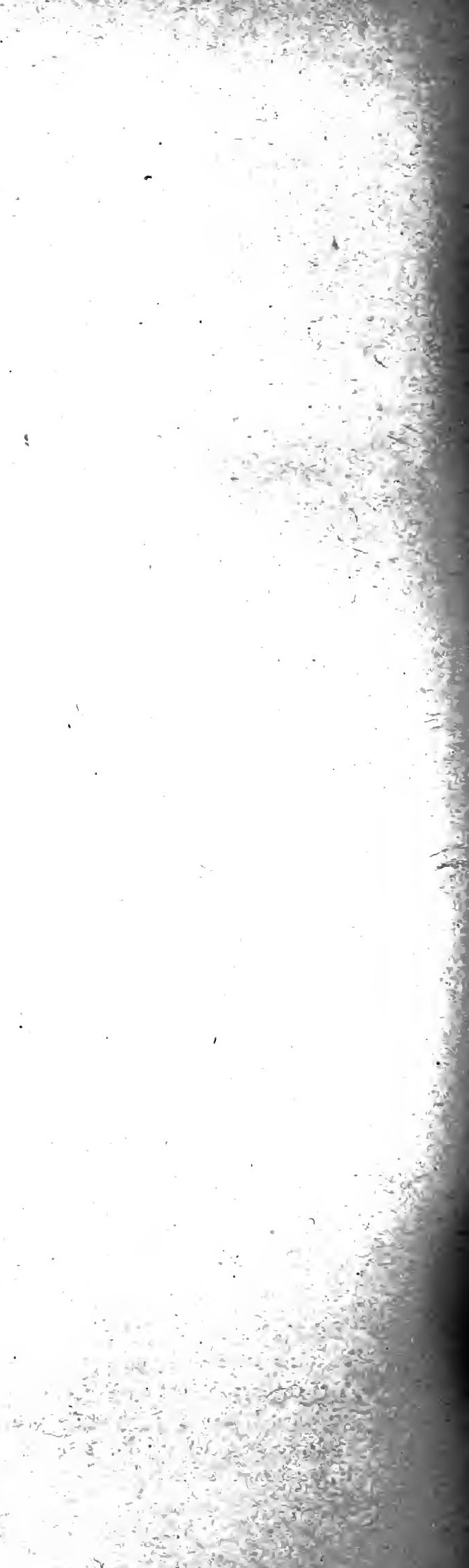


The advantage of using the larger sizes of the tubes lies in the fact that the folds of the mucous membrane of the urethra are smoothed out and the mouths of the follicles are exposed. With the smaller sizes, the part of the canal which lies at the bottom of the folds may escape contact with the medicament.

Nitrate of silver, to be efficient in the treatment of areas of chronic urethritis, should be used in strength of not less than a io per cent. solution, and it is often desirable to employ solutions as strong as 20 per cent. or 30 per cent. The same strengths, if applied as an injection, are not tolerated, and produce acute inflammatory reaction, whereas if the drug is applied by a cotton swab which is merely moistened with the solution, the result is beneficial. Moreover, thus used it is painless.

The treatment of chronic posterior urethritis consists in applying, by means of a syringe, a small quantity of one or another of the remedies mentioned below. Of these syringes for making the instillations, there are two sorts, one being that of Ultzmann, and the other that of Keyes. The tube of the Ultzmann instrument is fenestrated at its distal end by

FIG. 12

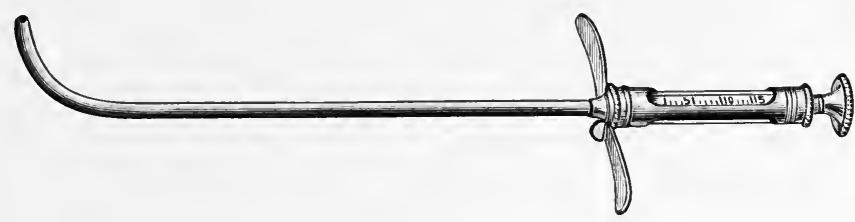

Keyes' deep urethral syringe.

four longitudinal windows near the tip; that of Keyes has a single opening only, which is placed at the extreme end of the tip. The form given to the nozzle of the syringe which fits into the other end of the tube in Keyes' instrument, is more convenient than in the other one (Fig. I2).

The applications in the anterior urethra are made in the following manner: The patient is directed to urinate. An endoscopic tube of a caliber of 26 to 28 Charrière scale is then passed into the urethra as far as the bulbomembranous junction. The obturator of the tube is removed. The tube is next slowly withdrawn, stopping it at each inflamed part of the urethra, and as each of these areas comes into view it is touched with the swab of cotton wound upon the end of the cotton stick and moistened in the solution, after having removed the surplus moisture from the swab with a bit of blotting paper. The whole of each of the inflamed parts should be thoroughly touched and brought into contact with the solution.

The treatment should be varied with different individuals, both with respect to the frequency of the applications and strength of the solutions. As a rule, the best results are obtained by making the applications every 
third or fourth day, and it is best to begin with weaker solutions and gradually increase their strength. The stronger applications should be reserved for the more obstinate cases of long-standing chronic inflammation, and it is generally unnecessary to apply them more than once or twice in the course of the treatment. Injections of mild solutions of permanganate of potash ( $\mathrm{I}$ to 6000) in the intervals are decidedly helpful.

As a rule, the duration of the treatment in the ordinary case in which the inflammation is neither deep-seated nor of long standing is from one to two months.

Two or three times during the course of it the urethra should be dilated by the passage of a full-sized sound, and massage of any thickened parts of it between the sound and the finger is often of advantage.

The measure of tolerance of each individual urethra to the frequency and strength of the applications should be established, and a universal rule should not be applied to all patients. Fit the treatment to the patient and not the patient to the treatment.

In our experience there is no substitute for nitrate of silver in the treatment which possesses anything like its value in cases of chronic urethritis.

Treatment of Posterior Urethritis.-There is an objection to the use of nitrate of silver in the posterior urethra which does not apply to its employment with the endoscope in the cases of chronic anterior urethritis, for if it is applied through a prostatic tube, the latter may do more harm than the application does good, while if the remedy is used in a larger quantity, as is the case when it is placed in the posterior urethra with a syringe, it is painful. It is, however, better to accept the latter evil rather than to lose the advantage that is to be gained by the use of the remedy in the form of instillations in the prostatic urethra. For this purpose, 4 to 8 minims of a solution of the strength of I per cent., subsequently increased to 5 per cent., or somewhat more, are placed in the posterior urethra by means of the Ultzmann or the Keyes syringe, both of which are excellently well suited for the purpose.

The sulphate of thallin in 3 per cent. solution, increasing it to 12 per cent. later, if its effect is beneficial, is advised by Keyes, more especially at the beginning of the treatment, and until the patient's tendency to reaction, or the contrary, has been learned.

Protargol has also given us satisfaction in the chronic form of the disease in the posterior urethra, and has the advantage of being painless. It may be begun in a strength of 4 per cent. solution and increased to Io per cent. if its employment is followed by improvement.

Treatment of Inflamed Follicles.-Inflamed follicles and gland ducts of the urethra, when their orifices are dilated, may be treated with direct application of pyrozone, or other medicament, by means of the delicate 
instruments devised for the purpose (Fig. I3). When it is seen by the endoscope that the orifices of these ducts are closed, and in consequence that they lack proper drainage, they can be laid open by means of the Kollman or other fine-bladed knife passed through the endoscopic tube (Fig. I4).

One of the most important of the conditions which the surgeon is called upon to treat locally in the urethra is that of peri-urethral abscess. The important matter involved in these cases is, whether or not to incur the danger of urethral fistula by opening the abscess externally in the cases in which there is already a communication with the urethra interiorly, or that of having the urine enter the cavity of the abscess by making an internal opening through which it may, perhaps, be admitted.

Fig. I3

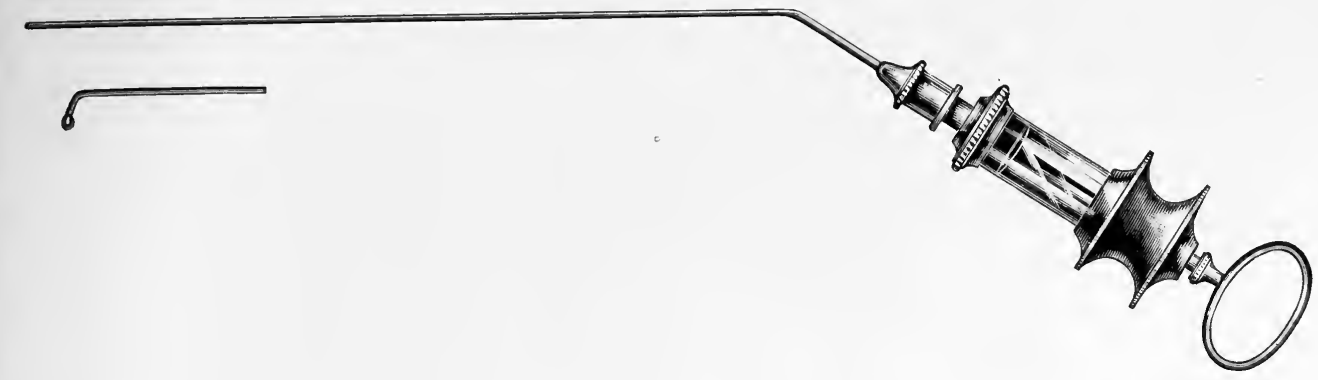

Tilden Brown's urethral syringe.

FIG. I4

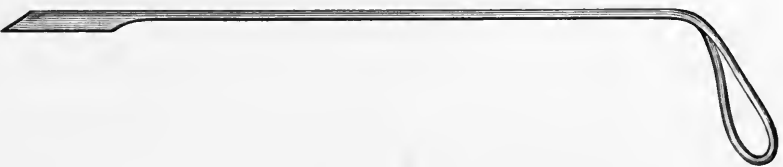

Knife for use in connection with the endoscope.

If the abscess is large, and if it communicates with the interior of the urethra by a small orifice, free external opening and proper aftertreatment of the wound, so that it shall heal from the bottom, will rarely result in urinary fistula. We do not sanction the internal opening of such abscesses, except the incision be of very limited extent and the abscess of comparatively small size. When this is the case, a small incision in the outer end of the duct or follicle will of ten result in cure without incurring the risk of having the urine enter the cavity of the abscess.

The after-treatment consists in the cleansing of the abscess cavity with injections of 25 per cent. pyrozone and in expressing its contents by pressure of the fingers upon the outside of it.

vol. I-6 
Stricture.-Stricture may be recognized by the whiter rim in the centre of the field, and by the harder tissue of which it is composed. (Plate XIV, Figs. 8 and 9.)

In cases in which the stricture has been cut by internal urethrotomy, the line of the former incision is easily recognized as a longitudinal white narrow area upon the roof of the canal.

The endoscope is often of service in detecting the orifices of strictures having eccentric openings, and in enabling the surgeon to enter the tip of an instrument into them.

\section{POLYP AND PAPILLOMA.}

Polyp and papilloma of the urethra are both rare conditions. As seen by the endoscope, they present somewhat the appearances shown in Plate XIV, Figs. Io and I I.

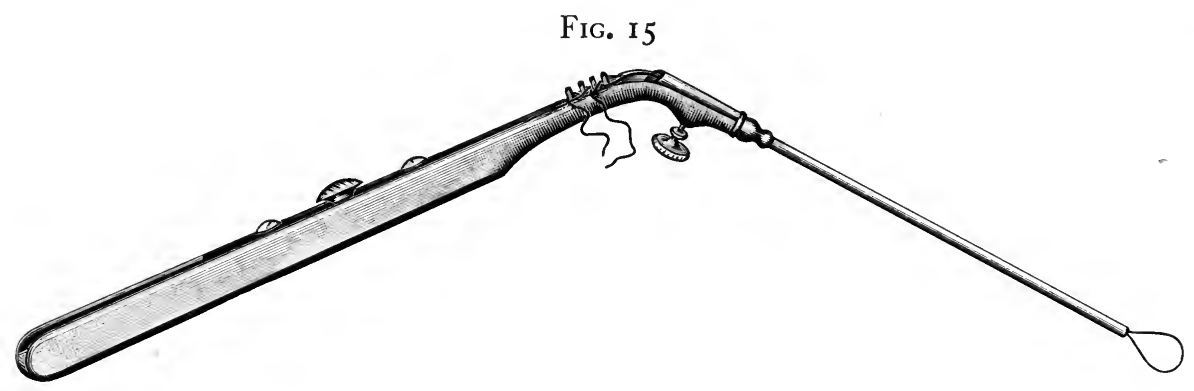

Urethral snare.

Fig. I6

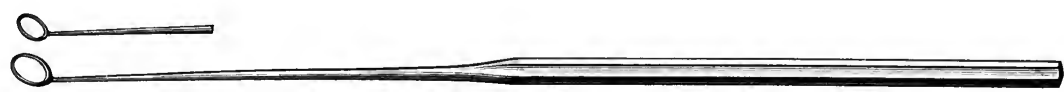

Urethral curette.

Treatment of Urethral Polypi.-Urethral polypi may be removed in most instances either with the fine snare or urethral curette (Figs. 15 and I6). The base of the growth after removal should be touched with nitrate-ofsilver stick, which is best applied by fusing a small quantity of it upon a probe and using the latter through an endoscopic tube, passing it into the urethra after it has been expanded by a Brown's urethral speculum.

Papilloma of the Urethra.-The removal of papillomata by curetting is attended by so much hemorrhage as to obscure the field of operation. To overcome this difficulty, Oberlander conceived the idea of breaking down the growths by applying friction to them with a tightly wound bit 
of cotton upon a tampon holder and rubbing the growths with it. A large-sized tube should be used. Bleeding is very much less free with this method of removal, and a few applications of the treatment usually suffice to reduce the growths to their bases, which may then be destroyed by caustics.

\section{Bibliography.}

I. Neisser. Centralbl. f. d. med. Wissensch., I879, Nr. 28.

2. Welander. Gaz. Med. de Paris, I884, p. 267.

3. Keyes. Genito-urinary Diseases, 1905, p. 55 .

4. White and Martin. Genito-urinary and Venereal Diseases, 6th edition.

5. Finger. Arch. f. Dermat., I 894, xxviii, 277.

6. Keyes. Op. cit.

7. Keyes. Op. cit. 


\section{H A P T E R I I I.}

\section{STRICTURE OF THE URETHRA.}

Definition.-In a general sense, stricture means any narrowing in the course of the urethra, irrespective of its nature or of its cause. Specifically, strictures are divided into: (I) Spasmodic. (2) Inflammatory. (3) Organic. (a) Gonorrhœal. (b) Traumatic. (c) Congenital.

Spasmodic Stricture.-Spasmodic stricture is a more or less temporary partial or total closure of the urethra produced by spasmodic contraction of some part of its own muscular fibers, which is rare, or of the compressor urethræ muscle outside of it, which is common.

Such spasm may be produced by a number of different agents, such as psychic influence, as in the familiar example of persons who are unable to urinate in the presence of others; an irritating quality of the urine; the use of irritating urethral injections; the passage of instrumen s into the urethra; the presence of stricture in the anterior urethra; irritation transmitted from a diseased condition of a neighboring part, such as hemorrhoids, or inflammation of the seminal vesicles.

Other and remote lesions are sometimes the cause of spasm. Keyes ${ }^{1}$ refers to two cases reported by Thompson and Brodie, in which the patients had periodic spasm in connection with malarial attacks, and were both cured by quinine.

Again, spasm is sometimes seen in connection with renal lesions, notably in cases of renal calculus.

Inflammatory Stricture.-This term is used to signify a temporary narrowing or closure of the urethra produced by acute inflammation, or by abscess formation in the urethra or peri-urethral glandular structures, respectively.

Organic Stricture.-Organic stricture is a narrowing of the urethral canal due to the formation of connective tissue in or around its mucous membrane, which connective tissue represents the end product of a chronic inflammatory process, almost invariably of gonorrhœal origin, or produced in the healing of an injury of the urethra, the stricture in that case being called traumatic.

Traumatic Stricture.-This variety of organic stricture is classed separately because of its being caused by a different agency to that which produces the great majority of strictures and because of its possessing certain special characteristics.

$(84)$ 
In most cases of traumatic stricture the injury is in the nature of a blow or crushing force applied to the urethra. The larger part of such injuries fall upon the perineal portion of the canal, and are incurred commonly by such accidents as falling astride of a wheel or fence, or by receiving a kick upon the perineum. Some of the less frequent forms of violence productive of the urethral lesion will be found in Nos. I, 2, and 3 of illustrative cases at end of this chapter.

The characteristics of traumatic stricture are referable to the nature and extent of the injury inflicted upon the urethra, and to the correspondingly large deposit of connective tissue produced in the course of healing at the site of the injury. Owing to this fact these strictures are very rapid in their formation, and are dense and non-dilatable. They are consequently difficult to cure.

Obliteration and Stricture of the Urethra (Congenital).-Among the congenital abnormalities of the urethra, stricture of the canal is sometimes observed. Obliteration of the canal in different degrees, from total obliteration to a localized obliteration of one or another part of it, also occurs.

Total and Partial Obliteration.-The complete obliteration of the urethra is a rare abnormality, but undoubted instances of it have been reported. In these, the urethra is represented by a small band in which, however, the elements of the urethral structure are demonstrable.

In the cases of partial obliteration of the canal, the closure may be situated in any part of it; cases have been reported in which there have been several points in the course of the urethra which have been obliterated.

Stricture of the urethra of congenital origin may occur at any point of the canal, even in the prostatic urethra.

Congenital narrowing of the meatus and of the urethra immediately behind it are not uncommon. Constrictions deeper in the canal are not so rare as might be supposed, and the less serious grades of the narrowings are often overlooked, or the symptoms resulting from them are referred to a wrong source.

According to Frisch and Zuckerkandl, ${ }^{2}$ the narrowing which has been observed in some cases at the point of division between the posterior limit of the fossa navicularis and the beginning of the spongy part of the urethra is frequently associated with congenital phimosis and narrowing of the meatus. In some cases it appears in the form of a hard constriction without the co-existence of either one of these associated conditions, and in still other instances it takes the form of a valve-like fold of the inner part of the lining of the canal, in the latter cases representing the remains of the septum which originally separated the two parts named. 
In the deeper portions of the canal, the same authors speak of the congenital narrowings, as follows:

These strictures have a circular form, or appear as folds and valves.

Of the first variety-a very rare condition-they cite, among other cases, that reported by Guibe, ${ }^{3}$ of a newborn male child who died sixteen hours after birth. Autospsy showed a circular stricture $5 \mathrm{~mm}$. in front of the veru montanum, which admitted only a steel knitting needle. The urethra posterior to it was enormously dilated and continuous with the bladder, so that there was no distinction between the two cavities. The bladder itself was hypertrophied, and trabeculated; both ureters were dilated and both kidneys showed cystic degeneration.

In the cases of obliteration, the subjects are either born dead or survive but a short time.

In these instances the bladder is found enormously dilated; occupies the whole of the abdominal cavity, and forms a tumor so great as to have produced rupture of the uterus in one case during parturition. The ureters and kidneys share in the dilatation. (See Cunningham's case at end of this chapter.)

Pathology of Stricture.-Tissue changes beginning in chronic gonorrhaal urethritis and ending in organic stricture. Finger ${ }^{4}$ gives the following description of the changes found by him in the tissues in the course of a long and careful study of them in their different stages.

"In some instances the epithelium may preserve its cylinder-cell character, but the more superficial layer of these cells is disorganized, exfoliated, and in a state of mucous degeneration.

"The stratum of transitional cells, which normally consists of one or two layers, is now converted into many.

"Numbers of pus cells are found between the layers of transitional cells and the cylinder cells. Another feature is the conversion of the cylinder to pavement cells.

"The subepithelial connective tissue is the seat of the essential changes characterizing the chronic inflammatory process, and it is upon the extent to which this particular part is affected that the subsequent course of the disease depends.

"The pathological process here consists primarily of a round-cell infiltration of greater or less extent, in the train of which the connective tissue takes on, in varying degree, a contractile character, and ultimately that of cicatricial tissue.

"The depth to which the infiltration extends also varies, in some cases being only superficial, in others invading adjacent structures, not infrequently the corpora cavernosa.

"The area occupied by the infiltration may be extensive, and the cell 
infiltration evenly distributed in the parts affected, but in most cases it manifests a preference for the portions of the urethra in which the lacunæ and glands are most numerous." (Finger, however, states that he has never seen an instance in which it was confined to a periglandular or perilacunar process.)

"The round cells which characterize the early stages of the infiltration subsequently show a large admixture of spindle cells, the latter being in part derived from them, and in part from the epithelioid cells.

"In some cases there is a rich new formation of capillary bloodvessels in the infiltrated area, and it is this factor which gives to the surface its papillary appearance, for which reason also it is often called granulation tissue.

"If granulation tissue has characterized the process and subsisted for a considerable period, contraction subsequently occurs, and the surface then becomes flattened and indurated. The degree of the change that takes place depends upon the extent and depth of the area involved in the first phase of the infiltration. In some cases it is limited to a small area and is superficial, while in others the reverse is the case.

"The lacunæ are involved in a process of a character similar to that just described. Owing to the infiltration around the lacunæ, the mouths of the latter are sometimes drawn open and the lacunæ are lifted up, giving the appearance of crater-like elevations upon the surface of the mucous membrane. When the infiltration goes on to the formation of connective tissue, the lacunæ ultimately atrophy and disappear.

"The glands of Littre, which are situated in the framework of the corpora cavernosa, and reach the surface of the mucous membrane by long, winding ducts, are involved by the infiltration which begins about the orifices of the ducts as a periglandular process, then advances to and around the glands themselves; next, the epithelial lining of the ducts is attacked, and a cell transformation similar to that described above takes place. This invasion moves onward, penetrates to the finest branches of the ducts, and finally pushes beneath the epithelial cells of the gland itself and destroys the latter by pressure."

One of the conclusions reached by Finger is that the areas of chronic gonorrhœal urethritis localize themselves by preference in the pendulous, bulbous, and prostatic portions of the canal; the membranous portion is, according to him, rather immune to chronic inflammation of this sort. In 32 cases he found but 2 in which that part of the urethra was affected, and in these the inflammation was of a superficial nature.

Kaersmaecker and Verhoogen, on the contrary, think that the membranous urethra is a frequent seat of the chronic inflammatory process, and that it attains a greater degree of severity there than Finger and 
others believe. They assert that when this part of the urethral mucous membrane is attacked, it splits into fissures, because of the fixation of this portion of the canal and because of the action of the compressor urethræ muscle upon it, and that in these fissures changes take place which end in the formation of connective tissue, thus frequently giving rise to stricture of that particular portion of the canal.

Total Occlusion of the Urethra Due to Injury from Chemical Agents.-Now and then this condition is met with, as in the example described in No. 9 of illustrative cases at end of this chapter. When a chemical agent of a sufficiently corroding character is injected into the urethra, there may result a sloughing of the whole inner lining of the canal and an excessive deposit of connective tissue, which is formed $\mathrm{n}$ the course of the healing of the injured area. This will not extend beyond the anterior urethra, in all probability, the deeper parts of the canal having been protected from contact with the fluid by immediate spasm of the compressor urethræ muscle. Contraction will be more rapid under such circumstances than with the usual forms of organic stricture of gonorrhœal origin, and will probably result in a total occlusion of the urethra, as in the writer's case, referred to above. Such a stricture is wholly unamenable to the ordinary measures of treatment.

Secondary Changes Due to the Presence of Stricture.-The secondary effects of stricture are produced in two ways, the first being by mechanical influence, and the second by infection.

I. Mechanical Effects.-The first evidence is seen in the form of an ampulla-like dilatation of the canal immediately behind the stricture. This is produced by the increased pressure upon the urethral walls by the urine meeting an obstacle in its outward course. As the stricture contracts, the same effect is extended and produces similar results in a wider area; the back pressure is presently felt by the bladder, upon which the demand for harder work in order to expel the urine is thus made. As a consequence, one of two things happens: he bladder meets the demand by an increase of its muscular apparatus, and undergoes a compensatory hypertrophy, or its walls fail to accomplish this, and become thin, flabby, and lose their contractile power, and, as it is called, atony of the bladder results.

Next in order, the ureters are subjected to the same influence, become dilated, and lose their propulsive force; finally, the pelvis of the kidney undergoes either a compensatory hypertrophy or dilatation, and, as a last step, the pressure brought to bear upon the interior of the kidney itself may produce atrophy of its parenchyma and the condition known as hydronephrosis. The existence of these conditions invites bacterial invasion of the respective parts affected by them, and it is not uncommon 
to have the invitation accepted. In this way there comes about the second of the tissue changes which are referable to the presence of stricture, viz.:

2. Infection Due to the Presence of Stricture.-Some of the products of chronic inflammation and a little urine are retained behind the stricture and serve to perpetuate the localized inflammation. These foci are points from which an extension of the inflammatory process at any time may, and not infrequently does, take place.

When this happens, any or all of the different parts of the genito-urinary tract may become involved. In the urethra, the follicles and perifollicular tissues and the glands communicating with its inner surface are often implicated, and there may result, owing to the closure of the mouths of the ducts and in obedience to the virulence or the contrary of the infecting organisms, suppuration of a severe and rapidly advancing type, or a circumscribed abscess of small size, very slow progress, which,

FIG. I7

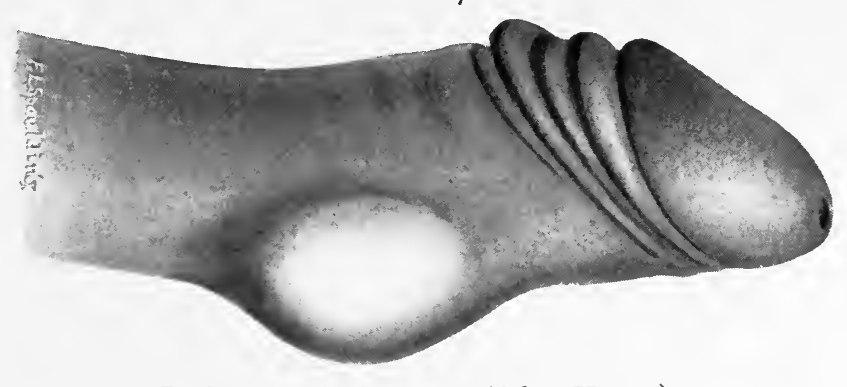

Peri-urethral abscess. (After Keyes.)

after reaching a certain point, may remain stationary for an indefinite period, giving rise to no constitutional disturbance, and, very possibly, being overlooked by the patient. The latter variety is frequently found in the anterior part of the urethra, and is often manifested in the form of hard nodules, which can be felt beneath the skin along the sides and the floor of the urethra. The name follicular abscess (Fig. I7) is usually given to these circumscribed forms of peri-urethral suppuration, the more important and serious ones, which frequently have their seat in the glands of Cowper, or of Littre, are generally classed as periurethral abscess, and will be included in the discussion of the part of the subject which comes under the next heading.

Urinary Extravasation or Infiltration.-Diffuse Phlegmonous INFLAMmation.- The theory entertained formerly with respect to the manner in which the condition ordinarily called urinary extravasation is produced was as follows:

Under the influence of hydrostatic pressure upon a localized area of 
the urethra already weakened by chronic inflammation, such as is found behind a stricture, the urethral wall finally gives way at this point, and thus provides an outlet for the escape of urine into the peri-urethral tissues; rapidly, if the rent is of large extent and the bladder forces the urine through it forcibly; slowly, if the contrary conditions prevail.

The following facts show that this explanation is inadequate:

I. The condition which is usually termed urinary extravasation occurs in some instances in the absence of stricture of the urethra, or in cases in which there is but a very slight constriction of the canal.

2. Also in some cases in which there is no opening communicating with the urethra.

3. In some cases the opening into the urethra is in front of the stricture.

4. Delbet and Albarran have shown that there is no urine in the extravasated fluids in some of the cases examined by them, and that the fluids which have been thought to be urine have been the products of an acute diffuse phlegmonous inflammation.

A suggestive fact is that many of these infiltrations and urinary abscesses connected with them originate in the glands of Littre and of Cowper, and are manifested as perineal abscess.

Motz and Bartrina ${ }^{5}$ attribute a most important role in the production of perineal suppuration to the glandular structures just mentioned.

They show that the glands of Littre, of Cowper, and the glands generally found near the bulbous urethra are the frequent starting points of peri-urethral and perineal suppurations and of the so-called condition, infiltration of urine, the microbes of the urethra being the infecting organisms, and finding their way to the glandular structure itself through the excretory ducts of the above-named glands.

They state that the resulting inflammations and suppurations appear in the following forms:

I. As an insidious suppuration, which does not produce symptoms, is of limited extent, and is usually detected at autopsy only.

2. As a perineal abscess, circumscribed, and giving rise to grave symptoms.

3. In the form of a diffuse phlegmonous inflammation, which rapidly exceeds the limits of the perineum and extends widely over a large area.

These suppurations may or may not break into the urethra. If they do, the urine enters the already infected district and aggravates the condition by engrafting upon it a secondary infection. The urinary element then becomes an important factor of the process, but is not, as has been supposed, the primary causal agent. Fig. I 8 illustrates the condition just described. 
In the cases in which no communication is established with the urethra by the breaking into it of the already existing suppuration from without, a diffuse phlegmonous process may take place, under which circumstances urine will not be present as a factor in the production of the condition. In 8 out of $\mathrm{r} 8$ specimens of perineal suppuration and so-called infiltration of urine examined by Motz and Bartrina, there were 3 in which there was no communication with the urethra. (See Nos. 4 and 5 of illustrative cases at end of this chapter.)

FIG. 18

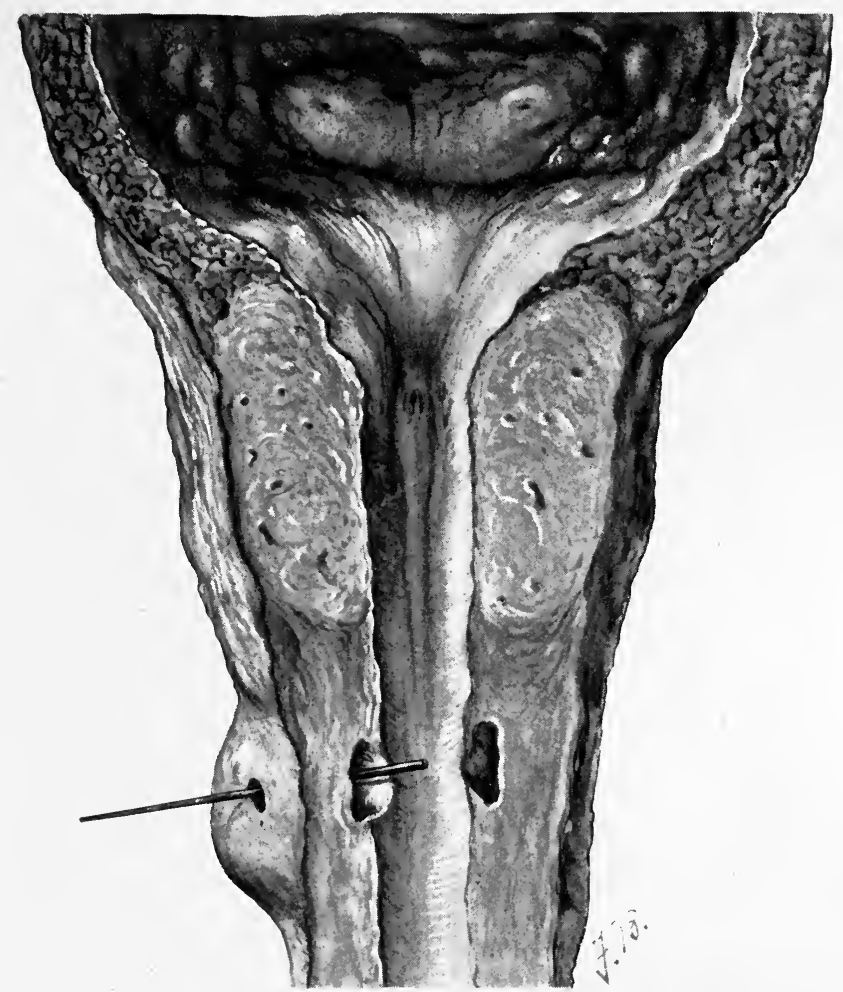

Urinary extravasation originating in abscess of Cowper's gland which ruptured into the urethra.

Anatomical Distribution of the Fluids in Cases of Urinary Infiltration.The course followed by the extravasated fluid through the tissues is determined by the anatomical arrangement of certain structures of the parts involved, in correspondence with which there are three varieties of extravasation produced.

I. That in which the communication with the urethra is in front of the anterior leaf of the triangular ligament.

2. That in which the process takes place at first between the two layers of the triangular ligament. 
3. That which has the opening behind the posterior leaf of the triangular ligament.

(a) In the first variety the fluid, after invading the spongy tissue immediately around the urethra, pursues one of two courses. The first and most frequent one is that in which it breaks through the fibrous sheath of the penis and makes its way into the areolar tissue of the scrotum, and from there upward above the symphysis pubis, its extension upward over the front of the body being limited only because of necrosis of the skin at some point, and the escape of the imprisoned fluid through the opening thus made.

\section{FIG. I9}

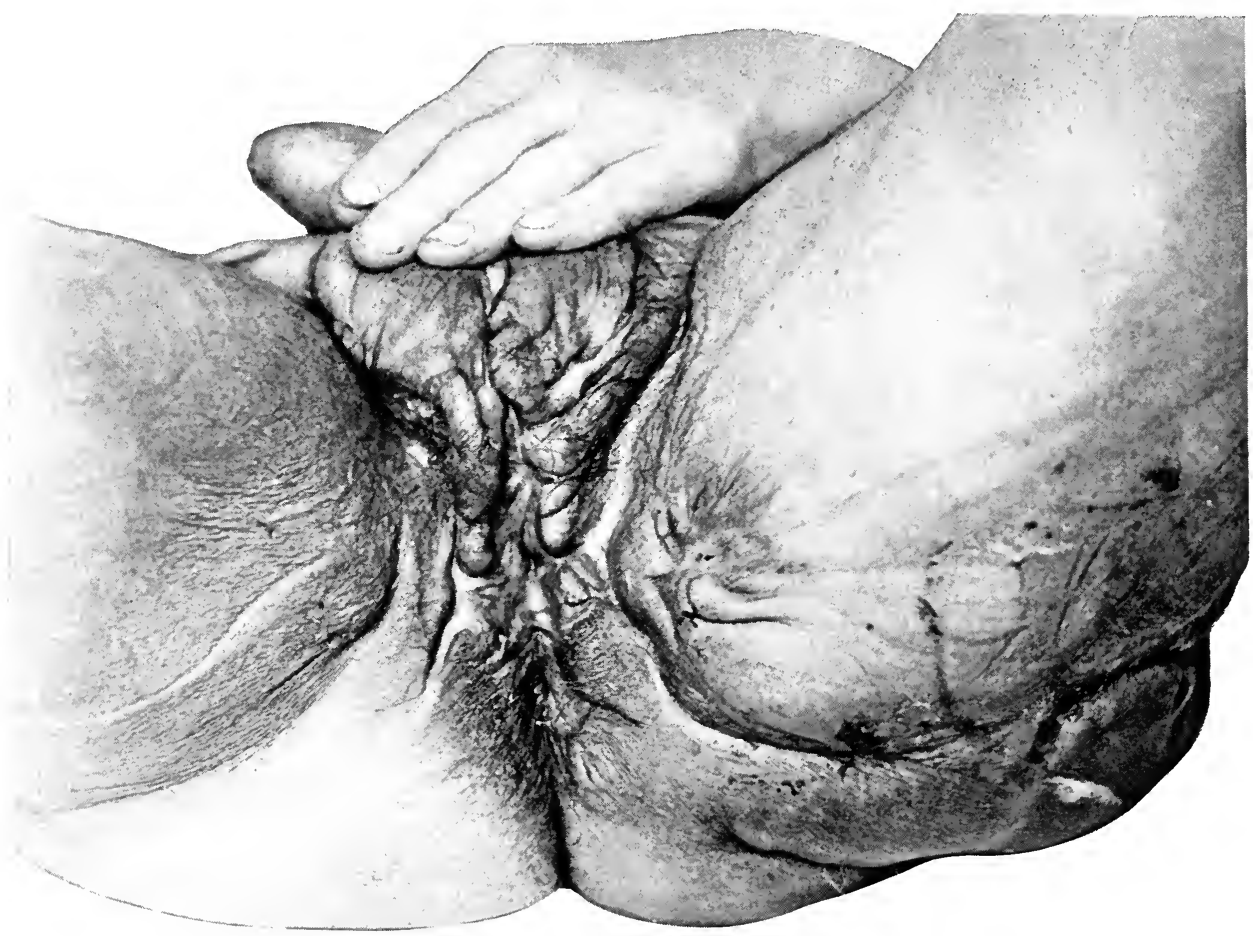

Showing results of extensive extravasation of urine.

Instead of doing this, the other course referred to may be taken, in which case the fluid remains within the envelope made by the fibrous sheath of the corpus spongiosum, works its way forward, and appears upon the dorsum of the penis, at first between the corpora cavernosa, from which point it extends backward beneath the integument of the penis.

(b) In the second variety, the extension of the fluids forward and backward is limited by the two layers of the tough triangular ligament. 
It cannot advance upward because of the union and insertion of these two layers above, consequently it takes the only road left, which is downward, toward the surface of the perineum, beneath which it appears as a more or less well-marked swelling. The fluid may then burrow as far as the tuberosities of the ischium and into the loose tissue of the ischiorectal fossæ, or it may push outward and break through the integument of the perineum. In some cases it extends as far laterally as the trochanters breaking through the skin at one or more points, and thus giving rise to the formation of fistulous tracts through which pus and urine may escape for an indefinite period if there is no surgical intervention. Such a case is illustrated in Figs. I9 and 20.

FIG. 20

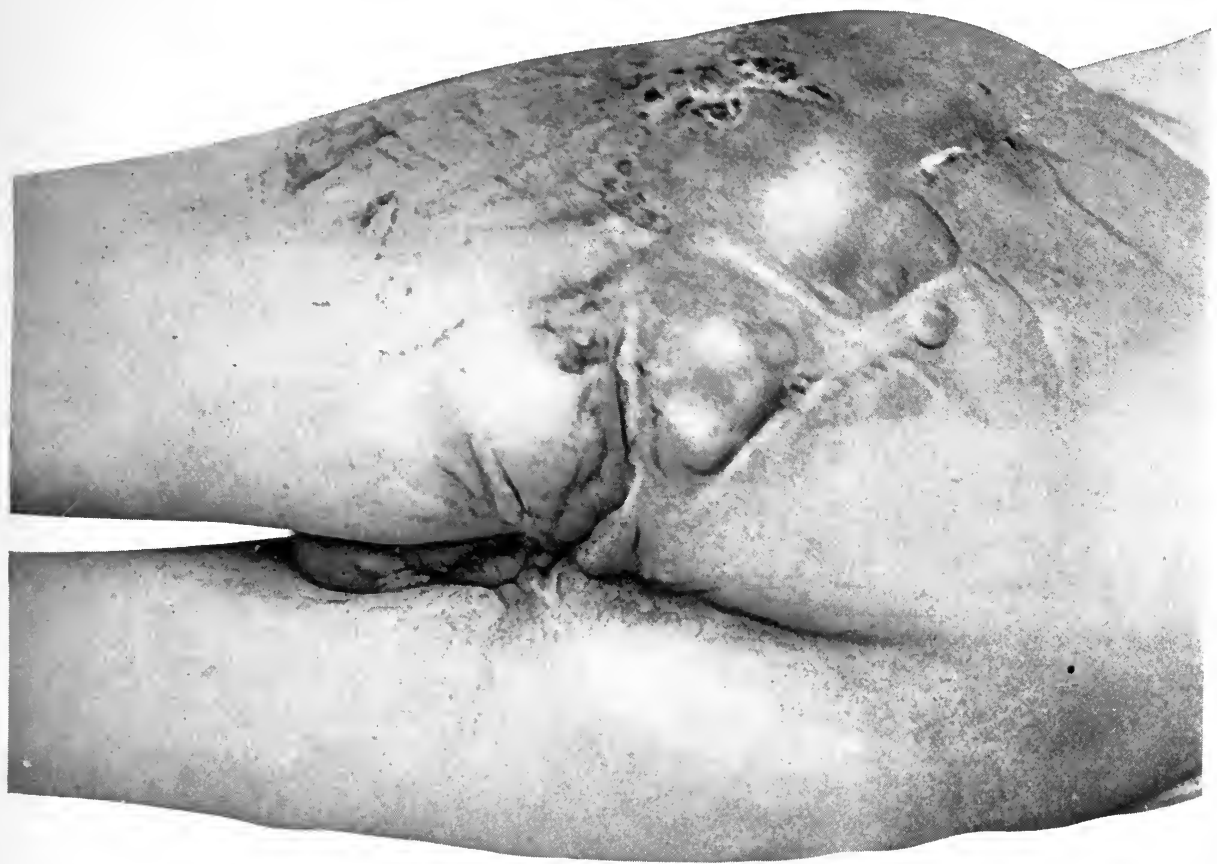

Showing results of extensive extravasation of urine.

(c) In this variety, the fluid makes its exit through an opening behind the posterior layer of the triangular ligament. Here the extravasation has access to the pelvis, and burrows around the rectum, into the space of Retzius, and perivesical tissues. The peritoneum may be perforated, allowing the entrance of the infiltrating fluid into the peritoneal cavity and causing a fatal peritonitis.

In those cases classed by Motz and Bartrina ${ }^{5}$ and De bet and Albarran as diffuse phlegmon, the rapidity of the invasion and the violence of the 
septic infection are extreme, and remarkably destructive of the parts involved in the process. Within a few hours, sometimes, from the beginning of the attack, gangrene of more or less of the integument of the scrotum and of the penis may occur, while rapid necrosis of the

FIG. 2I

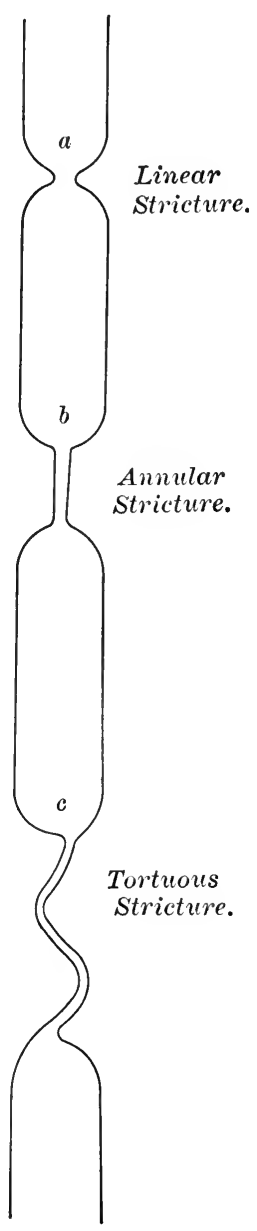

Diagram of different forms of stricture.
Fig. 22

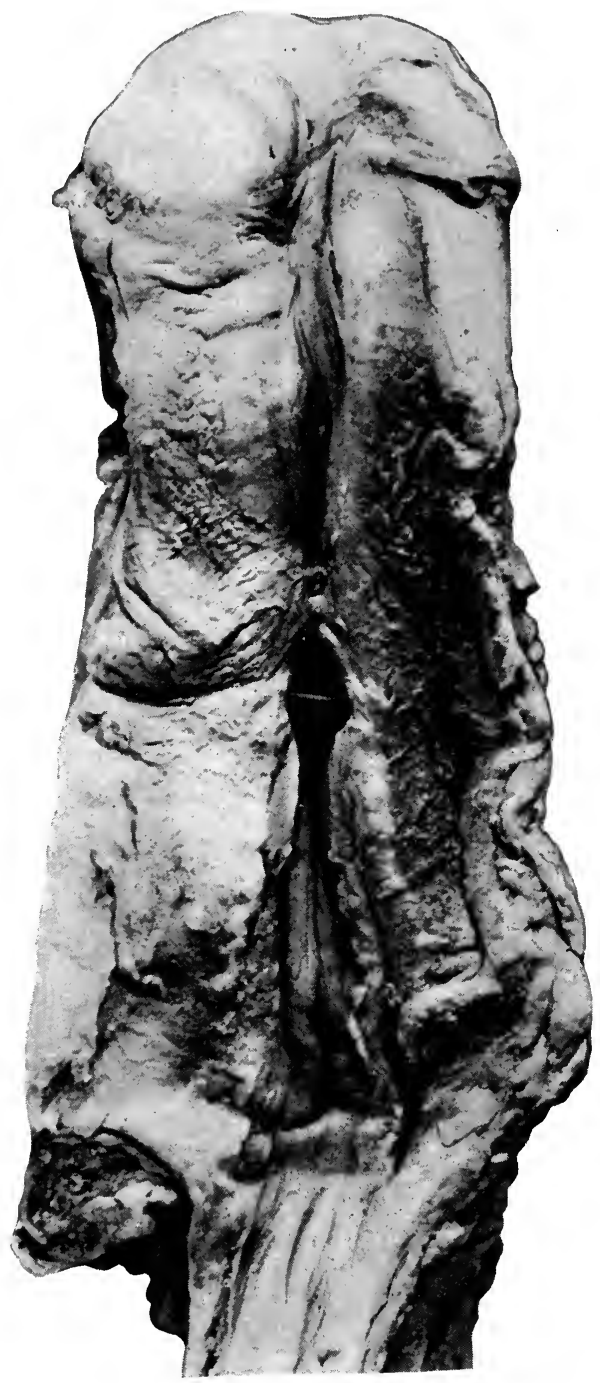

Specimen showing annular stricture.

urethra and the deeper tissues of the penis and perineum follows. (See No. 6 of illustrative cases at end of this chapter.)

Varieties of Stricture.-There are many descriptive terms applied to designate the special qualities of strictures. The only ones which are 
essential are the three following: (1) Linear. (2) Annular. (3) Tortuous.

Linear stricture is well represented by tying a thread around the outside of a rubber tube. When this is done, there is created on the inside of the tube a correspondingly narrow constriction, which will close the lumen of the canal, more or less, according to the tightness with which the thread is drawn (Fig. $2 \mathrm{I}, a$ ).

Annular stricture may be similarly imitated by substituting a bit of tape for the thread. This will produce a constriction of the inside of the tube, which will occupy a wider extent of it than that created by the thread (Fig. 21, b).

A tortuous stricture is one that is made by a greater deposit of connective tissue at some parts of the canal than at others. This condition compromises the caliber of the tube more at the parts where there is more connective tissue deposited than at those where there is a smaller amount; as a result, the passage presents a series of irregular narrowings and the canal becomes tortuous (Fig. 21, c).

Fig. 22 shows stricture of annular form in the urethra, which has been laid open to expose it to view.

Such terms as resistant, undilatable, impermeable, impassable, etc., explain themselves.

By resilient stricture is meant one that is elastic and springs backrecontracts - to its former size quickly, after being stretched or dilated by the passage through it of an instrument.

Strictures of wide caliber are spoken of without any special agreement as to the meaning of the term; as it is ordinarily used, it signifies those which are of the size of about 28 or more of the French scale.

Number of Strictures. - Cases have been reported in which as many as fourteen strictures have been found in one urethra, and there may be any number between this and a single stricture.

Sir Henry Thompson ${ }^{6}$ examined 270 specimens with reference to the numbers of strictures present in the individual cases, and found that there was but one in each of 226 of the number.

The writer's experience from clinical examinations does not agree with these observations. In 300 cases in which he examined the urethras of patients with stricture, the following were the numbers present:

In 198 (60.0 per cent.) there was but I stricture.

$$
\begin{aligned}
& \text { " } 73(24.0 \text { " ") " were } 2 \text { strictures. } \\
& \text { " I6 ( } 5.0 \text { " " }) \text { " " } 303 \\
& \text { " } 9 \text { ( } 3.0 \text { " " }) \text { " “ } 404 \\
& \text { “4 ( } 1.3 \text { ") " " } 45 \text { or more strictures. }
\end{aligned}
$$


Location of Strictures.-The tendency of strictures to occupy certain parts of the urethra in preference to others is conspicuous. According to the observation of Sir Henry Thompson, ${ }^{6}$ the sites of stricture in 270 specimens examined by him were as follows:

r. Within the part of the urethra beginning $I$ inch in front of and extending $\frac{3}{4}$ of an inch behind the bulbomembranous junction.

2. Between the anterior of these limits and a point $2 \frac{1}{2}$ inches behind the meatus

3. In the first $2 \frac{1}{2}$ inches from the meatus

215, or 67 per cent.

$5 \mathrm{I}$, or 16

54 , or 17

In I98 cases examined clinically by the writer, the following were found to be the sites of the constrictions:

I. Opposite the penoscrotal angle

2. In the bulbous urethra or at the bulbomembranous junction, and extend-

ing back into the membranous urethra .
Within the first inch and a half behind the

4. Between the latter point and the penoscrotal angle

5. Between the penoscrotal angle and the bulbous urethra

The points of the urethra at which strictures are seen to be most frequently seated are, roughly speaking-according to the last series of observations-those at which normally there are points of narrowing as compared with the parts immediately behind them. It is probable that the irritation produced by the increased friction and the retention of inflammatory products at these points is the reason for their being selected for the localization of the subsequently formed strictures.

Owing to the fact that most of the injuries of the urethra fall upon the part of it which corresponds to the perineum, it is in that part of the canal that the majority of traumatic strictures are found.

Stricture of the prostatic urethra is of the rarest occurrence. Keyes ${ }^{1}$ quotes as authentic examples of the condition in this part of the canal cases reported by Walsh, Leroy d'Etiolles, Ricord, Civiale, and Mastin.

There is a wide difference of opinion as to the existence or frequency of occurrence of stricture of gonorrhœal origin in the membranous urethra. It is usually held that stricture limited to the membranous urethra alone, and of gonorrhœal origin, does not occur, or but very rarely. That such strictures are rare is doubtless true, but that the membranous urethra is never the seat of stricture is far from being the fact. Both clinical observations and microscopic examinations prove the contrary. But it is probable that stricture of the membranous urethra alone is of the rarest occurrence. It is, as has been shown by Bazy and Decloux, ${ }^{7}$ an extension of the stricture into the membranous portion from the urethra immediately in front of the bulbomembranous junction, and not a stricture 
originating in and wholly confined to the membranous part of the canal. The writer has included five cases of stricture in his list of examinations of the location of the disease as being of the membranous urethra, but they are so called on the strength of what was seen at the time of operation, and it is impossible to determine the exact limits of a stricture under those circumstances.

Time of Formation of Stricture.-We have never seen an organic stricture form sooner than eight months after the beginning of an attack of gonorrhœa. Stricture may form at any time between this and twenty years. The average time is about three years after the gonorrhœa from which it results. With traumatic strictures it is much shorter.

Symptoms.-The symptoms of stricture are: (I) Gleet. (2) Changes in force, size, and form of stream. (3) Increased frequency of micturition. (4) Loss of sexual power.

GLEET.-The slight urethral discharge which persists after the subsidence of acute gonorrhœa, and which is called gleet, is one of the most constant symptoms of stricture. A gleet which continues longer than six months after the beginning of an attack of gonorrhœa should always excite a suspicion of beginning stricture formation.

Gleet may be present without stricture, and, conversely, stricture may exist without gleet. In the former case the discharge may represent the products of chronic inflammatory or suppurative processes in any of the following structures: the glands of Littre, of Cowper, of the prostate, the ejaculatory ducts, or seminal vesicles. The inflammatory products emanating from the glands which are within the membranous urethra, or behind it, are usually passed into the bladder, and appear only in the urine. This is not always true, however; when the compressor urethræ muscle is relaxed, they may show as a gleet exuding from the meatus. In a considerable number of cases in which gleet appears as a symptom, Cowper's glands and the glands of Littre are the sources of the discharge.

When none of the above-named structures is responsible for the gleet, it is produced from areas of chronic inflammation in the urethra, generally having the form of what is called granulation tissue, which lie immediately behind the stricture, the latter retaining a little of the discharge, and that in turn-being irritating-perpetuating the inflammatory area, so that the gleet is thus continued for long periods.

Changes in the Form, Force, and Size of the Urinary Stream.Even before the stricture is well organized, and has begun to contract, its presence is usually manifested by more or less well-marked changes in the form and manner of projection of the urinary stream.

Such changes are seen in connection with any condition of the urethra which affects the integrity of the parts which participate in the expulsion voL. I-7 
of the urine through the urethra, and therefore is not essentially characteristic of stricture.

The early changes are twisting or forking of the stream; later progressive diminution in its size, which finally leads to the passing of the urine drop by drop, and at length to complete retention-that is to say, inability to pass any urine at all. Patients are frequently very unobservant of these changes, and will sometimes declare that they are passing a full-sized stream of urine, when, as a matter of fact, the water is expelled guttatim only. Their statements should always be tested by the surgeon's own observation.

Concomitantly with the changes noted above there is diminution in the force with which the stream is projected, and dribbling of a few drops after the apparent completion of micturition.

InCREASEd Frequency of URination.-This symptom is due either to the passage backward of inflammation to the prostatic urethra, or to the inability of the bladder to wholly empty itself; as a consequence of the latter condition, a residual urine is formed, the presence of which causes an irritation of the bladder, because of the demand made upon it to expel its contents wholly, which demand it cannot meet.

The involvement of the bladder in the extension of the inflammatory process and the setting up of cystitis is another reason for increased frequency of urination.

Disturbances of the Sexual Function.-A certain number of patients with stricture suffer from more or less well-marked disturbance of the sexual function. This is manifested in various degrees of weakness of functional activity, up to complete impotence. That stricture is the causal agent in the production of the loss of sexual power is shown by the fact that, in a number of cases, there has been a restoration of functional power subsequent to operation upon the stricture.

Diagnosis.-Caliber of the Normal Urethra and Meatus, and the Means by Which They Are Estimated.-To determine the presence of stricture, we must know beforehand what the normal caliber of the urethra is. Upon this point there is a considerable divergence of opinion. The writer is a strong believer in the views with respect to urethral caliber maintained by the late Dr. Fessenden Otis, of New York, and regards the data upon which they were founded as accurate, having repeated the urethral measurements made by Otis, and arrived at the same conclusions reached by him. There is no other standard of measurements or way of estimating urethral caliber that is logical, and, as a matter of fact, surgeons who do not adopt Otis ${ }^{8}$ rule are entirely at sea. This is shown in various ways, for example: In a discussion upon the subject of internal urethrotomy, which occurred at the meeting of l'Asso- 
ciation Française d'Urologie, in I90r, Guillon ${ }^{9}$ brought forward as a new idea-which is certainly a singular claim in view of the fact that it has been recognized and universally accepted for many years in this countrythat the permanency of the results of internal urethrotomy depends upon dilatation of the canal at the time of the operation, and subsequently, being carried to a sufficiently high point. He then, after advocating carrying the dilatation up to high numbers, quotes Guiard's opinion, to the effect that it should be carried to 2I or 23 of the Charrière scale, which numbers Guillon agrees to as being the right degree of dilatation in most cases, but adds, that in some he thinks it too small, and that the dilatation should be carried higher. How much higher is not suggested. At this session of the Association no one referred to the rules of Otis, or suggested that the usual estimate of urethral caliber in this country had received any serious consideration among French surgeons.

The absence of a rational ground other than that adopted by Otis becomes obvious the moment the question is asked: Why should 23 of the French scale be taken as a measure of the size of the average urethra? Why not No. i 8 or 26 , or any other number that the surgeon may happen to like? There is no reasonable or convincing reply to be made to these questions. Formerly, the meatus used to be taken as the guide to the size of the urethra. One only needs to recall the very large number of meatuses which will not permit the passage of an instrument of more than I6, French scale, and of a good many in which the opening is no larger than 8 of the same scale and finally, the great variation in the size of different meatuses, to see the fallacy and impossibility of accepting this orifice of the urethra as a guide to the size of the latter.

Until the later years of his life, Sir Henry Thompson was content with the assertion that there could be no stricture in a urethra which would admit the passage through it of a No.I2 or I4 English-20 and 23 French - sounds. Here, again, is an entirely arbitrary choice of size.

In this country the rules of Otis with regard to urethral caliber are widely accepted. They are as follows:

I. There is a definite and constant relation between the circumference of the flaccid penis and the urethra which passes through it, which relation is a reliable means by which the caliber of the urethra can be readily determined in individual cases; it is as follows:

Circumference of Penis.

3 inches.

$\begin{array}{lll}3 \frac{1}{4} & \text { “ (average size). } \\ 3 \frac{1}{2} & \text { “ } \\ 3 \frac{3}{4} & \text { “ }\end{array}$

And so on.
Caliber of Urethra.

$30 \mathrm{~mm}$. French scale.

$32 \mathrm{~mm}$. " " " (average size).

$34 \mathrm{~mm}$. " " "

$36 \mathrm{~mm}$. " " "

And so on. 
From this it appears that the urethra increases in a ratio of $2 \mathrm{~mm}$. for every increase of $\frac{1}{4}$ of an inch in the circumference of the penis, and that the average urethra has a caliber of $32 \mathrm{~mm}$.

2. The size of the urethra varies in different individuals.

3. The size of the meatus varies greatly in different individuals. Its average size is $24 \mathrm{~mm}$. The size of the meatus cannot be taken as a guide to that of the urethra beyond it. The meatus is rarely as large as the urethra to which it belongs.

One question remains to be answered with respect to the measurement of the urethra: How are we to determine the degree of distensibility of the canal that is normal? There is no absolute standard or manner of deciding this point, but it is fair to assume, at the least, that the canal is not overdistended or stretched beyond its natural limits of expansion by the passage of a sound through it (after the incision of the meatus), that will enter the bladder with only the force of its own weight. This is, therefore, a safely conservative manner of estimating the natural distensibility of the urethra, and it is the one usually adopted, and it conforms to the rules of Otis.

The acceptance of Otis' rules for establishing the urethral caliber obliges us to recognize the necessity of determining the size of the urethra of each patient, and forbids the adoption of any one number, or one or two numbers, arbitrarily chosen, as the standard of size of the canal in all cases, as it is so frequent a custom to do in other countries.

Any narrowing of the urethra, due to connective-tissue formation, originating in a pathological process, and of a size that is less than the normal caliber of the special urethra in which it exists, and which is the cause of symptoms definitely characteristic of stricture, should be regarded as a stricture. Thus, a well-marked constriction in the course of a urethra of a normal caliber of 36 , which would not allow of the passage of a bougie à boule larger than 32 would be a stricture from the surgical point of view if there were symptoms referable to it, and in that case one demanding treatment.

It is true that there are a number of points of anatomical narrowing in the course of the perfectly normal urethra. Otis was criticised because he was said to have regarded such slight narrowings in all cases as being strictures. This is not, however, quite accurate, for he expressly stated that they were not to be regarded as strictures, except they were producing perfectly definite symptoms.

Mестотомy.-Except the urethrometer be used, the meatus must be cut in almost all cases in order to allow the detection of strictures which have a wider caliber than that of the urethral orifice. This is best done by an instrument which with certainty will divide the meatus in one cut 
to the desired number. Civiale's meatotome is one which has been for many years used for the purpose. The instrument of Gouley is shown in Fig. 23, and is similar to Civiale's. The degree of the separation of the ends of the blades is determined by the upright screw in the handle. The blades are introduced (closed) for about one-quarter of an inch beyond the meatus, opened and withdrawn quickly. The incision should be made through the floor of the meatus.

FIG. 23

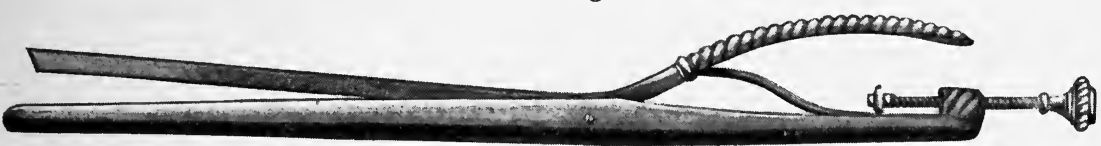

Falspaniding

Meatotorne.

FIG. 24

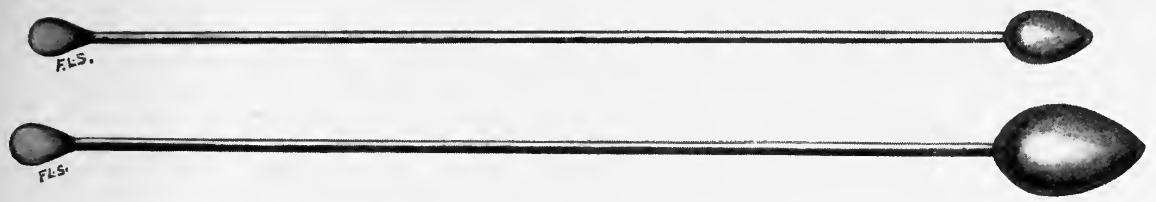

FL.Sprultino

Bougie à boule and diagram of same in contact with the posterior face of the stricture.

FIG. 25

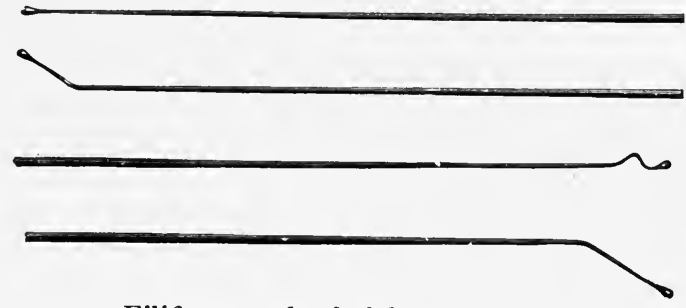

Filiform and whalebone bougies.

Diagnosis and Methods of Examination.-Diagnosis of stricture is made in part from the symptoms, but chiefly from instrumental examination of the urethra. For this purpose the following instruments are employed: (I) The bougie à boule, or acorn-tipped bougie. (2) The urethrometer. (3) Olivary and filiform bougies.

(4) Conical steel sounds. (5) The endoscope. (Figs. 24, 25, and 26.) 
Bougie à Boule.-The bougie à boule is the best instrument by which to determine the site, size, and number of strictures of the pendulous urethra. Its form is shown in Fig. 24 .

It is used for these purposes in the following way: The size just a little smaller than the meatus will admit is first used, and the bulb of the instrument is passed into the canal until its further progress is arrested by a narrowing which will not allow it to pass.

Successively smaller sizes of the instrument are then tried until one is found that will pass through the obstacle. This, after traversing the stricture, is slowly withdrawn until the abrupt shoulder comes in contact with the posterior surface of the stricture (see Fig. 24). The thumb nail is then placed on the shaft at the meatus and retained there while the instrument is being withdrawn; in this way the distance of the posterior face of the stricture from the meatus can be measured, it being represented by the length of that part of the shaft of the instrument which lies between the thumb nail and the shoulder of the acorn tip.

FIG. 26

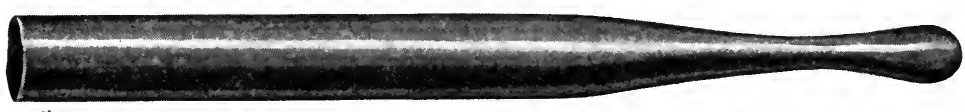

Olivary bougies, good and bad forms

In passing through the stricture from behind forward, the ball or acorn tip of the bougie encounters the obstacle, and then jumps suddenly over it, giving to the thumb and finger in so doing a characteristic sensation, which is unmistakable, and may be very well imitated by quickly drawing the tip of the instrument across the stretched web of the skin between the thumb and forefinger.

If there are strictures of smaller caliber posterior to the one in front, successively smaller sizes of the bougies will be tried in the same way as that just described.

When there is a narrowing due to the presence of granulation tissue and an infiltration of a limited part of the canal, this characteristic sensation of the bulb passing through the organic stricture is replaced by the feeling of resistance such as would be conveyed if the instrument were to be pushed through a bit of dough. There is usually greater sensitiveness at the part of the canal corresponding to an area of granulation tissue than elsewhere. 
The limitations to the use of the bougie à boule are seen when (I) the anterior of two or more strictures is of smaller caliber than those behind it; here the posterior ones will not be felt by a bulb that is small enough to pass through the anterior narrowing; (2) when the strictures to be examined are too narrow to admit the smallest of the bulbs of the bougie à boule.

The Urethrometer.-The most convenient form of urethrometer is that devised by the late Dr. Otis. The distal end of the instrument may be likened to an egg-beater when it is expanded. It is passed closed as far as the bulbomembranous junction of the urethra, then, by turning the screw head at the outer end, the rods expand into a form similar to that of the bulbous end of the bougie à boule. The expanding bars or bands are covered with a rubber cap in order to protect the urethral mucous membrane from being caught in the sharp angle made by their junction when they are opened, and thus being torn when the instrument is withdrawn. The rubber cap should be tested before being used, for in time it becomes hardened, and then ruptures easily when stretched. The arrow on the dial which travels across its face when the screw is turned records the degree of expansion of the bands at the other end.

The strictures are located and their caliber determined in the same manner as that described in connection with the bougie à boule, the only difference being that, instead of having to use a number of instruments, one suffices for all the different sizes of stricture and for estimating them. When the expanded end of the instrument is stopped in its passage outward, on being withdrawn, its size should be reduced by turning the screw head backward until it is small enough to pass the obstacle, and the number to which the arrow of the dial points is noted by the examiner. On the staff there is a sliding button, which is placed at that moment against the meatus, and by it the distance of the posterior face of the stricture from the meatus is measured, there being a scale of inches and subdivisions of inches on the staff.

The sizes of strictures which are too small to allow this instrument to be passed through them are est mated by the use of olivary bougies of successively smaller numbers until one is found which will pass (see Fig. 25). The bougies of this sort are made in all sizes, from that of the normal urethra down to the finest filiform.

Bougies.-Some of the filiform bougies are made with bayonet tips and curves of various sorts, for the purpose of enabling them to engage in the entrances of strictures which are eccentrically placed. We do not personally find any advantage in the employment of such forms, as compared with the straight ones. Fig. 25 illustrates some of the shapes of the ends of the instruments just referred to. 
Sounds.-The conical steel sounds in the sizes from 20 upward are used to explore the membranous urethra. These instruments in other than expert hands, in the sizes smaller than 20 , are less safe than the olivary bougies, and the latter are therefore advised when it is necessary to use these numbers.

The curve and taper of the steel sound are the result of careful calculation. Fig. 27 shows an instrument of this sort in which both of these qualities are embodied accurately. Fig. 28 is the so-called Beneque form of sound. Fig. 29 is the double taper sound, which has the advantage of not stretching the meatus after the largest part of it has passed beyond this point.

Fig. 27

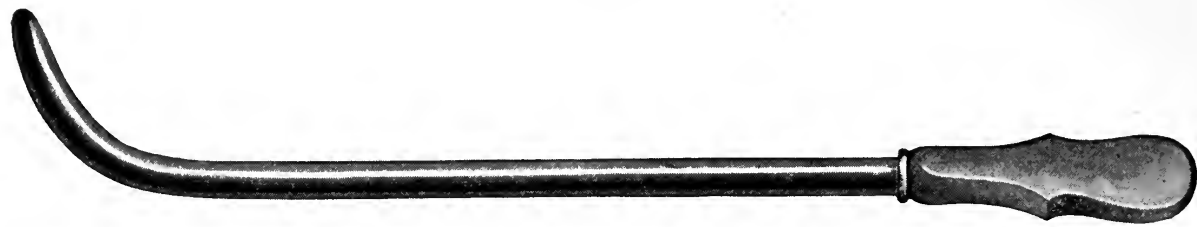

FIG. 28

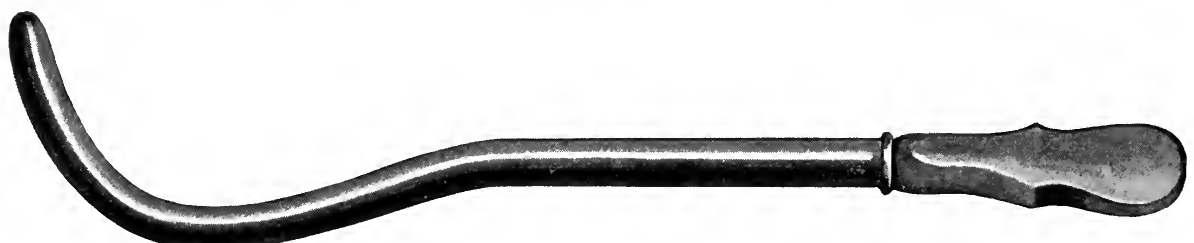

FIG. 29

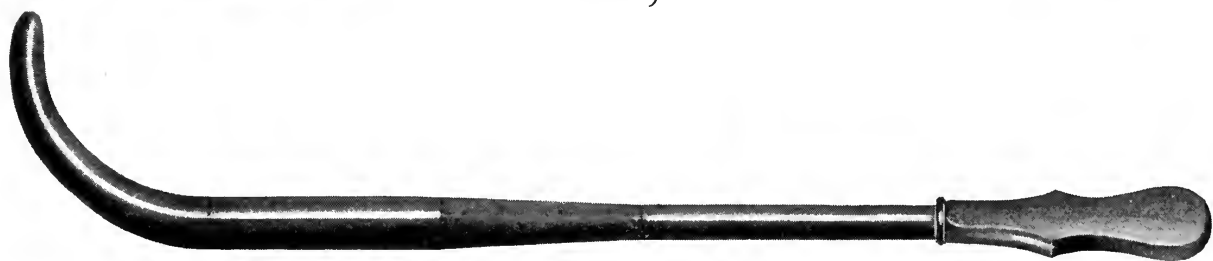

Figs. 27, 28, and 29.-Different forms of sounds.

Endoscope.-The endoscope is useful in detecting the early stages of stricture formation and the areas of chronic inflammation, at the sites of which stricture may subsequently form. It is also of value in locating the orifice of the stricture when the latter is placed eccentrically, and in thus enabling the surgeon to enter a bougie into it. (See section on Endoscopy.)

Difficulties Encountered in Examining Strictures.-I. The tip of a small bougie is often caught in the dilated mouths of the urethral follicles; also in false passages if such have been made by previous unskilful use of instruments. 
One of the methods advised to overcome the trouble arising from the catching of the tip of the instrument in follicles is to pass a number of filiform bougies into the urethra, and allow their points to engage in and thus occupy them all, so that finally one of the instruments will enter the orifice of the stricture, there being no more follicles for it to catch in.

2. In strictures having eccentric openings, the tip of the bougie failing to engage in the orifice may bend upon itself and return through the meatus. The specially formed tips of the filiforms already spoken of sometimes aid in overcoming this difficulty, but the endoscope is more useful for this purpose.

Spasm.-A frequent error in examining for stricture is the mistaking of spasm of the deep urethra for organic stricture.

Spasm will usually yield to the steady pressure of the tip of a large sound held gently and quietly against the part of the urethra which is spasmodically contracted. When the tip of the sound enters the spas-

Fig. 30

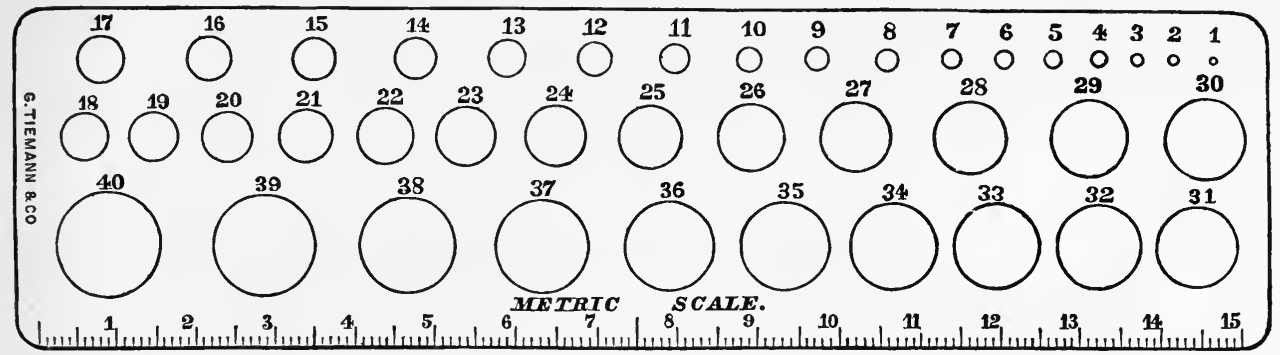

Scale of measurements for sounds.

modic area, a series of twitching movements, caused by the alternate contraction and relaxation of the compressor urethræ muscle, are conveyed to the hand holding the outer end of the instrument. When a sound enters the constriction made by organic stricture, no such sensation is felt, but instead, the instrument is grasped and held firmly.

Scales of Measurement of Urethral Instruments.-There are, unfortunately, several scales of measurement in use. Those which are best known are the so-called French scale of Charrière, and the English one. Besides these two are the American scale, for which there is no raison d'etre, and one or two other French scales. The only one of these which is satisfactory is that of Charrière (Fig. 30).

Treatment. ${ }^{1}$ - The object of all methods of treatment is to restore the strictured portions of the canal to the normal caliber of the particular

${ }^{1}$ For treatment of extravasation of urine, septic phlegmon, retention of urine, urethral fistula, false passage, see under heading Treatment of Complications. 
individual's urethra in which the stricture exists. The following methods have been emploved for this purpose: (I) Stretching. (2) Splitting. (3) Cutting through. (4) Cutting out. (5) Expansion by an electric current. These are called, respectively, dilatation, divulsion, urethrotomy, resection, and electrolysis.

Dilatation.-This method of treatment has been carried out in three ways: (I) Gradual dilatation. (2) Rapid dilatation. (3) Continuous dilatation. Of these different ways, only the first should be employed. The two others have been from time to time in vogue, and therefore must be briefly described.

Rapid Dilatation.-Rapid dilatation consists in the quick stretching of the strictured part of the canal by the passage through it of successively larger instruments-olivary bougies or steel sounds-at one and the same sitting. If the stricture is dense and resistant, this procedure will split it and thus becomes a divulsion and not a dilatation. If it yield very readily, the stretching of the stricture will be accomplished, it is true, but the passage of a number of sounds one after another in quick succession invites inflammatory reaction, and is, with previously unknown patients, an undesirable thing to do.

Continuous Dilatation.-Continuous dilatation is carried out by tying a catheter into the urethra and bladder and allowing it to remain there for a time-twenty-four hours-then replacing it with a larger size, and so on until the full size of the urethra has been reached. It invites inflammatory reaction and infection, and is a most undesirable method to employ.

Gradual Dilatation.-Gradual dilatation consists in the stretching of the strictured part of the canal by the passage through it of successively larger sizes of bougies or sounds, at intervals of varying lengths, until the constriction has been dilated to the normal caliber of the urethra in which it exists, or sometimes beyond the normal caliber.

Until the normal caliber has been restored to this part, the sounds should be passed at intervals of four or five days, unless there is some contra-indication to their employment. Thereafter the intervals between the sittings are lengthened, until the time at which the stricture begins to recontract is learned. The intervals will then be made a little shorter than that time.

The time during which strictures maintain the degree of dilatation to which they have been carried varies very much, and is dependent upon their special qualities, which have already been described. In the early stages from four to six months may sometimes pass after dilatation before there is recontraction. As the stricture becomes older, or if the original deposit of connective tissue was extensive, or if the stricture is resilient, recontraction will be rapid. 
Rules to be Observed in the Treatment by Gradual Dilatation.-Never undertake this, or any instrumental treatment of stricture, if there be an acute urethritis present.

Never continue it if constitutional symptoms-chills, fever, fainting, threatening symptoms of suppression of urine-follow the use of the instruments, or if they excite acute inflammatory reaction in the urethra.

Never use other than gentle manipulation.

Have the sounds warm when they are used.

Do not pass the instruments with the patient standing up.

Lubricate the instruments amply and use a sterile medium for the purpose.

Sterilize the instruments before using them; the steel sounds by heat. An alcohol flame is the most convenient manner of applying heat to these instruments. The webbing bougies may be boiled in a strong aqueous solution 'of rock salt for twenty minutes.

Irrigate the urethra, when possible, before passing the instruments, preferably with a I to 4000 solution of permanganate of potash.

Use olivary bougies until the stricture has been dilated as far as Nos. I 8 or 20, French scale, and the steel sounds from this upward.

Avoid making jerky, sudden movements; urge the sound steadily and gently forward; hold the handle lightly; do not grip it.

Do not try to gain too much at one time; on the other hand, do not have your patient come to you twice as often as is necessary.

Spasm of the Compressor Urethre Muscle.-Spasm of this muscle is often mistaken for organic stricture. It is more readily excited by the passage of a small instrument than of a moderately large one. It can ordinarily be overcome by quiet, steady pressure of the tip of the instrument.

In some cases the spasm does not relax, and the following measures are then useful: A hot compress held against the perineum, a hot sitz bath, hot rectal irrigation, an opium and belladonna suppository. These failing, it may be necessary to etherize the patient.

How to Pass Steel Sounds.-Preferably, stand on the patient's left. Enter the tip of the sound into the meatus with the shaft of the instrument in the line of Poupart's ligament and not much raised above the surface of the body. Next, push the instrument onward through the urethra directly toward the anal orifice, bringing the shaft at the same time gradually to the middle line of the body, but not lifting it much above the surface until the tip has reached the farther end of the bulbous urethra; this will be known by the fact that it is stopped at that point. The left hand holds the head of the penis between the thumb and forefinger during this movement, and follows the right hand across to the 
middle line of the body. On reaching the bulbous urethra and feeling the tip of the sound arrested there, withdraw it slightly, put the penis on

FIG. 31

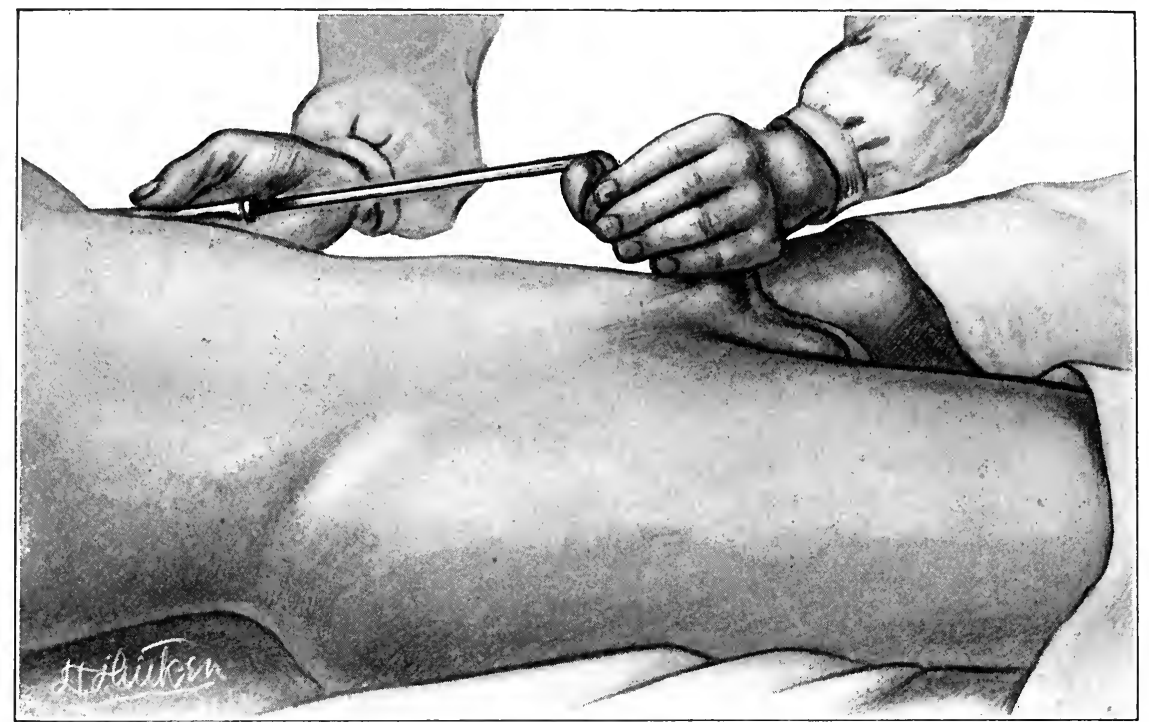

First step in passing the sound.

FIG. $3^{2}$

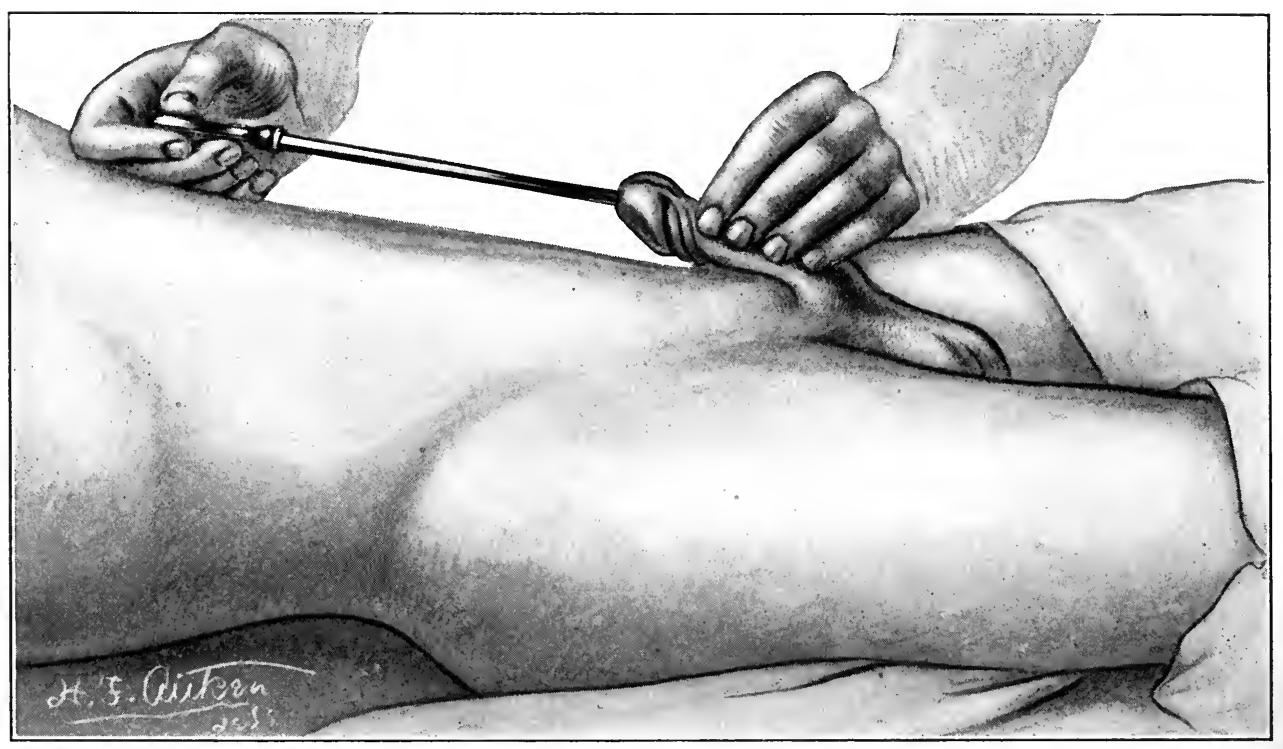

Second step in passing the sound.

the stretch by traction with the left hand, then bring the shaft of the sound directly forward over the symphysis pubis toward the space 
between the legs and simultaneously relax the suspensory ligament of the penis by pressure toward the feet, with the right hand placed just above the symphysis pubis. In making this movement the tip of the sound rises and follows the course of the membranous and the prostatic urethra into the neck of the bladder (Figs. 31, 32, 33, and 34).

If by the first examination it has been determined that there is no stricture beyond the anterior urethra, one should avoid passing the sound into the bladder at subsequent sittings, since one may sometimes carry

FIG. 33

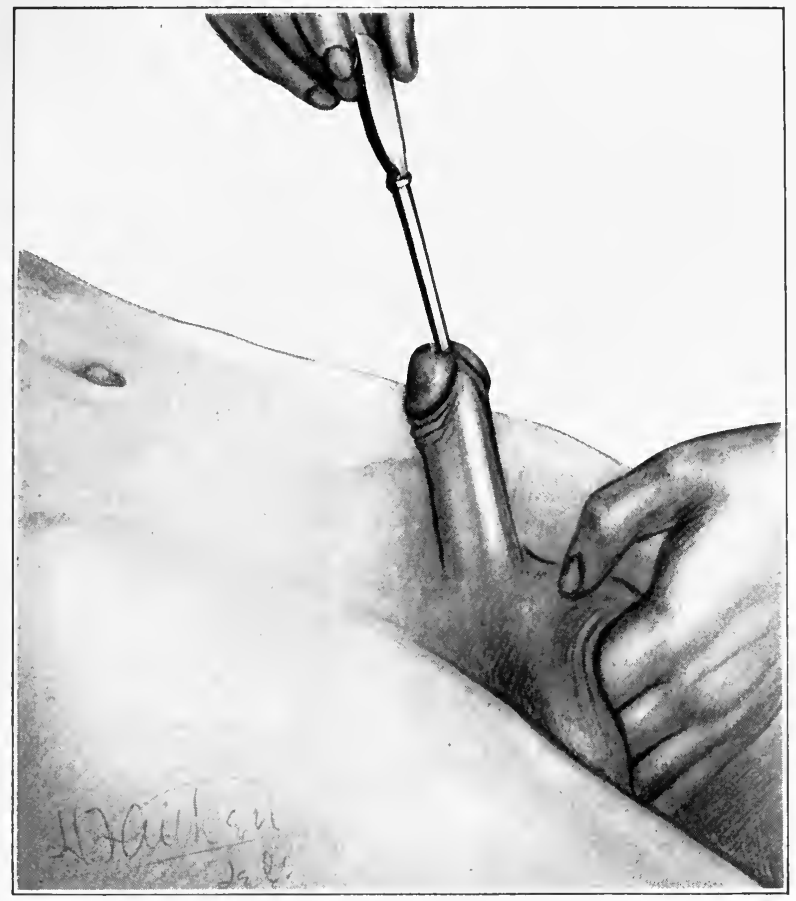

Third step in passing the sound.

infection into the deeper part of the canal and to the bladder; moreover, it is from the deep urethra that the impulses which in some cases produce shock or other nervous symptoms are propagated.

Anatomical Difficulties Met with in the Passing of Sounds.-There are three difficulties usually encountered by the beginner when passing sounds. The first is of his own making, and is created by the endeavor to pass the tip of the sound upward under the arch of the symphysis pubis, and, as he supposes, into the bladder, sooner than it should be done (see Fig. 35). As a consequence, the end of the instrument is brought against the anterior face of the triangular ligament, and its further progress is arrested. 'This difficulty will be avoided if the sound is carried directly 
onward and toward the anus until it stops, before any attempt is made to turn it upward into the membranous and prostatic urethra (Fig. 35).

The second obstacle is made by the natural dilatation in the floor of the bulbous urethra, owing to which the tip of the sound may press the somewhat slack mucous membrane ahead of it, and make a small pocket upon the urethral floor. If the sound is pushed on, there is danger of making a false passage. The obstacle is overcome by making the point and concave part of the curve of the sound cling to the roof of the urethra and by not pressing it downward upon the floor of the canal at this point, and by putting the penis on the stretch, or by lifting it upward by the fingers placed upon the perineum, as shown in Fig. 33 .

FIG. 34

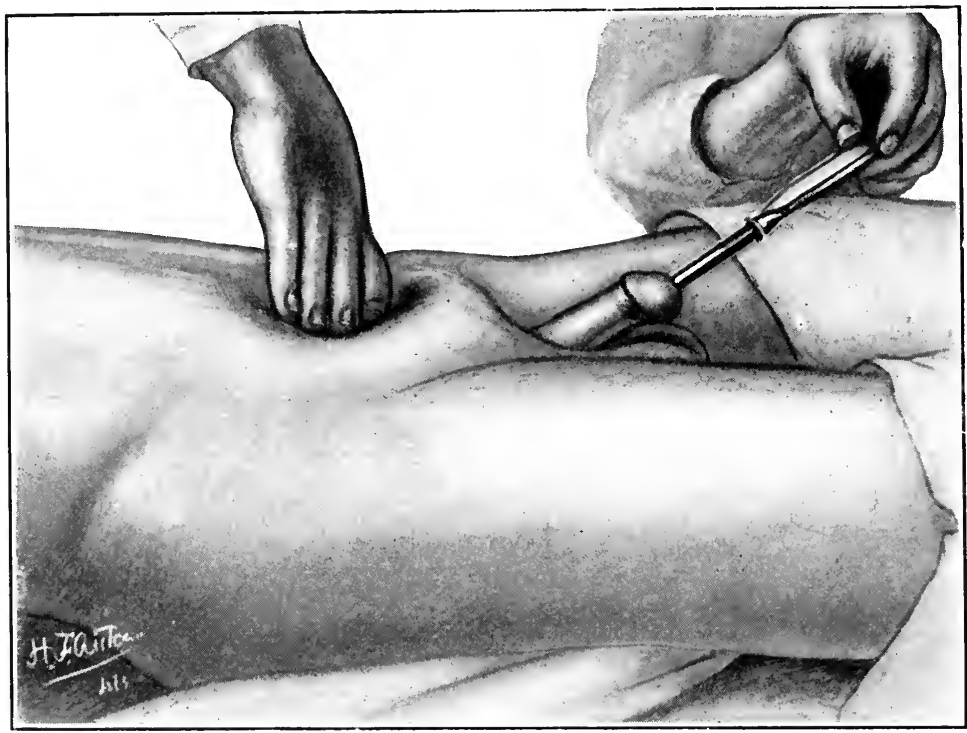

Fourth step in passing the sound.

The third difficulty is caused by the upward change in the direction of the urethra at the bulbomembranous junction as it passes to the bladder, and partly by the tension of the suspensory ligament of the penis, which opposes the downward excursion of the shaft of the sound when depressing it. Here, again, keeping the concave curve of the sound close to the roof of the canal will overcome the first difficulty, while the second one is avoided by relaxing the suspensory ligament by pressing with the fingers of one hand placed above the symphysis pubis toward the feet in a horizontal direction (Fig. 34).

Dilatation with Flexible Olivary Bougies.-The olivary bougie is a safer instrument than the steel sound, especially the smaller sizes of the latter. Its safety is owing to its flexibility and absence of the leverage 
which exists with the metal sound. The filiform sizes, if made of whalebone, may, however, easily make false passages if their tips become engaged in expanded follicles, and are then pushed forward. There is much difference in the quality of the olivary bougies. Those made by the French are much the best. The desiderata of the olivary bougie are:

FIG. 35

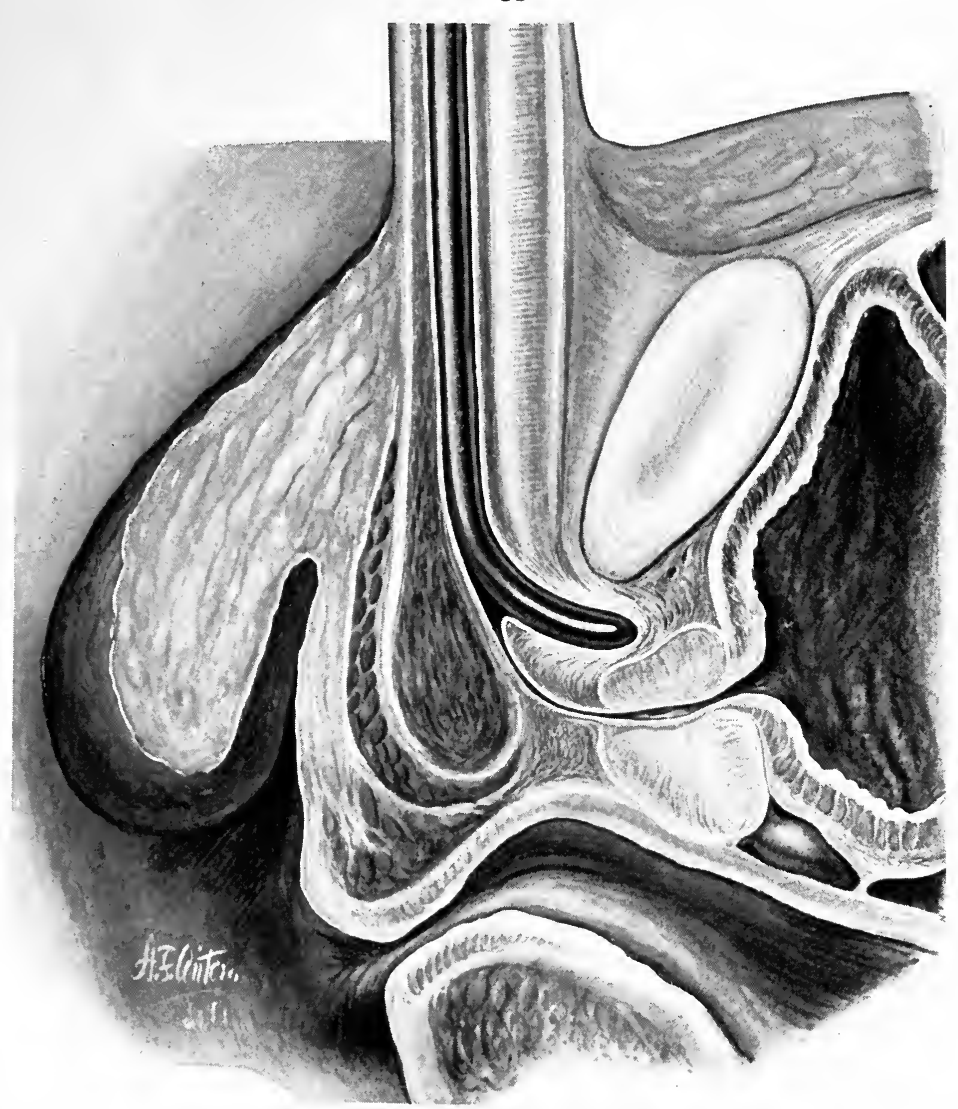

Showing error, in passing sound, of turning its point upward too soon.

I. That the neck be long, slender, and flexible, and the olive-shaped tip not too large. These characteristics appear in the first of the two instruments shown in Fig. 26. The second instrument of the same figure shows the contrary qualities, $i$. $e$., those which are undesirable in the form of the instruments.

2. The surface should be smooth and highly polished, and made of durable material.

3. The swell of the instrument after the narrow and flexible neck should be evenly graded.

4. The filiform sizes should not be too pliable. 
In cases of tight stricture, there is often as much difficulty in passing the next larger size after the withdrawal of the first filiform instrument Fig. 36

Fig. 37
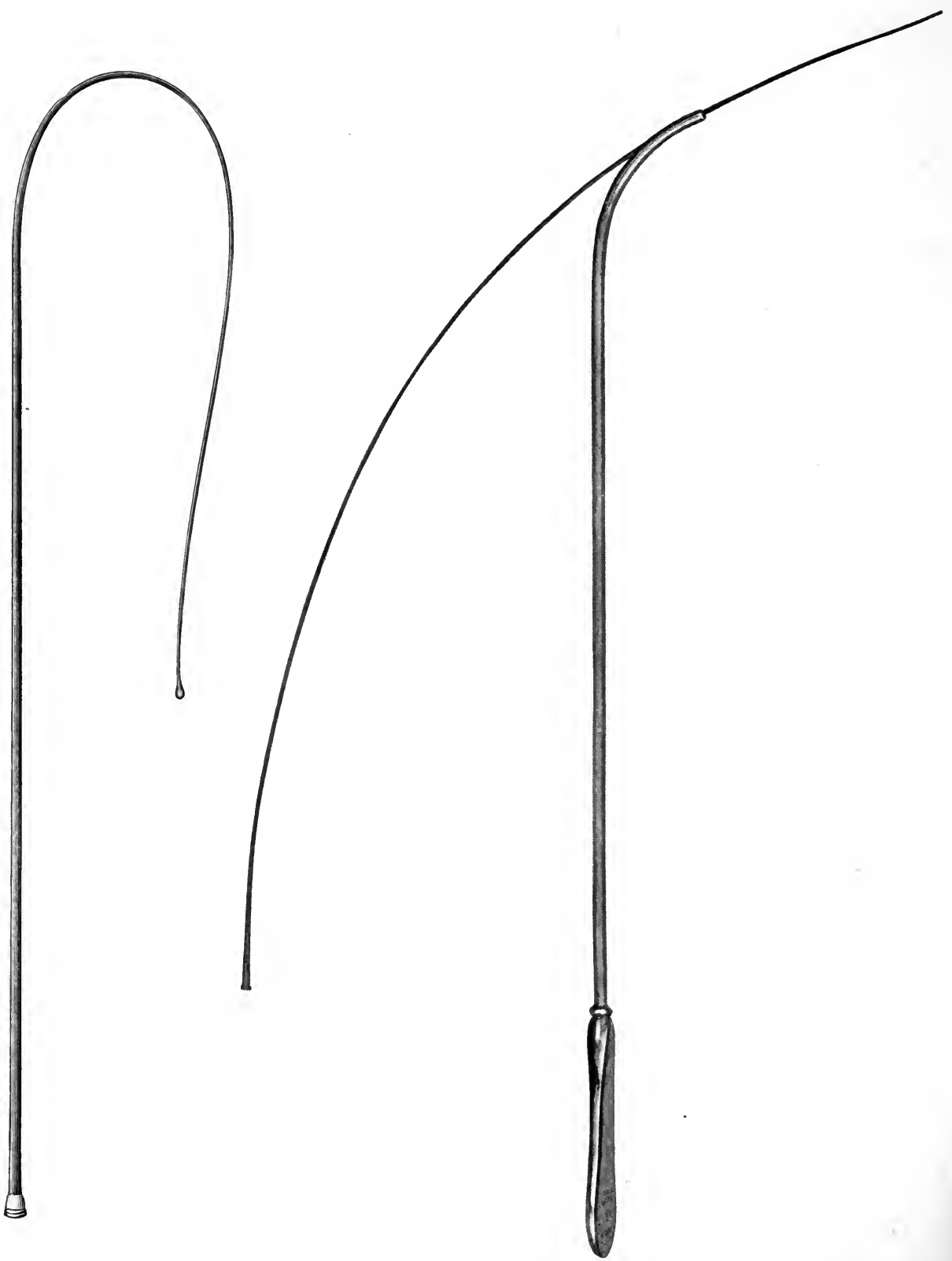

Harrison's urethral whip bougie. Gouley's tunnelled sound, with filiform guide.

as there was in inserting the latter. To overcome this difficulty, Harrison ${ }^{10}$ devised an instrument known as the whip bougie (Fig. 36). This most 
excellent device is a long bougie having the filiform size at one extremity and gradually increasing through several larger sizes to the other end, which enables the surgeon to dilate the stricture several numbers at once if the filiform end can be made to pass. The fine end of the bougie curls up in the bladder when the rest of the instrument is being pushed through the narrowing.

If not provided with the whip bougie, the same purpose may be served by the use of the staff of a Maisonneuve urethrotome attached to a filiform bougie.

The same end may also be gained by the employment of Gouley's tunnelled sound, which is represented in Fig. 37. This instrument is threaded onto a filiform bougie, which serves as a guide to the passage of the sound through the constriction.

Gouley's instrument is not without danger, which arises from the possibility of making a false passage with it in spite of its filiform guide.

If none of these special forms of instruments is at hand, and if the surgeon has succeeded in passing one filiform, it is much wiser to secure it in place and allow it to remain for twenty-four hours, rather than to remove it at once. When the bougie has been in the urethra for some length of time the stricture will usually be found to have expanded sufficiently to admit an instrument of from one to three sizes larger.

Kollmann's Dilator.-Figs. 38 , 39, and 40 show the form and mechanism of this instrument. It is used to accomplish the immediate-rapid or gradual-dilatation of strictures which are large enough to admit of its passage through them, or as a substitute for the steel sound in the treatment by gradual dilatation, in which case only a moderate degree of stretching of the stricture is attempted at each of several sittings.

The objections to the employment of Kollmann's instrument are that the dilatation is estimated by numbers read off on the dial plate, and not by the delicacy of touch, as is the case with the use of the steel sound. It is not applicable to strictures of small caliber. It must be said, however, that some surgeons like it better than the latter instrument, and it has the great advantage of permitting the strictured part of the canal to be widely dilated without necessitating a preliminary meatotomy and without painfully dilating the meatus.

Divulsion.-The technique of all the operations which follow will be found described in the chapter on Technique of Operations for Stricture of the Urethra.

The operation consists in splitting the stricture by suddenly forcing through it an instrument of a much larger size than the constricted part of the canal. The method lacks precision, and is open to other objections, which will be mentioned later in the text.

vor. I-8 
INTERNAL URETHRotomy.-The rules which must be observed in order to have success attend the performance of this operation are stated later in the chapter. Here we shall speak merely with reference to aseptic measures, and the question of immediate dilatation and the employment of a catheter à demeure in connection with the procedure. This we do largely because of the curious points of view of some of the French surgeons which were expressed in a discussion upon this operation by the members of l'Association Française d'Urologie in I90 I with reference to these special features, some of which have been so commonly employed in

FIG. $3^{8}$

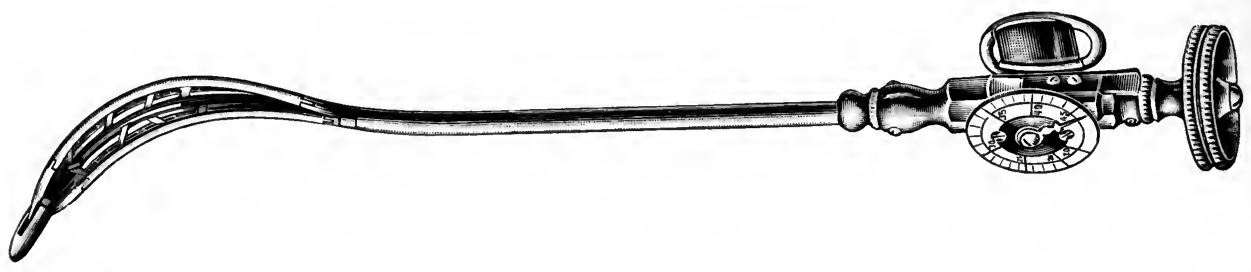

FIG. 39

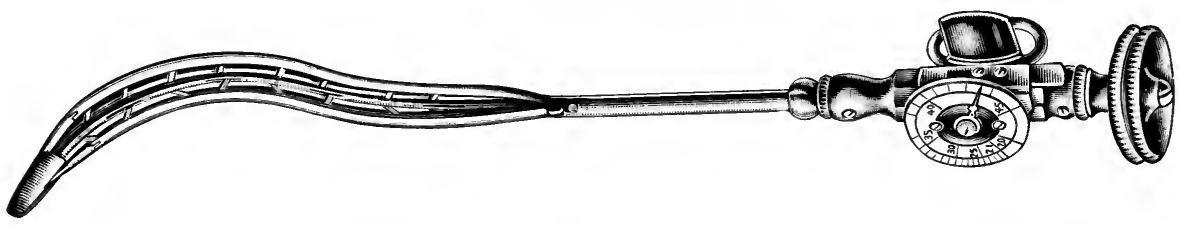

FIG. 40

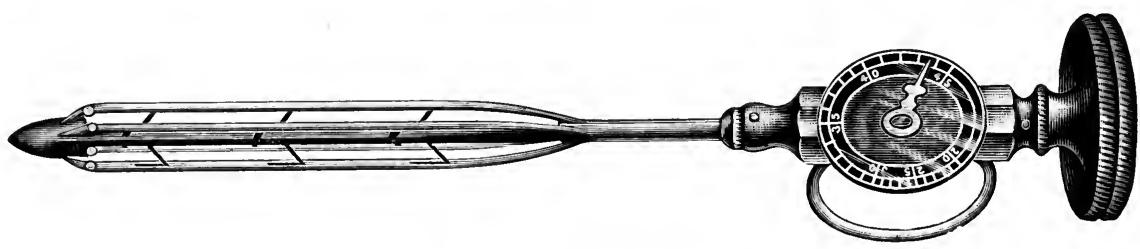

Figs. 38, 39, and 40.-Kollman's urethral dilators.

this country for many years, of which fact-that of omitting the catheter after the operation and the full dilatation of the urethra at the time of its performance-one of the members (Reynes ${ }^{11}$ ) did not appear to be aware.

Following him, Escat ${ }^{11}$ Albarran, ${ }^{11}$ Guiard, ${ }^{11}$ and Pousson ${ }^{11}$ all condemned the practice of immediate dilatation in connection with the operation. Pousson spoke thus: "The custom which consists in passing successive instruments of larger and larger sizes immediately after internal urethrotomy is detestable." He attributed the "frightfully disastrous results" reported by the surgeons of Bordeaux to the employment of this procedure. 
These adverse opinions to a step which is so commonly taken in this country without any ill results are difficult to understand. In 220 internal urethrotomies performed by the writer, with two deaths, neither of which could by any possibility be traced to the employment of the use of fullsized sounds in immediate connection with the operation, it has been his invariable practice to pass a sound of the size corresponding to the normal caliber of the urethra as the last step in the operation. The small mortality and the entire absence of untoward results in any of the other cases, is a direct contradiction to the views of the French surgeons quoted above. The writer's practice in this respect is, so far as he knows, that which prevails with American surgeons.

It is also customary, and in the writer's opinion essential, in order to obtain a permanent result and a cure by the operation of internal urethrotomy, to pass sounds of the size of the normal urethra every second day following the operation, until blood ceases to appear upon the withdrawal of the instrument.

With regard to the omission of the catheter à demeure after internal urethrotomy, the writer is equally convinced that it is good practice, and that no harm whatever results from it. The same personal experience in this respect as that to which he has referred in connection with immediate dilatation supports the correctness of the statement, since in none of his cases has he tied a catheter into the bladder following the operation, and no harm has ever come from having failed to do so.

Aseptic Precautions in Connection with Internal Urethrotomy.When strictures are not too narrow to permit of its being done, the urethra should be irrigated before doing the operation, and it should invariably be cleansed thus at the end of it, also after the first urination and after each time the sounds are passed subsequent to the operation.

The best fluids to employ are those which are soothing and cleansing. No attempt should be made to render the urethra sterile by the use of fluids of sufficient strength to be germicidal, if they are at all irritating, such as corrosive sublimate and carbolic acid. It is impossible to sterilize the canal, in the first place, and the irritation produced by such agents is far more productive of ill than anything else. The most useful of the preparations for the purpose of irrigation are solutions of permanganate of potash I to 4000,4 per cent. boracic acid, argyrol of 2 to 5 per cent. strength, and sterile saline solution.

The routine practice of using these fluids in the manner described does more to avert constitutional disturbance after the operation than any or all other measures. Internally, $7 \frac{1}{2}$ grain doses, thrice daily, of urotropin are valuable. 
External Perineal Urethrotomy.-This operation is employed under three conditions: (I) In connection with internal urethrotomy, for the purpose of supplying drainage from the bladder through the perineal incision, and thus avoiding the contact of the urine with the freshly cut surfaces made in the course of that operation. (2) In cases of strictures situated at and posterior to the bulbous urethra which are not suitable for internal urethrotomy alone, because of the greater liability to hemorrhage which is difficult to control, and to sepsis because of the lack of free drainage, which is not supplied so well by an internal urethrotomy as by the external operation in this situation. (3) It is absolutely demanded in all cases of urinary extravasation, in order to arrest further infiltration of the tissues, which it prevents by draining the urine from the bladder directly through the catheter tied into it through the posterior urethra.

RESECTION.-This method is appropriate in cases in which the perineal part of the urethra has been injured, and also in those in which very dense strictures exist that are intractable to the forms of treatment already mentioned.

Choice of Method.-It does not always lie in the surgeon's power to apply the best method of treatment to patients having stricture, for the reason that many of them decline urethrotomy, which would afford the best chance of a cure, or, at any rate, yield the most enduring results, and prefer gradual dilatation as long as it is possible to maintain sufficient patency of the urethra to enable them to pass any water at all, or else being unwilling to take the time away from their interests which may be required for the operation. It is partly on this account that gradual dilatation is the method far more frequently employed than any other, and partly because the general practitioner considers himself entirely capable of carrying out that method of treatment.

The following are the factors determining the choice of the method of treatment:

I. The safest of all methods of treatment is that of gradual dilatation.

2. The difference between the mortality attending internal urethrotomy properly performed, and limited to strictures situated in the penile urethra, and that of gradual dilatation is so slight that it may be disregarded if the urethrotomy offers advantages not obtainable by dilatation in other important respects.

3. Dilatation, with the exception of a very small proportion of cases, almost all of which are those of strictures of short duration, does not effect permanent cure.

4. Internal urethrotomy, properly performed, is the only method by which an importantly large number of cures can be obtained. 
5. Divulsion is neither more nor less safe than internal urethrotomy, and has the disadvantages of obtaining but a relatively small number of cures, and, in some instances, of producing strictures of greater density than those to relieve which it was performed. It is an operation which should have no place in the treatment of stricture.

6. The mortality of external perineal urethrotomy is far higher than any of the methods mentioned. This is not, however, because of its being intrinsically a more dangerous operation, but is owing to the fact of its being often performed in a much more dangerous class of cases than those in which the other methods are ordinarily employed. It is the best means to apply in all cases in which strictures of the bulbous urethra or posterior to it resist treatment by gradual dilatation. It is imperatively demanded in all cases in which there is urinary extravasation, and is thought by some surgeons (Reginald Harrison) to be a potent factor in averting constitutional disturbances when used for diverting the urine from the anterior urethra in connection with internal urethrotomy.

7. Resection is a valuable procedure in cases of very dense strictures of the perineal urethra which resist other forms of treatment.

8. Electrolysis has been proved conclusively to be useless in almost all, and productive of denser masses of connective tissue than originally were present in the strictures in some of the cases in which it is used. It should have no place in the treatment of stricture.

Operative Mortality.-Mortality of Gradual Dilatation.-There are no statistical compilations published in detail, so far as we are aware, by which to accurately gauge the operative mortality of this procedure, but there is such universal agreement among surgeons that we may accept the constantly repeated statement with regard to it as accurate, viz.: Now and then death follows the passage of a sound into the urethra. It is a rare occurrence, and it is probably fair to say that not more than 0.5 per cent. of all patients thus treated die.

\section{Mortality of Divulsion.}

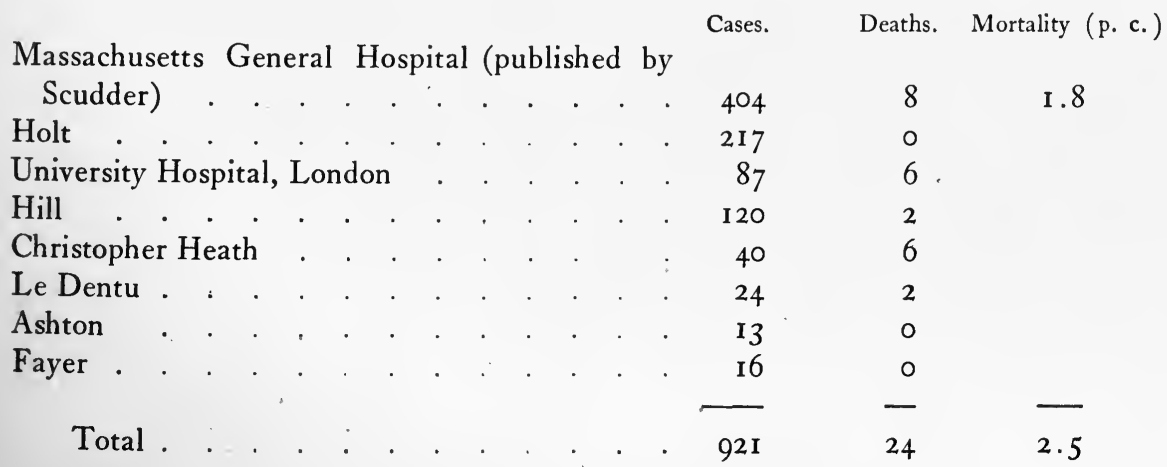


Mortality of Internal Urethrotomy.

\begin{tabular}{|c|c|c|c|c|c|c|c|c|c|c|c|c|c|c|c|}
\hline & & & & & & & & & & & & & Cases. & Deaths. & Mortality (p. c.) \\
\hline Tedenat & . & . & - & - & . & - & - & . & - & . & . & . & 650 & 0 & \\
\hline Pousson & . & . & . & . & . & . & - & . & . & . & . & . & 500 & 0 & \\
\hline Nicholich & . & . & . & . & . & . & . & . & . & . & . & . & 500 & I & \\
\hline Guyon . & . & . & . & . & . & . & - & . & - & - & . & . & 459 & 20 & \\
\hline Maisonneu & & . & . & . & . & . & . & . & . & . & . & . & 66 & 3 & \\
\hline Mallez & . & . & . & . & . & . & . & . & . & . & . & . & I80 & 2 & \\
\hline Le Dentu & . & . & . & . & . & . & - & . & . & . & $\therefore$ & . & 24 & 2 & \\
\hline Otis & . & . & . & . & . & . & - & . & . & 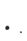 & . & . & 635 & 0 & \\
\hline Sir Henry & Tho & $\mathrm{mps}$ & son & & . & . & . & . & - & . & . & . & 430 & 6 & \\
\hline Mastin & . & . & . & . & . & . & . & . & - & . & . & . & 333 & o & \\
\hline Coulson & . & & & . & . & . & $\cdot$ & $\cdot$ & - & . & - & . & 206 & Io & \\
\hline Christopher & & eath & & . & . & . & - & . & - & - & - & - & 130 & 7 & \\
\hline Teevan & . & . & 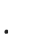 & . & . & . & . & - & . & . & . & . & 53 & 0 & \\
\hline Watson. & . & . & . & . & . & . & . & - & . & . & . & . & 220 & 2 & \\
\hline Brown . & . & . & . & . & . & . & $\cdot$ & - & . & ${ }^{\circ}$ & - & . & 300 & 0 & \\
\hline Total & & . & . & . & . & 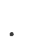 & • & • & • & . & & $\cdot$ & 4686 & 53 & I. I \\
\hline
\end{tabular}

The Mortality of External Perineal Urethrotomy. - Including the tables of Gregory and Heurteloupe, and adding others which the writer has collected, there is a total number of 2200 cases in which this operation was performed. The mortality attending it in this series is 9 per cent.

The Mortality Attending Resection.-The writer has collected I 50 cases in which resection was performed. Of 64 of these, the details are given farther on in the text. There are but 3 deaths in the series, or 2 per cent.

From the above computation, which may be accepted as a fair representation of the dangers encountered in the performance of operations and in the treatment of stricture by dilatation, it will be seen that the mortality attending every form of treatment, with the exception of external perineal urethrotomy, is a very small one, and that upon the score of danger alone there is comparatively little choice between gradual dilatation, divulsion, and internal urethrotomy.

With regard to external perineal urethro omy, it must be remembered that the class of cases in which this operation is employed includes all of the conditions which are dangerous per se. Thus, the cases of urinary extravasation, which contribute most to increasing the mortality, are all in this class. So, too, the chronic suppurations with fistulæ. In such conditions it is not a question of choice. External perineal urethrotomy is the only thing to be done.

Causes of Death.- The causes of death in connection with surgical treatment of stricture are: (I) Shock. (2) Sepsis and urinary fever. (3) Suppression of urine. (4) In rare cases, hemorrhage following urethrotomy. 
It is not very rare to see the milder manifestations of shock in connection with the use of an instrument-even the passage of sounds-in the urethra. They are much more noticeable when the sound traverses the deep urethra than when it is carried no farther than the bulb. The signs of moderate degrees of shock are faintness, lowered blood pressure, and slight nausea. These are more noticeable with the patient standing up than if he is reclining. In a few rare instances death has followed the passage of a urethral sound into the bladder. The writer has been so unfortunate as to have had this experience in one of his cases. In this instance, death followed eight hours after the passage of a steel sound. The patient became faint while the instrument was being used, and suffered from repeated recurrences of collapse, in one of which he died. There was entire failure to respond to the usual means employed for combating such symptoms.

The only explanation for death occurring so quickly after instruments being used in the urethra would seem to be that of the propagation of greatly depressing impulse to the circulatory and nerve centres from the peripheral nerves in the urethra. It is to be noted, however, that Albarran found large numbers of the Bacillus coli communis in the blood, as well as in the urethra, of one patient, whose death followed about twelve hours after an internal urethrotomy, so that it is possible that sepsis may play an important part in causing such deaths.

In other cases the nervous impulse appears to be telegraphed directly to the kidneys, causing the suspension of their function, through which death is brought about. In the larger number of these fatalities the kidneys have already been seriously incapacitated functionally. An interesting fact in this connection is that the suppression of the renal function is quite as liable to follow the gentle use of instruments for the purpose of dilating strictures as it is after the performance of an internal urethrotomy.

Sepsis.-Sepsis was formerly a frequent cause of death, especially in connection with internal urethrotomy, but it is now a very rare one, because of the modern methods of guarding against its occurrence. The cases in which it continues to play an important part are those of urinary extravasation. In these, the patient comes to the operating table already profoundly infected and often in a very critical condition. The operation of external urethrotomy has nothing whatever to do with the patient's death under such circumstances; on the contrary, it affords him the only chance of life. The manner in which the septic condition arises in these cases has been discussed more fully under the heading of extravasation of urine, in an early part of the chapter, to which we refer the reader. 
Except for the few deaths that are due to hemorrhage following urethrotomy, the other fatal conditions named are shared about equally between the operative methods of treatment.

Permanency of Results of the Different Methods of TreatMENT.-The writer's opinions with respect to the permanency of the results obtained by the different methods are positive, and are founded upon his own experience and confirmed by that of others. They are as follows:

Internal urethrotomy, either alone or in combination with external perineal urethrotomy, is the only method of treatment by which an important number of cures of urethral stricture can be obtained.

By cure is meant cessation of symptoms and the maintaining of the urethral caliber at the formerly strictured parts to within one or two sizes of the French scale, or at the full normal caliber secured at the time of the operation, and without the employment of instruments for the purpose after convalescence from the operation is complete.

This, however, is true only when the operation of internal urethrotomy is properly performed and under proper conditions. By this is meant: (I) That the stricture shall be divided in each case to the full size of the normal caliber of the urethra of the individual in whom it exists, the caliber being estimated according to the rules laid down by the late Fessenden Otis, of New York. (2) The meatus should always be cut to the size $2 \mathrm{~mm}$. larger than the caliber of the urethra. (3) The patency of the urethra to the full size to which its strictured portion has been enlarged by the incision must be maintained until the healing of the wound is complete (usually fourteen days), by the passage of a sound every second day following the operation, the instrument being of the size of the normal caliber of the particular patient's urethra in each case. (4) A catheter à demeure should not be used. (5) The operation should not be undertaken in the presence of urethritis of acute or subacute character. (6) The urethra must be irrigated freely at the time of the operation, immediately after the first urination following it, and when the sounds are passed every second day during the healing of the wound.

When these conditions are complied with, we may expect to obtain about 60 per cent. of permanent cures in all cases except those of very dense and extensive strictures situated at the bulbomembranous urethra, which are best treated by external perineal urethrotomy or by resection.

Apart from the latter forms no other method of treatment yields anything like so large a proportion of cures as that which is to be obtained by internal urethrotomy. 
The following tables represent the writer's personal experience in the treatment of stricture of the urethra previous to the year 1907. The first of the tables simply gives the number of the cases in which each of the methods was applied, and the operative mortality attending them. In the second table are given the results of internal urethrotomy operations at periods varying between three years and twenty-two years subsequent to the times at which the operations were performed in 96 of the whole number of patients whose subsequent histories have been known to the writer.

Of gonorrhœal origin

Cases.

Traumatic 542

Due to the action of caustic

Congenital

Total

Methods of Treatment.

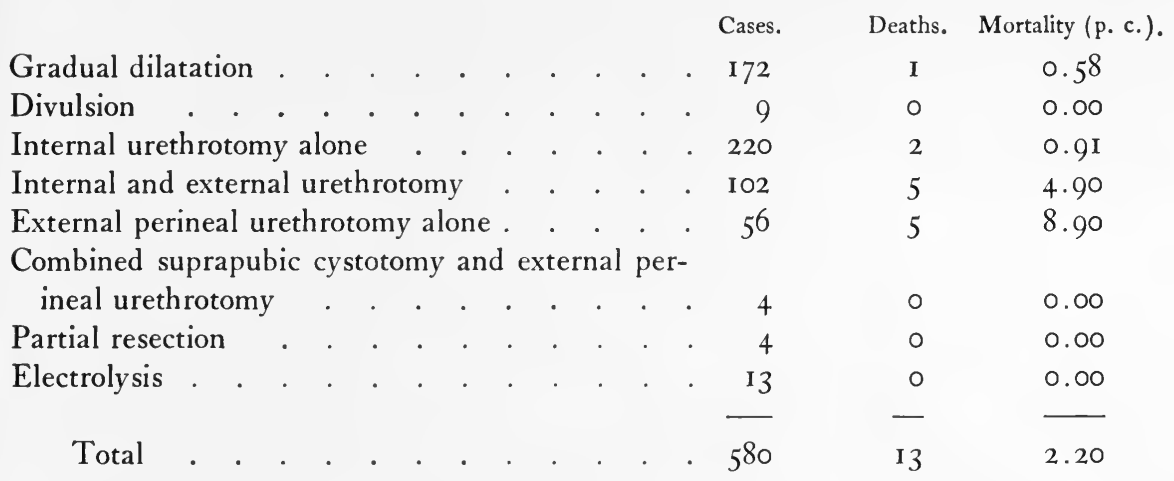

Subsequent Examinations.- There are altogether 96 patients who have afforded the opportunity for making examinations of the results of the operations of internal urethrotomy, done at previous times, varying from two to twenty-two years. Of these, there were 22 in which the intervals after the operations were less than two years, and these have not been included in the computation which follows:

There were 20 in which the operation was a failure so far as permanency is concerned, recontraction having taken place in varying degrees. In the remaining 54 cases cure was obtained, that is to say, in 55 per cent. The following table ${ }^{1}$ gives the results of the examinations:

${ }^{1}$ No instruments were used to maintain restored caliber of the urethra in any of these cases. 


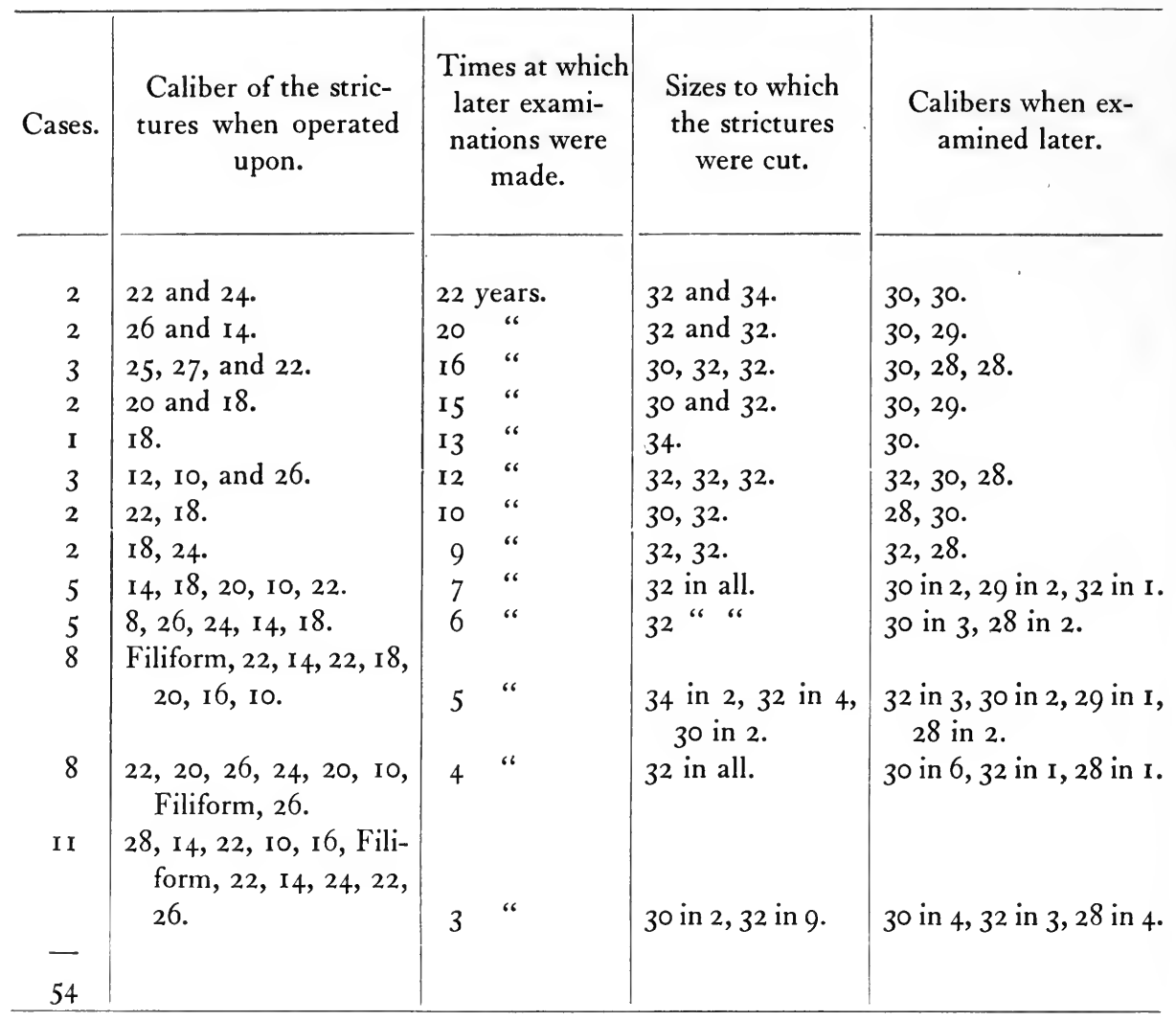

No recontraction whatever .

Cases.

Recontracted $4 \mathrm{~mm}$. in caliber .

I3

“" 3

Total 


\begin{tabular}{|c|c|c|c|}
\hline $\begin{array}{l}\text { Times elapsed } \\
\text { since operation. }\end{array}$ & Cases. & Use of sounds. & Recontraction or not. \\
\hline $\begin{array}{rc}\text { I2 } & \text { years. } \\
\text { I I } & \text { “ } \\
9 & \text { ، } \\
8 & \text { ، } \\
7 & \text { ، } \\
6 & \text { “ } \\
5 & \text { “ } \\
& \\
4 & \text { ، } \\
3 & \text { ، } \\
2 & \text { “ } \\
& \\
\text { I } & \text { ، }\end{array}$ & $\begin{array}{c}2 \\
\text { I } \\
\text { I } \\
\text { I } \\
\text { I } \\
\text { I } \\
2 \\
6 \\
3 \\
3 \\
6 \\
- \\
27\end{array}$ & $\begin{array}{l}\text { Yes. } \\
\text { No. } \\
\text { Yes. } \\
\text { Once in six months. } \\
\text { Yes. } \\
\text { Yes. } \\
\text { In I case, four months. } \\
\text { In I case, no sounds. } \\
\text { In I case, no; in } 5 \text { others, yes. } \\
\text { In I case, no; in } 2 \text { others, yes. } \\
\text { In I case, two months. } \\
\text { In } 2 \text { others, yes. } \\
\text { In } 4, \text { yes; in other } 2 \text {, for } 6 \text { and } 9 \text { mos. }\end{array}$ & $\begin{array}{l}28 \text { in both cases. } \\
\text { I6 } \\
2 \text { I } \\
22 \\
29 \\
30 \\
30 \\
26 \\
\text { I3, 25, 21, 25, I } 8,3 \text { I } \\
\text { Very small. 30, } 2 \text { I } \\
26 \\
26, \text { I I } \\
28,30,25,25,26,29\end{array}$ \\
\hline
\end{tabular}

It will be seen from this table that there were but 4 patients who did not use sounds at all. Recontraction took place in all these cases, as follows: $26, \mathrm{I} 6, \mathrm{I} 3$, and to a "very small size."

In 5 other cases, sounds were used but for a short time or at long intervals only. In 3 of these cases, cure may be properly said to have taken place; in the other 2, recontraction is noted to 26 and to 22 , respectively.

The remaining I 8 patients all used sounds subsequent to the operation. Even so, but 8 of them succeeded in maintaining the urethral calibers at sizes of 26 or more. Recontraction in various degrees between I I and 25 took place in the other Io, despite the use of sounds.

In other words, I I per cent. of cures by divulsion; 55 per cent. by internal urethrotomy; 6.9 per cent. by gradual dilatation.

Results of External Perineal Urethrotomy.-The later results of external perineal urethrotomy have been less fortunate in the writer's experience than those of the internal cutting operation so far as permanency of the results is concerned.

Of the I 2 cases in which the operation has been done by him, either alone or in combination with internal urethrotomy or suprapubic cystotomy, there are 23 in which subsequent examinations have allowed the results to be judged. In but 6 (or 26 per cent.) was cure effected.

The failure to cure strictures by this operation is not attributable to the operative method itself, but to the conditions associated with the stricture. We cannot look for cure in cases in which sloughing of the urethra 
has taken place in connection with urinary infiltration or diffuse septic phlegmon, or in which there is a great mass of connective tissue deposited in the perineum and urethral wall as a result of trauma.

It is possible, also, that the healing of the wound while in contact with dressings, drainage tubes, and while exposed to the surface, is an important factor in the production of connective tissue at the site of the incision during the reparative process.

Results of Resection.-Of the 64 cases of resection collected by the writer, there are but 13 in which subsequent examinations were made later than one year after the performance of the operations. In 5 of these the only statement made is that the urinary stream, or the urethral caliber, was normal. In the remaining 8 the results are stated as follows:

Three cases, examined one year after operation: calibers at sites of strictures were 24,26 , and 27 . In 3 others, examined at fourteen months, fifteen months, and two years, the calibers were 18,22 , and 23. In the last 2 cases the examinations were made at three, and six and one-half years; the calibers were 24 and 26.

In some of these cases the divided ends of the urethra were brought together and sutured; in others, the space between them was allowed to bridge over by granulation around a catheter. The results shown by these two methods did not appear to differ importantly.

\section{Cases Treated by Resection.}

\begin{tabular}{|c|c|c|c|c|}
\hline Operator. & Cases. & Deaths. & Urethra sutured or not. & Results. \\
\hline Lynn Thomas. & 2 & 0 & Not stated. & No detail. \\
\hline Manley. & 2 & 0 & $\begin{array}{l}\text { United by suture in } \\
\text { both. }\end{array}$ & Good. No detail. \\
\hline Keyes. & I & 0 & Plastic grafting. & $\begin{array}{l}\text { Cure. Seen one and one- } \\
\text { half years after operation. }\end{array}$ \\
\hline Guyon. & 9 & 0 & & \\
\hline Wile. & $\mathbf{I}$ & 0 & & \\
\hline Championniere. & I & 0 & & \\
\hline Jouon. & I & o & & \\
\hline Quenu. & $\mathbf{I}$ & 0 & & \\
\hline Despres. & I & ० & & Cure. \\
\hline & - & - & & \\
\hline & I9 & 0 & & \\
\hline
\end{tabular}

Seventeen of the above are quoted by Fenwick. The following 14 are quoted by Vignard: 
Cases Treated by Resection (Continued).

\begin{tabular}{|c|c|c|c|c|}
\hline Operator. & Cases. & Deaths. & Urethra sutured or not. & Results. \\
\hline Mollière. & 3 & 0 & $\begin{array}{l}\text { Urethra united by su- } \\
\text { ture in } 2 \text {; in I, not. }\end{array}$ & $\begin{array}{l}\text { Two cases sutured, seen } \\
\text { at I } 7 \text { mos. and } 8 \text { mos. } \\
\text { after operation. In both, } \\
\text { full stream. In I, caliber } \\
\text { called normal. In I not } \\
\text { sutured, caliber normal } \\
\text { at I } 4 \text { mos. }\end{array}$ \\
\hline König. & I & o & Not sutured. & $\begin{array}{l}\text { At Io mos., caliber normal. } \\
\text { No. not given. }\end{array}$ \\
\hline Heusner. & I & o & Sutured. & $\begin{array}{l}3 \text { years later, normal cal- } \\
\text { iber. No. not given }\end{array}$ \\
\hline Heuerteloupe. & I & o & Not sutured. & $\begin{array}{l}\text { At } 6 \frac{1}{2} \text { years, caliber main- } \\
\text { tained. }\end{array}$ \\
\hline Mayo Robson. & I & o & Sutured. & $\begin{array}{l}\text { At } 6 \text { mos., } 24 \text { French } \\
\text { passed easily. }\end{array}$ \\
\hline Loquin. & 2 & ० & Sutured. & $\begin{array}{l}\text { At I year, caliber normal; } \\
\text { I year, } 24 \text { caliber. }\end{array}$ \\
\hline Socin. & 2 & 0 & Sutured. & $\begin{array}{l}\text { At } 2 \text { years and at } 9 \text { mos., } \\
\text { I } 8 \text { and } 23 \text { calibers. }\end{array}$ \\
\hline Guyon (child of IO). & I & ० & Sutured. & I I mos., 24 caliber. \\
\hline Wahl. & I & o & Sutured. & Immediate result good. \\
\hline Heurteaux. & $\frac{I}{14}$ & $\frac{0}{0}$ & Sutured. & At 6 mos., I 4 caliber. \\
\hline Fuller. & 2 & ○ & Not sutured. & $\begin{array}{l}\text { At I year and at } 4 \text { mos., } \\
\text { calibers } 27 \text { and } 26 \text {. }\end{array}$ \\
\hline Platt. & I & o & Sutured. & $\begin{array}{l}\text { At I } 8 \text { mos., good. No de- } \\
\text { tails. }\end{array}$ \\
\hline Albarran. & 9 & o & $\begin{array}{l}\text { Sutured, 4; not su- } \\
\text { tured, } 5 .\end{array}$ & $\begin{array}{l}\text { In } 4 \text { sutured cases, at } 3 \\
\text { mos., Io mos., I } 4 \text { mos. } \\
\text { (time not stated in } 4 \text { th), } \\
\text { calibers } 50 \text { Beneque, } 22 \\
\text { Charrière, } 58 \text { Beneque; } \\
\text { fourth case, no number } \\
\text { given; } 3 \text { cases, not su- } \\
\text { tured, at } 5 \text { mos., imme- } \\
\text { diate, and } 6 \text { weeks, } 56 \text {, } \\
60 \text {, and } 44 \text { Beneque; } 2 \\
\text { cases, no data. }\end{array}$ \\
\hline Guyon. & 5 & 0 & $\begin{array}{l}\text { Sutured,2; not sutured, } \\
\text { I; urethra restored } \\
\text { from soft parts, } 2 .\end{array}$ & $\begin{array}{l}2 \text { sutured, at } 5 \text { mos. and at } \\
\text { end of several weeks, } \\
\text { calibers } 52 \text { and } 50 \text { Ben- } \\
\text { eque; I not sutured, at } \\
\text { I year, } 24 \text { Charrière; } 2 \\
\text { plastics, I lost sight of, I } \\
\text { at } 3 \text { mos., } 48 \text { Beneque. }\end{array}$ \\
\hline
\end{tabular}




\section{Cases Treated by Resection (Continued).}

\begin{tabular}{|c|c|c|c|c|}
\hline Operator. & Cases. & Deaths. & Urethra sutured or not. & Results. \\
\hline Delorme. & I & 0 & Sutured. & At 8 mos., 40 Beneque. \\
\hline Tilden Brown. & I & 0 & Sutured. & At 10 mos., 32 Charrière. \\
\hline Degas. & $\mathbf{I}$ & o & Not sutured. & $\begin{array}{l}\text { At } 3 \text { mos., caliber as at } \\
\text { time of operation. }\end{array}$ \\
\hline Legueu. & 8 & I & $\begin{array}{l}\text { Sutured, 7; I not su- } \\
\text { tured. }\end{array}$ & $\begin{array}{l}\text { I not sutured, at } 2 \text { mos., } \\
\text { caliber } 45 \text { Beneque. In } \\
6 \text { sutured, dates of re- } \\
\text { examinations I } 5 \text { mos., } 6 \\
\text { mos., } 2 \text { mos., } 3 \text { mos., } 3 \\
\text { mos., } 8 \text { mos., calibers } 25 \text {, } \\
48,42,50,45,45 \text { Beneque. }\end{array}$ \\
\hline Genouville \& Zadok. & $\frac{3}{64}$ & $\frac{0}{I}$ & $\begin{array}{l}\text { Partial suture in } 2 ; \\
\text { complete suture in } \mathrm{I} .\end{array}$ & $\begin{array}{l}\text { I mo., } 60 \text { Beneque; other } \\
\text { 2, detail inadequate. }\end{array}$ \\
\hline
\end{tabular}

While it is true that there are but one or two cases which could be regarded as cures in the sense of our definition, yet in a considerable number it has been possible to maintain the caliber of the new urethra sufficiently, by the periodic passage of sounds, to keep the patient's health good and to prevent the urethra from contracting. It should be remembered also that the operation has been reserved for such cases as those in which all other methods have failed. They have been the more extreme examples of extensive and dense connective-tissue deposits in and around the urethra which had rendered futile all other forms of treatment, so that we should not expect favorable results as compared with the less severe forms of the disease treated by external perineal urethrotomy, etc.

Results of Electrolysis.-In I 888 Tilden Brown ${ }^{12}$ published an interesting and convincing article, in which he showed conclusively that the method of electrolysis for the treatment of stricture resulted, in many instances, in a greater deposit of connective tissue than was originally present, and that the method was not only without benefit, but was frequently injurious.

At about the same time the writer gave a careful trial to the method in 20 cases of well-defined organic stricture of the urethra anterior to the bulbous portion. In 13 of these he was able to follow the results subsequently. Of these, there were 4 in which there was undoubtedly a greater tendency to more rapid contraction of the strictures than had been present previous to the application of the treatment. In the remainder no benefit whatever followed. In one of them an acute urethritis resulted. 
The experience of all specialists of note in this country has been similar to that above described. The method we consider one that is undesirable to employ.

Summary of Treatment with Reference to Choice of Method.-From the foregoing statements concerning the relative mortality, sphere of application, and permanency of the results of the different ways of treating stricture, it will be seen that no one of them is universally applicable to all cases, and that each of them has its particular advantage, and becomes the method of choice according to the special conditions presented by the individual patient.

From the standpoint of surgical indications alone, it is obvious that internal urethrotomy is the best treatment that can be applied to all strictures of the urethra which are situated within five inches from the meatus, for the reason that it is as safe in this class as any other method, and the only one that offers the prospect of a large proportion of cures in the sense in which we have used that term. It is an operation which should not, we believe, be applied to strictures more deeply seated than the point named above, because of the danger from sepsis and hemorrhage which is then involved. It is the only method that can be of benefit in cases of resilient strictures.

Gradual dilatation is the best method to apply to all strictures at or beyond five inches from the meatus which are suitable for its employment, by which we mean those that can be dilated within a reasonable time, and that will maintain the dilatation for not less than a week after each sitting, and in cases in which the patient does not show constitutional disturbance of importance during the course of the treatment.

Divulsion is a safe procedure, but is open to the objections which have already been stated, and has no corresponding advantages. It is a form of treatment which we believe is never desirable to employ.

External perineal urethrotomy is the safest and most satisfactory form of treatment in cases of stricture of the deeper urethra which are not amenable to gradual dilatation and which are not so dense as to demand resection. It allows hemorrhage to be controlled and supplies drainage, thus avoiding the danger of sepsis. It is the only operation that should be performed in cases of suppuration occurring from urinary extravasation, peri-urethral abscess, and diffuse septic phlegmon in connection with stricture.

It is a valuable adjunct to internal urethrotomy for the purpose of averting urinary fever. External perineal urethrotomy, or one of the special procedures which are modifications of it, is the only operation that it is possible to apply in cases of impermeable stricture of the deep 
urethra. These modifications or substitutes for the usual method of doing the external perineal operation are: Cock's operation, the open dissection of the perineum to expose the posterior urethra, and the suprapubic retrograde method.

Of these, we think Cock's operation should be discarded, while between the open perineal dissection and the suprapubic manner of approach there is but little to choose, and the surgeon may exercise his personal preference.

Resection should be chosen in cases of stricture of the perineal urethra, which are composed of, or surrounded by, such dense masses of connective tissue that they are not amenable to treatment by any other method.

Treatment of Complications.-Urinary Extravasation and Acute Diffuse Septic Phlegmon.-The essential points in the treatment of this complication are: promptness, free skin incisions extending throughout the entire area involved, the evacuation and cleansing of all pus cavities, and the removal of all necrotic tissue.

The urethra should always be laid open in the perineum by a median incision. From this avenue of approach it will be possible to reach and freely open all pus collections.

In the cases in which operation has been delayed, even but a few hours sometimes, gangrene of the skin and greater or less destruction of the urethra and surrounding parts often take place. The tissues involved may be black, and look as though they had been charred by heat. Sometimes a bit of the urethra two or three inches long will be found lying loose in the cavity of the abscess surrounding it, and can be removed in the form of a gangrenous tube. The ischiatic fossæ are often involved in the process and more or less of their contents will have been destroyed by the suppuration.

When there has been destruction of part of the urethra, a soft catheter should be passed into the bladder through the whole length of the canal as soon as the septic process has subsided, if the anterior urethra is not occupied by strictures, which, of course, might make it impossible to do this. The object of placing a catheter in the urethra is that the newly formed part of the canal which is made in the healing over of the space where a part of it has been destroyed may be moulded about the instrument into a direct and straight channel instead of a tortuous one, which might otherwise be formed.

RETENTION OF URINE.-The treatment of retention of urine due to urethral stricture is: (I) Palliative. (2) Operative.

I. Palliative Treatment.-Hot hip baths, belladonna and opium suppository, hot fomentations over the symphysis pubis and to the 
perineum, recumbent posture with hips elevated. Direct the patient to pass his urine into the bath if he finds it possible to do so while seated in the tub.

2. Operative Treatment.-If the conditions are favorable to its immediate performance, external perineal urethrotomy may be done at once. It is usually better, however, to aspirate the bladder above the symphysis pubis, and withdraw the larger part-not the whole-of its contents before operating upon the stricture. (See chapter on the Technique of Operations on the Bladder.) Suprapubic aspiration should be the first step in all cases in which it is known that the retention depends on the presence of a tight organic stricture. In order to determine whether or not this is the cause of the retention, the attempt should be made to pass a catheter into the bladder. It will sometimes be found that a large instrument will pass without difficulty, and that the retention is not dependent upon a tight stricture at all, but upon spasm. If a tight stricture is found to be the cause of the retention, the best instrument with which to try to enter the bladder is Harrison's "whip catheter" (Fig. 36). If the palliative measures have relieved the patient for the time being, the stricture should be operated upon as soon as may be thereafter.

Urethral Fistula.-Urethral fistula results from the establishment of a channel of communication between the urethra, on the one side, and the surface of the penis, perineum, or the rectum, on the other, the channel being produced by suppuration of the peri-urethral tissues or by rupture of the urethra behind a stricture, the escape of urine through the rent, and suppuration as a result of the accident; or, again, the suppuration may originate in one or another of the glandular structures which are in immediate relation with the urethra, and break into the latter, subsequently burrowing to the surface and making a channel between the two in that manner. In some cases the tracts thus made may be very long, and numerous. These features are dependent upon the direction taken by the fluids as they travel beneath the tissues on their way to the surface.

The condition has already been discussed under the heading of Extravasation of Urine and Diffuse Septic Phlegmon in the early part of the chapter.

The larger number of urethral fistulæ are in the perineum.

In recently formed tracts, the walls are soft and covered with granulations which bleed freely; later, this tissue is replaced by a tough contractile connective tissue, which often is so extensive as to occupy the entire space of the perineum and to extend as far back as the prostate. 
The chief danger connected with these fistulæ is in their becoming the starting points for infection and suppuration at any time, the results of which may be grave.

Fistulæ which open into the urethra behind strictures, as they almost always do, are perpetuated by the presence of the stricture, and cannot be healed so long as the obstruction remains. A prime essential to the cure of fistula, therefore, is the restoration of the strictured part of the canal to its normal caliber, or at least keeping it sufficiently patent to prevent the urine being forced into the fistulous tract by the intra-urethral pressure during urination.

This is best accomplished by the operation of internal urethrotomy for such strictures as are in the penile urethra, which are those responsible for the production of the urethral fistulæ we are here considering, and external urethrotomy or resection of strictures that are situated at or near the bulbomembranous junction.

The restoration of the normal caliber of the urethra at the site of stricture will frequently cure the fistula; when it does not it may be necessary to divert the urinary stream entirely from the urethra by suprapubic cystotomy, and to employ prolonged drainage through that channel. The less radical course of permanent drainage through the urethra by a catheter should be tried before resorting to the last-named means. In conjunction with, or previous to, either of the above methods of treatment, excision or incision of the fistulous tract and the milder procedure of incision of the external orifice of the tract, curetting its inner surface and packing it in such a way as to secure healing from the bottom, should be tried. (For description of operative methods see chapter on Technique of Operations on the Penis.)

False Passage.-A false passage may be made either by forcing an instrument through the urethral wall when passing it into the bladder, or by breaking into the urethra of a peri-urethral abscess from without. In some instances the false passage will pass out of the urethra at one point and reënter it at another (Fig. 4I).

The most frequent sites of false passages made with instruments are at the bulbomembranous junction and in the prostatic urethra, the latter being especially common in cases of prostatic hypertrophy. In connection with stricture, they are, of course, most commonly situated just in front of the anterior face of the narrowing, that is to say, at the point at which the instrument meets with resistance to its further passage through the canal.

When due to suppuration and breaking through of abscess, the opening of the false passage will almost invariably be behind the posterior face of the stricture. 
Consequences of False Passage.-The results of making false passages are: (1) Hemorrhage. (2) Extravasation of urine and suppuration in one form or another-peri-urethral abscess, or septic phlegmonous inflammation. (3) In some cases the establishment of a communication with the rectum.

A curious fact which has often been noticed is that urinary suppression and shock are less likely to occur in connection with the violence done to the urethra in making a false passage than when gentle manipulation of instruments has been employed. Not so, however, with the serious local or general septic conditions, for these are sometimes produced as the result of instrumental injury done to the urethral walls.

Urinary extravasation is not a very common sequence of false passages, in the anterior urethra at any rate, and less so than would be supposed with those of the deeper parts of the canal.

Among the difficulties resulting from the making of a false passage is that attending the subsequent passage of instruments through the urethra.

Hemorrhage in connection with false passage is not often of serious importance, though exceptionally it may continue for a considerable time. In many instances false passages heal spontaneously, but in the majority such is not the case.

The evidence that an instrument has made or has entered into

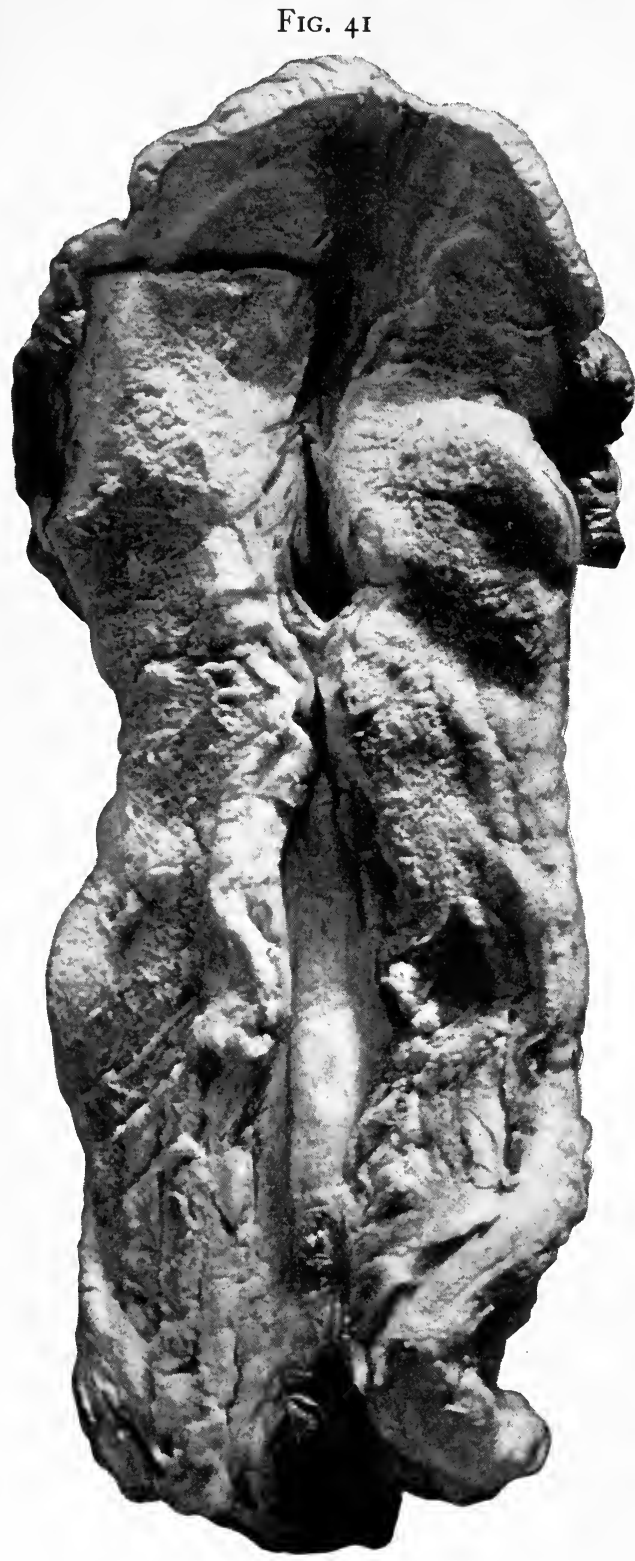

Showing false passage through urethral wall. a false passage is the sense that its tip has been arrested at the end of a blind pocket when the attempt is made to urge it onward through the urethra. If it is a sound that is being used, it will not be easy to rotate it from side 
to side. Blood flowing from the meatus, in conjunction with the other signs just mentioned, is another evidence of the occurrence of the accident, and if the false passage has been made in the deep part of the canal upon its floor, the tip of the sound will be felt by a finger in the rectum in such a way as to make it evident that there is a very thin layer of tissue separating the instrument from the bowel.

The treatment will depend upon the size, location, and extent of the false passage.

When the prostate, bladder, or rectum has been penetrated by an instrument, surgical intervention should be prompt, and should take the form of an external perineal urethrotomy, slitting up the false passage and packing and treating the wound afterward in such a way as to have it heal from the bottom.

When, on the contrary, the false passage is situated anterior to the bulb, or if it be of small size, the best treatment is no treatment at all, and especially to avoid all instrumental interference.

In the average case of the latter description there will be a moderate amount of bleeding for two or three days, followed probably by a slight purulent discharge for a week or two, and the passage will then heal.

Illustrative Cases-Two Cases of Stricture following Rupture of the Urethra Produced during Coitus.

CAsE I.-A young man and his partner in marriage, while both under the influence of liquor, indulged in coitus. The man failed to make a proper entrance, striking the head of the penis on the side of the vulva. He experienced a sharp pain upon so doing. A small quantity of blood flowed from the meatus immediately afterward. The patient experienced no difficulty in urination upon the first attempt after the accident. An hour later, however, a large swelling appeared in the perineum and scrotum, the surface of which was deeply discolored. Four days later the patient was admitted to the hospital, with high fever, rapid pulse, great prostration, and an infiltration of urine extending over the whole surface of the penis, scrotum, perineum, and upward over the symphysis pubis. The integument over the whole surface of the scrotum and penis was involved in a process of beginning gangrene. The patient's condition was critical.

Operation.-Free incisions were made through the gangrenous area. The subcutaneous tissue throughout the greater part of this was as black as though it had been charred by fire. A moderate amount of decomposed blood and urine and stinking pus were confined within this area, and were evacuated by the incisions. 
The urethra was opened by a median perineal incision. A rent half an inch in length was found upon the floor of the canal just in front of the bulbomembranous junction. The whole of the affected area was cleansed, sloughs were removed, a catheter was passed into the bladder through the whole length of the urethra three days later, at which time the involved area had become sufficiently freed from infective processes to make this safe to have done.

The skin of the whole penis and scrotum sloughed off. The bare surface left by this was later grafted by Thiersch's method of skin grafting. This was absolutely successful in its result, and secured to the patient a perfectly useful organ.

Six months later he again came to the hospital with a-firm traumatic stricture at the site of the ruptured urethra. An internal urethrotomy was performed, which opened the constricted part of the urethra to the size of 32 of the Charrière scale. The patient stated that his accident had not interfered with sexual relations after he was discharged from the hospital.

CASE II.-In this instance coitus took place with the woman upon the man. In coming down upon the head of the erect penis she struck upon it with almost her whole weight. Sharp pain immediately followed the injury, a profuse hemorrhage from the meatus took place at once and the penis became enormously distended with extravasated blood.

The patient was unable to pass any urine through the urethra upon making the attempt to do so an hour or two later. He then sought medical aid. A catheter passed easily through the urethra into the bladder. A laceration of the urethral wall had been made about two and one-half inches from the meatus. The outcoming stream of urine lifted the free edge of this rent and carried a bit of the urethral wall, which was large enough to occlude the canal, across to the other side of it. This was the cause of the retention. Stricture subsequently formed at the site of the injury; it was treated by internal urethrotomy, and with a good result.

CASE III. Rupture of urethra produced by a blow.-A sailor, while using a hand-winch, let the handle slip through his fingers, and in flying back it struck the penis upon the under surface, raised it upward, and crushed it against the bones of the symphysis. A moderate hemorrhage from the meatus ensued shortly afterward. Extravasation of urine took place immediately upon the first attempt to urinate after the injury. Retention was complete. The infiltration was of great extent, and involved the subcutaneous tissues of the abdomen far up above the umbilicus. Suppuration took place and pus finally broke through the surface in two places, one of them being at the site of the rupture of the 
urethra, which was at the point at which it had been crushed by the blow of the winch, the other through the integument of the abdomen, about two inches below the level of the umbilicus on the right side. Through these two openings the urine and pus escaped, and thus relieved the patient from his dangerous condition.

A dense stricture subsequently formed, about three inches from the meatus. It proved intractable to treatment by internal urethrotomy, and was submitted to resection about one year after the injury. The divided ends of the urethra were brought together by suture; union by first intention took place, and the urethra allowed the passage of a No. 26 sound readily three months subsequently. The patient was not seen later than this.

CASE IV.-Urinary abscess originating in suppuration of the prostate and brought about by rupture of the abscess into the urethra. There was no stricture of the urethra in this case. The communication with the urethra is stated by Motz and Bartrina to have been secondary to an abscess of the prostate gland.

CASE V.-Peri-urethral abscess originating in the glands of Littre, situated above the canal of the urethra. The abscess burrowed around and beneath the membranous urethra and appeared in the perineum. without having any communicating opening with the urethra.

CASE VI (Watson).-Acute diffuse phlegmonous inflammation of the penis; gangrene; necrosis of the perineal urethra; extravasation of urine. -A man, aged seventy years. Stricture, for many years, but had never had any serious trouble from it, because of having kept it open without difficulty by the use of bougies.

He was apparently in good health until forty-eight hours previous to entrance into the hospital; at that time there was some pain in the perineum, very soon followed by difficulty in voiding his urine. A few hours later there was a sudden swelling of the penis, scrotum, perineum, and lower part of the abdomen. Twenty-four hours after the appearance of the first symptom a dull-red spot appeared on the prepuce; this spread rapidly, and extended over an area of the size of a silver halfdollar. The perineum soon became involved in a similar process, which extended forward over the under surface of the scrotum. (Plate XV.) On admission to the hospital the pulse was 120 , feeble, and irregular, the tongue heavily coated, shallow respiration and pallor were present, and the patient was unconscious. There was marked emphysematous crackling beneath the skin of the perineum, under side of scrotum, and the prepuce was gangrenous.

Operation.-Incisions were carried through the skin of the entire area involved in the process. Those upon the penis gave exit to gas, a turbid 


\section{PLATE XV}

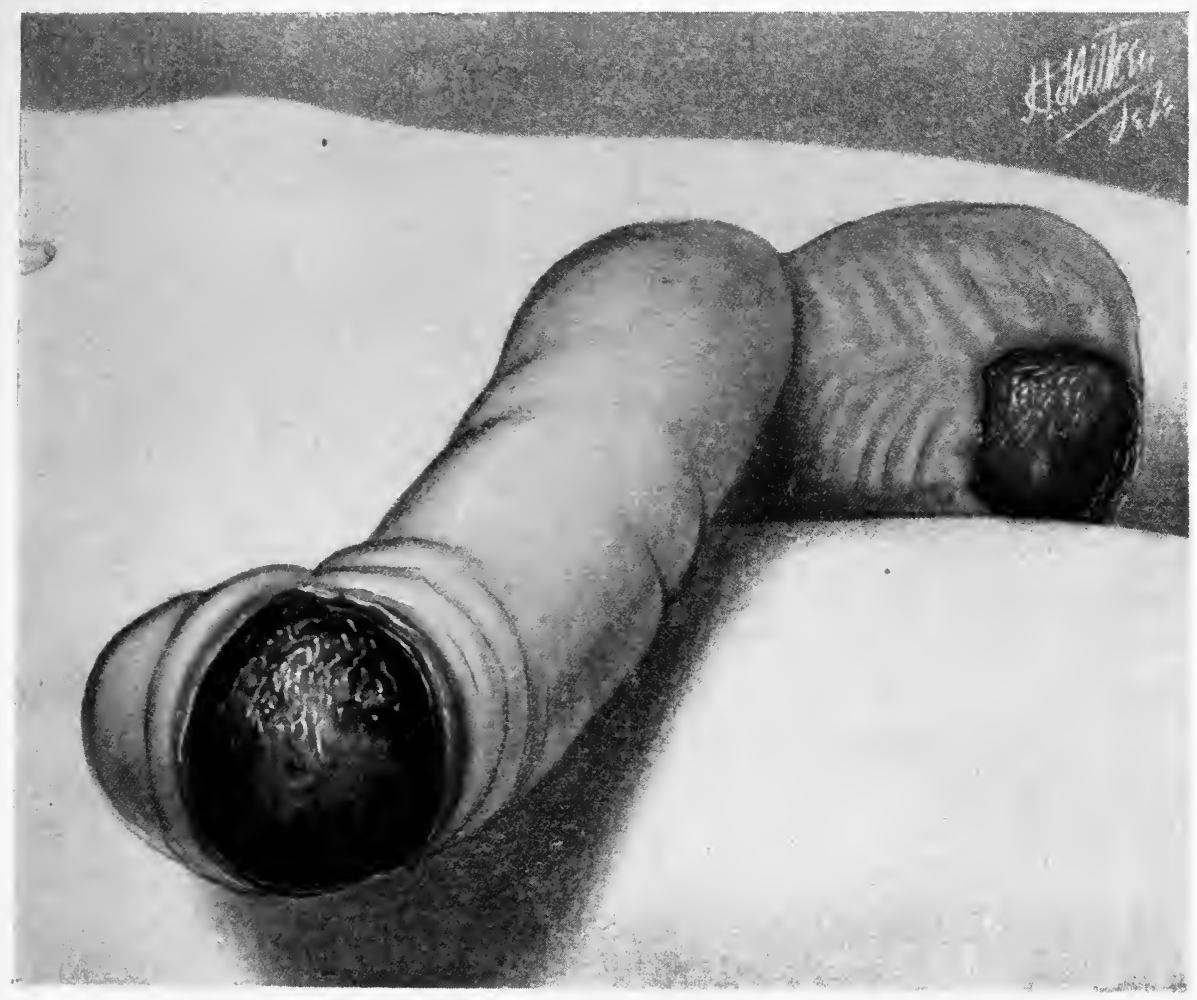

GANGRENE OF SKIN OF SCROTUII AND OF END OF PENIS, DUE TO EXTRAVASATION OF URINE. 
fluid, and a large quantity of foul-smelling pus and urine, and gas was evacuated through the median perineal incision. Slightly turbid fluid and gas were also evacuated through two incisions in the skin of the lower part of the abdomen. The perineal portion of the urethra was necrotic, black, and came away in the forceps upon slight traction. The areolar tissue of the ischiorectal fossæ was in part sloughing.

The entire area involved in the process was cleansed and packed with iodoform gauze. The patient's condition was critical for the first twentyfour hours, after which he slowly recovered.

CASE VII. Occlusion of the anterior urethra produced by injecting perchloride of iron to stop hemorrhage caused by the making of a false passage.-The patient had had an external perineal urethrotomy performed nine years previously upon a stricture of the bulbous urethra. Four years later the stricture had recontracted and he had retention of urine. While trying to relieve this condition a physician made several false passages in the urethra, and thereby caused severe hemorrhage. Becoming alarmed, the medical attendant injected perchloride of iron into the canal for the purpose of arresting bleeding. In consequence of this treatment the entire inner lining of the penile urethra sloughed and the whole length of that part of the canal subsequently became converted into a dense mass of connective tissue. Abscess of the deeper part of the urethra, spontaneous rupture through the perineum, and the establishment of a perineal fistula, which remained open for some months, resulted.

When the patient was first seen by the writer the fistula had been closed for a month, and twenty-four hours before first visiting him retention had taken place.

The immediate treatment was repeated suprapubic aspirations of the bladder. On the day following, the urethra was examined. The anterior part of it was wholly occluded, and no instrument could be passed through that portion of the canal. Perineal section without a guide was performed, and a permanent fistula in the perineum was established. The relief thus afforded kept the patient in good health and in comfort during the succeeding five or six years, after which he was lost sight of.

CASE VIII (Reported by Cunningham ${ }^{13}$ ).-Congenital occlusion of the urethra.-Both parents normal. When four months pregnant, the mother had a fall, and one month later was accidentally knocked down. Gestation proceeded, however, to full term.

When delivering the child, there was no difficulty in so doing until the head and shoulders had passed; it was then found impossible to deliver the body without using much force. 
The patient was etherized, and the child was finally delivered by the combined efforts of Drs. Percy and Hooker.

The fœtus was dead when born. Cunningham gives the following description of the specimen:

The Specimen.-The fotus measures $50 \mathrm{~cm}$. from crown of head to sole of foot, and $47 \mathrm{~cm}$. in circumference at level of umbilicus. The abdomen is enormously distended by a slightly lobulated mass. There is an intra-uterine amputation cicatrix of right leg a little below the knee. Otherwise the fotus seems normal. Incision of the amputation stump reveals a considerable amount of yellowish creamy pus just-beneath the cicatrix. Over the thorax is a large subcutaneous hematoma extending from the region of the suprasternal notch well down to the lower portion of the sternum.

The abdomen is filled by a large, fluctuant, coarsely lobular mass, extending from the pelvis, which it fills, almost up to the xiphoid cartilage. The intestines are crowded above this. No appendix is visible, and cecum is rudimentary, but there is a distinct Meckel's diverticulum. The liver, heart, lungs, and spleen present no abnormality.

Urethra.-A probe can be introduced through a normal meatus to the triangular ligament where the canal becomes imperforate.

Bladder.-The bladder is lobulated, and on section shows several imperfect partitions, suggesting a division of the viscus into chambers. The walls are very thin, and the partitions on the inner surface of the organ are seen as constrictions on the external sacculated surface.

Ureters.-The ureters are greatly dilated, their lumen being as large as that of the small intestine (Fig. 42). The normal points of narrowing, the "superior" and "inferior isthmus," are evident, although they are dilated in proportion to the remainder of the canals. There is no suggestion of a ureteral valve.

Kidneys.-The kidneys are fluctuant, cystic masses, which, on section, show the left organ to be a large, thick-walled cyst with a few smaller cysts connecting with the greater cavity. There is a little parenchyma at the upper pole. The right kidney is less destroyed, and is composed mostly of numerous small cysts and a little parenchyma at its upper pole (Fig. 42).

The rectum and anus are normal. The scrotum is also normally formed. The penis is normal in shape, rather large in size, and the skin suggests scrotal integument rather than that normally found as the penile skin.

The Genital Tract.-The testicles, epididymis, vas deferens, and seminal vesicles cannot be found. No trace of the gubernaculum can be detected. A dissection of the region normally occupied by the seminal 
vesicles, the inguinal and lumbar regions (the areas in which the testicle and epididymis are formed), and microscopic section of suspicious tissue, fails to reveal anything suggesting these structures.

The prostate is smaller than normal. The openings of the ejaculatory ducts cannot be found in the prostatic urethra. Serial microscopic sections of the gland from the vesical orifice to the apex show the urethra, but no ejaculatory ducts are present.

FIG. 42

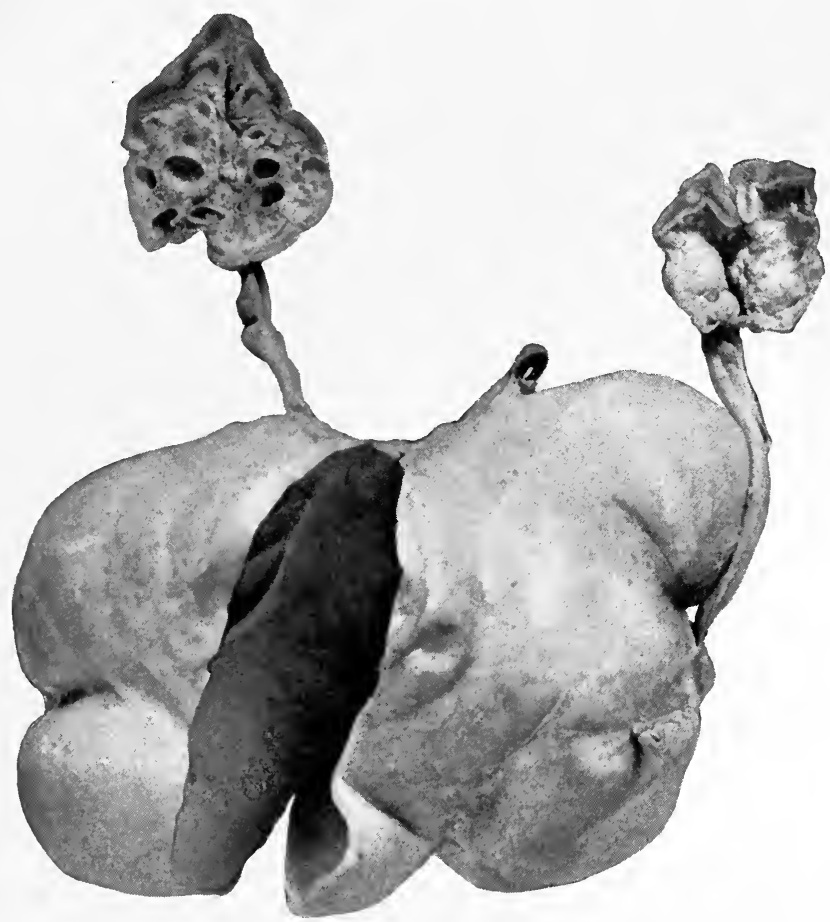

Bladder saccular, showing imperfect partitions within, and constriction on the external surface. The ureters dilated, with the superior isthmus plainly seen in the right ureter. The kidneys are dilated and cystic. (Cunningham.)

Consideration of the Case.-In addition to the urethral obliteration and its consequences the feature of special interest in this case is that of anorchism, or complete absence of the testicles, which is very rare indeed. The condition is confused with cryptorchism, from which it can only be differentiated by dissection. In the case here recorded, a careful dissection of the scrotum, inguinal and lumbar regions, and microscopic examinations of suggestive tissue failed to reveal any sign of testicular tissue, nor could any part of the seminal tract be discovered in gross or by the microscope. 
Jacobson states that, with absence of the testicle, the following developmental defects may exist:

I. Usually, absence of the epididymis and part of the vas deferens.

2. Exceptionally, entire absence of the seminal duct up to the vesicle.

3. Still more rarely, only the testicle is wanting.

4. The testicle may be present, and the vesicles, epididymis, and vas absent.

In this case, no evidence whatever of the seminal tract could be found. The prostate, however, was not abnormal; serial sections through the gland from the vesical orifice to the membranous urethra showed it to be normal in structure, but without the ejaculatory ducts.

In this respect there is but one other authentic case of anorchismFisher's case, much quoted, and being remarkable for the feminine qualities of the individual, who lived to be forty-five years old. After death a careful dissection revealed no evidence of testicular tissue.

\section{BiBLIOGRAPHY.}

I. Keyes. Surgical Diseases of the Genito-urinary Organs, New York, I905, p. I75.

2. Frisch and Zuckerkandl. Handbuch der Urologie.

3. Guibe. Ann. des Mal. des Org. Gén.-urin., ı896, p. I87.

4. Finger, Gohn, and Schlagenhaufer. Arch. f. Dermat. u. Syph., I894, xxviii, 277.

5. Motz and Bartrina. Ann. d. Mal. d. Org. Gén.-urin., Paris, I903, xxi, ı6or.

6. Thompson (Sir Henry). Clinical Lectures on Diseases of the Urinary Organs, Philadelphia, 1874, p. 56.

7. Bazy and Decloux. Ann. d. mal. d. Org. Gén.-urin., Paris, I903, xxi, 24I.

8. Otis. Stricture of the Male Urethra, New York, i88o, p. 89.

9. Guillon, Reynes et al. Assoc. Franc. d'Urol., Proc.-verb., 5e session, Paris, I90I, I902, 24I.

I0. Harrison. Lectures on Surgical Disorders of the Urinary Organs, 3d edition, London, I887.

II. Reynes et al. Compte rendu de l'Assoc. Franc. d'Urologie, I90I.

I2. Brown (F. Tilden). Journal of Cutaneous and Genito-urinary Diseases, New York, I888, vi, 244 .

13. Cunningham. American Journal of Dermatology and Genito-urinary Diseases, December, 1907, p. 485 . 


\section{H A P T E R I V. \\ TECHNIQUE OF OPERATIONS FOR STRICTURE OF THE URETHRA.}

\section{DIVULSION.}

Position of Patient.-Recumbent.

Instrument.-Bigelow's divulsor (Fig. 43).

In this operation the stricture is split open by means of the sudden application of force applied by a tapering metal sound, which is driven through the constricted part of the canal, being guided by a small rod which passes through the centre of the divulsing part of the instrument.

FIG. 43

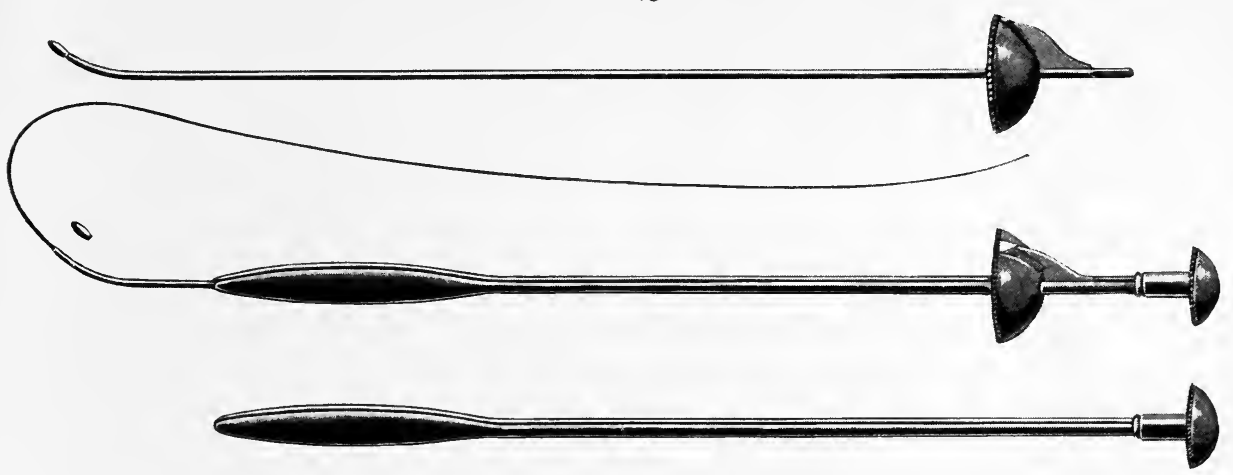

Bigelow's divulsor.

Operation.-If the stricture is so small as to allow the passage of a filiform bougie only, the filiform guide, to the outer end of which a metal cap is fitted, is first passed through the urethra. This being done, screw the rod of the Bigelow divulsor to the metal cap and pass the rod through the urethra, pushing the filiform guide ahead of it into the bladder.

Fit the divulsing sound (Fig. 43) upon the rod. Hold the latter steadily, and drive the divulsing metal forcibly through the stricture.

Withdraw the instrument from the urethra. Empty the bladder with a soft-rubber catheter. Irrigate the bladder. Irrigate the urethra thoroughly on withdrawing the catheter from the bladder.

The catheter may be tied in or not, according to the individual surgeon's views. We advise leaving it out. If it is to be tied in, the urethral irrigation should be done before the catheter is secured in place. 
The instrument may be retained in a number of different ways; the one devised by the writer (illustrated in Fig. 44) is a convenient one, and is carried out by slipping over the outer end of the catheter a piece of drainage tube about five inches long which has been cut in

FIG. 44

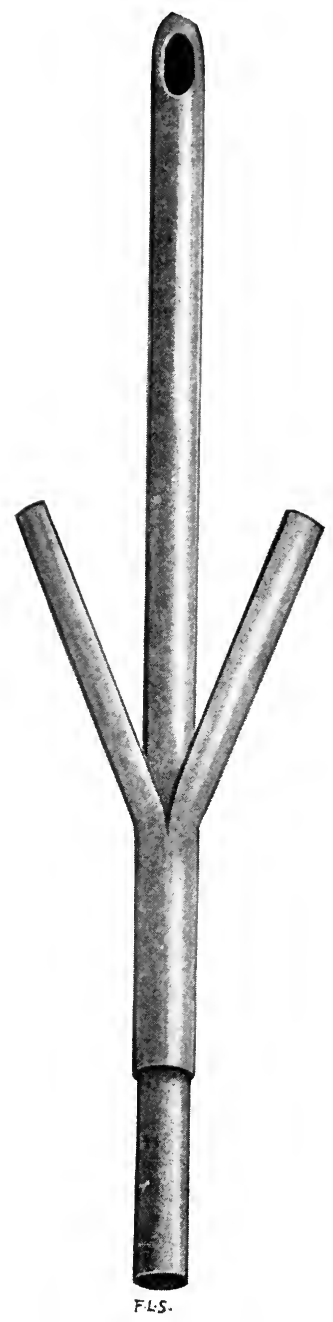

Author's manner of arranging catheter for tying it into the bladder. (Watson.) two longitudinally, except for an inch at the end, through which the catheter passes. The caliber of the catheter should be one size larger than the inner caliber of the tube, so that the instrument will bind upon the tube and be prevented from slipping in or out, once its proper place with reference to the tube has been determined. By stretching the catheter, the drainage tube can be readily slipped forward or backward upon it to whatever point may be desired. When this has been determined, all that remains to be done is to pass the catheter through the urethra until its end lies in the bladder and the meatus is pressed against the posterior part of the uncut end of the tube. The two arms of the tube, are now carried along the penis, one on either side, and are secured to it by two strips of adhesive plaster passed around the organ, at a short distance from each other.

The catheter should be retained for two days following the operation, or longer if it is desired to employ it for the purpose of treating cystitis in the cases in which that condition is present.

Comment.-Divulsion is, in our opinion, an objectionable and unscientific procedure. The rent made in the stricture is not in the control of the operator, either with respect to its extent or direction, and it results, in the healing, in a larger deposit of connective tissue than was originally present, in many instances.

\section{INTERNAL URETHROTOMY.}

There are two methods of doing this operation: one of them is adapted to strictures of small caliber, and the other to those having a caliber of 14 or more of the Charrière scale. There are also two ways in which the operation is done with reference to the direction given to the incision. In the first, the cut is made from before backward, and in the second, from behind forward. 
A great many instruments have been devised for the performance of internal urethrotomy. None of them are, in our judgment, as good as those of Maisonneuve and Otis, and we shall therefore confine ourselves to the description of these two operations.

Internal Urethrotomy by the Instrument of Maisonneuve.-Position of the Patient.Recumbent.

Instruments.-Maisonneuve urethrotome and filiform guide (Fig. 45).

The slot of the Maisonneuve instrument should be placed upon the upper surface of the shaft. It should not be carried up onto the curved end of the shaft, but should be stopped at a point about 7 inches from its outer end. The caliber of the staff corresponds to 7 of the Charrière scale. We do not like to use a knife blade which, together with the staff, exceeds a caliber of 24 of the Charrière scale. With very tight, dense strictures, we advise that the first incision be made with a blade which does not exceed I 5 of the Charrière scale.

Always test the strength of the attachment of the filiform guide to its metal cap, before employing it.

Operation. - Pass the filiform guide through the stricture. Attach the metal staff of the instrument to the guide by screwing the end of it into the metal cap upon the outer end of the filiform bougie. Pass the staff, with the guide thus attached, through the stricture. Enter the knife blade into the slot on the shaft. Draw the penis forward upon the staff and hold it steadily in this position, and in such a way that the incision will be made directly in the middle line of the roof of the urethra. Drive the knife through the stricture by a steady pushing movement. Withdraw the blade in the same line. Withdraw the shaft and guide. Irrigate the urethra through a small rubber

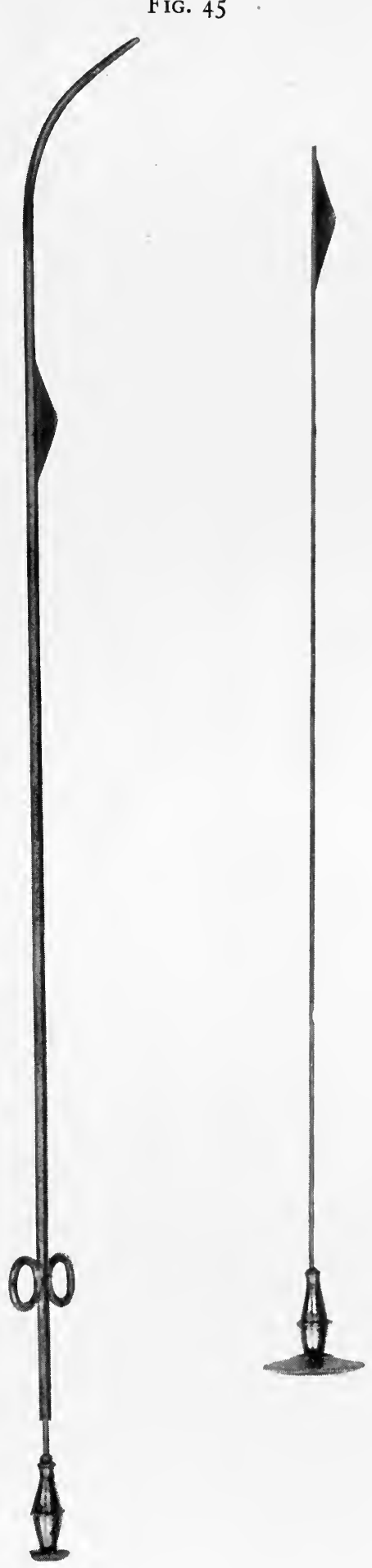

FIG. 45

Maisonneuve's urethrotome and guide. 
catheter. Pass a steel sound of the size of the normal urethra of the individual upon whom the operation is done.

Fig. 46 illustrates the instrument and its use in the urethra during the operation. ${ }^{1} \quad$ Note the position of the filiform bougie in the bladder.

Comment.-The great merit of this operation is that it can be applied to any stricture through which a filiform guide can be passed.

The upper angle of the knife blade is blunt. This avoids the danger of cutting the urethra in other than its constricted portions. The angle of the blade is an important feature, and should be that which is represented in Fig. 45 .

FIG. 46

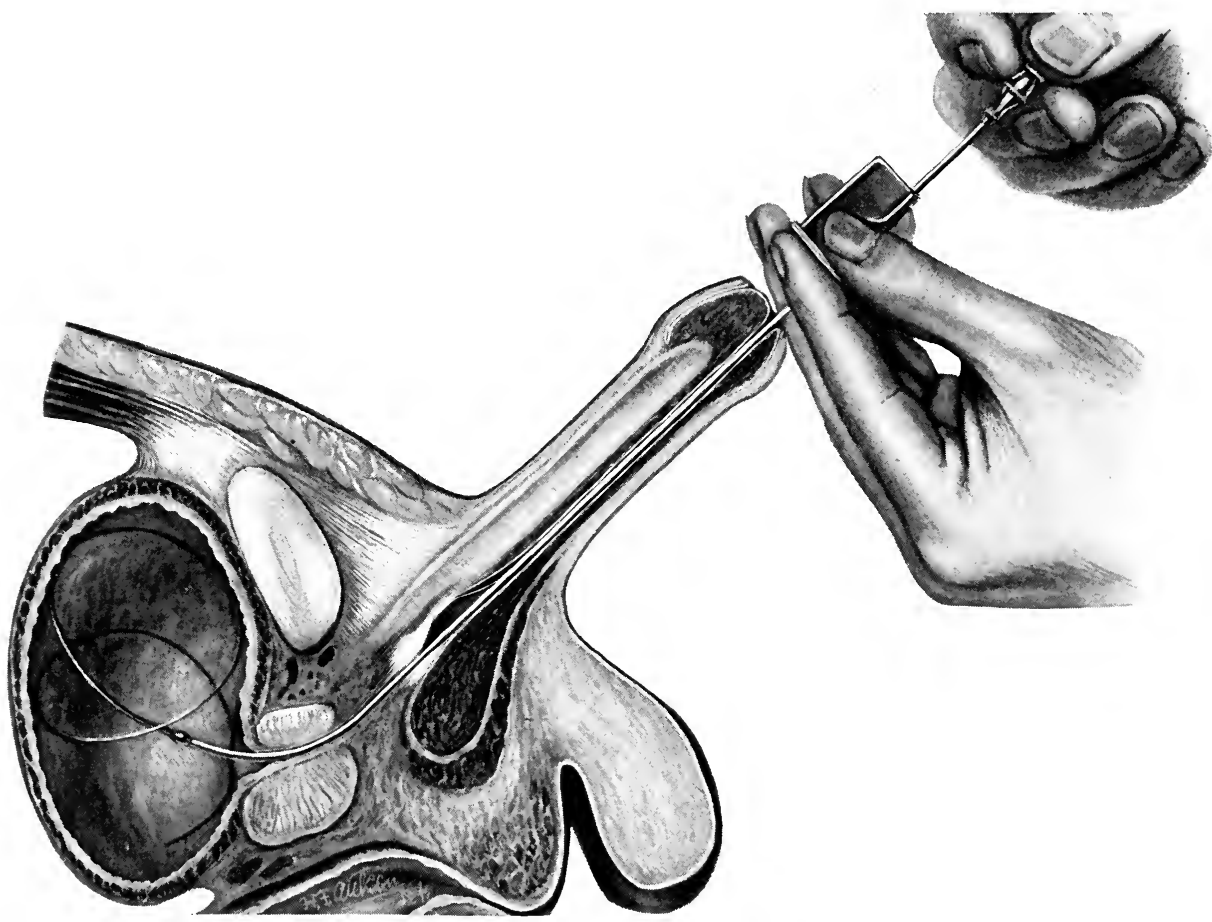

Maisonneuve's operation of internal urethrotomy.

Internal Urethrotomy with the Instrument of Otis.-The special feature of this operation is that of dividing the stricture by a longitudinal incision in the middle line of the roof of the urethra at the moment when the constricted part of the canal is stretched and made tense by means of

${ }^{1}$ Owing to an error of the author in not clearly explaining the position of the fingers of the left hand to the artist, they are incorrectly represented in this figure; they should be holding the head of the penis between the first and middle fingers, and the shield of the instrument should rest against the end of the glans. 
FIG. 47
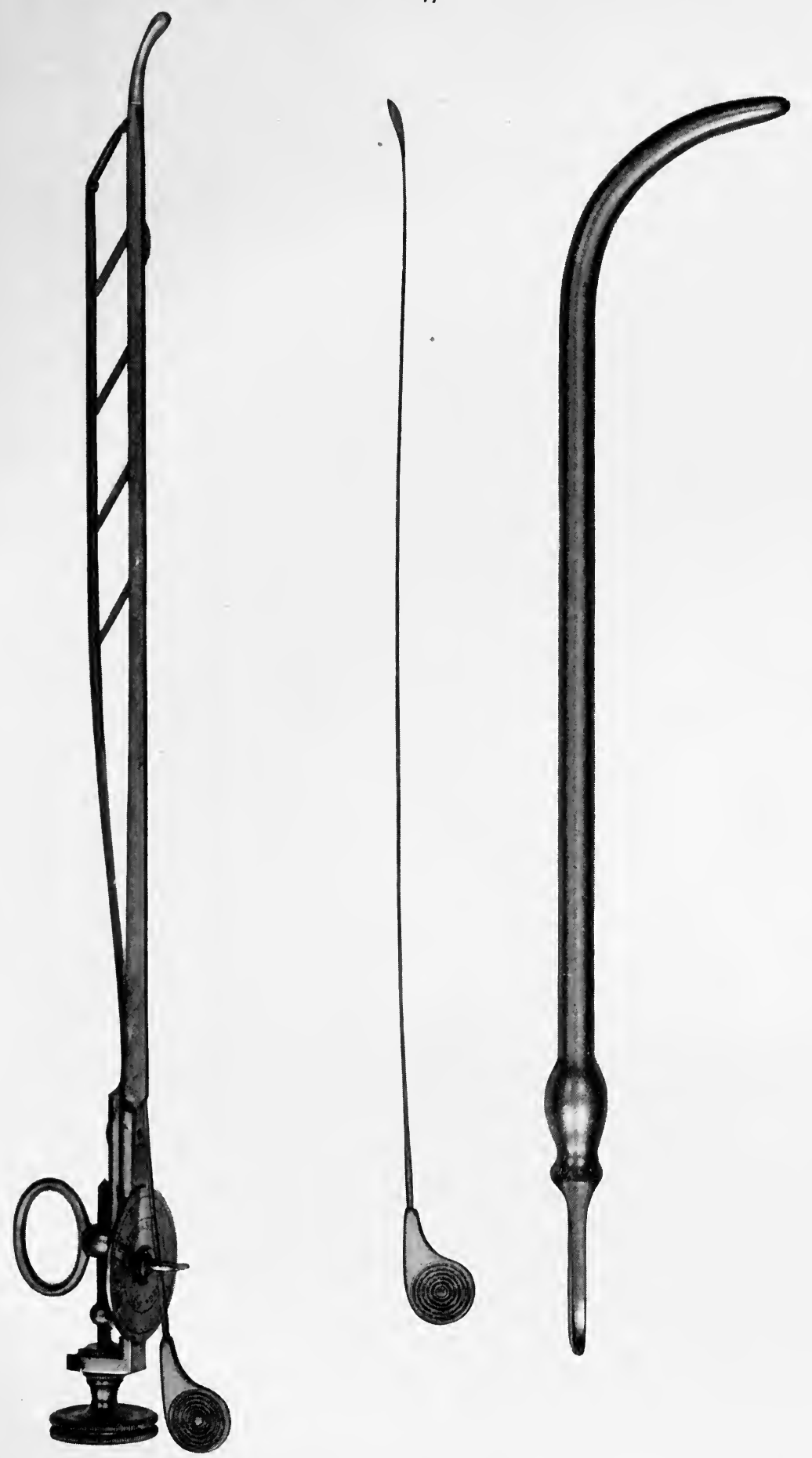

Otis' urethrotome and a steel sound. 
the separation of the two blades of the urethrotome, which are separated by a mechanism at the distal end of the shaft.

The instrument is too large to pass through strictures of smaller caliber than No. I4 of the French scale. In many instances, those which are narrower than this can be quickly dilated to the point which will allow the instrument of Otis to pass through them, and its employment is therefore less restricted than might, at first thought, appear to be the case.

\section{Position of Patient.-Recumbent.}

Instruments.-Bougies à boule; steel sounds; Otis' urethrotome; softrubber catheter to irrigate the urethra; meatotome (see Fig. 47).

Operation.-Meatotomy should be done as a preliminary step, and the location and extent of all strictures should have been determined by means of the urethrometer or the bougie à boule. (See chapter on Stricture of the Urethra.)

Pass the instrument of Otis, with its blades closed and with its knife already in position in the slot at the further end of the shaft, into the urethra until the concealed knife blade is a little beyond the posterior face of the stricture that is to be cut. Steady the penis on the instrument so that the shaft of the latter will be in a position that will insure the incision being made in the middle line of the roof of the canal. Separate the two arms of the instrument to whatever degree has been decided to be the proper one in each case, reading this point off upon the dial plate made for that purpose and attached at the outer end of the shaft. When this has been reached, draw the knife blade steadily along the slot until it has divided all the strictured tissue. Return the blade to the slot. Bring the two arms of the shaft again into approximation by turning the screw head in the direction opposite to that in which it was turned to separate them. Withdraw the instrument carefully.

The position of the instrument within the urethra, with its arms expanded and the knife in the position which it has when cutting through the stricture, is shown in Fig. 48.

The instrument should be withdrawn cautiously, else injury may be done to the mucous membrane of the urethra, because of catching a bit of it in the angle of the cross-bars between the arms as they are being closed, and a portion of the inner lining of the urethra torn off. If the instrument cannot be easily withdrawn, after its arms have been brought together again, it indicates that this accident has happened, and they should be at once slightly separated again, and the instrument pushed forward enough to liberate the pinched bit of mucous membrane caught between them, before making any further effort to withdraw it from the urethra. 
Irrigate the urethra. Empty the bladder and irrigate it also. Pass a full-sized sound.

After-treatment.-The urethra should be irrigated immediately after the first urination following the operation and after the first passage of the instruments subsequent to it.

FIG. 48

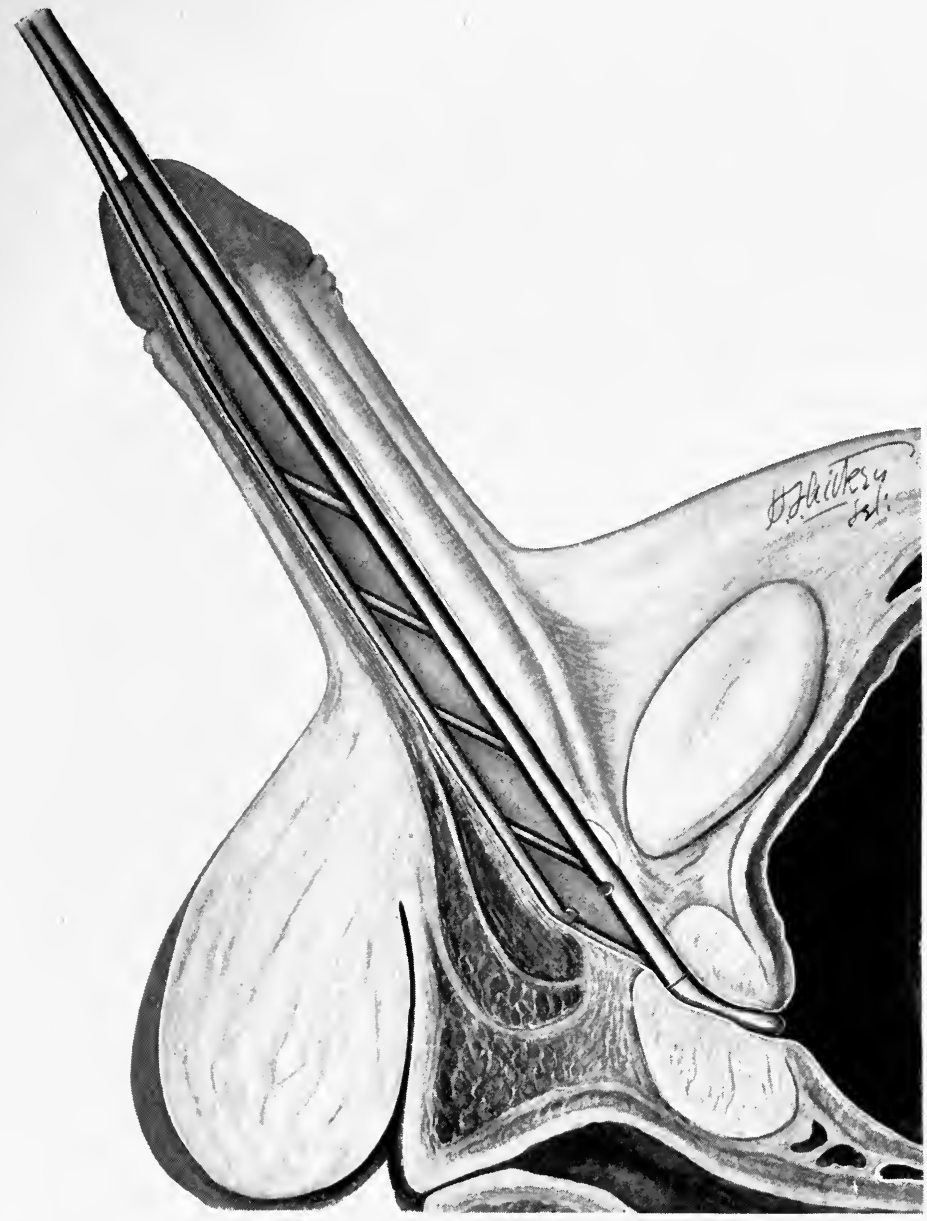

Otis' dilating internal urethrotomy. The instrument as it appears when the stricture is being cut.

We do not believe in tying a catheter into the bladder after this operation. Its presence in the urethra often gives rise to urethritis, and if this occurs, the success of the operation is very apt to be invalidated. The catheter does not prevent contact of urine with the freshly cut surfaces in the urethra, for more or less leaks along the sides of the instrument between it and the urethra, after the catheter has been retained for a few hours. 
The great preventive of urinary fever is thorough urethral irrigation employed in the manner described, and the internal administration of urotropin, which seems to give additional safety.

The meatotomy incision must be kept open until it is fully healed. For this purpose a small bit of absorbent cotton is to be laid between the cut surfaces. The cotton must be removed previous to each urination. If this is not done the urine may be sufficiently obstructed in its outflow to infiltrate the tissues through the urethrotomy incision.

The urethrotomy incision must be kept open during the process of healing. To this end, sounds of the normal caliber of the urethra must be passed through it every second day succeeding the operation, until no blood appears at the meatus upon withdrawing the instrument. This usually occurs on the sixth or seventh time of passing the sounds, and is a sign that the wound has healed.

Hemorrhage.-In no instance have we seen enough bleeding follow internal urethrotomy to cause us the least anxiety. We never cut a stricture by internal urethrotomy alone that is situated more than five and a half inches from the meatus, and rarely do so when it is more than five inches distant from it. The reason for this is the danger of concealed hemorrhage taking place from the deeper parts of the urethra-the blood passing backward into the bladder-when the cut is made deeper than this, and also because of the greater liability of septic absorption taking place from the cut surfaces when they are placed within the membranous urethra, owing to the failure of free drainage from that part of the canal as compared with the anterior part of it.

If it is necessary to arrest hemorrhage, the best manner of controlling it is by pressure applied firmly along the external surface of the perineum and the whole length of the anterior urethra. Such pressure is best applied by means of the arm of a well-applied T-bandage, resting the penis upon the symphysis pubis and upon a graduated compress above it, and drawing the arm of the T-bandage firmly upon the under surface of the organ. In case of failure to adjust such an arrangement as this successfully, the bleeding can readily be stopped by passing a good-sized soft-rubber catheter into the bladder and then bandaging the penis, with moderate pressure upon it.

The patient seldom need be confined to bed for more than two days. Personally, we prefer to have him stay there until the morning of the third day, which will be about sixteen hours or so after the first passage of the instruments following the operation, at which time the chill and succeeding fever which sometimes follow the operation are very apt to occur, if at all.

Should there have been a chill and fever or other untoward symptom, it is far wiser to keep the patient quiet for a longer time. 
Comment.-This operation has the great advantage of securing infinitely better and more enduring results than any other form of treatment thus far employed for the relief of stricture. It is attended by very little danger. These and kindred aspects of the subject are more fully discussed in the chapter on Stricture of the Urethra, when comparing the relative merits of the different methods of treatment of the malady.

\section{EXTERNAL PERINEAL URETHROTOMY-PERINEAL SECTION-THE BOUTONNIERE.}

\section{Position of Patient. - The same as for lateral lithotomy.}

Instruments (see Figs. 49 to 60).-Two scalpels: I with full belly, with narrow, straight blade; 2 tenacula; 2 curved Hagedorn needles, each carrying a stay suture; I Richter needle holder; I pair mediumsized, straight, blunt-pointed scissors; Wheelhouse staff; filiform bougies; catheter (soft rubber, and about No. 24 of the French scale); Watson's perineal hard-rubber button for tying catheter into the bladder after external perineal urethrotomy; I long fine probe; I director, bent at a distal end, as in the illustration; steel sounds; glover's needles and silkworm-gut sutures, or No. o chromicized catgut sutures; 6 pairs of artery forceps; 2 pairs of single-tooth forceps; irrigating jar or bag and connections for washing out the bladder.

Operation.-With a Guide.- The operation with a guide is one of the simplest and most rapidly performed in surgery. Without a guide, it is one of some difficulty and may demand a long time. Whenever a filiform guide can be passed through the stricture, proceed as follows:

Attach the Maisonneuve urethrotome to the guide, and divide with it all strictures in the canal of the urethra, in the manner described above when speaking of that operation. This is done with the patient in a horizontal position; then change the patient's position to that of lateral lithotomy. Pass a grooved curved staff into the urethra until the most prominent part of the curve lies in the perineal part of the canal. Have the staff held steadily by an assistant, so that its groove corresponds with the median raphé of the perineum, and have the staff pressed toward the surface, so that it is prominent and easily felt from the exterior of the perineum.

Incision.-From above downward, beginning a little above a point half-way between the margin of the anus and the scrotum, make an incision an inch and a half in length through the raphé. Continue dissection until the bulb of the urethra is exposed. Draw the penis well forward upon the staff. This will raise the membranous portion of the urethra into view. Change the scalpel thus far used for the 
narrow-bladed, straight bistoury. Place the nail of the forefinger in the groove of the staff at the point at which the latter occupies the membranous urethra. Using the finger nail as a guide, enter the point of the bistoury into the groove of the staff beside it, piercing the

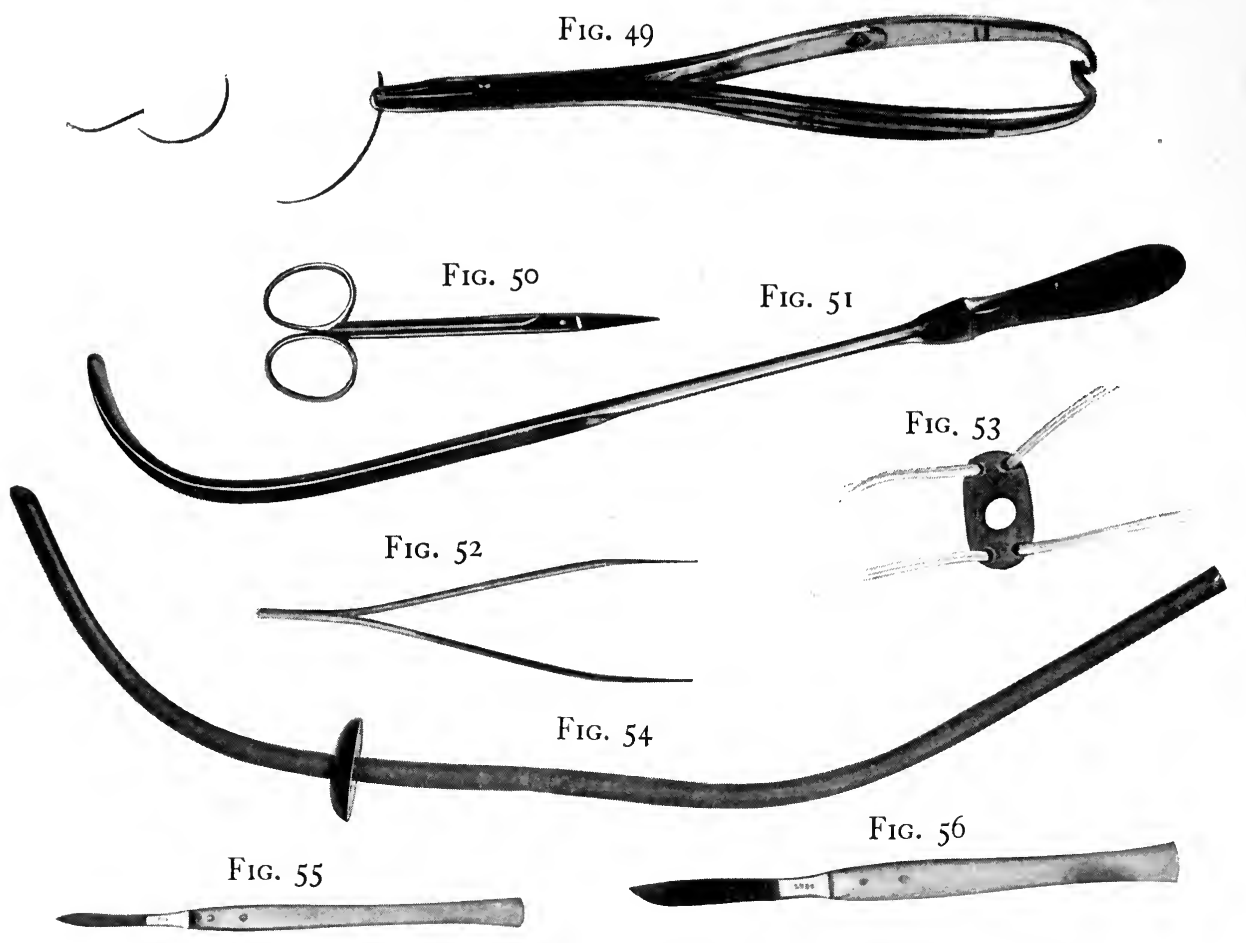

FIG. 57

FIG. $5^{8}$

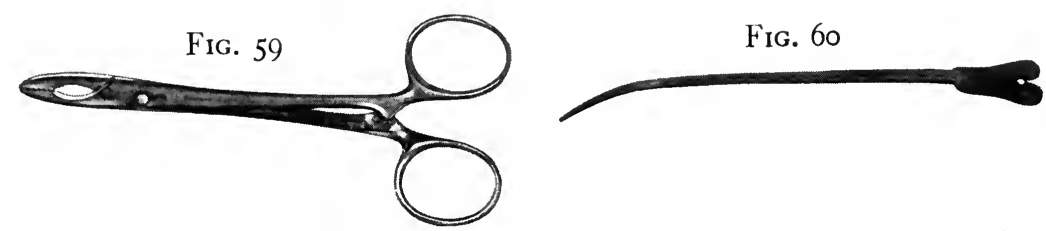

Instruments for the operation of external perineal urethrotomy.

membranous urethra in so doing. The cutting edge of the knife should be upward. Divide the anterior half of the membranous urethra and whatever extent of the canal in front of it is occupied by stricture tissue by sliding the knife blade forward along the groove of the staff, keeping 
the point always in contact with the latter (see Figs. I82 to I86, in the chapter on Technique of Operations on the Prostate).

Withdraw the staff until its tip is opposite the incision into the urethra, then turn it and bring it out externally through the latter. Draw the urethra upward by using traction upon the staff, the curve of which will occupy the upper angle of the urethral incision. The cut into the urethra is readily found when this is done. Pass a large steel sound into the bladder through the incision in the urethra, in order to be assured that the posterior limit of all stricture tissue has been thoroughly divided. The presence of the staff is rather inconvenient while doing this, and it may be withdrawn previous to passing the instruments, and the edges of the incised canal may be transfixed with stay sutures and drawn apart, if so desired, instead of using the grooved staff to lift up the urethra (see Fig. 6I).

Examine the anterior part of the canal with full-sized acorn-tipped bougies, to determine the presence of any further strictures that should be cut. If any are found, divide them with the Otis instrument in the manner already described.

Without a Guide.-The early steps of the operation are the same as in the preceding one described, until the passage of the staff. From this point the method changes, thus: instead of the curved staff, pass the straight one of Wheelhouse into the urethra until its further passage is arrested by the anterior face of the stricture. The instrument is held so that the groove in it faces toward the surface of the perineum.

Make the tip of the instrument prominent in the middle line of the perineum. Incise the skin and superficial fascia of the perineum directly over it. Using the finger nail as a guide, as in the last operation, lay open the urethra by an incision which enters the canal at the point in it corresponding to that occupied by the end of the groove in the staff, and extending upward from it one-half to three-quarters of an inch. Catch the divided edges of this incision a little above its lower end on either side, with toothed forceps, and place a stay suture in each of them. Draw the sides of the urethra apart and in an upward direction (see Fig. 6I). The Wheelhouse staff is then turned around so that its groove is in the opposite direction and the blunt hook which forms its tip faces outward. Draw the hook into the upper angle of the wound of the urethra and lift the incised canal in an upward direction by traction on the handle of the staff. (Personally, we do not find any advantage in this last step. The stay sutures display the interior of the divided urethral canal as well as it can be shown, and nothing further is gained by using the hook of the staff in the manner described.) Withdraw the staff.

The chief feature of the Wheelhouse staff is the blocking its tip so that 
the last quarter of an inch is not grooved. This prevents the incision of the urethra being carried to the actual end of the staff, and consequently, after withdrawal of the instrument and the lifting outward upon the stay sutures, there is a funnel-shaped pocket formed just anterior to the front face of the stricture. In most cases the orifice of the strictured part of the canal, the discovery of which is the key to the further successful

FIG. 6I

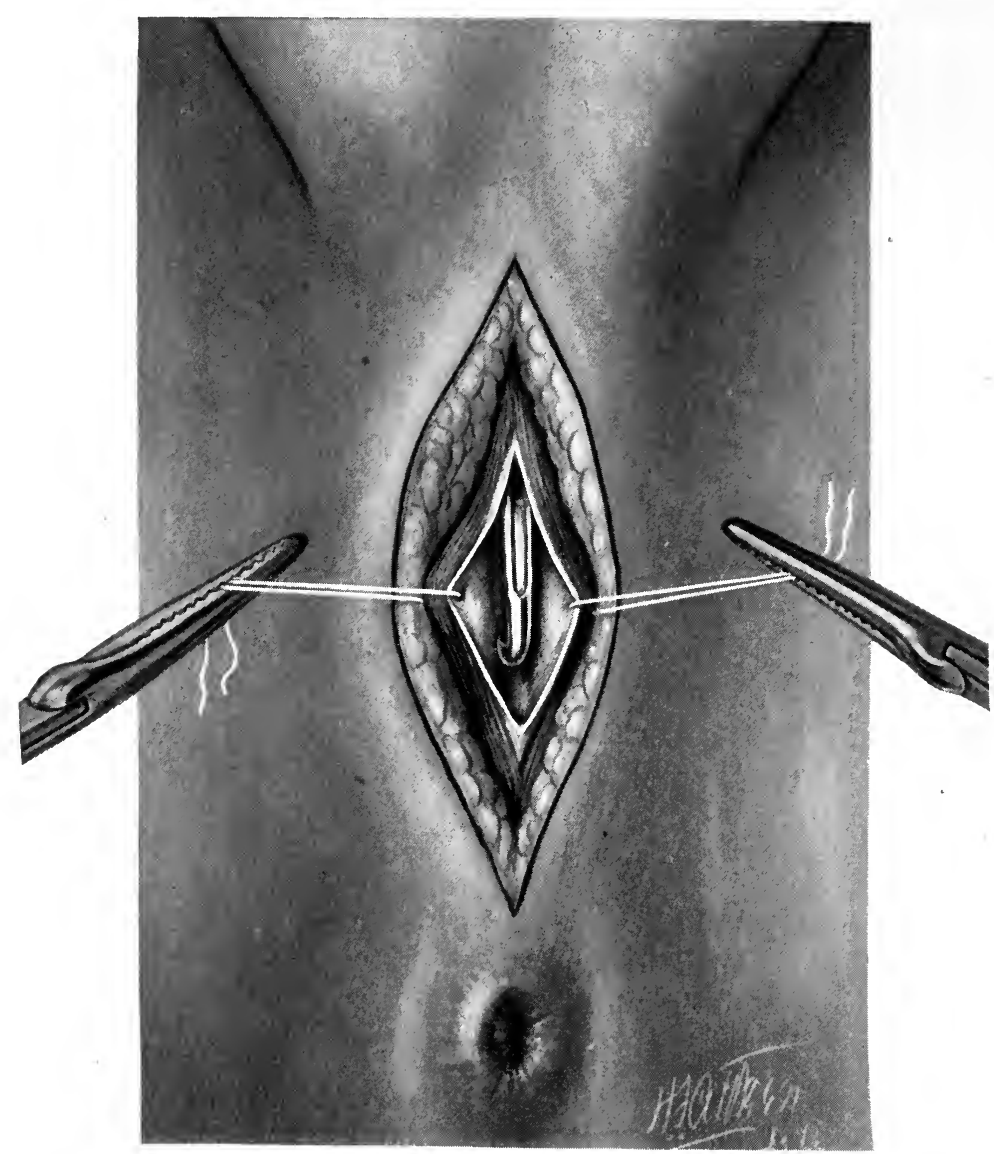

Exposure of the staff in the performance of external perineal urethrotomy without a guide and exposing the triangular cul-de-sac, at the end of which the orifice of the stricture lies.

prosecution of the operation, occupies the apex of this funnel, and it is at that point that the search for it should first be made. It happens, however, in some cases, that the orifice is placed elsewhere, and is then very likely to be found upon one or the other of the sides of the urethra. The search for it is made with a filiform bougie or with a fine probe. Its detection is made more easy if pressure upon the bladder from above the 
symphysis pubis is made, and some of the urine is thus forced through the strictured part of its canal.

When it is found proceed thus: Pass a filiform bougie or delicate probe into and through the constriction and lay that part of the canal open upon it as a guide. Be sure that all the constricted portion of the urethra has been divided.

After-treatment.-A soft-rubber catheter should be passed into the bladder through the perineal incision, and should be worn from twentyfour to forty-eight hours after the operation.

A convenient way of securing the catheter in place is by means of Watson's hard-rubber perineal button.

Other Methods.-When it is impossible to find the orifice of the strictured part of the canal, and when a reasonable length of time has been expended in the effort to do so, further attempts should not be made, and one or another of the following procedures should be resorted to:

Cock's Operation.-Place the tip of the forefinger of the left hand in the rectum and directly upon the apex of the prostate. Insert the blade of a straight, narrow bistoury in the median line of the perineum and push it directly forward and upward until its point enters the urethra just in front of the prostate. The finger tip in the rectum is used as a guide to the location of this point and to insure the safety of the bowel when making the cut. The cutting edge of the blade is held upward. As a rule, the flow of urine from the incision will announce that the urethra has been opened; when this does not happen the blade is withdrawn and a bougie catheter is passed into the wound to learn if the urethra has been successfully reached and opened. If the urine flows at first, pass a director through the incision into the bladder beside the knife blade and withdraw the latter, enlarging the outer incision with it in so doing. A catheter is then passed into the bladder through the wound, using the director as a guide, and the latter instrument is withdrawn. The catheter is then secured in place.

Comment.- The operation is rarely used, and is not to be recommended, the same purpose as that for which it is intended being attainable by better and safer means.

Retrograde Catheterism.-Open the bladder by the usual suprapubic incision, described under the heading Suprapubic Cystotomy in the chapter on the Technique of Operations upon the Bladder.

Introduce a conical steel sound through this incision into the bladder and pass it into the posterior urethra through the vesical outlet, until it is arrested by the posterior face of the stricture. Make the tip of the instrument prominent by pressing it toward the surface of the perineum, and lay open the urethra by cutting down upon the tip of the instrument 
through the median line of the perineum. If possible, pass a fine probe or filiform bougie through the stricture from behind forward and divide the strictured tissue upon it by tracing its course through the constricted part of the canal with a narrow-bladed, straight knife. In case this is found to be impossible, pass a sound or staff through the anterior urethra until its tip is arrested by the front face of the stricture, and then divide the tissue which intervenes between the tip of the posterior and that of the anterior sounds by an incision in the middle line.

\section{EXPOSURE OF THE URETHRA BEHIND THE STRICTURE BY OPEN DISSECTION.}

This method-said to have been employed by Dumarquay, ${ }^{1}$ and also by Forgue, ${ }^{2}$ and in 1888 by Le Dentu ${ }^{3}$ - of reaching and incising the urethra behind the stricture is advocated by some surgeons, and it certainly has the advantage of allowing the operator to see what he is about, and to reach the posterior urethra with certainty and ease. The only objection to it lies in the fact that it involves a large operation for the accomplishment of an object which can, with but few exceptions, be secured by a lesser one. The technique is the same as that described in the chapter on the Technique of Operations on the Prostate.

\section{SUTURE OF THE URETHRA AFTER PERINEAL URETHROTOMY.}

Instruments.-A pair of small, sharp-pointed scissors; two finetoothed forceps; full curved intestinal needles threaded with catgut; needle-holder; scalpel. These instruments are among those shown in Figs. 49 to 60 .

Position.-The patient's position is the same as in the operation just described.

Operation.-The suture may be applied in two different ways: (I) partial suture, or (2) complete suture.

With partial suture, only the inner or urethral part of the wound is closed. One row of stitches should be passed through the tissues immediately external to the edges of the divided floor of the canal and through the outer part of the wall of the latter. Care should be taken not to have the sutures pass through the inner surface of the mucous membrane.

In the complete suture, three lines of stitches are taken, beginning with the one just described, and setting the two others external to it; the last one includes the skin and closes the surface of the wound. 
Enough stitches should be taken in either method to secure a close union of the edges of the inner, or urethral, incision.

A catheter should be passed into the bladder from the meatus and secured in place. It should be retained there for about one week.

\section{RESECTION OF STRICTURE.}

The operation is said to date from I8I2. It has been but little employed until recently. The modern method of performing it is attributed to König, who successfully applied it to a stricture of the perineal portion of the urethra in I882. In this case the urethra was divided transversely at each end of the stricture, and the dense connective-tissue mass constituting the constriction was excised. The two ends of the divided canal were then brought together and united by sutures.

Since I89I the method has been widely applied by French surgeons, conspicuously by Guyon, Albarran, Poncet, Mollière. In Germany, Wölfler, and in America, Keyes, Cabot, and others have given attention to the subject.

Since $189 \mathrm{I}$ there have been at least 150 cases reported, 64 of which are tabulated in the chapter on Stricture, page I24.

Indications.- The operation is that of choice in cases of stricture of the urethra which are not amenable to other forms of treatment. The larger number of these are of traumatic origin and situated in the perineal urethra. Some are of gonorrhœal origin. Occasionally a stricture of this character is located in the anterior part of the canal. An example of such a one will be found in the list of illustrative cases at the end of the chapter on Stricture of the Urethra, page I34.

Technique.-Resection of the urethra may be performed in the following ways:

(I) Complete division of the urethra just in front of and just behind the stricture; removing the constricted part of the canal between the divided ends, and allowing the interval thus made in it to be reformed around a catheter which is passed into the bladder through the whole length of the urethra and retained there until healing is well advanced. (2) The same operation, except that the divided ends of the urethra are united by suture. (3) Leaving the roof of the urethra instead of completely dividing the latter, as is done in the first operation.

Instruments.-The same as those required for external perineal urethrotomy and for suture of the urethra.

Operation.--Expose the mass of indurated tissue, which includes also the strictured part of the urethra, by a long median longitudinal incision through the raphé of the perineum, or by a curved one, and the turning 
upward (or downward) of a flap which shall include the skin and superficial tissues of the perineum throughout two-thirds or more of its extent.

Carry the dissection through the intervening deeper structures down to the membranous urethra. Lay open the latter and determine the posterior limit of the stricture by introducing a sound or probe into the urethral incision and passing it forward.

Pass another sound or staff into the anterior urethra until its tip is arrested by the front face of the stricture in the perineum.

Catch the posterior part of the urethra at the point at which it was incised, with a pair of toothed forceps, draw it forward, and transfix it with a stay suture a little behind the face of the stricture at that point. Divide the urethra transversely just in front of the stay suture.

Place another suture through it just in front of the anterior face of the stricture and divide the urethra here in the same manner.

Dissect out the whole mass of connective tissue intervening between the two ends of the divided urethra.

Secure all bleeding points.

The two severed ends of the urethra are then to be united by an endto-end anastomosis. This can be done with little or no dissecting of the urethra from its surroundings beyond the points at which it has been cut across, provided there is not more than one inch separation between the divided ends. When this distance is greater it will be necessary to free the anterior segment more or less, in order to approximate them.

Traction upon a suture temporarily placed in the anterior divided end of the urethra aids the dissection.

Having freed the canal, proceed to suture the two ends together by one of the various stitches used for similar purposes elsewhere. Begin by placing the first stitch in the middle of the outer edge of the floor of the posterior divided end of the urethra and through the corresponding point of the anterior end of it. Place the successive stitches in the same way, at intervals in the circumference, until one-half of the latter has been united. Traction on each of the sutures after it has been placed and tied will rotate the urethra in such a way as to make the setting of the next stitch easier. When half of the circumference of the divided ends has been united, go back to the starting point at which the first stitch was placed and then complete the circle by closing the rest of it, taking the successive stitches in the opposite direction to that in which the first half were taken.

The suturing of the two ends of the canal together is made easier by having a catheter in the urethra while it is being done. 
After-treatment.-A catheter should be worn for the purpose of draining the bladder for from ten days to a fortnight after the operation.

The third manner of doing the operation differs only in leaving the roof of the canal intact. This method we have never tried, nor can we quite understand just what is meant by leaving the roof of the canal, when the part of the urethra upon which the operation is executed has no roof, floor, or sides, properly speaking. It is represented merely by a more or less distorted canal of very narrow caliber and tortuous course, buried in a mass of cicatricial tissue, in the cases in which resection is appropriate to apply at all. We can affirm that there is no essential advantage attaching to this manner of treating the canal, so far as success of the operation is concerned, since the methods already described are in many instances entirely satisfactory and successful.

Nogues $^{4}$ has urged the necessity of preserving the roof of the canal intact in order to avoid the springing apart of the ends of the canal, which they do if it be wholly divided. Nogues also urges complete closure of the perineal wound, and says that it is essential in order to secure good results, since the healing by granulation produces in the process a greater amount of connective tissue, and subsequent recontraction ensues, which can be avoided if healing by first intention of the wound is secured. The springing apart of the severed ends of the canal may be obviated by having placed sutures, one in each part of the canal close to where it is to be cut across, before dividing it; and the difficulty arising from this source, which is emphasized by Nogues, can thus be obviated.

\section{BIBLIOGRAPHY,}

I. Dumarquay. Union Méd., March I4, I853, p. IOI.

2. Forgue. Presse Méd., September 21, I903.

3. Le Dentu. Bull. Soc. de Chir., February I 5, I888, vol. xiv, p. I60.

4. Nogues. Ann. des Mals. des Org. Gén.-urin., I892, p. 707. 


\section{H A P T E R V.}

\section{TECHNIQUE OF OPERATIONS ON THE PENIS.}

\section{OPERATIONS FOR PHIMOSIS.}

Desiderata.-The three following points are the essential ones to be observed in all operations for phimosis: (I) To remove the constricted prepuce. (2) To leave the preputial orifice thus made sufficiently large to allow it to be easily retracted over the glans. (3) To avoid injury to the frenum.

Anatomical Data.-There are two things especially to be remembered in connection with the operations for phimosis: (I) That the prepuce is made up of an outer layer of integument and an inner one of mucous membrane. They are separated by a loose network of connective tissue, which allows the outer one to move freely upon the inner. A clamp placed upon the prepuce, as it is in certain of the operations for phimosis, holds the outer, but not the inner, layer of the prepuce in its blades; consequently, the incision which removes the part of the foreskin lying above the forceps does not pass through the inner layer of the prepuce. The latter, therefore, requires a second incision in order to divide it and to expose the glans. (2) The skin of the prepuce and of the penis is very loosely attached to the underlying structure, and can be drawn forward with great ease toward the preputial orifice. The inner layer of the prepuce, on the contrary, is firmly attached just behind the corona glandis. If care is not taken to catch the foreskin (when drawing it forward in order to apply the clamp, or preparatory to incising it when doing the operation for phimosis) just at the point of junction of the skin with the inner or mucous membrane layer of the preputial orifice, and to avoid pulling down the skin too far, the latter will roll inward beneath the mucous membrane, and a disproportionately large amount of the integumentary layer as compared with the inner layer will be divided, with the result that the skin will retract far backward toward the root of the penis when the incision is made through it in removing the foreskin, and will leave the underlying penis bared of its natural outer covering to a greater or less extent. The writer has once seen this unfortunate accident happen in the case of a patient who was operated upon by a young practitioner, and has therefore thought it worth while to take the space to give the 
above warning with regard to this particular part of the operation. The proper points upon which to place the forceps when drawing the foreskin forward are seen in Fig. 63.

Circumcision with Clamps.-Preparation.-The prepuce and glans should be thoroughly cleansed immediately before the operation. A probe or director should be swept over the surface of the glans to learn if there are any adhesions between it and the inner surface of the prepuce, and to break them down should they exist.

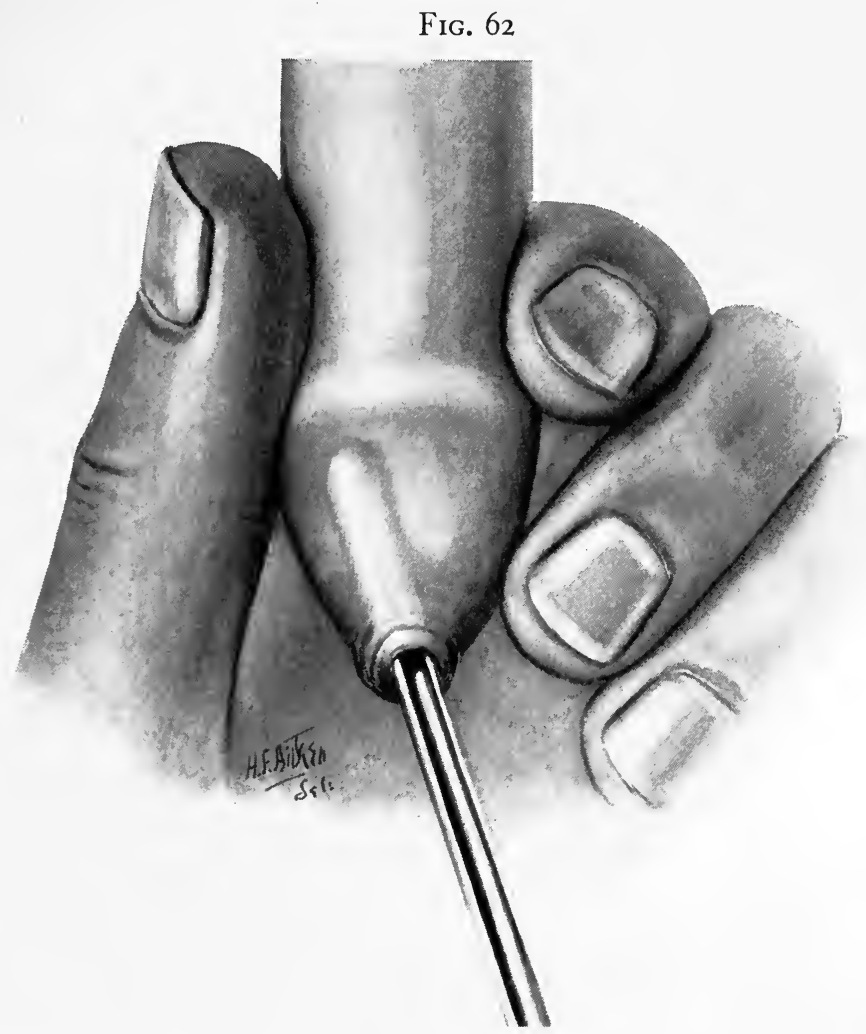

Showing a director passed through the phimosis opening and between the prepuce and glans, to destroy adhesions between the two.

The operation may be done under cocaine anesthesia in the cases of adults, but it is better to employ general anesthesia for children.

Instruments.-The instruments are shown in Figs. 62, 63, and 64 . One scalpel; I pair of straight scissors; I director; 2 pairs of singletoothed forceps; I clamp; straight surgical needles threaded with No. o catgut.

Operation.-Catch the prepuce with toothed forceps at the mid-points of its upper and lower margins and at the junction of its skin and mucous membrane, and draw it well forward beyond the tip of the glans penis. 
Adjust the blades of the clamp forceps upon the prepuce in the manner shown in Fig. 63, and close them just in front of the glans (note the angle of the blades of the clamp forceps with reference to the prepuce), and do not include the attachment of the frenum to the glans in the forceps.

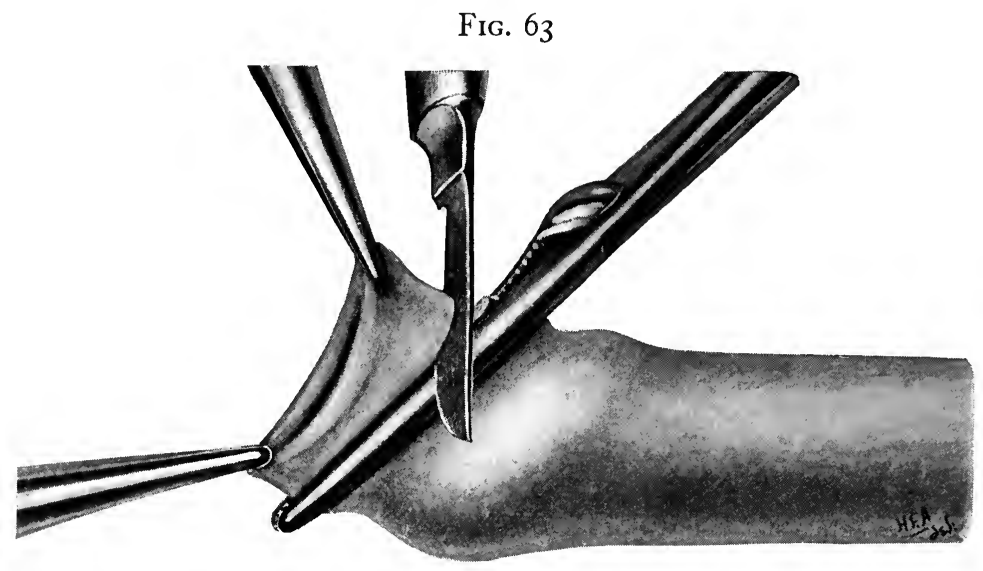

The free border of the prepuce is drawn forward by forceps. The clamp is placed at an angle which will preserve the frenum.

Divide the prepuce by an incision which passes just above and close to the upper margin of the blades of the clamp forceps, as shown in Fig. 63 .

Remove the clamp. The inner layer of the prepuce, still intact, is now exposed, as seen in Fig. 64, while the outer layer retracts, as shown in the same illustration.

\section{FIG. 64}

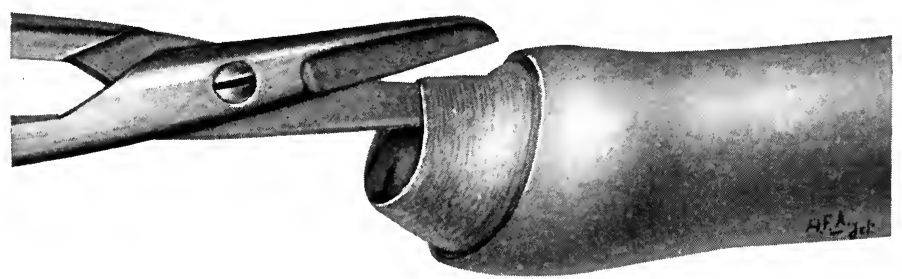

Showing the position of the scissors to divide the mucous membrane after the cutaneous surface of the prepuce has been cut away and the clamp removed.

Pass one blade of the scissors beneath the inner layer of the prepuce in the median line, and divide it as far back as the margin of the corona glandis.

Turn back the cuff of the mucous membrane thus made and unite its edges to those of the divided integument with interrupted catgut 
sutures. From six to eight sutures are usually required in the case of an adult; four or five ordinarily suffice for children. Whatever number may be needed to secure complete and perfect adaptation of the edges of the wound should be used (Fig. 65).

FIG. 65

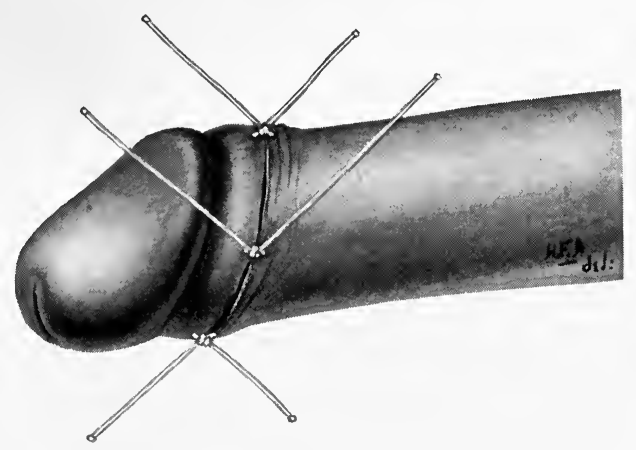

Showing the mucous membrane rolled back and sutured to the skin by fine catgut sutures. The knot has been tied and the ends left long.

The sutures are to be tied, leaving the ends long. Place a roll of sterilized or iodoform gauze around the penis and upon the wound and retain it in place by tying the long ends of the individual sutures together over the gauze (Fig. 66).

The dressing is not to be disturbed until the catgut sutures are absorbed, when it will drop off or can readily be detached from its place. The time at which this occurs is usually about a week after the operation.

FIG. 66

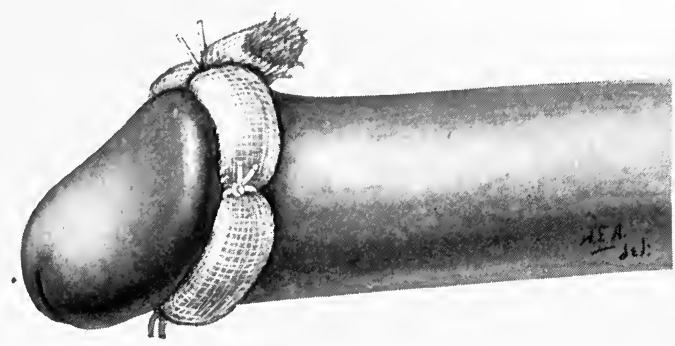

Showing the iodoform gauze covering the line of union and held in position by tying the long ends of the sutures over it.

The patient should remain in bed for the first day, after which he may get up and move about. The penis can be protected from friction by enveloping it in a dressing of absorbent cotton, held in place by a diaper. The steps of the operation are shown in Figs. 62, 63, 64, 65, and 66. 
Comment.-Hemorrhage rarely occurs. If it does, it should be controlled by firm pressure, without disturbing the dressing.

Occasionally there is a good deal of cedema of the area about the wound. If this becomes very pronounced, it may be reduced by multiple superficial punctures, made with a needle point, and by gently squeezing out the fluid from the tissues.

A better adaptation of the edges of the two incisions can be secured if the inner layer of the prepuce is trimmed off by a crescentic cut made through it with scissors anterior to and following the line of the corona glandis, between the end of the dorsal incision and the frenum, on either side.

This incision should not be made too close to the corona.

If the operation is done under cocaine anesthesia, the clamp is applied before injecting the drug beneath the skin. A I or 2 per cent. solution is employed, and is injected just above the upper margin of the blades of the clamp until enough has entered the subcutaneous tissue to infiltrate it along the line of the incision.

Previous to placing the clamp, a little of the cocaine solution may be thrown into the cavity of the prepuce.

Circumcision by a Single Dorsal Incision.-This procedure is applied more especially to the cases in which there is a chancroidal or inflammatory process beneath the prepuce, which it becomes necessary to have exposed in order to apply the proper treatment, and to supply free drainage of the preputial cavity. It is not a desirable method to employ under other conditions, for the reason that it leaves two redundant flaps, one on either side of the incision, and these may be a source of inconvenience and trouble with respect to coitus. If, however, these flaps are trimmed off at the time at which the dorsal incision is made, and the cut edges of the two preputial layers are united by suture, the operation becomes to all intents the same as the one first described.

If it is done in cases in which the inflammatory conditions referred to above are present, the edges of the incision should not be brought together by suture, except for the purpose of controlling hemorrhage from them.

Operation.-The operation consists in passing a director beneath the prepuce to the farthest end of the pocket formed by it, and in the mid-line of the dorsum of the foreskin. The prepuce is then slit up along this line to a point a little in front of the corona. Two pairs of forceps hold the prepuce steady while the cut is made.

Circumcision by Ventral and Combined Ventral and Dorsal Incisions.The operation may be done by a single ventral incision, instead of the dorsal cut just described, or by combined ventral and dorsal incisions. 
The latter procedure is known as "Remondino's operation." In this method the prepuce is slit in the middle line of the dorsal and also of the ventral surface of the prepuce. A flap is thus formed on either side of the glans. Each of the flaps is turned back and held in place by a dressing having the form of a Maltese cross, with a window cut in the centre to allow the head of the penis to pass through it. The arms of the cross are turned down over the flaps and the incisions, and the latter heal under one dressing.

Woodward's Operation.-This operation is designed for the purpose of relieving the preputial constriction, when it is accompanied by infection of the parts within the cavity of the foreskin (gonorrhœal balanitis, chancroid, etc.), without infecting the wound.

Operation.-Compress the glans until it is reduced to a size that will permit the prepuce to be drawn backward over it.

FIG. 67

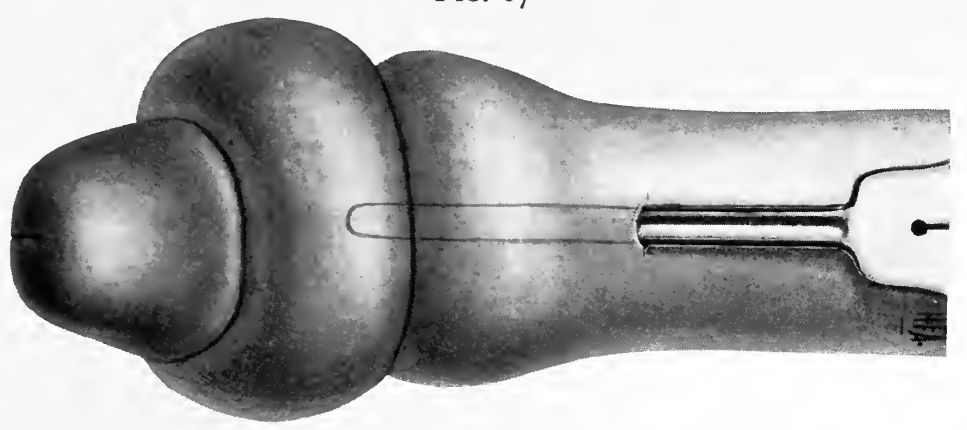

Showing director inserted beneath the constricting ring to be divided by the introduction of a tenotome.

The phimosis will then have been converted into a paraphimosis. Cleanse the glans and inner surface of the prepuce. Place a rubber tube around the root of the penis as a tourniquet.

Inject a 2 per cent. solution of cocaine into the subcutaneous tissue, one inch behind the corona glandis. Pick up the skin at this point and make an incision in it large enough to admit a director. Pass a grooved director through this incision and as far as the attachment of the mucous membrane of the prepuce to the glans. This will carry it beneath and beyond the preputial constriction (Fig. 67).

Pass a narrow, probe-pointed tenotome along the groove of the director -the blade lying flat and its cutting edge being horizontally directeduntil half the blade lies beneath the constricting ring of the prepuce. Turn the cutting edge upward and divide the constriction. The glans is then to be compressed again in order to allow the foreskin to be drawn forward over it, after the director and knife have been withdrawn. The small skin incision is closed by one or two sutures.

VOL. I-II 
Allis' Operation. ${ }^{1-M a k e}$ a circular incision around the end of the prepuce (Fig. 68). From the central point on the ventral surface

FIG. 68

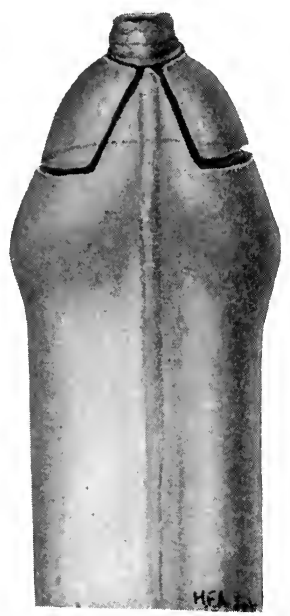

Showing the tip of the prepuce snipped off and the area of skin outlined which is to be removed, leaving the mucous membrane beneath.

FIg. 70

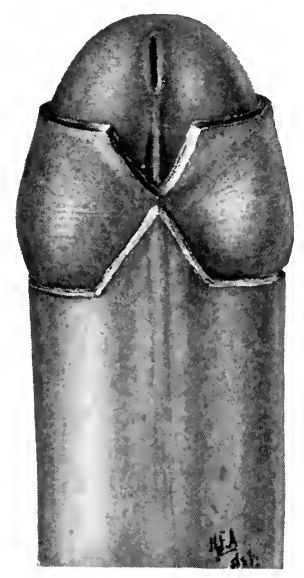

Showing the mucous membrane gaping from the relief of tension due to dividing it through the dotted line shown in Fig. 69.
FIG. 69

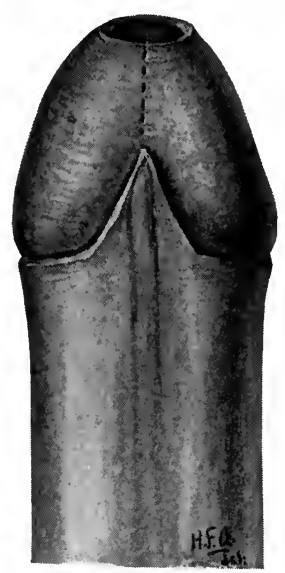

Showing the skin removed, leaving the raw surface of mucous membrane. The dotted line represents the line of division of the mucous membrane.

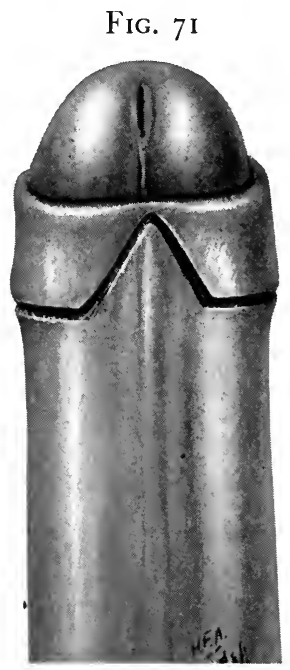

Showing the mucous membrane turned back and approximated to the skin to which it is to be sutured.

of the prepuce, and beginning at the circular cut just made, carry a diagonal incision downward and outward on either side of the median line, then backward, still diagonally, in a crescentic line; these incisions 
are continued over either side of the foreskin until they meet in the middle line of the dorsal aspect a short distance anterior to the corona glandis (Fig. 68). This gives the incisions on the ventral surface the form of an inverted V. The skin, but not the mucous membrane, is divided throughout these incisions.

The area of skin included within the line of these cuts is now dissected from the inner layer of the prepuce. Cut through the two layers of the prepuce transversely at the line marked by the first circular incision. This will remove the tip of the prepuce, which is in front of the circle. The inner layer of the prepuce is now exposed, as shown in Fig. 69, and the edge of the skin incision has retracted behind the corona.

Divide the mucous membrane of the prepuce in the middle line of the ventral surface, as indicated by the dotted line in Fig. 69. By so doing, the condition shown in Fig. 70 is produced. Turn back the cuff of the mucous membrane thus formed in the manner shown in Fig. 7I. Unite the edges of this cuff to those of the skin incision with interrupted catgut sutures (Figs. 68, 69, 70, 71).

\section{OPERATIONS FOR PARAPHIMOSIS.}

By Simple Incision.- Instrument.-A curved bistoury, such as that shown in Fig. 72.

Operation.-Draw down the tip of the glans in such a way as to arch the dorsal surface upward, and hold it in this position with the left hand. Pass the point of the bistoury through the mucous membrane just anterior to the constricting ring, at a point a little to one side of the median line, in order to avoid injury to the dorsal vein of the penis. Push the point of the knife through the constriction and divide the latter by cutting through all the tissue, including the skin. Draw the prepuce forward over the glans (see Fig. 72).

Subcutaneous Division.-Incise the skin of the dorsum of the penis, introduce a director, and push it forward beneath the skin and under the constriction. Pass a small, narrow-bladed tenotome on the director beneath the constricting ring, the blade being held horizontally. Turn the cutting edge of the blade upward and divide the constriction subcutaneously.

The point selected for the entrance of the knife should be posterior to the constricting ring and a little to one side of the median line of the dorsum of the penis (see Fig. 67).

When the swelling of the prepuce is very great, it can be reduced by making multiple superficial punctures in it with the point of a needle. 
FIG. 72

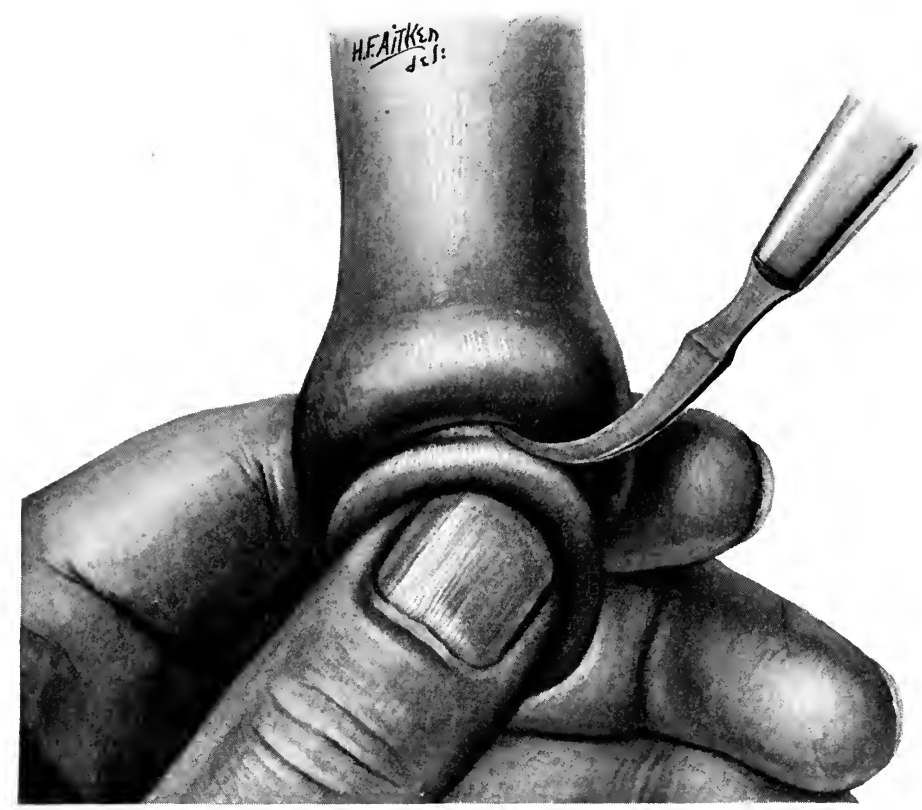

Showing the curved bistoury being introduced under the constricting ring to one side of the median line.

\section{AMPUTATION OF THE PENIS.}

I. Amputation through the Pendulous Portion of the Penis.-By Galvanocautery Wire.-Instruments.-Two pairs of fine-toothed forceps; one pair of scissors; straight needles, threaded with fine catgut sutures; galvanocautery wire écraseur.

Operation.-Pass a soft-rubber catheter into the urethra as far as the prostatic portion.

Place the wire of the cautery around the penis at a point at least $2 \mathrm{~cm}$. behind the diseased area.

Turn on the electric current and slowly tighten the wire until the penis and the catheter lying in the urethra are burned through.

Withdraw the catheter. Catch the edges of the mucous membrane of the urethra on either side with fine-toothed forceps and draw the urethra forward. Pass a number of catgut sutures through the edges of the urethra and suture it to the adjacent tissues a short distance outside of it. Patency of the newly formed orifice of the canal will be insured by making a short longitudinal cut through the middle line of the divided end of the urethra, above and below. 
Tie a catheter into the bladder.

The seared surface of the wound should be kept clean, and should heal under wet dressings. Sloughs should be removed as they form and become loose.

Fig. 73

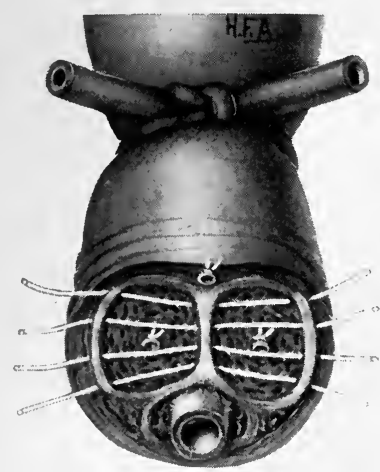

Showing the tourniquet in position. The diseased portion of the penis removed. The cuff of skin pushed back. The body of the penis divided. The vessels ligated. The sutules placed to bring together the fibrous sheaths of the corpora cavernosa. The urethra projecting for a short distance beyond the division of the body of the penis.

\section{Amputation by Transverse} Section (Humphrey's Operation).Instruments. - A large scalpel; I rubber tourniquet; straight needles, threaded with No. o catgut sutures; 2 pairs of fine-toothed forceps.
Fig. 74

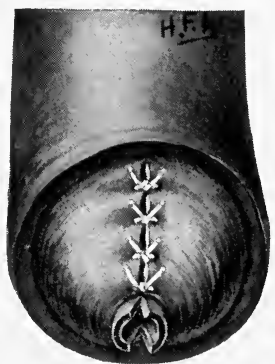

Showing the fibrous sheaths of the corpora cavernosa united by sutures and the projecting urethra divided.

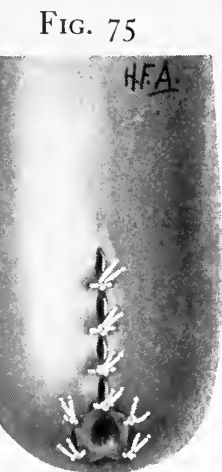

Showing the skin incision closed and the split urethra everted and sutured to the skin.

Operation.-Apply rubber tourniquet around the penis behind the proposed line of incision.

Carry a circular incision around the penis, through the skin only. Free the skin for half an inch and turn back the flap thus formed.

Divide the corpora cavernosa with another circular incision on a level with the line of attachment of the skin flap to the underlying tissues. Divide the corpus spongiosum and urethra about a quarter of an inch in front of the line of incision through the corpora cavernosa.

Pick up and tie the dorsal artery and vein.

Pass four or more catgut sutures transversely across the cut surfaces of the corpora cavernosa, through their envelopes on either side and through the fibrous septum, which lies between the two corpora. When these 
sutures have been placed, tie the two ends of each of them together. This closes the open ends of the sheaths of the corpora and compresses their blood spaces, thus checking hemorrhage.

The arteries of the corpora cavernosa should be tied previous to placing these sutures.

Remove the tourniquet. Make a dorsal incision through the middle line of the skin flap. Bring the edges of the circular part of the skin flap to the edges of the urethral orifice which projects beyond the surface of the now closed ends of the corpora cavernosa. Adapt the skin flap to the edges of the urethral orifice and suture the two structures together, incising the latter in the middle line of its circumference above and below before so doing, in order to guard against subsequent contraction. Unite the dorsal incision of the skin flap by interrupted sutures. Place a catheter through the urethra and in the bladder, and attach it by the contrivance shown in Fig. 76 . The tapes or strips of adhesive plaster which hold the button are placed external to the dressing, as is shown in Fig. 76 . Figs. $73,74,75$, and 76 show the steps of Humphrey's operation.

Fig. 76

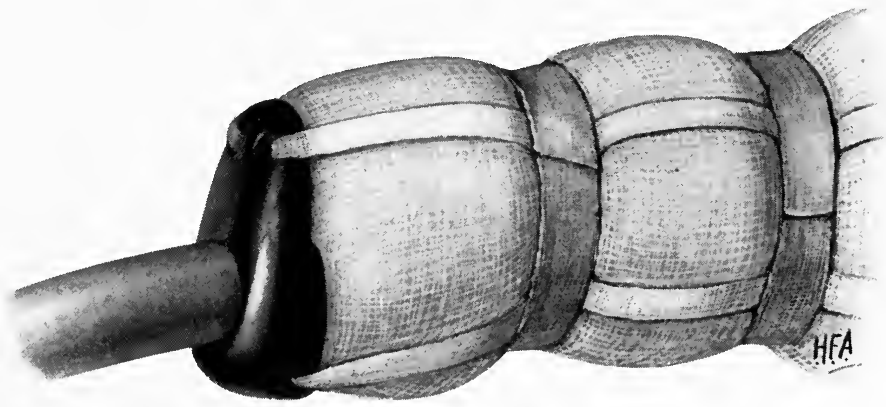

Showing the dressing of gauze held in position by adhesive plaster strips. A catheter is maintained in the bladder by means of a Watson button.

3. By Dorsal Flap Method.-Instruments.-Same as in last operation.

Operation.- The tourniquet being placed, an assistant draws forward the penis and holds it steadily while the operator proceeds as follows: Make a skin flap with a long dorsal upper and a short ventral lower circular incision, as seen in Fig. 78. Free the dorsal flap to its base and reflect it backward.

Transfix the penis with a narrow-bladed, straight bistoury passed between the corpora cavernosa and the corpus spongiosum. The knife is passed with the blade held horizontally. The transfixion is made on a line which will correspond to the base of the dorsal skin flap.

Separate the corpus spongiosum and urethra from the corpora cavernosa 
by cutting directly forward above the roof of the urethra and between it and the lower parts of the corpora cavernosa in the line of the long axis of the penis, and continuing the incision through the whole length of the anterior part of the organ.

Divide the corpora cavernosa by a perpendicular incision at right angles to the last one described close to the base of the dorsal flap and directed upward at right angles to the long axis of the penis.

FIG. 77

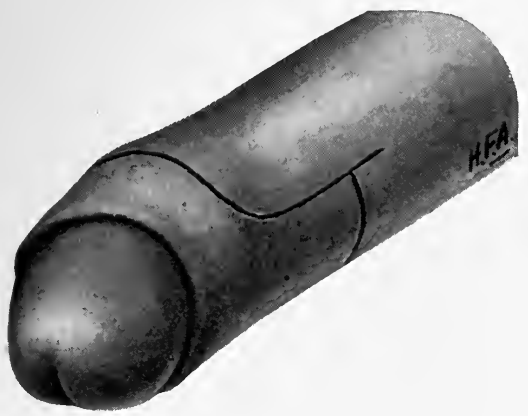

Dorsal flap method, showing the outline of the skin incision.

Divide the urethra and corpus spongiosum by an incision through them, about half an inch in front of the incision which divided the corpora cavernosa and directed at right angles to the long axis of the organ.

Ligate the dorsal vessels and the arteries of the corpora cavernosa. Remove the tourniquet, and, if necessary, control hemorrhage by means of transverse sutures passed through the cut ends of the corpora cavernosa and their sheaths.

Slit the newly made urethral orifice in the manner described in the last operation.

Make a small opening in the middle of the dorsal flap, a short distance behind its edge. Slip the end of the urethra through this hole, and attach the edges of the one to the other by interrupted catgut sutures. Tie a catheter into the bladder, as in the previous operations, and dress the wounds in the same manner as in those operations. (The steps of this operation are shown in Figs. 77,78 , and 79.)
FIG. 78

FIG. 79

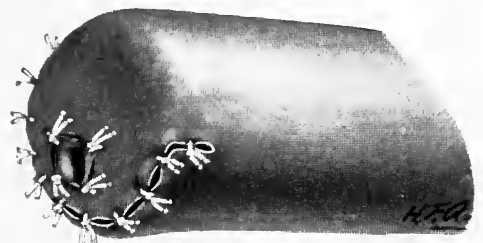

Dorsal flap method, showing the amputated stump with the split urethra brought through the slit in the dorsal flap, everted and sutured to the skin.

Dorsal flap method, showing the dorsal and ventral flaps. The body of the penis divided; the vessels ligated; the projecting urethra divided, and a slit in the dorsal flap for its reception.

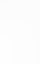

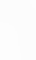


Comment.-Care should be taken to avoid wounding the urethra when separating it from the corpora cavernosa. The placing of a catheter in the canal enables the operator to do this.

\section{EXTIRPATION OF THE PENIS.}

Instruments.-Two scalpels; I pair curved, blunt-pointed scissors; I periosteum elevator; I needle-holder; curved and straight needles; catgut ligatures and sutures; 2 pairs of vulsellum forceps; 2 pairs of toothed forceps; 8 pairs of artery forceps; I steel sound; I soft-rubber catheter; I Watson's perineal hard-rubber button for retaining catheter in the bladder; 2 medium-sized retractors; 2 tenacula; 2 aneurysm needles.

Position of the Patient.-As for lateral perineal lithotomy.

Operation.-Split the scrotum in two longitudinally, following the line of the septum which separates its chambers, and carrying the incision down to the corpus spongiosum; the incision is then to be carried in an ellipse around the base of the penis, dividing the skin only.

Pass a sound through the urethra into the bladder for the purpose of defining the urethra. Clear the corpus spongiosum from adjacent structures-including the corpora cavernosa-beginning this separation about half an inch in front of the bulb of the corpus spongiosum, and working backward. The spongiosum and urethra should be cut across transversely before undertaking to free them in this way. They should be dissected from the surrounding structures as far back as the face of the triangular ligament.

The sound is, of course, to be withdrawn before dividing the urethra.

Pass a suture through each side of the proximal end of the divided urethra; leave the sutures long, and knot their ends so as to form a loop of each thread. This step is often useful in helping the operator to draw the severed urethra forward into place later in the operation.

Expose the suspensory ligament of the penis above the symphysis and divide it.

Isolate the bloodvessels of the dorsum of the penis and ligate them.

Clear the corpora cavernosa down to their attachments on either ramus of the pubic bone. Separate the crura from the bone on each side with a periosteum elevator, and by snipping through the tissues with scissors whenever it may be required. The two arteries of the crura are encountered during this step of the operation, and are to be tied.

Hemorrhage is severe during the separation of the crura and when exposing them. It may be in part avoided by carrying a ligature around the bloodvessels, which lie on the lateral aspects of the root of the penis 
and beneath the suspensory ligament, with an aneurysm needle and ligating them en masse, previous to dividing the attachments of the crura or to wholly exposing them.

Split the presenting end of the divided urethra and suture it to the edge of the incised scrotum with fine silk or catgut sutures, as may be preferred.

Fig. 80

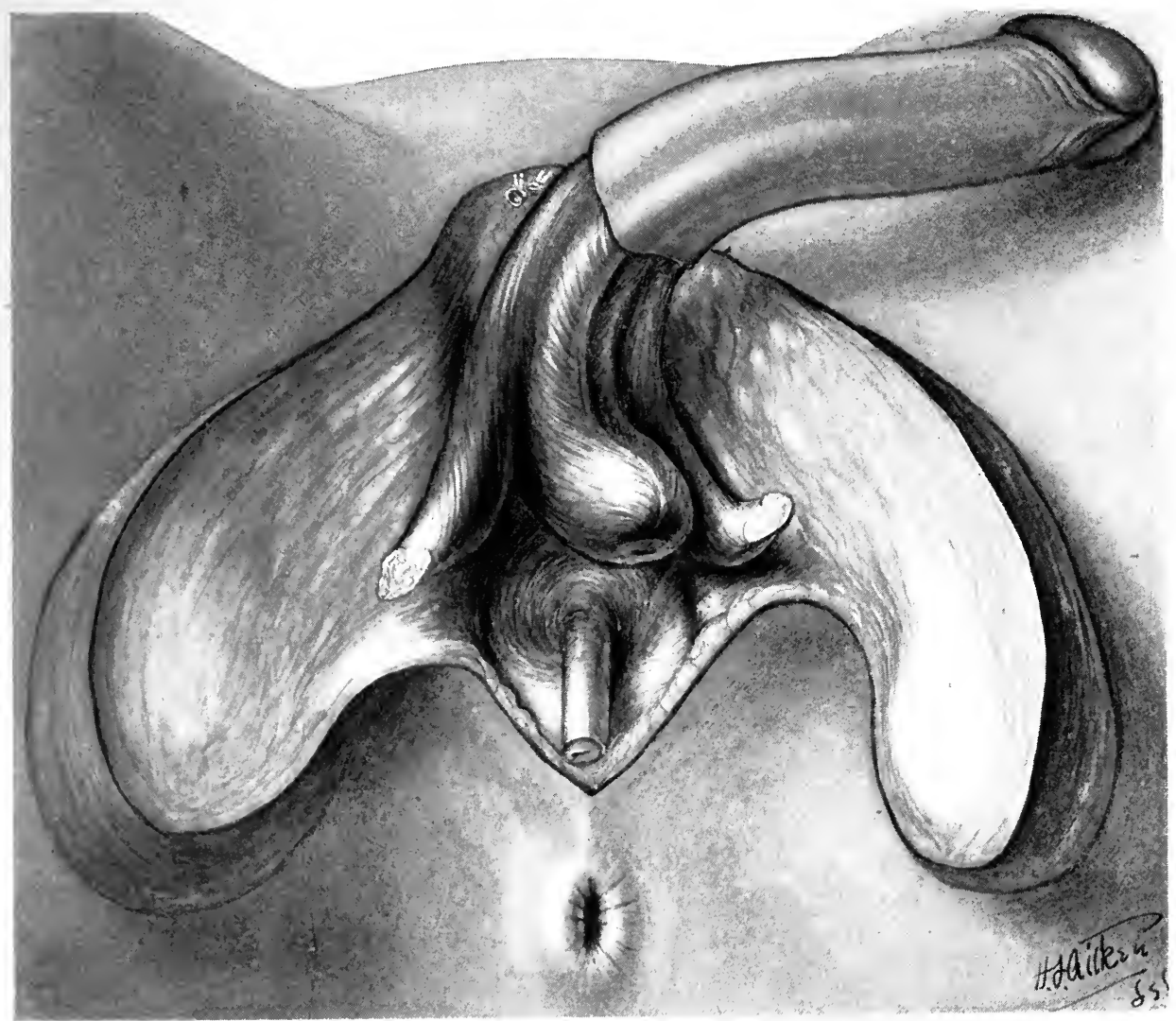

Extirpation of the penis, showing the scrotum split and the two halves separated; the dorsal arteries of the penis ligated; the urethra severed from the bulb; the crura freed and turned forward.

It is unnecessary to tie a catheter into the bladder, but it is desirable to do so in order to keep the dressings dry.

Close the scrotal incision up to the point of emergence of the cut end of the urethra (Figs. 80 and $8 \mathrm{I}$ ).

Inguinal nodes, when infected, should be removed.

Comment.-The newly made meatus does not tend to contract so readily as is the case with that made in the operation of partial amputa- 
tion. It is well, however, to pass a sound through it from time to time to insure its patency.

The disagreeable wetting of the thighs when urinating, which is so trying to the patient after the operation, may be avoided by applying a

Fig. 8I

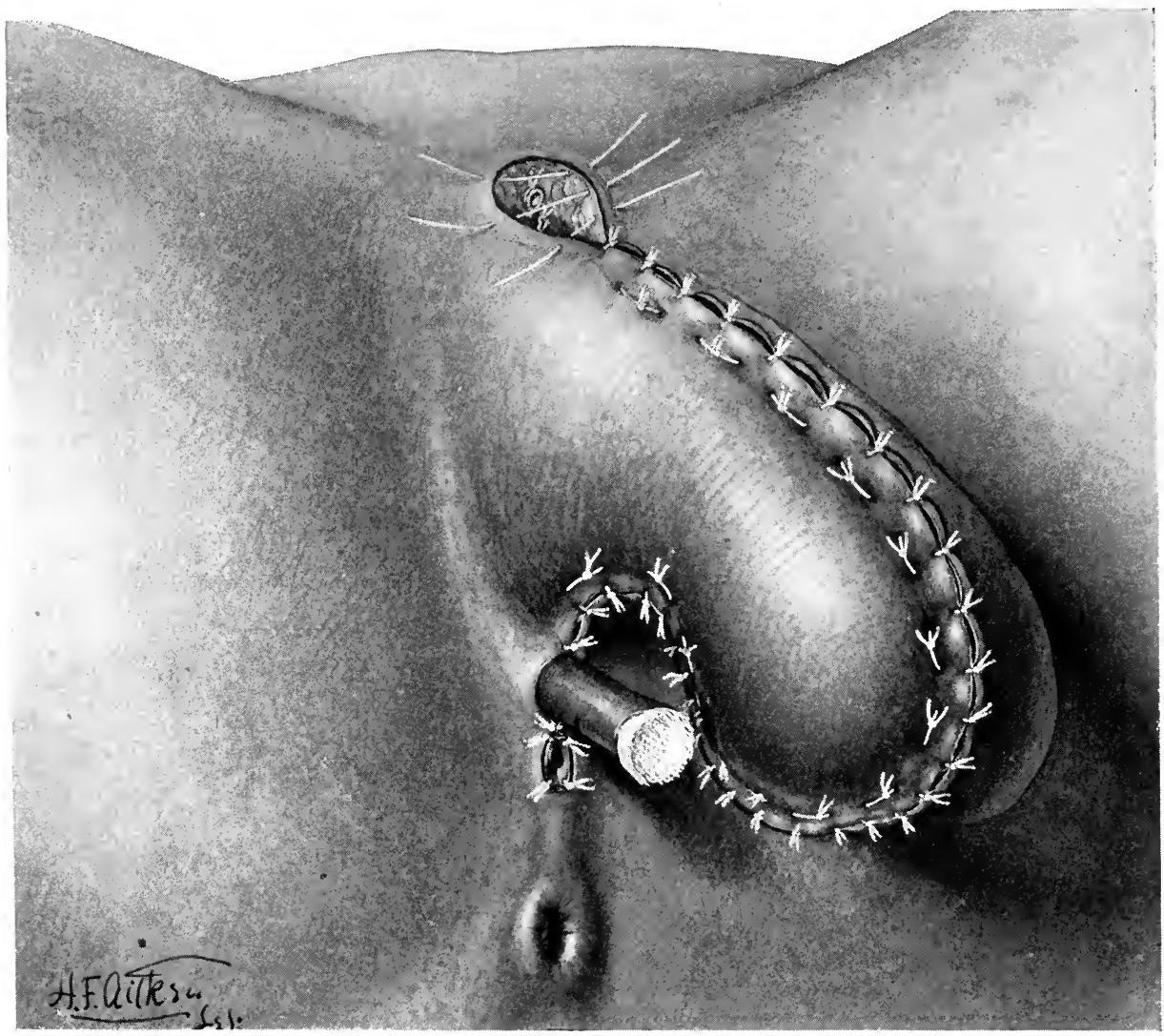

Extirpation of the penis, showing the two halves of the scrotum united by Siegle's method; the cigarette drain at the most dependent point of the wound; the split urethra everted and united to the skin.

small funnel to the surface of the scrotum and passing the urine through it and a short rubber tube attached to the other, or small, end of the funnel; in this way the urine can be kept from coming in contact with the parts lying adjacent to the end of the stumps of the urethra.

\section{TOTAL EMASCULATION OF PENIS.}

The removal of the testicles together with the penis is advised by a number of surgeons (Pantalin, ${ }^{2}$ Wheelhouse ${ }^{3}$ ), on the ground that the 
presence of the testicles without the penis, when the latter part only of the sexual organs has been removed, tends to reduce the patient to a state of despondency, or to cause him to become neurotic, owing to the constant suggestion of his lost power to have sexual intercourse, although the essential element of the male still remains to him.

We cannot share this opinion, in view of the large mortality which attends castration in elderly men, and which became so potent a factor in connection with the operation of castration in cases of prostatic hypertrophy, and in view also of the considerable number of patients who became insane or despondent after that operation in those cases.

The only difference between this and the last operation described lies in the ligation of the cords on either side at the level of the external inguinal rings (at which time the inguinal glands, if involved by the disease, should also be taken out) as a preliminary step to the removal of the penis and the testicles.

The testicles are separated from their attachments as in the usual operation of castration. The scrotum may advantageously be trimmed off so as to fit the urethral orifice better.

\section{PLASTIC OPERATIONS ON THE PENIS.}

The object of plastic operations is to cover over and repair losses of substance of the penis that have been brought about in one or another way.

By the Flap Method.-Instruments.-The same as in the operation for amputation of the penis.

The flap may be taken from the scrotum. It should be remembered that the flap in all plastics shrinks after being freed from the underlying tissues, and that the space to be covered over enlarges when its edges are separated from the adjacent tissues.

On this account the flap should be planned generously. Another point to be kept in mind is that the flap must be well nourished, and, that this may be provided for, the base must not be too narrow nor should the strip be too thin. The last point to be noted with reference to a flap operation is that it must be planned in such a way that there is no doubt but that the denuded space will be completely covered in.

Reich's Operation.-This operation is employed in cases in which the body of the penis is wholly denuded of skin.

Operation.- Refresh the edges and the surface of the denuded area. The latter is freshened by scraping it with a curette.

Make a transverse incision across the lower part of the scrotum, with its two ends equidistant from the central raphé. 
Make a circular incision around the base of the penis beyond the cicatricial part of the skin.

Dissect the intervening part of the scrotum between the first and second incisions from the underlying tissues. Pass the denuded penis through the incision immediately beneath its root, underneath the bridge of skin just made in the scrotum, and bring the head of it out through the lower opening.

FIG. 82

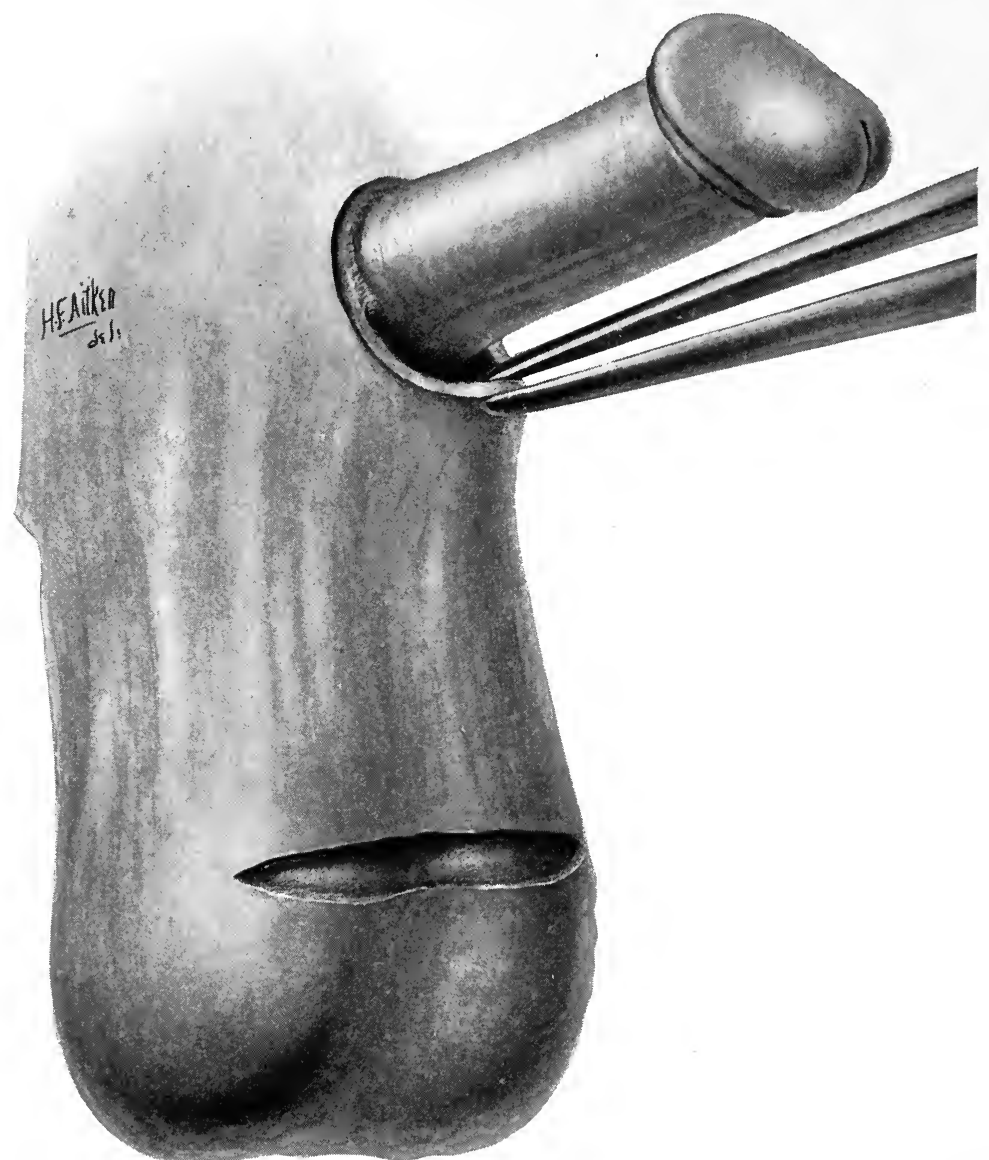

Showing the denuded penis; the fresh edge of skin at the root of the penis; the incision immediately under its base; the transverse incision in the scrotum.

Attach the margin of the upper incision to the base of the penis with catgut sutures and the edge of the upper part of the lower incision just behind the glans in the same way (Figs. 82 and 83 ).

Second Operation.-When healing has been firmly established, which is ordinarily in about three weeks, an incision is made through the skin of the scrotum on either side of the penis and parallel with it, extending 
from one to the other of the two former incisions. The dotted line in Fig. 83 shows this incision. The penis with the now adherent skin which covers it is lifted from the underlying surface, and the tissues connecting it with the latter are divided. The base of the flap is depended upon, in part, for its nourishment, and in part it is derived from the newly established system of vessels created between the under surface of the bridge of skin and the surface of the penis upon which it has been laid.

FIG. 83

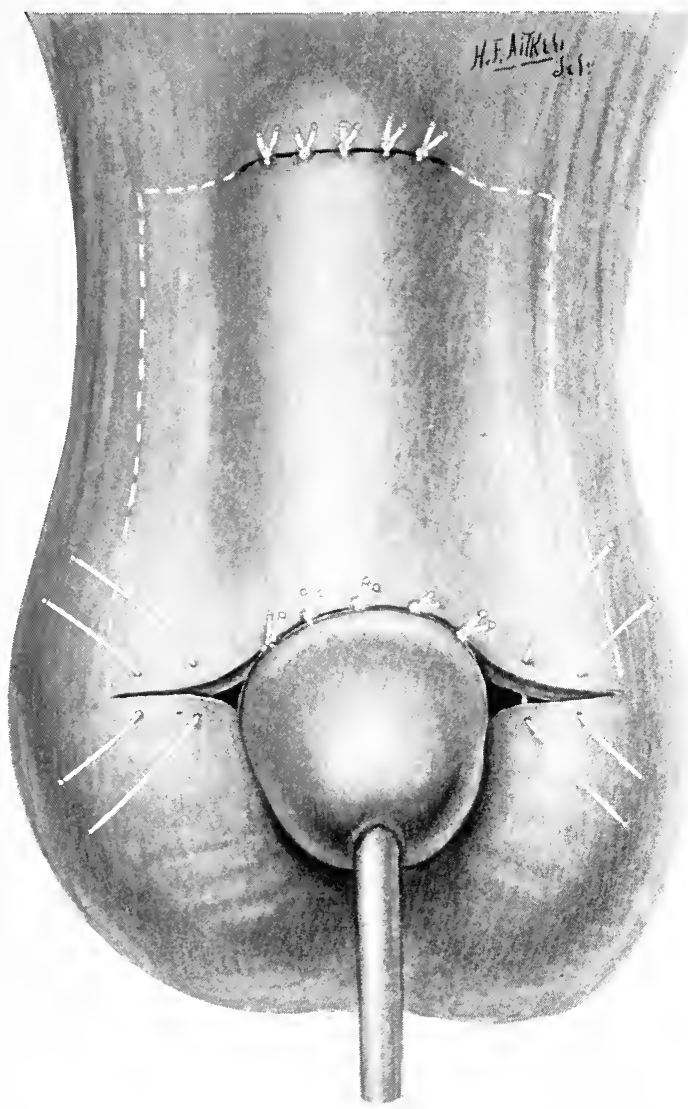

Showing the penis slipped under the bridge of skin between the two incisions. Both incisions closed by interrupted sutures. The dotted line represents the line of incision for the formation of flaps to cover the ventral surface of the organ at the second operation.

The edges of the flap, which have been freed in the manner just described, are now brought together beneath the body of the penis, and united to each other along the ventral surface.

Covering the Denuded Area by Thiersch's Skin Grafting.-The writer has had one case in which the entire penis and the anterior surface of the scrotum were wholly denuded by a virulent septic process produced by 
an infiltration of urine which proceeded from a rupture of the urethra near the bulbomembranous junction. This wide space he covered by the skin-grafting method of Thiersch, with the result that, when healing was complete, it was almost impossible to believe that the new skin was not the natural integument.

\section{OPERATIONS FOR HYPOSPADIAS.}

Preliminary Steps.-Cystitis and urethritis should be overcome, if present, before undertaking the operations.

The same is true of eczema in the cases in which epispadias is to be operated upon by the flap methods.

Hair should be removed from the parts about the field of operation.

Duplay's Operation.-Instruments.-Fine hemostatic forceps; I small tenotome; I small silver probe; 2 small tenacula; fine needles; fine catgut sutures; and some split shot to hold the sutures.

The penis may advantageously be attached to a board and held there by pins during the operation.

Operation.-This operation is suitable for the cases in which the hypospadias is situated just behind the glans. The defect is repaired by refreshing the edges of the opening and by approximating them to each other with silver sutures or by a pin. In the cases in which there is insufficient tissue to close the edges around a catheter passed into the urethra, cuts should be made into the glans on either side, as seen in Fig. $84(b)$, or upward, as in Fig. $84(a)$. The result will be that shown in Fig. $84(c)$.

Fig. 84
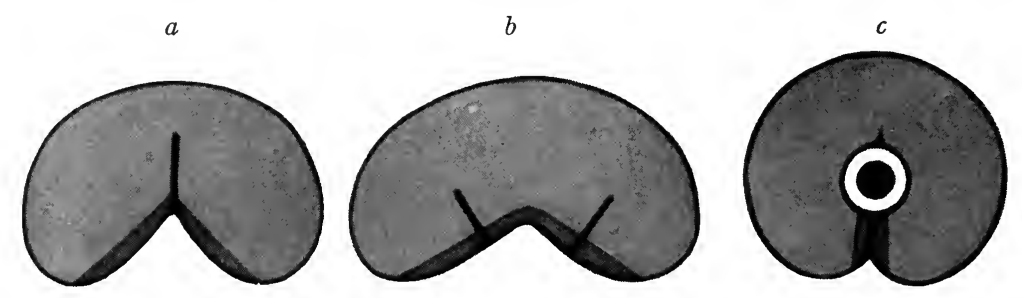

Duplay's operation for hypospadias.

A catheter should be retained in the bladder until union of the wounds is complete.

Beck's Operation.--The operation is especially suited to cases in which the abnormal opening of the urethra is situated but a short distance behind the glans (Figs. 85, 86, 87, and 88).

Operation.- Incise the opening of the canal and the tissue on either side of it sufficiently to allow the urethra to be easily drawn through the 
Fig. 85

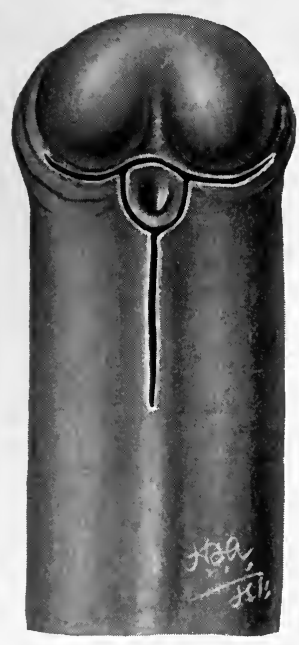

Beck's operation for hypospadias.

Fig. 87

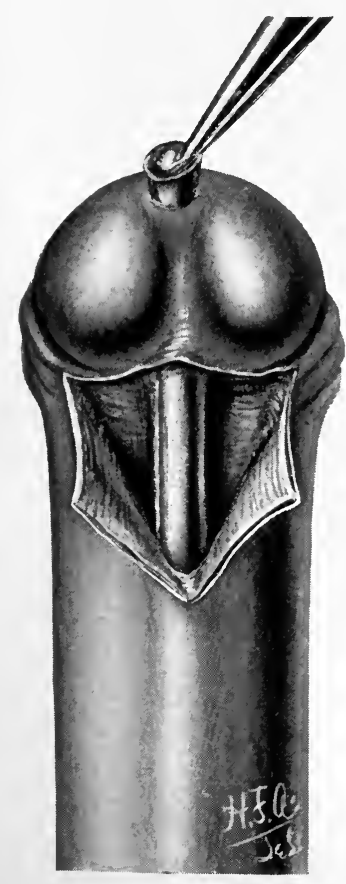

Beck's operation.
FIG. 86

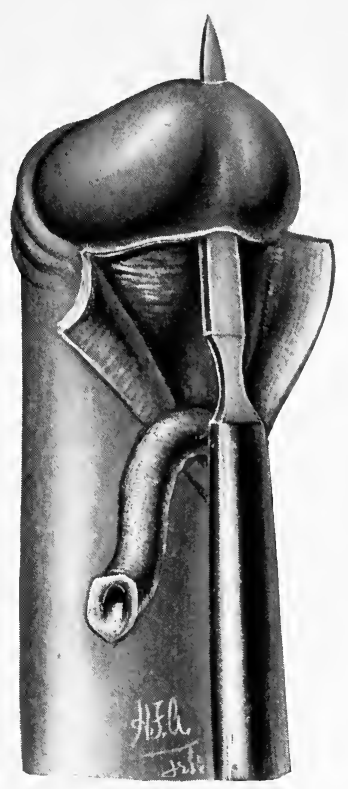

Beck's operation.

Fig. 88

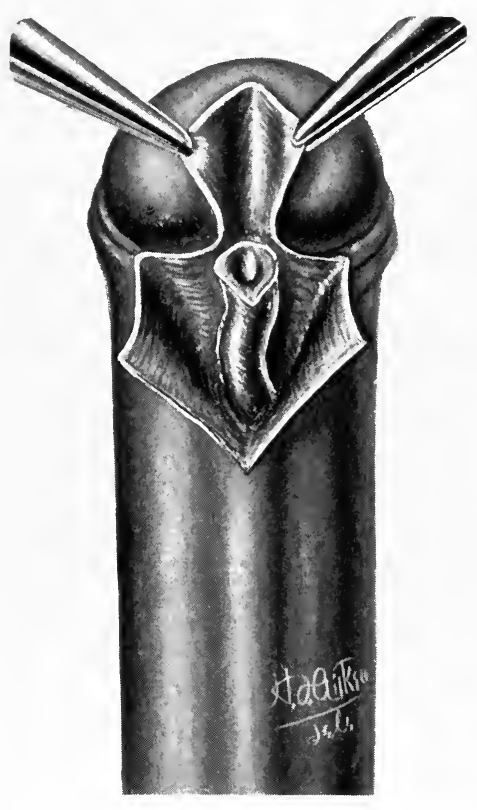

Beck's operation. 
opening thus made, and dissect a lateral skin flap from the ventral surface of the penis by carrying a median longitudinal incision downward in a

Fig. 89

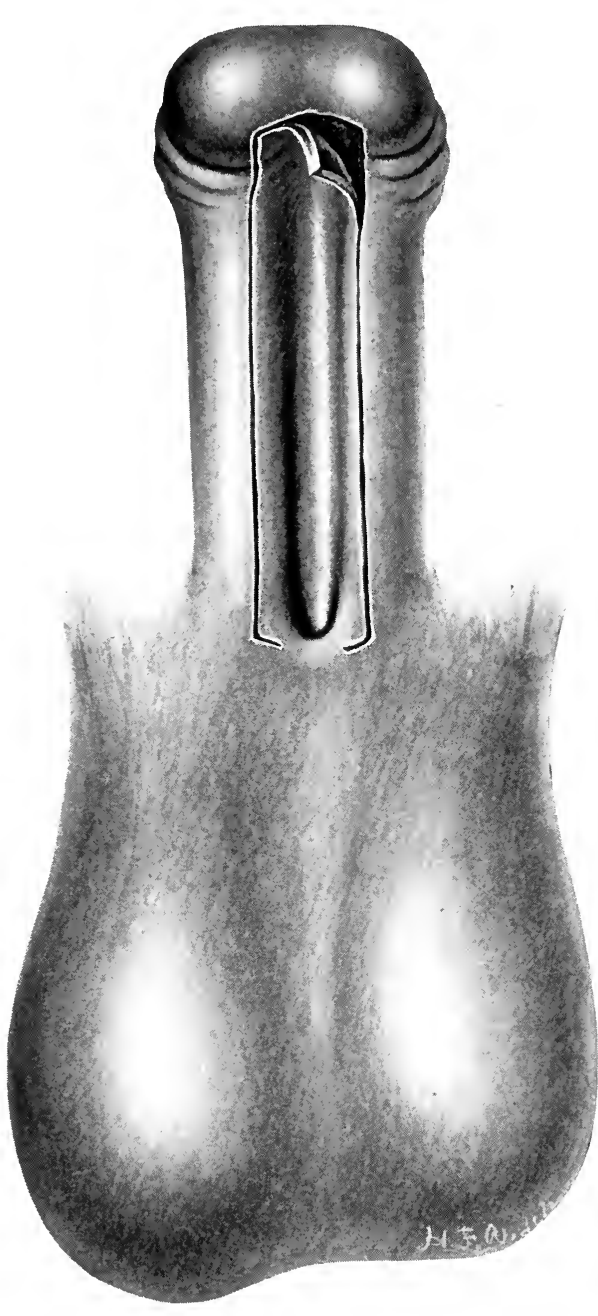

Beck's operation for scrotal hypospadias. First step.
FIG. 90

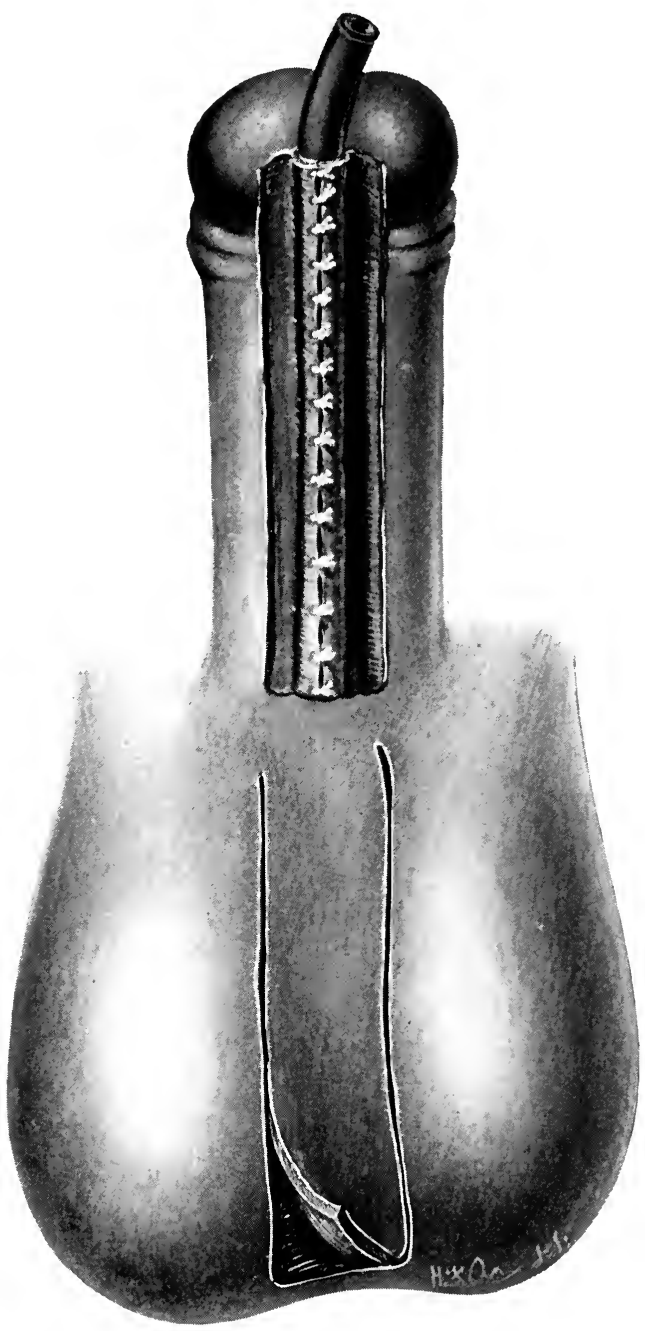

Beck's operation for scrotal hypospadias. Second step.

line corresponding to the course of the urethra, and by extending the first, or transverse incision outward over the sides of the penis.

Liberate the urethra from its anterior divided end backward as far as the lower angle of the lateral skin flaps (see Figs. 85 and 86).

Incise the ventral aspect of the glans by passing a straight bistoury 
through its substance in a line corresponding with the frenum and bringing it out in the mid-line of the glans, thus making a passage or tunnel. The end of the liberated urethra is then drawn through this tunnel (see Figs. 86 and 87 ) and attached to the surface of the glans by interrupted sutures placed through the end of the urethra and the margin of the orifice of the tunnel. The lateral skin flaps are then drawn across the under surface of the urethra and united by interrupted sutures.

Another manner of placing the end of the urethra in its new position is that shown in Fig. 88. In this method the ventral surface of the glans is cut through with a T-shaped cut, the free end of the urethra is drawn up to the top of it, and the sides of the incision in the glans are closed about it by uniting them to each other.

Beck's Operation for Scrotal Hypospadias.-Beck's operation makes a canal of double thickness by constructing it with two flaps instead of one. The first part of the operation consists in dissecting up a flap on either side of the median line of the part of the ventral surface of the penis, which should be occupied by the normal urethra, by bringing the edges of these flaps together and uniting them by sutures. Over the canal thus made a second flap is turned and its edges are united to the margins of the area denuded by making the first flaps. The

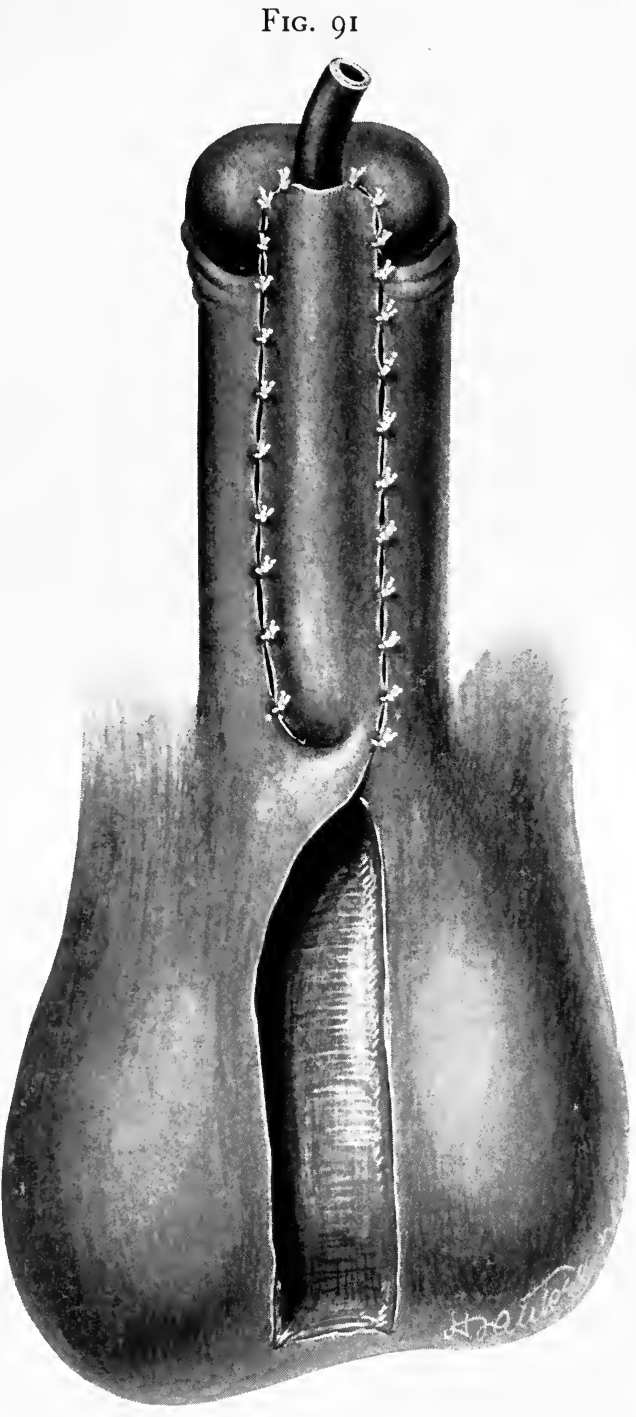

Beck's operation for scrotal hypospadias.

Third step. second flap is taken from the scrotum and its raw surface is apposed to that of the canal formed by the first flaps by twisting it at its base (Figs. 89, 90, and 9I).

Van Hook-Mayo Operation.-Draw forward the prepuce as in the operation for circumcision. Make two incisions, one inch apart, extendvol. I-I 2 
ing from the free border of the prepuce to its junction with the corona and extend them over the dorsum of the penis on either side of its lateral aspect (Fig. 92). Connect the further ends of these cuts by transverse incision, and dissect a flap of skin, thus marked out upon the dorsum of the penis, from the underlying tissues as far as the corona. Approximate the edges of the skin on either side of the denuded area thus made, and unite them with interrupted sutures, thereby covering over the exposed surface. Unite the edges of the prepuce and the flap from the dorsum so as to form a tube (Fig. 92).

FIG. 92

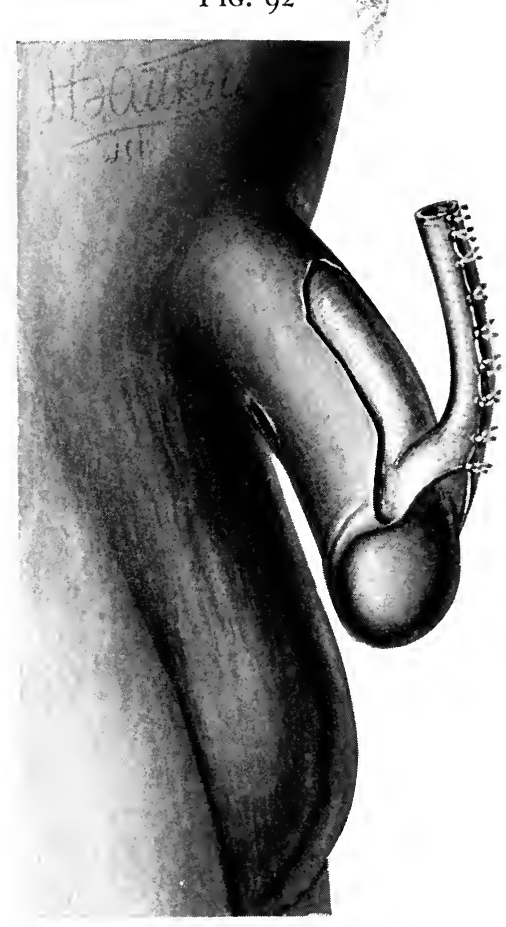

Mayo's operation for hypospadias. First step.
FIG. 93

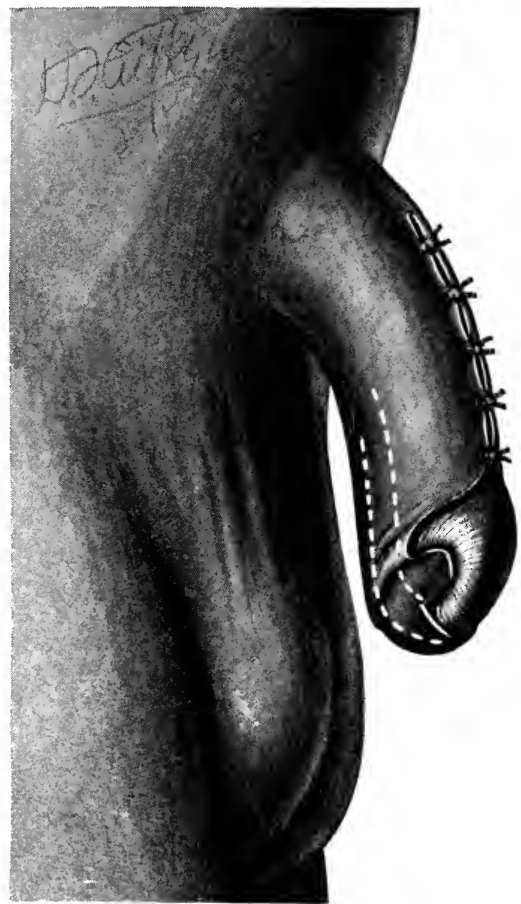

Mayo's operation for hypospadias. Second step.

Tunnel the penis upon its ventral surface as far as the hypospadiac opening with a straight, narrow bistoury, passing it through the middle of the glans and bringing the point of the knife out through the hypospadiac opening.

Turn the tube over, pass it through the tunnel thus made, and unite its end to that of the urethral orifice (Fig. 93).

Mayo allows ten days for the tube to become united to the bed that has been made for it, then cuts the pedicle just above the meatus and attaches the proximal end of the tube to the glans. 
A catheter is tied into the bladder through the posterior urethra, an external perineal urethrotomy incision being made for the purpose.

The hypospadiac orifice of the urethra should be liberated from its immediate surroundings and invaginated into the end of the tube of the new anterior part of the urethra (Figs. 92 and 93).

\section{OPERATIONS FOR EPISPADIAS.}

Zöller, ${ }^{4}$ in speaking of the operations for epispadias, divides them into two groups, as follows:

I. Those in which the defective urethra is reformed by uniting the freshened edges of the epispadiac groove.

2. Those in which this end is accomplished by plastic methods.

To these should be added the operation of Maydl, in which the ureters are transplanted.

Of the first group, the method of Duplay is the most important. It is carried out in four stages, thus:

Group I. Duplay's Operation.-Stage I.-A perineal fistula is formed, as a step preliminary to the first operation. This having been done, the operation proceeds thus:

Straighten the penis by dividing subcutaneously with a narrow-bladed, straight bistoury the bands of tissue which attach the organ to the anterior abdominal wall, or symphysis pubis. Enter the knife close to the os pubis and slowly and carefully divide the tissues spoken of until the object sought is attained. Hemorrhage, which may be free, is to be controlled by pressure.

The penis is afterward to be retained in its new position by strapping it down with strips of adhesive plaster. This corrected position will require to be maintained for several months, perhaps, before proceeding to the second operation.

Stage 2.- Make two denuded, narrow, quadrilateral areas, each about $5 \mathrm{~cm}$. wide, by removing a strip of skin of this width from a point just below the opening into the bladder to the distal end of the groove, on each side of it. A catheter is passed along the groove into the bladder and retained there.

Suture the edges of the denuded area on one side of the groove to those of its fellow on the other side, bringing them together above the catheter.

After-care.-The wound is dusted with iodoform and a dry dressing applied. The catheter should be retained until healing is complete.

Stage 3.-The treatment of the prepuce.

The treatment of the prepuce is to be undertaken when the wounds of the last operation are completely healed. 
A window is made in the prepuce and the latter is brought up onto the dorsum of the glans, which is passed through the incision in the prepuce. The edges of the anterior part of the newly formed roof of the canal created by the last operation are freshened and united to those of the transplanted prepuce.

Stage 4.-Closure of the opening into the bladder. The surfaces on either side of the opening of the bladder, which is still in communication with the surface, are freshened and united by sutures. A catheter should, in most instances, be retained in the canal and in the bladder. When healing is complete the catheter is withdrawn, and thereafter sounds of small caliber should be passed into the bladder.

Group 2. Plastic Operations.-Thiersch's Operation for Epispadias.Thiersch's method of operation is applicable to cases of penile epispadias. whether the bladder is extroverted or not, which is one point in its favor. The operation proceeds as follows:

Operation.-The first step consists in forming a new penile urethra. Two flaps are used for this purpose, each of them cut from the skin of the lateral aspects of the penis. (Flap I, Fig. 94, on left side of penis. Flap 2, Fig. 94, on right side of penis.)

The first flap is made by an incision well over on the side of the penis and extending from a point close to the base of the glans nearly to the symphysis pubis, in a line parallel with the furrow which lies in the median line of the dorsum of the penis between the two corpora cavernosa, and by a second and third cut, one at each end of this incision, at right angles to it, and carried nearly to the side of the furrow. The flap thus marked out is then dissected free.

Flap 2 is made by an incision parallel with the opposite side of the furrow from that on which the first one was made and close to the edge of the furrow. This incision extends, like the first one on the other side, the whole length of the body of the penis. Cross-cuts at right angles to it, one at each end, are carried outward over the side of the penis, and the flap thus marked out is dissected free and turned outward over the corpus cavernosum of the same side (Fig. 94). The edge of the first flap is then carried over across the furrow, in doing which its under surface will become turned uppermost, so that its raw surface will be apposed to the under or raw surface of the second flap when the latter is drawn over it. The edge of Flap $\mathrm{I}$ is to be sutured along the line of the base of the second one, and underneath that flap. The edge of Flap 2 is then carried across and (if too much tension is not thereby produced) above the raw surface of the first one, and is sutured to the margin of the skin from which the first flap was freed by the first incision of all (Figs. 94 and 95). 
In other words, the first flap is turned over so that the skin surface of it becomes the under one and its raw surface becomes the upper one, and is tucked under the second flap. The latter is then pulled over the first and attached to the opposite side of the denuded area made by dissecting off the first flap.

FIG. 94
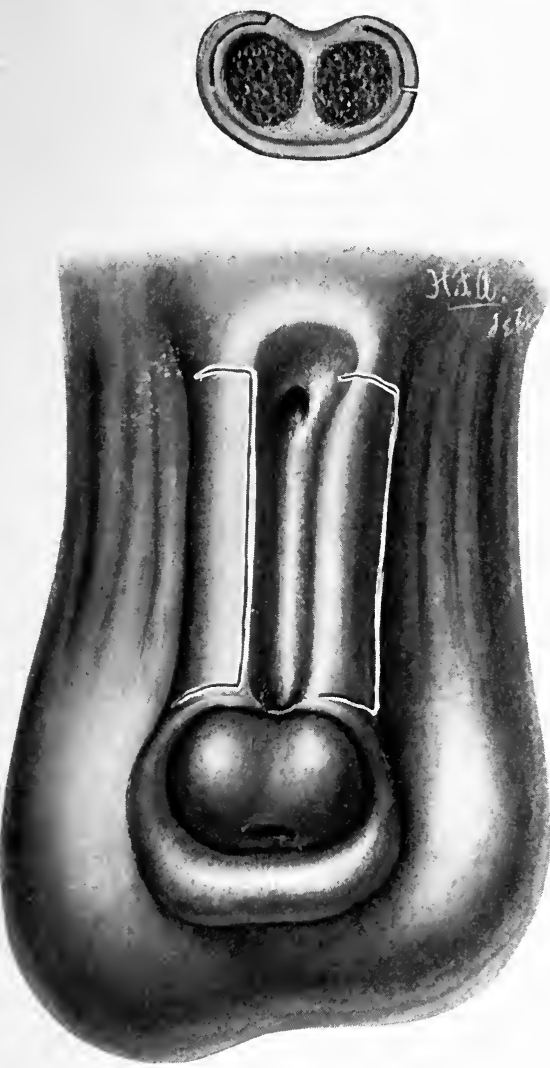

Thiersch's operation for epispadias. Showing the skin flaps as they appear on the penis and as they would appear in a cross-section of the organ.
FIG. 95
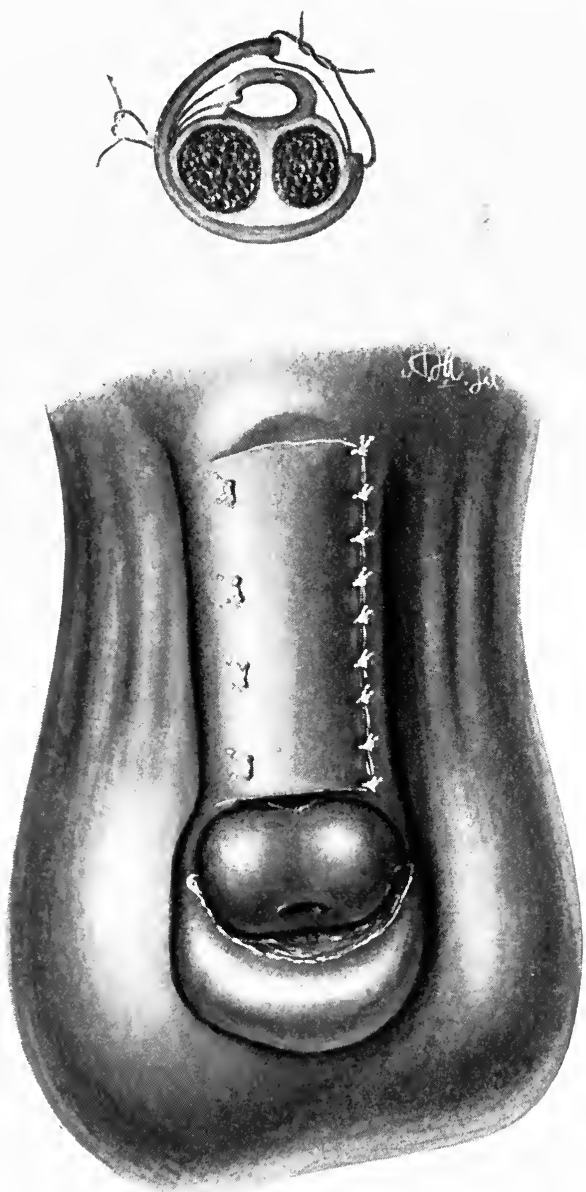

Manner of placing the flaps in Thiersch's operation and attaching them to form a new urethra, and showing the conditions as they appear on cross-section and on the penis.

A canal with double walls, one overlying the other, is thus formed. The two layers of the walls of the canal become welded into one when their apposed raw surfaces are grown together in the course of the process of healing. Figs. 94 and 95 illustrate the conditions just described. 
The next step is to form the roof of the small space which still remains defective and open at the anterior end of the newly constructed penile part of the canal. This step is taken only when the wounds resulting from the first operation have been thoroughly healed. It is accomplished by utilizing the prepuce for the purpose. A transverse incision is made through a part of its width; the glans is passed through this cut,

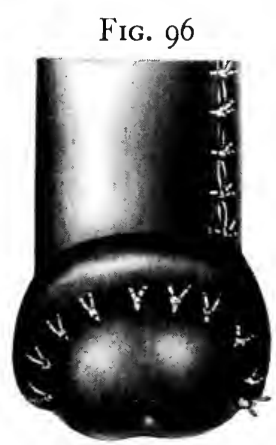

Third step of the operation, showing the glans after it has been slipped through incision in the prepuce, and also the latter sutured in place behind corona of glans. and the prepuce is united by its incised margin, which has been passed onto the dorsum of the penis, to the refreshed edge of the anterior ends of the flaps made at the time of the first operation (Fig. 96).

There still remains the posterior opening of the newly made canal to be closed. This is done by freshening the posterior edges of the canal made by the first operation. Next, two triangular flaps are made, one on either side of the base of the penis and extending upward over the anterior surface of the symphysis pubis. The upper end of the first flap is then turned down (thus bringing its under or raw surface uppermost), and its outer edge is attached to the freshened edges of the posterior end of the canal previously made at the first operation. The second flap is then slipped down over the raw surface of the first one until it entirely covers the upturned raw surface of the first flap, and their edges are approximated. The edges of the two flaps are then united to each other by suture. The upper end of the second flap will then lie in the angle of the denuded surface made by dissecting free the first flap.

Beck's Operation for Epispadias. ${ }^{5}$ - The first result obtained by Beck with this operation was a gratifying one. The patient was enabled to control urination, and could retain the urine in the bladder for four hours at a time. The operation has the distinctive feature of making the inner part of the new canal out of mucous membrane. It is done as follows:

Operation.-In cases of complete epispadias the principal feature of the operation consists in dissecting free a part of the tissue about the opening into the bladder and in transposing it in such a way as to form a new canal of mucous membrane.

Step 1.-The incision is made in the manner shown in Fig. 97, care being taken to include all the mucous membrane above the funnel-like opening of the bladder. If a sufficient amount of tissue is not secured without including a margin of the skin, the incision is carried in such a way as to include some of the integument, together with the mucous 
membrane. The tissue above the opening into the bladder is that from which the whole length of the roof of the new canal of the urethra is to be made. The part of it included by the cut must, therefore, be long enough to reach as far as the tip of the glans. The area being outlined, a funnel-like sac is dissected free. A finger should be placed within the opening into the bladder while this is being done, in order to avoid the danger of buttonholing the tissue that is to become the new urethra. The sac is drawn over this finger as it is freed, just as a glove might be. The sac-like structure should be at least two inches long.

FIG. 97

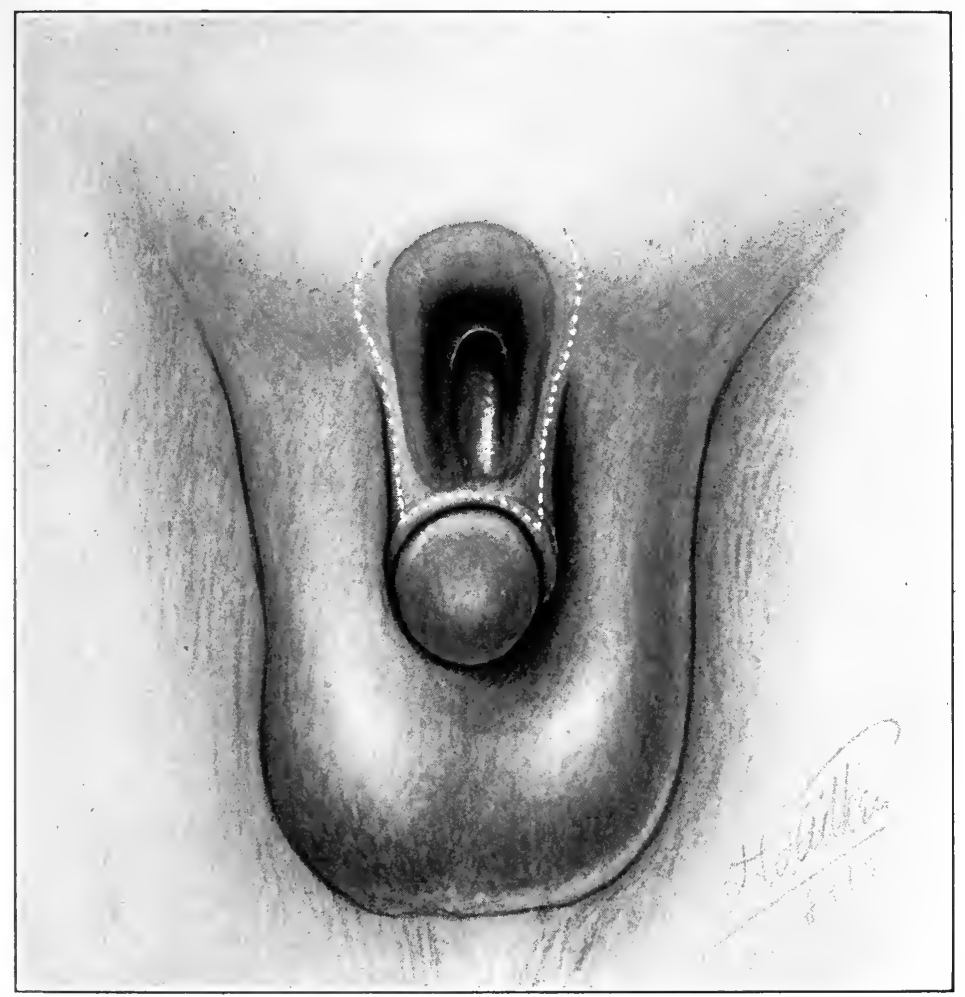

Beck's operation for epispadias. First step.

Step 2.- The anterior ends of the two longitudinal incisions are joined by a transverse cut made from one to the other of them. The floor of the gutter between the two corpora cavernosa is next dissected free from the underlying tissues (Fig. 98).

This dissection is carried to the under margin of the opening into the bladder. There have now been formed the roof and the floor of the canal which is about to become the new urethra. The tube forming this canal is next given complete form by uniting the edges of the first to the second 
FIG. 98

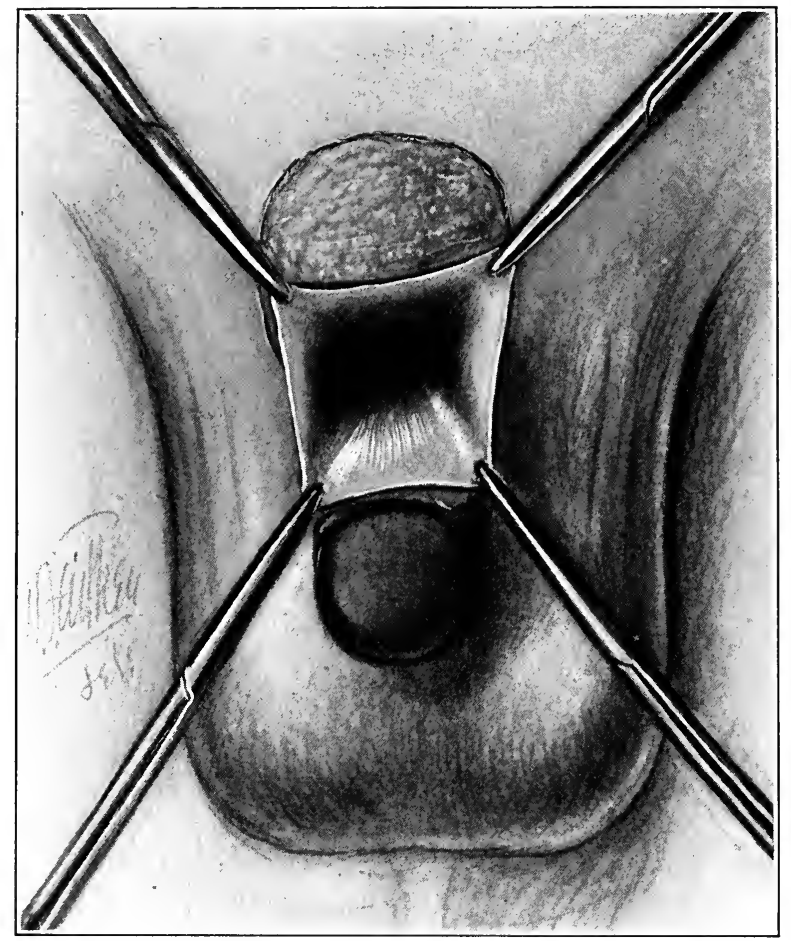

Beck's operation for epispadias. Second step.

FIG. 99

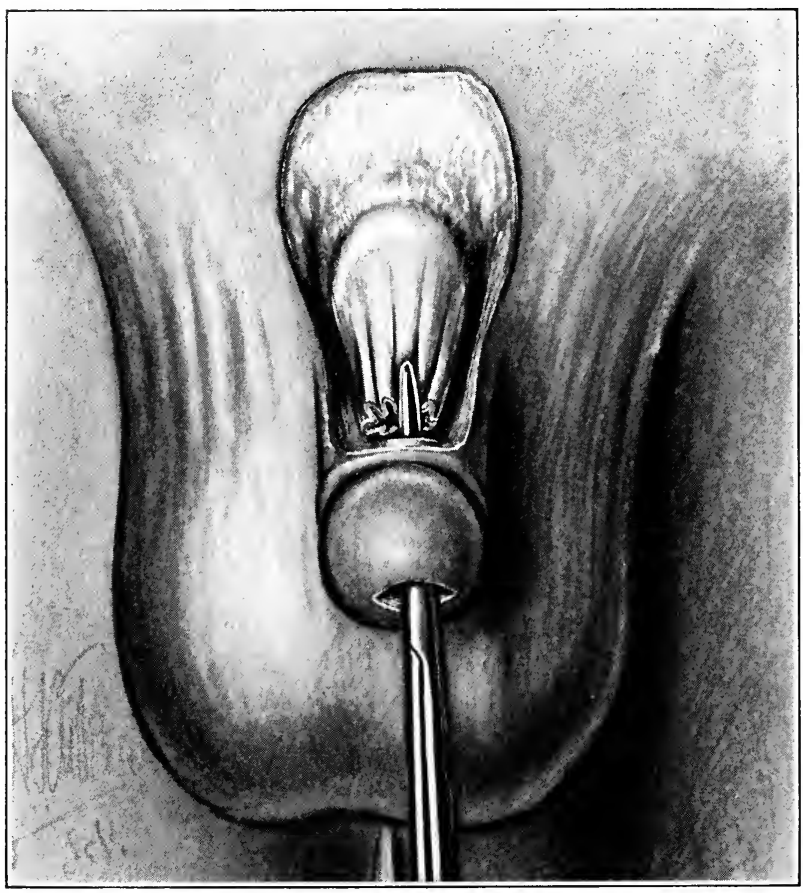

Beck's operation for epispadias. Third step. 
part of it. The posterior end of the tube is in direct continuity with the interior of the bladder while the anterior end of it is as yet unattached. The whole interior of the tube is composed of mucous membrane (Fig. 98).

Step 3.- The glans is now perforated by cutting or boring a channel through it, the outlet of which is to be made in the position at the tip of the glans which is ordinarily occupied by the meatus. Through this canal the end of the tube described above is drawn, and its margin is sutured to the newly made meatus or outlet of the channel through the glans just spoken of (Fig. 99).

\section{OPERATIONS FOR THE PURPOSE OF CLOSING URETHRAL FISTULA.}

Szymanowski's Operation.--The operation consists in closing the urethral defect by bringing its refreshed edges together and covering them over with a double skin flap, the two layers of which are apposed to each other and have raw surfaces. The operation is done as follows:

Flap I.-Make a crescentic incision on one side of the opening and with either end of it well beyond the opening. This flap should extend as far as possible toward the outer side of the perineum, in order to secure ample tissue to wholly cover over the hole in the urethra.

Dissect this flap free as far as the outer side of the urethral defect. Before doing this the epidermal layer of the flap should be scraped off with a curette. Make a second incision parallel with the direction of the urethra, and on its othe: side (and sufficiently far removed from the base of the flap upon the opposite side not to endanger the nutrition of the latter), extending from a point a little anterior to the anterior end of the first incision made to a point a little posterior to its other end.

From this incision the skin is dissected free from the subcutaneous tissue in such a way as to form a crescentic pocket corresponding in shape to the flap already described and sufficiently large to accommodate the latter.

The first flap is then turned back upon its base; its free edge is caught at several points by fine catgut sutures, which are first inserted at the base of the pocket opposite, then through the corresponding point on the edge of the flap, returned through the same edge at a point near that at which the needle was first passed through it, back again beneath the skin of the pocket, and out through its base at a point close to that at which the needle was first inserted. A sufficient number of sùtures, each of them placed in the manner just described, are to be set in the margin of the flap to insure its adaptation to the base of the pocket 
accurately when they are tied. The free ends of each one of these sutures will lie close together as they emerge through the closed side of the pocket. Tie together the ends of each of them.

Attach the free edge of the pocket to the margin of the skin on the opposite side of the denuded area made by dissecting the first flap. This is done by interrupted sutures set through the free margin of the pocket and that of the skin on the other side, in the same manner as that described when speaking of the sutures of the flap. When this last line of sutures is tied, the defect of the urethra will have been covered over by two layers

FIG. 100

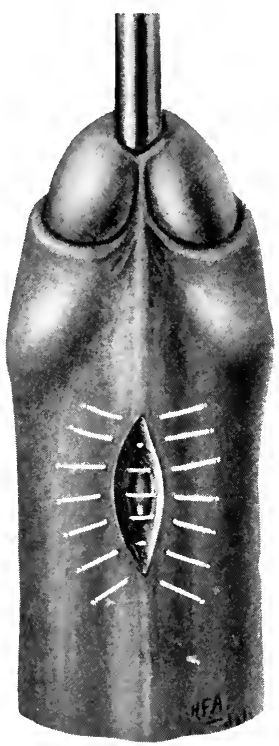

Method of closing the fistula by simple suture. The edges of the fistula are cut away and the fresh edges brought together over a catheter by interrupted sutures which do not pierce the lumen of the urethral canal.
FIG. IOI

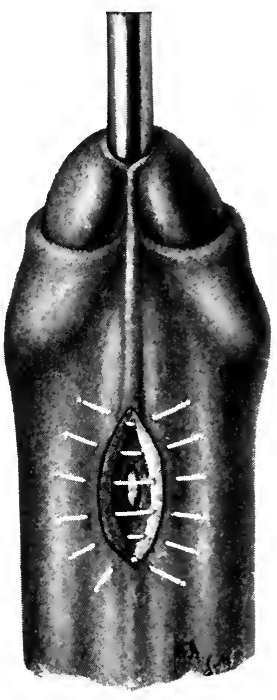

Showing the skin freed from the urethra after removing the fistulous tract. The wound is closed by interrupted sutures over a catheter, the sutures not piercing the urethra.

of integument, each of which has a raw surface to appose to the other, while the under surface of the flap has also been made raw and is thus prepared to adhere quickly to the under surface of the pocket, onto which it is drawn by the sutures. The margins of the urethral defect should be refreshed before the flap is made.

Closure by Simple Suture.-This operation consists merely in refreshing the edges of the urethral defect and then approximating them by a sufficient number of sutures to insure their adaptation. The sutures are best tied over a small roll of gauze or bit of round gutta-percha (Fig. I00). 
A catheter should be tied into the bladder through the urethra (Figs. IOO and IOI). It may be left there for ten days or a fortnight unless there are signs of urethral irritation due to it.

Simple Suture after Freeing the Skin on Either Side of the Defect.The only difference between this and the last operation lies in the fact that the skin is dissected from the underlying tissue around the urethral defect, in order that its margins may be more thoroughly approximated and for the purpose of uniting the edges of the skin more accurately (Fig. IOI).

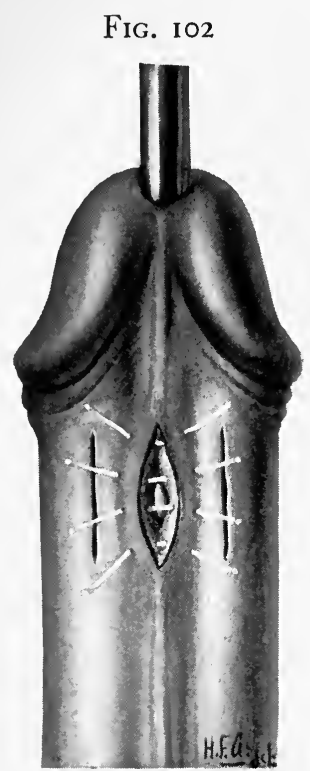

Dieffenbach's operation. Step I. Showing the fistulous tract removed; two parallel incisions through the skin. The skin between the fistula and each incision freed beneath from the underlying tissue. The sutures in position to close the fistula over a catheter.
FIG. I03

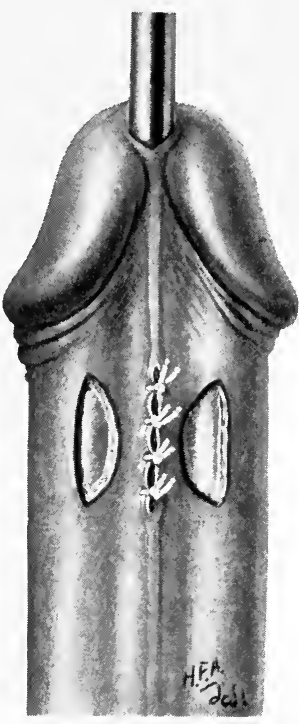

Dieffenbach's operation. Step 2. Showing the skin united closing the fistula. The gaping wounds on either side produced by the sliding inward of the skin. These wounds are closed by interrupted sutures.

Dieffenbach's Operation.--Refresh the margins of the urethral defect. Pass a catheter through the urethra as far as its deeper parts.

Make two incisions, one on either side of the urethral opening and parallel with the urethra. These incisions should be made at a little distance from the opening (Fig. I02).

Free the skin that lies between each of these incisions and their respective sides of the urethral defect, thus forming a bridge of skin on each side of it. The edges of these bridges and of the urethral opening are now brought together by sutures passed from the inner edge of the skin on one side, through the corresponding side of the urethral defect, through 
the opposite margin of the latter and the edge of the other skin bridge nearest the defect (see Fig. I03).

When these edges have been drawn together by tying the sutures, a denuded area is left on either side external to the bridge of skin. They may be closed over by suturing their outer margins of skin to the outer sides of the skin bridges, freeing the former in order to avoid too much tension being put upon the last sutures mentioned.

Lateral Flap Method.-An oblong area is denuded about the urethral defect. Next a skin flap of corresponding shape and size is made from the skin on one side of the area just denuded. A soft catheter, which

FIG. 104

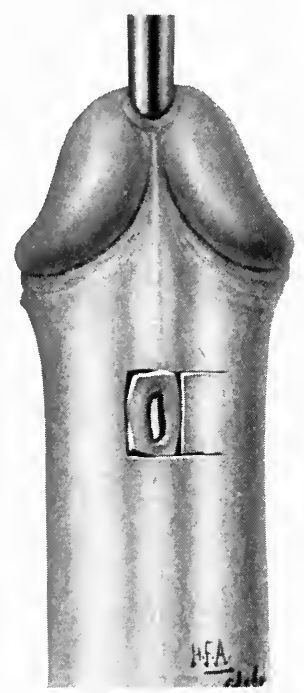

Lateral flap method. Step I. Showing the incision for removal of the fistula and the lateral flap outlined.
FIG. 105

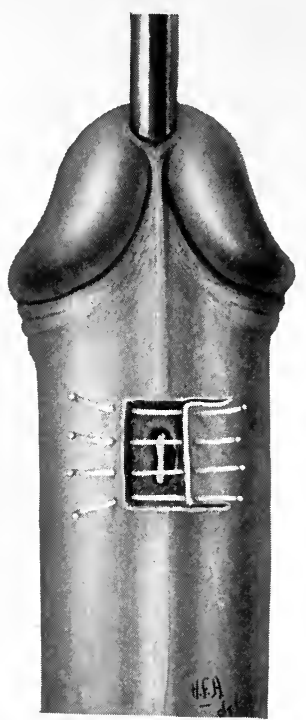

Lateral flap method. Step 2. Showing the rectangular denuded area and the lateral flap freed from the underlying tissue, and being brought into position over the fistulous opening by means of interrupted sutures.

moderately distends the urethra, is passed through its anterior portion. Sutures are next passed through the free edge of the skin flap, carried beneath the surface of the denuded area, and brought out through the free margin of the skin on the other side of it. The sutures are then tied and the catheter is passed on into the bladder and secured there. Figs. ${ }_{0} 0_{4}$ and 105 illustrate these steps.

Posterior and Lateral Flap Method.-The steps of this operation are shown in the accompanying illustrations, thus: In Fig. Io6 the lateral flaps are outlined. In Fig. I07 the margins of the fistulous tract have 
been removed and the flaps turned back. In Fig. I08 the posterior flap is seen freed from the underlying tissues and turned over the fistulous

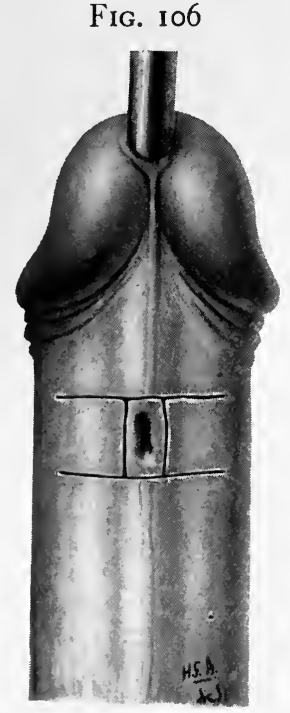

Posterior and lateral flap method. Step I. The incision to remove the fistulous opening and the lateral flaps outlined.

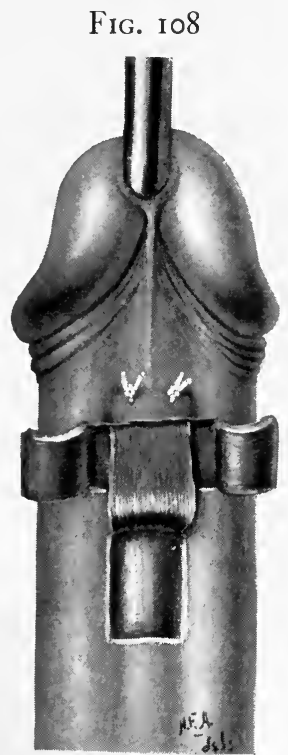

Posterior and lateral flap method. Step 3 . The posterior flap dissected up and the skin surface turned inward over the fistulous opening and sutured beneath the skin at the anterior end of the denuded area.

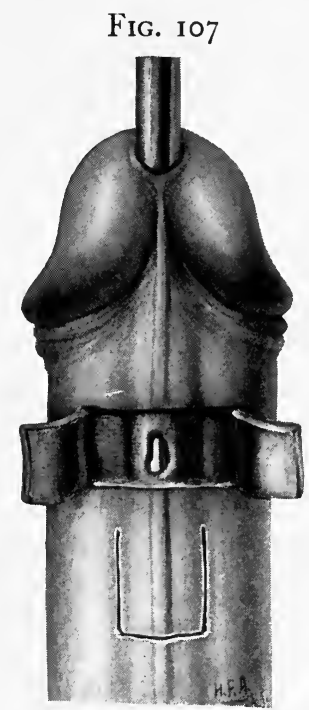

Posterior and lateral flap method. Step 2. The lateral flaps turned back and the posterior flap outlined.

FIG. 109

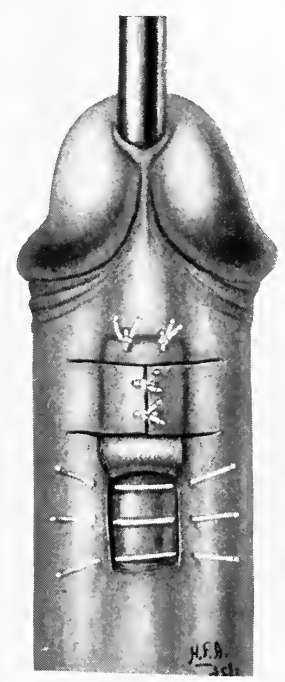

Posterior and lateral flap method. Step 4. The lateral flaps turned over the posterior flap and sutured together. The raw surfaces are in apposition. The sutures are placed to close the posterior denuded area. 
opening, with its skin surface facing inward and the flap sutured in place beneath the skin at the anterior end of the denuded area. Fig. Iog shows the lateral flaps drawn together and sutured over the posterior flap, the raw surfaces of both being in apposition.

\section{BiBLIOGRAPHY.}

I. Allis. Annals of Surgery, April, 1907.

2. Pantalin. Arch. Prov. de Chir., I 898 .

3. Wheelhouse. British Medical Journal, I886, vol. i, p. 187.

4. Zöller. Beit. z. klin. Chir., I894, Band xi, Heft I.

5. Beck. New York Medical Record, March 30, 1907. 


\section{H A P T E R V I.}

\section{THE SCROTUM.}

\section{ANATOMY OF THE SCROTUM.}

THE scrotum is the pendulous purse-like pouch beneath the penis in which the testicles and their adnexa are lodged.

Its outer surface is marked by a slightly elevated longitudinal ridge, which extends from the skin of the under surface of the penis above to that of the perineum below. The scrotal integument is darker in color than other parts of the integument, and is provided with a number of sebaceous glands. Its surface presents numerous horizontal rugæ. It is very elastic and is capable of being stretched in a remarkable degree. Large hernias and hydroceles show to what extent this may take place.

The dartos is the other component structure of the scrotum, and constitutes its tunica propria. This tunic is continuous with the superficial fascia of the groin, perineum, and penis, for which reason fluids which gain entrance to the dartos at any point may pass unimpeded beneath the skin of the whole of the area of these parts. Along the median line of the scrotum the tunic is reflected inward, and forms a firm partition between the two halves of the pouch, thus dividing it into two chambers, each of which contains one of the testicles and the part of its spermatic cord external to the inguinal canal.

The septum between the two sides of the scrotum made by the reflected dartos prevents fluid accumulated within one chamber from passing into the other one.

The dartos is made up of connective tissue and smooth muscular fibers, and possesses great contractility, on account of which property and its intimate connection with the scrotal skin it gives to the latter the corrugated markings which have been mentioned, and also lifts the testes up, when it is brought into action by the influence of cold, emotion, and bodily exercise. Warmth relaxes it, and this allows the testes to drop lower down, at the same time obliterating the rugæ on the skin of the scrotum. Upon its under surface the dartos is provided with a loose layer of areolar tissue, which is very vascular, and in the meshes made by which the fluid of œedema and extravasated blood can circulate freely. Owing to this arrangement, there are formed the extensive blood infiltrations which appear as ecchymotic areas on the scrotum, and which 
are very easily produced, moderate manipulation of the skin of the scrotum, such as occurs in operations about the parts, being enough oftentimes to discolor a large surface of the scrotum.

The Bloodvessels, Lymphatics, and Nerves of the Scrotum.-The arterial branches supplying the scrotum are derived from the superficial and deep external pudic, and one of them from the internal pudic. The veins empty into the saphenous and internal pudic. The lymphatics empty into the inguinal glands. The nerve branches are from the ilioinguinal, the two superficial perineal, the inferior pudendal, and the genital branch of the genitocrural. (See Plate II, Chapter I.)

\section{ABNORMALITIES OF THE SCROTUM.}

Abnormalities of the scrotum alone are rare, and are usually associated with congenital defects of the penis, such as are seen in cases of pseudohermaphroditism, in which the scrotum is cleft and resembles the labia majora of the female.

The scrotum is rudimentary or almost entirely absent in cases of unilateral and bilateral retention of the testes. In the unilateral defect, asymmetry of the scrotum is produced.

\section{INJURIES OF THE SCROTUM.}

Owing to the vascular structure of the scrotum, wounds and injuries which do not even break the skin may result in the formation of hematoma beneath the integument, and such slight injury as is inflicted by manipulation of the parts during operations upon them or neighboring structures is followed by more or less extensive ecchymosis. The structure of the inner part of the dartos, as has already been noted, favors the spreading of the fluids of œdema or extravasated blood widely through the subcutaneous scrotal tissue. Occasionally a hematoma is formed of such large size as to include the penis and to extend to the thighs and groin.

Treatment.-The hemorrhage of open wounds can be controlled by suture. In applying it, care should be taken to include the edges of the divided dartos and to avoid rolling in the skin, which is inclined to become inverted.

In the cases of subcutaneous hemorrhage, even compression applied with a bandage, elevation of the scrotum, and resting it upon an ice-bag placed upon the supporting shelf are often sufficient to arrest the bleeding. If not, incision may have to be practised, turning out of the clot, and ligating bleeding vessels. 
Fungus of the Scrotum.-This is not a frequent condition. It is produced by the prolapse of the tesicle through the scrotal wound, and the subsequent conversion of the exposed surface of the tunica albuginea into a fungus-looking mass by the growth upon it of exuberant granulation tissue, which, under these conditions, takes place. Adhesions are often formed, and bind the testis to the scrotum.

Treatment.-The treatment consists in reduction of the granulation tissue by application of the cautery, and allowing the surface to heal under mild antiseptic dressings.

\section{CUTANEOUS DISEASES OF THE SCROTUM.}

We do not regard these disorders as properly belonging to the sphere of this work, and shall consider them in the briefest possible manner consistent with the presentation of the most essential points connected with them.

Eczema.-Etiology.-Eczema of the scrotum is most commonly seen in persons of gouty or rheumatic diathesis, but may occur in anyone.

The condition is invited, augmented, and perpetuated by the friction and moisture of the parts and by uncleanliness. The skin of the scrotum is often excoriated, and is generally much thickened.

Symptoms.--The symptoms are those usual to the condition elsewhere in the body. Itching and burning, often intense, are present, and at times there may be a good deal of pain, arising from the excoriations and cracks of the surface. More or less exudation from the skin takes place.

Treatment.-One of the most essential features of the treatment is to prevent friction of the skin of the scrotum against that of the thighs, and to keep the surface dry. These ends are secured by wearing a well-fitting suspensory bandage with an inner layer of soft material interposed between it and the scrotum, and dusting the surface of the scrotum lightly with powders, among the best of which are stearate of zinc or zinc oxide, and starch powder and camphor mixed. An excellent salve, that may be of benefit in cases in which the application of powders does not suit the particular patient, is one made up of lanolin and lard in equal parts and carrying 2 per cent. of resorcin.

The itching may be controlled in most cases by the employment of weak solutions of carbolic acid, dabbed on the parts with a soft bit of gauze wet with it, or by a lotion recommended by White and Martin, which is as follows:

R-Picis liquidæ

Potassæ causticæ

Aqux.

$3 \mathbf{j}$

vol. $\mathrm{I}-\mathrm{r} 3$ 
Eczema Marginatum.-This condition is of parasitic nature, and, according to Keyes, is a combination of herpes tonsurans and intertrigo.

Its characteristic features are the peripheral advance and central healing. The active lesion has the form of a more or less crescentic line composed of "papules, vesicles, excoriations, and crusts" (Keyes). It always lies where the scrotum is in contact with the thighs.

Treatment.-Keyes recommends that a cooling lotion, such as dilute lead wash, be used at first in the cases in which there is a marked inflammatory element, and when this has subsided sufficiently, the employment of one or another of the parasiticide applications. Of these, he prefers corrosive sublimate in strength of $\mathrm{I}$ to 2000 . Iodine is also advised as a useful remedy. Whatever the measure adopted, it should be carried out for some time after an apparent cure has been effected. The applications must be kept continually in contact with the area involved in order to be efficacious.

Intertrigo.-Erythema intertrigo is most commonly seen in children who "wet themselves" and in elderly persons of uncleanly habits. Those who have gouty or rheumatic diathesis are more prone to it than others. The condition may be mistaken for a congenital syphilide when it occupies the buttocks.

Treatment.-The treatment consists in cleanliness and in the prevention of moisture and friction. In a few cases the employment of ointments and occasionally of astringent solutions of zinc or lead may be required. The most readily applied form of treatment is that of dusting upon the skin stearate of zinc or other similar powder. A suspensory bandage should be worn, and, if necessary, a layer of soft linen should be interposed between the chafing surfaces.

Pruritus.-Pruritus scroti is often observed in rheumatic, gouty, and diabetic subjects, and is commonly associated with pediculi pubis, although it may develop independently of these conditions. It often creates the impulse to masturbate.

Treatment.-The treatment should be chiefly systemic, directed to correcting rheumatism or gout and to diminishing the amount of sugar in the urine in cases in which diabetes is present. Prolonged hot bathing of the parts is useful, and Turkish and sulphur baths are often beneficial. Antipruritic remedies, such as thymol, tannic acid, and menthol, are of much use in relieving the disagreeable sensations.

Scabies.-Scabies is often carried to the genitals by the fingers. It is recognized by its usual characteristics and by its presence elsewhere, especially between the fingers. Scratching to relieve the itching produced by the parasite frequently leads to superficial ulcerations. In neglected cases eczema of the scrotum often results. 
Treatment.-A few applications of strong sulphur ointment will usually kill the parasite. Contact with the clothing that is infected by the parasite must be prevented, and such clothing should be disinfected.

Pediculi Pubis.-These parasites, which may be encountered upon any hairy part of the body, are especially apt to be found on the hair of the pubes and scrotum where their presence excites irritation, which may lead to eczema in neglected cases. They are usually found in abundance, and are visible to the naked eye. The lice infest the roots of the hairs, while their ova are firmly attached to the shafts of the hairs themselves, from which they are removed with difficulty. They may be present for some time without producing irritation, and are often discovered by accident.

Treatment.-While the infected part may sometimes be freed from the parasite by one or two applications of the ordinary mercurial ointment without cutting off the hair, the surest method is to shave the parts before applying the ointment. The mercurial treatment may be objected to because of its dirtiness. A few applications of a solution of $\mathrm{I}$ to 1000 corrosive sublimate, crude petroleum, or kerosene oil will destroy the parasite and its ova. Kerosene should be used cautiously, because it is irritating. An excellent method of treatment, when it is not desirable to remove the hair, is to keep the infested area wet with tincture of larkspur. One or two applications are, as a rule, sufficient to kill the parasite and destroy its ova. Mild soothing treatment should follow the application of any of the above-mentioned substances, because they are all irritating, and the skin of the part being delicate, inflammation is easily excited.

Steatoma or Sebaceous Cysts.-The glands of the scrotal skin are numerous, and often become enlarged by retained secretions. They are usually situated in the upper part of the scrotum, and may occasionally attain the size of a grape. (Plate XVI.)

Treatment.-The treatment consists simply in emptying the sac, either by squeezing out the contents or by incising the growth and scraping it out with a small curette. The tissue should be protected by a moist antiseptic dressing until healing has taken place.

Lupus.-Lupus is occasionally met with upon the scrotum, and should be distinguished from late syphilitic lesions, which it closely resembles. Lupus, as a rule, has a rigid border.

Treatment.-To be effective, excision of the affected part must be complete and thoroughly carried out. Good results are thus obtained.

Carcinoma of the Scrotum.-Cancer of the scrotum is usually of the epitheliomatous type, and has been described for years as being especially prone to develop in chimney-sweeps, and, in consequence is often known as "chimney-sweep's cancer." Its occurrence in those following this occu- 
pation is attributed to the irritation produced by the soot, but, inasmuch as the exposed parts of the body are more subject to irritation, and as those parts are not implicated as often as the scrotum, or "sweeps" with noticeable frequency as compared with other persons, this theory does not seem to be borne out by the facts.

The disease begins as a small epithelioid tubercle, which grows very slowly, and scabs form upon its surface. After ulceration takes place the growth advances more rapidly, and may extend over the whole scrotum, the penis, and perineum, and involve the testes and cords. In time the inguinal nodes are implicated, but they may not show any signs of enlargement until the growth has reached quite an advanced stage.

Treatment.-The only treatment that offers hope of cure is early and complete excision. If the inguinal glands are involved, they should be included in the parts excised. When the disease has extended to the testes, it is very doubtful if operation is warranted, except as a measure of relief.

\section{INFLAMMATIONS OF THE SCROTUM.}

Edema.--Besides the œdema of the scrotum, which occurs because of obstructed circulation in cases of cardiac, renal, and hepatic disease, it is also associated with acute and occasionally with chronic local or neighboring inflammations. It is constantly associated with acute epididymitis and orchitis of severe type, and is described as being seen in connection with chronic inflammation of the inguinal glands in some rare instances. It also occurs in connection with some cases of eczema of the scrotum.

Treatment.--The local treatment, when there is sufficient tension of the scrotal skin to demand it, should be the evacuation of the fluid of the œdema by multiple punctures made with a needle on either side of the median raphé of the scrotum, and not penetrating deeper than the integument. Manual pressure will then express the greater part of the fluid. After this has been accomplished the scrotum should be dressed with a wet mild antiseptic gauze dressing, and moderate pressure should be applied by a well-placed bandage. If there is but a slight degree of œdema, rest and elevation of the scrotum are ordinarily sufficient to overcome it.

Cellulitis and Abscess.-These forms of scrotal inflammation are usually due to urinary infiltration, but the condition known as acute septic phlegmon, in which there may be no urine in the tissues, and which is, nevertheless, usually mistaken for urinary extravasation, is one 


\section{PLATE XVI}

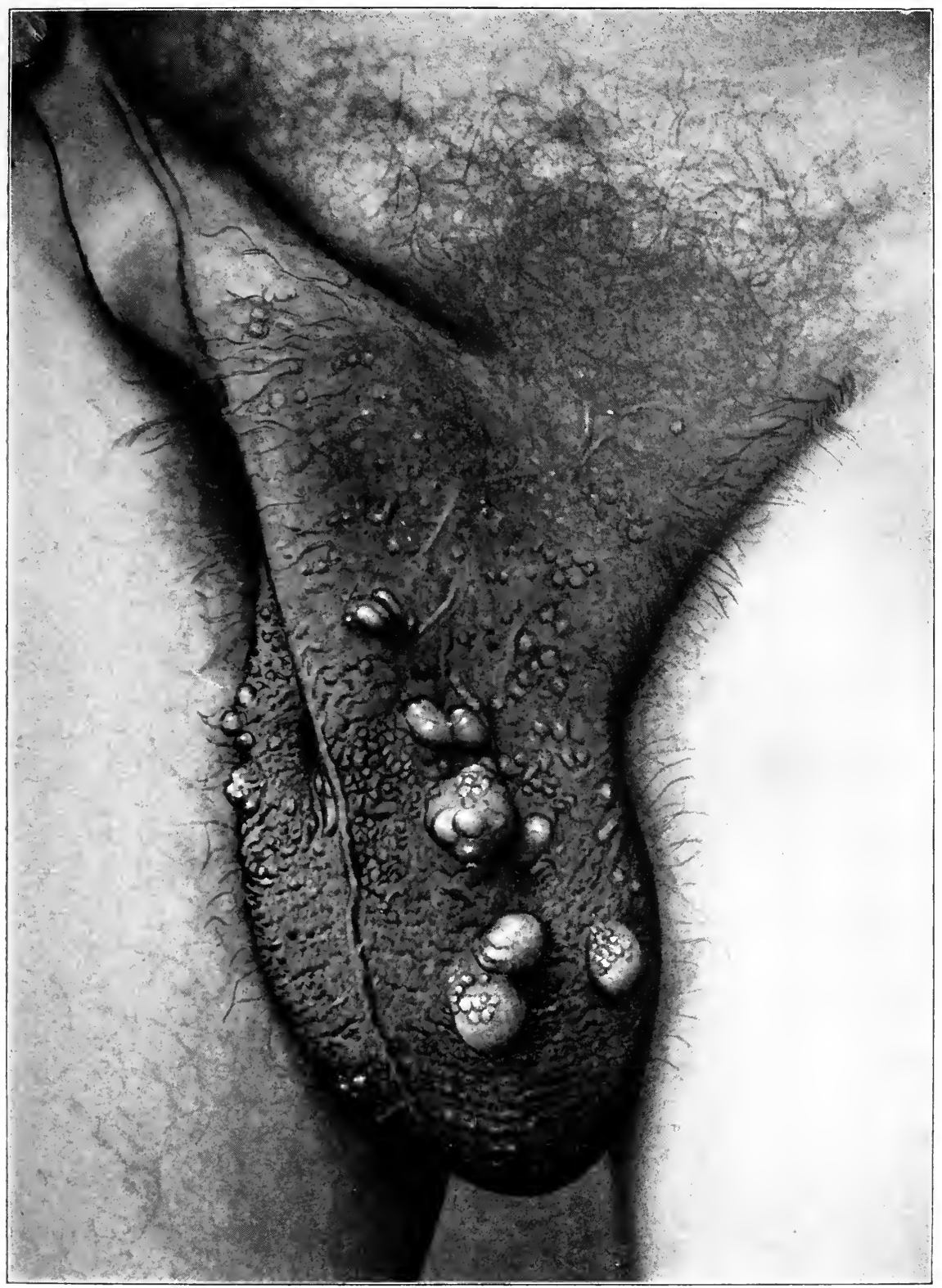

SEBACEOUS CYSTS (WENS) OF THE SCROTUM. (Taylor.) 

that is comparatively common and of much importance because of the virulence of the process. (It is discussed more in detail in the chapter on Stricture of the Urethra.)

External wounds in this part, as well as elsewhere, are liable to infection. There are no features of this condition in these cases that need special mention. The inflammatory conditions of the scrotum of this character are to be treated in accordance with the principles that govern them elsewhere in the body.

Emphysema.-Scrotal emphysema occurs in the course of general subcutaneous emphysema and locally from wounds in which gas has been generated by gas-producing organisms. It is also seen in connection with scrotal gangrene.

Treatment.--The gas should be evacuated by multiple superficial incisions and the wounds dressed with antiseptic poultices. When infection is due to aërogenous organisms, the part should be kept loosely covered with a dressing of hydrogen peroxide, one-half strength.

Elephantiasis of the Scrotum.-Elephantiasis scroti, or lymph scrotum, is rarely seen, except in tropical and subtropical countries. The sporadic cases, occasionally encountered in the colder climates, can usually be traced to a residence or a visit of the patient to the tropics.

Etiology.-Lymph scrotum results from obstruction of the lymphatics in the scrotal skin. It has been noted as occurring after extirpation of the inguinal nodes, but the etiological factor, in most cases, is the Filaria sanguinis hominis (Filaria Bancroftii, Filaria nocturna).

The life history of the filaria sanguinis hominis is extremely interesting. Briefly, it is this: The mosquito is the intermediate host; man the actual host. Female mosquitoes of the genus Culex ( $C$. fatigones) and, according to James (quoted by Manson), anopheles ( $A$. nigrorimus) draw the filarial embryos from an infected individual. The embryos undergo a metamorphosis, which, according to Manson, ${ }^{1}$ requires from sixteen to twenty days for completion. The parasite then, according to Low, ${ }^{2}$ enters the proboscis of the mosquito, and remains in that position until the insect feeds upon man, at which time the parasite enters the mediate host. It then finds its way into the lymphatic system, where it it remains and develops into the mature parent worm. The filarial embryos are to be found in the blood at certain periods, and at these times only. Upon the discovery of their presence in it, the diagnosis of the disease depends. Manson demonstrated that the filarial embryos are present in the peripheral circulation during the night. Their number reaches a maximum about midnight, when as many as 600 have been found in a single drop of blood, which, assuming that the parasites are equally distributed through the circulation, would indicate that there 
are from 40,000,000 to 50,000,000 in the blood current at that time. The number gradually decreases after midnight, and they usually disappear entirely by eight or nine in the morning. McKenzie ${ }^{3}$ has pointed out that if the patient sleeps by day and works by night, this habit of the filariæ changes correspondingly.

Pathology.-No pathological tissue changes are produced by the embryos, so far as is known; the parent worms, on the contrary, either directly or indirectly, obstruct the lymphatic circulation. While it is rare that the worms produce occlusion of the lymphatic channels, their presence results in secondary inflammatory changes, thickening their walls and narrowing their lumen. Thrombosis of the lymphatic channels causes an increased pressure in the occluded vessels, resulting in lymphatic varicosities and œdema. Great variation is shown in the manifestations, which correspond to the degree of engorgement of the lymphatics beyond the point of occlusion.

When lymph scrotum exists alone, which is seldom the case, the adult worm resides in the inguinal glands. When the parasite is in the thoracic duct, the scrotal œedema is but one of a number of manifestations of the disease. Others are as follows: (I) Dilated lymph vessels (lymphangiectasis). (2) Dilated lymph glands (varicose glands), which appear in the forms of scrotal œedema and elephantiasis. (3) Obstruction of cutaneous lymphatics. (4) Rupture of dilated lymph varices, which, according to the location of the point of rupture, will appear as chyluria, chylous ascites, or galactocele, or chylous diarrhœa. (5) Besides these, there may be the secondary conditions of pyogenic infection which have been engrafted upon the processes which characterize the filaria infection, viz., cellulitis, lymphangitis, and abscess.

Elephantiasis Scroti.-This condition results from obstruction of the lymphatics of the skin, and is usually accompanied by dilated lymph glands. Small varices may appear in the scrotal integument. Sometimes they rupture and allow an escape of lymph. Eczema of the integument is a frequent companion of the process, and cellulitis, abscess formation, and ulceration are often seen.

Symptoms.-Elephantiasis arabum usually begins as an erysipelatous inflammation of the scrotal integument, accompanied by a good deal of febrile disturbance. After the subsidence of the constitutional symptoms the lymph glands enlarge in the form of varices and the scrotal skin becomes brawny. Progressive thickening in the cutaneous and subcutaneous tissues, due to dilated lymphatics, enlarges the scrotum so that it may assume enormous proportions, enveloping and concealing the penis. Larrey mentions a case in which the tumor weighed 200 pounds, and others of great size are on record. 
Prophylaxis.-There seems to be no doubt that the filariasis is transmitted through the mosquito, wherefore any prophylaxis that is to be effective must insure against infection from this insect.

Changing the conditions which favor the breeding of the anopheles may succeed in banishing the disease from the locality which he infests. The culeces breed more readily in stagnant water, but may do so in any water. It becomes important, therefore, to make such collections of water inaccessible to these mosquitoes. Mosquito nets are another practical prophylactic measure. Individuals already infected with filaria are a menace to the communities in which they dwell, because of the opportunity offered by them to the mosquito to take the parasite of the disease from them and to transmit it to others.

So far as is known, the embryos are not harmful, and the treatment is therefore directed to the killing or removing the parent worm.

Medical Treatment.-Numerous drugs have been tried and recommended as being efficacious in curing the malady. They are one and all of but little value despite the claims now and then made for them.

Cathartics and diuretics are of value by reducing the tension in the lymphatics.

Operative Treatment.-To be anything more than palliative, the operation employed must remove the parent worm.

The thoracic duct, pelvic and abdominal lymphatics are inaccessible, and when the parent filariæ are in them, operation is unlikely to be of benefit. The number of cases in which surgical procedures have been employed is small. The success obtained by the writer in one case in which he operated, even though the parent worms were not found in the parts removed, has made him hopeful with regard to the possibility of cure offered by surgical intervention (Cunningham ${ }^{4}$ ).

\section{BiBLIOGRAPHY.}

I. Manson. Tropical Diseases, 1898 .

2. Low. British Medical Journal, I902, vol i, p. 196.

3. McKenzie. Lancet, August 27, I88 I, p. 398.

4. Cunningham. Annals of Surgery, October, 1906, p. 48 I. 


\section{CHA P T E R V I I.}

\section{THE VAS DEFERENS AND SPERMATIC CORD.}

\section{ANATOMY.}

The Spermatic Cords.-The spermatic cords are between $3 \frac{1}{2}$ and $4 \frac{1}{2}$ inches in length, the left a little longer than the right. They extend from the internal abdominal ring, where several structures converge to form them, along the inguinal canal to the globus minor of the epididymis. The cord is composed of the vas deferens, the remains of the funicular process (the habenula), the cremasteric muscle, the spermatic and cremasteric arteries, the artery of the vas deferens, the pampiniform plexus of veins, the genital branch of the genitocrural nerves, lymphatics, and sympathetic nerve fibers. (Plate XVII.) These structures are held together by areolar and connective tissue.

The Vas Deferens.- The vas deferens, the most important structure to the cord, is a continuation of the canal of the epididymis. It lies posterior to and a little to the inner side of the body of the cord, where it can be detected as a firm cord-like structure. At the internal abdominal ring the vas dips downward and inward and reaches the posterior surface of the bladder. It passes behind the ureter on the corresponding side, and here, becoming convoluted, runs parallel with it, and is separated from it by the seminal vesicle. (Plate XXI.) Just above the posterior border of the prostate the vas is joined by the duct of the seminal vesicle, forming a single canal-the ejaculatory duct. This canal and its fellow of the other side traverse the prostate and open into the prostatic urethra just below the end of and a little to either side of the verumontanum (Fig. I IO). The length of the ejaculatory ducts is about three-quarters of an inch. The vas is composed of a thin outer fibrous covering, beneath it a coat of longitudinal muscular fibers, an inner one of circular ones, and a lining membrane of columnar epithelium.

The Pampiniform Plexus of Veins.-This plexus is important because of the frequency with which its vessels become varicose, and in so doing give rise to the condition known as varicocele. The veins are arranged in two groups, one anterior and one posterior. The anterior set is by far the larger, and constitutes almost the whole of the plexus. The posterior group is small, and accompanies the vas. The vessels anastomose freely 
with one another; their valves are few and often defective. The veins of both groups finally unite into one vessel, the spermatic vein; that on the right side emptying into the inferior vena cava, and on the left, into the left renal vein.

The arteries are all of small caliber. The spermatic artery is derived from the aorta; the cremasteric from the epigastric; the artery of the vas from the superior vesical. The two former pursue a tortuous course through the cord; the latter is in direct relation with the vas.

FIG. IIO

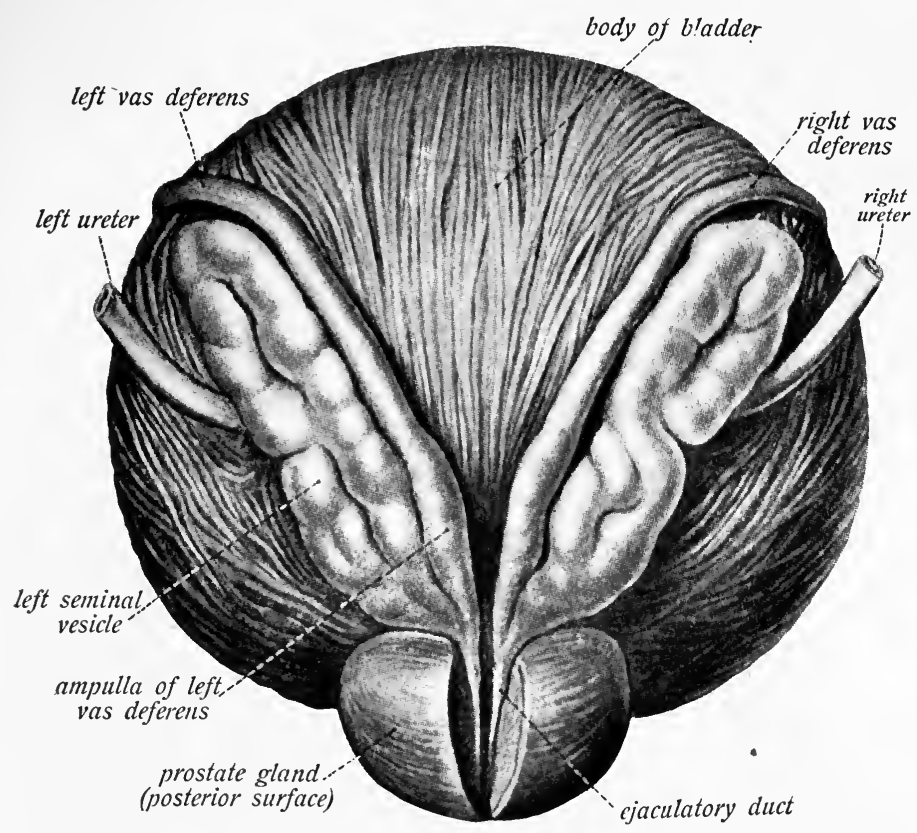

The urinary bladder with the seminal vesicles, the ampullæ of the vasa differentia and the prostate seen from behind and below. The prostate is partly divided longitudinally. (Sobotta.)

The Lymphatics.--The lymphatics, from four to eight in number, accompany the veins and drain into the nodes about the external iliac artery and the lower portion of the aorta.

The Nerves.-The sympathetic nerve filaments are derived partly from the spermatic and partly from the pelvic plexus. They accompany the artery of the vas deferens. The genito-crural nerve arises from the first and second lumbar nerves and supplies the cremasteric muscle with the genital branch. It is this nerve which is responsible for the cremasteric reflex, produced by stimulation of the crural branch, which supplies the anterior aspect of the thigh. 


\section{ABNORMALITIES.}

Anomalies of the vas deferens are extremely rare. The whole or part of the vas deferens may be absent. Curling mentions several such cases. The testicles, in some instances, have been normally placed in the scrotum when the vasa deferentia have been absent. The vasa may be fused together. The vas has been observed to open into the prostatic utricle and also into the ureters. These are early fœtal conditions which have persisted.

\section{INJURIES.}

Contusions.-Certain contusions may cause rupture of one or more veins of the cord and produce a hematoma. Swelling and ecchymosis are usually associated with the injury. The tumor formed by the hematoma has an elongated form and extends from the testicle below to the external abdominal ring above, and is confined to the cord. The bleeding is never severe, because of its being arrested spontaneously by the pressure exercised upon the collection of blood and the wounded bloodvessels by the fibrous sheath of the cord.

Treatment.-Rest in bed, elevation of the scrotum, and the application of cold by an ice-bag placed beneath the testicles. When the swelling begins to subside, the patient may be allowed to get up and go about, but the scrotum should still be supported by a well-fitting suspensory bandage.

Wounds.-Wounds of the cord are followed by profuse hemorrhage. If the vas is divided, its severed ends should be united. If the continuity of the vas is not reëstablished, the functional service of the testicle is lost. If the spermatic artery and the artery of the vas are divided by the incision, the testicle may become gangrenous; either artery alone may be cut without serious results necessarily occurring; if the nerve filaments supplying the organ are cut, the testis may atrophy.

Torsion of the Cord.- Torsion of the cord usually results from trauma or malposition of the testicle and causes gangrene of the organ, if the condition is not relieved. (See Chapter IV.)

\section{INFLAMMATION.}

Inflammation of the spermatic cord (funiculitis) is generally the result of an extension of gonorrhœal infection from the posterior urethra, and is ordinarily associated with epididymitis. It may also occur in connection with septic phlebitis, rheumatism, and trauma. 
Inflammation of the cord appears in both the acute and the chronic form. The former is usually seen in connection with a gonorrhœal infection, and the latter generally results from tuberculous infection.

The most important factor connected with acute inflammation of the cord is that it may extend to the peritoneum in the cases in which suppuration takes place, which occasionally occurs.

Treatment.-Incision and drainage of the pus should be carried out promptly in the cases in which suppuration occurs. If the process has extended to the peritoneum, the inguinal canal should be laid open and free exit for septic fluids should be made and maintained as long as may be required to insure the safety of the patient.

The vas occasionally becomes occluded from injury or acute inflammatory processes.

\section{TUMORS.}

Solid tumors of the spermatic cord are rare. Cystic tumors are comparatively common.

Solid Tumors.-Lipoma, fibroma, myoma, myxofibroma, sarcoma, and carcinoma have all been observed as primary tumors of the cord. Sarcoma and carcinoma of the cord occasionally produce metastases. Gumma rarely affects the cord.

Lipoma is the most common of the tumors of the cord. It has been asserted that it does not grow there as a primary tumor, and that it is secondary to the same condition in omental hernia; there seems to be no doubt, however, that it may and does originate in the cord itself, for it has been found there, independent of hernia. Lipoma may be situated in any part of the cord.

Histologically, such tumors may be simple lipoma or combined with myxoma and fibroma. There is a tendency for the tumor to send off finger-like projections, which penetrate between the structures of the cord. Lipomata are usually of small size, but occasionally are the reverse. Wilms has reported one case in which the tumor weighed twenty pounds. Nove Josserand ${ }^{1}$ another, in which the tumor weighed six and a half kilos, and mentions two others weighing fifteen.

Symptoms and Diagnosis.-The subjective symptoms are slight. The growth advances in a slow and painless manner, and is usually first noticed as a small swelling of firm consistency. It often has a more or less well-defined lobulated form; later it is soft, and when palpated may simulate closely the sense of fluctuation of an encapsulated collection of fluid. The swelling is not translucent. There is no impulse on coughing. 
Lipoma may easily be confused with omental hernia, and it is usually impossible to make a positive diagnosis without incision.

Treatment.-The treatment is wholly surgical, and consists in removing the tumor. If the growth is small, it can usually be separated from the cord by blunt dissection; if it is large; castration may be necessitated.

\section{VARICOCELE.}

Definition.-Varicocele is a dilatation of the veins of the spermatic cord-the pampiniform plexus.

It is a very common condition, and affects the left side only in the great majority of cases, although it is seen in the veins of the right cord in a small proportion of them.

Etiology.-The following features are conspicuous in connection with varicocele: (I) It is a condition which belongs to young adults. Elderly persons who have well-marked varicosities of the lower extremities are seldom subjects of varicocele. (3) It frequently disappears after marriage and under the influence of well-regulated sexual hygiene. It is almost always on the left side only.

The inferences which may be drawn from the first three of these facts are these: that congestion of the vessels of the parts produced by lack of well-regulated sexual habits and by frequently repeated sexual stimulation which is not gratified has something to do with the production of varicocele, and that the conditions which are responsible for the production of venous stasis and resulting varicose state of the veins of the lower extremity have nothing to do with the production of varicocele.

The fourth factor mentioned is the most interesting of them all. The occurrence of the condition on the left side only in so great a proportion of cases can only be explained upon the ground of its being dependent upon some constantly present causal elements. There is but one class of elements that present such constancy, namely, anatomical structures and their relations to the circulation of the parts under consideration.

The anatomical factors which are believed to be explanatory of the frequency of left-sided as compared with right-sided varicocele are as follows: (I) The column of blood which the left veins are required to support is longer than that in the veins of the right side, owing to two facts: the lower position of the left testicle than the right one, and the higher point at which the left spermatic vein enters the greater venous trunks than that at which the right one does-that is to say, the point of entrance of the left spermatic vein into the left renal vein is more distant from the left testicle than the point of entrance of the right 
spermatic vein into the vena cava is distant from the right testicle.

The entrance of the vein of the left side is at right angles to the renal vein, while that of the right vein is at an acute angle with the vena cava; hence the blood current in the former of these two large venous trunks exercises less suction upon the blood of the left spermatic vein than does that of the latter upon the blood of the right spermatic vein. (3) There is no valve at the junction of the left spermatic with the left renal vein. (4) The natural curve of the sigmoid flexure to the left subjects the vessels of the left spermatic cord to greater liability to be pressed upon by the bowel when it is distended, as it is, for example, in chronic constipation, than is the case with the vessels of the right cord.

These are constant factors which may one or all be influential in the production of varicocele on the left side. Others have been suggested, but their causal relation is too uncertain to give them an importance in the consideration of the etiology of the condition.

Symptomatically, varicocele may appear as the result of any condition causing intra-abdominal venous stasis. Especially is this likely to be the case from pressure exercised directly upon the left renal vein, as it may be in connection with some renal tumors, or tumors in the vicinity of the kidney. Guyon long since called attention to the occurrence of varicocele as a symptom of some malignant tumors of the kidney, and, more recently, Hochenegg ${ }^{2}$ has also recorded a case in which a varicocele developed in an elderly man in connection with a renal tumor.

Pathology.- The veins are elongated, dilated, and tortuous; their walls often show fatty degeneration and are thickened, in consequence of which they lose their elastic and contractile properties. The valves are defective, and phleboliths sometimes form in the lumen of the vessels.

The scrotum is usually relaxed, and the veins of its integument are apt to be enlarged.

Symptoms.-The symptoms of varicocele are usually very slight, and frequently it gives rise to none at all. In some cases there is more or less dull, dragging pain or ache. The patients with varicocele are among the most frequent victims of quacks, who excite their fears and reap a harvest from their credulity. Hypochrondriasis often originates in this way.

The external evidences of varicocele are relaxed scrotum and the peculiar character of the enlarged veins, which gives to the fingers the sense of handling a bag of earth-worms.

Diagnosis.-The diagnosis is simple, and rests upon the presence of the local conditions just named and upon the fact that the veins empty when the patient lies down and refill when he stands up.

Treatment.- The treatment in most cases consists in cold douches to the scrotum night and morning and in wearing a suspensory bandage. 
A regulation, which we do not hesitate to characterize as utterly absurd, exists in the army, which compels the recruiting officer to reject applicants for it if they have varicocele. It sometimes becomes desirable, on this account, or because the condition is causing pain or progressive atrophy of the testicle, which it does in connection with some large varicoceles, to cure it. This can be done by surgical procedures of one or another kind. (For the description of the technique of these operations, see the chapter on Technique of Operations on the Testicle, Vas, etc.)

The results of operations in cases of varicocele are excellent, and their application is attended by but little or no danger. Those which are of value are:

I. Subcutaneous ligation of the dilated veins.

2. Their ligation by the open method-incision of the scrotum-and the resection of a small part of the vessels at the same time.

3. Partial resection of the scrotum.

Our personal preference in the cases demanding operation is for the open method of ligation combined with resection of a portion of the veins, and approximating the severed ends, in order to raise the testicle and thus give it support. We have had uniformly good results from the employment of this method of treatment.

We have said that the operation carried with it very little danger; we should have added, if properly done, by which we mean if it be performed with rigid asepsis, and if the arterial blood supply is not cut off by the serious operative error of including all the arteries of the cord in the ligature. That this has been done by some surgeons is known. Probably it happens more often than is supposed, that one of the arterial branches is taken up in the ligature; the artery of the vas is, however, so intimately connected with that structure that it very rarely is accidentally ligated in performing the operation, and, unless it has been tied, the testicle will receive enough blood through it to escape becoming gangrenous.

\section{BibLIOGRAPHY.}

I. Nove Josserand. Lyon Méd., I897, vol. lxxxiv, p. 237.

2. Hochenegg. Zeitsch. f. klin. Med., July, I907. 


\section{H A P T E R V I I I.}

\section{THE TESTICLES.}

\section{ANATOMY OF THE TESTICLES.}

THE testicles are two ovoid glandular organs, the function of which is to produce spermatozoa. They are supported in the scrotum by the spermatic cords, each in its own chamber. (Plate XVII.)

The left testicle hangs somewhat lower than the right, owing to the greater length of its cord. In cases in which the abdominal viscera have been transposed it has been noted, in some instances, that the right testicle is lower than the left.

Each of the testes is made up of two distinct parts, the testicle proper and the epididymis, the latter being an adjunct of the former and concerned in the transmission but not in the production of the testicular product.

The Coverings of the Testis.-These are the tunica vaginalis and the tunica albuginea.

The Tunica Vaginalis.-This tunic is derived from the peritoneum, and envelops the testicle during its descent into the scrotum. When the migration of the organ is completed, the portion of this peritoneal reflection which covers the spermatic cord becomes obliterated near its upper part, converting the lower portion of it into a closed sac, in which the testicle lies. (See under the heading Descent of the Testis, and Fig. I I 15 .)

The tunica vaginalis has two layers, the first one of which envelops the testis and epididymis, attaching them to each other. From the posterior surface of the testis this layer is reflected onto the scrotum, where it becomes the parietal layer and the inner lining of the scrotum (see Figs. III and II2).

The surface of the tunica vaginalis is smooth and lined with endothelial cells. The space between the visceral and parietal portions of the tunic is known as the cavity of the tunica vaginalis.

The Tunica Albuginea.-This structure is the fibrous covering of the testicle proper. It is composed of white fibrous tissue and is reflected into the interior of the testis from its posterior border; this reflection, as it passes into the organ, is termed the mediastinum testis. It forms an incomplete vertical septum, which separates in the glandular part of the organ into partitions, which form a series of compartments, in which the 


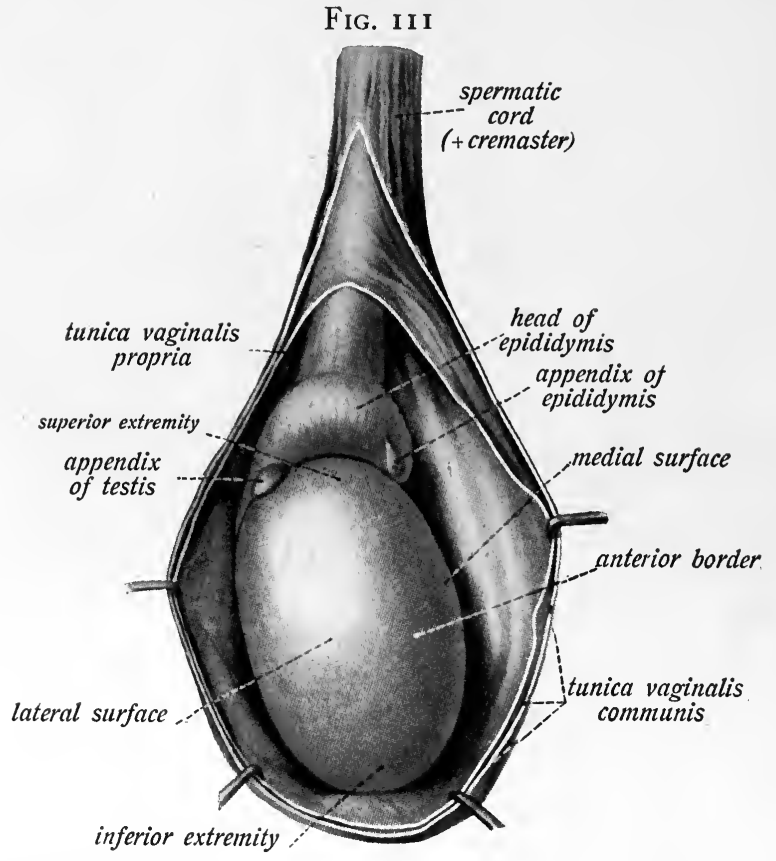

The testis and epididymis, with their investing membranes, seen from in front. (Sobotta.)

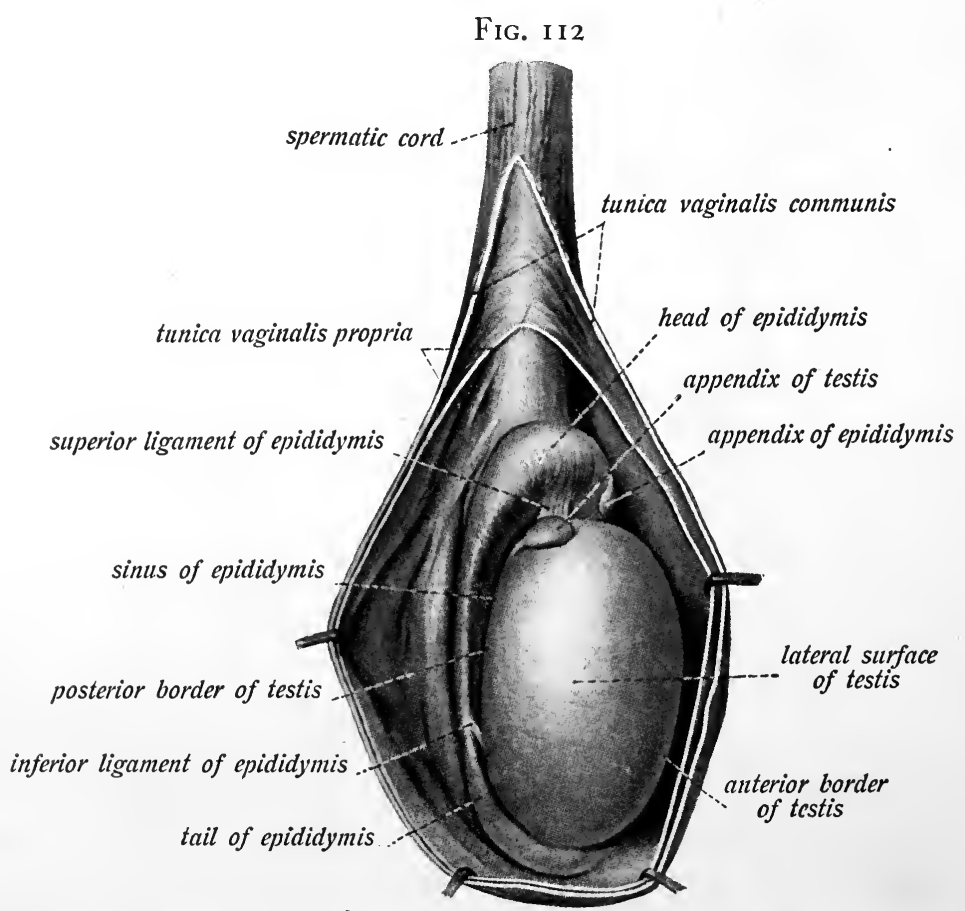

The testis and epididymis, with their investing membranes, seen from the lateral surface. (Sobotta.) 


\section{PLATE XVII}

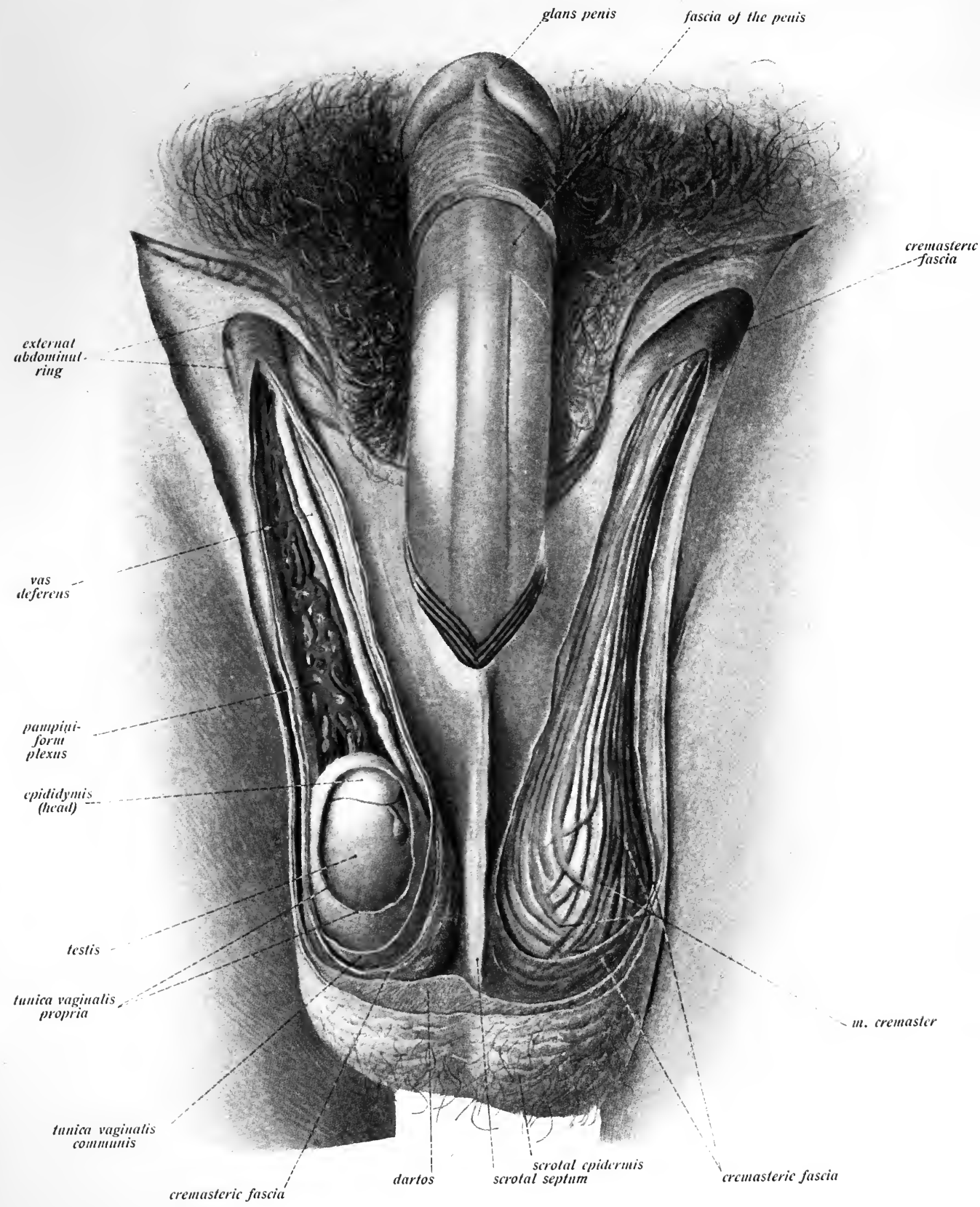

THE SCROTUM AND SPERMATIC CORD SEEN FROM IN FRONT. (Sobotta.)

Also shows the cremaster muscle, the coverings of the testes, the septum dividing them, and the accelerator urinæ muscle. 

separate lobules of the gland substance lie. The bloodvessels and the efferent vessels of the testis pass in and out of the organ, respectively, along the line of entrance of the mediastinum into the organ (Figs. I I3 and $\mathrm{II}_{4}$ ).

FIG. II3

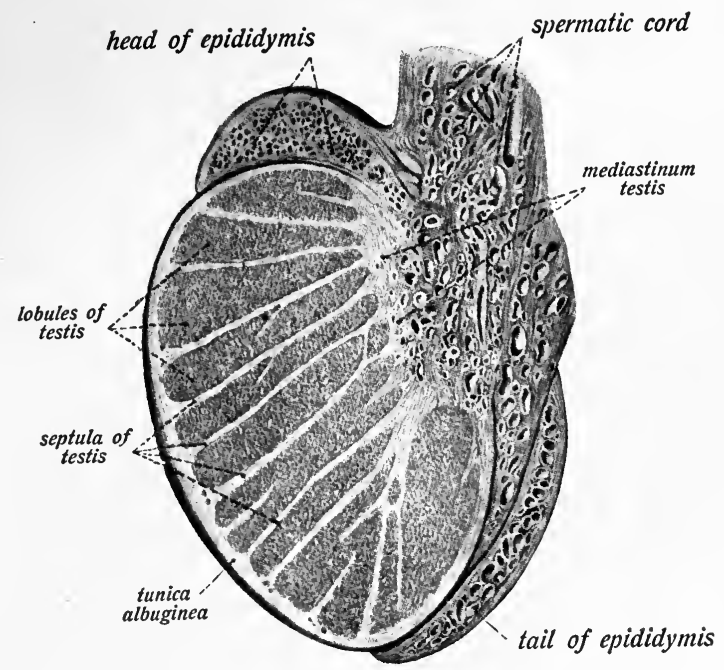

Longitudinal section of the testis and epididymis. (Sobotta.)

FIG. II 4

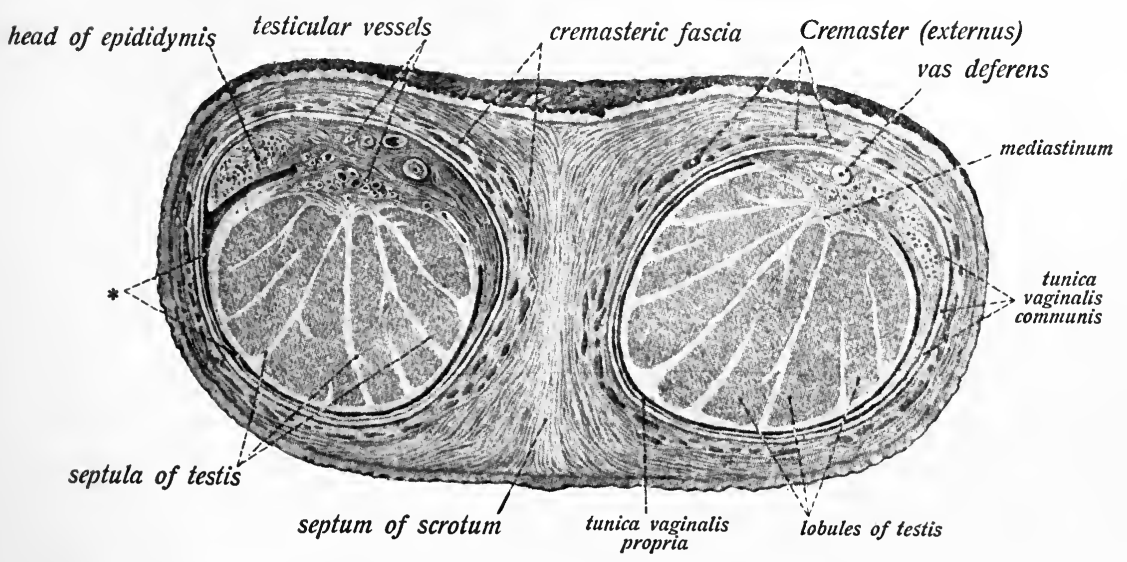

Transverse section of the scrotum and the two testes. Cavity of the tunica vaginalis propria. (Sobotta.)

It is to the pressure exerted upon the swollen testicle when it is inflamed by the dense unyielding fibrous tunica albuginea that the pain in cases of orchitis is due.

Tunica Vasculosa.-A third tunic is sometimes separately described under the above name. It consists of an inner lining of the tunica vol. I-I4 
albuginea, and is made up of delicate bloodvessels supported by a fine connective-tissue framework. This structure passes into the testicle and accompanies the different divisions of the tunica albuginea within the gland.

The Glandular Structure.-The gland tissue of the testicle is divided into a number of individual lobules, each of which occupies a separate compartment of conical shape; the bases of these chambers are directed toward the surface of the organ, their apices toward the mediastinum testis (see Fig. II3).

Each lobule contains from two to four tubuli seminiferi. These are the spermatozoa-producing structure. The length of each tubule, when uncoiled, is stated by Monod to be about sixteen feet. The lobules are convoluted, except at the apices, from which points they become straight, and enter the mediastinum, where they unite to form a body of twenty or thirty ducts - the rete testis. In the mediastinum they again become combined and form another set of larger ducts, from fifteen to twenty in number-the vasa efferentia. These penetrate the tunica albuginea and go to form the head or globus major of the epididymis (see Fig. II3).

The Epididymis.-The epididymis occupies a position on the top and posterior border of the testicles. It begins where the vasa efferentia pass through the tunica albuginea, and where, as has been said, the tubes become larger and form its head, or globus major. The parts of the vasa efferentia between the rete and the globus major are called the coni vasculosi, and empty into a single convoluted canal, which is the canal of the epididymis, forming the body of the organ. The body and remainder of the epididymis are connected with the testicle by the tunica vaginalis. The lower extremity of the epididymis - the tail or globus minor-is formed by the convolutions of the canal of the epididymis. From this point the canal becomes the vas deferens. The canal of the epididymis is lined with ciliated epithelium.

About the head of the epididymis there are several small protuberances, which are the remains of the Wolffian body and Müller's duct. They are known as the hydatids of Morgagni (see Figs. II I and II2).

The Blood Supply.-The blood supply is derived from the spermatic artery-a branch of the aorta-which joins the other structures of the spermatic cord at the internal ring. The lymphatics form a plexus around the seminiferous canals and the canal of the epididymis. They accompany the spermatic vessels and terminate in the lumbar nodesnot in the inguinal nodes.

Nerves.-The nerves are derived from the renal, aortic, and hypogastric plexuses of the sympathetic. 


\section{PHYSIOLOGY OF THE TESTICLE.}

The testicles remain in an undeveloped condition until the age of puberty, when a metamorphosis takes place in the mother cells of the spermatozoa. These cells undergo active division, and from them the matured spermatozoa are formed. There are other cells which disintegrate and form a nutritive fluid, with which the spermatozoa are mixed. The spermatozoa, collecting in the seminal tubules, are forced outward by the constant formation of others behind them. Entering the vasa efferentia and the canal of the epididymis, they are carried onward by the smooth muscle which surrounds these structures and by the ciliated epithelium which lines them. The glands in the ampulla of Henle contribute their secretion to the semen, which then enters the vas deferens, the ampulla at the further end of which is the chief storehouse for the spermatozoa. The ciliated epithelium here ceases, but the passage of semen is aided by the contraction of the muscles of its walls.

The seminal vesicles may be regarded as extensions or diverticula of the vasa deferentia, the chief if not the only function of which is to contribute the greater part of the fluid medium of the semen. The old belief that the seminal vesicles are storehouses for the spermatozoa is no longer maintained.

The semen is expelled into the urethra through the ejaculatory ducts, which are continuations of the vasa deferentia through the substance of the prostate.

Internal Secretion.- It is believed that the testicles contribute physiological elements beneficial to the metabolic processes of the body, but their secretion is not essential to life as are those of the thyroid and pancreas. It is supposed that the internal secretion of the testicle influences the general process of metabolism up to the time of puberty, but it is questionable if the influence persists subsequently.

Influence of Absence or Destruction of the Testicles.-Fisher ${ }^{1}$ describes the case of an anorchid, who died at the age of forty-five. His voice was of high pitch, and he always sang as a soprano. His manner was decidedly effeminate. He had no beard, and had never exhibited amorous propensities or desire for female society. After death, a careful dissection revealed no evidence of the testicular tissue. The cerebellum was the size of that of a female child. We are informed that the eunuchs of the Eastern harems, castrated before puberty, lose the sexual desire, while those who are castrated later often retain it, as well as their potency. 


\section{DESCENT OF THE TESTICLE.}

The epididymis and the testicle proper are separately developed in the fœtus. The exact nature of the embryonic tissue from which the testicle is formed is uncertain. It is situated in front of the Wolffian body, yet

FIG. II 5
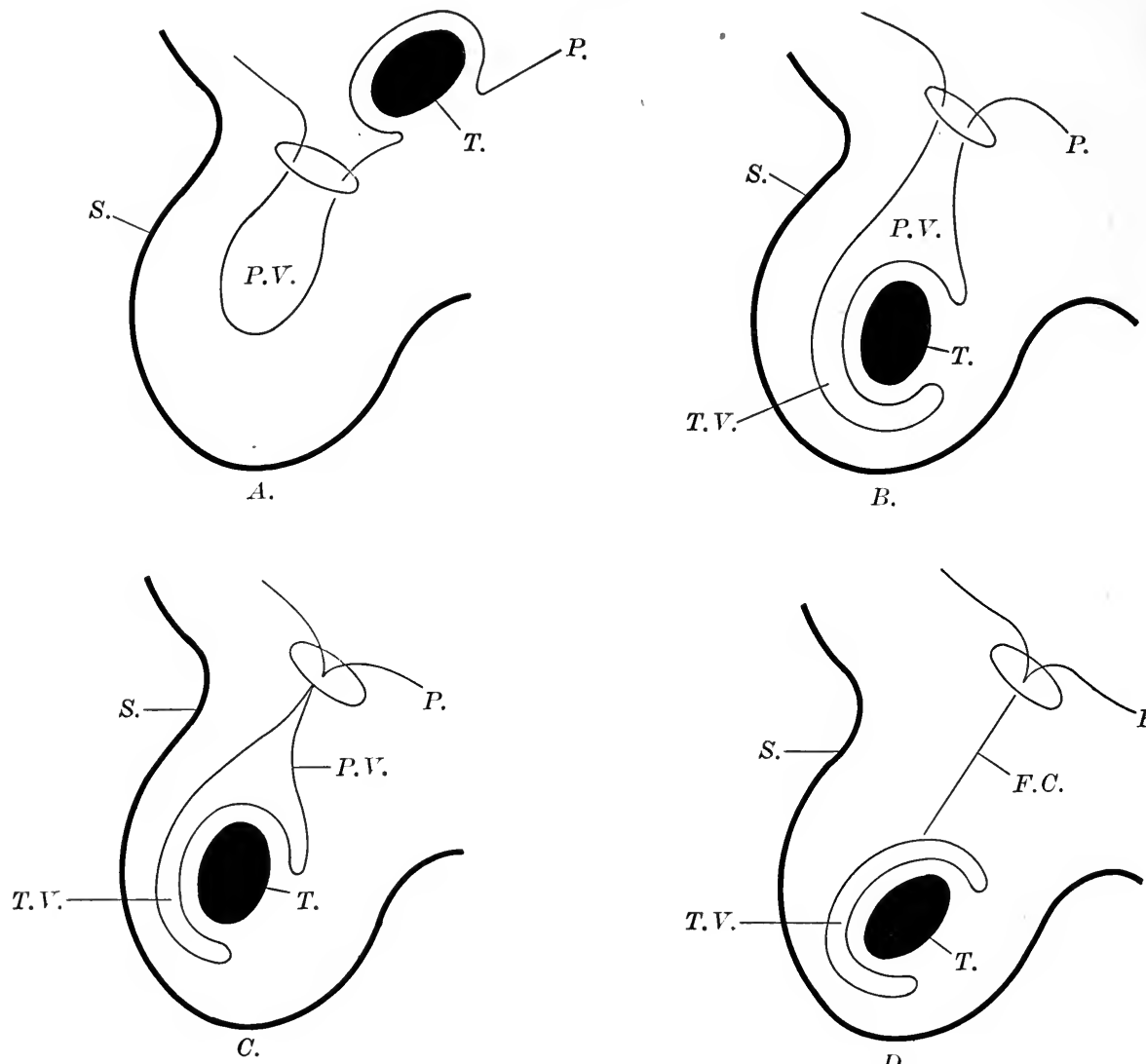

P. Peritoneum.

Processus vaginalis.

T. Testicle.

S. Scrotum.

T.V. Tunica vaginalis.

F.C. Fibrous cord or thread.

Inguinal Canal.

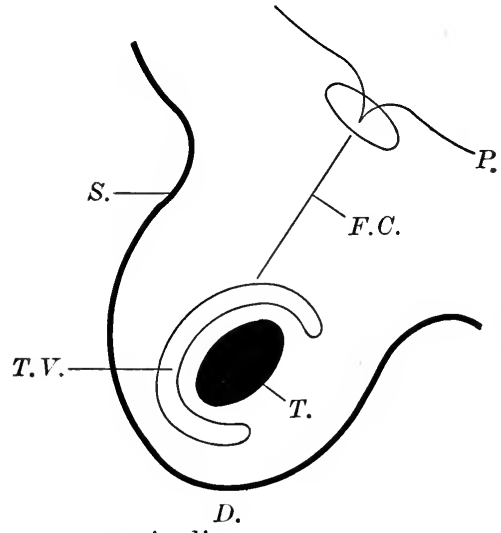

Diagram of the descent of the testicle.

apparently is separate from it. The epididymis is developed from the lower part of the Wolffian body.

The testes are at first placed at the back part of the abdominal cavity, behind the peritoneum, in front of and a little below the kidneys. At about the third month of fœtal life there is developed a slender fibrous 
band extending from the inguinal ring to the testicle and epididymis, to which it is attached. This is the gubernaculum testis.

The testis becomes invested by the peritoneum in a manner similar to that in which it envelops the colon, thus forming a testicular mesentery, or mesorchium, as it is called, which also covers in the gubernaculum. The gubernaculum develops into a cord of unstriped and striped muscular fibers, and, about the sixth month, extends to the bottom of the scrotum. The testicle also gradually descends during this time. A pouch of the peritoneum investing it is pushed onward by the organ in its descent, and, passing through the inguinal canal, finally reaches the bottom of the scrotum. By the eighth month the testis itself has arrived at the same position.

During the last month of fœetal life the upper part of the processus vaginalis begins to be obliterated. When completed, the communication with the peritoneal cavity is closed, leaving the testicle invaginated in its peritoneal sac, the tunica vaginalis. The fibrous cord formed by this obliterating process is known as the habenula (Fig. I I $5, A, B, C, D$ ).

There is some difference of opinion as to whether the gubernar' serves simply as a guide during the descent of the testicle, $r$ actually exerts a pulling force. Probably it acts in both $\mathrm{w}$. -

Keyes states that in from Io to 20 per cent. of all children the testicles are still in the abdomen at the time of birth, and that in most of the cases the testicle descends during the next few weeks.

No importance is attributed to the retention of the testicle until two years have passed, when, if it is still retained, the condition will probably be permanent unless relieved by surgical measures.

\section{ABNORMALITIES OF THE TESTICLE.}

The abnormalities of the testicle are the result of faulty development and incompleteness in the process of migration.

Monod and Terrillon have classified the abnormalities as follows:

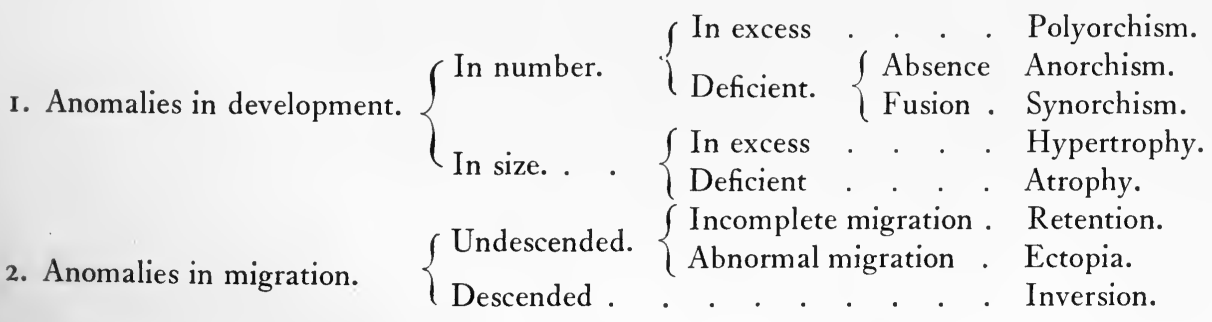

Polyorchism.-This condition is rare. Cases have been reported by Lane, ${ }^{2}$ Blasius, Haller. ${ }^{3}$ There appears to have been an hereditary 
tendency in Haller's case. Other instances reported have not been well proved.

Anorchism.-The complete absence of the testes is authentically recorded in two cases only. Cryptorchism is often mistaken for it. That total absence does sometimes occur, however, is shown by the cases reported by Fisher and Cunningham. ${ }^{4}$ In the latter's, the subject was a deformed child, in whom no evidence of testicles could be found upon careful dissection and microscopic examination of all suspicious tissue. (See under Illustrative Cases, at end of chapter on Stricture of he Urethra.)

Monorchism.-Monorchism, or absence of one testicle, is more often met with than is the condition of total absence of the organs.

Jacobson states that the following developmental defects may exist in association with the absence of one testicle:

I. Absence of the epididymis and of a part of the vas.

2. Exceptionally, entire absence of the seminal duct.

3. The testis may be present, and the vesicle, epididymis, and vas absent.

In Cunningham's case there was no evidence of the presence of the seminal duct.

Synorchism.-Fusion of the testicles has been recorded by Baillie, Ephemendes, Schurig, and Jacobson. The latter mentions Cruveilhier's and Lockwood's cases of intra-abdominal fusion of the testes-one in an adult and one in a fotus.

Atrophy of the Testis.-Atrophy of congenital origin occurs. It is almost always seen in cases of retained or ectopic testes. Sometimes the testes are of small size, even though naturally placed, but they are likely to develop under the influence of physiological activity.

Undescended Testis (Cryptorchism).--One or both of the testes in this condition are retained within the abdomen. It is termed monorchism when one of the organs is retained.

Frequency.-Marshall examined ro,80o English army recruits, and found 12 cases among them (I bilateral, 5 right-sided, and 6 left-sided). A proportion of $I$ in 900 .

Rennes found 6 cases among 3600 examined for the French army. None of these were bilateral. Odiorne and Simmons ${ }^{5}$ report 77 cases of undescended testis, 15 of which were cryptorchids.

In cryptorchids, the testes lie in the lumbar region near their original points of departure, or near the internal abdominal ring. Sometimes they are provided with a mesorchium, in which case the organs are freely movable. Occasionally the testis is lodged behind the pubic bone. 
The testicle may be retained in the inguinal canal or at the rings. When in the canal, it is exposed to pressure and injury, and, if inflamed, the condition is more serious than when the organ is in its natural position, owing to the unyielding canal in which it lies.

The descent of the vas and the epididymis into the scrotum, the testis being retained, has been recorded.

Aberrant migration of the testis is called ectopy. Here the organ may be found beneath the skin of the abdomen, on the front of the thigh, or in the perineum.

Influence of Retention upon the Function of the Organ.-The undescended testis almost always shows defective development. Griffith maintains that there has never been an authenticated example of spermatogenesis in an undescended testis. This extreme view is not shared by others.

Three different views have been held regarding the impairment of function of such testicles.

I. The retained testicle is imperfectly formed from the beginning, and its abnormality is a factor in its non-descent (Hunter).

2. The organ, although undescended, is normal until the age of puberty, and then fails to develop (Curling).

3. The testicle, although undescended, becomes a perfect organ at puberty, but then, owing to its faulty position, soon undergoes retrograde processes, resulting in partial or complete atrophy (Monod and Arthaud).

Griffiths, in experiments performed on dogs, has shown that, if normally descended testes be returned to the abdomen, they become soft and small, and never show active spermatogenesis.

Still another view, which seems to be true in a certain number of cases, has been supported by Bellingham, Smith, Monod, Arthaud, and others, who state that, occasionally, in undescended testicles, the function of spermatogenesis is established for a time, but that it persists for only a few years and is then lost. It is an undoubted fact that all double cryptorchids who have been reported to be the fathers of children have been very young men, and their children have been born within the first few years after the establishment of puberty. It is said by some observers that the function is never active after the age of puberty, but this does not seem to be true.

Prognosis of Undescended Testicle.-If the testicles have not descended at the end of two years, they are very unlikely to do so spontaneously later.

Complications and Conditions Incident to Undescended Testicle.-Acute inflammation may result from pressure upon the organ, or from extension of morbid processes from the urethra. 
The liability to injury of testes retained in the inguinal canal has been spoken of. If the testis is retained within the abdomen, and becomes inflamed, the symptoms to which it gives rise may simulate those of peritonitis. One case in which peritonitis actually did occur as the result of this condition has been recorded.

Attacks of inflammation are likely to result in a still further atrophy of the organ. The atrophy of the retained organ does not exempt it from the invasion of gonorrhœal infection. Murphy ${ }^{6}$ has recorded such a case, in which he demonstrated the presence of gonococci in the epididymis.

Hernia.-This is the most common complication of the retained testis. Eccles, in 48,000 hernia cases, found 854 imperfectly descended testes. Comer says that there is a hernial sac to be found in 70 per cent. of cases of retained testis. Inguinal hernia is more apt to become strangulated when in connection with an undescended testis. Sometimes the testis appears to act as an obstacle to the return of the intestine to the abdomen.

Malignant Disease of the Undescended Testis.-It is generally believed that the undescended testis is more liable to become the seat of malignant disease than the normal one. The disease, when it does occur, is invariably fatal and death takes place within a year after the appearance of the symptoms. Sarcoma is the most common of the tumors met with. It may attain a great size.

Treatment.-Pads or trusses, which are sometimes recommended, should never, in our opinion, be employed for the purpose of preventing the testicle from slipping into the ring or abdomen when it has been once brought outside of them.

The most favorable results from operations are obtained when they are done during childhood and prior to puberty.

If the testis is allowed to remain in an abnormal position until puberty, it is likely not to undergo the developmental changes incident to that period, and will probably remain functionless.

The operations that are performed for the relief of the condition are:

I. Orchidectomy.

2. Orchidopexy.

3. Replacement within the abdomen.

(For operative technique, see chapter on Technique of Operations on the Testes.)

InvERsion.-The organ may be inverted so that it hangs upside down. When in this position it is apt to lie high up in the scrotum and in the front of it. This fact should be remembered when operating upon hydrocele. 


\section{INJURIES OF THE TESTICLES.}

Dislocation (Luxation).- - The testicles are sometimes forced from their normal position as the result of injuries. Bruns ${ }^{i}$ reports the case of a man who was run over while lying on his back, and whose testicle was dislocated to a point over the pubes above the root of the penis. He mentions instances in which the testicle was dislocated into the inguinal canal, and one in which it was forced under the skin of the thigh. The importance attaching to the condition is the liability to atrophy of the dislocated organ, produced by direct injury to it, to its blood supply, or from pressure.

Treatment.-Provided the testicle cannot be replaced by manipulation, it should be cut down upon and returned to its normal position. If severely injured, castration may have to be performed. (For technique of the operations, see chapter on the Technique of Operations on the Testicles.)

Contusion.-Because of their protected position and great freedom of movement, contusions, as well as other injuries of the testes, are rare. They are usually produced by falling astride of some solid object, or by kicks or blows.

Ecchymosis of the scrotum is very easily produced by slight injury. On this account, a fighter's claim of a "foul blow" could not be substantiated unless ecchymosis was present to confirm it.

Hemorrhage into the body of the testicle is occasionally seen. The condition is illustrated in Fig. I 6 , which is from the case of a patient who received a kick from a horse upon the part.

Kocher records two deaths resulting from shock produced by blows upon the testicle.

Pain is always severe and is accompanied by a feeling of faintness and nausea, sometimes by convulsions.

Treatment.-If the injury is severe, the patient should be kept at rest in bed, the scrotum should be supported by a bandage and a light ice-bag should be placed beneath it.

Suppuration, which occasionally results, should be treated by incision and drainage, and may demand castration.

Wounds.-Incised, punctured, or gunshot wounds of the testis are occasionally met with.

If the tunica albuginea is incised and the substance of the organ protrudes through the opening in it, the wound should be carefully sutured.

Punctured wounds, if made, as they usually are, with a clean trocar, in tapping a hydrocele, rarely give trouble. 
Even in cases in which injury to the organ is extensive, it will rarely be necessary to sacrifice it.

Treatment.-The wound should be carefully cleaned and the protruding testicular tissue reduced if possible. If this cannot be accomplished, the tissue should be snipped off with scissors; but under no circumstances should it be pulled upon, as the tubules of the testicle will be drawn out, and may be irreparably injured. After reduction or removal of the hernia, the cut edges of the tunica albuginea should be closed over it by fine catgut sutures; the scrotal wound should also be closed and the scrotum elevated.

FIG. II6

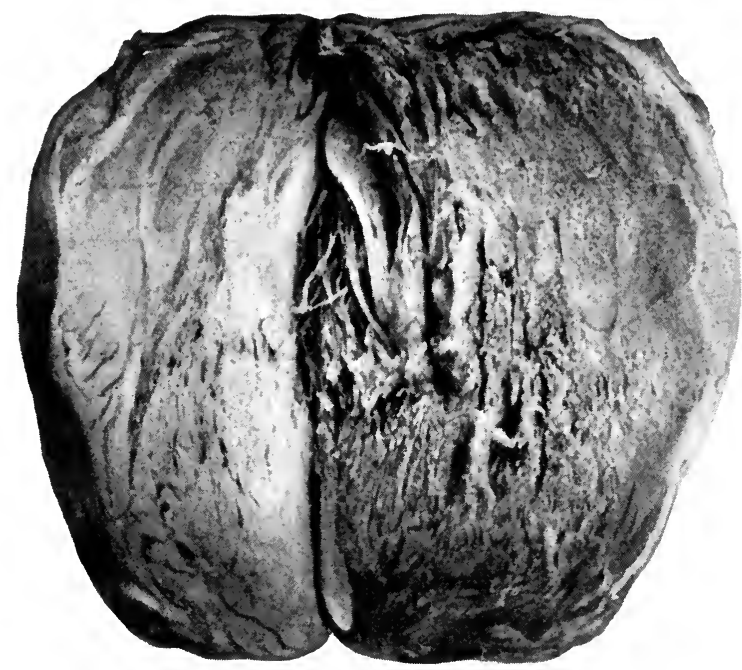

Hemorrhage within the testicle. The centre of the organ is occupied by partially organized blood clots. The tunica albuginea is much thickened, and there is little remaining testicular tissue.

Gangrene of the Testicle (due to torsion of the spermatic cord).Scudder ${ }^{8}$ reported 32 cases of gangrene of the testicle due to torsion of the cord. Since then, a few more have been published. In the majority of cases the right side is the one involved, and the condition is produced without any definite traumatism other than a quick wrench or strain. Malposition of the organ seems to be a predisposing factor, as in 10 of Scudder's cases the affected gland was retained in the inguinal canal, and in 5 under the pubes. In 2 other cases with which we are familiar there was incomplete descent of the organ. Rigby and Howard, ${ }^{9}$ in reporting 9 cases of torsion of the cord which they had observed, say that the predisposing factor of the condition is a congenital abnormality of the attachment of the testis to the spermatic cord. 
Pathology.-The cord is twisted upon itself in either an upward or outward direction; gangrene, which results, begins below the point of strangulation (Fig. II7). The testicle is odematous, congested, and presents areas of hemorrhage and necrosis; vaginal hydrocele or hematocele is usually associated with the condition. Even after the torsion has been relieved-in the cases in which gangrene does not occur-atrophic changes may follow in the gland tissue.

Symptoms. - The symptoms simulate those of strangulated hernia, from which the condition can be differentiated by the rather milder degree of the systemic manifestations and by the absence of intestinal obstruction. The inguinal region and the scrotum become enlarged, painful, and very tender. Nausea and vomiting are early symptoms; chills and fever develop as the changes in the gland become more pronounced.

Diagnosis. - The diagnosis rests chiefly upon the demonstration of intestinal patency, the exquisite tenderness of the strangulated cord and testicle, and the absence of an expansile impulse in the swelling, on coughing. In certain cases, in which these features cannot be demonstrated, immediate operation is the only means of determining the actual condition.

Treatment.-While, in some instances, it has been possible to untwist the cord without an open incision, this procedure can hardly be recommended as a desirable method of treatment, since, even when threatened gangrene is averted, torsion has recurred in the great majority of cases

Gangrene of the testicle due to torsion of the cord. The testicle and epididymis were gangrenous from a point just above the epididymis. Testicles and epididymis considerably enlarged from œdema. There were areas of hemorrhage and beginning necrosis on the surface and interior of the organs. There was associated hydrocele of the cord $(A)$ dependent upon the strangulation of the cord. (Scudder.)

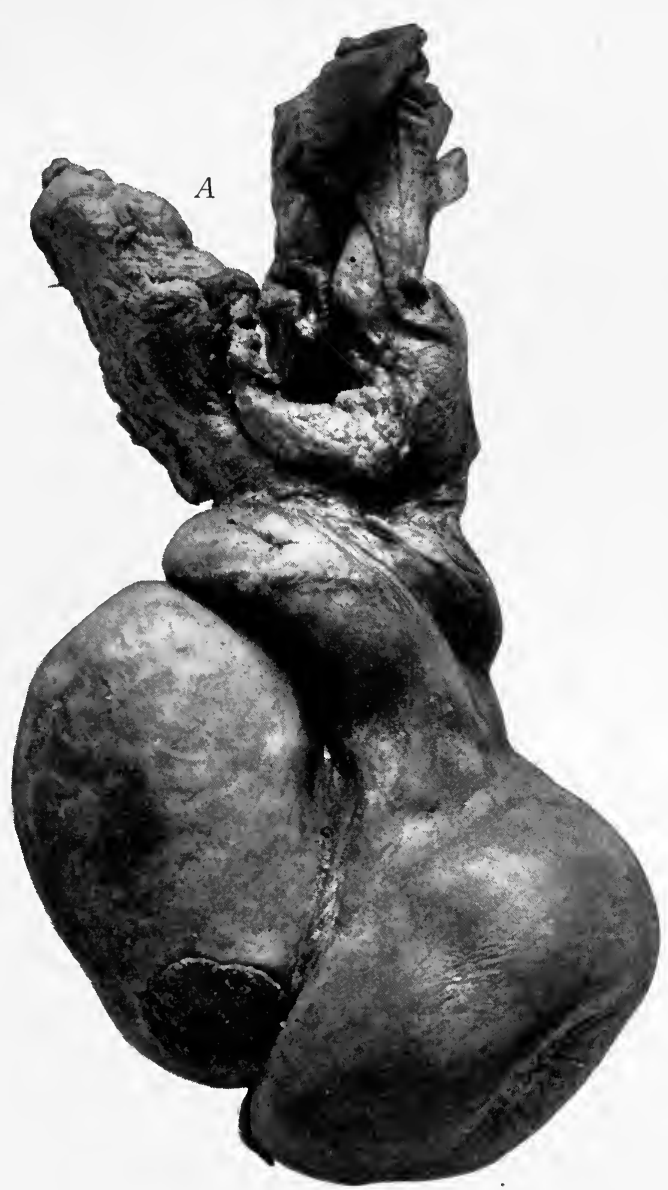


so treated, and atrophic changes are almost sure to result. Castration should be resorted to, removing the cord a short distance above the point of strangulation, unless the vitality of the organ is but little impaired and some form of fixation promises permanent relief, which is rarely the case.

\section{NEURALGIA OF THE TESTICLE.}

The term neuralgia of the testicle is applied to pain which occurs independently of inflammation or other lesion of the organ. It is rare and is usually secondary to some lesion elsewhere which causes pressure upon the nerves of the testicle.

Etiology.-The etiological factors are quite obscure. The condition usually occurs in neurotic individuals. It has been ascribed to sexual excess and masturbation. The existence and passage of a renal calculus usually cause severe neuralgic pains in the testicle, and should always be thought of in this connection. Neuralgia is also associated with varicocele in some instances, but this pain does not properly come under the heading of a pure neuralgia.

Symptoms.-The pain is usually unilateral, and radiates along the lumbar and sacral nerves. It is sometimes paroxysmal and sharp in character, and extends down the spermatic cord to the testicle; again, it may be a heavy, dragging sensation in the testicle itself. When in connection with the passage of a renal calculus it is severe.

Treatment.-Unless some definite cause be found, such as renal calculus, varicocele, or sexual excesses, the treatment is often without success. Sexual hygiene should be insisted upon. In some cases the mental impression produced by the passage of a cold sound, or the deep instillation of a weak solution of argyrol, is beneficial. If the pain is of a dull, dragging character, the patient should be directed to douche the genitals with cold water for five minutes night and morning. Alternating hot and cold douches have been recommended. A suspensory bandage should be worn.

\section{INFLAMMATIONS OF THE TESTICLE AND EPIDIDYMIS.}

Inflammations of the testicle attack either or both of the component parts of the organ, and usually remain confined to the part first affected. Inflammation of the epididymis (epididymitis) is far more common than inflammation of the secreting portion of the organ (orchitis), and is the commonest of testicular affections. The limitation of the inflammation to the part of the testicle involved may depend upon the blood supply, 
which is separate for each part of the organ. Inflammation beginning in the epididymis does occasionally invade the testicle proper, but the reverse is extremely rare. Following injury, both portions of the organ, epididymis and testis, usually enlarge simultaneously. In epididymitis, the adjacent tunica vaginalis becomes inflamed, and hydrocele may result; but in true orchitis, peri-orchitis does not occur, the extension of the process being prevented by the dense tunica albuginea.

Epididymitis.-Epididymitis is the most frequent of the testicular diseases and is important because of the liability to the occurrence of permanent secondary changes by which the functional power of the testicle is lost. Simultaneous bilateral epididymitis is extremely rare. Fournier ${ }^{10}$ states that he has never seen a case of double simultaneous epididymitis. Keyes has observed it twice, and we have met with it once. In the usual unilateral form, the healthy organ may be the seat of a subsequent infection or injury, and, as a consequence, complete sterility may result. Casper states that in his experience the majority of childless marriages, in which the husband is at fault, are due to bilateral epididymitis, and this is doubtless true.

Epididymitis occurs at any age, but is most commonly associated with early manhood, as the result of urethral inflammation. It is the most common complication of acute gonorrhœa; one attack predisposes to another, and incomplete or long-delayed resolution is frequent.

Etiology.-Inflammation of the epididymis depends upon four general factors, the relative frequency of which is as follows: (I) Inflammation of the posterior urethra (gonorrhoa, stricture, instrumentation, operation, local violence, prolonged sexual excitement). (2) Trauma (direct violence). (3) Infections from the blood current (tuberculosis, syphilis, gout, rheumatism). (4) Idiopathic.

I. Inflammation of the posterior urethra is responsible for the vast majority of infections of the epididymis. The specific organism is usually the gonococcus. While epididymitis generally occurs as a result of acute gonorrhœa, it may develop at any subsequent time as a postgonorrhœal complication, the infecting organisms still remaining in the mucous membrane, and becoming active and virulent because of lowered bodily resistance, or being produced by instrumentation in the deep urethra.

Non-virulent microörganisms, which normally inhabit the urethra, are known to exert pathogenic influences in the presence of urethral infection, and are often carried to the posterior urethra by the passage of instruments.

Following internal and external urethrotomy, divulsion, perineal and suprapubic prostatectomy, the posterior urethra often becomes the seat of 
inflammation, especially if there has been a previous gonorrhœa, and epididymitis occasionally results as a consequence. It is generally believed that posterior urethritis may be caused by prolonged sexual intercourse. We have seen a few cases in which this has occurred when there has been no previous urethral infection and in the absence of gonococci in the urethral secretion. The course of such cases is usually atypical. It is variously estimated that epididymitis occurs as a complication of gonorrhœa in from 20 per cent. to 30 per cent. of the cases. Instances have been recorded in which epididymitis has preceded the gonorrhœal discharge (Fourneaux, Jordan, Sturgis, Stansbury, Castleman, Vidal). We share the belief of Keyes with regard to these cases, viz., that they are not examples of fresh gonorrhœal infection, but of relapsing gonorrhœa, proceeding from re-infection in the prostate or deep urethra, and kindled into activity by excessive sexual intercourse or some other exciting cause. We have recently seen such a case, in which the original starting point was an infection of eight years before.

Epididymitis, as a gonorrhœal complication, seldom appears before the second week, and may occur at any time in the subsequent course of the attack, or as a postgonorrhœal sequela, without apparent exciting cause. The reason for its unilateral tendency is not clear.

2. Trauma, produced by direct violence, may cause epididymitis, and there is often an accompanying orchitis (epididymoörchitis). An epididymis previously the site of an acute gonorrhœal process has a predisposition to become inflamed on being injured, which would not otherwise exist.

3. Infections from the blood current are chiefly dependent upon a chronic disease elsewhere. Tuberculosis is by far the most common of these. (See chapter on Genito-urinary Tuberculosis.) Syphilis of the epididymis is extremely rare; the testicle is much more frequently attacked by it. Epididymitis is stated to occur as one phenomenon of gout and rheumatism, but it is a very rare manifestation.

4. Idiopathic Epididymitis.-Certain cases must be classified as idiopathic, since no etiological factors can be discovered to which the disease can be attributed.

Pathology.-Usually the acute process is most active in one part of the epididymis, most frequently in the tail or globus minor; next in the head, or globus major. The process may, however, involve the whole appendage. Malassez and Terrillon state that during the acute process the seminiferous tubules are swollen, their walls oedematous and infiltrated, and the epithelium is deprived of its cilia. The tubules may contain a mixture of pus and semen, and the connective tissue about them is œdematous and infiltrated. The inflammatory exudate gradually under- 
goes resolution and abscess formation is rare. The adjacent parts may become involved in the process, the testicle may be, but rarely is, invaded, and, according to Jacobson, the tunica vaginalis becomes inflamed, and results in acute hydrocele in one-third of the cases. Horttung, Columbia, Witte, Pizzini, and Kazwowski have demonstrated the gonococcus in the epididymis in the course of the acute stage.

In the reparative process connective tissue is deposited, and appears in the form of hard nodules at the site of the acute lesion. The condition is often permanent, and may form an enduring obstacle, which prevents the passage of the spermatozoa.

Symptoms (LocaL).-The local manifestations are pain, tenderness, swelling, heat, redness, and œdema of the skin of the scrotum, and, in some cases, the evidences of hydrocele.

The pain may be either in the groin or in the epididymis at first. It is more commonly located in the latter. When in the groin at the outset, it usually precedes by a few days its appearance in the testicle.

When allowed to hang, the increased weight of the inflamed organ produces a dragging pain along the course of the cord. Locomotion is distressing. Pain in the loin is not an infrequent accompaniment and may be due to traction upon the cord. Von Leyden has assumed it to be due to extension of the inflammation along the cord to the spermatic and renal nerve plexuses.

If the swollen cord becomes strangulated in the external ring, pain becomes intense. When the cord is involved extensively, the testicle may be drawn up to the external ring and the suffering is much augmented.

It must be remembered that the inflammation has reached the epididymis via the vas deferens, from the posterior urethra, and that the process invades the seminal vesicles in a large number of cases, according to Brönnum ${ }^{11}$ in about 80 per cent., a calculation based on his having found gonococci in the secretions of the seminal vesicles in that proportion of the patients examined by him.

In the instances in which this extension along the vas and into the abdominal cavity takes place, peritonitis may result. In this case, the temperature is very high, and the pain in the groin and lumbar region severe; eructations, nausea, hiccough, and vomiting are the symptoms characterizing the occurrence of peritonitis. All of these, however, may occur when there is no peritonitis present.

The course of the process in the epididymis is rapid. Swelling advances quickly, and soon becomes so great as to make it impossible to distinguish the epididymis from the testis itself. The scrotal skin becomes odematous and red, and an acute hydrocele is not infrequently formed. Following the rapid swelling of the next few days, the process remains 
stationary for about four or five more. The inflammation then gradually decreases, and its acute stage has usually terminated by the third week after the onset.

An accompanying inflammation of the tunica vaginalis, producing acute hydrocele, varies according to the severity of the inflammatory process. In some cases the fluid is poured out rapidly, and, as the swelling increases, pain becomes more pronounced, and may be so intense as to require to be relieved by tapping. The fluid may be entirely absorbed soon after the inflammation in the epididymis has become quiescent. The inflamed surfaces sometimes adhere to each other and obliterate the sac of the tunica vaginalis, or produce adhesions within it. Occasionally the fluid remains after the epididymitis has subsided. When the acuteness of the process has ceased, and the patient feels strong enough to be up and about, there may persist a dragging pain in the testicle during locomotion, which is sometimes unrelieved by suspension of the organ.

The gonorrhœal or gleety urethral discharge, when present, diminishes with the onset of the epididymitis, and may cease entirely, but usually returns as the inflammation in the epididymis subsides. Occasionally, however, the urethral discharge does not reappear.

Constitutional.- The constitutional symptoms in acute epididymitis vary with the virulence of the infection. There is the usual depression associated with pain, and the patient feels generally miserable. The temperature shows septic remissions, it is sometimes high, and disappears before the pain and swelling do.

Relapses.-Recurrences of pain in the epididymis are common, and an attempt should be made to keep the patient in the dorsal position until the acuteness of the symptoms has entirely abated. When the relapses do occur, they are milder than the first infection. Relapsing infections sometimes result in suppuration (Kocher, Finger, Albert).

Diagnosis. - The diagnosis of acute epididymitis, in cases in which there is a urethral discharge or a posterior urethritis from any cause, is simple. Palpation, although entirely unnecessary because of the typical picture presented, and to be avoided because of the severe pain it causes, will reveal that the epididymis is much harder than normal, and slightly uneven or nodular on its surface. The convex outline of the testicle may or may not be distinguishable. The only question in the diagnosis of the acute condition is whether or not the inflammation is in the epididymis or testicle. In the latter case, the general symptoms are more marked.

In chronic conditions, palpation is not so painful, and the site of the lesion can be more accurately determined.

Prognosis.-The prognosis regarding life is good. Fatal cases have, however, been recorded by Velpeau, Kocher, Finger, and Keyes. These 
were instances of peritonitis or purulent infection, and must be considered exceedingly rare. Suppuration is more usual when epididymitis is associated with prostatic hypertrophy. Sexual desire and potency are not diminished, but sterility of the affected organ may result. The seminal discharge is not visibly changed in quantity or general character, yet the spermatozoa may be absent. Benzla ${ }^{12}$ investigated the number of offspring begotten by the soldiers of the German army who had had gonorrhœa, and found that 10.5 per cent. of those who had had the disease without epididymitis were childless, while of those who had unilateral epididymitis, 23.4 per cent. were childless, and of those with bilateral epididymitis, 4I.7 per cent. were childless. Liegeois found that after unilateral epididymitis the spermatozoa were less in number, and of 28 cases of double epididymitis he found complete absence in 2I. Keyes states that patients who have recurrent attacks of epididymitis are less likely to be sterile than those who have but a single attack.

The location of the inflammation in the tail or body of the epididymis, where there is but one canal, is more likely to lead to sterility than if the process be situated in the head of the organ, where the canals are more numerous. Burnett ${ }^{13}$ says that, in many cases, sterility is averted by gradual absorption of the exudate which remains after the inflammation, but this resolution may require a long time.

Tuberculosis and neuralgia are prone to attack the indurated areas which remain in the epididymis after it has been the seat of inflammation.

Treatment (Prophylactic).- - It is an open question how much prophylaxis there may be in such measures as keeping the testicles suspended during an attack of gonorrhœa, although it is a measure that is usually strongly advised.

Such prophylaxis as is obviously to be obtained by the avoidance of urethral instrumentation during the acute infection, and of the employment of irritating injections, should be secured, and the same is true of exercising care in the use of urethral instruments at a later period, in order not to risk re-infection of the deep urethra. If any uneasiness or pain develops in the groin or testicle during the course of a urethritis, or after urethral instrumentation, the patient should be put to bed and the testicles well elevated.

The Treatment of Acute Epididymitis.- The following are the more essential measures to be observed: If the attack is a comparatively mild one, absolute rest in bed, elevation of the testes, and the application of ice may curtail it. If it is more severe in character, poultices, frequently renewed, are more efficacious in lessening pain. Morphine may be required to overcome the pain in the more serious forms of the condition.

An old-fashioned but often very useful remedy is the so-called tobacco VOL. I-I 5 
poultice, which is made by mixing a couple of teaspoonfuls of finely cut tobacco with a double handful of linseed meal. Multiple light applications of the actual cautery over the most painful part of the swelling may lessen the pain. The internal administration of salol, which is advised by some surgeons, has not been successful in our hands.

At the outset of the disease, guaiacol, locally applied, is highly commended by Keyes ${ }^{14}$ who says that a 50 per cent. glycerin solution of the drug, applied once or twice, may abort the attack. In this strength it may be used twice daily for two or three days. Care should be taken to avoid too frequent applications, inasmuch as the remedy is exceedingly irritating to the skin.

FIG. II 8

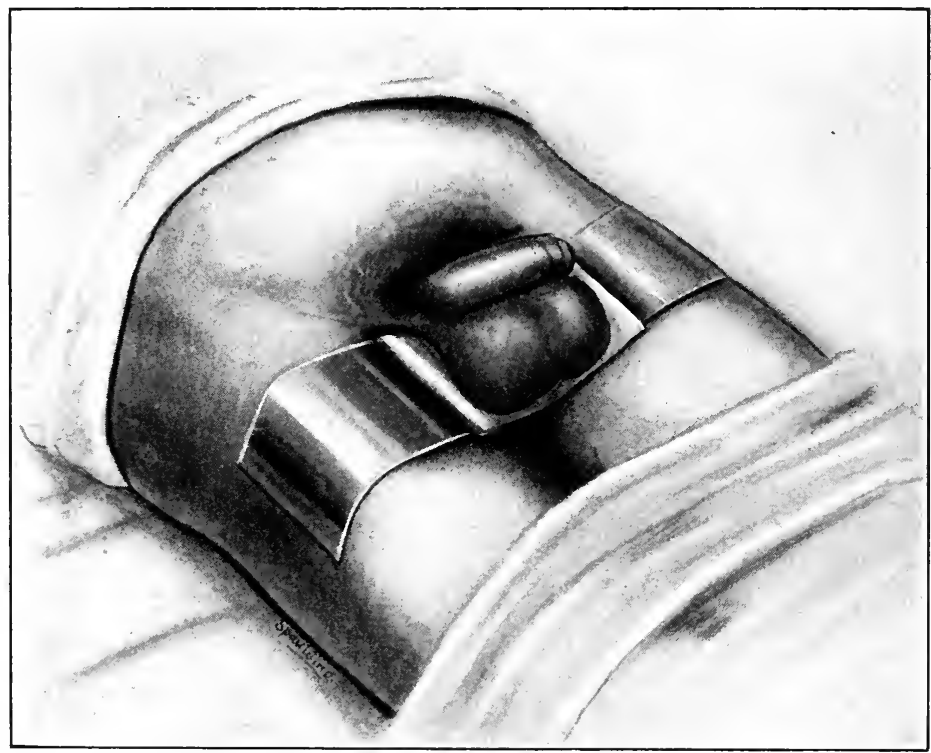

Manner of supporting the testicles. (Greene and Brooks.)

When an acute hydrocele is associated with the condition, it should be tapped. The pain may be considerably mitigated by this measure.

When suppuration takes place, the abscess should be promptly evacuated.

The testicles may be supported either by means of a large, soft handkerchief, or upon a well-adapted shelf (Fig. I i8).

In the later stages of epididymitis, when the acute inflammation has disappeared and only the hard nodules remain, their resolution may be promoted by the application of oleate of mercury.

Strapping and Suspension.- Strapping with a rubber bandage when the inflammatory process begins to decline, hastens the absorption 
of the inflammatory exudate, and thereby tends to avert the occlusion of the channels of the epididymis. The pressure exerted by the bandage causes an increased absorption through the superficial vessels, and at this stage the inflammatory œdema in the connective tissue about the parenchyma of the epididymis and the fluid in the canal itself is easily dislodged and more readily absorbed than at a later time. The rubber bandage suggested by Martin and recommended strongly by Keyes and others, is superior to the older method of strapping with adhesive plaster.

The Martin bandage consists of a piece of thin sheet rubber about eight inches long and two inches wide, more or less, according to the size of the organ. Chetwood has made the bandage more serviceable by adding a piece of adhesive plaster about one-quarter of an inch wide and four inches long to the end of it (Fig. I I9). The inflamed side of the scrotum is encircled with the bandage, the other testicle being pushed out of the way. It is important to apply gentle pressure above the greatest point of swelling or the bandage will slip off. The adhesive plaster strip prevents the bandage from unrolling (Fig. I20). It should not be applied so as to cause pain, and should be tightened every day in correspondence with the diminution of the swelling. Another method of applying compression is by the Zeissl-Langlebert suspensory bandage. It is strongly recommended by Casper, who has added to it several small hooks and eyes, in order to gain greater elevation. The bandage is FIG. II9

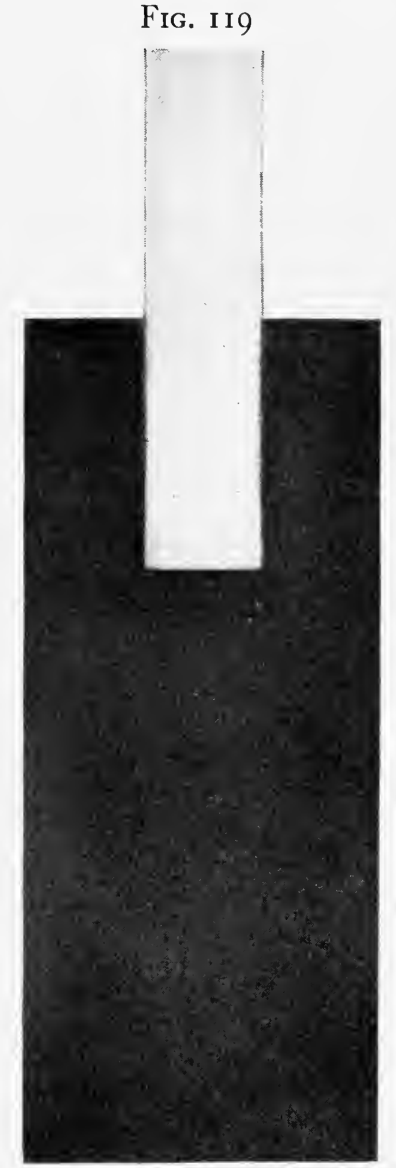

Bandage for strapping the testicle. padded with cotton, and the scrotum, having been anointed with lanolin, is placed within it and drawn upward toward the abdomen. More cotton should be added as the swelling declines. The pain is said to be, in large measure, overcome by this contrivance. The Horand-Langlebert suspensory bandage is also a satisfactory arrangement, and is especially recommended for those who will not consent to remain in bed as long as they should. The bandage is applied as follows: both testicles are enveloped in a thick layer of cotton-wool; a piece of gutta-percha tissue or oiled silk is placed outside of this, and the suspensory bandage over the 
whole. The dressing is adjusted so that the testicles lie on the front of the pubes, without tension on the cord, and a firm compression is exerted until the swelling has somewhat subsided, when the bandage should be renewed and adjusted. This degree of elevation and pressure is not possible during the early acute and very painful stages of the malady.

FIG. 120

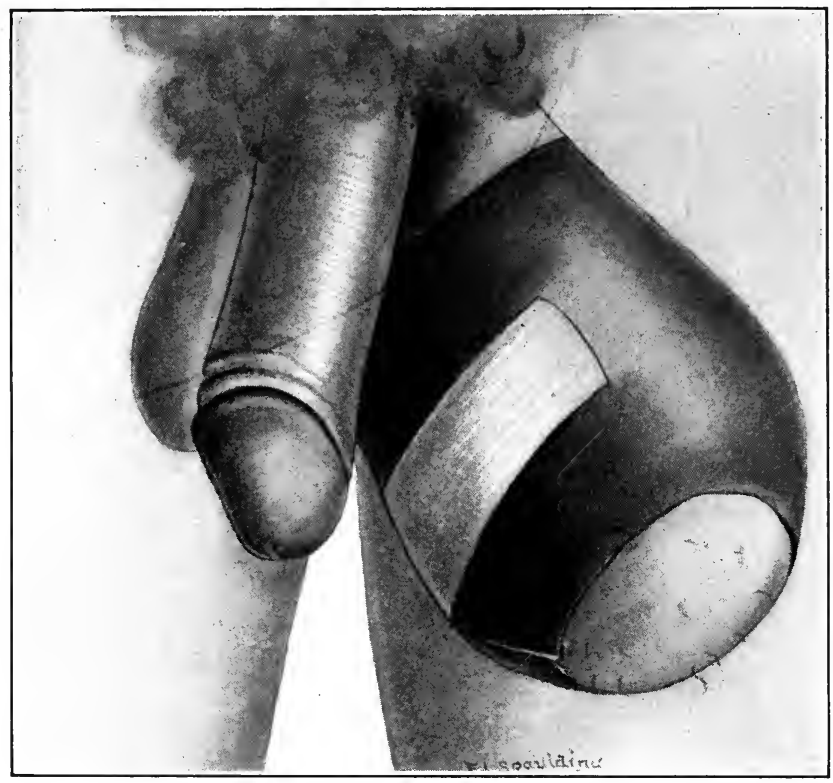

Bandage applied.

Urethral Treatment.-As previously stated, there should be no urethral treatment during the acute course of epididymitis.

It is unsafe to begin urethral instrumentation for at least a fortnight after the acute inflammation has subsided. The deep urethra should be treated by instillations of argyrol, 20 per cent.; the prostate and seminal vesicles should be emptied of their inflammatory exudates by massage.

Complications.-A co-existing reducible hernia may be rendered irreducible when attacked by inflammation of the cord. An extension of the inflammation to the peritoneum may result in peritonitis. A varicocele may be made worse by the gluing of the epididymis to it. Sterility has already been considered under Prognosis.

Recurrent Epididymitis.-Recurrence of the acute symptoms and condition takes place in a certain number of cases. Instrumentation is frequently the cause of a fresh outbreak. The wearing of a suspensory bandage, avoidance of active exercise, and the prolonged douching of the 
parts with hot water night and morning a fortnight or more after the subsidence of the attack, are the best and only prophylactic measures that can be taken to guard against relapse. It is stated by some observers that recurrent epididymitis is an evidence that the testicle is still functionally serviceable, since repeated attacks do not occur unless the canal of the vas deferens is patent. Succeeding attacks are of less severity and of shorter duration than the initial one.

In the cases of frequent recurrence surgical intervention may be demanded; that is to say, vasectomy or the establishment of drainage through the vas deferens after the manner recommended by Belfield. ${ }^{15}$

The division of the vas sacrifices the future functional capability of the testis, but the patient will be relieved from further attacks of inflammation. Chetwood has performed ten such operations, and the results have been promptly and permanently curative. In considering this operation, the prognosis should be somewhat guarded, because of the possibility of failure to prevent subsequent attacks, since they may be brought about by infective organisms which have been retained in the tissues of the epididymis. (For the technique of vasectomy, and irrigation and drainage through the vas, see chapter on Technique of Operations on the Testicles.)

Orchitis (Non-tuberculcus).-Orchitis is a relatively rare affection.

Etiology.-Primary orchitis may be due to traumatism, to inflammation elsewhere in the urinary tract, and to infection through the blood current.

Secondary orchitis is due to the extension of an epididymitis.

The etiological factors producing acute orchitis fall under four general headings: (I) Traumatism. (2) Extension of posterior urethral inflammation. (3) Hematogenous infection. (4) Idiopathic.

I. Traumatic Orchitis.-Injuries usually involve both the testicle proper and the epididymis (epididymo-orchitis). The undescended testicle may be the seat of inflammation arising from pressure exerted by the adjacent parts while the organ is retained within the inguinal canal. An organ displaced into the perineum or elsewhere is more liable than one that is in its normal position, to external violence and resulting traumatic orchitis. English has called attention to the possible casual relation to orchitis of the powerful contraction of the cremasteric muscle drawing the testicle upward and against the external inguinal ring, and so compressing the organ that inflammation of both the testicle and epididymis results. Velpeau suggested the possibility of producing the condition by compression of the cord by the abdominal muscles. Traumatic orchitis, however, is more frequently the result of a kick, blow, or falling astride some solid object. An important feature of traumatic orchitis is that the pressure exercised by the firm tunica albuginea may 
produce fatty degeneration of the secreting portion of the organ, and subsequent atrophy. Even slight blows, causing but moderate inflammation, may be followed by atrophy. Sometimes, when the trauma is great, the organ is totally destroyed by suppuration or gangrene.

2. Extension of Posterior Urethral Inflammation.-Extension of posterior urethral inflammation to the testicle, without including the epididymis, is an occasional cause of orchitis. Kocher mentions such an instance, and Hutchinson has recorded a case in which both testicles were inflamed under these circumstances. Casper states that the vas deferens and epididymis may escape, the inflammatory process being confined to the testicles. By far the most common associated condition is epididymitis; in fact, most cases of orchitis dependent upon inflammation of the posterior urethra are the result of primary inflammation of the epididymis and subsequent invasion of the testicles.

3. Infection of the Testicles through the Blood Current.Infection of the testicles through the blood current is rare, except in connection with mumps. Instances of infection, occurring in the course of pyemia, variola, scarlatina, malaria, and leprosy, have been recorded by Chiari, Simon, Kocher, Ziegler, and others. Kocher found typhoid bacilli in an infected testicle, occurring as a complication of typhoid fever. Keyes gives gout as an etiological factor, as does also Sir James Paget. Rheumatic orchitis is reported by McLeod and Bouisson, Vache, Dumont, and Reclus. Curling describes a patient suffering from acute rheumatism, in whom both testicles became inflamed and rapidly atrophied.

Syphilitic and tuberculous orchitis originate from blood infection, but because of their importance and special characteristics are considered separately.

Orchitis Due to Mumps.-This condition is most often seen in individuals about the age of puberty, though the complication may occur in still younger patients, and cases are reported in persons between the ages of forty and forty-five. It is, however, very rare in childhood. The orchitis of mumps develops during the decline of the parotitis, several days after the disappearance of the parotid inflammation. One and sometimes both testicles are involved. Cases are on record in which orchitis has preceded the parotitis, and, during epidemics of mumps, the occurrence of orchitis without the involvement of the parotid has been recorded by Kocher. The term metastasis, applied to signify transmission of the disease from the parotid to the testicle, is not correct, the orchitis being an independent manifestation of the disease. Ziegler's term, hematogenesis, expresses more exactly the character of the dual infection. The orchitis increases for three or four days, and resolution 
takes place in two or three weeks. The epididymis may or may not be involved; usually it is not. At the height of the inflammation there may be a mucopurulent discharge from the urethra, which disappears entirely upon the appearance of the inflammation of the testicle.

Suppuration seldom occurs, but in severe cases atrophy may follow. Osler states that if both organs are involved before puberty, the natural development is usually checked. Sexual vigor may be retained, however, even if the testicles are atrophied. The proportion of cases showing atrophy of the organ infected by mumps is large. Comby reports atrophy of the testis following the orchitis of mumps in Io3 out of 163 cases. Keyes states that orchitis occurs in about 5 per cent. of the cases of mumps in young adults.

4. Idiopathic Orchitis.-Idiopathic orchitis is the term applied to cases of the cause of which we are at present ignorant. Such cases are occasionally met with.

Symptoms and Diagnosis.-Local Symptoms.-The intensity of the symptoms in acute orchitis depends, in some measure, upon the cause. The acute orchitis of mumps and the idiopathic forms are the least severe. Gonorrhœal orchitis is more intense and of longer duration. The form that most frequently suppurates is that which results from instrumentation in cases of prostatic hypertrophy, deep urethral stricture, and operations done for the relief of these conditions. As it happens, the only cases in which we have seen suppuration have been of this character. Gardner cites examples of its occurrence after lithotomy, and Hutchinson has also recorded one instance of it.

Because of the firmness of the tunica albuginea and the acute sensitiveness of the organ, the symptoms of orchitis are entirely disproportionate in severity to the extent of the inflammation. The tunica albuginea prevents enlargement of the organ, and to this fact the intensity of the pain is due. It is excruciating, persists for about a week, as a rule, and then gradually subsides. The pain is not always confined to the testicle, but shoots up the cord to the back and loins, as it sometimes does in epididymitis. It is probably due to nerve reflex or to an actual neuritis. Keyes states that handling the inflamed organ may cause syncope. The scrotum is usually congested, and may show signs of inflammation, but there is seldom an effusion of serum into the tunica vaginalis, as in epididymitis. Sudden cessation of the pain in a severe case usually means that the tunica albuginea has ruptured, or that gangrene of the testis has taken place. The apparent enlargement of the testis is due, for the most part, to inflammatory thickening of the tunica albuginea. The shape of the organ is but little altered and the surface remains smooth. It is extremely tender to pressure, and if palpated with the patient under 
ether, it will be found to have lost its natural elastic quality. The epididymis cannot be distinguished from the testis when inflamed in association with it.

The symptoms are usually more severe in character than in the cases of epididymitis. This is true of both the general and local manifestations. The former are sometimes of very grave character, and conspicuous among them are vomiting, high fever, great prostration, and nervous symptoms.

Complications.-The complications and sequelæ of orchitis are as follows: (1) Suppuration. (2) Gangrene. (3) Hernia testis. (4) Atrophy.

I. Suppuration.-Suppuration occurs either as a single focus or in multiple form. A single central abscess is not common.

In whatever form the condition may occur, it proceeds to resolution and ultimate connective-tissue formation, with atrophy of the organ corresponding in extent to that of the area involved; or the pus works its way outward and forces a passage either to the surface of the scrotum or into the tunica vaginalis. Resolution is rare; the other course common. When the pus breaks through the surface of the scrotum the testicle protrudes through the opening as a pinkish or grayish mass; or, again, having a yellowish color (hernia testis). Later, the organ may become covered with exuberant granulations, and then often closely resembles cancer. The scrotal integument becomes adherent about the exposed surface of the testis. The condition just described is known as fungus testis.

The subsequent course is slow and results in greater or less destruction of the organ.

In some cases the multiple, and also the single, foci of pus pass through the substance of the testis and reach its surface by a number of channels, giving to it the appearance of a tuberculous process.

2. Gangrene.-Gangrene of the testicle in connection with orchitis is infrequent. When it occurs, the tunica albuginea becomes necrotic, ruptures, and, the tension being relieved, pain suddenly ceases. The testicle and the area of the scrotum which adheres to the organ before and after the gangrenous process reaches the surface, slough, and present a mass of necrotic, yellowish, dry, and soft tissue. There is little or no purulent discharge. The necrotic mass can be removed without pain.

Atrophy.-In the non-gangrenous form of orchitis atrophy of the organ results, in greater or less extent, after the subsidence of the acute inflammation. The organ gradually becomes soft and flabby, diminishes in size, and loses its natural sensitiveness. 
Treatment.-Acute Orchitis.-Absolute rest in bed, elevation of the testes, morphine to subdue pain when necessary.

Local measures are: Poultices (Keyes advises guaiacol as in epididymitis). Painting the skin of the scrotum over the testis with 25 per cent. solution of nitrate of silver may be of some benefit. Multiple punctures, practised by Vidal, and carried out by stretching the skin over the inflamed organ and making a number of small punctures through the tunica albuginea, for the purpose of relieving the tension and pressure of the latter upon the testis, is beneficial in stopping pain. If properly done, there is no fear of prolapse of the testicular substance through the openings. Vidal employed a narrow-bladed tenotome for the purpose; the punctures should be very small, and should not penetrate deeper than just through the tunic.

When suppuration occurs, the pus should be let out as soon as its presence is assured. The incision should be sufficiently small to avoid subsequent hernia of the testis through it. If it is desirable to have a free opening in order to cleanse the abscess cavity, it may be made at first and then partially closed immediately afterward, leaving only enough of it open to permit the insertion of a drain.

The scrotal skin is delicate, and especially prone to inflame; hence irritating dressings should be avoided.

If hernia does occur, its granulating surface should be cauterized by nitrate of silver stick, or snipped off with scissors. Properly applied pressure with gauze, maintained by adhesive straps, should be employed if the protrusion is small, and after it has been removed. If the surface of the fungus growth is clean, the edges of the surrounding tissue may be denuded, and united over the protruding mass.

If sinuses or fistula follow abscesses, they should be kept clean and occasionally touched with silver nitrate stick. If they fail to heal, they should be slit up, curetted, and packed with iodoform gauze. When these measures fail, or if the patient cannot give the condition proper care, and if the general health seems to be the cause of failure to heal, castration should be done. The same measure should be applied at once if there has been very extensive destruction of the organ.

The general health of the individual should receive careful consideration, and in all cases of orchitis the testicles should be supported by a suspensory bandage for some months after the attack has subsided.

Syphilis of the Testicle.-Syphilis of the testicle is a more common affection than is usually supposed. It may be conveniently discussed under two headings according to the part involved by the disease, as Syphilitic Epididymitis and Syphilitic Orchitis. 
Syphilitic Epididymitis.--The involvement of the epididymis alone by the syphilitic process is rare.

According to Taylor ${ }^{16}$ it is "usually a somewhat precocious manifestation of syphilis, occurring, in most cases, within the first six months, and sometimes as early as the second month."

Syphilitic Testis.-Syphilis of the testicle may be a tardy manifestation and appear late in the secondary or in the tertiary stage of the malady.

Of the syphilitic testis of the tertiary period, Keyes ${ }^{17}$ distinguishes two pathological forms: (I) The diffuse form (Fig. I2I). (2) The gummy

FIG. I2I

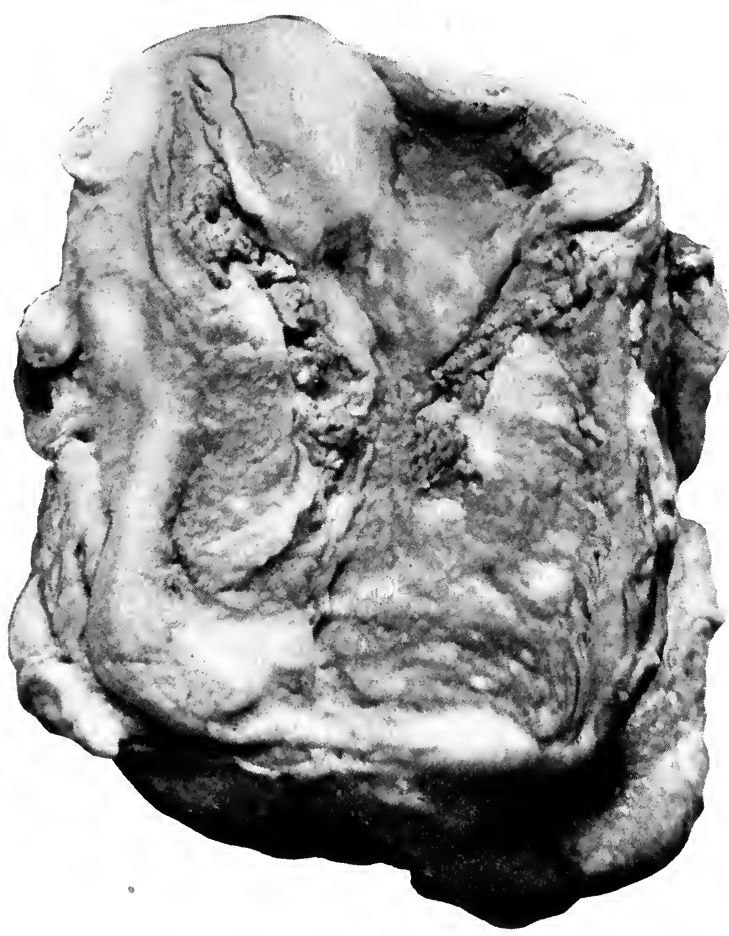

Syphilitic testicle of the diffuse interstitial type, with areas of calcification.

form (Fig. 122). The diffuse form he likens to the processes of interstitial character in interstitial nephritis, hepatitis, etc. He describes it as "A peculiar sort of chronic inflammation attacking the fibrous envelope and the septa of the organ.

"The process begins with hyperemia and a new-growth of connective tissue occurs in the stroma of the organ.

"This new tissue presses upon and gradually causes atrophy of the tubular structure.

"The gummy form is marked by the formation of nodules, usually 
multiple, which seem often to take their origin in the external tunic of a vessel or in the wall of a spermatic tubule (Lancereaux).

"The epididymis is not apparently involved in the diffuse orchitis, but with the more frequent gummatous process, the epididymis is involved in a characteristic manner."

Keyes states that he has never seen double syphilitic epididymoorchitis.

FIG. I22

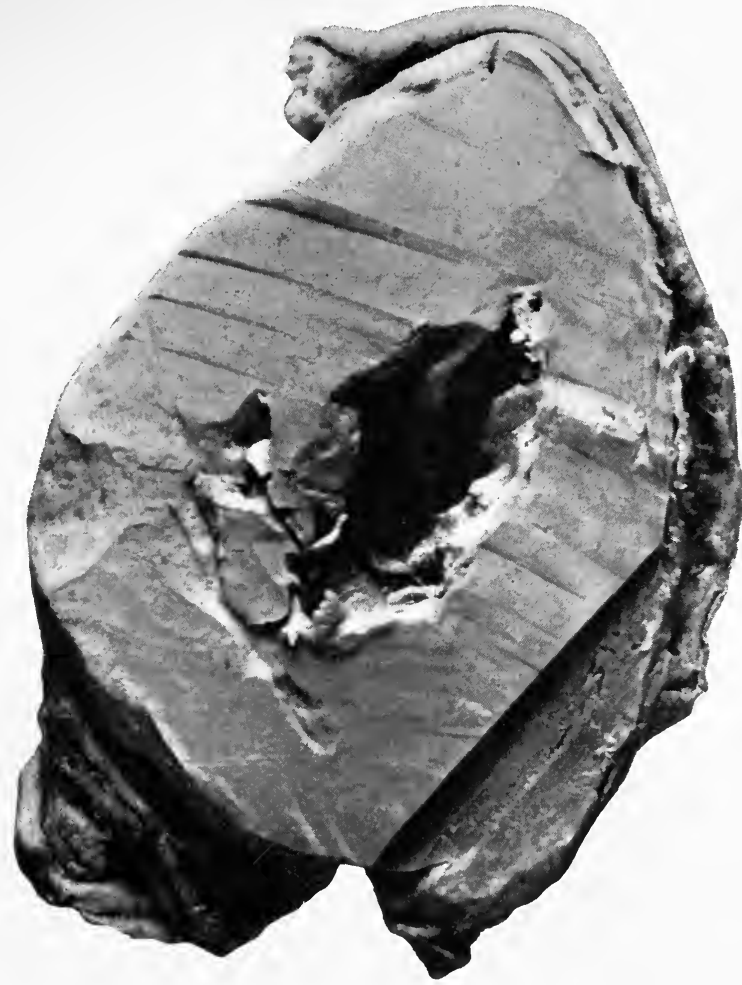

Gumma of the testicle, showing central necrosis. The tunica albuginea is much thickened and the visceral and parietal layers of the tunica vaginalis are adherent, obliterating its cavity.

Symptoms.-The first symptom is a slight, painless swelling of the testicle, the earliest stages of which usually escape the patient's notice. Its increase in size is gradual.

The characteristics of the enlargement of the organ are: absence of tenderness on pressure; as a rule, the surface is smooth and the enlargement is uniform and involves the whole of it; exceptionally, a part only of the testicle is implicated, the rest of the organ being normal; while in a few cases, instead of being smooth, the surface is nodular and irregular. 
The outline and character of the enlarged testis may be obscured by the fact that the epididymis is likewise involved, or by the co-existence of hydrocele.

The cord is not, as a rule, affected. The course of the disease is exceedingly slow.

Prognosis.-Keyes summarizes the prognosis thus: "The prognosis as to recovery is favorable, unless treatment has been delayed until complete atrophy or suppuration has taken place. The earlier the treatment is begun, the better the prognosis. Even when the disease has been of long standing, some of the parenchyma will probably have escaped atrophy and will perform its function when the pressure on the tubules is released."

Gummata disappear rapidly under antisyphilitic treatment, and, the intratesticular pressure being relieved, that portion of the testicle which has escaped atrophy resumes its normal function. The sexual appetite returns with the improvement in the organ, and Gosselin has found spermatozoa in the semen of patients cured of double syphilitic orchitis. The hereditary form of the disease may occur in the testicle at any age. Fournier reports a case of its occurring in the twenty-fourth year. It usually manifests itself within the first five years.

Treatment.- The treatment of syphilis of the testicle should be general, not local. Under antisyphilitic treatment the improvement is rapid and satisfactory. With the use of potassium iodide in increasing doses, gummata disappear, and the interstitial exudate becomes re-absorbed. Mercury, the chief factor in the treatment of syphilis, is used in combination or in alternation with the iodides. If hydrocele occurs, it should be tapped. As in all diseases of the testicle, a suspensory bandage should be worn while the patient is in the upright position.

\section{CYSTS AND NEW-GROWTHS OF THE TESTICLES.}

Cysts of the testicle should be distinguished from cystic disease or cystic degeneration of solid tumors, such as adenoma or sarcoma.

Cysts of the testicle may be situated either in the testicle itself or in the epididymis. They are due to distention of the tubules by retained secretion, and are frequently the result of antecedent inflammation, which obstructs the seminal canals. They are usually located at the junction of the rete vasculosum and the vasa efferentia, or near the head of the epididymis. The fibrous cyst wall is lined with columnar, cubical, or ciliated epithelium, according to the origin of the cyst. It is not unlikely that there are cysts lined with ciliated epithelium which are due to dilatation of remaining bits of the Wolffian ducts. 
The fluid contents may be clear or milky. In the latter instance, it is known as galactocele. When containing spermatozoa, it is called a spermatocele, and must have or have had at some time communication with a spermatic channel. Spermatocele of the hydatid of Morgagni, described by Luschka, is believed by Roth to be due to a dilatation with spermatic fluid of the aberrant diverticulum of the canal of the epididymis, which ends blindly in the hydatid.

Cysts situated in the head of the epididymis are generally met with in those individuals who have had previous inflammation of the part. They usually remain small, but sometimes increase gradually to a considerable size.

Cysts in the substance of the testicle are of slow growth, and may or may not appear on the surface of the organ. Occasionally they protrude from the surface of the gland as thin-walled sacs. Instances are recorded in which the cyst lay between the testicle and epididymis, the body of the latter having been separated from its attachment to the testicle (Fig. I23).

FIG. 123

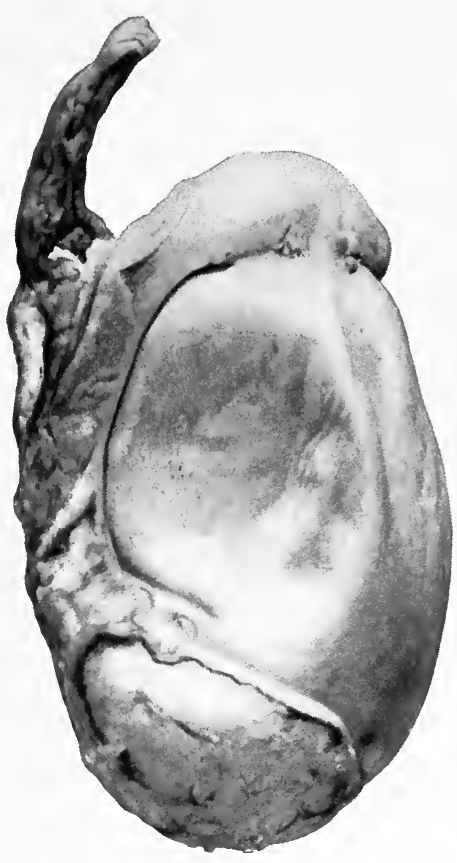

Cyst between testicle and epididymis, separating one from the other. (Warren Museum.)

\section{SOLID TUMORS OF THE TESTICLE AND EPIDIDYMIS.}

It is often impossible to distinguish, clinically, between the nonmalignant and the malignant tumors affecting the testicle. Tumors starting as benign growths may become malignant; thus, an adenoma may, by free proliferation of the stroma, become adenosarcoma.

Carcinoma and sarcoma are the most common tumors of the testicle. Adenoma is also a relatively frequent form. Teratomata have been reported a number of times, and rare examples of fibroma, enchondroma, osteoma, myoma, lipoma, myxoma, have also been recorded.

Adenoma.-Adenoma is found in both children and adults. The tumor assumes a variety of forms, and there is a decided tendency in them to undergo cystic degeneration. Such a condition is illustrated in Fig. I24. 
Billroth and Senftleben have observed striated muscle fibers in the stroma of this variety of the condition. The contents of the cysts is either clear or a blood-tinged viscid fluid; sometimes a yellowish, pulpy substance. Circumscribed patches of cartilaginous tissue are sometimes found in the walls of these growths.

Fig. 124

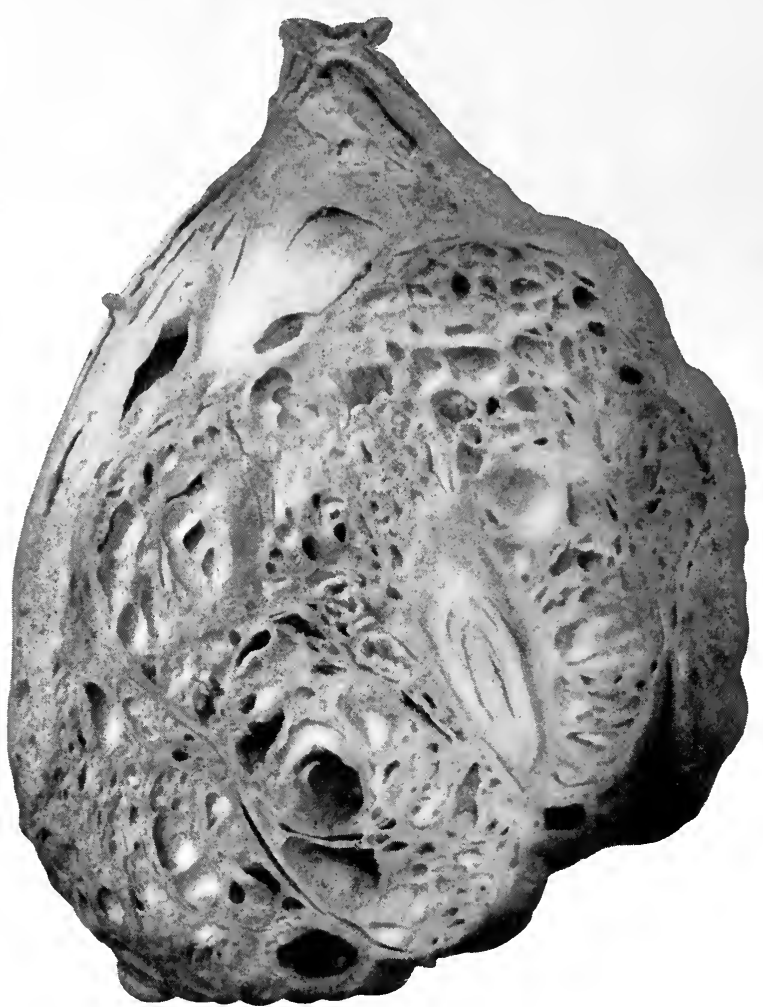

Cystadenoma of the testicle. Solid structures replaced by cysts, separated by irregular, thin, fibrous partitions. From a man, aged thirty-five years. Disease of four years' duration. (Warren Museum.)

Carcinoma.-Carcinoma is one of the commonest tumors of the testicle, but is less frequent than sarcoma. The disease occurs in childhood and old age, but is usually met with between the ages of thirty and fifty. It is generally unilateral. It occurs in two forms: (I) A soft, marrow-like growth (encephaloid or medullary carcinoma); and (2) a firm, compact tumor, with an abundant stroma (scirrhous carcinoma). The former variety is much more common than the latter.

In the second form the nodules exist chiefly in the rete testis and in the epididymis. The growth then involves the seminal canals and extends throughout the organ. In some cases areas of cartilaginous tissue are 
found. For these forms Ziegler has suggested the name chondrocarcinoma.

Sarcomatous changes sometimes co-exist with carcinoma of the testis. Metastasis takes place through the bloodvessels and the lymphatics of the cord, invading the deeper iliac and retroperitoneal nodes, which lie on a level with the kidneys. A special lymph node, which is said by Cuneo to be liable to be the seat of the malignant process, is one that he describes as lying close to the point at which the ureter crosses the iliac vessels.

The soft or medullary form of carcinoma grows more rapidly than the other or harder variety.

When the neoplasm breaks through the tunica albuginea, the ovoid shape of the tumor is lost, the growth becomes adherent to the wall of the scrotum, finally penetrates it, and appears on the surface in the form of a fungus-looking mass.

Prognosis.-The prognosis is always bad. Early metastasis, which is the rule in this condition, makes it especially so.

Treatment.-Early excision. The operation, to be of any value and to offer any hope of cure, must be done early, and must include the cord, the inguinal lymph nodes, and also the deeper intra-abdominal chain.

Sarcoma.-Sarcoma is rather more common than carcinoma of the testis. It rarely originates in the epididymis.

Almost every variety of sarcoma has been found in the testicle, including the lymphosarcoma, fibrosarcoma, angiosarcoma, melanotic sarcoma, and myxosarcoma. Kober, ${ }^{18}$ in a series of I I 4 cases of sarcoma of the testicle, made histological examinations in 75 , and found that the tumors were of the round-cell or mixed round- and spindle-cell varieties in 65 per cent. of them (see Fig. I25). The remainder were of the alveolar, cystic, or spindlecell types. In 7 I per cent. of Kober's cases the patients were between twenty and fifty years of age, but sarcoma is commonest in young persons.

Trauma appears to play an important part in its causation. Kober states that 43 per cent. of his series had a history of injury.

According to the special nature of their structure, the growths are of harder or softer consistency. They may attain very large size; more especially is this true if the tubules of the gland undergo cystic dilatation. When the neoplasm becomes cystic, portions of the tumor sometimes project from the cyst walls in the form of papillomata.

Sarcoma shows a tendency to fatty degeneration, and areas of hemorrhage are scattered throughout the neoplasm. Unlike carcinoma, sarcoma shows but little tendency to break through the tunica albuginea.

Metastasis takes place in the same manner as it does with carcinoma, but sarcoma shows a greater tendency to become widely disseminated, forming secondary deposits in the liver, kidneys, and brain. 
Prognosis. - The prognosis is bad.

Treatment.-The treatment consists in early castration (see chapter on Technique of Operations on the Testicles) and removal of such involved structures and nodes as are accessible, in cases in which there is no

FIG. 125

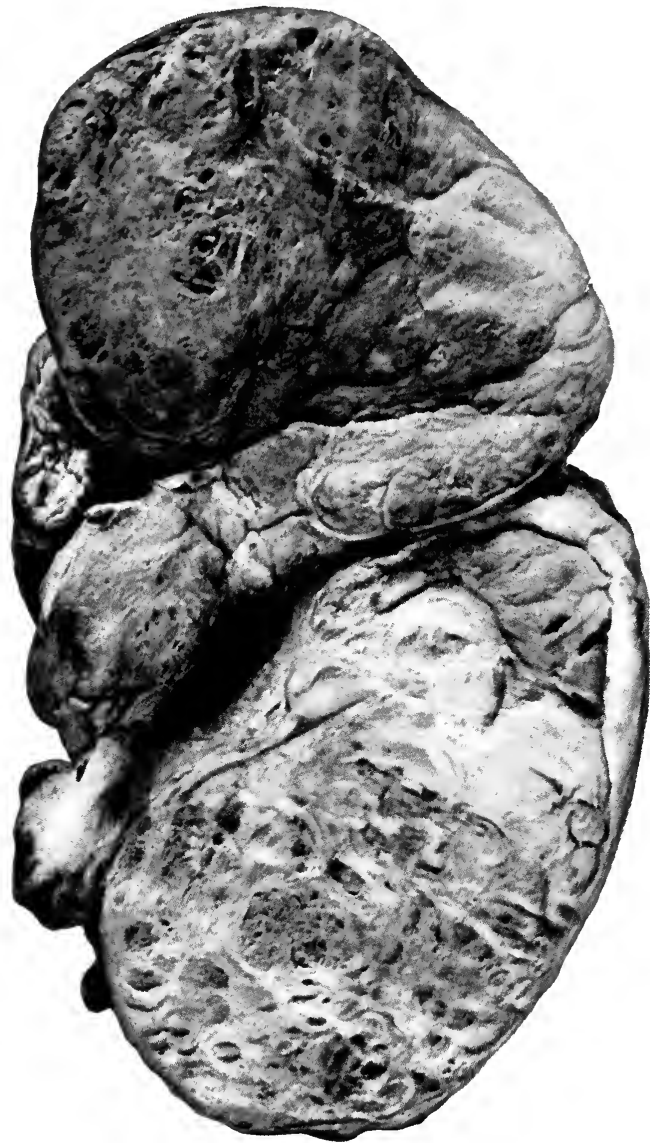

Large round-cell sarcoma of the testicle. Lobulated in character, and showing grayish-black mottling from hemorrhage and a slightly honeycombed appearance from cystic degeneration. (Warren Museum.)
FIG. I26

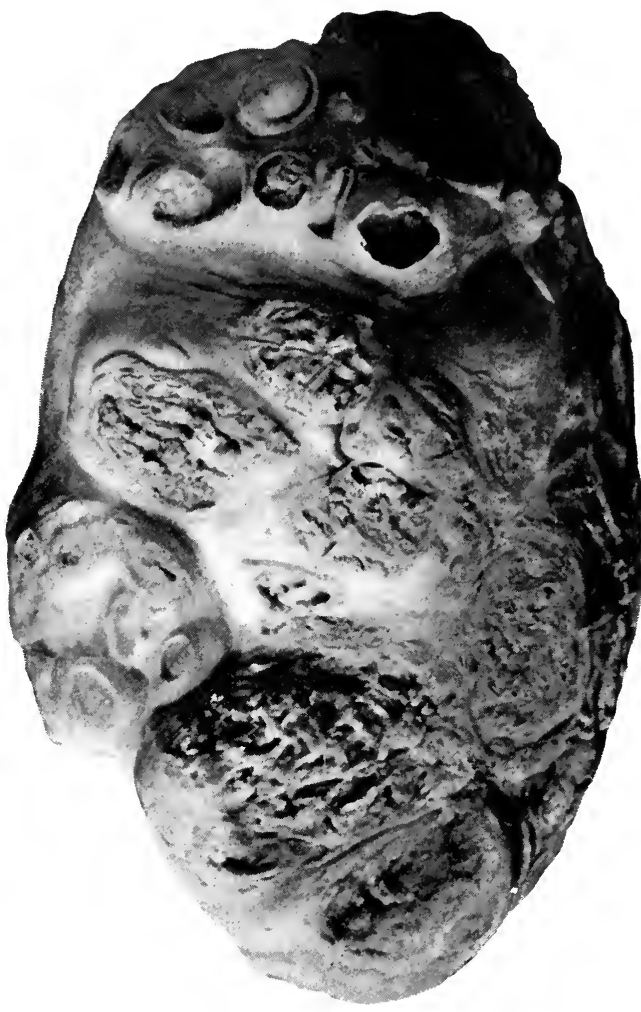

Teratoma of the testicle. The nodulous character of the growth is due to areas of hyaline cartilage, bone, fat, and connective tissue. The tumor contains chorio-epithelium. (Warren Museum.)

evidence of metastasis having already taken place. If the contrary is true, operative treatment is useless.

Teratomata.-Teratomata are congenital tumors originating from the three blastodermic layers. They have been considered rare, but the recent literature contains the reports of a number of such cases (Fig. I26). 
The existence of chorio-epithelium in the tumors is evidence of the very malignant nature of the growth. Many tumors which have been considered sarcoma or carcinoma really belong to the teratomata, inasmuch as they have contained chorio-epithelium. Frank has collected I9 authentic cases.

Fatal metastases occurred in 16 of Sturgis' cases. Hearn, of Philadelphia, has also had a case in which fatal metastasis occurred within a few months.

Teratomata have been reported as having first developed in the scrotal wall, later involving the testicle. They are usually noticed about the age of puberty, at which time they grow more rapidly, probably because of the increased activity of the testicle at this time. The growth may penetrate the scrotal skin and form sinuses through which the fœtal structures are discharged.

Diagnosis.-The diagnosis is made by the discovery of chorio-epithelium in the tumor.

Treatment.-The treatment consists in the removal of the growth. When the tumor is incorporated with the testicle, that organ must necessarily be removed with it; when the contrary is the case, the testis may, in some instances, be preserved.

Rarer Forms of Tumor.-Fibroma, enchondroma, osteoma, myoma, lipoma, and myxoma are the rarer of the testicular tumors. They present no clinically significant features, and are of interest chiefly because of their rarity. As a class, they are benign, but any of them may become malignant. Fibroma is the only member of this group which calls for special mention.

Fibroma.-Most circumscribed areas of fibrous tissue in the testicle are due to previous gonorrhøal infection, to injury, or to syphilis.

True fibromata of spontaneous origin have rarely been met with. Ziegler states that they originate in the rete testis and tunica albuginea, appearing as small nodules, some of them often being calcified.

Diagnosis. - The diagnosis of true fibromata, as stated by Ziegler, is difficult.

The diagnosis of the other form of fibrous tissue deposits is simplified by a knowledge of the history of the case.

Treatment.-All fibrous indurations of the testicle should receive antisyphilitic treatment, even if there be no clear history of syphilis in the case before being submitted to operation.

Diagnosis of Testicular Tumors in General.-As previously stated, it is frequently impossible to differentiate one tumor of the testicle from another. Two important points are to be remembered in connection with the diagnosis: (I) That the growth may be syphilitic, and, if so, it vol. $\mathrm{I}-\mathrm{I} 6$ 
will disappear under antisyphilitic treatment; and (2) that most testicular tumors are malignant, or likely to become so.

In all doubtful cases mercury and iodides should be given a fair trial. If no benefit follows their employment within a reasonable time (two months), operation is indicated. The aspirator may be used to determine whether the tumor is wholly solid, or if it contains fluid.

The following table of diagnostic features of syphilitic orchitis, encephaloid cancer and tuberculous disease of the testis, is taken from White and Martin. ${ }^{19}$

\section{Encephaloid Carcinoma}

\section{Syphilitic Orchitis.}

Syphilitic history.

Usually occurs at about twenty-five or thirty years of age.

Begins in the testicle.

Is situated primarily in the connective tissue.

Tends to fibrous overgrowth.

Slow in its progress.

Skin of the scrotum rarely involved.

Ulceration or suppuration rare.

Fistulæ rare.

A feeling of great weight, with only such pain as results from dragging on the cord.

Tumor very hard and uniform.

Skin of scrotum purplish, but unaffected.

Testicle of moderate size; rarely exceeds twice its normal diameter.

Painless on pressure.

Both testicles often affected.

Fungus rare.

\section{of Testicle.}

No history of any special condition.

Occurs at any age.

Begins in the body of the organ.

Begins by the deposit of small nodules in the seminiferous tubules.

Tends to formation of patches of softened, white, pultaceous material.

Rapid in its course.

Skin of the scrotum finally involved.

Ulceration and fungus common.

Fistulæ common.

Pain severe and lancinating in advanced stages.

Soft and fluctuating.

Network of large veins over surface of tumor.

Attains great size.

Painless on pressure.

Generally only one testicle affected.

Fungus always present in advanced stages
Tuberculous

Orchitis.

Tuberculous history.

Not often seen after thirty.

Begins in the epididymis.

Exists primarily in the tubules.

Tends to fatty, caseous, or purulent degeneration.

Slow in its progress.

Skin involved only just before the formation of abscess.

Suppuration common.

Fistulæ common.

Little pain.

At first hard, knotty, irregular.

Skin congested, but otherwise unaffected.

Of moderate size.

Often painful on pressure.

Often both testicles affected.

Fungus common. 
Encephaloid Carcinoma Syphilitic Orchitis.

No discharge or bleeding.

Lasts many years.

Curable.

No involvement of inguinal glands, as a rule. of Testicle.

Bleeds freely; offensive discharge.

Rarely extends beyond twenty months.

Usually fatal.

Inguinal, iliac, and lumbar glands and cord affected.

\section{Tuberculous}

Orchitis.

Less apt to bleed; discharge less offensive.

Lasts several years.

Generally incurable.

Usually no inflammation of glands.

\section{HYDROCELE.}

Definition.-The term hydrocele signifies an accumulation of the natural fluid secretion of the tunica vaginalis within its cavity, or a collection of serous fluid within the processus vaginalis when the latter has failed to become obliterated, as it should under normal conditions.

FIG. 127

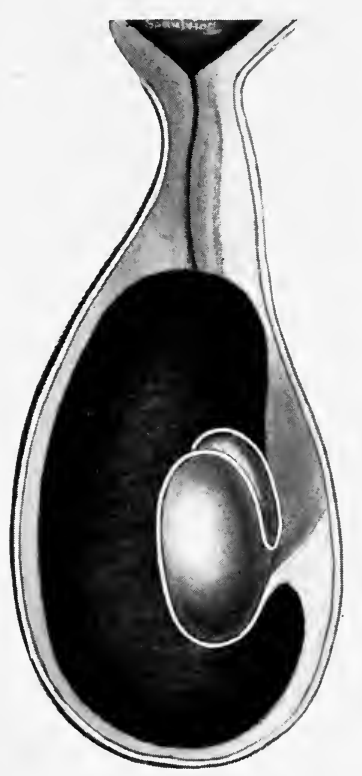

Hydrocele of tunica vaginalis.
FIG. 128

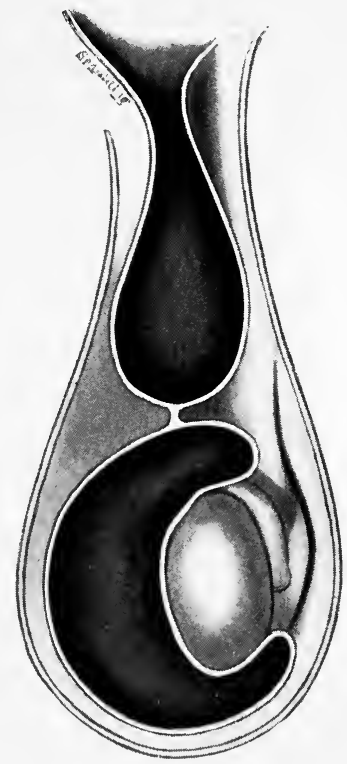

Hydrocele of the cord, opening into the peritoneal cavity.

Classification.-Hydroceles are classified in several different ways, thus: in accordance with their anatomical character; in accordance with their etiological factors; in accordance with their clinical character; and in accordance with certain special characteristics of form. Thus:

Hydrocele of the Tunica Vaginalis.-This is the common form of hydrocele. Here the fluid is confined within the closed cavity of the tunica vaginalis (Fig. I27). 
Hydrocele of the Cord.-This condition is represented by a collection of serous fluid in the tunic of the unobliterated processus vaginalis (see Fig. 128).

Encysted Hydrocele of the Cord.-A collection of serous fluid within a chamber formed by the unobliterated processus vaginalis, which has no communicating opening with either the abdomen above or the cavity of the tunica vaginalis below (Fig. I29).

Congenital Hydrocele.-In this form of the condition the cavity of the hydrocele communicates with the peritoneal cavity by an opening which varies in size, it sometimes being too small to permit the passage of more than a little of the hydrocele fluid, or to allow of the descent of a hernia (Fig. I30).

FIG. 129

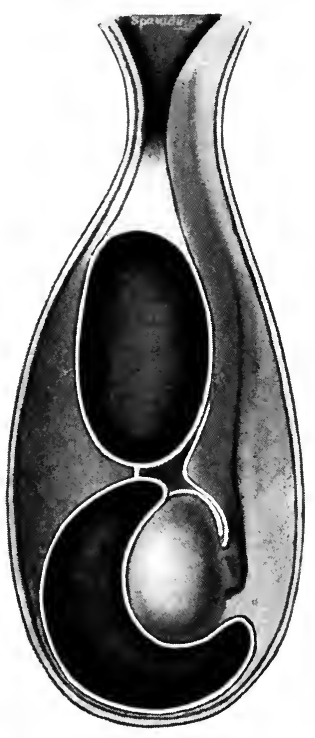

Encysted hydrocele of the cord.
FIG. I30

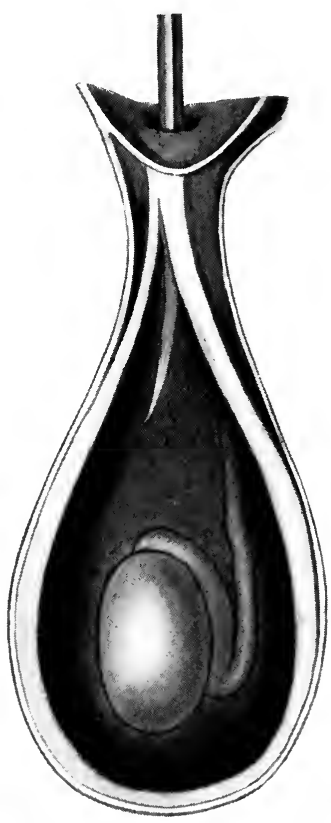

Congenital hydrocele.

Infantile Hydrocele.- - Here there has been no obliteration of the vaginal process, except at its uppermost point, and there results, in consequence, a hydrocele cavity which is continuous from the bottom of the tunica vaginalis to the point of the processus referred to.

Bilocular and Multilocular Hydrocele.-When the ordinary hydrocele of the tunica vaginalis and an encysted hydrocele of the cord co-exist, a sac is formed, which is slightly constricted at the line of junction of the two, which gives the appearance of a two-chambered cavity; this is known as bilocular hydrocele. When the cavity of a hydrocele is divided 
into a number of compartments by the formation of a series of partitions, the condition is called multilocular hydrocele.

Hydrocele of a Hernial Sac.-This name is given to an accumulation of serous fluid within an empty hernial sac.

Inguinal Hydrocele.-This is a hydrocele which occurs in connection with a retained testicle.

Hydrocele is further classified according to its clinical character, as acute and chronic hydrocele, and etiologically as symptomatic and idiopathic.

The terms purulent, fibrinous, and plastic are often joined to that of hydrocele to designate a special character of the fluid exudate. By its very meaning the word hydrocele can be applicable only to a watery collection of fluid, so that it is as much of a misnomer to speak of purulent hydrocele as it would be to call a hematocele a hydrocele. Such conditions as these can be best described as hydroceles which have taken on a different character, owing to the occurrence of certain special changes of a pathological nature, which will be mentioned later, but they cease to be hydroceles in the proper sense of the term.

Acute Hydrocele.-Etiology.-The rapid accumulation of serous fluid which receives the name of acute hydrocele is a manifestation of an underlying lesion, and is comparable to the serous pleuritic effusion which takes place in connection with certain pulmonary diseases. In the case of hydrocele, epididymitis is the most common lesion from which it develops. Other conditions from which it springs are trauma and infected wounds of the scrotal contents.

Occasionally an acute hydrocele develops in the course of a general systemic malady, e.g., pyemia and variola.

Pathology.--In the early stage of acute hydrocele the tunica vaginalis is hyperemic. Rapid secretion of serous fluid takes place from its surface and fills its cavity. Later, the exudate may contain fibrin, which adheres in flakes to the walls of the tunica, sometimes forming a plastic membrane marked by ridge-like elevations, producing ultimate and permanent adhesions between the visceral and parietal layers of the tunica (so-called fibrinous or plastic hydrocele).

Infection of an acute hydrocele, usually from septic wounds of the scrotum-rarely hematogenous-may result in suppuration. When this happens, the condition ceases to be a hydrocele, properly speaking. Suppuration may arise from puncture of a hydrocele with a dirty trocar.

In some cases, in which suppuration takes place and is associated with a fibrinous exudate, the surface of the tunica may be covered with granulation tissue, and its cavity is then likely to become obliterated by the adhering together of its sides. 
Course.-Simple, acute serous hydrocele reaches its height in from three to six days, and is accompanied by more or less fever during this period. The effusion may then gradually subside, as the inflammation with which it is associated declines, or it may remain in a chronic form.

The fibrinous or plastic form is a more severe process throughout, persisting indefinitely, and frequently resulting in thickening of the scrotal walls and obliteration of the sac of the tunic. In the latter case, spontaneous cure is brought about.

Symptoms.-The onset of acute hydrocele is sudden, and the swelling increases rapidly. The character of the symptoms is, in a great measure, determined by the nature of the underlying condition which causes it.

In the more severe cases, constitutional disturbance, fever, and depression are usually associated with the local manifestation. Pain, swelling, œdema, and reddening of the scrotal integument occur in varying degrees. The pain is not confined entirely to the swelling, but is often propagated along the cord to the lumbar region.

The scrotal swelling is pear-shaped, the smaller end above, the larger one below. If the sac has not become thickened by previous attacks, and if the fluid contents is serous, the tumor is translucent. This is the characteristic feature of all simple hydroceles.

Diagnosis.-In ordinary cases of acute hydrocele, the diagnosis is easily made by the translucency of the tumor, by its shape, and by the absence of impulse on coughing. When due to an acute epididymitis, and if the amount of the fluid is small, the tumor may lose its characteristic form, and the swollen epididymis may obscure its translucency. Careful examination will usually discover a part of the tumor lying in front of the testicle, which is more elastic to the touch and less hard than the rest of the mass. This is the part in which the hydrocele is located.

In those cases in which the hydrocele has undergone suppuration, the diagnosis may be impossible to make, except by drawing off some of the fluid with an exploring needle, and the same is true of the differential diagnosis between hematocele and a hydrocele which has thick walls or has undergone suppuration.

Acute hydrocele is usually distinguishable from a rapidly developing hernia by the absence of fever in the latter condition and its presence in the former one; by the shape of the tumor; by the presence or absence of translucency; and by the absence of impulse on coughing.

Rapidly developing cysts of the testicle or epididymis cannot, as a rule, be distinguished from hydrocele. From a clinical standpoint, it is not important to do so.

Prognosis.-The prognosis of acute hydrocele, in so far as life is concerned, is good. In cases of the fibrinous variety in which the cavity of 
the tunica becomes obliterated, the testicle may undergo atrophy, as a consequence of the pressure exerted upon it, and of impaired circulation.

In the cases in which suppuration takes place, there is but little danger of systemic infection. The pus works rapidly to the surface of the scrotum and is discharged spontaneously if not evacuated by incision.

Treatment.-Acute hydrocele may undergo spontaneous cure. The treatment should be directed chiefly to relieving the condition of which the hydrocele is the symptom.

In all cases-the mild as well as the severe ones-the scrotum should be elevated by laying it upon a shelf placed across the thighs; the patient should be kept in bed, and cold compresses should be applied to the parts; or, better, an ice-bag should be placed upon the shelf and the testicles rested upon it, several layers of some soft material being interposed between the scrotum and the ice-bag.

If there is a large amount of fluid, it should be withdrawn with an aspirating needle; otherwise this should not be done.

The patient should be allowed to go about, wearing a suspensory bandage, soon after the acute symptoms and the swelling begin to subside. If suppuration occurs, prompt incision, drainage, and irrigation should be practised.

Compression should not be applied to the tumor until the height of the disease has passed.

Chronic Hydrocele.-Etiology.-There is a good deal of divergence of opinion with regard to the etiology of chronic hydrocele. Some surgeons maintain that it invariably results from an underlying lesion, even though they may not be able to demonstrate the existence or the nature of that lesion. The inability to discover it is the basis for the opposite view, which is, that the exudate is of a purely idiopathic character.

The condition cannot be traced to passive congestion as a cause, and, except as the residue of an acute hydrocele, the chronic form of the malady does not appear to be the result of inflammatory processes. Klebs and Virchow, in their pathological examinations, failed to find evidence of inflammation in the tunica, and the fact that the disease occurs congenitally seems to indicate that such a process is not the underlying cause of it.

To say that the cause is a discrepancy between the secreting and absorbing powers of the tunica is nothing more than to explain the obvious, and explains nothing, since it fails to show why such disturbance of the normal balance of the two takes place.

In a few instances chronic hydrocele originates in the rupture of a cyst of the epididymis, ${ }^{20}$ the testis, or of the hydatid of Morgagni. Its origin has also been attributed to long-continued irritation of the tunica, the nature of which is unknown, and to remote injuries. 
The disease occurs at all times of life, and is equally frequent on both sides; sometimes both are simultaneously affected. As a rule, it is unilateral.

Pathology.-The changes in the tunica vaginalis, the amount and the character of the fluid, and their influence upon the condition of the testicle, vary according to the duration of the condition.

The Tunica Vaginalis.-In all cases of hydrocele there is a tendency for the tunica vaginalis to become thickened by connective-tissue proliferation. In long-standing cases the thickening may be great (Fig. I3I). The inner surface is often marked by ridges. The sac may

FIG. I3I

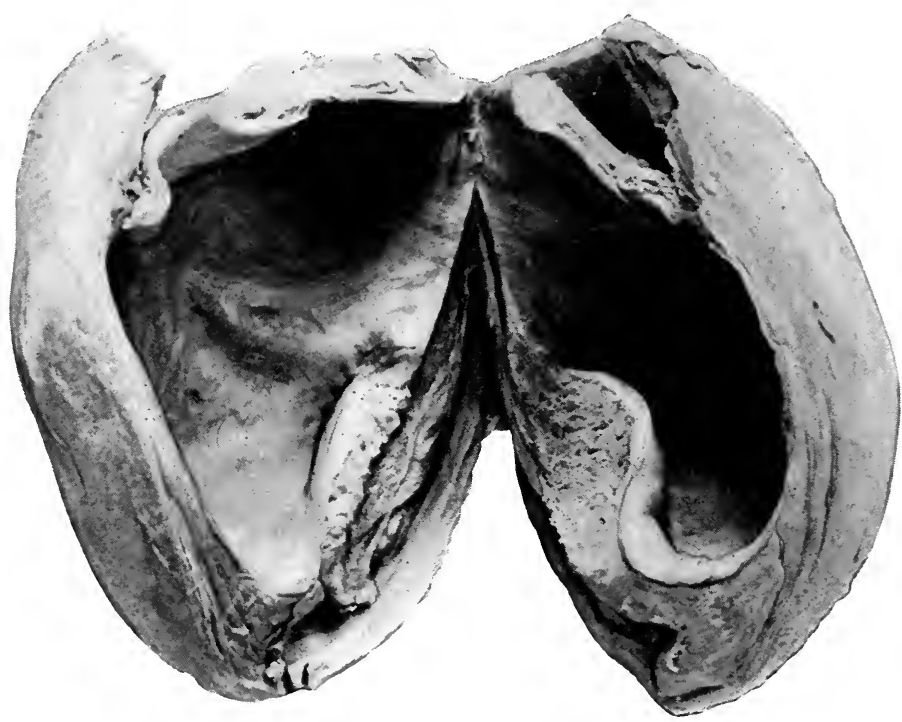

Hydrocele. The cavity of the tunica vaginalis is greatly dilated. Its walls are thick and fibrous. Note that the wall is separated from the scrotum in the upper left portion. The testicle is atrophied. (Warren Museum.)

become cartilaginous in character. Park ${ }^{21}$ has reported a case in which there was calcification of the tunic.

The epithelial lining of the tunica vaginalis shows no change in cases of hydrocele which have not been inflamed. In some of those which have been inflamed the cavity of the sac is obliterated as the result of the formation of inflammatory adhesions.

The Fluid.-The amount of the fluid varies greatly. Leigh ${ }^{22}$ mentions a hydrocele which weighed I20 pounds; Marsinna ${ }^{23}$ records a hydrocele which measured twenty-seven inches in length and seventeen inches in its transverse axis. Ordinarily, the fluid contents is but a few ounces. 
The character of the fluid is usually serous, but it may be serofibrinous. In common hydrocele the fluid is of a clear amber color, slightly viscid, and resembles ascitic or pleuritic fluid. Its reaction is neutral; specific gravity, I020 to 1026 , and it is rich in albumin, the addition of nitric acid often causing a coagulation of the entire fluid. Unlike ascitic fluid, hydrocele fluid contains fibrinogen and inorganic salts.

Occasionally, the fluid is found to be turbid or reddish brown, due to the presence of desquamated epithelial cells and leukocytes, or extravasated blood. Sometimes the fluid is opaque, from the presence of cholesterin; or milky, from the admixture of spermatozoa (spermatic hydrocele).

Concretions having a nucleus of the earthy phosphates, or carbonates, and covered with fibrin, are occasionally found, lying free in the cavity of a hydrocele. They are usually about the size of a small bean, and are single.

Spermatic Hydrocele.-As stated above, the condition is that of a hydrocele into the fluid of which spermatozoa have entered. This entrance is made either by the rupture of a spermatocele into the hydrocele or by the existence of a vas aberrans, which opens into the sac of the tunica vaginalis at the hydatid of Morgagni.

The Testicle in Cases of Hydrocele. - The testicle almost always lies behind the hydrocele sac (see Fig. 127). Exceptionally, it lies in front. (See Inversion of the Testicle.) It is important to remember this unusual location of the organ, and its position should be carefully determined previous to tapping a hydrocele, so that it may escape injury.

In cases of long-standing hydrocele, atrophy of the testis may result from the pressure of the fluid upon the organ, but atrophy is more often due to antecedent orchitis or epididymitis.

Symptoms.- Subjectively, the symptoms of chronic hydrocele are not distressing. The swelling takes place gradually and painlessly, and the chief discomfort is due to the weight of the tumor. Objectively, the characteristic feature of the hydrocele is the scrotal tumor. In its typical form, this is pear-shaped. The special kinds of hydrocele present corresponding variations of form of the character already described in the text.

Diagnosis.-If the tumor is translucent, there can be no doubt as to its character; if it is not, the aspirating needle will have to be used, in many instances, to determine the nature of the condition. The manner of examining the tumor in order to learn if it is translucent or not is as follows:

The patient stands beside a table upon which a lighted candle is placed so that its flame shall be on a level with the scrotal tumor, or near a window through which a strong light is coming. The examiner places 
himself on the other side of the patient, so that the latter is between him and the light. The scrotal tumor is then raised, in order that the light may strike fully upon the farther side of it, and the surgeon, holding it with one hand, presses with the other, one end of a tube firmly against the skin of the scrotum upon the side nearest him, and places his eye close to the other end of it. When the tumor is translucent, it is shown by the yellowish-red light which is transmitted to the end of the tube from the candle through the tumor. When it is not, no light is to be seen.

A moderate degree of thickening of the hydrocele sac or a slight turbidity of the fluid contents does not deprive the tumor entirely of its translucency.

Reducible hernia may be mistaken for a congenital hydrocele, and irreducible hernia for the ordinary form of hydrocele with a thickened sac. The presence of a tympanitic percussion note, of impulse on coughing, and the fact that the tumor extends into the inguinal canal are the distinctive features which characterize hernia, and are not present in hydrocele.

The testicular tumors are recognizable by their solid consistency, absence of translucency, irregularity of the surface in many cases, and by the history of their development.

Spermatocele and cysts of the epididymis are generally impossible to distinguish from hydrocele. Aspiration will, however, reveal the nature of the first of the conditions by the milky character of the fluid.

Prognosis.-Hydrocele threatens neither the good health nor the life of the patient. It is an inconvenience, and, if very large, is unsightly and causes embarrassment because of the inability to conceal it.

The prognosis with regard to recurrence after the fluid has been withdrawn by tapping, is not good in the case of adults. With children, one aspiration of the contents of the sac often results in cure.

It is seldom that cure is not effected by the method of treatment of injecting carbolic acid into the sac, and of radical treatment by excision of the sac.

Spontaneous cure, cure by means of applications to the skin of the scrotum, or by internal medication, very rarely takes place unless suppuration, followed by obliteration of the sac, occurs.

Treatment.-The only effective method of treatment is by surgical procedures of one or another kind. (For the technique of the surgical operations in cases of hydrocele see chapter on the Technique of Operations on the Testicle, etc.)

The surgical methods that are employed in cases of hydrocele are: (I) The palliative measure of tapping. (2) The radical ones of injection of the sac with irritating fluids (carbolic acid or tincture of iodine). 
Incision of the sac and drainage. (4) Excision of the sac. (5) Eversion of the sac. The results obtained by all of them are excellent. Cure rarely fails to follow the application of any one of the radical procedures. Tapping is an excellent temporary expedient, but, as has been said, it seldom cures the condition. The simplest and a very effective method of treatment is that of injecting the sac with carbolic acid or tincture of iodine.

Treatment by Injections of Iodine or Carbolic Acid into the SAc.-Of the two fluids, carbolic acid is to be preferred. Iodine injection is often painful, and is less certain in its results. (For manner of making the injections see chapter on the Technique of Operations on the Testicle.)

Little, if any pain, is caused by the injection. A few patients will complain of faintness if the procedure is carried out while they are standing upright, but this is unusual.

Rest in bed, with elevation of the scrotum, for one or more days is prescribed if much swelling follows the injection. The patient should not begin to go about until all pain has disappeared. It is rare to have severe pain follow the operation, but it does occasionally occur. It is to be combated by morphine and by the application of poultices to the scrotum. In a good many cases the patients do not feel pain at any time.

More or less redness and swelling of the scrotum is the rule. The fluid of the hydrocele sometimes re-accumulates in the sac shortly after the operation, in some instances. If the amount of the fluid that is thus poured out is enough to make it desirable to have it withdrawn, this should be done at any time after the first two or three days following the operation.

Obliteration of the sac by the formation of adhesions, brings about a cure in almost every case of simple hydrocele treated by this method. This is not true, however, of the cases in which any of the following conditions exists: (I) Much thickening of the hydrocele sac. (2) When the hydrocele is very large. (3) Hydroceles originating in other conditions (epididymitis). (4) Those in which there has been hemorrhage. In such as are the seat of inflammatory processes.

The procedure may also fail because of errors in the technique, such as not entirely emptying the sac before making the injection.

The method is as applicable to encysted or multilocular hydrocele (provided the operator makes sure that the injected fluid enters the separate sacs) as it is to simple hydrocele of the tunica vaginalis.

In the cases in which the conditions are present that make success by the method of injection improbable, the other radical measures may be successfully applied. 
Of the three radical methods of treating the hydrocele sac-incision and drainage, excision and eversion-we prefer the second, and do not approve of the first. The results obtained by excision of the sac of the hydrocele have been uniformly good in our hands, and we have, in conse-

FIG. I32

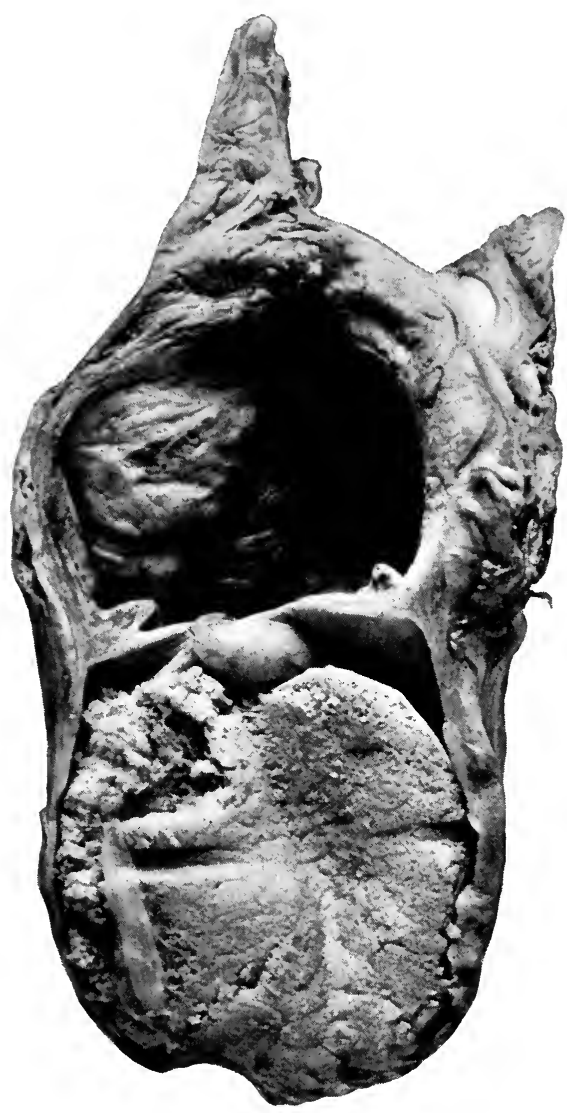

Encysted hydrocele of the cord. Note that the cavity is lying above the testicle along the course of the cord, but that the cord is continuous above the cyst. (Warren Museum.) quence, practised this method consistently in those cases in which injections are not appropriate, and in preference to that of eversion.

Encysted Hydrocele of the Cord.Etiology.-The condition has already been defined in an earlier part of the chapter. The etiology is obscure, as it is in many of the cases of the ordinary form of chronic hydrocele. In some instances the cavity of the sac represents an old hernial pouch of the peritoneum, which has been emptied of its bowel contents and become closed at its neck, so as to shut off its communication with the peritoneal cavity, and has then become distended by hydrocele fluid (Fig. 132).

Symptoms.-The subjective symptoms are generally insignificant. The objective ones differ but little from those of common hydrocele, except in the location of the accumulated fluid. There is a swelling, varying in size from that of a grape to that of a goose egg, situated between the external inguinal ring and the testis, with its long diameter parallel with the cord. The spermatic cord is situated slightly behind and internal to the tumor. Occasionally the effusion descends behind the testicle, displacing it forward, and it may extend into the inguinal canal.

Diagnosis.-Encysted hydrocele of the cord may readily be confused with congenital inguinal hernia. The hydrocele, however, is not reducible, nor does change of posture from the upright to the recumbent posture alter its character. The tumor is fluctuating and translucent, 
unless the sac is much thickened. The absence of tympany, impulse on coughing, reducibility of the tumor, and other characteristic evidences of hernia are absent in the case of hydrocele.

Treatment.-As with hydrocele of the tunica vaginalis, the treatment is wholly surgical. In children, simple tapping may result in a permanent cure, yet, even with them, the condition is prone to recur. Injection of the sac with carbolic acid is the best form of treatment in all simple cases.

Congenital Hydrocele.- Symptoms and Diagnosis.-This form of hydrocele is usually noticed soon after birth, and appears as a small swelling, extending from the inguinal canal to the bottom of the scrotum. If the fluid is in considerable amount, the testicle may not be distinguishable.

Hernia is often associated with this variety of the condition, and when the intestine is in the sac gives a tympanitic percussion resonance and gurgling of the intestinal gas and liquid contents, which serve to warn the examiner of its presence.

The characteristic feature of congenital hydrocele is the disappearance of the swelling upon pressure upon the scrotum. Translucency is present, unless it is obscured by the existence of an associated hernia.

Treatment.-Tapping and injection of the sac of the hydrocele are obviously improper measures to apply to this form of the condition, because of the communication with the peritoneal cavity. Spontaneous cure not infrequently takes place, and may sometimes be hastened by the wearing of a well-fitting truss.

Infantile Hydrocele.-Treatment.-The best treatment to apply to this form of hydrocele, if it recurs after tapping, is injection of the sac in the manner already described in connection with the common form of the condition.

\section{HEMATOCELE.}

The term hematocele is rather general, including all tumors of the tunica vaginalis, spermatic cord, and testis, which are, in part or wholly, composed of blood. More specifically, a hematoma of the tunica vaginalis is termed a vaginal hematocele; when in connection with the spermatic cord, an hematocele of the cord; and within the testicle itself, a parenchymatous hematocele. This latter condition is more often spoken of as a hematoma of the testicle.

Etiology.-The etiology of hematocele of the tunica vaginalis and of the cord is the same. The process may be either acute or chronic.

The acute form is by far the most common, and is due to trauma. The effusion of blood may follow the removal of a large amount of hydrocele fluid, the blood oozing from the capillaries, which rapidly become dis- 
tended on being relieved of the pressure, as sometimes occurs after emptying an overdistended urinary bladder. Hematocele may also result from injury of a bloodvessel by the trocar in tapping a hydrocele. Following operations within the scrotum, hematocele may result if all

Fig. 133

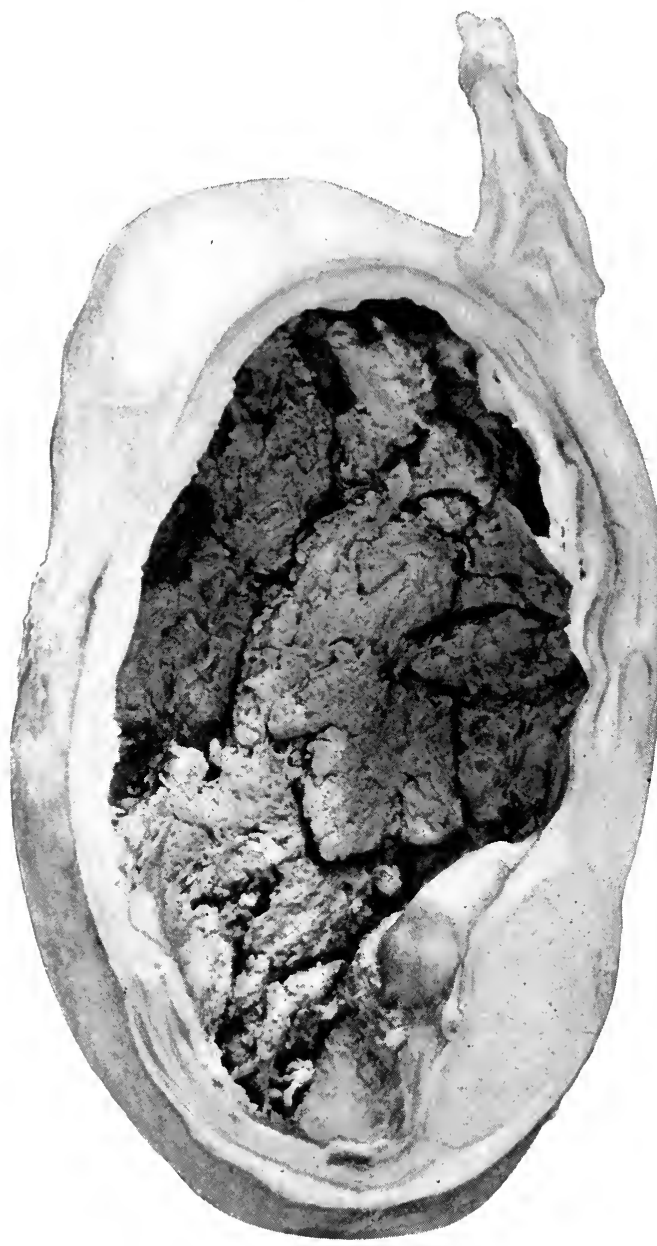

Hematocele. The walls of the tunica vaginalis are thickened; the cavity dilated and filled with old blood clot. Testicle atrophied. (Warren Museum.)

bleeding has not been arrested, or if the testicles are not supported by a firm bandage.

Acute hematoma develops rapidly, and the scrotal skin usually becomes discolored and occasionally œdematous.

The chronic form of hematocele is comparatively rare. There is generally some predisposing cause, such as hemophilia or scorbutus.

Hemorrhage may take place spontaneously in an already existing hydrocele, changing the character of the fluid.

Genouville and Pasteau ${ }^{24}$ report a case in which the circumference of the scrotum was 85 $\mathrm{cm}$., and the contents more than 9 liters.

Pathology.-If the fluid is not removed, changes take place in the sac, causing it to become thickened. There is not only a true hypertrophy of the walls of the tunica vaginalis, but the blood becomes clotted (Fig. I33), and may be deposited upon its inner surface in laminæ, in a manner similar to that seen in certain aneurysms. The testicle becomes atrophied because of the resulting pressure.

Symptoms.--In the acute form, the symptoms develop rapidly, and are dependent, in some degree, upon the etiological factors producing the condition. Pain and tension of the parts are always present, and the constitutional reaction may be severe. Objectively, the scrotum is 
enlarged, ecchymotic or œdematous, and tender. Crepitation can sometimes be felt. The tumor is not translucent. Suppuration rarely occurs; when it does, the evidences of inflammation appear.

In the chronic form of the malady, the subjective symptoms are slight, as a rule. The swelling develops painlessly and gradually. Objectively, the swelling is pear-shaped, as in the common form of hydrocele. The surface is smooth and the tumor is elastic to touch. Fluctuation can seldom be detected, because of the thickening of the sac. The tumor is never translucent.

Diagnosis.-In the acute form, the history will usually suffice to make the diagnosis clear. In the chronic form, the task is not so simple. The history may be of little service in distinguishing it from common hydrocele, and the absence of translucency is not a sufficiently reliable feature to trust.

Having proved that hernia is not present, the aspirating needle may be employed for diagnostic purposes, always remembering when using it that the testicle lies posteriorly in the scrotum and a little below its middle. The characteristic testicular pain may be elicited by pressure over this area. In making the diagnostic tapping, too small a trocar may fail to allow the exit of blood through it, and it is better to use a moderately large one on this account.

Prognosis.-The testicle may become atrophied if the intrascrotal pressure is not relieved.

The prognosis with respect to cure is good, provided the sac is removed and renewal of bleeding is guarded against.

Treatment.-In the acute form, the patient should be kept absolutely at rest in bed with the testicle elevated and supported by a tray. Cold compresses or an ice-bag should be applied until the swelling begins to diminish, when a suspensory bandage should be applied and the patient allowed to get up. If the swelling persists, open incision, evacuation of the sac, and its removal are the best measures to apply.

If suppuration occurs, immediate incision and drainage are indicated.

In the chronic form, no other treatment than opening and removing as much of the sac as possible is of value. If operation does not seem wise, because of the existence of hemophilia or scorbutus, these conditions should receive appropriate treatment, and the palliative measures of supporting and compressing the scrotum should be substituted for the radical surgical ones.

\section{SPERMATOCELE.}

Spermatocele is a cyst situated at the head of the epididymis, and having a fluid contents with spermatozoa in it. 
Etiology.-There is some difference of opinion with regard to the manner in which these cysts are developed, one view being that they originate in vasa aberrantia, or the hydatid of Morgagni, while the other is that they represent retention cysts merely, produced by occlusion at a point beyond the vasa efferentia, in consequence of which they dilate.

The cyst is usually situated behind the upper part of the testis, and at the junction of the epididymis and testis. It extends, as it increases in size, either downward or upward along the cord. In the former case, it may be situated extravaginally or intravaginally.

These cysts sometimes attain a large size.

Another variety is that which is seen in adult life in the form of multiple cysts, which frequently contain spermatozoa. They are connected with the epididymis, and their rupture may give rise to spermatic hydrocele.

Symptoms.-The subjective symptoms of spermatocele are of a mild character. The tumor is usually discovered by accident.

The Objective Symptoms.-The condition usually occurs in young adults, and is first noticed as a small, tense, elastic tumor at the upper and back part of the testicle. Its growth is very slow. The cyst is often too tense to allow fluctuation to be detected. If it is small, its translucency may be impossible to detect. The situation of the cyst is the important diagnostic feature under these circumstances.

Diagnosis.-This will depend chiefly upon the location of the cyst, which serves to distinguish it from the common form of hydrocele. It is often impossible to distinguish it from an encysted hydrocele of the cord, without aspiration of the fluid contents. The turbid appearance of the fluid of a spermatocele then shows at once the nature of the condition.

Treatment.-The treatment should be injection of carbolic acid into the sac, or excision of it. Tapping rarely results in a cure.

\section{GALACTOCELE.}

Galactocele (chylocele) is a collection of chyle in the cavity of the tunica vaginalis. The chyle is exuded from the lymphatics of the spermatic cord.

Etiology.-Chylous hydrocele is a rare condition, usually met with in the tropics. It is the result of an obstruction at some point in the lymphatic system which produces a stasis of lymph in the vessels distal to it. The essential etiological factor is almost invariably the Filaria sanguinis hominis. The pressure within the lymphatics of the cord may be so great as to cause them to rupture, with the result that the cavity of the tunica vaginalis is filled with chyle. Manson, in 62 cases of filariasis, found 6 in 
which there was chylous hydrocele. Flynn records 12 in a series of 50 individuals with filariasis. Filarial embryos have been demonstrated in chylous hydrocele fluid. Galactocele is frequently associated with other manifestations of filarial disease, such as lymph-scrotum or elephantiasis of the penis or of the lower extremities.

Symptoms.-Galactocele has but little translucency. Other than this and the presence of associated manifestations of filariasis, the symptoms do not differ from those of ordinary hydrocele.

Treatment.-To be anything more than palliative, the treatment must be directed to the removal of the parent worm. Simple tapping of a chylocele is of but little value. The sac quickly refills. In some instances the adult filariæ have been found in the lymphatics of the cord. The surgical treatment should include the excision of all dilated lymphatics of the cord. It is useless to remove the tunica vaginalis, for this structure does not participate in the disease.

\section{BiBLIOGRAPHY.}

I. Fisher. American Journal of the Medical Sciences, February, I839.

2. Lane. British Medical Journal, I894, ii, I24I.

3. Haller. Elementa Physiol. Corporis Humani.

4. Cunningham. American Journal of Dermatology and Genito-urinary Diseases, December, I907.

5. Odiorne and Simmons. Annals of Surgery, December, I904.

6. Murphy. Boston Medical and Surgical Journal, vol. clxiv, No. 2, p. 33.

7. Bruns. Mith. aus der Chir. Klin., Tübingen, I 884, iii, 483 .

8. Scudder. Annals of Surgery, I90I, xxxiv, 234.

9. Rigby and Howard. Lancet, May 20, 1907.

ı. Fournier. Blennorrhagie, Dict. de Méd. et de Chir. Prat., p. 2 I I.

II. Brönnum. Hospitalstidende, Copenhagen, No. 46, I907.

12. Benzla. Arch. f. Dermat. u. Syph., i 898, xlv, p. 33.

I3. Burnett. Morrow's System of Genito-urinary Diseases, vol. i, p. 9I4.

14. Keyes. Genito-urinary Diseases, 1905, p. 226.

I5. Belfield. American Association of Genito-urinary Surgeons, I 906.

I6. Taylor. Genito-urinary Diseases, I904.

17. Keyes. Genito-urinary Diseases, I905.

18. Kober. American Journal of the Medical Sciences, I899, cxvii, p. 535.

19. White and Martin. Genito-urinary and Venereal Diseases, I907.

20. London Lancet, I885, p. 748 .

2I. Park. Journal of Cutaneous and Genito-urinary Diseases, I895, xiii, p. 36 I.

22. Leigh. Ex. Citationes Quinque Oxonii, I697, p. 80.

23. Marsinna. Neue Med. Chir. Beobach., Berlin, 1796.

24. Genouville and Pasteau. Transactions of the French Urological Association, I 898 , viii, 445 . 
THE SEMINAL VESICLES.

\section{ANATOMY OF THE SEMINAL VESICLES.}

The seminal vesicles (Fig. 134) are two, lobulated pyriform pouches, each about two inches long, situated between the bladder and the rectum, and lying one on either side of the median line of the posterior surface of

FIG. I34

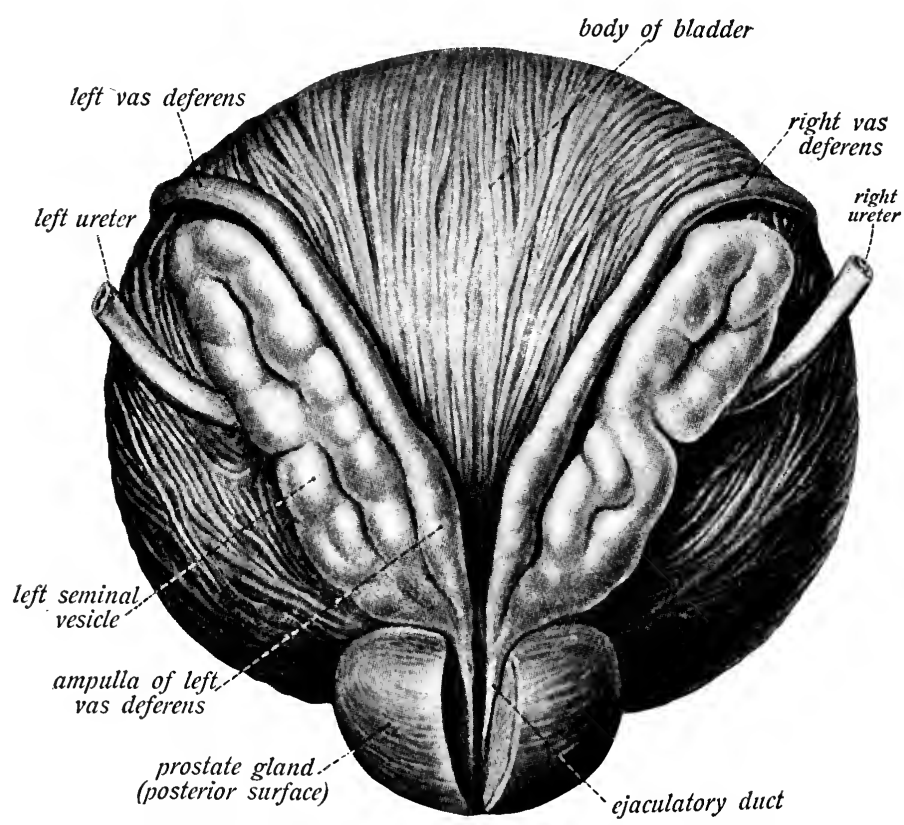

The urinary bladder with the seminal vesicles, the ampullæ of the vasa deferentia and the prostate seen from behind and below. The prostate is partly divided longitudinally. (Sobotta).

the former. They are closely connected with the bladder by their anterior surfaces, while posteriorly they are separated from the rectum by a portion of the vesical fascia, by areolar tissue, and by the recto-vesical fascia.

According to W. G. Richardson, ${ }^{1}$ in his admirable monograph on the Development and Anatomy of the Prostate Gland, the vesical fascia, as it descends from above over the posterior surface of the bladder, divides 
on reaching the seminal vesicles into two layers, one of which passes in front of them, interposing between their anterior surfaces and the bladder wall proper, while the other extends behind them. These two leaves of the fascia unite laterally on either side, close to the external borders of the vesicles, and thus form a compartment enclosing within it the vasa deferentia and the vesicles. Below, the fascia joins the capsule of the prostate, and is so closely connected with the vesicles in the lower part of their course as to make them appear to be attached to the prostate. The anterior leaf of the fascia is but loosely united to the bladder wall in its lower segment, so that it is easily separated from it, but is more adherent to the surfaces of the vesicles. There is no difficulty in separating the posterior layer from the rectum and its coverings. These facts have a practical bearing in operations for the removal of the vesicles.

Each vesicle lies to the outer side of the corresponding vas deferens, and to the inner side of each of the ureters, overlying, however, the latter in part. A layer of fibrous, fatty tissue intervenes between the ureters and the vesicles, joining them loosely together.

The body of each of the vesicles tapers from above downward, and terminates in a duct at its lower end, which joins with the vas deferens of its corresponding side to form the common ejaculatory duct of that side.

The ejaculatory ducts are each about three-quarters of an inch in length. They enter the prostate a little below its upper posterior border, and, traversing its posterior commissure in an upward and forward direction, terminate in two narrow orifices, one upon either side of the verumontanum. The portion of the prostate which is traversed by these ducts is largely made up of muscular and fibrous tissue, and is more or less definitely differentiated from the glandular structure of the organ. It has been said that in the condition of senile hypertrophy of the gland the ejaculatory ducts become more distinctly remote from the gland tissue proper, and it is therefore possible to enucleate the gland by the operation of prostatectomy without necessarily lacerating or destroying the ducts. This we know to be true, because of having demonstrated it in one postmortem examination after the performance of prostatectomy, and from clinical evidence in some of our cases in which the later results of the operation seem to indicate that the ducts have not been seriously injured, or have escaped all injury. Other observers have claimed to avoid all injury to the ducts when doing prostatectomy, and have brought forward evidence to substantiate the claim, e.g., Young, Goodfellow, and others.

The upper part of the seminal vesicles is covered by the peritoneum.

Structure.-Each seminal vesicle is in effect a single pouch composed of several convolutions which represent a number of pockets or sacs, all 
of them being held together by connective tissue in such a way as to become one body. Numerous ampullæ or diverticula are given off from the inner surface of the vesicle and are separated from each other by more or less well-defined partitions of connective tissue (Fig. 135). The interior of the vesicle is lined with mucous membrane, provided with epithelial cells, which secrete a fluid of a special character.

The walls consist of an outer fibrous coat, a central muscular one, which is made up of both circular and longitudinal fibers, and an inner lining, which is mucous membrane. Except the mucous membrane, these layers are not clearly defined.

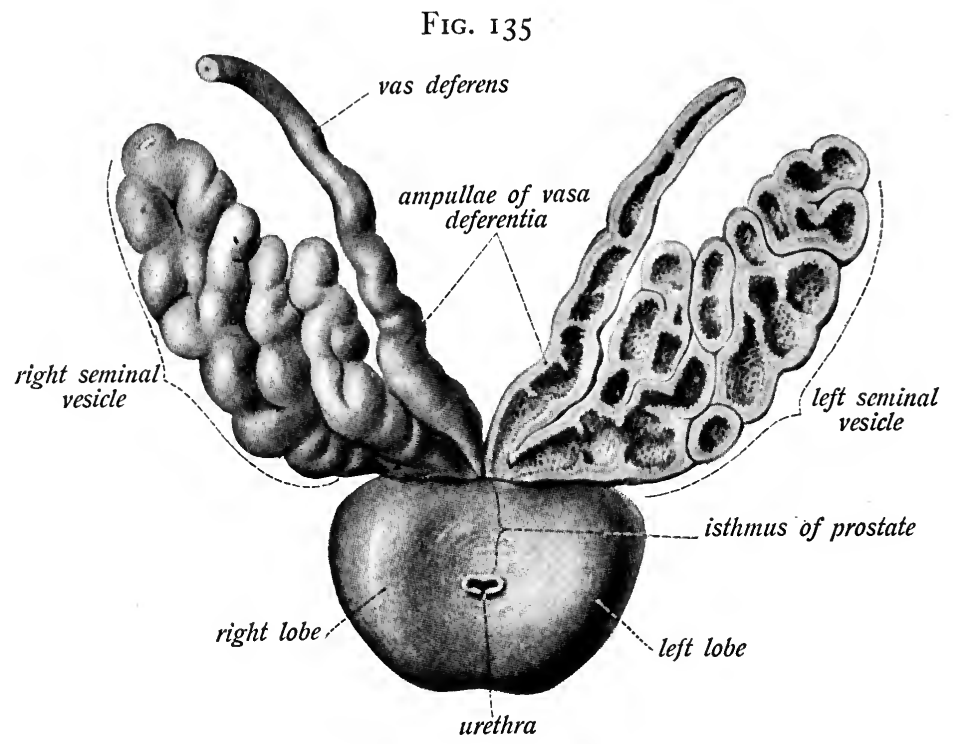

The prostate and the seminal vesicles seen from above and in front. The left seminal vesicle, together with the ampulla of the vas deferens, has been divided longitudinally and the urethra has been cut off close to its exit from the bladder. (Sobotta.)

Bloodvessels.-The arteries supplying the vesicles are derived from the artery of the vas deferens in part and in part from the inferior vesical and middle hemorrhoidal arteries.

The veins of the vesicle form a separate plexus, and are given off from the prostatic plexus. The ejaculatory ducts receive their blood supply from the vessels of the prostate.

\section{PHYSIOLOGY OF THE SEMINAL VESICLES.}

It was formerly believed that the seminal vesicles served merely as storehouses for the spermatozoa-laden testicular fluid during the intervals 
between the times of sexual congress and the accompanying ejaculation of the seminal fluid from the vesicles, until once again refilled by a further supply of the testicular product.

This view has long since been modified, and it is known that the vesicles contribute a special secretion, which is derived from the epithelial cells of the inner lining and some glandular bodies which occupy the mucous membrane. This secretion is a more or less essential part of the composite seminal fluid.

\section{ABNORMALITIES OF THE SEMINAL VESICLES.}

Malformations of the seminal vesicles are generally associated with congenital defects of other parts of the genito-urinary tract. They have but little clinical importance.

Absence of the Vesicles.-Absence of the vesicles is more frequently unilateral than bilateral. When but one vesicle is absent, there is apt to be absence of one or more of the following parts of the same side: the vas deferens, testis, ureter, and kidney.

In the cases of absence of both vesicles, the testes may be absent or atrophied, and the prostate is also reported to have been absent.

Fusion of the Vesicles.-This condition is recorded. When it occurs, the vesicles are fused together, and occupy the median line of the back of the bladder.

Multiple Vesicles.-The existence of multiple vesicles has been reported, but it has been denied by Guelliot and the accuracy of the observation doubted.

Unusual Sites of the Outlets of the Vesicles.-The vesicular outlets have been seen to occupy the following unusual sites: emptying into the ureters; the ejaculatory ducts passing along the urethral wall and opening upon the glans penis or at the external urinary meatus.

In some cases occlusion of the ejaculatory ducts has been observed.

\section{INJURIES OF THE SEMINAL VESICLES.}

Injuries of the vesicles are exceedingly rare, owing to their protected position. Keyes quotes Guelliot as stating that there is but one case of accidental injury of these bodies recorded. In that instance it was associated with a fracture of the ischium.

The writer has seen one instance of accidental injury of the vesicles. This occurred in the case of a sailor who fell from the yard of a vessel 
astride a belaying pin, which was set upright upon the rail of the ship's bulwarks. The blunt end of the pin struck the perineum and passed into the pelvis behind the bladder, lacerating the vesicles among other parts which were seriously damaged.

Injury to the ejaculatory ducts is commonly inflicted in the performance of the operations of perineal lithotomy. If both of them are involved in the injury, sterility, because of subsequent occlusion, takes place, and is a serious objection to such of the operations as those in which it is likely to happen, especially in the case of children or young adults.

\section{DISEASES OF THE SEMINAL VESICLES.}

Tuberculous disease is included in the chapter on Tuberculosis of the Genito-urinary Tract.

Vesiculitis, Spermatocystitis, Inflammation of the Vesicles.-The most common disease of the seminal vesicles is inflammation. It occurs in both acute and chronic forms. As a rule, the latter represents the sequel of the former condition. It may, however, occur independently of it as a chronic manifestation throughout.

Etiology.-Inflammation of the seminal vesicles originates almost invariably from an extension of an already existing inflammation in the posterior urethra along the ejaculatory ducts and so on to the vesicles themselves. Occasionally it arises from an inflammatory or suppurative condition of the prostate or bladder, and it is believed that it may be produced, in a certain number of cases, by hematogenous infection or by direct invasion of bacteria from the rectum. In the latter class, this belief rests chiefly upon the fact that cases have been observed in which there has been no posterior urethritis and in which the Bacillus communis coli has been found in the vesicle, apparently being, in these instances, the infecting organism, and having migrated from the bowel or been taken to the vesicle through the blood current.

In the large majority of cases the infection is of gonorrhœal nature. In a few it is due to other pyogenic organisms.

The vesicle may become involved in the course of acute gonorrhœal posterior urethritis or at any time during the progress of a chronic gonorrhœal inflammation in the same situation. In the latter case, the vesicular inflammation may proceed as a chronic extension of the original trouble, or it may assume an acute or subacute form from the beginning. It is usually seen in the milder or chronic guise.

The agent in the production of the infection of the deep urethra from which vesiculitis originates may be an instrument which is unclean or which is used roughly or unskilfully in the urethra. 
The chronic and also the subacute variety of the disease is sometimes caused by sexual excesses, in the absence of any specific infection. It is more often excited or renewed by such excess when there has been a preceding urethritis. The latter may have been apparently wholly quiescent for a long time past, or the patient may have believed that it no longer existed, while, as a matter of fact, there has persisted a small area of tissue which still harbors the residue of an old gonorrhoal inflammation, and from which there starts forth a new and active process, extending to the vesicle on one or both sides.

Freeman ${ }^{2}$ reports the occurrence of vesiculitis following typhoid, and, in the absence of any other demonstrable point of departure, it is believed that the infection here takes place hematogenously and as a sequela of the fever.

The exciting cause may be anything which lights up an old process in the posterior urethra or prostate-such things, for example, as bicycling and horseback riding. We have had one patient who produced four distinct attacks of vesiculitis of subacute character in this way by riding a horse. There was a long interval between the second and third attack, and there had been apparently an entire subsidence of all trouble before its recurrence on each of the four occasions. No other exciting cause could be traced in this instance. We do not believe that vesiculitis ever originates in such exercises as those just referred to, unless there has been a preceding inflammation at some time in the past either of the vesicle or the deep urethra, which, even though believed to have been cured, has, in fact, still lurked in some corner of the deep urethra or adjacent tissues.

Pathology.-As a rule, but one of the vesicles is involved in an inflammatory process at one time. Sometimes both are attacked simultaneously or in more or less quick succession.

The force of the inflammation is at first expended upon the interior of the vesicle, but very rapidly involves the whole structure, and in the acute form of the condition invariably extends to, or in some degree implicates, the adjacent perivesical tissue.

The occupation of the inner lining of the sac by the process results in a desquamation of its epithelial covering and a round-cell infiltration of the mucous membrane, which soon extends to the other parts of the vesicle, producing thickening of them. Outside the vesicle an exudation takes place, which clinically appears in the guise of a more or less extended œdema, plainly palpable by the examining finger in the rectum, and, when at all marked, obscuring the outline of the prostate and the vesicles so that they cannot be made out by touch. The inflammatory process, when acute, may be so pronounced in extent and in character as to attack 
the more distant parts of the neighborhood, sometimes involving the peritoneum in its ultimate progress. Suppuration may take place in the areolar tissue between the vesicles and the rectum, and a few instances have been reported in which pus has broken through into the peritoneal cavity with fatal result. Others have occurred in which septic absorption has taken place from such an area of suppuration, and the dissemination of the infection to other parts of the body has produced a general septicemia or pyemia.

Again, there may be an extension of the inflammation to the vas deferens and through it to the epididymis and testis. Here, however, it is usually difficult to attribute definitely the starting point of the malady in those parts to the vesicle, and it is generally the case that a posterior urethritis is responsible for the inflammation of the vas, epididymis, testis, and of the vesicle as well.

The outlet of the vesicle is very apt to become temporarily occluded by being plugged with the detritus and exudation resulting from the inflammation. Under these conditions, there is greater or less distention of the vesicle, and cases have been reported in which it has ruptured.

As a rule, the acute inflammatory process ends in resolution, and either the chronic form succeeds it or complete restoration to a normal state takes place. The continuance of the condition in a chronic form is certainly exceedingly common, and it is difficult to pronounce with any certainty that a restitutio ad integrum has taken place in any case whatever.

In the chronic form of the malady a more or less well-marked induration in, and sometimes about, the vesicle remains for a longer or shorter time. Some degree - often considerable - of distention of the sac or sacs remains, and there is a frequent obstruction of the ejaculatory ducts present in many instances. True stricture of the common duct is, however, not observed. The sac's interior lining is often the seat of one or more areas of granulation tissue, and a chronic thickening of the mucous membrane exists.

Ultzmann $^{3}$ pointed out thirty years or more ago certain special qualities presented by the spermatic fluid in cases of vesiculitis. In some, the fluid has a well-marked blue color from the admixture of indigo; a reddishbrown or yellowish color shows the admixture of blood and of pus in the fluid, and each of these color changes denotes the presence of an inflammatory process in the seminal vesicles.

Symptoms and Diagnosis.-Vesiculitis is generally associated with inflammations of the prostate or deep urethra, or both. It consequently becomes difficult to determine the precise source of the symptoms to which they give rise, especially as they closely resemble each other. 
Jacobson quotes Zeissl as asserting that there is one symptom which belongs exclusively to vesiculitis, namely, constant erections, which are often painful. We cannot say that this accords with our own experience, though the symptom, it is true, does frequently exist, and is associated with a good deal of sexual excitability. An evidence which we regard as more suggestive of the presence of vesiculitis is the occurrence of a dull pain immediately following defecation or semina emission, situated in or about the rectum and often accompanied by a sickening sense somewhat similar to that experienced by one who has received an injury of the testicle. Another sign of the condition belonging to inflammation of the vesicles, though no wise limited to it, is sexual excitability. This is pronounced in the earlier stages of the acute condition, and is noticeable from time to time in the course of the chronic form of the malady. The staining of the seminal fluid with colors (see under the heading of Pathology) or the presence of blood in it are exceedingly characteristic evidences of the condition. In the acute form, the physical examination gives pretty clear evidence of the presence of vesiculitis, though it is, in some respects, difficult or impossible to discriminate between it and prostatitis in the acute cases.

Acute Vesiculitis.-The onset of acute vesiculitis is ordinarily sharp, and the symptoms severe, and are: Fever, occasionally ushered in by a chill, coated tongue, and constipation. Frequent and painful urination and defecation are almost always present. Pain is augmented by both acts. Erections are frequent, sometimes constant. They are often painful. Pain in many cases radiates toward the hip, upward over the abdomen, and exceptionally even to the upper lumbar region. When in the last two localities referred to, it may simulate appendicitis, and perhaps might suggest renal colic. The pain is occasionally so severe as to imply the presence of peritonitis. That condition rarely occurs as a complication of the disease, but it may be closely simulated. In these instances a sign which is generally present when the process extends far upward, and especially when it involves the peritoneum, is tenderness on pressure upon the abdomen at a point about two inches within and below the anterior superior spinous process of the ilium.

Rectal examination supplies the most characteristic evidences of the existence of acute inflammation of the seminal vesicles. There is marked pain on the introduction of the finger, which comes upon a hot, tender, swollen area, most marked near the upper part of the prostate and above it, and extending beyond the reach of the finger. The outlines of the prostatic lobes and of the lower ends of the vesicles-ordinarily felt clearly-are lost; they are replaced by an almost even swelling, which has the feeling of an odematous tissue, and which is one in fact. If but 
one vesicle is involved, the swelling will be chiefly confined to the affected side; if both of them are implicated, this distinction does not exist. It is often impossible to determine whether the rectal conditions presented are connected with the prostate or not.

Chronic Vesiculitis.-The symptoms of the chronic form of the inflammation are as follows: Gleet. A gleety discharge appearing at irregular intervals, and especially when it is found, upon endoscopic examination, not to proceed from any urethral lesion, is very apt to be the product of a chronic inflammation of the seminal vesicles. This is a particularly important fact to bear in mind in order to avoid the error of assuming that all gleety discharges must be due to a chronic process in the urethra-areas of granulation tissue or stricture-and because the knowledge that they often do arise from the vesicle will aid in leading to the discovery of the true seat of the trouble

Microscopic examination of the discharge will also enable the surgeon in many instances to trace it to its true source. It contains spermatozoa - usually dead-pus, and, on standing, will show the spermatic crystals. Associated with these elements there are shreds of mucus, epithelial cells, and often more or less blood corpuscles. There is nothing essentially characteristic in any one of these per se with relation to the condition, but their association is very suggestive of it.

Pain.-The one almost pathognomonic sign of chronic vesiculitis is, as has been mentioned, the more or less frequent occurrence of a dull pain in and about the anus and rectum, accompanied by a sickening sense similar to that felt when the testicle is injured. This pain is always connected with defecation or with the ejaculation of the semen. It is not an invariable symptom, and in many cases it is absent. When present, it is exceedingly characteristic of chronic vesiculitis. It has been ascribed to cramp of the muscular fibers of the vesicle, excited by the sexual orgasm or the passage of feces. Whether or not this is the true cause, no one can positively assert.

Disturbance of Urination.-Frequent urination and more or less teasing desire to urinate is one of the symptoms frequently present in the chronic form of the malady. Inasmuch as there is almost always a certain degree of prostatitis or chronic posterior urethritis associated with the vesiculitis, it is not easy to assert that these urinary manifestations are to be referred to the condition of the vesicle exclusively, and in all probability each of the parts named, when implicated, has a share in their production.

Nervous Symptoms.-More or less well-marked nervous disturbances have been ascribed to the existence of vesiculitis. Such manifestations are, in our opinion, far more closely related to chronic inflammations of 
the deep urethra and of the prostate gland than to the same conditions involving the vesicles. The peculiar sexual hypochondria, so strikingly exemplified in patients with chronic prostatitis and obstinate inflammations of the prostatic urethra, we have not noted as being so conspicuous in cases in which the vesicles alone were the seat of the disease, or in which they were the predominating element when associated with the urethral and prostatic conditions named.

Sexual Symptoms.-In the earlier stages of a chronic vesiculitis, sexual desire is ordinarily stimulated; later, it is diminished, and finally, in a considerable number of the patients, it ceases altogether. The sexual power and ability to have complete erections is likewise, ultimately, in greater or less degree interfered with, and complete impotence may result.

Escape of Seminal Fluid from the Ejaculatory Ducts or Spermatorrhea.-This is a very common accompaniment of chronic vesiculitis, and is due to a loss of tonicity in the muscular fibers immediately connected with the ejaculatory ducts, and in part, to a greater or less thickening of their orifices, whereby they become more patent than normal, and permit the escape of the fluid from the vesicles and ampullæ of the vasa deferentia.

The escape of this fluid is often limited to the times of defecation and sometimes to the end of urination, when the last drops of the expelled urine will be found to contain a certain amount of the spermatic fluid. This gives to them a gummy consistency, which is easily appreciated by the touch. The patient is very apt to have a definite and unusual sensation of tickling, or a slight irritability in or along the urethra accompanying the passage of the last drops of the urine when the vesicular discharge is present.

The prostate very often gives out a similar discharge in cases of chronic prostatitis, and the two fluids are so much alike as to require microscopic examination to discriminate one from the other. The presence of the spermatozoa determines the source of the fluid when it comes from the vesicles and the vasa; their absence does not, however, exclude the vesicle as the place from which it proceeds.

Rectal Examination.-In chronic inflammation of the vesicle, the sac is usually harder, less elastic, and more irregular in contour than normal. It is usually enlarged, and can be more clearly felt in the rectum than when in its normal condition.

In some instances there is a considerable thickening of the perivesical tissues, and when this is the case, the outline of the vesicles is lost, and they are difficult to define by the finger in the rectum. It is hard to determine, without a good deal of practice, whether the enlargement of 
the vesicle felt by the finger is due to its being distended by a large amount of fluid, or if it be due to thickening of the walls of the sac.

Relapses in Cases of Urethritis and Epididymitis.-In some cases of inflammation of the deep urethra relapses are to be accounted for by recurring infections proceeding from the seminal vesicles.

Prognosis.-In the acute form of the disease, the prognosis is good, with but few exceptions. There is very rarely any danger to life. With respect to cure, however, the prediction is less assured, owing to the proneness to become chronic evinced by the condition, and to the persistence of it in the latter form in many instances.

In the chronic form of the malady the prognosis is usually good. The chief danger from the condition lies in the possibility which always exists in the presence of a focus of infection in the genito-urinary tract, of its making infectious excursions to other parts of it. With respect to cure, the prognosis depends largely upon the intelligence with which the condition is treated, and upon its recognition.

Treatment.-Acute Vesiculitis.-The treatment of the acute form of the malady consists in the following measures: Absolute rest in bed, hot rectal injections, provided they obviously give marked relief to the pain and swelling of the vesicles and the perivesical tissues; otherwise, they should not be employed after a failure to accomplish this purpose. We do not counsel the use of cold in the rectum, or in the form of hip baths at any stage of the acute condition. We advise against the employment of urethral irrigations during the same time. All local urethral treatment should be avoided. The only instrument that should enter the canal in an acute vesiculitis is a catheter, and only when its employment is necessitated by actual retention of urine. If this procedure becomes imperative for that reason, the urethra should be carefully irrigated beforehand with a I to 4000 solution of permanganate of potash, and a small soft-rubber catheter only should be used for the purpose of emptying the bladder.

Salol and phenacetin by mouth, and morphine and belladonna by rectum, in the form of suppository, are indicated to relieve pain. Rectal digital examinations and all local treatment in the rectum, except that of the hot rectal irrigations, should be carefully avoided. High enemata may become necessary to procure movements of the bowels, for constipation and the passage of hard feces over the inflamed area should be avoided.

Surgical Treatment.-This may be demanded because of suppuration, either of the vesicle or perivesical tissues. The evidence of the occurrence of this condition will usually be an additional rise of the patient's temperature, and either an extension of the area of pain or greater severity 
of it. The rectum becomes exquisitely sensitive to digital examinations, and in many instances they cannot be conducted without anesthesia. If there is such intense pain, suspicion of the formation of an abscess is often justified. A rectal examination should be made in the presence of this symptom, with the patient anesthetized, and with his permission beforehand to proceed to operation if, on examination, it becomes necessary to do so.

Pus arising in connection with the acute form of vesiculitis should be evacuated at the first available moment after its presence has been determined. The best manner of accomplishing this is by a perineal operation. We prefer that which allows free access to the abscess and free drainage through the perineum after it has been opened. These desiderata are best secured by laying open the space between the rectum and posterior surface of the bladder, as is done by the technique of Proust in connection with the operation of prostatectomy. (See chapter on Technique of Operations on the Prostate.)

Chronic Vesiculitis. - The chief objects to be sought in the treatment of the chronic form of the disease are: (I) To free the ejaculatory ducts and to drain the vesicles of their pathological secretions; (2) to bring about resolution of the exudate in the walls of the vesicle and the surrounding tissues.

The first as well as the second of these is secured by manipulations of the lower parts of the sacs and their ducts, which should include also the ampullæ of the vasa deferentia. These manipulations are made digitally, except in a few instances in which the vesicles are placed very high, or in which they are made less accessible than usual because of there being a narrow pelvic outlet and a very fat perineum. In such cases it is sometimes necessary to express their contents with an instrument of special form.

Manner of Applying Massage to the Vesicles.-The points to be observed in carrying out this treatment are these:

I. To apply sufficient pressure to thoroughly express the contents of the ducts and vesicles, while, at the same time, avoiding such force as to incur the danger of setting up further inflammation, which is liable to follow upon too violent manipulation.

2. To avoid too frequent applications of the treatment, while not making too long intervals, on the other hand, between the sittings.

3. To apply the manipulations intelligently, i. e., for the right length of time, in the right manner, and in accordance with the local conditions presented and the patient's subjective symptoms.

It is impossible to describe in words what is the right and what is the wrong amount of pressure to be exercised by the operator; that is a 
matter that everyone must learn for himself. If the two indications stated above be used as guides, viz., to secure the expression of the contents of the ducts and the lower part of the sacs, and not to excite renewed inflammatory action, the beginner will not go far wrong. To secure the first, the finger must reach sufficiently high up upon the posterior parts of the vesicles, and the movement made in "stripping" them is to be in the line of their course and from above downward. The first pressure should be stopped a little short of the prostatic urethra, the last ones should be carried over its entire length. The prostate should be passed across from its outer sides to its middle line in making the movement. The bladder, it is generally advised, should be full. This certainly does not bring the seminal vesicles nearer to the anal margin, or, in other words, it does not make them more accessible to the finger tip than when the bladder is empty; on the contrary, it raises them higher up in the pelvis, but it does give a firmer background for the finger to press against when massaging them; hence it is advantageous.

The position of the patient may be either recumbent, or, as it is usually advised, leaning forward across the rim of the table or over a chairback, or in the knee-elbow position upon the table. The best position for him to assume is that which will enable the individual surgeon to reach and massage the vesicles and their perivesical tissues most efficiently.

The vesicles will be found to be more readily reached if the bladder is pressed downward toward the rectum and perineum by the operator's free hand, from above the symphysis pubis. The urine should be voided immediately after the vesicles have been emptied of their contents, in order to note whether or not this has been successfully accomplished. The seminal fluid for the most part will be found in the urine in the form of gelatinous-looking masses of various sizes and a cloudy sediment which settles quickly. There is sometimes more or less pus mixed with the secretion of the sacs, and it may be streaked with blood. Some of the expressed fluid may appear at the meatus and a little blood also sometimes shows in the same spot.

The intervals between the sittings should ordinarily be about four days, but no hard-and-fast rule should be adopted in this respect. The times of the sittings should be regulated according to the indications furnished as the case progresses.

Relief of symptoms is generally obtained early in the course of the treatment. Cure rarely comes quickly, and the patient should be advised of this at the outset, and also that success will depend in large measure upon his care in following out the directions given, especially with reference to the regularity of his attendance. 
The general condition of the individual is of importance, and it should be supported by appropriate measures of hygiene.

Horseback riding, and bicycling should be prohibited, as should all violent exercises. Moderate exercises should be urged upon the patient, however. Except in the case of anemic and debilitated persons, alcohol should be interdicted.

\section{CALCIFICATION AND CONCRETIONS OF THE VESICLES AND DUCTS.}

Calcification of the vesicles, either alone or in association with the same condition of the vasa deferentia, has been observed, but is rare.

Etiology.-The causal factors in the production of this condition are: preceding inflammation of the sacs and ducts, and the changes incident to old age. Chiari ${ }^{4}$ records two cases of calcification of the vas deferens and one of calcification of the seminal vesicles. George ${ }^{5}$ reports two other instances of the same condition.

In one of George's cases the inferior third of the right vesicle was obliterated. Calcareous infiltration of the cellular substance and the outer wall of the vesicle was present, and the adjacent connective tissue was sclerotic. Chiari found that the application of sulphuric acid to the calcified areas was attended by the evolution of gas, and that crystals resembling those of calcium sulphate were formed.

Klebs, ${ }^{6}$ Rokitanski, $^{7}$ and Orth ${ }^{8}$ believe that the calcification of this part occurs as the result of chronic inflammatory processes.

Lallemand ${ }^{9}$ has recorded a case of suppuration, induration, and calcification of a previously chronically inflamed vesicle, and Guelliot records one following repeated inflammations of the vesicle in a tuberculous patient.

Calculi.-Calculi are usually without clinical importance. They are found at postmortem examinations, and are sometimes single, sometimes multiple, and may be as large as cherry stones. They are believed to result from the secretion of the vesicle which has been retained, and which becomes infiltrated with phosphate or carbonate of lime salts. The organic matter, which is about Io per cent. of the whole calculus, is composed of epithelium, detritus, mucus, and spermatozoa.

A distinction is sometimes made between seminal calculi and seminal concretions. According to Meckel, the latter are postmortem products. Fürbringer, however, believes them to be formed during life.

The concretions are usually multiple, and lie in diverticula of the vesicle, but do not obstruct the ejaculatory ducts. They resemble hard 
wax when cut with a knife. Casper ${ }^{10}$ states that they are insoluble in acetic acid, and are composed chiefly of mucus, spermatozoa, and some inorganic matter.

Symptoms.-Spermatic colic results from plugging of the ejaculatory duct by calculus, and the secretion of the vesicle may be prevented from escaping from it for the same reason.

Spermatic Colic.-This symptom occurs during sexual orgasm. The pain is severe, is located in the perineum and rectum, and radiates more or less to the glans penis, testicles, and sacrum. The pain usually subsides in a few moments, but may be sufficiently severe to produce faintness.

Occasionally, there is a more or less frequently repeated dull pain in the rectum, quite independent of coitus. This is apt to be accentuated by defecation and also by the bladder being overdistended.

Rectal examination will usually detect the presence of the calculus.

Treatment.-The pain may be relieved by hot sitz baths or rectal injections.

The breaking up of the stone in situ by pressure upon it from the rectum has been suggested. In one case in which we made the attempt to do this it was unsuccessful. More radical operative measures are seldom demanded, but in some cases the frequency and severity of the pain referred to above, or pus formation, might constitute indications for excision of the vesicle. This procedure, however, is usually reserved for cases of tuberculosis of the vesicles. (For the details of the operative treatment, see chapter on the Technique of Operations upon the Seminal Vesicles.)

\section{BibLiograPhy.}

I. W. G. Richardson. Development and Anatomy of the Prostate Gland. J. \& A. Churchill, London, I904.

2. Freeman. Denver Medical Times, I903, vol. xxvii, p. 577 .

3. Ultzmann. Neuropathien des männlichen Harn und Geschlectsapparates, Wien, 1879.

4. Chiari. Zeitsch. f. Heilk., Wien u. Leipzig, 1903, xxiv, 283.

5. George. Journal of the American Medical Association, I906, vol. xlvii, p. I03.

6. Klebs. Handb. der path. Anat., 1876 .

7. Rokitanski. Lehrb. der path. Anat., I86r.

8. Orth. Lehrb. der speciellen path. Anat., I893.

9. Lallemand. Des pertes seminales involuntaires, Paris, I836-I84I.

Io. Casper. Genito-urinary Diseases, p. 44I. 


\section{TECHNIQUE OF OPERATIONS ON THE TESTICLE, EPIDIDY- MIS, SPERMATIC CORD, AND SEMINAL VESICLES.}

\section{ORCHIDOPEXY.}

THE operation seeks to remove the testicle from the abnormal position in which it is placed and to fix it as nearly as possible in its normal position.

Bevan's Operation.-Instruments. - One scalpel; I pair straight scissors; artery forceps; curved and straight needles; catgut ligatures and sutures.

Operation.-Incision.-The oblique incision as for the operation for radical cure of inguinal hernia, extending from a little below the external inguinal ring upward and outward for three inches, and following the line of the inguinal canal.

Expose the fascia of the external oblique muscle and divide it in the line of the incision.

Divide the cremasteric and transversalis fasciæ. The peritoneal pouch enclosing the testicle is then exposed.

Open this pouch by a transverse incision. The sac may contain omentum or intestine. If this is the case, they are to be returned to the abdomen. Divide the vaginal process of the tunica above the testis.

Free the sac from the cord as far up as possible, and ligate it by transfixing it with a needle carrying a catgut ligature. The sac should be tied off as close to the internal inguinal ring as may be.

Close the vaginal process in which the testicle lies by a catgut pursestring suture. This covers the testicle and makes a sac for it (see Fig. I36).

Lift the testicle and its newly made envelope out of its bed and make downward traction on the cord. This manœuver makes tense the connective tissue bands of fibers which bind the testis and cord to the adjacent parts; they are to be divided with blunt-pointed scissors or broken down with forceps. This step should be carried out thoroughly and carefully.

The vas and its vessels are now exposed, but are too short to allow the testicle to be drawn down to its proper place. This is made possible by freeing them from all attachments to the peritoneum above the inner inguinal ring, as well as through the inguinal canal. This is gently done with the finger tip.

VOL. I-I 8 
It is to be remembered that the vessels and the vas separate at the internal inguinal ring, the vessels passing upward and inward while the vas passes downward and inward, and each of them must be followed in its own direction and freed. When it has been accomplished, the cord is usually lengthened by from one to three inches, and the testicle can be brought into the scrotum.

FIG. 136

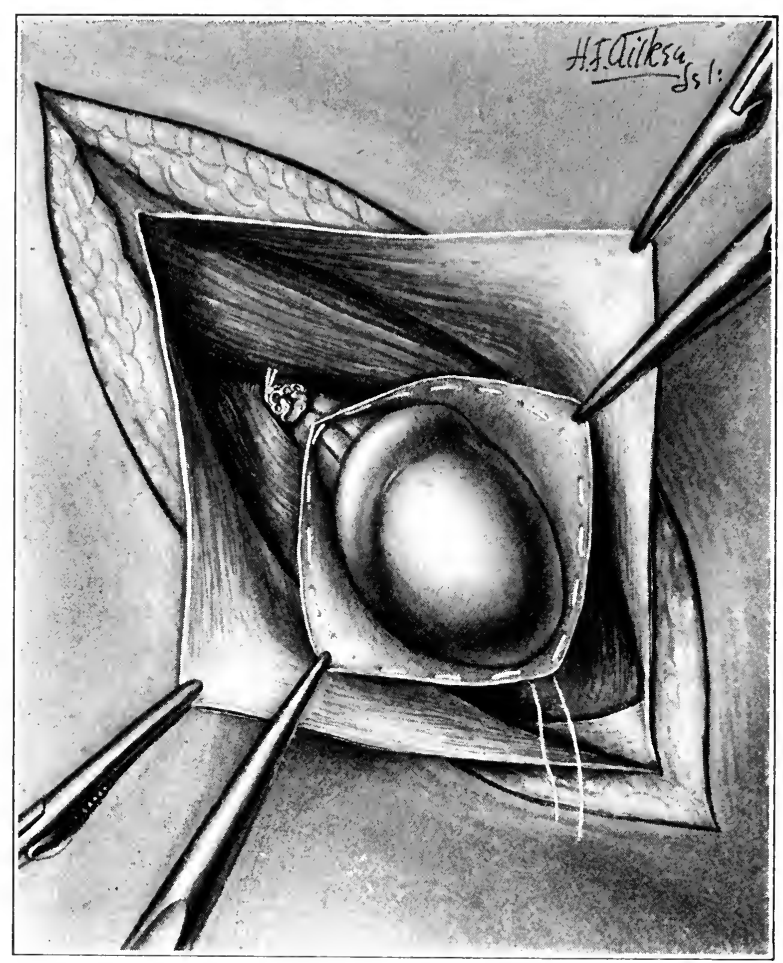

Bevan's operation. The inguinal canal opened and its coverings retracted. The peritoneal sac has been divided above the testicle, freed from the cord, transfixed, and ligated, and may be seen at the internal ring. The lower portion of the sac has been opened and a purse-string suture run so as to form a tunica vaginalis for the testicle.

In some cases the steps described above do not accomplish the end sought, and the cord still remains too short to allow the testicle to be brought down sufficiently. This will be found to apply especially to the testicles which are retained within the abdomen. When the testicle cannot be brought down, it is because the vessels of the cord oppose its descent, and it then becomes necessary to ligate them in two places and to divide them between the ligatures. The steps described above having been taken, proceed as follows (Fig. I37): 
Make a bed in the scrotum for the testicle by boring down into the scrotal cavity with the finger tip; the testicle is then drawn down into it and held in its place by a suture passed through its tunic and through the inner surface of the lowest part of the scrotum. It is prevented from retracting into the inguinal canal by placing a purse-string suture in the

FIG. I37

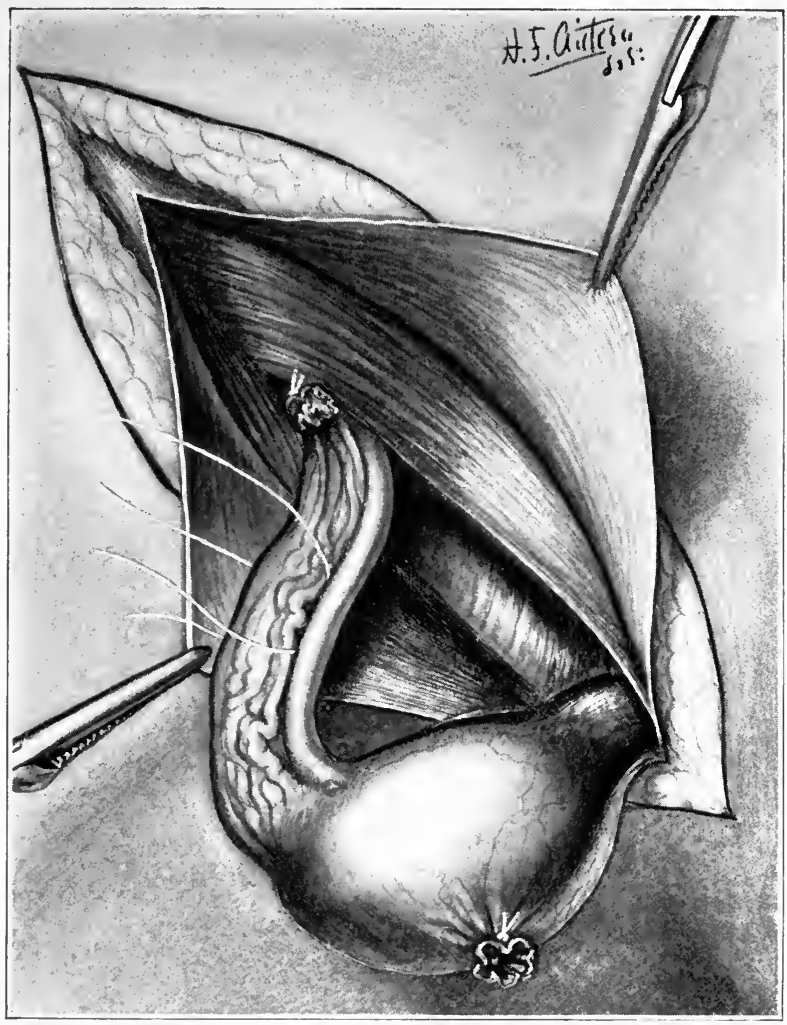

Bevan's operation. The purse-string suture tied, thus forming a new tunica vaginalis for the testicle. The cord has been lengthened by dividing the connective-tissue bands which normally pucker up the veins; the vas and spermatic vessels have been freed from their intrapelvic peritoneal covering by the finger passed through the internal abdominal ring. Two ligatures are shown in position for the division of the spermatic vessels, in case division of the connective-tissue bands does not allow the testicle to reach its normal position in the scrotum. If it is necessary to divide these vessels to accomplish this, the ligatures are tied and a division of all but the vas is made between them.

fascia of the external and internal oblique muscles below the level of the external inguinal ring. This suture is not to be drawn so tightly as to compress the cord, but partly closes the scrotal cavity above the testicle, making there a circle sufficiently small to effectually prevent the organ from rising upward beyond it. This tendency seldom exists if the steps described have been carefully carried out. 
The final steps of the operation consist in closing the wound, which is done with two rows of sutures, as in the operations for radical cure of

FIG. ${ }^{3} 3^{8}$

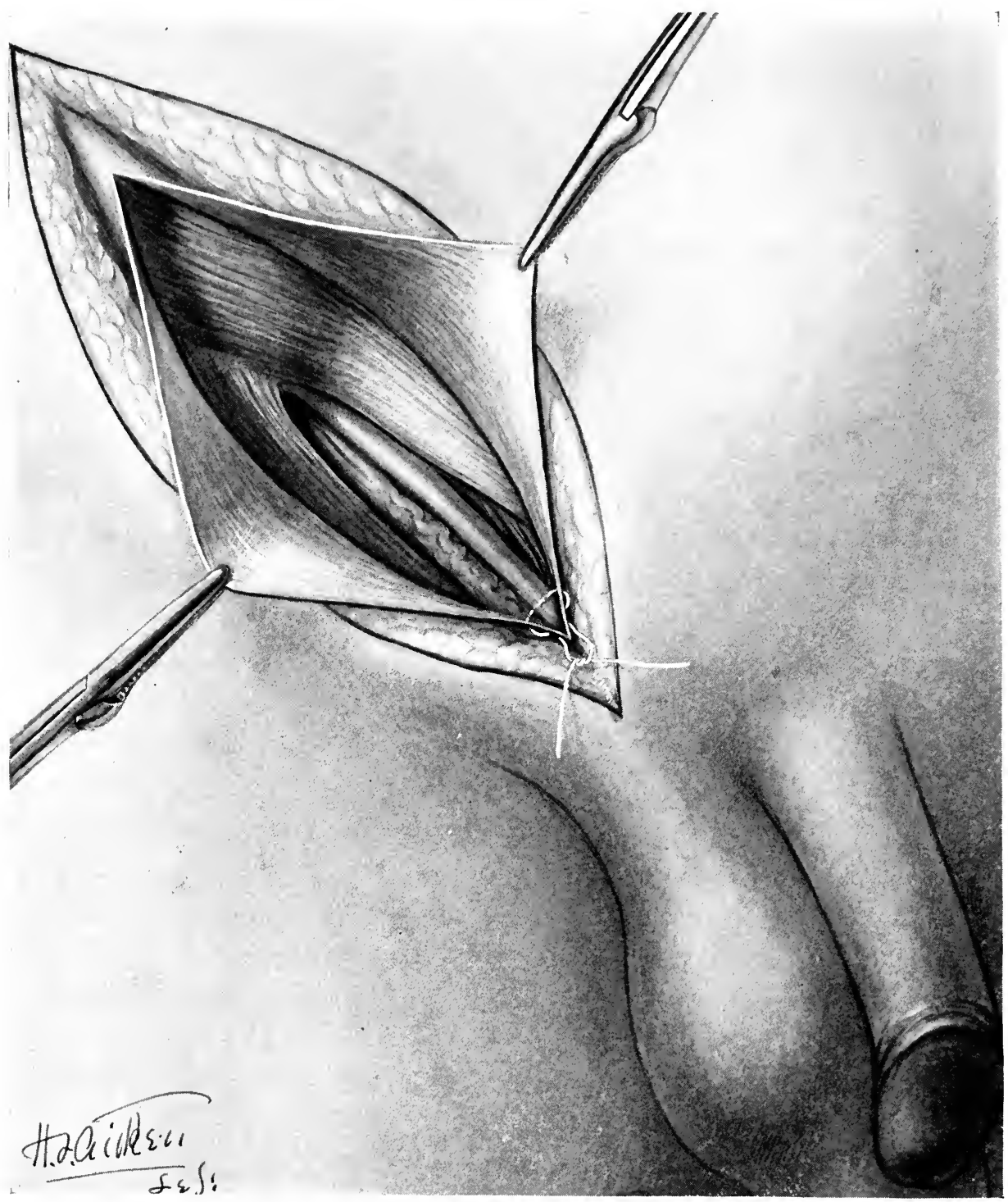

Bevan's operation. The testicle placed in its normal position in the scrotum and the purse-string sutures passing through the various layers of fascia at a point slightly below the normal position of the external ring, so as to prevent any retraction of the testicle.

inguinal hernia without transplantation of the cord. The steps of the operation are shown in Figs. 136,137 , and 138. 
Comment.-In some cases we have found the following measure to be useful in keeping the testicle in its new position and in preventing it from once more retracting into the inguinal canal.

Pass a suture through the lower surface of the tunic of the testis, carry it out directly through the bottom of the scrotum, leaving the ends long, and attach them to the inner side of the thigh with adhesive plaster. The leg is held still by attaching a long splint to it, in order to avoid too great traction being made upon the suture.

Beck's Operation.-Instruments.-The same as in the foregoing operation.

Operation.-The first steps of the operation are the same as in the preceding one. The distinctive feature of Beck's method of dealing with the undescended testicle is the manner in which he seeks to prevent the retraction of the organ after it has been brought down into the scrotum. This is done by turning a flap transversely across and above the cord and attaching the free end of it to the fascia of the opposite side (see Fig. I39). The flap is taken from the anterior and external side of the inguinal canal, and is composed of the fascia of the external oblique, the cremaster, and the transversalis muscles. When in position, the flap surrounds most of the circumference of the cord above the testicle in the form of a collar. The sutures by which the free end of the flap is attached are shown in the same illustration.

Comment.-The weak point in Beck's operation is the failure to provide for the lengthening of the cord in the cases in which the testicle has been retained within the abdomen. Unless this is done, the testicle cannot be brought down sufficiently to place it in its new position. Indeed, in the larger number of these cases it cannot be drawn out beyond the external inguinal ring.

Another point in Beck's operation which we do not like is that of sacrificing a part of the wall of the external inguinal canal, as is done in making the flap to retain the testis in its place. Moreover, it is unnecessary to do so, for it can be accomplished better by the means employed by Bevan in his method of operating, which involves no sacrifice of the integrity of any important structure in order to secure the same result.

There need be no hesitation about dividing the vessels of the cord, as is done in some cases in performing Bevan's operation, for it has repeatedly been demonstrated that the artery of the vas is capable of nourishing the testis.

The most essential feature of the operation is the lengthening of the cord. Upon the success or failure to secure this the result of the operation depends. 
Broca $^{1}$ reports the results of his operations in 79 cases of undescended testicles in young children. In 3 I of the cases the testicle was normal in both character and position at the end of one year after operation; in 35 the testis was normal, though the position was near the external inguinal ring. In 13 the testicle was atrophied.

FIG. 139

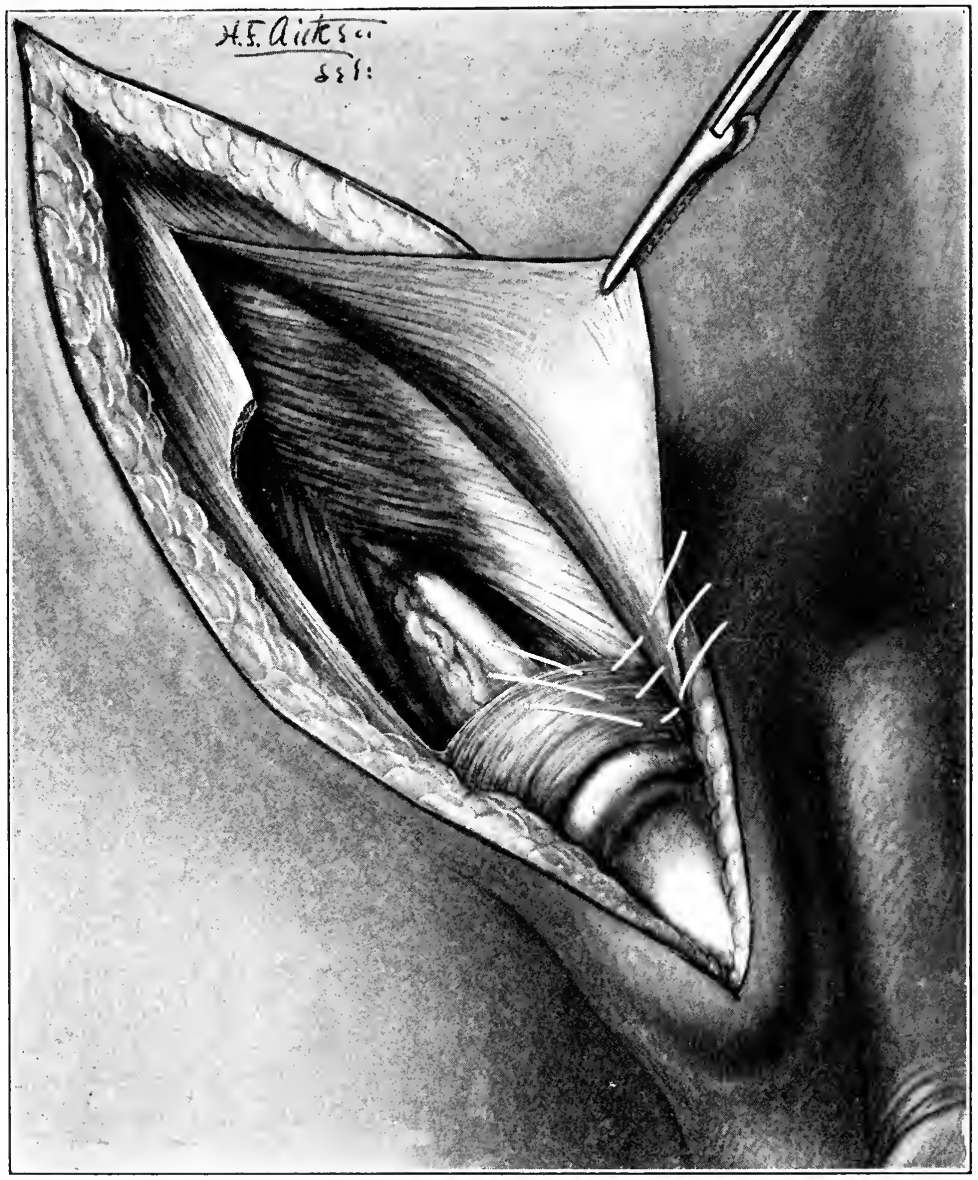

Beck's operation. The inguinal canal open. The cord has been lengthened by nicking the connective-tissue bands. The flap, three inches long and composed of the fascia of the external oblique, cremasteric, and transversalis muscles, has been cut from the outer edge of the wound. A cavity for the testicle has been made in the scrotum by the finger. The flap is seen turned over the cord just above the testicle and united in the region of the conjoined tendon, thus holding the testicle outside of the new external ring.

Coley reports a series of 38 cases, 27 of the patients being children. In 2 cases castration was performed. The results were satisfactory in almost all the others. 


\section{ORCHIDECTOMY (CASTRATION).}

Instruments.-One scalpel; I pair of straight scissors; catgut ligatures and sutures; straight needles; 4 artery forceps.

Operation.-In cases of double castration, we prefer to do the operation through a transverse incision made across the bottom of the scrotum, except in cases of malignant disease.

FIG. I40

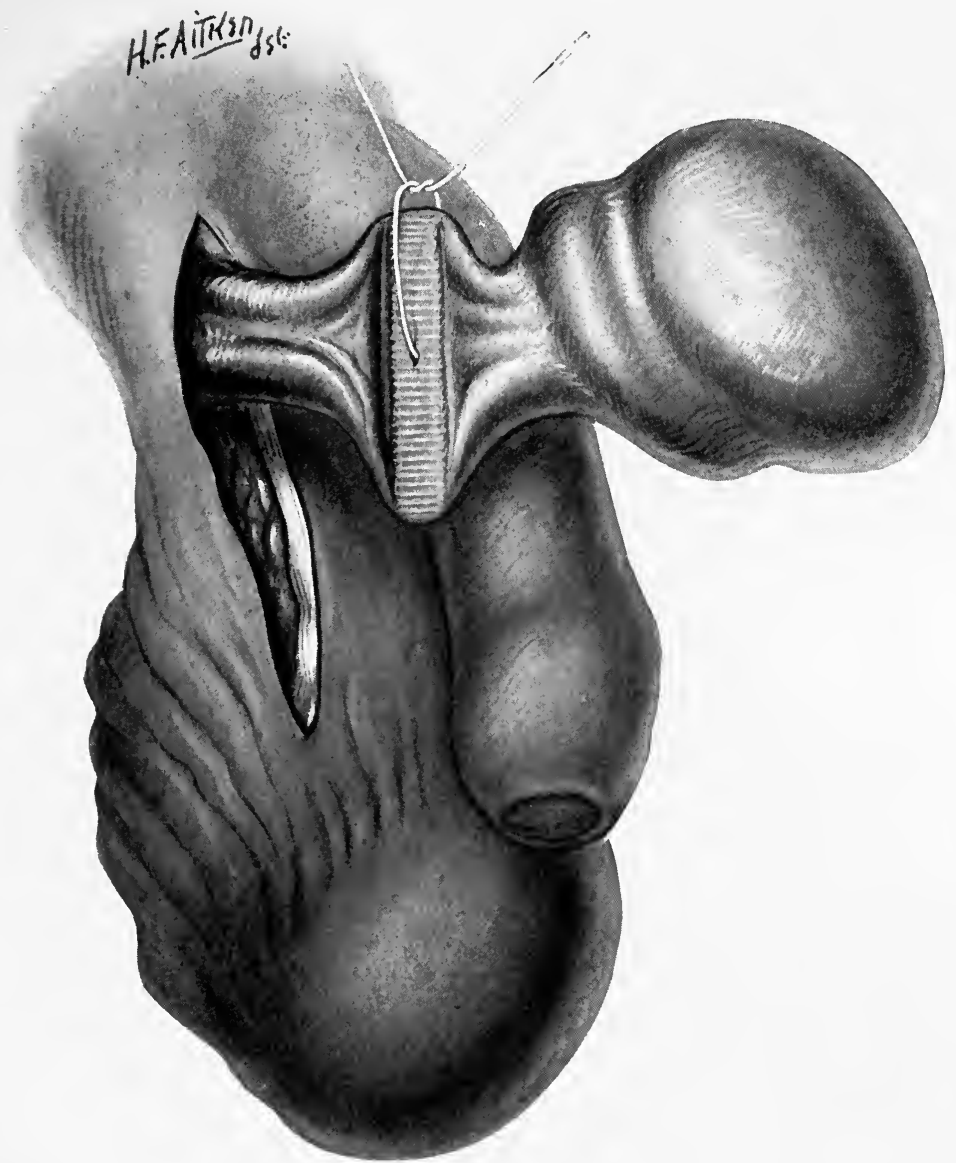

Orchidectomy. The incision beginning at the external abdominal ring and extending a short way into the scrotum, the testicle delivered, the cord crushed, and the suture transfixing the cord in the crushed area.

In bilateral castration, make an incision across the lowest part of the scrotum, laying open the tunica vaginalis of each testicle and exposing both organs. Retract the scrotum and push the testicles through the wound at the same time. 
Ligate the vessels and the vas of each side separately, taking particular care to avoid including the nerve in these ligatures. Do not divide the vasa deferentia or the vessels too close to the inguinal ring, but about midway between the testis and the ring. If the ligature is placed too high up, and the stump retracts within the ring, and, should the ligature slip off the vessels, troublesome hemorrhage will very likely occur.

When the operation is unilateral it is done as follows:

Operation.-Make an incision two inches long, beginning at the external inguinal ring, extending downward through the anterior surface of the scrotum, and laying open the tunica vaginalis. Express the testicle through the wound. Free the cord from the external ring downward.

Ligate the cord and its vessels in the same manner as that described in the preceding operation. Separate the testicle from its attachments by blunt dissection. Divide the cord below the ligature and remove the testicle and its adnexa. Close the wound in the scrotum without drainage.

The operation is sometimes modified by crushing the structures of the cord with a pair of strong forceps placed transversely across them. The clamp is then removed and a suture with ends of equal lengths is passed through the middle of the crushed part of the cord, and half of the latter is ligated with one strand of the ligature after cutting its loop, and the other half with the second part of it. The rest of the operation is then performed in the same manner as that described in the preceding one (see Fig. I40).

When castration is done in cases in which hernia testis exists, or in which the organ is adherent to the outer skin of the scrotum, the incision should be made in such a way as to pass around the diseased or adherent area, including it within an oval of sound skin in order that it may be removed together with the testicle. Drainage is sometimes desirable in this special class of cases.

Castration for Malignant Disease.-The operation differs in cases of malignant disease from that which is performed under other conditions, because of the necessity which is imposed of removing a greater amount of the structures.

Operation.-Make an incision in the same manner as is done in the operation for the radical cure of inguinal hernia.

Expose the external inguinal ring and divide the outer wall of the inguinal canal, carrying the incision a little beyond the internal ring.

Free the cord from its attachments at and immediately above the external ring, then lay open the floor of the canal. Follow the vas and the vessels of the cord as far as possible into the pelvis, exposing the field for this purpose by stripping back the peritoneum and holding it out of the way with a suitable retractor. 
Double ligate the spermatic vessels as low as possible and divide them between the two ligatures.

Tie the vas and divide it.

If the lymphatic glands into which the lymph vessels of the testis and its adnexa empty are involved, the operation for castration will be futile, for they, practically speaking, surround the aorta, and it is out of the question to completely remove them. The examination of these structures should be made at the time at which the deeper portions of the vessels of the cord and the vas are exposed, as just described.

There is one gland that should always be sought for and removed, as it is usually involved by the malignant process. It is that described by Cuneo, and lies upon the iliac vein at the point at which the ureter crosses this vessel.

The lower end of the ligated and divided vessels and of the divided vas are next brought out through the wound; the latter is extended more or less, according to circumstances, downward through the anterior aspect of the scrotum, and, without opening the coverings of the cord or testicle, the two structures are liberated from their attachments to the scrotal tissues, together with their immediate coverings, and are removed.

All areas of skin that are involved by the malignant process should be included in the parts taken away.

If the scrotum is involved in the disease, the inguinal glands will be implicated, and should be removed.

\section{OPERATIONS FOR HYDROCELE.}

The operation of acupuncture, the treatment by seton, and by incision and drainage of the sac are rarely, if ever, practised, and need only be named for the sake of saying that they are none of them desirable methods of treatment.

Tapping.-Preparation.-Make sure of the position of the testicle in the scrotum before tapping the hydrocele. This is the only preliminary step required.

Position of the Patient.-The operation is more readily performed with the patient standing up, but occasionally a nervous individual faints when the hydrocele is tapped, and it is, therefore, safer on general principles to have the patient lie down.

Operation.-Grasp the scrotal tumor made by the hydrocele between the thumb and fingers of the left hand, and make the scrotum tense by compressing the swelling near its neck and upon the posterior surface. The hand that holds the scrotum is placed behind it. 
Holding a trocar of rather small size with the tip of the forefinger in the position shown in Fig. I4I (the trocar is too large as it appears in this illustration), the operator thrusts it into the cavity of the tunica vaginalis in a diagonal and upward direction. The thrust should be made boldly; the point of the instrument is prevented from passing too far into the hydrocele by having the forefinger tip placed about an inch from the point of the instrument. The point at which the instrument should be entered is about the junction of the lower and middle thirds of the anterior surface of the scrotum (Fig. I4I).

FIG. I4I

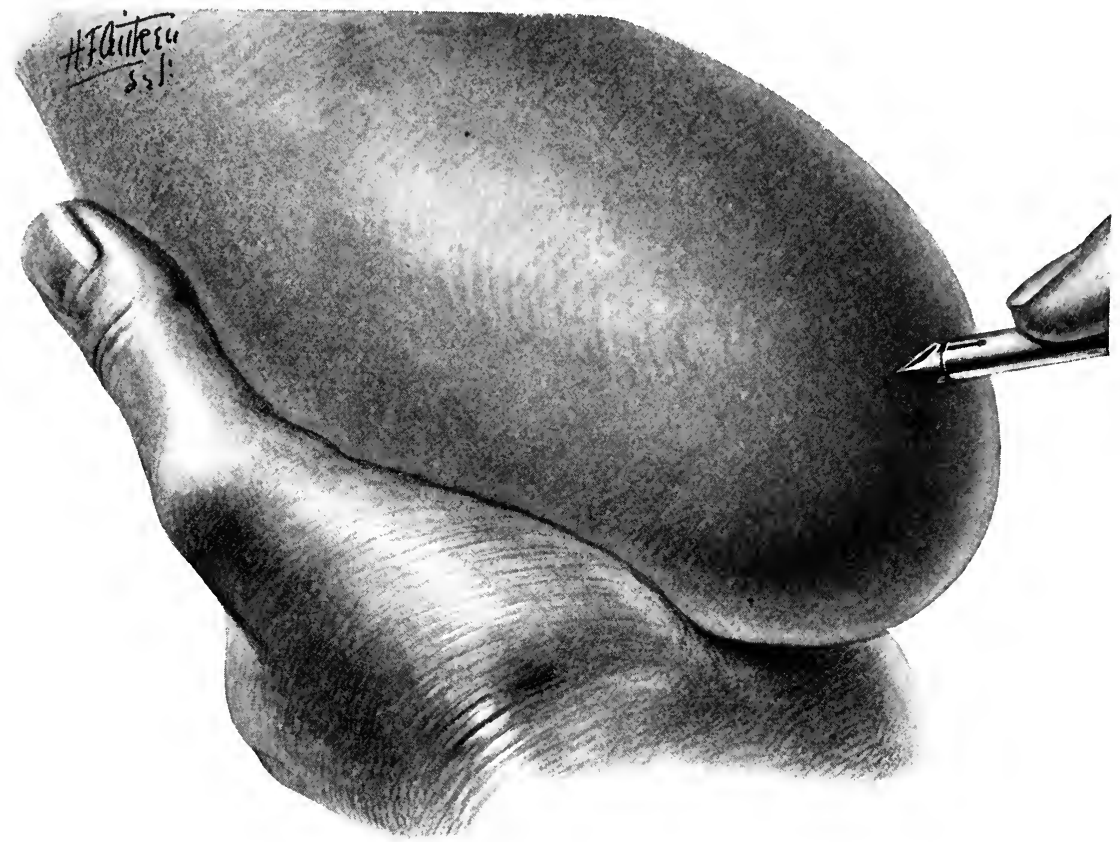

The hand grasping the posterior aspect of the scrotum, making the anterior surface tense; the trocar, guarded by the finger three-quarters of an inch from the point, about to be plunged into the hydrocele sac.

As soon as the operator is certain that the end of the cannula has entered well within the cavity of the hydrocele, the pointed stylet is withdrawn and the fluid escapes through the tube.

Comment.-The essential feature of the operation of tapping a hydrocele is to avoid injuring the testicle and the vessels of the cord. The first of these mishaps need never occur if care is taken to locate the position of the organ beforehand. This is readily done in all cases in which the fluid of the hydrocele is translucent.

When it is not so, however, there may be some difficulty in discovering just where the testis lies in the hydrocele cavity. Its natural position is 
at the back of the swelling and low down in the scrotum. It is very rarely placed elsewhere than in this position. The sense of touch and the detection by it of an area of increased resistance must be relied upon to locate the organ in the cases in which the fluid in the sac is not translucent. Wounding the vessels of the cord may happen, especially if the hydrocele is small, by pushing the trocar too far into the sac. Care should be taken to avoid this.

Injection of the Hydrocele Sac with Iodine or Carbolic Acid.-Of the two agents, carbolic acid gives more certain results, but both of them are excellent for the purpose of bringing about obliteration of the hydrocele sac.

Operation (with iodine).-An aspirating needle sufficiently large to insure the escape through it of the hydrocele fluid is thrust into the sac, its sharp pointed tip is withdrawn, and the hydrocele is completely evacuated. The cannula, which should be provided with a small syringe that can be fitted to its outer end, is not withdrawn from the sac. Two drams of the tincture of iodine are injected into the sac and brought in contact with its whole surface by gentle manipulation of the scrotum. The tube is then withdrawn.

With Carbolic Acid.-The treatment as it is carried out by Keyes is as follows: The needle of a hypodermic syringe is passed into the hydrocele sac and allowed to remain there. The hydrocele is then tapped with a trocar and completely emptied of its fluid, and the cannula of the trocar is withdrawn. A hypodermic syringe containing 50 minims of deliquesced crystals of carbolic acid is next attached to the needle and the contents of the syringe is injected into the sac and spread over its inner surface by manipulating the scrotum.

Comment.-With but few exceptions, the treatment by carbolic acid injection results in cure by obliteration of the sac, if it is rightly applied, and to the cases which are appropriate for it. These are the hydroceles which have not got thickened sacs.

There is, as a rule, more or less redness and swelling of the scrotum following the injection. Occasionally the patient feels a good deal of pain at the time, or soon after the application of the treatment. Sometimes there is a re-accumulation of the hydrocele fluid shortly after the injection has been given. If the quantity of it is large enough to make it desirable to have it withdrawn, this should be done.

Failure of the treatment is usually due to an improper selection of the cases, or to not having wholly emptied the sac before making the injection; also if it be applied to very large hydroceles, or those originating in an epididymitis which is still present.

Faintness may be felt when the injection is made, and it is therefore better to do the operation with the patient in the recumbent posture. 
The patient should be kept in bed until the inflammatory reaction, which is manifested by redness and swelling of the scrotum, has begun to subside. A suspensory bandage should be worn when he first begins to go about and until all swelling and redness have disappeared.

Excision of the Tunica Vaginalis (Von Bergmann's Operation).Instruments.-One scalpel; I pair of straight scissors; 2 pairs of singletoothed forceps; 6 pairs of artery forceps; straight needles carrying catgut sutures; catgut ligatures.

\section{FIG. ${ }^{42}$}

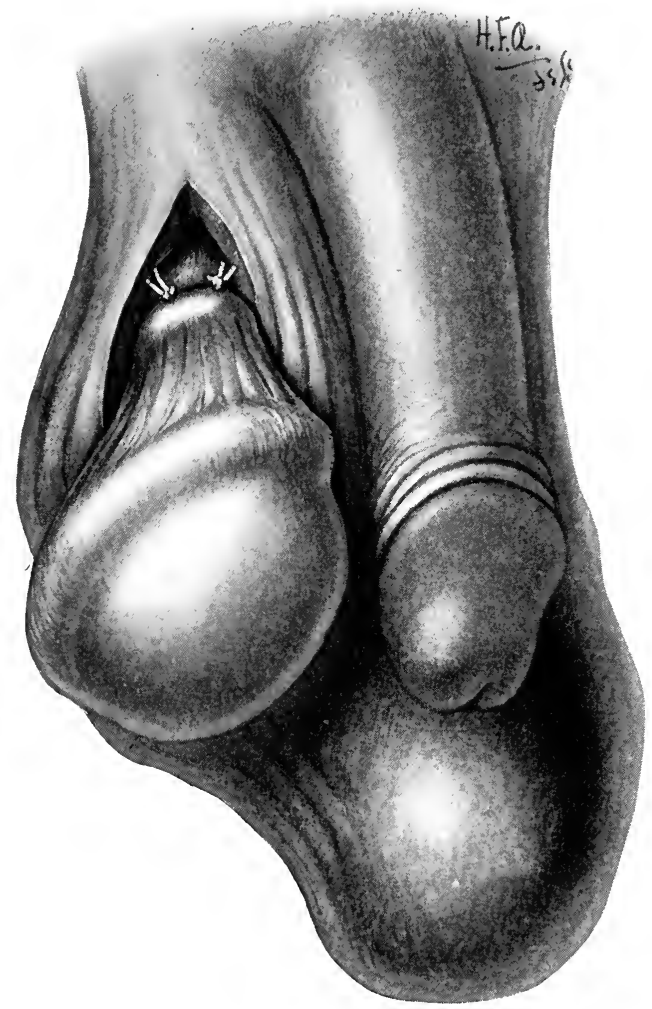

Eversion of the sac. The testicle brought out through the incision, the sac separated from the scrotum and turned inside out, rolled up, and caught above the testicle to the cord.

Operation.-Make an incision three inches long upon the middle line of the anterior surface of the scrotum, beginning it at the neck of the sac and carrying it downward. The tissues are to be divided to, but not through, the tunica vaginalis. Separate the sac of the hydrocele from the scrotal wall by blunt dissection, aided by "wiping" the tissues away from each other with the finger tips covered by a bit of gauze. Pick up and ligate bleeding vessels as they are met with. Puncture the sac and empty it of its fluid. 
Enter the forefinger into the sac through an opening made by extending the puncture wound. Separate the testicle from such adhesions as may exist between it and the parietal layer of the sac, then split the tunica vaginalis from end to end in the line of the incision already made in it.

Trim away the tunic as close to the testicle and the cord as can be done safely, and arrest bleeding from the cut edges of it by a continuous catgut suture applied to them. Close the outer wound without drainage.

Comment.-This operation has yielded uniformly good results in the writer's experience. It requires the patient to stay in bed for several days after it has been done. During this time the testicles should be elevated, and moderate pressure should be exercised upon them by a firmly applied bandage. Retention of urine occasionally follows the operation, and must be relieved by the passage of the catheter as often as may be required.

Eversion of the Tunica Vaginalis.-Instruments.-The same as in the operation last described.

Operation.-The tunic is exposed and separated from the adjacent tissues in the same manner as that described in the last operation, and the same steps are repeated until the sac has been laid open freely. The testicle is then pressed out through the incision, the sac is turned backward behind it and the cord, and its cut edges are brought together in that position. When the hydrocele sac is a large one, it should be trimmed off on either side of the incision, enough of it merely being left to insure its cut edges being united together in the manner just described (Fig. I42).

Comment.-Either of the above operations may be done with cocaine anesthesia, if it is thought preferable to perform them with this anesthetic.

\section{EPIDIDYMECTOMY.}

This operation has for its object the removal of disease which is confined to the epididymis and vas.

Instruments.-One scalpel; I pair of straight blunt-pointed scissors; 2 pairs of single-toothed forceps; artery forceps; straight needles carrying catgut sutures; catgut ligatures; I director.

Operation.-An incision is made in the scrotum, beginning just below the external inguinal ring and extending downward in a line corresponding to that of the cord. If there are areas of skin adherent to the cord or epididymis, or if fistulous tracts connect them with the surface, these should be embraced by the incision, which should then be carried around them on either side. 
Turn the testicle out through the incision in the scrotum, and incise the tunica vaginalis along the outer side of the line of junction of the epididymis with the testicle.

Separate the epididymis from the testicle by blunt dissection with a director or other appropriate instrument, beginning at the globus major, and loosening its attachment to the testis by nicking the tunica albuginea at this point. The rest of the epididymis can then be separated by blunt dissection without difficulty.

The bloodvessels which pass to the testicle lie along the inner side of the line of junction between the epididymis and testis, that is to say, on the side of the epididymis opposite to that along which the incision is made. These bloodvessels may be avoided by putting the epididymis on the stretch and by slowly separating the vessels from it, taking care not to cut or break through the layer of tissue in which they run on the inner surface of the epididymis.

The vas is separated from the rest of the structures of the cord by blunt dissection. When the external ring is reached, the fascia of the external oblique is to be incised and the vas freed from the inguinal canal as far up as the inner ring, where it is ligated and divided. If the vas is believed to be diseased beyond this point, an injection of an emulsion of iodoform, or other medicament, may be made into it before placing the ligature.

Unite the incision made in the fascia of the external oblique muscle with a continuous catgut suture, and close the wound with or without drainage, according to circumstances.

Partial Vasectomy and Union of the Testicle with its Fellow Organ.Pascale's Operation.-The object of this operation is to retain the function of the testicle from which the epididymis and vas deferens have been removed by uniting it with its healthy fellow organ.

Instruments.-The same as for the last operation described.

Operation.-Remove the diseased epididymis and vas in the manner described in the last operation. Break through the septum between the two testicles with a blunt instrument and expose the other testicle. The tunica albuginea of the other testicle is incised close to the Highmorian body and the rete of both testicles, thus exposed, are approximated and held together by catgut sutures passed through the edges of the incisions in the albuginea (Fig. I43).

Close the wound without drainage.

Epididymectomy with Anastomosis between the Vas Deferens and the Rete Testis.-Operation.-The epididymis is separated from the testis in the manner already described, and divided from the cord as near to the junction of the two as possible.

Split the divided end of the vas on one side, using a fine probe passed 
into its canal as a guide. Suture the edges of the divided end of the vas to those of the tunica albuginea on either side of the exposed area of the rete testis with interrupted Lembert sutures, so that the canal of the vas, which is widened by spreading out its end, shall be in contact with the rete. Close the outer wound without drainage (see Fig. I44).

Another method is that which is illustrated in Fig. 145.

FIG. 143

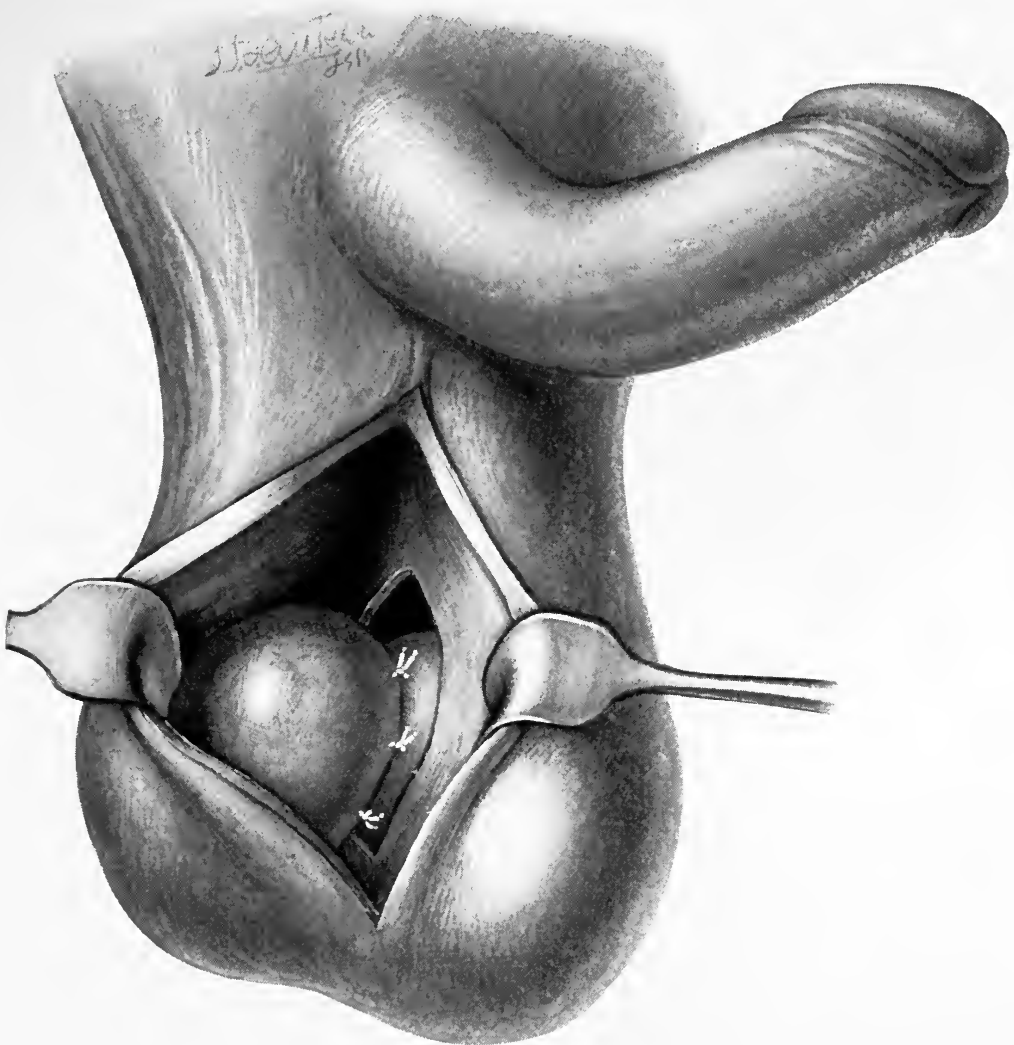

Pascale's operation. The testicle, devoid of the epididymus and vas, attached to its healthy mate in the region of the corpus Highmorianum by splitting through the scrotal septum.

\section{VASECTOMY.}

The term vasectomy, properly speaking, means excision of the entire vas deferens. The name partial vasectomy is applied to the operation by which a portion of the vas only is removed.

The total removal of the vas is sometimes combined with that of the testicle, and sometimes the seminal vesicle is taken out with it, or either one of the latter organs may be removed with part of the vas. 
Castration and Partial Vasectomy.-Instruments.-The same as for epididymectomy.

Incision.-The same as for inguinal hernia, and extended for two inches downward into the scrotum.

Operation.-Turn the testicle out through the wound. Separate any adhesions which attach it to adjacent parts. Lay open the inguinal canal.

FIG. I 44

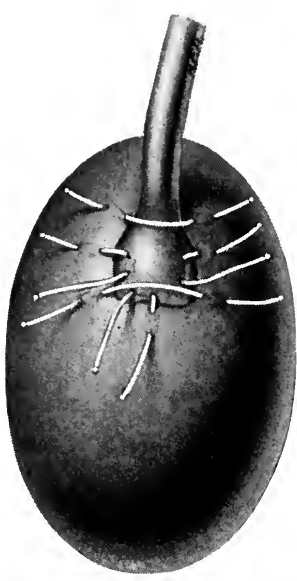

Epididymectomy with anastomosis between the vas deferens and the rete testis. The vas deferens split at its distal end and united to the rete testis by Lembert sutures, which include the tunica albuginea on either side so that the line of union will be buried when the sutures are tied.
FIG. 145

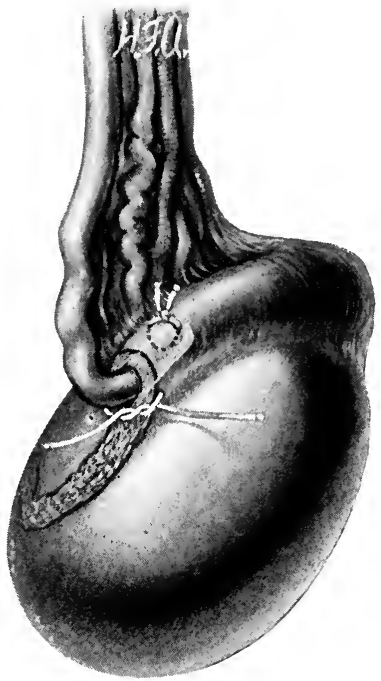

Anastomosis between the vas and epididymis after extirpation of the tail of the epididymis. The tail of the epididymis has been removed, the upper portion of the vas tunnelled, and the split end of the vas invaginated and held by the sutures.

Separate the vas from the rest of the cord at the internal inguinal ring and crush the cord with a pair of strong clamp forceps, after which transfix it with a suture having ends of equal lengths. Cut the loop of the suture and tie the cord by passing each part of the thread around one-half of it. Divide the cord and the vas. Remove both structures, together with the testicle. Tie the upper end of the vas. Close the outer wound without drainage (see Fig. I40).

Complete Vasectomy with Castration.-The operation differs from the one last described in that the whole of the vas is removed instead of a part of it.

It is simpler to remove the testicle and the cord together with the vas and to separate the last two structures in the manner described in the last 
operation before taking out the remaining part of the vas. The latter step is effected as follows:

Operation.-The patient should be in the Trendelenburg posture.

Divide the floor of the inguinal canal, lift the peritoneum and keep it up with a retractor, after separating the vas from it as far as it can be traced in its course in the pelvis. Search for the upper margins of the seminal vesicles, which are marked by a ridge upon the posterior surface of the bladder.

The vas is clamped and tied as close to this point as possible.

Removal of the Seminal Vesicle and Vas.-If the seminal vesicle is to be removed, the operation proceeds from the point at which the one last described stopped, thus:

Operation.-Break through the ridge above the tops of the seminal vesicles with the finger tip, aided, if necessary, by cutting the tissues with scissors. The vesicular bloodvessels are likely to be wounded in doing this, and if this happens it will probably be impossible to pick them up and ligate them, the wound must, therefore, be temporarily packed.

If the hemorrhage can be arrested in this way, or by pressure upon the bloodvessels at a point which does not interfere with the further steps of the operation, the latter proceeds as follows: the base of the diseased vesicle is freed from the peritoneum covering it on the one side, and from its attachments to the bladder on the other. In carrying out this part of the operation, the finger tip should be kept close to the vesicle, in order to avoid opening the peritoneal cavity. When freed, the base of the vesicle is to be grasped in the blades of a pair of forceps and drawn upward. It is then separated from the prostate and its other attachments, divided, and removed. Hemorrhage is to be arrested by packing. The wound is to be provided with drainage and closed throughout the remainder of its extent.

Removal of the Seminal Vesicle by Other Methods.-The operation just described presents difficulties too great to make it desirable, and, consequently, one or another of the following methods is to be preferred to it.

The Abdominal Approach to the Vesicles $\left(\mathrm{Young}^{2}\right)$.- In this operation the vesicle is exposed by a T-shaped incision, the perpendicular arm of the letter being in the middle line of the abdomen and the horizontal one being made by a transverse incision through the recti abdominis muscles across the end of the first cut. The peritoneum is not opened, but is stripped off the flaps formed in the manner just described, and also from the surface of the bladder, until the vesicles are exposed. They are then separated from their attachments to the bladder and the base of the prostate, ligated, divided, and removed.

vol. I-I9 
Removal through a Perineal Incision.-The vesicles may also be removed through the crescentic or inverted $\mathrm{V}$ perineal incisions, and, after separating the prostate and base of the bladder from the rectum after the manner of Proust's technique for prostatectomy. (See chapter on Technique of Operations on the Prostate.)

Young has removed the vesicles in this way together with the prostate in some cases of malignant disease of the latter. The description of the technique of this operation will also be found in the chapter on Technique of Operations on the Prostate.

Comment.-Of these operations, that which approaches the vesicles through the perineum is, in our judgment, the best, unless it be a combined suprapubic and perineal operation which, so far as we know, has not as yet been performed for this purpose.

Should such an operation be done, the peritoneum should be stripped from the bladder, or the vesicles should be reached through a laparotomy incision, and it would then, it seems to us, be very much easier to attack the vesicles by one hand passed through the suprapubic and the other through the perineal incisions than would be the case in any of the other methods to which we have referred. It should be said, however, that we have not attempted the removal of the vesicles in the manner just proposed.

Two other procedures are to be mentioned. One is the inguinal operation of Villeneuve, and the other the sacral route, which is favored by some surgeons, or its modified form, which gives access to the base of the bladder but without involving the bony structures, and which Rigby ${ }^{3}$ describes in connection with the removal of ureteral calculus impacted in the lower end of the canal.

None of these operations, however, have appealed to us as being as advantageous as the perineal, abdominal, or combined perineal and abdominal, of which we have just spoken, and we therefore do not describe them.

Comment.-The operations upon the vas deferens, epididymis, testicle, and seminal vesicle which have been described above are usually undertaken in cases of malignant or tuberculous disease of the parts. They do not, at best, offer a very brilliant prospect, yet in some instances they have been attended with success.

The operation of anastomosis of the vas and testicle should never, of course, be employed when the latter organ is the seat of tuberculous disease. When, however, the epididymis alone is involved, its removal, together with a part of the vas above the diseased area, is quite as likely to be curative as castration, as has been demonstrated by Bardenheuer and by Murphy. 
If the vas has been removed, leaving the testicle behind, the latter organ if not diseased may be united with its fellow.

Pascale claims that by this operation not only is the internal secretion of the organ retained, but the spermatozoa from the testicle which has been deprived of its vas and epididymis pass from it to the healthy vas of the other organ. He maintains that the bloodvessels and nerves of the rete of the testis from which its adnexa have been removed retain their normal powers, and enable the testis to continue its function when its secretion is given the opportunity, which is provided by his operation for the passage of spermatozoa from it.

In cases of tuberculosis in which the vas is involved to and beyond the internal inguinal ring, the seminal vesicle of the same side usually participates in the pathological process; the removal of the vas under these circumstances would be without benefit. In general, it may be said that any operation upon the parts in cases of malignant disease and tuberculosis will prove futile unless all of the diseased structures are removed.

\section{INCISION OF THE EPIDIDYMIS.}

Incision of the epididymis (or drainage of a suppurating focus or area of infiltration in the course of the epididymis).

Operation.-The line of junction of the epididymis with the testis is defined and exposed by an incision in the part of the scrotum and tunica vaginalis immediately overlying it. The testis and swollen epididymis are brought out through the incision; in cases in which the epididymis is infiltrated it should be punctured in numerous places with the point of a fine-bladed tenotome. If it is found that there is pus in the epididymis, it is to be let out by an incision instead of by the punctures. If there are tuberculous cavities in the epididymis, they are to be curetted, after being laid open, and may be filled with iodoform emulsion and drained. The cavity of the tunica vaginalis is to be thoroughly washed out with a weak solution of corrosive sublimate, followed by sterile saline solution or other cleansing fluid.

Comment.-Baermann ${ }^{4}$ concluded from a study of 28 cases which showed uniformly good results from this treatment in the hands of different operators, that the operation was indicated in all cases of acute epididymitis in which there is evidence of the occurrence of suppuration or much tension from infiltration of the epididymis, with or without associated hydrocele, and in cases in which there is pain from intra-epididymal tension.

He believes that experience has shown that relief of pain and a marked lessening of systemic infection can be obtained from the above described 
procedure in cases of acute gonorrhœal epididymitis, and that recovery is greatly hastened by its practice.

Drainage and Irrigation through the Vas.-Belfield ${ }^{5}$ advocates this procedure as a method of treatment of gonorrhœal and other infections of the seminal vesicle and in cases of recurrent epididymitis resulting from latent foci of infection in the seminal vesicles or deep urethra.

Instruments.-A subcutaneous syringe, small curved needles, small scalpel, fine silkworm-gut or horsehair sutures, blunt-pointed subcutaneous needle.

Operation.-Cocaine anesthesia. Press the vas into the cocainized area, which has been made directly over the most conspicuous part of the cord in the groin. Pass a silkworm-gut suture beneath the vas with a curved needle. Make an incision one-half an inch long over this part of the vas, through the scrotum. Make a small transverse cut in the vas, opening its lumen. Unite the edges of this incision to those of the scrotal incision with fine horsehair sutures, thus establishing a fistula, which may be maintained for as long a time as desired.

Through this opening the vas may be irrigated daily with small quantities of a ro per cent. solution of argyrol by means of the blunt-pointed subcutaneous needle and its syringe. Thirty minims is enough for the first injection. Later, the amount may be increased.

\section{OPERATIONS FOR VARICOCELE.}

Subcutaneous Ligature (Keyes).--Instrument.-The varicocele ligature needle shown in Fig. 146.

Operation.-The essential point in performing this operation is that the vas shall be separated from the dilated veins of the cord and excluded from the ligature which is to embrace the vessels. This is done by drawing the veins away from the vas and toward the outer side of the scrotum, and holding them there with the tip of the finger and of the thumb, and by pressing the veins against the skin; the finger tip intervenes between the vas and the vessels, and each of the structures is clearly distinguishable from the other.

Pass the needle, carrying a stout silk ligature, through the scrotum between the vas and the finger and thumb holding the veins. Disengage one strand of the ligature and draw its end out through the point of exit of the needle from the scrotum. Draw the needle back into the scrotum until its eye has reached the inner surface of the skin close to the point of entrance of the needle into the scrotum. Let go the hold of the finger and thumb which is keeping the vas separated from the veins and allow the latter to resume their natural place in the scrotum. 
Pass the needle again through the scrotum, this time in front of the veins, instead of behind them, as in its first passage, and bring its eye out of the same opening as that through which it first made its exit. Disengage the ligature from the eye entirely. Withdraw the needle. Tie the ligature tight, cut the ends short, and drop it into the scrotum.

It will be seen that the first strand of the ligature passes behind the veins and the second one in front of them, and that they are, therefore, necessarily included within the loop thus made, and will be firmly tied when the latter is drawn tight. The knot of the ligature can be readily slipped through the hole of exit made by the needle in the scrotum.

Fig. I 46

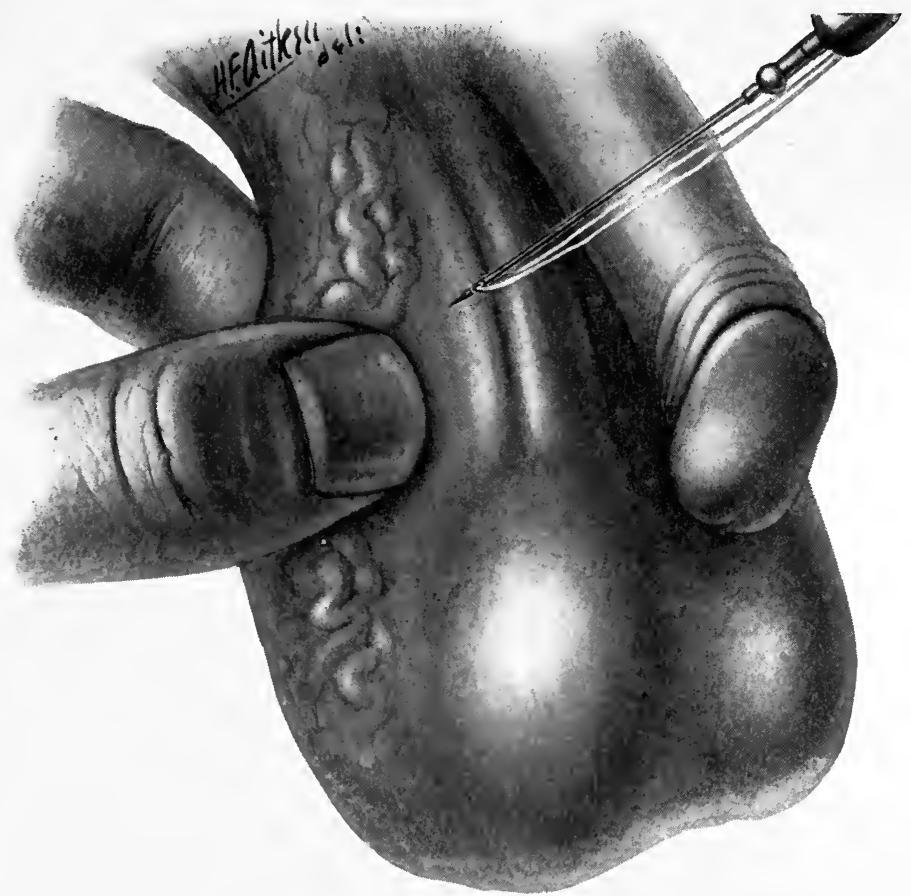

Subcutaneous ligature, showing all the veins brought against the scrotum and separated from the vas deferens by the thumb and forefinger. The mounted needle armed with silk about to enter the anterior anesthetized area.

Comment.-We have always preferred the open operation to the subcutaneous method of treating varicocele. By it the operator has the whole field of the operation under his eye, and can distinguish the tissues from each other and be more exact than in the procedure just described. Moreover, the latter does not provide the suspension for the testicle which the operation next to be described secures, and which is, in our judgment, a desirable feature to have embodied in the operative treatment. 
By the Open Method.-Instruments.-A scalpel; a pair of blunt-pointed scissors; catgut ligatures; a director.

Operation.-Open the scrotum by a two-inch incision through its anterior surface, and beginning at its upper part. Isolate the cord. Separate the vas from the veins. Pass two ligatures around the veins at a distance of an inch or rather more apart. Tie them tight-the lower one first-leaving one end of each of them long. Resect the portion of the veins between the two ligatures. Bring the divided ends of the stumps thus made together, by tying the ends of the strands left long to one another, thus shortening the cord and supporting the testicle (Fig. 147).

\section{FIG. 147}

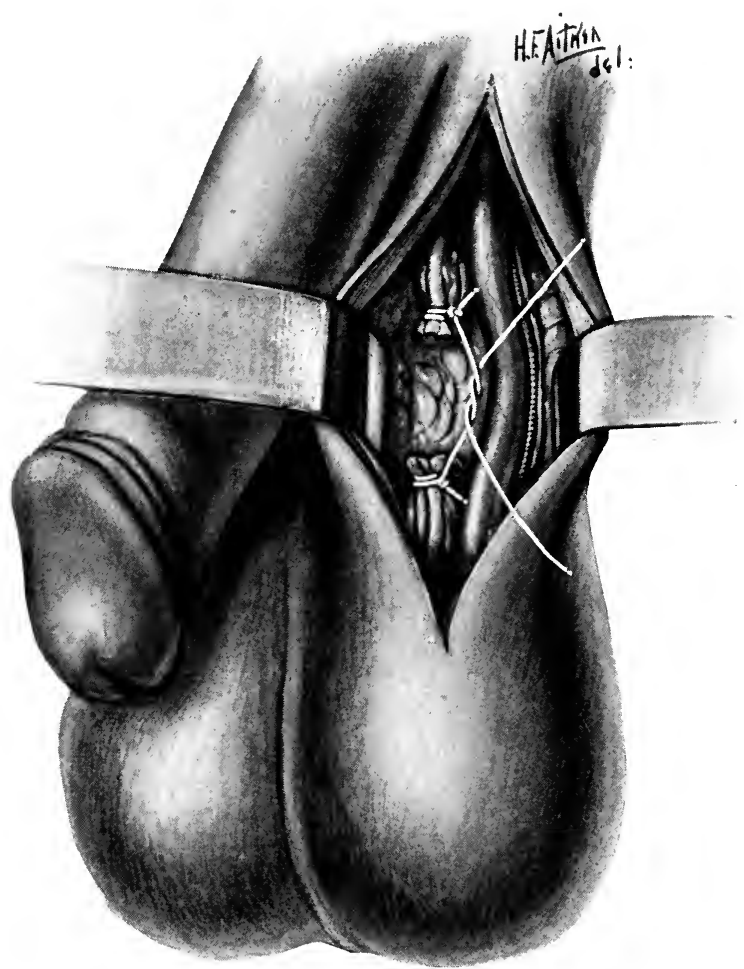

Open method, showing the vas deferens and spermatic vessels to the outer side of the vein stumps. A portion of the veins has been removed, and the two stumps are being united by the long ends of the ligatures.

Two other operations are practised for varicocele. One of them has the advantage of avoiding interference with the veins, and seeks to relieve the condition by a procedure which is, in effect, to supply a permanent suspensory bandage. This is done by shortening the scrotum. (See Technique of Operations upon the Scrotum.). The other operation demands a special instrument-an angiotribe. It is performed by 
opening the scrotum, bending the veins into a loop, and then burning through the base of this loop by the blades of the angiotribe, heated by an electric current. The outer end of the loop is cut across just above the instrument and removed. The veins thus compressd and burned through adhere together at the point at which the blades of the instrument have been applied, and their ends are firmly sealed. The outer wound is closed tight.

Comment.-We have never tried this operation. It has the disadvantage of requiring the operator to possess a special instrument, and does not seem to offer any advantage that is not shared by the open method and double ligation of the vessels.

The first of the two methods last described has the advantage of avoiding all direct interference with the veins. If its results are satisfactory, it is an operation which would appeal to our judgment as being preferable to the other methods. We cannot, however, speak of the late results of it from personal experience.

\section{INCISION OF ABSCESS OF THE SEMINAL VESICLES.}

Instruments.-Two scalpels, one of them having a straight narrow blade, with short cutting edge; one pair blunt-pointed scissors, artery forceps, long dressing forceps, retractors to spread open the incisions, a metal catheter, a director.

Fuller's Operation. ${ }^{6}$ - Position of Patient.-The patient is placed face downward with the front of the pelvis and the part of the abdomen immediately above it resting across a solid support.

Operation.-The perineum and the space between the bladder and rectum are opened and made accessible through an $\mathrm{H}$-shaped incision in the perineum, by division of the upper end of the external anal sphincter, and by blunt dissection with the finger tip in the same manner, except for the form of the outer incision, as that of the open method practised in removing the prostate. (See chapter on Technique of Operations on the Prostate.)

When the base of the bladder has been separated from the rectum, the blunt dissection is continued upward along its posterior surface until the seminal vesicles have been separated from the rectum. The area occupied by the abscess or by the inflammatory exudate is then exposed, and can be incised, and its contents evacuated.

The space opened by the operation is drained by a wick, and the outer wound, carefully including the margin of the anal sphincter in the sutures, is closed, except for sufficient space to allow the drain to pass through it. 


\section{RESECTION OF SCROTUM.}

Instruments.-Two long clamps; scalpel; scissors; straight needles threaded with silkworm or catgut.

Operation.-Press both testicles far up into the upper part of the scrotum. Apply clamp transversely to the skin beneath the testicles (Fig. I48). Place one row of sutures immediately above the clamp.

\section{FIG. 148}

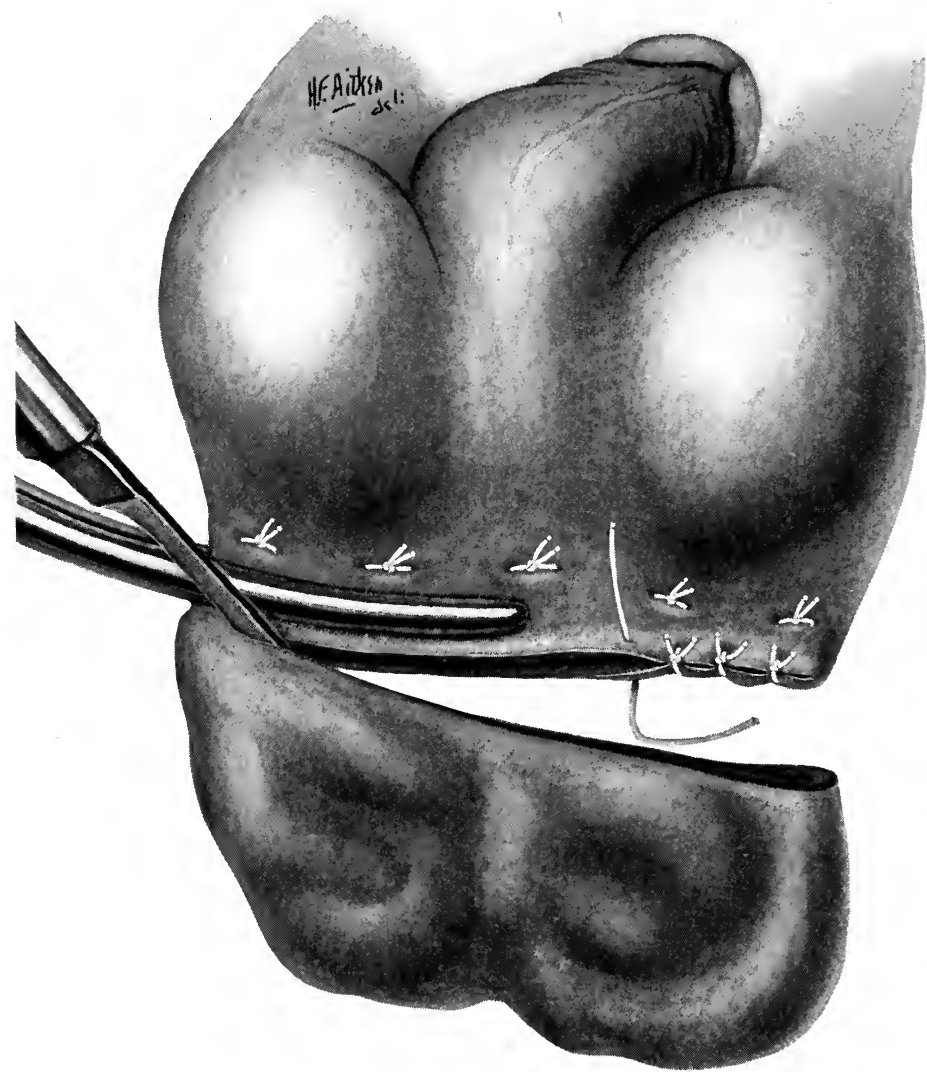

Showing the testicles forced up to the external rings, the position of the clamps, the through-and-through sutures above the clamp, and the edges brought together after the removal of the clamp.

Divide the scrotum transversely below the clamp. Remove the clamp. Bring the edges of the incision together by tying the previously set sutures.

The stitches are to be removed in from seven to ten days, or, if catgut is used, they are allowed to drop off.

The patient may be allowed to get up at any time after the fifth day. 


\section{EXCISION OF THE SCROTUM.}

This operation is performed especially in cases of lymph-scrotum. The incisions will be varied somewhat in accordance with the requirements imposed by the differences of the condition in individual cases. Occasionally the inguinal glands and the spermatic cords may be the seat of filarial disease, and should receive attention when such is the case. Under these conditions the incision may have to be more or less extended and carried upward to the inguinal region.

FIG. I 49

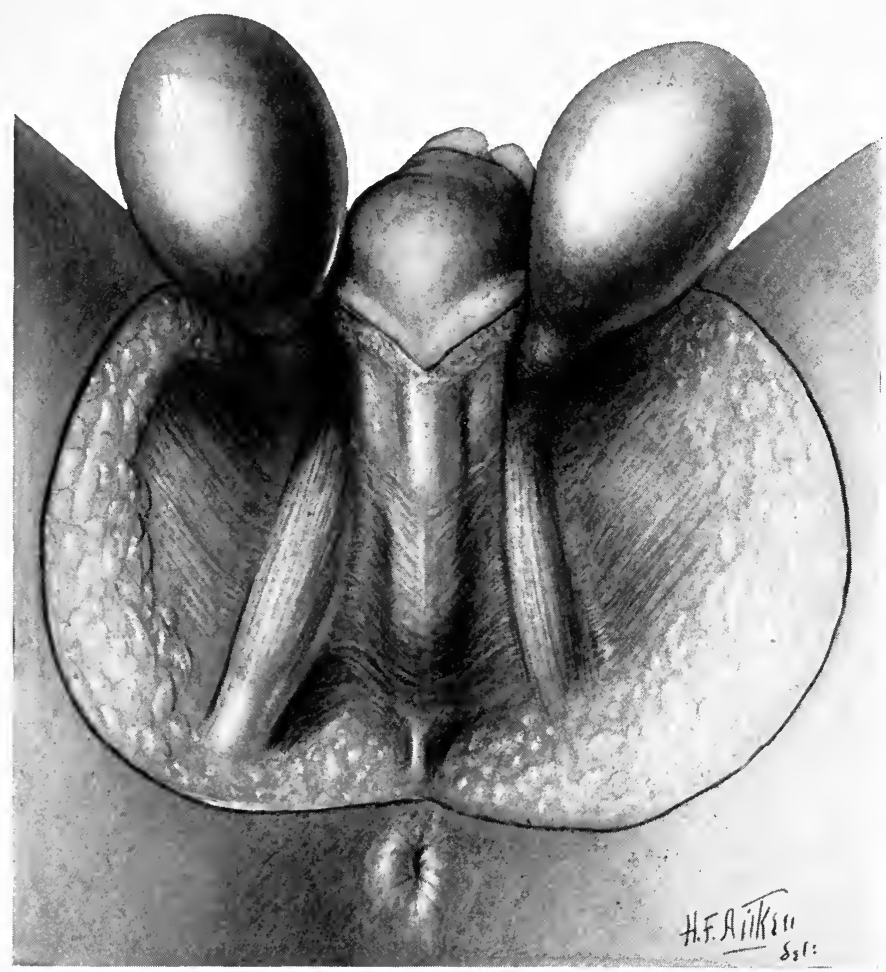

The scrotum removed; the testicles and the external muscles of the perineum exposed.

Instruments.-The same as for the last operation, and, in addition, several pairs of artery forceps.

Operation.-The incision begins at the junction of the scrotum with the thigh on one side, is carried upward across the base of the penis and back again in the corresponding line on the other side, until it meets the point from which it started, passing close to the upper margin of the anus on its way, making an oval area, which is included within it, and surrounding the scrotum. The testicles are forced upward toward the external abdominal rings and the diseased and infiltrated tissues included 
within the incision are dissected off, leaving the testicles and the whole space of the scrotum and the perineum denuded.

Bleeding vessels are to be ligated as they are divided. If the inguinal glands are involved, they should be removed, and the same is true of any lymphatic varicosities along the spermatic cords. They should be dissected out as far as the internal inguinal ring, dividing the fascia of the external oblique muscle in order to gain access to it.

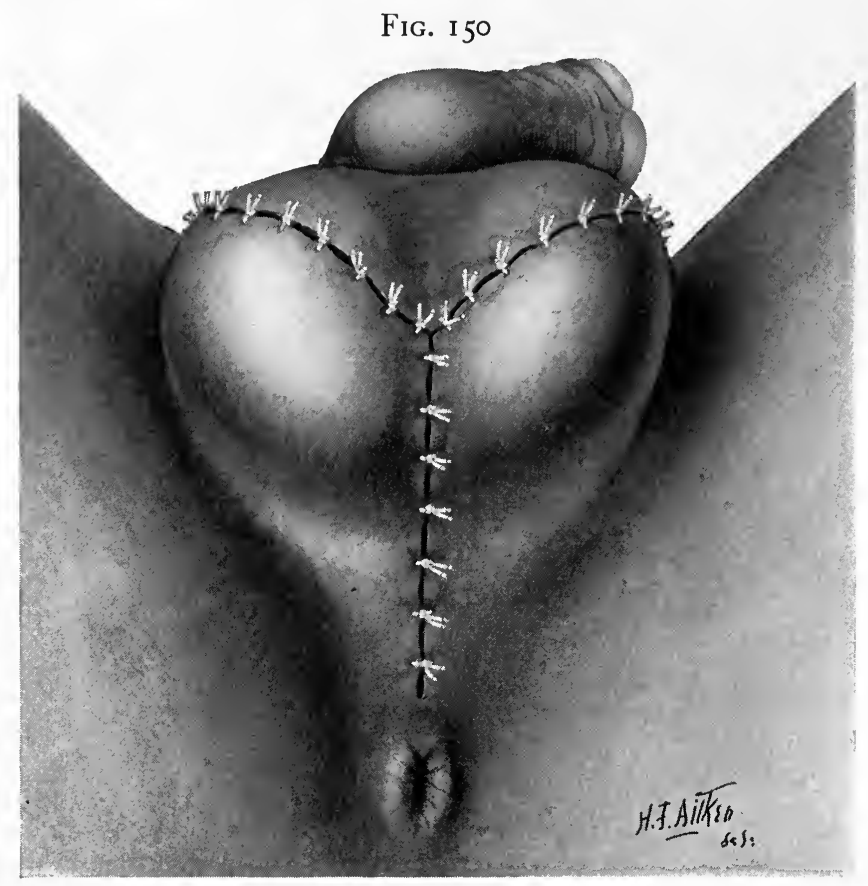

The scrotum is lifted from the thighs, drawn inward, and united in the median line, and the skin from above drawn down and united to the thigh flaps, thus covering the testicles.

Close the wound by interrupted sutures, beginning just in front of the anus, and bringing the edges of the incised skin together as far as this can be done (Fig. I50). If the bared area cannot be wholly covered in this way, Thiersch skin-grafting can be employed, or a plastic operation done.

\section{Bibliography.}

I. Broca. Gaz. hebdom., I899, vol. iv, p. 289.

2. Young. Annals of Surgery, October, 1900.

3. Rigby. Annals of Surgery, November, I907.

4. Baermann. Deut. med. Woch., I903, Nr. 40.

5. Belfield. Transactions of the American Association of Genito-urinary Surgeons, I 906, p. 63.

6. Fuller. Journal of the American Medical Association, January, I901, vol. xxxvi, p. 1228. 


\section{P A R T I I.}

THE PROSTATE AND BLADDER.

C H A P T E R X I.

THE PROSTATE.

\section{ANATOMY OF THE PROSTATE.}

Gross Anatomy.-Size and Shape.-The normal prostate has approximately the size and shape of a Spanish chestnut. Its length from apex to base is about one and one-half inches; its transverse diameter is a little more than this. It is three-quarters of an inch in thickness.

Weight.-Between 4 and 5 drams.

Lobes of the Prostate.-The prostate is composed of two lobes, which are joined together above and below the prostatic urethra, which passes through the gland from base to apex longitudinally.

The part joining the two lobes above the urethra is called the anterior, that joining them beneath it, the posterior commissure.

The upper ends of the lobes - the base of the prostate-are separated from each other by a slight interval, which can be felt by the finger in the rectum, as can also the furrow which corresponds to the line of the posterior commissure, and which is most marked near the base of the gland.

The urethra traverses the prostate in such a way that one-third of the gland lies above, and two-thirds below, the canal.

Surfaces.-The posterior surface of the gland is rather flattened; the anterior and lateral ones are more convex.

The Capsule and the Outer Fibrous Sheath of the Prostate.-Capsula Vera.-The true capsule of the prostate is a thin membrane which invests it, and is intimately connected with the outer surface of the gland.

From the inner aspect of this capsule numerous strands of connective tissue and involuntary muscle fibers extend inward and become continuous with the stroma of the prostate. From the outer surface of the capsule other connective-tissue fibers are given off and pass to the inner surface of the second or outer covering of the gland, which is known as its fibrous sheath, thus attaching these two membranes to each other. 
The attachment of the capsula vera to the outer surface of the gland is so intimate that it is very difficult to separate the two, and it cannot be done without tearing the gland substance and bringing away more or less of it, together with the capsule. The connection between the true capsule and the outer fibrous sheath is, on the contrary, much less intimate, and the two membranes can ordinarily be separated without difficulty.

These facts are of practical importance, since the looseness of the connection between the two membranes makes it possible to enucleate the gland readily, if the procedure be carried out in the space between them, while it becomes impossible to accomplish it if the attempt be made to do so by separating the capsula vera from the surface of the gland. Fig. I5I shows the capsula vera.

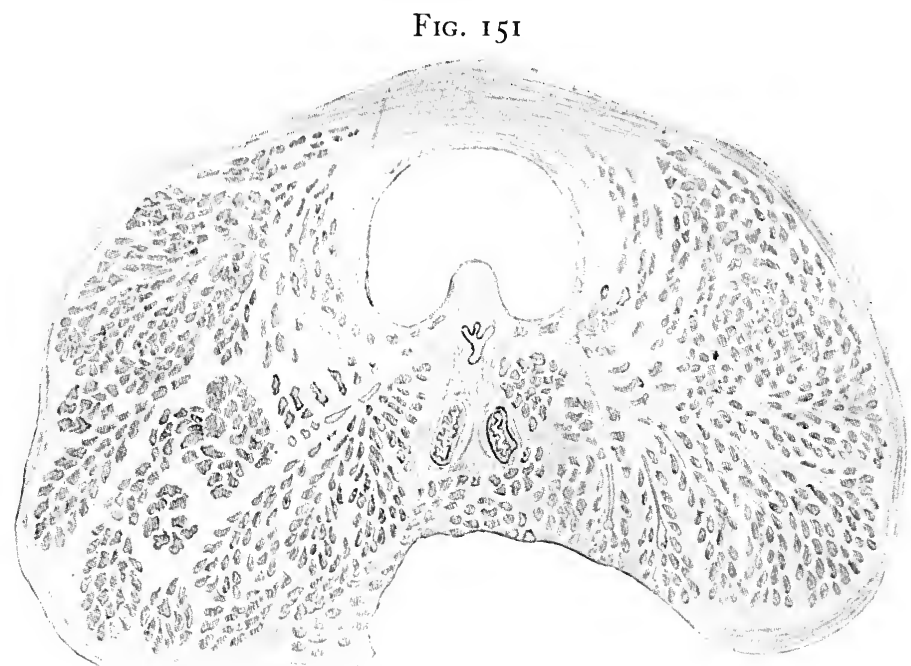

Transverse section through middle of human prostate, showing capsule, stroma, anterior commissure, arrangement of gland tissue in lobules, common ejaculatory ducts, and utricle. (From W. G. Richardson.)

The Fibrous Sheath.-This constitutes the second or outer envelope of the gland. It consists of a thick, firm layer of connective tissue, which surrounds the prostate. It is derived from the rectovesical fascia, with the inner layer of which it is continuous. The same layer of this fascia also goes to form the fibrous coat of the bladder, and is reflected onto the rectum. It overlies the seminal vesicles and the vasa deferentia posteriorly, and accompanies the ejaculatory ducts, forming their outer coat. In front, it blends with the inner leaf of the triangular ligament.

The outer fibrous sheath of the prostate is of much importance in connection with the operation of total prostatectomy, because of its forming and defining the external limits of the field of operation within which the procedure must be confined, and executed without doing injury to this 
sheath; also because of its affording protection to the rectum and the bladder during the enucleation of the gland; and finally, because the prostatic plexus of the veins runs through it, and will not be injured and give rise to hemorrhage, unless the sheath is torn.

Laterally, the outer sheath is in relation with the tissues of the ischiorectal fossæ, anteriorly with the puboprostatic ligament, the areolar tissue of the lower part of the prevesical space, and with the dorsal bloodvessels of the penis. Posteriorly, it is in relation with the areolar tissue lying between the prostate and the rectum. The latter connection is not a firm one, and allows the separation of the posterior surface of the sheath from the bowel without difficulty. This also has a practical bearing with relation to the removal of the prostate by the open or dissecting methods, as they are termed, since it is owing to the looseness of this connection that it is possible to expose the posterior surface of the gland with ease and to make it accessible to operative manipulations.

The sheath varies in thickness in different parts. In the condition of senile hypertrophy of the prostate it is usually thin where it invests the bases of the lobes, and interposes between them and the neck and floor of the bladder.

Whether or not it is always the case that the sheath is continued in the form of a partition between the enlarged middle lobe and the two lateral ones, in cases of prostatic hypertrophy, in such a way as to make a separate chamber for each of the three, as is well shown in Fig. I52, we do not know, but it certainly does so in a considerable number of the specimens which have been examined by us with reference to this point. The practical bearing of this fact is obvious, and it becomes evident that, in cases of three-lobe enlargement, it is better to remove the median enlargement separately, rather than to attempt to take it away, together with the lateral lobes, in one mass (see Fig. 152).

Ejaculatory Ducts and Utricle.-These ducts enter the prostate, one on either side of the median line of its posterior surface and near the upper margin of the gland. W. G. Richardson ${ }^{1}$ describes the utricle and the ejaculatory ducts in effect as follows:

Near the upper margin of the posterior surface of the prostate there is a depression; at the bottom of the depression is the opening of a canal, which descends obliquely downward and forward as far as the urethra, at the wall of which it stops, immediately under the highest point of the veru montanum. This canal is about one-eighth of an inch in diameter throughout, and in it the two common ejaculatory ducts and the utricle are found.

The latter is a narrow blind tube with the open mouth in the urethra and the closed end lying in the further extremity of the previously men- 
tioned canal. The utricle is about one-third of an inch in length. Its walls are composed of involuntary muscle fiber lined by areolar tissue, upon which lies the mucous membrane. External to the muscular coat there is a layer of connective tissue which surrounds the utricle. Except where the utricle perforates the urethra, it is nowhere, strictly speaking, in the prostate. It lies outside the capsule of the gland and is separated from the surface of the latter and wholly disconnected from its stroma.

The mouth of the utricle, which is slightly narrower than the rest of the pouch, opens upon the surface of the verumontanum a little anterior to its summit.

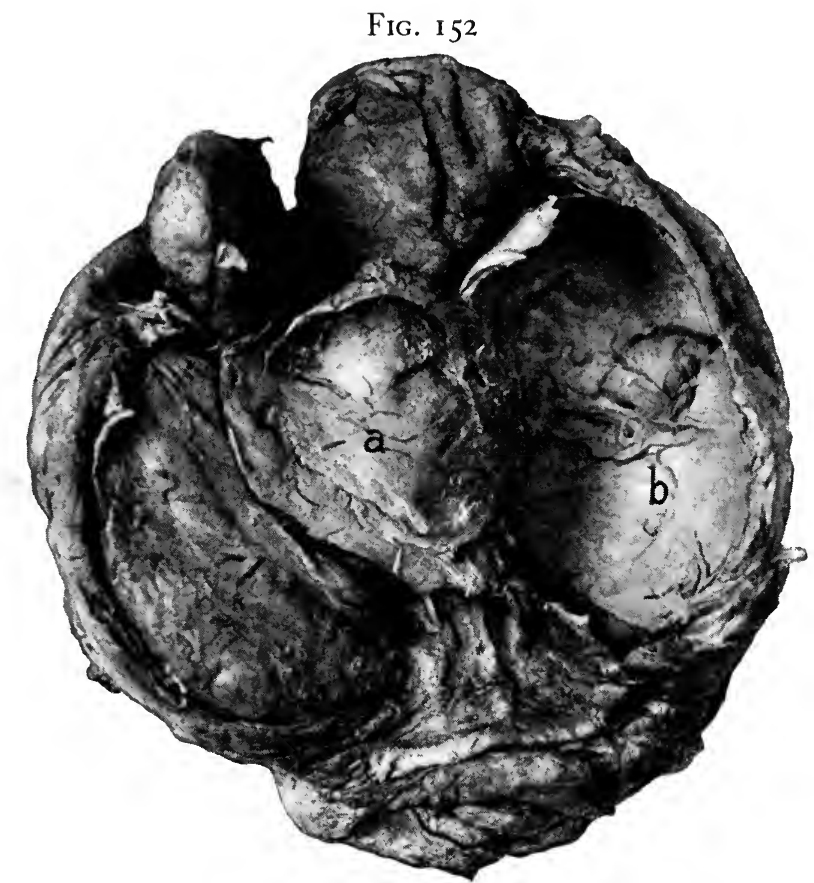

The prostatic urethra and middle lobe of the prostate cut in two longitudinally through their upper aspect in the middle line: $a$, the roof of the chamber of the left lobe turned back over the prostatic urethra; $b$, the interior of the chamber made by the outer sheath of the left lobe of the prostate from which the lobe has been removed; the separate chambers, in which each of the three lobes lie, are seen. (Watson.)

The ducts lie in the middle line of the depression in the posterior surface of the prostate already referred to, along which they pass in company with the utricle, in and upon the wall of which they lie, and upon the lower lip of the mouth of which they open into the urethra by two minute orifices, which are not much larger than to permit the passage of a hog's bristle. In their course through the posterior part of the prostate they do not traverse directly the gland itself, but, as is the case with the utricle, are separated from it by a distinct wall, which constitutes their 
outer coat. The separation of the utricle and ejaculatory ducts from the glandular substance of the prostate is shown in Figs. I 53 and I 54, which are taken from W. G. Richardson. ${ }^{1}$

FIG. I 53

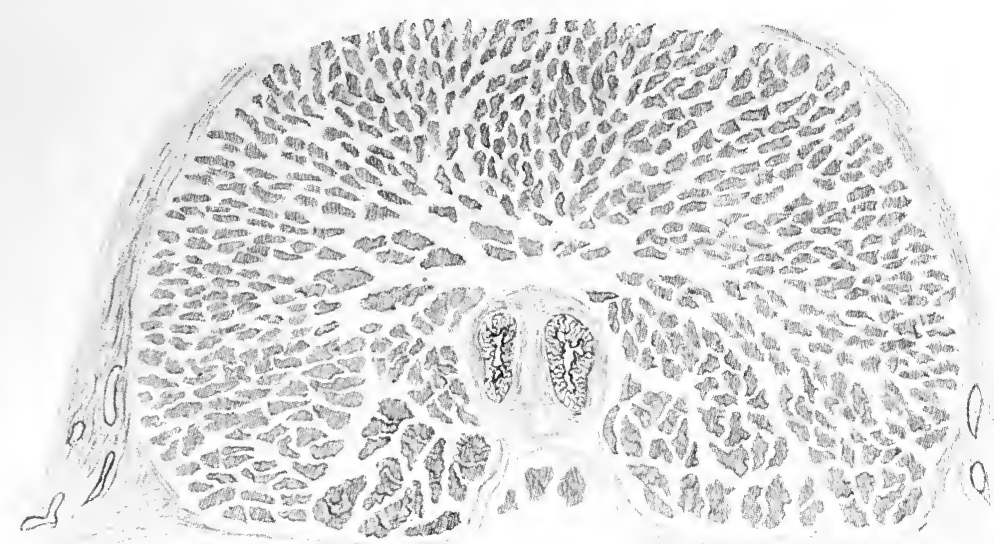

Coronal section of human prostate behind urethra, showing sheath, capsule, stroma, lobules of lateral lobes, separate lobules of middle lobes, and the ejaculatory ducts.

F1G. I 54

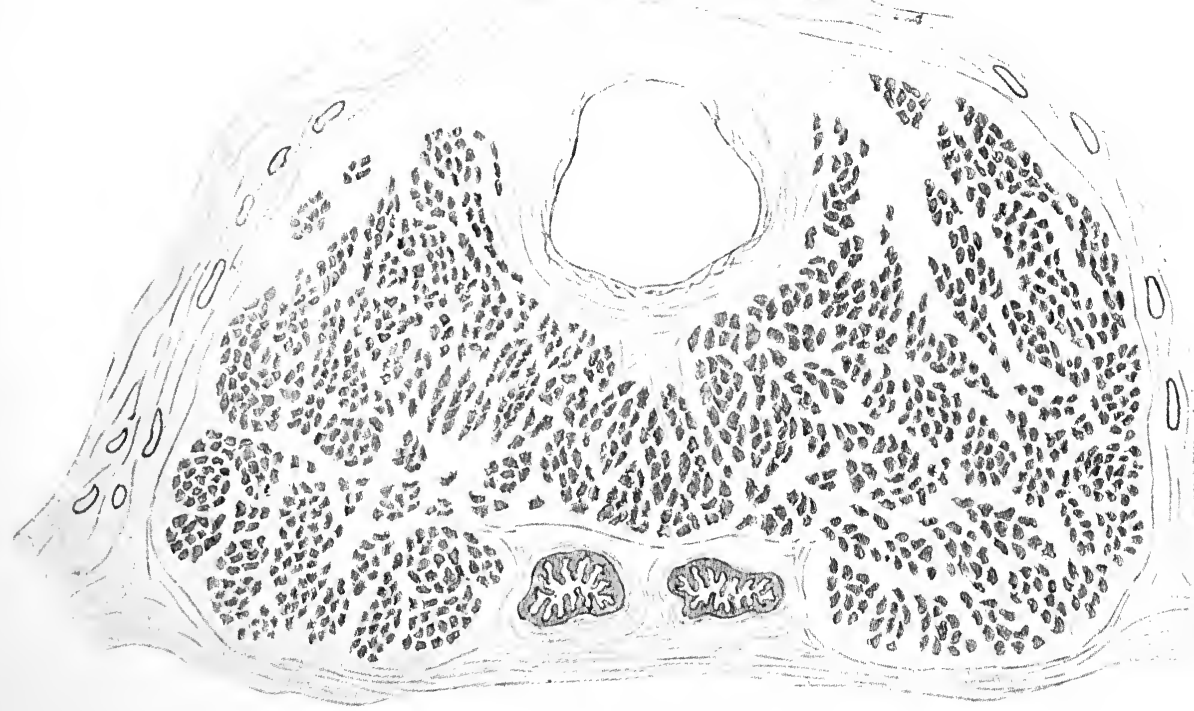

Transverse section through middle of human prostate, slightly magnified, showing capsule, stroma; sheath, anterior commissure, lobules of lateral lobes, separate lobules of middle lobe focussing on back of urethra, and the ejaculatory ducts. 
Structure of the Gland.-The gland is made up of the following elements: glandular tissue, with its mucous membrane and epithelial cells, connective tissue, and involuntary muscle-fiber stroma, bloodvessels, nerves, and lymphatics.

FIG. 155

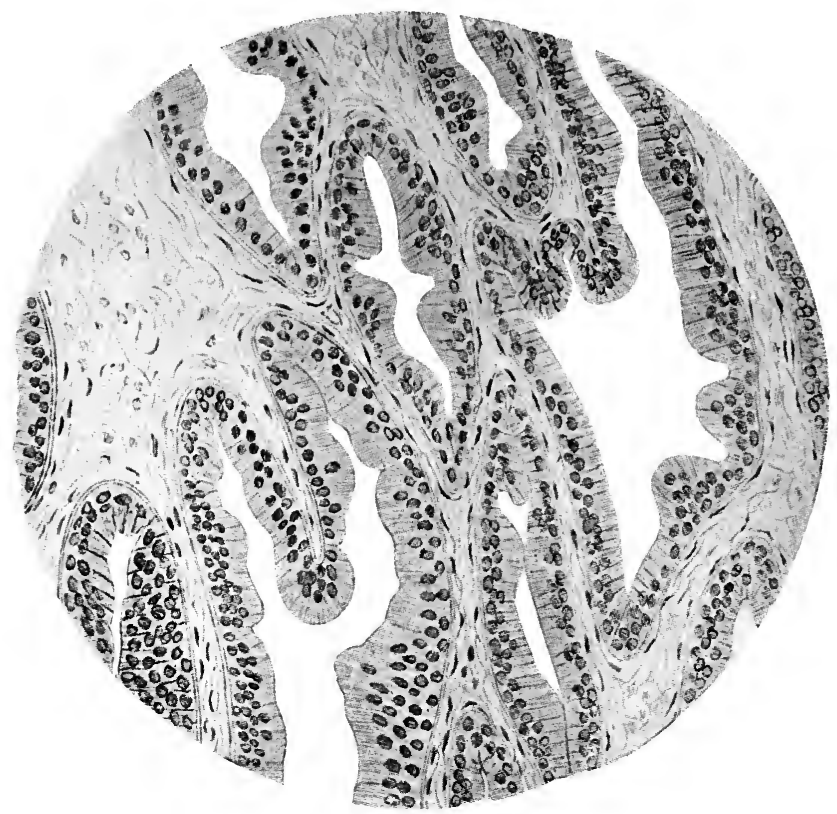

Microscopic section of human prostate, showing stroma, basement membrane, and arrangement of gland epithelium. Tubules mostly in longitudinal section. (W. G. Richardson.)

Fig. ${ }^{5} 6$

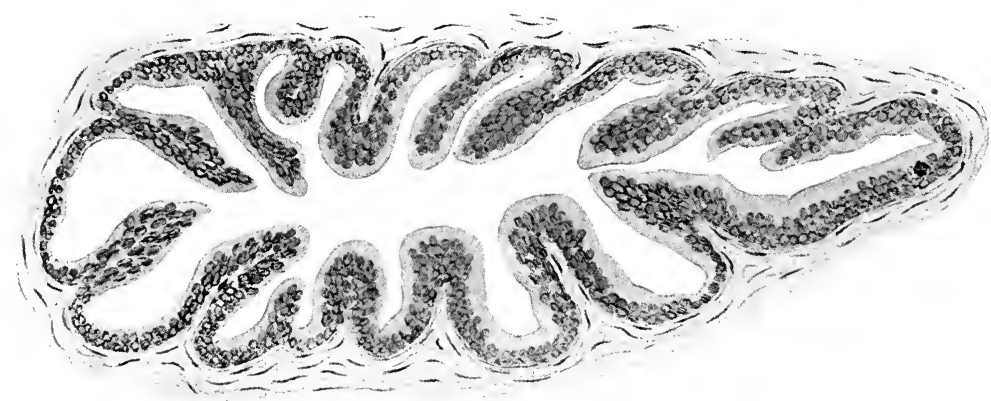

Tubule of human prostate cut in transverse section, showing connective tissue, basement membrane, and arrangement of gland epithelium. (W. G. Richardson.)

Gland Tissue and Stroma.-The glandular part of the structure is separated into different divisions by the partitions made by the stroma, which ramifies through it. Each of these divisions of the gland substance may be considered as an individual gland, and each is provided with a 
duct, these collectively pass to the urethra, upon the floor of which, on either side of the median ridge made by the prolongation of the middle lobe of the gland, they open by a number of small orifices. The structure of one of these glandular bodies is shown in Figs. I55 and 156 . The individual glandular lobules, which together compose the whole secreting substance of the prostate, are acinous and tubular in character.

The stroma, as already stated, consists of the connective tissue and involuntary muscular fiber given off from the inner surface of the capsula vera. It is larger in amount relative to the gland substance near the periphery and less so toward the central portions of the lobes.

The fibers of the stroma blend with the circular muscular ones of the prostatic urethra. They also interlace to form the greater part of the anterior and posterior commissures.

The importance of these last-named anatomical features with respect to the operation of prostatectomy is evident, for they show the difficulty of enucleating the gland without injuring the urethra, and, in fact, explain why it is impossible to do so in many instances without leaving a small portion of the inner lateral aspects of the lobes attached to the outer sides of the urethra.

Bloodvessels.- The arterial supply of the prostate is derived from the inferior vesicle through the vesicoprostatic artery, which is a branch of it, from a number of small branches of the internal pudic, and from the middle hemorrhoidal branch of the inferior vesical arteries.

The veins of the gland are most numerous in the plexus which is chiefly distributed through the outer fibrous sheath upon its lateral and anterior surfaces. It is on account of the anatomical distribution of this venous plexus that the posterior or urethral approaches are selected in operations for total enucleation of the prostate, in order to avoid the risk of wounding the vessels, and it is also on this account that care is taken to avoid tearing through the outer sheath during their performance.

The bloodvessels entering the substance of the gland itself are small in size and few in number. They are of no importance from a surgical point of view. Figs. 155 and 156 show the position of the veins in the outer sheath of the prostate.

Lymphatics.-The lymph channels issue from the surface of the gland, pass upward, and empty into the nodes which lie just below the external iliac vessels.

Nerves.-The sensory nerves of the prostate are derived from the tenth and eleventh dorsal, first, second, and third sacral, and the five lumbar nerves. The fibers supplying the gland substance and governing its secretory function are from the sacral plexus of the sympathetic nerves, and surround the external surface of the outer fibrous sheath.

vol. I-20 
Relations of the Prostate.-The prostate surrounds the urethra from the point of its emerging from the vesical outlet to the posterior limit of the membranous portion of the canal. At this point the apex of the gland rests upon the posterior layer of the triangular ligament.

The posterior surface of the prostate is in relation with the rectum, from the anterior wall of which it is separated by the outer fibrous sheath and by areolar tissue.

The apex of the gland is in relation with the upper portion of the levator ani muscle and the anal sphincter. At this point the gland and the rectum are brought into close apposition by the point of tendinous junction of the anal and perineal muscles in the centre of the perineum. This is important to remember in connection with the performance of the open or dissecting operations of total prostatectomy, for the severing of this tendinous point and division of the recto-urethralis muscle immediately behind it is the key to the successful performance of the operation, inasmuch as the so-called retroprostatic space is thereby opened and the posterior surface of the prostate is made accessible by the ability to separate the rectum from it, which is secured by this manœuver.

The two rounded ends of the lateral lobes of the gland which constitute its base lie one on either side of the vesical outlet and a little above the level of it. In cases of senile hypertrophy of the prostate, the growth of the gland is chiefly in the direction of its long axis, and it proceeds toward the interior of the bladder, producing in so doing a marked elongation of the prostatic urethra and more or less well-defined intravesical projections.

The Third Lobe of the Prostate.-It is generally stated that the third or middle lobe does not exist as an anatomical structure. It is true so far as the presence of any body of lobular form is concerned, but it is not true in the sense that there exists no gland tissue occupying the middle line of the vesical outlet and upper end of the prostatic urethra, for the contrary is the fact. At this point there exists a V-shaped area, its base directed upward toward the bladder, which rises slightly above the surface in the middle line and constitutes a wedge-shaped body interposing between the sides of the beginning of the prostatic urethra, which, consequently, take the form of depressions, and which represent the beginning of the prostatic sinuses upon this part of the floor of the urethra. Immediately beneath this $V$-shaped part of the surface lies a small mass of gland tissue, and this is the third or middle lobe of the prostate, which, in a certain number of cases of senile hypertrophy of the gland, becomes enlarged, and appears in the form of a tumor of greater or less size projecting into the interior of the bladder. 


\section{HYPERTROPHY OF THE PROSTATE.}

Definition.-The term hypertrophy of the prostate has been so long in use that we prefer to retain it even though it is not strictly correct, inasmuch as the changes taking place in the gland, although they are a hyperplasia of its normal tissues, represent a degenerative process, and not the increase of tissue growth, which is seen in some other organs when they are called upon to overcome an obstacle which is opposed to the natural performance of their function.

The hyperplasia of the prostatic tissues is only noted at or after middle life, and results in the enlargement of the gland, to which the name hypertrophy has been given.

\section{Fig. I 57}

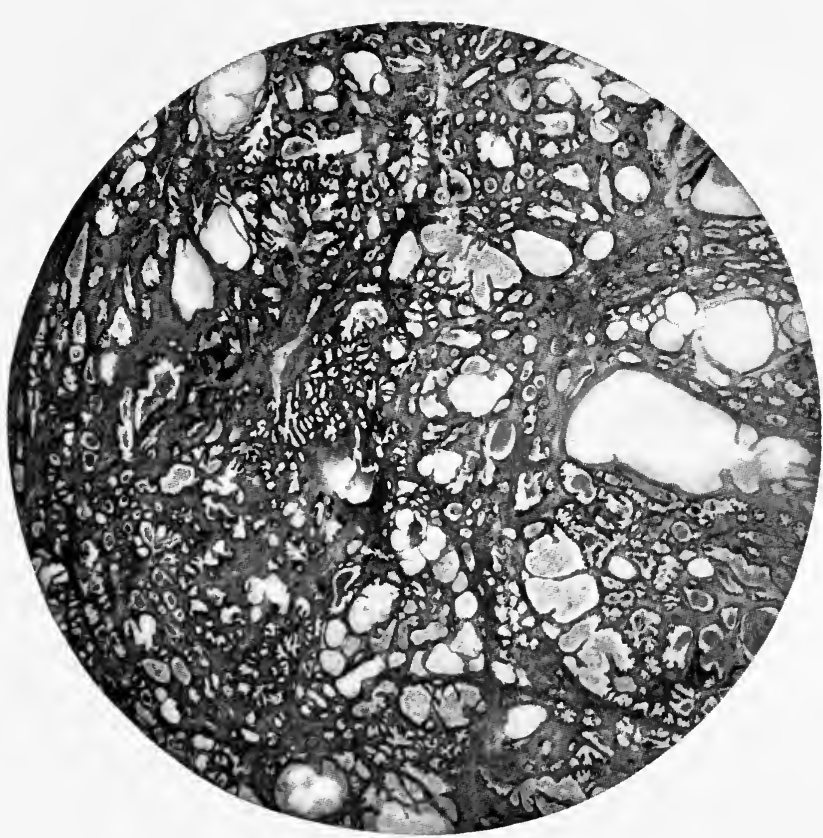

Shows general character of the process. The white parts, glandular overgrowth. The darker parts, muscle fiber, and a small amount of connective tissue. (From specimen of the author.)

Frequency.-It is generally estimated that about one in every five men after the age of fifty has more or less of this form of prostatic enlargement, and that about one in every twelve or so has more or less marked symptoms in consequence of it.

Etiology.-Among the many theories advanced to explain the occurrence of the senile enlargement of the prostate gland, the only one that can be 
said to bear close scrutiny, and that is common to all cases, is that of age. Sexual excesses, atheromatous changes, Ciechanowski's ${ }^{2}$ tentative explanation that the condition has its origin in chronic inflammation, usually of gonorrhœal nature, all fail to cover the ground, and are all inadequate. The best, perhaps, that can be said is that at or after middle life the greater number of persons exhibit a tendency to fibrous formations of one or another structure of the organism, and that among these a very common one is that of the change which affects the prostate, although the changes are not confined to the connective tissue of the gland.

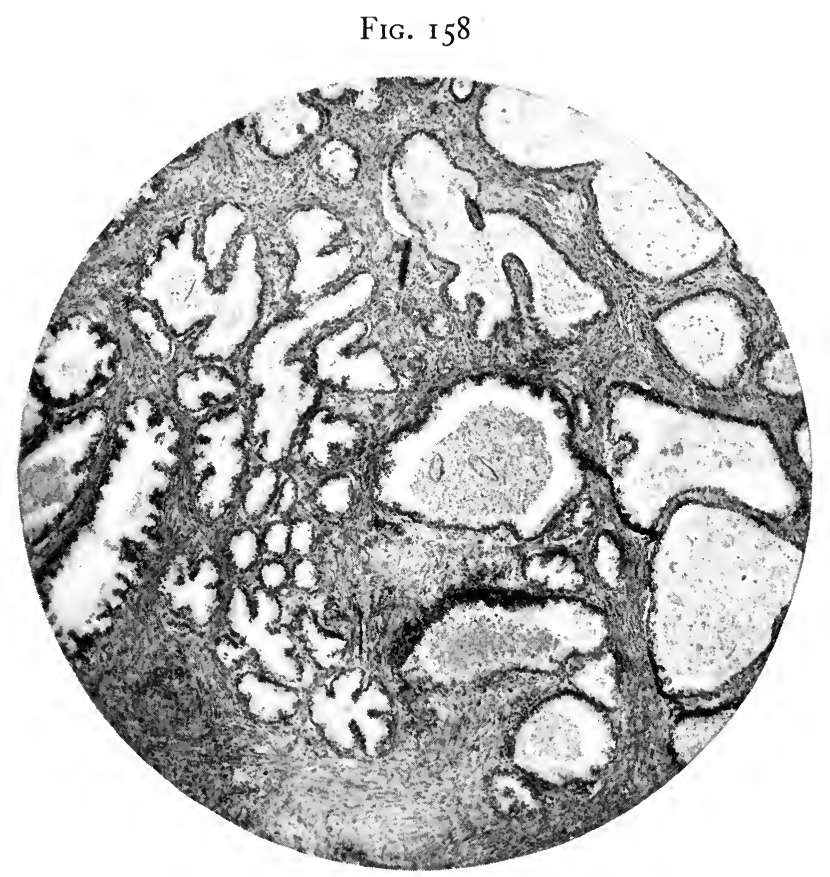

The same as Fig. 157. Higher power, lighter parts are glandular overgrowths. The darker ones, muscular fiber, and a small amount of connective tissue. (From specimen of the author.)

Pathology.-Microscopic Changes.-Hypertrophied prostates may be divided into two classes, according to the nature of the tissue changes taking place in the gland:

I. That in which the overgrowth of glandular tissue predominates.

2. That in which the overgrowth of connective tissue predominates. Apart from these two main groups, there are a small number of cases in which there is a marked increase in the muscular elements of the prostate, and a few instances in which there is believed, by some observers, to be an actual new formation of the acini, which condition would 
represent true adenoma. Others contend that the changes noted in the last-named group consist in the dilatation of already existing acini, produced by the blocking up of their ducts by the epithelial elements of the gland tubules which have undergone proliferation. Sometimes the acini are filled with masses of epithelial cells, which has led to the erroneous belief that the condition was cancer, when such was not the case.

The prostates in which there is a preponderance of the hyperplasia of the glandular elements are the larger and softer forms of the enlarged gland; those in which the connective-tissue elements are the conspicuous feature are the smaller and harder ones.

FIG. 159

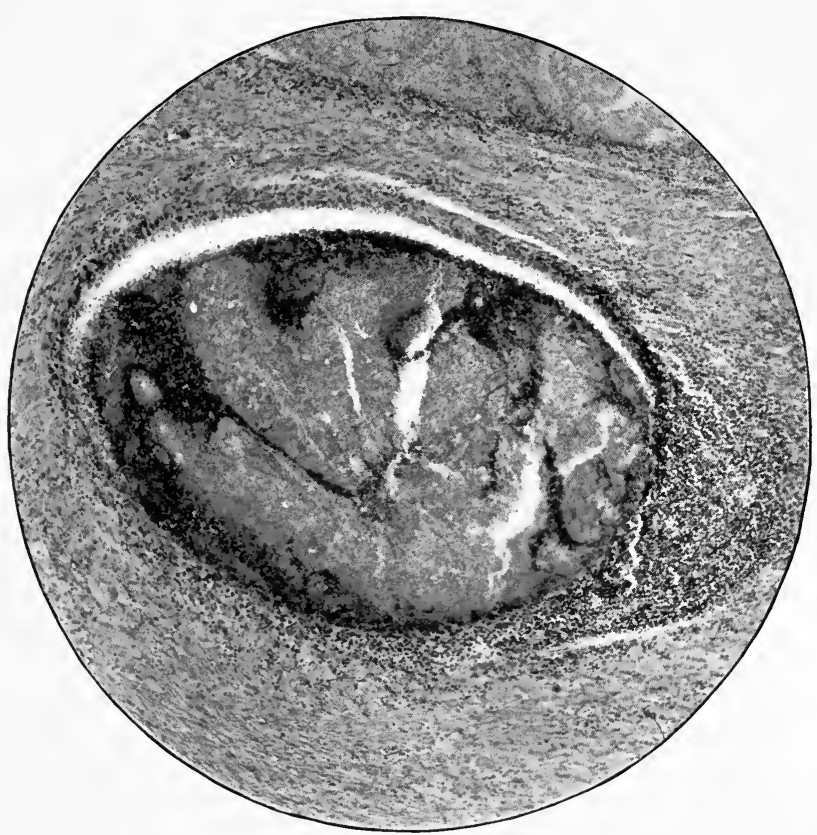

A single acinus blocked by granular-cell detritus and dilated. Surrounding tissue is muscular and connective-tissue fiber and fine round-cell infiltration immediately around the circumference of the dilated acinus. (From specimen of the author.)

Proof is lacking of the assertion that the hard prostates in which the connective-tissue overgrowth predominates represent a later stage of the condition which began as a glandular overgrowth.

Figs. 157, 158, and I 59 illustrate the microscopic appearances presented by some of the above-mentioned varieties of the condition.

Macroscopic Appearances.--The relative frequency with which the different parts of the gland are involved may be judged by the following figures, which, with the exception of the series of observations of Mansell Moullin, are taken from Keyes: ${ }^{3}$ 
Cases in Which the Median and Both Lateral Lobes are INVOLVED.

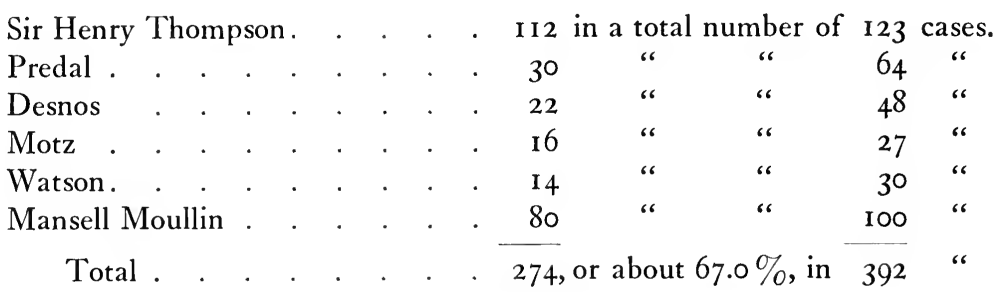

FIG. I60

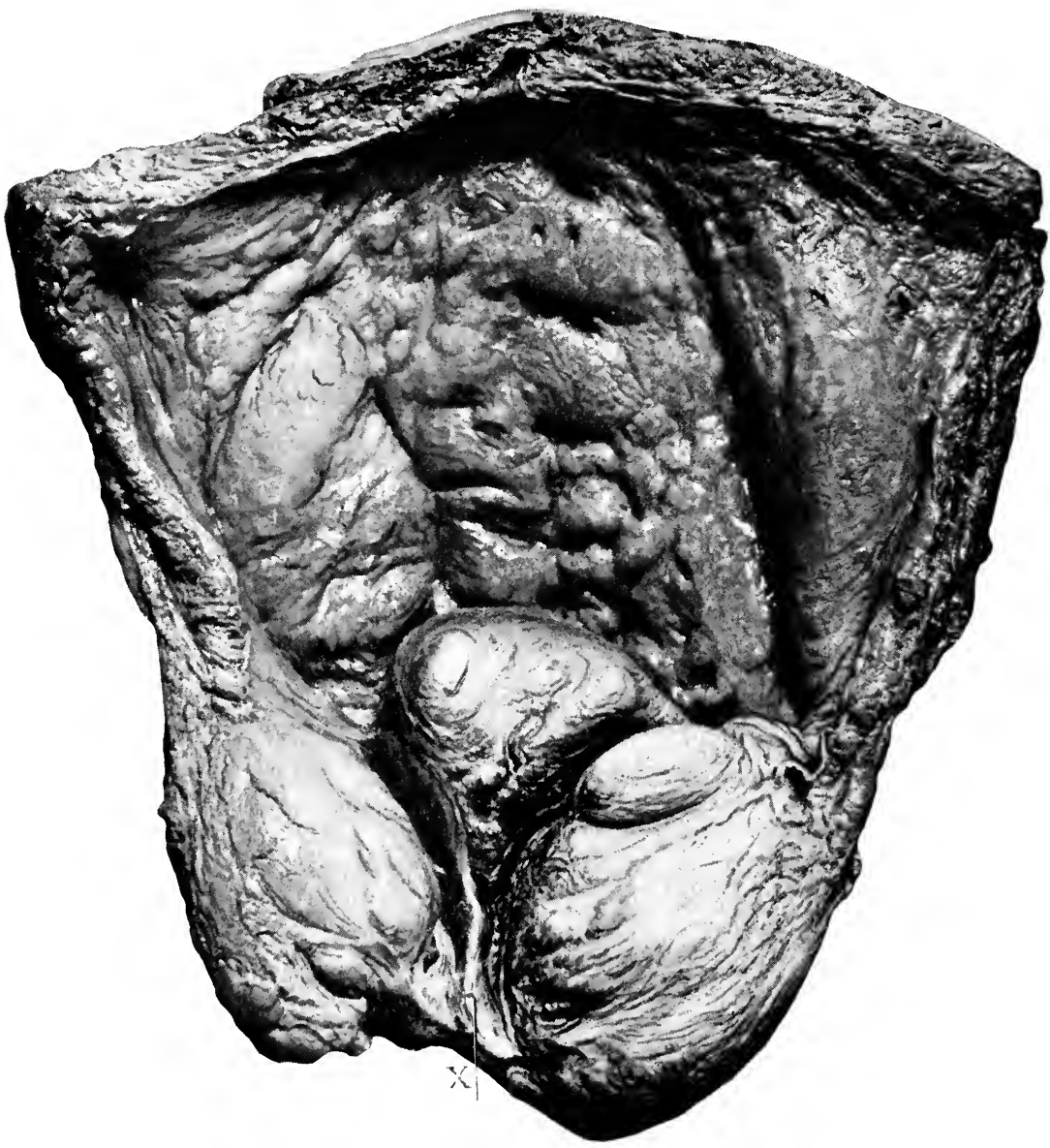

Hypertrophy of both lateral and the median lobes. The Y-shape taken by the prostatic urethra as it passes on either side the median enlargement to enter the bladder is well shown. Reduced $1 / 5$. (Watson.)

The middle lobe alone was enlarged in the same series of cases in I3 per cent. of the whole number, namely, in 52 . 
The lateral lobes only were involved in about $\mathrm{I} 3$ per cent., or $5 \mathrm{I}$ of all the cases.

In a smaller number, there is enlargement of one lateral and the median lobe, or of one lateral lobe only, and some few cases of irregular forms of

\section{Fig. I6I}

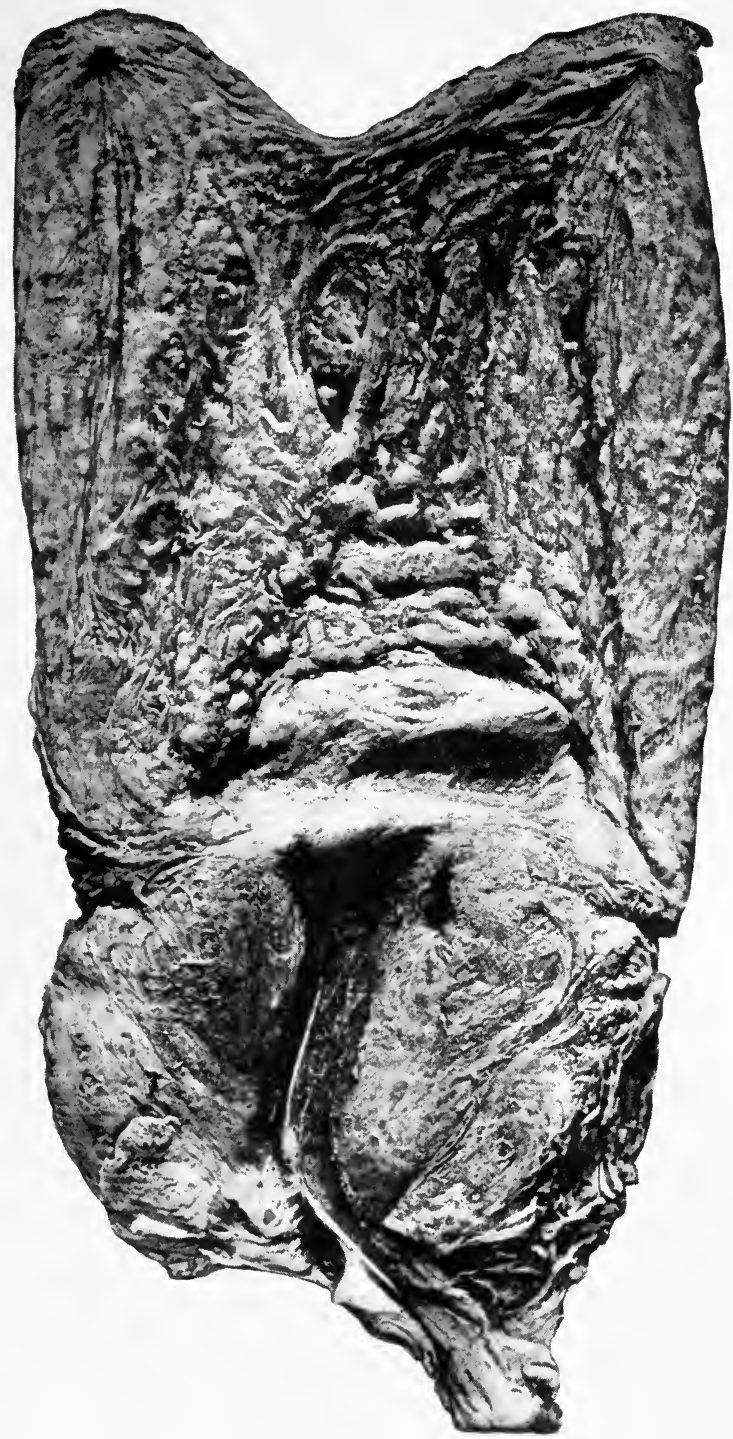

Bilateral hypertrophy. The two lateral lobes joined by a bridge or median bar, the so-called bar at the neck of the bladder. Reduced $1 / 4$. (Watson.)

enlargement. Examples of all the above-noted varieties are shown in Figs. I60 to I70, inclusive.

Bar at the Neck of the Bladder.-This term is applied to a modified form of hypertrophy which unites the intravesical portions of the enlarged 
lateral lobes, or which may occur in the absence of lateral hypertrophy. In that case the condition takes the form rather of a contracture of the neck of the bladder.

Size.-The hypertrophied gland may vary in size all the way from the small median enlargement shown in Fig. I69 to the enormous general hypertrophy illustrated in Fig. I70.

Changes in the Prostatic Urethra.-These are: (I) increase in the length of the prostatic urethra; (2) change in the direction of the canal; (3) widening of it; (4) narrowing of it.

FIG. I62

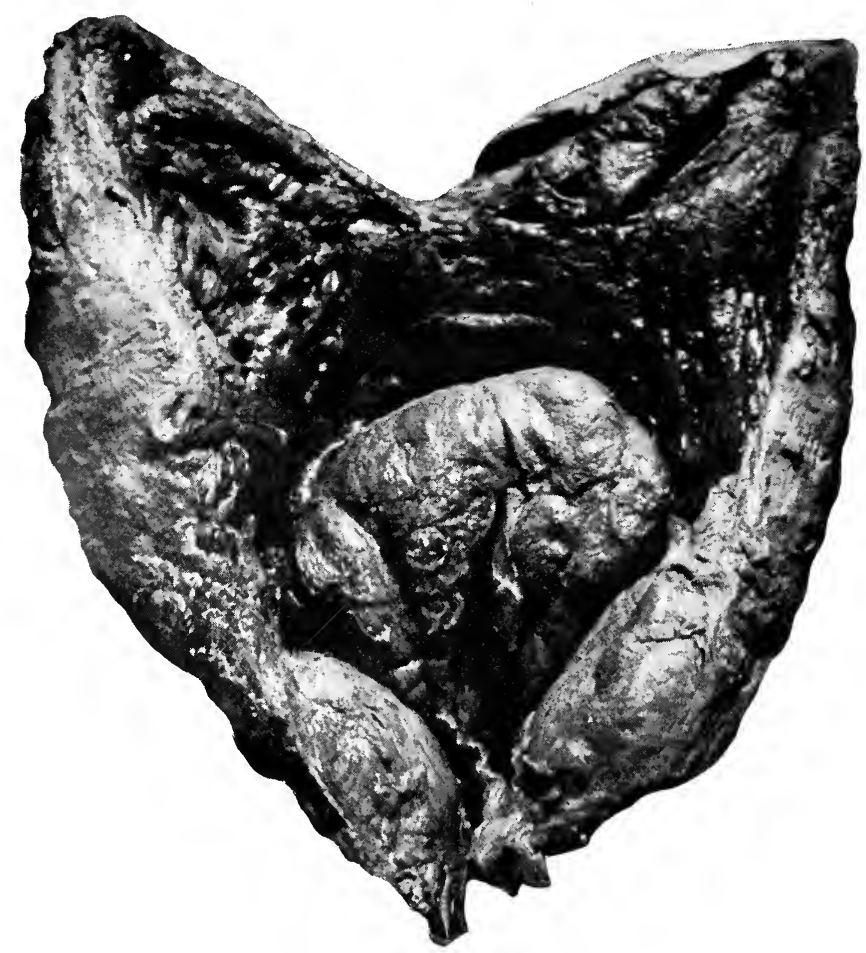

Hypertrophy of median lobe only. (Watson.)

I. All lateral-lobe enlargements lengthen the prostatic urethra more or less. This is owing to the direction taken by these lobes in the course of their growth, which is upward toward the vesical outlet as well as laterally. The fibers of the connective and muscular tissues of this part of the bladder are continuous with those of the urethra, so that the prostatic portion of the latter necessarily shares the elongation of the lateral lobes and takes the same direction.

2. Change in direction of the prostatic urethra is produced in one of three ways: the first being the upward turn given to it by the abrupt obstacle 
formed by a middle-lobe enlargement, as seen in Fig. I7I; the second being a deviation to one or the other side, produced by the greater enlargement of one or the other lateral lobe as compared to its fellow (see Fig. I64), the third being the branching in two directions caused by the passage of the canal on either side of the middle lobe near and at the vesical outlet, which gives to the urethra at this point the Y-shape shown in Fig. I60.

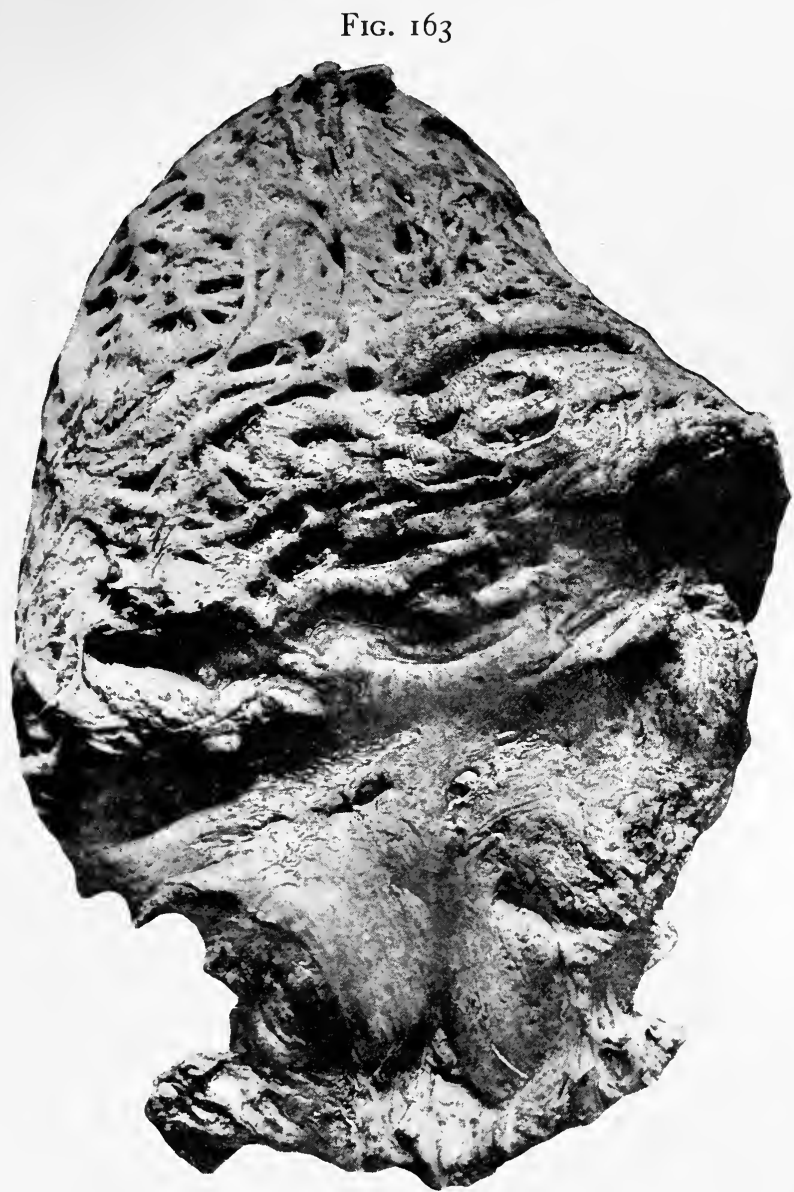

Hypertrophy of the lateral lobes only. (Watson.)

3. Widening of the prostatic urethra is produced in some cases and is difficult of explanation. Mansell Moullin ${ }^{4}$ speaks as follows with regard to this point: "When there is an upgrowth of adenomatous tissue in the posterior wall of the urethra, and when it is accompanied by uniform enlargement of the median and lateral lobes, it sometimes leads to permanent dilatation of the prostatic urethra. As it grows it spreads forward and outward as well as upward into the bladder, and holds the sides of the urethra apart. If they are rigid, the canal is kept open and 
a permanent cavity formed. This space is often capable of containing several drams of urine, and is sometimes of such a size that a shortbeaked sound can be rotated in it without difficulty."

4. Narrowing of the canal is produced by the encroachment upon it of the lateral lobes.

Prolongation of the median enlargement into the prostatic urethra in the form of a more or less well-marked ridge occurs in about two-thirds of the cases of median enlargement. It is well shown at $X$, in Fig. I60.

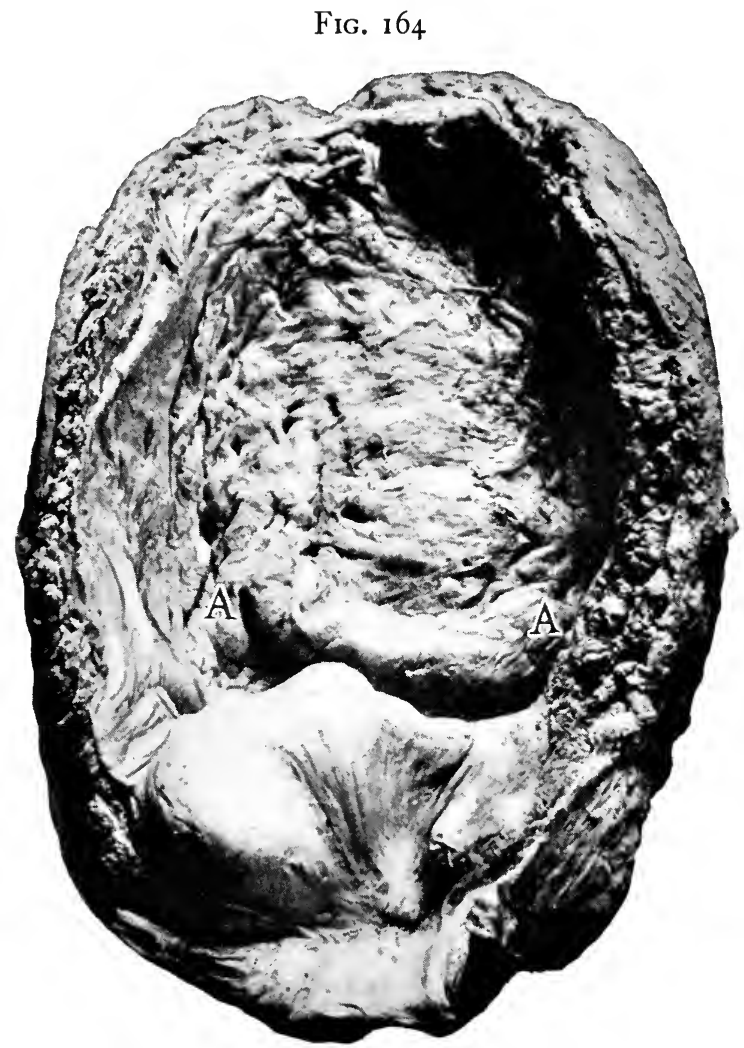

Irregular form of hypertrophy. Median and one lateral lobe: $A, A$, interureteral bar. (Watson.)

Changes in the Outer Fibrous Sheath.-These consist in a thickening of the structure and in the formation of more or less dense adhesions between the inner surface of the fibrous sheath and the outer surface of the gland in some instances. Both have a practical importance-the first because of the greater ease and safety with which the gland can be enucleated when it exists, and the second because it sometimes makes enucleation difficult or impossible. 
Secondary Changes in the Genito-urinary Tract, Due to Prostatic Hypertrophy.-These are primarily due to mechanical influences and are first manifested in the bladder. Subsequently, the ureters, renal pelves, and kidneys are involved.

Changes in the Bladder.-The bladder undergoes either hypertrophy or atrophy. The former change represents nature's effort to overcome the obstacle which the enlarged gland offers to the free escape of urine from the bladder. When it takes place, the bladder wall becomes thickened, loses its elasticity, and its interior capacity is more or less diminished. The increased thickness is due to an hypertrophy of the muscular fibers of the organ. The process is irregularly distributed in

FIG. 165

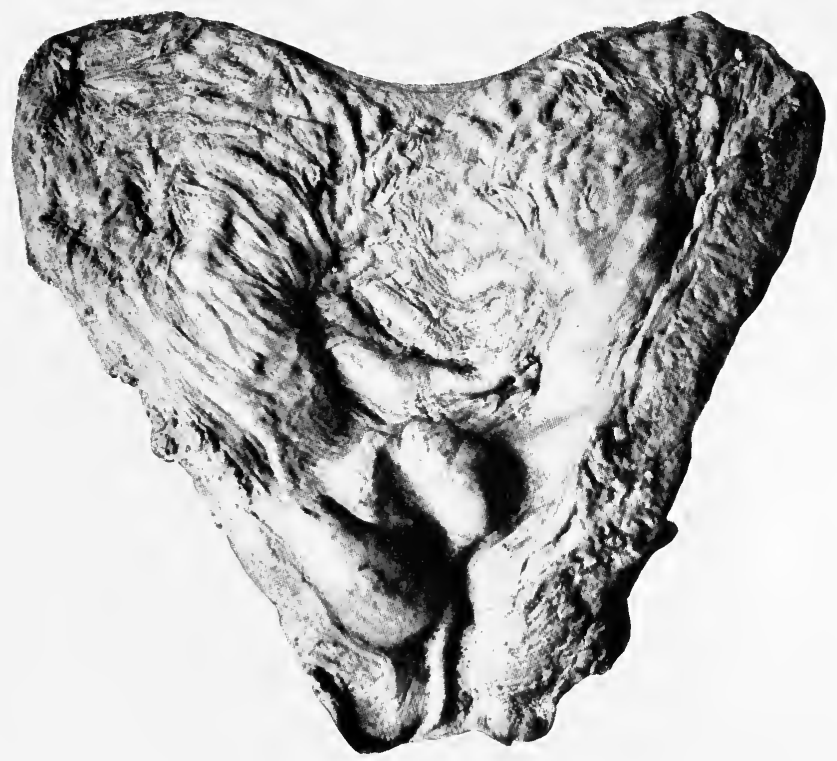

Irregular hypertrophy of middle and one lateral lobe. (Watson.)

such a way as to produce well-marked ridges on the inner surface of the bladder, with intervals between them. This peculiar trabeculation of the bladder is because of the anatomical arrangement of its muscular fibers. The weaker parts which constitute the intervals yield to the intravesical pressure put upon them to such a degree, in some instances, as to be pushed outward in the form of pouches, or, as they are called, saccules or diverticula. (See section on Diverticula of the Bladder.) In the pockets thus formed the urine becomes stagnant, undergoes alkaline decomposition, deposits its alkaline salts, and stone formation not infrequently results, under which circumstances the latter receives the name of "encysted calculus." (See chapter on Vesical Calculus.) 
Interureteral Bar.-This name is given to the bar formed of hypertrophied muscular fibers which extends between the two ureteral orifices. It is more marked and more resistant than any of the other bands of hypertrophied muscle fibers just noted above.

One of the characteristic forms sometimes exhibited in the bladder in these cases is that of a well-developed interureteral bar, with a pouching downward of the bladder above and below it, the weaker parts of the

FIG. I66

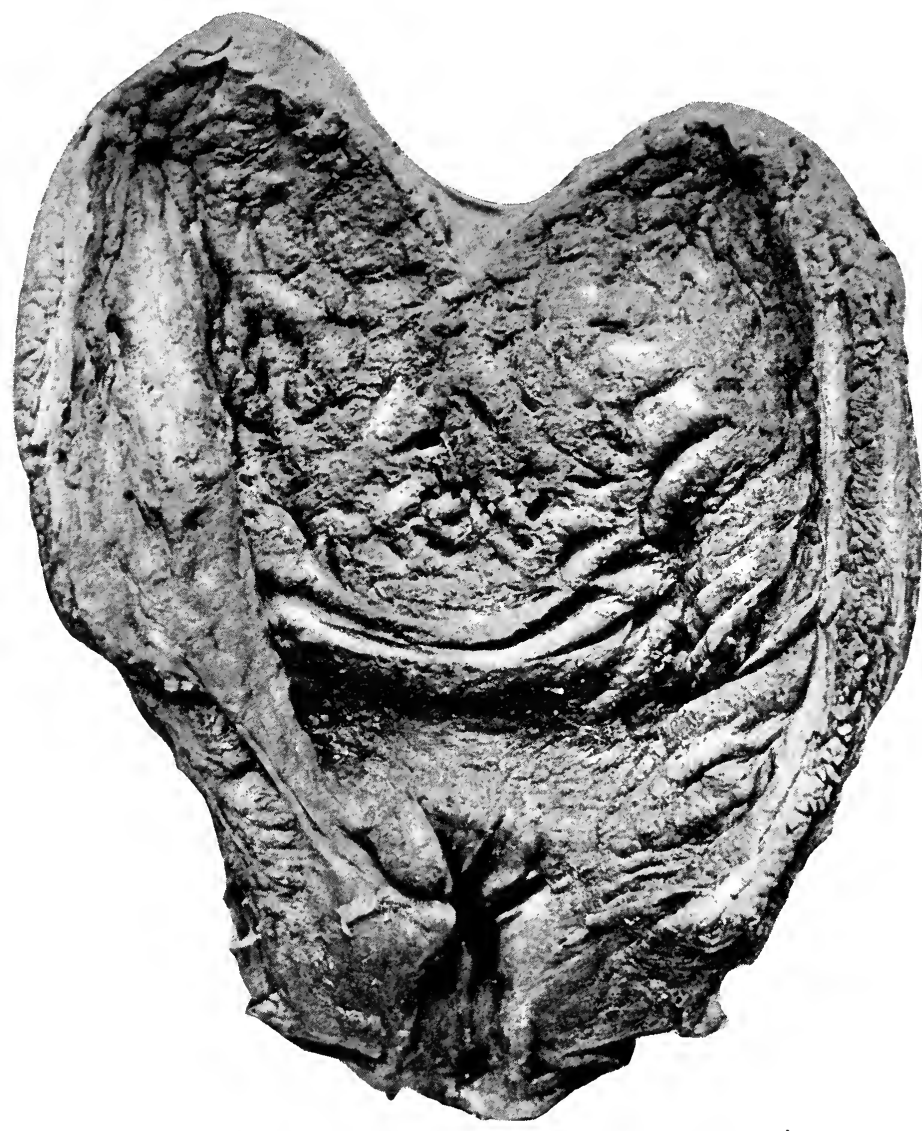

A rare form of irregular hypertrophy. (Watson.)

viscus in these places having been unable to support the strain put upon them by an increasing load of retained urine, while the stronger portion - that corresponding to the bar-still maintains its resistance to the pressure. This bar is well shown in Figs. 161,164 , and 166.

The mucous membranes of the hypertrophied bladders are always thickened and more or less congested or inflamed; the congestion is in part produced by the frequent calls made upon the organ to try to empty 
itself, and to the spasmodic activity of its muscular apparatus in response to these demands; congestion in turn invites inflammation, and the

FIG. 167

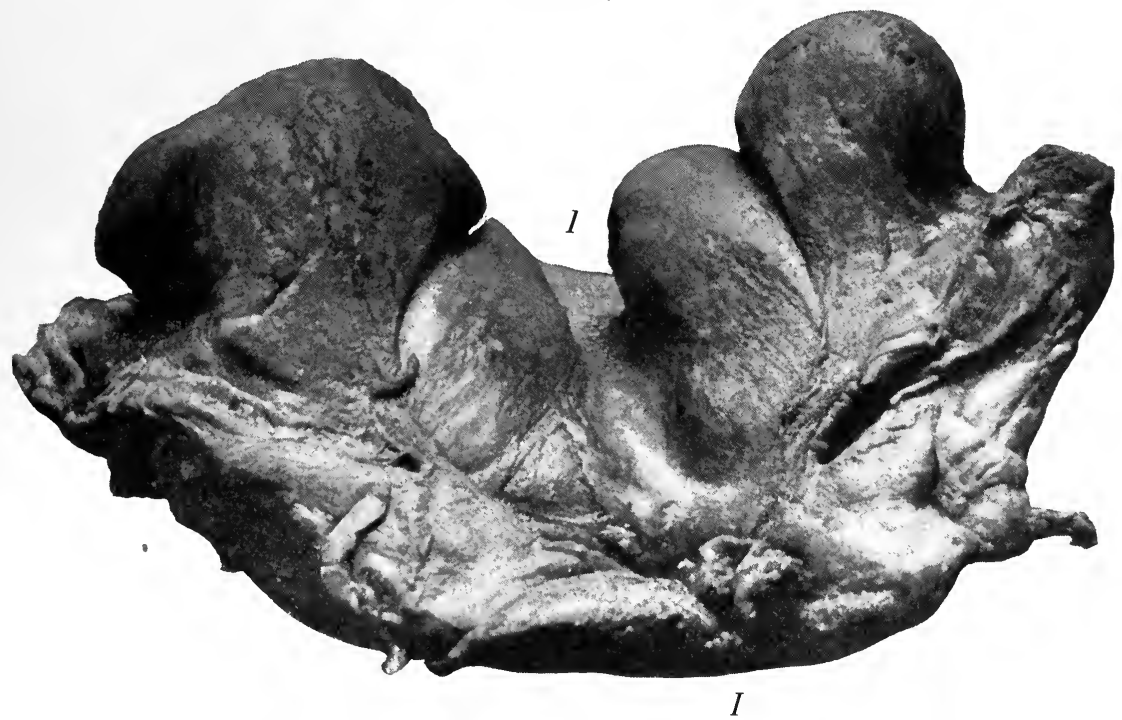

Isolated tumors springing from the lateral lobes of the prostate. Viewed from in front: $I I$, the prostatic urethra. (Watson.)

FIG. 168

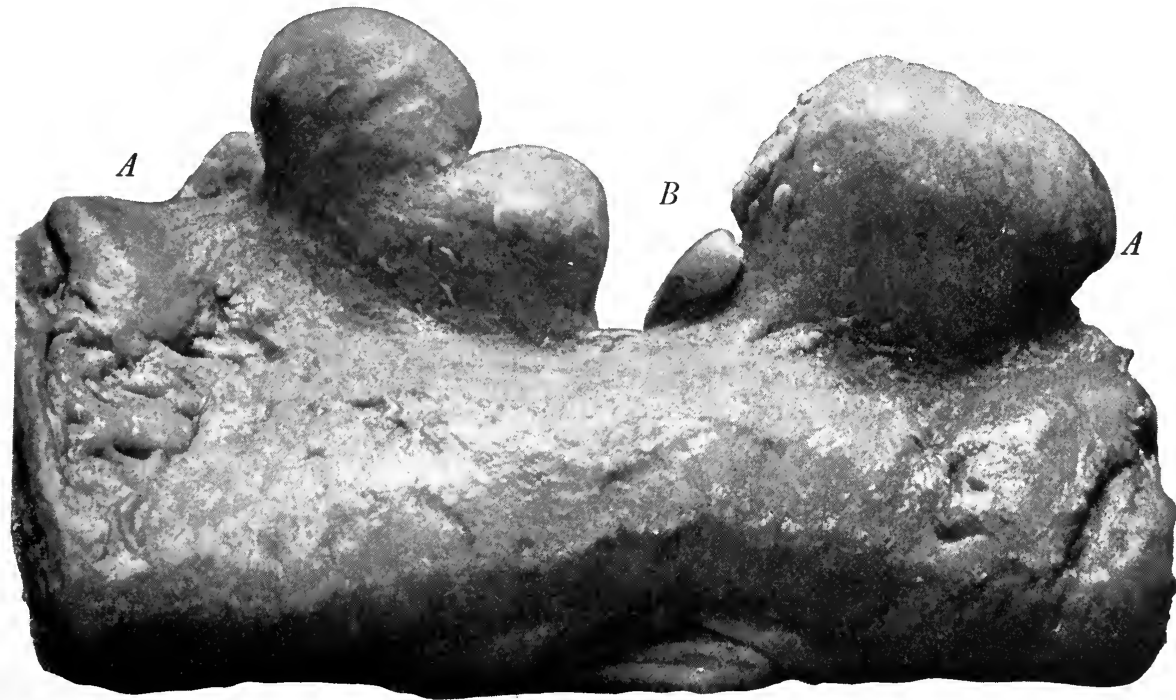

The same specimen as Fig. 167 , viewed from the interior of the bladder: $A A$, lateral tumors; $B$, beginning of prostatic urethra. (Watson.)

invitation is frequently accepted. Thus the hypertrophied bladders with the rigid walls, small capacity, and congested or inflamed mucous membranes are the ones that cause the greatest suffering to the patients. 
The opposite picture is presented in the cases in which atrophy of the muscular apparatus, instead of hypertrophy, takes place at first, or later, when further capability of muscular increase is exhausted.

These are the quiet bladders; they are those which distend gradually without calling the patient's attention imperatively to the dangers associated with them, which become known only because of the increasingly frequent necessity to micturate under which he finds himself, until he is voiding it guttatim and almost continuously, and finally has complete retention, at which time the quantity of urine of which the bladder is

FIG. 169

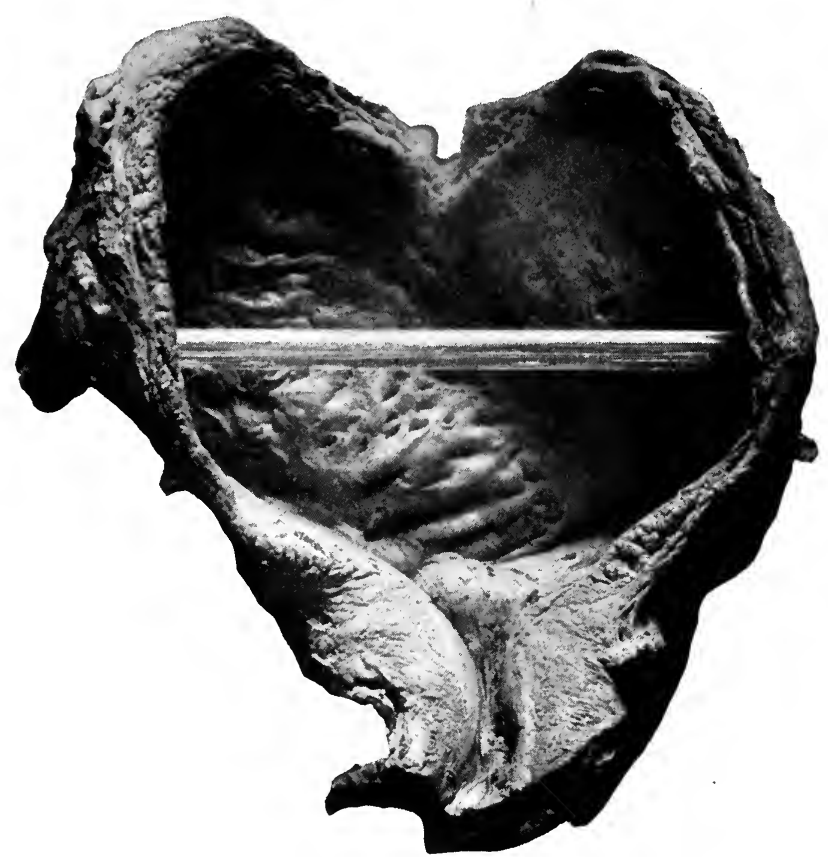

An example of very small middle-lobe enlargement, showing, however, secondary changes in the bladder quite as well-marked as in some cases of much larger hypertrophy. (Watson.)

unable to rid itself may have become enormous. Here the bladder walls are very thin, its mucous membrane is smooth and not thickened, as a rule, and-until (or unless) an instrument is passed into it-is generally not the seat of cystitis. Passive congestion of the venous circulation of the organ is usually very marked in these atonied bladders, and is due to the compression of the veins exercised by the large quantity of residual urine. This congestion exposes such bladders to bacterial invasion, but it occurs later than in those which are hypertrophied.

The Bas Fond of the Bladder.-This term is given to that part of the bladder which lies immediately behind and below the level of the vesical 
orifice-and, in the condition which we are considering-of the intravesical portions of the prostate as well. It has the form of a depression, or pocket, which bags downward (see Fig. I7I). It has much practical importance, for it is this portion of the bladder which harbors the part of

\section{Fig. 170}

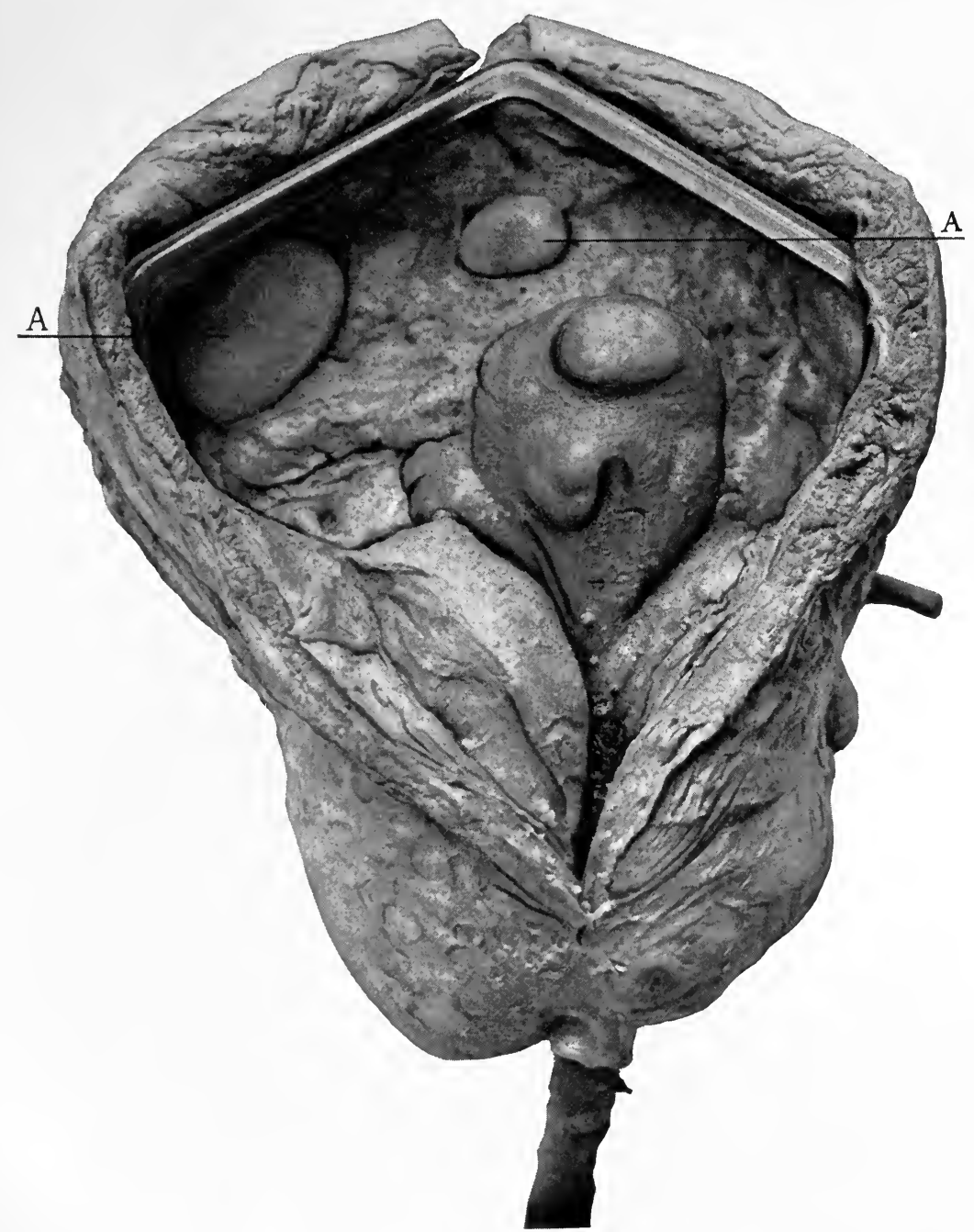

An example of very great general enlargement of the prostate and of a middle lobe reaching high up into the bladder. Two calculi $(A A)$ were present. The smaller one was encysted. (Watson.)

the urine which cannot be evacuated voluntarily, which is termed the "residual urine."

The bas fond may be regarded as a kind of diverticulum, differing only from the usual form of that condition in having a wide, instead of a 
narrow orifice. The urine retained in it is prone to become decomposed, alkaline urinary salts are then deposited, and calculus is very apt to be formed. This is the condition which, above all others, favors the formation of calculi in the bladder, and explains their frequent occurrence in elderly men. In certain cases of prostatic hypertrophy, the intravesical portions of the growth form a ledge or shelf overlying the lowest part of the bas fond, beneath which it sometimes happens that one or more small calculi become lodged, and are then wholly out of reach of either stone searcher, lithotrite, or evacuating tubes. In this situation

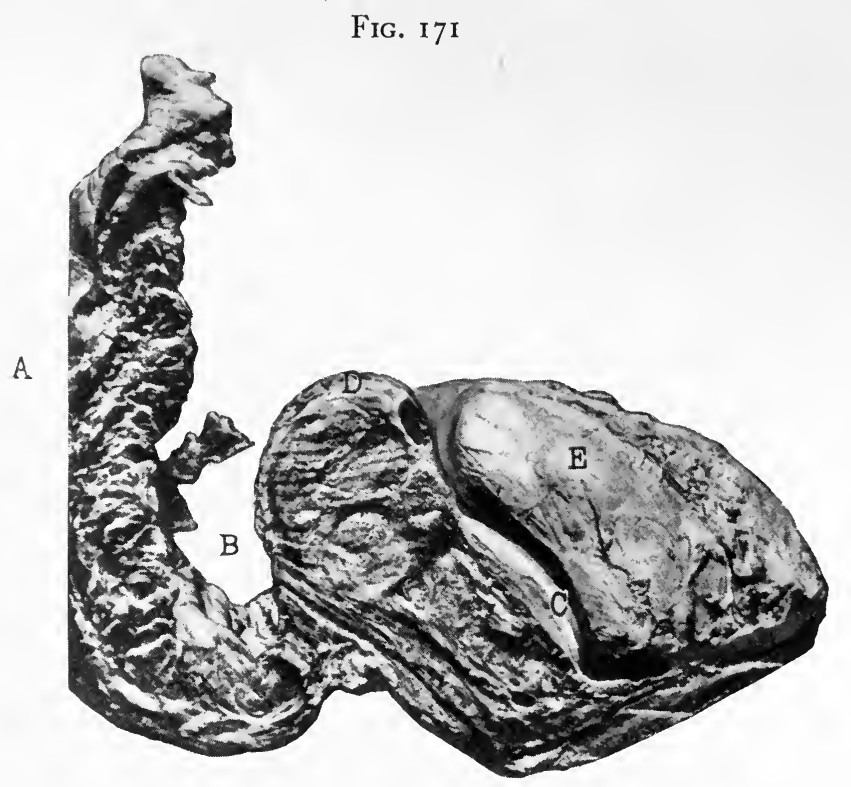

Median sagittal section of the bladder, median prostatic enlargement, and prostatic urethra, showing the abrupt upward course taken by the latter between its junction with the membranous urethra and the vesical orifice, also the greatly thickened wall of the bladder and the depression of its floor immediately behind the prostate: $A$, thickened bladder wall; $B$, postprostatic depression; $C$, prostatic urethra; $D$, median-lobe hypertrophy; $E$, one lateral lobe. (Watson.)

they do not always give rise to pain, and are then very likely to be overlooked. The finger should always be run in under this shelf in making digital explorations of the bladder in cases of suspected vesical calculi, to make sure that there are none there. (See chapter on Vesical Calculus.)

Changes in the Ureters and Kidneys.-The first effects upon the ureters and kidneys are, as in the case of the bladder, due to mechanical influences. As a result of these, the ureters and renal pelves may become dilated, and this dilatation may later be shared by the kidney and produce hydronephrosis of varying degrees. When the kidney is infected, the more serious condition of pyonephrosis is often created. 
It is noticeable, however, that prostatic hypertrophy, even of a serious grade, may exist for years without causing correspondingly grave changes of this kind in the kidneys or their pelves.

One of the effects of a large residual urine that has been present for a long time is to bring about a functional insufficiency of the kidneys, the only sign of which in the urine may be a diminishing amount of urea and other solid constituents. In a rough way this is fairly well estimated by the fact of the urine having a low specific gravity.

Symptoms and Diagnosis.-The symptoms of hypertrophy of the prostate are due to the secondary changes in the bladder and the kidneys. Those which are referable to the bladder are, for the most part, local; those arising from the kidney are chiefly systemic.

I. Symptoms Referable to the Changes in the Bladder.Nocturnal and Frequent Micturition.-The most characteristic subjective symptom of prostatic hypertrophy is the necessity of passing water during the night, when it is felt by a patient who has not hitherto experienced it. Just what the explanation of this phenomenon is we do not quite know. It is due in part probably to congestion of the vesical outlet, and in part to the presence of residual urine.

As the enlargement of the gland proceeds, there is an increased frequency of urination in the daytime as well as at night. If the bladder becomes hypertrophied or infected, these symptoms become more marked, and vesical tenesmus is associated with them. In the atrophied, distended, thin-walled bladders tenesmus and pain are absent, and the residual urine gradually increases until the condition known as "overflow bladder" is produced, the urine flowing guttatim, or almost constantly, in the more extreme examples of it. This condition is frequently mistaken for true incontinence by both patient and physician, and it is difficult to persuade the former that there is any urine contained in the bladder when it is constantly running away. True incontinence in cases of prostatic hypertrophy is exceedingly rare, and very frequent dribbling of urine should always lead to an examination of the bladder, which will almost invariably show it to be greatly distended.

Complete Retention.- This condition occurs in some cases in which there are hypertrophied, thick-walled bladders, but more often with the atonied, thin-walled ones.

A small median enlargement may cause complete retention quite as readily as some of the larger lateral hypertrophies.

Changes in the Urinary Stream.-These are in some respects characteristic. The more noticeable features are: lack of projection of the stream and the perpendicular direction it takes toward the end of micturition; diminution in size of the stream; dribbling of urine after the VOL. I-2I 
apparent completion of the act; and, in cases of very large residual urines and overdistended bladders, the passing of the urine guttatim.

Pain.-Pain is present in cases of hypertrophied prostate when any of the following conditions occur in connection with it: cystitis, vesical calculus, inflammation or abscess of the gland, abrasions or ulcerations on the prominent parts of the gland.

The pain due to the cystitis which is associated with prostatic hypertrophy has no special characteristic by which to distinguish it from that of cystitis produced by any other condition. The pain which occurs in the cases complicated by vesical calculus will have the character of that caused by the latter condition.

Pain which radiates to the anterior crural or sciatic regions should always excite suspicion of cancer of the gland.

Hematuria.-Blood is present in the urine in cases which are complicated by vesical calculus; in some of those in which cystitis supervenes; when the prostate has been injured by instruments, or when an ulcer has formed upon a prominent intravesical projection.

The writer has seen two cases in which such profuse and repeated hemorrhages from the latter source took place as to threaten the patients' lives and made operation imperatively necessary in order to save them. Ordinarily, hematuria is not a conspicuous or characteristic sign of hypertrophy of the prostate. When hematuria exists, the last part of the urine passed or drawn with the catheter will contain a larger proportion of blood than that which comes first.

2. Symptoms Referable to the Changes Occurring in the Kidney or Renal Pelvis.- The manner in which degeneration and destruction of the renal tissue are brought about has already been described. The symptoms resulting from the changes vary in degree, but are, for the most part, referable to progressive failure of the renal function, which proceeds pari passu with the degeneration of the renal parenchyma. Such other symptoms as occur are due to general sepsis produced by infection of the kidney.

The symptoms which proceed from insufficiency of the renal functions are included under the general heading of uremia, which complex condition they represent in varying degrees, in the milder cases being manifested by moderate disturbances of digestion, thirst, slightly furred tongue, and uneasy sleep; in the more severe forms, appearing as muscular twitchings, stupor, delirium, convulsions, and, finally, as uremic coma. Coincident with these evidences of uremia, the urine diminishes in quantity, and sometimes complete suppression occurs.

The most important sign of impending or already present renal insufficiency is the diminishing quantity of solids excreted by the kidneys. 
This is estimated by the usual tests for the quantity of urea and other solid constituents of the urine, and by the application of the more modern methods by which the capability of the renal function is tested, viz., phloridzin, cryoscopy, and the methylene-blue tests. (See chapter on Urinary Analysis.)

Casts and renal epithelium are often absent.

The symptoms of sepsis may proceed from cystitis or from an infected kidney, usually the latter. They are those which, in greater or less degree, characterize sepsis of other parts of the organism: rigors, chills, fever, dry and coated tongue, prostration, sweating, diarrhœa, etc. Suppurative cystitis may be present for a considerable time, in some cases for very long periods, without giving rise to septic symptoms of consequence.

Physical Examination.-Detection of Residual Urine.-This examination is made by passing a catheter into the bladder and drawing off whatever urine may remain in it immediately after the patient has passed all that can voluntarily be evacuated. The part which cannot be spontaneously voided is called the "residual urine."

A medium-sized soft-rubber catheter should, when possible, be used for this purpose. If it cannot be passed into the bladder, a smaller size of the Mercier coudé or bicoudé instrument should be tried next, and this failing to enter, a bougie catheter is to be used. Before using the catheter, the urethra should be irrigated with a solution of I to 4000 permanganate of potash, and the patient should be given three 7 grain doses of urotropin in the course of the preceding twenty-four hours. The catheter should be sterilized by boiling it in a saturated solution of ammonium sulphate, the head of the penis should be cleansed with a weak bichloride solution-I to 8000-and the surgeon should wear sterilized gloves, if he wish to be consistent in his efforts to secure aseptic catheterization. It should be remembered that it is impossible to wholly sterilize the urethra. We need not be surprised, therefore, at the fact that the bladder of the patient with prostatic hypertrophy very frequently becomes infected by the passage of the catheter, even though every precaution has been taken to prevent it.

Rectal Examination.-By palpation with the finger in the rectum, enlargement of the lateral lobes can always be detected, if present. The points to be borne in mind when making this examination are: (I) The length of the gland from apex to base. This cannot be estimated in cases of very extensive hypertrophy because of the fact that the enlargement of the lateral lobes always takes place longitudinally, and, when it has exceeded the limits of a moderate hypertrophy, the bases of these lobes, and the notch which separates them, are beyond the reach of most 
fingers. We can, however, when this is the case, always infer that the enlargement is more than moderate. (2) The hardness, softness, or elasticity of the gland to the touch. If very hard, and especially if the gland is fixed, suspicion of malignant disease of the prostate should always be aroused. (3) Congestion and inflammation of the gland are shown by the strong pulsation of the small arteries and the unnatural heat felt by the examining finger in the rectum. There is also a certain amount of œedema to be recognized around the outside of the gland. When this is present, the outline of the lateral lobes is difficult to make out with the finger tip, and the sensation conveyed is as of touching a doughy substance. (4) The extent to which the lateral lobes encroach upon the rectum. (5) Irregularities and areas of fluctuation upon the surface of the gland.

Examination of the Prostatic Urethra.-Length of the prostatic urethra, and changes of its direction or width, can be detected by means of a short-beaked sound such as is ordinarily used in examining for vesical calculus. Cunningham ${ }^{5}$ has devised a sound with notches upon one side, which can be turned against the floor of the prostatic urethra by turning the beak of the instrument over behind the base of the gland after passing it into the bladder (Fig. I72). The distance between the vesical outlet, against which the beak of the instrument rests, and the apex of the gland, which distance represents the length of the prostatic urethra, is measured by drawing the finger tip placed in the rectum over the notches upon the staff, which can thus be counted between these two points. Each of the notches and each distance between them is $\mathrm{I} \mathrm{cm}$., so that the number of notches traversed by the finger in this journey will give the whole length of the prostatic urethra (Fig. I73).

Lateral deviation of the beak of a sound as it passes through the prostatic urethra shows greater enlargement of the urethral aspect of one lateral lobe than of the other. If the deviation occurs just as the tip of the instrument nears the entrance of the bladder, it means that it has passed into one or the other of the divisions made by the projection of the median enlargement of the prostatic urethra, which has already been described. When a very marked obstruction is encountered in the prostatic urethra, and it can be overridden by lifting the point of the instrument well up, as it passes on into the bladder, or if there is much difficulty in rotating the beak across the floor of the bladder, after it has entered it, a median hypertrophy exists.

From what has been said it is evident that the diagnosis of prostatic hypertrophy is easily made. The presence of a residual urine, the detection of the enlarged gland by rectal palpation, and the symptom of nocturnal micturition, when combined in a patient of over fifty years of age, are sufficient to establish the diagnosis. 
In common with a number of other surgeons, we do not regard the cystoscopic examination as of much value, ordinarily, in making the diagnosis of hypertrophy of the prostate, but for some of the special

FIG. I72

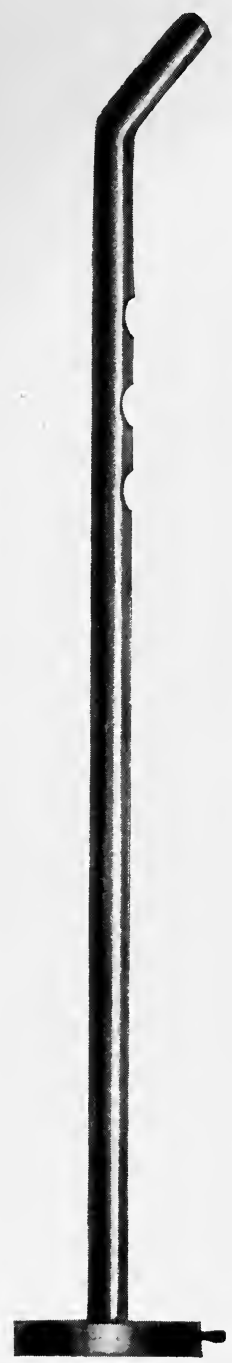

Cunningham's special instrument for determining the length of the prostatic urethra. It consists of a $2 \mathrm{~cm}$. Mercier beak, a shaft of 20 French which has three depressions: one, $3 \mathrm{~cm}$. from the point of union of the beak with the shaft; another, $5 \mathrm{~cm}$., and another $7 \mathrm{~cm}$., each depression and interval between being $\mathrm{I} \mathrm{cm}$. in length.
FIG. 173

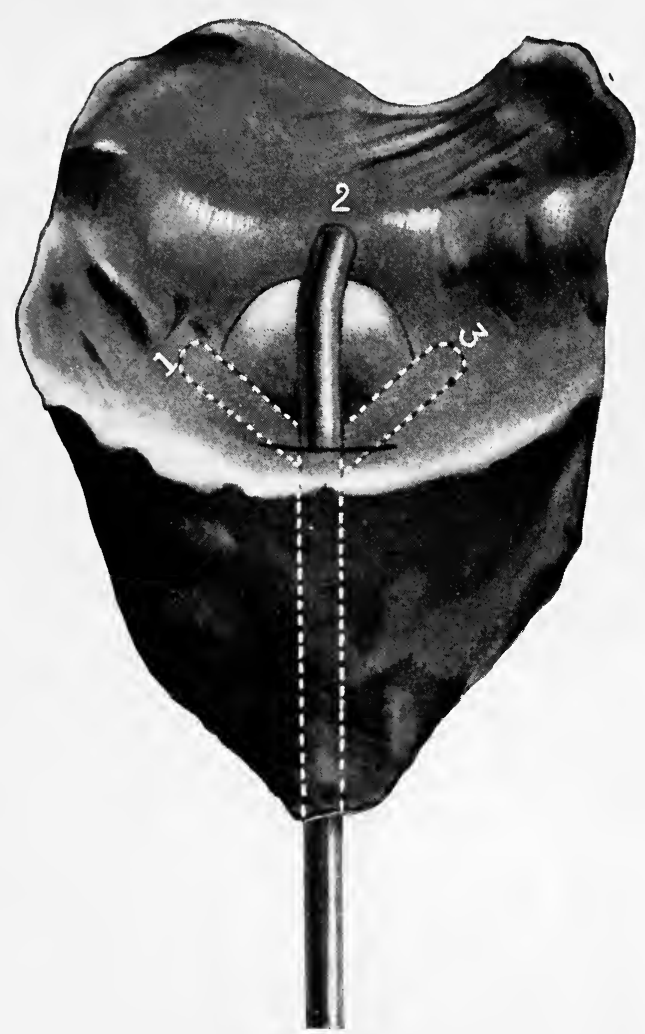

The method of determining the height and breadth of the intravesical projection of the median lobe. The instrument is seen in three positions. Position $\mathrm{I}$ : The beak is in the urethral cleft at the right of the median lobe; by keeping the beak on the surface of the gland and rotating the instrument, the shaft will be drawn inward as the beak surmounts the intravesical elevation and assumes position 2. The distance which it is drawn in is determined by counting the grooves in the instrument's shaft. Further rotation will cause the beak to assume position 3. The distance from position I to position 3 is the width of the lobe, and can be more or less accurately estimated by noting the arc through which the beak passes in going from position $\mathrm{I}$ to position 3. (Cunningham.) 
conditions, such as ulceration upon the intravesical portions of the surface of the gland, and for the detection of the secondary changes in the bladder that occur in connection with hypertrophy, the cystoscope is of decided value.

Treatment.-The treatment consists of: (I) hygienic, medicinal and palliative; (2) by the regular use of the catheter; and (3) operative measures.

Medicinal and Palliative Treatment.-The palliative methods of treatment are more or less useful in conjunction with, or as preparation for, treatment by the catheter or by operation. Their aim is to restore the patient's strength, lessen pain, and make him comfortable.

The following are among the more helpful of the measures employed for these purposes: hot hip baths, rest in bed, prostatic massage, mineral waters, and urotropin.

The application of these palliatives in combination is often successful in overcoming the frequent and painful urination which is the most distressing feature of the condition, and thus restores the patient's sleep and renews his strength. By reducing the bladder inflammation, they also contribute to the success of any subsequent operative procedure.

Hot hip baths should be given twice or thrice daily, should be continued for about ten minutes each time, and the temperature of the water should be maintained by adding more hot water during the bath. Only the hips should be immersed.

Rest in Bed.- - Rest in bed, with the hips slightly raised and supported by a hard hair pillow or air cushion, is very beneficial in reducing congestion of the prostate and quieting the inflammation of the bladder.

In some cases no benefit will be derived from these measures, and it must not be forgotten that confining an old man to bed may give rise to hypostatic pneumonia, and is, therefore, not without danger. This risk is much lessened if the following precautions are observed. Let the patient get up two or three times for a short time each day and walk about his room. Apply friction to the back over the lower part of the lungs. Prescribe massage for a short time every day or every second day. Keep the patient's feet warm. Do not allow constipation to occur.

Diet and Mineral Waters.-One of the best measures of treatment of the cystitis associated with prostatic hypertrophy is a milk diet, or an almost exclusively milk diet, the only articles of food allowed besides milk being dry toast, and once daily a little broiled fish. The milk should be taken as follows: Quantity in each twenty-four hours, two quarts. Dilute each tumbler of milk with Vals or Vichy water, in the proportion of one part water to three parts of milk. Have the milk slightly warmed. Sip it slowly. If given in this way, there are but few patients with whom milk 
does not agree. The tendency to constipation which often results from a milk diet should be combated.

Prostatic Massage.-Prostatic massage is exceedingly useful in diminishing the congestion, and with it the size of the enlarged gland and the residual urine. This is particularly true in the earlier stages of the malady, during which the congestive element is more marked than subsequently. The inexperienced practitioner will often be led to believe that a permanent disappearance of the enlargement has taken place. Such is not the fact, however, in any case of true hypertrophy.

Medicines.-There are but few medicines that are of much service. The following have proved the most useful in our hands:

Urotropin.-Urotropin in 7 grain doses thrice daily has been, in our experience, the most valuable medicine that we have tried for the purpose of reducing the amount of pus in the urine and for preventing ammoniacal decomposition. In small doses, it may be continued uninterruptedly for a long time without producing irritation or congestion of the kidney or bladder. If administered in 7 grain doses, its employment should be interrupted after a week; it may then be resumed for another week, after an interval of two days. Some patients are very sensitive to this drug, and in such cases it should not be employed.

Citrate and Acetate of Potash and Benzoate of Sodium.-Each of these remedies is of more or less value in rendering the urine less irritating, and in thus exercising a soothing effect on the bladder. The dose of each is from Io to 15 grains, thrice daily, in a half-tumblerful of water.

Increasing the Quantity of Urine.-If the bladder is being regularly emptied by the catheter, the quantity of urine should be increased by the plentiful drinking of water, which aids in washing out the pus and mucus. If the catheter is not being used, such an increase of the quantity of the urine only serves to augment the amount of the residual urine, and thus adds to the burden already imposed upon the bladder. If it is desired to increase the quantity of urine, it should be done by the administration of diluents and not by stimulating diuretics.

Catheter Treatment.-The Best Forms of Catheters.-The metal catheter we regard as an undesirable instrument, because of the danger of having false passages made by it, which may occur in the hands of inexperienced and unskilled physicians. It is, moreover, an unnecessary instrument, for it can accomplish nothing which cannot be attained by the use of safer and better forms of catheters. There are three kinds of catheters, one or another of which will meet all requirements. These are:

I. The soft-rubber catheter.

2. The single or double-elbow catheter of Mercier.

3. The bougie catheter (see Figs. I74 to I78). 
The Qualities that the Catheter Should Embody.-In the first and second kinds, the requisites are as follows: the eye should be as near as possible to the end of the instrument. There should be no cul-de-sac beyond the eye. The eye should be as large as the caliber of the tube permits, and its edges should be smooth and rounded.

The bougie catheter should not have the eye so near to the end as to destroy the proper taper of the instrument. The neck beyond the eye

FIG. I74

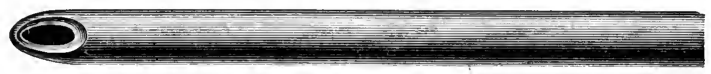

The soft-rubber catheter.

FIG. 175

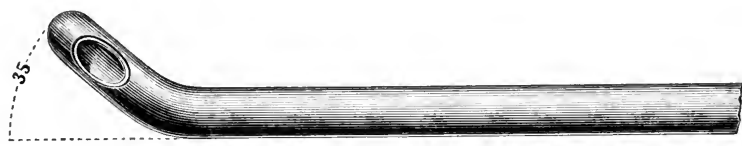

Mercier coudé catheter.

Fig. ${ }^{7} 76$

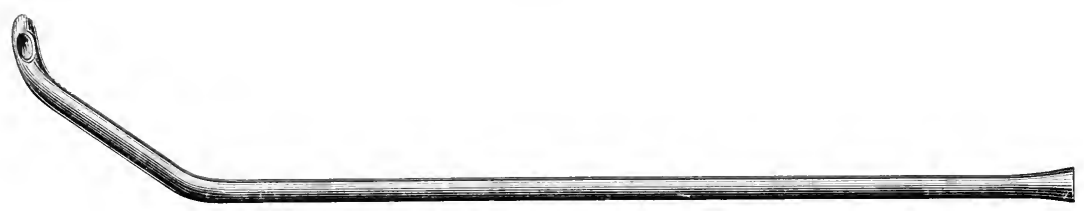

Mercier bicoudé catheter.

FIG. 177

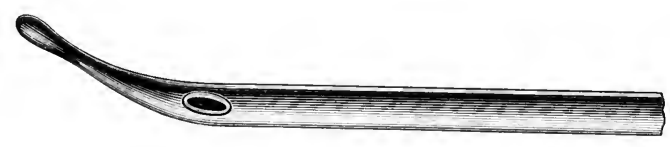

FIG. ${ }^{7} 78$

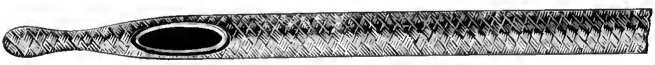

Bougie catheters.

should be delicate and flexible. The distal end should not be too large. The surfaces of the webbing catheters should be even and perfectly smooth. Instruments of the best quality preserve the fine polish of the surface after being used and boiled a good many times.

The soft-rubber catheter is the form which should be tried first. If it cannot be passed into the bladder, the Mercier instrument should be next tried, and this failing, the bougie catheter is to be used. 
The Mercier catheter is best suited for cases in which there is a median enlargement; the bougie catheter will often pass more readily in those in which the prostatic urethra is much narrowed by encroachment of one or both of the lateral lobes. Fig. I79 shows how well adapted the Mercier instrument is to surmount a prominent middle lobe.

When and How Often the Catheter Should be Used.-In the early stages of the malady, which, as we have seen, are usually marked by more or less congestion of the prostate, there may be but a small amount of FIG. I79

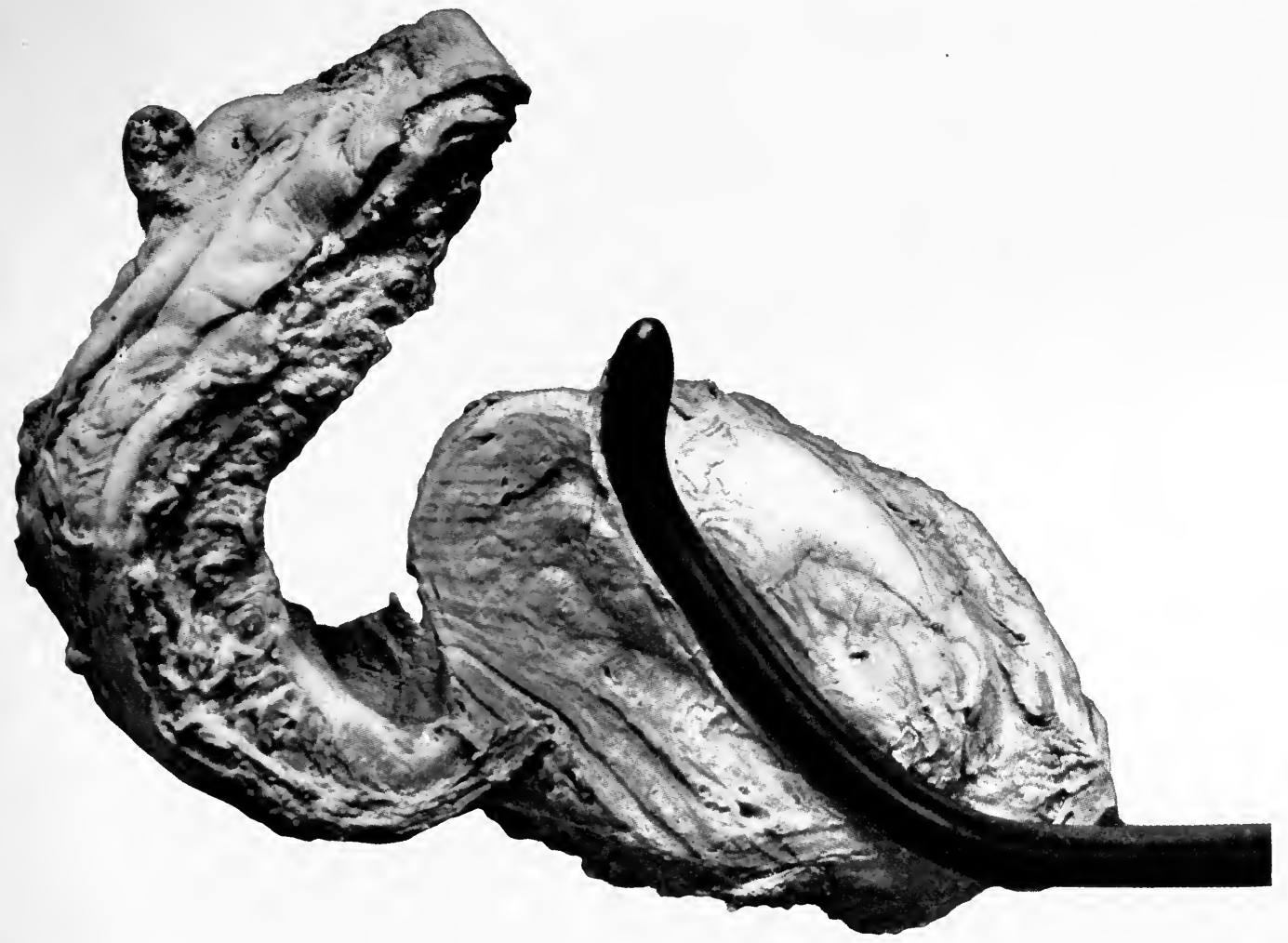

To illustrate the ease with which the Mercier bicoudé catheter surmounts obstructing middle lobes. (Watson.)

residual urine. Irritability of the bladder, however, is often pronounced. The use of the catheter at this time is more apt to be harmful than not, and, moreover, it is not often required. Instead of passing the instrument at the outset, the measures already mentioned on p. 326 , for overcoming congestion, should be employed first. If the gland does not diminish in size, and if the irritability of the bladder is not lessened under their employment, we may conclude that the symptoms are probably due to the presence of residual urine rather than to congestion of the prostate, and should then proceed to the use of the catheter. 
Indications for the Regular Use of the Catheter.-The two factors which determine the use of the catheter are: (I) the amount of residual urine; (2) the presence or absence of infection of the bladder. The character of the subjective symptoms also influences the decision.

A residual urine, even if its quantity does not exceed 2 ounces, calls for the use of a catheter once daily, if the bladder is infected and the patient suffering from marked vesical irritability.

A residuum exceeding 3 or 4 ounces, even though the urine is clear and the patient free from marked bladder irritability, calls for the use of the catheter each evening before retiring.

The Frequency with Which the Catheter Should be Used.-The catheter should be passed as seldom as possible. The number of times will be determined by the factors stated above; that is to say, the amount of residuum, the presence or absence of infection of the bladder, and, in addition, the measure of relief which the use of the instrument affords.

If the bladder is not infected or irritable, and if the residuum does not exceed 6 or 8 ounces, the single passage of the catheter daily will be sufficient in most instances. If relief is not afforded, if the single passage daily does not prevent an increase in the amount of residuum, or does not diminish it, if it be very large, or if the bladder is infected, it will probably be necessary to use the instrument twice daily at least, and very likely three or even four times.

When benefit does not follow so frequent a use of the catheter as this, harm rather than good will almost always result from using it more often.

Contra-indications to the Employment of the Catheter and Conditions Which Render its Use Impossible.-Whenever the use of the catheter fails to relieve distressing symptoms already present, or if its use results in the appearance of distressing symptoms, especially when, as is the rule, these symptoms are associated with infection of the bladder, this manner of treatment should be abandoned.

It should not be continued if the passage of the instrument is attended by hemorrhage, or if it causes pain of consequence.

It should not be carried on if inflammation of the prostatic urethra, of the prostate, of the epididymis, or testicle arises in the course of the treatment.

The use of the catheter is impossible in some cases owing to the form assumed by the hypertrophied lobes, $i . e$., when it is such as to make the prostatic urethra impassable.

Manner of $U$ sing the Catheter.-Pass the catheter gently. The number of patients who are killed or seriously injured by the unskilled use of the metal instruments is considerable, especially in cases of prostatic hyper- 
trophy, in which it is particularly easy to make false passages. The accident is most likely to occur in those in whom there is a well-marked median enlargement. The patient is usually suffering greatly, and embarrasses the physician by complaints; the doctor becomes worried, and, under the urgence of the patient, uses undue force and makes a false route, with resulting hemorrhage and infection.

Avoidance of metal catheters will do much to prevent such occurrences. It is impossible to bore a hole through the urethra with a soft rubber instrument, and this is the first one that should be tried. The best size to begin with is about 16 of the French scale. The soft-rubber catheter is passed by a series of short pushes, taking hold of the instrument about half an inch outside the meatus each time with the thumb and forefinger, and pressing it forward for that distance into the urethra. The penis should be held in a straight line and moderately stretched while this is being done.

The only thing to be remembered in passing the Mercier instrument is to keep its tip pointed directly upward in the median line.

The bougie catheter is passed by urging it gently onward and allowing it to follow whatever direction it may take. Its slender neck and flexibility make it especially suitable in the cases in which the course of the prostatic urethra is tortuous or narrow.

Lubricants.-Lubricants should be used liberally. Among the best of them are: sterilized olive oil, alboline, and a preparation made from Irish moss, which here passes under the name of crystal emollient.

Prophylaxis against Vesical Sepsis.-It is impossible to sterilize the urethra, but something may be done toward it, and the chance of infection may thus be lessened.

The measures to be taken have already been discussed on page 323 . They comprise the use of sterilized catheters, the wearing of sterile gloves, irrigating the urethra beforehand, and the bladder afterward, with solution of potassium permanganate of a strength of I part to 4000 or 5000 of water, or weak solution of argyrol or simple sterile saline solution, and the administration of urotropin beforehand.

Catheter life should not be begun without surrounding the patient with every safeguard against the serious consequences which attend it in many cases. In addition to the measures just referred to, he should not be exposed to cold, and should stay indoors for a few days when the treatment is begun.

Stimulants should be used sparingly, but not absolutely omitted if the patient has been in the habit of taking them regularly for a long while. It is also well to give a small dose of morphine just before the first use of the catheter. 
Treatment of Retention of Urine.-The following precautions should be observed when relieving retention of urine:

The bladder should not be emptied at once in any case in which there is a large quantity of urine in it. The withdrawal of a large amount, by suddenly taking off the pressure to which the capillary bloodvessels have been subjected, allows them to become distended, and thus disposes to marked congestion or inflammation of the mucous membrane of the bladder, which sometimes causes rupture of the small terminal bloodvessels, and intravesical hemorrhage results. This we have seen so abundant in two or three cases as to form large clots in the bladder. When this occurs it greatly complicates the already sufficiently grave condition. The treatment to be adopted under these circumstances is to break up the clot and remove it by means of a lithotrity evacuating apparatus.

If the blood clots are allowed to remain in the bladder, retention continues, and congestion is increased by the pressure exerted upon the veins. To obviate a repetition of the hemorrhage, the bladder should be in part refilled with sterile saline solution immediately upon withdrawing the blood clot from it, or a solution of adrenalin, I to 2000 or I to 3000 , may be injected into it. Such hemorrhage need never occur if the precaution is taken of not withdrawing the whole of a large quantity of urine in the first place. It should be taken away by repeated passages of the catheter, a moderate quantity at a time. In cases of overdistended bladders, we never withdraw more than I2 or 14 ounces at the first passage of the catheter.

In some cases there is very marked polyuria following the withdrawal of even that amount of urine, so that the bladder rapidly refills. Under these conditions, it may require several days before the balance of the secretion becomes more naturally established and before we can completely empty the viscus.

A safer way to begin the withdrawal of the contents of the bladder in the cases of overdistention with complete retention is by aspiration with a small needle introduced just above the upper margin of the symphysis pubis, and taking away but a portion of the urine each time that the

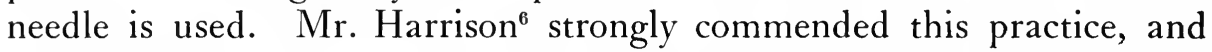
quoted several cases to show the harmlessness of the procedure, even though frequently repeated. In one instance it was done thirty-four times in the course of four or five weeks. The writer has confirmed the truth of this statement of Harrison in many cases, and we advise the method, instead of the use of the catheter in the first instance, as the best means of relieving distended bladders. It greatly lessens the chance of vesical infection, and it often happens that the patient will be able to 
pass the urine voluntarily after its employment, sometimes when but one or two aspirations have been made. The procedure should be supplemented by the internal administration of a small dose of morphine and by hot hip baths, directing the patient to void the urine while in the bath, if possible. Rest in bed for a day or two should always be enjoined. Two precautions should be observed in performing aspiration of the bladder. The needle used should not be a large one, and it should be inserted directly over the upper margin of the symphysis, in a direction at right angles to it; that is to say, in the horizontal plane, assuming the patient to be standing.

Continuous Drainage of the Bladder and Bladder Irrigations.-In cases in which the bladder becomes infected, or is already so when the catheter treatment is begun, and also with patients having defective renal function, continuous drainage of the bladder sometimes diminishes the one and improves the character of the other of these conditions. It is accomplished by tying a catheter into the bladder in one of three ways: (I) through the whole urethra; (2) through the deep urethra by the artificial opening made by an external perineal urethrotomy incision; (3) through a suprapubic cystotomy incision.

I. The first of these three methods is objectionable on account of its liability to excite urethritis. When this happens the catheter should be promptly removed and the inflamed urethra treated with irrigations of I to 4000 permanganate of potash solution. The catheter should be changed for a fresh one every twenty-four hours or so.

2. Continuous Drainage through the Perineum.-The operation of external perineal urethrotomy may be utilized for the purpose of establishing permanent drainage of the bladder through the posterior urethra. The operation per se is entirely harmless, especially if it be done under local anesthesia, and it requires but one or two minutes for its performance. (For manner of doing the operation and arranging the drainage see chapter on the Technique of Operations for Stricture of the Urethra.) The advantage of this method as compared with draining through the whole length of the urethra is that it avoids the occurrence of urethritis, to which the other is liable.

3. Suprapubic Drainage.-Sir Henry Thompson ${ }^{7}$ reported to the Clinical Society of London the case of a patient whose bladder had drained for a dozen years or so through a suprapubic cystotomy incision by means of a tube passed through a shield into the bladder, the other end being led to a portable urinal. The patient remained in good health and comfort and was relieved of the painful symptoms which had been previously caused by the enlargement of his prostate.

Harrison, ${ }^{8}$ Watson, ${ }^{9}$ and Cabot $^{10}$ have spoken of the advantages to be 
derived sometimes from improvement of the renal function which may result from long-continued drainage of the bladder.

Irrigation of the Bladder.-The following rules should be observed in connection with irrigation of the bladder:

I. All fluids used in irrigation should be of a temperature of not less than $105^{\circ} \mathrm{F}$. In cases in which the intravesical hemorrhage occurs, the prolonged irrigation of the bladder with a fluid at a temperature of $115^{\circ}$ to $120^{\circ} \mathrm{F}$. is serviceable in arresting the bleeding.

2. It is not only useless, but harmful, to employ irritating antiseptic fluids, such as corrosive sublimate, carbolic acid, creolin, etc., for the purpose of cleansing or disinfecting a bladder which is the seat of cystitis. There is no known antiseptic by which disinfection of the bladder can be accomplished. The best solutions to employ for the purpose of irrigation are the non-irritating cleansing fluids, and of these, the two most effective in our experience are permanganate of potash, I part to 4000 of water, and argyrol, beginning with a 2 per cent. solution and increasing the strength to 5 per cent. or io per cent. if it proves to be beneficial.

3. Attempts to dilate bladders with rigid walls and of small capacity by means of forcible distention, or by gradually increasing distention with successively larger quantities of fluid injections, we have always found to be harmful and to cause an increase of bladder irritability and inflammation.

It is better not to try to inject more fluid into the bladder than it can comfortably tolerate. Whatever that quantity may be in individual cases, it should be repeated until the returning fluid is clear.

Results of Catheter Treatment.-This is one of the most important and one of the least easily determined parts of the subject. No one has thus far published a statistical compilation of the results of catheter treatment with reference to the points which are the most important to know, and we have no means other than that of individual personal impressions by which to establish the proportion of cases in which catheter treatment is well tolerated, that in which it fails, and that in which it soon causes the death of the patient. Watson ${ }^{11}$ presents a short table on the results of catheter treatment with reference to mortality in 207 cases collected by himself, by Rovsing, and by Casper, in which the mortality was calculated from the same standpoint as that which would be used in computing the death rate attending surgical operations, namely, by reckoning all deaths following within one month of the beginning of the use of the catheter as operative or catheter deaths. The proportion he found to be a little less than 8 per cent. Impression for impression, our own as compared with others, we believe that if the time in which deaths 
considered as due to the catheter was extended to two months in the calculation of the mortality attending this treatment, the death rate would be found to be a good deal higher than 8 per cent., and if so, it would be larger than the mortality of either prostatectomy or the Bottini operation.

There is no means of telling beforehand with any certainty which patient will and which patient will not tolerate the catheter treatment, and this is the weakest point in connection with it. In a general way it is, of course, true that those least likely to tolerate well this or any other form of treatment are the patients with defective kidney function and overdistended bladders with chronic retention.

In these cases the course taken by the disease when treated by the catheter is far too frequently as follows:

Despite skilful catheterizeation, aseptic methods, and the employment of palliative measures of whatever kind, cystitis follows the use of the instrument, grows steadily worse, frequent and painful passing of the catheter becomes necessary, the urine is fetid or bloody, the kidneys become involved, a low form of septicemia and uremia arise, and under these combined conditions the patient, after some days or weeks of much suffering, dies.

The reverse and happier side of the picture of catheter treatment is seen in the cases in which, even though more or less cystitis follows its employment, the organism tolerates its use, the kidneys do not become infected, maintain their functional sufficiency, and the general bodily health remains good or becomes so if it has been previously poor. These favorable conditions, curiously enough, are sometimes met with in the cases of patients who neglect every rule of cleanliness and asepsis, whose catheters are never clean, and who often use the instruments roughly.

The Objections to the Catheter Treatment.-The objections to the catheter treatment may be briefly summarized thus: (I) It is never curative. (2) Even though it may have been tolerated by the patient for a greater or less length of time, that fact is never a guarantee that it may not at any future time fail to give relief or to save the patient's life, or spare him suffering. (3) In a considerable proportion of cases-probably not less than io per cent.-its employment results in the death of the patient; no one can foretell in which of them this result will and in which it will not occur. (4) It is always accompanied by the risk of having the patient attacked by one or another of the complications due to its use or incident to hypertrophy of the gland, viz., prostatic abscess or inflammation of the gland, epididymitis, orchitis, abscess of the testicle. (5) It is the cause of many deaths, because of its being put in practice by ignorant or unskilled medical men, few of whom hesitate to undertake it, and but few of whom are competent to do so. 
(6) In the cases in which it proves not to be tolerated the surgeon has been deprived of giving the patient the excellent chance of cure and good health which he might have promised had he been permitted to institute the operative treatment at first. In the face of this arraignment it is not surprising that many expert surgeons should have taken the stand that it is better to do away with catheter treatment altogether and to concentrate the attention on the operative methods and improving them.

The Operative Treatment of the Hypertrophied Prostate.Historical Resumé of the Principal Features Which Have Marked the Evolution of the Operative Treatment.-For special details of the history, see Watson ${ }^{12}$ and Deaver. ${ }^{13}$

We are tempted to a more extended survey of this part of the subject than in most of the other chapters of our work, because of its inherent interest, and more especially because of the fact that many of those who have more recently entered the operative field have been conspicuous for their ignorance or for ignoring much of the work that has been done in the past, and in some instances have put forward operative methods as original which had already been practised and described many years before.

The history of the operative treatment of the hypertrophied prostate may conveniently be divided into the three following periods:

I. That in which parts or whole of obstructing portions of the prostate were removed in the course of the performance of operations undertaken for other purposes, generally lithotomies, the removal usually being accidental; occasionally intentional, but incidental to the original operation. The beginning of this period is uncertain, but it covers the time, at any rate, between I639, in which year Corvillard is said to have removed a large mass, which may have been a part or the whole of the prostate, through a perineal incision, down to the time when, in 1856 , Mercier ${ }^{14}$ made the first systematic endeavor to remove the obstacle to urination offered by the enlarged prostate.

The procedures applied during this period were as follows: Crushing and tearing off the obstructing mass by forceps through a perineal cystotomy incision. Tunnelling the obstructing portions of the gland by an instrument passed through the urethra from the meatus (Lafaye, in 1766; reported by Chopart). The same manœuver at a later time by John Hunter, Brodie, Everard Horne, and others. This practice did not find favor, and was lost sight of until Harrison ${ }^{15}$ (I88I) revived interest in it for a time by the report of a case which he had successfully treated by tunnelling the obstructing gland through a perineal urethrotomy incision. Incision of the median or lateral portion of the obstructing gland by instruments passed through the urethra from the meatus 
(Leroy d'Etiolles ${ }^{16}{ }^{1} 832$ ); Guthrie, ${ }^{17}$ I 834 , the same through the perineal urethra; Leroy d'Etiolles, ${ }^{16}{ }^{1} 832$, by snaring the middle lobe by a ligature carrier passed through the urethra from the meatus. Removal of the middle lobe through a suprapubic cystotomy incision by Amussat, ${ }^{18}$ in I827, who cut it off with scissors. Division of the median enlargement through the external median perineal urethrotomy incision, by Sir William Blizzard, in 1806 . Sir William Fergusson, ${ }^{19}$ in 1848 , definitely advised the removal of prostatic obstructions in the performance of lateral perineal lithotomy, and took this additional step purposely in a case which he reported, saying that the procedure would not add materially to the dangers of the other operation.

Thus it will be seen that prior to 1856 , the time of Mercier's work, there had already been performed the procedures of tunnelling the prostate, snaring off its median hypertrophied portion, incision of the middle lobe, or "bar at the neck of the bladder" (Guthrie), that is to say, prostatotomy; the same manœuvers done through a median external perineal urethrotomy incision, also in the course of lateral or median lithotomy, and, in addition, avulsion or enucleation of parts of the gland with forceps or blunt dissectors, and excision with scissors of the median enlargement through a suprapubic cystotomy incision. Other measures, such as compression of the obstructions by stretching instruments introduced into the prostatic urethra, destruction of the gland by caustics, pressure upon it by hydraulic force with a water-bag passed into the prostatic urethra, and by special instruments of the form of elongated bougies, were also practised.

2. From Mercier (1856) to McGill (1887). This period is marked by the systematic efforts of certain surgeons to place the operative treatment upon a practical footing and to demonstrate its feasibility. It begins with Mercier and ends with McGill. The following procedures were developed in the course of it.

Mercier: Division of the median bar or lobe by means of a specially devised instrument passed through the whole length of the urethra from the meatus. Also, the removal of a U-shaped piece by another form of instrument. These instruments had short beaks, which were turned over behind the prostate after entering the bladder; the cutting blade of the first form of the two was then drawn out through the obstructing median portion of the enlarged gland and for a short distance of the prostatic urethra. The blade was then pushed back into its original position and the instrument was withdrawn, after its beak had once more been turned upward.

The second instrument was turned over in the same manner as the first, on entering the bladder. Its shorter blade was drawn out into the voL. I-22 
prostatic urethra and then driven home against the longer one, cutting out a U-shaped bit of the middle part of the gland in its passage back to its original place. The beak was then turned up and the instrument withdrawn, holding in its jaws the piece of the gland that had been cut out.

The former of these instruments was the forerunner of that of Bottini in its form and manner of action.

In 1874 and 1877 Bottini $^{20}$ adopted the same plan as that of Mercier, just described, modifying it by substituting a galvanocautery blade for the cutting one, and changing its form somewhat. This operation has remained in use up to the present time in the hands of a few surgeons, notably in those of Freudenberg, ${ }^{21}$ who is, however, today almost its only steadfast advocate. Freudenberg and, later, Young both modified the instrument of Bottini, and the former made the electric heating of the blade more exact, and also added to the original incision one or two diagonal lateral ones.

In 1873 Gouley practised the operation of Mercier, applying it, however, through an external perineal urethrotomy wound, as well as in the original manner of Mercier. Gouley was the first also to propose the total enucleation of the gland by the finger through an external perineal urethrotomy incision in the median line of the perineum. The following words, which we quote from his work, ${ }^{22}$ give, as it seems to us, perfectly explicit directions as to the manner of doing the operation. He says: "The surgeon should endeavor to give permanent relief by a procedure which will not add materially to the dangers of the preceding steps [he has been describing a partial prostatectomy done through an external median perineal urethrotomy incision 7 . He should explore the prostate through the artificial opening, and, if he should discover a median outgrowth or isolated tumors [lobes], he should dilate the prostatic sinus, or incise it laterally, and enucleate the lateral tumors; and, if there be a median enlargement, excise it," etc.

In 1870 Sir William Fergusson ${ }^{19}$ removed by enucleation, in the course of a lateral perineal lithotomy operation, a mass from the hypertrophied prostate. His prostatic operation, unlike that of Billroth ${ }^{23}$ (see Malignant Diseases of the Prostate) and that proposed by Gouley, was incidental to a lithotomy, but his words in reporting the case are interesting and suggestive: "I have ventured to put on record what some of my professional brethren may have hesitated to do, from fear that they may have been guilty in performing their operations of perpetrating some rough mechanism not in accord with the nicety of manipulation which is thought so essential in the performance of the master handiwork of surgery-lithotomy." 
In I878 and I88 I Bickersteth and Harrison, respectively, followed the example of Fergusson, removing, in the course of perineal lithotomy operations, masses of the hypertrophied prostate by enucleation.

In 1882 Leisrink ${ }^{24}$ repeated Billroth's operation in a case of malignant disease of the prostate, with an operative recovery, which did not follow the operation of Billroth. Leisrink employed the classic transperineal, prerectal incision of Cèlsus, dating back nearly 2000 years, and recently called Zuckerkandl's incision. Sir William Fergusson employed, as did other surgeons before and after him, for stone operations, the inverted $\mathrm{V}$ and inverted $\mathrm{Y}$ incisions, recently designated-though not by themselves-the incisions of Senn and of Young. In 1882 Ashhurst also operated through the perineum.

This may be called the tentative period of the operative treatment. Its features were: the introduction of the Bottini operation, the performance of total perineal prostatectomy by Billroth, the suggestion of Gouley to perform total prostatectomy by enucleation through a median perineal urethrotomy incision and through incisions in the sides of the prostatic urethra.

3. The third period, in which perineal and suprapubic prostatectomy became established, in which the indirect operations of castration and vasectomy were introduced, had a short-lived popularity, and then declined in favor- 1887 to the present time.

In $1887 \mathrm{McGill}^{25}$ launched and gained a hearing for the operation of total suprapubic prostatectomy, which Belfield and he simultaneously had been employing for a short time.

In the larger number of his cases, McGill performed partial operations only, but in some of them he removed the whole of the hypertrophied gland, and the operation in those cases was essentially the same as that which Freyer long afterward in I90 I endeavored to introduce as a new operation. The immediate followers of McGill were Mansell Moullin,, ${ }^{26}$ Atkinson, Mayo Robson, and a few others in England.

With the introduction of castration and vasectomy there was in England a pause in the practice of direct or total prostatectomies and an interregnum of several years, which ended there with the somewhat spectacular entrance of Mr. Freyer upon the scene.

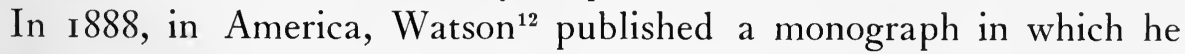
demonstrated: (I) That two-thirds of all hypertrophied prostates could be reached by a median external perineal urethrotomy incision, and partly or wholly removed through it. (2) That the remaining third were not within reach for removal through this incision, but could be readily taken away through the suprapubic route proposed by McGill and Belfield. He counselled the adoption of these two methods of radical 
operative treatment in all cases in which catheter treatment failed to relieve and at an early date in the course of that failure, and also urged that neither the perineal nor the suprapubic operation should be adopted to the exclusion of the other, but that each should be applied according to the indications presented by individual cases. He asserted that the Bottini should have but a limited application, and that it was less desirable than either of the other procedures named; finally, he stated that the perineal operation was that of choice in all cases in which the conditions permitted its performance, and that external perineal urethrotomy and digital exploration of the existing conditions through that incision should be the first step in all cases, the operation to be completed in whichever of the two ways-perineal or suprapubic - was thereby found to be the most suitable.

This was the writer's view at that time, and, with one exception, has been his view ever since, the exception being that, instead of two-thirds only of the cases being appropriate for the performance of perineal prostatectomy, experience has taught him that there are, at most, not more than 5 per cent. or 6 per cent. in which the gland cannot be readily removed through that a venue of approach.

McGill, with a small following, and Watson alone, in England and in America, respectively, remained for some years almost the only advocates of the high and the low operations of prostatectomy.

In 1890 Dittel ${ }^{27}$ added another suggestion, that of removing a triangular portion of each of the lateral lobes, and suggested a new method of approach from the perineum. The important point of Dittel's operation was the making of the incisions through which the parts of the gland were to be removed, in the posterior surfaces of the outer sheath, and of avoiding injury to the urethra, by placing them on either side the median line.

In 1892 Pyle $^{28}$ proposed to remove the entire prostate, lobe by lobe, through a perineal curved pre-rectal incision, and by exposing the prostate by blunt dissection. His proposal was to then enucleate the lobes through an incision in the posterior aspect of the outer sheath of the gland. This is essentially the same methed as that later put forward by some of the French surgeons.

In I 890 Goodfellow began to operate by finger enucleation through the sides of the prostatic urethra in the manner that had been suggested by Gouley, and as Watson also carried out the operation.

In I $_{94} \mathrm{Nicoll}^{29}$ proposed to remove the prostate, one lobe at a time, by a combined operation, the essential feature of which was, however, the perineal part of it, the suprapubic step being taken merely for the sake of making the gland accessible for finger enucleation by downward pressure 
upon it through the suprapubic cystotomy incision with the fingers of the other hand. Nicoll's operation avoided all injury to the floor of the bladder and sought to do so as well for the urethra, which it does not open. The gland was removed through the perineal incision.

In 1895 Alexander ${ }^{30}$ brought forward another combined operation in which the suprapubic step was the same as that of Nicoll, while the lower or perineal part of the operation was the same as that practised by Watson and Goodfellow, neither of whom had, however, at that time published descriptions of the manner in which they operated.

In 1895 Fuller $^{31}$ again advocated the suprapubic operation, adding the step of pressing the gland upward and thus making it more accessible by pressure with one finger in the rectum and the thumb on the perineum. He removed the lobes separately or in one mass, as the case might be, by finger enucleation, aided by a special form of scissors with serrated edges, in the same way as that which Freyer later adopted.

Castration and Vasectomy.-This, in effect, was the position of operative treatment when White ${ }^{32}$ came forward with the proposal to apply castration as a method of treatment for the cure of the disease. The results of the early operations performed in pursuance of this proposal were, in some cases, so striking and brilliantly successful as to encourage the hope that a simpler and less dangerous way out of the difficulties offered by the condition had been found. Not long afterward, however, it was shown by $\mathrm{Cabot}^{33}$ that the mortality attending castration was high, and subsequent computations of results served to confirm his statement. It was furthermore shown that there was a good deal of uncertainty in the later results, and the operation was ultimately abandoned by the majority of surgeons.

In the last edition of White and $\operatorname{Martin}^{34}$ (1905, p. 77 I) White expresses his opinion of the operation thus: "My present opinion is that castration and vasectomy are likely to occupy a more and more restricted field in the treatment of prostatic hypertrophy. In properly selected cases I still think that they are likely to have a low mortality, and that, when fully successful, they secure a return to a condition more closely resembling the normal than most of the other operations.

"The uncertainty of the results (castration and vasectomy), both immediate and remote, the fact that they are not free from danger, etc., combined to render their final status in the treatment of prostatic hypertrophy a matter of doubt."

Vasectomy was tried after the failure of castration to establish itself, but suffered a like fate to that of the other indirect operation.

These two methods served to postpone the further progress of the more radical procedures of prostatectomy, by diverting the attention of many 
surgeons from them. Those, however, who were already well confirmed in their belief in the value of prostatectomy, and had been practising it for a longer or shorter time, notably Mansell Moullin, Watson, Goodfellow, Nicoll, Alexander, Fuller, and others who from time to time became adherents of prostatectomy, were not diverted from that method.

Castration and vasectomy occupied the general surgical attention, however, from I894 to about I899, in which year some of the French surgeons, apparently for the first time, began to take an interest in the operations, and in the course of the next two or three years had produced a multiplicity of modifications of the perineal method which were marked by the technical completeness and finish which characterizes the work of that race. With this movement began the class of perineal methods which are called the open or dissecting operations.

Of the French perineal operations, those of Proust ${ }^{35}$ and Albarran ${ }^{36}$ are the most notable of the techniques. In both the prostatic urethra is split in two by an incision through its floor and the gland removed lobe by lobe or piecemeal; Proust ligating the ejaculatory ducts, Albarran not doing so; both of them closing the incision in the urethra by suture. They approached and exposed the gland by the transverse prerectal incision and by blunt dissection, dividing the central tendon of the perineum and the recto-urethral muscle to allow the rectum to be drawn backward and held by a retractor. These techniques were described in detail in I900-I902.

In I90 I Syms, and in 1902 De Pezzer, introduced special forms of tractors; in 1903 Young devised another and in I90I Fergusson still another, by the use of which the gland is brought down and made more accessible.

In 1903 Young, ${ }^{37}$ of Baltimore, became an advocate of the open or dissecting operations, and modified the technique of Proust by employing lateral capsular incisions. He also introduced a special manner of dealing with the middle lobe, and avoided injury to the ejaculatory ducts. (Pyle and Nicoll had, as has already been shown, introduced the step of preserving the prostatic urethra intact.)

In I90 I Freyer ${ }^{38}$ appeared upon the field with what he termed his new operation of suprapubic prostatectomy, which, as has already been said' differs not at all from the technique of Fuller. Mr. Freyer's claims to novelty have been so frequently shown to be without the least justification that there is no need to refer to this part of his connection with the subject. He might appropriately be described as a universal usurper of previously preëmpted prostatic privileges. Mr. Freyer has, in spite of this fact, done good service to the cause by establishing the suprapubic operation upon a more extended and better basis in England than it 
formerly occupied or would have been likely to occupy for a good while without his vigorous advocacy of its already known but little utilized advantages.

We refer any who may be interested in the controversy with regard to Mr. Freyer's claims to originality to the articles of Fuller ${ }^{39}$ and of Nicoll, ${ }^{40}$ and to a review in the Boston Medical and. Surgical Journal, January 3I, 1907.

In I90 I Parker Syms ${ }^{41}$ became an advocate of the perineal operation, done through the median external perineal urethrotomy incision, and introduced a rubber balloon tractor. Both Ferguson ${ }^{42}$ and Murphy ${ }^{43}$ have been valuable workers in the field.

Among the best single contributions hitherto published, in addition to those of the surgeons already mentioned, are those of the following: Escat, ${ }^{44}$ Proust, ${ }^{45}$ Deaver $^{46}$ (1905), and W. G. Richardson, ${ }^{47}$ of Newcastleon-Tyne, I904.

A number of miscellaneous methods of operating have recently been suggested, none of which, in our judgment, are of value. They are briefly noted in the article by Watson. ${ }^{48}$

Between I 895 and 1905 Bottini's operation found several enthusiastic advocates. Albarran, Willy Meyer, Bangs, Horwitz, and Young have each in turn been special advocates of the method, and each of them has in turn ceased to be so, or has retired the operation to a position of secondary importance. Freudenberg ${ }^{21}$ is at the present writing (1907) almost the only steadfast partisan of this method, which he advantageously modified. Chetwood ${ }^{49}$ has also employed a method similar to that of Bottini which he applies through the perineum.

When the writer first published his views upon the subject of the operative treatment (I888) he was unable to find more than 22 cases in which prostatectomy had been done. How widely the profession has since then adopted the practice will be seen by a glance at the statistical tables a little farther on in the chapter. This historical sketch includes, we believe, all the important operative steps, and we end it here and proceed to the discussion of the operative methods.

The Operative Methods.-No one method has established an exclusive monopoly among the operations undertaken for the relief of prostatic hypertrophy. Some have been discarded as the result of the last fifteen years' experience, and four are left from which to choose. These are:

I. The palliative operations for drainage.

2. The galvanocautery operation of Bottini and its modification by Chetwood.

3. Total perineal prostatectomy.

4. Total suprapubic prostatectomy. 
The choice of one or another of these surgical procedures should be determined in accordance with (I) their relative dangers; (2) their relative limitations; (3) the character of their results.

There are two things with regard to the operative dangers attending surgical procedures which we wish to know when considering which one of them we shall choose. The first is the actual operative mortality without regard to anything else, and the second is the special danger which attends each one of them, or to which one of them is more exposed, if at all, than another. These two factors we have, so far as possible, determined by a careful study of all available cases reported up to the present time (1907), and have embodied the results in the tables which are below.

\section{Operative Dangers.}

\begin{tabular}{|c|c|c|c|c|c|c|c|c|}
\hline & & & & & & Cases. & Deaths. & $\begin{array}{l}\text { Mortality. } \\
\text { Per cent. }\end{array}$ \\
\hline Palliative operations for drainage & . & . & . & . & . & I 46 & 49 & 33.0 \\
\hline Bottini operation & . & . & . & . & . & I 164 & 69 & 6.3 \\
\hline Total perineal prostatectomy & . & . & & . & . & 1000 & $6 I$ & 6. I \\
\hline Total suprapubic prostatectomy & . & . & . & . & 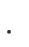 & 406 & $3^{8}$ & 9.6 \\
\hline Combined suprapubic and perineal & & . & . & . & . & I 57 & I3 & 8.2 \\
\hline
\end{tabular}

It is not without interest to note the different series of cases of the perineal and suprapubic operations which have been collected by different writers and to compare them with each other. The following are the most notable statistical compilations that have been presented of late years.

Mortality of Perineal Prostatectomy.

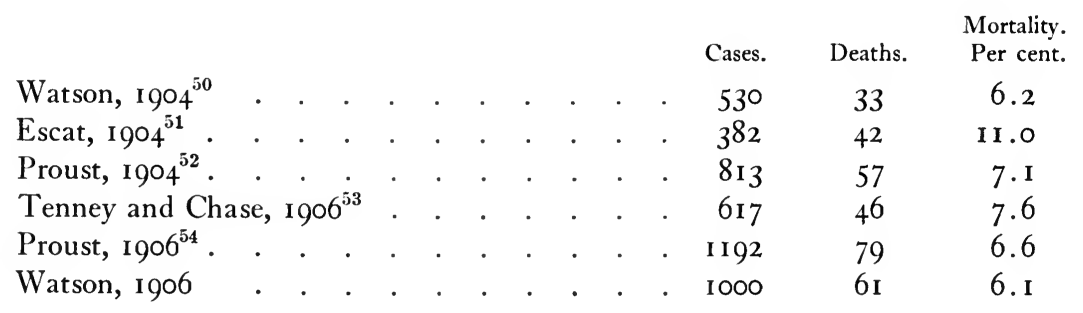

The approximate concurrence of these different writers is striking, and, so far as figures are of value in forming our judgment of such matters, they are very convincing on that account.

Escat's series may be assumed to give the least accurate estimate of the operative dangers attending this particular procedure, because its number is smaller than that of the others in the column. If we include 
Escat's cases, the average mortality percentage is approximately 7.6 per cent. for the whole number.

In addition to these series of large numbers of cases it is worth while to look at some of the results obtained by individual operators. For this purpose we have selected the following:

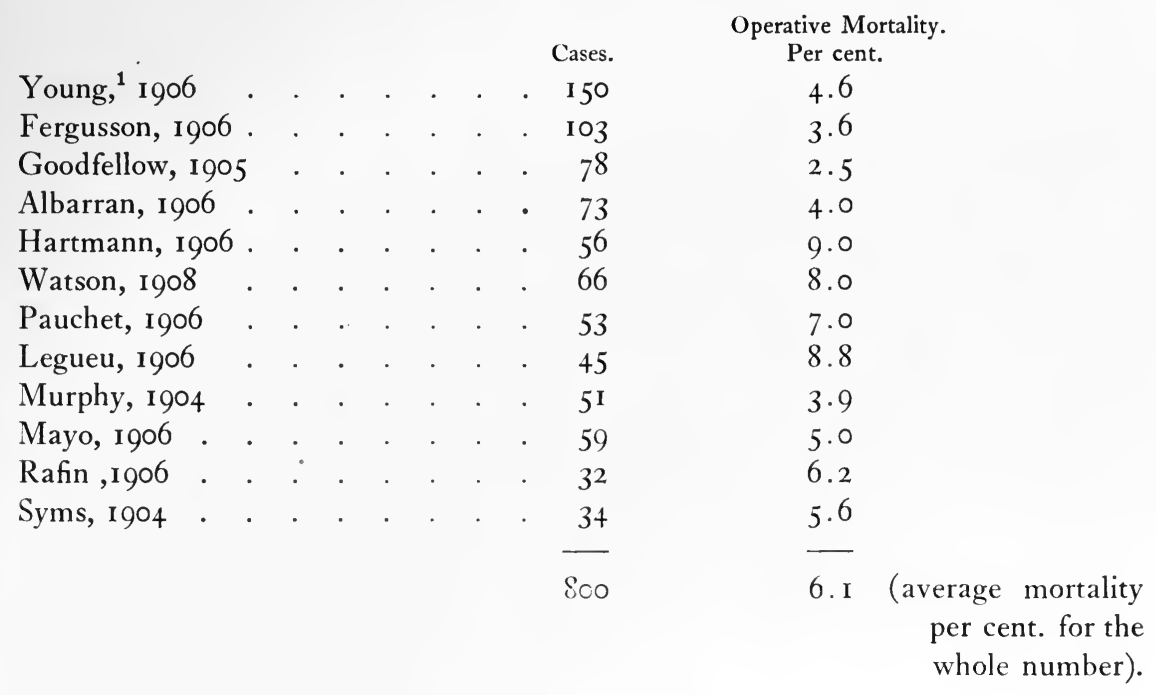

These personal series, representing the results of the work of twelve different operators, give an average of 6.I per cent. as the operative mortality for 800 cases, which is just the same as that shown in our most recent compilation of 1000 cases, and approximately the same as that found by Proust for a still larger number of cases, namely, i i92. We may accept this as being a fair estimate of the dangers of this operation at the present time. The mortality percentage will become less as more operators become skilled in the selection of their cases, in the technique of their operations, and in the after-care of the patients.

Operative Mortality of Total Suprapubic Prostatectomy.-The operative mortality of the high operations may be judged from the following series of collected cases:

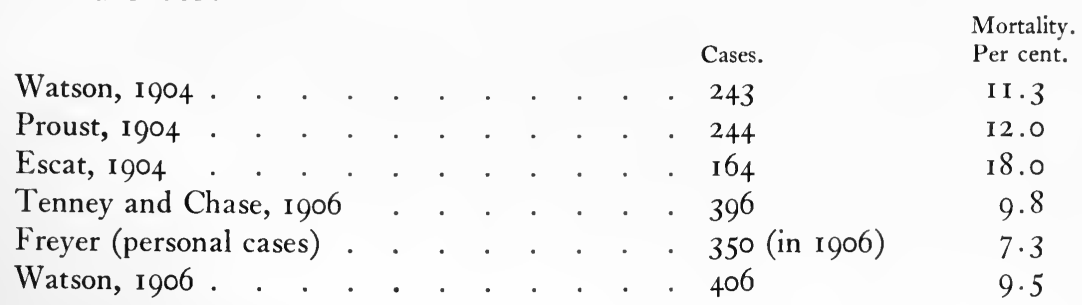

${ }^{1}$ In 1908 Young published a series of 128 consecutive perineal prostatectomies without a death. 
Of these different series of cases, the mortality percentage of that of Escat is undoubtedly too high to fairly represent the dangers of the operation at the time of this writing (1908). A more just view of the mortality immediately attending the operation will be gained by taking the average mortality percentage of the whole number of the cases of the different series. This would be II.3 per cent. Freyer's personal statistics, which show an operative mortality of 7.3 per cent., is a very fair estimate of the mortality which will probably be reached in the course of the next year or so; very possibly it may be lower yet. The death rate is decreasing steadily in this operation, but not in this operation alone. It is equally true of the perineal methods. The only operation that will not, in our belief, show an important lessening of mortality is the Bottini. There is no reason why it should do so, while there is good reason for a somewhat lower mortality being attained in connection with both the high and the low prostatectomy operations.

The ratio of the higher mortality percentage of the suprapubic operations than of the perineal methods is just about the same as it has always been, viz., a little less than twice as great.

Before passing to the consideration of the causes of death in the fatal cases of each of the operative methods of treatment, we give the results of our study of the reported cases of castration and vasectomy, and also of the mortality of catheter treatment, as estimated from a small number of cases, in which we have calculated it in connection with this form of treatment by the number of deaths occurring within one month after beginning the regular use of the instrument, which puts it upon the same basis as that of the operative treatment in this respect.

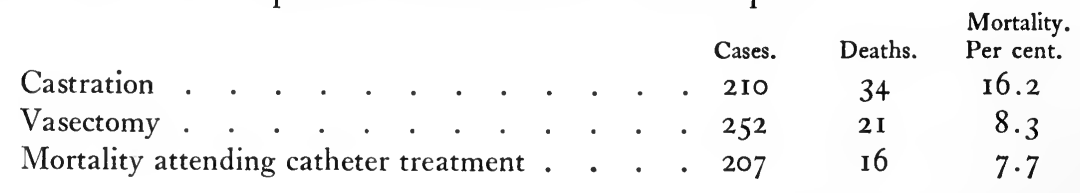

From the above computations we may draw the following conclusions: (I) That the smallest number of deaths attends the performance of the Bottini and total perineal prostatectomy operations. (2) That the mortality percentage of each of them is but little more than half as great as that of the suprapubic method. (3) That the best results, so far as operative mortality is concerned, in the latter operation have been obtained by Freyer, viz., 7.3 per cent., and the best in connection with the perineal operation, that of Young, as stated in the footnote on p. 345 . (4) That treatment by catheter is much more dangerous than by total perineal prostatectomy, if judged by the best results obtained by the latter; somewhat more so than is shown to be the case by the average mortality percentage of that operation, and about the same as that of the best 
results of suprapubic prostatectomy. Finally, that vasectomy has but a little less mortality than the suprapubic operation and a good deal larger one than either the Bottini or perineal operations, and that castration is attended with a far higher death rate than any of the other procedures, except the palliative operations for drainage.

It must be remembered with regard to the latter operations that they have almost invariably been performed in the worst class of cases, upon patients to whom relief had to be afforded by some means or other, and who were obviously too enfeebled to endure any radical procedure.

Dangers More or Less Special to Each Operative Method.-In order to estimate these, the writer analyzed the cases of the series already given which had been treated by the Bottini, by total perineal, and by total suprapubic prostatectomy. The analysis was made with reference to the causes of death in the fatal cases which occurred in connection with the performance of these three operations, respectively. It was seen that there were but four fatal factors that were of importance, namely, uremia, shock, sepsis, and postoperative pulmonary complications. Of these, uremia played by far the more important part, many more deaths being due to it than to any other one of the four conditions. The relative percentage of deaths in the fatal cases from each of these causes was as follows:

Bottini .

Total perineal prostatectomy

Total suprapubic prostatectomy

Bottini .

Total perineal prostatectomy

Total suprapubic prostatectomy

Bottini .

Total perineal prostatectomy

Total suprapubic prostatectomy

Bottini .

Total perineal prostatectomy

Total suprapubic prostatectomy

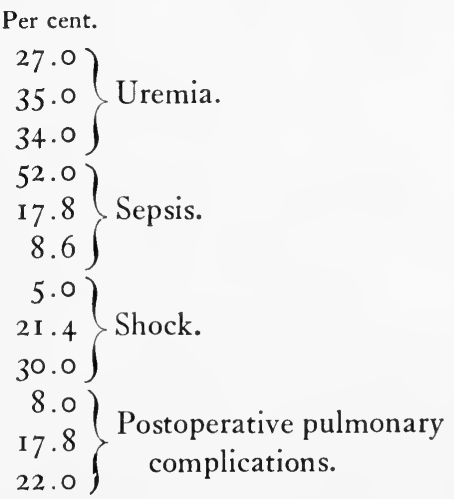

Among the deaths classed as due to postoperative pulmonary complications there were 5 of pulmonary embolism (Watson, 2; Young, I; Pousson, I; Murphy, I).

Such inferences as we may draw from the above data with reference to the causes of death in connection with each of the special operative methods are as follows:

I. That uremia is the most to be feared, and about equally to be feared in all the surgical operations, irrespective of their nature.

2. That the Bottini operation is more likely to be followed by septic infection and death from that cause than either of the other operations. 
3. Per contra, that the Bottini operation is less liable to be fatal from shock or postoperative pulmonary complications than either of the other methods of operative treatment.

In so far, then, as these data influence the choice, we should naturally prefer to avoid the Bottini operation in the case of patients having cystitis of severe grade, and should reserve it for the cases of patients with very weak hearts, pulmonary disease, or conditions predisposing markedly to it after operation, and to such as were especially exposed to the dangers of shock in connection with the performance of the operation of total prostatectomy.

Influence of Age upon the Mortality.-We are not inclined to attribute much importance to age per se in its influence upon our decision as to the performance or avoidance of operation, or with reference to the choice of one or another of the operative procedures. In a general way, it is, of course, true that the older the patient the greater his operative risk, but the bodily condition of each one is a much more potent factor than the mere fact of advanced age. Thus, other things being equal, we should take less risk in doing a prostatectomy on a patient of eighty years, whose renal function was still being capably performed, than on one of fifty-five whose kidneys were diseased.

In this connection, the tables published in 1906 by Tenney and Chase ${ }^{53}$ are of interest.

Tenney and Chase's tables of age with reference to its influence on the operative mortality are as follows:

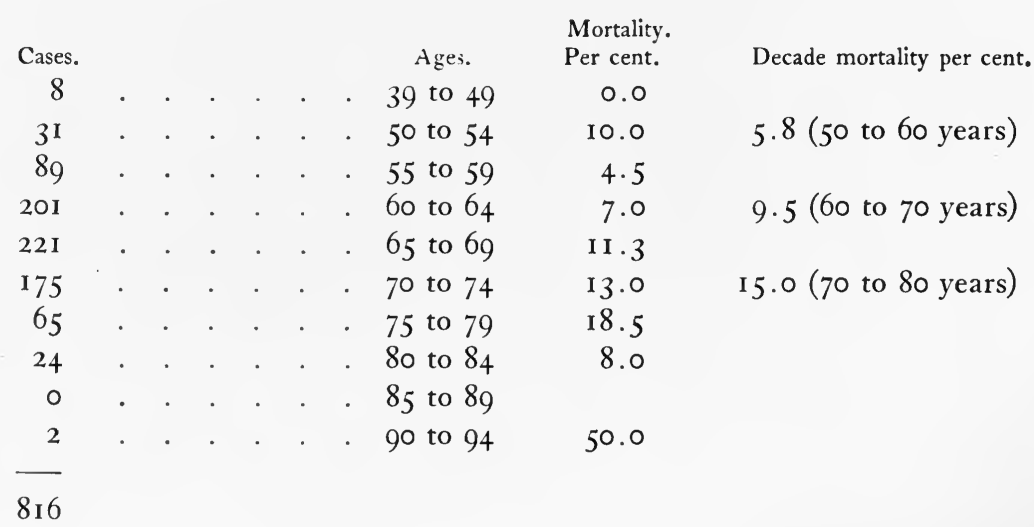

These figures show some curious contradictions, and that we may not, as already said, rely upon age per se as an essential factor influencing our decision. For instance, the mortality percentage of the cases of patients between fifty and fifty-five years of age was twice as great as that of those between fifty-five and sixty; it was also notably higher than the mortality percentage of the patients between sixty and sixty-five, and 
of those between eighty and eighty-five. So much depends upon how the cases are grouped in such calculations that the results of different ways of computing the same figures and the same cases may bring us to very different conclusions. It is far better to base our decision upon the results of physical examinations than upon any other data which are available to us.

The Influence of Occupation, Environment, etc., upon Mortality.-Too little attention has been given to this part of the subject. In the writer's personal experience, it has been the most potent of all the elements which enter into the question of the operative mortality. This is especially true of hospital practice, in which the surgeon often has this dilemma presented to him, viz., whether to subject the patient to the risks immediately involved in a prostatectomy, or to have him encounter the equally great, or even greater, probability of dying, though at a somewhat more remote time, that is involved in having him attempt to carry out the treatment by the catheter in uncleanly surroundings, with the dangers arising from lack of care and his own ignorance. Such patients cannot be permanently kept in a hospital; they are usually subject to attacks of complete retention; they are too ignorant, too careless, and too dirty to make it possible to institute permanent drainage of the bladder for their relief. Under these conditions, the writer has often been compelled to select the operative horn of the dilemma, and this course has been the chief factor in furnishing a relatively high mortality to the series of operations which he has personally performed.

The Influence of Delay and Previous Catheter Treatment upon Operative Mortality.-This is another element to which we owe a higher death rate than there should be in connection with the radical operations, and for it the medical wing of the profession is largely responsible. The medical attendant continues, far too frequently, to subject patients to catheter treatment until they are broken down and reduced to a condition in which surgical intervention must necessarily be attended by serious risk. In short, the patient is deprived of his best chance of having his life saved or prolonged.

Non-operative Deaths Occurring within a Comparatively Short Time after Operation.-Proust ${ }^{55}$ notes the cause of death in 7 cases in which it took place more than one month after the performance of the operation. Three of the cases were of Hartmann's, 3 of Murphy's, and I of Young's. One of the deaths does not appear to have had the least connection with the operation, the patient dying six months later of intestinal obstruction. The other 6 died of the following conditions, and at the following times after the operations: 


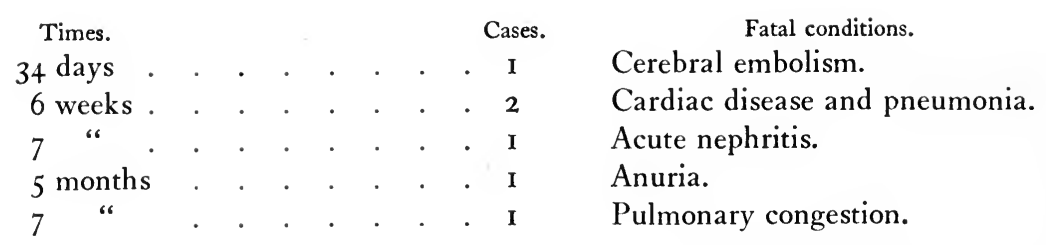

To these the writer may add another case from his own practice, in which death resulted two months after a total perineal prostatectomy, and was due to a lumbar abscess, for which the patient refused to be operated upon until it was too late to avert the fatal issue. No autopsy was permitted, and the origin of the very extensive suppuration was not determined. The collection of pus first pointed just above the iliac crest on the left side.

The point to be borne in mind in connection with operative treatment of patients is the risk which presents itself in this malady, as compared with that which is necessarily encountered in the cases of all old people with whatever surgical procedure it is necessary to employ with them. In the more serious forms of the disease under consideration, however, we are more often compelled to interfere than with other conditions. Retention of urine must be treated surgically in one way or another, whatever the patient's age, and irrespective of how desperately ill he may be at the moment.

Character of Results.-Cure.-In considering the results we use the term cure in the sense that the patients have been given freedom from pain, from frequent urination, and vesical tenesmus; that the bladder is either spontaneously emptied, or that the residual urine does not exceed 50 c.c.; that the urine does not contain more than a trifling amount of pus or blood, and that these conditions shall exist at a time not less than three months after the operation.

Good Results.-By the term good results we mean to designate the cases in which marked improvement has followed the operation, plus those in which cure has been obtained.

The following analysis of cases with reference to later results was made by the writer in $1904 .{ }^{11}$

\begin{tabular}{|c|c|c|c|c|}
\hline $\begin{array}{c}\text { Cures. } \\
\text { Bottini operation }\end{array}$ & . & $\begin{array}{l}\text { Cases. } \\
490\end{array}$ & $\begin{array}{l}\text { Cures. } \\
149\end{array}$ & $\begin{array}{c}\text { Per cent. } \\
30.4\end{array}$ \\
\hline Total perineal prostatectomy & . & 143 & 87 & 60.0 \\
\hline Total suprapubic prostatectomy & . & 53 & 35 & 66.0 \\
\hline
\end{tabular}

Good results.

Bottini operation . . . . . . . . . . . . . . . . . . 86.0

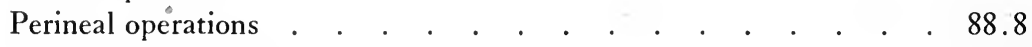

Suprapubic operations . . . . . . . . . . . . . . . . . . 
Limitations.-The Bottini Operation.-The Bottini operation cannot or should not be employed under the following circumstances:

When for any reason a satisfactory cystoscopic examination cannot be made beforehand; in cases in which the form or the size of a middle-lobe enlargement makes it difficult, dangerous, or impossible to apply the heated blade to it; in cases in which there is a well-marked purulent cystitis, and, therefore, a special liability to sepsis; and finally, in those in which there are associated calculi.

Total Perineal Prostatectomy.-In a small number of cases-perhaps in 5 per cent. of them-it is so difficult to reach and bring down the lobes in order to enucleate them, that it is better and safer to employ the suprapubic operation than to try to remove the gland by the perineal route. This is the only limitation attached to the performance of the perineal operation except that mentioned in the next paragraph.

Total Suprapubic Prostatectomy.-The only limitation to the performance of suprapubic operation is that which is due to firm adhesions uniting the outer surface of the gland with the inner one of the fibrous sheath, and this applies equally to the perineal operations.

Accidents, Complications, and Failures Attending Each of The Methods.-The Bottini Operation.-This operation is subject to certain accidents, complications, and to failure in a number of the cases. These may be presented by quoting the personal experience of Willy Meyer with the operation, in a series of 59 cases. In 4 , perineal abscess followed the operation; in 2 of them it was due to injury of the membranous urethra; in I case, recto-urethral fistula; in 6 cases, epididymitis; in 2 cases, orchitis, resulting in one of them in gangrene of the testis, and in the other in suppuration.

In the series of I I 64 Bottini operations collected by the writer in I904, 2.6 per cent. were followed by incontinence of urine. In 8 of the 69 fatal cases of this series the bladder wall was burned through by the blade of the instrument. In I I per cent. of the whole number of the operations failure to mitigate the symptoms resulted. In 13 per cent. of the cases the operation had to be repeated. In I case the bladder exploded.

In several cases the inner blade of the instrument has been bent during the operation, and could not be closed upon the outer one.

The Perineal Operations of Total Prostatectomy.-In 2 cases the peritoneum has been opened; once death resulted. In 2.7 per cent. of $53^{\circ}$ cases published by the writer, ${ }^{11}$ in 1904 , urethrorectal fistula resulted. In 3.5 per cent. of the cases in the same series, incontinence of urine followed the operations. Failure occurred in 7.4 per cent.

Escat, ${ }^{56}$ in 382 cases collected by him, found 12 , or 3 per cent., in which incontinence of urine followed the performance of the perineal operations, 
and lasted for a longer or shorter time. Similar figures are reported by Albarran, Proust, Goodfellow, and others.

If care be taken to avoid injury to the compressor urethræ muscle, which is the true vesical sphincter, as was long ago pointed out by Ultzmann, incontinence of urine will very rarely follow the operations. Ruggles $^{57}$ has recently again called attention to the importance of the compressor urethræ muscle in controlling the escape of urine from the bladder. The writer has in two instances inflicted extensive injury upon the vesical outlet, when doing perineal total prostatectomies. In neither instance did the patient have incontinence of urine. On the other hand, he has had three patients who have suffered for long periods from incontinence of urine, following perineal prostatectomy, and in these three cases absolutely no injury was done to the vesical outlet, but more or less damage was done to the compressor urethræ muscle.

It has been thought by some surgeons that the injury done to the nerve branches supplying the muscles which prevent the escape of urine from the bladder may account for some of the cases of incontinence of urine which have followed perineal prostatectomy. We do not know whether this is true or not, but we are very well convinced that interference with the compressor urethræ muscle, if not repaired in the course of the healing process, is the true reason for urinary incontinence in a good many instances, at any rate.

Epididymitis following the operation has been reported by some surgeons. The writer has never had this complication in connection with any of his own cases, and it is certainly a rare mishap.

Hemorrhage occasionally attends the performance of perineal prostatectomy, and in some instances in which the open or dissecting methods are employed there is a good deal of bleeding. It is readily controlled, however, and personally the writer has never had a case in which hemorrhage was of any consequence at all. When it happens, it is always due to tearing of the outer fibrous sheath, and this need not occur if proper care is taken to avoid it.

Stricture of the prostatic urethra has occasionally resulted from the operation. The instances of its occurrence are very few, however; we have seen it in four cases. Two of the patients required secondary operations to relieve the condition; in the other two instances the condition presented was not, properly speaking, stricture, but an obstruction due to the irregular course assumed by the prostatic urethra during the healing process. Proust, Czerny, and W. G. Richardson have each recorded a case in which stricture resulted from the operation.

Suprapubic Prostatectomy.-Incontinence of urine following the operation is rare. Fistula followed it in 2 per cent. of the 406 cases analyzed by the writer. 
Urinary infiltration of the tissues of the prevesical space in 2 per cent.

Peritonitis in I case.

The rectum was injured in 2 cases.

Hemorrhage of serious character in 5 cases.

Failure to relieve the symptoms occurred in 6.7 per cent. of the cases.

Advantages of the Different Operative Methods.-The Bottini Operation.-It does not require general anesthesia.

The patient can be up and about rather sooner than after the operations of total prostatectomy.

It is accompanied by less shock than is the case with the more radical measures.

It is less liable to be followed by postoperative pulmonary complications.

Its operative mortality is not high.

Total Perineal Prostatectomy.-Rapidity of performance.

It is a complete operation.

In successful cases it results in complete cure of the patient.

The smallest operative mortality as yet attained in connection with any of the operative methods has been in connection with total perineal prostatectomy.

It supplies free drainage of the bladder.

It has a small percentage of failures and a large one of cures.

It does not require previous cystoscopic examinations.

Total Suprapubic Prostatectomy.-It is a complete operation.

It has a large percentage of cures.

It has a small percentage of operative complications.

It is applicable to all varieties of the condition.

It does not require previous cystoscopic examination.

It affords a free space in which to work and a wide channel through which to withdraw the gland.

It is rapid of performance.

It supplies free drainage for the bladder.

Conclusions with Regard to Choice of Method.-The reasons for preferring one or another of the operative methods are, we think, clearly enough defined in individual cases. We should avoid becoming wedded to one operation exclusively, for the conditions presented in the special instances make sometimes one and sometimes another method the more desirable to apply.

In general, we may say that an operation which removes the whole gland is better than one which merely takes away a part of it, or that voL. $\mathrm{I}-23$ 
makes a channel through it, provided the more radical measure is not more dangerous, does not involve greater subsequent disabilities than the more conservative procedure, and if its results are as good.

We have shown that total perineal prostatectomy is not more dangerous than the Bottini operation, and we have seen that total suprapubic prostatectomy is attended by a higher operative mortality than either of. the other two.

This is a general statement only, however, and we must go farther in order to arrive at a just conclusion with regard to the respective merits of these three procedures. When this is done, it is seen that deficient drainage of the bladder and sepsis are largely responsible for the deaths following the Bottini operation; but little danger of death attaches to it on account of shock or postoperative pulmonary complications, and that exactly the contrary of these things is true of both perineal and suprapubic prostatectomy. Again, there is greater liability to incontinence of urine after the Bottini and the perineal operations than with suprapubic total prostatectomy, although the proportion of cases in which this complication occurs with the two former is a small one. And finally, that the proportion of cures is much larger with the total prostatectomies-both perineal and suprapubic - than with the Bottini operation, and that the latter is not so generally applicable as either of the former methods of treatment.

Our inference from the above data is that the Bottini operation should be limited to the cases in which the patients are especially liable to pulmonary and cardiac postoperative complications, and that it should not be employed in the cases in which there are well-marked septic vesical conditions.

The Choice between the Perineal and the Suprapubic Operations.The same fact that existed at the outset of the operative treatment of the condition remains true of the high and the low prostatectomies. The mortality of the former is decidedly greater than that attaching to the latter. This is true, whether we compare the series of the best results of the operations, respectively, obtained by certain special surgeons or those of each of the procedures as they appear in the hands of a large number of surgeons and are reckoned generally. As an offset to this disadvantage under which the high operation labors, it must be acknowledged that there is less liability to incontinence of urine after it than with the perineal method. The criticism which has been brought against the perineal operation, that the rectum is more liable to be injured in its performance than with the suprapubic method, is not sound, provided proper care is taken to avoid this accident when doing perineal prostatectomy. 
All the methods are about equally exposed to the one great danger connected with the performance of the prostatic operations in these cases, namely, that of uremia.

We conclude, therefore, that the perineal operation is to be preferred to the suprapubic in the great majority of cases, simply on the ground that it is a safer operation. In the few çases in which there is a very high reaching intravesical enlargement of the gland, the suprapubic operation is the method of choice.

The Choice of the Perineal Techniques.-We frankly confess to being prejudiced in favor of the method that we have employed for perineal prostatectomy from the time at which we first did our operations to the present, with such exceptions as we have made in order to become personally familiar with the performance of some of its modifications that have since been introduced, notably those of Nicoll, Proust, and of Young, from the essays of all of which we have invariably returned to the manner originally practised by us, with the conviction that the others had nothing to offer to us personally that was of greater advantage than the operation to which we were most accustomed.

The advantages of the method to which we refer, which is that of finger enucleation through an external perineal urethrotomy incision, are the greater simplicity and the rapidity with which it can be done, as soon as the finger of the operator has become trained to appreciate just what it is doing out of sight when accomplishing the enucleation. It must be admitted that this demands that the surgeon practising it should have performed the operation a number of times; but, on the other hand, it is the fact that the sense of touch, once trained, is, in the experience of all who have adopted this manner of enucleating the gland, far more accurate than that of sight, which is, for the most part, relied upon in the performance of the dissecting or open methods, as they are called. The rapid enucleation method by the sense of touch only requires on an average about six to eight minutes. We have done the operation inside of two minutes in one or two instances, and in a good many more in from four to six minutes, and under primary anesthesia.

Apart from the special steps and slight changes of one or another sort in the performance of the various methods, there is a word to be said with regard to the much more important question which remains to be considered, viz., the failure of the general practitioner to appreciate the fact that catheter treatment is quite as, and often more, dangerous than the radical operative procedures. The medical wing of the profession still continues to prepare patients for failure of the surgical treatment of the malady, by infecting the bladder and the kidneys with the catheter, or by permitting residual urine to accumulate to the point at which its presence proves injurious to the kidneys, and prepares it for receiving infection, 
thus greatly augmenting the dangers of surgical interventions of whatever sort.

It is to the overcoming of these things that surgical attention needs to be directed today much more than to devising trifling changes in the technique of this or that method of operation, or to contention concerning priority in having introduced one or another of the modifications or methods of operation.

Treatment of Prostatic Hypertrophy by the X-rays.-Recently this agent has been applied for the purpose of reducing the enlargement of the prostate. It is too soon to pronounce as to its merits, but it is important to record the few results that have come within our knowledge, for the desirability is great, of avoiding the operative dangers involved in the radical measures, comparatively slight though they have become, and if it can be done in this way the method of treatment will be of great value.

Tansard and Flieg ${ }^{58}$ report the results of the treatment upon two patients with whom they have tried it, and speak of other recorded cases thus:

"Carabelli and Luraschi were the first to try the Röntgen rays in the treatment of hypertrophy of the prostate in I904. They did not then publish the results, wishing to wait long enough to know if they were lasting. Two patients were submitted to the treatment. The rays were directed to the centre of the perineum only, the surrounding parts being encased in lead. The tube was held 20 to $25 \mathrm{~cm}$. from the surface.

"One patient was submitted to fifteen different applications of the treatment at intervals of two or three days at first, and later of sixteen days. Exposure three to seven minutes, I 5 amperes, 100 volts posometer of Brand: $\mathrm{N}^{\mathrm{e}} \cdot 3$.

"The other patient had ten sittings of ten minutes each in eleven days. Spontaneous urination was restored and the residual urine disappeared. One year later the cure was still maintained.

"Moskowicz, in April, I905, reported 3 cases in which he had employed the same treatment, applying it, however, through a short speculum introduced into the rectum, protecting all the parts except the most salient portion of the prostatic enlargement by sheet lead. Under this treatment one patient was apparently cured; in two others it gave rise to complications, epididymitis in one and a hemorrhagic cystitis in the other."

The authors themselves have treated two patients in this way, applying the rays to the surface of the perineum and protecting all but a circular area of $5 \mathrm{~cm}$. in diameter by a lead diaphragm, arranged so as to form a part of the cylinder through which the rays were conveyed. "The rays employed marked 7 on the radiochromometer of Benoist." 
One patient was cured. The treatments were continued for eight months, at intervals varying between a fortnight and one month, once being suspended for fifty-five days because of a dermatitis.

After the second treatment, the frequency of urination became less and there was a perceptible diminution in the size of the gland. The prostate was greatly lessened in size after the fifth treatment. Following the long interval necessitated by suspension of the treatment on account of the dermatitis, there was a moderate recurrence of the symptoms. Following the last treatment, at the end of the eight months, the prostate had become reduced to the normal size of the gland, and the patient was free from residual urine and all subjective symptoms.

The result was less satisfactory in the second case, the patient received, however, but four treatments.

\section{MALIGNANT DISEASE OF THE PROSTATE.}

Frequency.-Albarran ${ }^{59}$ reported that $\mathrm{I}_{4}$ out of $\mathrm{I}$ oo specimens examined by him, and that were believed to be benign enlargements of the prostate, showed cancerous degeneration.

Hawley ${ }^{60}$ gives the following data: Winiwarter found in 290 cases of carcinoma in male subjects $I$ in which the disease involved the prostate.

Heimann, in 20,544 cases of cancer recorded in Germany, found that 34 involved the prostate.

Young, ${ }^{61}$ as the result of a study of 40 cases of carcinoma of the prostate, concludes that the disease occurs in about Io per cent. of the cases of enlargement of the gland, usually classed as prostatic hypertrophy.

Greene and Brooks ${ }^{62}$ found that there were 3 cases of carcinoma of the prostate in 53 specimens of supposed benign enlargement.

Other observers hold similar opinions as to the relatively frequent occurrence of cancer of the prostate.

Sarcoma also has been reported as having its seat in the prostate in a few cases (Spanton, ${ }^{63}$ Pousson ${ }^{64}$ ).

Pathological Anatomy of Carcinoma of the Prostate.-The appearances noted in most of the cases reported by Young ${ }^{61}$ were those presented by adenocarcinoma. Three forms of pathological changes are described in connection with these cases: (I) Carcinoma simplex. (2) Tubular. (3) Medullary carcinoma. In two of the specimens it was shown that the process may begin in and for a long time probably remain confined to a small area of a benign adenomatous hypertrophy.

Hawley ${ }^{60}$ describes the microscopic appearances as follows: "Adenocarcinoma resembles very closely the non-malignant adenomatous 
growth found in the so-called hypertrophy. The alveoli in both may be distended with desquamated epithelial elements with central detritusbearing areas. In carcinoma, however, there exists usually less space between the alveoli, the cell nuclei stain deeper, and the lymph spaces often contain cancer cells.

"In all varieties may be found areas of round-cell infiltration, as in simple hypertrophy; also, nests of cancer cells invading the lymph spaces, the sheaths of the nerves, the bloodvessels and especially the veins. They are often completely filled, their walls having been penetrated.

"To the naked eye a cut surface shows in some cases little or nothing that suggests the presence of malignant disease in the gland; in others there are well-defined, usually round, nodules, or there may be a single focus of the process. The nodules, when multiple, are often scattered more or less generally throughout the whole of one, and sometimes of both, or all three lobes; they may, on the other hand, be confined to one lobe alone, more often the lateral than the middle. The process begins, in the larger number of the cases, centrally with respect to the lobe, or, at any rate, in the deeper rather than the peripheral parts of the gland.

"The size of the nodules varies between that of a pinhead to that of a goose egg.

"In appearance the small nodules are usually spherical in form and of a pale, reddish-gray color.

"The fibrous sheath is not involved until the last, and often may escape being invaded, and very often escapes being penetrated by the disease for years."

Propagation to Other Parts.-One of the interesting points connected with cancer of the prostate is the well-known fact that the primary focus of the process in the gland may remain throughout life, small and stationary as to development, yet most extensive and widespread metastases may result from it.

Another is that emphasized by Young ${ }^{61}$ with regard to the point at which the process passes beyond the confines of the gland and penetrates its capsule in its invasion of immediately contiguous parts by direct extension. This, he states, takes place where the sheath is thinnest; that is to say, over the bases of the lobes. Here the fascia of Denonvilliers is lacking. The disease proceeds, when it has broken through the sheath, to invade the inferior surface of the trigone and the lymphatics leading toward the lateral walls of the pelvis, the seminal vesicles, and the ends of the vasa deferentia.

According to Young, the first exit of the process from the gland is 
through the ejaculatory ducts and into the space immediately above the prostate, between the seminal vesicles and the bladder, and beneath the fascia of Denonvilliers.

Glandular Involvement.-In the 40 cases studied by Young there were I 5 in which there was glandular involvement: the deep pelvic glands in 4 ; the inguinal glands in 5 ; in 2, the iliac; in 2, the sacral; the axillary and the epitrochlear in I each.

Local Invasion.-There are different estimates of the frequency with which carcinoma of the prostate extends directly to other contiguous tissues. Hawley quotes Englehardt as having found it in 57 out of 100 cases examined by him. The bladder interior is not often invaded, and ulceration of its surface is rare in connection with cancer of the prostate. The most frequent direction of the local invasion is, as has been said, to the seminal vesicles. In some cases the disease involves the entire tissue from the exterior of the prostate and the bladder to the pelvic wall. Such was the case in the patient from whom the specimen shown in Plate XVIII was taken.

Progress of the Disease.-In so far as the primary local focus of the disease in the prostate is concerned, it is undoubtedly, in many instances, of very slow development, and it is chiefly because of the metastases that the malady is so serious. The remarkable disproportion between the latter manifestations of the condition and the primary focus has already been noted. The extent and dissemination of the secondary deposits is, in the majority of instances, so great as to cause death, on an average, in from six months to a year and a half.

Symptoms and Diagnosis. - In a good many cases the disease runs its course without suspicion of its existence being aroused by any local manifestations. In a large number, indeed in almost all cases in which the prostate was enlarged, the condition has been regarded as simple hypertrophy of the gland. Since Albarran drew attention to the frequency with which cancer takes place in the prostate, much greater care has been taken to make thorough microscopic examinations of such of the glands as have been removed by operation.

When local symptoms do occur, they are often such as arise in connection with ordinary hypertrophy of the gland, and consequently are frequently attributed to that condition alone.

The most characteristic evidences of the cancerous nature of the disease are radiating pain and extreme hardness of the gland, as detected by rectal touch.

- In addition to the two signs just noted, Young and others have drawn attention to the extension of the indurated area in the cases in which the prostate is very hard, and which they describe as taking place 
in an upward direction toward the seminal vesicles and between their ducts.

Young also regards hematuria in conjunction with pain and induration of the gland as peculiarly significant of the existence of cancer of the prostate, and we are in accord with this view.

The crstoscope is of comparatively little value in the diagnosis of cancer of the prostate, since but few of the cases show ulceration of the intravesical surfaces of the gland, which would be the only characteristic crstoscopic evidence of the nature of the enlargement. The surface of the cancerous prostate is sometimes perfectly smooth; in others it is nodular and uneven.

It should not be forgotten that in quite a number of instances the prostate is not at all enlarged.

The combination of the following symptoms is so suggestive as to warrant the diagnosis of cancer: pain, radiating to one or both the sciatic regions, to the sacrum, or the groin. The sense of very marked induration of the gland, felt when making rectal examination, especially if the indurated area extends upward upon the posterior surface of the bladder between and toward the seminal vesicles; ulceration of the intravesical surface of the base of the prostate; hematuria; and, in advanced cases, the extension of the indurated area laterally toward the pelvic wall.

Treatment.-The treatment by radical surgical measures will be futile, except in the cases in which an early diagnosis is made. When this has been done-and it is not very often possible-excision of the entire area invaded by the process, if it can be defined with reasonable certainty, is, we think, the proper course to pursue. This is best carried out by the method suggested and practised by Young. (For the description of the technique of this operation see chapter on the Technique of Operations upon the Prostate.)

The results of operative treatment thus far recorded are set forth in the cases which the writer has tabulated below, and all of which are included in Young's report, the latter being likewise set forth in the tables: 


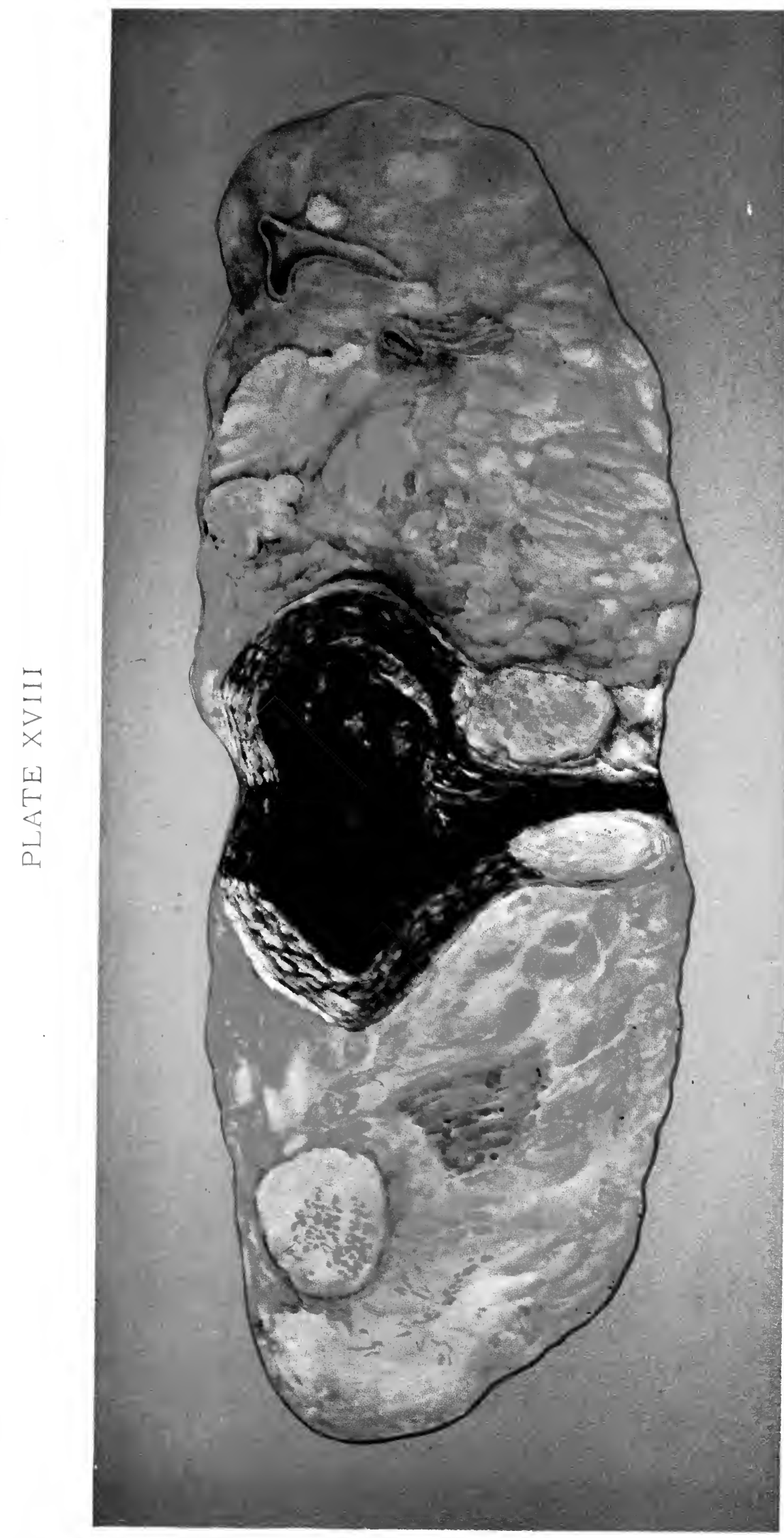


, 
Partial Operations for Malignant Disease of the Prostate.

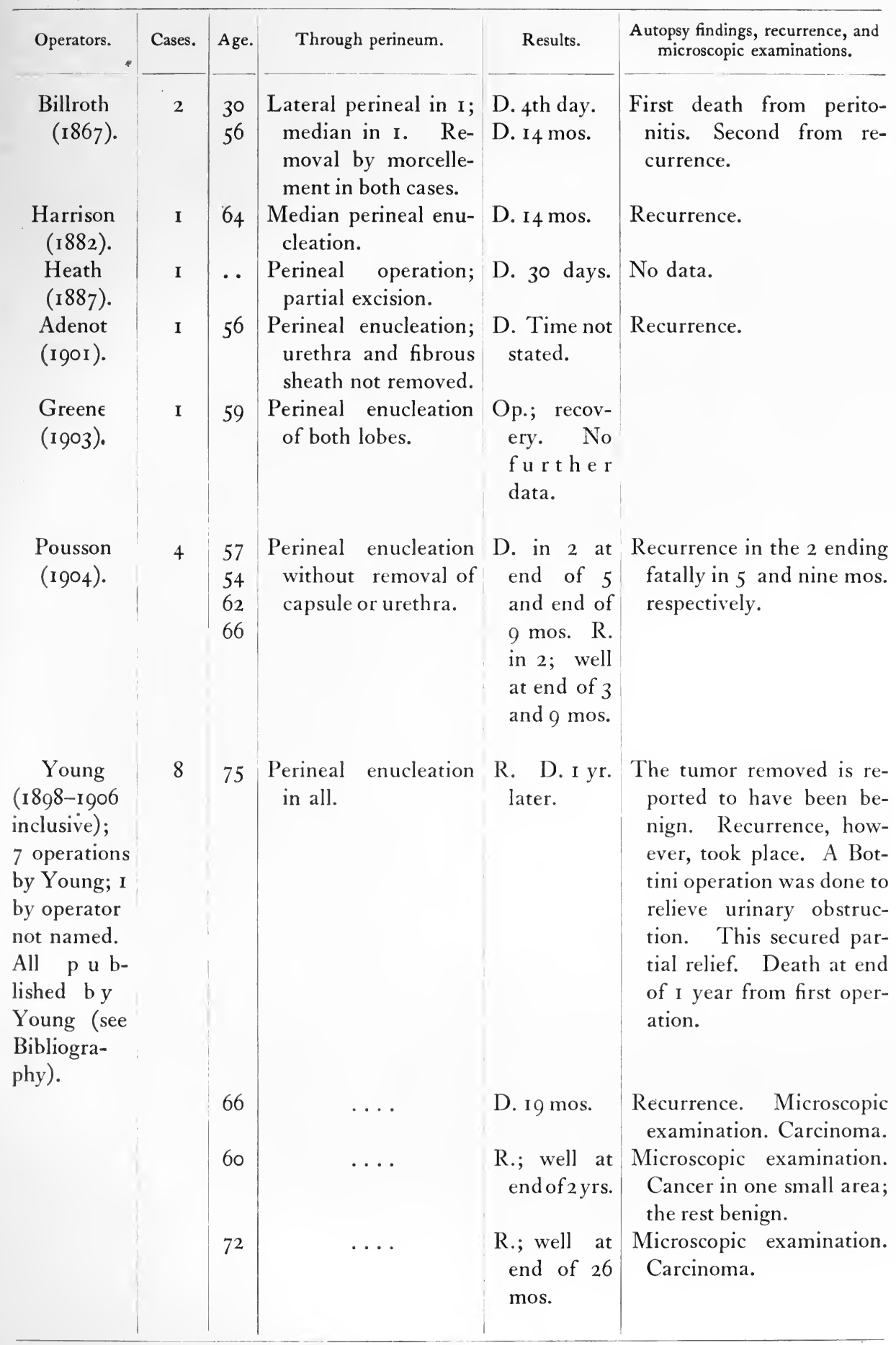


Partial Operations for Malignant Disease of the Prostate (Continued).

\begin{tabular}{|c|c|c|c|c|c|}
\hline Operators. & Cases. & Age. & Through perineum. & Results. & $\begin{array}{l}\text { Autopsy findings, recurrence, and } \\
\text { microscopic examinations. }\end{array}$ \\
\hline & & 78 & $\cdots$ & $\begin{array}{l}\text { R.; well at } \\
\text { end of ro } \\
\text { mos. }\end{array}$ & $\begin{array}{l}\text { Microscopic examination. } \\
\text { Adenocarcinoma. }\end{array}$ \\
\hline & & 64 & $\cdots$ & $\begin{array}{l}\text { R.; well at } \\
\text { end of } 6 \\
\text { mos. }\end{array}$ & $\begin{array}{l}\text { Microscopic examination. } \\
\text { Carcinoma. }\end{array}$ \\
\hline & & 76 & $\cdots$ & $\begin{array}{l}\text { D. Oper- } \\
\text { ative. }\end{array}$ & $\begin{array}{l}\text { Microscopic examination. } \\
\text { Carcinoma. }\end{array}$ \\
\hline & & 60 & .. & $\begin{array}{l}\text { R.; well at } \\
\text { end of con- } \\
\text { valescence. }\end{array}$ & $\begin{array}{l}\text { Microscopic examination. } \\
\text { Carcinoma. }\end{array}$ \\
\hline
\end{tabular}

Suprapubic Enucleation.

\begin{tabular}{|c|c|c|c|c|c|}
\hline $\begin{array}{l}\text { Belfield } \\
\text { ( } 1888)\end{array}$ & I & 48 & $\begin{array}{l}\text { Suprapubic excision, } \\
\text { curette and cautery }\end{array}$ & D., 5 mos. & Recurrence in 12 mos. \\
\hline $\begin{array}{l}\text { Czerny } \\
(\text { I } 889) \text {. }\end{array}$ & I & 42 & Curette and cautery. & No data. & \\
\hline $\begin{array}{l}\text { Parona } \\
\text { (I89r). }\end{array}$ & I & & $\begin{array}{l}\text { Excision of middle } \\
\text { lobe. }\end{array}$ & $\begin{array}{l}\text { D.; time not } \\
\text { stated. }\end{array}$ & Recurrence. \\
\hline $\begin{array}{l}\text { Von Frisch } \\
\text { (1898). }\end{array}$ & 2 & & $\begin{array}{l}\text { Excision of middle } \\
\text { lobe. }\end{array}$ & $\begin{array}{l}\text { R.; well at } \\
\text { end of I yr. }\end{array}$ & \\
\hline $\begin{array}{l}\text { Harrison } \\
\text { (1903). }\end{array}$ & 2 & $\begin{array}{l}64 \\
61\end{array}$ & $\begin{array}{c}\text { By morcellement. } \\
\ldots .\end{array}$ & $\begin{array}{l}\text { D., } 4 \text { mos. } \\
\text { D., I } 6 \text { mos. }\end{array}$ & Recurrence in both. \\
\hline $\begin{array}{l}\text { Jacobson } \\
\text { (I } 90 \mathrm{I}) \text {. }\end{array}$ & I & $6 I$ & $\begin{array}{l}\text { Enucleation of both } \\
\text { lobes. }\end{array}$ & D., 6 mos. & Recurrence. \\
\hline $\begin{array}{l}\text { Young } \\
\text { (I9or). }\end{array}$ & I & 67 & $\begin{array}{l}\text { Enucleation of both } \\
\text { lobes and prostatic } \\
\text { urethra. }\end{array}$ & $\begin{array}{l}\text { R.; d. } 4 \text { yrs. } \\
\text { later from }\end{array}$ & Retroperitoneal metastasis. \\
\hline $\begin{array}{l}\text { Cushing } \\
\text { (1 } 898) \text {. }\end{array}$ & I & & $\begin{array}{l}\text { Excision of a part of } \\
\text { the prostate. }\end{array}$ & Op.; death. & \\
\hline
\end{tabular}

Total Extirpation.

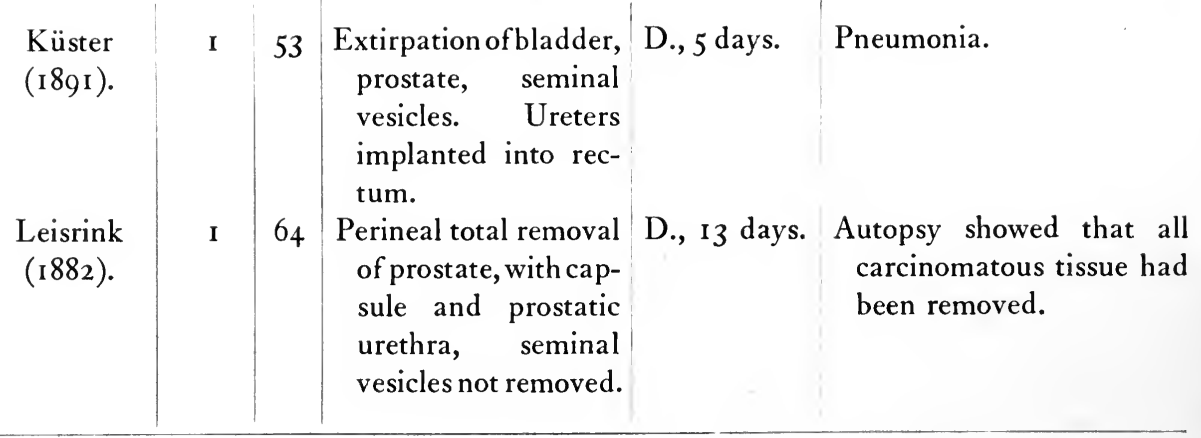


Total Extirpation (Continued).

\begin{tabular}{|c|c|c|c|c|c|}
\hline Operators. & Cases. & Age. & Through perineum. & Results. & $\begin{array}{l}\text { Autopsy findings, recurrence, and } \\
\text { microscopic examinations. }\end{array}$ \\
\hline $\begin{array}{l}\text { Czerny } \\
\text { (I889). }\end{array}$ & I & 47 & $\begin{array}{l}\text { Suprapubic and peri- } \\
\text { neal operation com- } \\
\text { bined. Prostate and } \\
\text { fibrous sheath re- } \\
\text { moved. }\end{array}$ & D., 9 mos. & \\
\hline $\begin{array}{l}\text { Fuller } \\
\text { (I } 898) \text {. }\end{array}$ & I & & $\begin{array}{l}\text { Removal of prostate, } \\
\text { sheath, deep urethra, } \\
\text { and bladder as far } \\
\text { as ureteral orifices; } \\
\text { seminal vesicles not } \\
\text { removed. }\end{array}$ & D., I I mos. & Recurrence. \\
\hline $\begin{array}{l}\text { Harris } \\
\text { (1902). }\end{array}$ & I & 53 & $\begin{array}{c}\text { Total extirpation of } \\
\text { bladder, prostate, } \\
\text { and seminal vesicles. }\end{array}$ & D., 2 mos. & $\begin{array}{l}\text { Pneumonia, metastasis to } \\
\text { glands about kidney. }\end{array}$ \\
\hline $\begin{array}{l}\text { Young } \\
(1904) \text {. }\end{array}$ & 6 & 70 & $\begin{array}{l}\text { All done by Young's } \\
\text { perineal technique, } \\
\text { by which the sheath } \\
\text { with prostate, the } \\
\text { vesical neck, and the } \\
\text { seminal vesicles are } \\
\text { removed in one } \\
\text { mass, and the di- } \\
\text { vided bladder is } \\
\text { stitched to the di- } \\
\text { vided urethra. }\end{array}$ & R.; d. 9 mos. & $\begin{array}{l}\text { Death followed litholapaxy } \\
9 \text { mos. after the first } \\
\text { operation. Small area of } \\
\text { cancerous tissue found in } \\
\text { pelvis. }\end{array}$ \\
\hline & . & 64 & $\ldots$ & $\begin{array}{l}\text { D., } 7 \text { weeks; } \\
\text { uremia. }\end{array}$ & \\
\hline (1904). & . & 65 & $\cdots$ & $\begin{array}{l}\text { R.; well at } \\
\text { end of I I } \\
\text { mos. }\end{array}$ & \\
\hline (1905). & . & 64 & $\ldots$. & $\begin{array}{l}\text { R.; well at } \\
\text { end of } \mathrm{I} \text { yr. }\end{array}$ & \\
\hline $\begin{array}{l}(1905) . \\
(1906) .\end{array}$ & $\begin{array}{l}\cdots \\
\cdots\end{array}$ & $\begin{array}{l}75 \\
67\end{array}$ & $\begin{array}{l}\cdots \\
\cdots\end{array}$ & $\begin{array}{l}\text { D., } 2 \text { hours. } \\
\text { R.; reported } \\
\text { at end of } 4 \\
\text { mo. as well. }\end{array}$ & \\
\hline
\end{tabular}

A point of considerable interest and importance is brought out in the course of the analysis of these cases, which is the relatively good results attending the performance of total enucleation of the prostate within its fibrous sheath, as compared with the operations which Young has done, in which the whole cancerous area, including the neck of the bladder and seminal vesicles, was excised.

In comparing, for instance, Young's 6 cases of total extirpation, in which he removed the prostate, together with its fibrous sheath, prostatic 
urethra, neck of bladder, seminal vesicles, and part of the vasa deferentia in one mass, there is no instance in which a patient had lived, at the time of his report, longer than one year following the operation. The time reported at which the patients were noted as being free from recurrence were, in this particular series of 6 cases, eleven months, four months, and one year. In I case, in which the patient died after a litholapaxy nine months after the prostatic operation, a small area of cancerous tissue was found in the pelvis at the autopsy. Two patients died shortly after operation. In Fuller's and Czerny's cases, the patients, although each of them had recurrence, lived for eleven and nine months, respectively. In the last two cases the seminal vesicles were not removed.

It appears, therefore, that we have thus far no warrant for asserting that the results of such extirpation as that proposed by Young have as yet been as successful as in the cases in which some of the patients have had long periods of immunity from recurrence following the ordinary intracapsular enucleation, as it is done in cases of hypertrophy of the gland. Thus, in one of Young's enucleations, in which he removed the gland suprapubically, the patient lived four years. In three other instances in which he removed the gland from the perineum by enucleation, the patients are reported as being well at the end of twenty-six months, twenty-four months, and nineteen months, respectively.

There was but one instance in which a patient has lived more than four years after any operation, and in this case the procedure was only the usual enucleation as done for the removal of the hypertrophied gland.

We are not in a position to say whether or not it may be shown later that the results of the complete operation, including all the parts removed, as in Young's six cases, will justify further efforts in this direction. It is true, however, in view of the inevitably fatal and, in many instances, suffering termination of the lives of the patients with this malady, that any procedure which tends to ameliorate their sufferings, even though it offers but a very small chance of permanent cure, should be employed, for we have no such prospect by the application of palliative measures.

\section{ACUTE PROSTATITIS, NON-TUBERCULOUS SUPPURATION OF THE PROSTATE.}

Etiology.-In the majority of cases the cause of acute prostatic inflammation and suppuration (abscess) is the backward extension of gonorrhœa from the anterior to the prostatic portion of the urethra, and thence to the substance of the gland via the openings of the gland ducts leading into the interior of the lobes of one or both sides. In other instances, the mechanical irritation of frequent catheterization or actual injury of the 
deep urethra by the unskilled use of instruments and infection following in its train are the causes. In a few cases inflammation and suppuration follow, or occur in the course of typhoid or other of the infective systemic fevers, being then brought about by the infection of the gland with the specific organism of the general malady, or by other microbian invasion from the urethra, which is invited by a lessened resistance of the prostatic tissues, which has been produced in the course of the illness.

Symptoms and Diagnosis.-The symptoms are (I) constitutional, and (2) local.

Constitutional Symptoms.-The constitutional symptoms are: fever, which may be high, sometimes $104^{\circ}$ to $105^{\circ} \mathrm{F}$; chills, which are apt to mark the beginning of pus formation in the acutely inflamed gland; marked prostration; and the other accompaniments of fever and sepsis, rapid pulse, thirst, and a coated tongue with red edges. Constipation is apt to be marked, and the chills may be recurrent.

Local Symptoms.-The urinary stream becomes small, as the swelling of the prostate increases, and retention occurs in many instances.

Rectal pain is always present. It may be of a dull, aching character, but, as a rule, it is very severe. Rectal examination with the finger causes it to become excruciating. There is a sense of throbbing pain and a feeling of fulness in the rectum, and rectal tenesmus is often present.

The physical signs are: enlargement, heat, and tenderness of the prostate on rectal examination with the finger, and, in the cases in which the abscess has already formed, an area of fluctuation may be detected.

Treatment.-Palliative.-Hot hip baths, hot poultices to the perineum, hot and prolonged irrigation of the rectum, with saline solutions administered through a small soft-rubber catheter, are valuable in bringing about resolution prior to the formation of pus, in hastening the suppurative process and advancing it to the point at which it can be felt by the finger in the rectum, and evacuated. When retention of urine occurs, a catheter should be tied into the bladder rather than to pass the instrument frequently, unless the condition is overcome by the first or second use of it.

Operative.-The only treatment that should be considered when pus has formed and can be made out by the examining finger is evacuating it by incision. We are decidedly of the opinion that it is wiser to make such an incision into the gland in cases in which, even though its actual presence cannot be demonstrated, the severity of the constitutional symptoms and very marked enlargement and tenderness of the gland indicate that pus formation has taken place.

In two instances the writer has failed to find pus in the gland upon making such an incision, but the operation gave complete relief in both, 
and within a few hours afterward pus was discharged through the incision. Puncture of the abscess through the rectum, which was formerly practised, should never be done.

In the writer's belief, much the best way to approach abscess of the prostate is through one or another of the perineal incisions which leaves the urethra intact. (See chapter on the Technique of Operations on the Prostate.) Immediate relief follows the evacuation of the abscess, and the constitutional disturbance quickly subsides.

If both lobes of the gland are involved, the abscesses, which at first will be distinct, are best approached by separate incisions; one on either side of the perineum, rather than through a single one, but at a later stage, when the pus has broken through intervening tissues and the two abscesses have become confluent, a single incision can be used to evacuate the pus cavity.

Some surgeons prefer to employ the curved prerectal perineal incision for the purpose of reaching and opening prostatic abscesses, but it has always seemed to the writer to be a needlessly extensive operation to accomplish what can be equally well compassed by a less radical procedure.

\section{Illustrative Cases.}

Case I.-Acute Prostatic Abscess Occurring in the Third Week of Gonorrhoe a and of Typhoid.-A man, aged thirty years, in the third week of gonorrhœal infection, and also of typhoid fever, suddenly developed severe pain in the region of the prostate; the temperature, which had been $102^{\circ}$ to $103^{\circ}$, rose to $104.5^{\circ}$, following a chill.

Rectal examination showed the left lobe of the prostate to be very sensitive, greatly enlarged, hot and pulsating. No fluctuation could be made out. Twenty-four hours later the right lobe of the gland became enlarged. Fluctuation was present in a small area in the left one.

Operation.-The patient, being in the lithotomy position, a sound was passed into the bladder. The classic lateral lithotomy incision was made in the perineum and carried through the deeper structures until the point of the knife came near the urethra. The forefinger of the left hand was then placed in the rectum, the tip of it resting upon the area of fluctuation in the left lobe of the prostate. The edge of the blade of the knife was then inclined laterally outward in order to avoid cutting either rectum or urethra, and its point was pushed directly onward into the left lobe of the gland until it reached the abscess cavity and opened it. About half an ounce of pus was evacuated. The abscess cavity was cleansed, the outer part of the wound was slightly enlarged, and a drainage tube was inserted. 
The temperature again fell to $102^{\circ}$, as it had been in the course of the typhoid fever before the prostatic inflammation and abscess occurred, but two days after the operation it again rose to $105^{\circ}$, and the patient was much prostrated; there was a return of the prostatic and rectal pain, and rectal examination showed that another abscess had formed in the lobe of the other side. This was treated in exactly the same manner as the first one had been, and was followed by an equally marked relief of both local and general symptoms.

The patient made thereafter an uninterrupted though rather slow recovery from his typhoid fever, and was discharged from the hospital at the end of eight weeks, well.

The perineal incisions healed in the course of about eight or ten days after each had been made, and there was no further sign of trouble from the prostate during his stay in the hospital. The gonorrhœal inflammation was nearly well also.

The bacteriological examination of the pus from the first abscess failed to show the presence of gonococci. The positive findings were of streptococci and Staphylococcus pyogenes aureus.

CASE II.-Illustrating the error of allowing the abscess to rupture spontaneously. A man, aged twenty-five years, in the fifth week of a gonorrhœa, was attacked by severe pain in the rectum. Examination showed the prostate to be greatly enlarged, exquisitely tender, and very hot. The patient absolutely refused the operation which was advised.

Five days later there was a sense of something having "given way," and a discharge of pus and blood took place from the rectum and from the urethra. The abscess had ruptured spontaneously in both directions. Later, a fistula was established between the deep urethra and the rectum as the result of expectant treatment.

\section{PROSTATIC CALCULUS.}

Prostatic calculi are either primary in the gland or are passed into the prostatic urethra from the bladder, become impacted there and then push their way into the prostate (see Fig. I 80). The former are the ones to which the term prostatic calculi may be properly applied. They are formed in the follicles of the gland out of the prostatic secretion which has become inspissated, and are most frequently seen in persons of between forty and sixty years of age. They are multiple and usually of small size, being really concretions, except in the cases in which, as sometimes happens, a number become packed together into a single mass. 
The calculi may attain so large a size as to gradually destroy the substance of one of the lobes and fill the entire space formerly occupied by it. In the case of the larger stones, they may penetrate the outer sheath of the gland, and occasionally produce fistula. In such instances the calculus lies in a diverticulum and may give rise to abscess and periurethral burrowing of pus. It is impossible to determine absolutely the manner of origin of these pouches. Some of them are doubtless made

FIG. I80

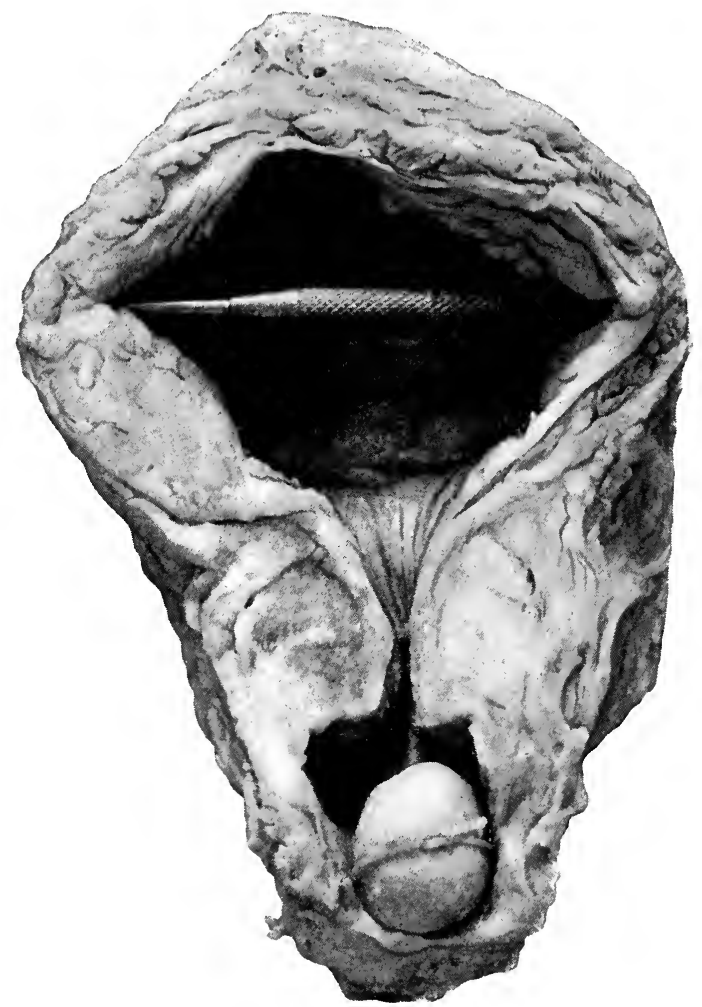

Calculus passed into the prostatic urethra from the bladder, and becoming impacted there formed a chamber for itself in which it remained for a considerable time, the urine being passed beside it. Not true prostatic calculus.

by the pressure of a calculus impacted in the posterior urethra, as in the case which is illustrated in Fig. I80, and others in all probability result from calculus formation and the increase in size of the stone, beginning originally in the cavity of a prostatic abscess or in a false passage instrumentally made.

The pouches in which the prostatic calculi are lodged vary in shape. In some instances the openings into them from the urethra are narrow, and in that case the calculus might readily escape detection by a sound 
passed through the'urethra. In others, the orifices are wide, and the calculi project more or less into the urethra, forming an obstacle to the passage of urine or instruments. Occasionally a calculus will have the form of a shirt-stud, one-half lying in the urethra and connected by a narrow isthmus with the other part, which is within the pouch, the mouth of which is small. Cases have been reported in which a similar but more extensive prolongation of prostatic calculus into the urethra and backward through the vesical orifice into the bladder has been found.

Symptoms.-The symptoms are: varying degrees of pain, dysuria, and frequency of urination; in some cases more or less hematuria and pyuria. Abscess may form in the prostate and burrow in the perineum, or upward, and discharge into the bladder, or, if the prostatic capsule is ruptured posteriorly, between the rectum and the bladder. This will rarely, however, be the case, for the reason that there is usually a sufficient outlet through the mouth of the pouch into the urethra to permit the escape of pus through it freely enough to avoid its taking any other direction. The diagnosis is made by a stone searcher in the urethra and by rectal examination.

Treatment.-The treatment consists in removing the stone, which is, in the great majority of cases, readily done through an external perineal urethrotomy incision. With large stones, and in those cases in which the stone extends to the bladder, the lateral lithotomy incision or suprapubic cystotomy may have to be employed. The opening into the urethra will close after the latter operation.

\section{Illustrative Cases.}

CASE I.-Calculus embedded in the left lobe of the prostate. The stone is shown in Fig. I $8 \mathrm{I}$.

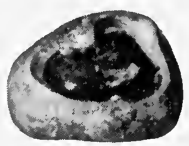

Six months prior to his first coming under observation, the patient, a man aged thirty years, had an attack of renal colic on the left side. A fortnight later he experienced some difficulty in passing his water. The dysuria gradually subsided, but he soon began to have painful and frequent urination, and blood was seen in the urine occasionally.

Rectal examination showed that the prostate was not enlarged, but a hard nodule could be felt in it, near the base of the left lobe. On VOL. I- 24 
passing a steel sound into the bladder a calculus could be felt with it, close to the vesical outlet.

Operation.--External perineal urethrotomy. Digital examination of the prostatic urethra detected a calculus, part of which protruded into the urethra, while the rest of it lay in a pocket in the left lobe of the prostate. The orifice of the pocket was enlarged by the tip of the finger, a pair of forceps was passed into it through the urethrotomy incision, the calculus was caught with the blades, and extracted without difficulty.

The cavity in which the calculus had rested was, in this case, evidently formed primarily by the pressure of the stone upon the side of the urethra, in which it had become impacted.

The calculus was composed of a central part of oxalates and an outer covering of phosphates.

The patient made an uneventful and complete recovery.

\section{BiBLIOGRAPHY.}

I. Richardson. The Development and Anatomy of the Prostate Gland, J. \& A. Churchill, London, I904.

2. Ciechanowski. Ann. des Mal. des Org. Gén.-urin., I9o I, xix, p. 536.

3. Keyes. Genito-urinary Diseases, I905.

4. Moullin. Enlargement of the Prostate, I894.

5. Cunningham. Annals of Surgery, April, 1905.

6. Harrison. Surgical Disorders of the Urinary Organs, 1887,3 d edition.

7. Sir Henry Thompson. British Medical Journal, November I 7, i887.

8. Harrison. Lettsomian Lectures, I887.

9. Watson. New York Medical Journal, June i6, i 894.

Io. Cabot. Boston Medical and Surgical Journal, I903, ii, 559.

I I. Watson. Operative Treatment of the Hypertrophied Prostate, Annals of Surgery, June, I904, p. 853 .

I 2. Watson. The Operative Treatment of the Hypertrophied Prostate, 1888.

13. Deaver. Enlargement of the Prostate Gland, Philadelphia, I905.

I4. Mercier. Recherches sur les Traitement des Maladies des Organes Urinaires, Paris, I 856 , pp. 36 and 213 .

I5. Harrison. British Medical Journal, December 24, I88I.

I6. Leroy d'Etiolles. Malgaigne's Surgery, i 846 , p. 504.

I7. Guthrie. Anatomy and Diseases of the Neck of the Bladder, London, I834, p. 252.

I8. Amussat. Leçons sur les Rétentions d'Urine et sur les Maladies de la Prostate, Paris, I832, p. 218.

I9. Sir W. Fergusson. London Lancet, January I, I 870.

20. Bottini. Arch. f. klin. Chir., I887, xxxi, I.

21. Freudenberg. British Medical Journal, i889.

22. Gouley. Diseases of the Urinary Organs, 1873 .

23. Billroth. Clinical Surgery. Translation of New Sydenham Soc., London, I881, p. 288.

24. Leisrink. Arch. f. klin. Chir., I882, Berlin, xxviii, 578 .

25. McGill. Treatment of Retention of Urine from Prostatic Enlargement, Leeds, I889; also Trans. Clin. Soc., London, I888, xxxi, 52. 
26. Mansell Moullin. Enlargement of the Prostate, I894; also London Lancet, December 5, 1903; also Operat.ve Treatment of the Enlarged Prostate, 1892.

27. Dittel. Wien. klin. Woch., 1890 . No. 18.

28. Pyle. New York Medical Record, I892, vol. xlii.

29. Nicoll. London Lancet, April I4, 1894.

30. Alexander. New York Medical Journal, i896, p. I71.

3I. Fuller. Diseases of the Genito-urinary System, 1900, and Journal of Cutaneous and Genito-urinary Diseases, I895, ii, 239.

32. White. Annals of Surgery, I 893 ; ibid., I 895.

33. Cabot. Annals of Surgery, September, 1896.

34. White and Martin. Genito-urinary and Venereal Diseases, I 897.

35. Proust. Comptes rendus de l'Assoc. Française d'Urologie, I 90 I.

36. Albarran. Ibid.

37. Young. Journal of American Medical Association, I903, ii, 999.

38. Freyer. London Lancet, January I9, I90 ; British Medical Journal, February I, 1902; Practitioner, London, June, I903.

39. Fuller. Annals of Surgery, April, I905.

40. Nicoll. British Medical Journal, August I I, 1906.

4I. Syms. New York Medical Record, I90 I, ii 35; also Annals of Surgery, I 902, i, 468; Journal of American Medical Association, 1904, ii, I378; Annals of Surgery, April, I905.

42. Ferguson. Transactions of the Southern Surgical and Gynecological Association, I90I, xiv, I47; Journal of American Medical Association, October I3, I906.

43. Murphy. Journal of American Medical Association, March 29, I902; ibid., I904, i, 1408 , and ii, I4.

44. Escat. Comptes rendus de l'Assoc. Française d'Urologie, 1904, p. 9.

45. Proust. Ibid.

46. Deaver. Op. cit.

47. W. G. Richardson. The Prostate Gland. J. \& A. Churchill, London, I904.

48. Watson. Annals of Surgery, June, I904, 843 .

49. Chetwood. Keyes, Genito-urinary Diseases, I905, p. 299.

50. Watson. Op. cit.

51. Escat. Comptes rendus de l'Assoc. Française d'Urologie, I904, p. 9.

52. Proust. Ibid., p. I 50.

53. Tenney and Chase. Journal of American Medical Association, May I 2, I 906.

54. Proust. Quoted by Tuffier. Traitement de l'Hypertrophie de la Prostate.

55. Proust. Comptes rendus de l'Assoc. Française d'Urologie, 1904, p. I 84

56. Escat. Comptes rendus de l'Assoc. Française d'Urologie, I904.

57. Ruggles. Annals of Surgery, April, I 905.

58. Tansard and Flieg. Annales des Mal. des Org. Gén.-urin., December I 5, 1906.

59. Albarran. Ann. des Mal. des Org. Gén.-Urin., I900.

6o. Hawley. Annals of Surgery, June, 1904.

6I. Young. Johns Hopkins Hospital Bulletin, October, I905.

62. Greene and Brooks. New York Medical Journal, October, I903; Journal of American Medical Association, April, 1902.

63. Spanton. Lancet, I882, vol. lxxvii.

64. Pousson. Ann. des Mal. des Org. Gén.-Urin., I904, p. 882. 


\section{CHA P T E R XII.}

\section{TECHNIQUE OF OPERATIONS UPON THE PROSTATE.}

\section{PROSTATECTOMY.}

Total Perineal Prostatectomy.-By Median Urethrotomy Incision and Finger Enucleation.-(Watson, Goodfellow, Pyle, Syms, Cunningham.)

Preparation of Patient.-Rectum cleared by injection, urotropin given for three days previously; bladder irrigated immediately before; subcutaneous sterile saline solution injection at the beginning or immediately before the operation begins; perineum shaved and cleaned; bladder empty.

Position of Patient.-Lateral lithotomy position, with hips slightly raised above the horizontal. Some surgeons prefer an exaggerated lithotomy posture. We neither employ it, nor advise it.

Instruments.-Grooved staff (the groove should be a broad one); I full-bellied and I straight and narrow scalpel; blunt-pointed, curved scissors; gum lance, or ivory nail cleaner, with sharp, curved end; 3 steel sounds, Nos. 30, 32, and 34; I pair of forceps to withdraw the gland when enucleated; artery forceps.

Incision.-The ordinary incision used in the operation for stricture of the urethra in the deeper part of the canal; that is to say, a median external perineal urethrotomy, exposing the bulb of the urethra and the anterior end of the membranous urethra.

Operation.-Pass the grooved staff into the bladder and make it prominent in the middle line of the perineum. If there is special difficulty in passing the sound into the bladder, because of the obstruction offered by the prostate, it should not be carried beyond the posterior end of the membranous urethra. This serves the purpose for which the sound is used just as well as though it were passed into the bladder.

Expose the bulbous and the anterior part of the membranous urethra by a median incision, as shown in Figs. 182 and 183 .

Open the posterior end of the membranous and anterior end of the prostatic urethra by a straight narrow bistoury, using the nail of the index finger, placed in the groove of the staff as a guide. The direction given in most writings is to make this cut into the urethra with the cutting edge of the knife upward and drawing the knife from behind forward. 
It is immaterial whether the cut be made in that or in the opposite direction, but the cutting edge of the knife should be directed upward. The cut should be long enough to admit the forefinger into the urethra easily (see Fig. 184).

FIG. 182

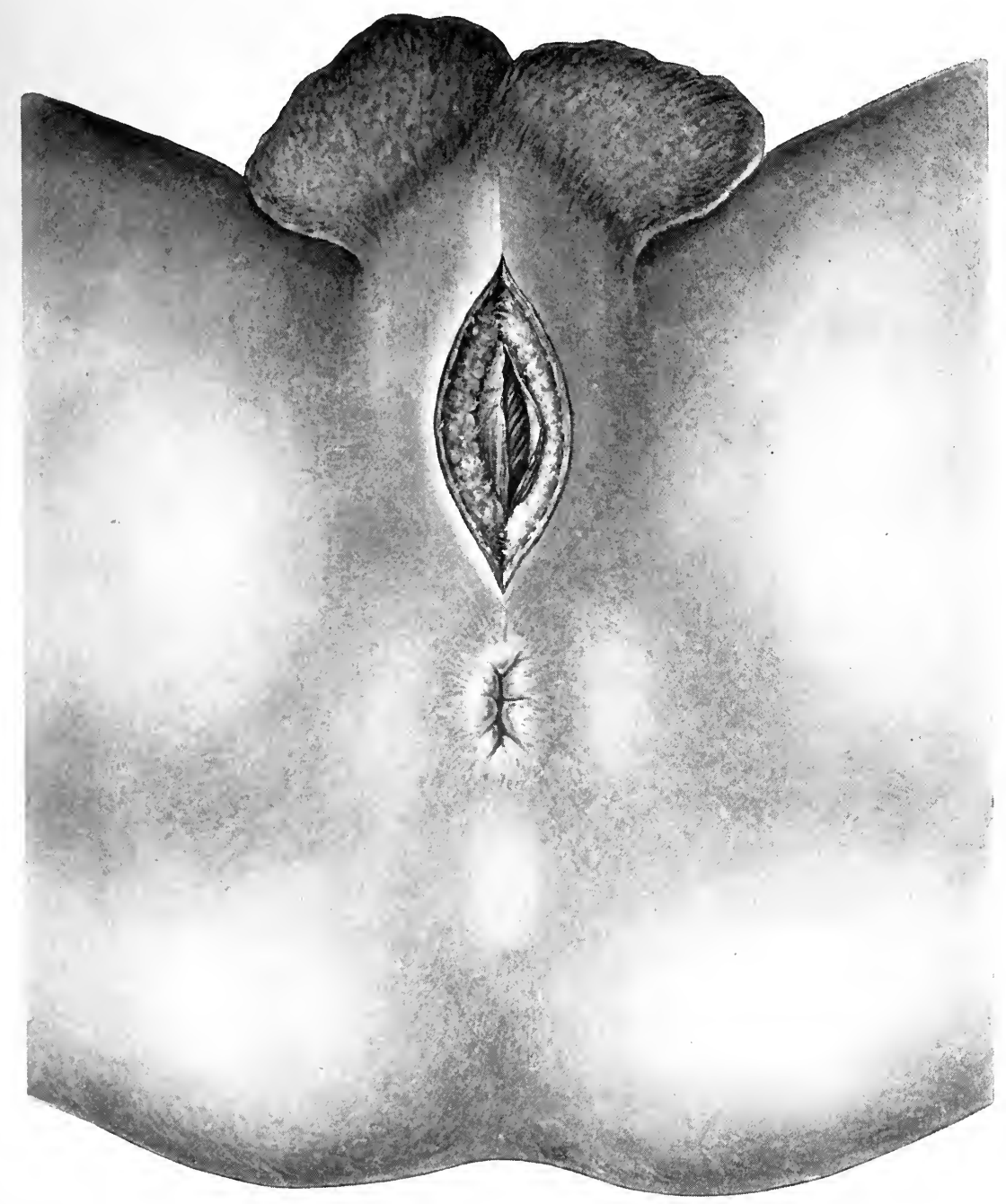

Perineal prostatectomy. Colles' fascia has been excised, exposing the bulb of the urethra. (Deaver.)

Withdraw the staff. Pass into the incision in the urethra and through the prostatic urethra into the bladder three steel sounds, the little finger, and the index finger in succession (see Fig. 185).

Push the prostate toward the surface of the perineum and maintain it firmly in this position by strong downward pressure upon the abdominal 
wall made by the hand of an assistant. This is the manœuver advised by Sir Henry Thompson in I 884 for making digital examinations of the interior of the bladder, and, if rightly done, it brings the gland down quite

FIG. I 83

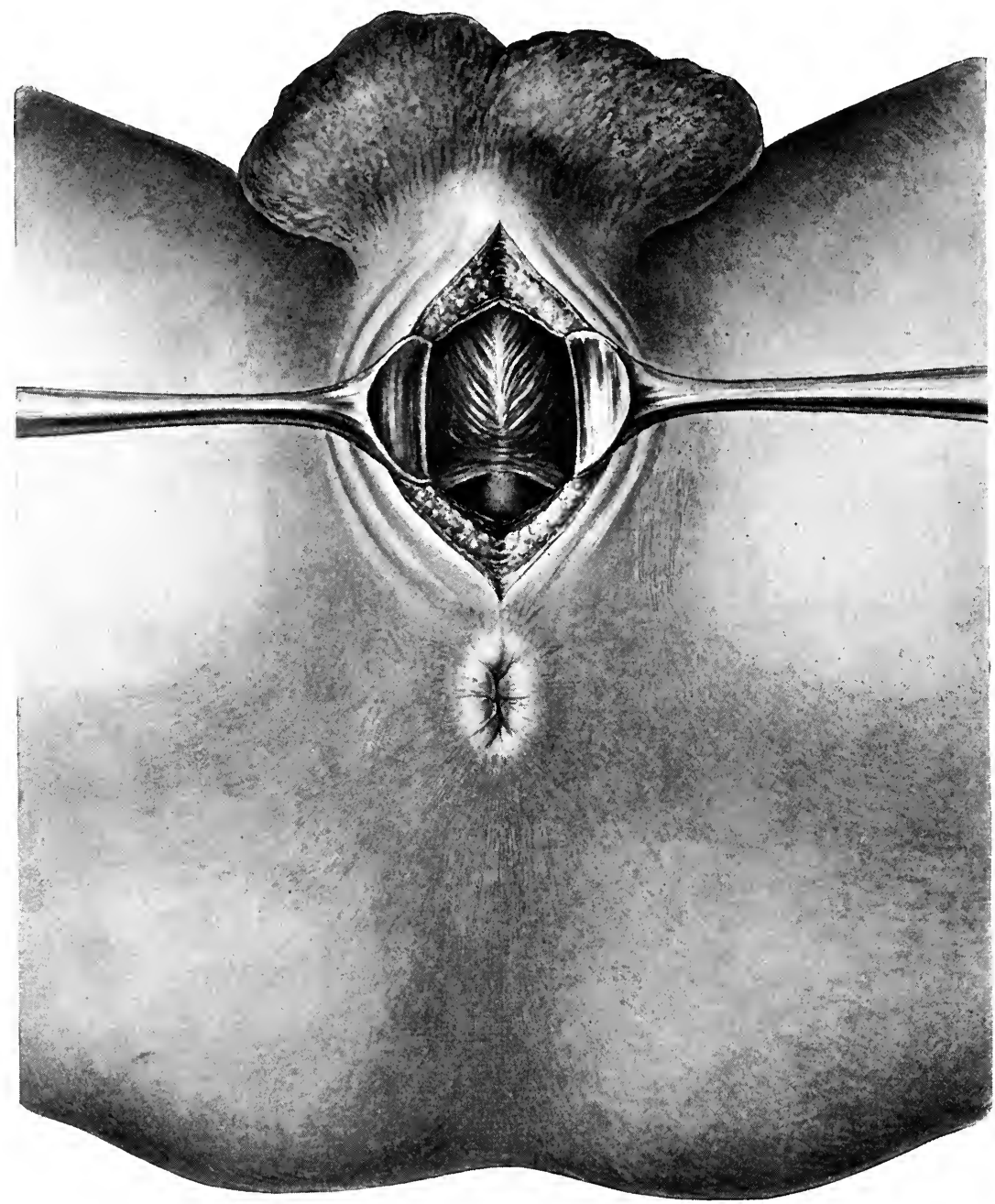

Perineal prostatectomy. By retracting the margins of the wound the membranous urethra, the transverse perineal muscles, and the anterior fibers of the levator ani are exposed, in addition to the bulb of the urethra. (Deaver.)

as far and maintains it in position quite as well, except in the cases of very fat patients, as any of the forms of prostatic tractors, and has the advantage of not taking up any room in the urethra or of requiring an extra hand to hold an instrument, as is the case with the tractors; more- 
FIG. I 84

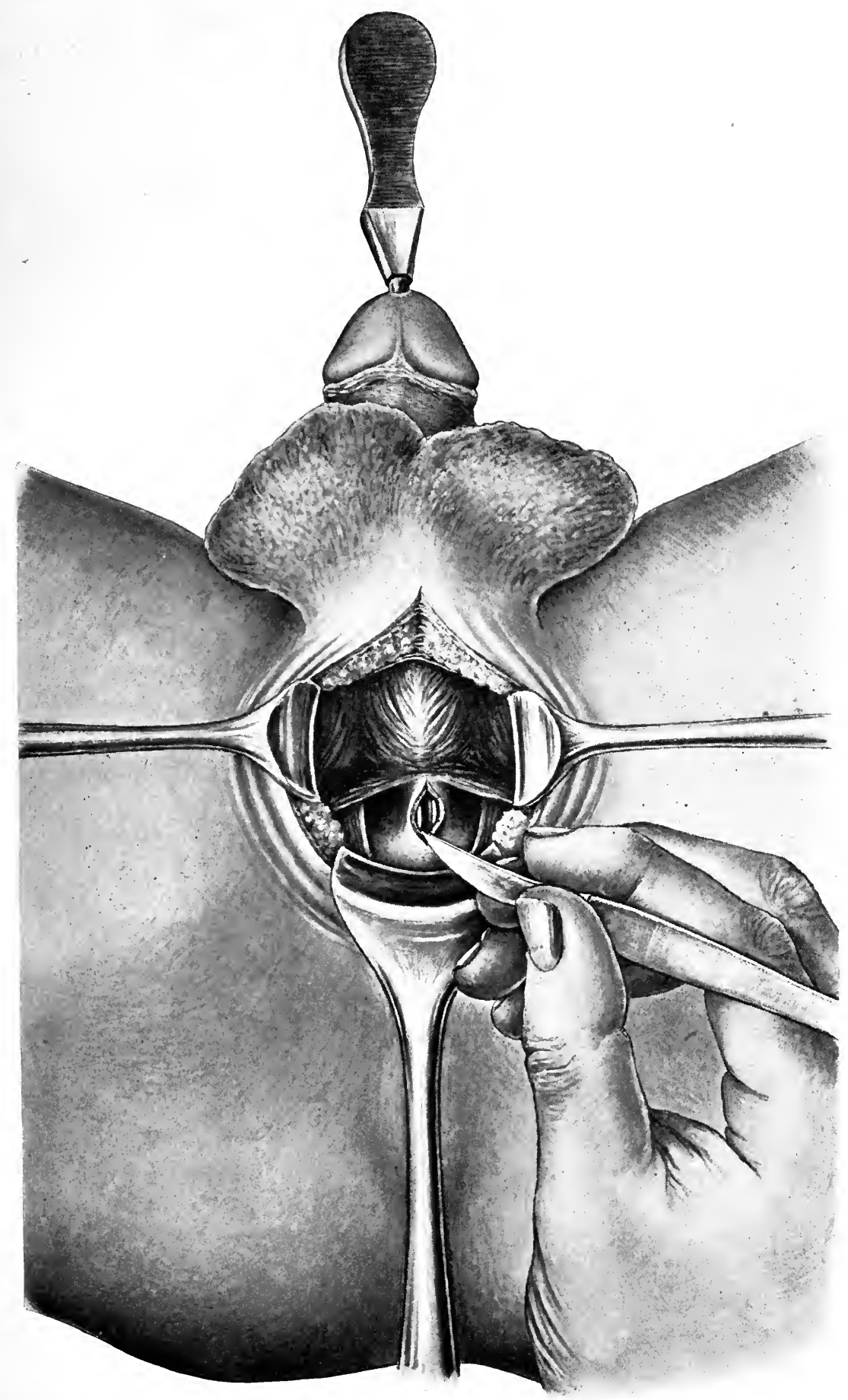

Perineal prostatectomy. The membranous urethra is opened on a grooved staff, being more fully exposed by retracting the rectal tissue downward. (Deaver.) 
over, it avoids the chance of breaking through the floor of the bladder, which is liable to happen when tractors are used (Fig. I86).

The index finger is pushed into the prostatic urethra and turned upward so that its dorsum rests on the floor of the canal (Figs. I85 and I86).

Press the finger tip against the side of the urethra above its floor and make an opening about midway in its length, through the urethra and all tissues intervening between it and the capsula vera of the lobe of that

FIG. 185

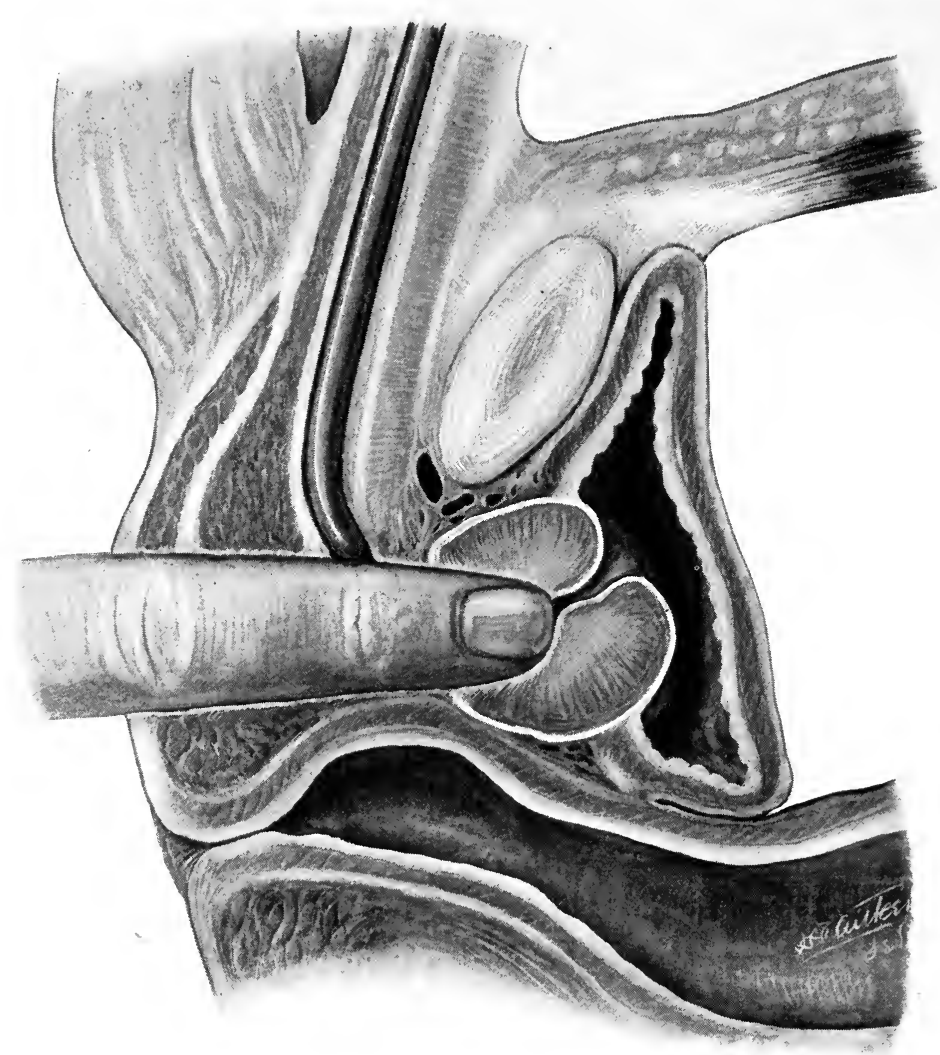

Finger introduced into the prostatic urethra and about to begin the enucleation. (Watson.)

side of the gland. This may be done in one or another of the three following ways: (I) Scratching through with the finger nail. (2) Passing a gum lance or other equally appropriate instrument upon the finger, turning its cutting edge against the side of the prostatic urethra, and making an opening there large enough to admit the tip of the finger. (3) By pushing the finger tip beneath the part of the lateral lobe which 
is most prominent within the urethra and then prying upward with it upon the under surface of the lobe to be enucleated first. This makes a rent through the side of the prostatic urethra above the level of its floor and admits the finger tip to the surface of the gland (Figs. I 86 and I87).

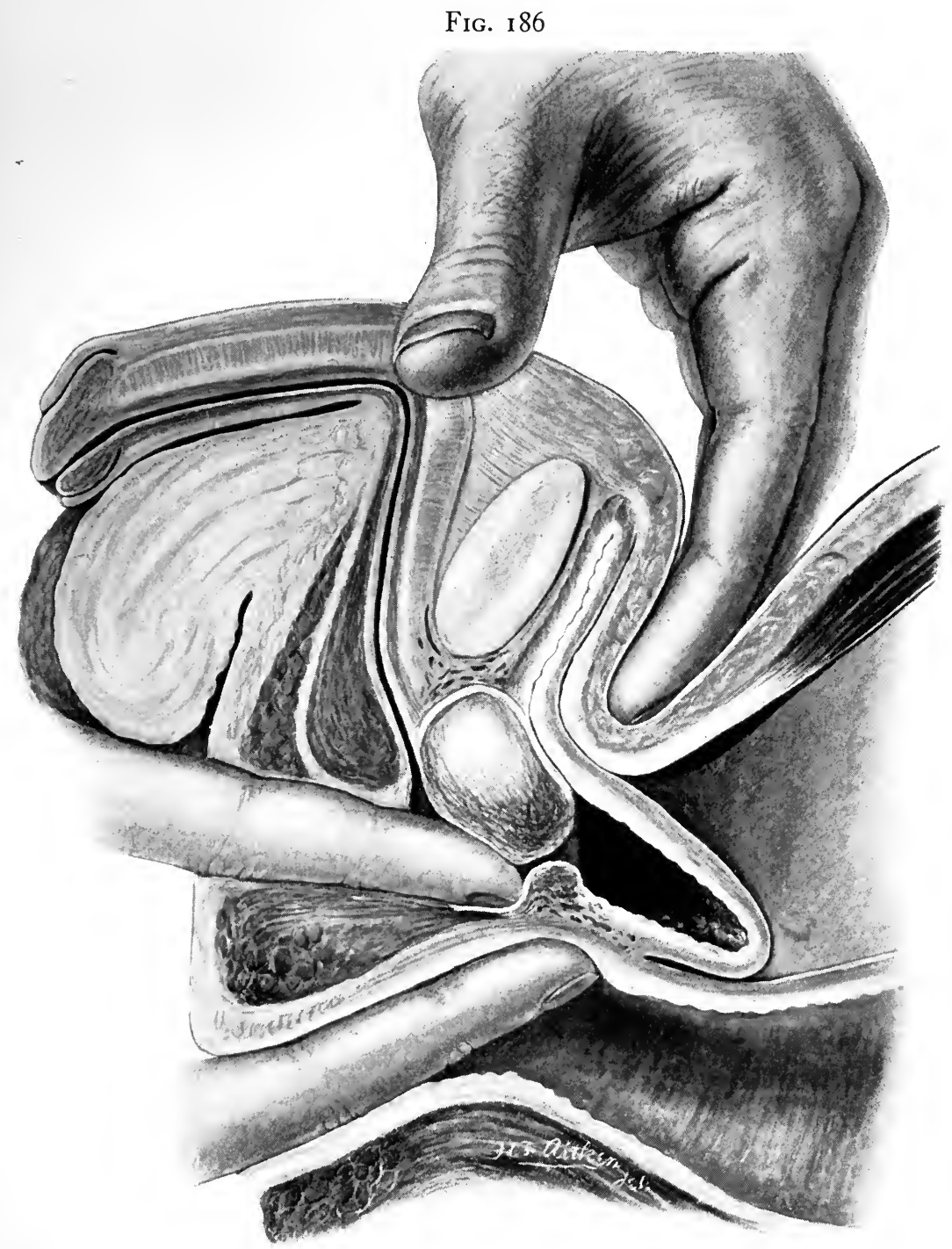

Enucleation through a median external perineal urethrotomy incision. (Watson.)

Proceed to enucleate thus: Pass the finger tip beneath the posterior surface of the lobe, between it and the outer fibrous sheath (Figs. I 86 and 187), and break down the fibers attaching it to the gland. Carry the finger over the outer and superior aspects of the lobe as far as the middle line above-anterior commissure. Go back and free the apex of the lobe 
from its attachment to the triangular ligament. Then separate the base of the lobe from the floor of the bladder and its outlet. If the gland is to be removed one lobe at a time, break through the anterior commissure and separate what remains attached to the prostatic urethra. This leaves the first lateral lobe free. Apply forceps to it and gently withdraw it through the external incision.

If the two lobes are to be removed in one mass, the finger tip passes over the anterior commissure, instead of breaking through it, and repeats

FIG. 187

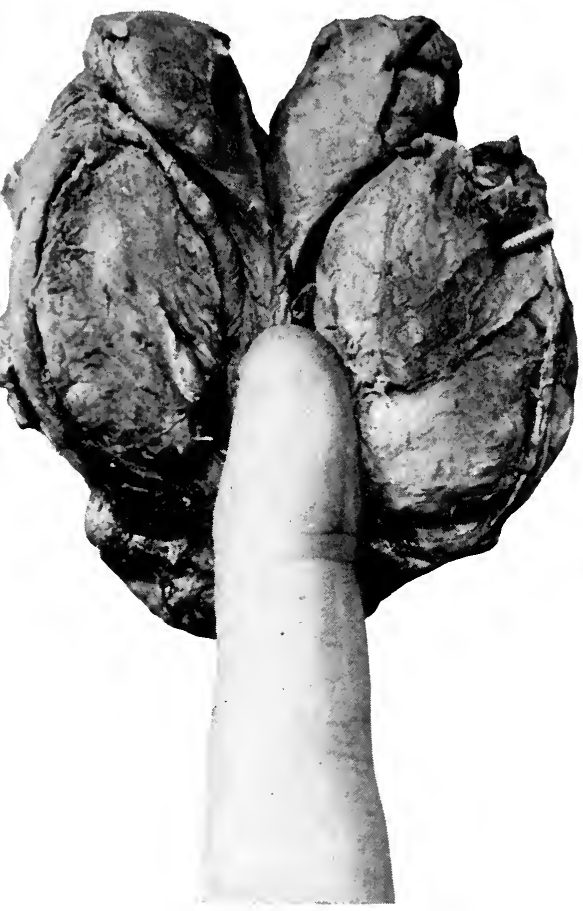

Finger enucleation. The finger tip is just beginning the enucleation of a lateral lobe through the side of the prostatic urethra. (Watson.)
FIG. I 88

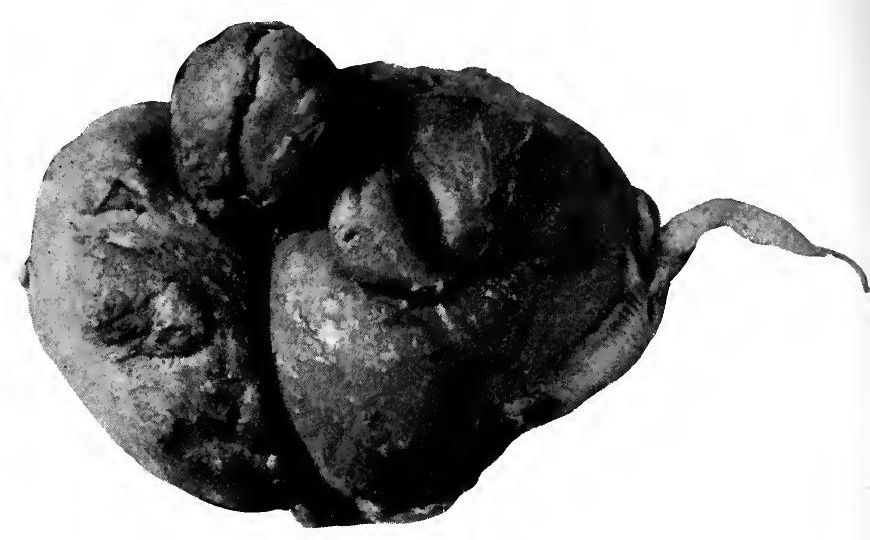

Prostate enucleated in one mass through a median external perineal urethrotomy incision. (Watson.)

the same manœuvers with the second lobe that were employed to free the first one. Care should be taken to avoid, so far as possible, laceration of the membranous urethra in withdrawing large masses through the external wound. Fig. I 88 is from a photograph of a prostate removed in one mass by the writer in this way in 1897 . Fig. 189 is from a photograph of a large prostate removed in two separate masses by the same method; Fig. I90, of another still larger one, which was removed by Cunningham through the perineal route. 
If a middle lobe is present, the mucous membrane covering it should be split, scratched, or cut through, either in the middle line above or along one side of the enlargement, and enucleated with the finger, cut away with scissors, or snared off. It can be brought within reach of the finger tip by downward pressure from above the symphysis upon the external surface of the abdomen, as in the case of the lateral lobes, or, after the latter have been removed, it is readily hooked down with the finger tip.

FIG. 189

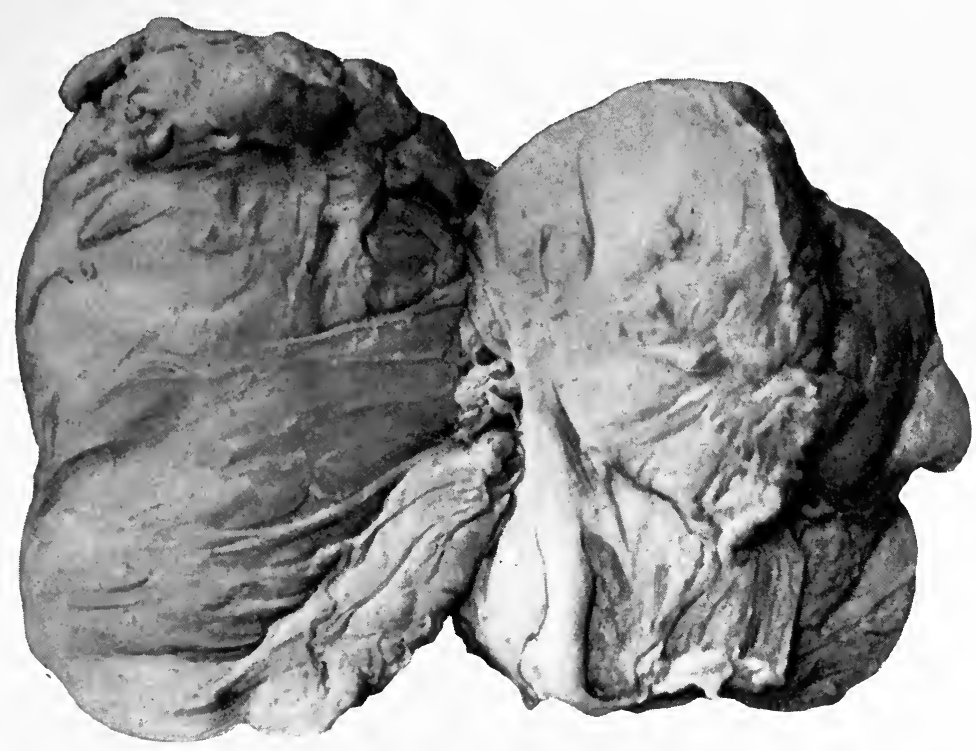

Two large lateral lobes removed by finger enucleation through the median perineal route. (Watson.)

Precautions.-The three most important points to be observed in doing prostatectomy in this way are these: (I) Avoidance of laceration of the membranous urethra. (2) Avoidance of injury to the rectum. Avoidance of hemorrhage.

I. The first accident can be avoided if the attempt is not made to withdraw too large masses through the external urethrotomy incision, and by using care when separating the apex of each lobe from its attachments.

2. With ordinary care, and by observing the precaution of putting one or two fingers of the hand not occupied with the enucleation into the rectum, and exercising counterpressure with them, that structure need never be injured. The laceration it sometimes receives is almost always due to downward pressure upon the upper surface of one or the other of the lateral lobes while they are being enucleated. 
3. Unless the outer fibrous sheath has been torn during the enucleation, there will never be hemorrhage of the least consequence in connection with this method of operating.

FIG. I90

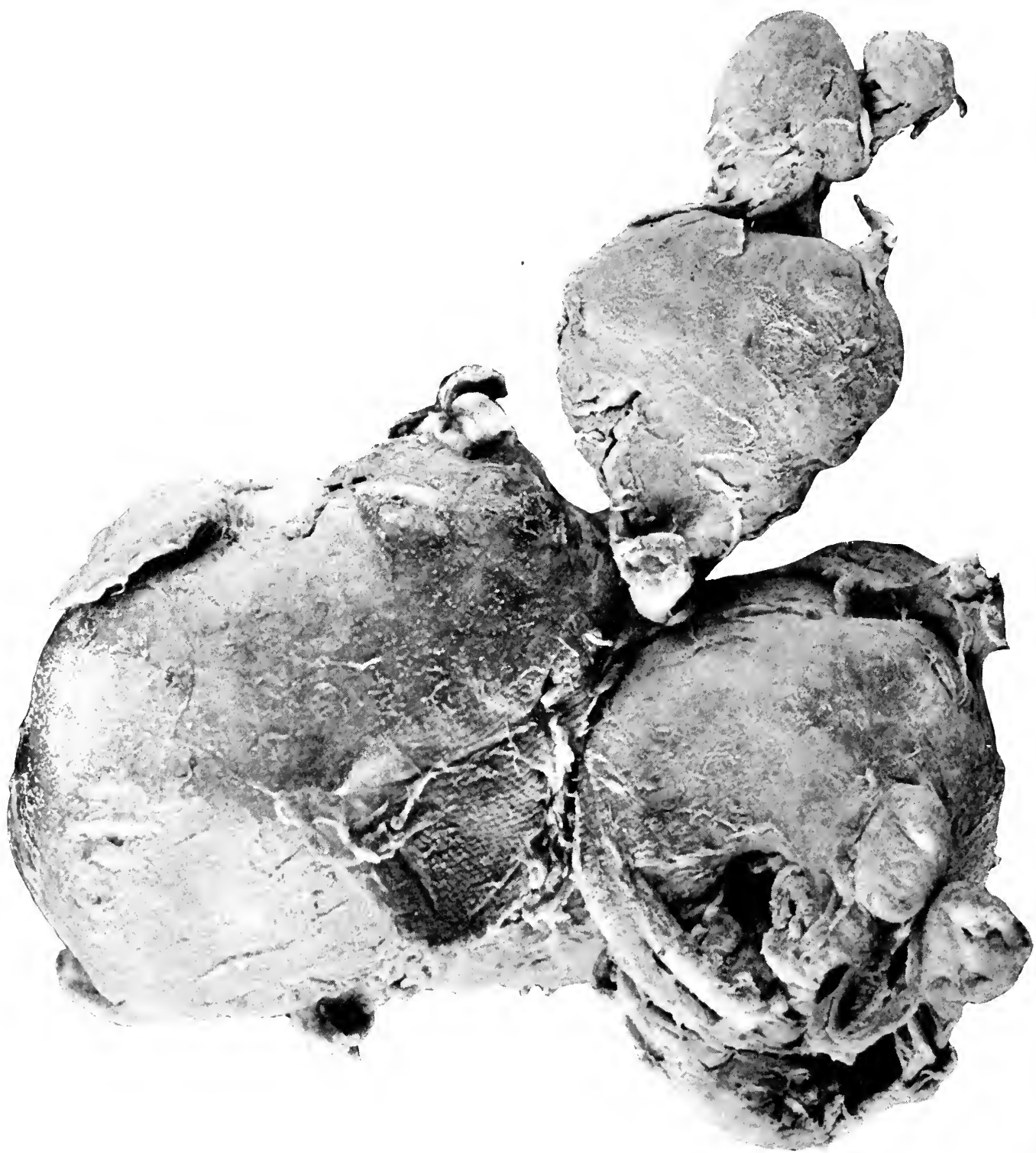

The prostate removed by finger enucleation through the median perineal route. Actual size. (Cunningham.)

Other points to be noted are these: Tough bands of fibers. which do not break down readily beneath the finger are better divided by scissors or blunt dissectors. 
If the suprapubic pressure is taken off after the gland has been nearly or wholly freed, the latter may easily be pushed back into the bladder, and prolong the operation because of the difficulty of catching and withdrawing it.

Parker Syms ${ }^{1}$ uses a balloon tractor (Fig. I9I) for the purpose of bringing the prostate within reach while carrying out the above-described technique. It is said to be useful for the purpose for which it was devised. We have not, as has been said, found that tractors bring the gland any better within reach than properly applied suprapubic pressure, except in the case of very fat persons.

FIG. I9I

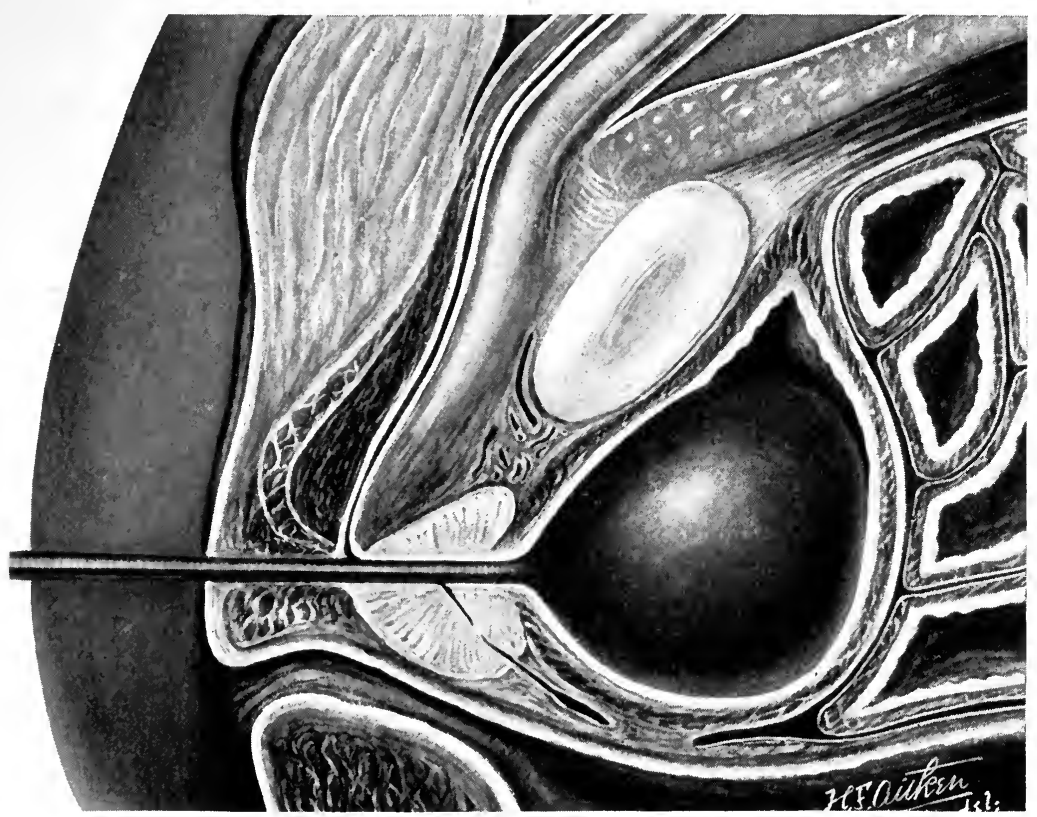

Syms' prostatic tractor in use. Its bulbous extremity has been expanded within the bladder, and the traction on the stem of the prostate is drawn down into the perineum.

Drainage of the Bladder.-The manner of arranging drainage which is employed by Young, and illustrated in Fig. 192, is an excellent one for maintaining continuous irrigation and drainage of the bladder after this or other of the perineal methods of prostatectomy.

We believe very strongly in the importance and value of drainage of the bladder and the large empty spaces created by the removal of the gland, despite the fact that Goodfellow's results have been so good without it.

Cases in Which Enucleation Cannot be Done.-In some cases complete enucleation cannot be carried out whatever the method employed. 
The prostates of which this is true are such as have the outer surface of the gland and the inner one of the fibrous sheath so intimately bound together by adhesions as to make it impossible to separate the one from the other without tearing through the sheath, and, in doing so, giving rise to serious hemorrhage by wounding veins which lie in it. Fig. I93 is an example of such a prostate. The gland in this case has been cut through

Fig. I 92

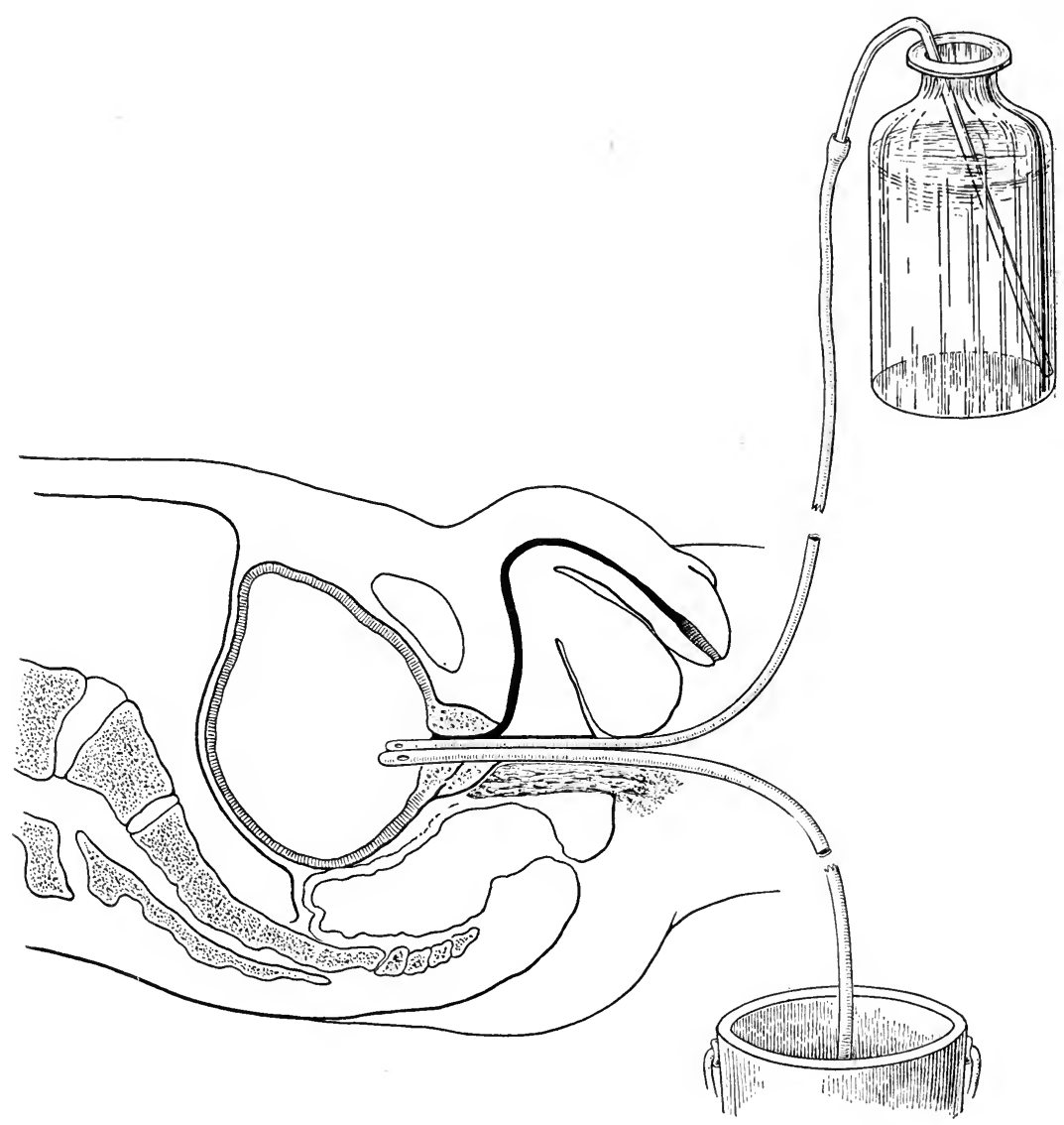

Diagram showing the use of continuous irrigation of the bladder, as employed by Young after perineal prostatectomy. (Deaver.)

its centre in a transverse direction, and the lower half is hanging downward, attached to the upper one by a thin bridge of undivided tissue. In this specimen it is difficult to distinguish between the outer sheath and the substance of the gland itself.

In such cases the only resource is to remove the gland by morcellement.

After-treatment.-We continue drainage for a variable length of time, according to the conditions presented in each case. If there is cystitis, 
the drainage and irrigation are maintained for ten days or a fortnight. Again, if there is much suppuration from the empty spaces created by the operation, drainage of them should be kept up for a longer time. Ordinarily, from four to six days is sufficient.

Packing.-Packing, except it be for the purpose of controlling hemorrhage, should not be employed. The writer has never had hemorrhage of any consequence attend the performance of this operation, and has found no need, therefore, for packing.

Passing of Sounds. - Sounds should be passed occasionally in the course of convalescence. We are in the habit of passing the first one on the sixth day after the operation, and thereafter every second day for the next fortnight.

Comment.-This operation has been criticised as being a blind and needlessly rough procedure, involving destruction of the ejaculatory ducts and the prostatic urethra. That it is done without the aid of sight is quite true. That the sense of touch, for those who employ this method, is more delicate, accurate, and reliable than that of sight, so far as they are personally concerned at any rate, is equally true. That the prostatic urethra is in part destroyed - sides and roof-is also true, but it is not true that the floor or the ejaculatory ducts are necessarily injured. The floor of the canal of the urethra is expressly preserved.

That the ejaculatory ducts are not always injured in the performance of this operation the writer is certain, having had the opportunity to

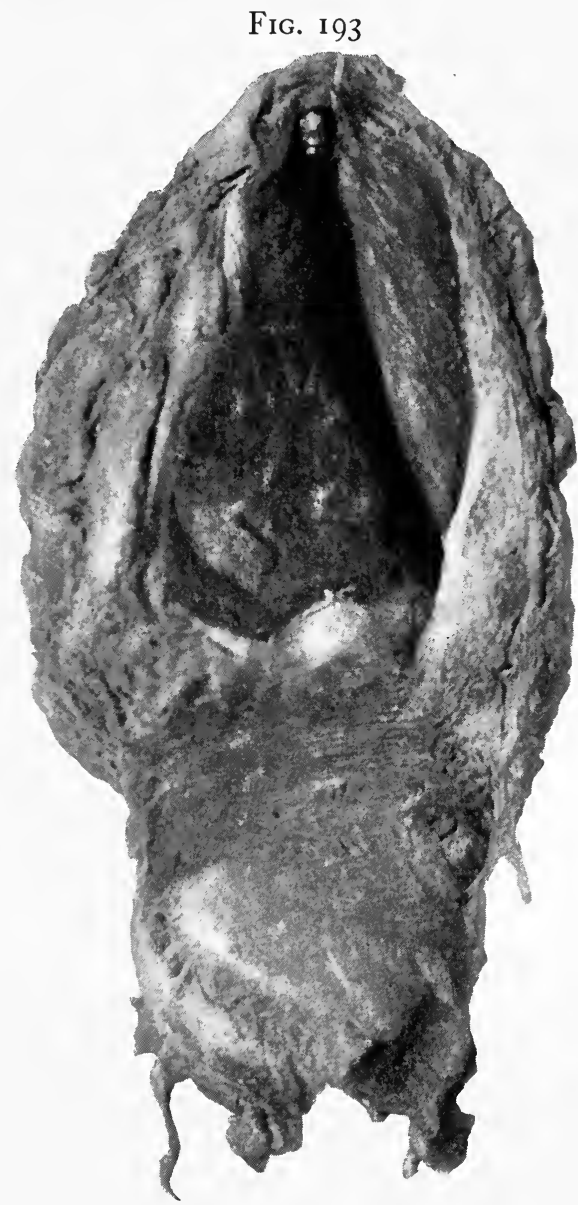

Specimen showing a prostate in which the attachments between the sheath and the surface of the gland are very intimate, and make enucleation difficult or impossible. (Watson.) examine a specimen of the parts from a patient who died on the fifth day after the operation, and finding them intact in that case, and having the same fact attested by several patients who have preserved both sexual power, and, so far as can be judged, natural ejaculation of the seminal 
fluid as well. Further evidence of their having been spared in connection with the operation is furnished by Goodfellow, ${ }^{2}$ who reports that the sexual power and natural ejaculation of the seminal fluid have been present in a number of his cases following the operation.

This method of operating was originally criticised in England by McGill, who said, in referring to Watson's ${ }^{3}$ advocacy of the perineal operation in I888, that the choice of operative method should not be made dependent upon the unusual length of one operator's finger, which McGill assumed to be necessary for its successful performance.

This dictum took root in England, and has been quoted from time to time by different surgeons as an argument against the selection of this perineal route for prostatectomy. That it was a mistaken criticism has been demonstrated ever since it was uttered, for the operation has now been done many times by surgeons with fingers of all sorts of lengths, and with entire success. The preference of English surgeons for the suprapubic method of removing the gland is largely due to the fact that they have made but little effort to familiarize themselves with the perineal techniques, and are, with but few exceptions, without experience in the matter.

The writer's preference for this method rests upon the following facts: The rapidity with which it can be performed, the ease with which, in the great majority of the cases, the gland can be removed in this way, the absence of hemorrhage attending its performance, the small amount of disturbance of the perineal structures that is involved in it.

Stricture as a Consequence of the Operation.-We have seen strictures of the prostatic urethra occur twice in our own series of perineal prostatectomies. In a very small number of the cases reported by other operators it has been noted as a result of the operation. There are but four instances, in our knowledge, in which this has happened, making, with the two in our own series, six altogether.

Open or Dissecting Methods of Perineal Prostatectomy.-Pyle's ${ }^{4}$ Technique (I892).-This surgeon's description of the operaton has been lost sight of, and, inasmuch as the more modern technical methods are, in many important respects, anticipated by it, it seems but just to quote him here. (Compare with technique of Young and Proust.)

"The prerectal crescentic incision (Fig. I94) is made, extending from one tuberosity of the ischium to the other. The insertion of the levator ani is exposed by dissection with the handle of the scalpel; this and the fascia overlying it are divided. The dissection proceeds between the bulb of the urethra and the rectum. Further blunt dissection between the urethra and the rectum exposes the prostate. The gland is then grasped with vulsellum forceps and enucleated with the finger through 
a median incision in the posterior surface of the fibrous. sheath. It is well to place a bougie in the urethra in order to avoid wounding that structure."

Proust's Technique ${ }^{5}$ (I90I).-Distinctive Features.-These are: Raising the pelvis of the patient high above the horizontal plane into an

FIG. I94

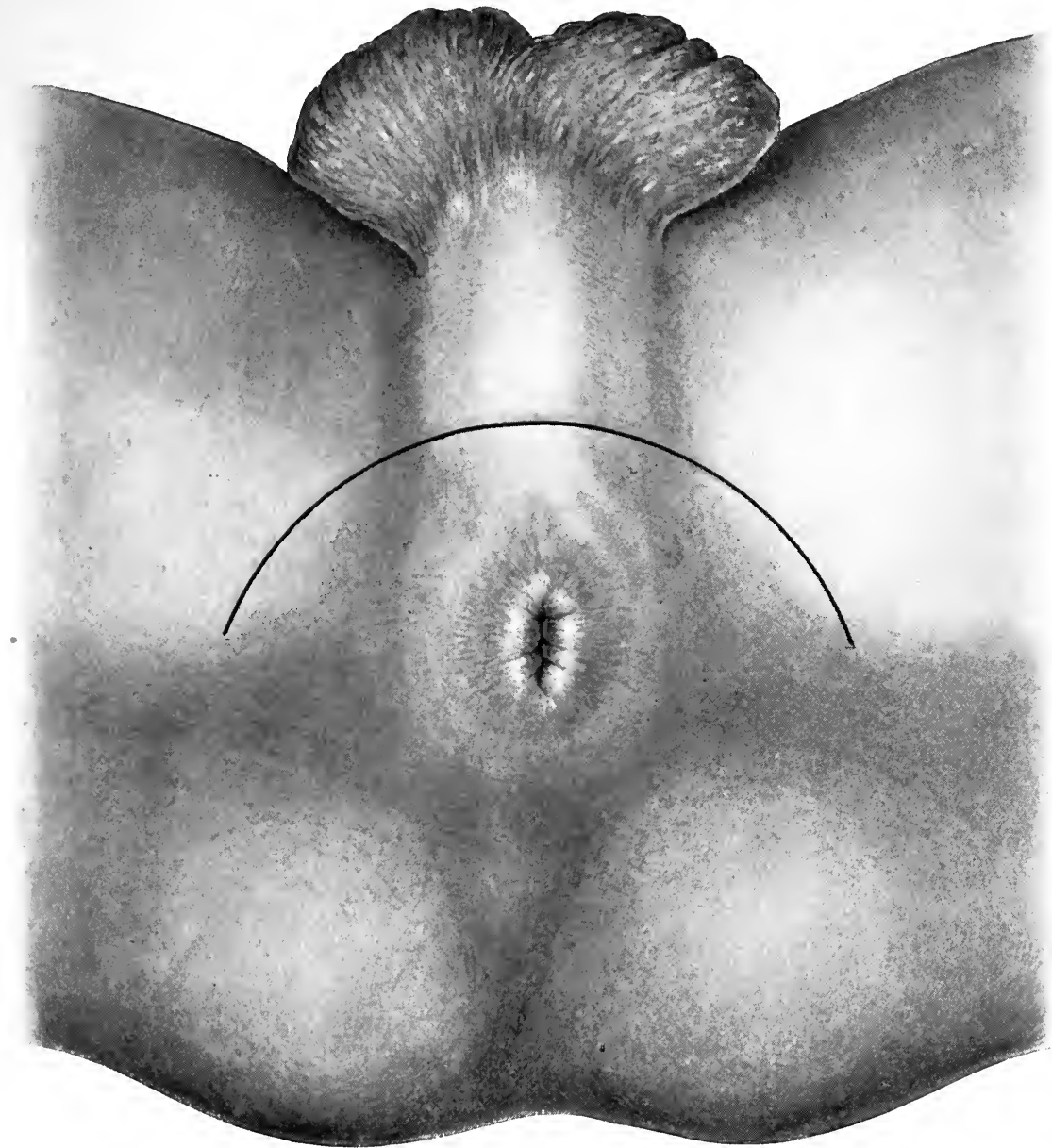

Perineal prostatectomy. (Proust.) The transverse perineal incision. (Deaver.)

extremely exaggerated lithotomy position, complete longitudinal division of the floor of the prostatic urethra, ligature of the ejaculatory ducts, exposure of the retroprostatic space by division of the central tendon of the perineum and urethrorectalis muscle, and by blunt dissection, separating rectum from prostate, closure of the incision of the prostatic urethra by suture.

vOL. I- 25 
Instruments.-The same as those already named for the first operation. In addition, a special form of bifid retractor, which Proust designed to lift the urethra upward away from the rectum (see Fig. 198).

FIG. 195

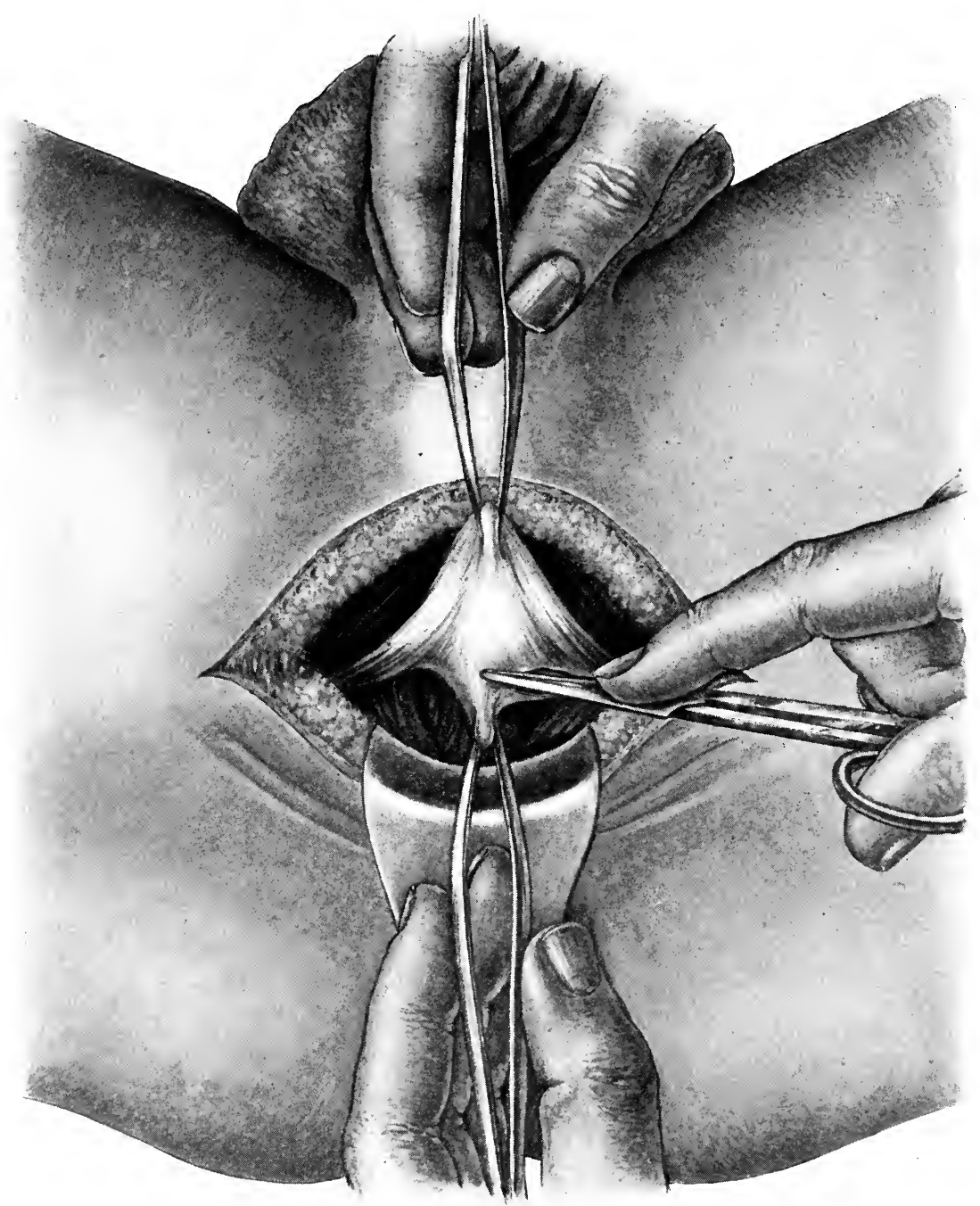

Perineal prostatectomy. (Proust.) After dividing the skin and separating the insertion of the sphincter ani from the perineal centre (which is raised by forceps in the right hand of an assistant), the recto-urethral muscle is exposed, and is now being divided with scissors, close to the membranous urethra. (Deaver.)

Incision.-The prerectal curved incision (see Fig. I94).

Operation.- Through the above-named incision the central tendon of the perineum is exposed and divided transversely (Fig. 195). 
Immediately behind this is the recto-urethralis muscle. This muscle is also divided transversely, and the space between the prostate and the

FIG. I96

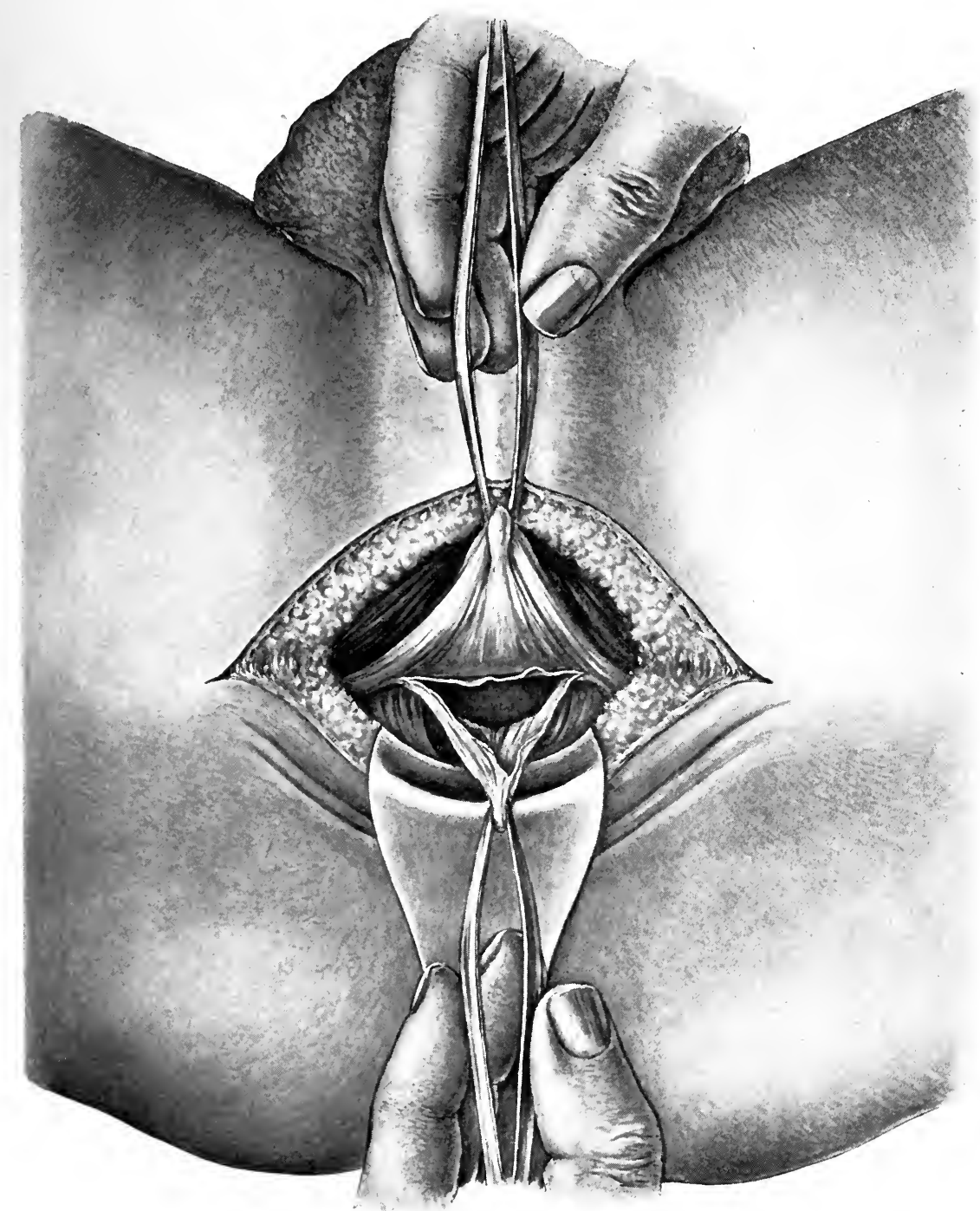

Perineal prostatectomy. (Proust.) The recto-urethral muscle has been divided, allowing the rectum to fall away from the anterior structures, and opening l'espace décollable rétroprostatique. (Deaver.)

rectum, called by Proust l'espace décollable rétroprostatique, is cleared by the finger tips (Figs. I 96 and I97).

This step is the keynote of the operation. It allows the rectum to be separated from the prostate and the space between them to be so freely 
opened as to give access to the posterior surface of the outer sheath of the gland.

The space is kept open by the use of the retractors in the manner shown in Fig. 198.

FIG. 197

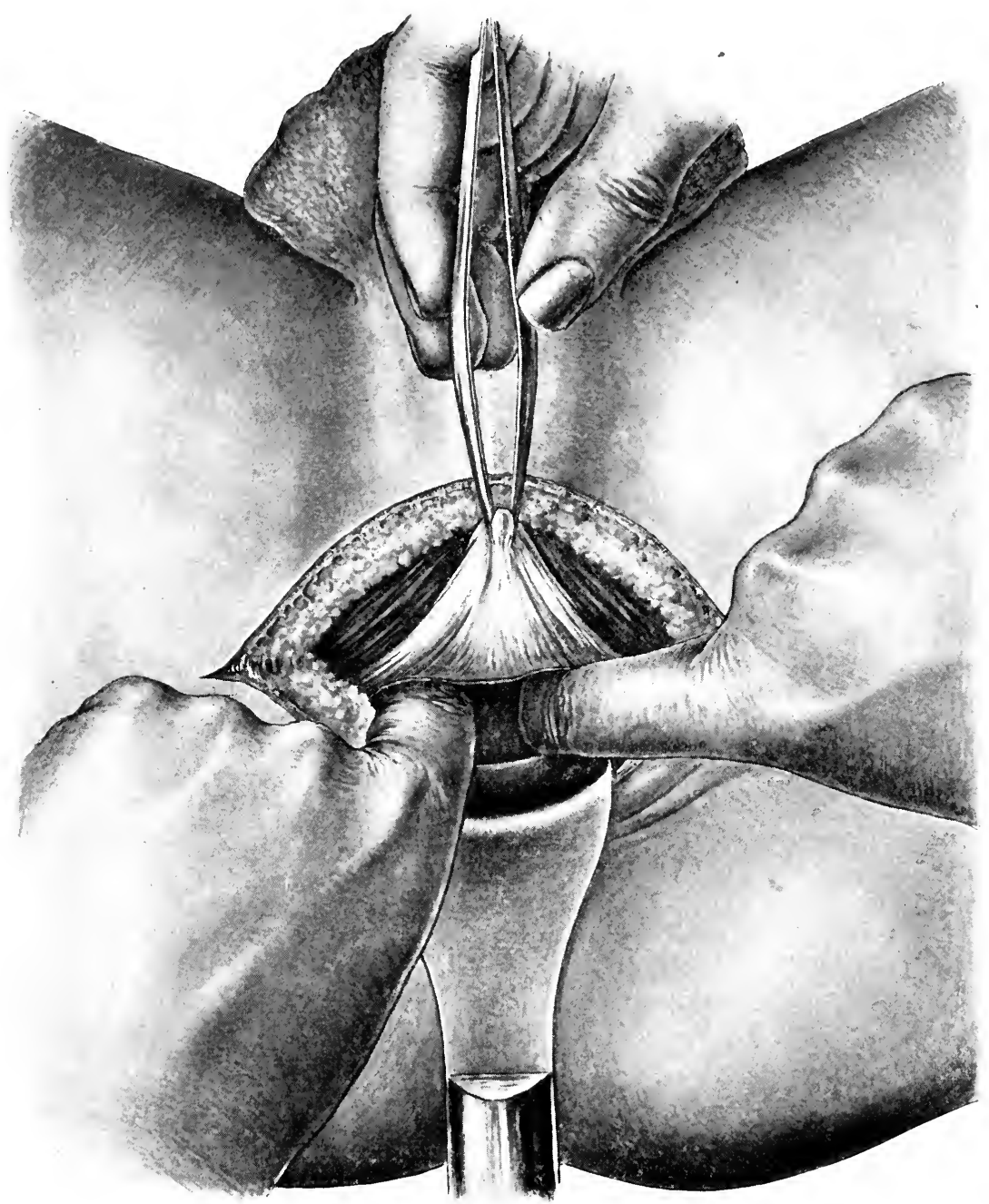

Perineal prostatectomy. (Proust.) The two index fingers of the operator are introduced between the two layers of the aponeurosis of Denonvilliers, and enlarge l'espace décollable rétroprostatique. (Deaver.)

A grooved staff is next passed into the bladder through the urethra, and the prostatic urethra is divided upon it by an incision in the median line.

The enucleation of each of the lateral lobes in succession is next carried out by separating one of them from the outer fibrous sheath with the finger 
tip or scissors, and then its fellow of the other side in the same way (see Figs. 199 and 200). The finger of the other hand is laid against the inner surface of the prostatic urethra to serve as a guide in order to avoid injury to the canal upon its lateral aspects. The lobe is cut as close as possible to the urethra without wounding the sides of the latter, after freeing the rest of the lobe from its attachments.

The ejaculatory ducts are found in the wound of the prostatic urethra, freed and ligated (see Fig. 20I).

FIG. ${ }_{19} 8$

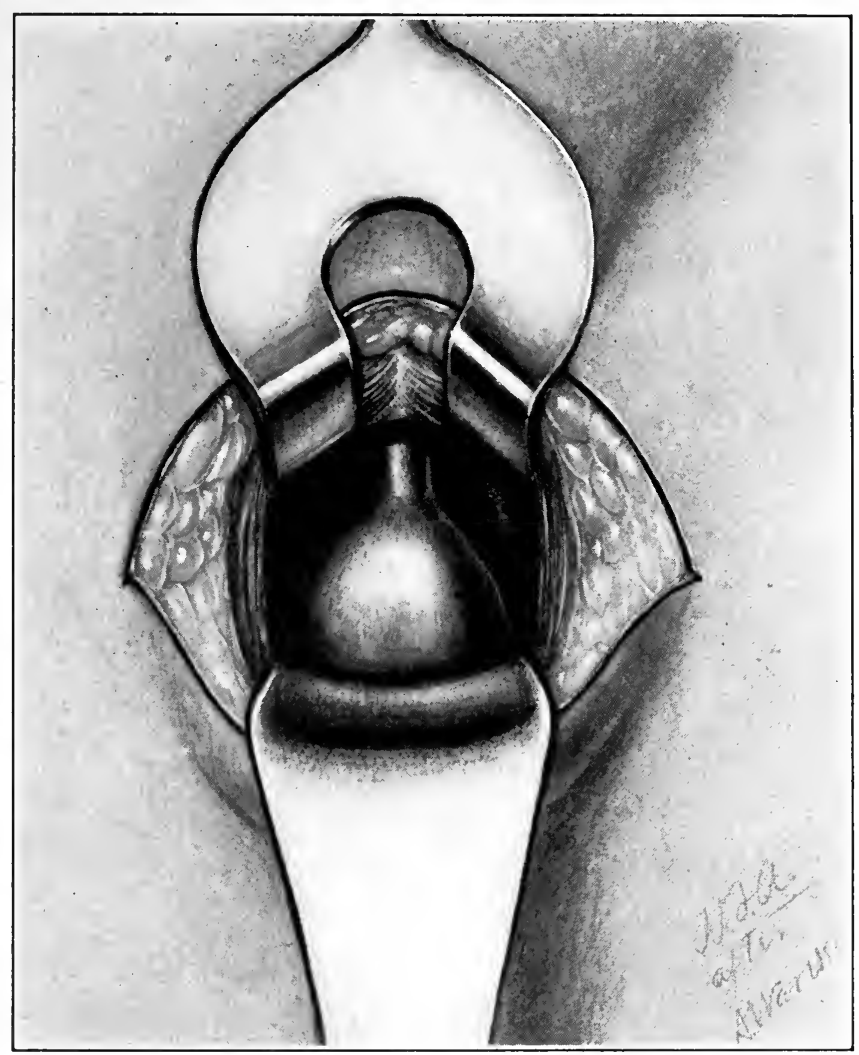

Proust's retractors in situ.

After the separation of the lobes the incision in the prostatic urethra is sutured with the intention of securing its union per primam (see Fig. 201).

Comment.-The ligature of the ducts is done with the intention of averting subsequent epididymitis. The writer has never had this complication in any of his own cases, and is therefore not disposed to regard this step as at all essential for the purpose for which it is designed. The weak point of this technique lies in the fact of incising the prostatic urethra and ligation of the ducts, for the only possible advantage of 
doing an open dissection is the preservation of these two structures, and since both are purposely compromised by Proust, this advantage, if it be one, is lost.

FIG. 199

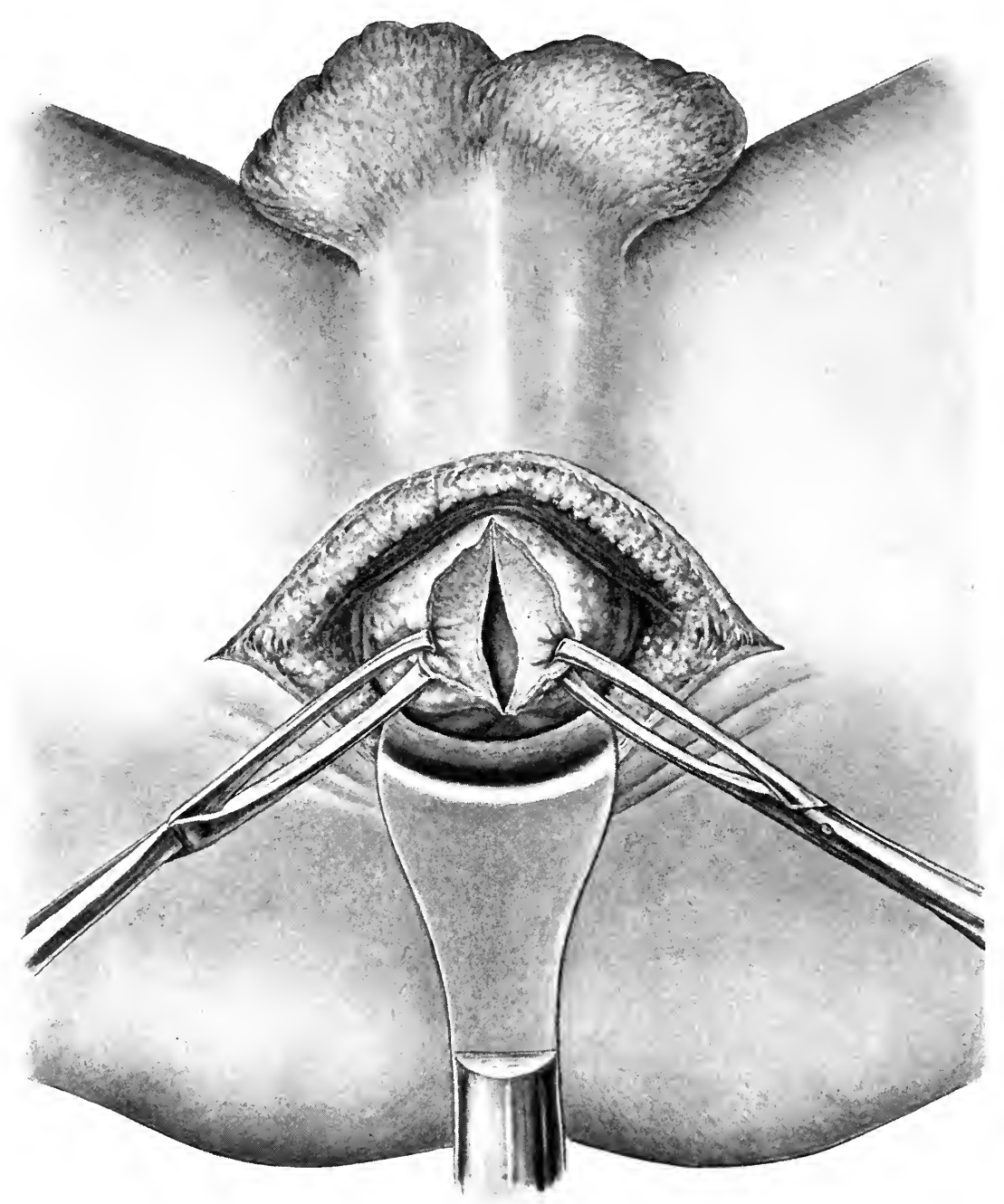

Perineal prostatectomy. (Proust.) Hemisection of the prostate along the floor of the urethra. (Deaver.)

Rydygier's modification ${ }^{6}$ consists in clamping the lobes close to and parallel with the prostatic urethra, after having freed them elsewhere, and cutting first one and then the other off just outside the clamps. $\mathrm{He}$ does not lay open the prostatic urethra, but gains access to the gland and enucleates it through the lateral incisions made in the sheath, as in Young's method. 
Young's Technique. ${ }^{7}$-The distinctive features of this technique are: the employment of a special form of prostatic tractor devised by Young, enucleating the gland through two lateral incisions in the fibrous sheath

FIG. 200

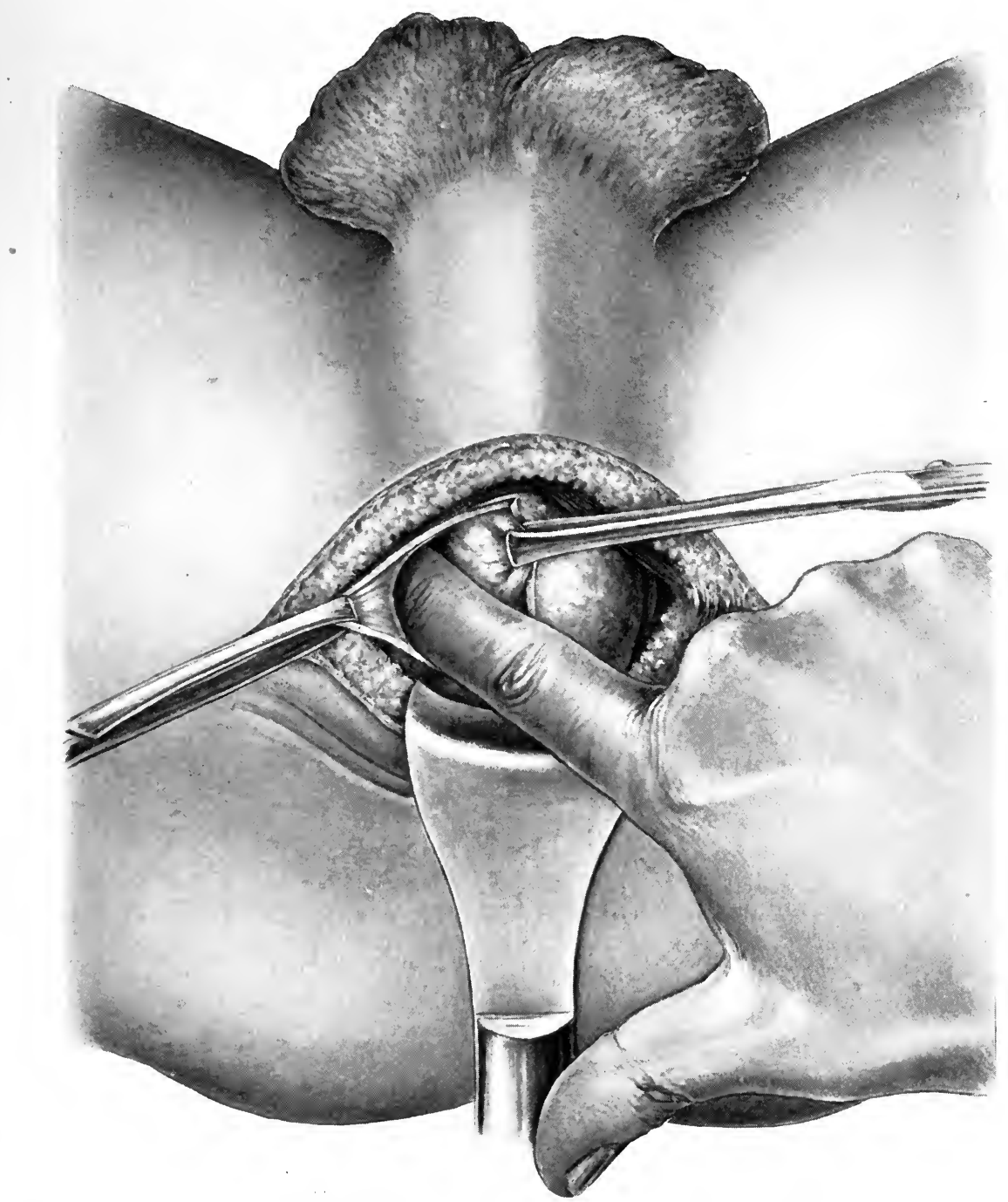

Perineal prostatectomy. (Proust.) The sheath of the prostate (the anterior layer of the aponeurosis of Denonvilliers) has been opened, and the surgeon's finger now detaches the sheath from the prostate by blunt dissection. The prostatic tractor sometimes employed by Proust is not shown. (Deaver.)

on its posterior surface outside the urethra and parallel with it, and the preservation of the ejaculatory ducts and prostatic urethra. Position of the Patient.-Slightly exaggerated lithotomy posture. 
Instruments.-Same as in the first of the perineal operations described, and, in addition, a special form of prostatic tractor (Figs. 202 and 203) similar in character to that of De Pezzer (Figs. 204 and 205); a blunt dissector and a special form of forceps for withdrawing the lobes; 3

FIG. 20I

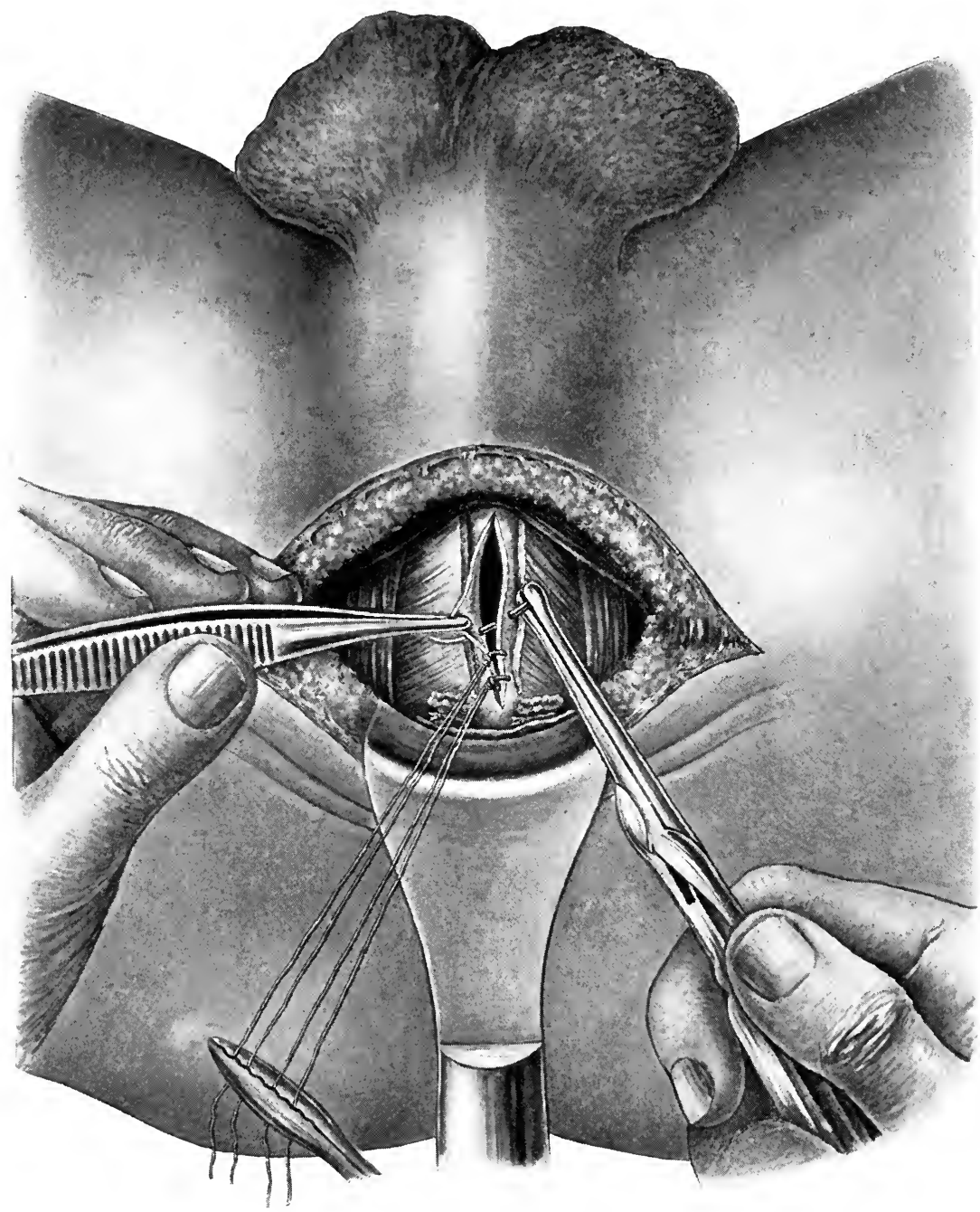

Perineal prostatectomy. (Proust.) The ejaculatory ducts have been ligated, and the urethra is now being sutured. (Deaver.)

retractors to spread open the retroprostatic space and keep back the rectum (see Figs. 207, 209, and 210).

Operation.-The superficial structures of the perineum are exposed by an inverted $\mathrm{V}$ or $\mathrm{Y}$ incision (see Fig. 206). 
FIG. 202

Young's prostatic tractor (open).

FIG. 203

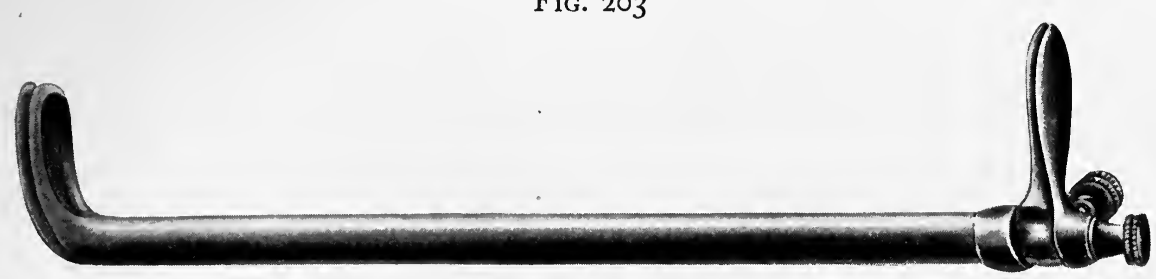

Young's prostatic tractor (closed).

FIG. 204

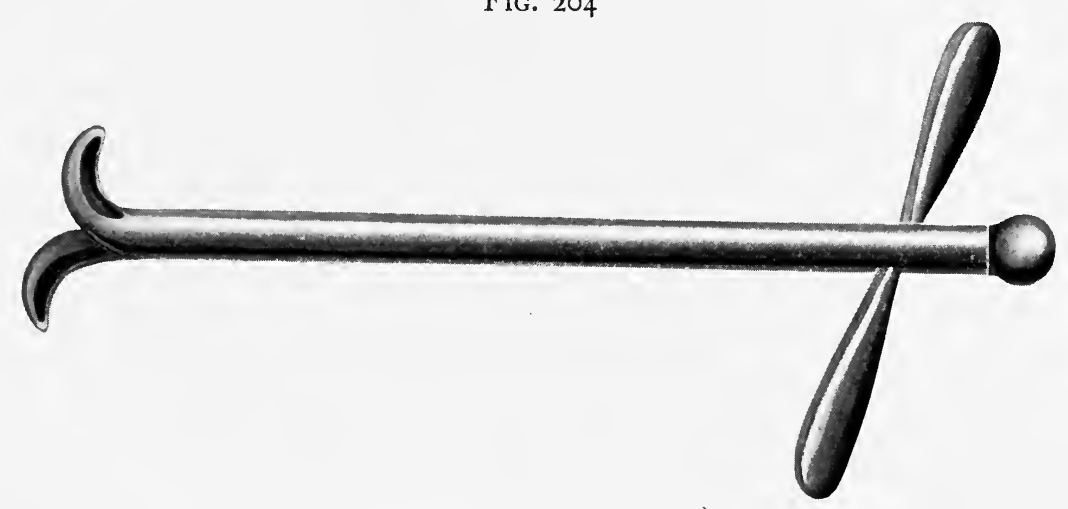

De Pezzer's tractor (open).

FIG. 205

De Pezzer's tractor (closed). 
The exposure of the posterior surface of the gland is made in the same manner as in Proust's technique. When the membranous urethra has been reached and exposed, it is opened on a grooved staff sufficiently to allow the tractor to be inserted into the bladder (Fig. 207). When it has been entered, its two blades are spread apart and drawn forward against the intravesical aspects of the lateral lobes (see Fig. 208), and the gland

FIG. 206

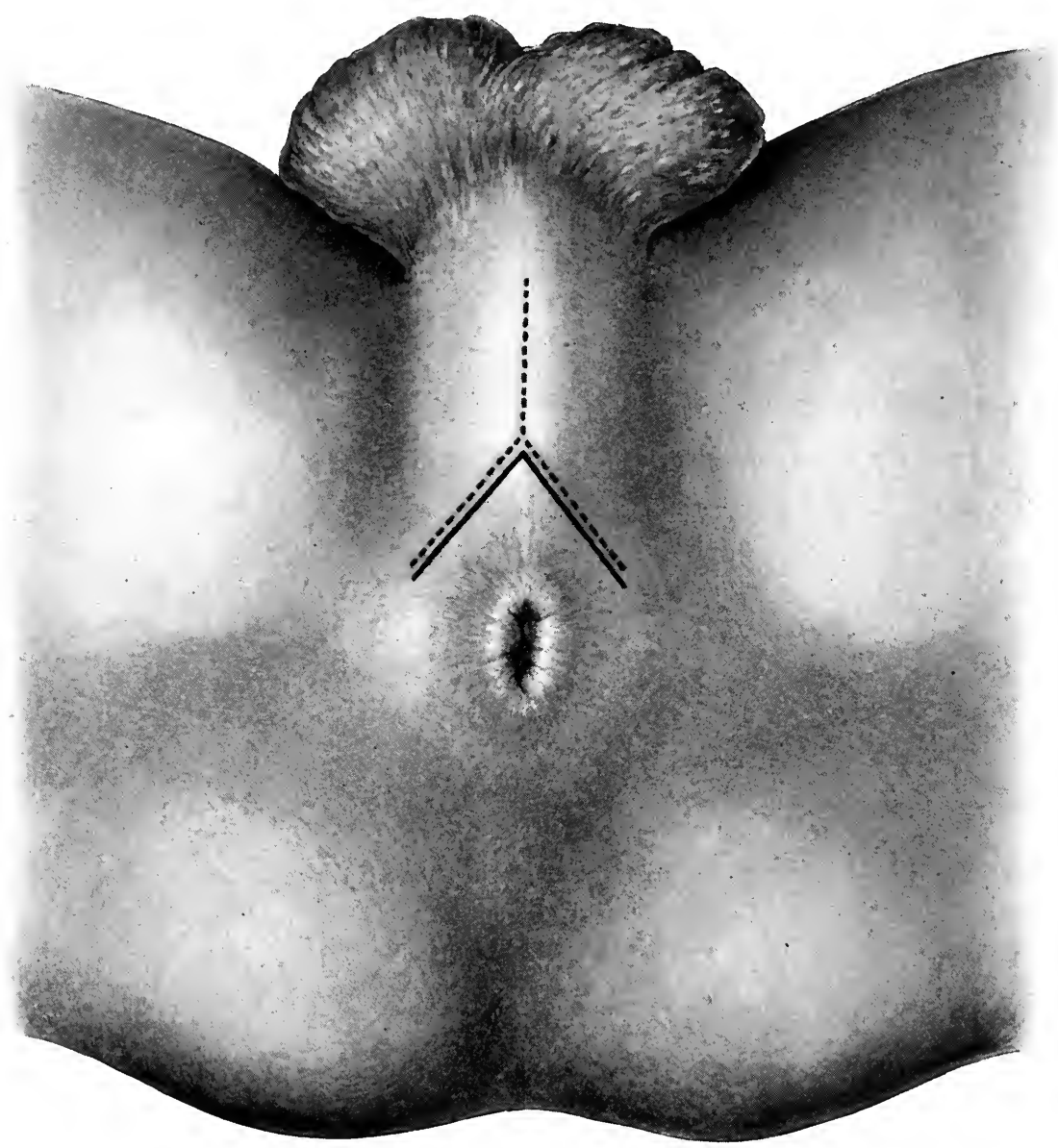

Skin incisions for perineal prostatectomy. (Deaver.)

is drawn toward the surface of the perineum, its posterior surface being at the same time tilted upward, and thus exposed (Fig. 207). The posterior surface of the prostate is then cleared by the fingers or handle of the scalpel, and lateral incisions on either side and parallel with the urethra are made through the posterior surface of the fibrous sheath of the prostate (Fig. 207). 
The enucleation is then carried out through each of the incisions in the fibrous sheath in turn, the lateral lobes being thus removed in sequence. The separation of the gland is effected with a blunt dissecting instrument (see Fig. 209) or with the finger tip. The object of the lateral incisions through the sheath is to avoid injury to the prostatic urethra and the ejaculatory ducts. The external parts of the lobes are freed first.

\section{FIG. 207}

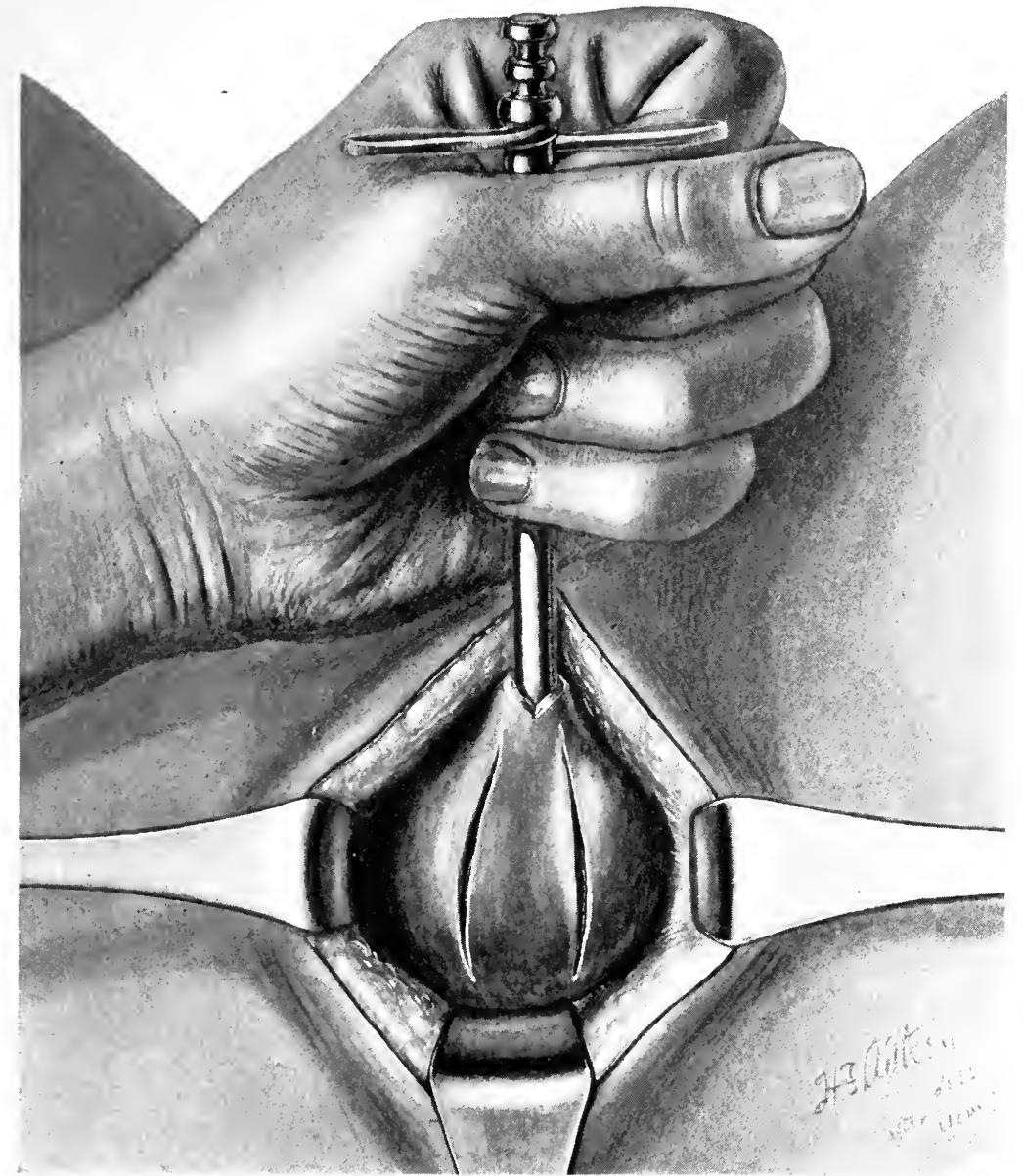

Young's technique: prostate brought down and lateral incisions made in capsule. (After Young.)

Traction with forceps (see Fig. 210) is made upon the lobes in turn after they have been freed sufficiently to allow of the forceps being advantageously placed upon them.

When the lobes have been removed there are two chambers, one on either side of the urethra and ejaculatory ducts, and it is claimed that neither of these structures is injured. 
The middle lobe, when present, is removed by turning the blades of the prostatic tractor within the bladder so that one of them is engaged

FIG. 208

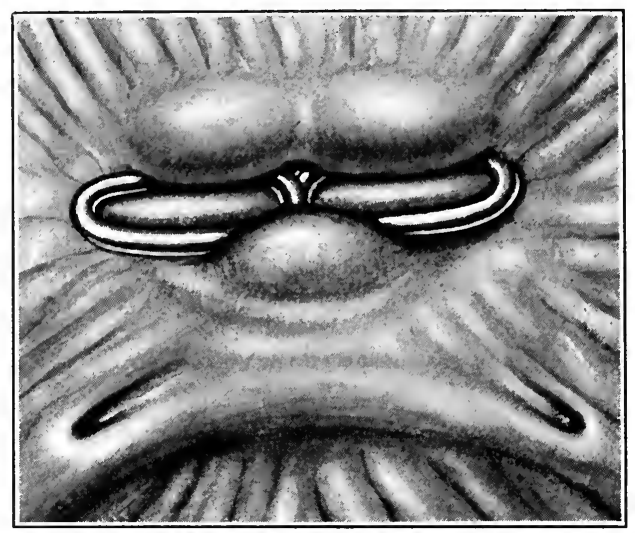

Young's tractor in situ in the bladder. (After Young.)

FIG. $2 \approx 9$

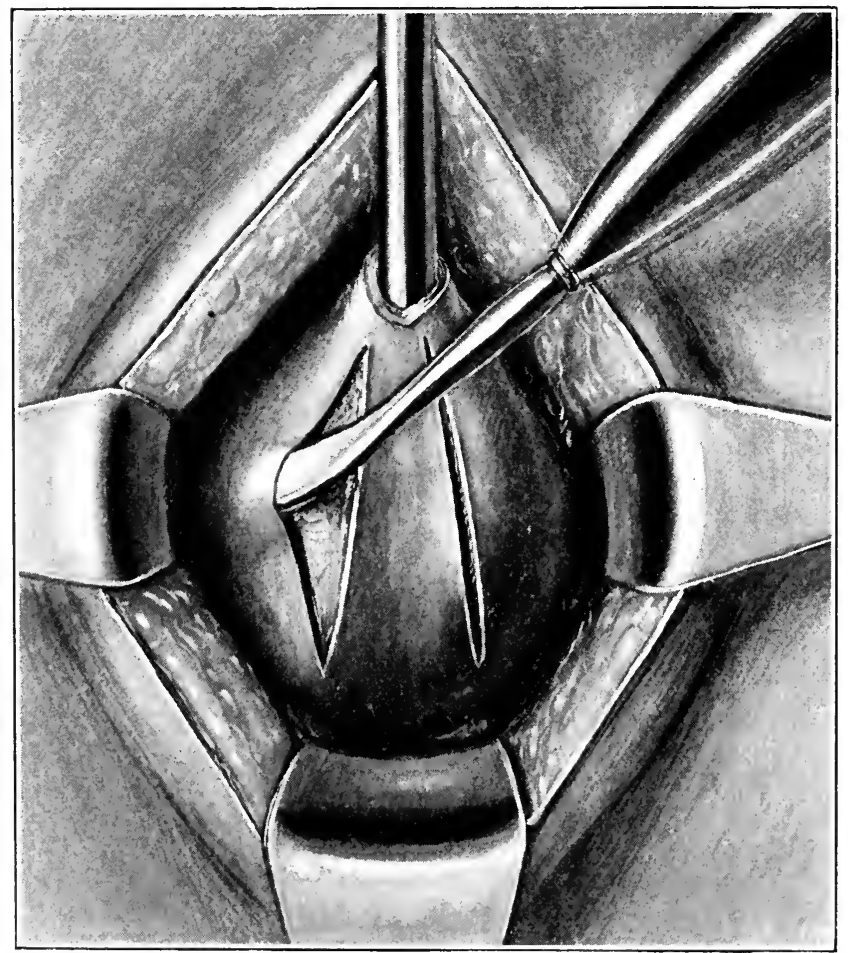

Young's technique: separation of capsule with blunt dissector. (After Young.)

behind the farther end of the lobe (Fig. 2II), and then drawing it down and sideways into one of the lateral pouches left by the enucleated lateral 
lobe on that side. It is then seized with forceps and enucleated through the same opening in the sheath by which that lateral lobe was removed (see Fig. 212). The outer wound is closed and the bladder drained in

Frg. 210

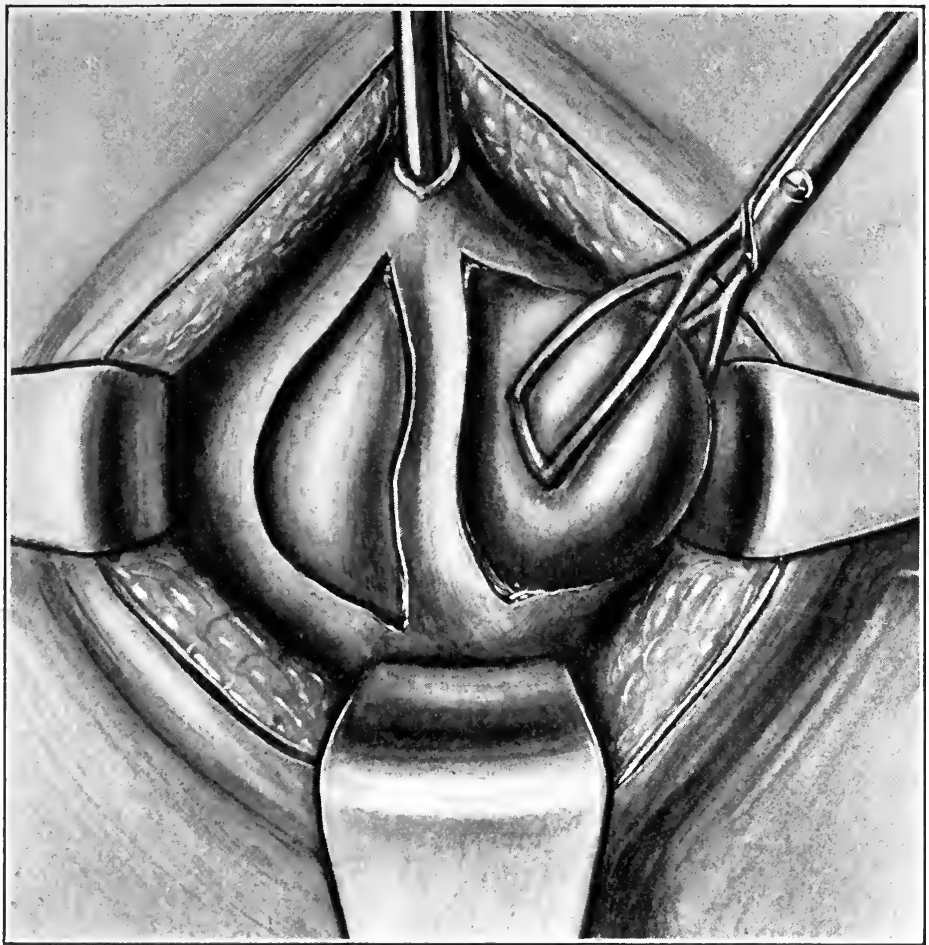

Young's technique: withdrawing lateral lobes after being enucleated. (After Young.)

FIG. 2 II

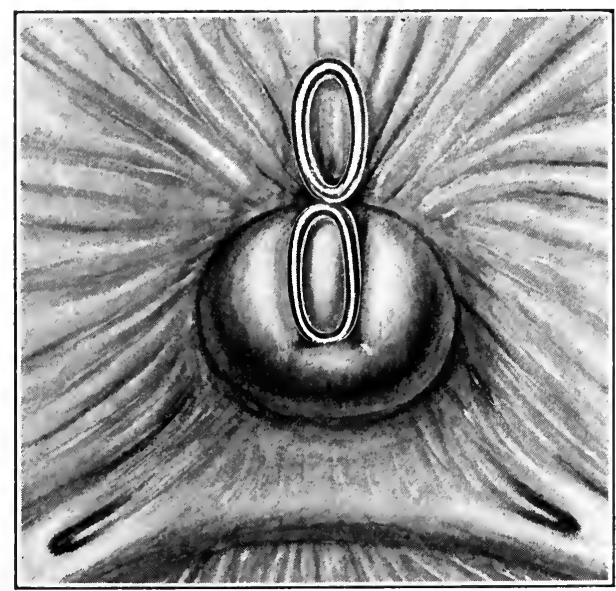

Young's technique: manner of applying blade of tractor to middle lobe. (After Young.) 
the manner shown in Fig. 20I. The lateral cavities are packed with gauze.

Comment.-The objection that we have to this, as to other open or dissecting methods, is primarily that they secure, so far as we may judge up to the present time, no better functional results with respect to the control of the micturition, and are attended with no smaller operative mortality, occupy a far longer time in their performance than the rapid finger enucleation, and involve a needlessly extensive division of the perineal structures.

FIG. 212

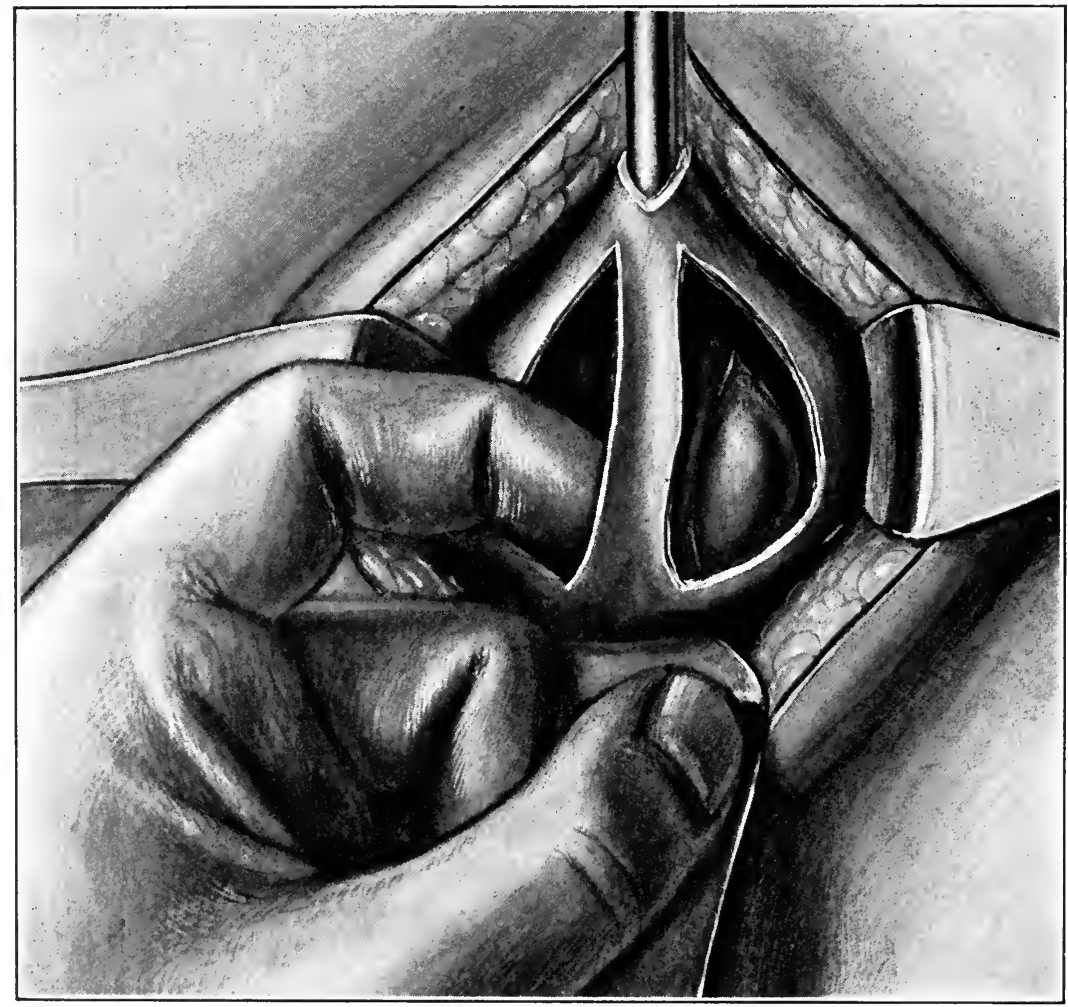

Young's technique: manner of removing middle lobe. (After Young.)

The greater length of time which these dissecting techniques demand should, theoretically, be a disadvantage. The results obtained by some operators-Young, Ferguson, etc.- do not seem to bear out this impression, however.

The use of a prostatic tractor we rarely find necessary or more effective in bringing the gland down than suprapubic pressure, except in the 
cases of stout patients; the tractor is then certainly useful, since suprapubic pressure fails to accomplish its object in such instances.

Precautions. - The tractors should be used with care, for otherwise there is always the chance of their tearing through the floor of the bladder and more or less of the prostatic urethra whenever too much force is used, especially when carrying out the separation of the vesical aspects of the lateral lobes, and, again, after they have been freed.

Ferguson's Technique. ${ }^{8}$ - The distinctive features of this technique are: a special form of tractor (Fig. 213) for depressing the prostate and making it accessible; placing fine catheters in the ejaculatory ducts through incisions in the vasa deferentia, which are exposed on either side of the body in the groins, and employing the instruments as guides to aid in locating the ducts and as a means of avoiding injury to them. When the canals are too small to allow the catheters to pass into them, he injects the prostatic urethra with colored fluid, forcing it into the ducts from the surface of the urethra.

The exposure and removal of the lateral lobes is effected in the same way as in the technique of Proust, except that the ducts are not ligated.

FIG. 213

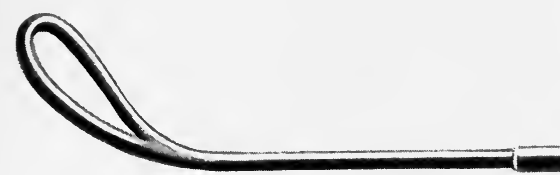

Ferguson's prostatic tractor.

Comment.-We cannot say that we are favorably impressed with the idea of the injection of colored fluids into the ducts. Ferguson himself says that this does not enable him to avoid injury to them, but informs him only that they have been injured when such is the case. There does not appear to us to be any very important advantage gained from this information. The use of the catheters we can quite understand being of service in tracing the course of the ducts in the cases in which they can be inserted into them, but this step also seems to us to be rather needless, and to prolong the operation without offering sufficient compensation to make it worth while.

Albarran's Technique. ${ }^{8}$-The distinctive feature of Albarran's technique is the removal of the lateral lobes by morcellement instead of each of them entire, or both in one mass. He dispenses with the use of tractors to make the gland more accessible, and, instead, employs for this purpose 
the tip of the index finger of one hand hooked over the intravesical portion of the lateral lobes, each in turn. The anterior parts of the lobes are first cut away, then the deeper ones (Fig. 214).

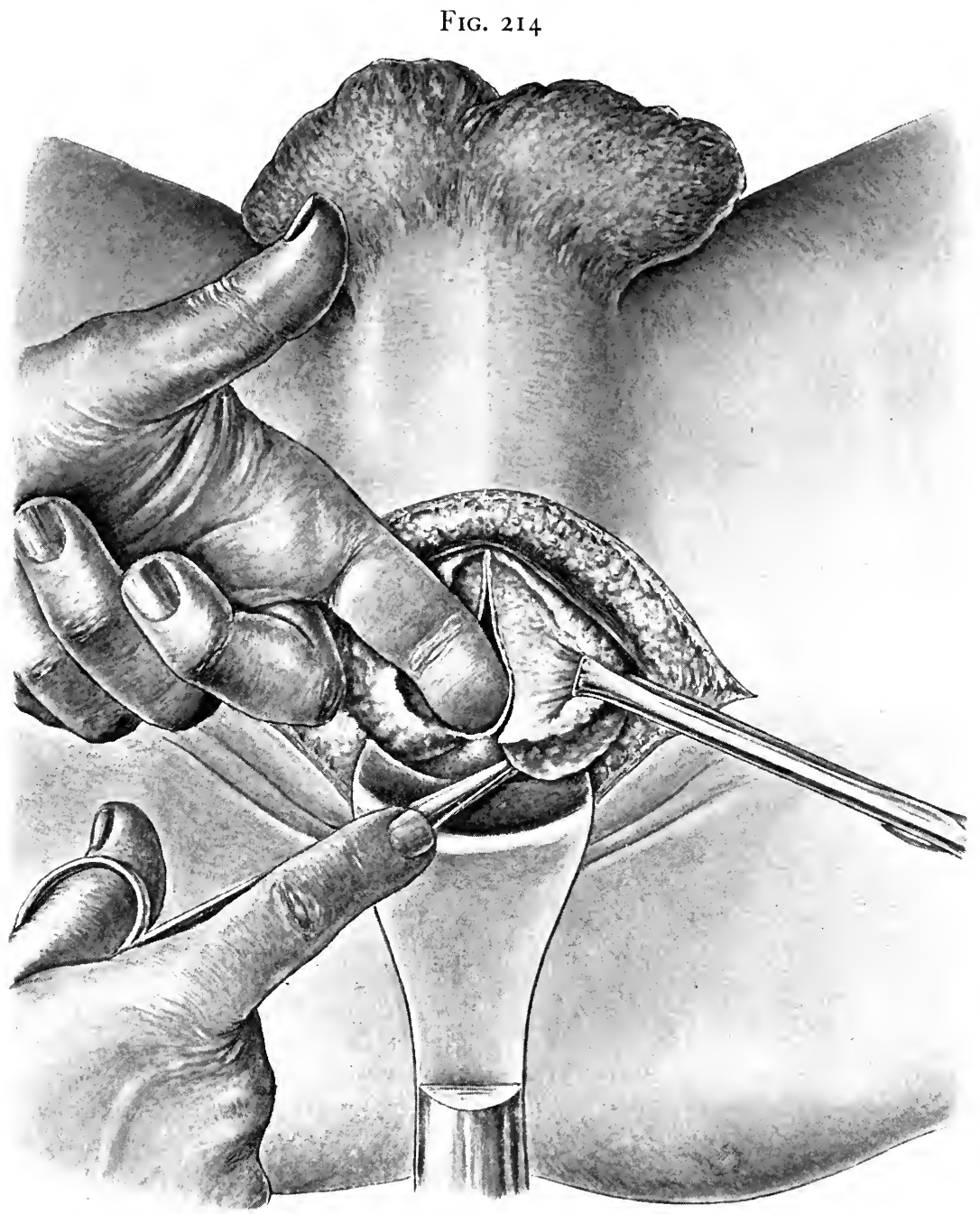

Perineal prostatectomy. (Albarran.) Each lobe of the prostate in turn is dissected free from the sides of the prostatic urethra, and the outer sheath all around for half its length. It is then cut off and the remaining part of the lobe is similarly dealt with. (Deaver.)

Comment.-The removal of the gland piecemeal may be of advantage in the case of very large hypertrophies, the withdrawal of which en masse would risk tearing of the membranous urethra or the rectum. Albarran claims that it is much easier to take away the more distant or vesical 
parts of the prostatic lobes after the anterior portions have been cut away by the scissors. Personally, we have never had occasion to employ this method of removal except in such a condition as that illustrated in Fig. I93.

Suprapubic Total Prostatectomy (McGill, Belfield, I887; Fuller, I895; Freyer, I901).-The suprapubic cystotomy incision having been made, the forefinger is passed into the bladder through it, and the interior of the viscus is explored. Calculi, if present, are removed, phosphatic incrustations scraped away, etc.

FIG. 215

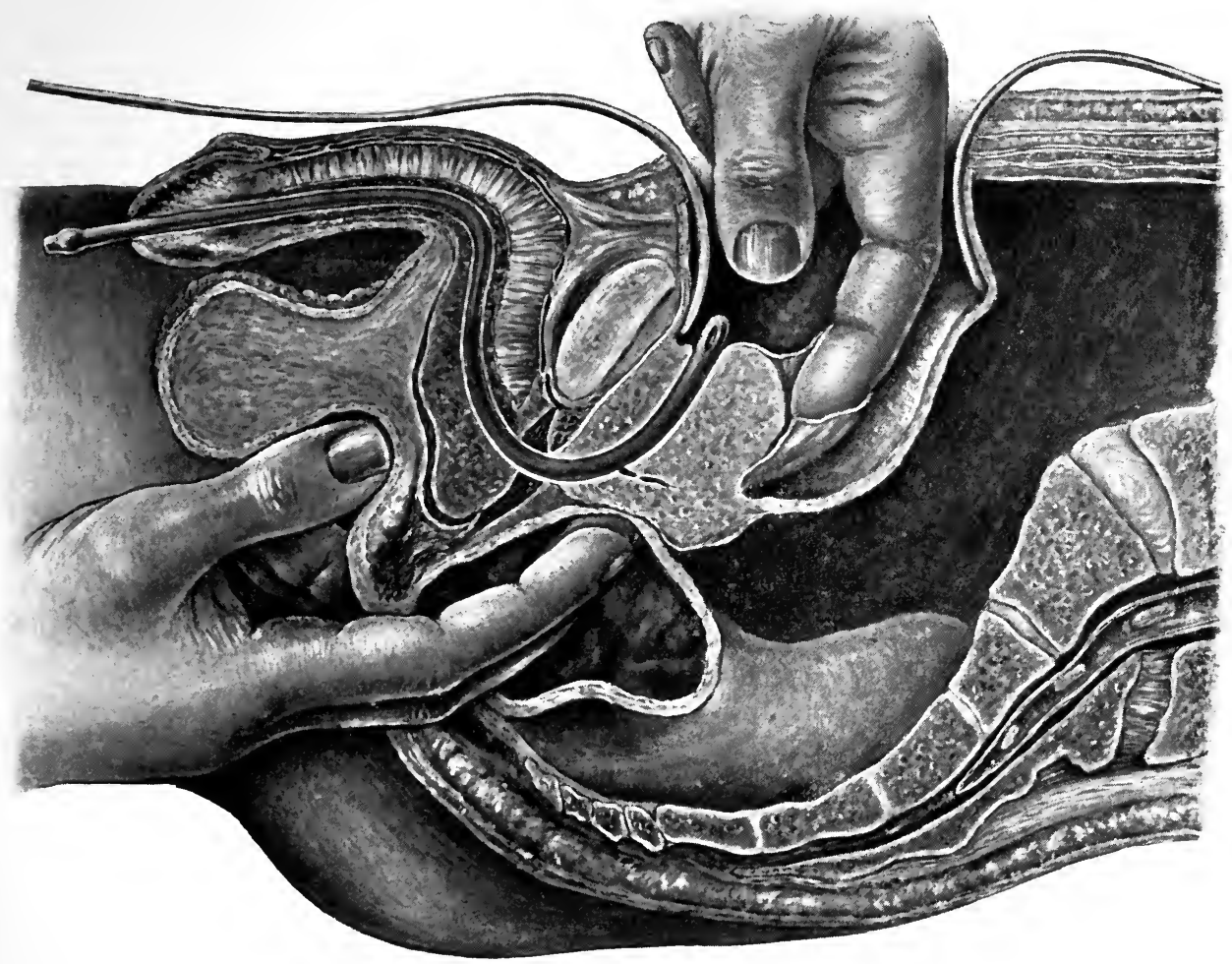

Suprapubic enucleation of the prostate. Counterpressure is made with the other hand in the perineum and rectum. (Deaver.)

Removal of Prostate.- Scratch an opening with the finger nail, or make it with a scalpel or scissors through the floor of the bladder over the most prominent part of the intravesical projection of the prostate on either side of the vesical outlet.

Insert the finger tip between the surface of the gland and the outer fibrous sheath and enucleate in the same manner as that described in the first of the perineal methods of performing prostatectomy (Fig. 215).

vol. $\mathrm{I}-26$ 
The gland may be removed in one mass, or lobe by lobe. It is immaterial which is done.

If in one mass, the prostatic urethra and the ducts will be destroyed. If care be taken and each lobe removed separately, the prostatic urethra and the ducts may be preserved, as has been described and demonstrated in connection with the perineal operation.

The enucleation is made more readily if the gland is raised and held fixed in this position while it is being done. This is accomplished by placing two fingers in the rectum and the thumb against the perineum and pressing the gland upward

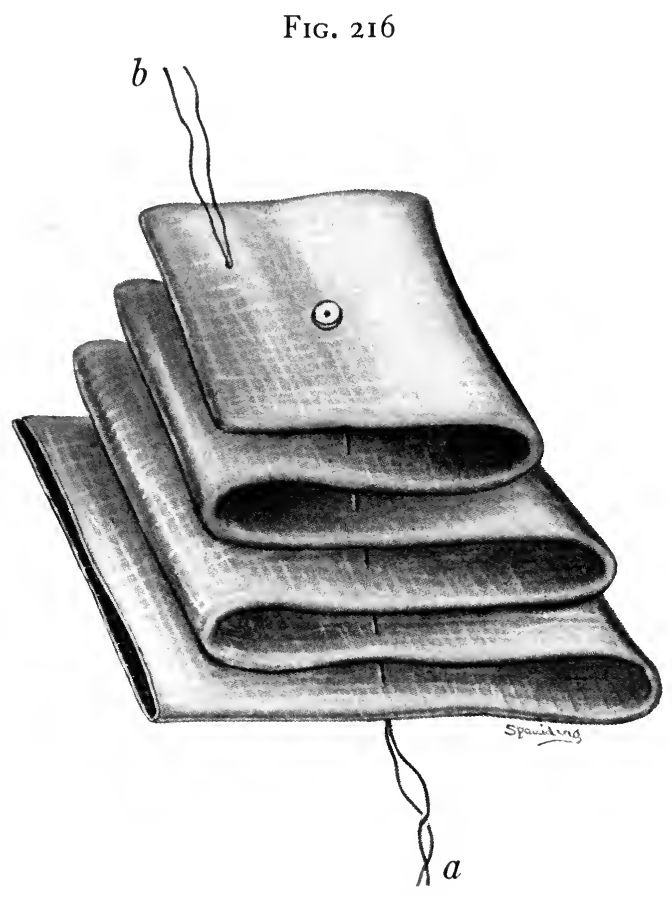

Cabot's tampon. and backward toward the surface of the abdomen (Fig. 216).

The middle lobe is removed readily by splitting the mucous membrane of the bladder overlying it and enucleating it, together with those lobes, or separately, afterward. If it be very high-reaching and large, it is better to remove the middle lobe before taking out the lateral ones.

If the outer fibrous sheath should be torn in doing the operation, and bleeding should occur, it is best controlled by pressure with a Cabot tampon (Fig. 216). The tampon consists of a long strip of sterilized gauze folded upon itself in the manner shown in Fig. 216. In order to apply it to the spaces made by the removal of the prostate, a perineal urethrotomy incision must be made. The thread $a$ is attached to a probe, which is then passed into the bladder through the suprapubic cystotomy incision and out again through the perineal opening made in the urethra, carrying the thread with it. The tampon is then pushed into the bladder, pressed firmly into the spaces made vacant by the removal of the gland, from some part of which the bleeding will be taking place, and maintained there by drawing tight the thread which has been passed through the perineal opening, and securing it on the surface of the perineum in whatever way the surgeon may prefer. Attaching it to a short bit of strong drainage tube or to a good-sized button which rests 
against the surface of the perineum is an easy way of doing this. The other thread of the tampon $b$, which is attached to the upper layer of the folded gauze only, is led out through the suprapubic incision.

When it is desired to remove the tampon, the lower thread is cut, and the upper one, $b$, pulled upon; in doing this, the gauze is unfolded bit by bit, and comes away easily, instead of with great difficulty, as would be the case were it necessary to take it out in one large mass without unfolding it.

After-treatment of Suprapubic Prostatectomy.-(For suprapubic drainage of the bladder, see section on the Bladder, chapter on Operative Technique.)

Comment.-The suprapubic operation has the advantage of being rapid of performance, and stands on equal terms with the first of the perineal methods described, in this respect. Like that method, it destroys a large part of the prostatic urethra. In some cases it may, however, be possible to preserve the prostatic urethra intact if the gland is removed lobe by lobe. If it is taken out in one mass we do not believe that it is possible to avoid injury to the prostatic urethra in the performance of the operation.

The floor of the bladder is necessarily injured in the suprapubic operation. In the perineal operations it is not damaged.

The enucleation of the gland in the suprapubic and the first of the perineal methods described is carried out in precisely the same manner, the only difference being that in the high operation it is taken out above and in the other below.

The suprapubic operation is, in our experience, rather more easily done than any of the other methods. It is less liable to be followed by incontinence of urine than the perineal procedures. It has a higher operative mortality attaching to it than the perineal operations have. It is neither more nor less liable to injure the rectum than are the perineal operations. There is no need of having this accident occur in either the high or the low operations.

The Combined Operation.-Nicoll's Technique ${ }^{10}$ (1894).-Incision.-As for suprapubic cystotomy. Then the inverted $\mathrm{V}$ or the curved prerectal perineal incision, the patient's posture having been changed to that for the lateral perineal lithotomy operation.

Operation.-The above steps having been taken, and the posterior surface of the prostate having been exposed, the gland is pushed toward the perineum by downward pressure with the tips of the fingers on the bases of the lobes within the bladder, and the operation proceeds as follows: The gland is attacked through the perineal incision. A sound is passed into the bladder. Open the outer fibrous sheath of the prostate by a 
median or crucial incision, made in its posterior surface. Through this incision enucleate each lateral lobe in turn with the tip of the finger, taking care to avoid opening the floor of the bladder or the prostatic urethra.

Nicoll says that he has succeeded in avoiding injury to either of these structures in 70 per cent. of his own cases. He uses the finger tip to enucleate the lobes, except in the cases in which there are especially tough bands of connective tissue attaching the gland to its outer sheath; in these instances he snips them across with a pair of blunt-pointed scissors.

Nicoll, ${ }^{10}$ in his recent publication, very properly calls attention to the fact that the practice of cutting off the gland in these hard adherent prostates as close as possible to the urethra and floor of the bladder, in order to save these structures intact, was customary with him long before it was again proposed as a new procedure by the Continental surgeons in 1902 and 1903 , and that he had already described it in 1894 , and again in 1898 .

Nicoll thus comments upon the question of the importance or the contrary of avoiding injury to the prostatic urethra:

"The submucous method (his own, which we have described above) was devised to obviate injury to the bladder and urethra at a time when it seemed essential to keep the (often putrid) urine from entering and lodging in the vacant prostatic capsule, with possible pyogenic toxemic effects, and when the preservation of the urethra appeared to be a sine qua non in securing the early restoration of normal micturition. Of late two facts of importance have been demonstrated by increasing experience.

"The first is, that many chronic catheter prostatics are immune with reference to putrid urine. The other is that the mucous membrane of the urethra is very readily reproduced after its removal by a prostatectomy."

These facts have of late led Mr. Nicoll to lean toward the removal of the gland in the ordinary suprapubic way, in view of the lack of necessity of for preserving the urethra intact.

Comment.-The perineal part of Nicoll's operation is its essential feature. The suprapubic step is quite unnecessary, and merely adds to the danger of the operation. His own results, which he divides into three sets of cases, are as follows as to operative mortality: 48 cases, 4 deaths, or 8 per cent.; 25 cases, 5 deaths, or 20 per cent.; and 38 cases, 2 deaths, or 5.5 per cent. A series of I I I cases and I I deaths, or Io per cent. mortality.

That is to say, the operative mortality is as high as that of suprapubic operation, while the perineal part of the procedure is as good, but no 
better, than that of the other methods already described. It antedated, however, all the operations which avoided injury to the prostatic urethra except that suggested by Pyle in I 892 .

Alexander's Technique ${ }^{11}$ (I895).-The first step of the operation is a suprapubic cystotomy. Through this incision the gland is pressed down toward the surface of the perineum by the tip of the finger, as is done in Nicoll's method.

The gland is then enucleated through the external perineal urethrotomy incision and in the same way as that in the first of the perineal operations described.

Comment.-Alexander, so far as we are aware, was the first to describe in detail the perineal step of this operation. It is the same as that practised by Watson and Goodfellow in their operations since I889 and I89I, respectively, but of which neither of them published a description prior to Alexander's.

What has been said about Nicoll's combined operations applies equally to that of Alexander, who, at the present time, has discarded the first of the steps and now removes the gland through the perineal route alone in the same manner as that described in the first of the perineal operations.

Special Steps.-Bryson ${ }^{12}$ and Guiteras ${ }^{13}$ both proposed to cut down suprapubically to, but not into, the bladder in order to exert downward pressure upon the gland. The step is irrational, and has never gained adherents.

\section{PROSTATOTOMY.}

The Operations of Bottini and of Chetwood.-Mercier was the author of the operations of prostatotomy and partial prostatectomy (I, division of, and 2, removal of a portion of the obstructing parts of the prostate). Mercier performed these operations by instruments of special forms,

FIG. 217

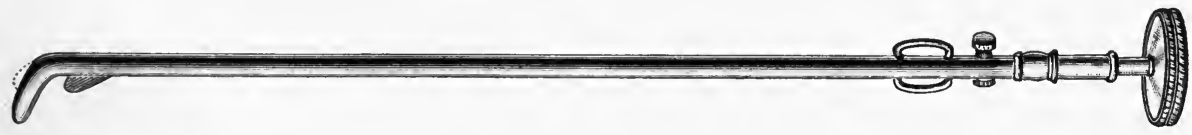

Mercier's instrument for dividing a bar at the neck of the bladder.

which were passed through the urethra from the meatus into the bladder (Figs. 217 and 218).

Gouley did them with the same instruments, passing them into the bladder through an external perineal urethrotomy incision. Bottini 
modified the operation of Mercier by performing it with a galvanocautery instrument, the heated blade of which divided the median portion of the obstruction (Figs. 219, 220, and 221). Watson modified this procedure by carrying out the second of Mercier's operations (partial prostatectomy) with a galvanocautery instrument, which was passed

FIG. 218

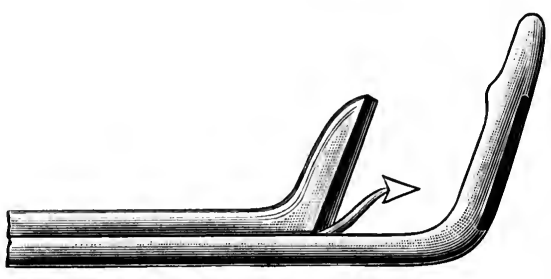

Mercier's prostatectotome.

FIG. 219

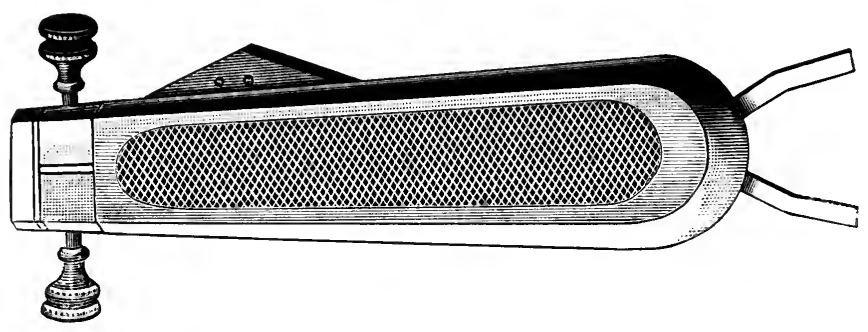

Bottini's apparatus, circuit breaker.

FIG. 220

FIG. 22I

Bottini's instrument.

through an external perineal urethrotomy incision into the bladder, and by which a U-shaped piece of the obstructing parts was burned out, and proposed to apply the same procedure through the suprapubic cystotomy incision. He abandoned the use of the instrument, preferring total prostatectomy (Fig. 222). 
Chetwood applies a modified Bottini instrument through the median external perineal urethrotomy incision.

Bottini's Operation. ${ }^{14}$ - Preliminary cystoscopic examination is essential to enable the cuts to be made safely and rightly.

FIG. 222

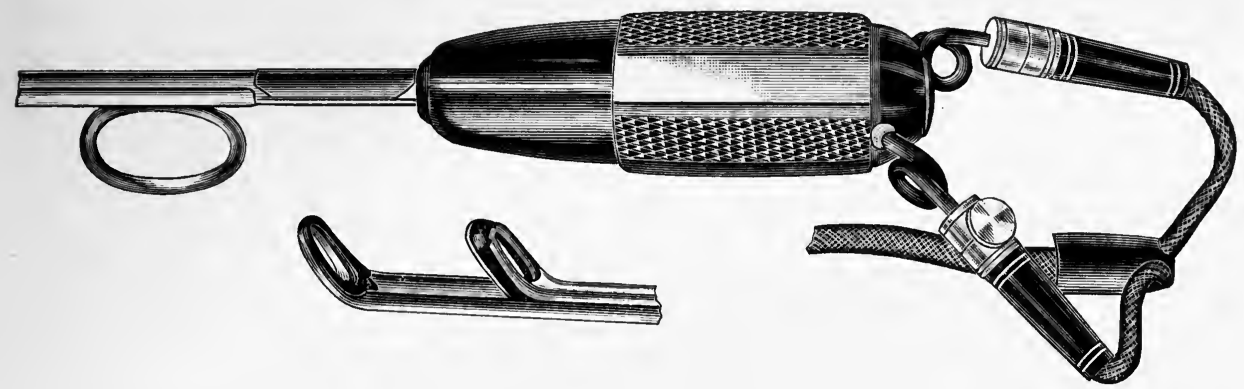

Author's galvanocautery prostatectotome, devised in 1888 and used by him in a few cases only, total prostatectomy having been preferred by him from the first.

FIG. 223

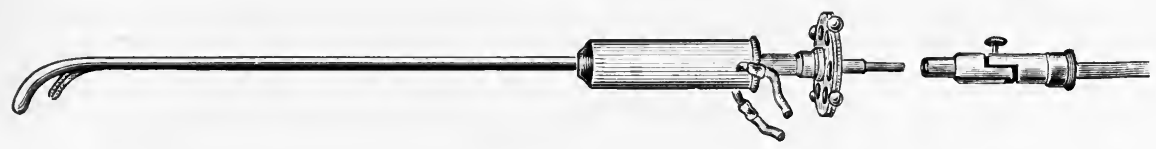

FIG. 224

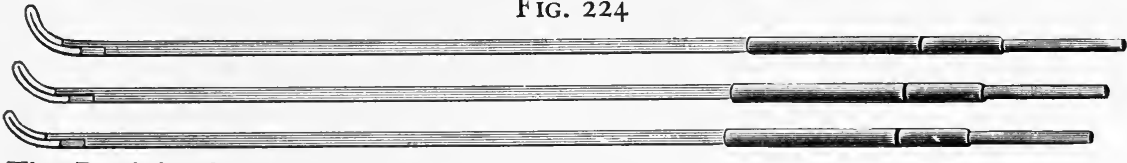

The Bottini galvanocautery modified by Young, who provided the instrument with blades of different lengths.

FIG. 225

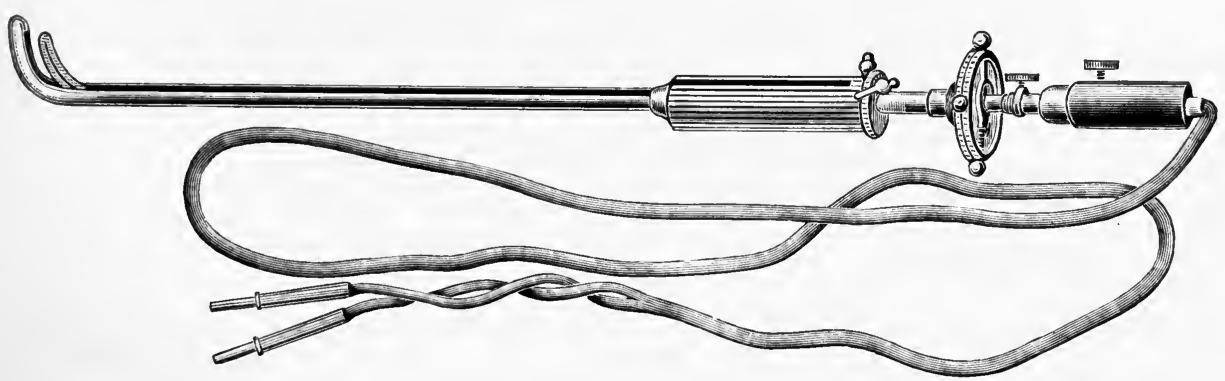

The Bottini galvanocautery as modified by Freudenberg.

Bottini's operation is the same as that of Mercier, except that it is done with a heated instead of a cold blade. Bottini, except in his later operations, divided only the median obstruction.

Freudenberg ${ }^{10}$ extended the scope of the operation by improving the instrument and applying the blade to the lateral as well as to the median 
lobe (see Figs. 225 and 226). Young ${ }^{16}$ (see Figs. 223 and 224) modified the Bottini instrument by using with it blades of different lengths, in order that the depth of the incisions might be adapted to the different conditions of the enlargements of the prostate which are encountered in different cases.

The instrument resembles a lithotrite in form, but instead of a crushing inner blade it is supplied with a thin plate of metal, which is heated by electricity and serves as a galvanocautery to burn through the obstructing part of the gland from within the bladder outward through the prostatic

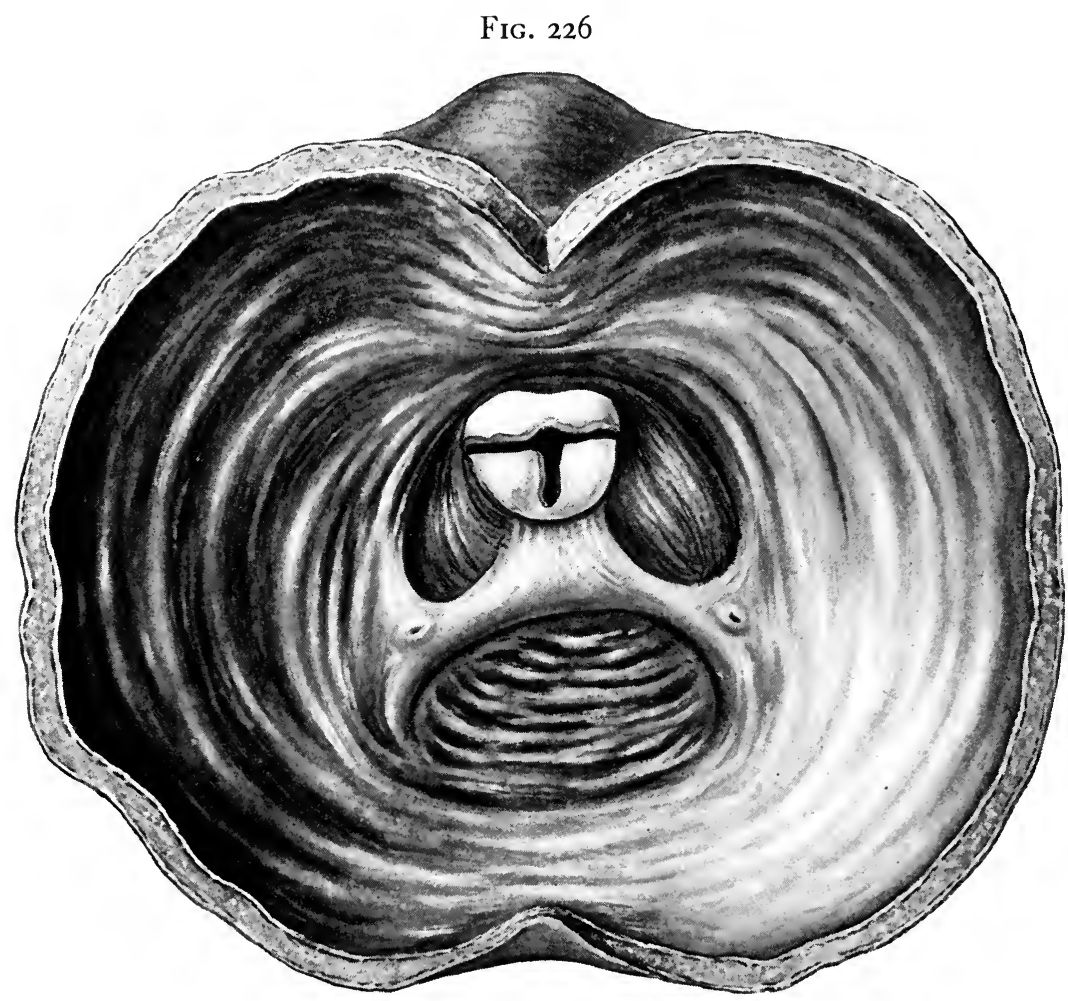

Bottini's incision within the bladder, as modified by Freudenberg. (Deaver, after Socin and Burckhardt.)

urethra, either in the median line of the obstructing middle lobe or in a diagonal or right angle direction through one or both of the lateral lobes, as may be required by the nature of the hypertrophy (Fig. 226). The instrument is passed into the bladder through the urethra from the meatus; its beak is then turned over against the posterior aspect of the intravesical projections of the prostate. The blade is then heated and drawn through the obstructing parts of the gland successively by means of a ratchet in the handle of the instrument (Figs. 225 and 227). 
The important points to be observed in the performance of the operation are these: the bladder should be washed as clean as possible beforehand and four or five ounces of the fluid left in it, or it may be distended with air. The operation was at first done with the bladder

FIG. 227
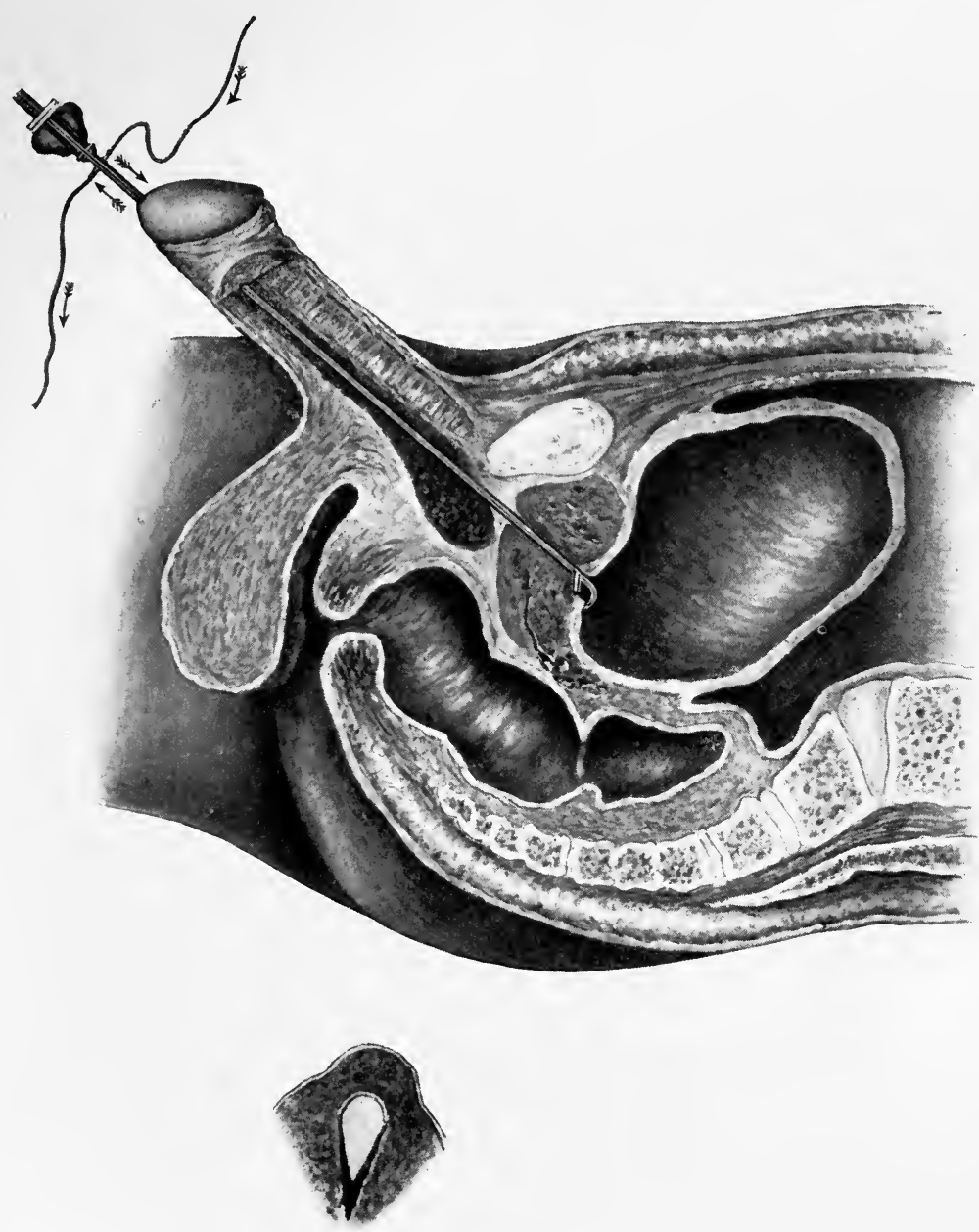

The Bottini instrument in use. Below is shown a transverse section of the prostatic urethra after its floor has been divided by the galvanocautery. (Deaver, after Socin and Burckhardt.)

empty; this was, however, abandoned, because of the danger of burning the walls by overheating the instrument, which was originally unprovided with a cooling apparatus. Later, Freudenberg devised a means by which a continuous current of cold water is kept flowing through the shaft of the instrument. If fluid is left in the bladder, difficulty arises, 
either from having the blade cooled so that it cannot be brought to the required degree of heat, or else the fluid becomes too hot. This led some surgeons to substitute air for fluid distention of the bladder. It must be noted that in one case, that reported by Rosenstein, ${ }^{17}$ the bladder which had been distended by air, exploded during the performance of the last incision in a Bottini operation. Upon doing laparotomy, the bladder was found to have been ruptured. The patient died a few days later.

When the blade is in position to make the cut through the prostatic obstruction, the current of electricity is turned on and fifteen seconds allowed to pass, in order to bring the blade to a white heat. One finger of an assistant or of the operator is passed into the rectum and serves as a guide and to steady the beak of the instrument during the passage of the blade through the gland. It is essential that the instrument be maintained in the same position through this entire manœuver, for the return passage of the burning blade to its original position should be in exactly the same channel as that which it has already burned through the obstruction. The heated blade is now drawn through the obstruction steadily and slowly at a rate of $\mathrm{I} \mathrm{cm}$. a minute. The length of the incision varies according to the extent of the hypertrophy in different cases. The channel burned through the lateral lobes is about $4 \mathrm{~cm}$. in the average case.

The blade should never be drawn out beyond the prostatic urethra. Injury to the membranous part of the canal is to be scrupulously avoided.

If the heated blade is moved too fast, it is liable to be bent, and then cannot be closed upon its fellow-the outer one-when it is desired to do so.

When the forward excursion of the burning blade has been made, the same route is traversed again in passing it back when closing the instrument.

When the (one or more) channels have been made through the obstructing parts of the gland, and it is desired to withdraw the instrument, the point of the beak is turned upward, the blades are shut together, the electric current turned off, the burning blade allowed to cool, and then withdrawn.

Chetwood's Operation. ${ }^{18}$-Operation.-Cleanse the bladder. A grooved staff is passed into the urethra and the latter is opened upon it in the membranous portion. The description given by Keyes ${ }^{19}$ says that a median perineal cystotomy is done.

The condition of the prostatic enlargements is explored through this incision with the finger. 
The instrument is then passed into the bladder and its beak turned over and drawn forward against one of the obstructing parts of the gland (Fig. 228).

If the obstruction is unilateral, the channel through it is burned by the heated blade in the middle of the lobe in an oblique direction; if median, the blade is made to pass through one or both sides of it.

If both lateral lobes are enlarged, the second one is burned through in the sáme way as was the first lateral one.

Fig. 228

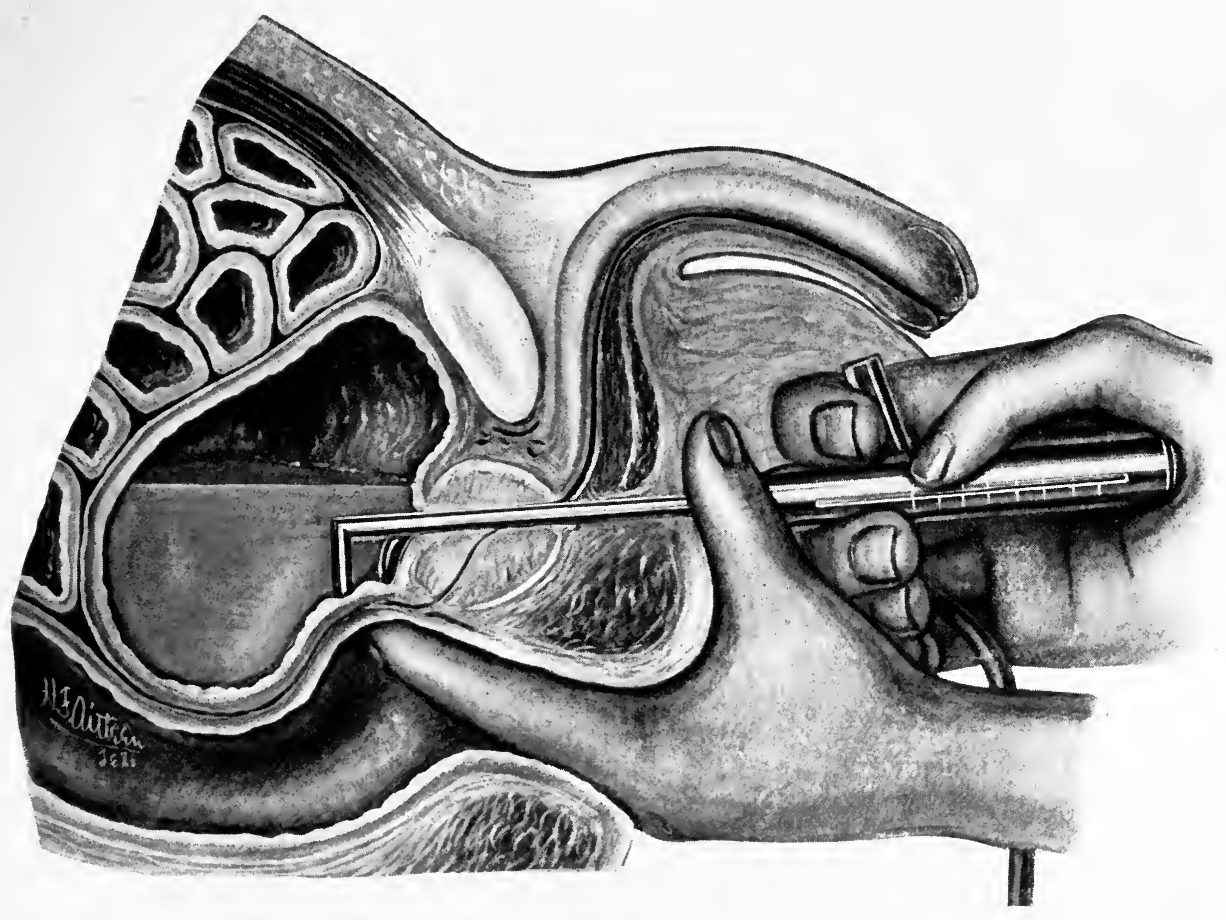

Chetwood's operation of perineal galvanoprostatotomy. (Keyes.)

If for any reason the procedure fails to bring the vesical outlet to the level of the floor of the bladder, it is advised that a partial median lobe prostatectomy by finger enucleation be done at once.

During the operation the staff of the instrument is kept cool by allowing a stream of water to flow through the urethra from the meatus. The instrument is provided with blades of different sizes, as in Young's modification of the Bottini instrument.

Comment.-The operation is, to all intents, a Bottini, done through a perineal cystotomy incision. We fail to see the necessity of making the perineal incision into the urethra into an extended cystotomy incision. 
The advantage claimed for the method as compared with that of Bottini is that it is more accurate, because of the finger exploration which is permitted through the perineal incision, which is undoubtedly true, and that it permits, if desired, the immediate performance of a partial prostatectomy in case of failure to correct the trouble by the Chetwood instrument.

The writer proposed and tried this method in $1888,{ }^{20}$ with an instrument of different form which he devised and which is shown in Fig: 222, and which was to be used either through an external perineal urethrotomy incision or through a suprapubic cystotomy incision. He very shortly afterward abandoned the use of the instrument and the method, it being inferior, in his belief, in all respects to perineal or suprapubic prostatectomy. The instrument was made in the form of a lithotrite, but its beak was expanded into the shape of a horseshoe, and the burning was done by a hot wire of the same shape, which was attached to the inner of the two blades. The channel which was burned through the obstructing median or lateral lobes was, therefore, of greater width than that produced by the Bottini.

\section{PERINEAL PROSTATOTOMY FOR ABSCESS OF THE PROSTATE.}

Abscess of the prostate may be reached and opened from the perineum in one or another of the following ways:

I. By an external perineal urethrotomy incision, and boring into the abscess with the finger tip, or cutting into it through the side of the prostatic urethra (Alexander ${ }^{21}$ ).

2. By the transverse prerectal curved perineal incision and open dissection through the perineal tissues.

3. By thrusting a narrow-bladed, straight bistoury into the abscess through the perineum, in front of the rectum and to the outer side of the urethra.

4. By the lateral perineal lithotomy incision or its equivalent, but without incising either urethra, bladder, or rectum.

I. This method is advocated by Alexander. It is done thus:

Operation.-The membranous urethra is opened by the usual external perineal urethrotomy incision. Through this cut dilate the prostatic urethra.

Keeping the forefinger in the prostatic urethra, pass alongside it a gum lance or similar suitable cutting instrument; turn its cutting edge against the lateral aspect of the prostatic urethra on the side of the lobe in which the abscess is located, and cut through the urethra into the gland and the abscess cavity. 
The same object may be accomplished by boring into the abscess with the finger tip or with the end of a steel sound.

2. (For description of this operation see Perineal Prostatectomythe open or dissecting methods.) The only difference that exists between the operations for total prostatectomy and the opening of prostatic abscess in this way is that in the former the gland is removed; in the latter the abscess cavity merely is opened. This step may be taken by incising the posterior surface of one or both lobes of the gland as may be required by the presence of an abscess in one alone or in both of them.

3. This is a method which has been successfully employed by the writer in ro cases. He does not know if it is practised by others or not.

Operation.-Pass a sound into the urethra and the forefinger of the left hand into the rectum as guides. The finger tip should rest on the area of fluctuation in the prostate, when it is well-defined enough to be appreciated by touch; otherwise, it is to be placed upon the apex of the prostate or just behind it.

Enter the point of a very narrow-bladed, straight bistoury about onehalf an inch outside the urethra and a little more than this distance in front of the upper margin of the anus.

Push the knife straight forward into the lobe of the prostate in which the abscess lies. The knowledge that the abscess cavity has been reached will be given by the freer movement of the tip of the knife as it enters it and by the flow of pus through the wound.

In withdrawing the blade, the outer part of the incision should be enlarged laterally in order to give to the track of the cut a V-shape, the spread of the arms of the letter being at the surface of the perineum, its point in the abscess cavity. This is for the purpose of insuring better drainage and to prevent premature closure of the orifice of the incision. The path of the incision may be spread open by a pair of straight dressing forceps. A gauze wick or drainage tube should be passed into the abscess cavity through the wound.

4. The following method of reaching a prostatic abscess is a favorite one with the writer, and seems to him to be the safest and least troublesome manner of evacuating it.

Operation.-Patient in lithotomy position.

Pass a steel sound into the bladder through the urethra, and have it held by an assistant.

Make the usual classic lateral perineal lithotomy incision on the side of the perineum corresponding to the lobe of the prostate in which the abscess is present. The incision should divide the skin and the more superficial structures of the perineum only.

Enter the forefinger tip into the wound and bore through the deeper 
tissues of the perineum with it until the peri-urethral tissue is reached and the sound can be felt lying within the canal of the urethra.

Continue the blunt dissection until the apex of the prostate is felt. Rest the tip of the finger upon this point, the back of it touching the urethra, in which the sound lies. Employing the tip of the finger as a guide, pass upon its anterior surface a narrow-bladed, straight bistoury into the lobe of the prostate and the abscess cavity.

Lusk $^{22}$ describes a similar method of approach to the prostatic abscess. Instead of passing through the anterior part of the deeper structures of the perineum, as is done by the lateral lithotomy incision, Lusk proposes (and has practised in one case which he reports in the article noted above) that the incision be made farther back, and slanted diagonally in a different direction; so that, instead of passing through the triangular ligament above the transverse border which marks its base or lower limit, he opens a fascial compartment farther back, just inside the inner border of the tuberosity of the ischium. The outer side of this compartment is represented by the tough obturator fascia. The apex of the fascial compartment is then punctured just beneath the transverse border of the triangular ligament, and the finger tip is passed upward and backward through this avenue of approach to the prostate, which is bulging forward into the path of the canal thus made. The incision is then made into the lobe of the gland which is the seat of the abscess, and its cavity opened and evacuated.

Comment.-This method of Lusk's we have never tried. It seems to us to be a very good one, but not to possess any advantages which make it preferable to the ordinary classic lateral lithotomy incision as a path of approach.

\section{OPERATIONS FOR MALIGNANT DISEASE OF THE PROSTATE.}

The technique which has been applied to the cases of cancer of the prostate may be considered under three headings, in accordance with the extent and character of the parts removed, thus:

I. Partial removals of the prostate, in which but a portion of one or both lobes has been removed by either the suprapubic or perineal routes.

2. Enucleation of both lobes without removing the fibrous sheath.

3. Removal of the gland with its sheath, the prostatic urethra, the vesical neck, with or without including the seminal vesicles in the operation.

The first case recorded is that in which Billroth ${ }^{23}$ removed a carcinomatous prostate from the perineum in 1867 by morcellement. This case ended fatally. In another instance the operation was followed by 
recovery, the patient living fourteen months and then dying of recurrence. Leisrink ${ }^{24}$ removed the gland in 1882 , together with its sheath, and united the divided ends of the vesical neck and the membranous urethra by suture.

A number of enucleations of the lobes within the fibrous sheath, and also excisions, curetting, and cauterizations were performed at different times between 1890 and 1900 .

FIG. 229

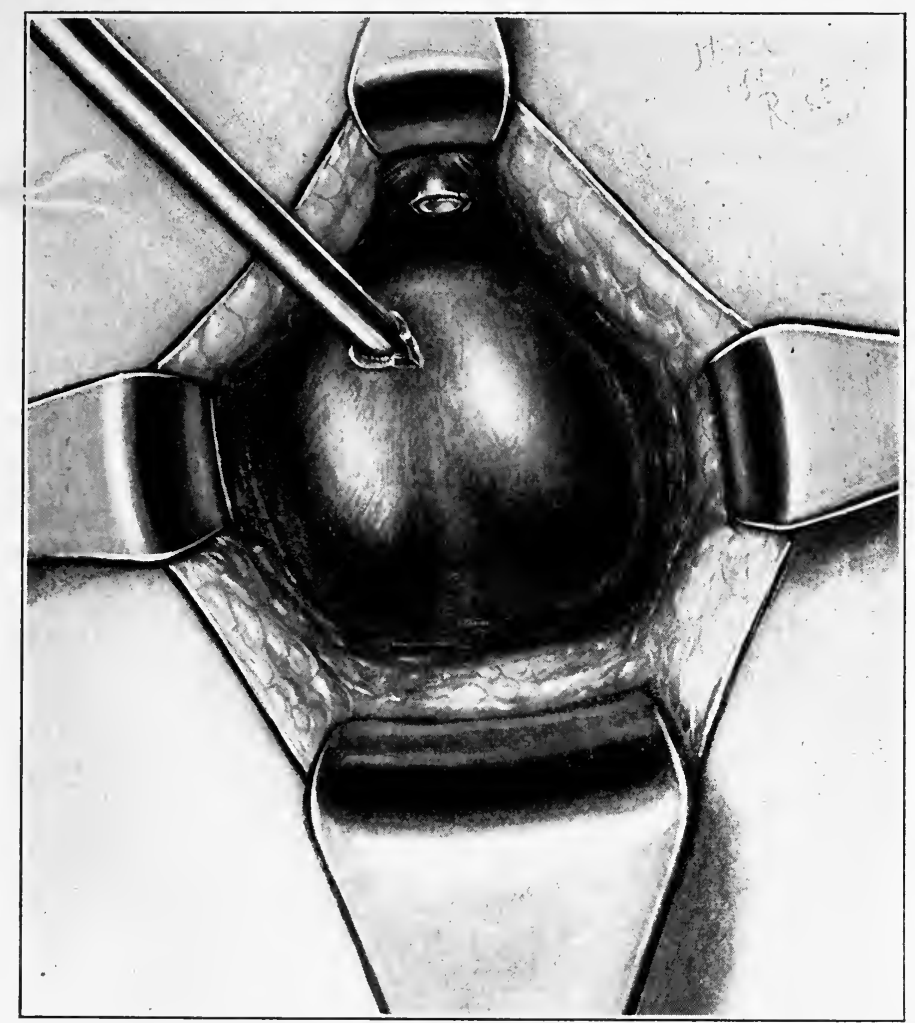

Young's technique for total excision of the prostate in cases of malignant disease. The prostate is separated from the surrounding tissues except posteriorly.

Küster ${ }^{25}$ removed bladder, prostate, sheath, and seminal vesicles in I $89 \mathrm{I}$ for malignant disease of the prostate and bladder. The patient died on the fifth day.

Fuller $^{26}$ removed prostate, sheath, and vesical neck in 1898 ; the patient lived for nine months without recurrence, and had control of urination. The disease then returned, and death occurred at the end of eleven months. In this instance there was no attempt made to unite the bladder to the divided urethra. The interesting feature in the case was the formation, in the course of healing, of a large channel between the 
divided bladder and urethra, which allowed free urination and readily admitted the passage of sounds of large caliber.

Young, ${ }^{27}$ in 1904 , modified the operation of Leisrink by including in it the removal of the seminal vesicles, which were not taken away in the latter's case, while, at the same time, he did not remove the whole bladder, as was done in the cases of Küster and Harris, in which the seminal vesicles were removed in conjunction with the more extensive operation. Young gave the operative treatment effective form, and its practical launching is to be credited to him.

Fig. 230

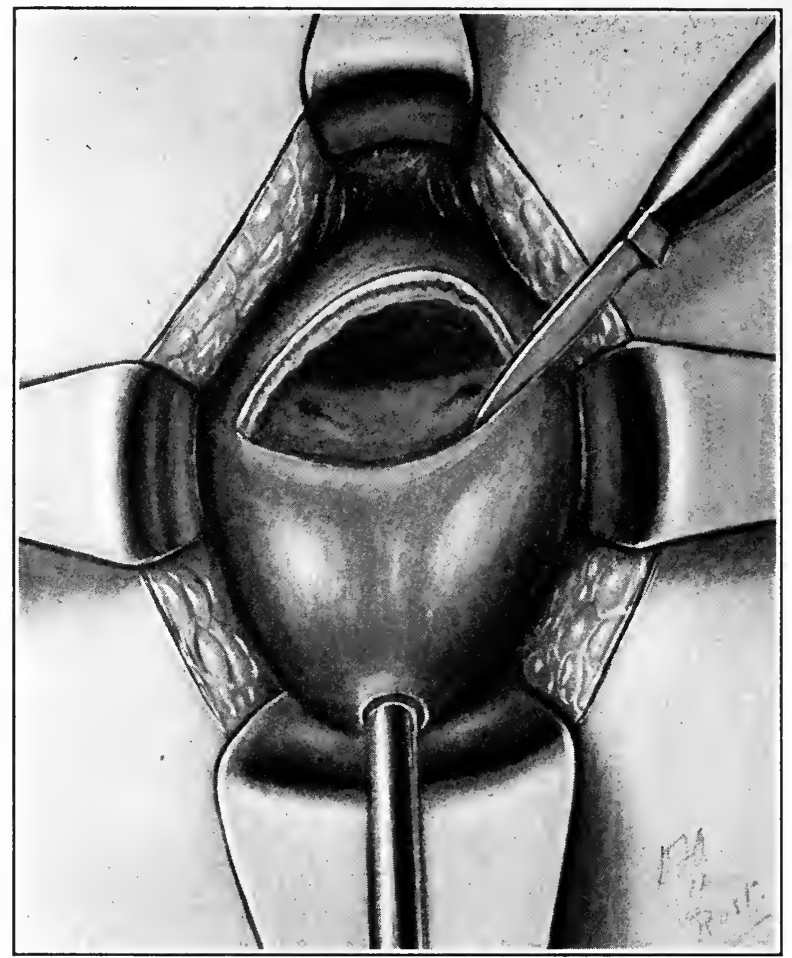

Young's technique: second step in operation of total excision of prostate.

These comprise the important steps thus far taken in the cases in which cancer of the prostate has been operated upon. The most thoroughly elaborated technique is that described by Young as follows: "An inverted V cutaneous incision in the perineum." (See Operations for the Hypertrophied Prostate.) "By blunt dissection the end of the bulb and central tendon are exposed, and the latter divided, exposing in turn the recto-urethralis muscle. . . . Urethrotomy upon a grooved staff, followed by the introduction of the prostatic tractor. "While traction is made upon this instrument, the rectum is separated 
from the prostatic capsule by blunt dissection until the entire posterior surface of the prostate is brought into view." (See Proust's technique.) "The tissues around the prostate" (in the case in connection with which the technique is described by Young) "were more hemorrhagic and the walls of the rectum more closely adherent to the capsule of the prostate than usual (see Fig. 229).

"Complete excision was carried out as follows: The handle of the tractor is depressed, thus exposing the membranous urethra anterior to it, where it is easily divided transversely, leaving a small stump of the

FIG. 23I

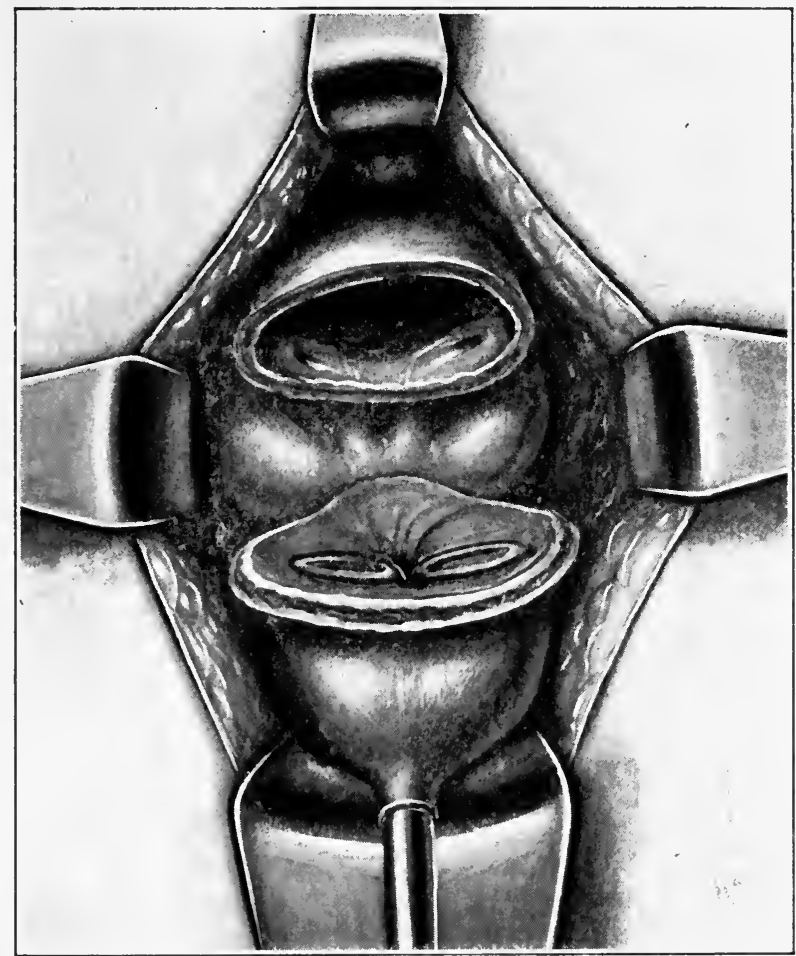

Young's technique: total excision of the prostate.

membranous urethra protruding from the posterior surface of the triangular ligament. By further depressing the handle of the tractor, the puboprostatic ligament is exposed, and easily divided by scissors, thus completely separating the prostate from all important attachments (except posteriorly). The lateral attachments, which are slight, are easily separated by the finger. The posterior surface of the seminal vesicles is then freed by blunt dissection, the now mobile prostate being drawn well out of the wound"' (Fig. 230). 
Young cautions against breaking through the fascia of Denonvilliers which he regards as an important barrier to the backward growth of the disease.

"The next step is to expose the anterior surface of the bladder, which is done by depressing the tractor and making strong traction. By this procedure the bladder is drawn down so close to the skin wound that it is easily incised at a point in the middle line about $\mathrm{I} \mathrm{cm}$. above the prostatovesical juncture (see Fig. 230).

FIG. 232

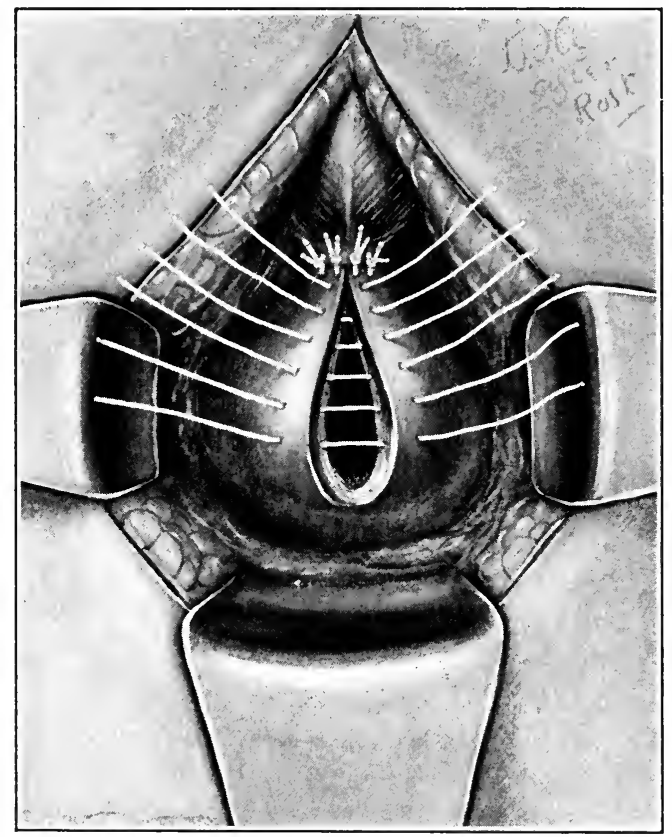

Final step of Young's operation for total excision of the prostate.

"By means of scissors the division is continued on each side until the trigone is exposed. After sponging out the wound, the ureters are easily found and the line of the incision is carried across the trigone, so as to pass about $\mathrm{I} \mathrm{cm}$. in front of the ureteral orifices (Fig. 23I).

"While still making traction, the base of the bladder is pushed upward thus exposing the anterior surface of the seminal vesicles and the adjacent vasa deferentia, all of which are freed by blunt dissection with the finger as high as possible, so as to remove, with the vesicles, as much circumjacent fat and areolar tissue as possible, on account of the lymphatics which they contain. The vasa deferentia, after being drawn down, are picked up on a small blunt hook and divided with scissors high up, care being taken that the ureters are not endangered. After division 
of the vasa, the seminal vesicles come down more readily and the deep adhesions are finally divided and the mass removed.

"The defect thus created is repaired as follows: The vesical opening, about $8 \mathrm{~cm}$. in diameter, sinks far back into the depths. The stump of the membranous urethra may have been obliterated by compression of the anterior retractor, so that it may be necessary to insert a soft-rubber catheter through the urethra from the meatus to discover it. The anterior wall of the vesical opening is caught with forceps and is easily drawn down to the membranous urethra, and the anastomosis is readily made with it (Fig. 232).

FIG. 233

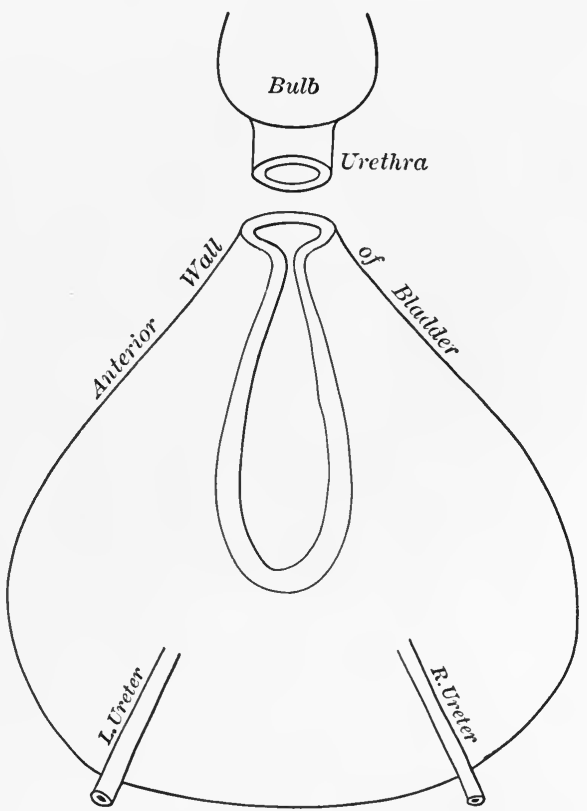

Diagram showing plan of vesico-urethral anastomosis. (Young.)

"The first suture is placed by inserting the needle through the triangular ligament above the urethra and out through the anterior wall of the membranous urethra; then through the anterior wall of the bladder in the median line, from within out, care being taken to include only the submucosa and muscle. This leaves the knot outside the junction of the two approximated edges. The thread is left long. Fig. 233 shows diagrammatically the plan of the vesical urethral anastomosis.

"Lateral sutures, including the peri-urethral muscular structures below, and two posterior sutures, complete the anastomosis of the membranous urethra with a small ring into which the anterior portion of the margin of the vesical wound has been fashioned by tying the sutures, as 
shown in Fig. 232. The remainder of the vesical wound is now closed with sutures.

"Alternate catgut and silkworm-gut sutures, left long, are the best."

\section{Bibliography.}

I. Parker Syms. New York Medical Record, I90I-02, p. 35.

2. Goodfellow. Communication read before the Academy of Medicine, California, April, I902.

3. Watson. Monograph: Operative Treatment of the Hypertrophied Prostate, I 888.

4. Pyles. Technique, New York Medical Record, I 892.

5. Proust. Comptes rendus de l'Assoc. Française d'Urologie, I90I.

6. Rydygier. Zentralbl. f. Chir., January 9, I904, p. 2.

7. Young. Conservative Perineal Prostatectomy, October, i 903.

8. Ferguson. Journal of American Medical Association, 1906.

9. Albarran. Ann. des Org. des Urin., February, I900; Comptes rendus de l'Assoc. Française d'Urologie, I90 I.

ı. Nicoll. British Medical Journal, August I I, I 906; London Lancet, April I4, I 894.

I I. Alexander. New York Medical Record, i895.

I2. Bryson. St. Louis Medical Record, February 22, 1902; Annals of Surgery, November, 1902.

13. Guiteras. Journal of American Medical Association, November 2, I90I.

I 4. Bottini. Arch. f. klin. Chir., I 877, xxi, I ; London Lancet, I885, i, 582.

15. Freudenberg. New York Medical Journal, February I2, I 898.

I6. Young. Journal of the Medical Society, January II, I902.

I7. Rosenstein. Deut. med. Woch., 1904, xxx, No. 39 .

I8. Chetwood. New York Medical Record, igor, lix, 767 .

I 9. Keyes. Genito-urinary Diseases, I905.

20. Watson. Op. cit., I 888; Annals of Surgery, June, I904.

21. Alexander. Annals of Surgery, December, 1905.

22. Lusk. Annals of Surgery, 1907 .

23. Billroth. Arch. klin. Chir., i 869, Band x, p. 568.

24. Leisrink. Ibid., I882, xxviii, 578 .

25. Küster. Ibid., I89 I, Band xlii, p. 864.

26. Fuller. Journal of Cutaneous and Genito-urinary Diseases, I898, p. $5^{8}$ I.

27. Young. Johns Hopkins Hospital Bulletin, October, 1905. 


\section{CHAPTER XIII.}

\section{CYSTOSCOPY.}

THE first attempts at cystoscopy were made by Bozzini, of Frankfort, in 1805 , with a mechanism consisting of an upright stand which carried a candle, a mirror which reflected the candlelight into a tube that was attached to the other side of the upright stand. From this beginning, the steps by which the present instruments were reached were as follows:

FIG. 234

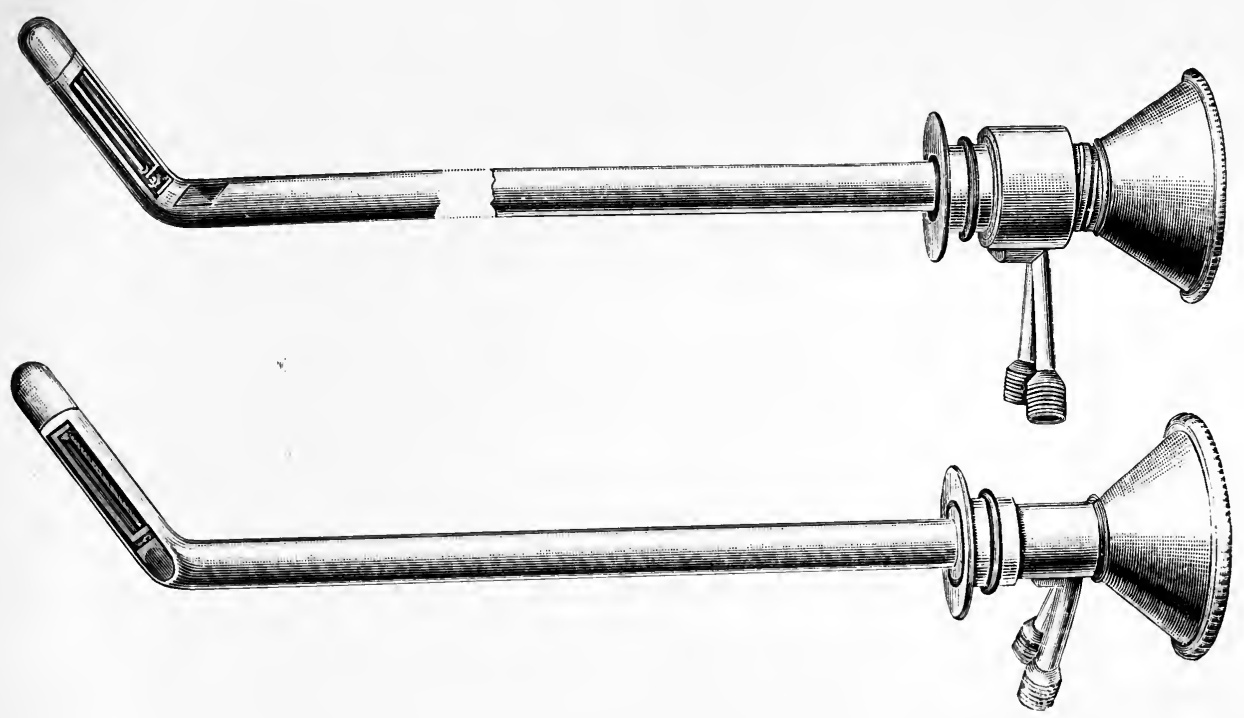

Nitze-Leiter platinum cystoscope.

The substitution of oil lamp and better mirrors in the place of candlelight and the reflector of Bozzini, which were introduced by Fisher, by Cruise, and by Desormeaux.

The instrument produced by Leiter, which embodied the ideas of Nitze. In this cystoscope a platinum wire loop placed in the beak of the instrument and heated by electric current illuminated the bladder. This and its later modifications were made in 1876, I 877 , and 1879 (Fig. 234).

The application of the Edison incandescent lamp to the cystoscope. These instruments required a cooling apparatus on account of the heat generated by the lamps. 
The introduction of the irrigating ureteral catheterizing cystoscope, by Brenner, in I 888 .

The replacing of the hot lamp by a cold one.

The addition of a second ureteral catheter, which permitted the two ureters to be simultaneously catheterized.

The addition of a mechanism which allows parts of the interior of the bladder to be photographed.

FIG. 235

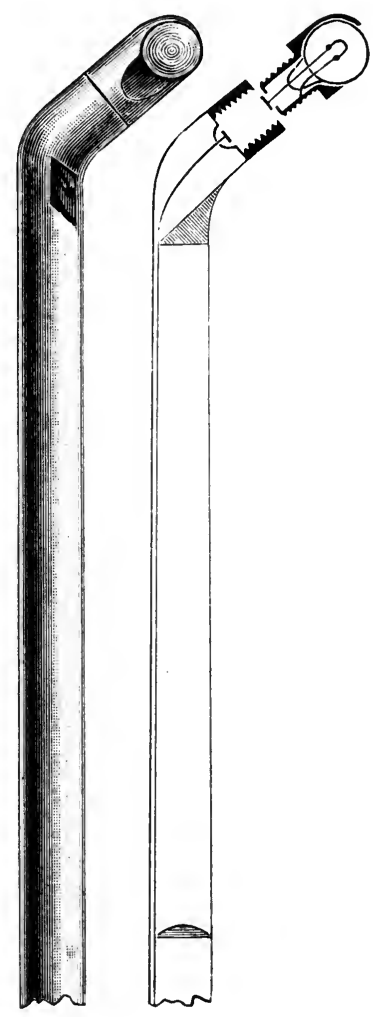

FIg. 236

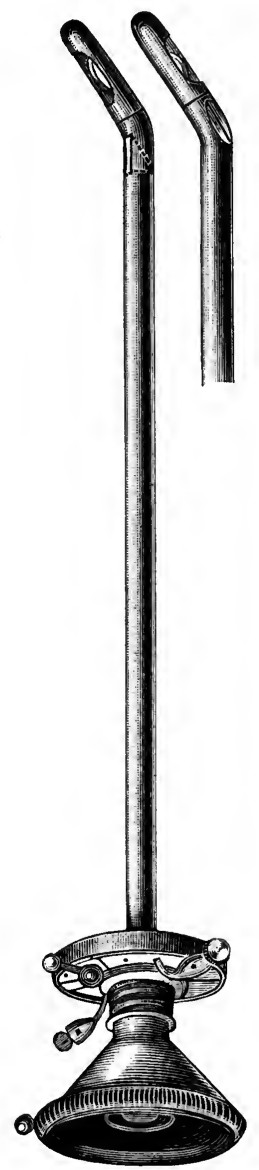

Nitze's incandescent lamp cystoscope. Two forms of Leiter's electric cystoscopes.

The addition of several different sorts of contrivances by which certain operations can be performed within the bladder under visual inspection.

The introduction of a lens and window by which a view of the vesical outlet and the immediately adjacent surface can be obtained.

The incandescent lamp instruments which were produced by Nitze and by Leiter almost simultaneously in 1887 , and the Brenner instrument, 
embody all the essentials which are to be found in the modern instruments of to-day (Figs. 235, 236, and 237).

Three other varieties of cystoscopes have been introduced. One is the cystoscope of Whitehead, which is of much larger caliber and carries a more powerful lamp than other instruments, and which is intended to be used through an external perineal urethrotomy incision. The second is an instrument intended to be used through a suprapubic cystotomy incision, and the third is for use in connection with air instead of water distention of the bladder.

We do not like any of these three kinds of cystoscopes. The objection to Mr. Whitehead's instrument is that it is unnecessary. The use of a cystoscope through a suprapubic cystotomy puncture is undesirable because, as was long ago demonstrated, a puncture of the bladder as in the second way proposed is much more dangerous than a free suprapubic cystotomy incision, and is therefore an objectionable step to take; while, on the other hand, it is an anomaly to use a cystoscope through a free suprapubic incision, since a direct view of the interior of the bladder can be obtained through such an incision, and the interior may be illuminated with a lamp of any sort that is suitable for the purpose.

The objection to the air distention and to the instrument devised to use with it is that it allows but a very limited field of vision and that the recognition of certain conditions of the bladder is not so easy as with water distention, $e . g$., tumors of the bladder, the villi of which often float in the fluid medium and give a most characteristic picture.

The essential factors to be remembered by the student in connection with cystoscopic instruments are that they are typified and may in a sense be classified in accordance with certain features which they have, thus: direct instruments, indirect instruments, catheterizing cystoscopes, irrigating cystoscopes, diagnostic cystoscopes, photographic cystoscopes, and operating cystoscopes.

There are two distinct types of instruments: (I) the convex, or direct; (2) the concave, or indirect. In the former, the window and the lamp are on the convex or outer surfare of the beak; in the latter, the lamp is on the inner or concave side of the beak, and the window is on the shaft, close to the junction of the latter with the beak and is also on the same side as the lamp.

With the direct instrument, the observer has a direct view of the part of the base and posterior wall of the bladder which is opposite the window. These are the only parts of the organ which can be inspected by it (Fig. 236).

With the indirect instrument the image is caught in an inverted form by a lens placed beneath the window; from this lens it is transmitted to 
two others near the ocular end of the instrument, by which it is righted again, and in that form passed on to still another lens in the ocular end, by which it is magnified, and comes to the eye of the observer after being thus modified (see Figs. 235 and 236).

With the indirect instrument a much larger extent of the bladder can be seen, and by turning its beak downward, depressing the shaft in order to do so, the base of the organ can be brought into view.

The ureter catheterizing cystoscopes are made in both the direct and indirect forms. The first one, that of Brenner, already referred to, carried but one ureteral catheter; later instruments were provided with two catheters, so that both ureters could be catheterized at the same time.

FIG. 237

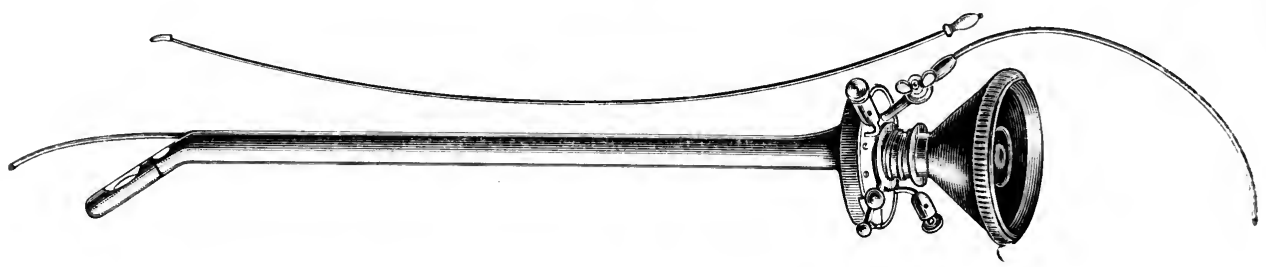

Brenner irrigating cystoscope.

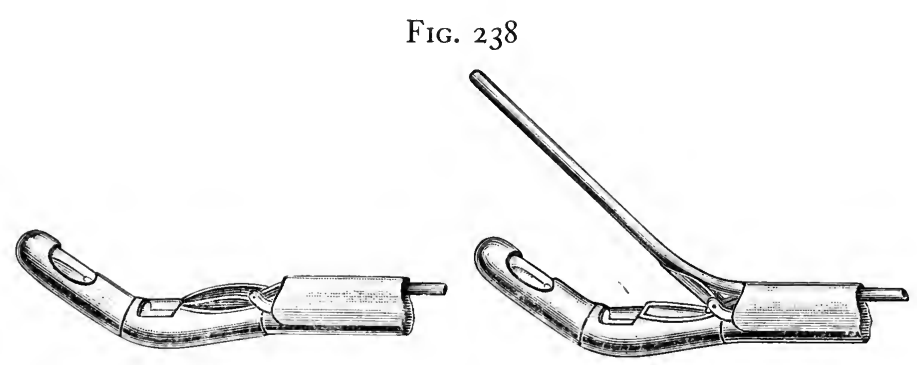

Beak of Albarran's ureter cystoscope.

The advantage of the direct as compared with the indirect is in the fact that, although the ureteral orifices are more difficult to find, yet, once found, it is easier to insert the catheters into them; while the contrary is true of the indirect instruments. The latter must be turned over so that the beak points downward, in order to bring the ureteral orifices into view and to insert the catheters into them (Figs. 237 and 238).

Irrigating Cystoscopes.-The irrigating cystoscopes differ in no respect from others, except in the addition of small canals, through which an ingoing and an outgoing current of water can pass, and which are included in the wall of the shaft of the instrument.

In most instances this contrivance is of but little value, except for the 
purpose of diminishing or increasing the volume of fluid in the bladder, for the channels are too small to allow of the passage through them of blood clots, of flakes of epithelium, or of thick pus.

In practice, we have always thought that time is saved, without any disadvantage being incurred, by removing the cystoscope from the bladder and then thoroughly irrigating the latter through a good-sized soft-rubber catheter, which cleans the viscus quickly and thoroughly; while, with a fine channel irrigating cystoscope, a long time is required; and, as we have said, the bladder cannot be cleansed by them when the contents are thick or when it contains clots, and it is precisely in those cases that the irrigation becomes necessary. The ordinary irrigating cystoscope, it should be said, will allow the exit of the fluid from the bladder when it is merely rendered cloudy by the admixture of slight quantities of mucus or pus, so that such instruments may be used to clear the fluid contents of such bladders as these without trouble, if the operator wishes to employ them at the expense of prolonging the examination.

Photographic Attachment to the Cystoscope.-The art of photographing the interior of the bladder, or rather of small parts of its interior, has been much advanced within the last few years. Its value consists in the securing of views for purposes of illustration, and as records of the actual conditions presented at certain times. Nitze contrived a circular disk, which is attached to the proximal end of the shaft of the cystoscope, and in the periphery of which are placed ten round apertures, each of which in turn can be brought in line with the visual axis of the shaft. Upon the disk is laid a gelatin bromide of silver plate, which is held in place by another disk with a screw attachment. In addition to this plate holder there is an ocular contrivance of special form, which is also attached to the proximal end of the shaft above the rotating disk.

The tube in which the lenses of this contrivance are placed can be pushed down so that the lower lens lies within the cavity of the shaft of the cystoscope and is thus in the visual line of the latter, and can then receive the image and transmit it to the eye of the operator; or the tube can be raised upward and drawn out of the canal of the shaft, thus leaving the latter unobstructed for the reception of the image upon the photographic plates. By rotating the disk of the photographic plate holder, ten pictures can be obtained in succession without making any change in the apparatus. The essential features of the contrivance are shown in Fig. 239, $a, b, c, d$.

Operating Cystoscopes.-Various forms of operating cystoscopes have been devised. The object of these contrivances is to snare or bite off small pedunculated tumors of the bladder, to crush small calculi which are so placed as to make the seizing of them in the jaws of a lithotrite 
without the aid of vision a matter of much uncertainty, and finally to insure the proper placing of the burning blade of the instrument in performing the operation of Bottini in cases of enlarged prostates.

That these various procedures can be carried out by those who have become especially skilled by long practice in the use of the cystoscope there is no doubt; whether it is important or especially desirable to employ this means for the purposes just mentioned is questionable.

FIG. 239
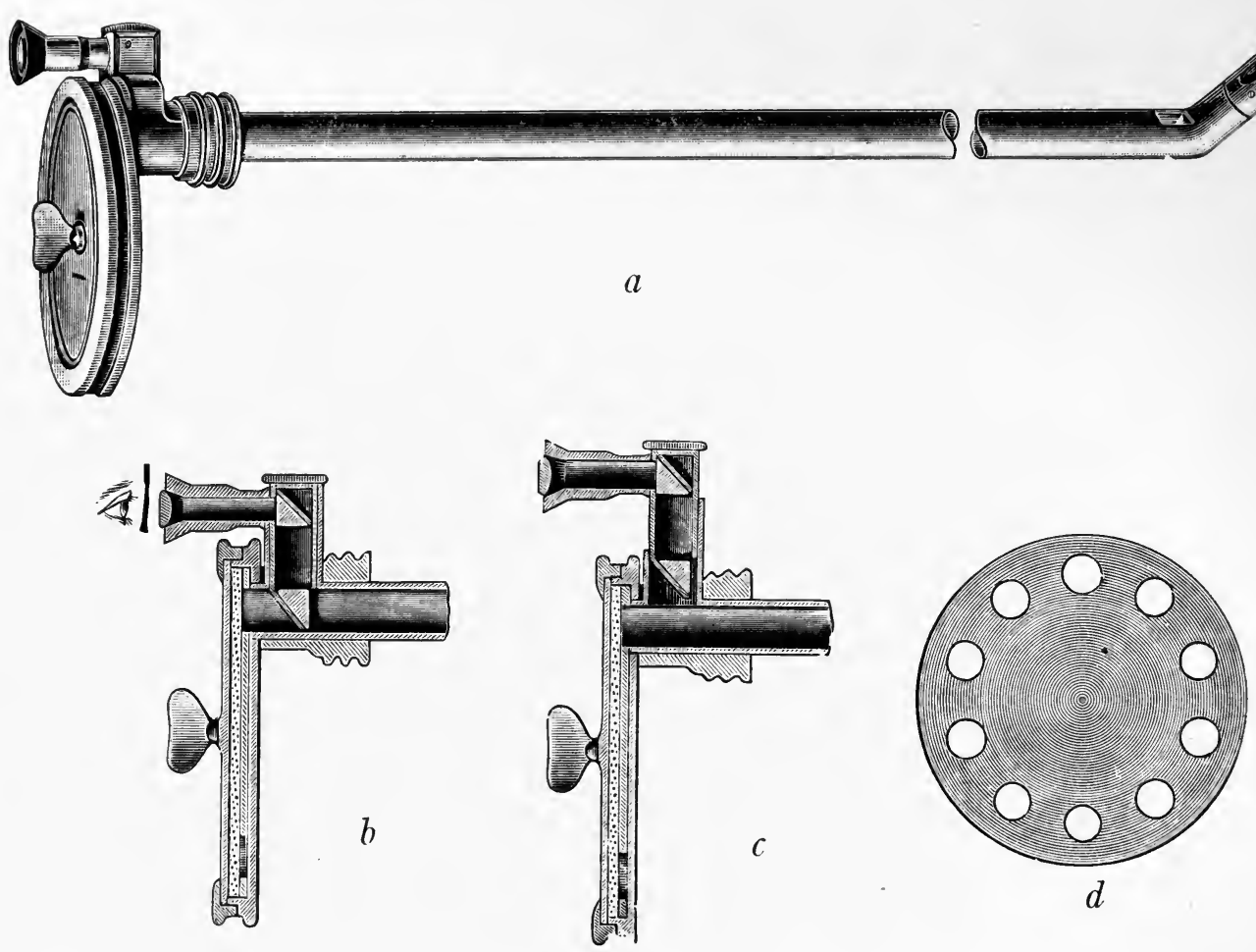

Photographic cystoscope.

This method of operating is open to the following criticism: In the case of small pedunculated tumors of the bladder, it is true that the growth may be caught in the loop of a wire snare or between the biting blades of an operating cystoscope, and taken away by a single operation. These growths are usually benign, and the procedure then suffices. When, however, the tumor is sessile, several "sittings" are necessary for the complete removal of the entire growth. Furthermore, this method of attack does not include in the removal a margin of the tissues around the growth, and, of course, it does not allow the performance of a partial resection of the bladder. Now, the number of single tumors of the 
bladder with long and delicate pedicles, which are the only ones that, in our judgment, are appropriate for removal by the operating cystoscope, is exceedingly small. The number of benign tumors among those which are multiple or broadly sessile is likewise small. To the latter class the procedure of removal with the operating cystoscope is, in our belief, utterly inadequate, and we strongly counsel against its employment. With the exception of the views of the late Dr. Max Nitze and a few other operators, the opinion of surgical experts of the present time is in accord with the above statements. (Further comment on this aspect of the subject will be found in the section on the Bladder, chapter on Tumors of the Bladder.)

With regard to the use of the operating cystoscope in cases of calculi which are placed in positions in the bladder from which it is difficult to extract them, or in which they are out of the reach of the blades of the lithotrite, or in those of encysted calculi, we see no advantage whatever in the employment of the instrument. The far more effective, quick, and equally safe way of dealing with such conditions as these is by the perfectly harmless procedure of making an external perineal urethrotomy incision and reaching and dealing with these special calculi through that route, employing digital exploration and the appropriate instruments for the purpose.

In the Bottini operation it is essential to use the cystoscope beforehand in order to determine the exact condition which the prostate presents, but except for this purpose the cystoscope is not required and cannot be utilized during the actual performance of the operation.

Cleansing of the Cystoscope.-It is a matter of a good deal of importance to know how to clean the cystoscope after it has been used, or rather to know what to avoid doing when cleaning it. Boiling, washing in alcohol and ether are not available for cleansing the cystoscopes, on account of the damage which is likely to be done to the lens settings. Carbolic acid, therefore, has become the most frequently employed medium for sterilizing and cleaning the instruments. It can be used in strong solutions for short applications, and in the weaker ones for long periods.

The instrument may stand as long as desired in I to 40 carbolic acid solution, and washed before placing it in this solution with a 90 per cent. solution. Ether or alcohol may be applied to all parts of the instruments except the lenses, and they are the best cleansing mediums to employ if this precaution is observed.

The ureteral catheters may be boiled in a concentrated aqueous solution of rock salt. The inner canals should be washed by forcing the same solution through them with a fine-pointed syringe. The contact surfaces of the instruments with those by which the electric current is transmitted from the battery should be kept clean and dry. They corrode easily. 
Manner of Making Cystoscopic Examinations.-In order to make a satisfactory cystoscopic examination the following conditions must be secured: (I) The fluid medium in the bladder must be clear. The meatus and urethral canal must be large enough throughout to admit the instrument readily through them and into the bladder. Spasm of the bladder must be overcome in order to permit it to contain a sufficient amount of fluid. (4) The quantity of fluid in the bladder must be not less than four and should be as much as six ounces. (5) The examination should be made painlessly, to accomplish which the posterior urethra, vesical orifice, and the bladder itself often require to, be anesthetized by the application of a cocaine solution. A solution of 2 per cent. is, as a rule, sufficient for this purpose; if not, stronger solutions may be employed-4 per cent. to 6 per cent. The latter will very rarely be required.

In a good many cases the meatus must be cut as a step preliminary to the cystoscopic examination, in order to allow the instrument to pass through it. Strictures, if present, must be dilated, and the urethral caliber made large enough to allow the cystoscope to pass easily into the bladder. These steps should be taken sufficiently long beforehand to allow time for the complete healing of the incisions.

Lubricating Medium.-The best lubricator is glycerin or liquid alboline; the latter is less slippery, but is nevertheless a good lubricator.

Position of the Patient.-The patient may be placed in a wholly, a partially, or a slightly recumbent posture, as the operator may desire. Most surgeons prefer the patient to be in a sitting position, with the body somewhat inclined backward, as when seated in an easy chair, and with the legs separated and supported by having the feet placed in stirrups, one on either side the examining table or chair. The operator places himself between the patient's legs, standing or sitting down, as he may find it most convenient. The patient's urethra should be on a level with the examiner's eye.

Insert a soft-rubber catheter, irrigating the urethra with sterile saline solution through it, as it enters, and empty the bladder. Irrigate the bladder until the returning fluid is perfectly clear, empty the bladder again, and inject into it an ounce or two of a 2 per cent. cocaine solution. In withdrawing the catheter, stop for a moment when its tip lies in the prostatic urethra, and let the contents of the instrument, which has been retained by pinching its end, run out into the urethra. After waiting for four minutes, irrigate the bladder once more and leave six ounces of the clear fluid in it.

Test the lamp of the instrument to be sure that it is in good working order. Lubricate the beak and shaft thoroughly with glycerin and pass it into the bladder. 
Turn on the current and begin the inspection of the inner surface of the bladder. If the indirect concave instrument is being used, make the first part of the examination with the beak pointed upward, rotating it first in one direction then in the other, in order to bring as much of the surface of the bladder as possible into view.

After examining all that can be seen with the instrument at one point, advance the beak, and repeat the movements of turning it from side to side. Next repeat the same process after having raised the tip of the instrument by depressing the shaft. Finally, turn the tip directly over, so that it points downward, and examine the base of the bladder and the orifices of the ureters. By carrying the shaft to one side, and then toward the other, in a horizontal plane, the beak will correspondingly be made to travel over a wider extent of the surface, and a larger view will thus be obtained. When the examination is completed, turn the tip of the instrument directly upward and withdraw it.

If the intention is to catheterize the ureters, and if the indirect instrument is employed for the purpose, the beak is turned over in the manner just described, so that the base of the bladder is brought into view. When the orifices have been located, the ureteral catheters are protruded beyond the end of the shaft sufficiently to bring them into view. The instrument is then held in such a way as to bring the point of the catheter directly over the ureteral orifice, and the instrument is advanced and its point is entered into the ureter. It should be pushed onward for a greater or less distance, according to the nature of the conditions which it is intended to study.

If the direct instrument is used, the ureteral orifices are located, the tip of the instrument is slightly raised, held directly above them, and the end of the catheter is inserted as described above.

Difficulties Encountered in Cystoscopy.-Certain conditions of the bladder and of the prostatic urethra constitute greater or less difficulties in making a cystoscopic examination. In some instances they make it impossible. Some forms of prostatic hypertrophy make the passage of the instrument or its rotation in the bladder so difficult as to render the examination useless. Trabeculation and diverticula in the bladder may make it impossible to recognize the ureteral orifice, or, when recognized, to insert a catheter into it. Conditions of the bladder in which the instrument causes hemorrhage to such degree that it is impossible to obtain a clear medium; and, again, the same is true of bladders in which there is so much pus as to constantly produce renewed cloudiness.

The kidneys may be the source of so much blood or pus which flows into the bladder as to make the examination impossible.

The wall of the bladder immediately beneath the vesical outlet may be so perpendicular in its direction that it is impossible to insert a ureteral 
catheter into the orifices of the ureters which cannot be brought into proper position for the purpose in such instances.

Unfortunately, it is, in some instances, precisely the conditions that we have named for the detection and for the study of which we most especially desire the aid of the cystoscope. If must be said, however, in general, that in the great majority of the diseases in which the cystoscope is valuable it can be used satisfactorily.

Cystoscopic Pictures Presented by the Normal Bladder and by Certain of the Pathological Conditions of the Organ.-The color of the normal mucous membrane of the bladder is yellow, shading slightly to a pale red. (Plate XIX, Fig. I.)

The vesical outlet presents the appearances shown in Fig. 2.

A typical picture presented by chronic cystitis is that shown in Fig. 3 .

Chronic cystitis showing mucopurulent mass surrounding the orifice of the ureter is seen in Fig. 4.

Ureteral cone-shaped orifice discharging blood is seen in Fig. 5 .

Fig. 6 shows the issuing of pus from the cone-shaped ureteric orifice.

Tuberculous ulceration at one stage of the disease is seen in Fig. 7 .

Figs. 8 and 9 show golf-hole.

Visual Fallacies.-Fenwick ${ }^{1}$ calls attention to the following fallacies, which may lead to errors in diagnosis:

The Ureteric Cone.-In the healthy bladder, when it is well distended, the ureteric orifices appear in the form of slits. In the partially distended bladder they appear as thick-lipped, pouting openings, and often also in the form of cones with a red color. These may strongly resemble a vesical tumor. (Plate XX.) The distinction can be made by the fact that the cone-like orifice of the ureter is subject to rhythmic contractions, sends out little jets of fluid periodically, and by its position in the bladder.

$R u g \mathscr{C}$, when viewed in profile, have somewhat the appearance of a vesical tumor. If the instrument is placed in such a way as to give a direct sight of them in face, the likeness ceases.

Adherent blood clot may, according to Fenwick, easily be mistaken for a dark oxalate of lime calculus.

Incrustations of vesical tumors are easily mistaken for calculus; the liability to error is increased by the hard substance that is felt by the tip of the instrument when it taps upon the incrusted surface. Fenwick points out the fact that usually small bits of the surface of the tumor will be seen showing at different points through the incrusting covering, and will thus allow the true nature of the condition to be recognized. 


\section{PLATE XIX}
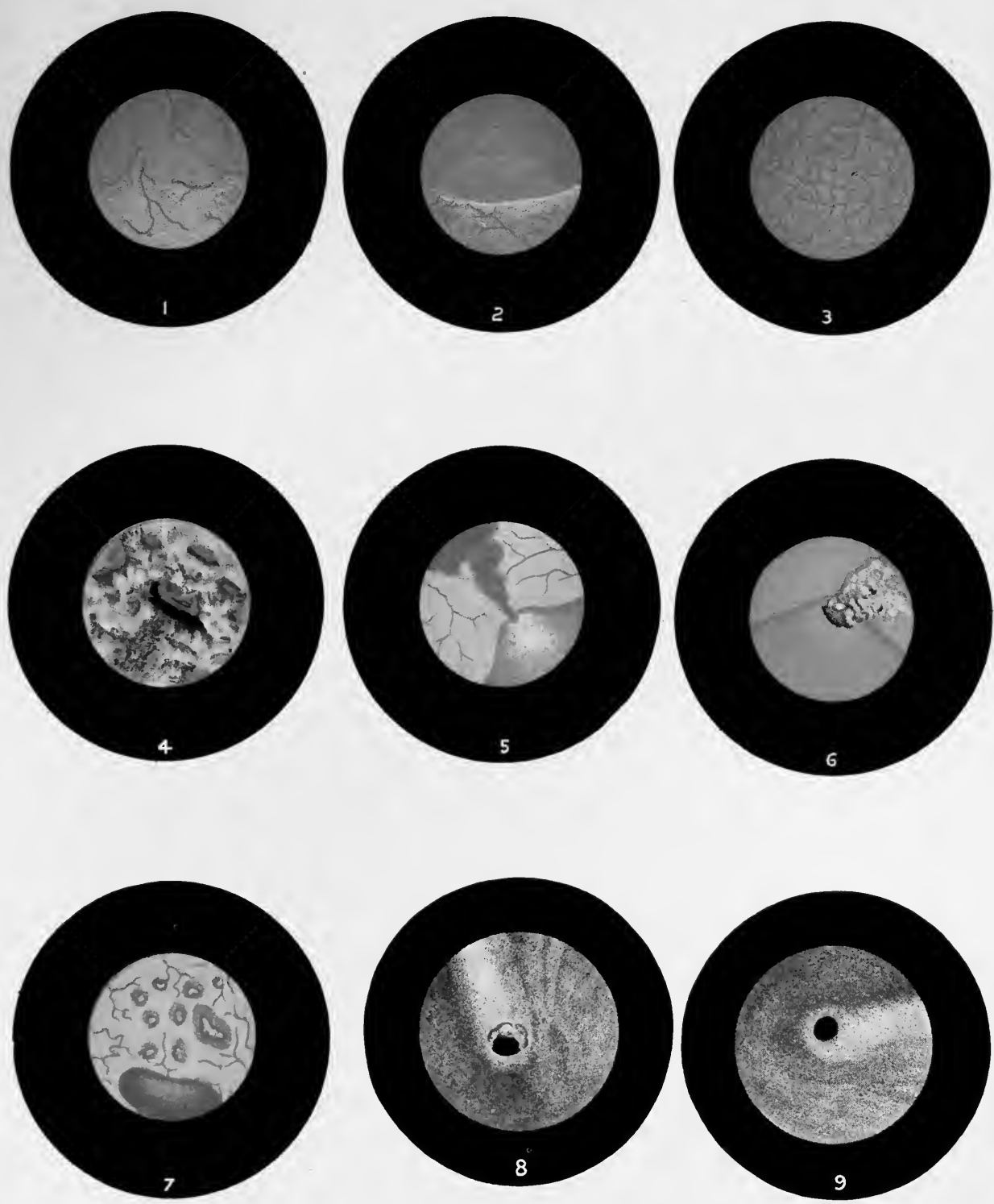

\section{CYSTOSCOPY. (Fenwick.)}

Fig. 1.-Color of normal mucous membrane of the tladder.

. 2.-Appearances of the normal vesical outlet.

“ 3.-Typical picture of chronic cystitis.

“ 4.-Chronic cystitis, with mucopurulent mass surrounding orifice of ureter.

“ 5.-Cone-shaped ureteral orifice, with blood issuing from it.

“ 6.-The same, with pus issuing from the ureteral orifice.

“7.-Tuberculous ulceration.

“ 8.-An open "golf-hole" ureteral orifice denoting dilatation of the ureter.

“ 9.-The same, with ulcerated edge. 





\section{CHA P TE R XIV.}

THE BLADDER.

\section{ANATOMY OF THE BLADDER.}

THE urinary bladder is a hollow, musculomembranous viscus, which serves as a reservoir for the collection of the urine from the ureters as they convey it from the kidneys.

FIG. 240

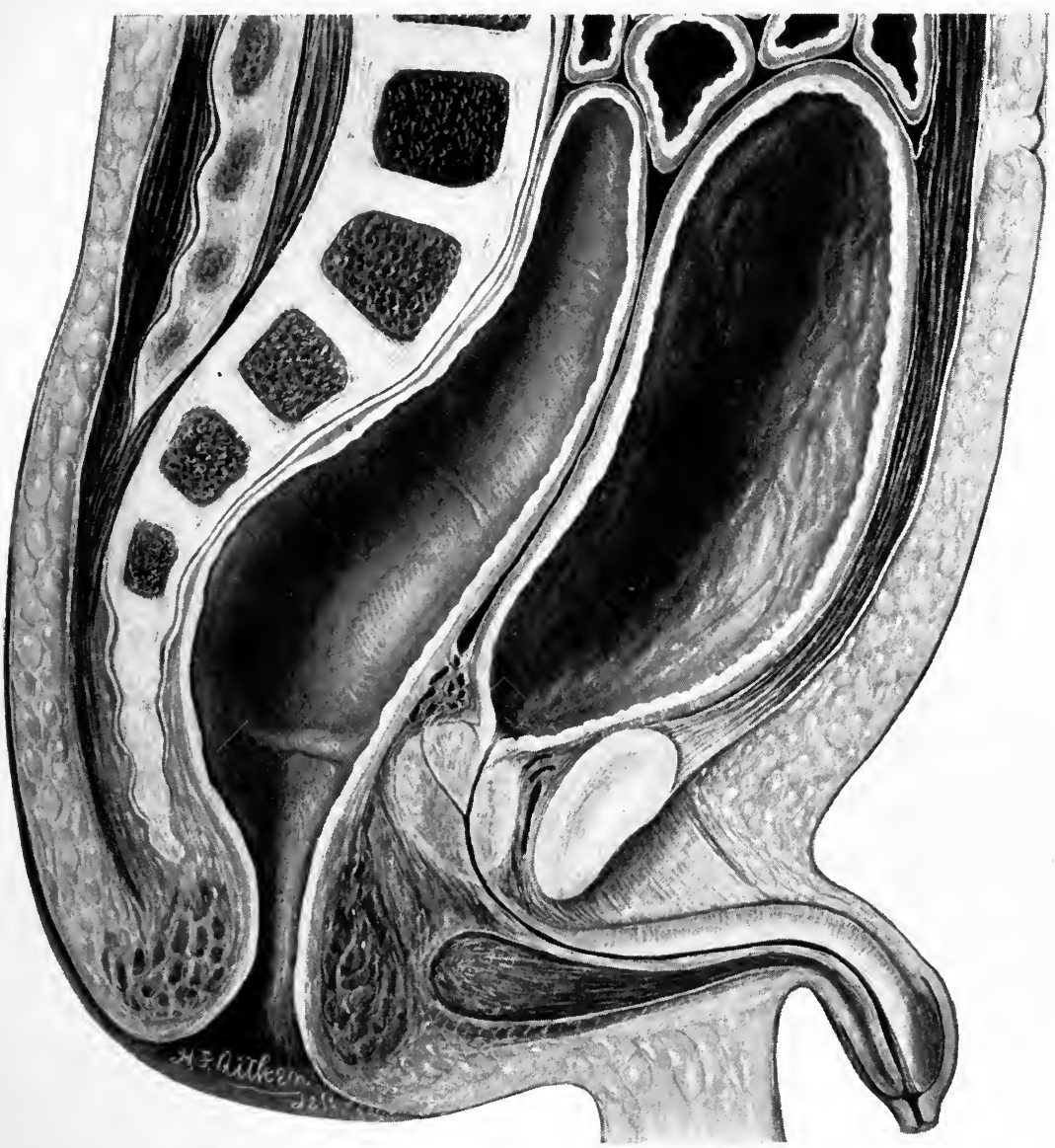

Showing the high position of the bladder in infancy. The height to which the bladder reaches is much too great; it should be one-half to two-thirds lower. 
Position and Form.--In the male subject the bladder lies between the rectum and the symphysis pubis; in the female it is placed between the uterus and the symphysis.

The position, form, and relations of the bladder vary according to the age, sex, and degree of distention of the organ. In infancy it has a - conical shape, and lies high in the hypogastrium (see Fig. 240). Later it sinks into the pelvis, becomes almost spherical in form, and slightly flattened anteroposteriorly.

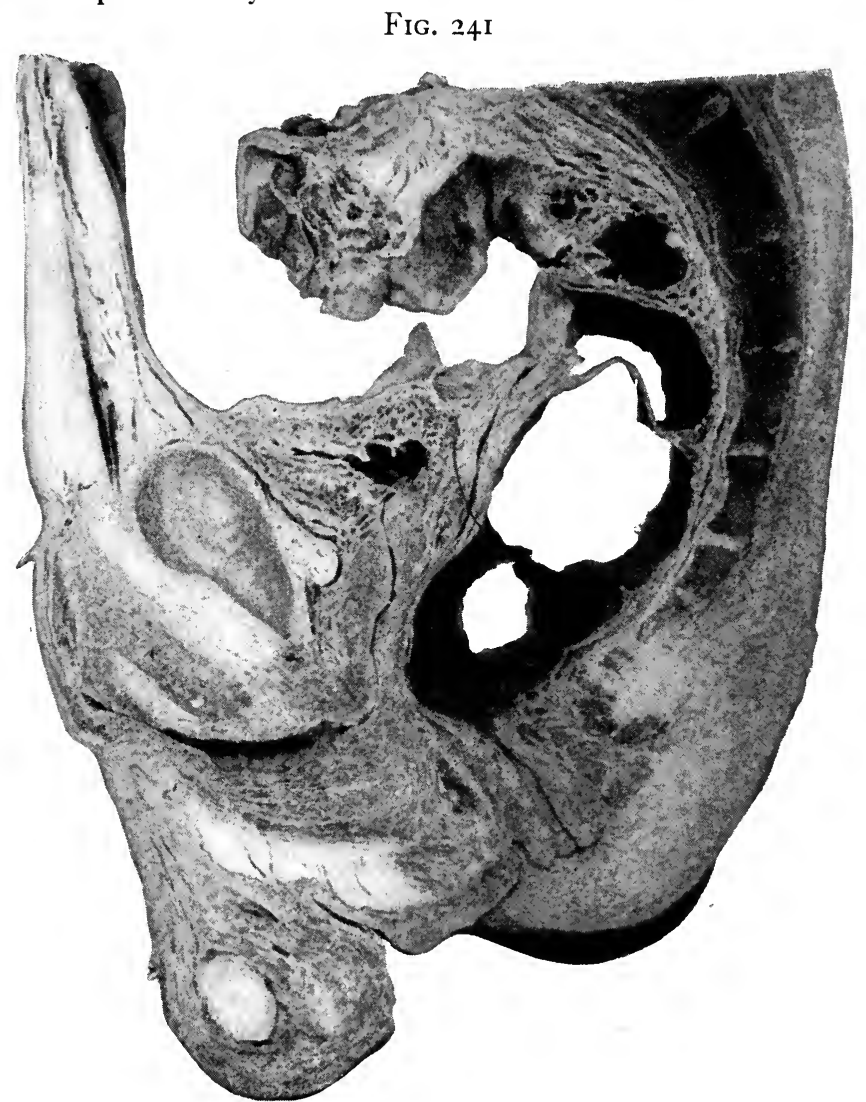

Showing the form and position of the bladder when empty.

When the bladder is empty its walls fall together and it takes the shape of a $\mathrm{V}$, roughly speaking, the anterior arm of the letter being somewhat shorter than the posterior one. The apex of the $\mathrm{V}$ is at the vesical orifice (Fig. 24I).

When normally distended (i.e., with a contents of about eight fluidounces) the bladder has an ovoid form and the position which is shown in Fig. 242.

As the organ becomes more distended, it rises above the pelvis into the abdominal cavity and becomes even more ovoid in contour; when 
extremely distended it may reach as high as the umbilicus. It is then readily palpable and can be seen in the lower part of the abdomen as a rounded tumor.

When rising in this manner, the peritoneal investment of the bladder shares in the upward movement, and its reflection upon the anterior wall is lifted (with not more than twelve ounces in the bladder) from two to two and a half inches above the upper margin of the symphysis pubis,

FIG. 242

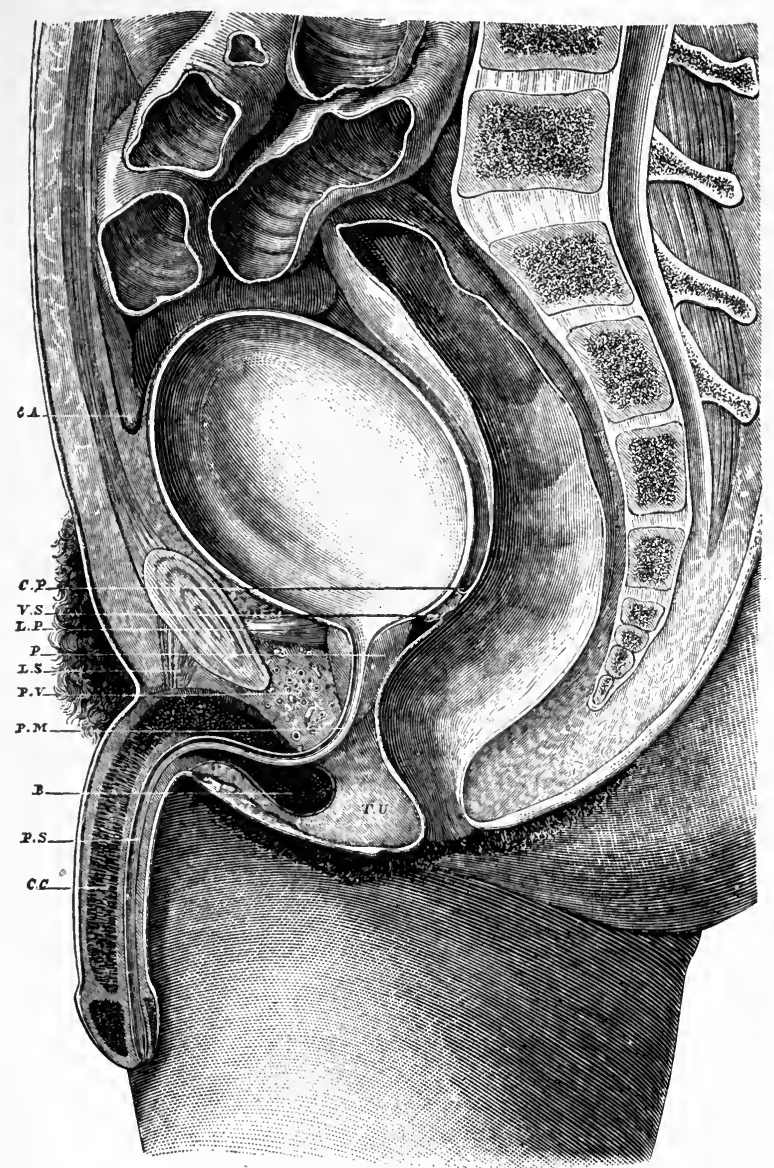

Oval form of distended bladder and Retzius' space. (Tillaux.)

leaving the area below it free from peritoneum. It is through this area, and because of its being below the peritoneal investment, that the incision into the bladder is made in the operation of suprapubic cystotomy. In like manner, the posterior reflection of the peritoneum is raised so that it stands from an inch to an inch and a half higher above the anal margin than when the organ is empty. In the latter position it is about two inches from that point. (See Figs. 243, 244 and Plate XXI.) 
Retzius' Space.-The space in front of the bladder below its peritoneal reflection, between the bladder and symphysis pubis, and downward beside the prostate is called the space of Retzius. It is practically obliterated when the organ is empty, but becomes evident, as the viscus rises, when distended. This space is important on account of its giving opportunity for the formation of abscess and because of being the site of infiltration and burrowing of urine and pus which may find lodgement there in connection with injury of the bladder or following operations upon it (see

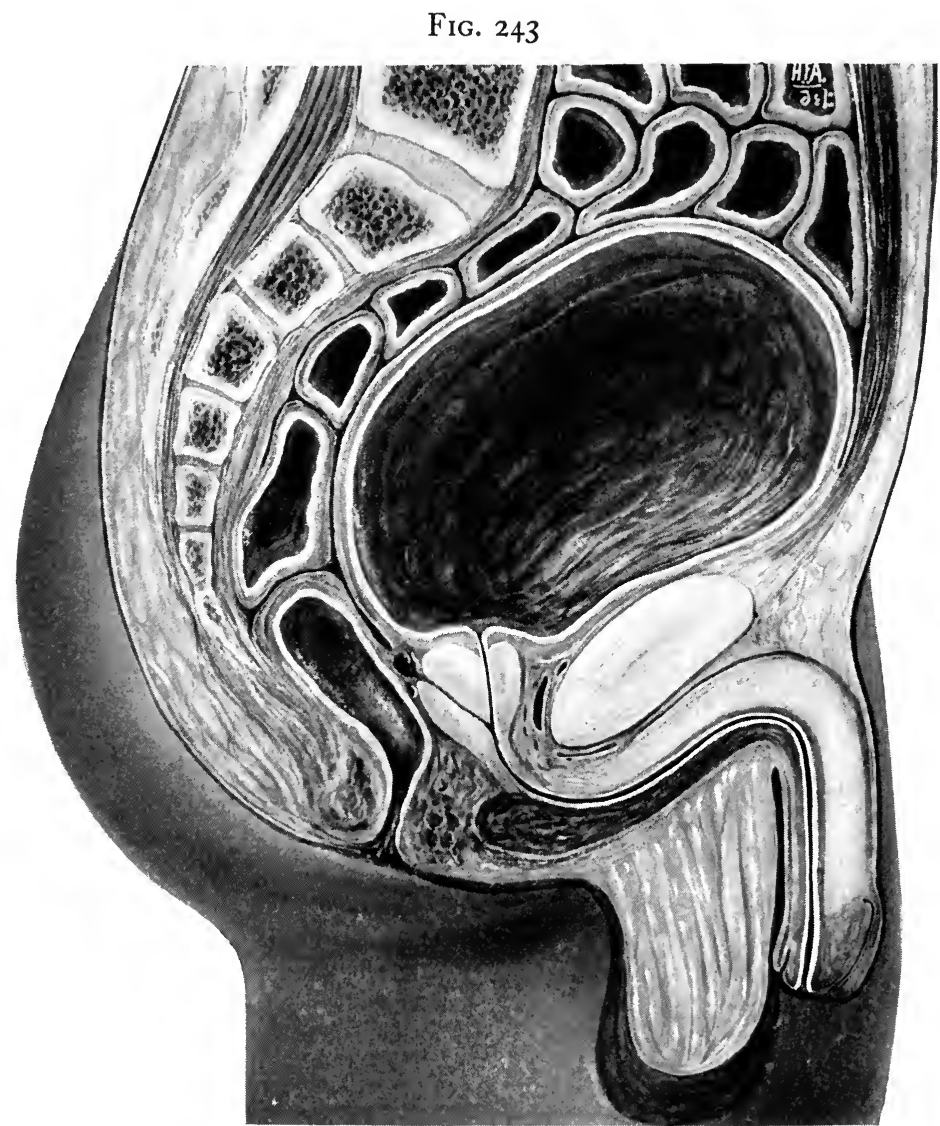

Bladder distended. Rectum empty. (After White and Martin.)

Fig. 242). This space is filled with areolar tissue. The fat which lies in this connective-tissue framework is the guide to the bladder when exposing it by suprapubic incision. The anterior wall of the viscus lies directly beneath this fat tissue, and the peritoneal foldis concealed by it.

Relations.-With the bladder empty, its anterior surface is in direct relation with the posterior aspect of the symphysis pubis. With the organ full, Retzius' space and its contents interpose between the two. 


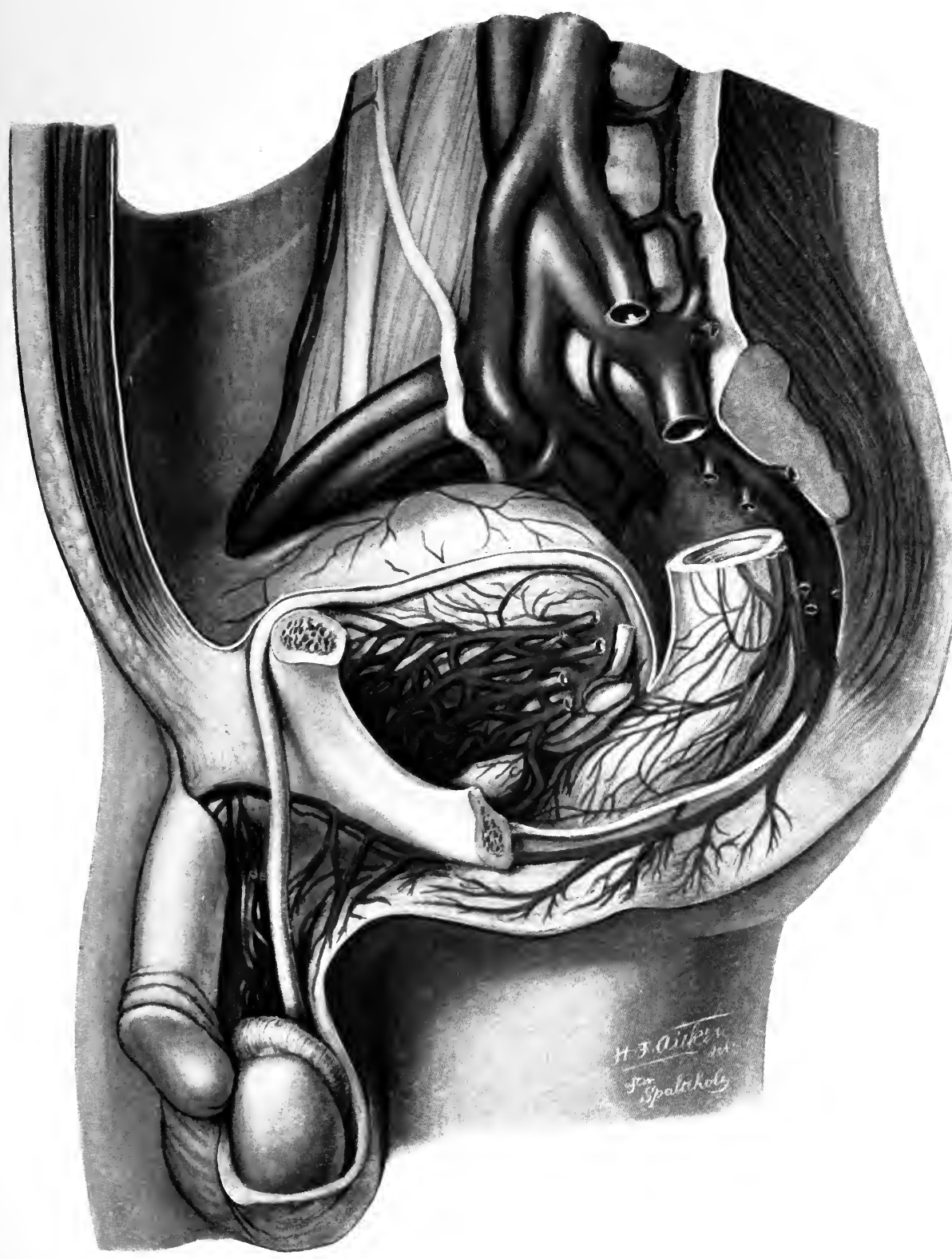

VESICAL PLEXUS OF VEINS; ARTERIAL SUPPLY AND RELATION OF THE PERITONEUM TO THE BLADDER; COURSE OF VAS DEFERENS AND POSITION OF SEMINAL VESICLES; COURSE OF THE URETER. 


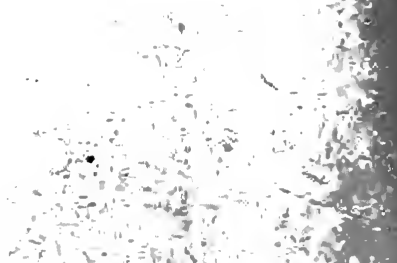
$\therefore$ (1s

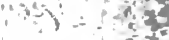


The upper part of the anterior surface, the whole of its superior surface, and the posterior aspect of it as far as a line on a level with the junction of the upper and middle thirds of the seminal vesicles are in direct relation with the peritoneum, which also extends downward over the lateral surfaces covering somewhat more than half of their extent. (Plate XXI.)

The posterior surface, except for the interposition of the peritoneum over the part of it described above, is in direct relation with the vasa

FIG. 244

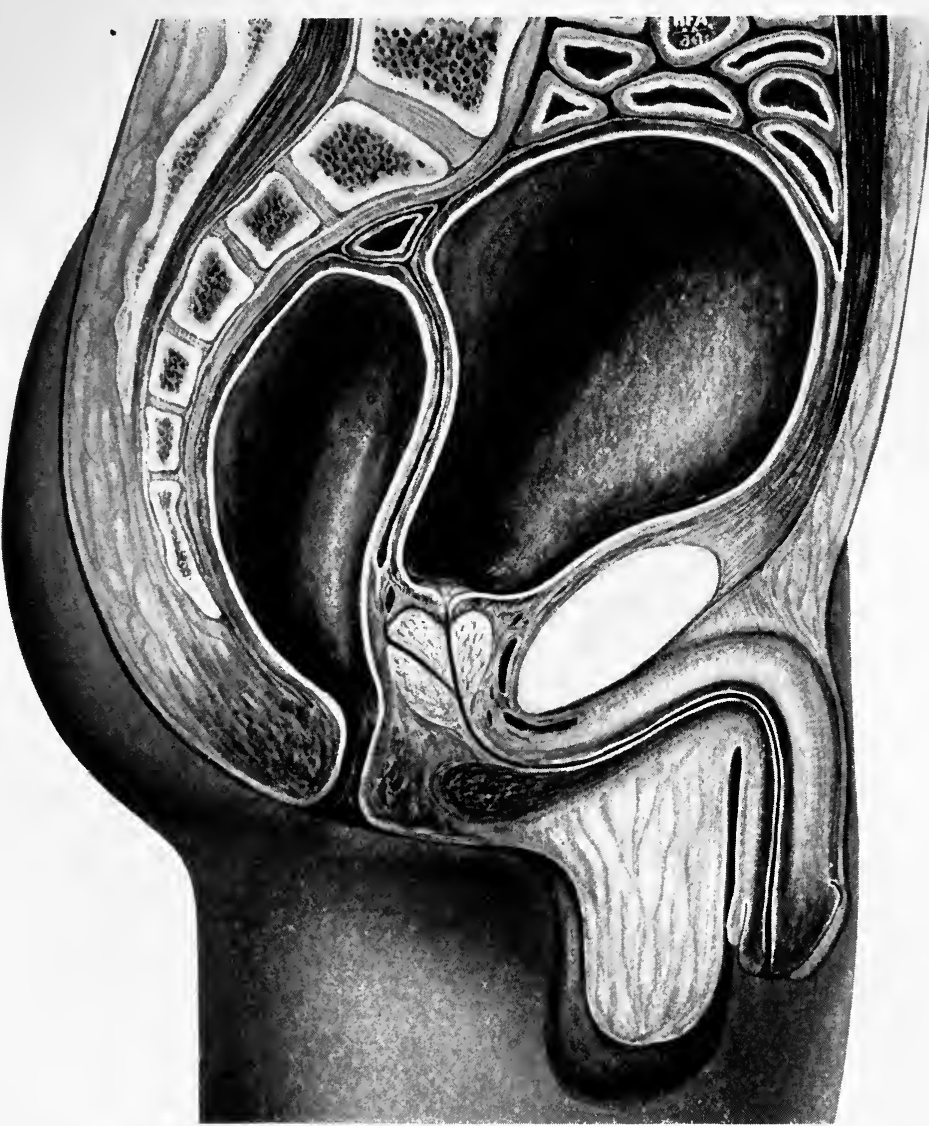

Bladder and rectum both distended. (After White and Martin.)

deferentia, the seminal vesicles, a short bit of the lower ends of the ureters, and, close to the vesical outlet, with the base of the prostate. The bladder is surrounded by the fat-containing areolar tissue, which fills a large part of the pelvic cavity.

Divisions of the Bladder.-The bladder is divided for purposes of description into: (I) The summit or apex. (2) The body. (3) The base. (4) The neck or cervix. These terms designate, respectively, the top, the 
middle, the floor, and the part immediately behind the vesical outlet. In the floor of the bladder is a triangular space, bounded above by the interureteral bar, below by the vesical outlet, and laterally by two lines extending from the outer ends of the bar, or base of the triangle, above to the apex of it at the vesical outlet below. This space is called the trigone. The interureteral bar (plica ureterica) is the thickest part of the organ, owing to the presence there of an aggregation of muscular fibers. Near either end of the bar there is a slit-like depression which represents the mouth of the ureter of each side.

Just above the vesical outlet, at the apex of the trigone, there is a slight, rounded elevation, which is produced by the presence immediately beneath the mucous membrane of a small mass of gland tissue. This represents the middle or third lobe of the prostate, which, in a certain proportion of cases of senile hypertrophy of the gland, shares in the enlargement of the lateral lobes, and becomes a tumor of greater or less size.

Supports of the Bladder.-The supports of the bladder are mainly derived from reflections of the pelvic fascia. The facial extensions which constitute the rectovesical fascia, and which support the bladder and maintain it in its position, are sufficiently loose in their connection with the organ to allow the changes of size and position which it undergoes in correspondence with its expansion when full and its contraction when empty. These supports are further supplemented by the urachus and the obliterated hypogastric arteries which extend from the summit of the bladder to the umbilicus, expansions of the pelvic fascia, which pass to the base of the bladder, and the vesicopubic muscle, which consists of a prolongation of the longitudinal muscular fibers of the bladder, and which connects the organ with the posterior surface of the symphysis pubis. These are termed the true ligaments of the bladder. In addition to them it receives some support from folds of the peritoneum which are known as its false ligaments.

The true ligaments all blend with the fibrous layer of the bladder. The support which serves the summit of the bladder consists of the urachus and the obliterated hypogastric arteries. The puboprostatic ligaments, which pass from either side of the symphysis to the prostate and anterior part of the neck of the bladder, maintain it anteriorly; the lateral expansions of the rectovesical fascia serve as lateral supports, and are distributed over the sides of the organ.

The false ligaments are the folds of the peritoneum which accompany the urachus and the obliterated hypogastric arteries above, the reflections of the peritoneum from the iliac fossæ on either side, and the reflection posteriorly of the peritoneum from the rectum.

Structure.-The bladder is composed of three coats or layers, and over a portion of its exterior surface there is added the investment of peritoneum. 
From within outward the layers are the mucous membrane, constituting the inner lining of the bladder; the muscular layer, which furnishes the greater part of the thickness of the bladder wall; and the fibrous or external layer, which is intimately blended with the muscular one.

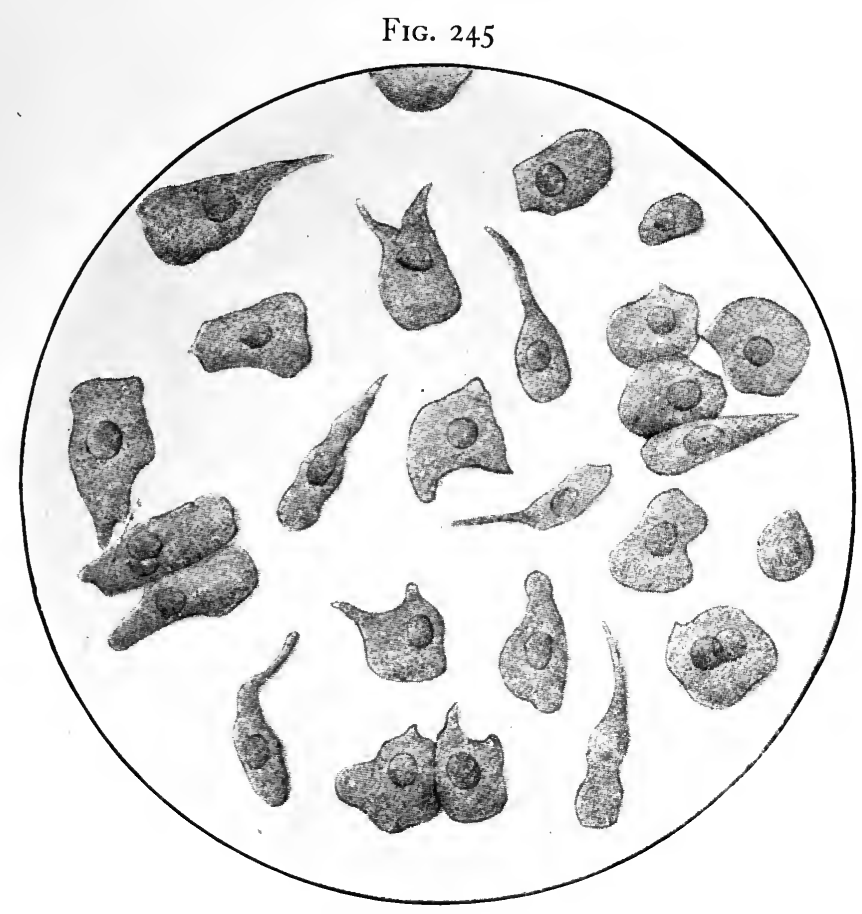

Epithelial cells of the bladder mucous membrane.

FIg. 246

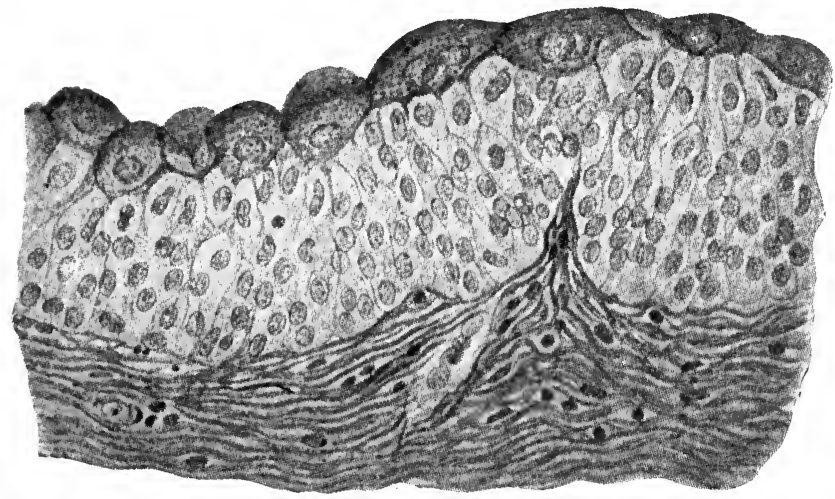

Vertical section of the vesical mucosa. (From Frisch and Zuckerkandl.)

The Mucous Membrane.-The mucous membrane of the bladder forms a soft and elastic lining of its inner wall. It is freely movable upon the underlying muscularis, except at the trigone, where it is thicker than 
elsewhere, and is more or less adherent. Elsewhere it can be readily peeled off from the tissue beneath. When the bladder is empty the mucous membrane falls into a series of folds, except over a small area upon the posterior surface above the vesical outlet. When the bladder is distended its inner lining becomes thinner and smooth. The mucous membrane of the bladder is continuous with that of the ureters and of the urethra.

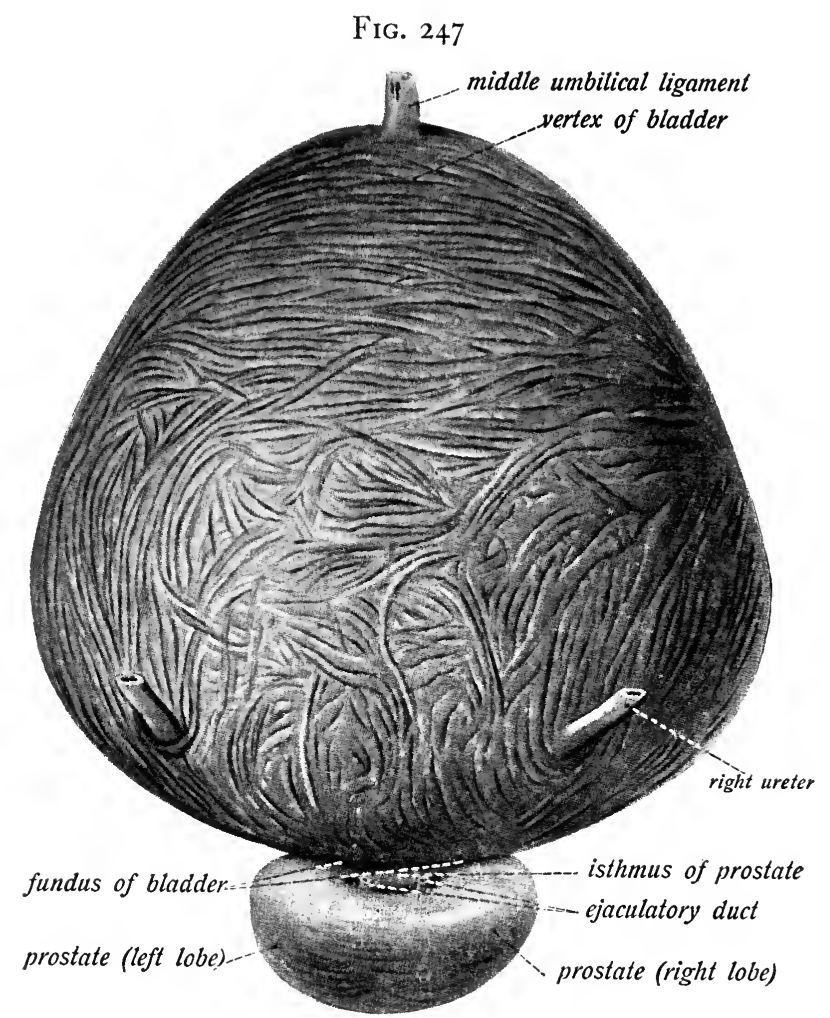

The urinary bladder and prostate seen from behind. The superficial musculature of the bladder has been removed. (Sobotta.)

The mucous membrane is composed of flat epithelial cells upon its surface, which are superimposed upon club-shaped cells beneath (see Figs. 245, 246, and Plate XXII). The superficial cells have one or more large nuclei, the cells of the deep layer have but one nucleus.

The Muscularis (Figs. 247 and 248). - The muscular structure of the bladder is divided into three layers. The inner layer is made up of longitudinal fibers, which extend from the apex to the vesical neck. These fibers are gathered into strands or bundles connected with each other by a series of diverging branches, so that a network is formed by them of alternating bands and interspaces, which give to the inner surface 


\section{PLATE XXII}

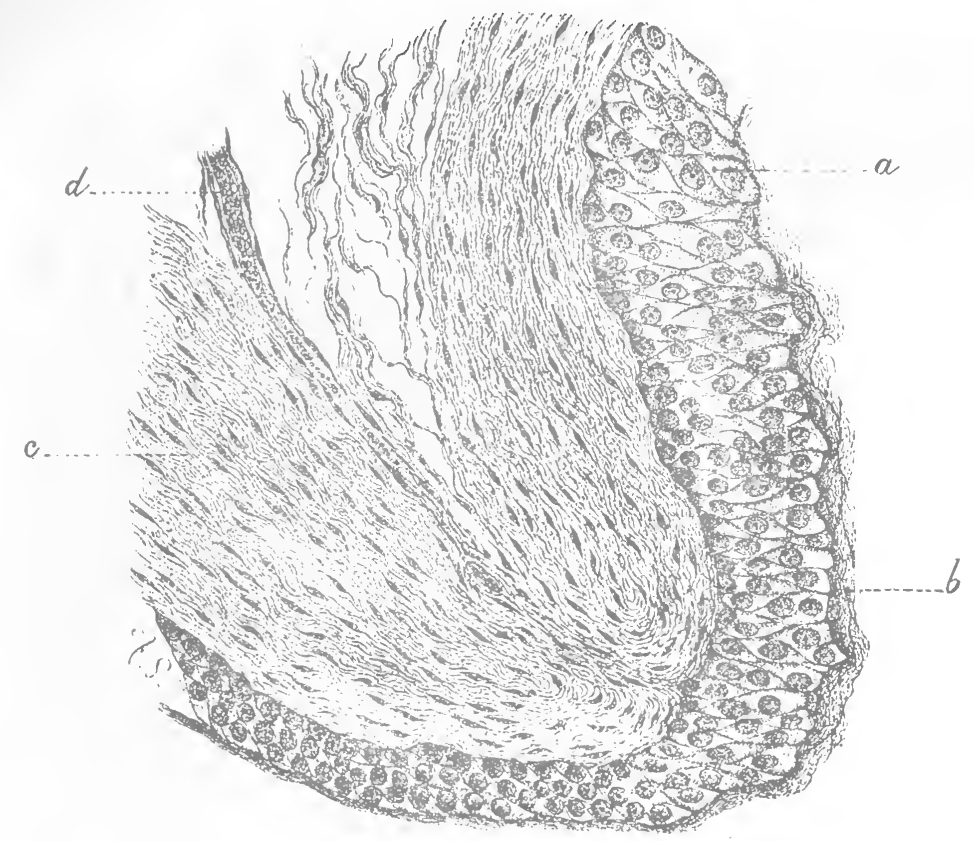

NORMAL MUCOUS MEMBRANE OF THE BLADDER. (Albarran.) 

of the bladder its reticulated appearance, and which, when the muscular bundles become hypertrophied, cause it to assume the still more exaggerated, trabeculated form which is so noticeable in cases in which there is an obstacle to the free escape of urine from the bladder. A further significance is given to the arrangement of these muscular fibers because it is owing to the existence of the interspaces that diverticula are formed by the pressure exercised upon the inner wall of the bladder by its fluid contents, when the conditions exist that prevent the free escape of the urine from the organ. The middle layer of muscle tissue is composed of

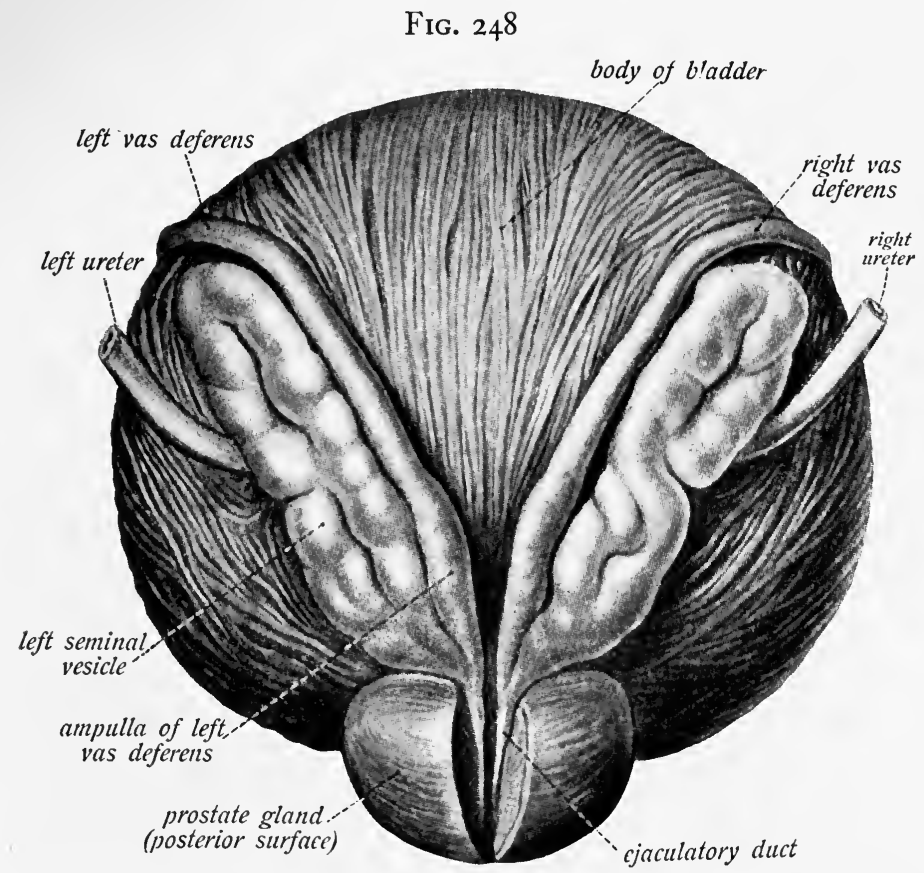

The urinary bladder with the seminal vesicles, the ampullæ of the vasa deferentia and the prostate seen from behind and below. The prostate is partly divided longitudinally. (Sobotta.)

circular fibers which completely cover the bladder. From this layer the internal vesical sphincter is derived and is formed by the strongest and most numerous of its muscular fibers. The outer muscle layer is composed of longitudinal fibers, some of which are prolonged anteriorly and go to form two bands which connect the bladder with the symphysis; others pass downward and spread over the sides of the prostate.

The Fibrous Layer.-This layer, which most writers describe as a part of the outer fascia of the bladder, is considered by Deaver as a separate structure. It covers the external surface of the organ and blends intimately with the fibers of the outer muscular layer. 
Bloodvessels. - The bladder derives its blood supply from branches of the internal.iliac arteries, which are: the superior vesical, which is distributed to the summit and sides of the organ; the middle vesical, which supplies the base of the bladder and the seminal vesicles; and the inferior vesicals, which go to the prostate, the trigone, and seminal vesicles.

These arterial branches enter the muscular coat of the bladder and terminate in capillaries which nourish the epithelium of the mucous membrane, and which are given off from the anastomosing network of arterioles lying immediately beneath the mucosa.

FIG. 249

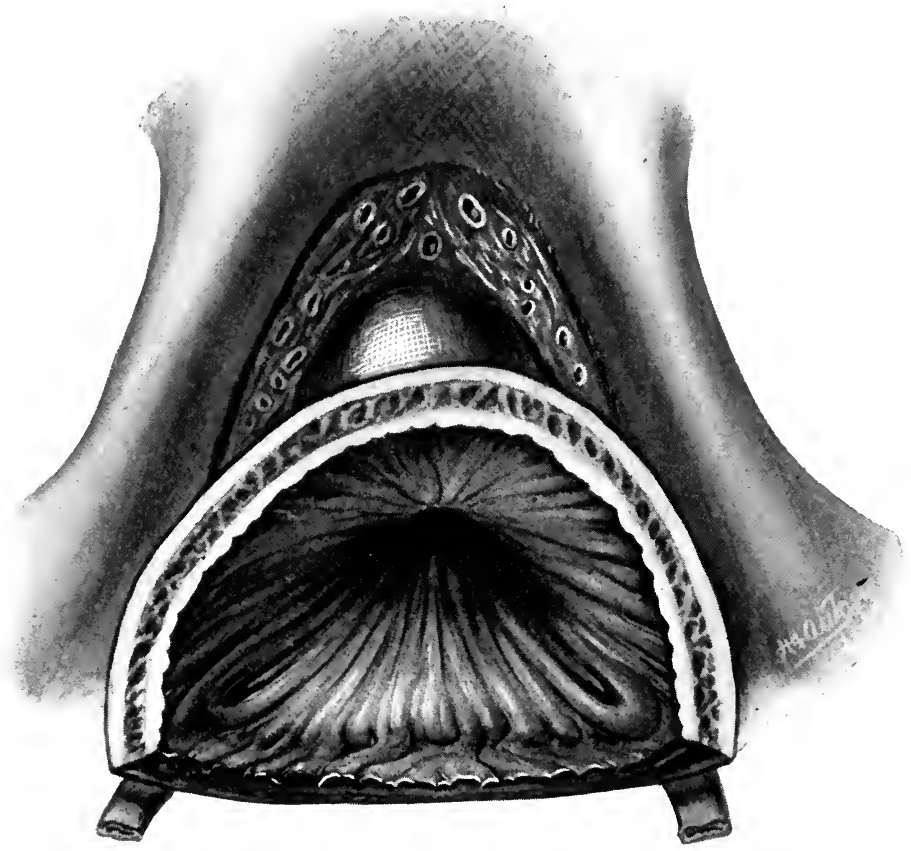

The venous plexus about the neck of the bladder and prostate.

The veins begin in a capillary system corresponding to that of the arteries, and pass outward through the muscular coat to the surface of the bladder, where they form anteriorly the puboprostatic plexus (plexus of Santorini), which lies immediately beneath the symphysis and extends backward on either side by communicating trunks to join the lateral plexus which covers the outer sides of the prostate and neck of the bladder (Fig. 249; see also Plate XXI), and which also anastomoses with the seminal plexus of veins, in this way forming a continuous set of vessels, which pass along the outer side of the prostate and neck of the bladder, and which connect with and empty into the hypogastric, hemorrhoidal, internal pudic, obturator, spermatic, and internal iliac veins. 
Nerves.- "The sympathetic nerves of the bladder are derived from the pelvic plexus and chiefly from the third and fourth sacral nerves. The plexus supplies the bladder, upon the side and base of which its branches accompany the vesical arteries" (Deaver).

Lymphatics.-The lymphatic system of the bladder takes its origin immediately beneath the mucous membrane, and passes through the muscular structure to reach the exterior of the organ.

The lymphatic network of the mucous membrane is shown in Fig. 250, which is taken from the classic work of Albarran upon Tumors of the Bladder, published in 1892.

FIG. 250

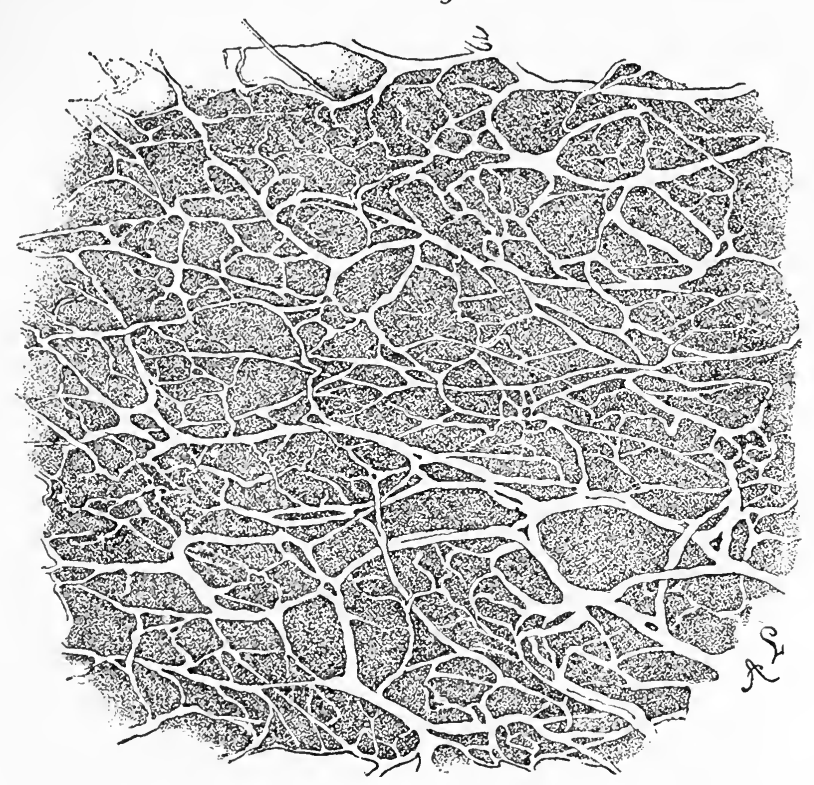

The lymphatics of the bladder mucous membrane near the trigonum. (Albarran.)

Albarran quotes the following description of the lymphatics from Gerota": "The lymphatics of the bladder are divided into two sets: (I) those of the mucosa, and (2) those of the muscularis. The former are especially developed about the trigone, but not there alone, for they are demonstrable upon the lateral aspects of the bladder as well.

"The lymphatics of the mucosa connect with those of the urethra, and together with them go to join the lymph glands of the lateral walls of the pelvis.

"The lymphatics of the muscularis arise in its most superficial parts in a delicate network, which combines to make larger vessels, which pass through the sides of the bladder, and some of which join the lymphatic glands in front of the symphysis. 
"The efferent trunks of the anterior and lateral lymphatics pass to the sides of the pelvis and enter the glands which are situated below the external iliac artery or those which are at the point of division of the hypogastric artery."

Papillæ.-The existence of papillæ in the bladder has been denied by Zuckerkandl and affirmed by Albarran (op. cit.), who says that, while sometimes lacking, they nevertheless are present in many cases, and who gives data with reference to this point which he obtained from a careful study of seven bladders.

Glands.-The existence of glandular elements in the mucous membrane of the bladder has been denied by Sappey, but it has been clearly demonstrated by Luschka, Henle, Krause, and Englisch.

These glands are most numerous about the vesical orifice, but are to be found elsewhere in the organ. They have the form of tubules or are racemose and open upon the surface by wide mouths. This fact has a bearing upon the occurrence of true adenomata, which some (Motz and others) have asserted to be always of prostatic origin and never to arise from the bladder itself.

\section{ANOMALIES OF THE BLADDER.}

The anomalies and abnormalities of the bladder are comparatively few in number, and are as follows:

I. Absence of the bladder.

2. Multiple bladder.

3. Patent urachus.

4. Exstrophy of the bladder.

5. Fusion of the bladder with other organs or structures.

Of these various conditions, the last but one is by far the most common. The others are rare.

Absence of the Bladder.-Gould and Pyle ${ }^{2}$ refer to cases reported by Blanchard, Blasius, Haller, Nebel, and Rhodius in which there was an absence of the bladder.

When this condition exists, the ureters terminate in unusual and abnormal positions. These we have dealt with in the chapter on Abnormalities, etc., of the Ureters, to which the reader is referred.

Multiple Bladder.-The same authors state that this is a more common condition than that of absence of the organ, and mention the following examples of it which have been among those reported: The case of a patient reported by Scibelli, of Naples. The specimen is shown in Fig. 25I, which is reproduced from Gould and Pyle's work. The patient 
from whom the bladder came died at the age of fifty-seven years, with symptoms of retention of urine. The illustration represents the normal bladder, and the supplementary bladders, with their respective points of entrance into the main viscus. As will be noticed, the ureters terminate in the supplementary bladders. (It is probable that the illustration represents dilatation of the ureters rather than triple bladder.)

It must be remembered that a number of the cases which have been recorded as examples of multiple bladders have undoubtedly been vesical diverticula (see section of Diverticula of the Bladder, in the chapter on Vesical Calculus and Diverticula).

Patent Urachus.-Persistent patency of the urachus is due to a failure of the normal complete obliteration of the communication between the bladder and the allantois. Under these conditions, the urine escapes from the umbilicus.

\section{FIG. 25I}

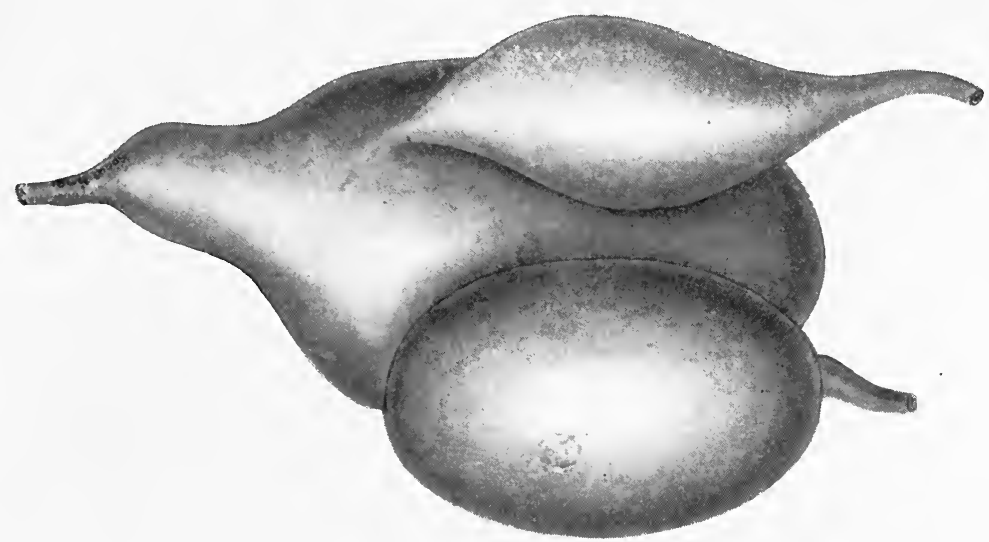

Triple bladder. (Scibelli.)

Treatment.-The treatment consists in removing an obstacle to the free escape of urine from the bladder, which is usually congenital stricture; or, if this does not result in cure, in excision of the sac and closure of the communicating opening in the bladder. This step is carried out through an abdominal incision.

Exstrophy of the Bladder.-In this condition there has been an arrest of development and a failure of union of the structures in the median line of the anterior aspect of the body which normally should take place. It is an arrest of development similar to that of harelip.

The usual form of the condition is that in which there is an absence of the anterior wall of the bladder, but there have been cases recorded in which the posterior wall has been the seat of the defect. With the vesical defect there is usually associated a corresponding deformity of the 
penis in which the roof of the urethra is absent and the canal of the conduit is represented by a gutter lying between the two corpora cavernosa, the condition being called epispadias. In the extreme examples there is a failure of the bones of the symphysis pubis to be fully developed. in consequence of which an interval occurs between them. Double inguinal hernia is also frequently observed, and is due to the weakening of the anterior abdominal wall coincident to the other defects of development mentioned.

FIG. 252

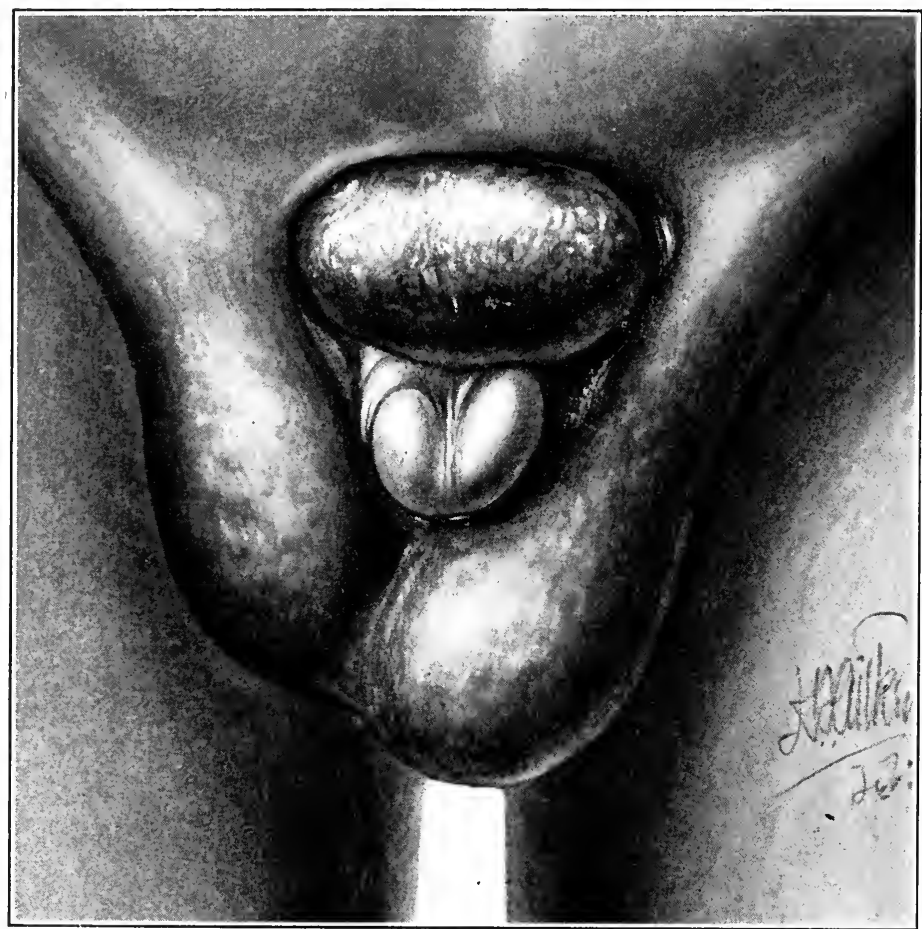

Exstrophy of the bladder. The exposed mucous membrane of the bladder is everted above the symphysis pubis and the dorsum of the penis which presents the condition of complete epispadias.

Exstrophy is more common in the male than in the female subject.

In appearance the condition presents an area-of a size varying in different individuals and in children as compared with adults_of red or purplish mucous membrane, with a wet surface, from which urine is constantly escaping (Fig. 252).

The exposed area is united to the surrounding skin by a more or less wide line of connective tissue.

The penis is deformed and undeveloped, and marked by the condition of epispadias already alluded to. 
In some instances the rectum or anus is connected with the surface of the exposed bladder.

The condition of exstrophy is a disgusting one, which renders the presence of the individual who has it disagreeable to others and almost intolerable to himself. It may be sometimes palliated, and can be radically remedied by surgical operation.

Treatment.-The palliative treatment consists in the wearing of a portable urinal, which collects the urine as it is poured onto the exposed surface of the bladder, and conducts it into a rubber bag placed conveniently for the wearer and attached to the thigh.

This contrivance is but a poor makeshift at the best, however, and it is well worth the risk and time involved in the surgical treatment to make the effort to thus correct the abnormal condition.

The surgical treatment is of two sorts: (I) that by which the effort is made to construct a new anterior wall for the bladder, which is done by one of a number of plastic operations, and (2) by diverting the urinary secretion from the bladder and by removal of the latter organ at the same time or subsequently.

The first of these two ways of overcoming the difficulty is tedious and liable to failure; the second is in greater or less degree a compromise.

The chief difficulty encountered in all plastic operations is not so much in the factor of supplying a new wall to the bladder, but in the inability to create an efficient sphincter, in the absence of which incontinence of urine continues as before, although its results are mitigated and it is more easily controlled. The element of inflammation of the exposed mucous surface is eliminated by a successful plastic operation.

The operations which divert the urinary stream are of three sorts: (I) Diverting the urine into the intestine (rectum), of which one method only should be employed (Maydl's operation). (2) Bringing the ureters to the skin (Harrison, ${ }^{3}$ Bottini, ${ }^{4}$ Bottomley) (see chapter on Operations on the Ureters). (3) Bilateral nephrostomy and the establishment of permanent lumbar renal fistulæ proposed by Watson ${ }^{5}$ in 1905 , as a step preliminary to total extirpation of the bladder in cases of vesical tumor. (For the technique of these operations, see chapters on Operative Technique, under the respective headings.)

The flap operations have been ingenious and numerous, but they all suffer from two factors which tend to cause failure, viz., the inability to construct a sphincter, and the very frequent failure of the union of the flaps, owing to the contact of urine with the wounds.

One objection to all forms of ureteral implantation in the rectum is the discomfort caused by the liquid stools which result from the presence of urine in the bowel. It should be said, however, that in some instances 
this is much less than would be supposed, and patients have been able to control the evacuations sufficiently to have intervals of as long as four to six hours between them.

To all but Maydl's operation there is a far greater objection, viz., the danger of renal infection, which takes place in connection with ureteral implantations in a seriously large proportion of the cases in which the ureters are attached within the bowel.

This obstacle to the success of the operations of that class is in great measure avoided by Maydl's method, which differs from others in that the bladder wall immediately surrounding the ureteral orifices is excised and implanted into the rectum together with the ureters; by this means the natural relations of the conduits with the bladder are not disturbed and protection against renal infection is afforded.

Still more advantage in this respect is gained by bringing the ureteral openings-after the canals have been divided-to the surface of the loins. When this is done, the natural road of escape of the urine from the kidneys is in part preserved, and the kidneys may be kept free from infection provided the implanted orifices of the ureters do not become contracted, and if the canals do not become narrowed at any point between the terminations on the skin and the renal pelves. Both of these accidents are, however, likely to occur in a certain proportion of the cases, in what proportion of them we do not know, because there have been but very few operations of the kind done as yet. The liability to have this constriction of the ureters take place will be rendered much less if small drainage tubes are continuously worn in the ureters, as has been practised in one case, in which Dr. F: Tilden Brown, of New York, adopted this manner of diverting the urinary stream on one side in a case of vesical tumor, and by nephrostomy on the other.

Watson's proposal to accomplish the diversion of the urinary stream by means of bilateral nephrostomy, etc., has the advantage of affording a freer drainage to the kidney, and of not being subject to the liability to have the drainage interfered with by malpositions of the kidney, pressure of constricting bands of adhesions, and contraction of the fistulous tract, all of which may occur in connection with ureteral implantation, as has already been suggested.

The patients who are submitted to either Bottomley's or Watson's plans of procedure can be kept dry and comfortable by wearing either of the apparatuses devised for the purpose by each of these surgeons in connection with the method of operating proposed by him. (See chapter on Operations upon the Kidney, in which Watson's device is illustrated.) 


\section{INJURIES OF THE BLADDER.}

Rupture of the Bladder.-Definition.-Laceration of the bladder, involving all its coats.

Etiology.-Rupture of the bladder may occur: (I) Spontaneously. (2) From muscular contraction of the abdominal parietes. (3) From external violence, such as blows and falls. The bladder is never ruptured when empty.

I. Spontaneous Rupture.-This probably never occurs unless there exists some pathological change in the organ, $e . g$., diverticula with very thin walls. The term spontaneous rupture is not strictly correct; it is applied to those cases in which the immediate cause of the accident is not traumatic, and in which the bladder is torn simply by the pressure of greater or less fluid contents upon the weak part of its wall. Cases have been reported in which the adult bladder has ruptured upon ten ounces of fluid being injected into it, and in a child five ounces have in one instance produced the same result.

2. Muscular Contraction.-Muscular contraction may, under the same conditions of the organ, rupture it.

3. Traumatic Rupture.-The injury may be produced by perforation with instruments roughly used, or, as is usually the case, by blows or falls. The force under such circumstances may be expended directly upon the abdominal wall or symphysis pubis immediately in front of the bladder, upon the lower part of the back, or upon the perineum and under surface of the pubic arch.

The character of the violence inflicted varies greatly. In some cases it is a crushing force, such as that described in the first of the illustrative cases (see illustrative cases, No. I), or as that applied when a man is caught between the buffers of railroad cars. In others, the patient falls astride of a beam or wheel; and again, it is a direct blow upon the symphysis or sacrum. Fractures of the pelvis, especially those which involve the rami of the pubic bones, are sometimes complicated by rupture of the bladder.

Pathology.-Ruptures of the bladder are usually divided into two classes: (I) those in which the wound is extraperitoneal, and (2) those in which it involves the peritoneal investment, and is intraperitoneal.

The relative proportion of these two forms of the injury is about 20 per cent. extraperitoneal to 80 per cent. intraperitoneal.

Location of the Laceration.-In 36 per cent. of 90 cases collected by Mitchell ${ }^{6}$ the laceration was upon the anterior wall.

Results of Rupture of the Bladder.-The immediate results of the accident will be extravasation of blood and urine into the prevesical 
space, or into the areolar tissue about the sides and posterior surface of the bladder in cases of extraperitoneal injury, and into the peritoneal cavity when the laceration is intraperitoneal.

If the extravasated fluids are not evacuated, sooner or later infection of the field occupied by them takes place; and, in the extraperitoneal variety of the injury, suppuration and burrowing of pus between the bladder and rectum and throughout the pelvis occurs if the patient lives long enough. In the intraperitoneal ruptures, septic peritonitis ensues. The length of time, however, before this condition arises is sometimes extraordinarily long. The liability to have it occur is much increased if the patient is catheterized.

Quervain $^{7}$ quotes Küster, König, Albarran, Schede, Petroff, and Edler as holding the belief that all patients without surgical intervention, who have extravasations of blood and urine in the peritoneal cavity, die of septic peritonitis. That this is not invariably true is shown by a case reported by Ledderhose, ${ }^{8}$ in which an intraperitoneal rupture of the bladder was found in a patient, seventeen days after injury, during an operation for the evacuation of a small extraperitoneal abscess. No sign of peritonitis was discovered during the entire time of the patient's convalescence.

Tuffier (quoted by Quervain, op. cit.) showed experimentally that urine does not produce peritonitis when introduced into the peritoneal cavity very gradually, or if repeated introductions of it are made, provided the intervals between them are sufficiently long.

Quervain's own experiments yielded similar results. If, however, the urine flows continuously into the peritoneal cavity, peritonitis generally results.

Israel and Grawitz found that in the case of dogs death did not result sooner than from four to five weeks from allowing urine to flow into the peritoneal cavity from the end of a divided ureter.

General septicemia not infrequently has its starting point in septic thrombi of the venous plexus surrounding the neck of the bladder, especially in cases of extraperitoneal rupture. Bartels states that 27 per cent. of the deaths in this class of cases is due to septic processes originating in this way.

Symptoms and Diagnosis.-In typical cases the diagnosis is easily made from the existence of the cardinal symptom, hematuria, in connection with the failure to find by the catheter more than a small quantity of urine in the bladder, and the presence of more or less shock, together with the history of accident of such a character as would be likely to cause the injury; finally, the presence of an increasing swelling immediately above the symphysis pubis. There are numerous cases, however, 
in which it is by no means easy to make the diagnosis. Hematuria may not occur; micturition may be undisturbed if hematuria is present; the blood may come from the kidney. It is not very uncommon to have patients with multiple injuries, as, for example, in No. I of the illustrative cases at the end of this section, in which the evidence at first pointed to a subparietal laceration of the kidney rather than to a rupture of the bladder. In intraperitoneal rupture the suprapubic tumor is usually lacking, and the true nature of the injury may not be shown in the earlier stages of the case, because of the absence of characteristic symptoms suggesting an intraperitoneal lesion; later, when enough urine has flowed into the abdominal cavity, its presence there as free fluid will give the clue to the real condition.

The cases in which the injury of the bladder is most likely to escape notice at first are those in which there is rupture of the urethra associated with it, as in No. 2 of the illustrative cases. The attention of the surgeon is concentrated upon the evident urethral injury, and it may be merely by chance that he is led to the detection of the graver lesion. In all cases in which great violence has been inflicted upon the perineum and lower part of the pubic arch or the rami of the pubic bones, the possibility of the coincidence of the vesical injury with that of a ruptured urethra should be borne in mind, and the surgeon should satisfy himself with regard to the existence of the lesion by exploring the interior of the viscus, which is readily done at the time of his operation upon the urethra.

Again, the probability of rupture of the bladder in connection with fractures of the pelvis should always be remembered and should lead to an examination of the viscus in order to determine the fact.

A subperitoneal rupture of the bladder in which the urine is not yet extravasated sufficiently to have lifted off the peritoneal investment of the organ may, for a good while, fail to present any symptoms characteristic of a vesical laceration, the only ones likely to draw attention to the bladder being pain in the lower part of the abdomen, and perhaps inability to spontaneously evacuate the bladder, both of which might occur in connection with fracture of the pelvis without the vesical injury.

The most essential diagnostic factor is the failure to obtain more than a small quantity of urine on the passage of the catheter, and the presence of more or less blood in the urine drawn. This evidence of vesical laceration will be present in the great majority of cases. If a specified quantity of fluid is injected into the bladder, and a less amount of it returns through the catheter, this is strong confirmatory evidence of the existence of the injury. This step should not be taken unless the diagnosis cannot be made without it, because of the liability to infection which is involved in it. If the patient's consent has been obtained to VOL. I-29 
the immediate performance of operation after the injection has been made, this objection no longer holds, inasmuch as the occurrence of time required for infection will not have elapsed.

The cystoscope is ordinarily of little use in cases of ruptured bladder. The inability to distend the bladder with fluid and to obtain a clear medium make its employment valueless.

Treatment.-The only excuse for neglecting to operate or for delaying to do so is great doubt as to the existence of the injury. There is no room for debate as to the course to be pursued in any case of rupture of the bladder in which the diagnosis can be made with even a moderate degree of certainty. It should be prompt surgical intervention as soon as possible after the diagnosis has been made.

The above statement is confirmed by the following table:

Cases of Intraperitoneal and Extraperitoneal Ruptures of the Bladder Treated Expectantly. Intraperitoneal.

Bartels (Arch f. klin. Chir., Band xxii) .

Ullman (Wien. med. Woch., i 887 , p. 749)

Cases. Deaths. Mortality.

$9+93$

I 43 I 4 I

Extraperitoneal.

Mitchell

Bartels

\begin{tabular}{|c|c|}
\hline 53 & $5^{I}$ \\
\hline 75 & 39 \\
\hline $3^{6}$ & 324 \\
\hline
\end{tabular}

Cases Treated Operatively.

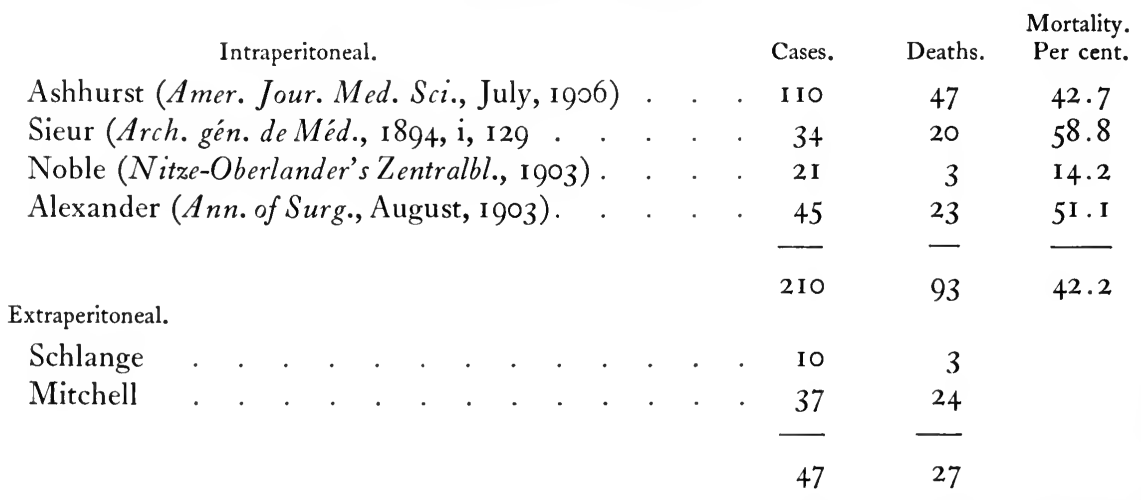

There need be no hesitation, furthermore, as to the nature of the first step of the operative treatment, which should be the exposure of the bladder extraperitoneally above the symphysis pubis. If the rupture is an extraperitoneal one, the opening of the peritoneal cavity will thus be avoided; if intraperitoneal, the abdominal cavity can be at once opened by extending the first incision upward as far as may be required. 
In the majority of the cases of extraperitoneal laceration, the wound will be seated in the anterior wall or neck of the bladder, since we know that the posteriorly or laterally placed injuries almost always involve the peritoneum.

Suture of the Wound, or Drainage, in Extraperitoneal Lacerations.When the wound is extraperitoneal, the choice of method of treating it lies between its total or partial suture and leaving it entirely unsutured.

The objection to total suture is the fact that the wound is not a clean cut, as it is in the case of an ordinary suprapubic cystotomy incision, but a wound with bruised and irregular edges, which afford poor holding ground for the stitches, and in which we cannot accurately estimate the extent of tissue involved beyond the edges, which may subsequently slough. The chance of securing healing by first intention is, therefore, not particularly good, and if it does not take place the dangers to the patient are distinctly increased by having applied it. On the other hand, the open treatment of the wound, or, better, the partial suture of it around a double drainage tube, which, if properly arranged, allows but little or no leakage, does not involve this risk, and, as a matter of fact, the patients do very well, the only disadvantage being a longer period of convalescence than that which occurs in the cases in which complete suture is done and in which it is successful.

If shock is severe at the time at which the operation is done, or occurs during its performance, all attempt to close the bladder incision had better be abandoned, and the surgeon had best rest content with having given a free exit to the extravasated urine rather than to incur the dangers connected with a more prolonged operation, which the placing of the sutures would necessitate. Otherwise than this, we prefer to treat the extraperitoneal cases by partial closure of the laceration and draining the bladder through two parallel rubber drainage tubes in the manner described in the chapter on Operations on the Bladder.

In Cases of Intraperitoneal Rupture.-Whenever it is known beforehand that there is an intraperitoneal rupture of the bladder, or when there is discovered to be one upon exposing the bladder suprapubically, the peritoneal cavity should at once be opened and the laceration sutured. (See chapter on Technique of Operations on the Bladder.) In this case the bladder is not opened suprapubically outside the peritoneum, as in an ordinary suprapubic cystotomy. The wound in it is approached through a laparotomy incision, and the rent is repaired. The suture which we personally prefer is the right-angle one of Cushing. (See chapter on Technique of Operations on the Bladder.) Whatever form of suture be employed, it must be one which inverts the edges of the laceration and closely approximates the peritoneal surfaces on either side of it. 
Before closing the abdominal incision the bladder should be filled with water through a catheter passed through the urethra, in order to test the suture and make sure that it is tight. If not, additional Lembert stitches should be applied to reinforce it. A catheter should then be passed through the urethra into the bladder and secured there.

The abdominal wound should be closed, except for sufficient space to allow the passage of a wick for drainage. The whole intraperitoneal field of operation should be carefully cleansed before closure of the external wound.

As in the case of other kindred intraperitoneal conditions, the mortality of this class of the cases of rupture of the bladder will be greatly lessened by the performance of the operation soon after the receipt of the injury, and increased in proportion to the length of the delay before surgical treatment is applied. The following figures bearing upon this point (quoted from Mitchell by Keyes ${ }^{6}$ ) are of interest in this connection:

\begin{tabular}{|c|c|c|c|c|}
\hline Operation. & Cases. & Cured. & Died. & $\begin{array}{l}\text { Mortality. } \\
\text { Per cent. }\end{array}$ \\
\hline Within twelve hours . . . & . $\quad$ I3 & 8 & 5 & $3^{8.4}$ \\
\hline Twelve to twenty-four hours & . 10 & 3 & 7 & 70.0 \\
\hline \multirow[t]{3}{*}{ Twenty-four to sixty-two hours } & . I I I & 3 & 8 & 72.7 \\
\hline & - & - & 一 & - \\
\hline & 34 & 14 & 20 & $5^{8.8}$ \\
\hline
\end{tabular}

The few cases in which long-delayed operations have saved the patients' lives are of interest, but do not justify delay in other instances, for they are merely the exceptions to the rule. Quick ${ }^{9}$ reports one in which he operated upon a patient whose injury had been received eleven days previously, recovery following the operation. This is the longest interval between the occurrence of the injury and the operation in which surgical intervention has been successful, so far as we know. Previous to this the longest time was six days in one case.

The following analysis of cases is taken from Mitchell: ${ }^{6}$

Analysis of Cases.

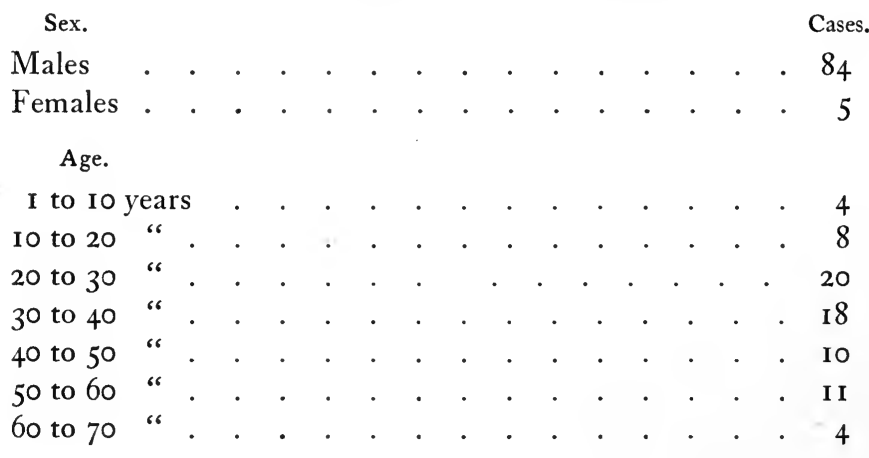


Causes.

Cases. Per cent.

Crushed by weight falling on body . . . . . . 23

Run over by wagon . . . . . . . . . . 25

Fall from a height . . . . . . . . . . . 22

Struck by an engine or car . . . . . . . . . 4

Crushed between wagons or cars . . . . . . . . . . 9

\section{Result.}

Whole number of cases

Whole number of deaths

Whole number of recoveries $\cdot \cdot \cdot \cdot \cdot 75$

Time of death.

First day . . . . . . . . . . . . . . . 9

Second day

Third day . . . . . . . . . . . . . . . . . 7

Fourth day . . . . . . . . . . . . . . . . . I0

Fifth day . . . . . . . . . . . . . . . . 5

Sixth day . . . . . . . . . . . . . . . . . $\quad .5$

Seventh day . . . . . . . . . . . . . . . 3

Total first week . . . . . . . . . . . 53

First week . . . . . . . . . . . . . . 53

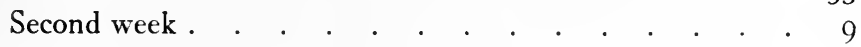

Third week . . . . . . . . . . . . . . . 2

Fourth week . . . . . . . . . . . . . . . . 4

Fifth week . . . . . . . . . . . . . . . . I

Sixth week . . . . . . . . . . . . . . . 2

Six months . . . . . . . . . . . . . . I

Fourteen months . . . . . . . . . . . . I

Time of Operation.

Time after accident.

Within twenty-four hours

" forty-eight hours

" four days . . . . . . . . 5

" two weeks . . . . . . . . 7

" three weeks
Total.

Deaths.

$$
7
$$$$
2
$$

5

2

Mortality. Per cent.

Bones fractured.

Multiple fracture . . . . . . . . . . . . . 42

Os pubis . . . . . . . . . . . . . . . . . . . 49

Sacrum . . . . . . . . . . . . . . . . 9

Ischium . . . . . . . . . . . . . . . . . . $\quad .99$

Ilium . . . . . . . . . . . . . . . . . 6

Separation of symphysis pubis . . . . . . . . . . $3^{\mathbf{I}}$

Separation of sacro-iliac synchondrosis . . . . . . . 10

Penetration of bladder by bone . . . . . . . . 20 


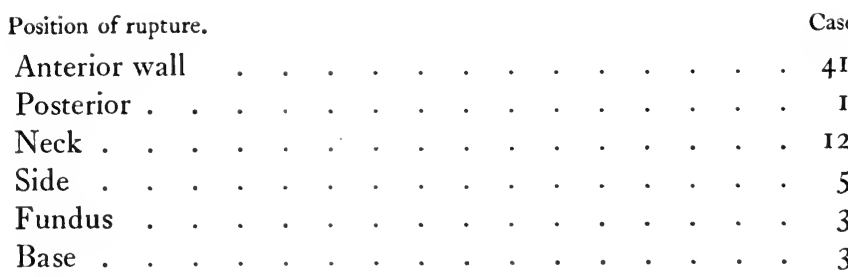

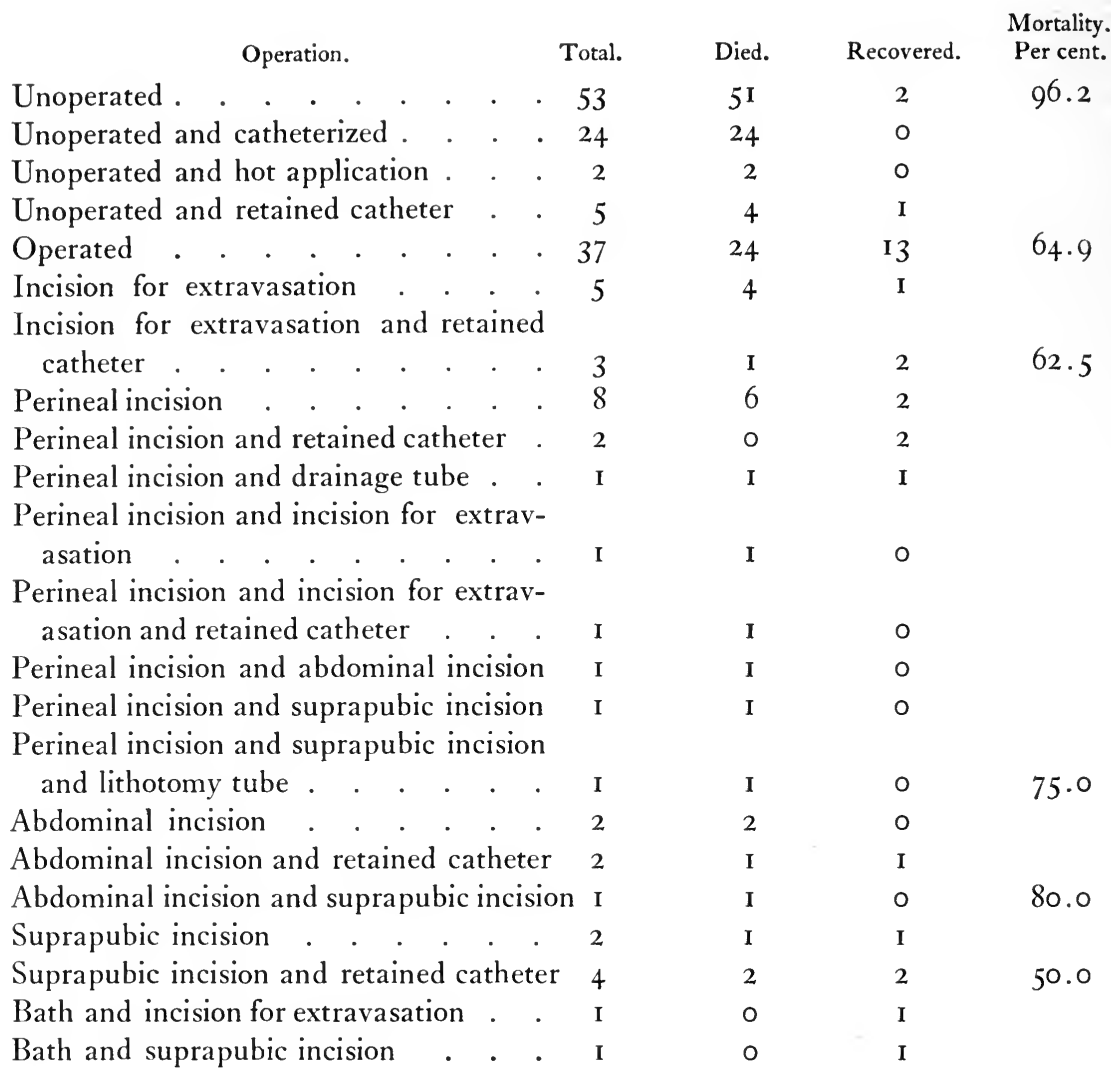

\section{Illustrative Cases of Rupture of the Bladder.}

The following cases are selected from those occurring in the practice of the writer:

CAsE I.-Contusion of abdomen; fracture of lower ribs on both sides; scalp wound; extraperitoneal rupture of the bladder; subcutaneous laceration of the recti abdominis muscles; extravasation of urine into Retzius' space, scrotum, and dorsum of the penis; rent in the bladder an inch and a half long in the direction of the long axis of the viscus and in the median line of its anterior surface, not extending into the peritoneum. Suture of the vesical wound. Recovery.

A man, aged about forty years, was caught between the casing and an 
elevator and crushed by the force which was applied to the front and back of the body simultaneously. When first brought to the hospital, immediately after the accident, his condition was as follows: Cyanotic; extremities cool; face pallid and pinched; on left side of head a contusion and a scalp wound; three lower ribs on left side and two lower ones on right were fractured; ecchymosis in the left loin; slight rigidity of the abdominal muscles, most marked over the lower third of the abdomen. A very large swelling occupied the suprapubic region, extending upward half-way to the umbilicus and downward over the dorsum of the penis and over the scrotum. Tenderness was also present over the same region. No fracture of the pelvis was made out.

Catheter passed, and drew bloody urine.

In the next hour and a half the swelling and rigidity in the lower abdomen became more marked; the patient then reacted from shock.

Operation (three hours after the accident).-The swelling at this time was nearly as high as the umbilicus. It was fluctuating. The whole abdomen was tense, and there was moderate distention and tympanites in the upper part of it. Dull percussion note over the swelling in the lower part. The abdomen was opened by a median incision four inches long, extending upward from the upper margin of the symphysis pubis.

Immediately upon opening the prevesical space, bloody urine ran out of the wound. It was seen that the recti abdominis muscles were torn completely across, an inch and a half above their insertions, and the severed ends had retracted, leaving an interval of at least five inches between them and the upper margin of the symphysis. The bladder was thus freely exposed to view at once on dividing the skin and subcutaneous tissue, beneath which was a very large collection of urine, which had been poured into the prevesical space from a rent on the anterior surface of the viscus about an inch and a half long and not involving the peritoneal investment of the organ. It was this collection of urine that had made the large tumor which was so conspicuous above the symphysis. The vesical wound was sutured with chromic catgut and the bladder was distended to test it. It did not leak. The ends of the recti muscles were far retracted within their sheaths, but the patient's condition was so critical, that no attempt was made to bring them down and attach them in their normal position.

The perivesical spaces were well drained in every direction, and the outer wound was partly closed by suture. The dorsum of the penis was scarified. A catheter was tied into the bladder through the urethra and retained there for one week.

A few drops of urine leaked through the healing abdominal wound on 
the seventh day. Some leakage continued for ten days, then ceased. The wound wholly healed at end of the eighteenth day.

Six weeks after the accident the patient walked into the writer's office and demonstrated the interesting fact that he could not only stand upright, but on lying down on his back on a table he could raise himself into a sitting posture without helping himself with his hands. While standing there was a large bulging outward of the abdominal wall above the symphysis pubis, but the space left vacant by the tearing across and retraction of the recti muscles had apparently been filled in enough to retain the intestines and prevent them from forming an unlimited hernia.

Patient stated that micturition was just as natural as it had been before the accident and operation.

Rupture of the Bladder (Extraperitoneal) and of Membranous URETHRA.

CASE II.-Nature of Injury.-Falling astride of a wooden rail. Time elapsed after accident before operation, four hours. No urine passed in this interval.

Condition at Time of Operation.-The patient, a man aged thirty-five years, was beginning to rally from shock three hours after the accident.

Physical Signs.-Extravasation of blood. The parts occupied by this were the perineum, the scrotum, the root of the penis, the front of the symphysis pubis, and the inner surface of each of the thighs for about three inches. The perineum was much swollen. Slight bleeding had been present from the meatus ever since the accident. Percussion above the symphysis pubis was resonant. Catheter could not be passed into the bladder because of the obstacle presented by ruptured membranous urethra.

Operation.-Trendelenburg posture. Bladder exposed by the usual median incision above the symphysis pubis. This was done partly because of the inability to pass a catheter into the bladder, and in order to trace the course of the ruptured urethra by retrograde catheterism from above.

On entering the prevesical space a quantity of extravasated urine and blood ran out through the wound, issuing from a rent in the anterior wall of the bladder, beginning at the vesical neck and extending upward to the insertion of the peritoneum. The vesical wound was closed by suture. A catheter was tied into the bladder through the urethra. Uninterrupted recovery. 


\section{CYSTITIS (INFLAMMATION OF THE BLADDER).}

Etiology.-The essential etiological factor of cystitis is infection by one or another variety of microörganism. The predisposing factor is congestion, and the factors productive of congestion are numerous and of different character. Among the agents which produce congestion of the bladder are the following: cantharides, turpentine, balsam of copaiba and of sandalwood oil, arsenic, and alcoholic stimulants when taken by mouth in too large doses; corrosive sublimate and carbolic acid when applied to the bladder directly in the form of vesical irrigations; vesical calculus and rough instrumentation within the bladder; retention of urine from any cause. This acts by compression of the veins of the vesical and prostatic plexuses, which produces passive congestion. An irritating quality of the urine, such as occurs in persons with phosphaturia, oxaluria, and a marked uric acid diathesis. Less directly, exposure to cold, rectal tenesmus, and straining at stool.

Classification.-Cases of cystitis are variously classified: (I) By descriptive terms. (2) According to the nature of the etiological factors. (3) In accordance with the character of their clinical course: as purulent, membranous, gangrenous, alkaline, and acid cystitis; as gonorrhœal, tuberculous, diphtheritic, and calculous cystitis; as acute and chronic cystitis.

Vesical Infection.-The bacteria found in the bladder in cases of vesical infection are as follows: (I) The Bacillus coli communis, the Bacillus tuberculosis, the typhoid bacillus, the pneumococcus, the gonococcus; these are found in acid urines. (2) In cystitis with alkaline urine, staphylococci, streptococci, streptobacillus anthracoides, and the bacillus proteus. The most frequently found are the staphylococcus ureæ liquefaciens and the diplococcus ureæ liquefaciens. The infection in alkaline cystitis is usually a mixed one.

Avenue of Invasion of Vesical Infection.-Infection of the bladder takes place through one or another of the following channels: (I) By direct extension from the posterior urethra. (2) By descending infection from the kidney. (3) Through the blood current. (4) By extension from adjacent structures or organs.

I. By Direct Extension.-In the majority of the cases of cystitis which originate in the urethra the gonococcus is the active microörganism of the infection. It is not necessary, however, that there should be a suppurative process present in the urethra, in order to have vesical infection produced from this site, for microörganisms which habitually reside in the urethra, and which are not ordinarily pathogenic, may become so in the bladder, provided there exist in it the conditions which 
favor the development of an infection, viz., congestion or a residual urine. The infection may extend spontaneously from the urethra or it may be conveyed to the bladder by instruments passed through the urethra.

The bladder may become infected by gonorrhœal or other urethral microörganisms which proceed from a latent focus of long standing, chronic urethral inflammation, the presence of which may be entirely overlooked, and which may be causing no symptoms. On this account such foci are a constant menace.

2. Descending Infection from the Kidney.-As in the case of infection extending from the urethra, so, too, when there is a descending one from the kidney the bladder must be ready to receive and develop it; otherwise, it is unlikely to effect a foothold there. This is shown by the length of time which may pass without infection of the bladder taking place, in cases of tuberculous kidneys, which are constantly pouring infected urine over the vesical mucous membrane. Such immunity is seen only when the bladder is in a normal condition; otherwise, infection is probable.

The importance of recognizing the source of the vesical infection in such cases is great, since treatment of cystitis without including or preceding it by treatment of the renal condition is futile.

3. Hematogenous Infection.-Whether or not cystitis which develops in the course of certain general maladies-such as septicemia and pyemia, and some of the systemic fevers, typhoid, pneumonia, malaria-takes place by invasion of the bladder directly through the blood current, or from the passage of the microörganisms or their toxins into it from the kidney with the urine, is largely a matter of conjecture. The fact that such infections do occur in association with these diseases in some instances, and the presence of the characteristic microörganisms of one or another of them in the bladder, is well recognized.

4. Extension from Adjacent Structures.-Whether infection of the bladder from the rectum can take place by direct migration of pathogenic organisms through the intervening tissues is a question which still remains undecided. Both clinical and microscopic observations appear, however, to make the occurrence of invasion by this route probable. Thus the association of cystitis in some instances with ulcerative lesions of the rectum and the demonstration in these cases of the presence of the Bacillus coli communis in the bladder and in the tissues which have more or less direct connection with both bladder and rectum seem to suggest the strong probability of the infection of the bladder having been produced by such a migration as we have mentioned. The relatively frequent occurrence of cystitis in cases of typhoid, and the discovery of the typhoid 
bacillus in the bladders of these patients, is another suggestive fact which seems to point in the same direction. Bliss $^{10}$ found that there were $3^{\mathrm{I}}$ cases out of 3 II of typhoid fever in which there was bacilluria or cystitis and in which the typhoid bacillus was found in the urine. Richardson estimated the frequency of bladder infection in typhoid fever to be from $2 \mathrm{I}$ to 25 per cent., and Horton-Smith ${ }^{11}$ found 28 per cent. of bladder infections in 25 cases of typhoid fever. Bliss believes that the infection occurs after the second week of the disease, and has noted it in one case as late as the one hundred and eighteenth day.

It is, of course, equally probable that this infection may take place through the blood current, or via the kidney, as by direct extension from the bowel to the bladder.

Pathology.-Acute Cystitis.-In the beginning there is congestion of the mucous membrane of the floor of the bladder, associated with œdema and dilatation of the bloodvessels of the same parts. Epithelial cell desquamation occurs soon afterward and pus formation shortly follows. The process gradually extends and may involve the entire mucosa. In the very acute cases, more or less hemorrhage takes place from the capillaries. In some instances small cysts and abscesses develop upon the surface and, after rupturing, are converted into ulcers.

In the more extreme forms of acute inflammation, local areas of necrosis are seen. Occasionally the whole lining of the membrane of the bladder becomes gangrenous. In the severe grades of the condition the muscular walls may participate in the process, and gangrene of the bladder wall with perforation is reported to have occurred. The walls of the organ become thickened and infiltrated and the immediately adjacent tissues external to the bladder may be involved (pericystitis). The reparative process under such circumstances is slow and the condition is very likely to become chronic. When the lesions are limited to the mucous membrane, resolution is ordinarily complete and may be comparatively rapid.

Chronic Cystitis.-When the process becomes chronic, the muscular structure of the bladder is always implicated. The mucous membrane changes its normal color and becomes of a dull bluish tinge, or paler than normal, and is frequently marked by small red dots, which represent capillary hemorrhage. Ulcerations with mucopus or phosphatic incrustations upon their surfaces are sometimes present. The mucosa as well as the muscular structure becomes thickened, and the inner lining of the bladder assumes the characteristic appearance of trabeculation which is produced by the special arrangement of the muscular fibers. (See Anatomy of the Bladder.) Rapid proliferation of the cells of the mucosa, leading to the development of the peculiar condition known 
as leukoplasia vesicæ is produced in some cases. (See chapter on Tumors of the Bladder.)

Heymann ${ }^{12}$ has called special attention to the fact that the cylindrical epithelium is changed into the squamous variety in a very large proportion of these cases. Heymann and also Ravisini ${ }^{13}$ and Breck ${ }^{14}$ have suggested the possibility of metaplasia being an etiological factor of malignant disease of the bladder. The proliferation process invades the submucous tissues in the form of large alveoli.

Hallé and Motz ${ }^{15}$ give an admirable description of the condition.

The wall of the bladder in cases of chronic inflammation may be an inch in thickness. The thickening is due to an increase of the muscular bundles and in lesser degree to the changes in the mucosa.

There is often a marked infiltration of the perivesical areolar tissue, and later, more or less connective-tissue forms.

The adjacent peritoneum may be involved in the process, and as a result becomes adherent to the prevesical tissue, and sometimes to the posterior aspect of the symphysis pubis. The latter condition is of practical importance because of the danger which it involves of cutting into the peritoneal cavity when doing a suprapubic cystotomy.

Pus formation may take place in the perivesical tissue. Perivesiculitis on the posterior aspect of the bladder causes the contour of the prostate and seminal vesicles to be obscured.

Bierhoff ${ }^{16}$ describes a peculiar form of cystitis, or a condition which he says he has found associated with cystitis in some instances, in which parts of the mucous membrane of the bladder are occupied by circumscribed vesicles varying in size from a pea to a millet seed, and which he states are produced by localized elevations of the epithelium by an underlying serous exudate. He has given the name "œdema bullosum" to the lesion. He has met with the condition in 15 cases in which it appeared as an accompaniment of catarrhal cystitis in 4 , of purulent cystitis of the vesical neck in I, of urethritis in I, of gonorrhœal cystitis in I, of ulceration produced by instrumentation in 2, of cystocele in I, of carcinoma in 4, of pyosalpinx in I. (This condition was described by Albarran and others long before.)

The conditions known as membranous, gangrenous, and diphtheritic cystitis are not essentially different in character, but only in degree. The last term is not intended to imply the presence or causal relation of the Klebs-Loeffler bacillus of diphtheria in the process which takes place in the bladder. It is applied to the cystitis in which false membrane is formed upon the inner surface of the bladder, and is cast off in a manner similar to that which is seen in cases of laryngeal and tracheal diphtheria. The urine is exceedingly ammoniacal in cystitis of this character. 
In membranous cystitis there is a marked exfoliation of the mucous membrane of the bladder. The condition usually occurs in connection with prolonged parturition, and is due to injury of the organ by the longcontinued pressure of the fœtal head upon it.

Gangrenous cystitis represents the extreme degree of vesical inflammation. The process may involve the mucosa only, or it may include the muscular structure as well, and may even cause perforation of the bladder.

Symptoms and Diagnosis.-AcuTE Cystitis.-The symptoms of acute cystitis are frequent and painful urination, fever, pain, and tenderness in the perineum and over the symphysis pubis, hematuria, pyuria, and an excess of mucous and bladder epithelial cells.

The urine may be either acid or alkaline in the early stages of an acute cystitis, and sometimes remains acid throughout its course. If the bladder becomes acutely inflamed when there is an obstruction to the free escape of urine from it, the reaction is alkaline.

A characteristic feature of the severer grades of acute cystitis is vesical tenesmus and the squeezing out of a few drops of pure blood from the bladder at the end of micturition. The blood is forced from the dilated capillaries of the congested mucous membrane by the violent contractions of the bladder.

With the exception of the alkaline reaction of the urine, all the other symptoms just enumerated may be present in cases of acute inflammation of the posterior urethra.

Chronic Cystitis.- In chronic cystitis the symptoms are less violent. Frequency of urination is present, though less marked. The urine contains large quantities of mucus and pus, and its reaction is alkaline. In many instances the mucus is so thick that it forms in ropy masses, and in certain cases in which a large amount of fibrin also appears in the urine, the whole of it, after being passed, is converted into a gelatinous semisolid substance, so thick that it will not flow from the glass containing it except in one mass. To this condition Ultzmann gave the name fibrinuria. He found it to be present in four cases of vesical cancer.

In chronic cystitis and in the declining stage of the acute condition the frequency of urination may be little more than normal.

The error in diagnosis most commonly made is that of mistaking pyelitis, pyelonephritis, or pyonephrosis for cystitis. It is a very frequent custom with general practitioners who lack the more exact means for making the diagnoses in these cases to assume that a patient is suffering from cystitis when he has frequent and more or less painful or uncomfortable urination, and a purulent and alkaline urine.

The following are the distinctive features by which the renal conditions 
may be distinguished from cystitis: Pyelitis and pyelonephritis, when not associated with cystitis, have an acid reaction of the urine, and the sediment settles slowly to the bottom of the vessel into which the urine is poured. In all cases of cystitis in which there is decomposition of urine the reaction is alkaline and the sediment sinks rapidly to the bottom of the vessel in which it is placed. Quick settling of the urinary sediment takes place in cases of cystitis in which the reaction of the urine is acid as well as those in which it is alkaline. Renal elements are lacking in cases of cystitis, and are present, in most instances, in cases of pyelitis and pyelonephritis. In cystitis there is marked desquamation of the epithelial cells of the bladder, while this feature is lacking in the suppurative renal conditions which are under discussion. The percentage of albumin in cases of the renal conditions mentioned is relatively high as compared with that associated with cystitis.

In cystitis, pain, if present, is localized above the symphysis, in the perineum, and along the urethra to the head of the penis, during micturition, while in the renal conditions pain and tenderness, if they occur are located just below the lower border of the twelfth rib on one or both sides.

The history of the case will supply, or fail to supply, a good reason for believing that cystitis exists. In addition to these distinctive features there are still more certain diagnostic methods that may be employed. These are the cystoscope and the ureteral catheter. The use of the latter instrument, however, is undesirable because of the danger of infecting the kidney when it is employed in the presence of cystitis.

The suppurative conditions of the kidney or the renal pelvis are demonstrated by the discovery of a urine coming from one or both of the ureters which contains pus or blood and renal elements.

In the cases in which the cystoscope and ureteral catheter cannot be used, the diagnosis must rest upon the features of the kidney conditions and of cystitis which we have already mentioned.

The differential diagnosis between cystitis and inflammation of the posterior urethra is best made by the so-called three-glass test. The urine is divided into three parts as it is passed, by catching it in three separate vessels. In cases of posterior urethritis, the first part of the urine contains a larger amount of pus and mucus than the second, and the third contains none at all, or very much less than either of the other parts passed. In cystitis all three parts contain pus and mucus, and the last part contains as much, at any rate, and generally much more than either of the two preceding ones.

In cases of posterior urethritis there is, or has been, an inflammation of the anterior urethra, while in many of the cases of cystitis the 
inflammation of the bladder arises without the occurrence of urethritis at all; when this is the case there need be but little question as to the diagnosis of cystitis, in so far as the differentiation between it and posterior urethral inflammation is concerned. In cases of cystitis which originate from an extension of a posterior urethritis, inflammation of the bladder may be limited to the area immediately about the vesical outlet. In these instances it may be difficult to discriminate between the vesical and the urethral lesion.

Treatment and Prognosis.- The treatment of cystitis varies in accordance with the special etiological factors which underlie the malady in individual cases. If calculus has produced the vesical inflammation, removal of the stone from the bladder is often the only measure of treatment that will be necessary to effect a cure. If an hypertrophied prostate or stricture of the urethra is responsible for the cystitis, these conditions must be treated appropriately before the inflammation of the bladder can be successfully dealt with.

So, too, with cystitis which originates in an infection that has descended from the kidney, the renal lesion must be remedied before we can hope to overcome the disease in the bladder.

The reader will find further reference to these special features under the headings and in the chapters in which they are individually discussed.

The treatment of the inflammation of the bladder per se, apart from that which is directed to the special etiological factors to which it is secondary, and which have just been mentioned, consists of the following measures: (I) Internal medication. (2) Local applications to the bladder. (3) Operative treatment. (4) Opsonic treatment. (5) General hygienic and supporting measures.

I. Internal Medication and Remedial Measures.-The indications to be fulfilled in cases of acute cystitis are: to lessen the congestion of the mucous membrane of the bladder, to overcome the activity of the invading microörganisms, and to remove the field offered for their development that is afforded by the presence of a decomposed residual urine.

These objects are accomplished by the employment of the following measures: by rest; hot hip baths; rendering the urine bland and nonirritating, which is best accomplished by drinking large quantities of water; by the administration of diluents and demulcents, $e$. $g$., flaxseed tea, and alkaline diuretics which act moderately, and among the best of which are the citate or acetate of potash. By overcoming pain and spasm of the bladder. This can be done by the use of morphine and belladonna suppositories in proportion of $\frac{1}{4}$ grain of the former and $\frac{1}{6}$ grain of the latter, or by combining hyoscyamus with the acetate of potash, taken internally. 
Casper ${ }^{15}$ recommends the following formula for diminishing pain and vesical tenesmus, to be used in the form of a rectal injection: antipyrine, I.O; laudanum, Io to 20 drops; water, $\mathrm{I} \frac{1}{2}$ fluidrams.

In cases of gonorrhœal cystitis, the balsams of copaiba and sandalwood oil are beneficial, especially in the declining stages of the disease, and if not given in too large doses.

Hot compresses, frequently renewed, and hot rectal injections of salt water (about two tablespoonfuls of rock salt to a pint of water at a temperature of $I I 5^{\circ}$ ) are helpful in allaying spasm of the acutely inflamed bladder. The injections should be administered with a soft-rubber catheter, not through a stiff tube, and the application of heat in this way should be prolonged if it is to be beneficial. Short applications of hot water to the rectal mucous membrane do not allay the vesical congestion, but if they are repeated often enough at each sitting, good does result from them.

When in bed the patient's hips should be elevated by resting them on a hard pillow.

The above measures constitute the remedial and palliative treatment which is appropriate for acute cystitis.

For the declining stages of acute cystitis and for chronic cystitis the principal effort is directed to rendering the urine in the bladder aseptic. The best remedies at our command for this purpose are the ammonium salts of formaldehyde (hexamethylenetetramin), urotropin, etc. They are too irritating to be used in the acute stages of the disease, and even in some of the chronic cases are not well borne, especially if prolonged; in small doses, however, they are almost always well tolerated and may be continued for considerable lengths of time without harm. When the urine is already acid, the hyperacidity of it produced by the liberation of formalin from these preparations is the cause of the irritation created by them, and under these conditions the urine should be made neutral or alkaline when employing the drug. One excellent preparation at present on the market is a tablet $n$ which cystogen is combined with the tartrate of lithia. The ordinary dose of urotropin is from 7 to ro grains thrice daily. If the bladder becomes irritable during its employment, the dosage should be cut down to 3 grains, or even less, and for two or three days it should be omitted altogether. Hematuria has been caused by the usual doses of urotropin, and so, too, has albuminuria. Omit the drug if they occur. Hygienic treatment is of importance in the cases of chronic cystitis. The action of the bowels should be carefully regulated, the skin should be kept active, and the patient should live outdoors as much as possible, care being taken to avoid his being chilled. Bodily exercise should be of moderate character. 
Local Applications to the Bladder.-Instillations.-In the acute stages of cystitis local applications are used with the intention of destroying the infecting organisms that have invaded the bladder. They are administered in the form of instillations or irrigations. The advantage of the treatment by irrigations as compared with that by instillations lies in the fact that the products of the inflammation are withdrawn from the bladder, and in the application of the remedial agent to the mucous membrane as well, while with instillations the latter of these ends only is secured.

Whichever method of treatment is selected, the drug that is employed for it should be used thoroughly and without hesitation. Both measures are painful, but the pain may be avoided by preceding the bladder application with a 2 per cent. or 4 per cent. cocaine instillation in the posterior urethra and vesical neck. This should be administered by a small softrubber catheter passed only as far as the anterior part of the prostatic urethra, from which point the fluid will flow over the rest of the urethra and into the bladder.

The drugs usually employed for making instillations in the bladder in cases of acute cystitis are argyrol and protargol.

The bladder should be emptied immediately before making the application. These two drugs are used in Io per cent. solutions, and are applied by the Keyes or Ultzmann deep urethral syringe; 5 to 40 drops is the quantity used in making the instillations. Desnos ${ }^{17}$ recommends the use of protargol every second day in this manner. In some cases this strength of the solution is too great, and it should be weakened and the intervals between the applications should be lengthened.

In the declining stages of the inflammation, silver nitrate is more beneficial than either argyrol or protargol. It has, however, the disadvantage of being painful, which is not the case with either of the other remedies. Silver nitrate is used in strengths of from $\frac{1}{10}$ of I per cent. to $\frac{1}{2}$ of I per cent.

In cases in which there is an acute gonorrhœal urethritis present in the anterior urethra this treatment should not be applied.

Irrigations.-It is impossible to sterilize the bladder by means of local applications of germicidal drugs; at best, the most that can be hoped for is that the activity of the microörganisms will be lessened. It may be laid down as a general principle that it is not only useless but harmful to attempt to destroy the infecting microörganisms by any antiseptic which is irritating in character, such as corrosive sublimate or carbolic acid; the exception to this rule is nitrate of silver. The cleansing, soothing medicaments are far preferable to those just mentioned, protargol and argyrol in solutions of from 5 per cent. to Io per cent. are among those vor. $\mathrm{I}-30$ 
which are beneficial. A preliminary irrigation of the bladder with sterile saline solution, followed by a 5 per cent. argyrol solution, is often admirable in its effect. In the more quiescent stages of the condition, oxycyanide of mercury is found by some surgeons to be excellent.

In the chronic form of cystitis, alphozone, in a $\mathrm{I}$ to Iooo solution, has proved in our experience of much service as a local application.

There are two ways of employing bladder irrigations: (I) By forcing the fluid into the urethra under the pressure of a column of water from three to five feet in height, which is enough to overcome the resistance of the compressor urethræ muscle in most cases. The injection is passed into the meatus through a nozzle which only enters a short distance into the urethra. (The Keifer nozzle is one that is especially well suited for the purpose.) (2) In the second of the ways of irrigating the bladder, the fluid is injected through a soft-rubber catheter.

When there is an acute inflammation in the posterior urethra, the first is the better of the two methods of irrigating the bladder; when there is a chronic process in the posterior urethra, the second is the better.

The bladder should not be distended by the irrigating fluid to the point of being painful in any case, and even when no pain is felt by the patient it is best not to inject a large amount of fluid, but to stop at six ounces, letting it return again at once, and repeating the injections until the returning fluid is clear. Boric acid (4 per cent.) solution or sterile saline solution may be thus used as a preliminary cleansing wash, and when completed, a couple of drams of a 5 to ro per cent. argyrol solution are to be thrown into the bladder and the catheter is withdrawn, leaving the medicament in the bladder to be passed out with the next urination.

Irrigation has the advantage of emptying and cleansing the bladder, and hence is to be preferred to instillations for all cases in which the inflammation has extended beyond the vesical neck.

Such irrigations of the bladder may be given twice daily in cases of chronic cystitis with foul urines.

Continuous Drainage.-In cases of chronic cystitis, and especially those in which there is a residual urine, or in which the renal pelvis or kidneys are involved in the suppurative process, or in which the kidneys are, for any reason, functionally inadequate, whe her infected or not, continuous drainage through a catheter tied into the bladder through the urethra is in some instances beneficial to both the vesical and the renal conditions. The catheter should be of soft rubber.

Opsonic Treatment.- The experience with this method of treatment is as yet limited.

Ohlmacher ${ }^{18}$ has reported a case of cystitis and pyelonephritis caused by the Bacillus coli communis, in a tabetic patient. The urine was 
loaded with thick gelatinous pus. There were daily chills, with rise of temperature to $103^{\circ}$. The kidney was enlarged and palpable; the phagocytic index was one-third less than normal; a serum was made by isolating the bacillus coli from the urine; the patient was inoculated with this serum at intervals of from five to ten days, receiving five doses altogether. Improvement of the patient's condition was apparent from the time of the first injection; the phagocytic index rose and the temperature did not reach $100^{\circ}$; the chills ceased three days later. At the time of Ohlmacher's publication of the case, which was seven weeks after the last injection had been given, the patient's general condition was greatly improved and there was but a small amount of thin pus in the urine.

Surgical Treatment.-In some cases of chronic cystitis which fail to receive benefit from the methods of treatment already enumerated prolonged suprapubic drainage with a double tube may be tried with advantage. The measure, however, is one that is usually employed in connection with prostatectomy or the operations for removal of vesical tumors and calculus, and is considered at greater length in connection with those special operations.

\section{Bibliography.}

I. Gerota. Anat. anz., xii, 4, 5, p. 89 .

2. Gould and Pyle. Anomalies and Curiosities of Medicine, I 897.

3. Harrison. Lancet, I 897 .

4. Bottomley. Journal of American Medical Association, July I3, 1907.

5. Watson. Annals of Surgery, December, 1905.

6. Mitchell. Annals of Surgery, I898, p. I5I.

7. Quervain. Deut. Zeit. f. Chir., I90I-I902, lxii, 58.

8. Ledderhose. Verhandl. der Deut. Ges. f. Chir., I902.

9. Quick. Annals of Surgery, 1907, p. 94.

I0. Bliss. Edinburgh Med. Jour., I902, vol. liv, p. 35 I.

I I. Horton-Smith. London Lancet, April I 4, 1900.

I2. Heymann. Centralbl. f. Krank. d. Harn. u. Sex. Org., I9o6, xvii, I 77.

13. Ravisini. Ibid., I903, xiv, 255.

14. Breck. Wien. med. Presse, i 896, Nos. 36 and 37.

15. Hallé and Motz. Ann. des Mal. des Org. Gén.-urin., I902, xx, I7.

16. Bierhoff. Medical News, New York, 1900, vol. lxxvii.

17. Desnos. Rev. Gén. de Chir. et Thérap., I904, lxxvii, 508.

I8. Ohlmacher. Journal of American Medical Association, xlviii, 575. 


\section{H A P T E R X V. \\ VESICAL CALCULUS AND DIVERTICULUM OF THE BLADDER.}

\section{VESICAL CALCULUS.}

Definition.-A stone-like body in the bladder composed of crystals of the urinary salts, held together by a colloid material, found under certain conditions in the urinary tract.

Manner in Which Vesical Calculus is Formed (see chapter on Renal Calculus).- Ord, Rainey, and Carter have demonstrated that two elements must co-exist in the urinary tract in order to have calculus form in it, and that the presence of crystals alone is not enough to bring it about. These two elements are the crystals of the urinary salts and a colloid substance the presence of which in the urinary tract is dependent upon conditions but imperfectly understood. According to Carter, this material causes the deposit not of actual crystals, but of bodies which he designates as submorphous, which have the property not only of adhering to adjacent surfaces, but also to each other in laminar series.

The presence or absence of the colloid material in individual cases may explain the occurrence in some of them of rapid calculus formation, and in others its tardy appearance, despite the fact that in the latter as well as in the former there may have been crystals deposited in the urine for long periods.

Primary and Secondary Calculi.-Primary calculi are those which are formed in acid urines. The larger number of these are of renal origin, and subsequently descend to the bladder. Secondary calculi are those which are formed in alkaline urines and originate in the bladder itself in the majority of cases. Stones of uric acid, the acid urates, oxalate of lime, xanthin, cystin, and indigo belong to the first class; those composed of the triple phosphates, the urate of ammonia, amorphous phosphates, to the second.

The conditions in the bladder which favor calculus formation are: (I) inability to completely empty itself, and (2) vesical infection. The most frequent causes of the former of these two conditions are urethral stricture and prostatic hypertrophy. They also often give rise to vesical 
infection by bringing about the local conditions of the bladder which render it particularly prone to bacterial invasion. The cystitis which results from the infection of such bladders causes alkaline decomposition of the urine and thus leads to the deposit of the crystals of the triple phosphates which, with the colloid material already referred to, sooner or later form calculi in bladders which do not wholly empty themselves.

The distinction should be clearly made between the calculi composed of the triple phosphates and those consisting of the amorphous phosphates. The latter do not appear in the urine as the result of the conditions which are active in the formation of secondary vesical calculus, but are precipitated because the urine is alkaline or neutral when secreted in the kidney, and its alkalinity, therefore, is not dependent upon the existence of vesical infection and alkaline decomposition of urine in the bladder.

Etiology.-(The etiology of primary calculi will be found in the chapter on Renal Calculus, to which the reader is referred for this part of the subject.)

Chemical Nature of Vesical Calculus.-Primary calculi often have the same chemical composition throughout. Less frequently this is the case with the secondary calculi. In most instances the stone is composed of alternating layers of different kinds of urinary salts, those most commonly met with being uric acid and triple phosphates or the urates in successive layers.

Classification.-Calculi may be classified according to the nature of the salts which predominate in their composition, thus:

I. Uric acid and acid urates.

2. Oxalate of lime.

3. Phosphatic.

4. Cystin.

5. The rare calculi of xanthin, urostealith, indigo.

Frequency.-The relative frequency with which the different varieties of stones occur may be judged from the following table, taken from Sir Henry Thompson. ${ }^{11}$

\section{Male Adults.}

Uric acid

Uric acid and phosphates .

Phosphates .

Oxalates and urates

Oxalates.

Oxalates and phosphates .

Cystin

Total . 


\section{Female Adults.}

Uric acid . . . . . . . . . . . . . . . . . . Io

Uric acid and phosphates. . . . . . . . . . . . . . . 2

Phosphates . . . . . . . . . . . . . . . . . . 2

Total . . . . . . . . . . . . . . . . . . . $\mathrm{I}_{4}$

\section{Children.}

Uric acid

Urates and phosphates

Oxalate .

Oxalates and urates

Phosphates .

Total

Total, 1007 cases.

From the above it will be seen that $5^{6}$ per cent. of the whole number were uric acid stones.

\section{Tests of the Chemical Nature of Calculi.}

Combustible. $\left\{\begin{array}{l}\text { Without } \mathrm{fl} \text { a } \mathrm{m} \mathrm{e}\left\{\begin{array}{c}\text { Murexide test, with ammonia } \\ \text { purple red, with potash purple } \\ \text { and odor. } \\ \text { violet. } \\ \text { Murexide test with ammonia } \\ \text { yellow, with potash orange. }\end{array}\right. \\ \text { Non-combustible. } \\ \text { The powder burns with pale blue flame and smell } \\ \text { of asafoetida. }\end{array}\right.$

Characteristics.-The oxalate of lime calculi are the hardest of the stones, the amorphous phosphates and cystin are the softest, and between these two extremes stand the urates, uric acid, and mixed stones. Pure uric acid calculi are in some instances friable and not very hard; ordinarily they are hard. The urate stones are soft. Those composed of cystin are very soft and crumbly.

According to Ultzmann, when calculi are solitary and freely movable in the bladder, their form is largely determined by their chemical composition. Pure urate stone has a long oval shape, the pure oxalate is more spherical, the form of the mixed stone will be inclined to follow that which is characteristic of the chemical composition of the salt predominating in it. 
The shape is modified by the pressure of calculi upon each other when more than one is present.

Very curious forms are at times assumed by encysted calculi. Some of the features of the different kinds of calculi are shown in Fig. 253.

FIG. 253

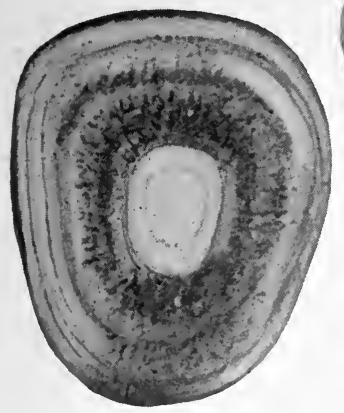

D

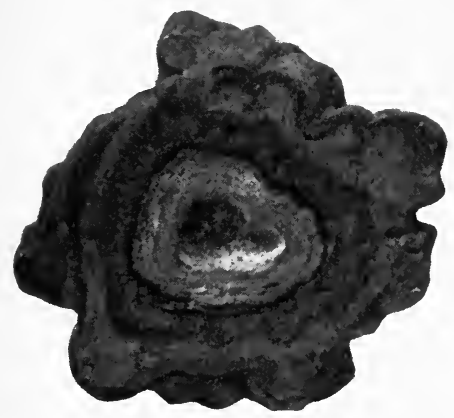

C

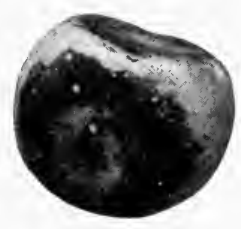

A
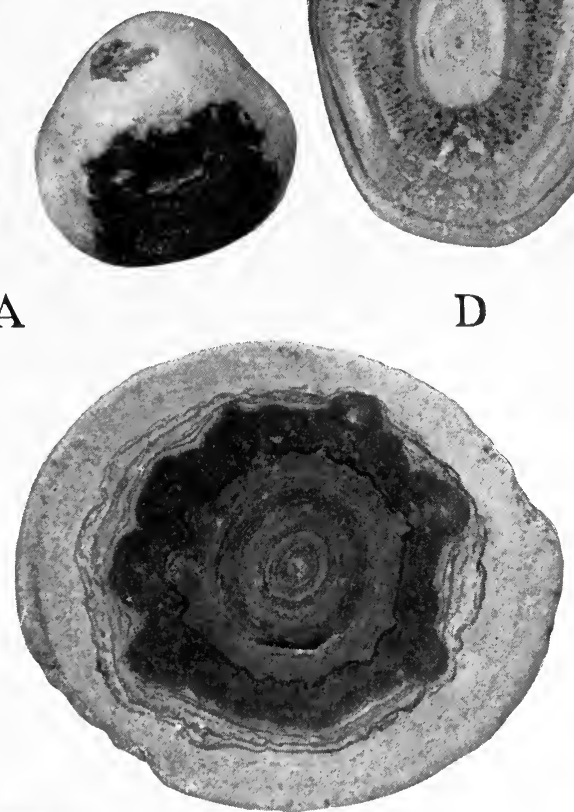

B

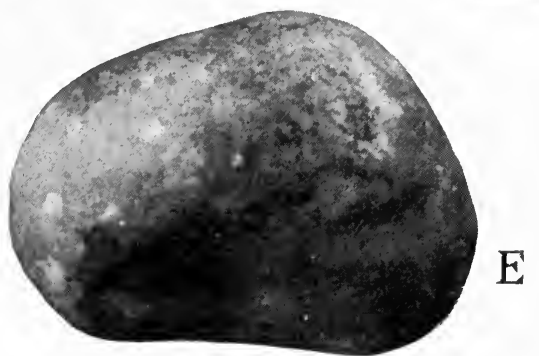

$A$, four oxalate calculi incrusted with phosphates; $B$, oxalate centre, outer layers phosphates; $C$, pure oxalate stone (mulberry calculus); $D$, phosphate and urate calculi; $E$, urate calculus.

Color.-Uric acid, reddish brown. Oxalate of lime, gray to almost black. Phosphates, dirty grayish or yellowish white. Urates, yellowish or yellowish red. Cystin, pale yellow. 
Surface.-In its typical form oxalate of lime has the most characteristic surface of any of the calculi. It is composed of a number of small elevations, owing to which and to its color it has been called the "mulberry calculus."

Phosphates.- Smooth, or roughened by fine irregularities, which give it a gritty surface. In some instances the surface is thrown into more or less irregular nodules of larger size.

Urates and Uric Acid.-Smooth surfaces.

Size.-Only such calculi as are too large to be passed spontaneously from the bladder through the urethra are included under the term of calculus; those smaller than this are usually called concretions, gravel, or sand. This definition would exclude those of less and include those of more than 20 grains in weight, approximately.

The weight of calculi varies from 20 grains to many ounces. The following table gives the weights of some of the largest stones that have been reported. They are divided into three series, the first including those not removed by operation, the second those in which the patients recovered after the removal of the stones by operation, and the third those in which death followed operation for the removal of the calculi.

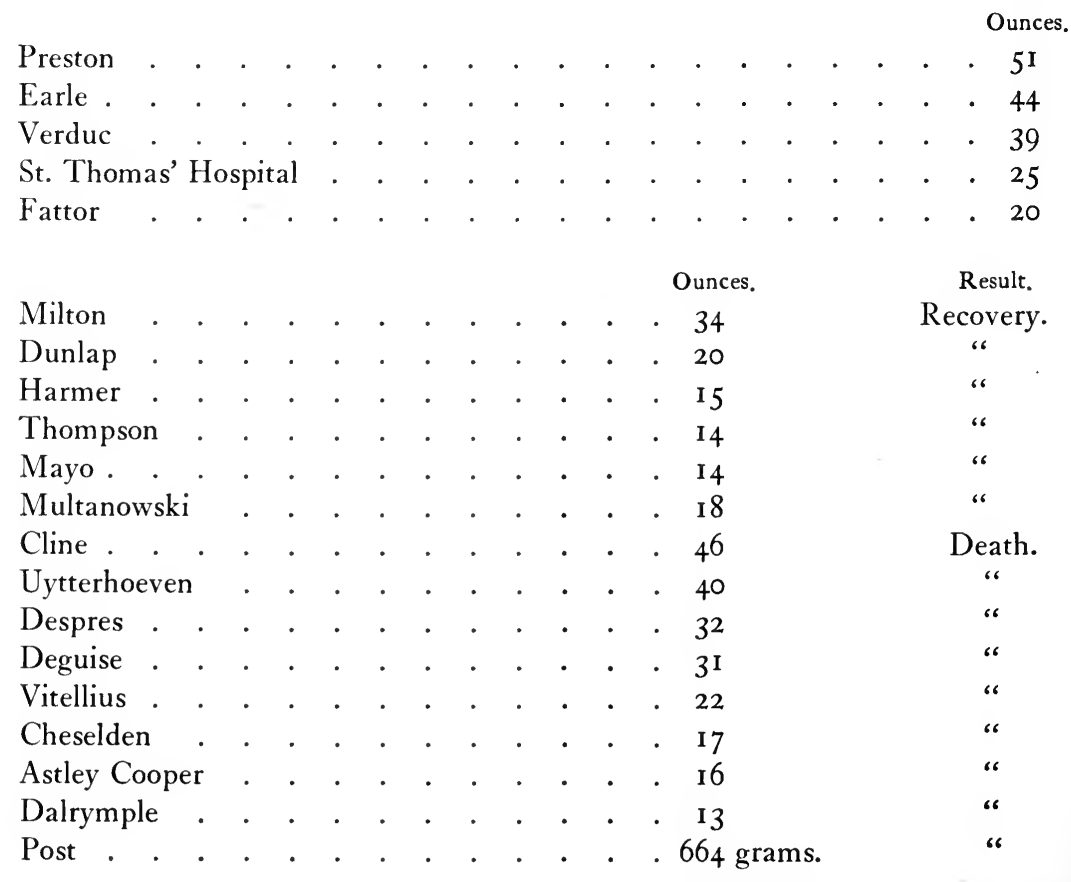

Fig. 254 is from a photograph, showing the actual size of a vesical calculus weighing $66_{4}$ grams, removed by Dr. Abner Post, of Boston, by a suprapubic lithotomy. 
Encysted Calculus.-Diverticula of the bladder not infrequently harbor calculi. The stones under these circumstances assume very odd forms, which correspond, as a rule, to the conformation of the pocket in which they lie. In many instances one part of the calculus rests within and the other projects outside of the pouch into the interior of the bladder, the stone in such cases usually having a more or less dumb-bell shape, owing to the fact of its being constricted at the point at which the neck of the sac surrounds it (see Figs. 255 and 256).

In other cases, a part of a calculus will rest in a sort of depression or wide-mouthed cavity, generally upon the floor of the viscus, and is then

\section{FIG. 254}

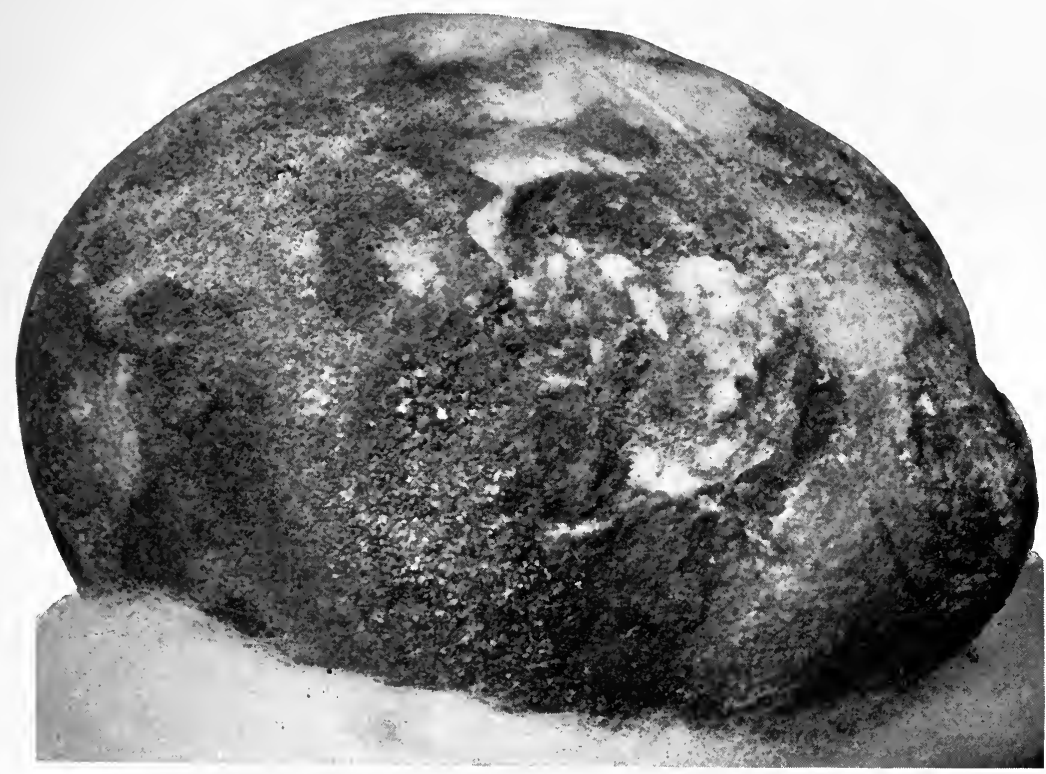

Stone removed from the bladder by Dr. Abner Post of Boston. Weight, 664 grams. Actual size.

apt to be firmly attached to the sides of it. Such stones as these are often very difficult to deal with on account of their intimate connection with the bladder.

Encysted calculi offer special obstacles both because of the difficulty of detecting them and the impossibility of removing them.

In a few instances, calculi are found lying partly within the prostatic urethra or a pouch in the prostate itself and partly in the bladder (see Fig. 257).

Number.-Cases have been reported in which as many as 300 or 400 stones have been found in the bladder. It is rare, however, to find more 
than five or six. The presence of facets on one calculus suggests the co-existence of others. The practical bearing of this fact in connection with the removal of calculi is obvious (see Fig. 253, $A$ ).

Nuclei.-Uric acid is said to form the nucleus of from 60 per cent. to 80 per cent. of all calculi, but it may consist of any of the urinary salts.

FIG. 255

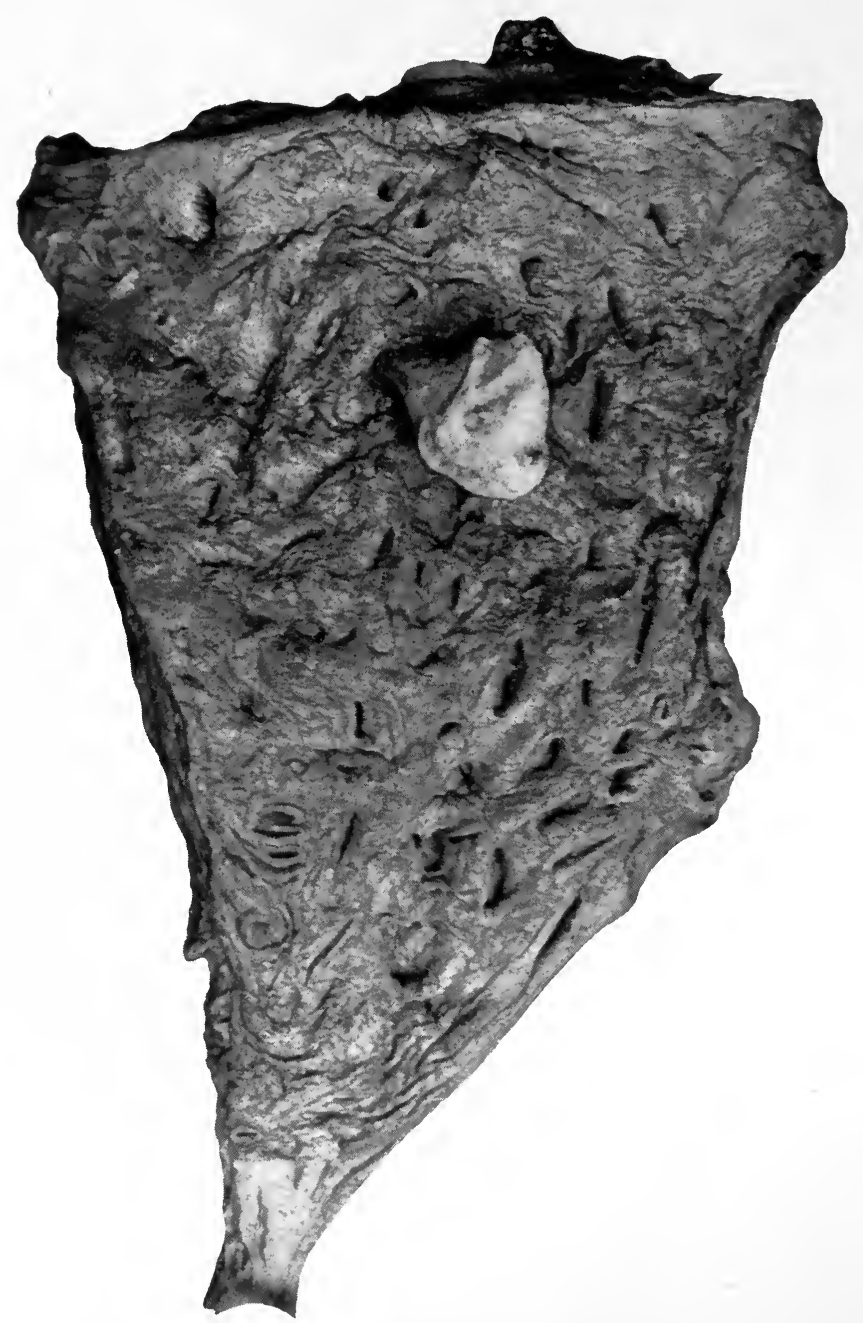

Portion of bladder with encysted calculus, from a specimen in author's possession.

Foreign Bodies as Nuclei.-A great variety of substances have been reported as constituting the nuclei of vesical stones. Many of these have been introduced into the bladder, especially in the case of women, in the endeavor to procure sexual sensation. 
FIG. 256

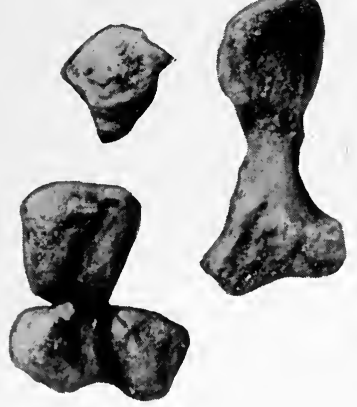

Calculi removed from a, double chambered diverticulum. Unusual forms of calculi.

FIG. 257

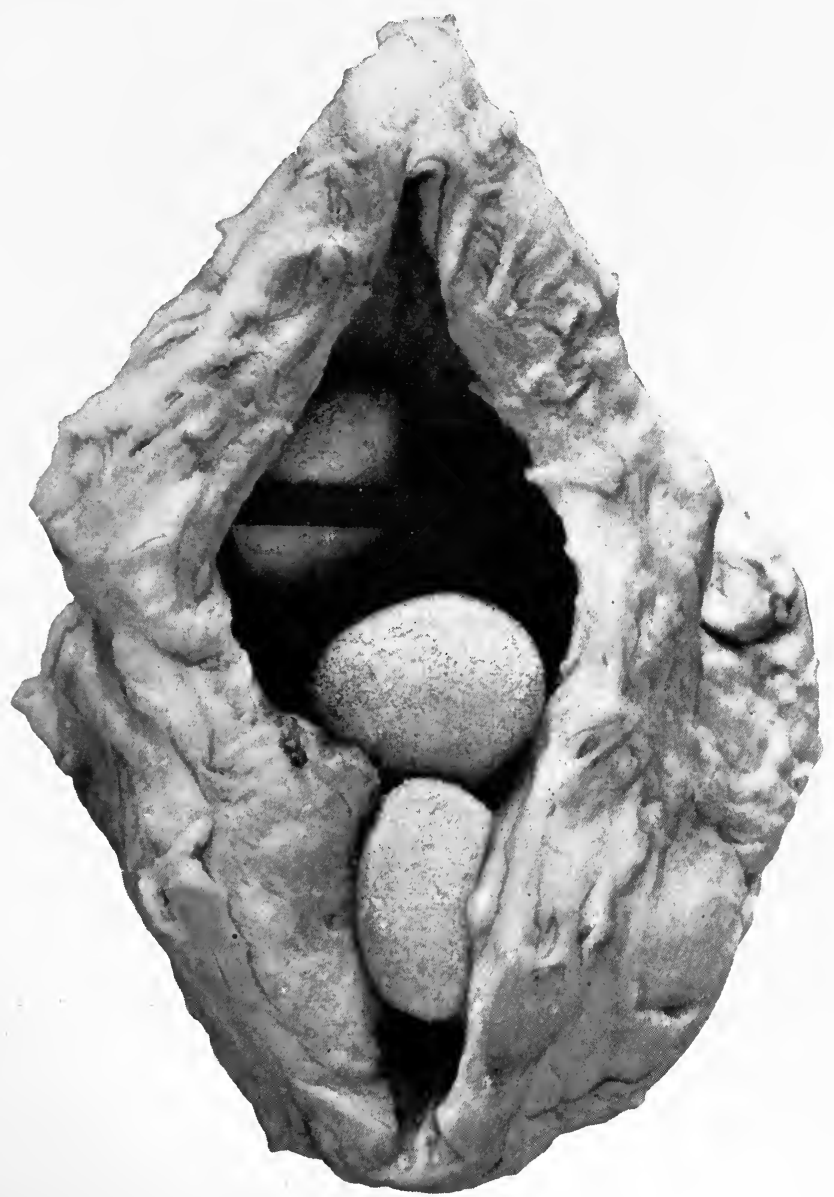

Calculi lying in the bladder. One is impacted in the prostatic urethra. (Warren Museum.) 
Figs. 258, 259, and 260 illustrate some of the articles which have been found as the nuclei of vesical stones. Among them is the mouthpiece of a pipe found in the bladder of a patient of Reginald Harrison. The patient declared that he had swallowed the mouthpiece some time before,

FIG. 258

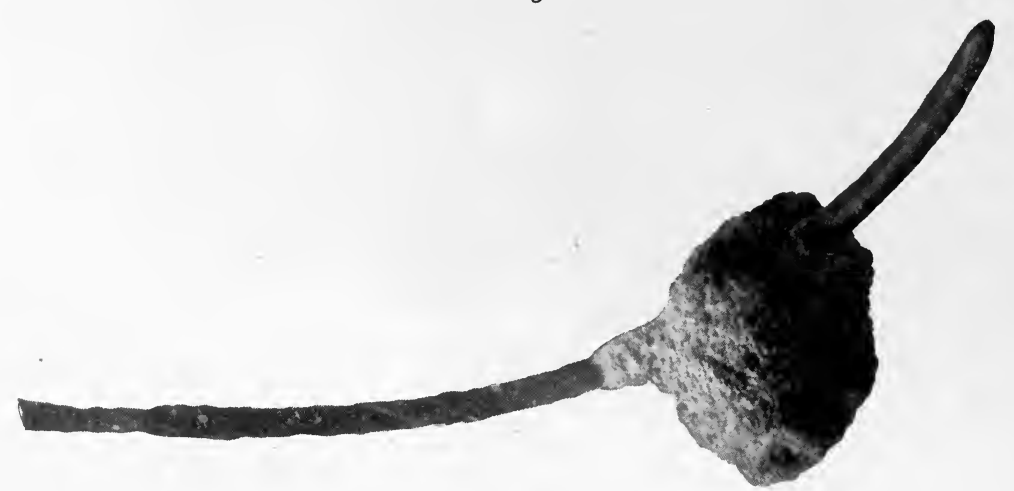

Calculus formed about a bit of catheter in the bladder. (Warren Museum.)

FIG. 259

Needle armed with a knob of sealing-wax. (Harrison.)

FIG. 260

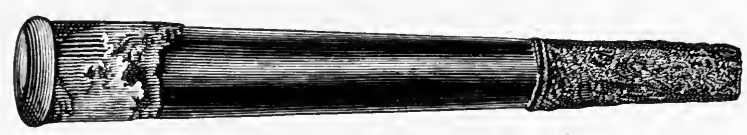

Mouthpiece of pipe removed from the bladder. (Harrison.)

and stoutly adhered to this story. It is possible that it may have reached the bladder by ulcerating through the intestinal and vesical walls. Several other examples of similar curious occurrences are to be found in medical literature.

Spontaneous Fracture of Stone.-This interesting phenomenon is not infrequently observed by the physicians practising at some of the Continental health resorts. Debout d'Estrees and Francon, of Contrexeville and Aix les Bains, respectively, have told the writer that it is not an infrequent occurrence in their experience.

Two theories are advanced to explain the occurrence of spontaneous fracture of stone. One, that it is due to mechanical, and the other that it is brought about by chemical action. That a stone may spontaneously break in the absence of mechanical injury is clearly shown by one of the writer's cases. Whether or not the breaking of the part of the stone in 
FIG. 26I

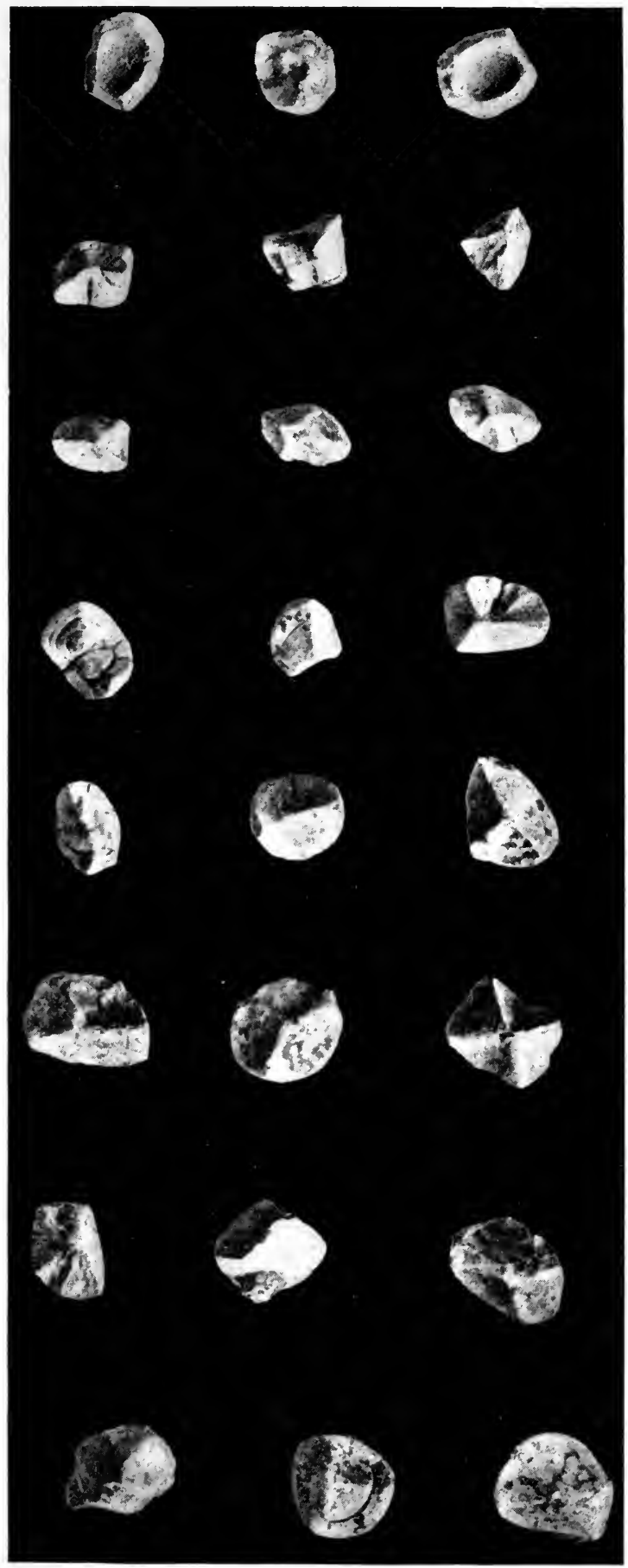

Spontaneous fracture of stone in the bladder. (Watson.) 
this case, which lay within a diverticulum, was caused by chemical action, and, if so, by what kind of chemical influence, is not clear. Ord and Rainey have suggested that this may be brought about by some peculiar condition of the calculus, owing to which its colloid framework has rapidly imbibed fluid, causing it to swell and thus fracture the stone (Figs. 26I and 262).

Pathology.-The pathological changes in the urinary tract are all secondary to the presence of calculus, and are primarily such as result

FIG. 262

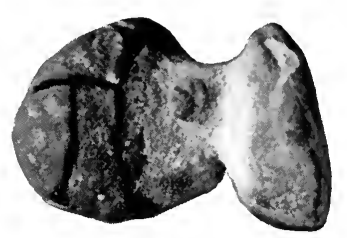

Spontaneous fracture of stone in the bladder. The fractured end of this calculus lay within a diverticulum and was protected from mechanical injury. (Watson.) from the mechanical irritation set up by it. The bladder is naturally the first structure to suffer. The changes produced in it are those of congestion, infection, and inflammation. In some cases it is a good while before these appear in a well-marked degree. This is in large part due to the character of the surface of the calculus. A smooth uric acid stone formed in an acid urine may be present for some time before giving rise to cystitis of importance, while a rough oxalate stone will, on the contrary, do so very shortly. (The tissue changes which occur in cystitis are described under the heading Pathology in the chapter on Cystitis.) The secondary conditions to which vesical calculus may give rise affect the kidneys, and are due to the interference with the performance of their function: (I) by obstructing the outflow of the urine, and (2) by infection, to which they are rendered liable because of the presence of infection of the bladder, in consequence of which pyelitis and pyelonephritis occur in a certain proportion of the cases.

Symptoms and Diagnosis.- The two cardinal symptoms of vesical calculus are pain and hematuria.

Pain.-The occurrence of actual pain is usually preceded for variable periods by vesical irritability, which is manifested in the form of frequent urination and more or less discomfort attending the act.

It should not be forgotten that bladder irritability is often due to the presence of a renal lesion or condition causing renal irritation, the sensation produced by it being the same as that which is commonly associated with disturbances of the bladder. It is likewise important to bear in mind the fact that a urine which contains an abundant crystalline precipitate will give rise to the same feeling that is produced by calculus in some cases.

In the great majority of cases, pain of a severe or distressing character is produced by the presence of stone in the bladder. There are a few 
instances in which it is but slight, and it may be entirely absent. Its absence may be accounted for sometimes by the fact that the calculus is lodged beneath an overlying intravesical projection of an enlarged prostate in such a way that it does not come in contact with the more sensitive part of the bladder, namely, its outlet. The writer recalls one case in which the patient had never been conscious of any pain whatever, and in whose bladder there were found postmortem a number of caluli situated beneath a projecting prostatic lobe.

The characteristics of pain due to vesical calculus are these: (I) Its increase on exercise, and from jolting movements, e. $g$., driving over a rough road, riding a hard-gaited horse. (2) Its being most severe at the end of the act of urination. The pain is most marked in the case of children, because of the greater sensitiveness of their bladders. They dread having to urinate, and will often postpone the act as long as possible, knowing how greatly their suffering will be increased as the bladder becomes empty.

The nature of the pain is that of an intense desire to urinate in most cases; in some it has the character of a dull ache located in the perineum and about the anus. It often causes straining at stool, thus giving rise to hemorrhoids and sometimes to partial prolapse of the rectum.

Exceptionally, the pain radiates to the sacral, sciatic, and anterior crural regions. This should always cause suspicion of malignant disease of the prostate, however, as it is more often seen in connection with that condition than with vesical calculus. Some patients have periods of freedom from pain, which fact may be due to a change in the reaction of the urine and to a corresponding change in the surface of the calculus, whereby it becomes smoother and thus less irritating.

Blood.-Blood in the urine may be in microscopic quantities only; occasionally it is entirely absent, for a time at any rate; more often it is present in considerable amount; exceptionally there is profuse bleeding.

The special features of hematuria due to vesical calculus are as follows: (I) The amount of blood is increased by exercise. (2) It is most abundant in the last part of the urine passed as compared with the first half. (3) Except when the blood has been retained a long while in the bladder - as in cases of enlarged prostate-it has the character of fresh blood. (4) It is associated with pain of the nature just described above.

It is true that most of the features noted as being indicative of the existence of vesical calculus may be present in cases of prostatic hypertrophy in which there is an eroded surface of a prominent projection of the gland, and in some cases of tumor of the bladder, but both of these conditions lack, with but few exceptions, the feature of increase of both 
pain and blood with exercise, which is so distinctive of vesical calculus in the great majority of instances.

Sudden Stoppage of the Urinary Stream.-This symptom, which some writers state to be of frequent occurrence, is, according to Sir Henry Thompson, a rare one. When present, it is thought to be due to the spasmodic contraction of the vesical sphincter, called forth by the stone being pushed down into the outlet of the bladder as the organ empties itself.

Priapism and Pulling upon the Foreskin.-These are often evidences of the presence of stone in the bladder in the case of children.

Evidences Furnished by the Urine.-The urine from the bladders of patients with calculus cystitis shows no features which serve to distinguish it from that which is seen when it arises in connection with other vesical diseases; it does, however, often present characters which are, at any rate, suggestive of calculus. These are: a persistent crystalline sediment; an increase of the number of blood corpuscles, when blood is present in microscopic quantity only, after exercise as compared to that seen when the patient has been at rest. Cabot calls attention to the fact that the sudden disappearance of a brickdust sediment which has been abundant and persistent marks the beginning formation of vesical calculus in some instances.

Ultzmann considered the long-continued presence in the urine of the rarer forms of some of the urinary crystals-uric acid and oxalates especially - to be indicative of the formation of stone within a short time.

Physical Examination.-The actual proof of the existence of stone in the bladder is furnished, with but few exceptions, by one or another of the methods of physical examination. These are as follows: (I) $X$-ray examination. (2) The exploration of the bladder with the stone searcher. (3) The cystoscope. (4) Rectal and bimanual palpation. (5) Digital exploration of the bladder through an external median perineal urethrotomy incision.

X-ray Examination.-Skiagraphs will demonstrate the presence of all but the pure uric acid calculi, or in about one-half of the cases, if we take the relative numerical proportion in which the different calculi occur as that which we have quoted from the series of observations of Thompson. As a matter of fact, the number which can be demonstrated by the $x$-ray examination will prove to be a good deal larger than this. Indeed, Kümmel asserts, in speaking of renal calculi, that with the improved methods which he employs he can detect in the kidney any stone of any size or chemical composition.

The advantage of this means of demonstrating the presence of stone is that it obviates the necessity of passing instruments into the bladder for 
the purpose of directing it, and that the size of the calculus can be estimated.

The Stone Searcher.-The usual way of detecting stone in the bladder is by passing the instrument known as the stone searcher and feeling the calculus with its tip. The form of searcher that is well suited to the purpose is that designed by Thompson, and illustrated in Fig. 263. There are two sizes of this instrument, one having a caliber of about 16 of the French scale, for adults, and the other corresponding to No. Io of the same scale, for children.

A general idea of the size of the stone can be obtained by passing the end of the searcher along its sides. Its mobility can also be judged by pressing first on one and then on the other side of it with the tip of the searcher. So, too, can multiple calculi be detected, by pressing the beak of the instrument against the calculus upon which it has first rested and then rotating it to one side so that it will strike upon a second one if present. A better way of determining the presence of multiple calculi is by the lithotrite. This is done by seizing the stone first felt between the blades of the instrument and rotating it one way and then the other, so that it will strike against other calculi if present on either side.

FIG. 263

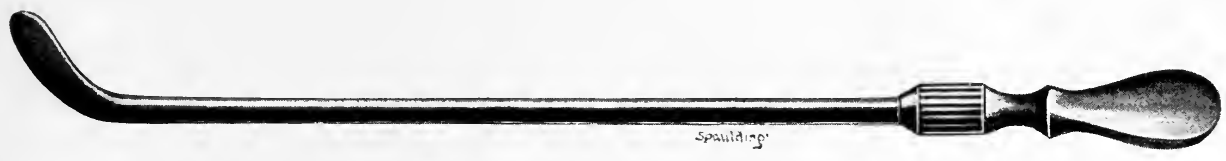

Thompson's stone searcher.

For purposes of demonstration, the sound made by the tapping of the end of the instrument against the stone within the bladder may be made audible by attaching a sounding board to its other extremity.

The bladder should contain from four to six ounces of fluid when the examination for stone is made.

Before completing the examination, and especially in cases in which there is an enlarged prostate, the tip of the searcher should be turned over so that it rests on the floor of the viscus behind and beneath the base of the gland, for it is in this depression that calculi very frequently lie. A finger in the rectum will aid in the detection of the stone when this is being done.

Sources of Error in Sounding for Stone.-I. Calculi which lie beneath intravesical projections of an enlarged prostate may be impossible to detect with the searcher, and are frequently overlooked (Fig. 264).

2. The operator may believe that he has entered the bladder with the instrument when in fact he has not done so, but is deceived by a distended voL I. -3 I 
prostatic urethra, which exists in some cases of prostatic hypertrophy, and which permits the free rotation of the sound. Such a dilated prostatic urethra may contain as much as four to six drams of fluid.

3. Phosphatic incrustation of the lining of the bladder gives a very deceptive sensation to the fingers holding the handle of the searcher, especially is this the case when the deposit of the salts occupies a prominent ridge of a trabeculated bladder.

4. The calculus may be covered with soft, sticky mucus, and then will give a characteristic sensation by which the fingers will recognize it.

5. The calculus may be encysted and then wholly escape detection, even though the searcher touches the spot on the interior of the bladder immediately behind which it lies.

Examination with the Evacuating Apparatus.-There is no better means of detecting the presence of small calculi than by the use of the

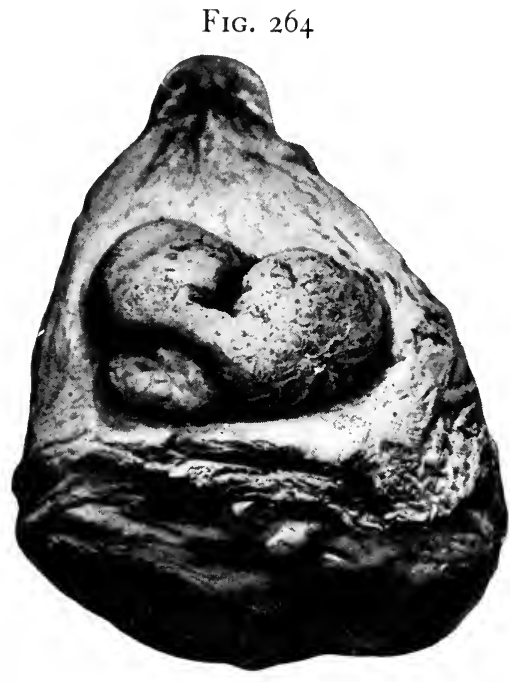

A calculus lodged beneath an intravesical projection of the prostate. (Watson.) evacuating tube and bulb. The tube is passed into the bladder, its tip pressed down upon the floor as near the base of the prostate as may be conveniently done. This is for the purpose of making a depression into which the calculus will be readily sucked by the outflowing stream, and the to-and-fro current of water which is set in motion by the alternate compression and relaxation of the rubber bulb attached to the tube. When this is done, the calculus is drawn sharply against the mouth of the tube, giving to it a quick stroke, which is accompanied by an audible click. In the case of women and children, the calculus if not a very small one can often be detected by bimanual palpation with the fingers of one hand upon the abdomen above the symphysis, and the forefinger of the other one in the rectum or in the vagina.

The Cystoscope.-The cystoscope is not often needed for the purpose of detecting vesical calculus, but is invaluable in cases of encysted stone and in some of those in which the stone is lodged beneath a projecting intravesical lobe of an hypertrophied prostate.

If none of these means succeed in detecting the stone, and there is good reason to believe it to be present, there remains digital exploration of the bladder. This may be done through an ordinary external median perineal urethrotomy incision. 
Treatment.-The treatment of vesical calculus is: (I) Preventive. (2) Operative.

Preventive Treatment.-(See chapters on Renal Calculus and on Cystitis.)

Operative Treatment.-(For description of the operative technique see chapter on Technique of Operations on the Bladder.)

The operative treatment of vesical calculus consists in the removal of the stone from the bladder by means of one of a number of the cutting operations, or by crushing it within the bladder and removing the fragments by a suction apparatus. These procedures are named as follows:

I. Lithotomy (perineal and suprapubic).

2. Lithotrity or partially crushing the calculus within the bladder at each of a number of sittings and allowing the debris to be passed out voluntarily.

3. Litholapaxy or crushing and removing the calculus at one sitting.

4. Perineal litholapaxy, the same operation performed through an external perineal urethrotomy incision and dilated prostatic urethra.

Bilateral and Lateral Perineal Lithotomy.-These operations have, practically speaking, been abandoned because of the fact that rapid lithotrity and suprapubic lithotomy have given so much better results and are free from the objections to which both of the formerly widely practiced methods are open. We shall, therefore, omit them from the discussion of the choice of operative methods to which we will now proceed.

The Choice of Operative Method.-The following are the determining factors with respect to the choice of operation:

I. The operative mortality $(a)$ as influenced by the age of the patient; (b) as influenced by the size of the calculus.

2. The character of the results of the different operative procedures; that is to say, with reference to permanency of cure or recurrence.

3. The presence or absence of prostatic hypertrophy.

It is manifestly misleading to compare surgical procedures when applied, in one instance, to elderly or old men, and in another to young adults or vigorous children; hence the separation of the cases which we have made in our subjoined analysis, so far as possible, into different classes with reference to the age of the patients.

It has not been possible for us to determine numerically in just what proportion of the cases prostatic hypertrophy has existed, because of the lack of definite statement with regard to this point in many of the reports. It can, of course, be asserted that the condition has not been present in any of the younger patients, and, equally, we may assume it to have existed in a considerable number of those over fifty years of age; but just 
what that number in the latter class is we do not know, hence we have not attempted to make any computation with reference to this particular point, and limit ourselves to the statement of our own personal belief with regard to the influence which is exercised upon the choice of operation by the existence of an obstructing prostate, which is, that litholapaxy should not be selected or persisted with in cases in which there is an enlargement such as to make the passage of the instruments unusually difficult or to incur the danger of injuring the projecting parts of the gland. The other effect that this condition has upon the choice of method is the greater liability to recurrence of calculus with litholapaxy because of overlooking fragments of the stone which has been crushed, owing to their being tucked away beneath intravesical projections of the gland (see Fig. 264). With regard to the influence exercised upon the choice of operation by the size of the stone, it may be said that, although very large calculi have been and can be successfully operated upon by litholapaxy, the fact of unusually large size is unfavorable to the success of that operation, especially if the stone is very hard.

One other special condition which makes litholapaxy undesirable and almost necessarily unsuccessful, is that of encysted calculus, because of the impossibility in many cases of reaching and crushing the portion of the stone which lies within the pouch of a diverticulum. It is true that this feat has been successfully accomplished by some surgeons, e. $g$., Freyer, ${ }^{1}$ but we do not advise the application of the method to this particular class of cases, because of the unusual difficulties presented by them.

We have collected a large number of cases in which the operations of litholapaxy, of lateral, of median perineal, and of suprapubic lithotomy have been performed, and have analyzed them with reference to the operative mortality per se, with reference to the ages of the patients, and the operative mortality; with regard to the proportion of recurrences, and finally, there is a list of a number of operations performed upon patients with calculi of unusually large size given in the earlier part of the chapter.

The total number of cases is as follows:

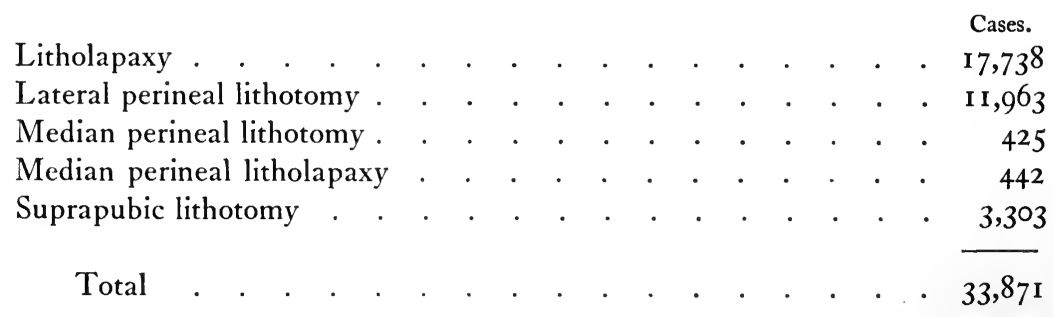

The following comparison of the methods with each other is made, first, with regard to the operative mortality with reference to the ages 
of the patients, when such a comparison was possible; that is to say, we have compared the cases in which each of the different procedures was applied, under three different headings: (I) That in which the patients were between the ages of one and fifteen years. (2) That in which the ages were between sixteen and fifty. (3) That in which the patients were over fifty years of age. The second table gives the operative mortality irrespective of the age of the patients.

\section{Operative Mortality.}

Median perineal lithotomy Suprapubic lithotomy Median perineal litholapaxy Lateral perineal lithotomy . Litholapaxy

\begin{tabular}{|c|c|c|c|c|c|}
\hline \multicolumn{2}{|c|}{ Ages 1 to 15 . } & \multicolumn{2}{|c|}{ Ages 16 to $5^{\circ}$} & \multicolumn{2}{|c|}{ Ages over 50} \\
\hline Cases. & Mortality. & Cases. & Mortality. & Cases. & $\begin{array}{l}\text { Mortality } \\
\text { Per cent. }\end{array}$ \\
\hline $5^{I}$ & I 5.6 & 64 & I $5 \cdot 5$ & 93 & $29 \cdot 7$ \\
\hline I I I I & $7 \cdot 4$ & 489 & I I . 2 & 378 & $25 \cdot 4$ \\
\hline 203 & I. 4 & 66 & $4 \cdot 5$ & & \\
\hline I 372 & $3 \cdot 3$ & 415 & $7 \cdot 9$ & 94 & 20.2 \\
\hline 2518 & I. 7 & 719 & I. 6 & 3395 & $4 \cdot 4$ \\
\hline
\end{tabular}

Mortality Irrespective of Age.

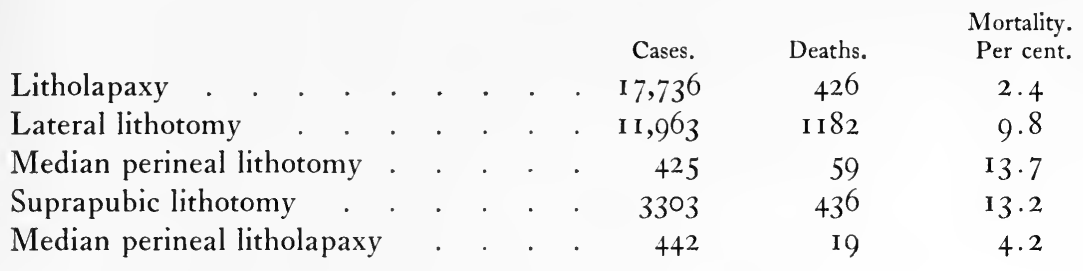

Percentage of Recurrence.

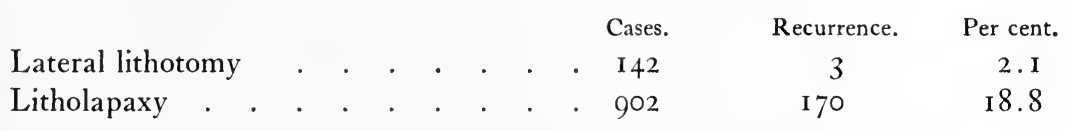

Another point of interest is with regard to the cases of suprapubic lithotomy in which partial or complete suture of the bladder incision was made. The following figures are taken from our compilation of suprapubic lithotomies:

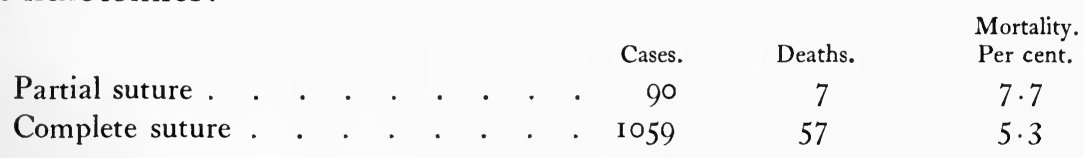

The proportion of primary unions in I059 cases was 454 (42.8 per cent.).

So far as these statistical calculations are concerned, they tell the following story: That the operative mortality of litholapaxy, at whatever age it is performed, is much less than that attending any of the other surgical procedures. The nearest approach to so small an operative mortality as that method exhibits is in connection with lateral perineal 
lithotomy in patients under fifteen years of age; yet, even so, it is three times as large as that of the operation of Bigelow.

The most dangerous of the procedures, at whatever age performed, as it appears in this series, is median perineal lithotomy, but the number of cases is a small one from which to judge, and undoubtedly gives an. erroneous impression.

The mortality of all the operations, as is well known, is much higher after fifty years of age than at any other time. A point of some interest is that primary union of the bladder incision after suprapubic cystotomy was secured in less than half of the cases. Also, that the death rate of suprapubic lithotomy in the cases in which suture was applied was less than one-half that attending the open method of treating the wound.

One other point of interest with regard to the operative mortality is the small percentage of deaths in connection with the operation of median perineal litholapaxy as compared with any of the others except litholapaxy performed in the usual way.

Character of Results.-With regard to recurrence, the data which are available to us are too few to allow of any inference being drawn in connection with other operations than litholapaxy. In this procedure it is seen to be nearly 19 per cent. in 902 cases. This undoubtedly represents a very high estimate of the number, and it should not be taken as that which occurs, except in connection with elderly men and the conditions which exist in that class of patients favoring recurrence of calculus whatever may have been the operation performed, unless, indeed, total prostatectomy is combined with it, under which circumstances the proportion of recurrences is very small. More than two-thirds of the 902 cases from which the computation of recurrence is made were of men over fifty years.

Conclusions with Respect to the Choice of Operative Method.-From what has been stated above with regard to the three factors which determine the choice of operation, we may accept the following things as being established:

I. Litholapaxy is the operation of choice, if the operator is skilled in its performance, except in the presence of one or other of the following conditions: (a) Calculi of unusually large size-six or more ounces-or of unusual hardness. (b) Calculi which are so closely attached to the wall of the bladder that there is danger of catching the mucous membrane in the jaws of the lithotrite when seizing the stone. (c) Cases of encysted stone, or those in which fragments or small calculi are lodged benneath projecting lobes of an hypertrophied prostate and cannot be dislodged and thus made accessible. (d) Cases in which an hypertrophied prostate 
is of such form as to make the passage of the instrument difficult and dangerous.

2. Perineal litholapaxy is preferable to litholapaxy done in the ordinary way, in cases in which prostatic hypertrophy makes the latter operation undesirable, for the reason just stated above.

One great advantage of doing the operation through the perineal urethrotomy incision is that digital exploration of the bladder is made possible, and that it allows the operator to remove the prostate at the same time if it is desirable to do so.

3. Suprapubic lithotomy is the operation of choice in cases of very large calculi; in those of encysted stone and in those in which small calculi or fragments of stone are lodged beneath an intravesical projection of an enlarged prostate.

4. Lateral and bilateral perineal lithotomy no longer hold a place in the surgical treatment of calculus of the bladder.

5. In cases in which there is but little or no cystitis present, the vesical incision of the suprapubic cystotomy should be closed by suture, the abdominal wound should also be closed except for a small space to provide for drainage in case of failure of the bladder suture to hold.

6. In cases of very large calculi, the suprapubic cystotomy incision should be enlarged in one or another of the ways specified in the chapter on the Technique of Operations on the Bladder.

\section{Illustrative Case.}

Calculi Encysted in a Rare Form of Vesical Diverticulum.-The patient, a man, aged seventy years, had already been operated upon three times for vesical calculus-twice by lithotrity, once by a suprapubic lithotomy. The first operation was fifteen years before the time at which he came under the care of the writer. After each of the operations there had been a period of freedom from the symptoms from which he had been suffering; then they returned. On each occasion they were characteristic of the condition.

Fourth Operation.-The ordinary external perineal urethrotomy incision was made, and through it and the dilated posterior urethra the bladder was explored digitally.

Projecting from the mouth of a diverticulum, a little above the right lobe of the prostate and on the lateral aspect of the bladder, a calculus was felt by the finger tip. Its outer part, as was seen later, had been crushed at the time of the last operation-a litholapaxy-about three months before. The neck of the stone was firmly grasped by the ring-like orifice of a diverticulum, in the sac of which the farther end of the calculus lay. 
After some difficulty the tips of a pair of curved forceps were inserted inside the mouth of the diverticulum and the latter was stretched open by separating them. The calculus could then be readily extracted from its pocket.

Two other small stones lay in the interior of the sac, and were also removed. It was then thought that all of them had been taken away, when the tip of the finger, as it passed over the inner surface of the sac, detected upon the farther side of it a second opening. Upon dilating this, it was found that it led into another pocket, in which there were two more small calculi.

The orifices of the two diverticula were cut slightly at four different points and then widely stretched by the blades of a pair of forceps.

The patient made an uninterrupted recovery. Two years later he returned with symptoms of his former trouble, and was again operated upon by the writer's colleague, Dr. George Monks, who removed five calculi weighing in all one-half ounce. Four of these calculi were free in the bladder, the other one was one-half inside and one-half outside of the orifice of the diverticulum, from which the writer had taken the other calculi previously.

\section{DIVERTICULA OF THE BLADDER.}

Definition.-The terms diverticula, saccules, pouches, pockets are given somewhat indiscriminately to congenital anomalies of the bladder, to the depression behind the prostate in the floor of the bladder, to that behind the trigone in which calculi are sometimes lodged, and to other forms of pouches or pockets existing in the bladder.

Strictly speaking, the term diverticulum is meant to apply to the form of sac, pouch, or pocket which is an acquired condition resulting from the pushing outward of the mucosa and submucosa of the bladder through an interspace between muscular bundles of the bladder wall, which exists because of the peculiar arrangement of the muscular fibers of the organ, and which yields to an abnormal intravesical pressure, such as is produced in conditions which prevent the free escape of the urine from the viscus, e.g., stricture of the urethra and prostatic hypertrophy.

The distinction is sharply made by some of the German writers between the pouches which are formed in this manner and the congenital form of the condition, the difference being that in the walls of the acquired form there are no muscular fibers found, while in the latter variety fibers of one or all of the muscular coats of the bladder may exist. 
In the congenital variety there is none of the muscular bundle hypertrophy of the bladder such as that just described, and there are to be found in the wall of the diverticulum either the whole or some part of the muscular fibers which normally belong to the bladder wall itself. A further evidence of the congenital variety of the condition is thought by Englisch and some of the other German investigators to be the presence of the ureteral orifice in the diverticulum itself; but, as Young ${ }^{2}$ suggests, this fact does not seem to be conclusive of the congenital nature of the pocket, for it is readily conceivable that the ureteral orifice should be drawn into the diverticulum as the latter increases in size.

Both of these two forms of diverticulum-for it seems less confusing to include them under one heading-are distinct from the congenital malformation of the bladder in which the organ is divided into two chambers by a transverse or an upright partition, each one of the two chambers in the latter case being provided with one ureteral orifice. The latter conditions are always of congenital origin and constitute the so-called "double bladder."

In the cases in which there are very large diverticula, it may be difficult to distinguish them from this congenital anomaly of the organ.

Location.-Gouley ${ }^{3}$ says that in a large number of specimens examined by him the diverticula were, in the great majority, seated on the lateral aspects of the organ.

Number.-Diverticula may be single or multiple. Gouley refers to a case reported by Civiale in which the diverticula were so numerous as to give to the bladder the appearance of a bunch of grapes.

Size.-Diverticula vary from the size of a pea to that of a tumor, which fills the whole pelvis and lower part of the abdomen. The latter have in some instances represented the so-called phantom tumors that are reported from time to time.

The explanation of the rarity of rupture of the walls of the diverticula is thought to be the increase of the thickness of the walls of the pouch which takes place progressively in correspondence with the growth of the pocket itself.

Fig. 265 shows on the left of the interior of the bladder four mouths of a diverticulum with four separate chambers all enclosed within one wall. On the right is the mouth of another single diverticulum.

The condition known as "pouch or pouching" of the bladder is to be distinguished from that of diverticulum or saccule of the organ. The term is usually applied to the depression which occurs immediately behind the interureteral bar or just beneath the base of the prostate on the floor of the bladder. The importance of this depression is considerable, in that it affords a space for the residual urine which collects in cases of 
prostatic hypertrophy, and by its decomposition causes a precipitation of the alkaline salts and thus offers the opportunity for calculus formation.

Pathological Changes.-Diverticula, even when of large size, do not always give rise to symptoms or to serious consequences, and may remain undetected through life. The factor which causes trouble is cystitis when it occurs in the diverticulum.

As a rule, diverticula do not wholly empty themselves, and even when the bladder is not inflamed, decomposition of the urine often takes place in the sac, and thus decomposition, crystalline deposit, and calculus

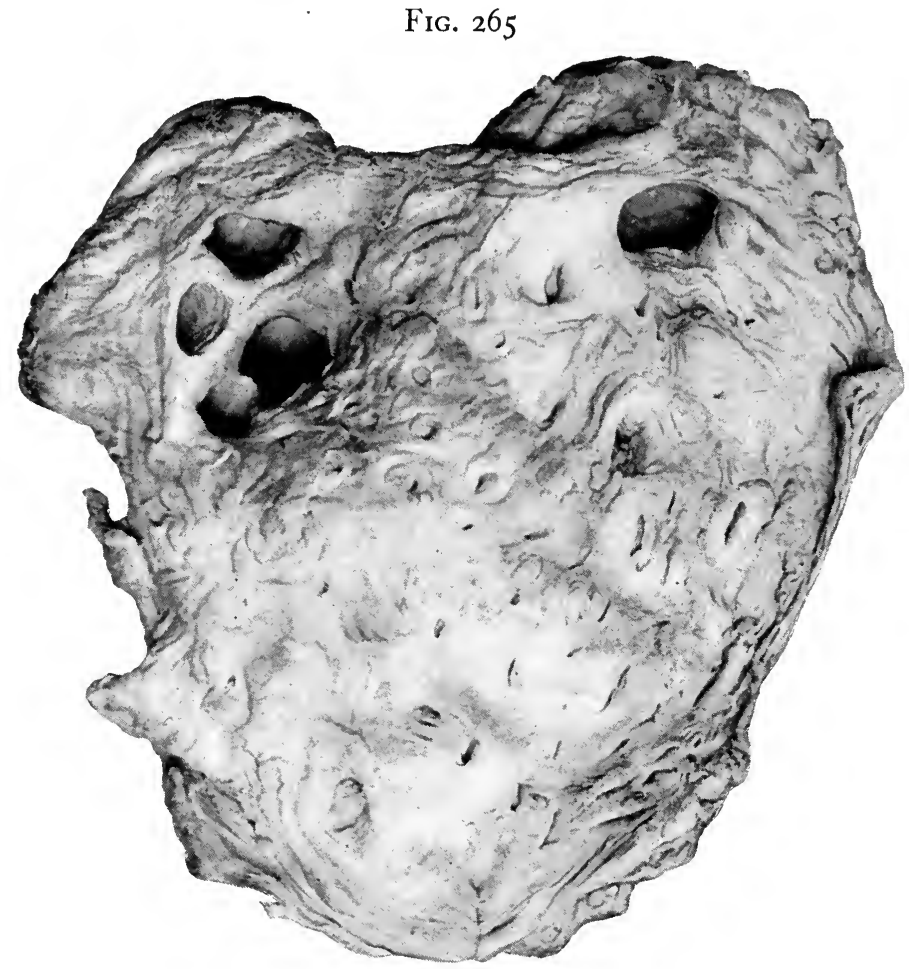

Diverticula seen from within the bladder.

formation not infrequently occur; the condition known as encysted calculus is produced in this way, the stone being retained within the pocket, or partly within and partly projecting into the bladder cavity from the mouth of the diverticulum.

Unless there is a septic infection of the diverticulum, it rarely gives rise to any trouble, but when infection does take place the consequences may be serious. The first condition resulting from such an infection is the passage of the inflammation into the bladder itself and the setting up in it of an obstinate cystitis. 
Symptoms and Diagnosis.-The symptoms of diverticulum, when present at all, are obscure, and but for the cystoscope the diagnosis would often be very difficult.

Guthrie mentioned what he called "the fluttering blow." Gouley refers to his description thus: "A sensation which is felt by the surgeon while he is drawing off the urine by means of a catheter, the end of the instrument being struck several times convulsively by the flapping emptied sac."

This sign is, as Gouley remarks, entirely unreliable, and is frequently felt by the fingers when using the catheter. It is what Bigelow used to call the "fish-bite," which is felt when using the evacuating tube after crushing a vesical calculus.

\section{FIG. 266}

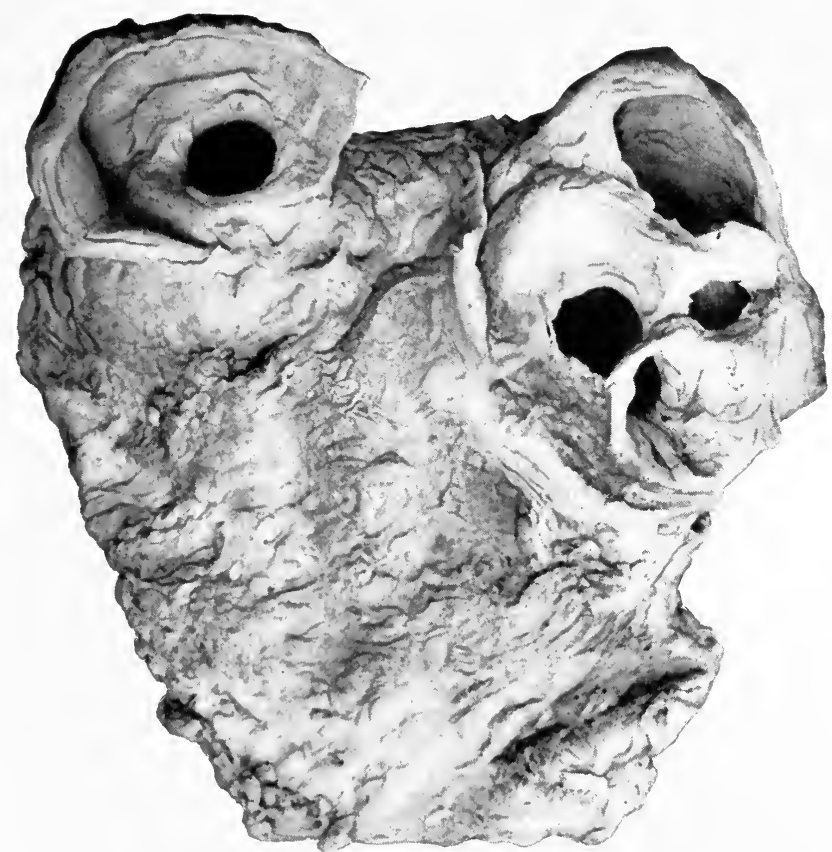

The same diverticula seen from outside the bladder after being cut across transversely.

A more reliable sign of the existence of a diverticulum is that of the sudden refilling of the bladder with urine immediately after it has been emptied by a catheter. This will only be noticed in connection with diverticulum of considerable size.

In the cases in which diverticula are inflamed and pus is produced within them, the sudden appearance of more or less pus in the urine, alternating with a clear urine, is suggestive. So, too, when a diverticulum is large enough to form a palpable abdominal tumor, will its sudden 
disappearance, especially if accompanied by a rapid influx of urine into the bladder, be suggestive of the presence of a diverticulum.

A still more characteristic sign of diverticulum is that of having the end of the catheter enter into and empty a considerable amount of urine from what appears to be a second chamber in the bladder, after the surgeon believes that he has entirely emptied the viscus with the instrument.

All of these signs, as well as the usual ones of dysuria and pyuria when the diverticulum is inflamed, are unnecessary for the diagnosis when the cystoscope is available, for they are unreliable and none of them absolutely characteristic, while the cystoscopic findings are positive. Plate XXIII, from Fenwick, gives a sufficiently good impression of the appearances of the orifices of the diverticula in certain cases.

Treatment.-Except they give rise to well-defined symptoms, diverticula of the bladder require no treatment. It is, however, far from correct to think that they are innocent and harmless, for the records of postmortem examinations are marked by many instances in which the condition has resulted fatally.

In a few instances, prolonged drainage of the diverticulum has been reported as having proved successful, but it is too uncertain and too little effective to accomplish this result often and should not be relied upon. The only treatment that can be adequate is surgical, and, when possible, it should be radical. It is all the more essential to abolish the sac of the diverticulum because of its affording lodgement for calculus, and because of the inadequacy of the ordinary methods for its extraction under these conditions. A diverticulum which has harbored calculus once is exceedingly prone to do so a second time, and recurrence after one or more removals of the stones is by no means unusual. The radical surgical methods which have been employed for removing diverticula are the following: (For the description of the technique of the operations see chapter on the Technique of Operations on the Bladder.)

I. Division of the septum between the bladder and the diverticulum.

2. Stretching the orifice of the diverticulum.

3. Suture of the orifice of the diverticulum.

4. Excision of the sac.

Of these various procedures, excision of the sac is the only one which appeals to us as being efficient. The procedure of throwing the bladder and the diverticulum into one cavity, which has been done by Young, ${ }^{2}$ by cutting through the septum dividing the two chambers, is not free from danger, since it is not always possible to define the limits of the attachment between the two, and consequently incurs the risk of cutting beyond the limits of it and of thus opening them into the adjacent external parts. Moreover, it does not do away with the sac. 


\section{PLATE XXIII}

FIG. 1

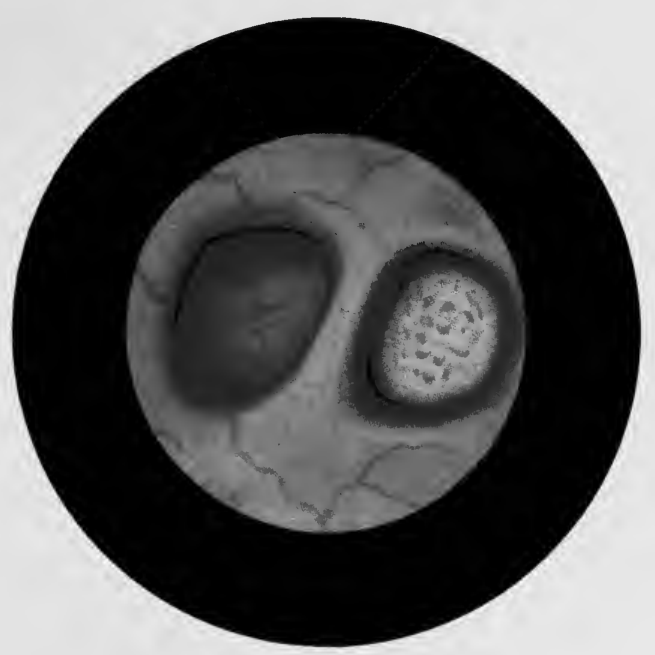

CYSTOSCOPIC APPEARANCE OF THE MOUTHS OF TWO DIVERTICULA, ONE CONTAINING A CALCULUS. (Fenwick.)

FIG. 2

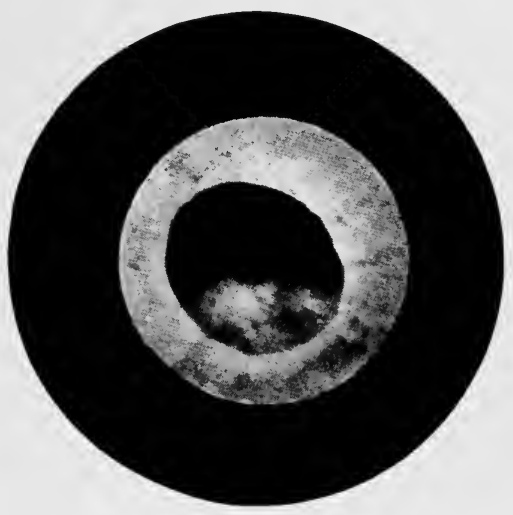

CYSTOPHOTOGRAPH OF A CONGENITAL DIVERTICULUM OF THE BLADDER. (Fenwick.) 

In a number of cases, the sac has been adherent to important structures by its external surface, so that it would necessitate opening the abdominal cavity in order to safely excise it. Thus the iliac vein and the intestine have been involved in adhesions with the exterior of the sacs of diverticula.

The cystoscope enables the surgeon to determine the site and to estimate the size of the diverticulum.

Whenever the sac is so situated as to be accessible, it should be excised in the cases in which the condition is giving rise to symptoms which seem to demand that radical measures be applied.

\section{BiBLIOGRAPHY.}

I. Freyer. The Modern Treatment of Stone in the Bladder by Litholapaxy.

2. Young. Johns Hopkins Hospital Reports, I906, vol. xiii.

3. Gouley. Surgery of the Genito-urinary Organs, New York, I907. 


\section{CHA P TE R XVI.}

\section{TUMORS OF THE BLADDER.}

The Origin of Tumors.-According to Cohnheim's theory, which still remains the one most widely accepted, a portion of one of the embryonic tissues remains in its primitive condition in some one part of the organism instead of proceeding to complete development, and from this embryonic focus a tumor subsequently grows under the influence of causes the nature of which is unknown.

The occurrence of tumors whose cellular elements do not resemble in one or more of their characteristics those of the normal embryonic tissue of that part of the body in which the tumor is situated, but which do typify those of tissues which normally exist in some other parts of the body, is explained by the supposition that, in the process of fotal development, a bit of one of the embryonic layers is imprisoned, nipped off, or, as it is termed, included in the territory of one of the other layers, and that the tumor which subsequently arises from such an inclusion has cellular elements which are characteristic of the latter. The existence of epithelial tumors in parts of the body in which epithelium does not normally exist is one of the facts which seems to support this supposition.

These theories do not explain why, after being dormant for periods of many years, the cellular elements of the tissue at the site of the growth should suddenly display extraordinary reproductive activity, or, as it is termed, proliferation, and give birth thereby to what we call a tumor, and possess the power of sending forth and establishing colonies of offsprings by what is known as the process of metastasis, or change of place, which colonies exhibit a tendency to a strenuous life similar to that of the parents.

To account for this phenomenon, the traumatic or irritation hypothesis, which includes as one of its forms the parasitic theory, is offered.

According to this conjecture, the impulse to increased cellular activity is called forth by injury or prolonged or frequently repeated irritation, the latter of which may be of mechanical, chemical, or parasitic nature. This idea is supported by such facts as the more or less frequent occurrence of tumors at the sites of injuries, and the preference shown by certain forms of neoplasms for occupying parts of the body which are especially subjected to mechanical irritation, such as the lips, pylorus, the lower end of the rectum, etc. 
A series of cases of vesical tumors which were reported by Rehn, Leichtenstern, ${ }^{1}$ Wendel, ${ }^{2}$ occurring with notable frequency among the employés of some of the aniline dye factories of Germany, are suggestive and interesting. In connection with these tumors, attention was called to the possible causal influence in their production of the constantly repeated chemical irritation to which the patients' urinary tracts were subjected.

The parasitic theory rests in part upon the interpretation of the nature of certain globular bodies found embedded in the cellular elements of a number of tumors, which are difficult of explanation, and have been thought by some observers to be of a parasitic nature, because of their resemblance to certain spore-bearing forms of protozoa.

According to Albarran, ${ }^{3}$ these may exist in any part of the tumor's structures, and are not confined to the epithelial cells. When in the latter, the appearance is that of a cell with this hyaline wall and spore contents lying within the epithelial cell itself. These bodies are termed psorosperms by the French. Their parasitic nature is both asserted and denied by numerous observers, but, as has been shown by Councilman and others, they are not confined to cancerous growths. The view of those who deny their parasitic nature is that they represent the products of cell degeneration or secretion, and, on the whole, the weight of evidence seems to favor this opinion.

The spores which these bodies are thought to resemble are those of the coccidium, concerning which there are some interesting observations.

They have been successfully grown in both wet sand and in water. They have not, however, been proved to be an element in cancer by which it can become contagious. Suggestive observations, such as the transfer of cancer by human beings from one locality to another; the auto-inoculation of cancer in an individual; the cases of repeated occurrence of cancer in connection with certain houses, while suggestive of the contagiousness of cancer, are not proof of its actual occurrence.

One of the more important recent documents relative to this question is that published by the Croft Cancer Commission from the Surgical Department of the Harvard Medical School in I902. ${ }^{4}$ In this the conclusions are summarized thus:

"The lesion produced by coccidium oviforme is essentially a process of chronic inflammation, and is not analogous to the lesion seen in cancer.

"The lesion in molluscum contagiosum is characterized by certain changes in the epidermis, is not due to the action of protozoön, and is not analogous to cancer.

"The so-called blastomycetes (saccharomycetes) of Sanfelice and Plimmer are torulæ. 
“The lesions produced by these blastomycetes (torulæ) are essentially nodules of peculiar granulation tissue, are not cancerous, nor in any sense true tumors.

"Blastomycetes are not constantly present in human cancers.

"The peculiar bodies seen in the protoplasm of cancer cells are not parasites, nor the cause of the lesions, but are probably, in part at least, typical stages of the process of secretion by glandular epithelium."

Structural Classification of Vesical Tumors.-Tumors of the bladder represent three principal tissues, viz., epithelium, connective tissue, and muscular tissue, the first being derived from the embryonic entoderm and the last two from the mesenchyma of the mesoderm. Classified as they represent these different structures, the tumors correspondingly fall into three principal groups as follows:

I. Tumors Arising from Epithelial Tissue.-Papilloma, adenoma, carcinoma, simple cysts.

2. Tumors Arising from Connective Tissue.-Sarcoma, myxoma, fibroma, angioma.

3. Tumors Derived from Muscular Tissue.-Myoma.

4. Heterotopic Tumors.-These tumors represent fœtal inclusions, and are composed of tissues which represent those of the embryonic layers which anatomically and normally do not belong to the parts of the organism in which the tumors have their seat. The following have been observed in the bladder:

Cholesteatoma.-Representing a portion of the ectoderm.

Chondroma.-Representing cartilage.

Rhabdomyoma.-Representing striped muscular tissue.

Dermoid Cysts.-Representing a number of different tissues.

Parasitic Tumors.-Represented in the bladder by one case only-an hydatid cyst.

Subdivisions and Synonyms.-Papilloma.-Synonyms.-Papilloma is called by a number of different names, which are descriptive of its gross appearances in different cases; thus fimbriated papilloma, villous papilloma, cauliflower tumor, lobulated papilloma, etc. Again, it is called, when its appearance is not referred to, benign epithelial papilloma; and, again, when connective tissue enters largely into its structure, fibropapilloma.

Carcinoma.-Synonyms.-Epidermoid cancer, epithelioma, and various descriptive terms, such as scirrhus, encephaloid, cauliflower, etc.

Subdivisions.-Alveolar, cylindrical-cell, lobular, reticulated.

Myoma.-Synonym.-Leiomyoma.

Myxoma.-Synonym.-Mucoid polyp. 
Sarcoma.-Subdivisions.-Round-cell, spindle-cell, mixed-cell, giantcell lymphosarcoma, alveolar sarcoma, fibrosarcoma, myxosarcoma, myosarcoma, angiosarcoma, chondrosarcoma, osteoid chondrosarcoma.

Heterotopic, also called Heterogenous or Heteroplastic Tumors.

Cholesteatoma.-Synonyms.-Leukoplakia vesicæ, epidermization of the bladder, cancroide.

FIG. 267

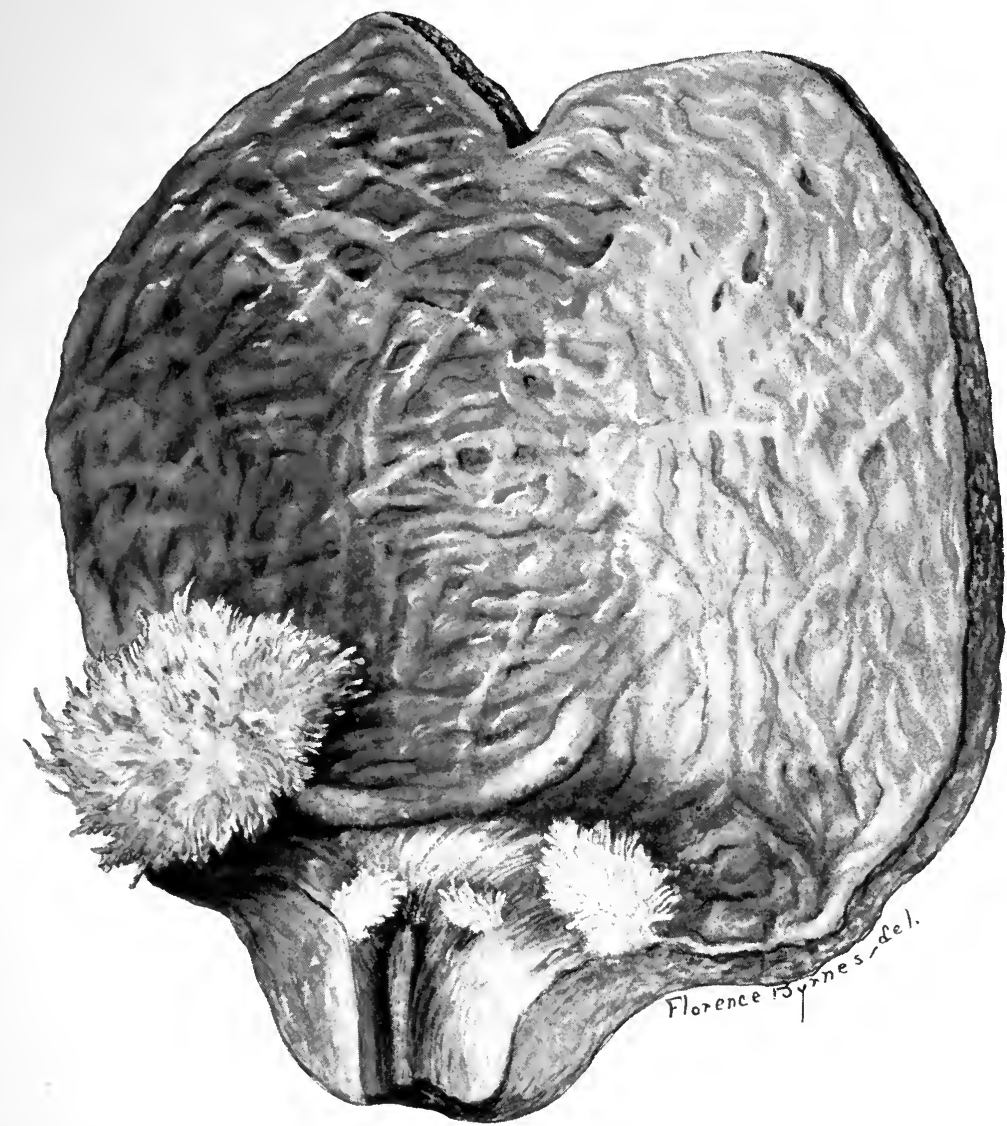

Interior of the bladder with four benign papillomas growing on its surface and showing the typical villous surface often seen in these neoplasms.

The terms benign and malignant have a purely clinical significance. While not scientifically accurate, they are convenient, and so firmly rooted by custom that it is not worth while to try to displace them.

Special Characters of Different Neoplasms of the Bladder.-Wide variation exists in the forms and the surfaces of the different vesical tumors, as well as in their microscopic features. No one form or character of surface is absolutely distinctive of any one of the special varieties. 
Papilloma.-As ordinarily used, this term designates a benign tumor, either sessile or with a well-marked pedicle, single or multiple, having a surface made up of branching arms, which terminate in finger-like projections, villi, or papillæ, and structurally composed of a central connective-tissue stem or axis, accompanying which is a loop of bloodvessels, and externally a covering of one or more layers of epithelial cells with large nuclei. The neoplasm is essentially an epithelioma with growth proceeding outward instead of inward by infiltration, as is the case with carcinoma.

When the terminal tufts of the villi are long, they float outward in the fluid in which the tumor is submerged like the filaments of certain

FIG. 268

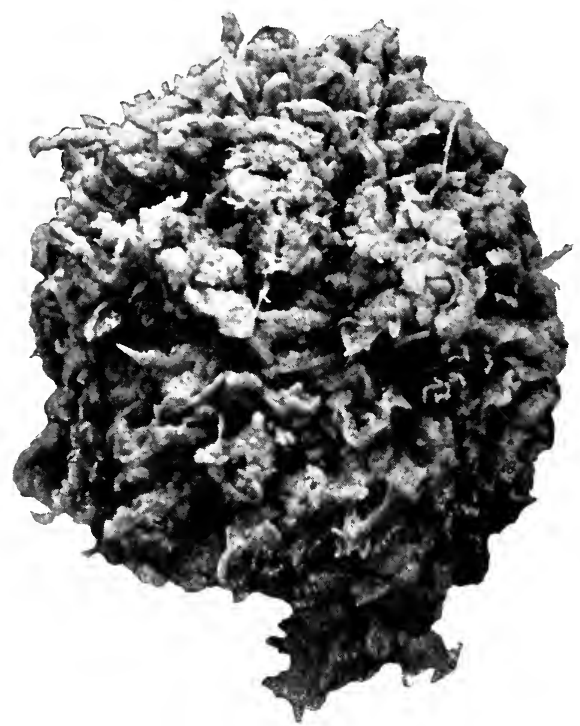

Benign papilloma of the bladder. sea-weeds. In other examples, the papillæ are more closely bunched together and shorter, and then resemble the surface of the cauliflower. In a rare form, which has been described by Thompson, ${ }^{5}$ separate villi spring directly from the inner surface of the bladder.

The pedicle of the papilloma is more or less hard, according to the relative amount of connective tissue entering into its composition.

The base of the growth is freely movable upon the underlying muscularis. This distinguishes it from carcinoma, and is an important feature clinically.

The macroscopic appearances of the typical form of papilloma are shown in Figs. 267 and 268. The general appearances presented by single villi, partly denuded of their epithelial covering in one, and covered in the other of the two, as seen with a low power, are illustrated in Fig. 269. The structure of the papillæ or villi and their relation with the main body of the tumor are shown in Fig. 270.

In Fig. 27I are shown the lacunæ, or spaces in which cell elements, detritus, or colloid material are frequently found.

Transition from Benign to Malignant Character of Papilloma.-Sir Henry Thompson, ${ }^{5}$ in his original work on Tumors of the Bladder, asserted that papilloma in some instances underwent change of a cancerous nature, and gave an illustration of what he termed "a transitional form" of vesical neoplasm in support of this statement. Since then the 
same assertion has been repeated. The occurrence of such change is denied by other observers.

Albarran ${ }^{3}$ speaks upon the subject as follows:

"The fact of the existence of a tumor demonstrates per se that there is in the cells which constitute it a considerable proliferating power.

FIG. 269

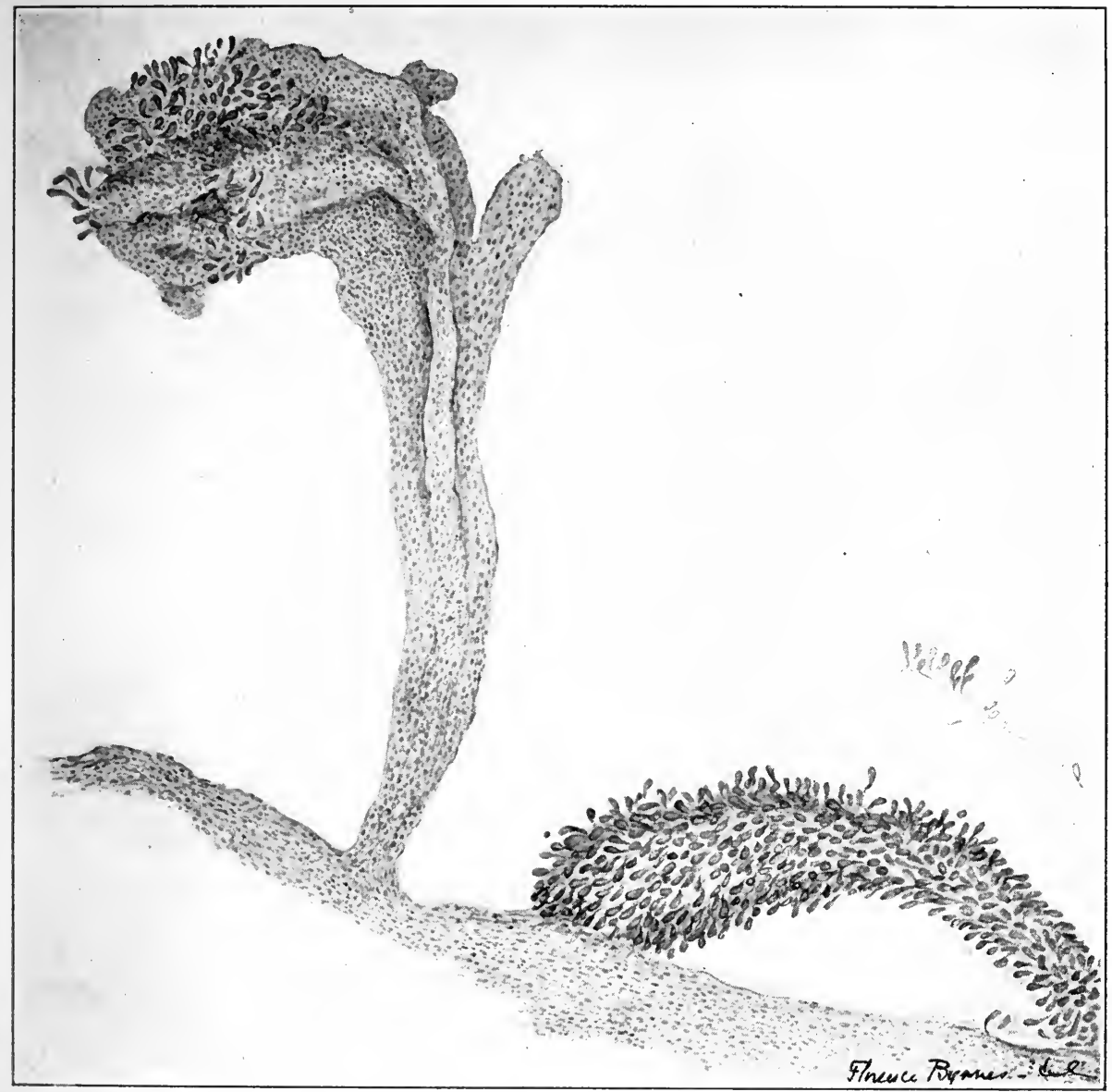

Villi of benign papilloma. One denuded in part of its epithelial covering. The other preserving it.

"In the case of the bladder, it is remarkable how often there are found tumors the cells of which exhibit the different stages of direct or indirect division. I have more frequently seen this division by 'budding' (bourgeonnement) than by kariokinetic division.

"One very often sees epitheliomas of the bladder whose cells exhibit in parts nests of 'budding' cells.

"In benign tumors, the cellular activity is more moderate than in the 
malignant varieties, and the cell proliferation produces elements of typical form. But it is quite possible that at a given moment, under the influence of an unknown cause, a benign tumor may become transformed into one of malignant character.

"These facts are difficult to explain except upon the supposition that a new tumor is developed upon the preëxisting one. This theory is all the more probable in that we already know that the formation of the original tumor is in itself an indication of the tendency to proliferation of the cellular elements of the organism.

FIG. 270

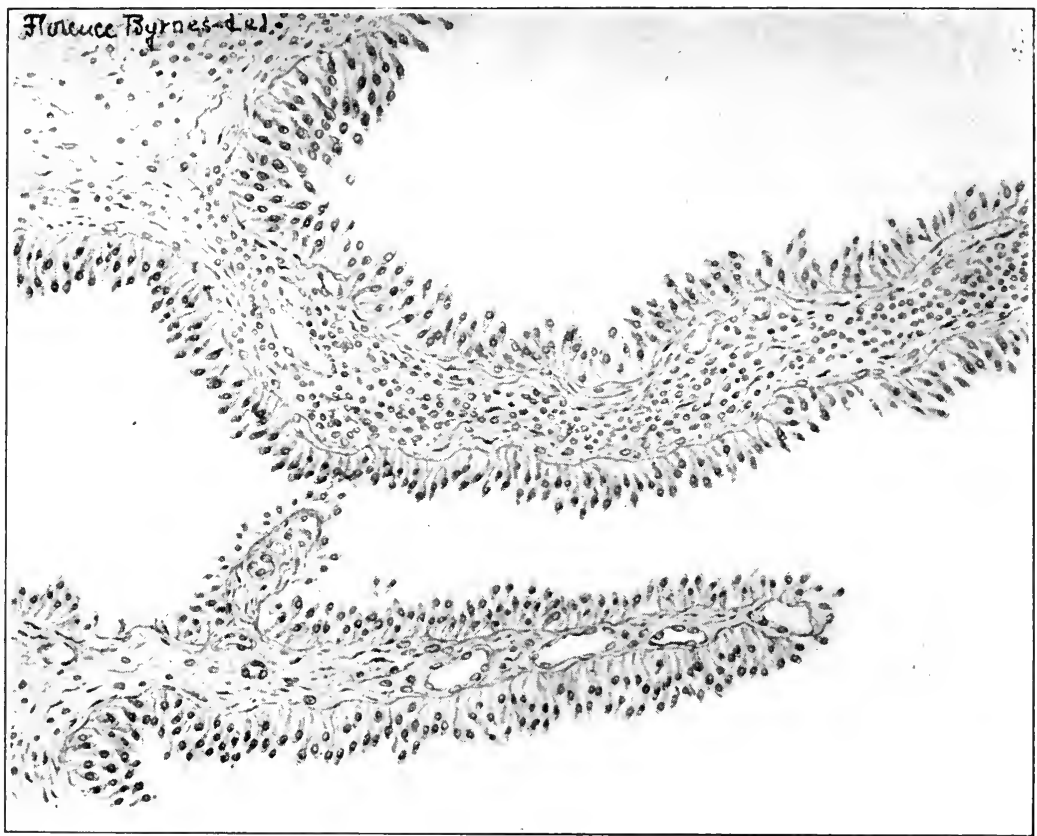

Villi of benign papilloma, showing epithelial covering central stem and the connection of the villi with the base of the tumor (longitudinal section).

"As a matter of fact, the transformation of benign to malignant tumor takes place quite frequently in neoplasms of the bladder.

"Almost invariably the secondary (recurrent) tumor of malignant character is of the same type as that of the primary growth; that is to say, an epithelial benign neoplasm, in such case, is replaced by an epithelial tumor of cancerous nature.

"Occasionally this rule is broken, and it is a tumor of different character type which becomes implanted in the place of the first one. Thus one observer has seen vesical myoma undergo cancerous degeneration upon its surface (Virchow), and, again, both cancerous and sarcomatous 
degeneration have simultaneously occurred in a myoma of the bladder" (Billroth ${ }^{6}$ and Gussenbauer).

Wendel ${ }^{2}$ describes a specimen in which he found typical carcinomatous structure in the terminal portion of a growth, all other parts of which, including the base, were typical of benign papilloma.

Just what the proportion of cases is in which a tumor of benign character, in the first instance, has subsequently undergone cancerous change, or in which, having been removed, it has been replaced by a recurrent neoplasm of that nature, we do not know. Certain well-authenticated examples of the latter occurrence are, however, recorded, as well as others

FIG. 27I

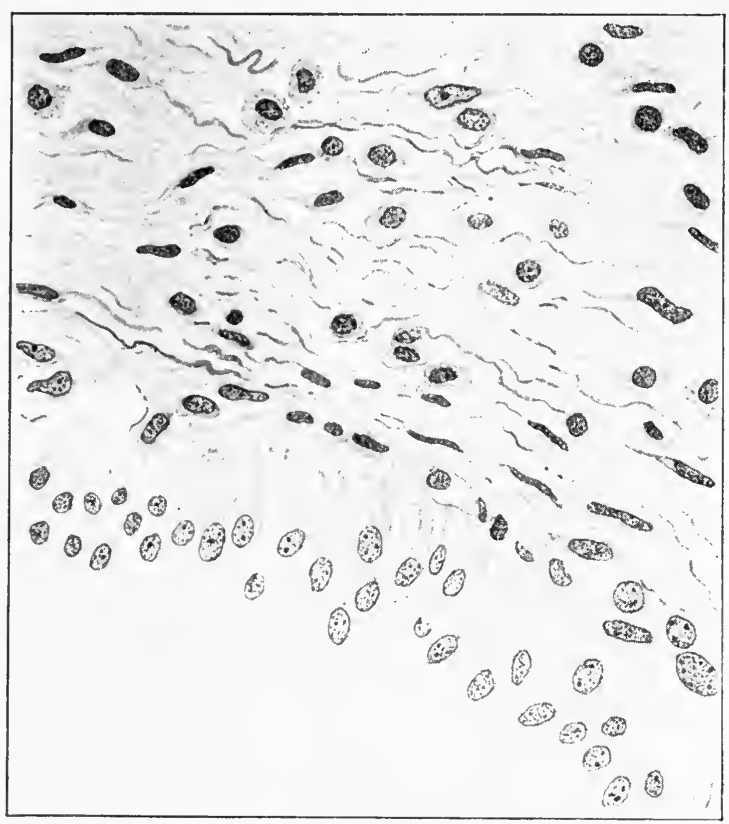

Microscopic appearances of single villus on longitudinal section near its base.

in which the recurrent neoplasm has been of a benign nature and identical with that of the primary tumor. As examples of the former, may be mentioned three instances in a total number of 15 benign papillomata in which the recurrent tumors were carcinoma in each case (Sutter ${ }^{7}$ ).

Motz $^{8}$ found one recurrent carcinoma following the removal of benign papilloma, and four benign recurrent papillomata following the removal of four primary tumors of the same nature; that is to say, in one of five cases, recurrence took place in the form of cancer.

In ten observations of recurrent tumors following the removal of benign papillomata, personally communicated to Rafin, ${ }^{9}$ there was but one 
instance in which the recurrent neoplasm was of cancerous nature. The others were all benign papillomata; that is to say, of the same nature as the original tumors.

Other instances than that mentioned by Wendel $^{2}$ are reported, in which small areas of so-called typical cancerous structure existed, the rest of the growth being typical of benign papilloma. The fact, however, that should be remembered is this, that there is no cell form or character which per se is positively characteristic - in the sense of being specificof cancer. The other elements which enter into the question, and are distinctive one way or the other, must be taken into account. The great importance of examining all parts of vesical tumor of apparently benign character is emphasized by the observations to which we have referred.

Whether because cancerous structure and features are often overlooked, and tumors, in consequence, are pronounced benign when in fact they are of malignant character, or for other reasons as yet undetermined, it is conspicuously true that the proportion of cases is very great in which recurrent tumors of malignant nature follow upon the removal of those said to have been benign; and this fact justifies the view expressed by Albarran, ${ }^{3}$ viz., "All vesical tumors are malignant or likely to become so;" and, in less degree, warrants that of Clado, ${ }^{10}$ that "Recurrent tumors following upon removal of benign papilloma are always in the form of malignant epithelioma."

The practical bearing of these statements and of the data given above upon the treatment of the disease will be discussed under the heading of Treatment.

The Development of Benign Papilloma.-Wendel ${ }^{2}$ thus describes the evolution of this variety of tumor: "The tumor begins in a series of proliferations of the epithelium at some point of the mucous membrane of the bladder. As this advances, it assumes the form of minute, hollow projections, the walls of which correspond in thickness with that of the superficial layer of the bladder epithelium, and are composed of cells identical with those of the latter. The next step consists in an increased cellular activity of the connective tissue immediately adjacent to the newly formed projections just mentioned, and also of the minute bloodvessels of the same part. Pari passu, with the development of the projections, a fine-cell infiltration of the connective tissue takes place, and presently the latter takes on an increased growth, pushing into the hollows of the projections and pressing them forward until a well-defined papillary or villous appearance is assumed.

"Up to this point there are no bloodvessels present in the projections, but now they too advance into the hollows of the latter, and finally assume the form of loops extending the whole length of the centre of the 
projections, and, together with the connective tissue, constitute the central axis of the papilla. The outer surface of this formation is invested by a greater or less number of layers of epithelial cells.

"The coalescence of several such individual structures as that just described constitutes a stem, on the summit of which the villi rest, the whole representing the typical benign papilloma."

Adenoma.- Rafin ${ }^{9}$ tabulates eleven cases of vesical adenoma in which operations were done. (See Table XI, p. 525.)

Rochet and Martel ${ }^{11}$ confirm the assertion already made by Albarran, ${ }^{3}$ that adenoma of the bladder does occur independent of the prostate, which fact has been denied by others. Rochet and Martel describe two forms of adenoma which have been demonstrated to have originated from the glandular structure of the bladder itself. One form they designate as "diffuse," the other as "circumscribed." In a case of their own, the tumor was of the former character and occupied the whole inner surface of the bladder in the form of papillary vegetations.

The circumscribed form appears as a single, pedunculated, lobulated tumor, sometimes having a smooth and at other times a villous surface.

Simple Cysts.-Albarran ${ }^{3}$ refers to them as follows:

"These have been described by Rokitannsky and Klebs, the latter calling them herpes of the bladder. Limbeck gave a detailed account of them in 1887 . Vincent and Laboulbene have reported cases of very large cysts of this nature. Ordinarily they are small, and have been likened in appearance to the fresh egg of the sturgeon, and are scattered over the inner surface of the bladder, sometimes in large numbers. They have a contents of viscid fluid, and in one case Limbeck found crystals of carbonate of lime.

"A very large cyst of somewhat similar character was seen by Segond and Thiercelin, who reported it in I89I. It protruded from the vulva, arose from the muscular layer of the bladder, and had a connective tissue wall and a serous contents.

"The inner lining of the cysts, as they usually appear, seems to be provided with an epithelial covering of its own, while its outer surface is supplied with another layer of epithelium, the latter being that of the vesical surface itself. A section made through the whole bladder wall and passing through the cyst, therefore presents on inspection these two layers of epithelial cells, one on either side of a delicate partition of connective tissue which represents the cell wall." Albarran believes that these cysts originate in the glands of the bladder mucous membrane.

Carcinoma.-Broad-based, hard, sessile tumors, especially if the surface be ulcerated, and, above all, if they cannot be moved freely over the 
FIG. 272

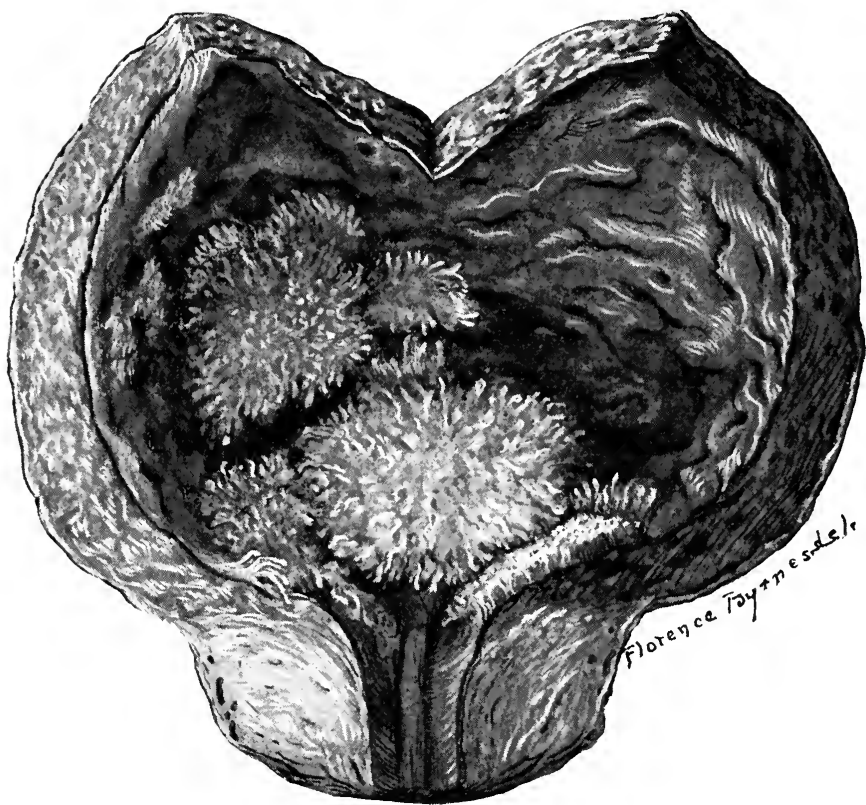

Carcinoma of the bladder, closely resembling benign papilloma.

FIG. 273

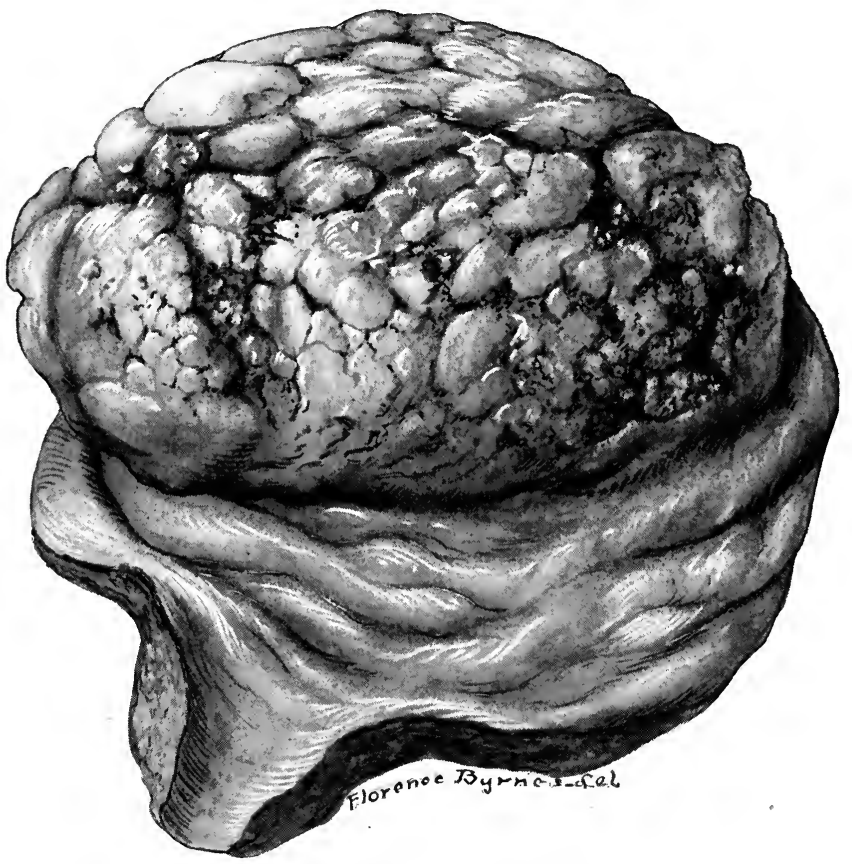

Carcinoma of the bladder. 
deeper layers of the underlying bladder wall, are, in all probability, of a cancerous nature. When they have reached a sufficiently advanced point of development, and if they are situated on the posterior wall of the bladder, they can be detected by rectal examination.

Carcinoma presents widely different appearances in individual cases. It has in some instances a close resemblance to, and on the surface may be identical with, benign papilloma (Fig. 272). Some of the variations in appearance are well shown in Figs. 273 and 274.

The microscopic appearances, as seen with low and high power respectively are shown in Figs. 275 and 276 .

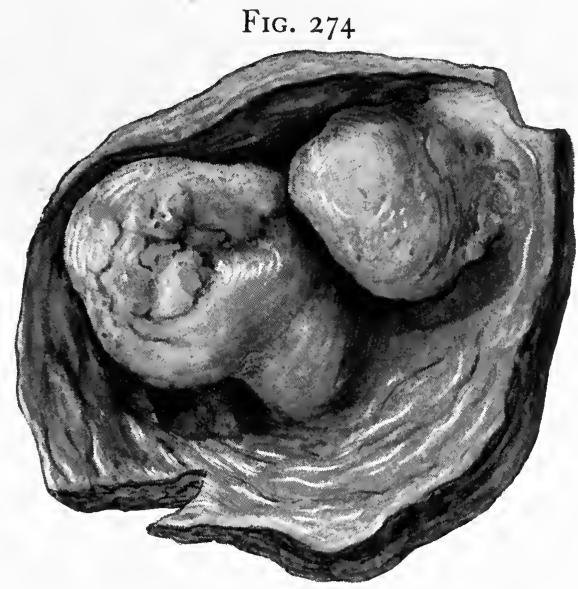

Carcinoma of the bladder obstructing the orifices of both ureters.

Sarcoma.-Macroscopically, these tumors appear under varied forms. They may be pedunculated or sessile; the surface is sometimes villous,

FIG. 275

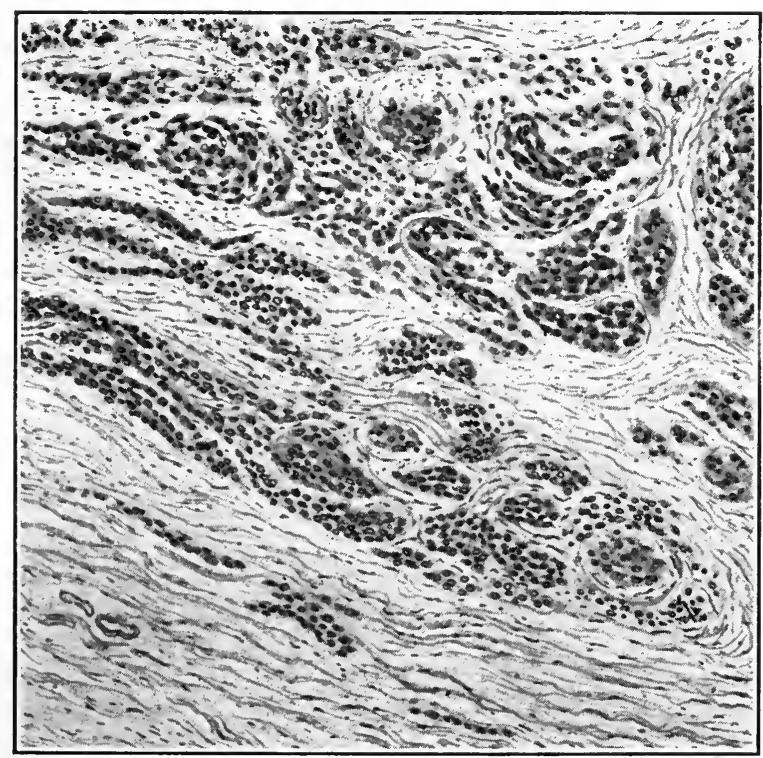

Microscopic appearance of carcinoma of the bladder. Low power.

and, again, it may be smooth. Ulceration of the surface is rare. The tumor is usually soft. It may or may not invade tissues adjacent to the bladder. 
The spindle-cell variety of sarcoma is the most frequent. Round-cell and polymorphous-cell sarcomata are also reported. Illustrative cases I and 2, at the end of chapter, illustrate remarkable and unique varieties of chondrosarcoma.

Myxoma.-Macroscopically, in its pure form, myxoma is a soft, gelatinous tumor, resembling the mucous polyp of the nose. When connective tissue enters into its composition, it becomes of firmer consistency. It is lobulated in form, and its surface is, in some instances, smooth; in others, lobulated.

Pure myxoma is rare. Histologically, the larger part of the growth is seen to consist of round cells, embedded in a granular, nearly amorphous intercellular substance, in which a small number of delicate connectivetissue fibers lie. The growth is rich in bloodvessel supply.

\section{FIG. 276}

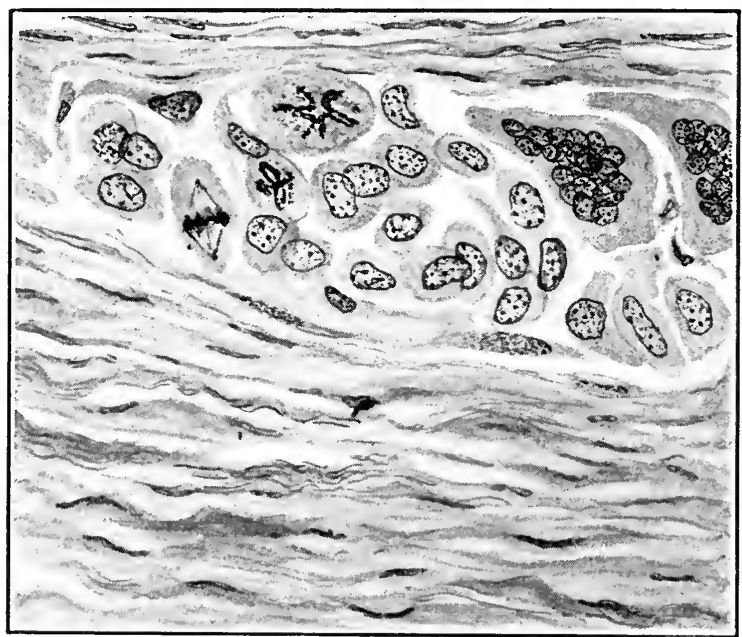

Microscopic appearance of carcinoma of bladder. High power.

Angioma.-Albarran ${ }^{3}$ describes the appearances of this variety of tumor thus: "The body of the growth is formed of large sinuous capillaries with ampulla-like dilatations. The vessels are separated from each other by a small amount of connective tissue. They are continued toward the surface as far as the epithelial covering. The cells of the epithelial surface are the same in form as those of the normal mucous membrane of the bladder."

Myoma.-These growths originate in the muscular tissue of the bladder wall. In some cases they are limited to that layer; in others, they push forward into the interior of the organ; and again, they may develop outwardly, exceed the wall of the bladder, and become eccentric tumors. 
An interesting example of the latter condition is reported by Pollailon, ${ }^{12}$ who, at the time of operation, found a tumor which he supposed to be a pedunculated uterine fibromyoma. It was seen, however, that it had originated in the muscular wall of the bladder and had extended beyond its limits in such a way as to cause the mistake.

Myoma of the bladder is composed of unstriped muscular fibers and an intermuscular stroma of connective tissue of varying amounts.

Cholesteatoma (Dermoid Epithelioma, Epidermization of the Bladder, Leukoplakia Vesica, Cancroide).-This very interesting condition of the bladder was described by Rokitannsky. Cases have been reported by Marchand, Bruchmar, ${ }^{13}$ Cabot, ${ }^{14}$ and a number of others. The condition probably represents a growth arising from an inclusion of embryonic epidermal tissues. The distribution varies greatly in different instances. In one of the cases the surface of the mucous membrane of the entire urinary tract was occupied by it. To the eye the surface of the affected areas has a pearly lustre. The mucous membrane is greatly thickened and indurated. In some instances there is an extraordinarly abundant desquamation of the superficial cells of the inner surface of the bladder, so that the organ may be filled with a mealy, granular, or putty-like mass, which is made up almost entirely of the desquamated epithelium.

Hallé $^{15}$ reports 13 cases, 9 of which were personal observations. Of the 13 , there were 4 in which the process extended beyond the bladder, the ureters and renal pelvis being involved.

Histologically, the cases which Hallé and others have reported have shown considerable variation. In some, the structure of normal epidermis in all its layers was found; in others, some of them were lacking; and, again, there was a horny layer resting on a basal one of polygonal cells. The epidermal structure was very thick; there was an extremely abundant superficial cell desquamation and also a very rich cell proliferation in its deeper portion.

In Cabot's case, the diseased surface could be peeled off with the fingers as a thick membrane, leaving a smooth surface beneath, the bladder wall after its removal being soft and supple. (See Nos. I5, I6, and 17 of illustrative cases at end of this chapter.)

Dermoid Cysts.- Several examples of these tumors have been observed as primary growths in the bladder; in other cases, the cysts have originated elsewhere and ruptured into the organ. Cases of primary dermoid have been reported by Le Dentu, Martini, ${ }^{16}$ Boucher, and Hallé. (See No. 3 of illustrative cases at end of this chapter.)

Hydatid Cyst. - The only case in which this condition has been authentically demonstrated as originating primarily in the bladder is that 
reported by Le Sauvage. Postmortem examination showed several cysts in the abdomen and one whose walls were represented by a portion of the bladder wall externally, muscular fibers of which were in direct continuity with those which entered into the structure of the inner or cyst wall itself.

Rhabdomyoma.-Albarran ${ }^{3}$ and Livio $^{17}$ have each described a tumor of this character. (See No. I8 of illustrative cases at end of this chapter.)

Propagation of Malignant Tumors.-Propagation of cancer of the bladder occurs through the lymphatics of the organ. The lymph glands outside the bladder most frequently infected are those of a group situated close to the point of division of the common iliac artery into its internal and external branches. Other lymph nodules involved are those which lie in the perivesical fat tissue. In this situation they are often difficult to detect and are frequently overlooked at the time of operation.

The noteworthy feature in connection with the extension of cancerous growths beyond the bladder is the very long period which may elapse, so far as can be determined, between the appearance of the tumor in the bladder and its propagation elsewhere. In a few instances, fifteen or twenty years have been thought to have passed before this has taken place. The tardiness noted in this respect is, perhaps, owing to the somewhat limited supply of lymphatics in the bladder and lack of the direct connection between them and the lymph nodes outside the organ. That such communication, however, does exist, appears to have been fully demonstrated by the injection of colored liquid into the bladder lymphatics and its appearance in the lymph structures that have been referred to.

How large a territory may be invaded by direct extension of the process to adjacent structures was seen in one of the author's cases, in which the operation was performed six years after the first appearance of the symptoms. (See No. 5 of illustrative cases at end of this chapter.)

Secondary Changes in the Tumor.-These are: ulceration and necrosis, fatty or colloid degeneration, and incrustation with phosphatic salts. Beyond the limits of the tumor itself, cystitis and pericystitis.

More remote changes are: dilatation of the ureters, the renal pelvis, and the kidney, and, if infection follows, pyonephrosis.

Ulceration and Necrosis.-These are produced by invasion of bacteria, disturbance of nutrition caused by vasomotor influence, or they may be also due to disproportionately rapid development of the cellular elements as compared with the bloodvessels supplying the tumor, and to the consequent failure of its nutrition. 
Cystitis.-This is a very constant companion of tumors of the bladder and almost invariably occurs in the later stages of the disease. It originates in bacterial invasion, to which the lowered resistance of the tissues renders them peculiarly liable.

Pericystitis.-Pericystitis is not an infrequent accompaniment of tumor of the bladder and the cystitis co-existing with it. It may arise through direct migration of bacteria through the bladder wall. Clinically, it appears as an area of increased resistance about the lower portion of the bladder, obscuring posteriorly the anatomical outlines of the seminal vesicles and sometimes of the prostate. Occasionally it terminates in suppuration in Retzius' space.

Hydronephrosis and Pyonephrosis.-(See chapter on these subjects.) Albarran found eight examples of hydronephrosis in eighty-eight personal observations of cases of vesical tumor.

Duret, Pasteau, ${ }^{18}$ Nicolich, Frasier, and a number of others report cases of the same conditions in connection with vesical tumors which involved the ureteral orifices.

Duret calls attention to the fact that there are some cases of tumor in which the growth has been at, or close to, the ureteral orifice, and in which no such secondary changes in the renal pelvis or kidney were present; also to a case seen by him in which there was hydronephrosis on the side opposite to that on which the ureter was obstructed by vesical tumor, but none on the same side.

These facts make him doubt the influence attributed to the presence of the tumor about the ureteral orifices in the production of the renal conditions referred to. Despite the view of Duret, there is no doubt as to there being a considerable number of cases in which the existence of hydronephrosis and pyonephrosis is directly referable to tumors of the bladder at or close to the ureteral orifices. Rafin ${ }^{9}$ speaks of the occurrence of renal retention as rare, despite the frequency of the ureteral orifices as the seat of tumors. He cites six cases only as examples: those of Hasenmayer, Krahn, Reverdin, Spangaro, Burckhardt, and Nicolich (bilateral hydronephrosis in the two last).

Frequency.-The relative frequency of vesical tumors to those occurring in other parts of the body is suggested by the statistics of Gürlt, who found 66 tumors of the bladder out of a total number of i6,637 tumors of all sorts, or 3.9 per cent. Küster ${ }^{19}$ found Io in a total of I 308 of all sorts, or 7.6 per cent. At the Necker there were 55 tumors of the bladder found among I 50 male patients, between I888 and I89 I. These cases were all in the service of the genito-urinary diseases.

Albarran $^{3}$ gives the following figures with reference to the relative frequency of the different varieties of the tumors of the bladder: 


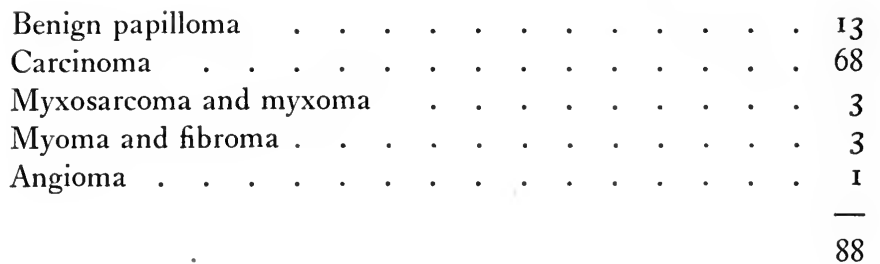
3 - 88

BAzY.

Carcinoma . . . . . . . . . . . . .

Fibroma . . . . . . . . . . . .

\section{Dittel.}

\begin{tabular}{|c|c|c|c|c|c|c|c|c|c|c|c|c|c|c|}
\hline Carcinoma & • & & $\cdot$ & $\cdot$ & * & & $\cdot$ & $\cdot$ & $\cdot$ & $\cdot$ & 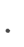 & • & - & 7 \\
\hline Papilloma . & 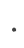 & . & . & . & . & . & . & . & 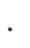 & . & . & . & . & . \\
\hline Myoma . & . & . & . & . & $\cdot$ & . & $\cdot$ & . & . & - & - & - & 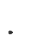 & . \\
\hline Sarcoma & . & . & $\cdot$ & $\cdot$ & . & . & . & . & & . & . & - & . & I \\
\hline \multicolumn{15}{|c|}{ IVERSON. } \\
\hline Carcinoma & 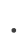 & - & $\cdot$ & $\cdot$ & $\cdot$ & $\cdot$ & . & . & • & . & - & . & . & - \\
\hline Papilloma . & . & . & . & . & . & . & . & . & . & . & - & $\cdot$ & $\cdot$ & 3 \\
\hline Sarcoma . & . & . & . & . & 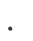 & . & . & . & & . & . & . & . & I \\
\hline
\end{tabular}

ISRAEL.

Carcinoma . . . . . . . . . . . . . . . $\quad$. 12

Papilloma . . . . . . . . . . . . . . 2

Carcinoma, ı00; papilloma, 21; fibroma, 2; myxoma, 3; sarcoma, 2; myoma, 3; angioma, $\mathbf{I}$.

As compared with the earlier series of statistics, it is noticeable that the more recent ones show an increasingly large proportion of carcinoma relative to benign growths; thus, Sir Henry Thompson ${ }^{5}$ reported 60 benign out of a total number of 100 cases; Conner, ${ }^{20} 8_{\text {I }}$ out of 107 ; Clado, ${ }^{10}$ Io6 out of 208; Pousson, ${ }^{21}$ I 38 out of 205 . Cases reported since the series of Albarran, tabulated above, all go to confirm the view that much the larger number of these neoplasms are of malignant nature. Reports upon the nature of tumors based upon cystoscopic appearances are not to be relied upon.

Sex.-The relative frequency of vesical tumors in men and women is variously estimated as 14 per cent. to 20 per cent. in women and 80 per cent. to 86 per cent. in men.

Age.-The earliest age at which vesical tumor has been reported is in the case observed by Martini, ${ }^{16}$ which was that of a child who died ten days after birth, and in whose bladder a dermoid cyst was found postmortem.

As elsewhere in the body, cancer of the bladder occurs more frequently after middle life. 
Myxoma and sarcoma are more frequent in childhood than at other periods of life. Of 32 cases of vesical tumor occurring in children, collected by Steinmetz, ${ }^{22}$ I 3 were myxoma and I3 sarcoma.

Location.-The lower third of the bladder is the seat of the tumor in about two-thirds of all cases. Tumors are much more frequently seated on the posterior than on the anterior wall of the organ. Fenwick ${ }^{23}$ pointed out that 43 per cent. of papillomata were situated close to the ureteral orifices.

Duration.-Albarran ${ }^{3}$ gives the following figures as to the length of time that elapsed in 162 cases of vesical tumors, from the appearance of the first symptom to that of the first observation by the surgeon. The following are some of the figures presented by him irrespective of the nature of the tumors:

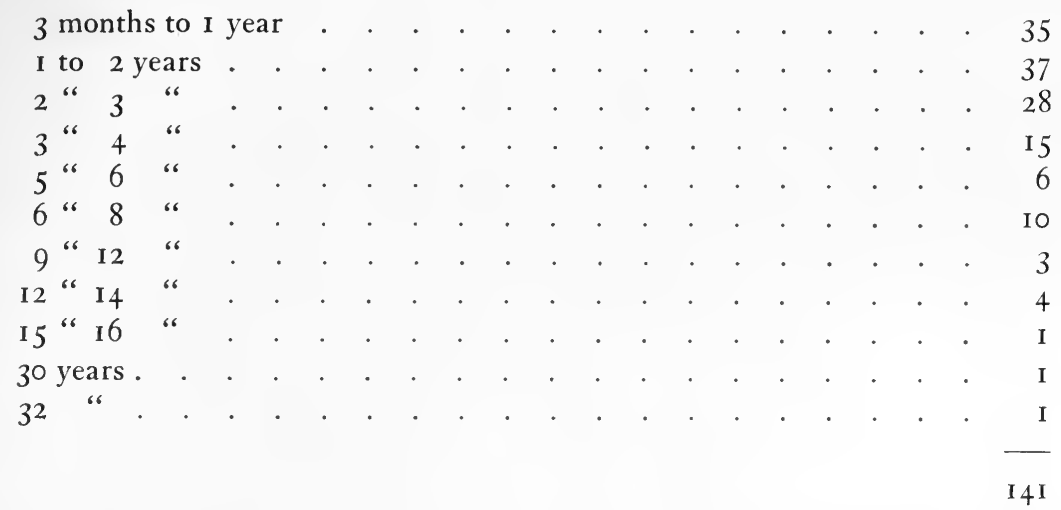

Considered with regard to the special nature of the tumors, the following figures resulted:

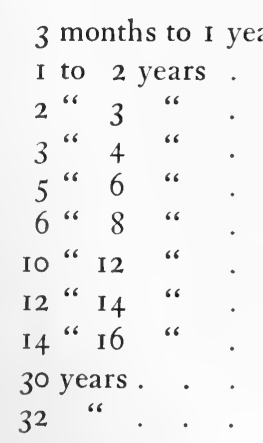

\begin{tabular}{|c|c|}
\hline Papilloma. & Carcinoma. \\
\hline I I & 23 \\
\hline 8 & 22 \\
\hline 12 & I 4 \\
\hline 9 & 4 \\
\hline 4 & 2 \\
\hline 4 & 6 \\
\hline$\circ$ & I \\
\hline I & 3 \\
\hline I & 0 \\
\hline I & I \\
\hline o & I \\
\hline 一 & 一 \\
\hline 5I & 77 \\
\hline
\end{tabular}

One of the striking features connected with tumors of the bladder, which has been commented upon by various writers, is the unusual length of time that intervenes in a considerable proportion of cases of carcinoma between the appearance of the first symptom and the extension 
of the disease by contiguity to adjacent tissues, or by metastasis to distant ones.

Sarcoma shows a greater rapidity of growth than any other form of tumor.

Myoma is relatively slow in its progress.

In I 5 out of the I4I cases which were mentioned above, the average time was somewhat less than three years.

Symptoms and Diagnosis.-Hematuria.-Hematuria is the cardinal symptom of tumors of the bladder. In cases in which it appears in typical form it presents characteristic features. These are:

The spontaneity of the occurrence of hematuria. It bears no relation to exercise or other exciting cause for which the patient is in any way responsible. In a great number of cases the hemorrhage is produced by the breaking off or necrotic separation of villi from the surface of some part of the tumor, which, being very vascular, gives rise to a much more extensive bleeding than would seem to be natural from so limited a surface and such small bloodvessels. The fact of the blood being mixed with urine gives it the appearance of being in larger quantities than is really the case.

Abundance of the blood is its other specially characteristic feature in the majority of cases. The benign papillomata, as a rule, bleed more profusely than the malignant growths.

Progressively increasing frequency of the attacks of hematuria as the malady advances is also characteristic.

Occasionally hematuria, in varying degree, is present throughout the entire course of the disease. In a few cases it is wholly absent.

The length of the intervals between the attacks of bleeding varies greatly. Seven years have been known to elapse between the first and second appearance of blood in the urine, and in many instances there are intervals of several months during the earlier stages of the condition. In one or two cases there has been but a single attack of bleeding and that has been the first and only symptom of the disease.

If at all abundant, the color of the blood is bright, unless it is retained in the bladder for a considerable time, owing to stricture of the urethra, or to prostatic hypertrophy, which prevents the bladder from emptying itself.

The so-called "terminal" character of the hematuria is also suggestive. This means that there is a larger amount and brighter color of blood in the last than in the first part of the irrigating fluid used when washing out the bladder, or in the urine when voluntarily passed.

Instrumental manipulations in the bladder are very likely to cause profuse hemorrhages. 
Hematuria is the first symptom in the majority of cases. Albarran found it to be so in 148 out of 200 cases. Pain and the disturbance of micturition were the first symptoms in 52 .

With reference to the special kind of tumor and the initial symptom, Albarran found in the same series of cases that 57 out of 62 of benign papilloma began with hematuria, 5 with pain. In 70 cases of carcinoma out of a total of I00, hematuria was the first symptom noted in 18 , painful urination in II. In 9 cases of myoma, 3 began with hematuria, 6 with painful urination.

The rapidity with which death may result from repeated and frequently recurring hemorrhages is seen in the cases cited at the end of the chapter. (See Nos. 6, 7, and 8 of illustrative cases at end of this chapter.)

Pain.-Pain on micturition will, of course, be present when cystitis supervenes; otherwise, in cases of benign tumors, unless they are seated near the neck of the bladder, pain does not ordinarily occur. Pain is almost always associated with the existence of a cancerous tumor in the later stages of its development, at any rate. This pain is usually severe and is not confined to the act of micturition. It frequently radiates to the sciatic and anterior crural regions. Pain is not notably increased by movement.

Evidence Furnished by the Urine.-The only absolute evidence of the existence of a tumor of the bladder furnished by the urine is the discovery in it of bits of the growth which have been shed from the surface and which are usually to be found upon careful and repeated examinations with the microscope. These bits of the tumor represent one or several of the villi, which show unmistakably characteristic forms and structure, or the contrary, according to whether they are necrotic and denuded of the epithelial covering or not. The diagnosis of tumor of the bladder is justified upon the strength of such a bit of tissue as this alone if seen in its characteristic form.

Nothing can be inferred from these fragments in the urine with regard to the nature of the tumor. They only inform us of its existence, for, as has been already pointed out, any variety of tumor may have a villous surface.

Epithelial Cells and Pus in the Urine.-A certain amount of significance attaches to the frequent and long-continued presence in the urine of polymorphous cells, the larger part of which are fusiform in shape and have large nuclei. It is true that these cells do not differ essentially from the normal epithelium of the deeper layers of the bladder mucous membrane, but in no other condition do they appear in such large numbers and so persistently as in connection with tumors.

Fibrinuria.-Ultzmann ${ }^{24}$ has called attention to a condition of the urine in which it contains a very large quantity of exceedingly viscid, vor. I-33 
tenacious mucus, which converts it into a thick, jelly-like, fibrinous mass, adhering so firmly to the surface of the vessel containing it that the latter can be turned upside down without causing it to run out. He has never seen this condition except in three cases of cancer of the bladder.

Physical Examination.-Palpation.-When a tumor of the bladder has reached a large size, and if it is of firm consistence, it can be readily felt by bimanual palpation with the finger of one hand in the rectum, and those of the other above the symphysis pubis.

Tumors which have infiltrated the posterior wall of the bladder can usually be appreciated by the finger in the rectum. When they feel hard to the touch, it is safe, in the majority of cases, to assume that they are cancerous. In fact, this assumption is fairly warranted, if such infiltration can be felt at all. The only source of confusion is that which may be due to the presence of cystitis or pericystitis. The latter condition is present not infrequently, and cystitis almost always occurs in the later stages of the disease.

Instrumental Exploration.-Except with the cystoscope, instrumental examination of the bladder in cases of suspected tumor should be avoided as much as possible, on account of the liability to excite cystitis and hemorrhage. In a few cases serious bleeding has followed such examinations. A soft-rubber catheter may be used to demonstrate the terminal character of the hematuria which has already been spoken of.

Prognosis.-Tumors of the bladder, irrespective of their nature, cause the death of the patients in all cases, practically speaking. Those which are structurally benign, by repeated hemorrhages, or by secondary conditions created by their presence-cystitis, renal infections, and sepsis; those which are malignant, by extension to adjacent structures, or to distant ones by metastasis, and the secondary systemic conditionssepsis and renal infection-which result from them.

The few exceptions to the rule of fatality have been in a small number of cases, in which spontaneous expulsion of benign papillomata has taken place and in which cure has apparently followed. (See Illustrative Cases Nos. 9 to 14 inclusive.)

The duration of life after the appearance of the first symptom, has been discussed in an earlier part of the chapter.

It is so variable that it is impossible to predict what it will be in any given case. It should be noted, however, that the average term of life from the time of the appearance of the first symptom is about three years in cases of benign papilloma and myoma, and rather less in cases of carcinoma; while in those of sarcoma and myxoma it is much shorter, as a rule. Again, it should be remembered that in some of the cases of carcinoma, as well as in those of benign growths, 
there has been an extraordinarily slow evolution of the disease. It will be recalled that we have laid stress upon the fact that it is impossible to determine with certainty the nature of a tumor by means of cystoscopic examinations.

Treatment.-Palliative.-Palliative treatment of vesical tumors has a limited but useful sphere. Its objects are as follows: To stop hemorrhage, relieve retention due to clots in the bladder; to diminish cystitis, relieve pain, and thus restore the patient's strength.

It is applicable in cases which are inoperable, or to prepare patients for radical operations.

The best means for arresting hemorrhage, in the writer's experience, has been prolonged irrigation of the bladder with cleansing solutions as hot as the patient can comfortably bear them; $125^{\circ}$ is usually well tolerated.

If there is retention due to clots filling the bladder, it is best relieved by breaking up the mass with an evacuating tube large enough to allow of their being drawn through it.

While it is true that the pressure of the clots tends to stop bleeding, it also increases venous congestion, and this tends to augment it. This is well shown by the appearance of a distended bladder when it is exposed in a suprapubic operation, and by the disappearance of the congestion as soon as the bladder is emptied.

Immediately after the clots have been removed, the bladder should be irrigated with a hot boric or permanganate of potash solution. The familiar illustration used to show the effect of prolonged application of hot water is that of a washerwoman's hands, which, after long immersion, present a white and shrivelled appearance of the surface. This may be produced similarly in the bladder by long-continued hot irrigation.

The hemorrhage which proceeds from ulcerated surfaces of tumors, or which is associated with cystitis, may often be restrained or stopped by these irrigations. This is also true of the pain arising from the same condition. Desnos has reported a few cses in which irrigation of the bladder with 4 per cent. boric acid, followed immediately by one of I per cent. of methylene blue, was followed by a disappearance of the tumor. He does not assert that the treatment produced this effect, but reports the observations as being interesting and suggestive.

Operative Treatment.-The best means at our disposal for deciding what method to adopt in the operative treatment of cases of tumors of the bladder is by the study of the surgical results obtained by the different operative procedures that have hitherto been employed in connection with these cases. The data which are set forth in the subjoined tables are derived from two sources, viz., the articles published by $\mathrm{Rafin}^{9}$ and by Watson. ${ }^{25}$ Each of these communications contained the cases of all 
sorts previously reported in the literature of the subject; in addition, there was a considerable number of others which were personally communicated to Rafin. The tables given in this chapter are composed of Watson's series, plus the cases personally reported to Rafin, and represent the largest and most comprehensive statistical compilation published previous to 1907. The two series of Rafin and Watson include all others of importance which were previously published, as well as a large number supplied to the former by individual surgeons.

The total number of cases of the series is 679 . The numbers of the special tumors are as follows:

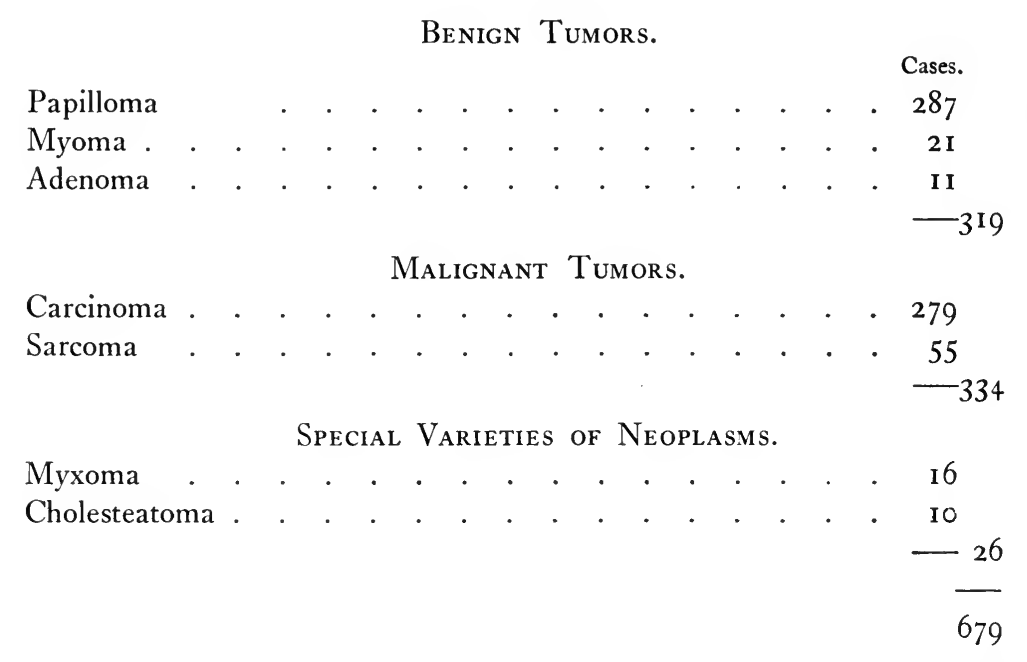

(For operative technique see chapter on Technique of Operations on the Bladder.)

The operative measures employed for the removal of tumors of the bladder are as follows:

I. Operations performed through the whole length of the urethra by means of specially devised instruments (the operating cystoscope of Nitze, ${ }^{26}$ etc.).

2. The removal of the tumor by means of specially devised instruments through an external perineal urethrotomy incision (Sir Henry Thompson ${ }^{5}$ ).

3. Suprapubic operations without resection of the bladder. By curetting, galvanocautery snare, or special forms of instruments (e. $g$., Watson' ${ }^{27}$ scissors cautery), or by excision of the tumor with knife or scissors.

4. By partial resection of the bladder.

5. By total extirpation of the bladder.

Surgical Results.- The cases in the tables which follow are analyzed with reference to: (I) The operative mortality of the different surgical 
procedures as applied to the special kinds of tumors individually.

The percentage of recurrences and non-recurrences following each of the surgical procedures in each special class of tumor. (3) An analysis of the cases irrespective of surgical method with reference to the general percentage of cures and of surgical failures shown in benign tumors and malignant tumors. Under the term cure are included cases in which no recurrence took place at the end of three or more years. The term surgical failure is applied to operative mortality plus recurrence. The computation of cures and of recurrences are, of course, made only from the cases in which the subsequent histories of patients surviving operation were known.

Table I.- $(A)$ Operative Mortality of Benign Tumors. Suprapubic Operations, not Resections. Total Cases, 3ig.

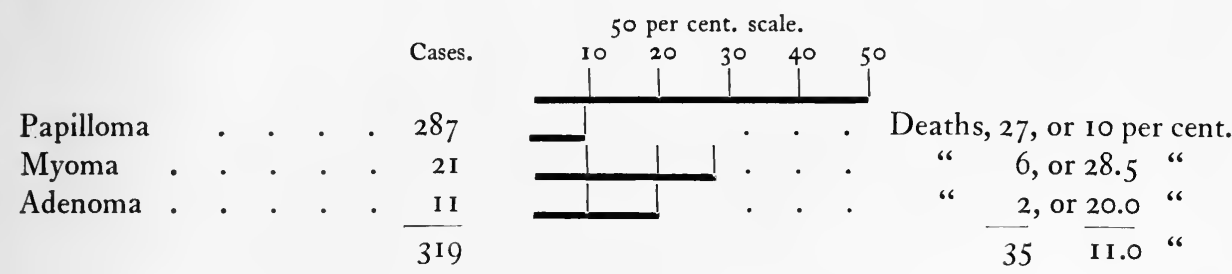

(B) Malignant Tumors. Total Cases, 334.

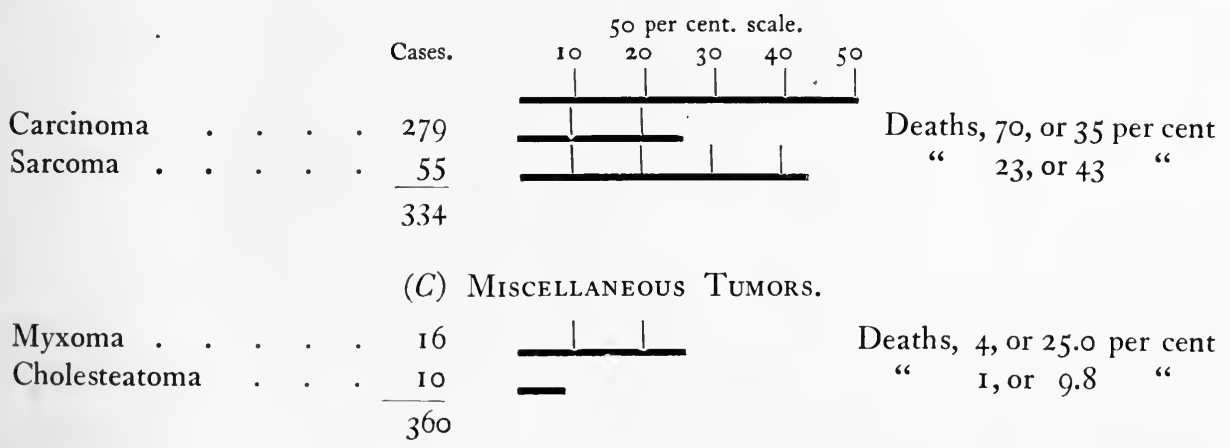

Table II.-Operative Mortality. Partial Resection.

Malignant Tumors.

Carcinoma . . . . $\quad 96$ Deaths, 21, or 21.8 per cent.

Table III.-Operative Mortality. Total Extirpation. Cases. 100 per cent. scale.

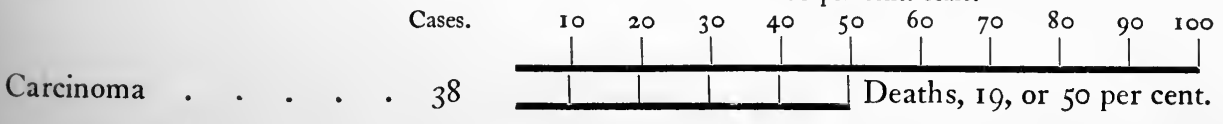


Table IV.-Recurrence and Non-recurrence. Suprapubic Operations, NOT RESECTIONS.

(Computed from patients with postoperative histories known.)

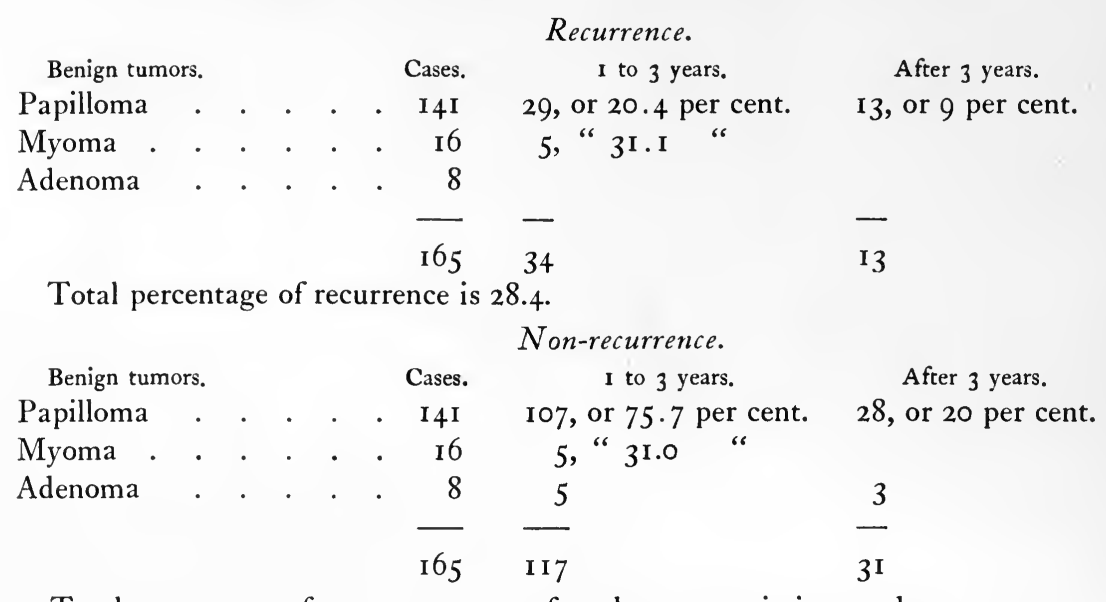

Total percentage of non-recurrence after three years is just under 20 per cent.

Benign tumors.

Recurrence.

Non-recurrence.

No data.

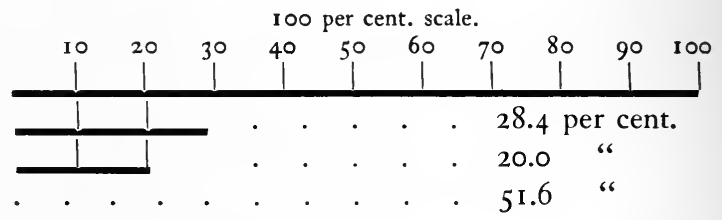

Table V.-Carcinoma. Recurrence and Non-recurrence.

Recurrence.

Surgical methods. Cases. Suprapubic, not resections $\quad \mathbf{2} 5$ 1 to 3 years. After 3 years.

Partial resections . . . 50

Total extirpation . . . 16

$$
82 \text { pts., or } 65.0 \text { per cent. }
$$

$\begin{array}{rrrr}29 & \text { “ } & \text { “ } & 58.0 \\ 2 & 6 & \text { “ } & \text { I } 2.5\end{array}$

$$
\text { I9I II3 }
$$

Suprapubic, not resections $\quad$ I25

Non-recurrence.

Partial resections . . . 50

Total extirpation . . . 16

I9I

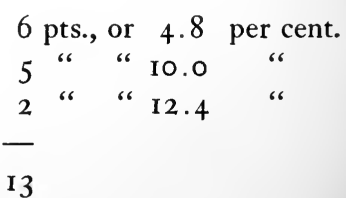

13

Myxoma and sarcoma have, with two exceptions (both of them cases of sarcoma) recurred and caused the death of the patients. One patient with sarcoma, which was removed by Czerny, had had no recurrence at the end of fifteen years. In one other case the patient was alive and well at the end of one and a half years.

In the Io cases of cholesteatoma tabulated by Rafin, in which operations have been done, there were 2 operative deaths; the longest 
period in which any of the surviving patients are reported as alive and well was three years. Recurrence took place in 2. Data are lacking in 3. One patient was alive and well at the end of two years, and I at the end of four months. (See also additional cases in Illustrative Cases Nos. 15, 16, and 17 at end of chapter.)

Table VI.-Cures Calculated with Reference to Number of Patients whose Histories Were Known Subsequent to Operation. Surgical Failure Considered as Operative Deaths, Plus Recurrences.

Benign Tumors.

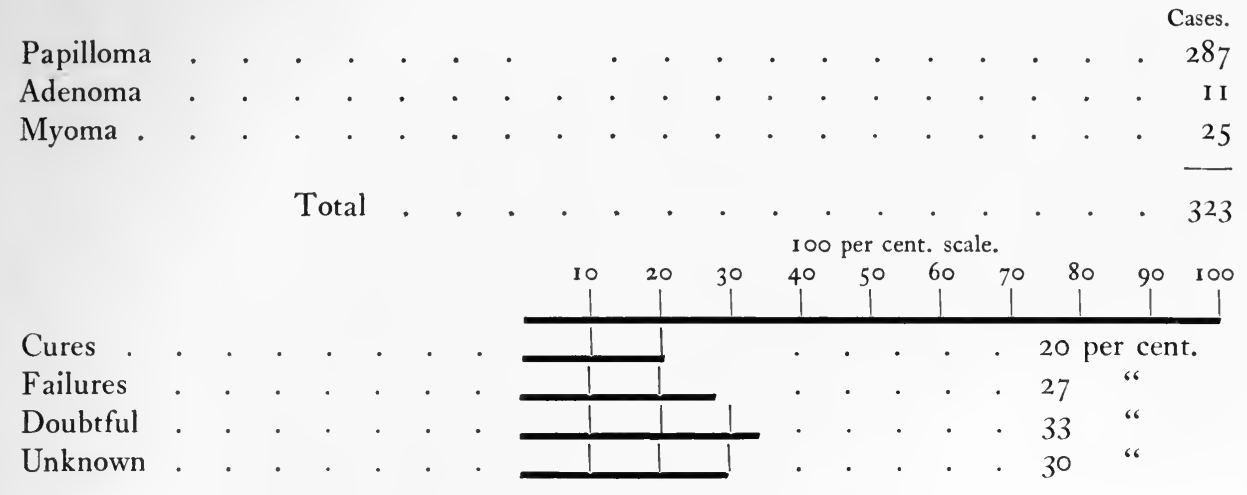

Malignant Tumors. Carcinoma. Total Cases, 279.

Cures

Failures

Doubtful

Unknown

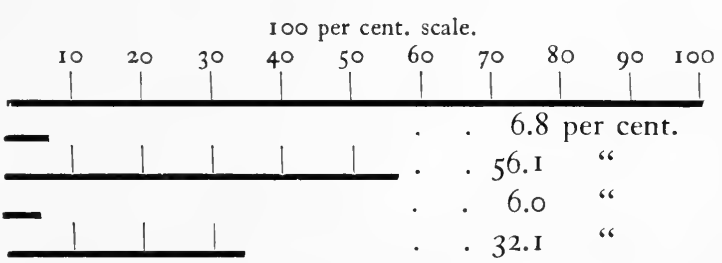

Sarcoma.-Cases, 55. Cures, I. Less than I per cent.

Мүхома.-Cases, i $6 . \quad$ ures, o.

Сholesteatoma.-Cases, io. Cures, 2. 20 per cent.

\section{CAUSES OF DEATH.}

Table ViI.-Benign Papilloma. Suprapubic not Resections.

Deaths, 27. Causes stated in $2 \mathrm{I}$.

Causes of death.

Postoperative pulmonary complications Scale of 2 I total deaths.

Sepsis

Renal infection

Cardiac failure

Hemorrhage

Shock

Peritonitis

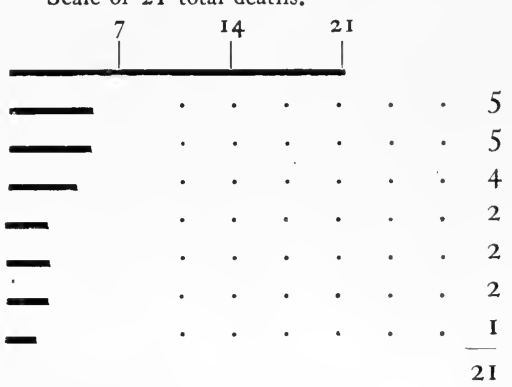




\section{Malignant Tumors. Carcinoma. Partial Resections.}

Deaths, 2I. Causes stated in 2I.

Scale of 2 I total deaths.

Causes of death.

Renal infection

Peritonitis

Shock

Pulmonary complications

Metastasis

Urinary infiltration

Hematuria

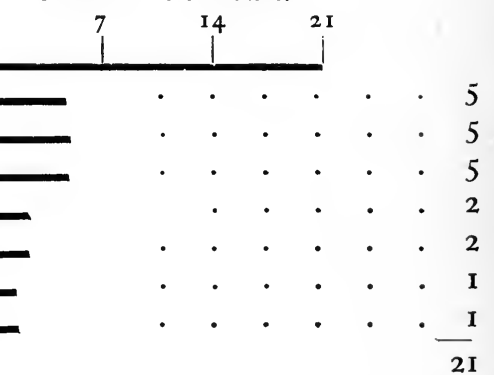

Table Vili.-Total Extirpation of Bladder in Cases of Vesical Tumors.

\begin{tabular}{|c|c|c|c|c|c|c|c|}
\hline $\begin{array}{l}\text { Operator and } \\
\text { date. }\end{array}$ & $\dot{z}$ & 遂 & 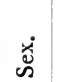 & $\begin{array}{l}\text { Disease and } \\
\text { situation. }\end{array}$ & Operation. & $\begin{array}{l}\text { Ureteral im- } \\
\text { plantation. }\end{array}$ & $\begin{array}{l}\text { Result, recovery, } \\
\text { death. }\end{array}$ \\
\hline $\begin{array}{l}\text { Bardenheuer } \\
\text { (1887) }\end{array}$ & I & $\cdots$ & M. & Carcinoma. & $\begin{array}{l}\text { Bladder ex- } \\
\text { tirpation. }\end{array}$ & $\begin{array}{l}\text { Left without } \\
\text { any implan- } \\
\text { tation; death } \\
\text { not referable } \\
\text { to ureteral } \\
\text { implantation. }\end{array}$ & $\begin{array}{l}\text { Died I } 4^{\text {th }} \text { day; } \\
\text { uremia, hydro- } \\
\text { nephrosis (both } \\
\text { sides). }\end{array}$ \\
\hline $\begin{array}{l}\text { Pawlik } \\
\text { (1888). }\end{array}$ & I & $5^{6}$ & F. & “ & Extirpation. & Vaginal. & $\begin{array}{c}\text { R.; well for } 15 \\
\text { years. }\end{array}$ \\
\hline $\begin{array}{l}\text { Küster } \\
(\mathbf{I} 89 \mathrm{I}) .\end{array}$ & I & 53 & M. & “ & “ & Rectal. & $\begin{array}{l}\text { D. } 5^{\text {th }} \text { day, pneu- } \\
\text { monia; kidney } \\
\text { pelves, ascend- } \\
\text { ing infection. }\end{array}$ \\
\hline $\begin{array}{l}\text { Kümmel } \\
\text { (I } 890) .\end{array}$ & I & 60 & F. & “ & “ & $\begin{array}{l}\text { One through } \\
\text { urethra by } \\
\text { ureteral cath- } \\
\text { eter; other } \\
\text { not drained. }\end{array}$ & $\begin{array}{l}\text { Died of shock } \\
\text { next day. }\end{array}$ \\
\hline $\begin{array}{l}\text { Kossinski } \\
\text { (I } 894) .\end{array}$ & I & $\ldots$ & F. & " & “ & $\begin{array}{l}\text { Vaginal im- } \\
\text { plantation. }\end{array}$ & $\mathrm{R}$. \\
\hline $\begin{array}{l}\text { Tuffier } \\
\text { (I 895). }\end{array}$ & I & 40 & M. & “ & " & Rectal. & $\begin{array}{l}\text { R.; died I4 mos. } \\
\text { later. Details } \\
\text { of death lack- } \\
\text { ing. }\end{array}$ \\
\hline $\begin{array}{l}\text { Giordano } \\
\text { (1 } 895) \text {. }\end{array}$ & I & 60 & $\mathrm{~F}$. & “ & \begin{tabular}{|} 
Extirpation. \\
Ligature of \\
internal ili- \\
acs; hyster- \\
ectomy, ne- \\
phrolithot- \\
omy, sym- \\
physeotomy.
\end{tabular} & Rectal. & $\begin{array}{l}\text { D. by shock in } 24 \\
\text { hours. }\end{array}$ \\
\hline
\end{tabular}


Total Extirpation of Bladder in Cases of Vesical Tumors (Continued).

\begin{tabular}{|c|c|c|c|c|c|c|c|}
\hline $\begin{array}{l}\text { Operator and } \\
\text { date. }\end{array}$ & $\dot{z}$ & 范 & 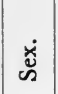 & $\begin{array}{l}\text { Disease and } \\
\text { situation. }\end{array}$ & Operation. & $\begin{array}{l}\text { Ureteral im- } \\
\text { plantation. }\end{array}$ & $\begin{array}{l}\text { Result, recovery, } \\
\text { death. }\end{array}$ \\
\hline $\begin{array}{l}\text { Chalot } \\
\text { (1898). }\end{array}$ & I & 45 & F. & $\begin{array}{l}\text { Carcinoma; } \\
\text { also of ute- } \\
\text { rus. }\end{array}$ & $\begin{array}{l}\text { Hysterectomy. } \\
\text { Ligature of } \\
\text { internal ili- } \\
\text { acs; extir- } \\
\text { pation of } \\
\text { bladder. }\end{array}$ & Rectal. & $\begin{array}{l}\text { R.; alive at end } \\
\text { of I year. }\end{array}$ \\
\hline $\begin{array}{l}\text { Vasilief } \\
\text { (I895). }\end{array}$ & I & 30 & M. & Carcinoma. & Extirpation. & $\begin{array}{l}\text { Angle of ab- } \\
\mathrm{d} \text { o m in a l } \\
\text { wound. }\end{array}$ & $\begin{array}{l}\text { R.; well } 4 \text { mos. } \\
\text { later. }\end{array}$ \\
\hline $\begin{array}{l}\text { Giordano } \\
\text { (1897). }\end{array}$ & I & 50 & F. & Sarcoma. & $\begin{array}{l}\text { Total extir- } \\
\text { pation of } \\
\text { bladder and } \\
\text { abd ominal } \\
\text { hyster ec- } \\
\text { tomy. }\end{array}$ & $\begin{array}{l}\text { Into sigmoid } \\
\text { flexure. }\end{array}$ & $\begin{array}{l}\text { D. I2th day: pye- } \\
\text { lonephritis. }\end{array}$ \\
\hline $\begin{array}{l}\text { Turetta } \\
\text { (1897). }\end{array}$ & $\mathbf{I}$ & 33 & M. & Carcinoma. & Extirpation. & Rectal. & $\begin{array}{l}\text { D. I6th d a y ; } \\
\text { acute acending } \\
\text { renal infection. }\end{array}$ \\
\hline $\begin{array}{l}\text { Schede } \\
(\mathbf{I} 898) \text {. }\end{array}$ & I & 55 & M. & “ & “ & “ & $\begin{array}{l}\text { D. 3d day of } \\
\text { shock. }\end{array}$ \\
\hline $\begin{array}{c}\text { Winiwater } \\
\text { reported by } \\
\text { Hogge ( } 1898 .\end{array}$ & I & 45 & M. & “ & $\begin{array}{l}\text { Removal of } \\
\text { bladder and } \\
\text { extern a l } \\
\text { genitals. }\end{array}$ & “ & $\begin{array}{l}\text { R.; no recur- } \\
\text { rence } 3 \text { years } \\
\text { later; patient } \\
\text { in comparative } \\
\text { comfort. }\end{array}$ \\
\hline $\begin{array}{l}\text { Krause } \\
\text { (I899). }\end{array}$ & I & 17 & M. & $\begin{array}{l}\text { Tumor of } \\
\text { b l a d d e r } \\
\text { compress- } \\
\text { ing l e f t } \\
\text { uterer. }\end{array}$ & Extirpation. & “ & $\begin{array}{l}\text { R. } 3 \frac{1}{2} \text { mos. later } \\
\text { was in excel- } \\
\text { lent health. }\end{array}$ \\
\hline $\begin{array}{l}\text { Lund } \\
\text { (1902). }\end{array}$ & I & 57 & M. & Carcinoma. & “ & “ & $\begin{array}{l}\text { D. 4th day of } \\
\text { uræmia, acute } \\
\text { ascending renal } \\
\text { infection. }\end{array}$ \\
\hline $\begin{array}{l}\text { Harris } \\
(1901) \text {. }\end{array}$ & I & 53 & M. & “ & $\begin{array}{l}\text { Extirpation, } \\
\text { except a } \\
\text { small bit of } \\
\text { vertex of } \\
\text { bladder. }\end{array}$ & $\begin{array}{r}\text { Sutured to sur- } \\
\text { face of supra- } \\
\text { pubic wound. }\end{array}$ & $\begin{array}{l}\text { D. } 2 \text { mos. later of } \\
\text { pneumonia. }\end{array}$ \\
\hline $\begin{array}{c}\text { Mayo Robson } \\
\text { (1903). }\end{array}$ & I & 42 & F. & “ & Extirpation. & $\begin{array}{l}\text { Vaginal im- } \\
\text { plantation. }\end{array}$ & $\begin{array}{l}\text { D. I3th day of } \\
\text { uræmia. }\end{array}$ \\
\hline
\end{tabular}


Total Extirpation of Bladder in Cases of Vesical Tumors (Continued).

\begin{tabular}{|c|c|c|c|c|c|c|c|}
\hline $\begin{array}{l}\text { Operator and } \\
\text { date. }\end{array}$ & $\dot{z}$ & $\underset{\dot{\alpha}}{\dot{\alpha}}$ & $\dot{\grave{d}}$ & $\begin{array}{l}\text { Disease and } \\
\text { situation. }\end{array}$ & Operation. & $\begin{array}{l}\text { Ureteral im- } \\
\text { plantation. }\end{array}$ & $\begin{array}{l}\text { Result, recovery, } \\
\text { death. }\end{array}$ \\
\hline $\begin{array}{l}\text { Woolsey } \\
\text { (1903). }\end{array}$ & I & 37 & M. & Carcinoma. & Extirpation. & $\begin{array}{l}\text { One ureter rec- } \\
\text { tal implanta- } \\
\text { tion, the other } \\
\mathrm{d} \text { r a i n e d } \\
\text { through the } \\
\text { sup ra pubic } \\
\text { wound with } \\
\text { ureteral cath- } \\
\text { eter. }\end{array}$ & $\begin{array}{l}\text { R.; did well for } \\
\text { six weeks, then } \\
\text { failed and died } \\
3^{\frac{1}{2}} \text { mos. after } \\
\text { operation; as- } \\
\text { cending renal } \\
\text { infection. }\end{array}$ \\
\hline $\begin{array}{l}\text { Kayser } \\
\text { (1903). }\end{array}$ & I & 37 & M. & “ & “، & $\begin{array}{l}\text { Neck of blad- } \\
\text { der. }\end{array}$ & D. \\
\hline $\begin{array}{l}\text { McCosh } \\
\text { (I903). }\end{array}$ & I & 50 & F. & " & “ & $\begin{array}{l}\text { Ureters } \mathrm{c} \mathrm{u} \mathrm{t} \\
\text { across I inch } \\
\text { from bladder } \\
\text { and cut ends } \\
\text { allowed t o } \\
\text { drain in t o } \\
\text { retroperito- } \\
\text { neal pelvic } \\
\text { tissue. }\end{array}$ & $\begin{array}{l}\text { R.; I } 5 \text { mos. later } \\
\text { no recurrence; } \\
\text { leading an ac- } \\
\text { tive life. }\end{array}$ \\
\hline “" & I & $5^{\circ}$ & F. & “ & “ & The same. & $\begin{array}{l}\text { R.; I } 3 \text { mos. later } \\
\text { no recurrence. } \\
\text { Leading an ac- } \\
\text { tive life. }\end{array}$ \\
\hline $\begin{array}{c}\text { Geo. Tully } \\
\text { Vaughan } \\
\text { (I903). }\end{array}$ & I & 32 & M. & Sarcoma. & $\begin{array}{l}\text { By combined } \\
\text { suprapubic } \\
\text { and peri- } \\
\text { neal opera- } \\
\text { tion. }\end{array}$ & Sigmoid. & $\begin{array}{l}\text { D. at end of oper- } \\
\text { ation; shock } \\
\text { a n d hemor- } \\
\text { rhage. }\end{array}$ \\
\hline $\begin{array}{l}\text { Lindner } \\
\text { (1895). }\end{array}$ & I & 32 & M. & Carcinoma. & Extirpation. & $\begin{array}{l}\text { Urethral; ure- } \\
\text { ters did not } \\
\text { drain. }\end{array}$ & $\begin{array}{l}\text { D.; operative. } \\
\text { Cancer of kid } \\
\text { ney associated } \\
\text { with that of } \\
\text { bladder. }\end{array}$ \\
\hline $\begin{array}{l}\text { Zeller } \\
(1896) \text {. }\end{array}$ & I & 56 & F. & “" & “ & Vaginal. & $\begin{array}{l}\text { D. } 24 \text { hours from } \\
\text { shock. }\end{array}$ \\
\hline $\begin{array}{l}\text { Gopel } \\
(1897) \text {. }\end{array}$ & I & 67 & M. & “ & $\begin{array}{l}\text { Combined su- } \\
\text { prapubic \& } \\
\text { perineal op- } \\
\text { eration. }\end{array}$ & Urethral. & D. from shock. \\
\hline $\begin{array}{c}\text { Bardenheuer } \\
\text { (I898). }\end{array}$ & I & $5^{I}$ & M. & Carcinoma. & $\begin{array}{l}\text { Suprapubic } \\
\text { extirpation. }\end{array}$ & $\begin{array}{l}\text { Abandoned in } \\
\text { wound. }\end{array}$ & $\begin{array}{l}\text { D. } 2 \text { d day; no re- } \\
\text { nal lesion. }\end{array}$ \\
\hline Bardenheuer. & I & 35 & M. & " & $\begin{array}{l}\text { Combined op- } \\
\text { eration. }\end{array}$ & $\begin{array}{l}\text { Abandoned in } \\
\text { wound. }\end{array}$ & $\begin{array}{l}\text { Lived } 4 \text { mos.; } \\
\text { death due to } \\
\text { renal infection. }\end{array}$ \\
\hline
\end{tabular}


Total Extirpation of Bladder in Cases of Vesical Tumors (Continued).

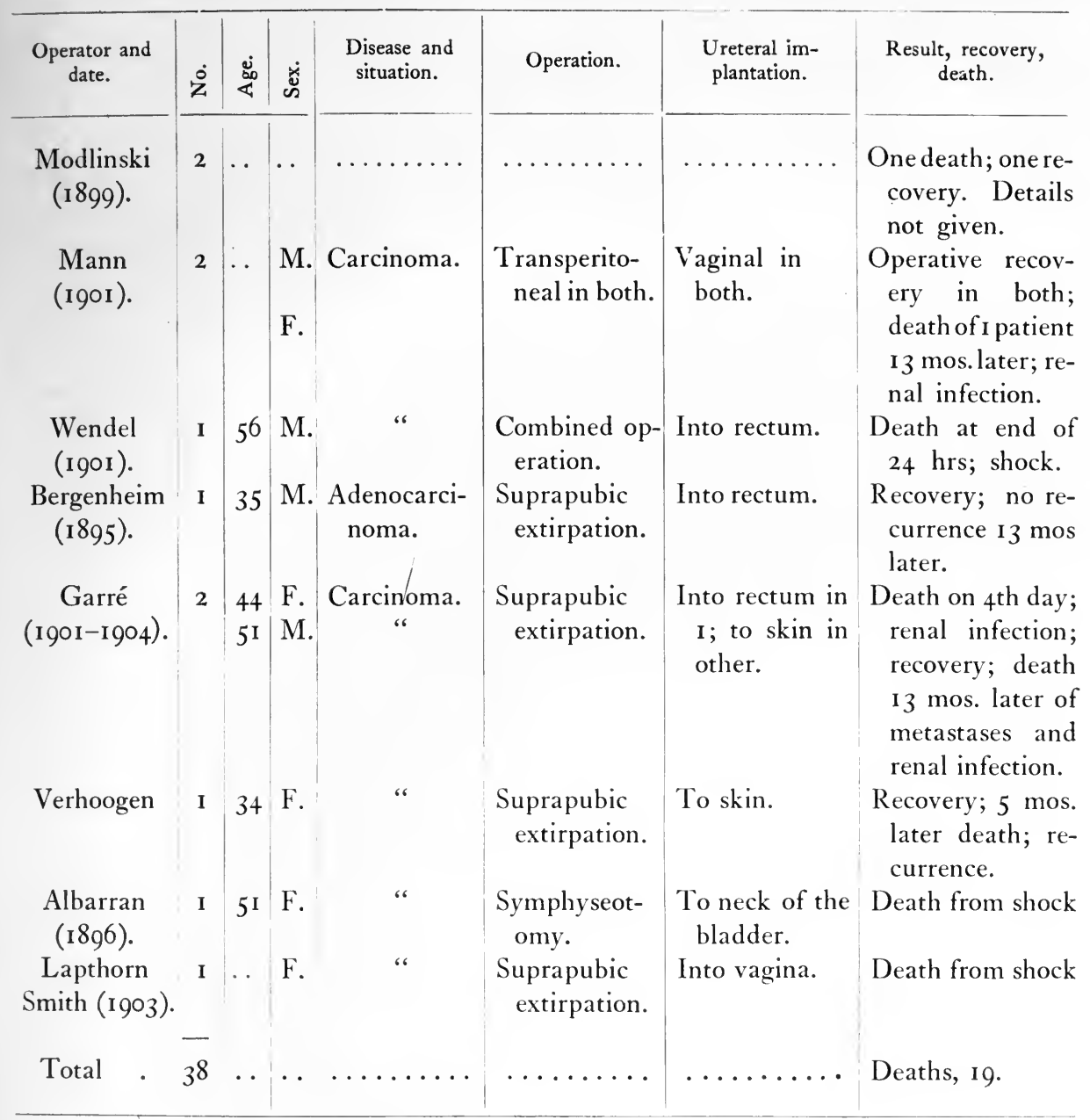

Total number of operations, 38 ; operative deaths, I9; mortality, 50 per cent. Deaths due to shock, 9; due to renal infection, 7; due to pneumonia, 2. Later deaths, 8. (Causes of death, see below.)

There were 19 operative deaths and 19 recoveries. Of the latter, the times during which the cure was maintained without recurrence were as follows: Pawlik's ${ }^{28}$ case, fifteen years; Hogge's ${ }^{29}$ case, four years; McCosh's 2 cases, fifteen and thirteen months; Bergenheim's (Rafin ${ }^{9}$ ) case, thirteen months; Chalot's ${ }^{30}$ case, one year; Vasilief's ${ }^{31}$ case, four months; Krause's ${ }^{32}$ case, three and a half months. 


\section{CAUSES OF DEATH.}

Table IX.-Carcinoma. Total Extirpation.

Deaths, 19. Causes stated in 18 .

Causes of death.

Shock

Ascending renal infection

Pneumonia

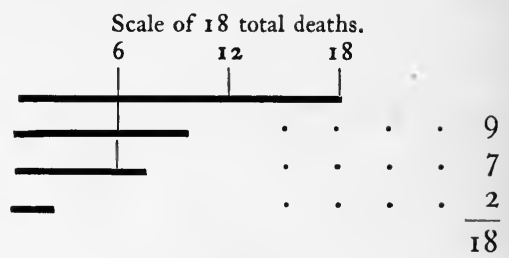

In 8 later deaths.

Ascending renal infection

Recurrence

Pneumonia

Due to ascending renal infection, altogether, I2. Of these $\mathbf{1 2}$, there were Io deaths, for which ureteral implantations were directly responsible.

Shock and ureteral implantation are, therefore, the two fatal factors in connection with the operation of total extirpation.

Table X.-Twenty-five Cases of Myoma.

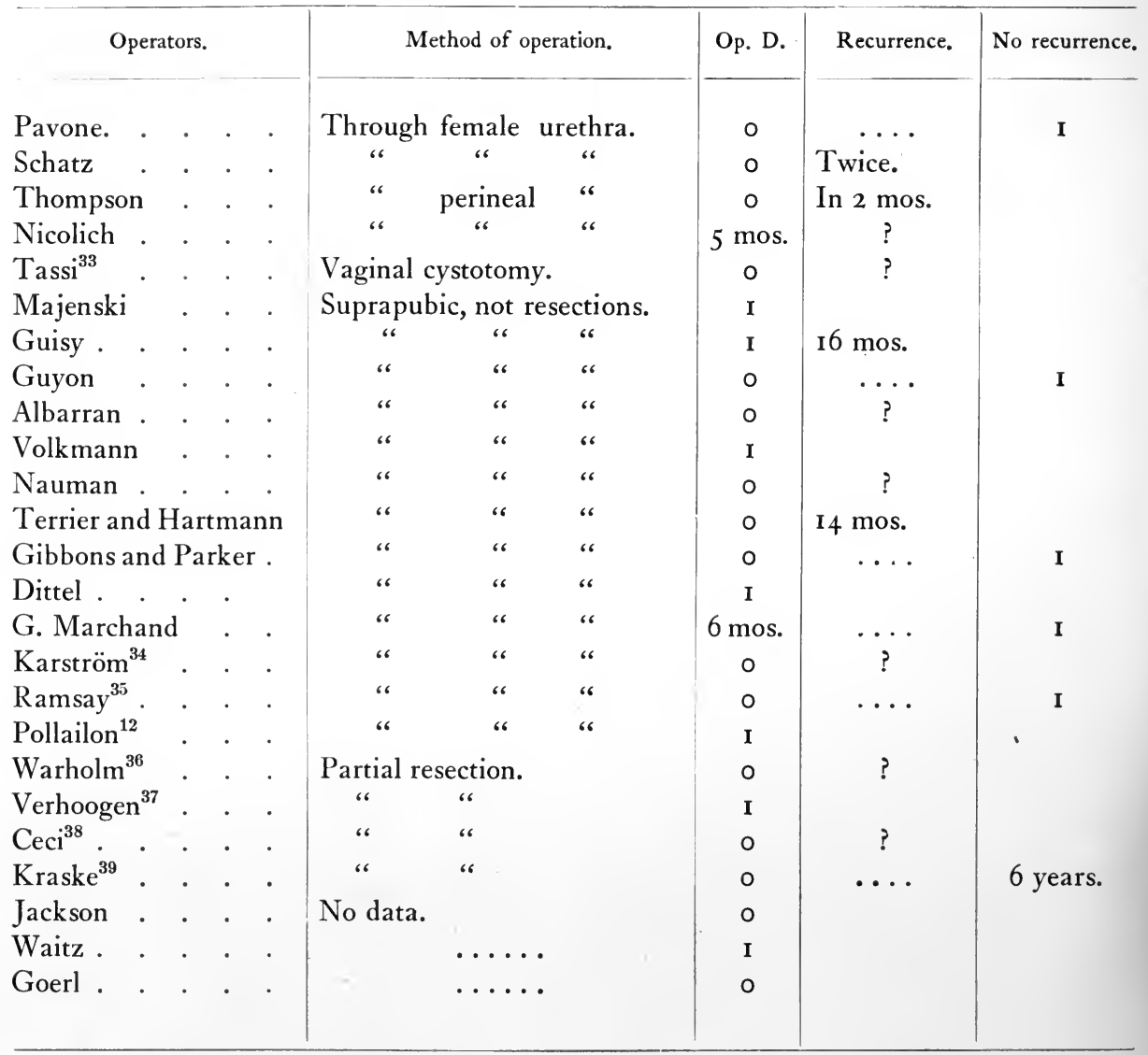




\section{Table XI.-Operations in Cases of Vesical Adenoma.}

(Translated from Rafin).

\begin{tabular}{|c|c|c|c|c|}
\hline Operators. & $\begin{array}{l}\text { Age } \\
\text { and } \\
\text { sex. }\end{array}$ & Operative method. & Character of tumor. & $\begin{array}{l}\text { Results and comment, } \\
\text { immediate and remote. }\end{array}$ \\
\hline Kaltenbach. ${ }^{40}$ & F. & Suprapubic. & $\begin{array}{l}\text { Papillary adenoma the } \\
\text { size of a walnut. }\end{array}$ & Recovery (operative). \\
\hline Cahen. ${ }^{41}$ & $\begin{array}{l}\text { M. } \\
42\end{array}$ & $\begin{array}{l}\text { Suprapubic ex- } \\
\text { ploratory. }\end{array}$ & $\begin{array}{l}\text { Autopsy, adenomata, } \\
\text { small disseminated } \\
\text { tumors. }\end{array}$ & $\begin{array}{l}\text { Death on } 2 \mathrm{~d} \text { day; ure- } \\
\text { mia. }\end{array}$ \\
\hline Lavaux. $^{42}$ & $\begin{array}{l}\text { M. } \\
36\end{array}$ & $\begin{array}{l}\text { Perin e al re- } \\
\text { moval. }\end{array}$ & $\begin{array}{l}\text { Adenoma seated at } \\
\text { vesical neck. }\end{array}$ & R.; well 6 years later. \\
\hline Audry. ${ }^{43}$ & $\begin{array}{l}\text { M. } \\
5^{6}\end{array}$ & $\begin{array}{l}\text { Suprapubic ex- } \\
\text { ploratory. }\end{array}$ & $\begin{array}{l}\text { Short pedicle, soft } \\
\text { masses filling the } \\
\text { whole bladder. }\end{array}$ & R.; Died 8 mos. later. \\
\hline Wittzack. ${ }^{44}$ & $\begin{array}{c}\text { M. } \\
58\end{array}$ & $\begin{array}{l}\text { Langenbuch's } \\
\text { operation. }\end{array}$ & $\begin{array}{l}\text { Near right ureteral ori- } \\
\text { fice; tumor size of a } \\
\text { cherry, sessile; ade- } \\
\text { noma. }\end{array}$ & \\
\hline Rochet and Martel. ${ }^{45}$ & $\begin{array}{l}\text { M. } \\
48\end{array}$ & $\begin{array}{l}\text { Suprapubic cu- } \\
\text { rettage. }\end{array}$ & $\begin{array}{l}\text { Small tumors scat- } \\
\text { tered over bladder. }\end{array}$ & $\begin{array}{l}\text { Died } 36 \text { hours later; } \\
\text { pyelonephritis. }\end{array}$ \\
\hline Malherbe. ${ }^{46}$ & $\begin{array}{l}\text { M. } \\
64\end{array}$ & Suprapubic. & $\begin{array}{l}\text { Size of a chestnut; } \\
\text { seated on anterior } \\
\text { wall of neck of blad- } \\
\text { der; adenoma. }\end{array}$ & $\begin{array}{l}\text { R.; died } 4 \text { years later } \\
\text { of cancer of rectum } \\
\text { without local vesical } \\
\text { recurrence. }\end{array}$ \\
\hline Motz. ${ }^{47}$ & M. & Suprapubic. & $\begin{array}{l}\text { Form and size of little } \\
\text { finger; adenoma. }\end{array}$ & $\begin{array}{l}\text { R.; well at end of } 2 \\
\text { years. }\end{array}$ \\
\hline Arcoleo. ${ }^{48}$ & $\begin{array}{l}\text { M. } \\
79\end{array}$ & Perineal. & $\begin{array}{l}\text { Size of pear; ade- } \\
\text { noma. }\end{array}$ & R. \\
\hline Albarran. & $\begin{array}{l}\mathrm{F} . \\
3^{2}\end{array}$ & Suprapubic. & $\begin{array}{l}\text { Small tumor close to } \\
\text { orifice of the ureter; } \\
\text { adenoma. }\end{array}$ & $\begin{array}{l}\text { R.; well at end of } 4 \\
\text { mos. }\end{array}$ \\
\hline Nicolich (unpublished) & $\begin{array}{l}M . \\
62\end{array}$ & Suprapubic. & $\begin{array}{l}\text { Size of walnut; tri- } \\
\text { gone adenosarcoma. }\end{array}$ & R.; well 5 years later. \\
\hline
\end{tabular}

There are two surgeons, Nitze ${ }^{26}$ and Kümmel, ${ }^{49}$ whose operative results differ so notably from those obtained by others that they deserve special mention. In $1906 \mathrm{Kümmel}^{49}$ reported the results of his operations in 7 cases of benign and in 30 of malignant tumors of the bladder. There was I operative death in the 7 benign cases, and the other 6 patients were alive, well, and free from recurrence at the end of three, five, nine, fifteen, eighteen, and nineteen years respectively.

There were 10 operative deaths in the series of 30 patients having malignant tumors; 5 of the 20 survivors are reported to have been alive, well, and free from recurrence at the end of six and a half, eight, fifteen, 
sixteen, and sixteen years. The operations were suprapubic, and the tumors were removed by excision.

The patients operated upon by $\mathrm{Nitze}^{26}$ with his operating cystoscope up to IgOI were stated by him in a letter to Pollak (which is referred to by $\mathrm{Rafin}^{9}$ in his resume in 1905) to be 84 . Four of the tumors were found to be carcinomata, and the cystoscopic operations were not continued. The neoplasms in the remaining 80 cases were benign. There were no operative deaths. Recurrence took place in but 7 cases. In 4 of them the recurrence was in situ; in 3 others the growths appeared at points of the bladder other than that occupied by the original one. The number of recurrences other than those just mentioned is not given in the article of Rafin, but is implied in what he says, namely, that recurrence did not take place in the remainder of the cases; that is to say, there was no recurrence in 9I per cent. of them.

Apart from the unusually fortunate results obtained by these two surgeons, the sum total of surgical experience is far from encouraging. In so far as the results in the cases of benign tumors are concerned, the writer knows of no such unfortunate record in the same class of neoplasms elsewhere in the body.

The following facts partially explain this very marked failure:

I. A considerable number of the tumors reported to have been benign were probably not so.

2. The striking tendency which has been shown by tumors originally benign to recur in malignant form.

3. The recurrence of benign tumors in its original form, but elsewhere in the bladder.

Bad as the surgical results have been, it should be remembered that they are, at any rate, much better than those obtained by any other manner of treatment, inasmuch as the disease, in whatever form it appears, is always fatal when not submitted to radical operation, causing death in the cases of benign tumors by repeated hemorrhages or sepsis, and, in those of malignant ones, in the same manner as that in which they bring it about elsewhere in the body.

So far as the statistics indicate, we may say that radical operations save life in at least 20 per cent. of patients with benign growths, and in 4 to 6 per cent. of those with malignant neoplasms.

Can the surgical results be improved?

There would seem to be but one way in which this may be done, namely, by the earlier application of more radical methods than it has been customary to employ.

In 1905 the writer ${ }^{25}$ proposed the following plan, with a view to securing better results from surgical intervention in these cases: 
I. That the only operation that offers reasonable hope of success in cases of carcinoma of the bladder is total extirpation of the organ, That this operation should be performed in all instances of this disease in which it is proper to do any radical operation at all; that is to say, when there is reasonable ground for believing that the pathological condition has not as yet exceeded the limits of the bladder itself.

2. In view of the very large number of cases in which, after the removal of tumors believed to be benign, recurrence takes place in the form of malignant neoplasms, that total extirpation of the bladder should be done in these cases also whenever recurrence does take place, or, at least, if there is more than one recurrence, for this fact per se, even when the recurrent tumor does not possess the absolute character of a malignant growth, is strong presumptive evidence of the appearance of, or change to, a malignant tumor in the near future, and it is safe to assume that further recurrence, and in malignant form, will occur.

3. That in any case in which total extirpation of the bladder is to be done, it should be performed as a secondary procedure to a preliminary operation having for its object the diverting of the urinary secretion from its usual channels, and that this preliminary operation should be a simultaneously performed bilateral nephrostomy, tying the ureters high up near the renal pelvis and establishing permanent lumbar urinary fistulæ, in preference to any form of ureteral implantation.

4. That in the inoperable cases of vesical tumor in which there is suffering due to its presence, the diverting of the urine in the manner proposed should be done as a measure of relief, much in the same way that lumbar colostomy is done in some cases of rectal cancer.

Ureteral implantation in connection with total extirpation of the bladder, besides the dangers of renal infection wheh it invites, is also objectionable because of its prolonging the operation for removal of the bladder, and thereby increasing shock. Renal infection is invited by ureteral implantation because the orifices of the implanted canals or some point between them and the renal pelvis are very prone to contraction. This results in preventing free drainage of the kidney, and that in turn, as we know, is a potent factor in the occurrence of infection of the kidney.

Maydl's operation is an exception to this statement because of the preservation in its performance of the part of the bladder wall immediately about the ureteral orifices; but as this should not be preserved in cases of malignant disease, and as it is impossible to do so because of the situation of the neoplasm in a large number of all cases, we cannot avail ourselves of the advantages offered by it, except in the instances 
in which the tumor is situated upon the summit or anterior wall, and these are not many.

The following data have been collected by the writer for the purpose of comparing, so far as possible, the dangers connected with nephrostomy and ureteral implantation. The data do not show this at all conclusively, but they are at least suggestive and interesting.

\section{Cases of Vesical Tumor.-Treatment of the Ureters when} Divided in Operating for the Removal of Tumor.

I. Total Extirpation of the Bladder- 38 Cases.

\begin{tabular}{|c|c|c|c|c|c|}
\hline Treatment of ureters. & Op. R. & Op. D. & Causes of death. & $\begin{array}{l}\text { Due to ureteral } \\
\text { implantation. }\end{array}$ & Later D. and causes of. \\
\hline Abandoned in wound & 2 & 2 & $\begin{array}{l}\text { Pneumonia } \\
\text { Shock, }\end{array}$ & & \\
\hline Sutured into intestine & 5 & IO & $\begin{array}{l}\text { Renal infection, } \\
\text { Shock, } \\
\text { No data, }\end{array}$ & 4 & $\begin{array}{l}\text { I at } 3 \frac{1}{2} \text { mos., renal } \\
\text { infection; I at } 14 \\
\text { mos., renal infec- } \\
\text { tion. }\end{array}$ \\
\hline Sutured to skin. & 3 & I & Renal infection, & $\mathbf{I}$ & $\begin{array}{l}\text { I at I3 mos., renal } \\
\text { infection; I at } 6 \\
\text { mos., recurrence. }\end{array}$ \\
\hline Sutured into vagina. & 3 & 2 & $\begin{array}{l}\text { Shock, } \\
\text { Pneumonia, }\end{array}$ & & \\
\hline Sutured to urethra. & $\begin{array}{r}\mathrm{I} \\
- \\
\mathrm{I}\end{array}$ & $\begin{array}{r}4 \\
- \\
19\end{array}$ & $\begin{array}{l}\text { Renal infection, } \\
\text { Shock, }\end{array}$ & $\begin{array}{r}2 \\
- \\
7\end{array}$ & \\
\hline
\end{tabular}

Without data, 5. Total number, 38 .

Deaths due to ureteral implantation, Io.

\section{Operators Reporting the Above Cases.}

Bardenheuer, 2; Pawlik, I; Albarran, I; Modlinski, 3; Küster, I ; Wassilief, I; McCosh, 2; Giordano, 2; Chalot, I; Kossinski, I ; Kümmel, I; Viljaminow, I; Bergenheim, I; Mann, I; Verhoogen, I; Vaughan, I; Woolsey, I; Schede, I; Turetta, I; Hogge, I; Krause, I; Kayser, I; Lund, I; Mayo Robson, I; Gopel, I; Harris, I; Tuffier, I; Lindner, I; Zeller, I; Garré, 2; Wendel, I. 
II. Partial Resections-23 Cases.

\begin{tabular}{|c|c|c|c|c|c|}
\hline Treatment of ureters. & & Op. R. & Op. D. & Causes of death. & $\begin{array}{l}\text { Deaths due to ureteral } \\
\text { implantation. }\end{array}$ \\
\hline $\begin{array}{l}\text { Implanted into bladder } \\
\text { Abandoned in the wound }\end{array}$ & . . & $\begin{array}{c}0 \\
- \\
12\end{array}$ & $\begin{array}{l}2 \\
- \\
\text { I I }\end{array}$ & $\begin{array}{l}\text { Shock, } \\
\text { Renal infection, } \\
\text { Infiltration of ur. } \\
\text { Peritonitis, } \\
\text { Peritonitis, } \\
\text { Cellulitis, }\end{array}$ & $\frac{\text { I }}{5}$ \\
\hline
\end{tabular}

Total, 23. Deaths, II, or 47.8 per cent.

Deaths due to ureteral implantation, 5, or 45.4 per cent.

The surgeons reporting these cases are:

Israel .

Czerny

Albarran .

Bardenheuer .

Schuchard

Helferich

Giordano

Schilling

Verhoogen

Rafin

Rehn

Küster

Mikulicz

Berg

Vincini

Implantation into bladder, 21. Abandoned in wound, 2.

III. Vesical Tumor.-Operations other than Partial Resection or Total Extirpation.

Cases, II. Operative deaths, none. Later, 2.

Implantation into bladder, II. Later deaths, 2. Due to ureteral implantation in one, perhaps; not so in the other.

In 7 other cases there was an opportunity to test the patency of the ureter at various times subsequent to operation. In one of these the ureter was apparently obliterated and the kidney was believed to have undergone atrophy. The patient was, however, in good health. 
The operators in these cases were: Routier, Pernice, Krug (case observed eight years after operation), Kalabin, Rafin, Wertheim, Frantz (5 cases). Total, I I.

Summary of the Treatment of the Ureter in all Cases in Which it Has Been Divided in Connection with the Operations of Suprapubic Excision of the Tumor, Partial Resection of the Bladder Including the Growth, and Total Extirpation of the Bladder in Cases of Vesical Tumor.

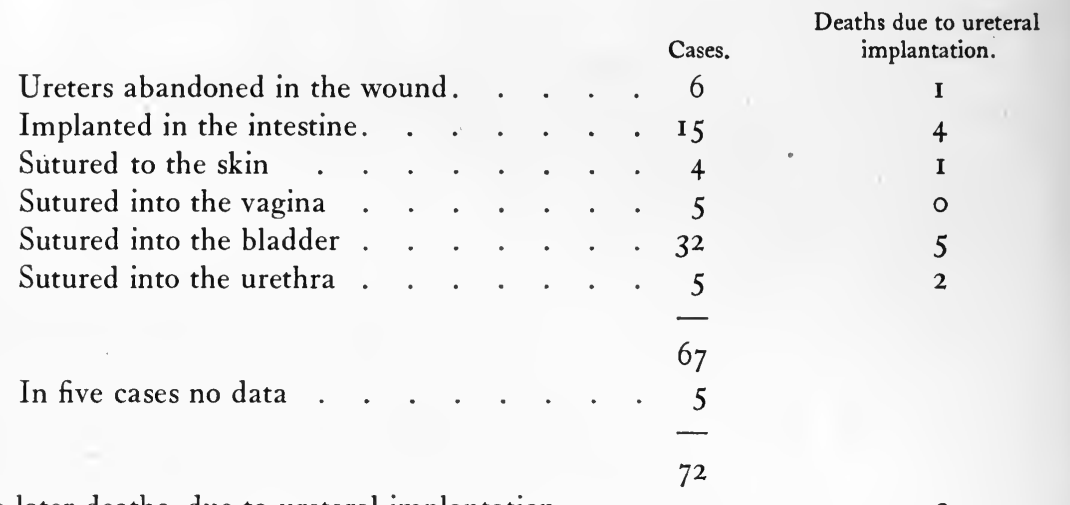

Three later deaths, due to ureteral implantation . . . . . . . I6

Total number of cases, 72. Total operative deaths, 30. Later deaths, 8 .

Total number of deaths due to ureteral implantation, I6, or 42.1 per cent.

In order to make the comparison between the results of nephrotomy and ureteral implantation the writer has collected the following results of nephrotomy:

Results of Nephrotomy.

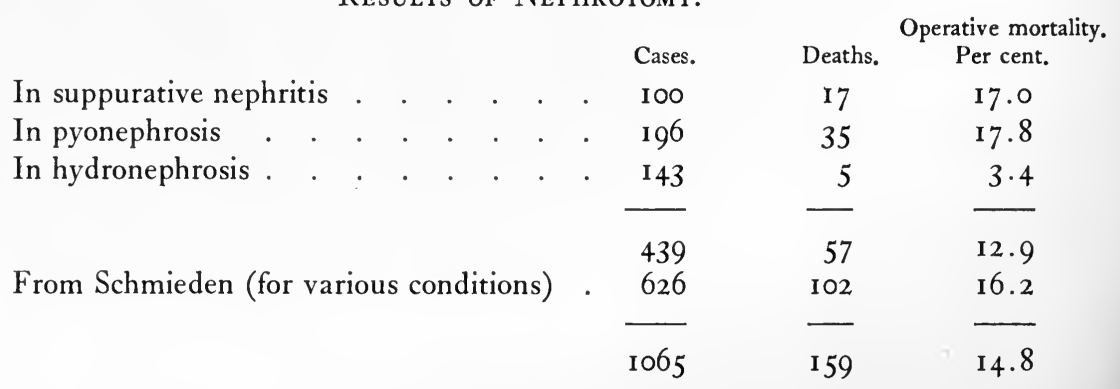

From the above tables of ureteral implantation and nephrotomy, respectively, we can obtain a fairly just idea of the operative mortality attending these procedures in the conditions in connection with which each of them was done. In making the comparison, it must be noticed that we have separated as rigidly as possible the deaths which were 
attributable to ureteral implantation from those which were due to other causes in the cases in which the ureteral implantation was done as one step of the whole surgical procedure.

The two objections to the plan proposed by the author which naturally suggest themselves are:

I. That the shock attending the simultaneous performance of bilateral nephrotomy, as a preliminary operation, will be quite as great as that involved in performing the ureteral implantation simultaneously with the operation of extirpation of the bladder.

2. That a permanent renal fistula in both loins will be a condition so intolerable to the patient that it might be just as well to allow him to die of the effects of the tumor as to secure a longer term of life for him at the cost of so distressing a condition.

All the evidence in our possession indicates that the first objection is groundless. So far as the writer is aware, three of his own cases are among the very few in which both kidneys have been incised at one sitting. In addition to them, there is one case reported by Legueu, ${ }^{50}$ in which he did nephrotomy and decapsulation upon both kidneys at one sitting for the relief of nephritis with hematuria, in a patient of eleven years of age, with a successful result. In none of these four cases was shock serious at the time of operation. One of the author's own patients, who had had obstructive anuria for three days and well-marked uræmia for twenty-four hours, died three days after double nephrolithotomy, done at one sitting (see chapter on Renal Calculus, Illustrative Cases), but not as the result of shock. In the two other cases, the patients made excellent recoveries, and shock was not at all conspicuous during or after the operations.

Further than this, surgical experience shows that shock is not a serious element in unilateral nephrotomy nor in bilateral decapsulation or nephropexy.

The importance of the second objection depends wholly upon whether or not the surgeon is capable of arranging renal drainage in the loin in such a way as to make the patient not only comfortable but to allow him to lead an active life and to have no one conscious of his infirmity.

To do this means to have it so arranged that the patient can be kept dry, and this end is secured by the contrivance devised by the writer in I $906,{ }^{51}$ and described in the chapter on Technique of Operations on the Kidney.

One further objection might be raised, which is, that kidney infection is just as likely to result after nephrostomy and permanent renal drainage as after ureteral implantation. The reply to this is that such is not the case. We may summarize the universal experience of surgeons with 
regard to this matter in the words of Albarran. ${ }^{3}$ Speaking of renal infection in connection with the existence of permanent renal drainage in the loin by nephrostomy, he says: "In these cases one is struck by the absolute immunity which the incised and exposed renal tissue enjoys. The open kidney remains indifferent to contacts and pressures. Foreign bodies which are introduced into or remain in it-drains, gauze, etc.-do it no harm.

"The interior of the organ may be curetted, injections of medicated fluids may be made into it, all without injury. The urine continues to be abundantly secreted and remains practically normal in character. All of which things show that nephrotomized kidneys are but little liable to infection, and under such circumstances but very slight changes are produced in their parenchyma."

Bottomley, of Boston, ${ }^{52}$ applied the plan proposed by Watson for tumors of the bladder in a case of exstrophy of the bladder, by adopting the step of diverting the urinary secretion from the field of the bladder operation as a preliminary to the performance of the latter, but modified the procedure by substituting ureteral implantation in the loin after the manner employed by Harrison, ${ }^{53}$ who, however, sacrificed one kidney and transplanted the end of the ureter of the other kidney into the skin of the loin.

Harrison did not remove the bladder, and the sacrifice of one of the kidneys has always seemed to the writer to have been needless. Bottomley's operation differed from Harrison's in that he transplanted both ureters to the surface of the loin and did not sacrifice the kidney.

In May of the same year (I906) Rovsing ${ }^{54}$ operated upon a patient by totally removing the bladder and implanted the ends of both of the divided ureters into the surface of the loin at the same time as that at which the bladder was removed, but not as a preliminary step. The operation was done in a case of a malignant tumor of the bladder. Subsequently, he repeated the operation in two other cases in which the bladder was the seat of similar conditions. The feature of Rovsing's operations was the removal of the bladder without opening it by a suprapubic cystotomy incision, as is usually done prior to shelling it out from the peritoneal investment. One of Rovsing's patients died shortly after the operation, the cause of death being atrophy of both kidneys and cardiac disease. The other two were alive and well at the time of the reports of the cases.

With regard to the technique of the removal of the bladder, the procedure employed by Rovsing is similar to that of Clado in that the bladder is not opened. Watson ${ }^{51}$ (1905) proposed to open the peritoneal cavity and to divide the peritoneal investment of the bladder after so 
doing, and then to peel the peritoneum from the bladder on either side this peritoneal incision, while Rovsing does not open the peritoneum at all, except it be done accidentally. Watson's idea was to facilitate the performance of the bladder operation and to allow of the more rapid removal of the organ, which he believed could be better accomplished if the peritoneal cavity were opened and a freer access was thus gained to the operative field by exposing the bladder intra-abdominally, without, however, opening the bladder itself, and, consequently, not risking infection of the peritoneum in any important degree.

For the reasons that have been presented above, and from the evidence that has been given, it would seem reasonable to look for better results from operative intervention if instituted according to the proposed plan of the writer.

Up to the present time there has been, so far as the author is informed, but one case in which his plan has been in some measure adopted. This was the case of Dr. Bottomley, already referred to.

Conclusions with Regard to Surgical Treatment.-It seems to us that the lesson taught by the surgical experience in the treatment of tumors of the bladder thus far is a clear one, and that it is this. In cases of small benign papillomata with well-marked pedicles, and in the rare cases of adenoma, we may look for about 60 per cent. of recovery to follow the simple removal of the tumor, if a margin of sound mucous membrane about the base of the pedicle is taken away with the growth. That about 20 per cent. of cures only can be expected in the cases of benign papilloma which are broadly sessile or which are multiple.

In these cases, the first operation for their removal may be simple excision. If recurrence takes place, however, it is highly probable that the recurrent tumor is malignant. This probability is rendered greater if recurrence takes place in the form of multiple growths, and becomes almost a certainty if it is found at the time of operation that the base of the tumor is not readily movable upon the underlying muscularis, and if, upon previous examination by the rectum, a welldefined sense of resistance is felt by the finger over the area occupied by the tumor. Positive knowledge of the nature of the tumor can only be gained by a careful microscopic examination of the specimen in its entirety subsequent to its removal; consequently when operating we must rely chiefly upon the evidences of malignancy that have just been mentioned to determine the nature of the tumors and to decide upon the manner in which we are to treat them.

We believe it to be of but little use to remove by ordinary excision tumors which present such evidences of malignancy as have been described above and in an earlier part of the text. 
There are two courses open to us with respect to the manner of treating such tumors as are evidently of malignant nature, or which are open to suspicion of being so. (We are not now speaking of the neoplasms which it is obviously improper to attempt to remove radically because of their having become propagated beyond the limits of the bladder itself.) We may either leave the disease to pursue its natural evolution, or we may attempt to obtain a cure by radical surgical procedure. The former course is one in favor of which there is something to be said. If such a patient is not suffering; if his vitality is not being seriously impaired by repeated hemorrhages; if the bladder has not as yet become infected, and if he, nevertheless, in all probability has a malignant growth in the bladder, there is a fair chance that his general condition may remain good for a number of years, and the local condition may not become a suffering one for a good while.

The best prospect that we can offer to a patient by any plan of radical operation in such a case, even when the tumor is in its earlier stages of development, is 4 or 5 per cent. of chance of cure or prolongation of life. This small chance is to be set over against the certainty of death sooner or later if the disease is allowed to proceed without interference.

It seems to us from a surgical standpoint that we should advise such patients to undergo radical operation. We should, at any rate, present the facts to the patient as they are stated above, and let him decide what shall be done.

If the decision is in favor of surgical intervention, we believe that it should take the form of the operation of total extirpation according to the plan proposed by the writer.

In cases in which there is reason to believe that metastasis has taken place, or that there has been a propagation of the pathological process beyond the limits of the bladder, all radical procedures are useless and should not be undertaken. In such cases we advise that nothing be done until such a time as suffering from the local conditions in the bladder begins; we then counsel that the urinary secretion be wholly diverted from the bladder by the performance of bilateral nephrostomy, tying off both ureters near the renal pelvis, and the establishment of permanent renal fistula in both loins, using this procedure as suggested for the purpose by the writer, much in the same manner as colostomy is employed as a palliative measure in cases of inoperable rectal cancer.

It is hopeless to attempt radical cure by any known surgical means in cases of sarcoma and myxoma.

Cure will follow radical operation in a small percentage of the cases of myoma and cholesteatoma. 
The fact that, practically speaking, all tumors of the bladder, irrespective of their nature, cause the death of the patient sooner or later, and that death will, in most instances, be preceded by a period of suffering, should be remembered when advising with regard to the treatment to be employed.

If radical measures are not to be used, the palliative steps advised in the earlier part of the text will be found to be valuable under the conditions in which their employment is indicated.

\section{Illustrative Cases.}

CASE I (Beneke ${ }^{55}$ ).-Osteochondrosarcoma of the Bladder.-The patient was a man, aged seventy-two years. The symptoms were frequent and painful micturition and hematuria for the past seven years.

Operation.-Suprapubic cystotomy. The tumor was found to be large and filled the whole bladder. It arose by a distinct pedicle, which was situated near the orifice of the left ureter. The pedicle was ligated and the tumor was removed.

Examination of the specimen showed the following features: The tumor was of bony hardness except upon its surface, which was composed of soft villi, and presented a cauliflower appearance.

On section, it was seen to be composed of a central mass $3 \mathrm{~cm}$. in width. It was traversed by bloodvessels in a way that suggested very closely the arrangement of the Haversian canals, for they passed through a series of distinctly marked channels of that character.

From this central bony stem branches of fibrous and cartilaginous tissue radiated to the periphery, finally being lost in the nodular surface of the tumor. Deposits of lime salts in narrow lines were seen in various parts of the bony part of the growth.

Microscopically, the soft parts of the neoplasm were seen to be made up of fibrous tissue richly infiltrated with leukocytes. From the central part of the tumor, and following the course of the bloodvessels, was a thin layer of tissue resembling cartilage. The cellular elements of the tumor were spindle cells, closely packed together, and had very large nuclei. Numerous mitoses were present. In some even of the superficial parts of the tumor, bony or cartilaginous tissue existed. True hyaline cartilage was present, arranged in long lines, or else as shot-like bodies grouped closely together.

Eight weeks later the tumor recurred. Death followed soon after.

The autopsy showed that the inner surface of the bladder, which, at the time of the operation, had had but the single tumor, was now studded over with numerous small growths. These all had the same character as that of the original one. 
CASE II.-Chondrosarcoma.-Shattock ${ }^{56}$ reports the result of the examination of a tumor of the bladder from a patient who was operated upon by Thompson. The microscopic findings were as follows:

"The tumor was composed, in part, of well-developed cartilaginous, and, in part, of sarcomatous tissue. The latter showed both spindle and round cells. The typical evolution of the cartilage capsules could be traced in the specimen."

CAse III (Martini ${ }^{16}$ ).-Dermoid Cyst.- "A newborn child dying ten days after birth. The bladder did not communicate with the urethra, but opened through the abdominal wall at the umbilicus and also by another opening into the rectum. There was but one ureter, which opened upon the anterior wall of the bladder, the inner surface of which was covered by normal mucous membrane. The posterior wall, on the contrary, consisted of skin from which hair grew, projecting into the interior of the bladder. This specimen furnished an absolute demonstration of the inclusion of a fœtal structure belonging to another part of the organism.

"The chief point of interest in this case noted by Albarran is the failure of complete closure of the fœetal cloaca, in association with a probable inclusion of a portion of the external envelope."

CAse IV $\left(\right.$ Livio $\left.^{17}\right)$. - Rhabdomyoma.-A child, aged thirteen years, who died of the effects of repeated hemorrhages from the bladder.

Autopsy showed on the lower part of the posterior wall of the bladder several tumors resembling in form very delicate papillomata, with long fine pedicles of a dark-red color.

Microscopically, they were seen to consist of fibers of striated muscular tissue of embryonic type. The fibers were continuous with the muscular layer of the bladder.

CASE V (Watson ${ }^{57}$ ) illustrates the inability to determine by microscopic examination of the fragments of the tumor passed in the urine what the true nature of the growth is, and also shows the extraordinary extension of the disease that may take place in some cases of carcinoma by direct invasion of the adjacent structures outside the bladder and by metastasis to other parts of the body. Also, the slow evolution and painless progress of disease that sometimes occurs.

A man, aged sixty years. The first symptom was hematuria six years before he sought medical advice. A little blood had been present almost constantly in the urine ever since its first appearance.

Two months before seeking advice, for the first time the urine began to contain larger amounts of blood; between that and the time of his first consulting the surgeon there was progressively frequent and profuse hematuria, and finally, retention of urine was caused by a hemorrhage so abundant as to fill the bladder with clots. 
The diagnosis was made by the discovery in the urine of typical villi cast off from the surface of the tumor. Microscopically, these showed nothing suggestive of malignant nature of the tumor.

The posterior wall of the bladder felt by the finger in the rectum was thickened. Bimanual palpation detected nothing resembling a tumor.

Operation, January 20, I 884 . The bladder was explored digitally through an ordinary external perineal urethrotomy incision. Its interior was found to be covered with multiple papillomatous tumors. There was apparently but little infiltration of the bladder wall, as judged by the sense of touch by the finger.

The whole surface of the bladder was curetted, and large masses of the tumors thus detached were withdrawn or washed out. There was but little bleeding in connection with the operation.

The patient was relieved of pain, and the hemorrhages ceased for the most part. A month later, however, marked cachexia was noticeable, and, after gradual loss of strength, the patient died in a little less than four months.

Autopsy.-Two-thirds of the bladder was occupied by infiltrating carcinoma, which presented a villous surface. On the right side the disease had extended beyond the bladder, involved all the tissues of the pelvis as far as and through the acetabulum, which was destroyed by it to such a degree that the head of the right femur had forced its way through the opening thus made in the pelvic bone and lay within the pelvis and a cavity formed by suppuration of the cancerous disease, which made a continuous space between the bladder and the opening in the right acetabulum.

The right ureter was much dilated, and the right kidney was hydronephrotic. There were metastatic nodules of cancer in the lungs and in the liver.

Cases in which death resulted from hemorrhage from benign papilloma of the bladder, one in which the same thing happened from an angioma, and another from a carcinoma of the bladder.

CASE VI (Riesman).-A man, aged forty-six years, for fourteen months had suffered pain in lower part of back and abdomen. No pain on urination. Had more or less frequent hematuria during the same period. Was admitted to hospital on account of an attack of hematuria, and died in consequence of the intravesical bleeding on the following day. Autopsy showed prostatic hypertrophy. A papilloma with thick pedicle was situated close to the orifice of the left ureter. Hemorrhage had proceeded from this tumor and had caused the patient's death.

CASE VII.-Riesman quotes another case of a patient who was in the care of Dr. Pepper in $187 \mathrm{I}$, in which death was also owing to hemorrhage 
from a papilloma of the bladder of the size of a walnut. In this instance there was continuous bleeding for ten days preceding death.

CASE VIII.-Another similar occurrence is reported in $185^{\circ}$ by Wardell, in the case of a patient with a malignant tumor of the bladder, and a fourth, which Langhaus reported in I879, was the case of a patient who died of intravesical bleeding from an angioma of the bladder.

CASE IX (Ultzmann ${ }^{24}$ ).-Cases of Spontaneous Expulsion of Tumors of the Bladder.-Three cases of spontaneous expulsion of papilloma of the bladder. In the first of these the patient was a man, aged fortynine years, who had had persistent hematuria for many months. After the expulsion of the tumor, which occurred in four separate pieces, the the urine was perfectly normal and free from blood during the following year, at the end of which time the patient was lost sight of.

CASE X.-A man, aged sixty years. Hematuria, painful and frequent micturition for nearly one year. Five separate masses of a papilloma were expelled by the patient after an instrumental examination of the bladder. Cessation of the previous symptoms and no return of them. This patient remained perfectly well and free from all symptoms during the seven years in which he was under observation.

CAse XI.-A man who had suffered for a long while from persistent hematuria was treated by Ultzmann with daily injections into the bladder of a weak nitrate of silver solution. On the fourteenth day of this treatment he passed several bits of a villous tumor of the bladder spontaneously. This patient, who was seventy years of age, was also relieved entirely of his symptoms, and at the end of two years reported that he had had no return of them and had been perfectly well in the interval.

Desnos reports four cases in which he personally had the opportunity to note the conditions following the spontaneous expulsion of tumors from the bladder.

CASE XII.-The cystoscope showed a tumor of the size of a walnut situated on the left side of the bas fond of the bladder. It had a welldefined pedicle. Soon after this examination the patient passed a large fragment of the tumor.

Examination showed the growth to have been diminished one-half in size. Soon afterward a second mass was passed. Cystoscopic examinations made thereafter showed that the whole tumor had disappeared. There was entire freedom from symptoms for a period of nine months following the expulsion of the last mass. Then there was a return of hematuria and the cystoscope showed that there was a recurrence of the tumor in situ, but that it had a shorter pedicle and the surface was not villous, as it had been on the first occasion. 
CASE XIII.-A tumor of moderate size was seen on the middle of the posterior wall of the bladder. It was sessile. A large fragment was spontaneously passed soon after, and in the course of the next few days several smaller ones. Hematuria ceased after a short time, and cystoscopic examination showed that the tumor had almost wholly disappeared. There was freedom from symptoms for one year, then the patient had a recurrence, which Desnos was able to observe with the cystoscope, and watched its progress for some time afterward. The other two cases were of similar character.

CASE XIV.-(Watson.) A man, aged thirty-six years. The patient had noted the first symptom (hematuria) three years before seeking advice. The attacks had become more frequent as time passed. He had not had dysuria. Characteristic bits of a papilloma were found on microscopic examiadtion of the urine. The patient was submitted to a perineal operation, by which a tumor with a well-marked and firm pedicle was removed by a snare, and the base curetted. The hematuria ceased for one year, then returned. A second time the perineal operation was done, and three other papillomatous growths were removed by curette and the bases thoroughly scraped. None of these three tumors occupied the same part of the bladder as the original one; the former was on the right side of the trigone; those found at the second operation were near the fundus Entire freedom from all symptoms was secured by this operation for eighteen months. Hematuria then returned. A third operation was undertaken-suprapubic-and two more tumors were found, this time on the posterior and anterior wall, respectively. As before, these tumors also bore all the marks of being benign papillomata. The operation was entirely successful in its immediate results and for the next fourteen months, at the end of which the patient again had several attacks of profuse hematuria. During one of them he passed, while in the surgeon's office a papillomatous mass as large as the last joint of the thumb. After this he was lost sight of. Microscopic examination showed these tumors in every instance to be benign papillomata.

Case XV (Marchand, I878).-Cholesteatoma (Cancroide, Leukoplakia $V$ esica).-A boy, aged fourteen years. Cystitis for seven years. The following appearances were found postmortem:

The mucous membrane of the bladder, urethra, ureters, and renal pelves, also portions of the lower surface of the diaphragm, were occupied by a process described as true epidermization.

The epithelial structure of the mucous membrane was found, on microscopic examination, to accurately resemble that of the epidermis, having the same forms, varieties, and successive arrangement of cells- 
including flat cells with keratohyalin-and giving off horny desquamating flakes.

CASE XVI (Bruchmar ${ }^{13}$ ).-A man, aged thirty-six years, who had had suprapubic cystotomy performed twice for the relief of severe bladder symptoms, finally died some time after the second operation. Autopsy showed that the whole of the mucous membrane of the bladder was in similar condition to that described in the preceding case, except that its surface in places was elevated, owing to the presence of projecting papillary growths. The interior of the bladder was filled with a grayishwhite, mealy mass, consisting of desquamated debris of the inner lining of the bladder.

CAse XVII $\left(\right.$ Cabot $\left.^{14}\right)$. - A man, aged forty years. Cystitis of five years' duration. Suprapubic cystotomy. The posterior wall of the bladder was of a yellowish-white color, and felt stiff to the finger. The surface of this area was rough. The rest of the bladder wall was normal in appearance.

The diseased surface could be peeled off with the fingers in a thick membrane, leaving a smooth surface beneath, which bled but slightly. The wall of the bladder after its removal felt soft and supple.

Recovery followed; but, for the following year, white, flaky masses of desquamated epithelium, which had been seen in the urine previous to the operation, continued to be present, though in much less degree, after which they disappeared, and only a slight cloudiness of the urine remained, which was due to mucus. The patient entirely regained his health.

Case XVIII (Fuller $\left.{ }^{58}\right)$.- Partial Resection of the Bladder.-A man, aged sixty-nine years. Suprapubic exposure of the bladder. The prostate and both anterior and posterior walls of the bladder adjacent to the vesical outlet were occupied hy carcinomatous growth.

The prostate was separated from the urethra by cutting across the latter at the junction of the membranous and prostatic parts through a perineal incision. The gland and the bladder as high up as the orifices of the ureters were freed from their attachments, and the latter was divided just below the ureteral mouths. The edges of a suprapubic cystotomy incision, which had also been made, were sutured to the abdominal wound. No attempt was made to close the rest of the wound of the bladder, or to attach it to the urethra below. The perineal and suprapubic incisions were left open. The former healed at the end of one month and the latter at the end of two months. The communication between the posterior end of the divided urethra and the part of the bladder wound, which had been left open when resecting that organ, was kept open by the frequent passage of sounds. A wide channel, constituting a new urethra, was formed between these two points. 
The patient lived in comparative comfort for eleven months, at the end of which he died of recurrence of the growth.

CASE XIX (Viljaminow ${ }^{59}$ ).-A woman, aged forty-nine years, presented herself with a tumor of the bladder, which was easily felt above the symphysis pubis.

Operation.-Bladder exposed by transverse suprapubic incision. A large tumor was found occupying its anterior wall. The peritoneal cavity was opened in separating the adhesions between it and the symphysis pubis.

Two-thirds of the bladder-its whole anterior wall and part of the summit-was resected. The posterior portion, with the ureteral orifices, was separated from its attachments, raised up, and united to the external wound. The latter and the edges of the bladder incision were not closed.

Convalescence was uninterrupted. At the end of six weeks only a small fistula remained in the site of the wound.

CASE XX (Tuffier ${ }^{60}$ ).-Total Extirpation of the Bladder.-Extirpation of the bladder was done as follows:

A previously made suprapubic wound was enlarged at its lower end by a transverse incision carried as far as the inguinal ring on each side.

The peritoneum was stripped from the bladder by the fingers, beginning on the anterior wall. The vesical arteries were ligated and divided during this process. The ureters, when exposed, were ligated and divided.

The lower part of the bladder was then separated from its attachments, divided transversely at its outlet, and raised upward into the wound. What remained of the peritoneum was then removed, and the bladder, thus freed, was taken out.

A catheter was tied into each ureter and the ureters were implanted in the rectum, the catheters being retained in them and led out of the anus.

The patient made a good recovery and remained well for fourteen months. He then died. Cause of death was not stated.

CASE XXI (Winiwarter, reported by Hogge ${ }^{61}$ ).- Total Extirpation of the Bladder, Prostate, Seminal Vesicles, Penis, and Testes.-Duration of the disease, two years. The patient was a man, aged forty-seven years.

The prostate and membranous urethra were exposed by a perineal incision and separated from their attachments. The wound was then tamponed and the symphysis pubis was exposed by a suprapubic incision. The penis was separated by division of the suspensory ligament, and the corpora cavernosa were divided at the insertions of the crura.

Symphyseotomy was then done. Sharp hemorrhage occurred at this point from the plexus of Santorini, but was easily controlled by tampon. 
The peritoneum was next stripped off from the front, then from the back, and then from the sides of the bladder.

The divided urethra, prostate, and $\mathrm{b}$ adder were lifted up through the wound; the ureters were then ligated and cut. The spermatic cords were removed-after being ligated and cut-together with the scrotum and testes. The corpora cavernosa were taken away by separating them from their. skin covering. Finally, the bladder was taken out.

Ureters were implanted in the rectum. Bones of the symphysis were reunited in their proper place by suture.

The patient recovered, and lived for five and a half years.

\section{BIBLIOGRAPHY.}

I. Leichtenstern. Deut. med. Woch., I898, xxiv, 709.

2. Wendel. Mitt. d. Grenzgeb. d. Med. u. Chir., Jena, 1900, vi, 15.

3. Albarran. Ann. d. Mal. d. Org. Gén.-urin., Paris, I897, xv, 785.

4. Report of the Croft Cancer Committee, Harvard Medical School, Boston, 1902.

5. Sir Henry Thompson. Tumors of the Bladder, London, I 884.

6. Billroth and Gussenbauer. Arch. f. klin. Chir., Berlin, I875, xviii, 4I I.

7. Sutter. Centralbl. f. d. Krankh. d. Harn. u. Sex. Org., Leipzig, 1902, xiii, 185.

8. Motz. Assoc. Franc. d'Urol., Proc. verb., Paris, I 898, iii, 347.

9. Rafin. Assoc. Franc. d'Urol., Proc. verb., 1905, Paris, I906, ix, I.

Io. Clado. Traité des tumeurs de la vessie, Paris, 1895 .

I I. Rochet and Martel. Gaz. hebd. d. méd. et chir., Paris, I898, 337.

I2. Pollailon et Legrand. Ann. d. Mal. d. Org. Gén.-urin., Paris, I888, vi, 604.

13. Bruchmar. Prag. med. Woch., I898, xxiii, 525.

I4. Cabot. American Journal of the Medical Sciences, I89I, ci, I35.

I 5. Hallé. Ann. d. Mal. d. Org. Gén.-urin., Paris, I896, xiv, 48 I.

I6. Martini. Arch. f. klin. Chir., Berlin, I894, xvii, 449.

17. Livio.

18. Pasteau. Assoc. franc. d'Urol., Proc. verb., Paris, I 899, iv, 565.

19. Küster. Arch. f. klin. Chir., Berlin, I89ı, xlii, 864 .

20. Conner. Transactions of the American Surgical Association, I890, viii, 21.

21. Pousson. Ann. d. Mal. d. Org. Gén.-urin., Paris, I885, iii, 528.

22. Steinmetz.

23. Fenwick. Operative and Inoperative Tumors of the Bladder, London, I90I.

24. Ultzmann. Krankheiten der Harnblase, Stuttgart, i 890.

25. Watson. Annals of Surgery, Philadelphia, 1905, xlii, 805 .

26. Nitze. Centralbl. f. d. Krankh. d. Harn. u. Sex. Org., Leipzig, ı 896, vii, 377, 470.

27. Watson. Lancet, London, I 890 , ii, 809 .

28. Pawlik. Wien. med. Woch., I89ı, xli, I8I4.

29. Hogge. Ann. d. Mal. d. Org. Gén.-urin., Paris, I902, xx, 375.

3o. Chalot. Independ. Méd., Paris, ı 896, ii,297.

31. Vasileff. Russk. Chir. Arch., St. Petersburg, I 895, i, 569.

32. Krause. Ann. d. Mal. d. Org. Gén.-urin., Paris, 1903, xxi, 770.

33. Tassi. Bull. d. R. Accad. med. di Roma, I 886, xii, 296.

34. Karström. Centralbl. f. Chir., Leipzig, I896, xxiii, I074.

35. Ramsay. Philadelphia Medical Journal, I900, vi, 86. 
36. Warholm. Hygiea, Stockholm, I893, lv, 323.

37. Verhoogen. Centralbl. f. d. Krankh. d. Harn. u. Sex. Org., Leipzig, I895, vi, I 32.

38. Ceci. Ann. d. Mal. d. Org. Gén.-urin., Paris, I903, xxi, I 44I.

39. Kraske. Centralbl. f. Chir., Leipzig, I904, xxxi, Beilage, I22.

40. Kaltenbach. Arch. f. klin. Chir., I 884.

4I. Cahen. Virchow's Archiv, 1888.

42. Lavaux. Chir. con. d. Voies urin., I892.

43. Audry. Mercredi Méd., I 893 .

44. Wittzack. Centralbl. f. d. Krankh. d. Harn. u. Sex. Org., I 894.

45. Rochet and Martel. Thesis of Bridoux, I 898 .

46. Malherbe. Cong. Franc. d'Urologie, I 897.

47. Motz. Ibid., I899.

48. Arcoleo. Soc. de Chir., I902.

49. Kummell. Deut. med. Woch., I887, xiii, 123.

50. Legueu. Assoc. Franc. d'Urol., I904, Proc. verb., Paris, I905, viii, 682.

51. Watson. Annals of Surgery, September, 1907.

52. Bottomley. Journal of the American Medical Association, July I3, I 907.

53. Harrison. Lancet, London, I897, p. I09I.

54. Rovsing. Annals of Surgery, December, 1907.

55. Beneke. Arch. f. path. Anat., Berlin, I900, clxi, 70.

56. Shattock. Transactions of the Pathological Society of London, I 886-87, xxxviii, I 83 .

57. Watson. Boston Medical and Surgical Journal, I884, cxi, 409.

58. Fuller. Journal of Cutaneous and Genito-urinary Diseases, 1898, vol. xvii.

59. Viljiaminow. Rev. de Chir., February, I 897.

6o. Tuffier. Ibid., I898, p. 277.

6r. Winiwater. Ann. des Mal. des Org. Gén.-urin., Paris, ı898, No. ı6, p. 838. 


\section{CHAPTER XVII.}

\section{TECHNIQUE OF OPERATIONS ON THE BLADDER.}

\section{SUPRAPUBIC ASPIRATION OF THE BLADDER.}

Instruments (Fig. 277). - A trocar of the caliber corresponding to No. 8 of the French scale, and about three inches long for thin persons, four or five for stout people.

Position.-The patient should lie upon the back.

Operation.-The operation is done by plunging the trocar into the bladder immediately above the upper margin of the symphysis pubis and in the median line of the body. The direction in which the trocar is entered should be at right angles with the upper margin of the symphysis. The motion made in entering the instrument should be a steady forward thrust. The trocar traverses the skin, the space between the insertions of the recti muscles, the prevesical space, and the anterior

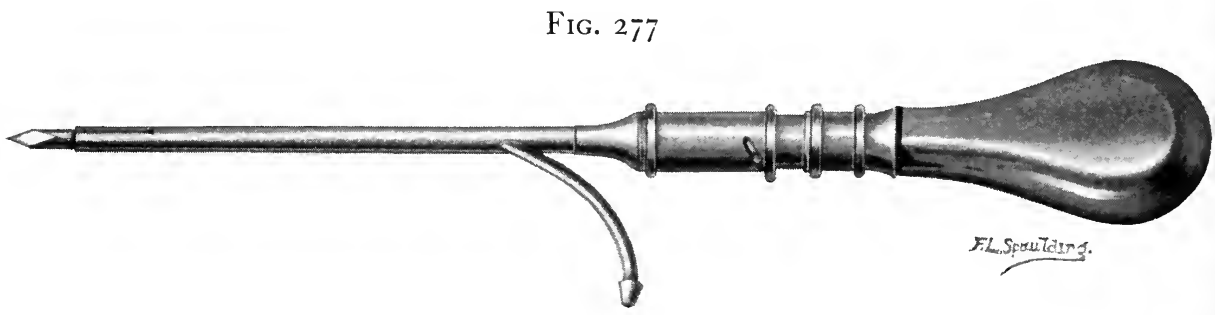

Trocar for suprapubic puncture of the bladder.

wall of the bladder below the insertion of the peritoneum upon its anterior surface. The point of the instrument should be entered well within the bladder, else it may slip out of it as the latter contracts while emptying.

In withdrawing the cannula when the bladder has been emptied, it should be done with a quick motion.

If the needle is too long and is thrust too far into the bladder, or if the right direction is not given to it, the prostate or the posterior wall of the bladder may be injured.

When the bladder is greatly overdistended, its whole contents should not be withdrawn at once, but by repeated aspirations. 


\section{SUPRAPUBIC CYSTOTOMY (EPICYSTOTOMY, SECTIO ALTA).}

Instruments.-Two scalpels; I pair of blunt-pointed scissors; 2 pairs of single-toothed forceps; I pair of dissecting forceps; 6 artery forceps;

FIG. 278

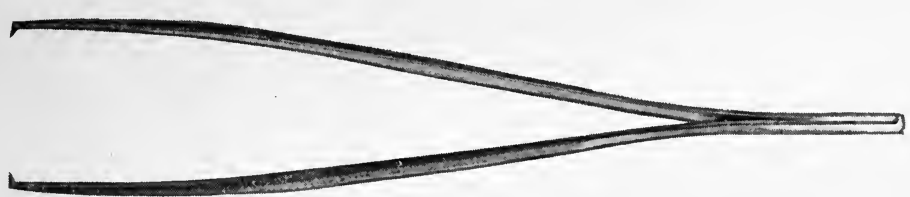

FIG. 279

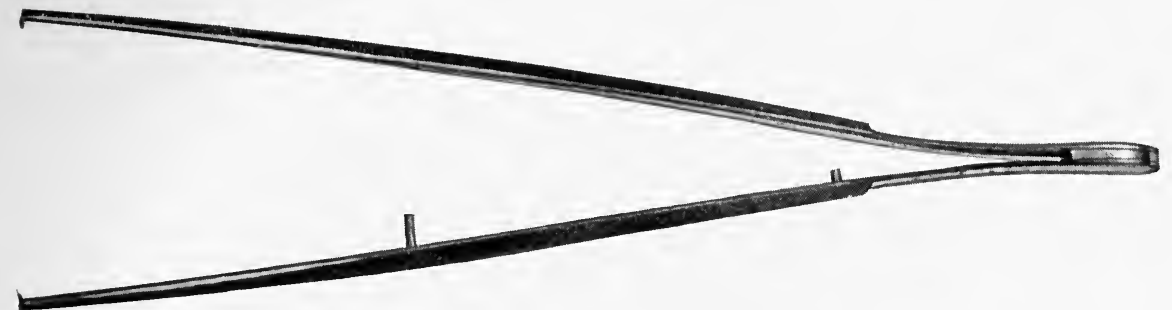

FIG. 280

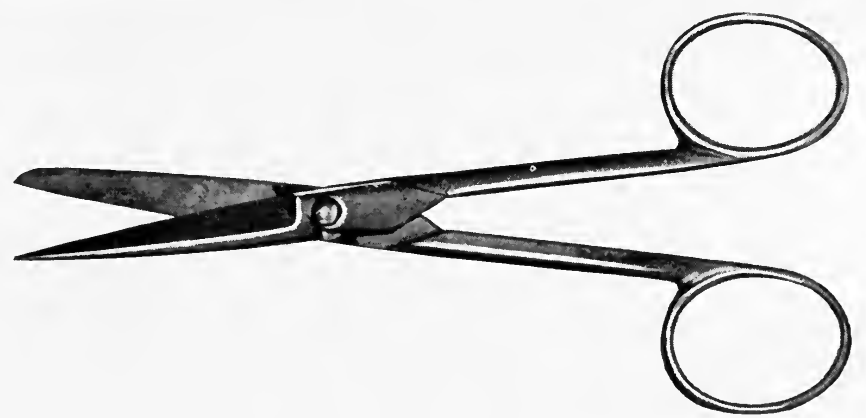

FIG. 28I

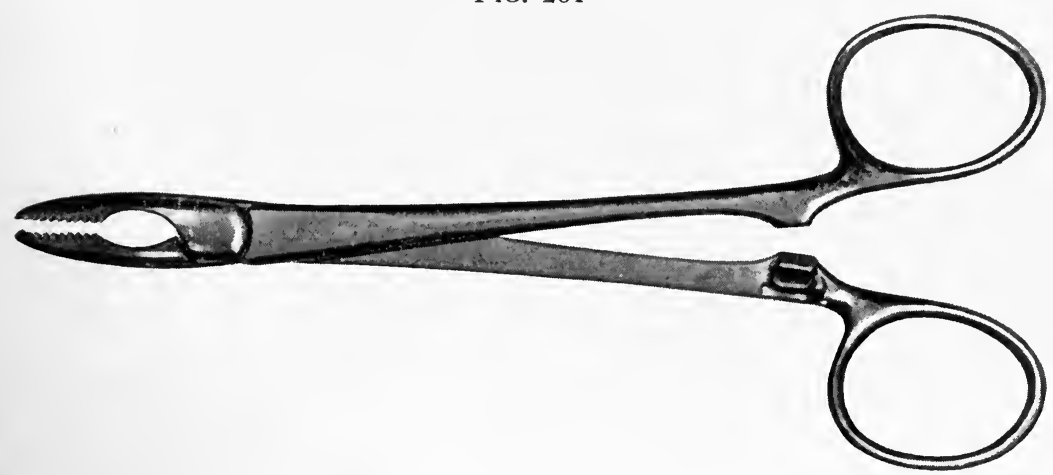

Instruments for suprapubic cystotomy.

3 retractors if it is desired to inspect the interior of the bladder, or Watson's speculum may be used in connection with a cystoscope lamp, VOL. I-35 
the latter instrument being passed through the urethra; I tenaculum; 2 full-curved Hagedorn needles; several smooth, round, full-curved needles; straight needles; catgut sutures and a few silk sutures; needleholder (Figs. 278 to 284). If Petersen's technique is employed, a rectal colpeurynter is required (Figs. 285 and 286).

FIG. 282

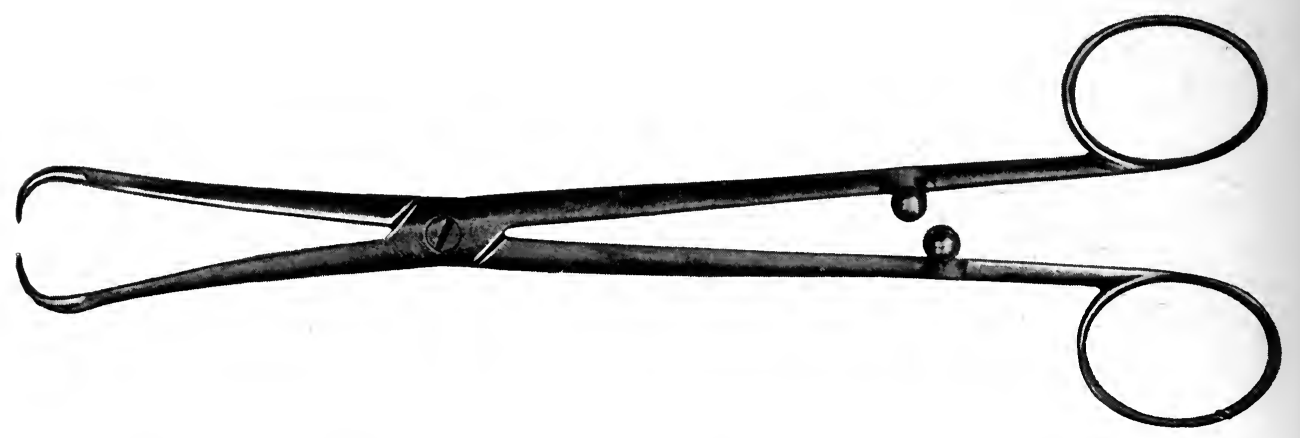

Fig. 283

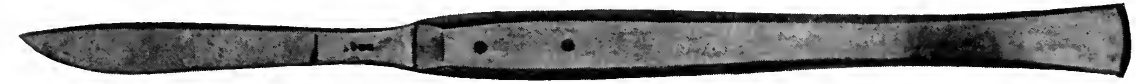

FIG. 284

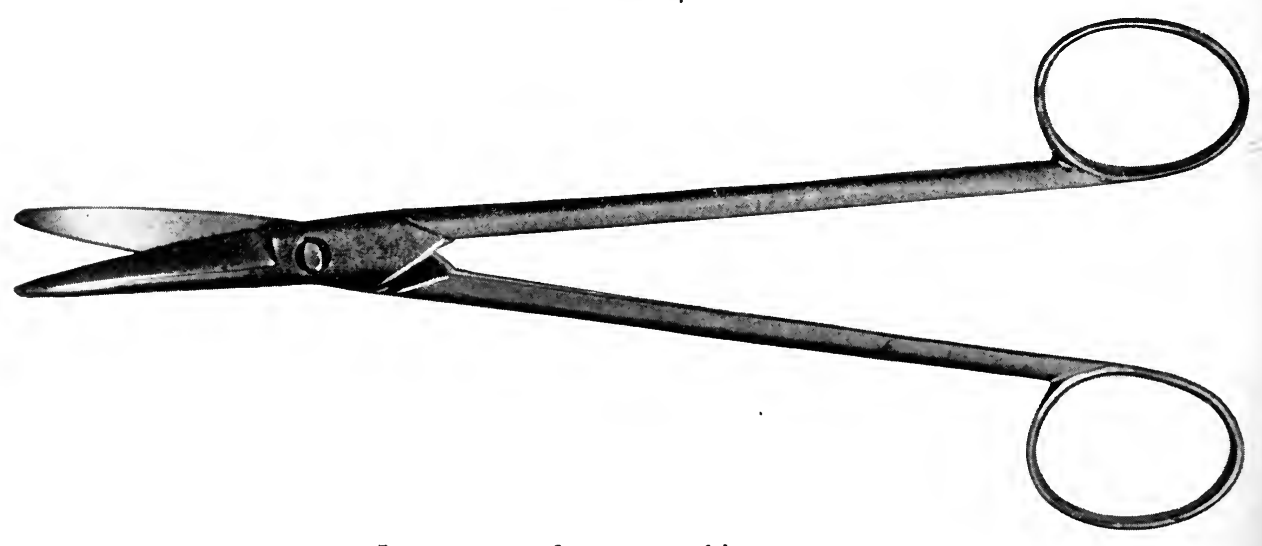

Instruments for suprapubic cystotomy.

Position of Patient.-At first, flat on the back; later, Trendelenburg posture (Fig. 287).

Operation (with rectal and bladder distention-Petersen's technique). - Grease the rectal bag, fold it longitudinally, and insert it into the rectum. Irrigate the bladder, and, after leaving eight to ten ounces of a sterile saline solution in it at the completion of the irrigation, withdraw the catheter. Inject eight ounces of warm water into the rectal bag. 
The best elevation of the bladder above the symphysis is obtained by distending the rectal bag first and the bladder afterward. Some surgeons prefer air distention for both the rectal bag and the bladder. We do not advise it.

Under the influence of the distention, the bladder rises out of the pelvis and is ordinarily lifted sufficiently high to expose above the symphysis from two to four fingers' breadth of the anterior surface of the organ below the peritoneal insertion. (See Anatomy of the Bladder.)

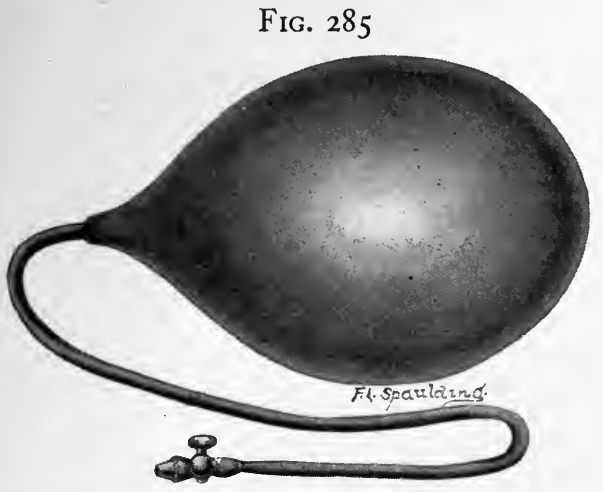

FIG。 286

Rectal colpeurynter.

FIG. 287

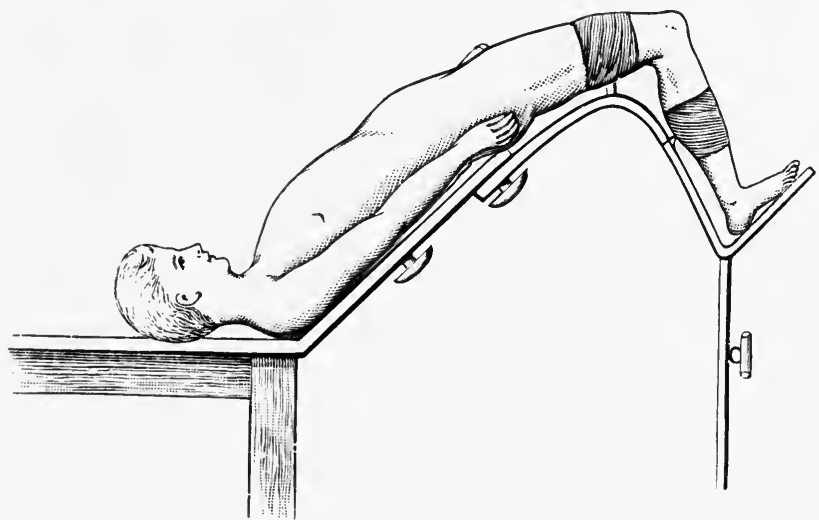

Postural method of raising the bladder. (Trendelenburg.)

The majority of surgeons no longer use the rectal distention, finding that the organ is made sufficiently accessible by the employment of the Trendelenburg posture. Personally, we prefer to have the patient flat upon his back until the prevesical fat has been exposed, and then to raise him to the Trendelenburg posture.

Incision.-Median line, beginning at the upper margin of the symphysis and extending upward three inches toward the umbilicus. The incision is to be lengthened if more room is required (see Fig. 288). 
Divide the skin and fascia, and continue between the insertions of the recti and the pyramidales muscles until the prevesical fat is exposed.

Immediately beneath this lies the bladder and the peritoneal reflection upon its anterior surface. These may be exposed in either one of two ways: By cutting through the fat tissue with blunt-pointed scissors, which is the better way, or by drawing it toward the upper angle of the wound with the finger tip and retaining it there with a retractor. This

FIG. 288

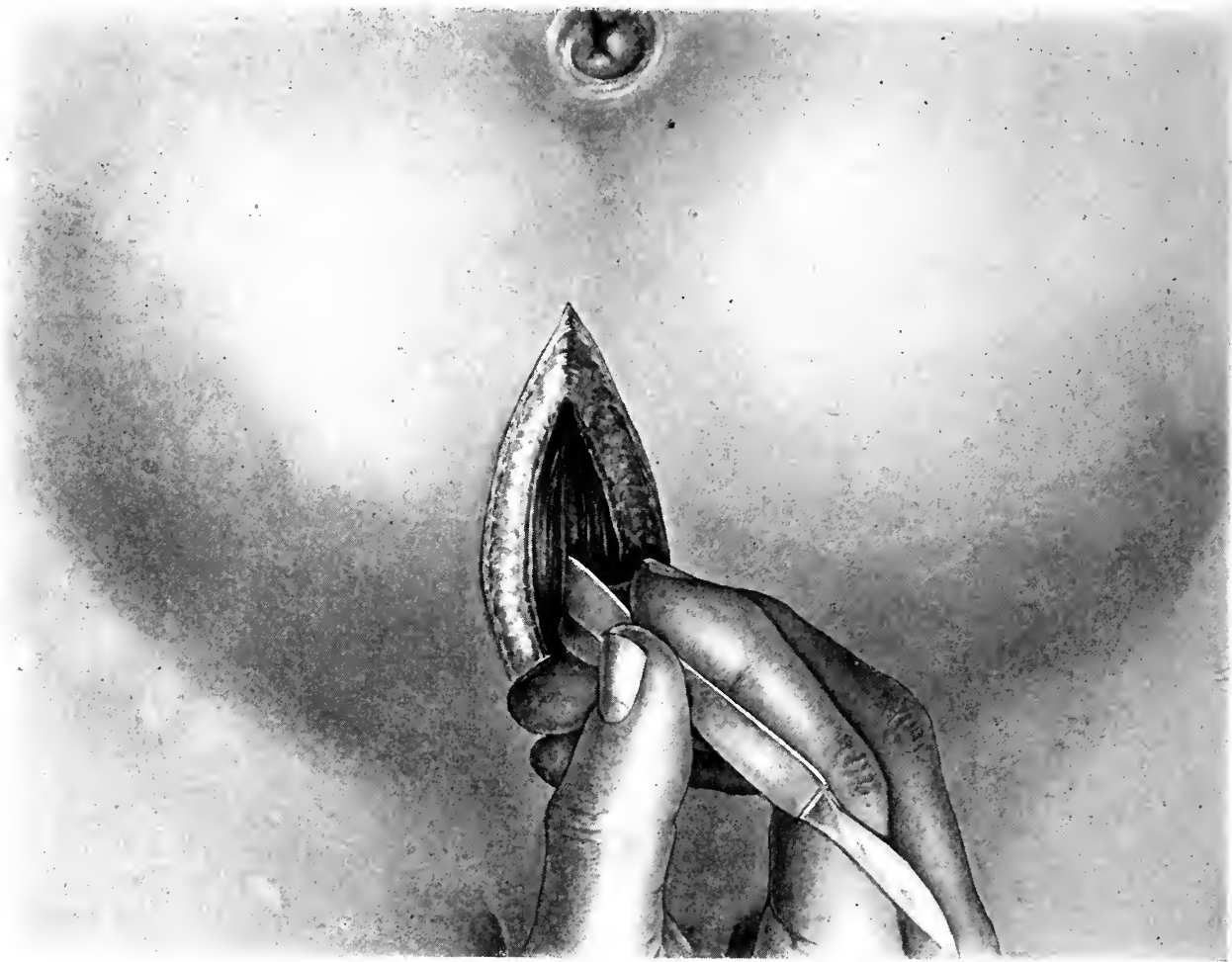

Suprapubic operation. Separation of the fibers of the rectus muscle with the handle of the scalpel. (Deaver.)

is the less desirable plan, because it disturbs the tissue occupying the prevesical space, and there is, therefore, greater chance afforded for urinary infiltration and infection in the spaces thus formed behind the symphysis.

The peritoneal insertion on the front of the organ is next to be sought. When found, a tenaculum is passed through the wall of the bladder immediately beneath it, and the bladder is lifted upward and toward the surface of the abdomen. 
The tip of a metal staff that has been previously passed into the bladder through the urethra is now pressed upward against the anterior vesical wall, and allows the operator to identify the bladder by feeling it with his finger.

Pierce the bladder just below the tenaculum with the straight, narrowbladed scalpel - the cutting edge of the blade toward the symphysisand incise the anterior wall downward longitudinally in the median line for a longer or shorter distance, as the conditions may require (Fig. 289). The urine rushes out immediately upon the entrance of the knife into the

FIG. 289

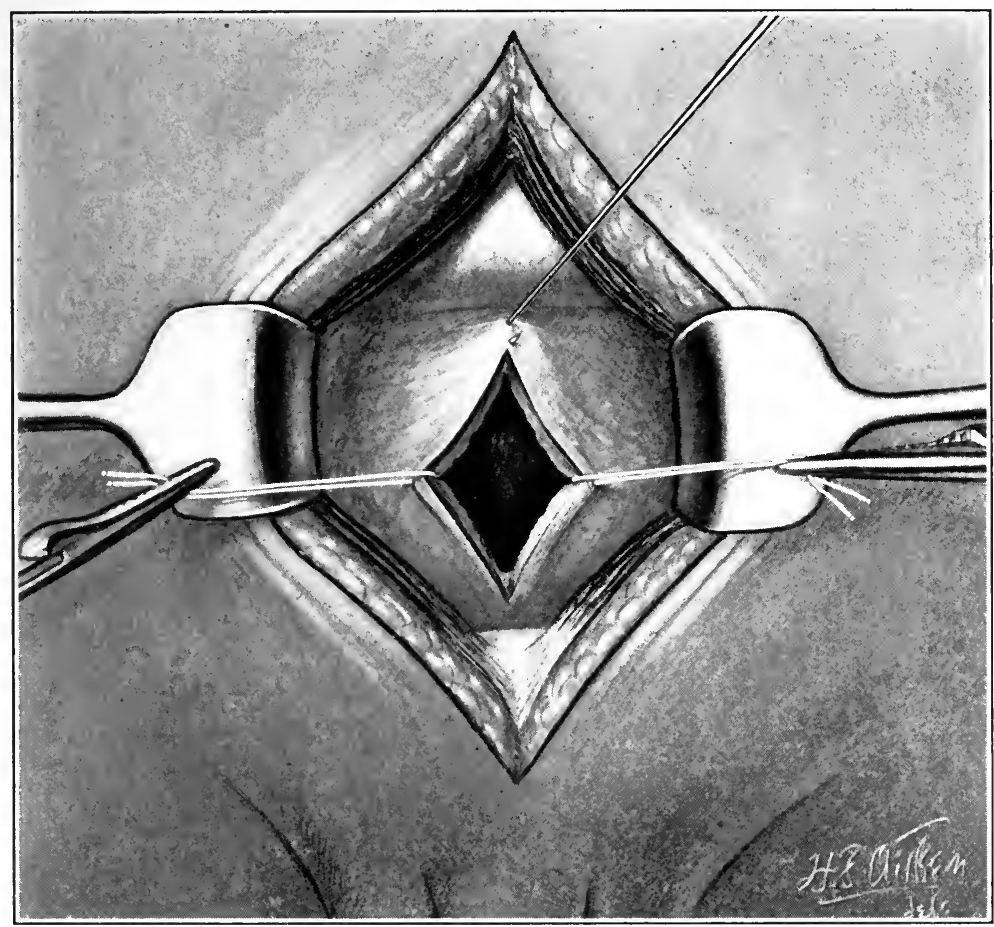

Opening the bladder by a longitudinal incision below the peritoneal reflection.

bladder. Pass a stay suture through either side of the bladder incision and the corresponding points on the sides of the abdominal wound, or catch their ends with forceps (Fig. 289). These sutures are for the purpose of keeping the organ raised and the sides of the incision separated.

If an intravesical operation is to be done, the bladder incision may be temporarily united to that of the abdomen at several points. Some surgeons prefer to have no sutures attaching the bladder to the abdominal incision. 
Remove the tenaculum as soon as stay sutures have been placed, and remove the staff as soon as the bladder incision is made.

Some surgeons prefer to place the sutures previous to opening the bladder. This may be done with a round, full-curved needle passed

FIG. 290

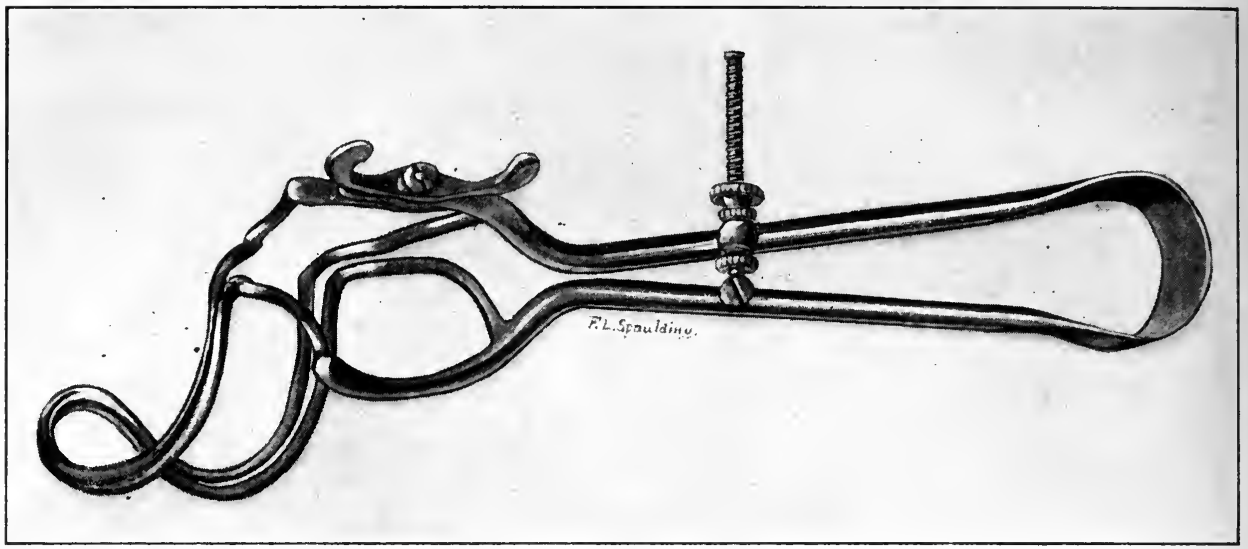

FIG. 29I

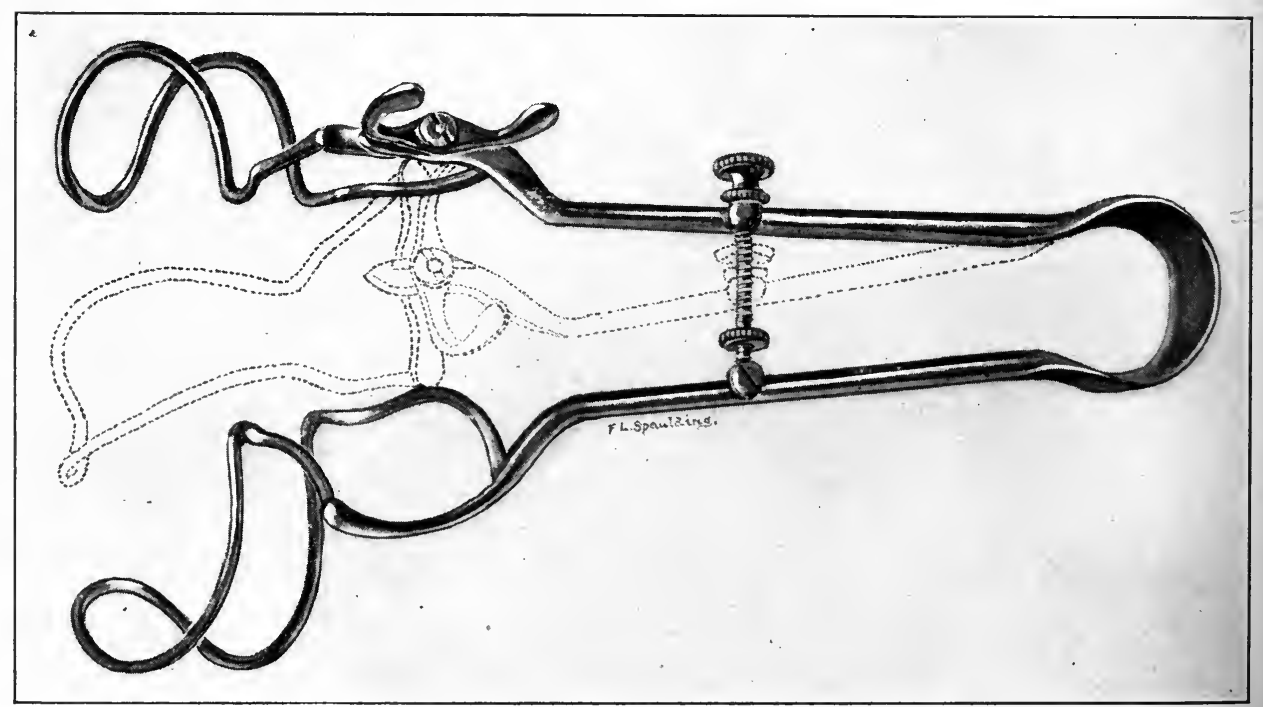

Watson's bladder speculum closed and open.

through the bladder wall on either side and a little below the tenaculum. The advantage of doing this lies in the greater ease with which the stitches can be placed before the bladder has been opened than afterward.

If the intention is only to establish drainage, the vesical incision should be no longer than is sufficient for the passage of the drainage tube or 
FIG. 292

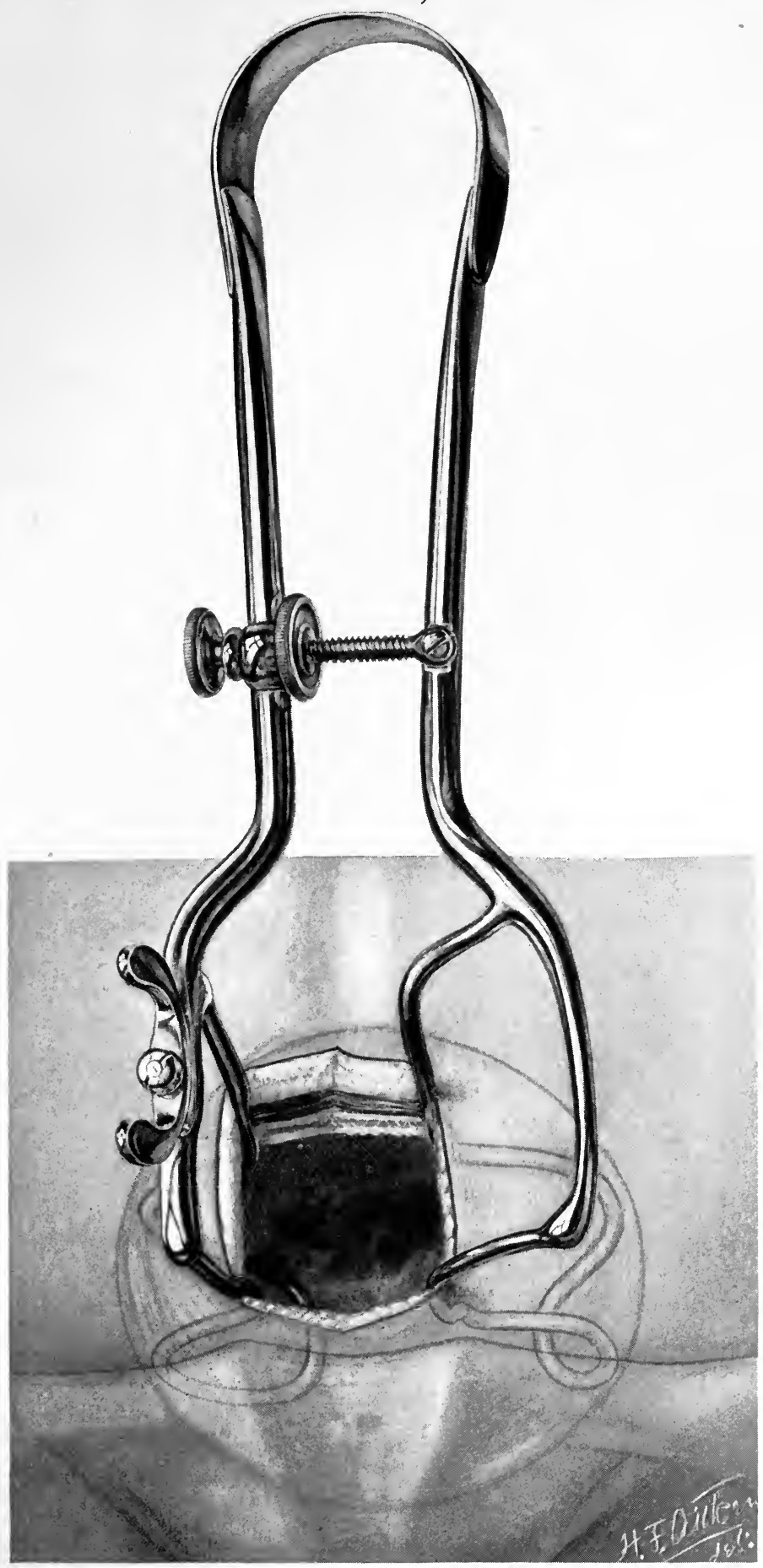

Watson's bladder speculum in situ. 
tubes through it, around which, after they have been attached to its edges, the incision should be closely sutured.

Visual Examination of the Interior of the Bladder.-This should be conducted with the bladder walls well separated from each other and with the interior illuminated by an electric lamp, or, in default of one, by sunlight reflected from a mirror and thrown into the bladder. The most satisfactory illumination is obtained by the use of the cystoscopic lamp, the instrument being passed through the urethra into the bladder and moved about as the operator may wish.

Devices for Separating the Walls of the Bladder.-The walls of the bladder may be held apart by one or another of a number of different kinds of retractors. The features which should be embodied in such instruments are: The parts of the blades which lie in the bladder must be curved in such a way as to press outward upon its sides and to separate them widely. There must be at least two, and preferably three, such blades or retractors, in order that the interior of the bladder shall be spread open and allow a clear view of it to be obtained.

These three requirements are, we think; best fulfilled by such an instrument as Watson's bladder speculum, which is shown in Figs. 290 and 29I. Fig. 292 shows the speculum in the bladder with the blades separated as they appear in Fig. 29I. The manner in which the instrument is used is sufficiently well shown in these three figures. Its blades are introduced closed, the inner one is then rotated and the bladder walls are thereby separated as seen in Fig. 292; the screw head upon the bar midway on the handles of the instrument prevents the recti muscles from pressing the blades together as they otherwise might do.

Digital Exploration of the Bladder.-This manœuver is very easy of execution, and is done by inserting the forefinger into the bladder through the cystotomy incision and palpating all parts of the interior of the organ.

Modifications of Suprapubic Cystotomy.-When for any reason more room is required, the incision may be enlarged in one of the following ways:

Transverse Skin Incision.-In very fat persons the skin incision may be advantageously made in the manner shown in Fig. 293. This is made in the form of a transverse crescentic cut with the convexity downward, its midpoint just above the upper margin of the symphysis, and by dissecting up a broad flap over the outer surface of the muscular layer of the abdominal wall, in the form of an apron. The space between the recti muscles is then easily found.

Modifications of the Incision in the Abdominal Muscles by Transverse Division of a Part of One or Both of the Recti Muscles.-A slight incision into the edge of the inner side of one or both of these muscles, about 
midway in the longitudinal cut through the linea alba, gives a much freer access to the interior of the bladder: It is rarely necessary to make an extensive division of the muscles.

Division of the Insertion of the Recti Abdominis Muscles.-The insertions of the recti are exposed by the external transverse incision and divided a little above the upper margin of the symphysis. The space opened by

FIG. 293

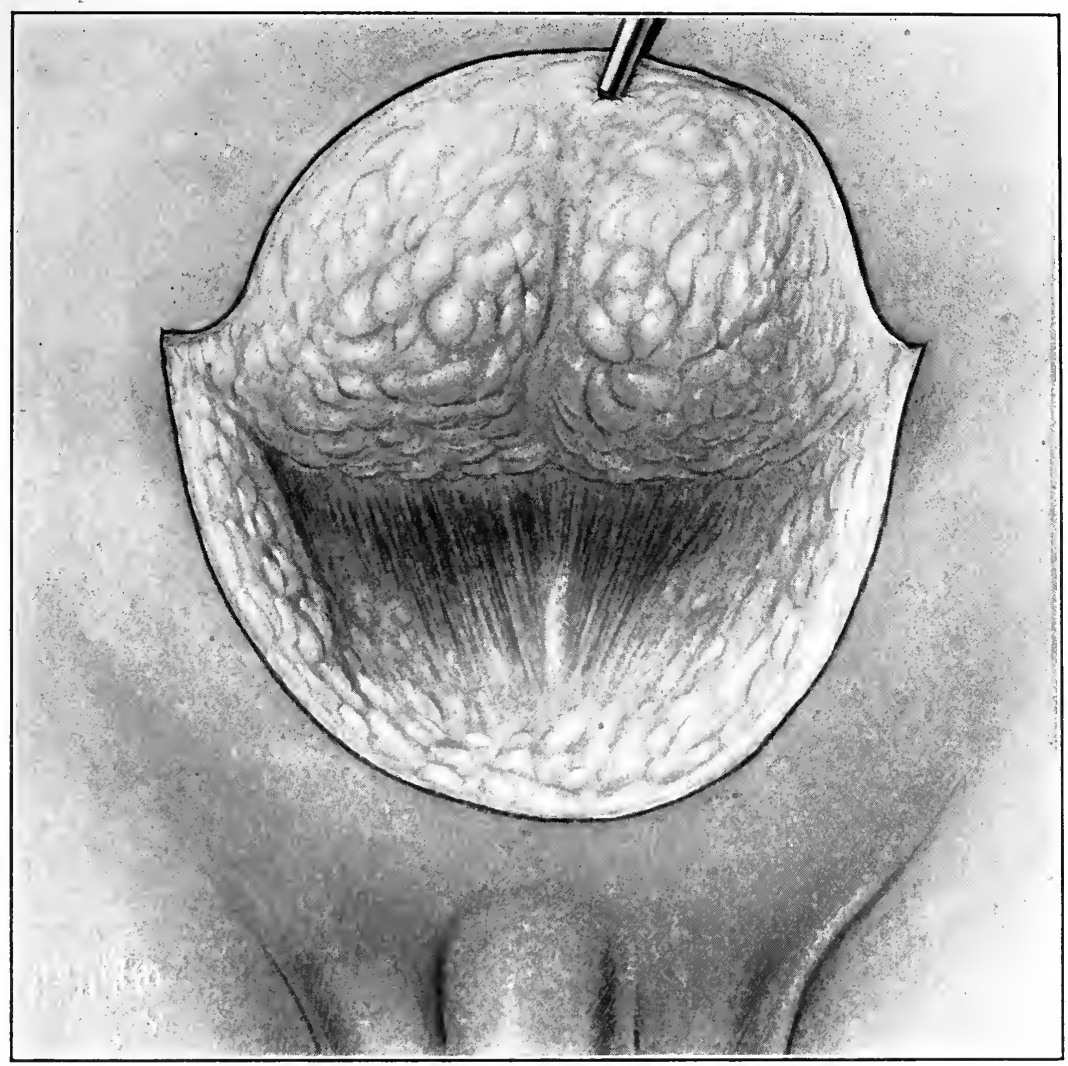

Transverse incision for suprapubic cystotomy.

the retracting of the muscles is a large one; the objection to this method is the difficulty in uniting the severed ends of the muscle subsequently.

If any such procedure is employed, the following method is to be preferred on account of the greater ease with which the lower ends of the recti muscles can be restored to their proper place.

Osteoplastic Resection of Symphysis Pubis.-The anterior surface of the symphysis pubis and the lower ends of the recti muscles are exposed by the curved transverse incision just above the upper margin of the 
bone. The sides of the incision are drawn apart so as to fully expose the symphysis, which, after division of the suspensory ligament of the penis, is partly sawed through a little beyond the insertion of the rectus muscle on either side; the part of the bone included between these two cuts is then divided with a chisel, separated from its attachment, and, together with the insertions of the recti muscles, is turned up over the lower part of the abdomen, exposing the prevesical space and offering a free avenue of approach for the further operative steps. When the latter have been completed, the resected bit of the symphysis with the insertions of the

FIG. 294

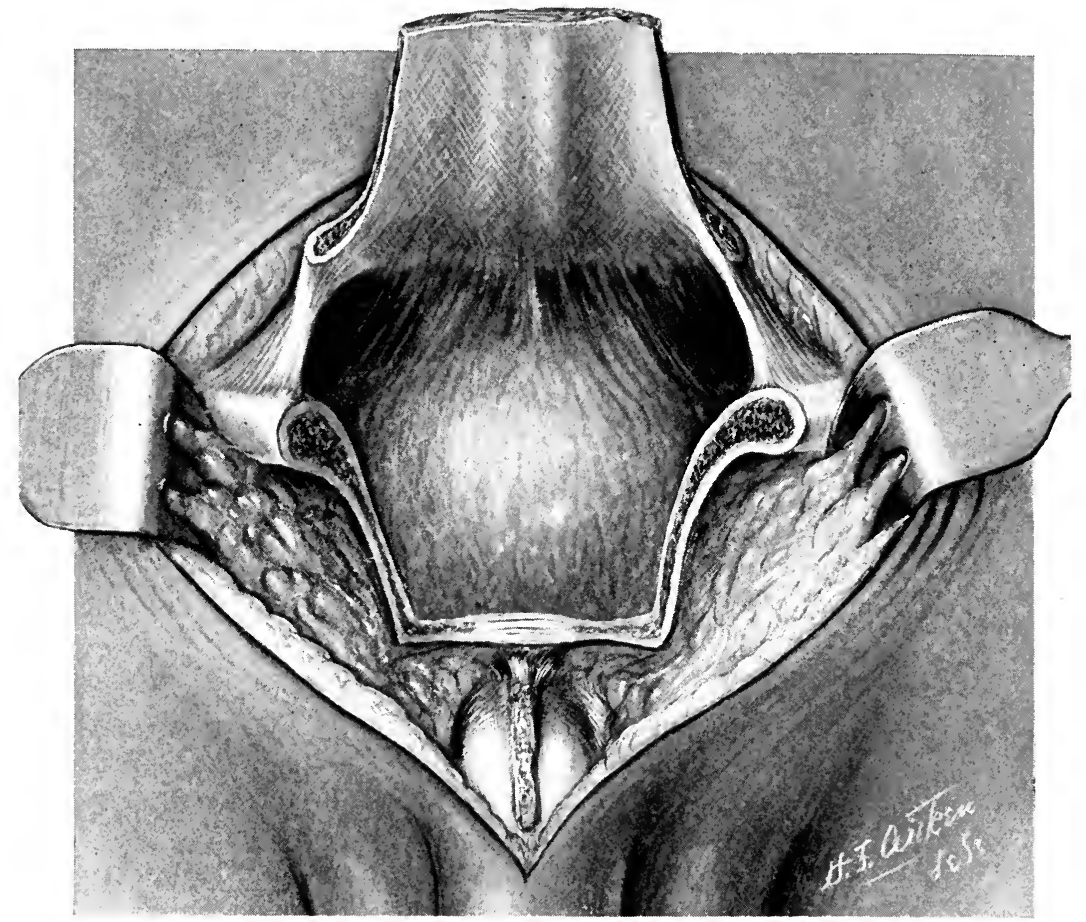

Osteoplastic resection of the symphysis pubis, the flap turned up and the bladder exposed.

recti muscles is returned to its original position and retained there by wire or kangaroo tendon sutures passed through the resected bit of the symphysis and the part of it from which it was temporarily removed (Figs. 294 and 295).

Modifications of the Bladder Incision.-The bladder incision is modified in one or another of the following ways for the purpose of obtaining more room:

The Transverse Cystotomy Incision.-This incision has the advantage of giving a somewhat larger space than the ordinary longitudinal one, 
and of being placed throughout its whole length higher up on the wall of the bladder. It calls for no special description other than to say that it is made immediately below the peritoneal reflection on the anterior surface of the viscus and transversely to its long axis.

The Unilateral, Bilateral, and T-shaped Incisions.-A good deal more room can be obtained by adding even one transverse incision to the ordinary longitudinal one, and a much wider entrance into the bladder is gained if two lateral cuts are made.

FIG. 295

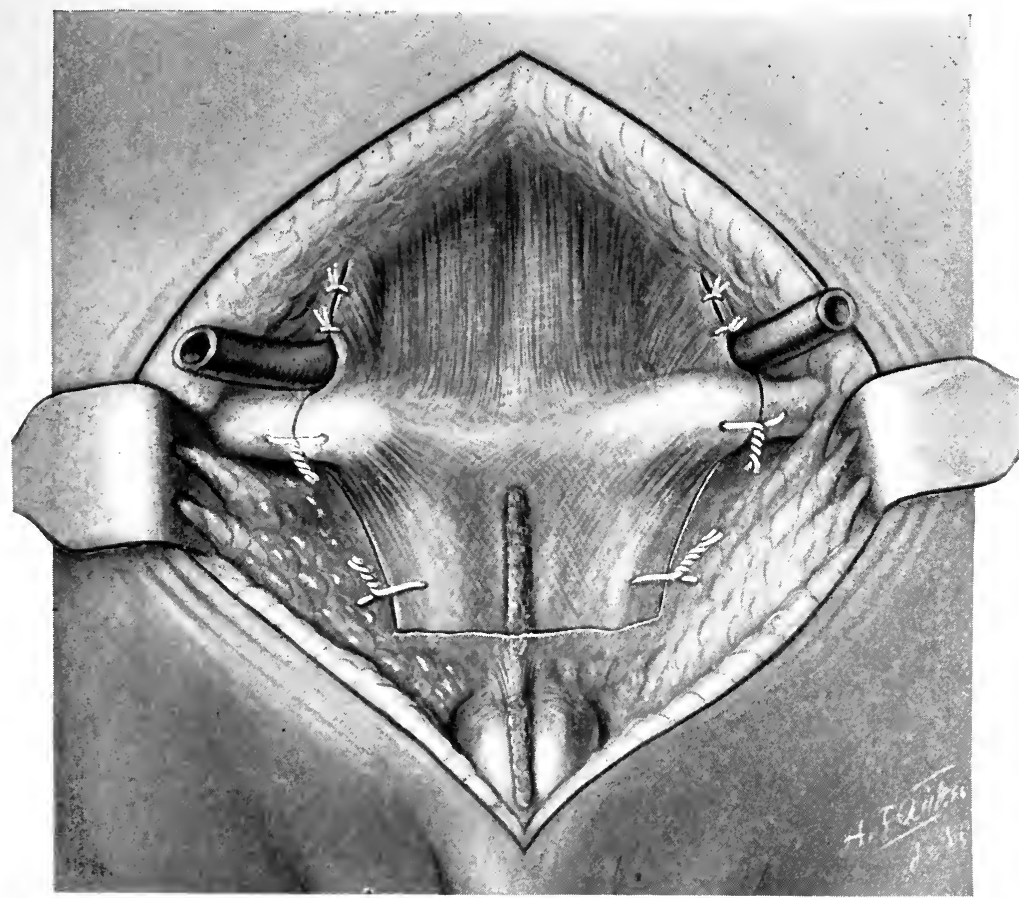

Osteoplastic resection of the symphysis pubis, the flap returned to its original position and sutured to the bones on either side the symphysis pubis.

We prefer to place the lateral incision or incisions at the upper end of the longitudinal one rather than lower down in its course. If two lateral cuts are made at the upper end of it, the whole incision has a T-shape. The advantage of placing the lateral cut or cuts high up is the greater ease with which suture can be applied later to close the wound.

Drainage of the Bladder after Suprapubic Cystotomy.-Temporary Drainage.-Temporary drainage is arranged by means of one or two rubber tubes or catheters attached to the edges of the vesical and abdominal incisions in order to prevent their escape from the bladder, and in such a way that their intravesical ends rest lightly upon the lowest part 
of its floor. The part of the catheters or drains which project outside the abdominal incision are passed through the dressing. Glass tubes five or six inches long are inserted into the outer ends of the drainage tubes, and long rubber tubes are attached to the other extremities of the glass ones and led into a receptacle upon the floor, tied to the side of the bed, or placed between the patient's legs (Fig. 296). The tubes should be attached to each other by a suture just above the skin. If one tube only is used, we prefer the catheter of De Pezzer (Figs. 297 and 298).

FIG. 296

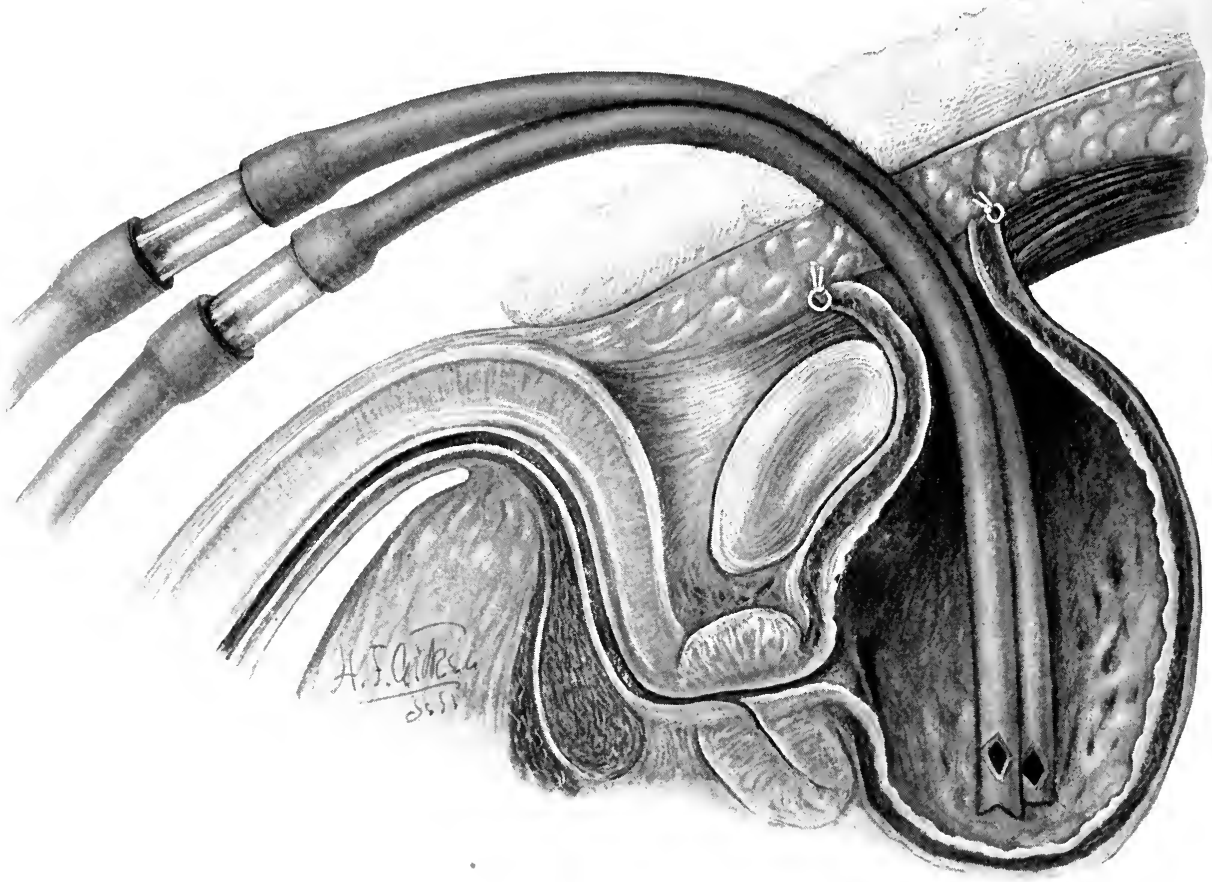

Method of suprapubic drainage of the bladder after cystotomy.

The advantage of De Pezzer's tube is that its openings in the end which rests in the bladder are raised slightly off the surface by the rounded projection at that extremity of the tube. The advantage of the double tubes lies in the opportunity they give for freer and more effective irrigation of the bladder, the inflowing stream passing through one and the outflow taking place through the other of them.

The essential features of good bladder drainage are:

I. Close adaptation of the bladder incision around the tubes.

2. Keeping the intravesical ends of the tubes clear of clots and other obstructions.

3. Efficient siphonage. 
The manner of securing these ends proposed by Gibson, and illustrated in Figs. 299 to 302, is excellent, and is as follows:

A drainage tube is inserted into the bladder and the edges of the incision are sutured tightly around it. Pass two interrupted Lembert sutures transversely through the bladder wall above the tube and two

FIG. 297

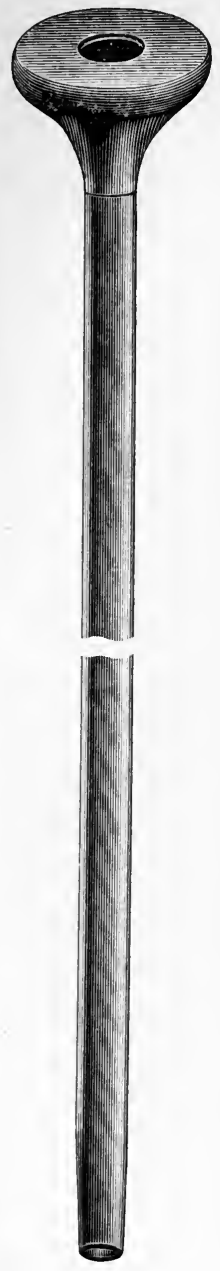

De Pezzer's self-retaining catheter.
FIG. 298

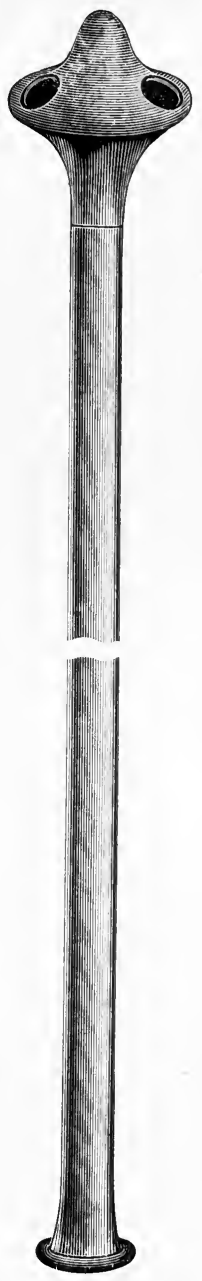

De Pezzer's self-retaining catheter.

others below it. Tie these sutures and cut the ends short (see Figs. 299 and 300).

Place a second row, alternating with the sutures of the first one. Tie and cut them short (see Figs. 300 and 301 ).

The tube is then enclosed within a canal the sides of which are closely adapted around it in the manner shown in the diagram (see Fig. 302). 
Permanent Drainage.-Permanent drainage may be carried out by: (I) Making an artificial channel-so-called urethra-from the bladder

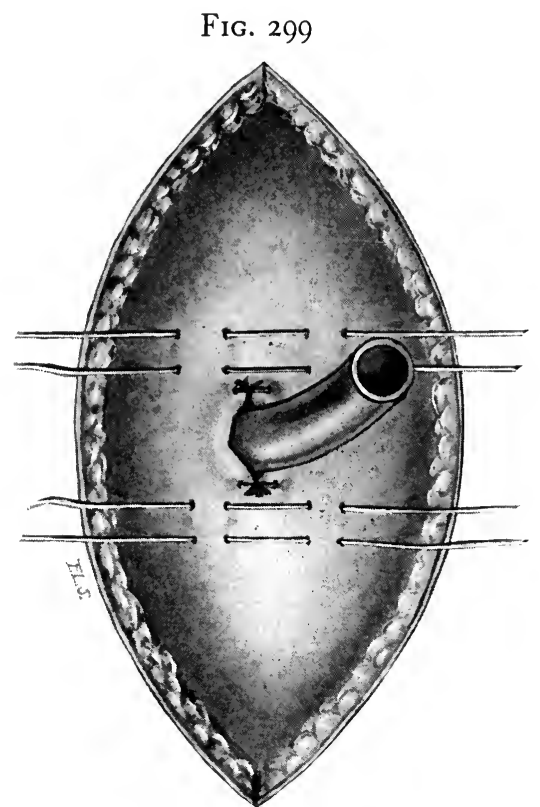

FIG. 30I

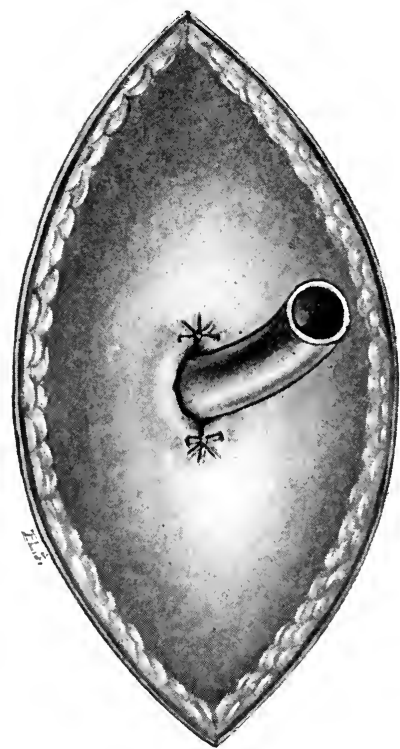

FIG. 300

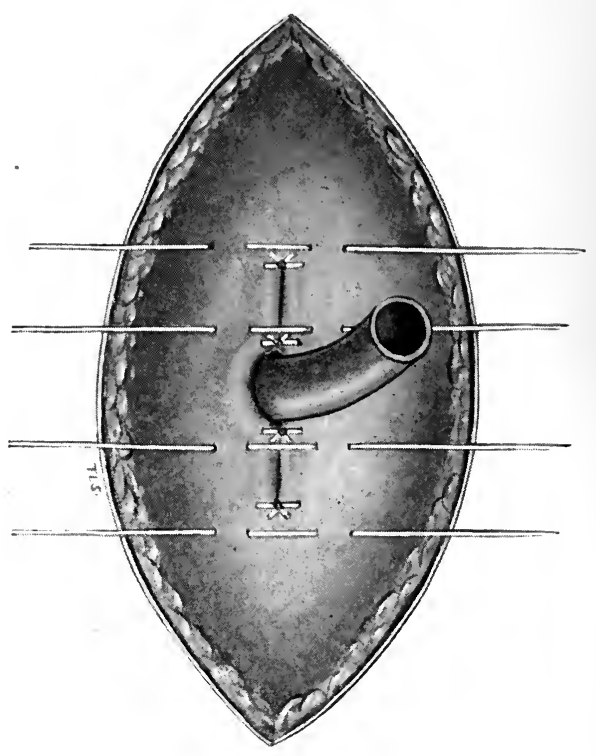

FIG. 302

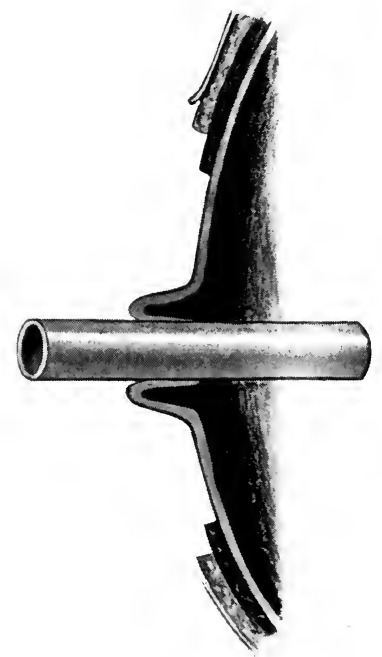

Gibson's method of suprapubic drainage.

to the surface of the abdomen, as proposed by Hunter McGuire, ${ }^{1}$ of Richmond. (2) Arranging it with an apparatus permanently worn by 
the patient. There are several which have been devised for the purpose. That which we prefer is illustrated in Fig. 303.

FIG. $3 \circ 3$

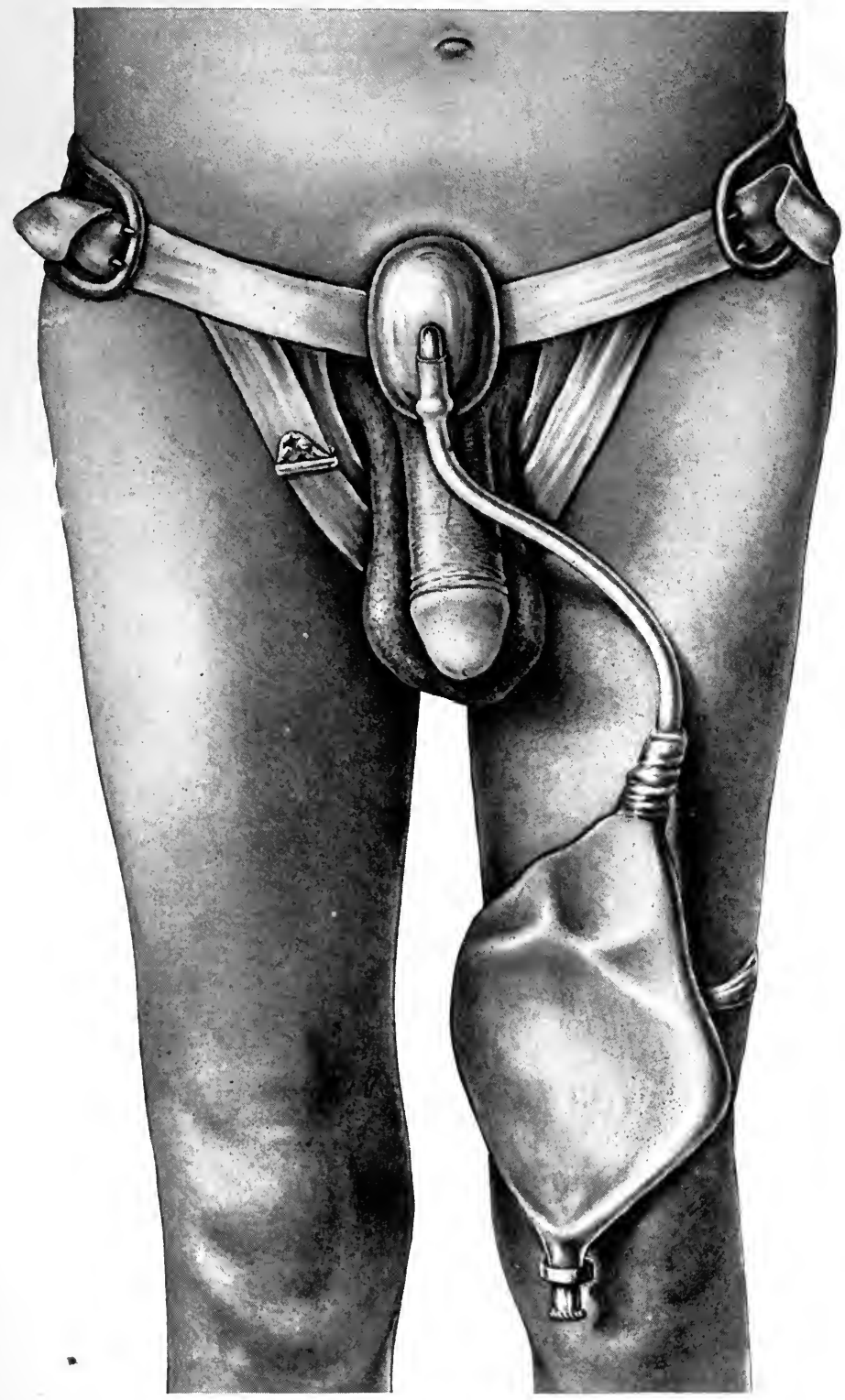

Stevenson's suprapubic tube in place, with urinal attached. (Deaver, after Da Costa.)

With proper care, this arrangement can be worn without distress to the patient and without being disagreeable to others on account of the odor. 
Bladder Suture.-Immediate closure of the bladder incision was introduced by Lotzbeck in 1838 .

The following are among the most important points to be noted in connection with suture of the suprapubic cystotomy incision.

Contra-indications.-Any conditions which make drainage of the bladder desirable. These are: (I) Cystitis. (2) Phosphatic incrustations. (3) Postoperative hemorrhage. (4) Obstruction to the natural flow of the urine from the bladder (prostatic hypertrophy, stricture of the urethra).

When none of the above conditions is present, immediate closure of the suprapubic incision is desirable because of the shorter period of convalescence and the avoidance of leakage of urine into the prevesical space and through the abdominal wound, which are secured by it, when the incision unites per primam.

The particular method of suture that is used is of no great importance so long as it is one which insures close and water-tight approximation of the sides of the incision. An objection to the purse-string suture is that it sacrifices an unnecessarily large area of the bladder wall. Certain other sutures are open to the objection that they demand too long a time for their application.

We prefer either the ordinary interrupted stitch or the continuous rightangle Cushing suture.

Suture in Cases of Rupture of the Bladder.-If the laceration is extraperitoneal, and situated upon the anterior wall, the Cushing suture referred to above, or an interrupted Lembert suture, may be applied in one or another of the ways just described. If intraperitoneal, proceed in one of the two following ways:

I. Suture applied from within the bladder through a suprapubic cystotomy incision.

2. Suture applied from the outside of the bladder through a laparotomy incision.

Position of Patient.-Trendelenburg posture.

Operation.-Expose the bladder as described under the heading of suprapubic cystotomy. Locate the laceration. A cystoscopic lamp introduced through the urethra may help to do this if there is difficulty in finding it. If the rent is in the anterior wall of the bladder, its edges should be caught with tenacula or toothed forceps and drawn up toward the surface of the abdomen. They can then be readily sutured in the manner already described. If the mouth of either ureter is involved in the wound, the ureter should be divided and transplanted into another part of the bladder. If the rent be upon the posterior aspect, the bladder must be incised through its anterior surface in order to reach the lacer- 
ation. When this has been done, lift up the edges of the rent with tenacula, catch them in the blades of a right-angle forceps in such a way as to close the laceration throughout its whole length, and suture the wound just beneath the blades of the forceps, or with a continuous suture over and over the blades; in the latter manner of applying the suture, withdraw the forceps and close the laceration by tying the two free ends of the thread to each other. Take up the slack of the thread before so doing.

If the laceration of the bladder is to be approached and closed from within the peritoneal cavity, a laparotomy incision should be made by extending the ordinary cut used for suprapubic cystotomy. Invert the edges and close the vesical laceration by applying continuous or interrupted sutures through the peritoneal investment and bladder wall, not including the mucosa, and by approximating the peritoneal surfaces when tying the threads; or the bladder may be opened through its anterior surface, the edges of the laceration caught by clamp forceps from within the bladder, and by applying the suture to close the wound in the first of the ways described above.

\section{OPERATIONS FOR VESICAL CALCULUS.}

Lithotomy.-References to cutting operations for stone in the bladder are found in the early writings of India. At the time of Hippocrates there was a class of men who "cut for stone," but who did not practise any other surgical procedure. This custom prevailed, indeed, until comparatively recent times, the "art" being handed down, in some instances, from father to son. The Hippocratic oath requires that lithotomy be left to those who make a special business of it. The early cutting operations were all done from the perineum.

The first accurate written description of such methods is that of Celsus, nearly 2000 years ago. He says that it should be performed upon children between nine and fourteen years of age, when medicines fail to relieve them.

Celsus" operation was known as "cutting on the gripe," and is described in effect thus: The middle and forefinger of the left hand are inserted into the rectum and the stone is drawn toward the surface of the perineum and held there firmly.

A crescentic prerectal transverse incision is made across the perineum (see Operations on the Prostate-Perineal Prostatectomy), and the neck of the bladder is exposed by further incisions and by blunt dissection in the line of the first cut. These are somewhat less in width than the outer incision. The neck of the bladder is then laid open by a transverse vol. $\mathrm{I}-36$ 
incision corresponding to the height of the convexity of the outer wound. The bladder incision should be large enough to let the stone pass through it without tearing the structures involved in the operation. Celsus warns that the dangers of the operation are much increased if this is not done.

If the stone is too large to be pressed out through the wound, recourse must be had to the method of one named Ammonius, who was a "cutter for stone." This method consisted in the following steps:

An instrument of special form and suitable for the purpose of holding the stone steady, so that it should not recoil and injure the bladder while being struck, was passed in through the perineal and bladder wounds, and the stone was grasped by it and held. An iron instrument with a small blunt end was then placed against the stone, which was broken into fragments by striking on the other end of the metal rod with a hammer.

Lateral Perineal Lithotomy.-The next step was that made and described by Paulus of Aegina. The first part of the operation is the same as that of Celsus. It then proceeds as follows: "We take the instrument called the lithotome and between the anus and the testicles-not, however, in the middle of the perineum, but on one side, toward the left buttockwe make an oblique incision, cutting down direct toward the stone where it protrudes, so the external incision may be wider, but the internal not larger than just to allow the stone to fall through it. Sometimes, from the pressure of the finger or fingers at the anus, the stone starts out readily at the same time that the incision is made, without requiring extraction; but if it does not start out of itself, we must extract it with forceps called the stone extractor."

In I543 Marianus Sanctus de Barletta introduced for the first time the guide or staff upon which the cut into the bladder was made. The details of this method are of interest. The operation did not involve an incision of the bladder itself, as those which preceded it had done. It was, in effect, a median external perineal urethrotomy incision, dilatation of the prostatic urethra followed, and the stone was then grasped between two thin metal rods passed into the bladder through the urethral incision, and was withdrawn by forceps passed between the thin metal rods, or "ductores." The cut into the membranous urethra was made upon a grooved staff previously passed through it into the bladder from the meatus. This method was known as the apparatus major, because of the large number of instruments required for its performance; the operation of Celsus was, in contradistinction, called the apparatus minor.

In the book called The Physician of Mydvai, written in the thirteenth century, the "lithotomy position" of the patient is described. It differs in no essential from that which is used to-day.

The operation of Marianus was practised by Pierre Franco, who lived 
in the sixteenth century, and whose treatise on surgery was published in Lyons in 1556. Franco was the most noted of the "cutters," and is credited with being the originator of the suprapubic operation for stone. This method he carried out for the first time in the case of a child of ten years of age, when, having made the attempt to extract the calculus from the bladder by the perineal operation, and failing to do so, he thereupon made a suprapubic incision into the bladder and withdrew it through that route. The patient recovered. This operation was done in 1560 .

The perineal methods just described were practised more or less frequently by priests and by laymen, conspicuous among the "cutters" being Frère Jacques Beaulieu, a priest, and Rau, a barber.

The English surgeon Cheselden was the first to place the lateral operation of lithotomy upon a sound anatomical and accurate basis, and at the end of his career had done 2 13 lateral lithotomies with a mortality of 5 per cent. only. This was prior to 1732 ; I0 5 of these operations were performed upon children. This operator carried the incision completely through the left lobe of the prostate laterally.

With slight modifications, the modern operation of lateral lithotomy is the same as that practised by Cheselden.

Crichton endeavored to secure union of the perineal incision by first intention, and claimed to have succeeded in so doing in many instances.

The more notable of the further steps in the development of the operations of perineal lithotomy are: (I) The bilateral cut made through the prostatic urethra and both lobes of the prostate, advocated by Dupuytren; and (2) the median method of Dolbeau, in which the stone was extracted through the dilated neck and deep urethra, which were entered by way of the ordinary external perineal urethrotomy inc sion made in the membranous part of the canal, and in which the deep urethra and the neck of the bladder were stretched but not cut. If the stone was too large to remove whole without damage to those structures, it was crushed before being extracted.

Suprapubic Lithotomy.-This operation originated, as already stated, with Pierre Franco about I560. Franco extracted a stone of the size of a hen's egg in his first case, and subsequently performed this operation in several other cases. He did not, however, recommend it as a routine practice. He recognized at once the importance of lifting the bladder upward and bringing it and the stone within it near the anterior surface of the abdomen and above the symphysis. (This is one of the many old manœuvers that have recently been put forward as new, in connection with the operation of suprapubic prostatectomy.) He accomplished this by pressing upward with the fingers in the rectum and by pressure on the perineum. 
In I $5^{8}$ I Rousset, of Montpellier, sought to do the same thing by filling the bladder with water.

The operation was but little employed until revived by Douglas and by Cheselden in England in 1723 .

Between $175^{8}$ and 1778 Frère Come practised it regularly. He introduced an instrument called the sonde à dard. This was a cannula with a long curve, inside of which there was a stylet with a sharp barbed point, which was concealed until the instrument had been passed into the bladder, when, the tip being pressed against its front wall, the dard was thrust through the open end of the cannula and transfixed the bladder. The front wall of the bladder could then be lifted upward above the symphysis, and the dard served as a guide in opening it when making the suprapubic incision.

Among the curiosities in the literature of suprapubic cystotomy is the case of Jean Doot, of Amsterdam, who, assisted by his apprentices, and using a shoemaker's knife, cut a large stone from his own bladder, and lived to tell the tale to his children afterward.

Frère Côme recorded his performance of Ioo suprapubic lithotomies with 19 deaths. This record is quite as good as that of most of the operators down to recent times.

The larger mortality attending this, as compared with the perineal method, which was chiefly owing to the septic infection of the prevesical space and of the peritoneum and the wounding of the latter, caused a decline in the popularity of the operation to such a degree that between $I 840$ and $I 880$ it was practically abandoned. In the latter year it was again revived through the reading of a paper at the International Medical Congress at Copenhagen by Petersen, in which he urged the strict application of antiseptic measures to the operation, and introduced a new feature, viz., that of raising the insertion of the peritoneum upon the anterior bladder wall, above the symphysis pubis. This he accomplished by means of distention of the rectum by a rubber bag into which from eight to twelve ounces of water were injected, and by filling the bladder with an equal quantity of fluid. When this is done, the peritoneal fold of the front of the bladder is lifted from two to four fingers' breadth above the upper margin of the symphysis, and can then easily be avoided when cutting into the organ.

This technique had for a time a vogue, then it was found that the Trendelenburg posture would accomplish practically the same thing, or, at any rate, would make the bladder readily accessible, and the method of Petersen has been, for the most part, abandoned.

The other important step in connection with the suprapubic operation is that of suture of the vesical incision for the purpose of securing its 
union by first intention. This part of the subject we have discussed under the separate heading of Suture of the Bladder, earlier in this chapter.

Finally, there is the step of entering the bladder through its peritoneal investment, for the purpose of extracting very large stones, and because of the knowledge that there is-if infection does not occura better chance of union by first intention of peritoneal surfaces than of those of the front wall of the bladder which are not invested with peritoneum. This was done by Rydygier in I887; subsequently, Harrington, ${ }^{3}$ of Boston, also operated in this manner, and, again, Post, of Boston, reported a case in which this step was taken.

(The further steps relating to bladder drainage and to bladder suture are treated under those separate headings in the earlier part of this chapter.)

Lithotrity.-According to Ultzmann, this procedure dates far back. He quotes Olympios, of Athens, as having shown that it was done in the ninth century. Statements are made in the biography of the Holy Theophanes which are to the effect that instruments were introduced into the bladder through the urethra from the meatus, for the purpose of crushing stone in the bladder and for removing it afterward.

Benedetti and Sanctorius also described crushing of stone in the bladder in the sixteenth century. The results of such isolated attempts as these, and a few more that are recorded, were so discouraging as to cause them to be abandoned and to be forgotten, and it was not until I 8 I 3 that we hear of the procedure again.

It was then once more suggested by Gruithuisen. This was a suggestion only, however, for its author did not practise it himself. It was not until I 8 I 8 that the young French surgeon Civiale published his work on Lithotrity, which may be taken as the starting point of the development of this method of treating vesical calculus.

Elderton, in Scotland, in I8rg, Amussat in I822, and Leroy d'Etiolles, in the same year, all described instruments or suggested the employment of this method. None of them, however, did the operation on the living subject. It was on January I3, I 824, that for the first time Civiale actually crushed a calculus in the bladder of a patient by an instrument passed through the whole urethra. This he did in the following way: A hollow tube or catheter, with its distal end open, and carrying a rod provided with three arms, was passed into the bladder and the stone felt by its tip, by which the stone could be caught and held. The breaking of the stone was accomplished by means of a sharp-pointed stylet, which was also passed through the tube, and which was made to 
bore into the stone between the blades or arms of the rod holding it. When this was done the fragments were allowed to fall out of the arms by loosening them. The separate bits of the calculus were then picked up and broken one by one in the same manner. The patient passed voluntarily such sand and fragments as were small enough to go through the urethra. Civiale operated twelve times in this way with eleven successful results.

In 1827 Eisenstein, in Australia, and in 1831 , Pirogoff, in Russia, introduced the method to their respective countries.

In England it was performed for the first time by Heurteloupe, July, I829, at Westminster Hospital. Dupeyre did the first one in America in $\mathrm{I} 83 \mathrm{I}$.

The first improvement in the mechanical detail of the operation was made by Weiss, in London, who substituted a two-bladed crushing instrument, somewhat similar to the modern lithotrites in form, for that of Civiale. The manner of crushing the stone, which had been by blows of the hammer upon the outer end of the instrument, was soon superseded by substituting the screw power for the other and cruder means. The screw force was at first applied by a ratchet and pinion mechanism, but this was presently improved by Civiale, who introduced a handle in the form of a wheel at the upper end of the shaft. Sir Henry Thompson again advantageously modified the instrument by giving to it a fenestrated blade, a cylindrical handle, and a more convenient mechanism for locking and unlocking the blades.

During the earlier period of the development of the operation, no attempt was made to withdraw the fragments from the bladder They were passed out by the voluntary urination of the patient after the operation.

The first definite effort to have the fragments evacuated as soon as the stone had been crushed was made by using scoop-shaped instruments; then the first suggestion of accomplishing the object by suction appeared. This was made by Sir Philip Crampton, of Dublin, in 1846 . The mechanism by which he proposed to do it was a glass chamber, resembling in form a soda-water bottle. Suction power was produced by exhausting the air within this chamber, and the connection with the bladder was made by means of a tube provided with a stop-cock.

Cornay later suggested the use of a rubber bulb, attached to a wash bottle, and in 1855 Clover introduced a far more practical instrument, which consisted of a large rubber bulb surmounting a glass chamber, into which the fragments collected when sucked out from the bladder.

These contrivances were one and all ineffective and inadequate, and the custom and the teaching up to 1878 was, that the "sittings" for 
crushing the stone should be as short as possible, and repeated as often as necessary, in order to get rid of it.

Litholapaxy.-Then came, in 1878 , the step which revolutionized the treatment of vesical calculus by lithotrity. This was the introduction by Professor Henry J. Bigelow, of Boston, of the operation of rapid lithotrity-lithotrity in one sitting; or, as he named it, "litholapaxy."

FIG. 304

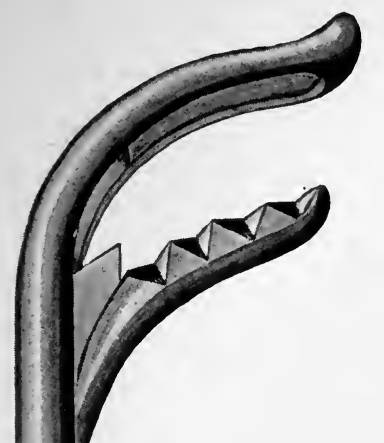

FIG. 306

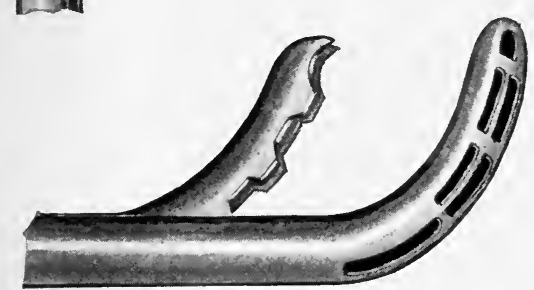

FIG. 305
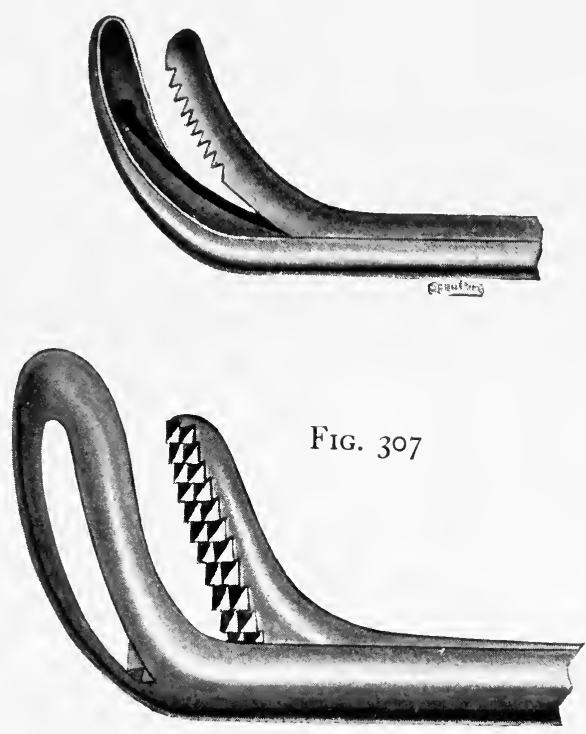

Different forms of lithrotrites.

The crushing blades of the lithotrites underwent various modifications -at first solid, later fenestrated in different ways, and in the instrument of Bigelow being provided upon the inner blade with a series of planes placed at angles with each other. Some of the forms of the lithotrites are shown in Figs. 304 to 307. The above sketch includes the more important steps taken than in the course of the gradual evolution of the different operative methods of dealing with vesical calculus, and we now proceed to the description of their technique.

\section{PERINEAL LITHOTOMY.}

Lateral Perineal Lithotomy.-This operation consists in opening the prostatic urethra and vesical orifice by an incision extending obliquely 
across the surface of the left side of the perineum and reaching the prostatic urethra by further and somewhat shorter incisions in the line of the first one; section of the prostatic urethra, a part of the prostate, the vesical outlet, and the extraction of the stone by forceps passed through this incision.

FIG. 308

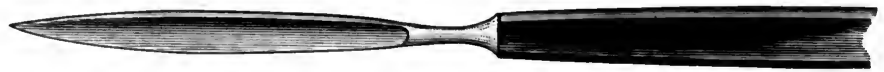

Lithotomy knife.

FIG. 309

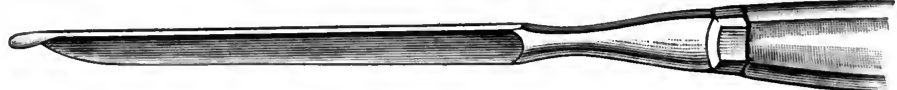

Blizzard's probe-pointed knife.

FIG. 310

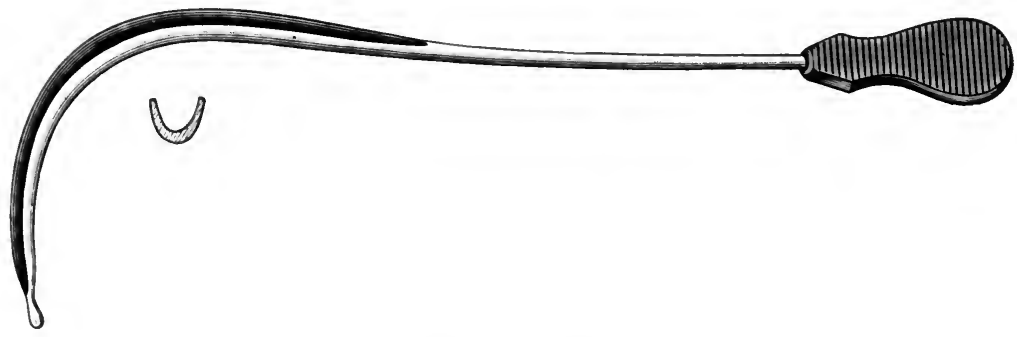

Lithotomy staff.

FIG. 3 II

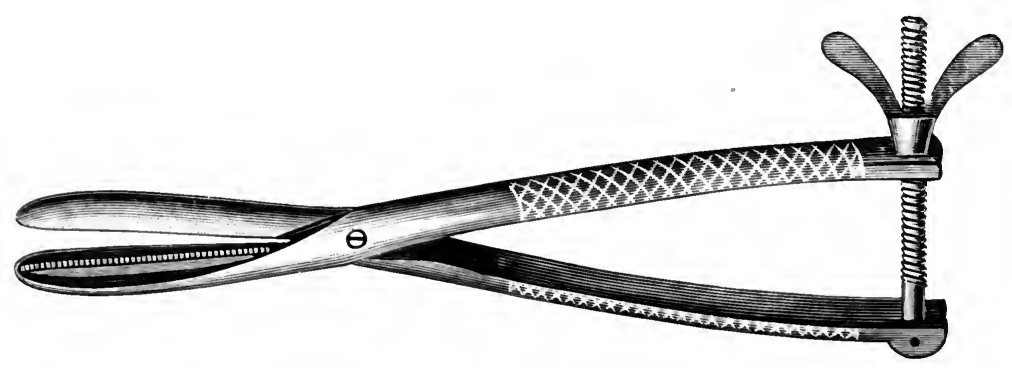

Dolbeau's lithoclast.

Instruments (Figs. 308 to 3I4).-Two scalpels: I for the external incision and $\mathrm{I}$ with a straight blade of but moderate width and with probe point; I staff with the groove upon its right lower aspect; I pair of stone extracting forceps; I lithoclast and irrigation apparatus and catheter; I scoop. 
Preliminary Steps.-Inject about eight ounces of sterile saline solution into the bladder after irrigating it. Be sure to feel the stone with a searcher or to have seen it with the cystoscope immediately previous to doing the operation.

Position.-The position is the so-called "lithotomy position." Thighs flexed on the body, separated from each other; the knees bent at a convenient angle. This position of the legs is maintained by a yoke or other device for the purpose; patient lies on his back, with the hips slightly raised.

FIG. 312

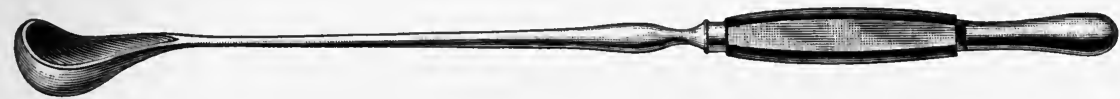

Lithotomy scoop.

FIG. 3I3

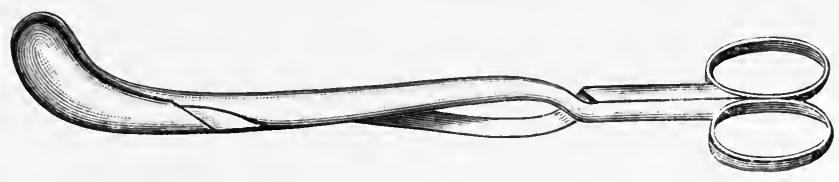

Stone-extracting forceps, curved blades.

FIG. 3I 4

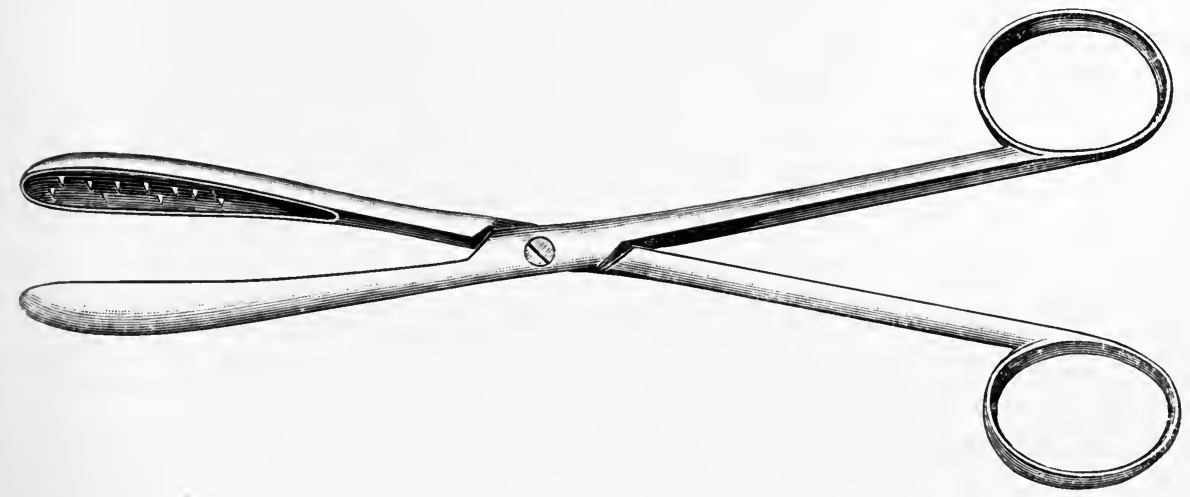

Stone-extracting forceps, straight blades.

The buttocks should be drawn well down over the lower end of the table and the perineum should be on a level with the operator's eyes as he sits facing the end of the table.

Landmarks.-The median raphé of the perineum, the left ischial tuberosity, the anus, the central tendinous point of the perineum, the apex of the left lobe of the prostate. 
Operation.-Pass the grooved staff into the bladder, and have it held steadily by an assistant in the median line, and hooked well up against the lower surface of the arch of the symphysis.

Incision.-The incision begins one-third of an inch from the median raphe of the perineum and on the left side of it, one to one and a half inches in front of the margin of the anus, and is carried obliquely across the left side of the perineum, and terminates about half to three-quarters of an inch to the inner side of the left ischial tuberosity. This incision is best limited at first to the division of the skin and subcutaneous tissue. It should then be carried on through the deeper perineal structures. The finger tip is then used to clear away the loose cellular tissue still interposing between it and the membranous urethra.

Hold the knife at a slightly oblique angle, enter its point into the groove of the staff, using the finger nail as a guide for the purpose. Push the knife blade steadily onward into the bladder, taking care to keep its point firmly pressed into the groove of the staff while so doing.

The gush of urine announces the entrance of the knife into the bladder. In withdrawing the blade, turn it outward and increase the extent of the incision by lowering the handle slightly. Enter the finger into the bladder beside the staff. Withdraw the latter, keeping the finger in the bladder. Introduce the forceps with the blades closed and use the finger as a guide to the opening into the viscus and to the stone. Withdraw the finger. Open the blades of the forceps. Press one of them against the side of the bladder and make a rotary, sweeping motion with the other. Close the blades, and the stone will usually be found to have been grasped by them.

If it can be done conveniently, adjust the stone in the blades with the finger tip, so that its shortest diameter shall be presented to the wound while it is being extracted. In withdrawing the forceps and the stone the handles of the former are to be given a somewhat downward direction, and should be moved from side to side to facilitate the extraction of the calculus.

In cases in which the stone is too large to be withdrawn through the ordinary incision, extend the latter by turning the cutting edge of the knife outward toward the side of the prostate, and by cutting more deeply into the left lobe of the gland when withdrawing the blade. The bladder should be thoroughly irrigated after the stone has been extracted.

After-treatment.-With children, no drainage of the bladder is required, nor with adults, except when it may be desirable to keep the wound open to relieve cystitis. The wound usually unites readily and rapidly. Fistula is very rare, although with elderly people it is occasionally a long time before the incision entirely closes. 
Complications.-Hemorrhage arises from one or both of the following sources: The artery of the bulb on the left side, which is often divided, and the prostatic plexus of veins in case the incision has been extended too far laterally and has involved the outer fibrous sheath of the prostate. The artery of the bulb, when cut, can always be picked up and tied. The venous hemorrhage is best controlled by compression, which should be applied by the device known as the canule à chemise (Figs. 3I 5 and 316), or by means of an inflatable rubber bag, which is inserted into the wound while collapsed and then inflated with air. The latter contrivance was devised by Buckston Browne, and called by him "the air canule à chemise" (Fig. 317).

FIG. 315

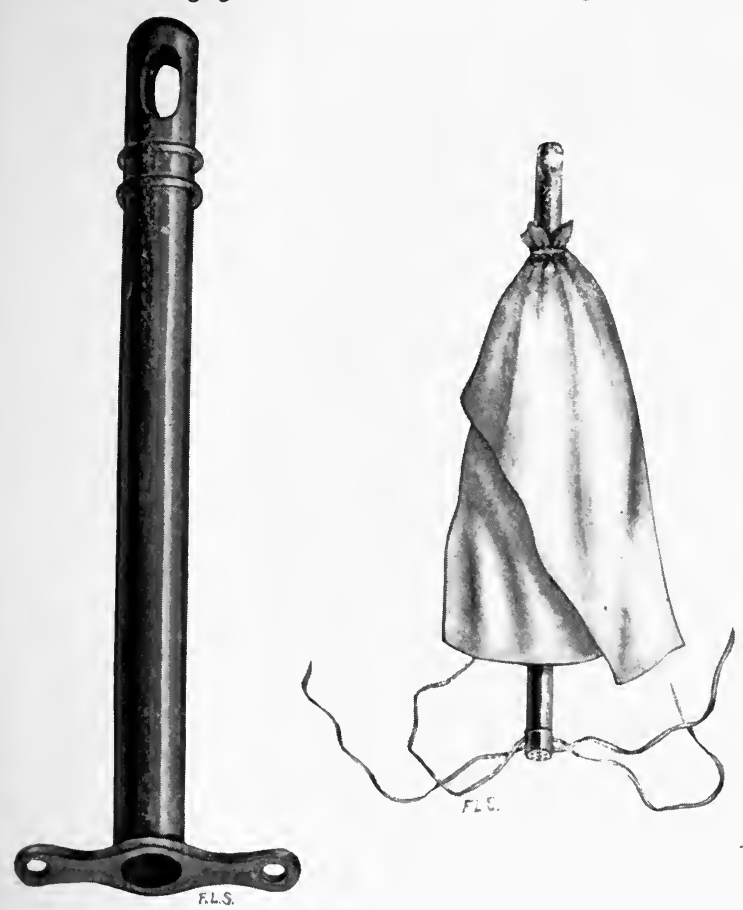

Canule à chemise.
FIG. 3 I 7

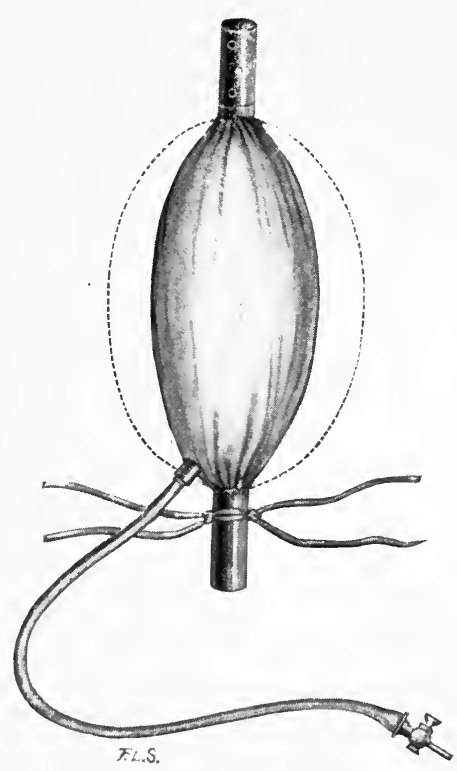

Buckston Browne’s air cannula.

In the ordinary canule à chemise the tube which drains the bladder has attached to it a bit of compress near its inner end. The tube, together with this compress, which has the shape of a circular tent, is passed through the wound so that the end of the tube shall lie within the bladder. Gauze wicks are then packed around the tube and beneath the compress until sufficient pressure has been made to arrest the hemorrhage.

Encysted Calculus.-Encysted calculus may be overlooked; or, if the part of it which projects into the bladder is grasped by the blades of 
the forceps, this outer portion may be broken off, leading the operator to believe that he has extracted the whole stone; or, again, he may do injury to the bladder wall by nipping it where it surrounds the calculus at the orifice of the pouch. Yet, again, the inclosed part of the stone may be too large to be withdrawn through the neck of the pocket and thus prevent the extraction of the whole calculus.

In some cases the mouth of the diverticulum may be incised by one or more slight cuts sufficiently to permit the withdrawal of the stone from it. The same result may, at times, be gained by dilating the orifice. Still another way of overcoming the difficulty is by crushing the calculus within its pocket and then removing the fragments. Finally, recourse may be had to suprapubic cystotomy and the stone removed through that avenue of approach.

A Stone Too Large to Pass through the Incision.-Under these circumstances the incision must be enlarged, or the stone must be crushed before it can be extracted. The second of these two ways of overcoming the difficulty is far the best and safest.

FIG. 3 I 8

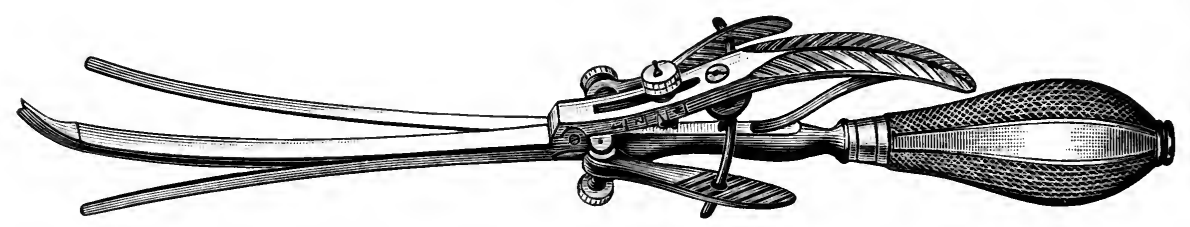

Dupuytren's double lithotome caché.

Incontinence of Urine.-This complication now and then follows the operation. It is best avoided by not cutting the membranous urethra too extensively and by letting the wound heal from the bottom.

Injury to the Rectum.-This will rarely, if ever, occur if the grooved staff along which the blade of the knife passes is hooked up well against the under surface of the symphysis and if the blade of the knife is slanted in an oblique direction. The point of the blade should never be allowed to leave the groove of the staff while making the incision into the bladder.

Bilateral Perineal Lithotomy.-Dupuytren introduced this method, which consisted in the substitution of two lateral incisions for the single one just described. The technique was as follows:

The patient's position is the same as in the last operation.

Instruments.-A staff, with groove in the middle line instead of on the side, as it is in the lateral operation. The only special instrument other than those used in the lateral lithotomy is a lithotome (Fig. 318), with two concealed blades, which are to be separated when the incision is made. 
Operation.-Expose the membranous urethra by the crescentic prerectal incision, crossing the perineum from one tuberosity of the ischium to its fellow on the other side, and by finger dissection.

Open the membranous urethra-on the groove of the staff, which has been previously passed-sufficiently to admit the lithotome. Withdraw the staff. Pass the lithotome into the bladder so that its tip lies just within the vesical outlet and touching the stone. Separate the blades of the lithotome and withdraw the instrument, cutting into each of the lateral lobes of the prostate in so doing. Through the opening thus made introduce the blades of forceps and extract the calculus in the same manner as that already described in connection with the lateral operation.

Giviale's bilateral operation differed from that of Dupuytren with respect to the outer incision, which he made an ordinary external median perineal cystotomy, and in the form of the lithotome he used, which was straight instead of being curved.

Median Perineal Lithotomy.-This operation is attributed to Dolbeau. The incision is that of external median perineal urethrotomy. The prostatic urethra is then dilated instrumentally, and the calculus withdrawn through it without making any further incisions, or if too large to pass through the incision, it is crushed and then withdrawn in fragments.

\section{SUPRAPUBIC LITHOTOMY.}

Instruments.-The same as for suprapubic cystotomy, and, in addition, the following: scoop, stone-crushing and stone-extracting forceps.

Position of Patient.-Same as for suprapubic cystotomy.

Preliminary Steps.-Pass a soft-rubber catheter and irrigate the bladder. At the end of the irrigation inject ten ounces of a 5 per cent. argyrol solution into the bladder. Pass a staff with long curve into the bladder, and if the urine escapes around it, tie a piece of tape about the penis sufficiently tight to prevent it.

Operation.-Incision the same as for suprapubic cystotomy; to be modified as already directed in case the stone is too large to be extracted through the ordinary cut.

It is a safer procedure to crush the stone with forceps made for that purpose and to withdraw it in fragments, if the calculus is of unusually large size, rather than to extend the incision through the peritoneal investment and attempt to extract the stone whole. If, however, it is decided to extend the incision, the best way of doing so is by adding two lateral cuts to the median one in the manner described under the heading of Modifications of the Suprapubic Cystotomy Incision. 
The extraction of the stone, if it be a small one, may be made by passing the forefingers of each hand through the incision in the bladder, grasping the calculus between their tips and then bringing them out with the stone held between them.

When the calculus is caught between the blades of forceps, it should be so adjusted that its shortest diameter will present in the wound, and thus most readily pass through it. The fingers take up more space than the blades of the forceps, and the latter are, therefore, to be preferred for the purpose of extracting the stone, unless it be a small one.

After the removal of the stone from the bladder the interior of the organ should be carefully examined to make sure that no other calculi remain in it, that an encysted stone has not been overlooked, and that there is no other intravesical condition which demands attention. The best means of doing this is to illuminate the interior of the bladder with a cystoscopic lamp, after having separated the sides of the organ from each other by Watson's bladder speculum or other appropriate instrument.

Digital exploration should also be made. Special attention should be given to the part of the bladder beneath intravesical portions of an enlarged prostate, when such are present, for small calculi or fragments are very apt to be lodged there.

Treatment of the Wound.- The incisions of the bladder and abdominal wall may be treated in one or the other of the following ways: By (I) complete suture; (2) partial suture; (3) without any suture. (The manner of applying the suture and that of arranging for drainage have already been described.)

Drainage and bladder irrigations should be prolonged if there is a severe or long-standing cystitis. When such is not the case, it should be continued for as short a time as possible. Complete suture of the bladder incision is to be preferred to drainage when there is no cystitis.

The patients should be gotten out of bed as soon as possible after the operation. It is not necessary to confine them there until the wounds are wholly closed. The long stay in bed is especially to be avoided in the case of elderly people.

\section{LITHOLAPAXY (RAPID LITHOTRITY, LITHOTRITY IN ONE SITTING).}

Previous to the introduction of this method of dealing with vesical calculus by the late Professor Henry J. Bigelow, of Boston, it had been customary to remove the stone by repeated crushings of a small part of it at separate sittings and to allow the patient to pass from the bladder spontaneously the debris thus made. 
FIG. 3I9

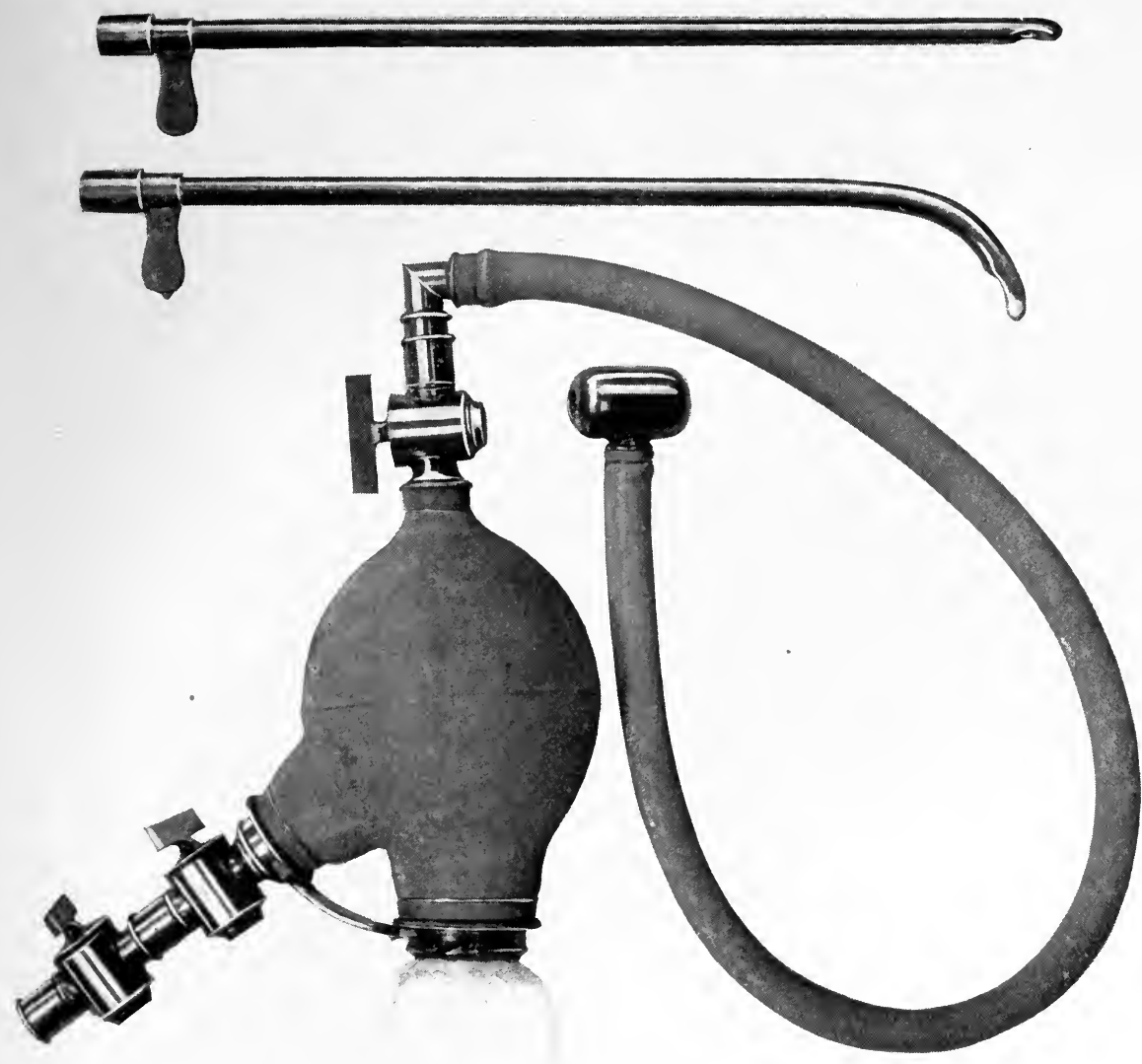

Bigelow's evacuating apparatus.

FIG. 320

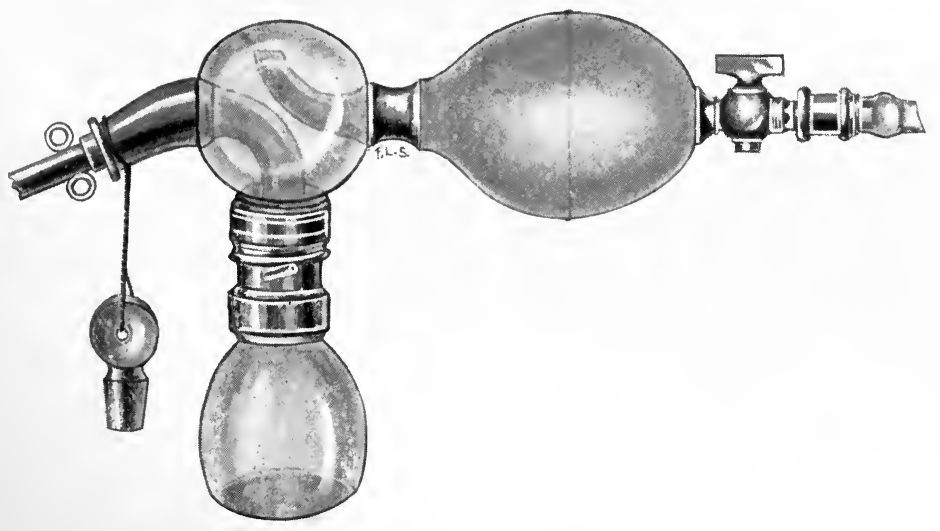

Otis' evacuating pump. 
The mortality attending the operation when done in this way was a seriously high one, and in the cases which were not fatal, constitutional symptoms were of frequent occurrence.

FIG. 32I

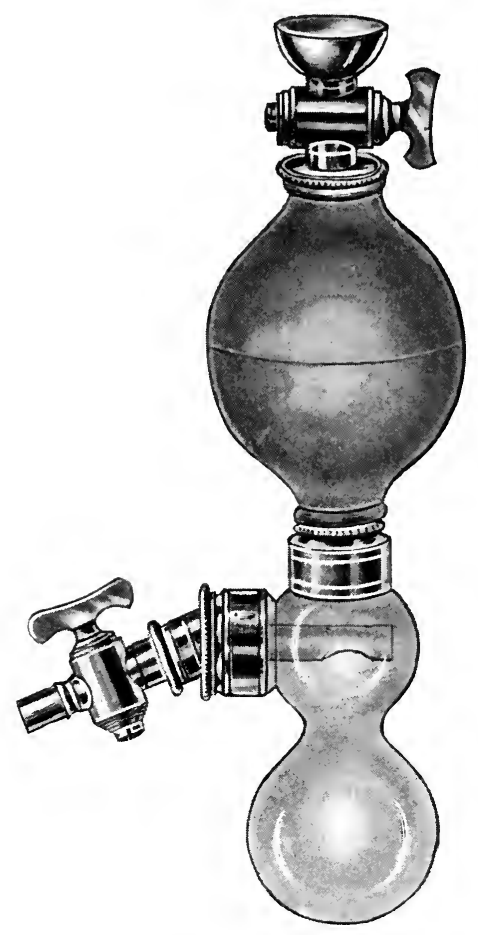

Keegan's evacuating pump.
FIG. 322

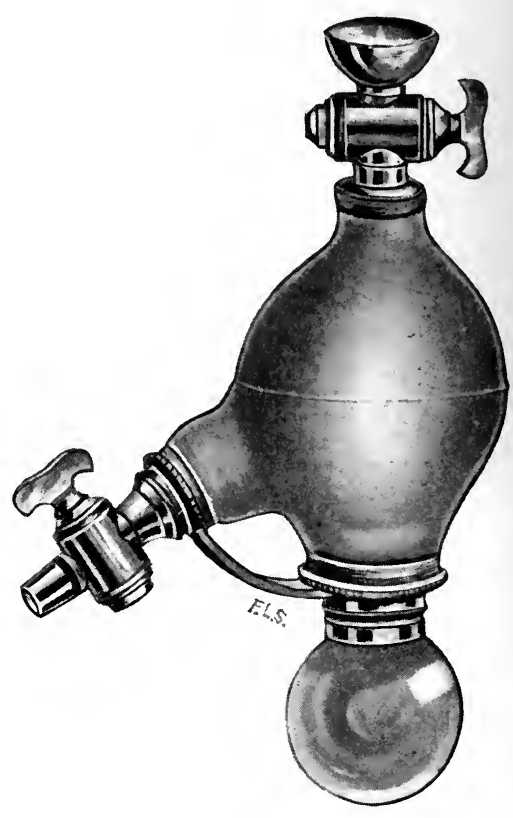

Freyer's evacuating pump.

FIG. 323

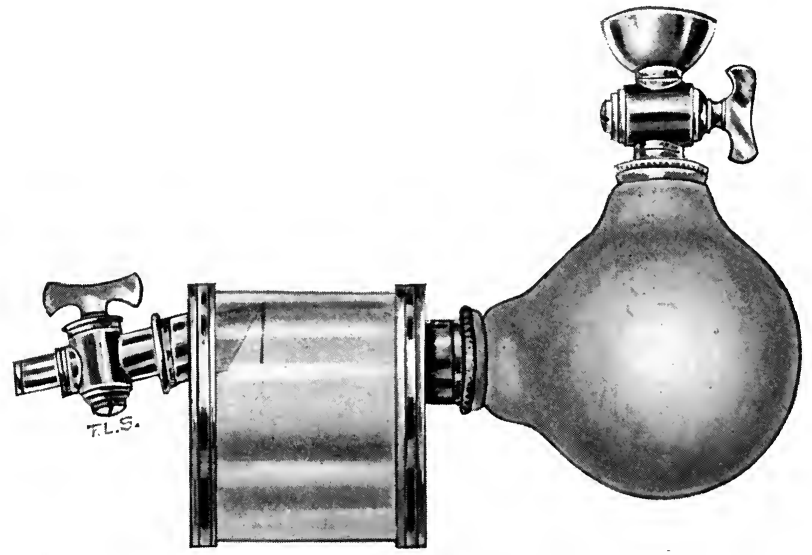

Thompson's evacuating pump.

Bigelow conceived the idea that both these results were due to the prolonged and repeated irritation of the bladder by the sharp particles 
and fragments which remained in it for a greater or less length of time after each crushing, and that they might be averted if the stone was completely crushed and removed at a single operation. He believed that the prolonged use of instruments in the bladder which would be required in some cases in order to accomplish this result would prove to be less harmful than leaving the debris of the crushings in the organ.

FIG. 324

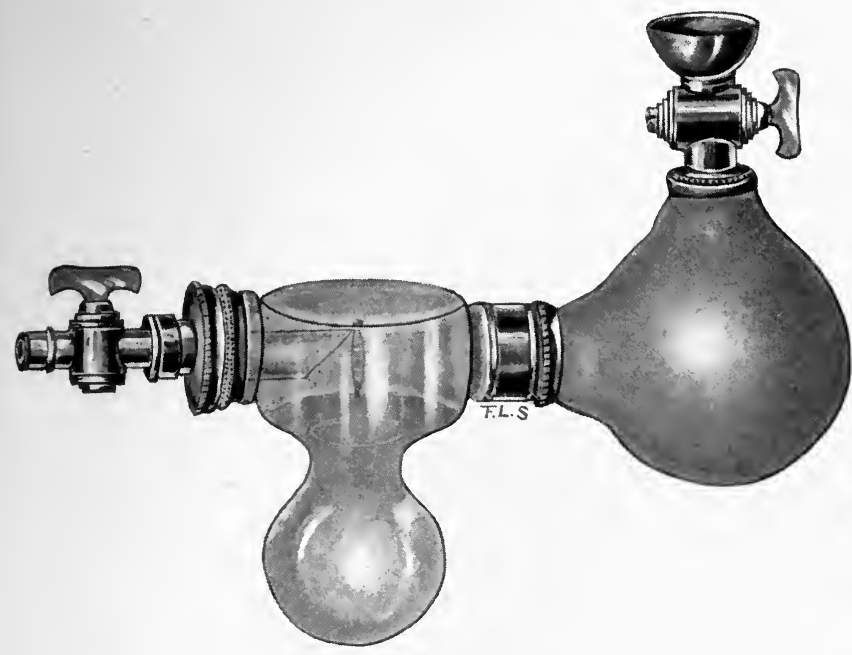

Golding Bird's evacuating pump.

To carry out this new conception of the operation, it was necessary to devise crushing instruments of greater power than those hitherto in use, and to construct an apparatus which would enable the operator to rapidly and wholly evacuate the bladder of sand and fragments.

One of the essential features of the evacuating apparatus was that of tubes of much larger size than any that had hitherto been employed, and a pump-evacuating bulb-which would prove efficient in withdrawing the debris and in catching it in such a way as to prevent its being sent back into the bladder again by the ingoing stream of water.

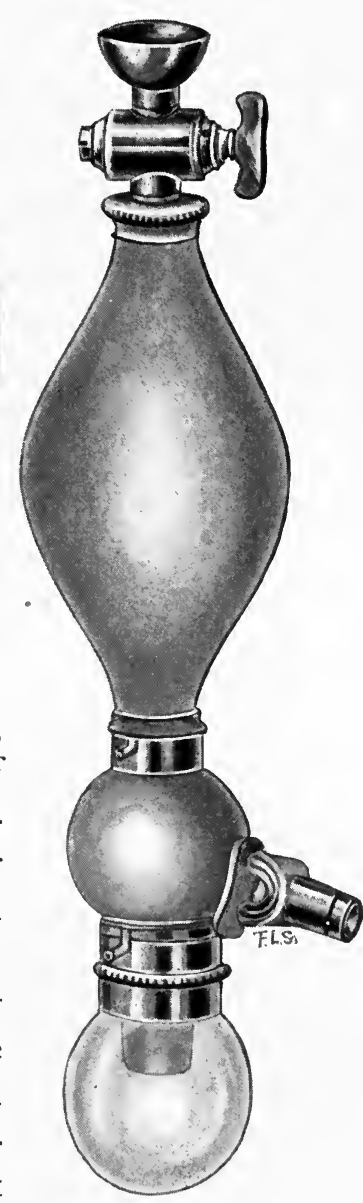

Evacuating pump.

Otis, of New York, had already demonstrated that the normal size of the urethra was much larger than had previously been believed, and that the size of the meatus was no guide to that of the urethra behind it. $\mathrm{He}$ showed that the caliber of the average adult urethra corresponded to $32 \mathrm{~mm}$. of the Charrière scale, and that but few were less than $30 \mathrm{~mm}$. It was clear, then, that after cutting the meatus, tubes at least as large as vor. I- 37 
28 or 29 of the French scale could be used without difficulty for adults, and Bigelow, accordingly, had the tubes made of these sizes. The evacuating apparatus is illustrated in Fig. 3 I9.

The whole mechanical device conceived and constructed by Bigelow is beautiful, and although a great many modifications of it have been introduced, none has improved upon it in any important respect. Figs. 320 to 325 illustrate some of the modified evacuating apparatuses which have been conceived and employed.

Instruments (Fig. 326).-For adults, the 2 larger sizes of the Bigelow lithotrites (Nos. 28 and 29); for children, the smaller sizes made especially for patients of that age (Nos. I2 to 22 French); the straight and curved

FIG. $3^{26}$

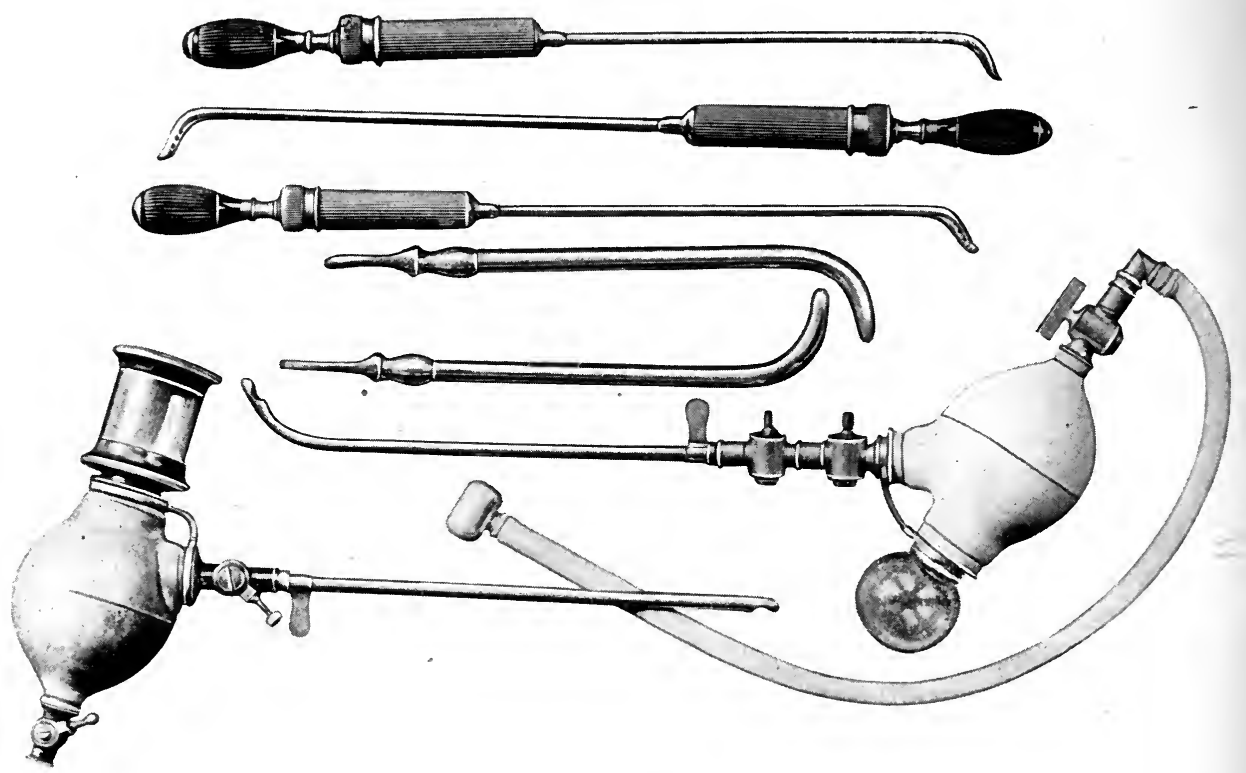

Instruments for Bigelow's litholapaxy.

Bigelow evacuating tubes; the evacuating pump and its connections; a soft-rubber catheter for injecting and irrigating the bladder; metal rod, to dislodge fragments of stone if caught in the tube during the evacuation; a piece of tape to tie around the penis, in order to prevent the escape of fluid from the bladder while crushing the stone.

Position of Patient.-Flat upon the back.

Preliminary Steps.-Irrigate the bladder with sterile saline solution and leave eight ounces of the fluid in the bladder. Cut the meatus if necessary and dilate stricture if present.

Operation.-Pass the lithotrite into the bladder, taking care not to injure the deep urethra, especially in cases of prostatic hypertrophy. 
Use the beak of the instrument as a searcher for the purpose of locating the stone and estimating its size.

Hold the instrument so that the tip of its beak points straight upward in the middle line of the bladder. Open the blades, turn them over to the side of the bladder in which the stone is believed to lie, and close them. The stone will probably have been grasped between them; if not, bring the tip of the beak back into the middle line (Figs. 327 and 328). Open the blades again and repeat the same manœuver upon the other side of the bladder. Never fail to bring the beak of the instrument upward and pointing in the middle line before separating the blades for

FIG. 327

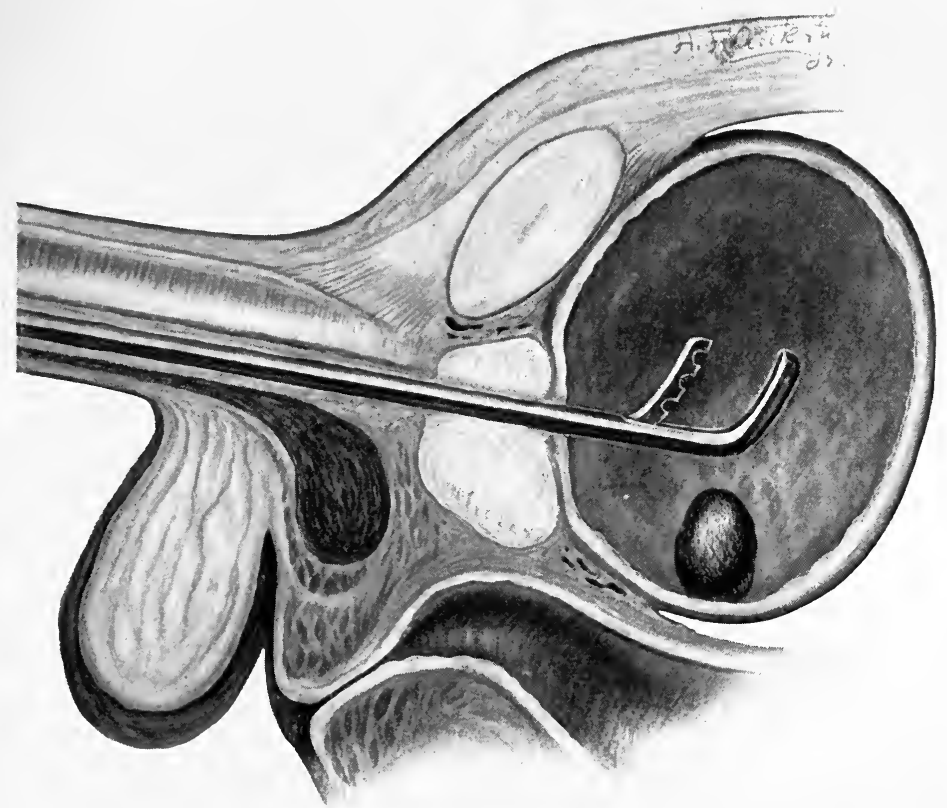

Lithotrite opened.

another trial at grasping the stone. If this is not done, the inner blade will almost certainly push the calculus before it, and thus prevent its being caught when the blades are closed.

When the stone has been grasped, lock the instrument by turning the metal rim immediately beneath its handle from left to right, or away from one's self, as a corkscrew would be used when entering the cork. Do not crush until the beak has been again rotated upward and until the instrument has been moved to and fro in order to give assurance that a bit of the bladder wall is not nipped by the blades. When this has been done, crush the stone (see Fig. 329). If the instrument cannot be moved freely 
within the bladder, in all probability a bit of the vesical wall has been caught, and the blades should be slightly separated in order to let it free again before beginning to crush. When there is a fragment directly

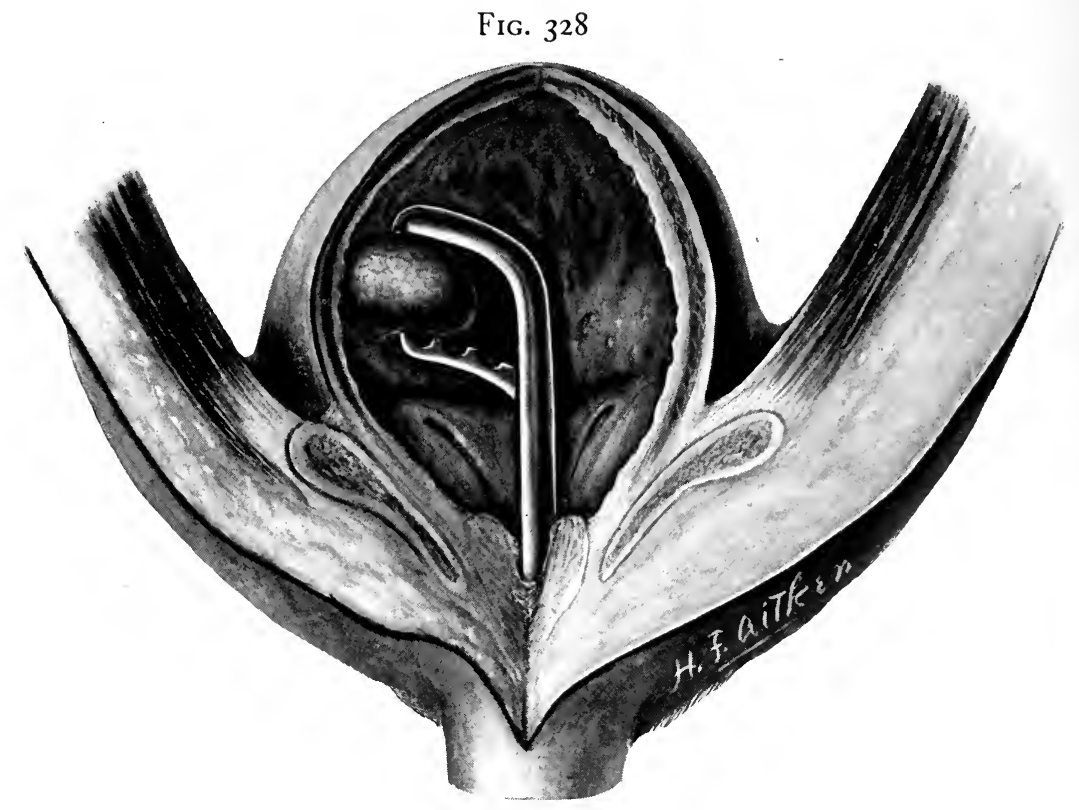

Lithotrite closed on stone.

FIG. 329

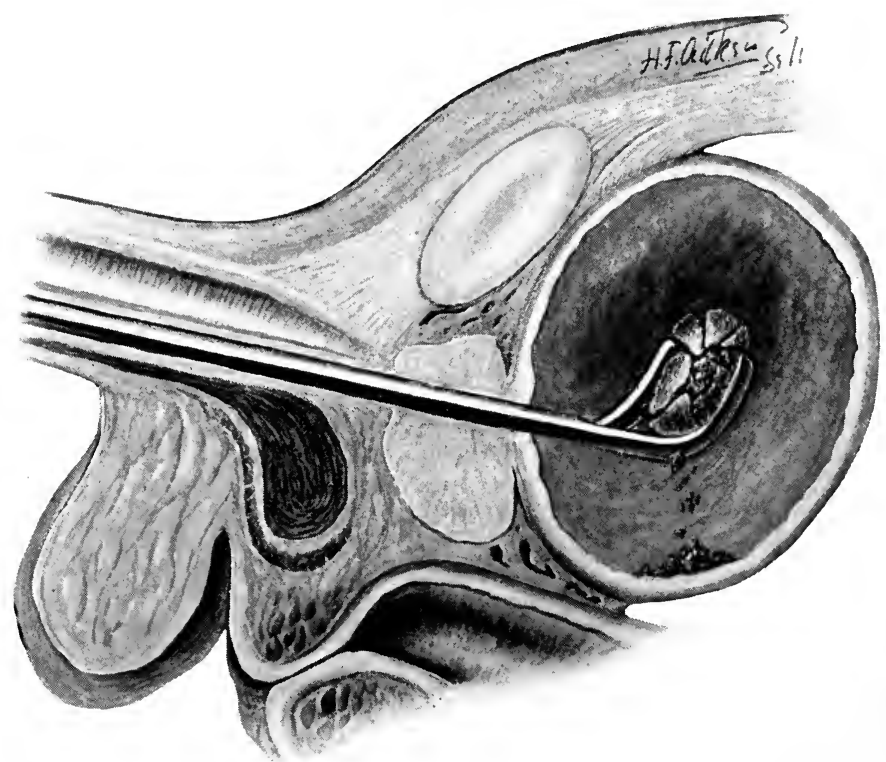

Lithotrite crushing stone. 
beneath the projecting lobe of an enlarged prostate, the instrument must be turned over, as in Fig. 330. Throughout the whole crushing, be sure and drive the inner blade hard home against the outer one every time that the stone or its fragments are caught and crushed; otherwise, the two blades may become separated by having the debris remain between them, and it may then be impossible to withdraw the lithotrite because of their being too widely separated.

Continue to crush the fragments until it becomes difficult to find new ones, then withdraw the instrument and begin the evacuation. Pass the evacuating tube and empty the bladder through it. Attach the bulb

FIG. 330

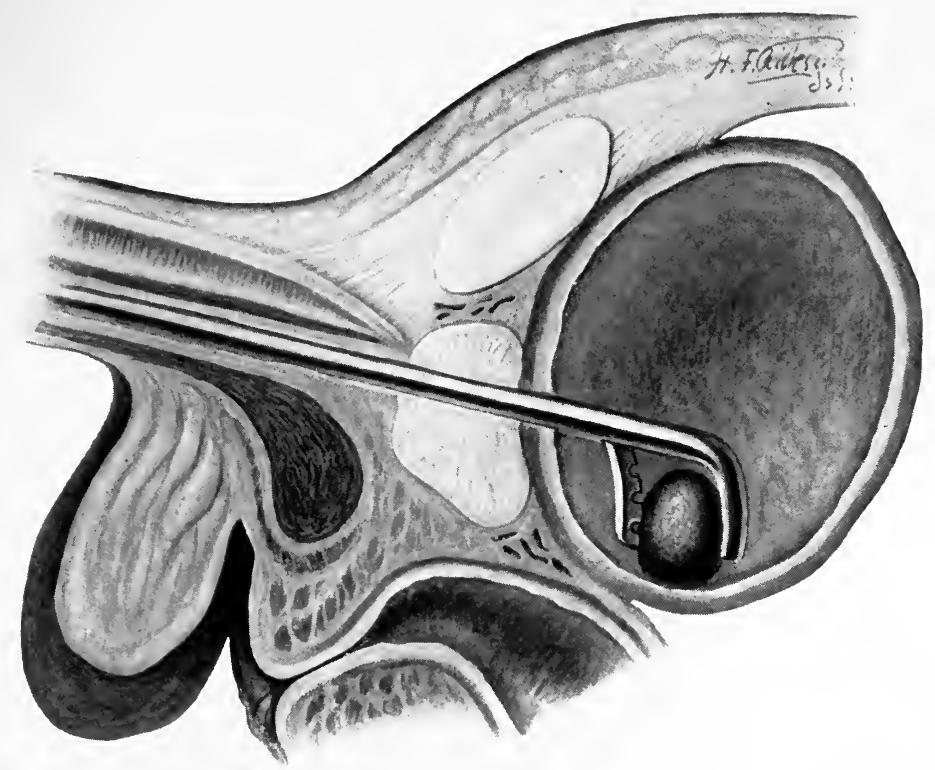

Lithotrite turned over behind prostate.

and from it fill the bladder with from six to eight ounces, more or less, according to circumstances. Refill the bulb by the tube on the top of it and begin the evacuation.

In using the evacuating apparatus, the following rules should be observed:

I. The straight tube is passed safely by pushing it forward in a perpendicular line until its tip is arrested at the bulbomembranous junction of the urethra; the penis is then to be stretched forward on the tube, the latter brought somewhat below the horizontal plane and passed onward into the bladder by a series of gentle corkscrew-like movements, lowering the outer end of the tube toward the floor while making them. 
In cases of prostatic hypertrophy we prefer the curved tube, because of its greater safety and the greater ease with which it can be passed.

The advantage of the straight tube is that the fragments can be more rapidly evacuated by it than by the curved one.

2. The quickest evacuation of the fragments is secured by pressing the end of the tube gently downward upon the floor of the bladder just behind the base of the prostate. This creates a funnel-shaped depression,

FIG. 33I

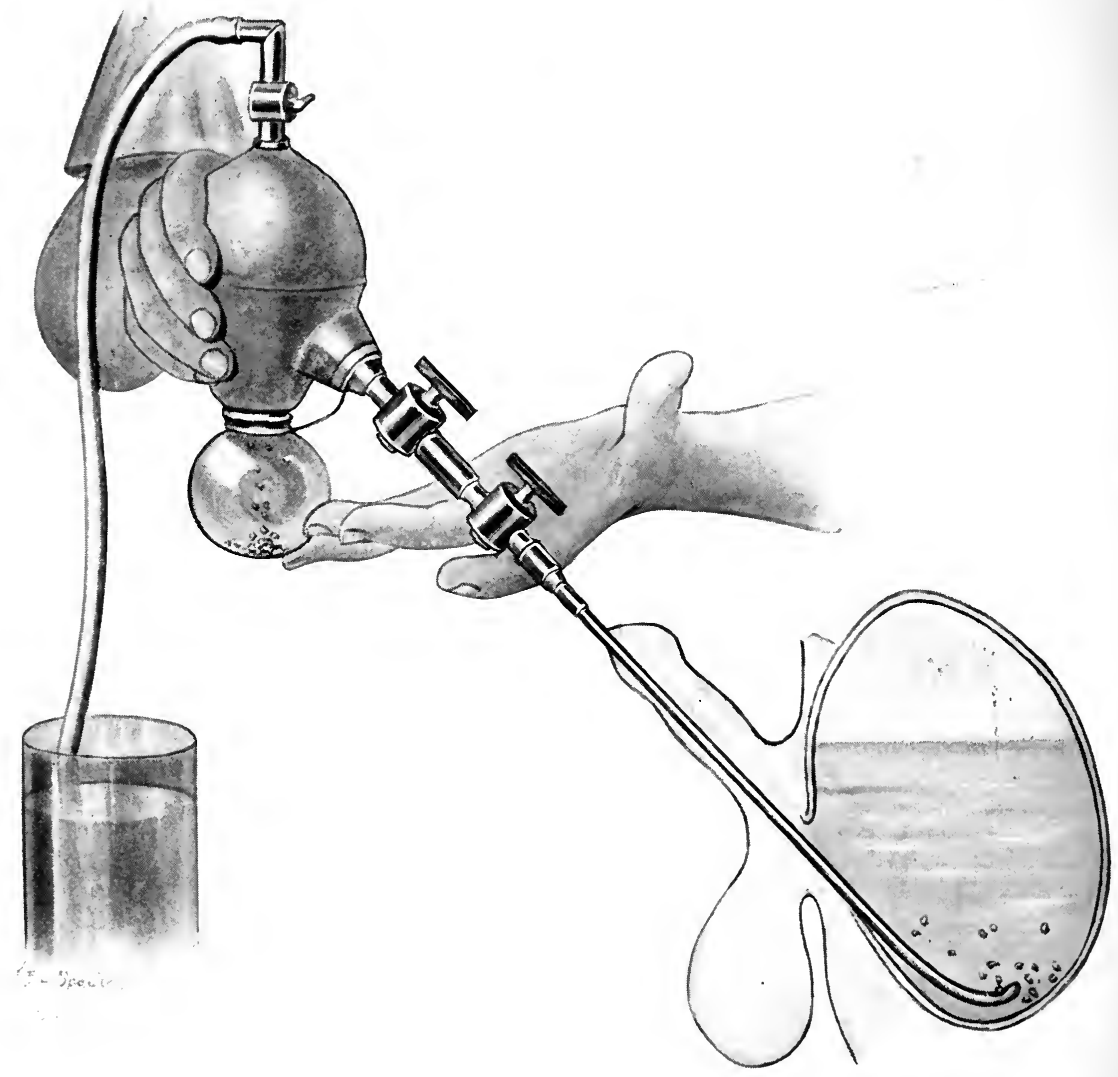

Bigelow's evacuating apparatus withdrawing fragments of calculus from the bladder.

into which the calculus debris and fragments naturally tend to gravitate and to become focussed (Fig. 33I).

3. At whatever point within the bladder the end of the tube may be at which it is found that the fragments and sand continue to be sucked out in abundance, keep it there until this ceases to be the case. "Stay where the fishing is good," is a well-known maxim of fishermen, and it might well be applied to this part of the operation of litholapaxy. 
4. In cases in which fragments are lodged beneath a salient intravesical projection of an hypertrophied prostate, they may sometimes be disengaged by raising the hips and by turning the end of the evacuating tube so that its mouth directly faces the prostate and then giving to the bulb a short sharp pressure and immediately relaxing the hold upon it.

If these manœuvers fail to remove the fragment from this locality, the urethra should be opened by an external perirenal urethrotomy incision in the ordinary way, the finger should be passed into the bladder, and the fragment dislodged by it. If it cannot be done in this way, the only thing left to do is a suprapubic cystotomy and the extraction of the calculus by that road.

5. Care should always be taken in withdrawing the evacuating tube that a fragment is not lodged in its mouth and projecting from it. When such is the case, it must be removed from the tube by driving it back into the bladder with the metal rod which is made for the purpose. The operator will be warned beforehand of the presence of a fragment in the mouth of the tube by the slower return of the back-flow current of the water through it and the failure of the bulb to quickly. expand as it should do at that moment; also, at the moment of the passing of the tube's mouth through the bladder orifice, by the obvious fact that it is being caught and detained there. The operator should at once desist from any attempt to withdraw the tube in the presence of either of these occurrences, and should remove the fragment before doing anything further.

6. In compressing the bulb of the evacuator, the movement should be deliberate and not too quickly repeated, else the debris does not have time to reach the trap beneath the bulb and may be washed back into the bladder again. The bulb should be held in the manner shown in Fig. 33I.

7. When the bladder has not been sufficiently distended with fluid, its walls, which are then slack, are liable to be sucked into the orifice of the tube with the back-flow of the fluid. This fact is announced by a series of trembling, jerky sensations conveyed to the hands of the operator. They resemble, and were spoken of by Professor Bigelow as, "the fish bite." When this happens, more fluid should be injected into the bladder. This can be done without disconnecting the evacuating bulb from the tube, there being a rubber tube connecting with the top of the bulb by which it can be filled, so that it is only necessary to turn the switch connecting the bulb with the evacuating tube so as to shut off the connection between the two, after having emptied part of the contents of the bulb into the bladder, and then to refill the bulb by the tube at its summit. 
8. The evacuation should be continued as long as there is a fairly free return of the sand and fragments into the trap of the bulb. When this ceases, time will be saved by discontinuing the evacuation and by resuming the crushing. Bits of the stone too large to pass into the tube give notice of the fact by the sharp click which they make in striking the mouth of it as the return flow of the fluid draws them against it.

After-treatment.-The after-treatment in most cases is simple. Drainage of the bladder is not often required. Occasionally retention of urine follows the operation, and it will then be necessary to tie a catheter into the bladder. In the cases in which there is a severe cystitis the catheter may advantageously be used for continuous drainage of the bladder for a longer or shorter time, as may be indicated. Irrigations of the bladderpreferably with 5 per cent. argyrol, or I to 4000 permanganate of potash solutions-are the best means by which to relieve cystitis.

The bladder should a ways be examined with the evacuating apparatus and cystoscope before discharging the patient, to make sure that no fragment has been left behind. This examination is best made about a week or ten days after the operation.

Difficulties, Complications, Dangers, Objections, Advantages.-Enlarged Prostate.-The most common difficulty encountered in the performance of litholapaxy arises from the enlarged prostate. This condition is an obstacle to the ready performance of the operation in two respects: (I) Because of rendering the passage of the instruments into the bladder difficult and, in some cases, dangerous; and (2) because of the lodging of fragments of small calculi beneath overhanging intravesical projections of the gland, where they may escape detection and from which it may be difficult or impossible to remove them.

Injury to the prostatic urethra is one of the most serious of the accidents that can occur. It should never happen in the hands of a skilled lithotritist. The second of the two difficulties, that of leaving a fragment in the bladder, may make the operation a failure, and may happen to anyone, no matter how skilled he may be.

Size and Character of the Stone.-Very large stones may be successfully removed by litholapaxy, but the difficulties presented by them, especially if they are hard, may make lithotomy preferable. The large size of the calculus may cause any or all of the following difficulties: It may be impossible to pass the blades of the lithotrite between the bladder wall and the stone. The stone may be too large to allow the blades of the lithotrite to grasp it between them and then to lock the instrument in order to crush. The stone may be so hard that it cannot be crushed, though this is rare. Encysted Stone.-If such a stone lies in a narrow-mouthed diverticulum, litholapaxy cannot be successfully applied to it. If the pouch has a wide 
mouth, the stone may be crushed in the chamber of the diverticulum itself and washed out, which has been accomplished by Freyer, ${ }^{4}$ or it may, if not too tightly held in the diverticulum, be withdrawn from it into the bladder and then crushed more safely and readily.

The crushing of a calculus in a diverticulum is not without danger. This is because of the possibility of catching the rim of the mouth of the pouch with the blades of the lithotrite, and again because of the likelihood of tearing through its wall when thin, as it sometimes is. Personally, we prefer to cut for encysted stone rather than to crush it, as Freyer has done.

Stricture of the Urethra.-This is a complication that is of but little consequence in any but the cases of very dense and narrow strictures. We do not like to employ internal urethrotomy in these cases in connection with the operation, except in the manner to be referred to presently. Divulsion we think an objectionable method per se, and if the stricture cannot be readily dilated, we prefer to substitute perineal for the ordinary litholapaxy, treating the stricture, if it is situated in the perineal urethra, by an external urethrotomy. If there is a stricture of the anterior urethra as well, we should always divide it by an internal urethrotomy in preference to any other method, but only after the stone has been removed, and not as a preliminary step to that operation. This is because of the greater liability to the occurrence of constitutional symptoms than would ordinarily be the case, caused by the irritation of the raw surface of an internal urethrotomy incision by sand and instrumental movements in the urethra during the performance of litholapaxy, were the urethrotomy to be done as a preliminary step to that operation.

Litholapaxy in Children.--Keegan ${ }^{5}$ first demonstrated the success of this operation in male children. In 1896 Freyer $^{4}$ had done the operation upon 165 male children. There was no death in the first II 9 of these cases, and but 2 in the whole number.

The only changes required in the operation are reductions in sizes of the lithotrites and evacuating tubes. According to Freyer, instruments of from 12 to 17 of the French scale are suitable for children between five and twelve years of age, and 17 to 22 for boys of from thirteen to seventeen years of age.

The youngest patient upon whom Freyer operated was eighteen months old. In this case he employed a lithotrite having the size of No. Io and an evacuating tube of No. I I of the French scale.

Both Keegan and Freyer recommend a lithotrite with a completely fenestrated blade. Both regard the use of the unfenestrated blades as "dangerous and unwarrantable."

The only point of special difference in the performance of the operation 
in children and in adults is that greater delicacy must be employed in all the instrumentation in the case of the former.

The operation in children is more tedious than in adults, partly because of the greater delicacy required in the manipulations, partly owing to the necessarily weaker and smaller lithotrites and the small caliber of the evacuating tubes; the latter feature makes it necessary to crush the stone finer before it can be made to pass through the tubes, and, again, the current through them is weaker and slower than it is in the larger ones used with adults. Notwithstanding these objections, the operation has had quite as brilliant a record as it has gained for itself with older persons.

Perineal Litholapaxy.-This operation consists in the removal of vesical calculus through the ordinary median external perineal urethrotomy incision after crushing it into fragments small enough to permit of their being withdrawn through the wound in the blades of the forceps, or washed out through an evacuating tube. The former manner of accomplishing the operation is, to all intents, Dolbeau's operation; the latter is the Bigelow operation done through a perineal urethrotomy incision : nstead of through the whole urethra.

Harrison, and again Forbes Keith, ${ }^{6}$ have both urged the adoption of this method of operating in certain cases, viz., those of patients with prostatic hypertrophy. The crushing instrument of Harrison (Fig. 332) is well suited for the purpose of breaking the stone into fragments.

There is absolutely no need, in fact there is decided objection, to doing this operation as is sometimes directed; by Keyes, for example, who says that after opening the membranous urethra at the apex of the prostate the prostatic urethra and the vesical outlet are to be divided by an incision through the floor of the urethra and extended into the bladder.

The stone can perfectly well be removed through the ordinary urethrotomy incision, after being crushed.

Instruments (Fig. 332).-Two scalpels; grooved staff; large steel sounds; Harrison's stone-crushing forceps; Bigelow's evacuating apparatus; I pair of stone-extracting forceps; soft-rubber catheter.

Operation.-Open the membranous urethra upon a grooved staff, previously passed from the meatus into the bladder. Withdraw the staff. Dilate the prostatic urethra. Pass the crushing instrument-Harrison's forceps or largest size Bigelow lithotrite-through the external urethrotomy incision into the bladder. Grasp the stone between the blades in the manner already described and crush it. Withdraw the fragments by stone-extracting forceps, or remove them with an evacuator when they have been made small enough for the purpose. If extracted with the forceps, be careful not to allow edges of the fragments to project beyond the blades and thus tear the urethra in their passage through it. 
Comment upon the Different Methods.-In considering the advantages and disadvantages of the different operative methods of treating vesical calculus, one fact stands out conspicuously before all others, which is, that at whatever age litholapaxy is done, its operative mortality is distinctly less than that of any other procedure (see chapter on Vesical Calculus). Other things being equal, therefore, we should naturally give the preference to this method whenever possible. It has, however, certain limitations, and they are such that it cannot be applied to all cases alike. We have seen the obstacles and the difficulties which may

Fig. 332

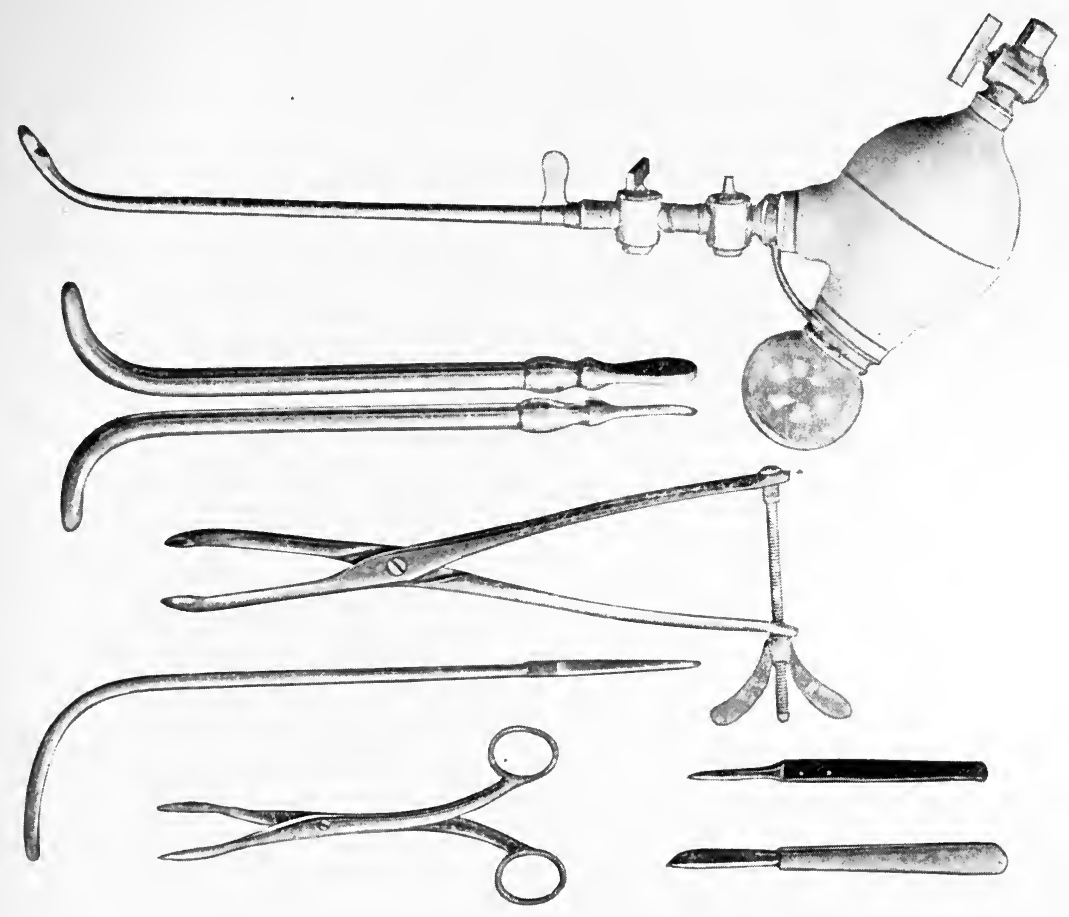

Instruments for perineal litholapaxy and Harrison's lithoclast.

interfere with its successful performance, and in the presence of one or more of them a cutting operation should be preferred to litholapaxy. In the absence of such difficulties and complications as have been specified, this operation is unquestionably the one of choice. It requires, however, natural dexterity and some practice before it can be well and safely done. This explains why so many surgeons select suprapubic lithotomy in its stead. The latter operation does not demand unusual skill, and is consequently within the reach of all ordinarily good surgeons.

In the cases in which difficulty in introducing the instruments used 
in litholapaxy occurs because of prostatic enlargement, perineal litholapaxy is, in our belief, the best substitute for litholapaxy as done in the ordinary manner; but in cases of encysted calculus, or those in which it is probable that there will be left behind in the bladder beneath an intravesical projection of an hypertrophied prostate one or more fragments after the crushing by litholapaxy, the suprapubic method of lithotomy is that of choice. Lateral and bilateral lithotomy are practically abandoned to-day except in the case of the former method for children, in which its mortality has always been low, and for this reason, and the lack of requisite skill to perform the crushing operation, a good many surgeons still cling to it. Practically speaking, there are but three operations which require to be known at the present time for the treatment of vesical calculus: litholapaxy, perineal litholapaxy, and suprapubic lithotomy. Each has its place, and of the three, litholapaxy is the most valuable and the best in all cases which are appropriate for it and in the hands of a surgeon skilled in its performance.

There is one special advantage attaching to perineal litholapaxy, and that is that it allows the prostatic urethra and prostate to be explored digitally, and offers the opportunity to remove the prostate by the perineal route if it be thought desirable to do so. The same is true of the suprapubic lithotomy, but the higher mortality attending that method of operating limits its applicability in some measure.

\section{OPERATIONS FOR REMOVAL OF VESICAL TUMORS.}

Removal of the Tumor through the Urethra.-The Procedure of Nitze. -Nitze devised a special form of operating cystoscope, which consisted of the usual cystoscopic tube, to the distal end of which was added a pair of cutting blades, which could be closed or opened by means of a mechanism at the other end of the shaft.

The instrument is introduced with the blades closed. Once within the bladder, they are opened and the tumor is located. When it is found, the end of the instrument is pressed against the tumor or the bladder immediately adjacent to its base, and the latter is caught between the blades and bitten through by them. The tumor is then withdrawn, together with the instrument.

Removal of the Tumor through an External Perineal Urethrotomy Incision.-This method of attacking tumors of the bladder, which was advocated by Sir Henry Thompson, ${ }^{7}$ is no longer employed, and calls for passing notice only. The operation was performed by making a digital exploration of the interior of the bladder through an external perineal urethrotomy incision, as its first step. The tumor having been located 
by this means, forceps with biting blades were passed into the bladder, and, guided by the finger, the base of the growth was seized between the blades and bitten through or twisted off.

Removal of the Tumor through the Suprapubic Cystotomy Incision, without Resection of the Bladder.-This operation consists in removing the tumor by means of one or another special kind of instrument, through a suprapubic cystotomy incision, which may be the ordinary longitudinal one, or, if the conditions demand a wider field of approach, it may be gained by modifying the operation in one or another of the ways described under the heading Modifications of Suprapubic Cystotomy.

The removal of the tumor may be accomplished by a wire snare, by scissors, or by the cautery. We prefer the galvanocautery scissors of Watson for the purpose (Fig. 333).

This instrument has a platinum wire upon the upper margin of the inner aspect of one of its blades, which can be heated to any degree that may be desired by the current of an electric battery, to which it is

FIG. 333

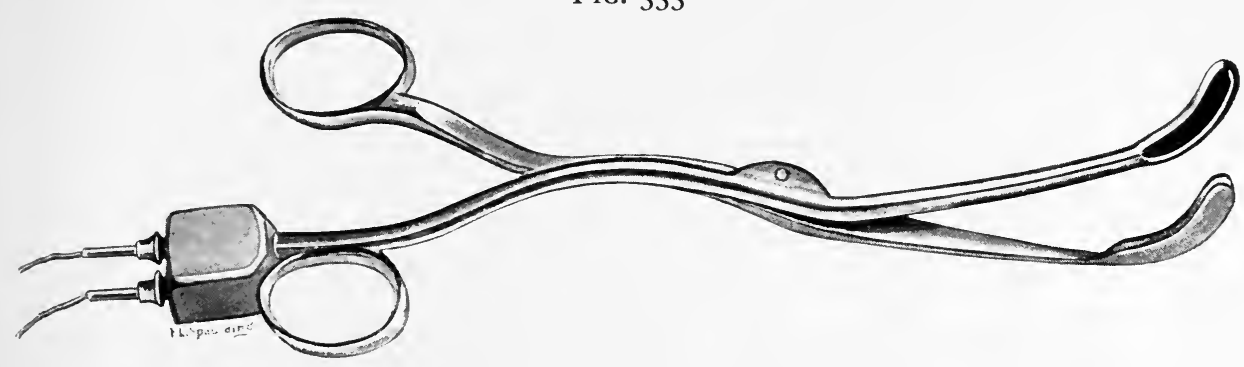

Watson's scissors cautery.

connected by two insulated wires attached to one of the handles of the scissors cautery. The base of the growth is seized with the blades of the instrument and burned through with the heated wire (see Fig. 334). The space left open by the operation may be allowed to remain so or may be closed by suture. The latter is the preferable manner of dealing with the wound resulting from the removal of the growth. Whatever may be the method employed for taking away the tumor, a margin of the sound mucous membrane around the base of it should be included in the part to be removed.

The mucous membrane is normally movable upon the underlying tissues of the bladder, and, in the case of benign growths of the organ, the base of the growth can be moved upon the structure beneath it. This is the principal evidence of the benign character of the tumor, by which it may be distinguished at the time of operation. All tumors of which this is not true should be operated upon by resection or total extirpation of the bladder and not by simple excision. 
Partial Resection.-The first partial resection of the bladder was done by Morton ${ }^{8}$ in 1879 . The operation was carried out through the vesicovaginal septum. The patient survived, and was cured.

The first abdominal resection of the bladder was done by Sonnenburg, ${ }^{9}$ in 1884 . The patient died five weeks later. The interesting observation is recorded in connection with this operation, that a new bladder was

FIG. 334

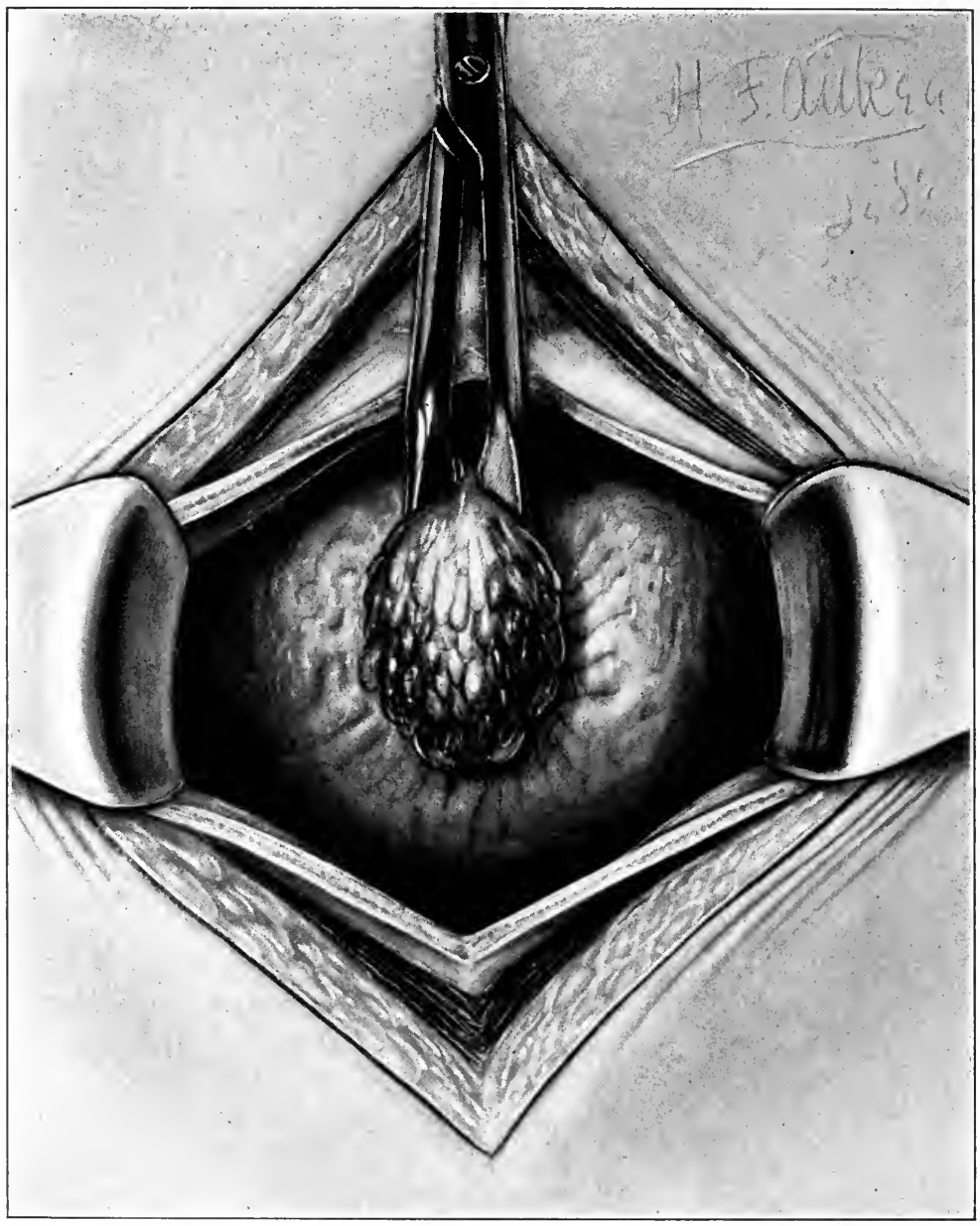

Removal of tumor of the bladder with Watson's cautery.

in process of formation, and that it was in part already functionally capable. It was being formed between the peritoneum on one side and the remnant of the bladder on the other.

Instruments.-The same as in the operation last described.

Clado's Operation.-Expose the bladder by a suprapubic cystotomy incision. Open the bladder; pass a pair of forceps through this incision; 
seize the base of the growth with them and draw it upward. When this is done, the form of a fold is given to the adjacent part of the bladder.

Pass a row of catgut sutures through the base of this fold, behind the blades of the forceps, and through the entire thickness of the invaginated bladder wall.

Divide the fold of the bladder and base of the tumor close to the upper surface of the blades of the forceps.

Remove the forceps and tie the sutures.

Close the cystotomy incision and cleanse the field of operation.

Open the abdominal cavity by extending the original incision of the abdominal wall and the peritoneum. Seek the site of the infolded bladder incision and place a second row of sutures through it external to the bladder and including the peritoneum.

Close the abdominal incision, except at its lower angle, which is left open to allow the passage of a drainage wick.

Comment.-The operation of Clado is applicable to the cases in which the tumor does not involve the floor of the bladder or the ureteral orifices.

Resection of the Symphysis Pubis.-Permanent resection of the symphysis as a method of giving freer access to the bladder for the subsequent operative manœuvers was proposed by Helferich, and was afterward employed by Küster, Israel, Allesandri, and Heydenreich; but one of the patients upon whom it was practised survived.

Temporary resection has been employed by three or four surgeons. (The operation is described and figured earlier in this chapter.)

Partial Resection of the Bladder in Cases in which its Floor is Involved by the Tumor.-(For technique of the operation when the orifices of the ureters are not involved by the disease see chapter on the Technique of Operations on the Prostate.)

In the cases in which the orifices of the ureters are involved, one or both of the ureters must be divided and transplanted. (The technique of these operations will be found in the chapter on the Technique of Operations upon the Ureters.)

The bladder part of the operation is essentially the same as that of the lower part of the operation of total extirpation, and may be incorporated together with it in the description.

\section{TOTAL EXTIRPATION OF THE BLADDER.}

First done by Bardenheuer in 1887 ; the patient did not live. The first successful case was done in the following year, I888, by Pawlik. Pawlik's operation was carried out in the following manner. 
A woman, aged fifty-six years, had been operated upon for a small vesical tumor by Pawlik one year before. Recurrence took place. The bladder was extirpated by two distinct operations. In the first of these the ureters were implanted in the upper end of the vagina. When these wounds had healed and the urinary stream had been successfully diverted, the second operation was carried out thus:

The organ was filled with ten ounces of iodoform emulsion; it was then exposed above the symphysis, and stripped of its peritoneal investment. The contents of the bladder was evacuated. The anterior wall of the vagina was incised, the bladder drawn through this incision, cut across just above its outlet, and removed.

The posterior end of the urethra was then connected by suture with the anterior wall of the vagina in front, and its posterior wall behind.

A reservoir was thus formed out of the vagina, into the upper end of which the ureters entered, and the lower end of which was in continuity with the urethra. The woman recovered, and lived for fifteen years.

Between the time of Bardenheuer's operation and the present year (I907) forty operations of total extirpation of the bladder have been done. (See table of these cases in chapter on Tumors of the Bladder.)

The changes that the operation has undergone have related for the most part to the treatment of the ureters. These have been implanted in the intestine, in the vagina, brought out onto the surface and attached there, either on the front of the abdomen or in the loin, and they have been abandoned in the operative field.

The bladder has been exposed more freely by temporary resection of the symphysis pubis, and, again, by division of the insertions of the recti muscles. A crescentic transverse incision has been employed, and finally, in a few cases, a combined suprapubic and perineal operation has been done. In one case, total emasculation was added to the operation for extirpation of the bladder.

In I905 Watson proposed bilateral lumbar nephrostomy and tying off the ureters close to the renal pelves as a preliminary step to total extirpation of the bladder, and in 1907 Bottomley proposed the implantation of both ureters to the loin-as was done by Le Dentu, Pozzi, ${ }^{10}$ Wassiljew, and others-as a preliminary step to total extirpation of the bladder. (See chapter on Tumors of the Bladder.)

The Operation of Total Extirpation of the Bladder.-Instruments.Two scalpels; 2 pairs of blunt-pointed scissors, one of them curved and with long handle; 3 abdominal retractors; 3 pairs of strong right-angle clamp forceps; 2 vulsellum forceps; 2 aneurysm needles; I needle-holder; full-curved round needles; full-curved Hagedorns; straight glover's 
needles; 2 pairs of toothed forceps; Nos. I and 2 chromicized catgut ligatures; Nos. I and 2 chromicized silk ligatures; artery forceps.

Position.-Moderate Trendelenburg.

Preparatory Measures.-Bladder to be emptied, thoroughly irrigated, and about eight ounces of sterile saline solution to be left in it. It is

FrG. 335

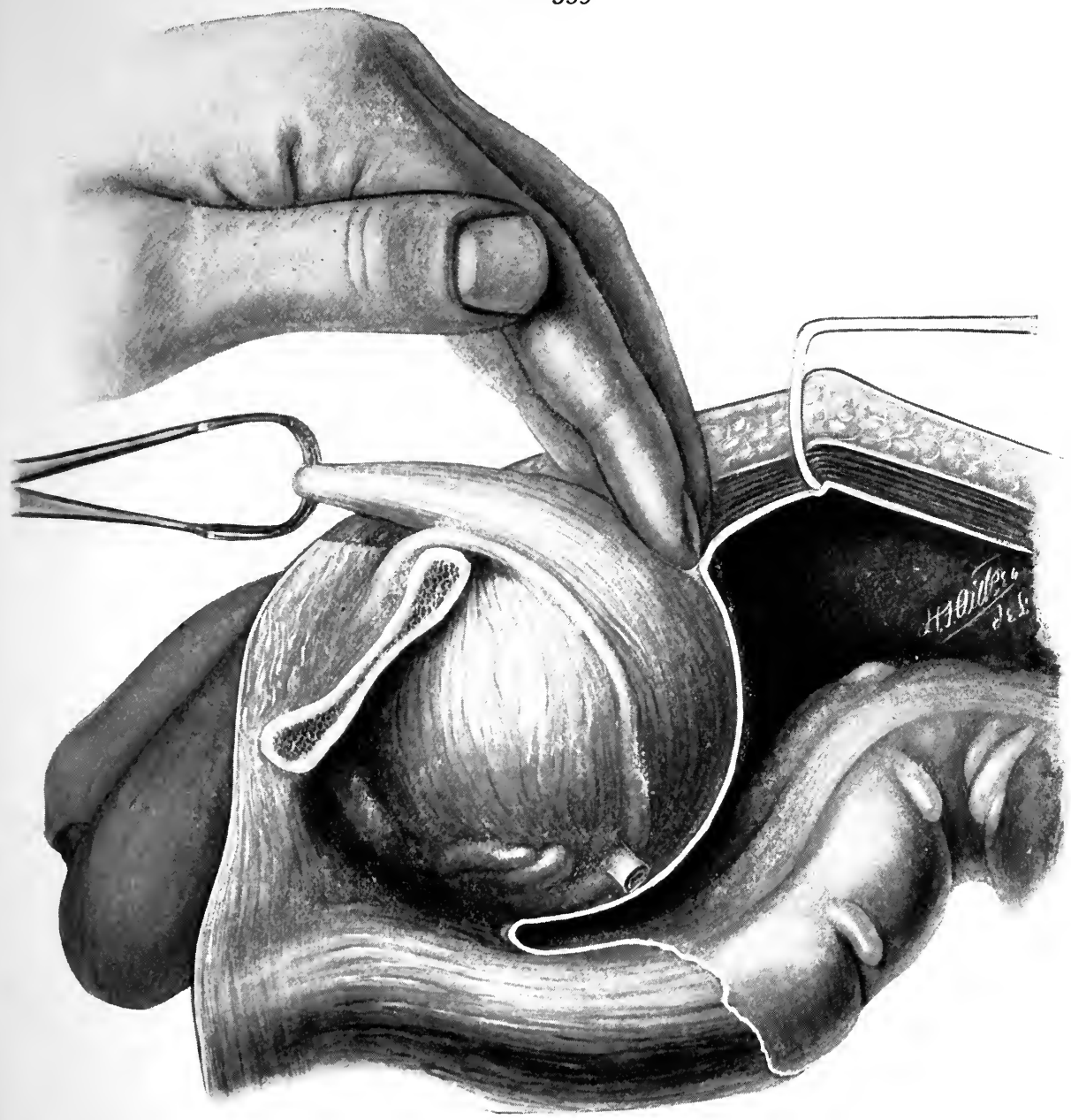

Total extirpation of the bladder. First step.

convenient to have a staff with a long curve passed into the bladder through the urethra before beginning the operation.

Operation.--Incision.- The median or transverse abdominal incisions. (See Suprapubic Cystotomy.)

Expose the anterior surface of the bladder and strip off its peritoneal investment as far as can conveniently be done. Draw down the bladder vol. $\mathrm{I}-38$ 
as this manœuver proceeds, in order to make it more accessible. Continue until the ureters have been reached. These, it will be remembered, have already been tied off at their upper ends in the first operation, if Watson's plan is carried out.

Apply a ligature with an aneurysm needle to each ureter as high up as can be done, and divide them. Draw the bladder upward as far over the symphysis as possible, complete the removal of its peritoneal invest-

FIG. 336

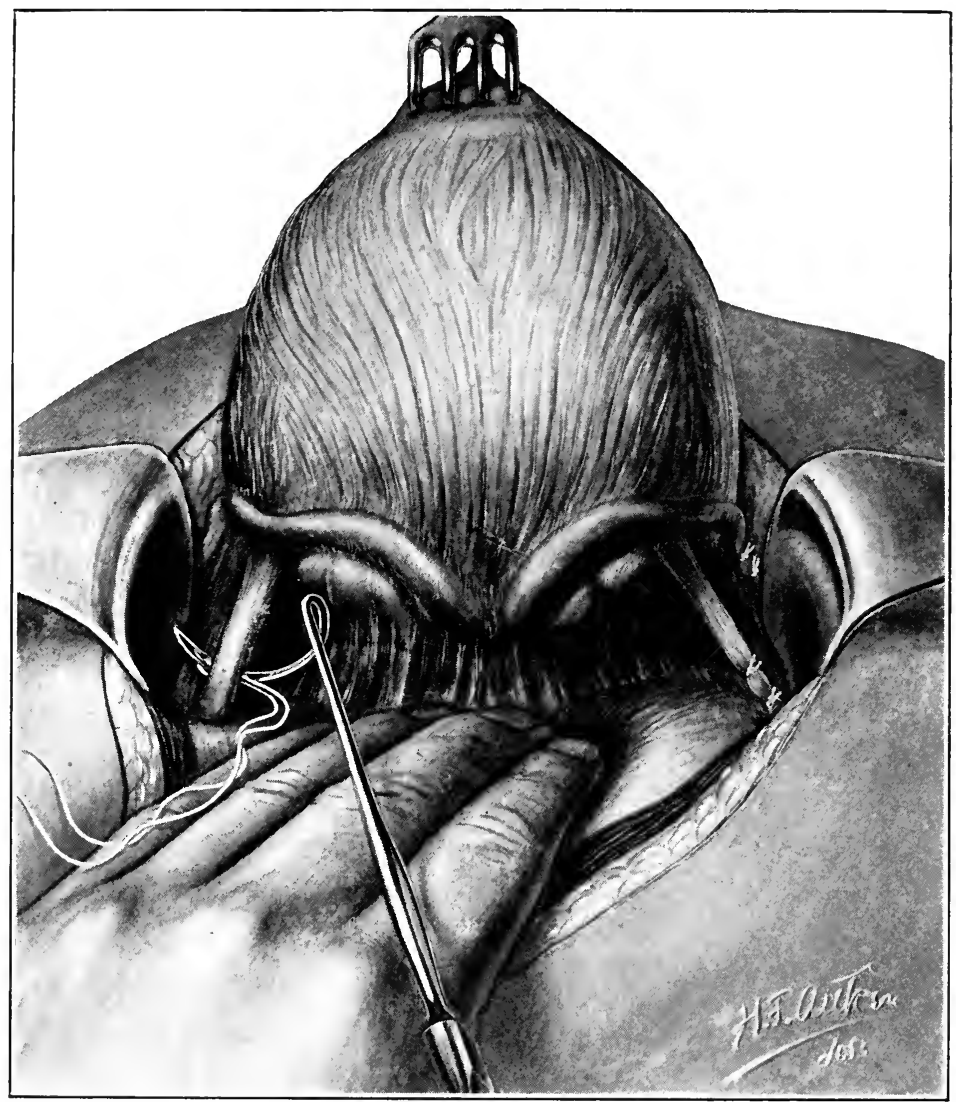

Total extirpation of the bladder. Second step.

ment, and separate its surface and that of the prostate from their connections with the rectum posteriorly.

Lift the bladder well up with vulsellum or other appropriate forceps, and with a curved needle held in the needle-holder pass a double ligature of No. 2 chromicized catgut through the median line of the posterior surface of the lowest part of the neck cf the bladder, letting the needle emerge through the anterior wall at a corresponding point. Cut the 
ligature in two at the eye of the needle. Ligate the neck of the bladder in two halves, including each half in one part of the double ligature.

Divide the bladder by a transverse incision just above the ligatures, and draw the organ out through the wound. Sponge the field of operation, dry and cleanse it.

Make a free counteropening in the median line of the perineum, and pass a gauze drain through it as far up as the peritoneum. Close the abdominal incision tight.

(The steps of the operation are shown in Figs. 335, 336, 337.)

Fig. 337

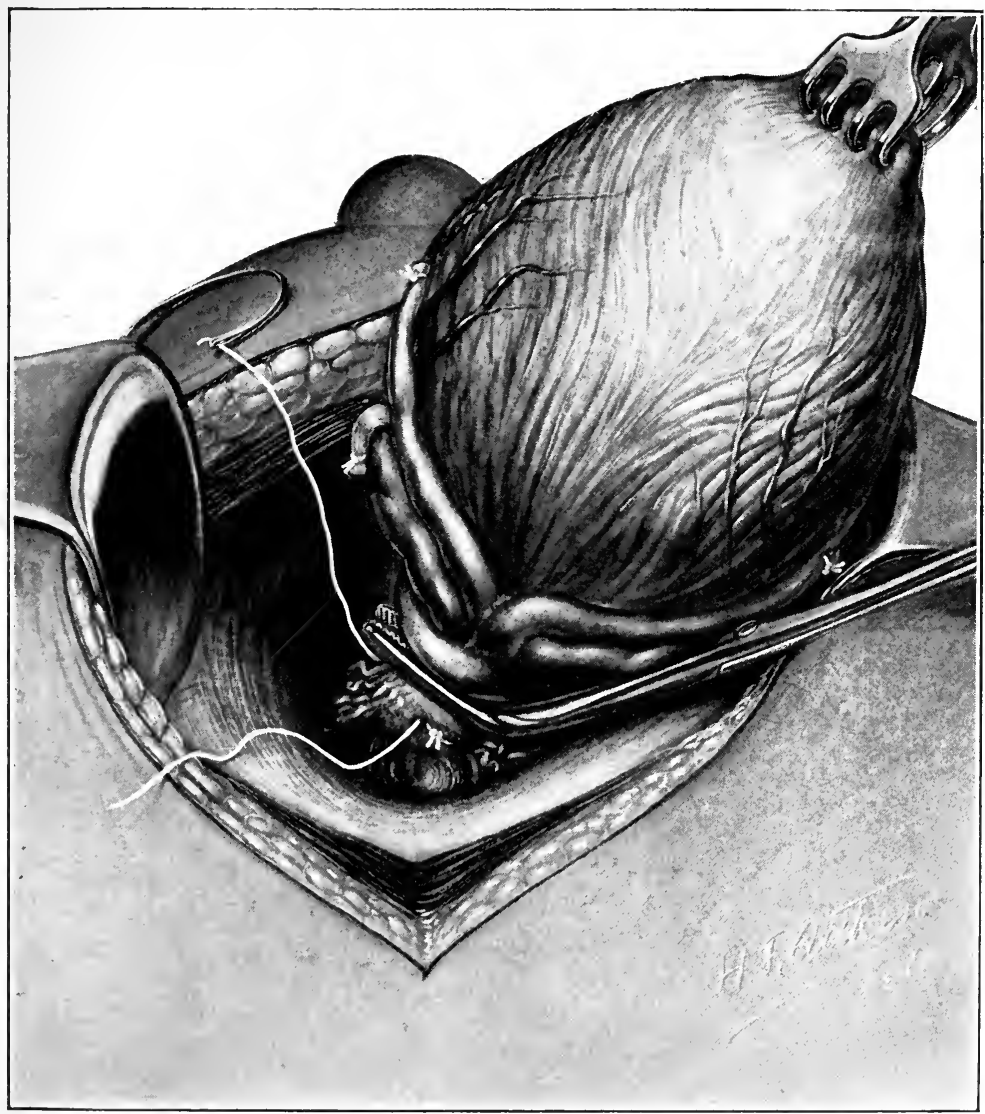

Total extirpation of the bladder. Third step.

If the prostate and (or) seminal vesicles are involved, they should also be removed. In this case the vasa deferentia must be ligated, preferably near the inguinal ring, divided, and removed with the bladder.

The prostate can be removed with the bladder by simply changing the site of the ligatures from the neck of the latter, where they are placed in 
the operation described above, and by passing them through the urethra at the apex of the prostate, tying each one around one-half of the tissues lying immediately to the outer side of each one respectively, including the bloodvessels supplying them.

Comment.-This method of doing the operation, in so far as the manner of dealing with the ureters is concerned, was originally proposed in 1905 by the writer, but he has thus far had no opportunity to put it in practice. The arguments in favor of it will be found in the text of the chapter on Tumors of the Bladder.

The advantages of the method are obvious. The difficult part of the operation of extirpation of the bladder as usually performed consists in the manner of dealing with the ureters. Not only does the time required for their implantation, no matter how it be done, greatly increase the dangers of the operation, but a large proportion of the deaths which have occurred subsequently have been due to the infection of the kidney arising because of insufficient drainage, brought about by contraction of the impaired orifices of the ducts. Both of these dangers will be eliminated in the proposed procedure. Moreover, the performance of the operation upon the bladder will be much facilitated by not having to stop to deal with the ureters, except to tie and cut them off, in order to free the bladder and allow its removal, and shock will be correspondingly diminished.

\section{OPERATIONS UPON DIVERTICULA OF THE BLADDER.}

The operations which have thus far been proposed or performed upon diverticula of the bladder are:

I. Stretching of the orifice.

2. Closure of the orifice by suture (Pousson ${ }^{11}$ ).

3. Ligature and excision of the sac $\left(\right.$ Alexander $\left.{ }^{12}\right)$.

4. Incision and suture of the septum between diverticula and bladder $\left(\right.$ Young ${ }^{13}$ ).

5. Anastomosis between diverticulum and bladder (Ljungren, I897).

The writer does not feel certain of the date of the first excision of a vesical diverticulum, but the case of Alexander above noted is the first, so far as he has been able to learn.

In that instance a diverticulum of the bladder protruded from the vulva. It contained a calculus. The diverticulum, after being incised and the calculus removed, was excised after having been ligatured around its neck. The patient recovered.

Lenander, in 1896 , endeavored to divide the septum between the 
diverticulum and the bladder by passing a suture through the septum and then ligating it tightly, the intention being to have the ligature cut its way through the partition. This failed, and he then attached a pair of forceps to the partition and left them clamped upon it. This manœuver produced necrosis, and thus did accomplish the object.

The patient died, but not as the result of the operation.

Young sought the same end by the procedure described by him as follows, in connection with a case which he reports:

"Suprapubic cystotomy. Just back of the interureteral bar was the orifice of a subtrigonal diverticulum. A second diverticulum lay to the outer side of the ureteral orifice. Fig. $33^{8}$ shows the positions of the diverticula and their orifices.

FIG. 338

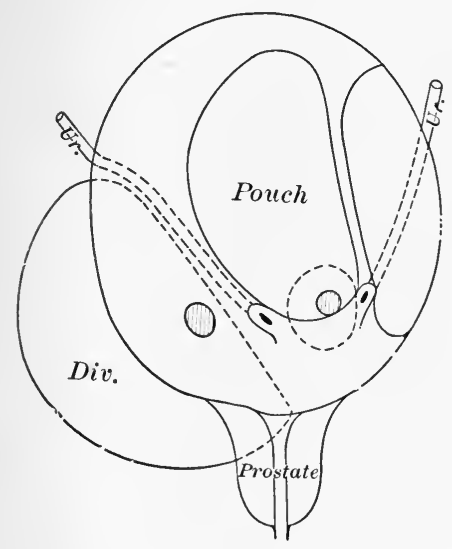

Diagram showing bladder and position of diverticula and pouches. (Young.)
Fig. 339

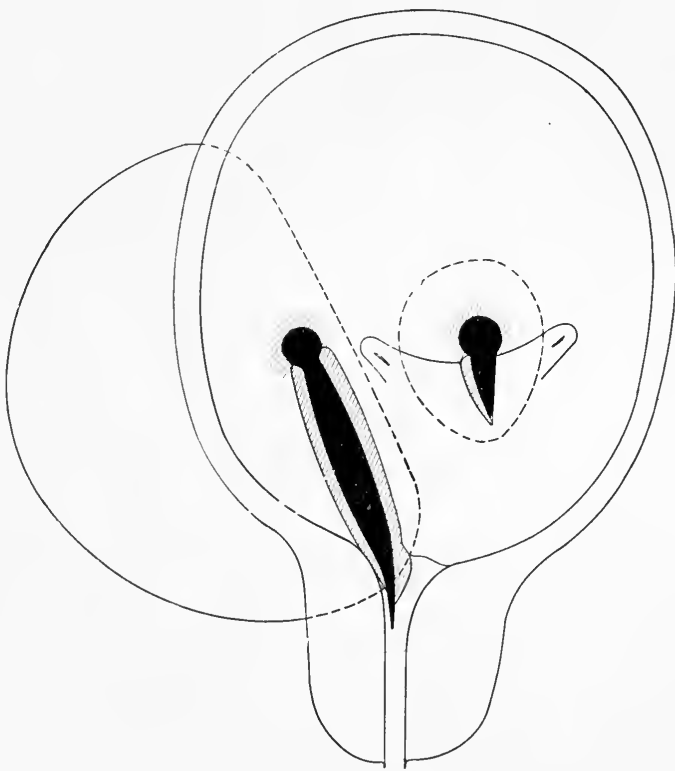

Showing the division of septa made at operation.

(Young.)

"The larger of the diverticula pushed the floor of the bladder to one side and obstructed the escape of urine from the vesical outlet. It was therefore decided to attack this one.

"In order to do this (to divide the septum between the diverticulum and the bladder) my prostatic tractor, closed, was introduced into the orifice of the diverticulum, and the blades separated . . . and by drawing the base of the bladder into the wound with the tractor it was an easy matter to divide the septum. 
"The incision was carried in a line between the orifice of the diverticulum and the urethra, so that the posterior portion of the urethra was made to enter directly into the diverticulum.

"The edges of the wound were then sutured together with six interrupted sutures of catgut on each side, thus uniting the mucous membrane of the diverticulum and the bladder on each side of the incision and throwing the two cavities together."

A similar procedure was carried out upon the second diverticulum, a single suture being in this case placed in the angle of the wound.

The suprapubic cystotomy incision was closed around a small drainage tube, after placing the end of a catheter in the larger of the diverticula and leading it out through the urethra. The outer wound, except the skin, was also closed around the drainage tube with silver sutures.

A fistula above the symphysis persisted for three months, then closed.

More than a year later, the patient reported as being well and comfortable, though he used a catheter at night once, on retiring, to empty the bladder. The viscus contained about 300 c.c. of residual urine.

Fig. 339 shows the lines of the incisions between the bladder and the diverticula.

Comment.-One point is to be remembered when dealing radically with diverticula of the bladder, which is, that they may be exteriorly adherent to very important structures, such as the intestine or pelvic bloodvessels. This is another reason, when they lie in parts of the bladder covered by the peritoneum, for not attacking them from within the viscus primarily.

In the excision of the sacs of diverticula in some of the cases in which they were removed by Young, ${ }^{13}$ the operations were done by a suprapubic extravesical extraperitoneal procedure. The peritoneum was pushed back from the front wall of the organ, and the diverticulum was cut across after the application of a suture about its neck where it joined the bladder, the suture being drawn tight prevented any leakage of urine.

In another instance the ureter had to be transplanted by Young into another part of the bladder. In one case he separated the inner lining of the diverticulum from its outer one, working from with $n$ the bladder, and then, after removing it, he cut off the outer coat of the sac.

When the peritoneal investment of a diverticulum can be stripped off such a procedure as that of Young would be of decided advantage, because it avoids opening the peritoneum. 


\section{Bibliography.}

I. McGuire. Bi-monthly Bulletin, University College of Medicine, Richmond, Va., I897, ii, I83; I898, iii, 37 I.

2. Celsus. Lib. vii, cap. xxvi.

3. Harrison. Annals of Surgery, October, 1893.

4. Freyer. Litholapaxy, I8g6.

5. Keegan. Indian Medical Gazette, 1885.

6. Keith. Lancet, London, September 30, 1893.

7. Sir Henry Thompson. Tumors of the Bladder, I 884.

8. Morton. Transactions of Chirurgical Society, May 9, I879.

9. Sonnenburg. Berlin. klin. Woch., I887, p. 834 .

10. Pozzi. Ann. des Mal. des. Org. Gén.-urin., I891, p. 539.

I I. Pousson. Bull. et Mém. de la Soc. de Chir., Paris, I900, p. i I03.

12. Alexander. Liverpool Medico-chirurgical Journal, July, I884.

13. Young. Johns Hopkins Hospital Reports, I906. 



\section{INDEX TO AUTHORS.}

\begin{tabular}{|c|c|}
\hline 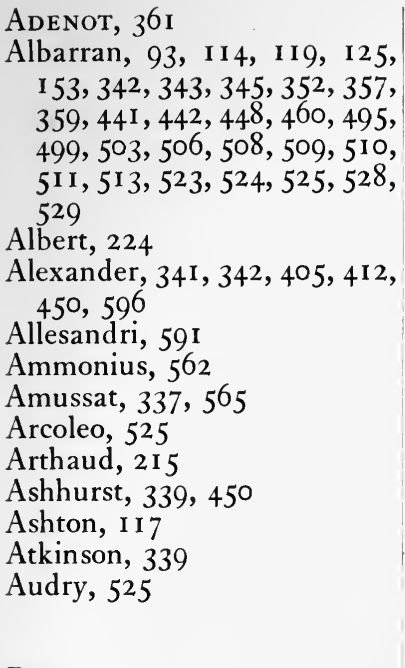 & $\begin{array}{l}\text { Bliss, 459 } \\
\text { Blizzard, 337 } \\
\text { Bottini, 338, 347, 407, 445 } \\
\text { Bottomley, 445, 446, 533, } 592 \\
\text { Boucher, 507 } \\
\text { Bouisson, 230 } \\
\text { Boutelier, 23 } \\
\text { Bozzini, 42 I } \\
\text { Breck, 460 } \\
\text { Brenner, 422 } \\
\text { Broca, 278 } \\
\text { Brodie, 336 } \\
\text { Brooks, 357 } \\
\text { Brown, I I } \\
\text { Brown, F. Tilden, I 26, 446 } \\
\text { Browne, Buckston, 57 I } \\
\text { Bruchmar, 507, 540 } \\
\text { Bruns, 2 I 7 } \\
\text { Bryson, 405 } \\
\text { Burckhardt, 509 } \\
\text { Burnett, 225 }\end{array}$ \\
\hline
\end{tabular}

Baermann, 291

Baillie, 2 I 4

Bangs, 343

Bardenheuer, 290, 520, 522, 528, 529, 591, 592

Barney, 50, 5I, 52

Bartels, 448, $45^{\circ}$

Bartrina, 91, 93

Bazy, 96

Beaulieu, Frère Jacques, 563

Beck, 24, I 74, I82, 277

Belfield, 229, 292, 339, 362, 4 I

Bellingham, 215

Benedetti, 565

Beneke, 535

Beneque, 104

Benzla, 225

Berg, 529

Bergenheim, 523, 528

Bevan, 273

Bickersteth, 339

Bierhoff, 460

Bigelow, Henry J., 567, 574, $576,578,583,586$

Billroth, 238, 338, 339, 36r, 4I 4 , 5OI

Blake, J. Bapst, 38

Blanchard, 442

Blasius, 2 I 3,442

Cahen, 525

Carabelli, 356

Carter, 468

Casper, 221, 227, 230, 272, 334,464

Castleman, 222

Ceci, 524

Championnière, 124

Charrière, 577

Chase, 344, 345, 348

Cheselden, 472, 563, 564

Chetwood, 229, 343, 407, 410

Chiari, 230, 27I

Chopart, 336

Ciechanowski, 308

Civiale, 96, 101, 565, 566, 573

Clado, 502, 5 10, 590, 591

Cline, 472

Clover, 566

Cock, 128

Cohnheim, 494

Coley, 278

Columbia, 223

Come, Frère, 564
Conner, 5 Ic

Cooper, Astley, 472

Cornay, 566

Corvillard, 336

Coulson, i 8

Councilman, 495

Crampton, Philip, 566

Crichton, $5^{6} 3$

Cruise, 42 I

Cruveilhier, 2 I 4

Cuneo, 239

Cunningham, I 35, I36, I99, 2I 4, 324, 372, 378

Curling, 21 5,230

Cushing, 362, 45 1,560

Czerny, 27, 352, 362, 363, $364,518,529$

Dalr ymple, 472

Deaver, 336, 343, 373, 374, $375,439,44 \mathrm{I}$

d'Estrees, Debout, 476

Савот, I 53, 333, 34I, 480, d'Etiolles, Leroy, 96, 337, $5^{6} 5$

de Barletta, Marianus Sanctus, 562

De Pezzer, 342, 392

Decloux, 96

Degas, 126

Deguise, 472

Delbet, 93

Delorme, I 26

Desnos, 310, 465, 515, 538, 539

Desormeaux, 42 I

Despres, 124, 472

Dieffenbach, 187

Dittel, 340, 524

Dolbeau, 27, 563, 573, 586

Doot, Jean, 564

Douglas, 564

Duckworth, 35

Ducrey, 43
Dumarquay, 23, I 52

Dumont, 230

Dunlap, 472

Dupeyre, 566

Duplay, 26, 1 74, 179

Dupuytren, 563, 572, 573

Duret, 509 
EARLE, 472

Edison, 42 I

Edler, 448

Eisenstein, 566

Elderton, 565

Englehardt, 359

Englisch, 442.

English, 229

Ephemendes, 2 I 4

Escat, I I 4, 343, 344, 345, 346, $35^{\mathrm{I}}$

FATTOR, 472

Fayer, I I 7

Fenwick, I 24, 430, 492, 5 I I

Ferguson, 343, 398, 399

Fergusson, Sir William, 337, $33^{8}, 339,342,345$

Finger, 87, 224

Fisher, 2 I I, 2 I 4, 42 I

Flieg, $35^{6}$

Flynn, 257

Forgue, I 52

Fourneaux, 222

Fournier, 43, 44, 72, 73, 22 I, 236

Franco, Pierre, 562, 563

Francon, 476

Frank, 24I

Frantz, 530

Frasier, 509

Freeman, 263

Freudenberg, 338, 343, 407, 409

Freyer, 339, 34I, 342, 345, $346,401,484,585$

Frisch, 85

Fuller, I25, 295, 34I, 342, $343,363,364,401,415$, 540

Fürbringer, 27 I

GaRdner, 23 I

Garré, 523, 528

Genouville, I 26, 254

George, 27 I

Gerota, 44I

Gibbons, 524

Giordano, 520, 521, 528, 529

Goerl, 524

Goodfellow, 259, 340, 34I, $342,345,35^{2}, 372,3^{81}, 3^{84}$, 405

Gopel, 522, 528

Goschler, 23

Gosselin, 236

Gould, 442

Gouley, I I I, I I 3, 338, 339, $340,405,489,49 \mathrm{I}$

Grawitz, 448
Greene, 357, 36I

Gregory, 1 I 8

Griffith, 215

Gruithuisen, 565

Guelliot, 26I, 27 I

Guiard, 99, I I 4

Guibe, 86

Guillon, 99

Guisy, 524

Guiteras, 405

Gürlt, 509

Gussenbauer, 50 I

Guthrie, 337, 491

Guyon, I 18, 124, I25, I 53, 205,524

HAGEDORN, 592

Hallé, 460, 507

Haller, 21 3, 442

Harmer, 472

Harrington, 565

Harris, 23, 363, 41 6, 521,528

Harrison, I I 2, 332, 333, 336, $339,361,362,445,5^{86}$

Harrison, Reginald, i I 7,476

Hartmann, 345, 349, 524

Hasenmayer, 509

Hawley, 357, 359

Hearn, 24I

Heath, 36 I

Heath, Christopher, I I 7, I I 8

Heimann, 357

Helferich, 529, 59I

Henle, 442

Heuerteloupe, 125, 566

Heurteaux, 125

Heusner, I 25

Heydenreich, 59I

Heymann, 460

Hill, I 7

Hochenegg, 205

Hogge, 52I, 523, 528, 54I

Holt, I 7

Hooker, 136

Horand, 227

Horne, Everard, 336

Horton-Smith, 459

Horttung, 223

Horwitz, 343

Howard, 218

Hunter, 215

Hunter, John, 336

Hutchinson, 30, 230, 23I

ISRAEL, 448, 529, 59I

JACK SON, 524

Jacobson, 21 4, 223, 265, 362

James, I 97

Jordan, 222
Josserand, 203

Jouon, I 24

Kaersmaecker, 87

Kalabin, $53^{\circ}$

Kaltenbach, 525

Kaposi, 49

Karstrom, 524

Kauffmann, 24

Kayser, 522

Kazwowski, 223

Keegan, 585

Keith, Forbes, 586

Keyes, 27, 55, 64, 70, 72, 73, $74,79,96,124,153,194$, 2 I 3, 22 I , 222, 224, 225, 226, 227, 230, 23 I, 233, 234, 235, $236,261,283,292,309,410$, $45^{2}, 465,586$

Klebs, 27 I, 503

Knapp, 73

Kober, 239

Kocher, 21 7, 224, 230

Kollmann, I 3

König, I 25, I 53, 448

Kossinski, 520, 528

Krahn, 509

Kraske, 524

Krause, 442, 52 I, 523, 528

Krug, 530

Kümmel, 480, 520, 525, 528

Küster, 362, 4I 5, 4I6, 448, $509,520,528,529,59 \mathrm{I}$

LABOULBENE, 503

Lafaye, 336

Lallemand, 27I

Lancereaux, 235

Lane, 2 I 3

Langhaus, $53^{8}$

Langlebert, 227

Lavaux, 525

Le Dentu, I I 7, I I 8, I 52, 507, 592

Le Sauvage, 508

Ledderhose, 448

Legueu, I 26, 345

Leichtenstern, 495

Leigh, 248

Leisrink, 339, 362, 41 5, 416

Leiter, 42 I, 422

Lenander, 596

Liegeois, 225

Limbeck, 503

Lindner, 522, 528

Livio, 508,536

Ljungren, 596

Lockwood, 214

Loquin, 125

Lorthior, 24 
Lotzbeck, 560

Loumeau, 24

Low, 197

Lucke, 26

Lund, 521,528

Luraschi, 356

Luschka, 237, 442

Lusk, 4I 4

Lydston, 24, 42

Lynn, Thomas, 124

$\mathrm{McCosh}, 522,523,528$

McGill, 337, 339, 340, 384, 401

McGuire, Hunter, $55^{8}$

McKenzie, 198

McLeod, 230

Maisonneuve, I I3, I 8

Majenski, 524

Malassez, 222

Malecot, 39

Malherbe, 525

Mallez, i 8

Manley, 124

Mann, 523, 528

Manson, I 97,256

Marchand, 507, 539

Marchand, G., 524

Marshall, 27, 2I 4

Marsinna, 248

Martel, 503, 525

Martin, 58, 193, 227, 242, 34I

Martini, 507, 510, 536

Mastin, 96, I I 8

Mayo, I 77, I 78, 345, 472

Meckel, 27I

Mercier, 336, 337, 405

Mikulicz, 529

Milton, 472

Mitchell, $447,45^{\circ}, 45^{2}$

Modlinski, 523, 528

Mollière, I25, I 53

Monks, George, 488

Monod, 210, 21 3, 215

Morton, 590

Moskowicz, 356

Motz, 91, 93, 310, 442, 46o, 501,525

Moullin, 309, 310, 3I3 339, 342

Multanowski, 472

Murphy, 2I6, 290, 343, 345, 347,349

Nauman, 524

Nebel, 442

Neisser, 55

Nicolich, I 1 8, 509, 524, 525

Nicoll, 340, 341, 342, 343, $355,403,404,405$
Nitze, 42I, 422, 425, 427, 516, Ravisini, 460 $525,526,588$

Noble, 450

Nogues, 155

ODIORNE, 214

Ohlmacher, 466

Olympios, 565

Ord, 468,478

Orth, 27I

Otis, 98, 99, 100, 103, 1 8 , I 20, 577

Paget, 35

Paget, Sir James, 230

Pantalin, I 70

Park, 248

Parker, 524

Parona, 362

Pascale, 286, 287, 29I

Pasteau, 254, 509

Pauchet, 345

Paulus, 562

Pavone, 524

Pawlik, 520, 523, 528, 59 I

Pepper, 537

Percy, 136

Pernice, 530

Petersen, $5^{6} 4$

Petroff, 448

Pirogoff, 566

Pizzini, 223

Platt, I 25

Pollailon, 507, 524

Pollak, 526

Poncet, I 53

Post, 472, 565

Pousson, I I 4, I I 8, 347, 357, 361,596

Pozzi, 592

Predal, 3 Iо

Preston, 472

Proust, 269, 290, 342, 343, $344,345,349,352,355,384$, $385,386,387,390,394,399$, $4 \mathrm{I} 7$

Pyle, 340, 342, 372, 405, 442

Quenu, I 24

Quervain, 448

Quick, $45^{2}$

RAFIN, 345, 50I, 503, 509, $515,516,518,523,526,529$, 530

Rainey, 468, 478

Ramsay, 524

Rau, 563

Sieur, $45^{\circ}$
Reclus, 230

Rehn, 495, 529

Rennes, 24, 27, 214

Reverdin, 509

Revolat, 23

Reynes, I I 4

Rhodius, 442

Richardson, 459

Richardson, W. G., 258, 30 I, $303,343,35^{2}$

Ricord, 48,96

Riesman, 537

Rigby, 2 I 8

Robson, Mayo, 125, 339, 521, 528

Rochet, 503, 525

Rokitannsky, 503, 507

Rokitanski, 27I, 507

Rovsing, 334, 533

Rosenstein, 4IO

Roth, 237

Rousset, $5^{64}$

Routier, 530

Ruggles, $35^{2}$

Rydygier, 390, 565

SAnctorius, 565

Sappey, $44^{2}$

Schatz, 524

Schede, 448, 52 I, 528

Schilling, 529

Schlange, $45^{\circ}$

Schuchard, 529

Schurig, 214

Scibelli, 442

Scudder, I22, 2 I 8

Segond, 503

Senftleben, 238

Senn, 339

Shattock, 536

Simmons, 2 I 4

Simon, 230

Smith, 24, 2 I 5

Smith, Lapthorn, 523

Sobotta, 208

Socin, 125

Sonnenburg, 590

Spangaro, 509

Spanton, 357

Stansbury, 222

Sturgis, 222, 24I

Sutter, 50 I

Syms, 342, 343, 345, 372, 38I

TANSARD, $35^{6}$

Tassi, 524

Taylor, 48, 49, 234

Tedenat, I I 8 
Teevan, I 8

Tenney, 344, 345, 348

Terrier, 524

Terrillon, 213, 222

Theophanes, 565

Thiercelin, 503

Thiersch, I80

Thomas, Lynn, 124

Thompson, 524

Thompson, Sir Henry, 95, 96, I I 8, 310, 333, 374, 469, 472, 480, 48I, 498, 510, 516, Von Bergmann, 284 566,588

Tuffier, $448,520,528,54 \mathrm{I}$

Turetta, 52I, 528

Ullman, $45^{\circ}$

Ultzmann, 79, 264, 46I, 465 , $470,480,5^{1} 3,53^{8}, 5^{6} 5$

Unna, 42, 43

Uytterhoeven, 472

VACHE, 230

Van Hook, I 77

Vasilief, 521, 523

Vaughan, 528

Vaughan, George Tully, 522

Velpeau, 224

Verduc, 472 529

Vidal, 222, 233

Vignard, I 24

Villeneuve, 290

Vincent, 503

Vincini, 529

Virchow, 500

Vitellius, 472

Volkmann, 524
Von Bergmann, 284

Von Frisch, 362

Von Leyden, 223

WAHL, 125

Waitz, 524

Walsh, 96

Wardell, 538

Warholm, 524

Wassilief, 528

Wassiljew, 592 $589,592,594$

Weiss, 566

Welander, 55
Verhoogen, 87, 523, 524, 528, Wendel, 495, 501, 502, 523,

Viljaminow, 528, 54I

Watson, I1 $5,118,134,310$, $334,336,339,340,341,342$, $343,344,345,347,372,376, Z_{\text {ADok, }} 26$ $384,405,406,445,446,5$ I 5, Zeissl, 227, 265 $5 \mathrm{I} 6,526,533,536,539,574$, Zeller, 522, 528 528

Wertheim, 530

Wheelhouse, I 70

White, $58,193,242,341$

Whitehead, 423

Wile, I24

Willy Meyer, 343, 351

Wilms, 203

Winiwarter, 357, 52 I, 54I

Witte, 223

Wittzack, 525

Wolfler, I 53

Woolsey, 522, 528

Wright, 23

Young, 259, 289, 290, 338, $339,342,343,345,346,347$, $349,350,357,358,359,360$, $361,362,363,364,381,384$, $391,398,408,411,416,417$, $4 \mathrm{I} 8,489,596,597$

Ziegler, 230, 239, $24 \mathrm{I}$

Zöller, I 79

Zuckerkandl, 85, 339, 442 


\section{INDEX TO VOLUME I.}

A

AbNormalities of penis, 23

of scrotum, 192

of seminal vesicles, 261

of testicle, 213

of development, 213

of migration, 214

of vas deferens, 202

Abscess of prostate, 364

perineal prostatotomy for, $4 \mathrm{I} 2$

of seminal vesicles, incision of, 295

Fuller's operation of, 295 position of painstruments for, 295

of scrotum, 196

Absence of bladder, 442

of penis, 23

of seminal vesicles, 26I

Acne of penis, 36

Adenoma of bladder, 503

operations in, table of, 525

of testicles, 237

Albarran's ureter cystoscope, 424

Alexander's technique of combined operation on prostate, 405

Allis' operation for phimosis, 162

Alveolar cylindrical-cell carcinoma of bladder, 496

sarcoma of bladder, 497

Amputation of penis, 164

Angioma of bladder, 506

Angiosarcoma of bladder, 497 of testicle, 239

Annular stricture of urethra, 95

Anomalies of bladder, 442

Anorchism, 214

Arteries of corpora cavernosa, I8, 19 of penis, 20 dorsal, 20

of vas deferens, $20 \mathrm{I}$

Aspiration of bladder, suprapubic, 544

Atrophy of testicle in orchitis, 232

\section{B}

Balanitic hypospadias, 24

Balanitis, 40 chronic, 40
Balanitis, complications of, $4 \mathrm{I}$

diagnosis of, 40

from chancroidal ulcer, 40

from herpes, $4 \mathrm{I}$

from syphilitic chancre, 40

etiology of, 40

symptoms of, 40

treatment of, $4 \mathrm{I}$

Balanoposthitis, 40

Beck's operation for epispadias, 182

for hypospadias, I 74 scrotal, 177

for orchidopexy, 273

Benign epithelial papilloma of bladder, 496

Bevan's operation for orchidopexy, 273

Bigelow's divulsor, I39 evacuating apparatus, 575

instruments for litholapaxy, 578

Bilateral perineal lithotomy for vesical calculus, 483

Bilocular hydrocele, 244

Bird's evacuating pump, 577

Bladder, absence of, 442

adenoma of, 503

operations for, table of, 525

anatomy of, $43 \mathrm{I}$

angioma of, 506

anomalies of, 442

bar at neck of, in hypertrophy of prostate, 3I I

bas fond of, in hypertrophy of prostate, 318

bloodvessels of, 440

calculi of, 468 . See Vesical calculus.

cancroid of, 539

carcinoma of, 496,503 synonyms of, 496

cholesteatoma of, $496,497,507,539$

chond roma of, 496

chondrosarcoma of, 535

cysts of, dermoid, 496, 507, 536

hydatid, 507

simple, 503

diverticula of, 488

definition of, 488

diagnosis of, 49 I

location of, 489

number of, 489

operations on, 596

size of, 489

symptoms of, 49I 
Bladder, diverticula of, treatment of, 492 divisions of, 435

double, 489

drainage of, after suprapubic cystotomy, 555

in perineal prostatectomy, $38 \mathrm{I}$ epithelioma of, 496

examination of, in suprapubic cystotomy,

$55^{2}$

exstrophy of, 443

treatment of, 444

extirpation of total, 54I, 59 I

incision in, 593

instruments for, 592

operation of, 592, 593

position in, 593

preparatory measures in, 593

form of, $43 \mathrm{I}$

glands of, 442

in hypertrophy of prostate, 315

drainage of, 333

irrigation of, 333,334

inflammation of, 457. See Cystitis.

injuries of, 447

ligaments of, 436

lymphatics of, 44I

mucous membrane of, 437

multiple, 442

muscles of, $43^{8}$

muscular contracture of, 447

myoma of, 496, 506

operations for, table of, 524

myxoma of, 496,506

nerves of, $44 \mathrm{I}$

operations on, 544

for calculus, $56 \mathrm{I}$

litholapaxy, 565, 574

in children, 585

perineal, 586

lithotomy, 56I perineal, bilateral, $57 \mathrm{I}$

lateral, 562, 567

suprapubic, 563,573

lithotrity, 565

in diverticula of, 596

extirpation of, total, 591, 592

suprapubic aspiration, 544

cystotomy, 545

for tumors, 588

removal through an external perineal urethrotomy incision, 588

through suprapubic cystotomy incision, 589

through urethra, 588

osteochond rosarcoma of, 535

papillæ of, 442

papilloma of, 496,498

benign, development of, 502

synonyms of, 496

patent urachus, 443

treatment of, 443
Bladder, position of, 43I

pouching of, 489

relations of, 431, 433

resection of, 540

partial, 590

instruments for, 590

when floor involved by tumor, 591

rhabdomyoma of, 496, 508, 536

rupture of, 447

definition of, 447

diagnosis of, 448

etiology of, 447

extraperitoneal, 447

drainage in, $45 \mathrm{I}$

suture of wound in, $45 \mathrm{I}$

illustrative cases of, 454

intraperitoneal, 447

drainage in, $45^{I}$

suture of wound in, $45 \mathrm{I}$

location of, 447

pathology of, 447

results of, 447

spontaneous, 447

symptoms of, 448

traumatic, 447

treatment of, $45^{\circ}$

sarcoma of, 497,505

structure of, 436

supports of, 436

suture of, in suprapubic cystotomy, 560

tumors of, 494

age in, 509, 510

arising from connective tissue, 496

from epithelial tissue, 496

causes of death in, table of, 524

classification of, structural, 496

cystitis in, 509

derived from muscular tissue, 496

diagnosis of, $5^{12}$

duration of, 509, 5 I I

examination in, instrumental, $5^{\mathrm{I}} 4$ physical, $5^{\text {I } 4}$

fibrinuria in, $5^{1} 3$

frequency of, 509

hematuria in, $5^{12}$

heterotopic, 496, 497

hydronephrosis in, 509

illustrative cases of, 535

location of, 509, $5 \mathrm{II}$

malignant, propagation of, 508

necrosis in, 508

nephrotomy in, results of, 530

operations on, 588

origin of, 494

pain in, $5 \mathrm{I} 3$

parasitic, 496

partial resection in, table of, 520,528

pericystitis in, 509

prognosis of, 5 I 4

pyonephrosis in, 509

recurrence of, 518 
Bladder, tumors of, secondary changes in, 508 sex in, 509, 510 special characters of, 497 spontaneous expulsion of, $53^{8}$ subdivisions of, 496 symptoms of, $5^{\mathrm{I} 2}$ synonyms of, 496 total extirpation in, table of, 520,528 treatment of, 515 operative, $5^{\mathrm{I}} 5$ conclusions with regard to, 533 results of, ${ }_{5} \mathrm{I} 6$ palliative, 515 ulceration in, 508 urine in, $5^{1} 3$

Blizzard's probe-pointed lithotomy knife, 568 Blood supply of testicles, 210 in vesical calculus, 478

Bloodvessels of bladder, $44^{\circ}$ of prostate, 305

of scrotum, 192

of seminal vesicles, 260

Bottini, galvanocautery operation of, in hypertrophy of prostate, 343 accidents in, $35 \mathrm{I}$ advantages of, 353 complications of, $35 \mathrm{I}$ failures of, $35 \mathrm{I}$ limitations of, 35I prostatectotome, modified by Freudenberg, 407

prostatectotome, 406

by Young, 407

Bougie à boule, I01, 102

catheter, 328

Bougies, filiform, IOI olivary, 102

whalebone, IOI

Boutonnière in stricture of urethra, 147

Brenner irrigating cystoscope, 424

Brown's urethral syringe, $8 \mathrm{I}$

Bubo, chancroidal, 49

Buck's fascia, I9

Bulb of corpus spongiosum, i 8 arteries of, 20

Bulbocavernous muscle, 22

\section{C}

Calculi, prostatic, 367 symptoms of, 369 treatment of, 369

of seminal vesicles, $27 \mathrm{I}$ etiology of, $27 \mathrm{I}$ symptoms of, 272

Calculus, vesical, 468 treatment of, 272

blood in, 478 characteristics of, 470
Calculus, vesical, chemistry of, 469

classification of, 469

color of, 47 I

definition of, 468

diagnosis of, 478

encysted, 473

in lateral perineal lithotomy, $5^{1} 7$ in litholapaxy, $5^{84}$

etiology of, 469

examination in, cystoscopic, 482 physical, 480

with evacuating apparatus, 482 $x$-ray, 480

formation of, 468

frequency of, 469

illustrative cases of, 487

nuclei of, 474

foreign bodies as, 474

number of, 473

operations for, $56 \mathrm{I}$

litholapaxy, 565, 574 in children, 585 perineal, 487,586

lithotomy, 56I perineal bilateral, $483,57 \mathrm{I}$ lateral, $483,562,567$ suprapubic, $487,562,573$

lithotrity, 565

pain in, 478

pathology of, 478

priapism in, 480

primary, 468

secondary, 468

size of, 472

sounding for stone in, errors in, $48 \mathbf{I}$ spontaneous fracture of, 476

stone searcher in, $48 \mathrm{I}$

surface of, 472

symptoms of, 478

treatment of, 483 operative, 483 preventive, 483

urinary system in, 480

urine in, 480

Cancer, epidermoid, of bladder, 496

of penis, 50. See Carcinoma.

Cancroid of bladder, 497, 507, 539

Capsula vera of prostate, 299

Carcinoma of bladder, 496, 503 synonyms of, 496

of penis, 50

age in, $5^{\circ}$

duration of, $5^{\mathrm{I}}$

etiology of, $5^{\circ}$

glandular involvement in, 52

metastases in, 52

occupation in, 50

pathology of, 52

phimosis in, $5 \mathrm{I}$

point of origin of, 5 I

treatment of, 52

venereal disease in, $5 \mathrm{I}$ 
Carcinoma, of prostate, 357 frequency of, 357

of scrotum, I 95 treatment of, 196

of testicles, 238 encephaloid, 238 medullary, 238 prognosis of, 239 scirrhus, 238 treatment of, 239

Castration, 279

bilateral, 279

complete vasectomy with, 288 operation of, 289

for malignant disease, 280

in hypertrophy of prostate, 34I

partial vasectomy and, 288

incision in, 288

instruments for, 288

unilateral, 280 operation of, 288

Catheter treatment of hypertrophy of prostate, 327

contraindications for, $33^{\circ}$

indications for, 330

manner of use of, 330

when and how often used, 329

Catheterism, retrograde, in stricture of urethra, I 5 I

Catheters, best forms of, 327

bougie, 328

Mercier bicoudé, 328 coudé, 328

soft-rubber, 328

Cauliflower carcinoma of bladder, 496

tumor of bladder, 496

Cavernositis, 35

chronic, 35

treatment of, 35

treatment of, 35

Cellulitis of penis, 34 treatment of, 34

of scrotum, 196

Chancre, diagnosis of, from chancroid, 46 syphilitic, diagnosis of, from balanitis, 40

Chancroid, 43

appearances of, 45

complications of, 49

bubo and, 49

development of, 44

diagnosis of, 45

from chancre, 46

from herpes, 46

discharge from, 45

duration of, 45

etiology of, 43

location of, 43

lymphangitis and, 49

paraphimosis and, 50

phimosis and, 50

progress of, 45
Chancroid, prophylaxis of, 48 treatment of, 48

Chancroidal ulcer, diagnosis of, from balanitis, 40

Chetwood's operation for prostatotomy, 405, 410

Chimney-sweep's cancer, 195

Cholesteatoma of bladder, 496, 497, 507, 539

Chond rocarcinoma of testicle, 239

Chondroma of bladder, 496

Chondrosarcoma of bladder, 497, 536

Chylocele, 256

Cicatricial phimosis, treatment of, $3 \mathbf{I}$

Circumcision by combined ventral and dorsal incisions, 160

Allis' operation, I62

Woodward's operation, I6I

by single dorsal incision, $\mathrm{I} 6 \mathrm{O}$

by ventral incision, $\mathrm{I} 60$

with clamps, I 57

instruments for, 157

operation of, 158

preparation for, 157

Civiale's operation of bilateral perineal lithotomy, 573

Clado's operation of partial resection of bladder, $59^{\circ}$

Cock's operation in external perineal urethrotomy, I 5 I

Colic, spermatic, 272

Compressor urethræ muscle, spasm of, in stricture of urethra, 107

Condylomata acuminata, 36

Congenital hydrocele, 244, 253

diagnosis of, 253

symptoms of, 253

treatment of, 253

phimosis, 30

Connective tissue, tumors of bladder arising from, 496

Contusions of cord, 202 treatment of, 202

of penis, 28 treatment of, 28

of testicles, 2I 7 treatment of, 217

Cord, spermatic. See Spermatic cord.

Corpora cavernosa, I 7 arteries of, I $8,19,20$

fibrous sclerosis of, 35 treatment of, 35 inflammation of, 35

Corpus spongiosum, 18

bulb of, I 8 arteries of, 20

Crura of penis, I 7

Cryptorchism, 214

Cutaneous affections of penis, 36

Cystitis, 457

acute, $459,46 \mathrm{I}$

chronic, $459,46 \mathbf{I}$ 
Cystitis, classification of, 457

diagnosis of, $46 \mathrm{I}$

differential, 462

from inflammation of posterior urethra, 462

diphtheritic, 460

etiology of, 457

essential, 457

predisposing, 457

gangrenous, $46 \mathrm{I}$

membranous, 46I

pathology of, 459

prognosis of, 463

suppurative, in hypertrophy of prostate,

323

symptoms of, 46I

treatment of, 463

local applications in, 465 continuous drainage, 466 instillations, 465

medicinal, 463 irrigations, 465

opsonic, 466

surgical, 467

in tumors of bladder, 509

vesical infection in, 457

avenue of, 457

by direct extension, 457

descending infection from kidney, 458

extension from adjacent structures, $45^{8}$

hematogenous infection, $45^{8}$

Cystoscope, Albarran's ureter, 424

Brenner's irrigating, 424

cleansing of, 427

difficulties encountered in use of, 429

examinations with, $42 \dot{8}$

lubricating medium, 428

position of patient in, 428

Leiter's electric, 422

Nitze-Leiter platinum, 42 I

Nitze's incandescent lamp, 422

operating, 425

Whitehead's, 423

Cystoscopic examination in vesical calculus, 482

Cystoscopy, 42I

irrigating, 423

photographic attachment to, 425

Cystotomy, suprapubic, 545

drainage of bladder after, 555 examination of bladder in, digital,

incision in, 547

$$
\begin{gathered}
55^{2} \\
\text { visual, of interior, } 55^{2}
\end{gathered}
$$

instruments for, 545

modifications of, $55^{2}, 553$

operation of, 546

position of patient in, 546

suture of bladder in, 560
Cystotomy, suprapubic, suture of bladder in rupture of bladder, 560

Cysts of bladder, dermoid, 496, 507, 536

$$
\text { hydatid, } 507
$$

simple, 503

of epididymis, 237

of scrotum, sebaceous, 195

of testicle, $23^{6}$ treatment of, 195

\section{D}

DARTOS of penis, I 9

of scrotum, I9I

De Pezzer's prostatic tractor, 393

self-retaining catheter, 557

Dermoid cyst of bladder, $496,507,536$

Descent of testicle, 2I 2

Diabetic phimosis, treatment of, $3 \mathrm{I}$

Dieffenbach's operation for closure of urethral fistula, 187

Dilatation in stricture of urethra, I06 continuous, I06 gradual, I06 mortality of, 117 results of, 122

rapid, I06 with flexible olivary bougies, I IO

Diphtheritic cystitis, 460

Dislocation of penis, 29 symptoms of, 29 treatment of, 29

of testicle, 217 treatment of, 217

Dissecting method of perineal prostatectomy, 384

Diverticula of bladder, 488

definition of, 488

diagnosis of, $49 \mathbf{I}$

location of, 489

number of, 489

operations on, 596

pathology of, 490

size of, 489

symptoms of, 490

treatment of, 492

Divulsion, I 39

instruments in, 139

operation in, I 39

position of patient in, I 39

in stricture of urethra, I 13 results of, 122

Dolbeau's lithoclast, 568

Dorsal artery of penis, 20

Double penis, 24

Drainage of bladder in hypertrophy of prostate, 333

continuous, in cystitis, 466

suprapubic, in hypertrophy of prostate, 333

voL. I-39 
Drainage through perineum in hypertrophy Epididymis, 210 of prostate, 333

Duplay's operation for epispadias, 179 for hypospadias, 174

Dwarfing of penis, 23

\section{E}

Есснумоsis of scrotum, 2 I 7

Eczema of penis, 36

of scrotum, 193

etiology of, 193

marginatum, 194 treatment of, 194

symptoms of, I93

treatment of, 193

Ejaculatory ducts, 30 I, 302

Electric endoscopes, 75

Electrolysis in stricture of urethra, results of, I 26

Elephantiasis of penis, $3^{8}$ treatment of, $3^{8}$

of scrotum, 197 etiology of, I 97

filaria sanguinis hominis in, 197

pathology of, 198

prophylaxis in, 199

symptoms of, 198

treatment of, medical, I 99 operative, 199

Emasculation of penis, 170

Emphysema of scrotum, 197 treatment of, 197

Encephaloid carcinoma of bladder, 496 of testicles, 238

Enchondroma of testicle, 24I

Encysted calculus in lateral perineal lithotomy, $57 \mathrm{I}$ in litholapaxy, 584

hydrocele of cord, 244,252 diagnosis of, 252 symptoms of, 252 treatment of, 253

vesical calculus, 473

Endoscopes, 75

electric, for anterior urethra, 75 for prostatic urethra, 75

knife for use in connection with, 8 I

manner of using, 75

in stricture of urethra, 104

Endoscopy, 74

in stricture of urethra, $\mathrm{IO}_{4}$

Enucleation, finger, in perineal prostatectomy, 372

Epicystotomy, 545

Epidermization of bladder, 497, 507

Epidermoid cancer of bladder, 496

Epididymectomy, 285

instruments for, 285

operation of, 285

with anastomosis between vas deferens and rete testis, 287

cysts of, 237

incision of, $29 \mathrm{I}$

inflammations of. See Epididymitis.

operations on, 273

tumors of, 237

Epididymitis, 22 I

complications of, 228

diagnosis of, 224

etiology of, 22 I

following perineal prostatectomy in hypertrophy of prostate, $35^{2}$

pathology of, 222

prognosis of, 224

recurrent, 228

relapses in, 224

symptoms of, 223

constitutional, 224

local, 223

treatment of, 225

of acute, 225

prophylactic, 225

strapping and suspension in, 226

urethral, 228

Epididymo-orchitis, 229

Epispadias, 26

frequency of, 26

operations for, I 79

Beck's, I 82

Duplay's, I 79

plastic, I 80

'Thiersch's, I 80

symptoms of, 27

treatment of, 27

varieties of, 26

Epithelial papilloma of bladder, benign, 496

tissue, tumors of bladder arising from, 496

Epithelioma of bladder, 496

Erector penis muscle, 22

Evacuating pumps, 575, 576, 577

Exstrophy of bladder, 443

treatment of, 444

Extirpation of bladder, total, 54I, 591

incision in, 593

instruments for, 592

operation in, 592, 593

position in, 593

of penis, I $68,54 \mathrm{I}$ preparatory measures in, 593

of seminal vesicles, $54 \mathrm{I}$

Extraperitoneal rupture of bladder, 447

drainage in, $45^{\mathrm{I}}$

suture of wound in, $45^{1}$

F

Ferguson's prostatic tractor, 399

Fibrinous hydrocele, 245

Fibrinuria in tumors of bladder, 513

Fibroma of testicle, 24I 
Fibroma of testicle, diagnosis of, 24I treatment of, 24I

Fibropapilloma of bladder, 496

Fibrosarcoma of bladder, 497 of testicle, 239

Fibrous sclerosis of corpora cavernosa, 35

Filaria sanguinis hominis in elephantiasis of scrotum, 197

Filiform bougies, ror

Fimbriated papilloma of bladder, 496

Fistula, urethral, operations for closure of, I 85 in stricture of urethra, treatment of, 129

Follicles of urethra, inflammation of, treatment of, 80

Foreign bodies as nuclei of vesical calculus, 474

Fracture of penis, 28

etiology of, 28

prognosis of, 29

symptoms of, 28

treatment of, 29

of vesical calculus, spontaneous, 476

Freyer evacuating pump, 576

Fuller's operation for abscess of seminal vesicles, 295

Fungus of scrotum, 192

Funiculitis, 202 treatment of, 193

treatment of, 203

Fusion of seminal vesicles, $26 \mathrm{I}$

\section{G}

Galactocele, 237, 256

symptoms of, 257

treatment of, 257

Galvanocautery operation of Bottini in hypertrophy of prostate, 343

accidents in, $35 \mathrm{I}$

advantages of, 353

complications of, $35 \mathrm{I}$

failures of, $35^{\mathrm{I}}$

Gangrene of penis, 35

limitations of, $35 \mathrm{I}$ treatment of, 36

of testicles, 218 diagnosis of, 219

in orchitis, 232

pathology of, 219

symptoms of, 219

treatment of, 219

Gangrenous cystitis, 46 I

Genito-urinary tract in hypertrophy of prostate, 315

Giant-cell sarcoma of bladder, 497

Gibson's method of suprapubic drainage, 558

Glands of bladder, 442

Glandular hypospadias, 24

Glans penis, I 8
Gleet in stricture of urethra, 97

in vesiculitis, 266

Gonococcus in urethritis, 55

tests for, 56,57

culture, 58

Gram's, 56

Gonorrhœal infection of eye, urethritis and, 72

symptoms of, 72

treatment of, 72

rheumatism, urethritis and, 69

characteristics of, 70

course of, 70

diagnosis of, 70

treatment of, 7 r

Gouley's tunnelled sound, with filiform guide, I 12

Gout of penis, 35

Gram's test for gonococcus in urethritıs, 56

\section{$\mathrm{H}$}

HARRISON's urethral whip bougie, II 2

Hematocele, 253

acute, diagnosis of, 255

etiology of, 253

pathology of, 254

prognosis of, 255

symptoms of, 254

treatment of, 255

chronic, diagnosis of, 255

etiology of, $25+$

pathology of, 254

prognosis of, 255

symptoms of, 255

treatment of, 255

of cord, 253

parenchymatous, 253

vaginal, 253

Hematogenous infection in cystitis, $45^{8}$

Hematuria in hypertrophy of prostate, 322

in tumors of bladder, $5 \mathrm{I} 2$

Hemorrhage following perineal prostatectomy in hypertrophy of prostate, $35^{2}$

in internal urethrotomy, 146

in lateral perineal lithotomy, $57 \mathrm{I}$

into testicles, 2 I 7

Hermaphroditism, 24

Hernia, undescended testis and, 2 I 6

Hernial sac, hydrocele of, 245

Herpes progenitalis, $4 \mathrm{I}$

diagnosis of, 42

from balanitis, $4 \mathrm{I}$

from chancroid, 46

etiology of, $4 \mathrm{I}$

symptoms of, 42

treatment of, 43

zoster of penis, $3^{6}$

Heterogeneous tumors of bladder, 497

Heteroplastic tumors of bladder, 497

Heterotopic tumors of bladder, 496, 497 
Horny growth of penis, 38 treatment of, $3^{8}$

Hydatid cysts of bladder, 507

Hydatids of Morgagni, spermatocele of, 237 Hydrocele, 243

acute, 245

$$
\text { course of, } 246
$$

diagnosis of, 246

etiology of, 245

pathology of, 245

prognosis of, 246

symptoms of, 246

treatment of, 247

bilocular, 244

chronic, 247

diagnosis of, 249

etiology of, 247

fluid in, 248

pathology of, 248

prognosis of, 250

symptoms of, 249

treatment of, 250

carbolic acid in, 25I

iodine in, $25 \mathrm{I}$

classification of, 243

congenital, 244, 253

diagnosis of, 253

symptoms of, 253

treatment of, 253

of cord, 244

encysted, 244, 252

diagnosis of, 252

symptoms of, 252

definition of, 243 treatment of, 253

fibrinous, 245

of hernial sac, 245

infantile, 244, 253

treatment of, 253

inguinal, 245

injection of sac with carbolic acid, 283 with iodine, 283

multilocular, 244, 245

operations for, $28 \mathrm{I}$

position of patient, $28 \mathrm{I}$

preparation for, 28I

tapping, 28I

plastic, 245

spermatic, 249

testicle in, 249

of tunica vaginalis, 243,248

Hydronephrosis in tumors of bladder, 509

Hypertrophy of prostate, 307

bar at neck of bladder in, 3I I

bas fond of bladder in, 318

bladder in, 315

drainage of, 333

irrigation of, 333,334

castration in, $34 \mathrm{I}$

definition of, 307

diagnosis of, 32 I

drainage through perineum in, 333
Hypertrophy of prostate, etiology of, 307

examination in, physical, 323 of prostatic urethra, 324 rectal, 323

fibrous sheath in, 314

frequency of, 307

galvanocautery operation of Bottini in, 343

accidents in, $35 \mathrm{I}$

advantages of, 353

complications of, $35 \mathrm{I}$

failures in, $35 \mathrm{I}$

genito-urinary tract in, 315

hematuria in, 322

interureteral bar in, 316

kidneys in, 320

micturition in, frequent, $32 \mathrm{I}$

nocturnal, 32 I

mortality in, influence of age on, 348

of delay on, 349

of occupation on, 349

of environment on, 349

of previous catheter treat-

pain in, 322 ment on, 349

pathology of, 308

macroscopic, 309

microscopic, 308

perineal prostatectomy in, 343

accidents in, $35 \mathrm{I}$

advantages of, 353

choice between suprapubic and, 354

of technique in, 355

complications of, $35 \mathrm{I}$

epididymitis following, $35^{2}$

failure of, $35 \mathrm{I}$

hemorrhage following, $35^{2}$

limitations of, $35 \mathrm{I}$

mortality of, 343

stricture of prostatic urethra following, $35^{2}$

prophylaxis against vesical sepsis in, $33 \mathrm{I}$

prostatic urethra in, 3 I 2

residual urine in, 3I9

detection of, 323

retention of urine in, 321

size of, 3 I 2

treatment of, 332

suppurative cystitis in, 323

suprapubic drainage in, 333

prostatectomy in, 343

accidents in, $35 \mathrm{I}$

advantages of, 353

choice between perineal and, 354

complications of, $35^{2}$

failure in, 353

limitations of, $35^{\mathrm{I}}$

mortality of, 345

symptoms of, $32 \mathrm{I}$ 
Hypertrophy of prostate, treatment of, 326 catheter, 327

lubricants for, $33 \mathrm{I}$

objections to, 335

results of, 334

medicinal, 326

operative, 336 results of, $35^{\circ}$

palliative, 326

$x$-ray, 356 :

ureters in, $32 \mathrm{I}$

urinary system in, $32 \mathrm{I}$

Hypospadias, 24

vasectomy in, $34 \mathrm{I}$

balanitic, 24

consequences of, 26

frequency of, 24

glandular, 24

operations for, 174

Beck's, I 74

Duplay's, I 74

penile, 24

Van Hook-Mayo, I 77

scrotal, 25

operations for, Beck's, 177

perineoscrotal, 25

penoscrotal, 25

treatment of, 26

varieties of, 24

Interureteral bar in hypertrophy of prostate, $3 \mathrm{I} 6$

Intraperitoneal rupture of bladder, 447

drainage in, $45 \mathrm{I}$

Irrigating cystoscope, 423

suture of wound in, 45I

Irrigation of bladder in hypertrophy of prostate, 333, 334

in treatment of cystitis, 465

\section{J}

JANET's method of treatment of urethritis, 64

\section{$\mathbf{K}$}

Keegan evacuating pump, 576

Kidneys in hypertrophy of prostate, 320 infection from, in cystitis, $45^{8}$

Kollmann's dilator, I I3, I I 4

Keyes' deep urethral syringe, 79

subcutaneous ligature operation for varicocele, 292

\section{L}

LAteral perineal lithotomy for vesical calculus, 483

Leiomyoma of bladder, 496

Leiter's electric cystoscope, 422

INCONTINENCE of urine in lateral perineal

lithotomy, 572

Infantile hydrocele, 244,253 treatment of, 253

Inflammation of bladder, 457. See Cystitis. of cord, 202

treatment of, 203

of corpora cavernosa, 35

of epididymis, 221. See Epididymitis.

of follicles of urethra, treatment of, 80

of penis, 33

of scrotum, 196

of seminal vesicles, 262

of testicle, 229. See Orchitis.

undescended testis and, 215

of urethra, 54. See Urethritis.

diffuse phlegmonous, 9I

of posterior, diagnosis of, from cystitis, 462

of vas deferens, 203

Inflammatory paraphimosis, 32

phimosis, 30 treatment of, $3 \mathrm{I}$

stricture of urethra, 84

Inguinal hydrocele, 245

Instillations in treatment of cystitis, 465

Intertrigo of scrotum, 194 treatment of, I 94

Leukoplakia vesicæ, 497, 507, 539

Ligaments of bladder, 436

Linear stricture of urethra, 95

Lipoma of cord, 203

diagnosis of, 203

symptoms of, 203

treatment of, 204

of testicle, 24I

Lithoclast, 568

Litholapaxy, 574

advantages of, $5^{84}$

after-treatment in, $5^{84}$

in children, 585

complications of, $5^{84}$

dangers of, 584

difficulties of, 584

encysted stone in, 584

enlarged prostate in, 584

history of, 565

instruments for, 578

objections to, 584

operation of, 578

perineal, $5^{86}$

instruments for, 586

operation of, 586

for vesical calculus, 487

position of patient in, 578

preliminary steps in, 578

size and character of stone in, 584 
Litholapaxy, stricture of urethra in, 585

Lithotomy, 561

knife, 568

perineal, bilateral, 572

instruments for, 572

operation of, 573

lateral, 567

Civiale's, 573

after-treatment of, 570

complications of, $57 \mathrm{I}$

encysted calculus in, 571

hemorrhage in, $57 \mathrm{I}$

history of, 562

incision in, 570

incontinence of urine in, 572

injury to rectum in, 572

instruments for, 568

landmarks for, 569

operation of, $57^{\circ}$

position in, 569

median, 573

preliminary steps in, 569

for vesical calculus, bilateral, 483

lateral, 483

scoop, $5^{69}$ results of, 486

staff, 568

suprapubic, 573

history of, 563

instruments for, 573

operation of, 573

position in, 573

preliminary steps in, 573

treatment of wound in, 574

for vesical calculus, 487

Lithotrites, 567

Lithotrity, history of, $5^{6} 5$ rapid, 574

Lobular carcinoma of bladder, 496

Lobulated papilloma of bladder, 496

Lupus erythematosus of penis, 36

of scrotum, 195

treatment of, 195

Lymph scrotum, I97

Lymphangitis, chancroid and, 49

of penis, 34 treatment of, 34

urethritis and, 67

treatment of, 67

Lymphatics of bladder, $4+\mathrm{I}$

of penis, $2 \mathrm{I}$

of scrotum, 192

of testicles, 210

of vas deferens, 201

Lymphosarcoma of bladder, 497

of testicle, 239

\section{M}

Maissonneuve's urethrotome and guide, I 4 I

Meatotome, IO I

Meatus urinarius, i $\$$
Medullary carcinoma of testicles, 238

Melanotic sarcoma of testicle, 239

Membranous cystitis, $46 \mathrm{I}$

urethra, 2 I stricture of, 96

Mercier bicoudé catheter, 328 coudé catheter, 328 prostatectotome, 406

Micturition in hypertrophy of prostate, 321

Mixed-cell sarcoma of bladder, 497

Monorchism, 214

Mucoid polyp of bladder, 496

Mucous membrane affections of penis, 36

Multilocular hydrocele, 244, 245

Multiple bladder, 442

Muscles of bladder, 438

Muscular contracture of bladder, 447 tissue, tumors of bladder derived from, 496

Myoma of bladder, operations for, table of, 524

of testicle, $24 \mathrm{I}$

Myxoma of bladder, 506

Myxosarcoma of bladder, 497

\section{N}

Necrosis in tumors of bladder, 508

Nephrotomy in tumors of bladder, results of, 530

Nerves of bladder, 44I

of penis, 21

of prostate, 305

of scrotum, 192

of testicles, $2 \mathrm{IO}$

of vas deferens, $20 \mathrm{I}$

Neuralgia of testicle, 220

etiology of, 220

symptoms of, 220

treatment of, 220

Nicoll's technique in combined operations on prostate, 403

Nitze-Leiter platinum cystoscope, 42 I

Nitze's incandescent lamp cystoscope, 422 method of removal of tumor of bladder through urethra, 588

\section{O}

Obliteration of urethra, stricture and, 85

Occlusion of urethra due to chemical agents, 88

Edema bullosum, 460

of scrotum, 196 treatment of, 196

Olivary bougies, 102

Opsonic treatment of cystitis, 466

Orchidopexy, 273.

Beck's operation, 277

instruments for, 277 
Orchidopexy, Bevan's operation, 273 incision in, 273 instruments for, 273

instruments for, 279 operation in, 279

Orchitis, atrophy in, 232 complications of, 232 diagnosis of, 23 I due to mumps, 230 gangrene in, 232 idiopathic, $23 \mathrm{I}$ non-tuberculous, 229 primary, 229 secondary, 229 traumatic, 229

posterior urethral inflammation and, $23^{\circ}$ sequela of, 232

suppuration in, $23^{2}$

symptoms of, $23 \mathrm{I}$

local, $23 \mathrm{I}$

syphilitic, 234

treatment of, 233

Osteochondrosarcoma of bladder, 535

Osteoid chondrosarcoma of bladder, 497

Osteoma of testicle, 24I

Otis' evacuating pump, 575 urethrotome, I 43

\section{$\mathbf{P}$}

Papille of bladder, 442, 496, 498

Papilloma of bladder, benign, development of, 502 synonyms of, 496

of penis, 36

etiology of, 36

pathology of, 36

treatment of, $3^{8}$ operative, 38

of urethra, 82 topical applications, 38

Paraphimosis, 32

chancroidal, $5^{\circ}$

inflammatory, 32

operations for, $\mathrm{I} 63$

by simple incision, $\mathrm{I}_{3} 3$

by subcutaneous division, $\mathbf{I}_{3}$

symptoms of, 32

treatment of, 32

palliative, 33

Parasitic tumors of bladder, 496

Parenchymatous hematocele, 253

Pascale's operation of epididymectomy, 285 of partial vasectomy, 285

Patent urachus, 443 treatment of, 443

Pediculi pubis of scrotum, 195 treatment of, 195

Penis, abnormalities of, 23

absence of, 23

acne of, 36
Penis, amputation of, I64

by dorsal flap method, $\mathrm{I} 66$ operation in, 16\%

through pendulous portion, 164 operation in, $\mathrm{I} 64$

by transverse section, 165 operation in, 165

anatomy of, 17

arteries of, 20

dorsal, 20

balanitis of, 40

chronic, 40

complications of, $4 \mathrm{I}$

diagnosis of, 40

from chancroidal ulcers, 40

from herpes, 4 I

from syphilitic chancre, 40

etiology of, 40

symptoms of, 40

treatment of, $4 \mathrm{I}$

balanoposthitis of, 40

cancer of, 50. See Carcinoma.

carcinoma of, 50

age in, $5^{\circ}$

duration of, $5 \mathrm{I}$

etiology of, $5^{\circ}$

glandular involvement in, 52

metastases in, 52

occupation in, 50

pathology of, $5^{2}$

phimosis in, $5 \mathrm{I}$

point of origin of, $5 \mathrm{I}$

treatment of, 52

venereal disease in, $5^{1}$

cavernositis of, 35

chronic, 35

treatment of, 35

treatment of, 35

cellulitis of, 34

treatment of, 34

chancroid of, 43

appearances of, 45

bubo and, 49

complications of, 49

development of, 44

diagnosis of, 45

from chancre, 46

from herpes, 46

discharge from, 45

duration of, 45

etiology of, 43

location of, 43

lymphangitis and, 49

paraphimosis and, 50

phimosis and, 50

progress of, 45

prophylaxis of, 48

treatment of, 48

condylomata acuminata of, $3^{6}$

contusions of, 28

treatment of, 28

crura of, 17 
Penis, cutaneous affections of, 36 dartos of, 19

diseases of, 30

dislocation of, 29

symptoms of, 29

treatment of, 29

double, 24

dwarfing of, 23

eczema of, 36

elephantiasis of, 38 treatment of, 39

emasculation of, total, I 70

epispadias of, 26

frequency of, 26

symptoms of, 27

treatment of, 27

varieties of, 26

erection of, physiology of, 22

extirpation of, $\mathrm{r} 68,54 \mathrm{I}$

instruments for, $\mathrm{I} 68$

operation in, 168

position of patient in, 168

fracture of, 28

etiology of, 28

prognosis of, 29

symptoms of, 28

treatment of, 29

gangrene of, 35

treatment of, 36

gout of, 35

hermaphroditism, 24

herpes progenitalis, $4 \mathrm{I}$

diagnosis of, 42

etiology of, $4 \mathrm{I}$

symptoms of, 42

treatment of, 43

zoster of, 36

horny growths of, 38

hypospadias of, 24

treatment of, 38

balanitic, 24

consequences of, 26

frequency of, 24

glandular, 24

penile, 24

scrotal, 25 penoscrotal, 25 perineoscrotal, 25

treatment of, 26

varieties of, 24

inflammation of, 33

injuries of, 27

lupus erythematosus of, 36

lymphangitis of, 34

treatment of, 34

lymphatics of, $2 \mathrm{I}$

mucous membrane, affections of, 36

nerves of, 2I

operations on, 156

amputation, $16_{4}$

circumcision by combined [ventral and dorsal incisions, $\mathrm{I} 60$
Penis, operations on, circumcision by a single dorsal incision, I6o

by ventral incision, 160

with clamps, 157

for closing urethral fistula, 185

extirpation, I68

plastic, I 7 I

total emasculation, 170

papillomata of, 36

etiology of, 36

pathology of, 36

treatment of, 38

operative, 38

topical applications, $3^{8}$

paraphimosis of, 32

inflammatory, 32

symptoms of, 32

treatment of, 32

palliative, 33

phimosis of, 30

acquired, 30

cicatricial, treatment of, $3 \mathrm{I}$

complications of, $3 \mathbf{I}$ treatment of, $3^{1}$

congenital, 30

diabetic, treatment of, $3 \mathrm{I}$

inflammatory, 30 treatment of, $3 \mathrm{I}$

reflex nervous disturbances in, 30

symptoms of, 30

treatment of, $3 \mathrm{I}$

physiology of, 22

prepuce of, 20

psoriasis of, 36

scabies of, 36

secondary function of, 22

structure of, 17

tuberculosis of, 39

treatment of, 39

veins of, $2 \mathrm{I}$

verrucæ of, 36

warts of, hard, 37

soft, $3^{6}$

venereal, 36

wounds of, 27

treatment of, 27

Penoscrotal hypospadias, 25

Pericystitis in tumors of bladder, 509

Perineal litholapaxy, 586

instruments for, 586

operation of, 586

for vesical calculus, 487

lithotomy, bilateral, 572 instrument for, 572 operation of, 573

lateral, 567

Civiale's, 573

after-treatment of, 570

complications of, $57 \mathrm{I}$

encysted calculus in, 57 I

hemorrhage in, $57 \mathrm{I}$

history of, 565 
Perineal lithotomy, lateral, incision in, 570 incontinence of urine in, 572 injury to rectum in, 572

instruments for, 568

landmarks for, 569

operation of, 570

position in, 569

median, 573

preliminary steps in, 569

for vesical calculus, bilateral, 483

$$
\text { lateral, } 483
$$

results of, 486

prostatectomy, 372

for abscess of prostate, 412

dissecting method, 384

in hypertrophy of prostate, 343

accidents in, $35 \mathrm{I}$

advantages of, 353

choice between suprapubic and, 354

of technique in, 355

complications of, $35 \mathrm{I}$

epididymitis following, $35^{2}$

failures in, $35^{\mathrm{I}}$

hemorrhage following, $35^{2}$

limitations of, $35 \mathrm{I}$

mortality of, 343

stricture of prostatic urethra following, $35^{2}$

open method, $3^{8} 4$

Proust's technique, 385

Pyle's technique, 384

Rydygier's operation, 390

total, 372

Young's technique, 39I

after-treatment of, 382

drainage of bladder in, $38 \mathrm{I}$

incision in, 372

instruments in, 372

by median urethrotomy incision

and finger enucleation, 372

operation in, 372

packing in, 383

passing of sounds in, 383

position of patient in, 372

precautions in, 379

stricture after, 384

when enucleation cannot be done, $3^{81}$

section in stricture of urethra, 147

urethrotomy, external, i I6, I 47

mortality of, 118

suture of urethra after, 152

Perineoscrotal hypospadias, 25

Perineum, drainage through, in hypertrophy of prostate, 333

Phimosis, 30

acquired, 30

in carcinoma of penis, $5 \mathrm{I}$

chancroidal, 50

cicatricial, treatment of, $3^{1}$

complications of, $3 \mathrm{I}$
Phimosis, complications of, treatment of, 3I congenital, 30

diabetic, treatment of, $3 \mathrm{I}$

inflammatory, 30

treatment of, $3 \mathrm{I}$

operation for, 156

anatomical data, 156

circumcision by combined ventral and dorsal incisions, 160

by single dorsal incision, 160

by ventral-incision, 160

with clamps, I 57

reflex nervous disturbances in, 30

symptoms of, 30

treatment of, $3 \mathrm{I}$

Plastic hydrocele, 245

operations on penis, I I I

flap method, I 7

Reich's operation, I I I, I 72

Polyorchism, 21 3

Thiersch's skin-grafting, I 73

Polyp of urethra, 82

$$
\text { treatment of, } 82
$$

Prepuce of penis, 20

Priapism in vesical calculus, 480

Prostate, abscess of, 364

perineal prostatotomy for, $4 \mathrm{I} 2$

anatomy of, 299

bloodvessels of, 305

calculi of, 367. See Prostatic calculi.

capsule of, 299

enlarged, in litholapaxy, $5^{84}$

fibrous sheath of, 299, 300

gland tissue of, 304

hypertrophy of, 307

bar at neck of bladder in, 3 II

bas fond of bladder in, 3 I 8

bladder in, 315

drainage of, 333

irrigation of, 333,334

castration in, $34 \mathrm{I}$

definition of, 307

diagnosis of, $32 \mathrm{I}$

drainage through perineum in, 333

etiology of, 307

examination in, physical, 323

of prostatic urethra in, 324

rectal, 323

fibrous sheath in, 314

frequency of, 307

galvanocautery operation of Bottini in, 343

accidents in, $35 \mathrm{I}$

advantages of, 353

complications in, $35 \mathrm{I}$

failures in, $35 \mathrm{I}$

limitations of, $35 \mathrm{I}$

genito-urinary tract in, 315

hematuria in, 322

interureteral bar in, $3 \mathrm{I} 6$

kidneys in, 320

micturition in, frequent, $32 \mathrm{I}$ 
Prostate, hypertrophy of, micturition in, Prostate, malignant disease, diagnosis of, 359

nocturnal, 32 I

mortality in, influence of age on, 348

of environment on, 349

of delay on, 349

of occupation on, 349

of previous catheter treat-

pain in, 322

ment on, 349

pathology of, 308

macroscopic, 309

microscopic, 308

perineal prostatectomy in, 343

accidents in, $35^{\mathrm{I}}$

advantages of, 353

choice between suprapubic and, 354

of technique in, 355

complications of, $35 \mathrm{I}$

epididymitis following, 352

failure in, 35I

hemorrhage following, 352

limitations of, 35 I

mortality of, 344

stricture of prostatic urethra following, $35^{2}$

prophylaxis against vesical sepsis in, $33^{1}$

prostatic urethra in, 312

residual urine in, detection of, 323

retention of urine in, $32 \mathrm{I}$

size of, $3^{12}$

treatment of, $33^{2}$

suppurative cystitis in, 323

suprapubic drainage in, 333

prostatectomy in, 343

accidents in, $35 \mathrm{I}$

advantages of, 353

choice between perineal, and, 354

complications of, 352

failure in, 353

limitations of, $35 \mathrm{I}$ mortality of, 345

symptoms of, $32 \mathrm{I}$

treatment of, 326

catheter, 327

objections to, 335

results of, 334

lubricants in, $33 \mathrm{I}$

medicinal, 326

operative, 336 results of, $35^{\circ}$

palliative, 326

$x$-ray, 356

ureters in, 320

urinary system in, $32 \mathrm{I}$

vasectomy in, 34I

lobes of, 299

third, 306

lymphatics of, 305

malignant disease of, 357 frequency of, 357

operation for, 4I4

pathological anatomy of, 357

progress of, 359

symptoms of, 359

treatment of, 360

nerves of, 305

non-tuberculous suppuration of, 364

diagnosis of, 365

etiology of, 364

symptoms of, 365

treatment of, 365

constitutional, 365

local, 365

operative, 365

palliative, 365

operations on, 372

combined, 403

Alexander's technique, 405

Nicoll's technique in, 403

special steps in, 405

prostatectomy, 372

prostatotomy, 372

relations of, 306

sarcoma of, 357

shape of, 299

size of, 299

stroma of, 304

structure of, 304

surfaces of, 299

weight of, 299

Prostatectotomes, 406, 407

Prostatectomy, 372

perineal, dissecting method, 384

in hypertrophy of prostate, 343

accidents in, $35 \mathrm{I}$

advantages of, 353

choice between suprapubic, and, 354

of technique in, 355

complications of, $35 \mathrm{I}$

epididymitis following, $35^{2}$

failures in, $35 \mathrm{I}$

hemorrhages following, $35^{2}$

limitations of, $35 \mathrm{I}$

mortality of, 343

stricture of prostatic urethra following, $35^{2}$

open method, 384

Pyle's technique, 384

Proust's technique, 385

Rydygier's modification, 390

Young's technique, 39I

total, 372

after-treatment in, 382

drainage of bladder in, $38 \mathrm{I}$

incision in, 372

instruments in, 372

by median urethrotomy incision and finger enucleation, 372 
Prostatectomy, perineal, total, operation in, 372 packing in, 383

passing of sounds in, 383

position of patient in, 372

precautions in, 379

preparation of patient in, 372

stricture after, 384

when enucleation cannot be done, 381

suprapubic, in hypertrophy of prostate, accidents in, $35 \mathrm{I}$

advantages of, 353

choice between perineal, and, 354

complications of, 352

failures in, 353

limitations of, 351

total, $40 \mathrm{I}$ mortality of, 345

after-treatment in, 403

removal of prostate in, $4 \mathrm{OI}$

Prostatic calculi, 367

symptoms of, 360

treatment of, 369

massage in hypertrophy of prostate, 327

tractor, De Pezzer's, 393

Young's, 393

urethra, 2 I

electric endoscope for, 75

examination of, 77

in hyperthophy of prostate, 3I2

stricture of, 96

after perineal prostatectomy, 384

following perineal prostatectomy in hypertrophy of prostate, 352

Prostatitis, acute, 364

diagnosis of, 365

etiology of, 364

symptoms of, 365

constitutional, 365

local, 365

treatment of, 365

operative, 365

palliative, 365

Prostatotomy, 372, 405

Bottini's operation, 405

Chetwood's operation, 405, 410

perineal, for abscess of prostate, 4 I 2

Proust's operation in perineal prostatectomy, 385

distinctive features of, 385

incision in, 386

instruments for, 386

Pruritus of scrotum, I 94 operation in, 386

Psoriasis of penis, 36 treatment of, 194

Pyle's operation in perineal prostatectomy, 384

Pyonephrosis in tumors of bladder, 509

\section{R}

Rectum, injury to, in lateral perineal lithotomy, 572

Reich's operation on penis, I 71, I 72

Resection of bladder, partial, 540, 590, 59I

instruments for, 590

in stricture of urethra, I $16, I_{53}$

after-treatment in, 155

indications for, 153

instruments for, 153

mortality of, I 8

operation of, 153

results of, 124

technique of, 153

of symphysis pubis, $59 \mathrm{I}$

Residual urine in hypertrophy of prostate, 3 I 9 detection of, 323

Retention of urine in hypertrophy of prostate, 32 I

treatment of, 332

in stricture of urethra, treatment of, I 28

operative, 129

palliative, 128

Reticulated carcinoma of bladder, 496

Retrograde catheterism in stricture of urethra, I 5 I

Retzius' space, 432

Rhabdomyoma of bladder, 496, 508, 536

Rheumatism, gonorrhœal, urethritıs and, 69

Round-cell sarcoma of bladder, 497

Rupture of bladder, 447

definition of, 447

diagnosis of, 448

etiology of, 447

extraperitoneal, 447

drainage in, $45 \mathrm{I}$

suture of wound in, $45 \mathrm{I}$

illustrative cases of, 454

intraperitoneal, 447

drainage in, $45 \mathrm{I}$

suture of wound in, $45 \mathrm{I}$

location of, 447

pathology of, 447

results of, 447

spontaneous, 447

symptoms of, 448

traumatic, 447

Rydygier's operation in perineal prostatectomy, 390

\section{$\mathrm{S}$}

SARcoma of bladder, 497,505

of prostate, 357

of testicles, 239

melanotic, 239

prognosis of, 240

treatment of, 240

undescended testis and, 216

Scabies of penis, 36 
Scabies of scrotum, 194 treatment of, 195

Scirrhus carcinoma of bladder, 496 of testicles, 238

Sclerosis, fibrous, of corpora cavernosa, 35

Scrotal hypospadias, 25

Beck's operation for, 177

penoscrotal, 25

perineoscrotal, 25

Scrotum, abnormalities of, 192

abscess of, 196

anatomy of, 191

bloodvessels of, 192

carcinoma of, 195

treatment of, 196

cellulitis of, 196

cutaneous diseases of, 193

dartos of, I 19

ecchymosis of, 217

eczema of, 193

etiology of, 193

marginatum, 194 treatment of, 194

symptoms of, 193

treatment of, I 93

elephantiasis of, 197

etiology of, 197

filaria sanguinis hominis in, 197

pathology of, 198

symptoms of, 198

prophylaxis of, 199

treatment of, medical, 199 operative, 199

emphysema of, 197

treatment of, 197

excision of, 297

instruments for, 297

operation of, 297

fungus of, 193

treatment of, 193

inflammations of, 196

injuries of, 192

treatment of, 192

intertrigo of, 194 treatment of, 194

lupus of, 195 treatment of, 195

lymphatics of, 192

nerves of, 192

œdema of, 196 treatment of, 196

pediculi pubis, 195

pruritus of, 194 treatment of, 195

treatment of, 194

resection of, 296

instruments for, 296

operation of, 296

scabies of, 194

treatment of, 195

sebaceous cysts of, I 95

treatment of, I 95
Scrotum, steatoma of, 195

treatment of, 195

Sebaceous cysts of scrotum, I 95

Sectio alta, 545

treatment of, 195

Seminal vesicles, abnormalities of, 26I abscess of, incision of, 295

Fuller's operation of, 295 position of painstruments for, 295 tient in, 295

absence of, 26r

anatomy of, $25^{8}$

bloodvessels of, 260

calculi of, $27 \mathrm{I}$

etiology of, $27 \mathrm{I}$

symptoms of, 272

treatment of, 272

diseases of, 262

extirpation of, $54 \mathrm{I}$

fusion of, 26I

inflammation of, 262

injuries of, 26r

multiple, 26r

operations on, 273

physiology of, 260

removal of, 289

structure of, 259

Sepsis in stricture of urethra, 1 I 9

Septic phlegmon, acute diffuse, in stricture of urethra, treatment of, 128

Septum pectiniforme, 18

Sexual function, disturbances of, in stricture of urethra, 98

Soft-rubber catheter, 328

Sounds, passing of, in perineal prostatectomy, $3^{8} 3$

Spasm of compressor urethræ muscle in stricture of urethra, 107

Spermatic colic, 272

cord, anatomy of, 200

contusions of, 202

treatment of, 202

hematocele of, 253

hydrocele of, 244

encysted, 244, 252

diagnosis of, 252

symptoms of, 252

treatment of, 253

inflammation of, 202

treatment of, 203

injuries of, 202

lipoma of, 203

diagnosis of, 203

symptoms of, 203

treatment of, 204

operations on, 273

torsion of, 202

tumors of, 203

solid, 203

veins of, dilatation of, 204. See Varicocele. 
Spermatic cord, wounds of, 202

hydrocele, 249

Spermatocele, 237,255

diagnosis of, 256

etiology of, 256

of hydatid of Morgagni, 237

symptoms of, 256

treatment of, 256

Spermatocystitis, 262

Spermatorrhœa in vesiculitis, 267

Spindle-cell sarcoma of bladder, 497

Spongy urethra, 2I

Spontaneous rupture of bladder, 447

Stone extracting forceps, 569 searcher, $48 \mathrm{r}$

Stricture of urethra, 84

annular, 95

causes of death in, I 18

complications of, treatment of, 128

congenital, 85

definition of, 84

diagnosis of, 98

endoscopy in, 82

examination of, gor

bougie à boule in, 101,102

bougies in, IOI, 102

difficulties encountered in, 104

endoscope in, 104

meatatome in, ror

sounds in, 104

passing of, 107

anatomical difficulties in, 109

urethrometer in, 103

false passage in, 130

gleet in, 97

$$
\text { consequences of, } 13 \mathrm{r}
$$

illustrative cases of, I 32

infection due to, 89

inflammatory, 84

linear, 95

in litholapaxy, 585

location of, 96

mechanical effects of, 87

in membranous urethra, 96

number of, 95

obliteration of urethra by, 85

partial, 85

total, 85

occlusion of, total, due to chemical

agents, 88

operations for, 139

boutonnière in, 147

divulsion in, 139

exposure of urethra behind stricture by open dissection, 152

perineal section in, 147

resection of stricture in, 153

retrograde catheterism in, $15 \mathrm{I}$

suture of urethra after perineal urethrotomy, $\mathbf{I}_{52}$
Stricture of urethra, operations for, urethrotomy, external perineal, I 47 internal, 140

organic, 84

pathology of, 86

prostatic, 96

after perineal prostatectomy, 384

following perineal prostatectomy in hypertrophy of prostate, 352

resilient, 95

spasm of compressor urethræ muscle in, 107

secondary changes due to, 87

sepsis in, I 19

septic phlegmon in, acute diffuse, treatment of, 128

sexual function in, disturbances of, 98

spasmodic, 84

symptoms of, 97

time of formation of, 97

tortuous, 95

traumatic, 84

treatment of, 105

choice of method of, Ir6

dilatation in, 106

continuous, I06

gradual, Io6 mortality of, II 7 results of, 122

rapid, Io6

with flexible olivary bougies, I ro

divulsion in, I 13

results of, I 26

electrolysis in, results of, 126

operative mortality in, II 7

permanency of results of, 120

resection, I 16

mortality of, II 8

results of, 124

summary of, 127

urethrotomy, external perineal, I 6 mortality of, I I 8

internal, I I 4 results of, 123

urethral fistula in, treatment of, 129 urinary stream in, 97

urination in, increased frequency of, 98

urine in, extravasation of, treatment of, 128

retention of, treatment of, 128 operative, I 29

varieties of, 94 palliative, 128

of wide caliber, 95

Suppuration of prostate, non-tuberculous, $3^{64}$ diagnosis of, 365 
Suppuration of prostate, non-tuberculous, etiology of, 364 symptoms of, 365 constitutional, 365 local, 365

treatment of, 365 operative, 365 palliative, 365

of testicle in orchitis, 232

Suppurative cystitis in hypertrophy of prostate, 323

Suprapubic aspiration of bladder, 544 instruments in, 544 operation in, 544 position in, 544

cystotomy, 545 drainage of bladder after, 555 examination of bladder in, digital, 552 incision in, 545 visual, of interior, $55^{2}$

instruments in, 545

modifications of, 552, 553

operation of, 546

position of patient in, 546

suture of bladder in, 560 in rupture, 560

drainage in hypertrophy of prostate, 333

lithotomy, 573

instruments for, 573

operation of, 573

position in, 573

preliminary steps of, 573

treatment of wound of, 574

for vesical calculus, 487

prostatectomy in hypertrophy of prostate, 343 accidents in, $35 \mathrm{I}$ advantages of, 353 choice between perineal and, 354 complications of, 352

failures in, 353 limitations of, $35 \mathrm{I}$

total, 4 OI mortality of, 345

after-treatment in, 403 removal of prostate in, $40 \mathrm{I}$

Suspensory ligament of penis, I 9

Symphysis pubis, resection of, 591

Synorchism, 214

Syphilis of testicle, 233

diffuse form, 234

gummy form, 234

prognosis of, 236

symptoms of, 235 treatment of, 236

Syphilitic chancre, diagnosis of, from balanitis, 40

epididymitis, 234

orchitis, 234

Szymanowski's operation for closure of urethral fistula, 185

Teratoma of testicle, 240

diagnosis of, $24 \mathrm{I}$

treatment of, 24I

Testicles, abnormalities of, 213

of development, 2I3

of migration, 214

absence of, influence of, 2 I I

adenoma of, 237

anatomy of, 207

angiosarcoma of, 239

atrophy of, 2I 4 in orchitis, 232

blood supply of, 2 IO

carcinoma of, 237,238

encephaloid, 238

medullary, 238

prognosis of, 239

scirrhus, 238

treatment of, 239

chondrocarcinoma of, 239

contusion of, $2 \mathrm{I} 7$ treatment of, 217

cysts of, 236

descent of, 2 I 2

destruction of, influence of, 2 I I

dislocation of, 217 treatment of, $2 \mathrm{I} 7$

enchondroma of, 24I

fibroma of, 24I diagnosis of, 24I

treatment of, 24I

fibrosarcoma of, 239

gangrene of, 2 I 8

diagnosis of, 219

in orchitis, 232

pathology of, 219

symptoms of, 219

treatment of, 219

glandular structure of, 2 Io

hemorrhage into, 217

in hydrocele, 249

infection of, through blood current, $23^{\circ}$

inflammations of, 229. See Orchitis.

injuries of, $2 \mathrm{I} 7$

internal secretion of, 2 I I

lipoma of, 24I

lymphatics of, 2 Io

lymphosarcoma of, 239

myoma of, 24I

myxoma of, 24I

myxosarcoma of, 239

nerves of, 210

neuralgia of, 220

etiology of, 220

symptoms of, 220

treatment of, 220

new-growths of, 236

operations on, 273

osteoma of, 24I

physiology of, 2 I I 
Testicles, sarcoma of, 239

melanotic, 239

prognosis of, 240

treatment of, 240

suppuration of, in orchitis, 232

syphilis of, 233

diffuse form, 234

gummy form, 234

prognosis of, 236

symptoms of, 235

treatment of, 236

teratomata of, 240

diagnosis of, 24I

treatment of, 24I

tumors of, 237

diagnosis of, 24I

undescended, 2 I 4 differential, 242

complications of, 2 I 5

frequency of, 2I 4

hernia and, 216

inflammation and, 215

influence of, on function of organ, 215

inversion in, 216

prognosis of, 215

sarcoma and, 2 I 6

treatment of, 2 I 6

wounds of, $2 \mathrm{I} 7$

treatment of, 2 I 8

Testis, coverings of, 207

tunica albuginea, 207

vaginalis, 207

vasculosa, 209

Thiersch's operation for epispadias, I 80

skin grafting in operations on penis, I 73

Thompson's evacuating pump, 576

stone searcher, $48 \mathrm{r}$

Torsion of cord, 202

Traumatic rupture of bladder, 447

Tuberculosis of penis, 39

treatment of, 39

Tubuli seminiferi, 2 Io

Tumors of bladder, 494

age in, 509, 510

arising from connective tissue, 496

from epithelial tissue, 496

causes of death in, table of, 524

classification of, structural, 496

cystitis in, 509

derived from muscular tissue, 496

diagnosis of, 512

duration of, 509, 5 I I

examination in, instrumental, $5 \mathrm{I} 4$ physical, 5I4

frequency of, 509

fibrinuria in, $5^{1} 3$

hematuria in, 5 I 2

heterotopic, 496, 497

hydronephrosis in, 509

illustrative cases of, 535

location of, 509, 5I I
Tumors of bladder, malignant, propagation of, 508

necrosis in, 508

nephrotomy in, results of, $53^{\circ}$

operations for, 588

origin of, 494

pain in, $5 \mathrm{I} 3$

parasitic, 496

partial resections in, table of, 520, 528

pericystitis in, 509

prognosis of, 5 I 4

pyonephrosis in, 509

recurrence of, 518

secondary changes in, 508

sex in, 509, 510

special characters of, 497

spontaneous expulsion of, 538

subdivisions of, 496

symptoms of, 5 I 2

synonyms of, 496

total extirpation in, table of, 520,528

treatment of, 5I 5

operative, 5 I 5

conclusions with regard to, 533

results of, $5 \mathrm{I} 6$

palliative, 5 I 5

ulceration in, 508

urine in, $5 \mathrm{I} 3$

of cord, 203

of epididymis, 237

of testicles, 237

diagnosis of, 24I differential, 242

Tunica albuginea, 207

vaginalis, 207

eversion of, 284 instruments for, 284 operation of, 284

excision of, 284

hydrocele of, 243,248

vasculosa, 209

\section{U}

Ulcer, chancroidal, diagnosis of, from balanitis, 40

Ulceration in tumors of bladder, 508

Undescended testis, 2 I 4

Ureters in hypertrophy of prostate, $32 \mathrm{I}$

Urethra, 2I, 54 anterior, electric endoscope for, 75

follicles of, inflammation of, treatment of, 80

inflammation of, 54. See Urethritis. diffuse phlegmonous, 89

membranous, 2I stricture of, 96

normal, appearances of, 76 color of, 77 
Urethra, papilloma of, 82

polyp of, 82

treatment of, 82

posterior, inflammation of, diagnosis of,

from cystitis, 462

prostatic, $2 \mathrm{I}$

electric endoscope for, 75

examination of, 77

in hypertrophy of prostate, 312

stricture of, 96

after perineal prostatectomy, 384.

following perineal prostatectomy in hypertrophy of prostate, 352

removal of tumor of bladder through, 588

Nitze's method, 588

spongy, 21

stricture of, 84

annular, 95

causes of death in, I 18

complications of, treatment of, 128

congenital, 85

definition of, 84

diagnosis of, 98

endoscopy in, 82

examination of, IOI

bougie à boule in, IOI, 102

bougies in, IOI, IO2

difficulties encountered in use of, 104

endoscope in, 104

meatotome in, IOI

sounds in, 104

passing of, 107

anatomical difficulties

in, 109

subsequent, I 2 I

urethrotometer in, 103

false passages in, 130

gleet in, 97

consequence of, 13 I

illustrative cases of, 132

infection due to, 89

inflammatory, 84

linear, 95

in litholapaxy, 585

location of, 96

mechanical effects of, 87

number of, 95

obliteration of urethra by, 85 partial, 85 total, 85

occlusion of, total, due to chemical agents, 88

operations for, $\mathrm{I} 39$

boutonnière, in, 147

divulsion, 139

exposure of urethra behind stricture by open dissection, I 52
Urethra, stricture of, operations for, perineal section, I 47

resection of stricture, 153

retrograde catheterism, $I_{5} \mathrm{I}$

suture of urethra after perineal urethrotomy; I 52

urethrotomy, external perineal, 147

organic, 84

internal, 140

pathology of, 86

resilient, 95

secondary changes due to, 87

sepsis in, II 9

septic phlegmon in, acute diffuse, treatment of, 128

sexual function in, disturbances of, 98

spasm of compressor urethræ muscle in, 107

spasmodic, 84

symptoms of, 97

time of formation of, 97

tortuous, 95

traumatic, 84

treatment of, 105

choice of method of, I 16

dilatation in, 106

continuous, 106

gradual, 106 mortality of, II 7 results of, I 22

rapid, Io6

with flexible olivary bougies, I IO

divulsion in, I 13

results of, 122

electrolysis in, results of, 126

operative, mortality in, I I 7

permanency of results of, I2O

resection in, I 16

mortality of, I 8

results of, 124

summary of, 127

urethrotomy, external perineal, I 6 mortality of, I 18 results of, 123 internal, II 4

urethral fistula in, treatment of, 129

urinary stream in, 97

urine in extravasation of, treatment of, 128

retention of, treatment of, 128 operative, 129 palliative, 128

urination in, increased frequency of, 98

varieties of, 94

of wide caliber, 95

suture of, after perineal urethrotomy, 152

Urethral curette, 82 
Urethral fistula, operations for closure of, I 85 Dieffenbach's, I 87 lateral flap method, $\mathbf{1} 88$ posterior and lateral flap method, 188

by simple suture, 186,187 Szymanowski's, 185

in stricture of urethra, treatment of, 129

instruments, scale of measurement of, 105 snare, 82

syringe, 63

$$
\text { Brown's, 8I }
$$

Keyes', 79

Urethritis, acute, 54 complications of, 65

definition of, 54

endoscopy in, 74

etiology of, 54

gonococcus in, 55

tests for, 56,57

culture, $5^{8}$

Gram's, 56

gonorrhœal infection of eye and, 72 symptoms of, 72 treatment of, 73

rheumatism and, 69 character of, 70 course of, 70 diagnosis of, 70 treatment of, 7I

incubation in, 59

lymphangitis and, 67

treatment of, 67

pathological anatomy of, 58

pathology of, 54

posterior, 68

treatment of, 68

symptoms of, 59

treatment of, 60, 74. See Endoscopy.

argyrol in, 63 .

general medicinal and hygienic, 60

irrigation of urethra by catheter, 64

Janet method, 64

chronic, 74 local, 62

appearances of, 78

posterior, treatment of, 79,80 treatment of, 78

granular, 78

Urethrometer, I03.

Urethrotomes, Maissonneuve's, I 4I Otis', I 43

Urethrotomy, external perineal, I I6, I47 after-treatment in, $I_{5} \mathrm{I}$

Cock's operation in, I 5 I

instruments for, 147

mortality of, I 18

operation in, 147
Urethrotomy, external perineal, operation in, incision in, 147

with guide, I 47

without guide, 149

position of patient in, 147

results of, 123

incision, external perineal, removal of tumor of bladder through, $5^{88}$

internal, I I 4, I $_{4} 0$

by instrument of Maissonneuve, I 4I operation, I $4 \mathrm{I}$ position of patient, I4I

of Otis, I 42 after-treatment in, 145

hemorrhage in, 146

operation, I 44 position of patient, $\mathbf{I} 44$

median, in perineal prostatectomy, 372

perineal, suture of urethra after, I 52 instruments in, 152 operation in, 152

Urinary extravasation, 89

infiltration, 89

anatomical distribution of fluids in, 9I

system in hypertrophy of prostate, $32 \mathrm{I}$

in vesical calculus, 478

Urination, increased frequency of, in stricture of urethra, 98

Urine, extravasation of, in stricture of urethra, treatment of, 128

incontinence of, in lateral perineal lithotomy, 572

residual, in hypertrophy of prostate, 3 I 9 detection of, 323

retention of, in hypertrophy of prostate, $32 \mathrm{I}$

treatment of, 332

in stricture of urethra, treatment of, I 28

operative, 129

palliative, 128

in tumors of bladder, $5^{\mathrm{I}} 3$

in vesical calculus, 480

Urotropin in hypertrophy of prostate, 327

Utricle, $30 \mathrm{I}$

\section{V}

Vaginal hematocele, 253

Van Hook-Mayo operation for hypospadias, I 77

Varicocele, 204

diagnosis of, 205

etiology of, 204

operations for, 292

open method, 294 instruments for, 294 operation of, 294

subcutaneous ligature, 292

pathology of, 205

vol. I -40 
Varicocele, symptoms of, 205 treatment of, 205

Vas deferens, abnormalities of, 202 anatomy of, 200 arteries of, $20 \mathrm{I}$ drainage through, 292 inflammation of, 203 irrigation through, 292 lymphatics of, $20 \mathrm{I}$ nerves of, $20 \mathrm{I}$ pampiniform plexus of veins of, 200 removal of, 289

Vasectomy, 287 complete, with castration, 288 operation of, 289 in hypertrophy of prostate, $34 \mathrm{I}$ partial, castration and, $\mathbf{2 8 8}$ incision in, 288 instruments for, 288 operation of, 288

and union of testicle with its fellow organ, 287

Veins, pampiniform plexus of, 200 of penis, 21

Venereal disease in carcinoma of penis, $5^{\mathrm{I}}$

Verrucæ of penis, 36

Vesical calculus, 468

blood in, 479

characteristics of, 470

chemistry of, 469

classification of, 469

color of, $47 \mathrm{I}$

definition of, 468

diagnosis of, 478

encysted, 473

etiology of, 469

examination in, cystoscopic, 482 physical, 480

with evacuating apparatus, 482 $x$-ray, 480

formation of, 468

frequency of, 469

illustrative cases of, 487

nuclei of, 474

foreign bodies as, 474

number of, 473

operations for, $56 \mathrm{I}$

litholapaxy, 565, 574 in children, 585

perineal, 586

lithotomy, 56r perineal, bilateral, $57 \mathrm{I}$ lateral, 562,567 suprapubic, 562, 573

pain in, 478 lithotrity, 565

pathology of, 478

perineal litholapaxy in, 487

lithotomy in, bilateral, 483

lateral, 483

results of, 486

priapism in, 480
Vesical calculus, primary, 468

secondary, 468

size of, 472

sounding for stone in, error in, $48 \mathrm{I}$

spontaneous fracture of, 476

stone searcher in, $48 \mathrm{I}$

suprapubic lithotomy in, 487

surface of, 472

symptoms of, 478

treatment of, 483

operative, 483

preventive, 483

urinary system in, 480

urine in, 480

infection in cystitis, 457

avenue of, 457

descending infection from kidney, $45^{8}$

by direct extension, 457

extension from adjacent structures, $45^{8}$

hematogenous infection,

Vesiculitis, 262 $45^{8}$

acute, diagnosis of, 264

etiology of, 262

pathology of, 263

prognosis of, 268

rectal examination in, 265

symptoms of, 264, 265

treatment of, 268 surgical, 268

chronic, diagnosis of, 264

etiology of, 263

gleet in, 266

nervous symptoms in, 266

pain in, 266

pathology of, 264

prognosis of, 268

rectal examination in, 267

relapses in, 268

spermatorrhœa in, 267

symptoms of, 264, 266 sexual, 267

treatment of, 269

urination in, 266

Villous papilloma of bladder, 496

Von Bergmann's operation of excision of tunica vaginalis, 284

\section{W}

WARTS of penis, hard, 37 soft, 36

venereal, 36

Watson's bladder speculum, $55^{1}$

galvanocautery prostatectotome, 407

Whalebone bougies, IOI

Whitehead's cystoscope, 423

Woodward's operation for phimosis, I6I 
Wounds of cord, 202

of penis, 27

treatment of, 27

\section{$\mathbf{X}$}

$\mathrm{X}$-RAY examination in vesical calculus, 480 in hypertrophy of prostate, $35^{6}$
$\mathbf{Y}$

Young's operation in perineal prostatectomy, 39I

operation in, 392

position of patient in, 39

prostatic tractor, 393

technique in perineal prostatectomy, 



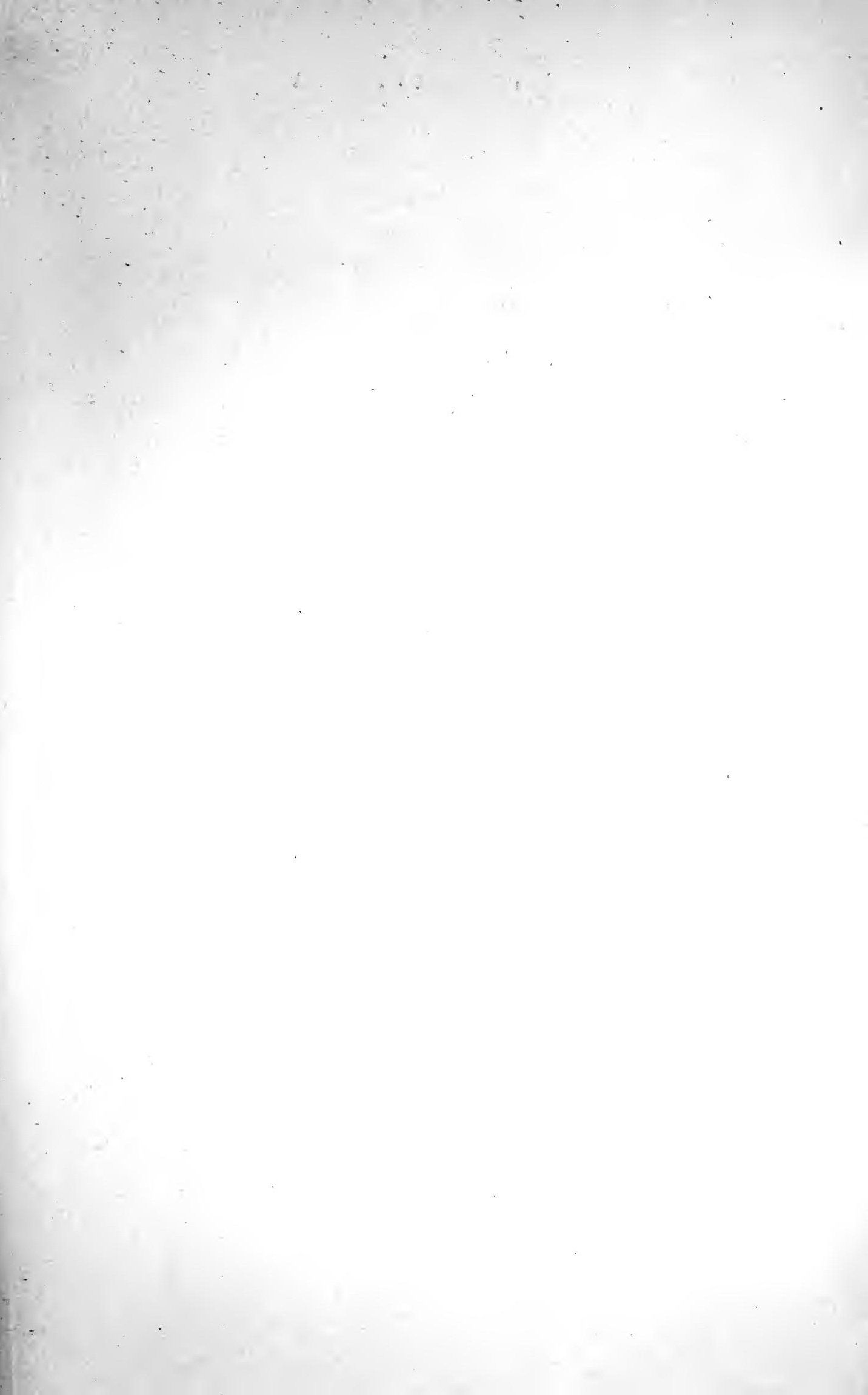




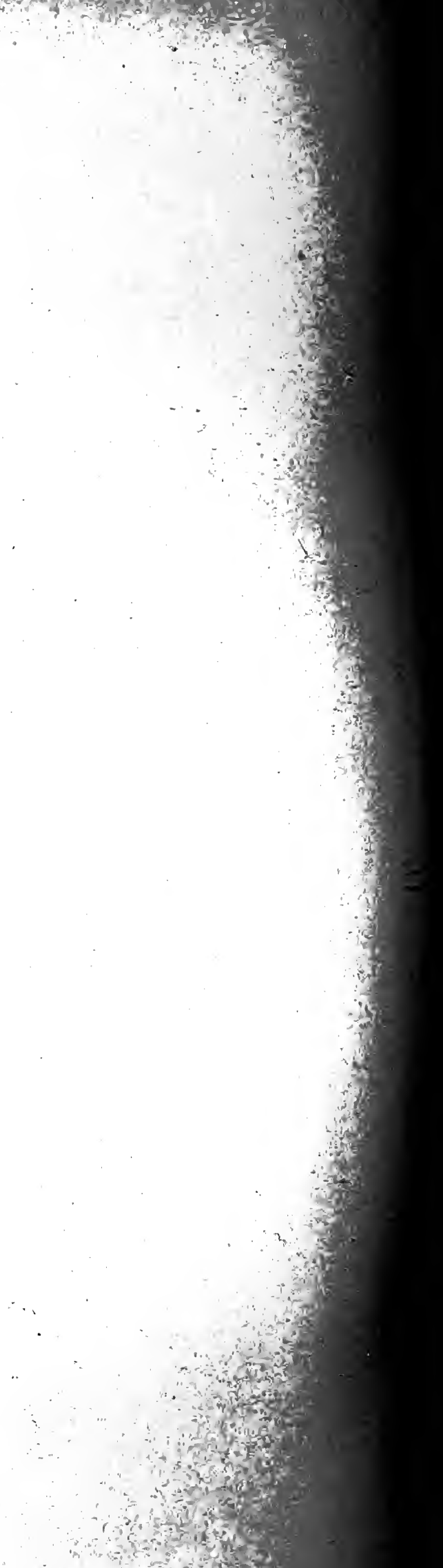






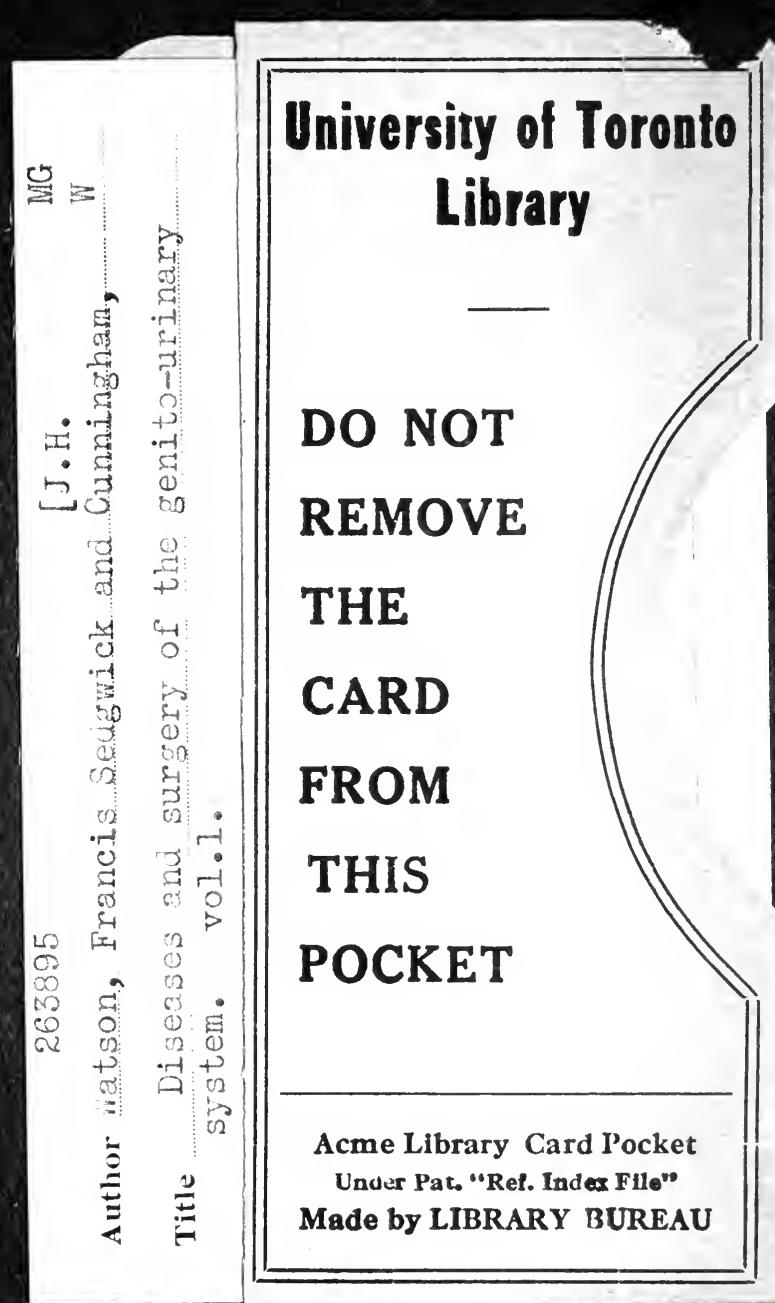


